\title{
Analytical Data Report for Grab Samples Collected From Operable Unit BP-5 B Well (C5859/C6226)
}

Michael Lindberg

September 2008

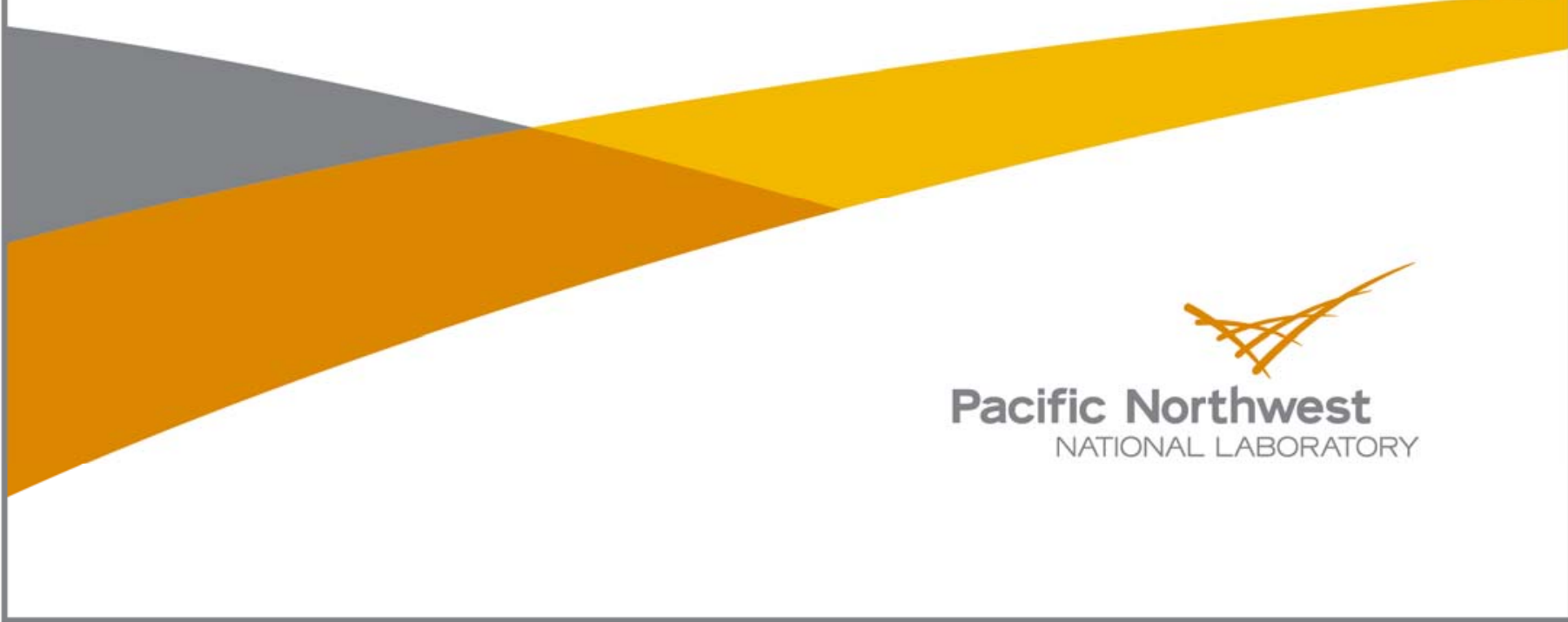


09/29/08 15:32

To: Dana Widrig

From: Michael J. Lindberg

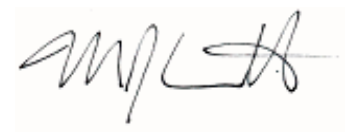

Environmental Sciences Laboratory

Energy and Environment Directorate, Pacific Northwest National Laboratory

Subject: Analytical Data Report for Grab Samples Collected From Operable Unit BP-5 B Well (C5859/C6226), Sample Delivery Group ESL070013, SAF Number F08-007

This letter contains the following information for sample delivery group ESL070013

- Cover Sheet

- Narrative

- Analytical Results

- Quality Control

- Geologic Logs

- Geologic Photos

- Chain of Custodies 


\section{Introduction}

Between December 20, 2007 and February 26, 2008 grab samples were received from Operable Unit BP-5 B Well (C5859/C6226) for geochemical studies.

\section{Analytical Results/Methodology}

The analyses for this project were performed at the 325 building located in the 300 Area of the Hanford Site. The analyses were performed according to Pacific Northwest National Laboratory (PNNL) approved procedures and/or nationally recognized test procedures. The data sets include the sample identification numbers, analytical results, estimated quantification limits (EQL), and quality control data.

\section{Quality Control}

The preparatory and analytical quality control requirements, calibration requirements, acceptance criteria, and failure actions are defined in the on-line QA plan “Conducting Analytical Work in Support of Regulatory Programs” (CAW). This QA plan implements the Hanford Analytical Services Quality Assurance Requirements Documents (HASQARD) for PNNL.

\section{Definitions}

Dup Duplicate

RPD Relative Percent Difference

\section{Sample Receipt}

Samples were received with a chain of custody (COC) and were analyzed according to the sample identification numbers supplied by the client. All Samples were refrigerated upon receipt until prepared for analysis.

All samples were received with custody seals intact unless noted in the Case Narrative.

\section{Holding Times}

Holding time is defined as the time from sample preparation to the time of analyses. The prescribed holding times were met for all analytes unless noted in the Case Narrative.

\section{Analytical Results}

All reported analytical results meet the requirements of the CAW or client specified SOW unless noted in the case narrative. 


\section{Case Narrative Report}

\section{Hold Time:}

No discrepancies noted.

\section{Preparation Blank (PB):}

QC Sample 8F11003-BLK1 failed criteria for Zinc in ICP-OES Vadose-AE.

$\mathrm{MDL}=0.0770 \mathrm{ug} / \mathrm{g}$

$\mathrm{MRL}=0.0770 \mathrm{ug} / \mathrm{g}$

Result $=0.115 \mathrm{ug} / \mathrm{g}$

Criterion $=1 \mathrm{x}$ MRL

The analyte was detected in samples and QC Blank. The concentration of the QC blank was less than $5 \%$ of the sample concentrations there should be no impact to results as reported.

QC Sample 8D28003-BLK1 failed criteria for Barium in ICP-OES Vadose-WE.

$\mathrm{MDL}=0.00879 \mathrm{ug} / \mathrm{g}$

$\mathrm{MRL}=0.00879 \mathrm{ug} / \mathrm{g}$

Result $=0.0312 \mathrm{ug} / \mathrm{g}$

Criterion = $1 \times$ MRL

The analyte was detected in samples and QC Blank. The concentration of the QC blank was less than $5 \%$ of the sample concentrations there should be no impact to results as reported.

\section{Duplicate (DUP):}

Duplicate RPD for Uranium 238 (40.8\%) was above the acceptance limit (35) in 8D15001-DUP2 for ICPMS-Tc_U-WE Potential analytical preparation error of the duplicate. Duplicate was in control for all other elements. There should be not impact to data as reported

Duplicate RPD for Aluminum (46.4\%) was above the acceptance limit (35) in 8D28003-DUP1 for ICP-OES Vadose-WE The concentration of the sample was less than 10 times the MDL. The $+/-35 \%$ criteria does not apply.

Duplicate RPD for Barium (59.3\%) was above the acceptance limit (35) in 8D28003-DUP1 for ICP-OES Vadose-WE The concentration of the sample was less than 10 times the MDL. The +/- $35 \%$ criteria does not apply.

Duplicate RPD for Aluminum (102\%) was above the acceptance limit (35) in 8E01006-DUP1 for ICP-OES Vadose-WE All other QC associated with the batch were in limits. Duplicate failure may be due to sample heterogeneity. There should be no impact to sample data as reported.

Duplicate RPD for Calcium (43.3\%) was above the acceptance limit (35) in 8E01006-DUP1 for ICP-OES Vadose-WE All other QC associated with the batch were in limits. Duplicate failure may be due to sample heterogeneity. There should be no impact to sample data as reported.

Duplicate RPD for Antimony 121 (42.3\%) was above the acceptance limit (35) in 8E01009-DUP2 for ICPMS-RCRA-WE The concentration of the sample was less than 10 times the MDL. The +/- $35 \%$ criteria does not apply.

\section{Laboratory Control Samples (LCS):}

The Blank spike failed criteria for silver. It was determined that silver is not stable in the nitric acid digestion as performed. Silver is not reported for acid extracted samples.

\section{Post Spike (PS):}

Post-Spike Recovery for Sodium (NR) was outside acceptance limits (75-125) in 8D28003-PS1 for ICP-OES Vadose-WE The native sample concentration was greater than 5 times the spike concentration. There should be not impact to data as reported. 


\section{Case Narrative Report}

\section{Post Spike (PS):}

Post-Spike Recovery for Calcium (70.1\%) was outside acceptance limits (75-125) in 8D28003-PS1 for ICP-OES Vadose-WE The native sample concentration was greater than 5 times the spike concentration. There should be not impact to data as reported.

Post-Spike Recovery for Magnesium (70.7\%) was outside acceptance limits (75-125) in 8D28003-PS1 for ICP-OES Vadose-WE The native sample concentration was greater than 5 times the spike concentration. There should be not impact to data as reported.

Post-Spike Recovery for Sodium (NR) was outside acceptance limits (75-125) in 8E01006-PS1 for ICP-OES Vadose-WE The native sample concentration was greater than 5 times the spike concentration. There should be not impact to data as reported.

Post-Spike Recovery for Calcium (NR) was outside acceptance limits (75-125) in 8E01006-PS1 for ICP-OES Vadose-WE The native sample concentration was greater than 5 times the spike concentration. There should be not impact to data as reported.

Post-Spike Recovery for Potassium (43.3) was outside acceptance limits (75-125) in 8E01006-PS1 for ICP-OES Vadose-WE The native sample concentration was greater than 5 times the spike concentration. There should be not impact to data as reported.

Post-Spike Recovery for Magnesium (7.79) was outside acceptance limits (75-125) in 8E01006-PS1 for ICP-OES Vadose-WE The native sample concentration was greater than 5 times the spike concentration. There should be not impact to data as reported.

Post-Spike Recovery for Sodium (NR) was outside acceptance limits (75-125) in 8E01006-PS1 for ICP-OES Vadose-WE The native sample concentration was greater than 5 times the spike concentration. There should be not impact to data as reported.

Post-Spike Recovery for Aluminum (241\%) was outside acceptance limits (75-125) in 8E12002-PS2 for ICP-OES Vadose-AE The native sample concentration was greater than 5 times the spike concentration. There should be not impact to data as reported.

Post-Spike Recovery for Calcium (350\%) was outside acceptance limits (75-125) in 8E12002-PS2 for ICP-OES Vadose-AE The native sample concentration was greater than 5 times the spike concentration. There should be not impact to data as reported.

Post-Spike Recovery for Magnesium (199\%) was outside acceptance limits (75-125) in 8E12002-PS2 for ICP-OES Vadose-AE The native sample concentration was greater than 5 times the spike concentration. There should be not impact to data as reported.

Post-Spike Recovery for Aluminum (NR) was outside acceptance limits (75-125) in 8F10003-PS1 for ICP-OES Vadose-AE The native sample concentration was greater than 5 times the spike concentration. There should be not impact to data as reported.

Post-Spike Recovery for Barium (66.6\%) was outside acceptance limits (75-125) in 8F10003-PS1 for ICP-OES Vadose-AE The native sample concentration was greater than 5 times the spike concentration. There should be not impact to data as reported.

Post-Spike Recovery for Calcium (NR) was outside acceptance limits (75-125) in 8F10003-PS1 for ICP-OES Vadose-AE The native sample concentration was greater than 5 times the spike concentration. There should be not impact to data as reported.

Post-Spike Recovery for Potassium (NR) was outside acceptance limits (75-125) in 8F10003-PS1 for ICP-OES Vadose-AE The native sample concentration was greater than 5 times the spike concentration. There should be not impact to data as reported.

Post-Spike Recovery for Magnesium (NR) was outside acceptance limits (75-125) in 8F10003-PS1 for ICP-OES Vadose-AE The native sample concentration was greater than 5 times the spike concentration. There should be not impact to data as reported.

Post-Spike Recovery for Manganese (NR) was outside acceptance limits (75-125) in 8F10003-PS1 for ICP-OES Vadose-AE The native sample concentration was greater than 5 times the spike concentration. There should be not impact to data as reported.

Post-Spike Recovery for Sodium (31.1\%) was outside acceptance limits (75-125) in 8F10003-PS1 for ICP-OES Vadose-AE The native sample concentration was greater than 5 times the spike concentration. There should be not impact to data as reported.

Post-Spike Recovery for Aluminum (NR) was outside acceptance limits (75-125) in 8F11003-PS1 for ICP-OES Vadose-AE The native sample concentration was greater than 5 times the spike concentration. There should be not impact to data as reported.

Post-Spike Recovery for Barium (64.6\%) was outside acceptance limits (75-125) in 8F11003-PS1 for ICP-OES Vadose-AE The native sample concentration was greater than 5 times the spike concentration. There should be not impact to data as reported.

Post-Spike Recovery for Calcium (NR) was outside acceptance limits (75-125) in 8F11003-PS1 for ICP-OES Vadose-AE The native sample concentration was greater than 5 times the spike concentration. There should be not impact to data as reported. 


\section{Case Narrative Report}

\section{Post Spike (PS):}

Post-Spike Recovery for Potassium (NR) was outside acceptance limits (75-125) in 8F11003-PS1 for ICP-OES Vadose-AE The native sample concentration was greater than 5 times the spike concentration. There should be not impact to data as reported.

Post-Spike Recovery for Magnesium (NR) was outside acceptance limits (75-125) in 8F11003-PS1 for ICP-OES Vadose-AE The native sample concentration was greater than 5 times the spike concentration. There should be not impact to data as reported.

Post-Spike Recovery for Manganese (NR) was outside acceptance limits (75-125) in 8F11003-PS1 for ICP-OES Vadose-AE The native sample concentration was greater than 5 times the spike concentration. There should be not impact to data as reported.

Post-Spike Recovery for Sodium (27.6\%) was outside acceptance limits (75-125) in 8F11003-PS1 for ICP-OES Vadose-AE The native sample concentration was greater than 5 times the spike concentration. There should be not impact to data as reported.

Post-Spike Recovery for Uranium (484) was outside acceptance limits (75-125) in 8E01017-PS1 for ICPMS-Tc_U-AE

The native sample concentration was greater than 5 times the spike concentration. There should be not impact to data as reported.

\section{Matrix Spike (MS):}

No discrepancies noted.

\section{Other QC Criteria:}

No discrepancies noted. 


\section{DISCLAIMER}

This report was prepared as an account of work sponsored by an agency of the United States Government. Neither the United States Government nor any agency thereof, nor Battelle Memorial Institute, nor any of their employees, makes any warranty, express or implied, or assumes any legal liability or responsibility for the accuracy, completeness, or usefulness of any information, apparatus, product, or process disclosed, or represents that its use would not infringe privately owned rights. Reference herein to any specific commercial product, process, or service by trade name, trademark, manufacturer, or otherwise does not necessarily constitute or imply its endorsement, recommendation, or favoring by the United States Government or any agency thereof, or Battelle Memorial Institute. The views and opinions of authors expressed herein do not necessarily state or reflect those of the United States Government or any agency thereof.

\section{SAMPLES INCLUDED IN THIS REPORT}

\section{BP 5 OU, C5859/C6226 B-Well VZ}

\begin{tabular}{|c|c|c|}
\hline $\begin{array}{l}\text { HEIS No. } \\
\text { B1RKL1 }\end{array}$ & $\begin{array}{l}\text { Laboratory ID } \\
0802002-02\end{array}$ & $\begin{array}{l}\text { Matrix } \\
\text { SOIL }\end{array}$ \\
\hline B1RKL6 & 0802002-09 & SOIL \\
\hline B1RKM1 & 0802002-17 & SOIL \\
\hline B1RKM8 & 0802002-31 & SOIL \\
\hline B1RKW7 & 0802003-02 & SOIL \\
\hline B1RLJ6-3 & 0802003-03 & SOIL \\
\hline B1RKW8 & 0802003-04 & SOIL \\
\hline B1RLJ7-4 & 0802003-05 & SOIL \\
\hline B1RKN7 & 0802005-03 & SOIL \\
\hline B1RKP3 & 0802005-09 & SOIL \\
\hline B1RKP9 & 0802005-15 & SOIL \\
\hline B1RKR5 & 0802005-21 & SOIL \\
\hline B1RKR9 & 0802005-25 & SOIL \\
\hline B1RKT3 & 0802005-29 & SOIL \\
\hline B1RKT7 & 0802006-07 & SOIL \\
\hline B1RKV1 & 0802006-11 & SOIL \\
\hline B1RKV5 & 0802007-04 & SOIL \\
\hline B1RKV9 & 0802007-12 & SOIL \\
\hline B1RKW2 & 0802008-04 & SOIL \\
\hline B1RKW4 & 0802008-08 & SOIL \\
\hline B1RKW5 & 0802009-02 & SOIL \\
\hline B1RKW6 & 0802009-04 & SOIL \\
\hline B1TFV6 & 0802028-03 & SOIL \\
\hline B1TFV8 & 0802028-05 & SOIL \\
\hline B1TFW0 & 0802028-07 & SOIL \\
\hline B1TFW2 & 0802028-09 & SOIL \\
\hline B1RKX0 & 0802028-11 & SOIL \\
\hline B1RKX3 & 0802028-17 & SOIL \\
\hline B1RKX5 & 0802028-23 & SOIL \\
\hline B1RKX7 & 0802028-29 & SOIL \\
\hline
\end{tabular}

\begin{tabular}{|c|c|}
\hline $\begin{array}{l}\text { Date Collected } \\
12 / 18 / 07 \quad 13: 40\end{array}$ & $\begin{array}{l}\text { Date Received } \\
12 / 20 / 07 \quad 13: 30\end{array}$ \\
\hline 12/19/07 07:49 & 12/20/07 13:3 \\
\hline 12/19/07 10:08 & $12 / 20 / 07 \quad 13: 30$ \\
\hline 12/20/07 10:31 & $12 / 20 / 07 \quad 13: 3$ \\
\hline 1/15/08 08:50 & $1 / 16 / 08 \quad 09: 30$ \\
\hline 1/15/08 09:10 & 1/16/08 $09: 30$ \\
\hline 1/15/08 09:33 & 1/16/08 09:30 \\
\hline 1/15/08 $10: 30$ & 1/16/08 $09: 30$ \\
\hline 12/26/07 11:24 & 1/2/08 13:10 \\
\hline 12/27/07 08:02 & 1/2/08 $13: 10$ \\
\hline 12/27/07 10:44 & 1/2/08 $13: 10$ \\
\hline 12/28/07 08:03 & 1/2/08 $13: 10$ \\
\hline 12/28/07 10:04 & 1/2/08 $13: 10$ \\
\hline $12 / 28 / 07 \quad 13: 50$ & 1/2/08 $13: 10$ \\
\hline $1 / 2 / 08 \quad 10: 26$ & $1 / 3 / 08 \quad 14: 00$ \\
\hline $1 / 2 / 08 \quad 14: 25$ & $1 / 3 / 08 \quad 14: 00$ \\
\hline 1/3/08 09:55 & $1 / 4 / 08 \quad 10: 50$ \\
\hline 1/3/08 13:30 & $1 / 4 / 08 \quad 10: 50$ \\
\hline 1/4/08 09:10 & $1 / 7 / 08 \quad 14: 28$ \\
\hline 1/4/08 13:45 & $1 / 7 / 08 \quad 14: 28$ \\
\hline 1/7/08 09:20 & $1 / 10 / 08 \quad 08: 15$ \\
\hline 1/7/08 $11: 10$ & 1/10/08 $08: 15$ \\
\hline 2/11/08 $10: 10$ & 2/14/08 $14: 00$ \\
\hline 2/11/08 $13: 24$ & $2 / 14 / 08 \quad 14: 00$ \\
\hline 2/12/08 $12: 50$ & $2 / 14 / 08 \quad 14: 00$ \\
\hline 2/12/08 13:20 & 2/25/08 $15: 15$ \\
\hline 2/13/08 $13: 40$ & 2/25/08 $15: 15$ \\
\hline 2/19/08 13:13 & 2/26/08 $13: 50$ \\
\hline 2/19/08 15:10 & 2/26/08 $13: 50$ \\
\hline /20/08 $11: 18$ & 2/26/08 $13: 50$ \\
\hline
\end{tabular}


The following analyses were performed on the following samples included in this report:

Metals 1:1 DI Water Extract by ICPMS

Metals Acid Extract by ICPMS

Alkalinity, Titrimetic ( $\mathrm{pH} 4.5)$

Anions By Ion Chromatography

GEA No Preparation

Geological Description

Metals 1:1 Water Extract by ICPOES

Metals Acid Extract by ICPOES

Moisture Content

pH of Waters By Electrode

Specific Conductance

Tc_U Acid Extract by ICPMS

Tc_U 1:1 DI Water Extract by ICPMS

Total Alpha Total Beta Acid Extract By LSC 


$\begin{array}{lll}\text { HEIS No. } & \text { Laboratory ID } & \text { Matrix } \\ \text { B1RKL1 } & 0802002-02 & \text { SOIL } \\ \text { B1RKL6 } & 0802002-09 & \text { SOIL } \\ \text { B1RKM1 } & 0802002-17 & \text { SOIL } \\ \text { B1RKM8 } & 0802002-31 & \text { SOIL } \\ \text { B1RKW7 } & 0802003-02 & \text { SOIL } \\ \text { B1RLJ6-3 } & 0802003-03 & \text { SOIL } \\ \text { B1RKW8 } & 0802003-04 & \text { SOIL } \\ \text { B1RLJ7-4 } & 0802003-05 & \text { SOIL } \\ \text { B1RKN7 } & 0802005-03 & \text { SOIL } \\ \text { B1RKP3 } & 0802005-09 & \text { SOIL } \\ \text { B1RKP9 } & 0802005-15 & \text { SOIL } \\ \text { B1RKR5 } & 0802005-21 & \text { SOIL } \\ \text { B1RKR9 } & 0802005-25 & \text { SOIL } \\ \text { B1RKT3 } & 0802005-29 & \text { SOIL } \\ \text { B1RKT7 } & 0802006-07 & \text { SOIL } \\ \text { B1RKV1 } & 0802006-11 & \text { SOIL } \\ \text { B1RKV5 } & 0802007-04 & \text { SOIL } \\ \text { B1RKV9 } & 0802007-12 & \text { SOIL } \\ \text { B1RKW2 } & 0802008-04 & \text { SOIL } \\ \text { B1RKW4 } & 0802008-08 & \text { SOIL } \\ \text { B1RKW5 } & 0802009-02 & \text { SOIL } \\ \text { B1RKW6 } & 0802009-04 & \text { SOIL } \\ \text { B1TFV6 } & 0802028-03 & \text { SOIL } \\ \text { B1TFV8 } & 0802028-05 & \text { SOIL } \\ \text { B1TFW0 } & 0802028-07 & \text { SOIL } \\ \text { B1TFW2 } & 0802028-09 & \text { SOIL } \\ \text { B1RKX0 } & 0802028-11 & \text { SOIL } \\ \text { B1RKX3 } & 0802028-17 & \text { SOIL } \\ \text { B1RKX5 } & 0802028-23 & \text { SOIL } \\ \text { B1RKX7 } & 0802028-29 & \text { SOIL }\end{array}$

\begin{tabular}{|c|c|}
\hline Date Collected & Date Received \\
\hline 12/18/07 13:40 & $12 / 20 / 07 \quad 13: 30$ \\
\hline $\begin{array}{lll}12 / 19 / 07 & 07: 49\end{array}$ & $12 / 20 / 07 \quad 13: 30$ \\
\hline $\begin{array}{lll}12 / 19 / 07 & 10: 08\end{array}$ & $12 / 20 / 07 \quad 13: 30$ \\
\hline 12/20/07 10:31 & $12 / 20 / 07 \quad 13: 30$ \\
\hline 1/15/08 08:50 & 1/16/08 09:30 \\
\hline 1/15/08 09:10 & 1/16/08 09:30 \\
\hline 1/15/08 09:33 & 1/16/08 09:30 \\
\hline 1/15/08 10:30 & $1 / 16 / 08 \quad 09: 30$ \\
\hline 12/26/07 11:24 & 1/2/08 $13: 10$ \\
\hline 12/27/07 08:02 & 1/2/08 $13: 10$ \\
\hline $12 / 27 / 07 \quad 10: 44$ & 1/2/08 13:10 \\
\hline 12/28/07 08:03 & 1/2/08 $13: 10$ \\
\hline 12/28/07 10:04 & 1/2/08 $13: 10$ \\
\hline 12/28/07 13:50 & 1/2/08 $13: 10$ \\
\hline 1/2/08 10:26 & 1/3/08 $14: 00$ \\
\hline $1 / 2 / 08 \quad 14: 25$ & 1/3/08 $14: 00$ \\
\hline 1/3/08 09:55 & $1 / 4 / 08 \quad 10: 50$ \\
\hline 1/3/08 $13: 30$ & $1 / 4 / 08 \quad 10: 50$ \\
\hline 1/4/08 09:10 & $1 / 7 / 08 \quad 14: 28$ \\
\hline 1/4/08 $13: 45$ & $1 / 7 / 08 \quad 14: 28$ \\
\hline 1/7/08 09:20 & 1/10/08 08:15 \\
\hline 1/7/08 $11: 10$ & 1/10/08 08:15 \\
\hline 2/11/08 10:10 & 2/14/08 14:00 \\
\hline 2/11/08 13:24 & $2 / 14 / 08 \quad 14: 00$ \\
\hline 2/12/08 12:50 & $2 / 14 / 08 \quad 14: 00$ \\
\hline 2/12/08 13:20 & $2 / 25 / 08 \quad 15: 15$ \\
\hline $2 / 13 / 08 \quad 13: 40$ & 2/25/08 15:15 \\
\hline 2/19/08 13:13 & 2/26/08 13:50 \\
\hline 2/19/08 15:10 & $2 / 26 / 08 \quad 13: 50$ \\
\hline 2/20/08 $11: 18$ & 2/26/08 $13: 50$ \\
\hline
\end{tabular}




\section{Wet Chemistry}

\begin{tabular}{|c|c|c|c|c|c|}
\hline $\begin{array}{l}\text { Alkalinity } \\
\text { Lab ID }\end{array}$ & $\begin{array}{c}\text { CaCO3 ( } \\
\text { HEIS No. }\end{array}$ & $\begin{array}{l}\text { Methoc } \\
\text { Results }\end{array}$ & $\begin{array}{c}\text { 20B } \\
\text { EQL }\end{array}$ & Analyzed & Batch \\
\hline 0802002-02 & B1RKL1 & 4.93E1 & $2.54 \mathrm{E} 1$ & $2 / 14 / 08$ & 8B29001 \\
\hline 0802002-09 & B1RKL6 & $5.10 \mathrm{E} 1$ & $2.55 \mathrm{E} 1$ & $2 / 14 / 08$ & 8B29001 \\
\hline 0802002-17 & B1RKM1 & $5.00 \mathrm{E} 1$ & 2.53E1 & $2 / 14 / 08$ & 8B29001 \\
\hline 0802002-31 & B1RKM8 & $4.61 \mathrm{E} 1$ & $2.53 \mathrm{E} 1$ & $2 / 14 / 08$ & 8B29001 \\
\hline 0802003-02 & B1RKW7 & 2.08E2 & $2.70 \mathrm{E} 1$ & $2 / 14 / 08$ & 8B29001 \\
\hline 0802003-04 & B1RKW8 & $1.60 \mathrm{E} 2$ & 2.53E1 & $2 / 14 / 08$ & 8B29001 \\
\hline 0802005-03 & B1RKN7 & 4.70E1 & 2.54E1 & $2 / 14 / 08$ & 8B29001 \\
\hline 0802005-09 & B1RKP3 & 4.84E1 & 2.53E1 & $2 / 14 / 08$ & 8B29001 \\
\hline 0802005-15 & B1RKP9 & 4.77E1 & 2.53E1 & $2 / 14 / 08$ & 8B29001 \\
\hline 0802005-21 & B1RKR5 & 4.99E1 & 2.53E1 & $2 / 14 / 08$ & 8B29001 \\
\hline 0802005-25 & B1RKR9 & 4.15E1 & $2.53 \mathrm{E} 1$ & $2 / 14 / 08$ & 8B29001 \\
\hline 0802005-29 & B1RKT3 & 4.46E1 & $2.53 \mathrm{E} 1$ & $2 / 14 / 08$ & 8B29001 \\
\hline 0802006-07 & B1RKT7 & $4.76 \mathrm{E} 1$ & 2.53E1 & $2 / 14 / 08$ & 8B29001 \\
\hline 0802006-11 & B1RKV1 & $6.08 \mathrm{E} 1$ & $2.82 \mathrm{E} 1$ & $2 / 14 / 08$ & 8B29001 \\
\hline 0802007-04 & B1RKV5 & 4.68E1 & 2.53E1 & $2 / 14 / 08$ & 8B29001 \\
\hline 0802007-12 & B1RKV9 & 5.07E1 & 2.53E1 & $2 / 14 / 08$ & 8B29001 \\
\hline 0802008-04 & B1RKW2 & $1.24 \mathrm{E} 2$ & $2.53 \mathrm{E} 1$ & $2 / 14 / 08$ & 8B29001 \\
\hline 0802008-08 & B1RKW4 & $3.88 \mathrm{E} 2$ & $2.53 \mathrm{E} 1$ & $2 / 14 / 08$ & 8B29001 \\
\hline 0802009-02 & B1RKW5 & $1.71 \mathrm{E} 2$ & $2.53 \mathrm{E} 1$ & $2 / 14 / 08$ & 8B29001 \\
\hline 0802009-04 & B1RKW6 & 2.33E2 & 2.53E1 & $2 / 14 / 08$ & 8B29001 \\
\hline 0802028-03 & B1TFV6 & $1.79 \mathrm{E} 2$ & 2.36E1 & $3 / 31 / 08$ & 8C31002 \\
\hline 0802028-05 & B1TFV8 & $2.45 \mathrm{E} 2$ & 2.34E1 & 3/31/08 & 8С31002 \\
\hline 0802028-07 & B1TFW0 & $7.54 \mathrm{E} 1$ & 2.35E1 & $3 / 31 / 08$ & 8C31002 \\
\hline 0802028-09 & B1TFW2 & 8.16E1 & $2.36 \mathrm{E} 1$ & 3/31/08 & 8C31002 \\
\hline 0802028-11 & B1RKX0 & $1.04 \mathrm{E} 2$ & 2.35E1 & $3 / 31 / 08$ & 8C31002 \\
\hline 0802028-17 & B1RKX3 & $1.12 \mathrm{E} 2$ & 2.35E1 & $3 / 31 / 08$ & 8C31002 \\
\hline 0802028-23 & B1RKX5 & 1.17E2 & 2.36E1 & $3 / 31 / 08$ & 8C31002 \\
\hline 0802028-29 & B1RKX7 & 4.71E1 & 2.35E1 & 3/31/08 & 8C31002 \\
\hline
\end{tabular}




\section{Wet Chemistry}

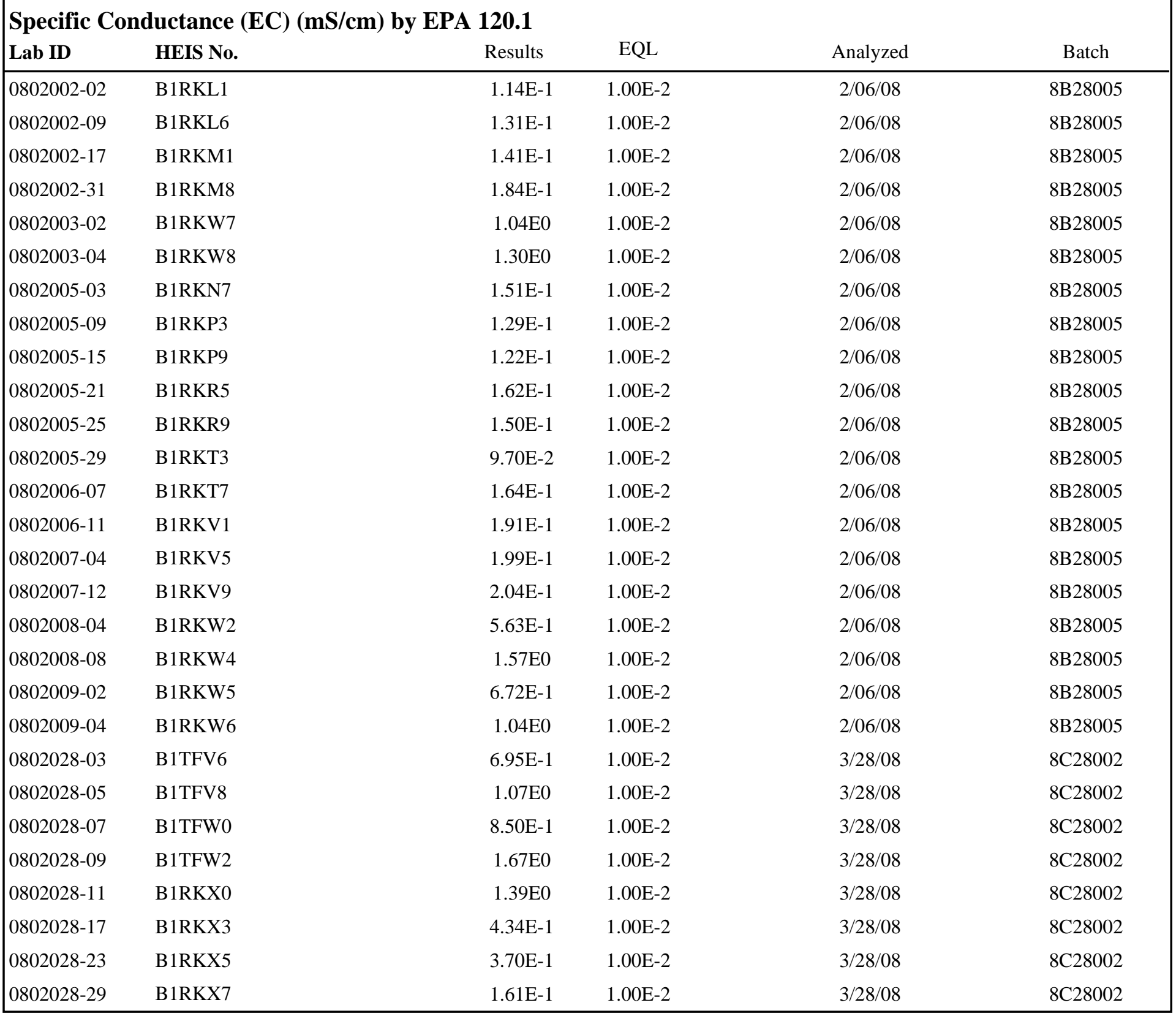




\section{Wet Chemistry}

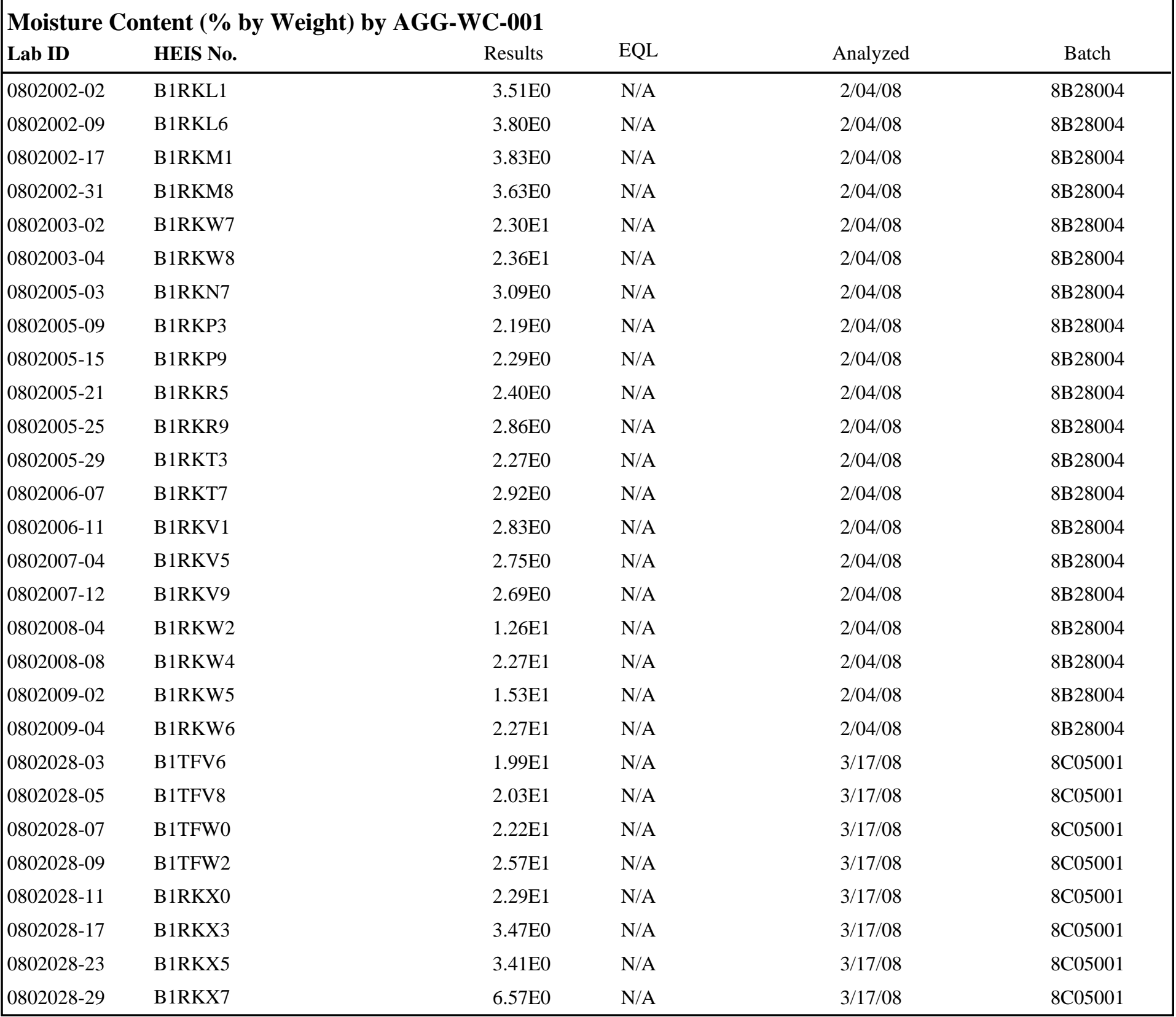




\section{Wet Chemistry}

\begin{tabular}{|c|c|c|c|c|c|}
\hline $\begin{array}{l}\text { pH (pH Ul } \\
\text { Lab ID }\end{array}$ & $\begin{array}{l}\text { ) by AGG } \\
\text { HEIS No. }\end{array}$ & Results & EQL & Analyzed & Batch \\
\hline 0802002-02 & B1RKL1 & 8.04E0 & N/A & $2 / 06 / 08$ & 8B28005 \\
\hline 0802002-09 & B1RKL6 & $8.20 \mathrm{E} 0$ & N/A & $2 / 06 / 08$ & 8B28005 \\
\hline 0802002-17 & B1RKM1 & 8.13E0 & N/A & $2 / 06 / 08$ & 8B28005 \\
\hline 0802002-31 & B1RKM8 & $7.90 \mathrm{E} 0$ & N/A & $2 / 06 / 08$ & 8B28005 \\
\hline 0802003-02 & B1RKW7 & 8.68E0 & N/A & $2 / 06 / 08$ & 8B28005 \\
\hline 0802003-04 & B1RKW8 & 8.53E0 & N/A & $2 / 06 / 08$ & 8B28005 \\
\hline 0802005-03 & B1RKN7 & 7.95E0 & N/A & $2 / 06 / 08$ & 8B28005 \\
\hline 0802005-09 & B1RKP3 & 8.03E0 & N/A & $2 / 06 / 08$ & 8B28005 \\
\hline 0802005-15 & B1RKP9 & $7.98 \mathrm{E} 0$ & N/A & $2 / 06 / 08$ & 8B28005 \\
\hline 0802005-21 & B1RKR5 & 8.03E0 & N/A & $2 / 06 / 08$ & 8B28005 \\
\hline 0802005-25 & B1RKR9 & $7.81 \mathrm{E} 0$ & N/A & $2 / 06 / 08$ & 8B28005 \\
\hline 0802005-29 & B1RKT3 & 8.01E0 & N/A & $2 / 06 / 08$ & 8B28005 \\
\hline 0802006-07 & B1RKT7 & 8.03E0 & N/A & $2 / 06 / 08$ & 8B28005 \\
\hline 0802006-11 & B1RKV1 & 8.09E0 & N/A & $2 / 06 / 08$ & $8 B 28005$ \\
\hline 0802007-04 & B1RKV5 & 7.91E0 & N/A & $2 / 06 / 08$ & 8B28005 \\
\hline 0802007-12 & B1RKV9 & $7.98 \mathrm{E} 0$ & N/A & $2 / 06 / 08$ & 8B28005 \\
\hline 0802008-04 & B1RKW2 & $8.80 \mathrm{E} 0$ & N/A & $2 / 06 / 08$ & 8B28005 \\
\hline 0802008-08 & B1RKW4 & $9.31 \mathrm{E} 0$ & N/A & $2 / 06 / 08$ & 8B28005 \\
\hline 0802009-02 & B1RKW5 & $9.10 \mathrm{E} 0$ & N/A & $2 / 06 / 08$ & 8B28005 \\
\hline 0802009-04 & B1RKW6 & 8.92E0 & N/A & $2 / 06 / 08$ & 8B28005 \\
\hline 0802028-03 & B1TFV6 & 8.84E0 & N/A & $3 / 28 / 08$ & 8C28002 \\
\hline 0802028-05 & B1TFV8 & $9.40 \mathrm{E} 0$ & N/A & $3 / 28 / 08$ & 8C28002 \\
\hline 0802028-07 & B1TFW0 & 7.98E0 & N/A & $3 / 28 / 08$ & 8C28002 \\
\hline 0802028-09 & B1TFW2 & 7.92E0 & N/A & $3 / 28 / 08$ & 8C28002 \\
\hline 0802028-11 & B1RKX0 & 8.30E0 & N/A & $3 / 28 / 08$ & 8C28002 \\
\hline 0802028-17 & B1RKX3 & 9.33E0 & N/A & $3 / 28 / 08$ & 8C28002 \\
\hline 0802028-23 & B1RKX5 & $9.15 \mathrm{E} 0$ & N/A & $3 / 28 / 08$ & 8C28002 \\
\hline 0802028-29 & B1RKX7 & $8.21 \mathrm{E} 0$ & N/A & $3 / 28 / 08$ & 8C28002 \\
\hline
\end{tabular}




\section{Anions by Ion Chromatography}

\begin{tabular}{|c|c|c|c|c|c|c|c|}
\hline CAS \# & Analyte & Results & Units & EQL & Analyzed & Batch & Method \\
\hline HEIS No. & B1RKL1 & \multicolumn{3}{|c|}{ Lab ID: $\quad$ 0802002-02 } & & & \\
\hline $16984-48-8$ & Fluoride & $2.81 \mathrm{E}-1$ & ug/g dry & $2.01 \mathrm{E}-1$ & 2/06/08 & 8E05002 & AGG-IC-001 \\
\hline 16887-00-6 & Chloride & $1.44 \mathrm{E} 0$ & ug/g dry & $5.02 \mathrm{E}-1$ & 2/06/08 & 8Е05002 & AGG-IC-001 \\
\hline 14797-65-0 & Nitrite & $<1.00 \mathrm{E} 0$ & ug/g dry & $1.00 \mathrm{E} 0$ & 2/06/08 & 8E05002 & AGG-IC-001 \\
\hline 14797-55-8 & Nitrate & 2.24E0 & ug/g dry & $1.00 \mathrm{E} 0$ & 2/06/08 & 8E05002 & AGG-IC-001 \\
\hline 14808-79-8 & Sulfate & 9.96E0 & ug/g dry & $1.50 \mathrm{E} 0$ & 2/06/08 & 8E05002 & AGG-IC-001 \\
\hline $14265-44-2$ & Phosphate & $<1.50 \mathrm{E} 0$ & ug/g dry & $1.50 \mathrm{E} 0$ & 2/06/08 & 8E05002 & AGG-IC-001 \\
\hline HEIS No. & B1RKL6 & \multicolumn{3}{|c|}{ Lab ID: $\quad$ 0802002-09 } & & & \\
\hline 16984-48-8 & Fluoride & $3.29 \mathrm{E}-1$ & ug/g dry & $2.01 \mathrm{E}-1$ & 2/06/08 & 8E05002 & AGG-IC-001 \\
\hline $16887-00-6$ & Chloride & 1.62E0 & ug/g dry & $5.03 \mathrm{E}-1$ & 2/06/08 & 8E05002 & AGG-IC-001 \\
\hline $14797-65-0$ & Nitrite & $<1.01 \mathrm{E} 0$ & ug/g dry & $1.01 \mathrm{E} 0$ & 2/06/08 & 8E05002 & AGG-IC-001 \\
\hline 14797-55-8 & Nitrate & 3.39E0 & ug/g dry & $1.01 \mathrm{E} 0$ & 2/06/08 & 8E05002 & AGG-IC-001 \\
\hline 14808-79-8 & Sulfate & 1.39E1 & ug/g dry & $1.51 \mathrm{E} 0$ & 2/06/08 & 8E05002 & AGG-IC-001 \\
\hline 14265-44-2 & Phosphate & $<1.51 \mathrm{E} 0$ & ug/g dry & $1.51 \mathrm{E} 0$ & 2/06/08 & 8E05002 & AGG-IC-001 \\
\hline HEIS No. & B1RKM1 & \multicolumn{3}{|c|}{ Lab ID: $\quad$ 0802002-17 } & & & \\
\hline 16984-48-8 & Fluoride & $2.68 \mathrm{E}-1$ & ug/g dry & $2.00 \mathrm{E}-1$ & 2/06/08 & 8E05002 & AGG-IC-001 \\
\hline 16887-00-6 & Chloride & 3.51E0 & ug/g dry & $5.00 \mathrm{E}-1$ & 2/06/08 & 8E05002 & AGG-IC-001 \\
\hline $14797-65-0$ & Nitrite & $<1.00 \mathrm{E} 0$ & ug/g dry & $1.00 \mathrm{E} 0$ & 2/06/08 & 8E05002 & AGG-IC-001 \\
\hline 14797-55-8 & Nitrate & 4.32E0 & ug/g dry & $1.00 \mathrm{E} 0$ & 2/06/08 & 8E05002 & AGG-IC-001 \\
\hline 14808-79-8 & Sulfate & $1.79 \mathrm{E} 1$ & ug/g dry & $1.50 \mathrm{E} 0$ & 2/06/08 & 8E05002 & AGG-IC-001 \\
\hline $14265-44-2$ & Phosphate & $<1.50 \mathrm{E} 0$ & ug/g dry & $1.50 \mathrm{E} 0$ & 2/06/08 & 8E05002 & AGG-IC-001 \\
\hline HEIS No. & B1RKM8 & \multicolumn{3}{|c|}{ Lab ID: $\quad$ 0802002-31 } & & & \\
\hline 16984-48-8 & Fluoride & $2.84 \mathrm{E}-1$ & ug/g dry & 2.00E-1 & 2/06/08 & 8E05002 & AGG-IC-001 \\
\hline 16887-00-6 & Chloride & 1.33E1 & ug/g dry & $5.00 \mathrm{E}-1$ & 2/06/08 & 8E05002 & AGG-IC-001 \\
\hline $14797-65-0$ & Nitrite & $<1.00 \mathrm{E} 0$ & ug/g dry & $1.00 \mathrm{E} 0$ & 2/06/08 & 8E05002 & AGG-IC-001 \\
\hline 14797-55-8 & Nitrate & 8.37E0 & ug/g dry & $1.00 \mathrm{E} 0$ & 2/06/08 & 8E05002 & AGG-IC-001 \\
\hline 14808-79-8 & Sulfate & $1.82 \mathrm{E} 1$ & ug/g dry & $1.50 \mathrm{E} 0$ & 2/06/08 & 8E05002 & AGG-IC-001 \\
\hline $14265-44-2$ & Phosphate & $<1.50 \mathrm{E} 0$ & ug/g dry & $1.50 \mathrm{E} 0$ & $2 / 06 / 08$ & 8E05002 & AGG-IC-001 \\
\hline HEIS No. & B1RKW7 & \multicolumn{3}{|c|}{ Lab ID: $\quad$ 0802003-02 } & & & \\
\hline 16984-48-8 & Fluoride & $1.01 \mathrm{E} 1$ & ug/g dry & 2.13E0 & 2/08/08 & 8E05002 & AGG-IC-001 \\
\hline $16887-00-6$ & Chloride & $1.94 \mathrm{E} 1$ & ug/g dry & 5.34E0 & 2/08/08 & 8E05002 & AGG-IC-001 \\
\hline $14797-65-0$ & Nitrite & $<1.07 \mathrm{E} 1$ & ug/g dry & 1.07E1 & 2/08/08 & 8E05002 & AGG-IC-001 \\
\hline $14797-55-8$ & Nitrate & $1.58 \mathrm{E} 2$ & ug/g dry & $1.07 \mathrm{E} 1$ & 2/08/08 & 8E05002 & AGG-IC-001 \\
\hline 14808-79-8 & Sulfate & $1.58 \mathrm{E} 2$ & ug/g dry & $1.60 \mathrm{E} 1$ & 2/08/08 & 8E05002 & AGG-IC-001 \\
\hline 14265-44-2 & Phosphate & $<1.60 \mathrm{E} 1$ & ug/g dry & $1.60 \mathrm{E} 1$ & 2/08/08 & 8E05002 & AGG-IC-001 \\
\hline HEIS No. & B1RKW8 & \multicolumn{3}{|c|}{ Lab ID: $\quad$ 0802003-04 } & & & \\
\hline $16984-48-8$ & Fluoride & $<2.00 \mathrm{E} 0$ & ug/g dry & $2.00 \mathrm{E} 0$ & 2/08/08 & 8E05002 & AGG-IC-001 \\
\hline 16887-00-6 & Chloride & $2.36 \mathrm{E} 1$ & ug/g dry & $5.00 \mathrm{E} 0$ & 2/08/08 & 8E05002 & AGG-IC-001 \\
\hline $14797-65-0$ & Nitrite & $<1.00 \mathrm{E} 1$ & ug/g dry & $1.00 \mathrm{E} 1$ & 2/08/08 & 8E05002 & AGG-IC-001 \\
\hline 14797-55-8 & Nitrate & $3.25 \mathrm{E} 2$ & ug/g dry & $1.00 \mathrm{E} 1$ & 2/08/08 & 8E05002 & AGG-IC-001 \\
\hline $14808-79-8$ & Sulfate & $1.76 \mathrm{E} 2$ & ug/g dry & $1.50 \mathrm{E} 1$ & $2 / 08 / 08$ & 8Е05002 & AGG-IC-001 \\
\hline $14265-44-2$ & Phosphate & $<1.50 \mathrm{E} 1$ & ug/g dry & $1.50 \mathrm{E} 1$ & 2/08/08 & 8E05002 & AGG-IC-001 \\
\hline HEIS No. & B1RKN7 & \multicolumn{3}{|c|}{ Lab ID: $\quad$ 0802005-03 } & & & \\
\hline $16984-48-8$ & Fluoride & $2.10 \mathrm{E}-1$ & ug/g dry & $2.01 \mathrm{E}-1$ & 2/06/08 & 8E05002 & AGG-IC-001 \\
\hline $16887-00-6$ & Chloride & $1.10 \mathrm{E} 1$ & ug/g dry & $5.01 \mathrm{E}-1$ & 2/06/08 & 8E05002 & AGG-IC-001 \\
\hline $14797-65-0$ & Nitrite & $<1.00 \mathrm{E} 0$ & ug/g dry & $1.00 \mathrm{E} 0$ & 2/06/08 & 8E05002 & AGG-IC-001 \\
\hline $14797-55-8$ & Nitrate & 8.12E0 & ug/g dry & $1.00 \mathrm{E} 0$ & 2/06/08 & 8E05002 & AGG-IC-001 \\
\hline 14808-79-8 & Sulfate & 1.13E1 & ug/g dry & $1.50 \mathrm{E} 0$ & 2/06/08 & 8E05002 & AGG-IC-001 \\
\hline 14265-44-2 & Phosphate & $<1.50 \mathrm{E} 0$ & ug/g dry & $1.50 \mathrm{E} 0$ & 2/06/08 & 8E05002 & AGG-IC-001 \\
\hline
\end{tabular}




\section{Anions by Ion Chromatography}

\begin{tabular}{|c|c|c|c|c|c|c|c|}
\hline CAS \# & Analyte & Results & Units & EQL & Analyzed & Batch & Method \\
\hline HEIS No. & B1RKP3 & \multicolumn{3}{|c|}{ Lab ID: $\quad$ 0802005-09 } & & & \\
\hline $16984-48-8$ & Fluoride & 3.67E-1 & ug/g dry & $2.00 \mathrm{E}-1$ & 2/06/08 & 8E05002 & AGG-IC-001 \\
\hline 16887-00-6 & Chloride & $3.32 \mathrm{E} 0$ & ug/g dry & $5.00 \mathrm{E}-1$ & 2/06/08 & 8Е05002 & AGG-IC-001 \\
\hline 14797-65-0 & Nitrite & $<1.00 \mathrm{E} 0$ & ug/g dry & $1.00 \mathrm{E} 0$ & 2/06/08 & 8E05002 & AGG-IC-001 \\
\hline 14797-55-8 & Nitrate & 2.12E0 & ug/g dry & $1.00 \mathrm{E} 0$ & 2/06/08 & 8E05002 & AGG-IC-001 \\
\hline 14808-79-8 & Sulfate & 1.24E1 & ug/g dry & $1.50 \mathrm{E} 0$ & 2/06/08 & 8E05002 & AGG-IC-001 \\
\hline $14265-44-2$ & Phosphate & $<1.50 \mathrm{E} 0$ & ug/g dry & $1.50 \mathrm{E} 0$ & 2/06/08 & 8E05002 & AGG-IC-001 \\
\hline HEIS No. & B1RKP9 & \multicolumn{3}{|c|}{ Lab ID: $\quad$ 0802005-15 } & & & \\
\hline 16984-48-8 & Fluoride & 2.13E-1 & ug/g dry & 2.00E-1 & 2/06/08 & 8E05002 & AGG-IC-001 \\
\hline $16887-00-6$ & Chloride & $1.47 \mathrm{E} 0$ & ug/g dry & $5.01 \mathrm{E}-1$ & 2/06/08 & 8E05002 & AGG-IC-001 \\
\hline $14797-65-0$ & Nitrite & $<1.00 \mathrm{E} 0$ & ug/g dry & $1.00 \mathrm{E} 0$ & 2/06/08 & 8E05002 & AGG-IC-001 \\
\hline 14797-55-8 & Nitrate & $1.57 \mathrm{E} 0$ & ug/g dry & $1.00 \mathrm{E} 0$ & 2/06/08 & 8E05002 & AGG-IC-001 \\
\hline 14808-79-8 & Sulfate & $1.68 \mathrm{E} 1$ & ug/g dry & $1.50 \mathrm{E} 0$ & 2/06/08 & 8E05002 & AGG-IC-001 \\
\hline 14265-44-2 & Phosphate & $<1.50 \mathrm{E} 0$ & ug/g dry & $1.50 \mathrm{E} 0$ & 2/06/08 & 8E05002 & AGG-IC-001 \\
\hline HEIS No. & B1RKR5 & \multicolumn{3}{|c|}{ Lab ID: $\quad$ 0802005-21 } & & & \\
\hline $16984-48-8$ & Fluoride & 3.23E-1 & ug/g dry & $2.00 \mathrm{E}-1$ & $2 / 06 / 08$ & 8E05002 & AGG-IC-001 \\
\hline $16887-00-6$ & Chloride & $1.92 \mathrm{E} 0$ & ug/g dry & $5.00 \mathrm{E}-1$ & 2/06/08 & 8E05002 & AGG-IC-001 \\
\hline $14797-65-0$ & Nitrite & $<1.00 \mathrm{E} 0$ & ug/g dry & $1.00 \mathrm{E} 0$ & 2/06/08 & 8E05002 & AGG-IC-001 \\
\hline 14797-55-8 & Nitrate & 2.09E0 & ug/g dry & $1.00 \mathrm{E} 0$ & 2/06/08 & 8E05002 & AGG-IC-001 \\
\hline $14808-79-8$ & Sulfate & $2.78 \mathrm{E} 1$ & ug/g dry & $1.50 \mathrm{E} 0$ & 2/06/08 & 8E05002 & AGG-IC-001 \\
\hline $14265-44-2$ & Phosphate & $<1.50 \mathrm{E} 0$ & ug/g dry & $1.50 \mathrm{E} 0$ & 2/06/08 & 8E05002 & AGG-IC-001 \\
\hline HEIS No. & B1RKR9 & \multicolumn{3}{|c|}{ Lab ID: $\quad$ 0802005-25 } & & & \\
\hline 16984-48-8 & Fluoride & $2.18 \mathrm{E}-1$ & ug/g dry & 2.00E-1 & 2/06/08 & 8E05002 & AGG-IC-001 \\
\hline 16887-00-6 & Chloride & $1.68 \mathrm{E} 0$ & ug/g dry & $5.00 \mathrm{E}-1$ & 2/06/08 & 8E05002 & AGG-IC-001 \\
\hline $14797-65-0$ & Nitrite & $<1.00 \mathrm{E} 0$ & ug/g dry & $1.00 \mathrm{E} 0$ & 2/06/08 & 8E05002 & AGG-IC-001 \\
\hline 14797-55-8 & Nitrate & 3.03E0 & ug/g dry & $1.00 \mathrm{E} 0$ & 2/06/08 & 8E05002 & AGG-IC-001 \\
\hline 14808-79-8 & Sulfate & 4.00E1 & ug/g dry & $1.50 \mathrm{E} 0$ & 2/06/08 & 8E05002 & AGG-IC-001 \\
\hline $14265-44-2$ & Phosphate & $<1.50 \mathrm{E} 0$ & ug/g dry & $1.50 \mathrm{E} 0$ & $2 / 06 / 08$ & 8E05002 & AGG-IC-001 \\
\hline HEIS No. & B1RKT3 & \multicolumn{3}{|c|}{ Lab ID: $\quad$ 0802005-29 } & & & \\
\hline 16984-48-8 & Fluoride & 2.07E-1 & ug/g dry & 2.00E-1 & 2/06/08 & 8E05002 & AGG-IC-001 \\
\hline $16887-00-6$ & Chloride & $<5.00 \mathrm{E}-1$ & ug/g dry & $5.00 \mathrm{E}-1$ & 2/06/08 & 8E05002 & AGG-IC-001 \\
\hline $14797-65-0$ & Nitrite & $<1.00 \mathrm{E} 0$ & ug/g dry & $1.00 \mathrm{E} 0$ & 2/06/08 & 8E05002 & AGG-IC-001 \\
\hline $14797-55-8$ & Nitrate & $1.02 \mathrm{E} 0$ & ug/g dry & $1.00 \mathrm{E} 0$ & 2/06/08 & 8E05002 & AGG-IC-001 \\
\hline 14808-79-8 & Sulfate & $9.04 \mathrm{E} 0$ & ug/g dry & $1.50 \mathrm{E} 0$ & 2/06/08 & 8E05002 & AGG-IC-001 \\
\hline 14265-44-2 & Phosphate & $<1.50 \mathrm{E} 0$ & ug/g dry & $1.50 \mathrm{E} 0$ & 2/06/08 & 8E05002 & AGG-IC-001 \\
\hline HEIS No. & B1RKT7 & \multicolumn{3}{|c|}{ Lab ID: $\quad$ 0802006-07 } & & & \\
\hline $16984-48-8$ & Fluoride & $<2.00 \mathrm{E} 0$ & ug/g dry & $2.00 \mathrm{E} 0$ & 2/08/08 & 8E05002 & AGG-IC-001 \\
\hline 16887-00-6 & Chloride & $<5.00 \mathrm{E} 0$ & ug/g dry & $5.00 \mathrm{E} 0$ & 2/08/08 & 8E05002 & AGG-IC-001 \\
\hline $14797-65-0$ & Nitrite & $<1.00 \mathrm{E} 1$ & ug/g dry & $1.00 \mathrm{E} 1$ & 2/08/08 & 8E05002 & AGG-IC-001 \\
\hline 14797-55-8 & Nitrate & $<1.00 \mathrm{E} 1$ & ug/g dry & $1.00 \mathrm{E} 1$ & 2/08/08 & 8E05002 & AGG-IC-001 \\
\hline 14808-79-8 & Sulfate & 3.67E1 & ug/g dry & $1.50 \mathrm{E} 1$ & 2/08/08 & 8Е05002 & AGG-IC-001 \\
\hline $14265-44-2$ & Phosphate & $<1.50 \mathrm{E} 1$ & ug/g dry & $1.50 \mathrm{E} 1$ & 2/08/08 & 8E05002 & AGG-IC-001 \\
\hline HEIS No. & B1RKV1 & \multicolumn{3}{|c|}{ Lab ID: $\quad$ 0802006-11 } & & & \\
\hline $16984-48-8$ & Fluoride & $<2.23 \mathrm{E} 0$ & ug/g dry & 2.23E0 & 2/08/08 & 8E05002 & AGG-IC-001 \\
\hline $16887-00-6$ & Chloride & $<5.57 \mathrm{E} 0$ & ug/g dry & 5.57E0 & 2/08/08 & 8E05002 & AGG-IC-001 \\
\hline $14797-65-0$ & Nitrite & $<1.11 \mathrm{E} 1$ & ug/g dry & $1.11 \mathrm{E} 1$ & 2/08/08 & 8E05002 & AGG-IC-001 \\
\hline $14797-55-8$ & Nitrate & $<1.11 \mathrm{E} 1$ & ug/g dry & $1.11 \mathrm{E} 1$ & 2/08/08 & 8E05002 & AGG-IC-001 \\
\hline 14808-79-8 & Sulfate & 4.69E1 & ug/g dry & 1.67E1 & 2/08/08 & 8E05002 & AGG-IC-001 \\
\hline 14265-44-2 & Phosphate & $<1.67 \mathrm{E} 1$ & ug/g dry & 1.67E1 & 2/08/08 & 8E05002 & AGG-IC-001 \\
\hline
\end{tabular}




\section{Anions by Ion Chromatography}

\begin{tabular}{|c|c|c|c|c|c|c|c|}
\hline CAS \# & Analyte & Results & Units & EQL & Analyzed & Batch & Method \\
\hline HEIS No. & B1RKV5 & \multicolumn{3}{|c|}{ Lab ID: $\quad$ 0802007-04 } & & & \\
\hline $16984-48-8$ & Fluoride & $<2.00 \mathrm{E} 0$ & ug/g dry & $2.00 \mathrm{E} 0$ & 2/08/08 & 8E05002 & AGG-IC-001 \\
\hline 16887-00-6 & Chloride & $<5.00 \mathrm{E} 0$ & ug/g dry & $5.00 \mathrm{E} 0$ & 2/08/08 & 8Е05002 & AGG-IC-001 \\
\hline 14797-65-0 & Nitrite & $<1.00 \mathrm{E} 1$ & ug/g dry & $1.00 \mathrm{E} 1$ & 2/08/08 & 8E05002 & AGG-IC-001 \\
\hline 14797-55-8 & Nitrate & $<1.00 \mathrm{E} 1$ & ug/g dry & $1.00 \mathrm{E} 1$ & 2/08/08 & 8E05002 & AGG-IC-001 \\
\hline 14808-79-8 & Sulfate & 5.10E1 & ug/g dry & $1.50 \mathrm{E} 1$ & 2/08/08 & 8E05002 & AGG-IC-001 \\
\hline $14265-44-2$ & Phosphate & $<1.50 \mathrm{E} 1$ & ug/g dry & $1.50 \mathrm{E} 1$ & 2/08/08 & 8E05002 & AGG-IC-001 \\
\hline HEIS No. & B1RKV9 & \multicolumn{3}{|c|}{ Lab ID: $\quad$ 0802007-12 } & & & \\
\hline 16984-48-8 & Fluoride & $<2.00 \mathrm{E} 0$ & ug/g dry & $2.00 \mathrm{E} 0$ & 2/08/08 & 8E05002 & AGG-IC-001 \\
\hline $16887-00-6$ & Chloride & $<5.00 \mathrm{E} 0$ & ug/g dry & $5.00 \mathrm{E} 0$ & 2/08/08 & 8E05002 & AGG-IC-001 \\
\hline $14797-65-0$ & Nitrite & $<1.00 \mathrm{E} 1$ & ug/g dry & $1.00 \mathrm{E} 1$ & 2/08/08 & 8E05002 & AGG-IC-001 \\
\hline $14797-55-8$ & Nitrate & $<1.00 \mathrm{E} 1$ & ug/g dry & $1.00 \mathrm{E} 1$ & 2/08/08 & 8E05002 & AGG-IC-001 \\
\hline 14808-79-8 & Sulfate & 5.07E1 & ug/g dry & $1.50 \mathrm{E} 1$ & 2/08/08 & 8E05002 & AGG-IC-001 \\
\hline 14265-44-2 & Phosphate & $<1.50 \mathrm{E} 1$ & ug/g dry & $1.50 \mathrm{E} 1$ & 2/08/08 & 8E05002 & AGG-IC-001 \\
\hline HEIS No. & B1RKW2 & \multicolumn{3}{|c|}{ Lab ID: $\quad$ 0802008-04 } & & & \\
\hline 16984-48-8 & Fluoride & 7.38E0 & ug/g dry & $2.00 \mathrm{E} 0$ & 2/08/08 & 8E05002 & AGG-IC-001 \\
\hline 16887-00-6 & Chloride & 6.77E0 & ug/g dry & $5.00 \mathrm{E} 0$ & 2/08/08 & 8E05002 & AGG-IC-001 \\
\hline $14797-65-0$ & Nitrite & $<1.00 \mathrm{E} 1$ & ug/g dry & $1.00 \mathrm{E} 1$ & 2/08/08 & 8E05002 & AGG-IC-001 \\
\hline 14797-55-8 & Nitrate & 3.01E1 & ug/g dry & $1.00 \mathrm{E} 1$ & 2/08/08 & 8E05002 & AGG-IC-001 \\
\hline 14808-79-8 & Sulfate & $1.07 \mathrm{E} 2$ & ug/g dry & $1.50 \mathrm{E} 1$ & 2/08/08 & 8E05002 & AGG-IC-001 \\
\hline $14265-44-2$ & Phosphate & $<1.50 \mathrm{E} 1$ & ug/g dry & $1.50 \mathrm{E} 1$ & 2/08/08 & 8E05002 & AGG-IC-001 \\
\hline HEIS No. & B1RKW4 & \multicolumn{3}{|c|}{ Lab ID: $\quad$ 0802008-08 } & & & \\
\hline 16984-48-8 & Fluoride & $5.24 \mathrm{E} 1$ & ug/g dry & $2.00 \mathrm{E} 0$ & 2/08/08 & 8E05002 & AGG-IC-001 \\
\hline 16887-00-6 & Chloride & $1.24 \mathrm{E} 1$ & ug/g dry & $5.00 \mathrm{E} 0$ & 2/08/08 & 8E05002 & AGG-IC-001 \\
\hline $14797-65-0$ & Nitrite & $<1.00 \mathrm{E} 1$ & ug/g dry & $1.00 \mathrm{E} 1$ & 2/08/08 & 8E05002 & AGG-IC-001 \\
\hline 14797-55-8 & Nitrate & $1.30 \mathrm{E} 2$ & ug/g dry & $1.00 \mathrm{E} 1$ & 2/08/08 & 8E05002 & AGG-IC-001 \\
\hline 14808-79-8 & Sulfate & $1.48 \mathrm{E} 2$ & ug/g dry & $1.50 \mathrm{E} 1$ & 2/08/08 & 8E05002 & AGG-IC-001 \\
\hline $14265-44-2$ & Phosphate & $<1.50 \mathrm{E} 1$ & ug/g dry & $1.50 \mathrm{E} 1$ & $2 / 08 / 08$ & 8E05002 & AGG-IC-001 \\
\hline HEIS No. & B1RKW5 & \multicolumn{3}{|c|}{ Lab ID: $\quad$ 0802009-02 } & & & \\
\hline 16984-48-8 & Fluoride & 2.16E1 & ug/g dry & $2.00 \mathrm{E} 0$ & 2/08/08 & 8E05002 & AGG-IC-001 \\
\hline $16887-00-6$ & Chloride & $9.07 \mathrm{E} 0$ & ug/g dry & $5.00 \mathrm{E} 0$ & 2/08/08 & 8E05002 & AGG-IC-001 \\
\hline $14797-65-0$ & Nitrite & $<1.00 \mathrm{E} 1$ & ug/g dry & $1.00 \mathrm{E} 1$ & 2/08/08 & 8E05002 & AGG-IC-001 \\
\hline $14797-55-8$ & Nitrate & 4.30E1 & ug/g dry & $1.00 \mathrm{E} 1$ & 2/08/08 & 8E05002 & AGG-IC-001 \\
\hline 14808-79-8 & Sulfate & 8.23E1 & ug/g dry & $1.50 \mathrm{E} 1$ & 2/08/08 & 8E05002 & AGG-IC-001 \\
\hline 14265-44-2 & Phosphate & $<1.50 \mathrm{E} 1$ & ug/g dry & $1.50 \mathrm{E} 1$ & 2/08/08 & 8E05002 & AGG-IC-001 \\
\hline HEIS No. & B1RKW6 & \multicolumn{3}{|c|}{ Lab ID: $\quad$ 0802009-04 } & & & \\
\hline 16984-48-8 & Fluoride & $2.60 \mathrm{E} 1$ & ug/g dry & $2.00 \mathrm{E} 0$ & 2/08/08 & 8E05002 & AGG-IC-001 \\
\hline 16887-00-6 & Chloride & $1.50 \mathrm{E} 1$ & ug/g dry & $5.00 \mathrm{E} 0$ & 2/08/08 & 8E05002 & AGG-IC-001 \\
\hline $14797-65-0$ & Nitrite & $<1.00 \mathrm{E} 1$ & ug/g dry & $1.00 \mathrm{E} 1$ & 2/08/08 & 8E05002 & AGG-IC-001 \\
\hline 14797-55-8 & Nitrate & $9.71 \mathrm{E} 1$ & ug/g dry & $1.00 \mathrm{E} 1$ & 2/08/08 & 8E05002 & AGG-IC-001 \\
\hline $14808-79-8$ & Sulfate & 1.33E2 & ug/g dry & $1.50 \mathrm{E} 1$ & 2/08/08 & 8E05002 & AGG-IC-001 \\
\hline $14265-44-2$ & Phosphate & $<1.50 \mathrm{E} 1$ & ug/g dry & $1.50 \mathrm{E} 1$ & 2/08/08 & 8E05002 & AGG-IC-001 \\
\hline HEIS No. & B1TFV6 & \multicolumn{3}{|c|}{ Lab ID: $\quad$ 0802028-03 } & & & \\
\hline $16984-48-8$ & Fluoride & $1.48 \mathrm{E} 0$ & ug/g dry & $2.01 \mathrm{E}-1$ & 3/29/08 & 8C28006 & AGG-IC-001 \\
\hline $16887-00-6$ & Chloride & $9.17 \mathrm{E} 0$ & ug/g dry & $5.02 \mathrm{E}-1$ & 3/29/08 & 8C28006 & AGG-IC-001 \\
\hline $14797-65-0$ & Nitrite & $<1.00 \mathrm{E} 0$ & ug/g dry & $1.00 \mathrm{E} 0$ & 3/29/08 & 8C28006 & AGG-IC-001 \\
\hline $14797-55-8$ & Nitrate & 4.70E1 & ug/g dry & $1.00 \mathrm{E} 0$ & 3/29/08 & 8C28006 & AGG-IC-001 \\
\hline 14808-79-8 & Sulfate & 1.07E2 & ug/g dry & $1.51 \mathrm{E} 0$ & 3/29/08 & 8C28006 & AGG-IC-001 \\
\hline 14265-44-2 & Phosphate & $3.24 \mathrm{E} 0$ & ug/g dry & $1.51 \mathrm{E} 0$ & 3/29/08 & 8C28006 & AGG-IC-001 \\
\hline
\end{tabular}




\section{Anions by Ion Chromatography}

\begin{tabular}{|c|c|c|c|c|c|c|c|}
\hline CAS \# & Analyte & Results & Units & EQL & Analyzed & Batch & Method \\
\hline HEIS No. & B1TFV8 & \multicolumn{3}{|c|}{ Lab ID: $\quad$ 0802028-05 } & & & \\
\hline $16984-48-8$ & Fluoride & $3.26 \mathrm{E} 1$ & ug/g dry & 1.99E0 & 3/31/08 & 8C28006 & AGG-IC-001 \\
\hline 16887-00-6 & Chloride & $1.08 \mathrm{E} 1$ & ug/g dry & $4.98 \mathrm{E}-1$ & 3/29/08 & 8C28006 & AGG-IC-001 \\
\hline 14797-65-0 & Nitrite & $<9.97 \mathrm{E}-1$ & ug/g dry & 9.97E-1 & 3/29/08 & 8C28006 & AGG-IC-001 \\
\hline 14797-55-8 & Nitrate & 5.67E1 & ug/g dry & $9.97 \mathrm{E}-1$ & 3/29/08 & 8C28006 & AGG-IC-001 \\
\hline 14808-79-8 & Sulfate & 1.17E2 & ug/g dry & $1.50 \mathrm{E} 0$ & 3/29/08 & 8C28006 & AGG-IC-001 \\
\hline $14265-44-2$ & Phosphate & $1.71 \mathrm{E} 0$ & ug/g dry & $1.50 \mathrm{E} 0$ & 3/29/08 & 8C28006 & AGG-IC-001 \\
\hline HEIS No. & B1TFW0 & \multicolumn{3}{|c|}{ Lab ID: $\quad$ 0802028-07 } & & & \\
\hline 16984-48-8 & Fluoride & $<2.00 \mathrm{E}-1$ & ug/g dry & 2.00E-1 & 3/29/08 & 8C28006 & AGG-IC-001 \\
\hline $16887-00-6$ & Chloride & 2.64E0 & ug/g dry & $5.01 \mathrm{E}-1$ & 3/29/08 & 8C28006 & AGG-IC-001 \\
\hline $14797-65-0$ & Nitrite & $<1.00 \mathrm{E} 0$ & ug/g dry & $1.00 \mathrm{E} 0$ & 3/29/08 & 8C28006 & AGG-IC-001 \\
\hline 14797-55-8 & Nitrate & 1.84E1 & ug/g dry & $1.00 \mathrm{E} 0$ & 3/29/08 & 8C28006 & AGG-IC-001 \\
\hline 14808-79-8 & Sulfate & $1.40 \mathrm{E} 1$ & ug/g dry & $1.50 \mathrm{E} 0$ & 3/29/08 & 8C28006 & AGG-IC-001 \\
\hline 14265-44-2 & Phosphate & $<1.50 \mathrm{E} 0$ & ug/g dry & $1.50 \mathrm{E} 0$ & 3/29/08 & 8C28006 & AGG-IC-001 \\
\hline HEIS No. & B1TFW2 & \multicolumn{3}{|c|}{ Lab ID: $\quad$ 0802028-09 } & & & \\
\hline 16984-48-8 & Fluoride & $<2.01 \mathrm{E}-1$ & ug/g dry & $2.01 \mathrm{E}-1$ & 3/29/08 & 8C28006 & AGG-IC-001 \\
\hline 16887-00-6 & Chloride & 3.12E0 & ug/g dry & $5.02 \mathrm{E}-1$ & 3/29/08 & 8C28006 & AGG-IC-001 \\
\hline $14797-65-0$ & Nitrite & $<1.00 \mathrm{E} 0$ & ug/g dry & $1.00 \mathrm{E} 0$ & 3/29/08 & 8C28006 & AGG-IC-001 \\
\hline 14797-55-8 & Nitrate & $5.90 \mathrm{E} 1$ & ug/g dry & $1.00 \mathrm{E} 0$ & 3/29/08 & 8C28006 & AGG-IC-001 \\
\hline 14808-79-8 & Sulfate & 4.23E1 & ug/g dry & $1.51 \mathrm{E} 0$ & 3/29/08 & 8C28006 & AGG-IC-001 \\
\hline $14265-44-2$ & Phosphate & $<1.51 \mathrm{E} 0$ & ug/g dry & $1.51 \mathrm{E} 0$ & 3/29/08 & 8C28006 & AGG-IC-001 \\
\hline HEIS No. & B1RKX0 & \multicolumn{3}{|c|}{ Lab ID: $\quad$ 0802028-11 } & & & \\
\hline 16984-48-8 & Fluoride & $<2.00 \mathrm{E}-1$ & ug/g dry & 2.00E-1 & 3/29/08 & 8C28006 & AGG-IC-001 \\
\hline 16887-00-6 & Chloride & 2.73E0 & ug/g dry & 4.99E-1 & 3/29/08 & 8C28006 & AGG-IC-001 \\
\hline $14797-65-0$ & Nitrite & $<9.98 \mathrm{E}-1$ & ug/g dry & $9.98 \mathrm{E}-1$ & 3/29/08 & 8C28006 & AGG-IC-001 \\
\hline 14797-55-8 & Nitrate & 3.60E1 & ug/g dry & $9.98 \mathrm{E}-1$ & 3/29/08 & 8C28006 & AGG-IC-001 \\
\hline 14808-79-8 & Sulfate & $1.78 \mathrm{E} 1$ & ug/g dry & $1.50 \mathrm{E} 0$ & 3/29/08 & 8C28006 & AGG-IC-001 \\
\hline $14265-44-2$ & Phosphate & $<1.50 \mathrm{E} 0$ & ug/g dry & $1.50 \mathrm{E} 0$ & $3 / 29 / 08$ & 8C28006 & AGG-IC-001 \\
\hline HEIS No. & B1RKX3 & \multicolumn{3}{|c|}{ Lab ID: $\quad$ 0802028-17 } & & & \\
\hline 16984-48-8 & Fluoride & $<2.00 \mathrm{E}-1$ & ug/g dry & 2.00E-1 & 3/29/08 & 8C28006 & AGG-IC-001 \\
\hline $16887-00-6$ & Chloride & $<5.00 \mathrm{E}-1$ & ug/g dry & $5.00 \mathrm{E}-1$ & 3/29/08 & 8C28006 & AGG-IC-001 \\
\hline $14797-65-0$ & Nitrite & $<1.00 \mathrm{E} 0$ & ug/g dry & $1.00 \mathrm{E} 0$ & 3/29/08 & 8C28006 & AGG-IC-001 \\
\hline $14797-55-8$ & Nitrate & $4.56 \mathrm{E} 0$ & ug/g dry & $1.00 \mathrm{E} 0$ & 3/29/08 & 8C28006 & AGG-IC-001 \\
\hline 14808-79-8 & Sulfate & 4.06E0 & ug/g dry & $1.50 \mathrm{E} 0$ & 3/29/08 & 8C28006 & AGG-IC-001 \\
\hline 14265-44-2 & Phosphate & $<1.50 \mathrm{E} 0$ & ug/g dry & $1.50 \mathrm{E} 0$ & 3/29/08 & 8C28006 & AGG-IC-001 \\
\hline HEIS No. & B1RKX5 & \multicolumn{3}{|c|}{ Lab ID: $\quad$ 0802028-23 } & & & \\
\hline $16984-48-8$ & Fluoride & $<2.00 \mathrm{E}-1$ & ug/g dry & $2.00 \mathrm{E}-1$ & 3/29/08 & 8C28006 & AGG-IC-001 \\
\hline $16887-00-6$ & Chloride & $<5.01 \mathrm{E}-1$ & ug/g dry & $5.01 \mathrm{E}-1$ & 3/29/08 & 8C28006 & AGG-IC-001 \\
\hline $14797-65-0$ & Nitrite & $<1.00 \mathrm{E} 0$ & ug/g dry & $1.00 \mathrm{E} 0$ & 3/29/08 & 8C28006 & AGG-IC-001 \\
\hline 14797-55-8 & Nitrate & 3.33Е0 & ug/g dry & $1.00 \mathrm{E} 0$ & 3/29/08 & 8C28006 & AGG-IC-001 \\
\hline $14808-79-8$ & Sulfate & 3.43E0 & ug/g dry & $1.50 \mathrm{E} 0$ & 3/29/08 & 8C28006 & AGG-IC-001 \\
\hline $14265-44-2$ & Phosphate & $<1.50 \mathrm{E} 0$ & ug/g dry & $1.50 \mathrm{E} 0$ & 3/29/08 & 8C28006 & AGG-IC-001 \\
\hline HEIS No. & B1RKX7 & \multicolumn{3}{|c|}{ Lab ID: $\quad$ 0802028-29 } & & & \\
\hline $16984-48-8$ & Fluoride & $<2.00 \mathrm{E}-1$ & ug/g dry & $2.00 \mathrm{E}-1$ & 3/29/08 & 8C28006 & AGG-IC-001 \\
\hline $16887-00-6$ & Chloride & $<5.00 \mathrm{E}-1$ & ug/g dry & $5.00 \mathrm{E}-1$ & 3/29/08 & 8C28006 & AGG-IC-001 \\
\hline $14797-65-0$ & Nitrite & $<1.00 \mathrm{E} 0$ & ug/g dry & $1.00 \mathrm{E} 0$ & 3/29/08 & 8C28006 & AGG-IC-001 \\
\hline $14797-55-8$ & Nitrate & $1.26 \mathrm{E} 0$ & ug/g dry & $1.00 \mathrm{E} 0$ & 3/29/08 & 8C28006 & AGG-IC-001 \\
\hline 14808-79-8 & Sulfate & $1.60 \mathrm{E} 0$ & ug/g dry & $1.50 \mathrm{E} 0$ & 3/29/08 & 8C28006 & AGG-IC-001 \\
\hline 14265-44-2 & Phosphate & $<1.50 \mathrm{E} 0$ & ug/g dry & $1.50 \mathrm{E} 0$ & 3/29/08 & 8C28006 & AGG-IC-001 \\
\hline
\end{tabular}


Total Metals by PNNL-AGG-ICP-AES/Water Extract

\begin{tabular}{|c|c|c|c|c|c|c|c|}
\hline CAS \# & Analyte & Results & Units & EQL & Analyzed & Batch & Method \\
\hline HEIS No. & B1RKL1 & \multicolumn{3}{|c|}{ Lab ID: $\quad$ 0802002-02 } & & & \\
\hline 7429-90-5 & Aluminum & $1.32 \mathrm{E}-1$ & ug/g dry & $8.61 \mathrm{E}-2$ & 2/07/08 & 8E01006 & PNNL-AGG-ICP-AES \\
\hline $7440-39-3$ & Barium & $1.75 \mathrm{E}-2$ & ug/g dry & $8.82 \mathrm{E}-3$ & 2/07/08 & 8E01006 & PNNL-AGG-ICP-AES \\
\hline $7440-70-2$ & Calcium & 7.06E0 & ug/g dry & $3.88 \mathrm{E}-1$ & 2/07/08 & 8E01006 & PNNL-AGG-ICP-AES \\
\hline $7440-48-4$ & Cobalt & $<9.63 \mathrm{E}-2$ & ug/g dry & $9.63 \mathrm{E}-2$ & $2 / 07 / 08$ & 8E01006 & PNNL-AGG-ICP-AES \\
\hline $7440-47-3$ & Chromium & $<3.34 \mathrm{E}-2$ & ug/g dry & $3.34 \mathrm{E}-2$ & 2/07/08 & 8E01006 & PNNL-AGG-ICP-AES \\
\hline $7440-50-8$ & Copper & $<8.07 \mathrm{E}-2$ & ug/g dry & 8.07E-2 & $2 / 07 / 08$ & 8E01006 & PNNL-AGG-ICP-AES \\
\hline 7440-09-7 & Potassium & $4.51 \mathrm{E} 0$ & ug/g dry & 2.34E0 & 2/07/08 & 8E01006 & PNNL-AGG-ICP-AES \\
\hline 7439-95-4 & Magnesium & 2.43E0 & ug/g dry & 8.37E-2 & 2/07/08 & 8E01006 & PNNL-AGG-ICP-AES \\
\hline 7439-96-5 & Manganese & $<1.72 \mathrm{E}-2$ & ug/g dry & $1.72 \mathrm{E}-2$ & $2 / 07 / 08$ & 8E01006 & PNNL-AGG-ICP-AES \\
\hline $7440-02-0$ & Nickel & $<9.36 \mathrm{E}-2$ & ug/g dry & $9.36 \mathrm{E}-2$ & $2 / 07 / 08$ & 8E01006 & PNNL-AGG-ICP-AES \\
\hline $7440-62-2$ & Vanadium & $<4.54 \mathrm{E}-2$ & ug/g dry & $4.54 \mathrm{E}-2$ & $2 / 07 / 08$ & 8E01006 & PNNL-AGG-ICP-AES \\
\hline $7440-66-6$ & Zinc & $<9.30 \mathrm{E}-2$ & ug/g dry & $9.30 \mathrm{E}-2$ & 2/07/08 & 8E01006 & PNNL-AGG-ICP-AES \\
\hline $7440-23-5$ & Sodium & 7.36Е0 & ug/g dry & $6.71 \mathrm{E}-1$ & $2 / 07 / 08$ & 8E01006 & PNNL-AGG-ICP-AES \\
\hline HEIS No. & B1RKL6 & \multicolumn{3}{|c|}{ Lab ID: $\quad$ 0802002-09 } & & & \\
\hline 7429-90-5 & Aluminum & $1.93 \mathrm{E}-1$ & ug/g dry & 8.63E-2 & $2 / 07 / 08$ & 8E01006 & PNNL-AGG-ICP-AES \\
\hline $7440-39-3$ & Barium & $1.77 \mathrm{E}-2$ & ug/g dry & 8.84E-3 & $2 / 07 / 08$ & 8E01006 & PNNL-AGG-ICP-AES \\
\hline $7440-70-2$ & Calcium & $6.73 \mathrm{E} 0$ & ug/g dry & $3.89 \mathrm{E}-1$ & 2/07/08 & 8E01006 & PNNL-AGG-ICP-AES \\
\hline $7440-48-4$ & Cobalt & $<9.66 \mathrm{E}-2$ & ug/g dry & $9.66 \mathrm{E}-2$ & $2 / 07 / 08$ & 8E01006 & PNNL-AGG-ICP-AES \\
\hline $7440-47-3$ & Chromium & $<3.35 \mathrm{E}-2$ & ug/g dry & 3.35E-2 & $2 / 07 / 08$ & 8E01006 & PNNL-AGG-ICP-AES \\
\hline $7440-50-8$ & Copper & $<8.09 \mathrm{E}-2$ & ug/g dry & 8.09E-2 & 2/07/08 & 8E01006 & PNNL-AGG-ICP-AES \\
\hline 7440-09-7 & Potassium & 4.71E0 & ug/g dry & $2.34 \mathrm{E} 0$ & 2/07/08 & 8E01006 & PNNL-AGG-ICP-AES \\
\hline 7439-95-4 & Magnesium & 2.37E0 & ug/g dry & 8.39E-2 & 2/07/08 & 8E01006 & PNNL-AGG-ICP-AES \\
\hline 7439-96-5 & Manganese & $<1.72 \mathrm{E}-2$ & ug/g dry & $1.72 \mathrm{E}-2$ & $2 / 07 / 08$ & 8E01006 & PNNL-AGG-ICP-AES \\
\hline $7440-02-0$ & Nickel & $<9.39 \mathrm{E}-2$ & ug/g dry & $9.39 \mathrm{E}-2$ & 2/07/08 & 8E01006 & PNNL-AGG-ICP-AES \\
\hline $7440-62-2$ & Vanadium & $<4.56 \mathrm{E}-2$ & ug/g dry & $4.56 \mathrm{E}-2$ & $2 / 07 / 08$ & 8E01006 & PNNL-AGG-ICP-AES \\
\hline $7440-66-6$ & Zinc & $<9.33 \mathrm{E}-2$ & ug/g dry & $9.33 \mathrm{E}-2$ & $2 / 07 / 08$ & 8E01006 & PNNL-AGG-ICP-AES \\
\hline $7440-23-5$ & Sodium & 1.09E1 & ug/g dry & 6.73E-1 & 2/07/08 & 8E01006 & PNNL-AGG-ICP-AES \\
\hline HEIS No. & B1RKM1 & \multicolumn{3}{|c|}{ Lab ID: $\quad$ 0802002-17 } & & & \\
\hline $7429-90-5$ & Aluminum & $1.54 \mathrm{E}-1$ & ug/g dry & $8.59 \mathrm{E}-2$ & $2 / 07 / 08$ & 8E01006 & PNNL-AGG-ICP-AES \\
\hline $7440-39-3$ & Barium & 1.19E-2 & ug/g dry & $8.80 \mathrm{E}-3$ & $2 / 07 / 08$ & 8E01006 & PNNL-AGG-ICP-AES \\
\hline $7440-70-2$ & Calcium & 7.38E0 & ug/g dry & 3.87E-1 & $2 / 07 / 08$ & 8E01006 & PNNL-AGG-ICP-AES \\
\hline $7440-48-4$ & Cobalt & $<9.61 \mathrm{E}-2$ & ug/g dry & $9.61 \mathrm{E}-2$ & 2/07/08 & 8E01006 & PNNL-AGG-ICP-AES \\
\hline $7440-47-3$ & Chromium & $<3.33 E-2$ & ug/g dry & $3.33 \mathrm{E}-2$ & $2 / 07 / 08$ & 8E01006 & PNNL-AGG-ICP-AES \\
\hline $7440-50-8$ & Copper & $<8.05 \mathrm{E}-2$ & ug/g dry & 8.05E-2 & 2/07/08 & 8E01006 & PNNL-AGG-ICP-AES \\
\hline 7440-09-7 & Potassium & 4.89E0 & ug/g dry & 2.33E0 & 2/07/08 & 8E01006 & PNNL-AGG-ICP-AES \\
\hline 7439-95-4 & Magnesium & $2.75 \mathrm{E} 0$ & ug/g dry & 8.35E-2 & 2/07/08 & 8E01006 & PNNL-AGG-ICP-AES \\
\hline 7439-96-5 & Manganese & $<1.71 \mathrm{E}-2$ & ug/g dry & $1.71 \mathrm{E}-2$ & 2/07/08 & 8E01006 & PNNL-AGG-ICP-AES \\
\hline $7440-02-0$ & Nickel & $<9.34 \mathrm{E}-2$ & ug/g dry & $9.34 \mathrm{E}-2$ & $2 / 07 / 08$ & 8E01006 & PNNL-AGG-ICP-AES \\
\hline $7440-62-2$ & Vanadium & $<4.53 \mathrm{E}-2$ & ug/g dry & 4.53E-2 & 2/07/08 & 8E01006 & PNNL-AGG-ICP-AES \\
\hline $7440-66-6$ & Zinc & $<9.28 \mathrm{E}-2$ & ug/g dry & $9.28 \mathrm{E}-2$ & 2/07/08 & 8E01006 & PNNL-AGG-ICP-AES \\
\hline $7440-23-5$ & Sodium & 1.06E1 & ug/g dry & $6.69 \mathrm{E}-1$ & 2/07/08 & 8E01006 & PNNL-AGG-ICP-AES \\
\hline HEIS No. & B1RKM8 & \multicolumn{3}{|c|}{ Lab ID: $\quad$ 0802002-31 } & & & \\
\hline $7429-90-5$ & Aluminum & $1.92 \mathrm{E}-1$ & ug/g dry & $8.58 \mathrm{E}-2$ & $2 / 07 / 08$ & 8E01006 & PNNL-AGG-ICP-AES \\
\hline $7440-39-3$ & Barium & $1.75 \mathrm{E}-2$ & ug/g dry & $8.79 \mathrm{E}-3$ & 2/07/08 & 8E01006 & PNNL-AGG-ICP-AES \\
\hline $7440-70-2$ & Calcium & $1.07 \mathrm{E} 1$ & ug/g dry & 3.87E-1 & 2/07/08 & 8E01006 & PNNL-AGG-ICP-AES \\
\hline $7440-48-4$ & Cobalt & $<9.60 \mathrm{E}-2$ & ug/g dry & $9.60 \mathrm{E}-2$ & 2/07/08 & 8E01006 & PNNL-AGG-ICP-AES \\
\hline $7440-47-3$ & Chromium & $<3.33 \mathrm{E}-2$ & ug/g dry & $3.33 \mathrm{E}-2$ & 2/07/08 & 8E01006 & PNNL-AGG-ICP-AES \\
\hline $7440-50-8$ & Copper & $<8.04 \mathrm{E}-2$ & ug/g dry & $8.04 \mathrm{E}-2$ & 2/07/08 & 8E01006 & PNNL-AGG-ICP-AES \\
\hline
\end{tabular}


Total Metals by PNNL-AGG-ICP-AES/Water Extract

\begin{tabular}{|c|c|c|c|c|c|c|c|}
\hline CAS \# & Analyte & Results & Units & EQL & Analyzed & Batch & Method \\
\hline HEIS No. & B1RKM8 & \multicolumn{3}{|c|}{ Lab ID: $\quad$ 0802002-31 } & & & \\
\hline $7440-09-7$ & Potassium & 5.77E0 & ug/g dry & 2.33E0 & 2/07/08 & 8E01006 & PNNL-AGG-ICP-AES \\
\hline 7439-95-4 & Magnesium & 3.66E0 & ug/g dry & 8.34E-2 & $2 / 07 / 08$ & 8E01006 & PNNL-AGG-ICP-AES \\
\hline 7439-96-5 & Manganese & $<1.71 \mathrm{E}-2$ & ug/g dry & $1.71 \mathrm{E}-2$ & $2 / 07 / 08$ & 8E01006 & PNNL-AGG-ICP-AES \\
\hline $7440-02-0$ & Nickel & $<9.33 \mathrm{E}-2$ & ug/g dry & $9.33 \mathrm{E}-2$ & $2 / 07 / 08$ & 8E01006 & PNNL-AGG-ICP-AES \\
\hline $7440-62-2$ & Vanadium & $<4.53 \mathrm{E}-2$ & ug/g dry & 4.53E-2 & $2 / 07 / 08$ & 8E01006 & PNNL-AGG-ICP-AES \\
\hline $7440-66-6$ & Zinc & $<9.27 \mathrm{E}-2$ & ug/g dry & $9.27 \mathrm{E}-2$ & $2 / 07 / 08$ & 8E01006 & PNNL-AGG-ICP-AES \\
\hline $7440-23-5$ & Sodium & 1.29E1 & ug/g dry & $6.69 \mathrm{E}-1$ & 2/07/08 & 8E01006 & PNNL-AGG-ICP-AES \\
\hline HEIS No. & B1RKW7 & \multicolumn{3}{|c|}{ Lab ID: $\quad$ 0802003-02 } & & & \\
\hline $7429-90-5$ & Aluminum & $<9.16 \mathrm{E}-2$ & ug/g dry & $9.16 \mathrm{E}-2$ & $2 / 07 / 08$ & 8E01006 & PNNL-AGG-ICP-AES \\
\hline $7440-39-3$ & Barium & 3.03E-2 & ug/g dry & $9.38 \mathrm{E}-3$ & 2/07/08 & 8E01006 & PNNL-AGG-ICP-AES \\
\hline $7440-70-2$ & Calcium & 4.76E0 & ug/g dry & 4.13E-1 & $2 / 07 / 08$ & 8E01006 & PNNL-AGG-ICP-AES \\
\hline $7440-48-4$ & Cobalt & $<1.02 \mathrm{E}-1$ & ug/g dry & $1.02 \mathrm{E}-1$ & 2/07/08 & 8E01006 & PNNL-AGG-ICP-AES \\
\hline $7440-47-3$ & Chromium & $<3.55 \mathrm{E}-2$ & ug/g dry & $3.55 \mathrm{E}-2$ & 2/07/08 & 8E01006 & PNNL-AGG-ICP-AES \\
\hline $7440-50-8$ & Copper & $<8.58 \mathrm{E}-2$ & ug/g dry & $8.58 \mathrm{E}-2$ & 2/07/08 & 8E01006 & PNNL-AGG-ICP-AES \\
\hline 7440-09-7 & Potassium & 4.65E0 & ug/g dry & $2.48 \mathrm{E} 0$ & $2 / 07 / 08$ & 8E01006 & PNNL-AGG-ICP-AES \\
\hline $7439-95-4$ & Magnesium & 2.67E0 & ug/g dry & 8.90E-2 & $2 / 07 / 08$ & 8E01006 & PNNL-AGG-ICP-AES \\
\hline $7439-96-5$ & Manganese & $<1.83 \mathrm{E}-2$ & ug/g dry & 1.83E-2 & 2/07/08 & 8E01006 & PNNL-AGG-ICP-AES \\
\hline $7440-02-0$ & Nickel & $<9.96 \mathrm{E}-2$ & ug/g dry & $9.96 \mathrm{E}-2$ & 2/07/08 & 8E01006 & PNNL-AGG-ICP-AES \\
\hline $7440-23-5$ & Sodium & 2.33E2 & ug/g dry & 7.14E-1 & $2 / 07 / 08$ & 8E01006 & PNNL-AGG-ICP-AES \\
\hline HEIS No. & B1RKW8 & \multicolumn{3}{|c|}{ Lab ID: $\quad$ 0802003-04 } & & & \\
\hline $7429-90-5$ & Aluminum & $<8.58 \mathrm{E}-2$ & ug/g dry & $8.58 \mathrm{E}-2$ & 2/07/08 & 8E01006 & PNNL-AGG-ICP-AES \\
\hline $7440-39-3$ & Barium & 3.84E-2 & ug/g dry & 8.79E-3 & 2/07/08 & 8E01006 & PNNL-AGG-ICP-AES \\
\hline $7440-70-2$ & Calcium & $1.08 \mathrm{E} 1$ & ug/g dry & 3.87E-1 & 2/07/08 & 8E01006 & PNNL-AGG-ICP-AES \\
\hline $7440-48-4$ & Cobalt & $<9.60 \mathrm{E}-2$ & ug/g dry & $9.60 \mathrm{E}-2$ & 2/07/08 & 8E01006 & PNNL-AGG-ICP-AES \\
\hline $7440-47-3$ & Chromium & $3.72 \mathrm{E}-2$ & ug/g dry & 3.33E-2 & 2/07/08 & 8E01006 & PNNL-AGG-ICP-AES \\
\hline $7440-50-8$ & Copper & $<8.04 \mathrm{E}-2$ & ug/g dry & 8.04E-2 & $2 / 07 / 08$ & 8E01006 & PNNL-AGG-ICP-AES \\
\hline 7440-09-7 & Potassium & 5.84E0 & ug/g dry & 2.33Е0 & $2 / 07 / 08$ & 8E01006 & PNNL-AGG-ICP-AES \\
\hline $7439-95-4$ & Magnesium & 7.32E0 & ug/g dry & 8.34E-2 & $2 / 07 / 08$ & 8E01006 & PNNL-AGG-ICP-AES \\
\hline 7439-96-5 & Manganese & $<1.71 \mathrm{E}-2$ & ug/g dry & $1.71 \mathrm{E}-2$ & 2/07/08 & 8E01006 & PNNL-AGG-ICP-AES \\
\hline $7440-02-0$ & Nickel & $<9.33 \mathrm{E}-2$ & ug/g dry & $9.33 \mathrm{E}-2$ & $2 / 07 / 08$ & 8E01006 & PNNL-AGG-ICP-AES \\
\hline $7440-23-5$ & Sodium & 2.56E2 & ug/g dry & $6.69 \mathrm{E}-1$ & 2/07/08 & 8E01006 & PNNL-AGG-ICP-AES \\
\hline HEIS No. & B1RKN7 & \multicolumn{3}{|c|}{ Lab ID: $\quad$ 0802005-03 } & & & \\
\hline 7429-90-5 & Aluminum & $2.32 \mathrm{E}-1$ & ug/g dry & $8.60 \mathrm{E}-2$ & $2 / 07 / 08$ & 8E01006 & PNNL-AGG-ICP-AES \\
\hline $7440-39-3$ & Barium & $1.91 \mathrm{E}-2$ & ug/g dry & 8.81E-3 & $2 / 07 / 08$ & 8E01006 & PNNL-AGG-ICP-AES \\
\hline $7440-70-2$ & Calcium & $9.50 \mathrm{E} 0$ & ug/g dry & $3.88 \mathrm{E}-1$ & 2/07/08 & 8E01006 & PNNL-AGG-ICP-AES \\
\hline $7440-48-4$ & Cobalt & $<9.63 \mathrm{E}-2$ & ug/g dry & $9.63 \mathrm{E}-2$ & 2/07/08 & 8E01006 & PNNL-AGG-ICP-AES \\
\hline $7440-47-3$ & Chromium & $<3.34 \mathrm{E}-2$ & ug/g dry & $3.34 \mathrm{E}-2$ & 2/07/08 & 8E01006 & PNNL-AGG-ICP-AES \\
\hline $7440-50-8$ & Copper & $<8.06 \mathrm{E}-2$ & ug/g dry & 8.06E-2 & $2 / 07 / 08$ & 8E01006 & PNNL-AGG-ICP-AES \\
\hline 7440-09-7 & Potassium & 4.47E0 & ug/g dry & 2.33Е0 & $2 / 07 / 08$ & 8E01006 & PNNL-AGG-ICP-AES \\
\hline $7439-95-4$ & Magnesium & 2.92E0 & ug/g dry & 8.36E-2 & $2 / 07 / 08$ & 8E01006 & PNNL-AGG-ICP-AES \\
\hline 7439-96-5 & Manganese & $<1.72 \mathrm{E}-2$ & ug/g dry & $1.72 \mathrm{E}-2$ & 2/07/08 & 8E01006 & PNNL-AGG-ICP-AES \\
\hline $7440-02-0$ & Nickel & $<9.36 \mathrm{E}-2$ & ug/g dry & $9.36 \mathrm{E}-2$ & 2/07/08 & 8E01006 & PNNL-AGG-ICP-AES \\
\hline $7440-62-2$ & Vanadium & $<4.54 \mathrm{E}-2$ & ug/g dry & $4.54 \mathrm{E}-2$ & 2/07/08 & 8E01006 & PNNL-AGG-ICP-AES \\
\hline $7440-66-6$ & Zinc & $<9.30 \mathrm{E}-2$ & ug/g dry & $9.30 \mathrm{E}-2$ & 2/07/08 & 8E01006 & PNNL-AGG-ICP-AES \\
\hline $7440-23-5$ & Sodium & $1.01 \mathrm{E} 1$ & ug/g dry & $6.71 \mathrm{E}-1$ & 2/07/08 & 8E01006 & PNNL-AGG-ICP-AES \\
\hline HEIS No. & B1RKP3 & \multicolumn{3}{|c|}{ Lab ID: $\quad$ 0802005-09 } & & & \\
\hline $7429-90-5$ & Aluminum & $3.15 \mathrm{E}-1$ & ug/g dry & $8.58 \mathrm{E}-2$ & 2/07/08 & 8E01006 & PNNL-AGG-ICP-AES \\
\hline $7440-39-3$ & Barium & $1.24 \mathrm{E}-2$ & ug/g dry & 8.79E-3 & 2/07/08 & 8E01006 & PNNL-AGG-ICP-AES \\
\hline
\end{tabular}


Total Metals by PNNL-AGG-ICP-AES/Water Extract

\begin{tabular}{|c|c|c|c|c|c|c|c|}
\hline CAS \# & Analyte & Results & Units & EQL & Analyzed & Batch & Method \\
\hline HEIS No. & B1RKP3 & \multicolumn{3}{|c|}{ Lab ID: $\quad$ 0802005-09 } & & & \\
\hline $7440-70-2$ & Calcium & 7.02E0 & ug/g dry & $3.87 \mathrm{E}-1$ & 2/07/08 & 8E01006 & PNNL-AGG-ICP-AES \\
\hline $7440-48-4$ & Cobalt & $<9.60 \mathrm{E}-2$ & ug/g dry & $9.60 \mathrm{E}-2$ & 2/07/08 & 8E01006 & PNNL-AGG-ICP-AES \\
\hline $7440-47-3$ & Chromium & $<3.33 \mathrm{E}-2$ & ug/g dry & 3.33E-2 & 2/07/08 & 8E01006 & PNNL-AGG-ICP-AES \\
\hline $7440-50-8$ & Copper & $<8.04 \mathrm{E}-2$ & ug/g dry & 8.04E-2 & $2 / 07 / 08$ & 8E01006 & PNNL-AGG-ICP-AES \\
\hline 7440-09-7 & Potassium & 4.85E0 & ug/g dry & 2.33Е0 & 2/07/08 & 8E01006 & PNNL-AGG-ICP-AES \\
\hline 7439-95-4 & Magnesium & $2.20 \mathrm{E} 0$ & ug/g dry & $8.34 \mathrm{E}-2$ & 2/07/08 & 8E01006 & PNNL-AGG-ICP-AES \\
\hline $7439-96-5$ & Manganese & $<1.71 \mathrm{E}-2$ & ug/g dry & $1.71 \mathrm{E}-2$ & $2 / 07 / 08$ & 8E01006 & PNNL-AGG-ICP-AES \\
\hline $7440-02-0$ & Nickel & $<9.33 \mathrm{E}-2$ & ug/g dry & $9.33 \mathrm{E}-2$ & 2/07/08 & 8E01006 & PNNL-AGG-ICP-AES \\
\hline $7440-62-2$ & Vanadium & $<4.53 \mathrm{E}-2$ & ug/g dry & 4.53E-2 & 2/07/08 & 8E01006 & PNNL-AGG-ICP-AES \\
\hline $7440-66-6$ & Zinc & $<9.27 \mathrm{E}-2$ & ug/g dry & $9.27 \mathrm{E}-2$ & $2 / 07 / 08$ & 8E01006 & PNNL-AGG-ICP-AES \\
\hline $7440-23-5$ & Sodium & $1.04 \mathrm{E} 1$ & ug/g dry & $6.69 \mathrm{E}-1$ & 2/07/08 & 8E01006 & PNNL-AGG-ICP-AES \\
\hline HEIS No. & B1RKP9 & \multicolumn{3}{|c|}{ Lab ID: $\quad$ 0802005-15 } & & & \\
\hline $7429-90-5$ & Aluminum & $2.72 \mathrm{E}-1$ & ug/g dry & $8.59 \mathrm{E}-2$ & $2 / 07 / 08$ & 8E01006 & PNNL-AGG-ICP-AES \\
\hline $7440-39-3$ & Barium & $1.49 \mathrm{E}-2$ & ug/g dry & $8.80 \mathrm{E}-3$ & 2/07/08 & 8E01006 & PNNL-AGG-ICP-AES \\
\hline $7440-70-2$ & Calcium & 7.28E0 & ug/g dry & $3.87 \mathrm{E}-1$ & 2/07/08 & 8E01006 & PNNL-AGG-ICP-AES \\
\hline $7440-48-4$ & Cobalt & $<9.61 \mathrm{E}-2$ & ug/g dry & $9.61 \mathrm{E}-2$ & 2/07/08 & 8E01006 & PNNL-AGG-ICP-AES \\
\hline $7440-47-3$ & Chromium & $<3.33 \mathrm{E}-2$ & ug/g dry & 3.33E-2 & 2/07/08 & 8E01006 & PNNL-AGG-ICP-AES \\
\hline $7440-50-8$ & Copper & $<8.05 E-2$ & ug/g dry & 8.05E-2 & $2 / 07 / 08$ & 8E01006 & PNNL-AGG-ICP-AES \\
\hline $7440-09-7$ & Potassium & 3.65E0 & ug/g dry & 2.33Е0 & $2 / 07 / 08$ & 8E01006 & PNNL-AGG-ICP-AES \\
\hline 7439-95-4 & Magnesium & $2.38 \mathrm{E} 0$ & ug/g dry & 8.35E-2 & 2/07/08 & 8E01006 & PNNL-AGG-ICP-AES \\
\hline $7439-96-5$ & Manganese & $<1.72 \mathrm{E}-2$ & ug/g dry & $1.72 \mathrm{E}-2$ & $2 / 07 / 08$ & 8E01006 & PNNL-AGG-ICP-AES \\
\hline $7440-02-0$ & Nickel & $<9.34 \mathrm{E}-2$ & ug/g dry & $9.34 \mathrm{E}-2$ & 2/07/08 & 8E01006 & PNNL-AGG-ICP-AES \\
\hline $7440-62-2$ & Vanadium & $<4.54 \mathrm{E}-2$ & ug/g dry & $4.54 \mathrm{E}-2$ & $2 / 07 / 08$ & 8E01006 & PNNL-AGG-ICP-AES \\
\hline $7440-66-6$ & Zinc & $<9.28 \mathrm{E}-2$ & ug/g dry & $9.28 \mathrm{E}-2$ & 2/07/08 & 8E01006 & PNNL-AGG-ICP-AES \\
\hline $7440-23-5$ & Sodium & $8.20 \mathrm{E} 0$ & ug/g dry & $6.70 \mathrm{E}-1$ & $2 / 07 / 08$ & 8E01006 & PNNL-AGG-ICP-AES \\
\hline HEIS No. & B1RKR5 & \multicolumn{3}{|c|}{ Lab ID: $\quad$ 0802005-21 } & & & \\
\hline $7429-90-5$ & Aluminum & 2.93E-1 & ug/g dry & $8.58 \mathrm{E}-2$ & $2 / 07 / 08$ & 8E01006 & PNNL-AGG-ICP-AES \\
\hline $7440-39-3$ & Barium & $1.55 \mathrm{E}-2$ & ug/g dry & 8.79E-3 & 2/07/08 & 8E01006 & PNNL-AGG-ICP-AES \\
\hline $7440-70-2$ & Calcium & 8.94E0 & ug/g dry & $3.87 \mathrm{E}-1$ & 2/07/08 & 8E01006 & PNNL-AGG-ICP-AES \\
\hline $7440-48-4$ & Cobalt & $<9.60 \mathrm{E}-2$ & ug/g dry & $9.60 \mathrm{E}-2$ & $2 / 07 / 08$ & 8E01006 & PNNL-AGG-ICP-AES \\
\hline $7440-47-3$ & Chromium & $<3.33 \mathrm{E}-2$ & ug/g dry & 3.33E-2 & $2 / 07 / 08$ & 8E01006 & PNNL-AGG-ICP-AES \\
\hline $7440-50-8$ & Copper & $<8.04 \mathrm{E}-2$ & ug/g dry & $8.04 \mathrm{E}-2$ & 2/07/08 & 8E01006 & PNNL-AGG-ICP-AES \\
\hline $7440-09-7$ & Potassium & 5.56E0 & ug/g dry & 2.33E0 & 2/07/08 & 8E01006 & PNNL-AGG-ICP-AES \\
\hline $7439-95-4$ & Magnesium & 2.84E0 & ug/g dry & $8.34 \mathrm{E}-2$ & 2/07/08 & 8E01006 & PNNL-AGG-ICP-AES \\
\hline $7439-96-5$ & Manganese & $<1.71 \mathrm{E}-2$ & ug/g dry & $1.71 \mathrm{E}-2$ & $2 / 07 / 08$ & 8E01006 & PNNL-AGG-ICP-AES \\
\hline $7440-02-0$ & Nickel & $<9.33 \mathrm{E}-2$ & ug/g dry & $9.33 \mathrm{E}-2$ & 2/07/08 & 8E01006 & PNNL-AGG-ICP-AES \\
\hline $7440-62-2$ & Vanadium & $<4.53 \mathrm{E}-2$ & ug/g dry & 4.53E-2 & 2/07/08 & 8E01006 & PNNL-AGG-ICP-AES \\
\hline $7440-66-6$ & Zinc & $<9.27 \mathrm{E}-2$ & ug/g dry & $9.27 \mathrm{E}-2$ & $2 / 07 / 08$ & 8E01006 & PNNL-AGG-ICP-AES \\
\hline $7440-23-5$ & Sodium & 1.31E1 & ug/g dry & $6.69 \mathrm{E}-1$ & $2 / 07 / 08$ & 8E01006 & PNNL-AGG-ICP-AES \\
\hline HEIS No. & B1RKR9 & \multicolumn{3}{|c|}{ Lab ID: $\quad$ 0802005-25 } & & & \\
\hline $7429-90-5$ & Aluminum & $2.52 \mathrm{E}-1$ & ug/g dry & $8.58 \mathrm{E}-2$ & $2 / 07 / 08$ & 8E01006 & PNNL-AGG-ICP-AES \\
\hline $7440-39-3$ & Barium & $1.51 \mathrm{E}-2$ & ug/g dry & 8.79E-3 & $2 / 07 / 08$ & 8E01006 & PNNL-AGG-ICP-AES \\
\hline $7440-70-2$ & Calcium & 1.16E1 & ug/g dry & 3.87E-1 & $2 / 07 / 08$ & 8E01006 & PNNL-AGG-ICP-AES \\
\hline $7440-48-4$ & Cobalt & $<9.60 \mathrm{E}-2$ & ug/g dry & $9.60 \mathrm{E}-2$ & 2/07/08 & 8E01006 & PNNL-AGG-ICP-AES \\
\hline $7440-47-3$ & Chromium & $<3.33 \mathrm{E}-2$ & ug/g dry & 3.33E-2 & 2/07/08 & 8E01006 & PNNL-AGG-ICP-AES \\
\hline $7440-50-8$ & Copper & $<8.04 \mathrm{E}-2$ & ug/g dry & $8.04 \mathrm{E}-2$ & 2/07/08 & 8E01006 & PNNL-AGG-ICP-AES \\
\hline 7440-09-7 & Potassium & 4.55E0 & ug/g dry & 2.33E0 & 2/07/08 & 8E01006 & PNNL-AGG-ICP-AES \\
\hline 7439-95-4 & Magnesium & $3.48 \mathrm{E} 0$ & ug/g dry & 8.34E-2 & 2/07/08 & 8E01006 & PNNL-AGG-ICP-AES \\
\hline
\end{tabular}


Total Metals by PNNL-AGG-ICP-AES/Water Extract

\begin{tabular}{|c|c|c|c|c|c|c|c|}
\hline CAS \# & Analyte & Results & Units & EQL & Analyzed & Batch & Method \\
\hline HEIS No. & B1RKR9 & \multicolumn{3}{|c|}{ Lab ID: $\quad$ 0802005-25 } & & & \\
\hline 7439-96-5 & Manganese & $<1.71 \mathrm{E}-2$ & ug/g dry & $1.71 \mathrm{E}-2$ & 2/07/08 & 8E01006 & PNNL-AGG-ICP-AES \\
\hline $7440-02-0$ & Nickel & $<9.33 \mathrm{E}-2$ & ug/g dry & $9.33 \mathrm{E}-2$ & 2/07/08 & 8E01006 & PNNL-AGG-ICP-AES \\
\hline $7440-62-2$ & Vanadium & $<4.53 \mathrm{E}-2$ & ug/g dry & 4.53E-2 & $2 / 07 / 08$ & 8E01006 & PNNL-AGG-ICP-AES \\
\hline $7440-66-6$ & Zinc & $<9.27 \mathrm{E}-2$ & ug/g dry & $9.27 \mathrm{E}-2$ & $2 / 07 / 08$ & 8E01006 & PNNL-AGG-ICP-AES \\
\hline $7440-23-5$ & Sodium & 1.10E1 & ug/g dry & $6.69 \mathrm{E}-1$ & $2 / 07 / 08$ & 8E01006 & PNNL-AGG-ICP-AES \\
\hline HEIS No. & B1RKT3 & \multicolumn{3}{|c|}{ Lab ID: $\quad$ 0802005-29 } & & & \\
\hline $7429-90-5$ & Aluminum & $2.81 \mathrm{E}-1$ & ug/g dry & $8.58 \mathrm{E}-2$ & $2 / 07 / 08$ & 8E01006 & PNNL-AGG-ICP-AES \\
\hline $7440-39-3$ & Barium & $1.38 \mathrm{E}-2$ & ug/g dry & $8.79 \mathrm{E}-3$ & $2 / 07 / 08$ & 8E01006 & PNNL-AGG-ICP-AES \\
\hline $7440-70-2$ & Calcium & 5.55E0 & ug/g dry & $3.87 \mathrm{E}-1$ & 2/07/08 & 8E01006 & PNNL-AGG-ICP-AES \\
\hline $7440-48-4$ & Cobalt & $<9.60 \mathrm{E}-2$ & ug/g dry & $9.60 \mathrm{E}-2$ & 2/07/08 & 8E01006 & PNNL-AGG-ICP-AES \\
\hline $7440-47-3$ & Chromium & $<3.33 \mathrm{E}-2$ & ug/g dry & 3.33E-2 & $2 / 07 / 08$ & 8E01006 & PNNL-AGG-ICP-AES \\
\hline $7440-50-8$ & Copper & $<8.04 \mathrm{E}-2$ & ug/g dry & 8.04E-2 & $2 / 07 / 08$ & 8E01006 & PNNL-AGG-ICP-AES \\
\hline 7440-09-7 & Potassium & 3.23E0 & ug/g dry & 2.33Е0 & $2 / 07 / 08$ & 8E01006 & PNNL-AGG-ICP-AES \\
\hline $7439-95-4$ & Magnesium & $1.73 \mathrm{E} 0$ & ug/g dry & 8.34E-2 & 2/07/08 & 8E01006 & PNNL-AGG-ICP-AES \\
\hline 7439-96-5 & Manganese & $<1.71 \mathrm{E}-2$ & ug/g dry & $1.71 \mathrm{E}-2$ & $2 / 07 / 08$ & 8E01006 & PNNL-AGG-ICP-AES \\
\hline $7440-02-0$ & Nickel & $<9.33 \mathrm{E}-2$ & ug/g dry & 9.33E-2 & $2 / 07 / 08$ & 8E01006 & PNNL-AGG-ICP-AES \\
\hline $7440-62-2$ & Vanadium & $<4.53 \mathrm{E}-2$ & ug/g dry & 4.53E-2 & 2/07/08 & 8E01006 & PNNL-AGG-ICP-AES \\
\hline $7440-66-6$ & Zinc & $<9.27 \mathrm{E}-2$ & ug/g dry & $9.27 \mathrm{E}-2$ & 2/07/08 & 8E01006 & PNNL-AGG-ICP-AES \\
\hline $7440-23-5$ & Sodium & 7.31E0 & ug/g dry & $6.69 \mathrm{E}-1$ & $2 / 07 / 08$ & 8E01006 & PNNL-AGG-ICP-AES \\
\hline HEIS No. & B1RKT7 & \multicolumn{3}{|c|}{ Lab ID: $\quad$ 0802006-07 } & & & \\
\hline $7429-90-5$ & Aluminum & $2.08 \mathrm{E}-1$ & ug/g dry & $8.59 \mathrm{E}-2$ & $2 / 07 / 08$ & 8E01006 & PNNL-AGG-ICP-AES \\
\hline $7440-39-3$ & Barium & $1.54 \mathrm{E}-2$ & ug/g dry & $8.80 \mathrm{E}-3$ & 2/07/08 & 8E01006 & PNNL-AGG-ICP-AES \\
\hline $7440-70-2$ & Calcium & 1.07E1 & ug/g dry & $3.87 \mathrm{E}-1$ & 2/07/08 & 8E01006 & PNNL-AGG-ICP-AES \\
\hline $7440-48-4$ & Cobalt & $<9.61 \mathrm{E}-2$ & ug/g dry & $9.61 \mathrm{E}-2$ & 2/07/08 & 8E01006 & PNNL-AGG-ICP-AES \\
\hline $7440-47-3$ & Chromium & $<3.33 \mathrm{E}-2$ & ug/g dry & 3.33E-2 & 2/07/08 & 8E01006 & PNNL-AGG-ICP-AES \\
\hline $7440-50-8$ & Copper & $<8.05 \mathrm{E}-2$ & ug/g dry & 8.05E-2 & $2 / 07 / 08$ & 8E01006 & PNNL-AGG-ICP-AES \\
\hline 7440-09-7 & Potassium & 4.23E0 & ug/g dry & 2.33Е0 & $2 / 07 / 08$ & 8E01006 & PNNL-AGG-ICP-AES \\
\hline $7439-95-4$ & Magnesium & 3.34E0 & ug/g dry & 8.35E-2 & $2 / 07 / 08$ & 8E01006 & PNNL-AGG-ICP-AES \\
\hline $7439-96-5$ & Manganese & $<1.71 \mathrm{E}-2$ & ug/g dry & $1.71 \mathrm{E}-2$ & 2/07/08 & 8E01006 & PNNL-AGG-ICP-AES \\
\hline $7440-02-0$ & Nickel & $<9.34 \mathrm{E}-2$ & ug/g dry & $9.34 \mathrm{E}-2$ & $2 / 07 / 08$ & 8E01006 & PNNL-AGG-ICP-AES \\
\hline $7440-62-2$ & Vanadium & $<4.53 \mathrm{E}-2$ & ug/g dry & 4.53E-2 & $2 / 07 / 08$ & 8E01006 & PNNL-AGG-ICP-AES \\
\hline $7440-66-6$ & Zinc & $<9.28 \mathrm{E}-2$ & ug/g dry & $9.28 \mathrm{E}-2$ & 2/07/08 & 8E01006 & PNNL-AGG-ICP-AES \\
\hline $7440-23-5$ & Sodium & $1.05 \mathrm{E} 1$ & ug/g dry & $6.69 \mathrm{E}-1$ & 2/07/08 & 8E01006 & PNNL-AGG-ICP-AES \\
\hline HEIS No. & B1RKV1 & \multicolumn{3}{|c|}{ Lab ID: $\quad$ 0802006-11 } & & & \\
\hline $7429-90-5$ & Aluminum & 3.17E-1 & ug/g dry & $9.56 \mathrm{E}-2$ & $2 / 07 / 08$ & 8E01006 & PNNL-AGG-ICP-AES \\
\hline $7440-39-3$ & Barium & $1.89 \mathrm{E}-2$ & ug/g dry & $9.79 \mathrm{E}-3$ & 2/07/08 & 8E01006 & PNNL-AGG-ICP-AES \\
\hline $7440-70-2$ & Calcium & 1.27E1 & ug/g dry & $4.31 \mathrm{E}-1$ & 2/07/08 & 8E01006 & PNNL-AGG-ICP-AES \\
\hline $7440-48-4$ & Cobalt & $<1.07 \mathrm{E}-1$ & ug/g dry & $1.07 \mathrm{E}-1$ & $2 / 07 / 08$ & 8E01006 & PNNL-AGG-ICP-AES \\
\hline $7440-47-3$ & Chromium & $<3.71 \mathrm{E}-2$ & ug/g dry & $3.71 \mathrm{E}-2$ & $2 / 07 / 08$ & 8E01006 & PNNL-AGG-ICP-AES \\
\hline $7440-50-8$ & Copper & $<8.96 \mathrm{E}-2$ & ug/g dry & 8.96E-2 & $2 / 07 / 08$ & 8E01006 & PNNL-AGG-ICP-AES \\
\hline $7440-09-7$ & Potassium & 6.33Е0 & ug/g dry & 2.59E0 & 2/07/08 & 8E01006 & PNNL-AGG-ICP-AES \\
\hline 7439-95-4 & Magnesium & 3.82E0 & ug/g dry & $9.29 \mathrm{E}-2$ & 2/07/08 & 8E01006 & PNNL-AGG-ICP-AES \\
\hline $7439-96-5$ & Manganese & $<1.91 \mathrm{E}-2$ & ug/g dry & $1.91 \mathrm{E}-2$ & $2 / 07 / 08$ & 8E01006 & PNNL-AGG-ICP-AES \\
\hline $7440-02-0$ & Nickel & $<1.04 \mathrm{E}-1$ & ug/g dry & $1.04 \mathrm{E}-1$ & 2/07/08 & 8E01006 & PNNL-AGG-ICP-AES \\
\hline $7440-62-2$ & Vanadium & $<5.05 \mathrm{E}-2$ & ug/g dry & $5.05 \mathrm{E}-2$ & 2/07/08 & 8E01006 & PNNL-AGG-ICP-AES \\
\hline $7440-66-6$ & Zinc & $<1.03 \mathrm{E}-1$ & ug/g dry & $1.03 \mathrm{E}-1$ & 2/07/08 & 8E01006 & PNNL-AGG-ICP-AES \\
\hline $7440-23-5$ & Sodium & 1.66E1 & ug/g dry & 7.45E-1 & $2 / 07 / 08$ & 8E01006 & PNNL-AGG-ICP-AES \\
\hline HEIS No. & B1RKV5 & $\mathbf{L}$ & b ID: & 2007-04 & & & \\
\hline
\end{tabular}


Total Metals by PNNL-AGG-ICP-AES/Water Extract

\begin{tabular}{|c|c|c|c|c|c|c|c|}
\hline CAS \# & Analyte & Results & Units & EQL & Analyzed & Batch & Method \\
\hline HEIS No. & B1RKV5 & \multicolumn{3}{|c|}{ Lab ID: $\quad$ 0802007-04 } & & & \\
\hline 7429-90-5 & Aluminum & 4.42E-1 & ug/g dry & $8.58 \mathrm{E}-2$ & 2/07/08 & 8E01006 & PNNL-AGG-ICP-AES \\
\hline $7440-39-3$ & Barium & $2.28 \mathrm{E}-2$ & ug/g dry & 8.79E-3 & $2 / 07 / 08$ & 8E01006 & PNNL-AGG-ICP-AES \\
\hline $7440-70-2$ & Calcium & $1.40 \mathrm{E} 1$ & ug/g dry & 3.87E-1 & 2/07/08 & 8E01006 & PNNL-AGG-ICP-AES \\
\hline $7440-48-4$ & Cobalt & $<9.60 \mathrm{E}-2$ & ug/g dry & $9.60 \mathrm{E}-2$ & $2 / 07 / 08$ & 8E01006 & PNNL-AGG-ICP-AES \\
\hline $7440-47-3$ & Chromium & $<3.33 \mathrm{E}-2$ & ug/g dry & $3.33 \mathrm{E}-2$ & 2/07/08 & 8E01006 & PNNL-AGG-ICP-AES \\
\hline $7440-50-8$ & Copper & $<8.04 \mathrm{E}-2$ & ug/g dry & 8.04E-2 & $2 / 07 / 08$ & 8E01006 & PNNL-AGG-ICP-AES \\
\hline 7440-09-7 & Potassium & $5.44 \mathrm{E} 0$ & ug/g dry & 2.33E0 & 2/07/08 & 8E01006 & PNNL-AGG-ICP-AES \\
\hline 7439-95-4 & Magnesium & $3.81 \mathrm{E} 0$ & ug/g dry & $8.34 \mathrm{E}-2$ & 2/07/08 & 8E01006 & PNNL-AGG-ICP-AES \\
\hline 7439-96-5 & Manganese & $<1.71 \mathrm{E}-2$ & ug/g dry & $1.71 \mathrm{E}-2$ & $2 / 07 / 08$ & 8E01006 & PNNL-AGG-ICP-AES \\
\hline $7440-02-0$ & Nickel & $<9.33 \mathrm{E}-2$ & ug/g dry & 9.33E-2 & $2 / 07 / 08$ & 8E01006 & PNNL-AGG-ICP-AES \\
\hline $7440-62-2$ & Vanadium & $<4.53 \mathrm{E}-2$ & ug/g dry & 4.53E-2 & $2 / 07 / 08$ & 8E01006 & PNNL-AGG-ICP-AES \\
\hline $7440-66-6$ & Zinc & $<9.27 \mathrm{E}-2$ & ug/g dry & $9.27 \mathrm{E}-2$ & 2/07/08 & 8E01006 & PNNL-AGG-ICP-AES \\
\hline $7440-23-5$ & Sodium & 1.27E1 & ug/g dry & $6.69 \mathrm{E}-1$ & $2 / 07 / 08$ & 8E01006 & PNNL-AGG-ICP-AES \\
\hline HEIS No. & B1RKV9 & \multicolumn{3}{|c|}{ Lab ID: $\quad$ 0802007-12 } & & & \\
\hline 7429-90-5 & Aluminum & 3.77E-1 & ug/g dry & $8.58 \mathrm{E}-2$ & $2 / 07 / 08$ & 8E01006 & PNNL-AGG-ICP-AES \\
\hline $7440-39-3$ & Barium & $2.89 \mathrm{E}-2$ & ug/g dry & 8.79E-3 & $2 / 07 / 08$ & 8E01006 & PNNL-AGG-ICP-AES \\
\hline $7440-70-2$ & Calcium & 1.37E1 & ug/g dry & $3.87 \mathrm{E}-1$ & 2/07/08 & 8E01006 & PNNL-AGG-ICP-AES \\
\hline $7440-48-4$ & Cobalt & $<9.60 \mathrm{E}-2$ & ug/g dry & $9.60 \mathrm{E}-2$ & 2/07/08 & 8E01006 & PNNL-AGG-ICP-AES \\
\hline $7440-47-3$ & Chromium & $<3.33 \mathrm{E}-2$ & ug/g dry & 3.33E-2 & $2 / 07 / 08$ & 8E01006 & PNNL-AGG-ICP-AES \\
\hline $7440-50-8$ & Copper & $<8.04 \mathrm{E}-2$ & ug/g dry & $8.04 \mathrm{E}-2$ & 2/07/08 & 8E01006 & PNNL-AGG-ICP-AES \\
\hline 7440-09-7 & Potassium & $5.16 \mathrm{E} 0$ & ug/g dry & 2.33E0 & 2/07/08 & 8E01006 & PNNL-AGG-ICP-AES \\
\hline 7439-95-4 & Magnesium & 3.61E0 & ug/g dry & 8.34E-2 & 2/07/08 & 8E01006 & PNNL-AGG-ICP-AES \\
\hline 7439-96-5 & Manganese & $<1.71 \mathrm{E}-2$ & ug/g dry & $1.71 \mathrm{E}-2$ & $2 / 07 / 08$ & 8E01006 & PNNL-AGG-ICP-AES \\
\hline $7440-02-0$ & Nickel & $<9.33 \mathrm{E}-2$ & ug/g dry & $9.33 \mathrm{E}-2$ & 2/07/08 & 8E01006 & PNNL-AGG-ICP-AES \\
\hline $7440-62-2$ & Vanadium & $<4.53 \mathrm{E}-2$ & ug/g dry & 4.53E-2 & $2 / 07 / 08$ & 8E01006 & PNNL-AGG-ICP-AES \\
\hline $7440-66-6$ & Zinc & $<9.27 \mathrm{E}-2$ & ug/g dry & $9.27 \mathrm{E}-2$ & $2 / 07 / 08$ & 8E01006 & PNNL-AGG-ICP-AES \\
\hline $7440-23-5$ & Sodium & 1.43E1 & ug/g dry & $6.69 \mathrm{E}-1$ & 2/07/08 & 8E01006 & PNNL-AGG-ICP-AES \\
\hline HEIS No. & B1RKW2 & \multicolumn{3}{|c|}{ Lab ID: $\quad$ 0802008-04 } & & & \\
\hline $7429-90-5$ & Aluminum & 4.44E-1 & ug/g dry & $8.58 \mathrm{E}-2$ & 2/07/08 & 8E01006 & PNNL-AGG-ICP-AES \\
\hline $7440-39-3$ & Barium & $1.64 \mathrm{E}-2$ & ug/g dry & $8.79 \mathrm{E}-3$ & 2/07/08 & 8E01006 & PNNL-AGG-ICP-AES \\
\hline $7440-70-2$ & Calcium & 2.10E0 & ug/g dry & 3.87E-1 & $2 / 07 / 08$ & 8E01006 & PNNL-AGG-ICP-AES \\
\hline $7440-48-4$ & Cobalt & $<9.60 \mathrm{E}-2$ & ug/g dry & $9.60 \mathrm{E}-2$ & 2/07/08 & 8E01006 & PNNL-AGG-ICP-AES \\
\hline $7440-47-3$ & Chromium & $<3.33 E-2$ & ug/g dry & $3.33 \mathrm{E}-2$ & $2 / 07 / 08$ & 8E01006 & PNNL-AGG-ICP-AES \\
\hline $7440-50-8$ & Copper & $<8.04 \mathrm{E}-2$ & ug/g dry & 8.04E-2 & 2/07/08 & 8E01006 & PNNL-AGG-ICP-AES \\
\hline 7440-09-7 & Potassium & 2.77E0 & ug/g dry & 2.33E0 & 2/07/08 & 8E01006 & PNNL-AGG-ICP-AES \\
\hline 7439-95-4 & Magnesium & $6.75 \mathrm{E}-1$ & ug/g dry & $8.34 \mathrm{E}-2$ & 2/07/08 & 8E01006 & PNNL-AGG-ICP-AES \\
\hline 7439-96-5 & Manganese & $<1.71 \mathrm{E}-2$ & ug/g dry & $1.71 \mathrm{E}-2$ & 2/07/08 & 8E01006 & PNNL-AGG-ICP-AES \\
\hline $7440-02-0$ & Nickel & $<9.33 \mathrm{E}-2$ & ug/g dry & $9.33 \mathrm{E}-2$ & $2 / 07 / 08$ & 8E01006 & PNNL-AGG-ICP-AES \\
\hline $7440-62-2$ & Vanadium & $<4.53 \mathrm{E}-2$ & ug/g dry & $4.53 \mathrm{E}-2$ & 2/07/08 & 8E01006 & PNNL-AGG-ICP-AES \\
\hline $7440-66-6$ & Zinc & $<9.27 \mathrm{E}-2$ & ug/g dry & $9.27 \mathrm{E}-2$ & 2/07/08 & 8E01006 & PNNL-AGG-ICP-AES \\
\hline $7440-23-5$ & Sodium & 1.18E2 & ug/g dry & $6.69 \mathrm{E}-1$ & $2 / 07 / 08$ & 8E01006 & PNNL-AGG-ICP-AES \\
\hline HEIS No. & B1RKW4 & \multicolumn{3}{|c|}{ Lab ID: $\quad$ 0802008-08 } & & & \\
\hline $7429-90-5$ & Aluminum & 3.57E0 & ug/g dry & $8.58 \mathrm{E}-2$ & $2 / 07 / 08$ & 8E01006 & PNNL-AGG-ICP-AES \\
\hline $7440-39-3$ & Barium & $9.20 \mathrm{E}-2$ & ug/g dry & $8.79 \mathrm{E}-3$ & 2/07/08 & 8E01006 & PNNL-AGG-ICP-AES \\
\hline $7440-70-2$ & Calcium & $2.25 \mathrm{E} 0$ & ug/g dry & 3.87E-1 & 2/07/08 & 8E01006 & PNNL-AGG-ICP-AES \\
\hline $7440-48-4$ & Cobalt & $<9.60 \mathrm{E}-2$ & ug/g dry & $9.60 \mathrm{E}-2$ & 2/07/08 & 8E01006 & PNNL-AGG-ICP-AES \\
\hline $7440-47-3$ & Chromium & 7.07E-2 & ug/g dry & $3.33 E-2$ & 2/07/08 & 8E01006 & PNNL-AGG-ICP-AES \\
\hline $7440-50-8$ & Copper & $<8.04 \mathrm{E}-2$ & ug/g dry & $8.04 \mathrm{E}-2$ & 2/07/08 & 8E01006 & PNNL-AGG-ICP-AES \\
\hline
\end{tabular}


Total Metals by PNNL-AGG-ICP-AES/Water Extract

\begin{tabular}{|c|c|c|c|c|c|c|c|}
\hline CAS \# & Analyte & Results & Units & EQL & Analyzed & Batch & Method \\
\hline HEIS No. & B1RKW4 & \multicolumn{3}{|c|}{ Lab ID: $\quad$ 0802008-08 } & & & \\
\hline 7440-09-7 & Potassium & 2.73E0 & ug/g dry & $2.33 \mathrm{E} 0$ & 2/07/08 & 8E01006 & PNNL-AGG-ICP-AES \\
\hline 7439-95-4 & Magnesium & 7.23E-1 & ug/g dry & 8.34E-2 & 2/07/08 & 8E01006 & PNNL-AGG-ICP-AES \\
\hline 7439-96-5 & Manganese & $1.10 \mathrm{E}-1$ & ug/g dry & $1.71 \mathrm{E}-2$ & 2/07/08 & 8E01006 & PNNL-AGG-ICP-AES \\
\hline $7440-02-0$ & Nickel & $<9.33 \mathrm{E}-2$ & ug/g dry & $9.33 \mathrm{E}-2$ & $2 / 07 / 08$ & 8E01006 & PNNL-AGG-ICP-AES \\
\hline $7440-62-2$ & Vanadium & $1.36 \mathrm{E}-1$ & ug/g dry & 4.53E-2 & 2/07/08 & 8E01006 & PNNL-AGG-ICP-AES \\
\hline $7440-66-6$ & Zinc & $<9.27 \mathrm{E}-2$ & ug/g dry & $9.27 \mathrm{E}-2$ & 2/07/08 & 8E01006 & PNNL-AGG-ICP-AES \\
\hline $7440-23-5$ & Sodium & 3.64E2 & ug/g dry & $6.69 \mathrm{E}-1$ & 2/07/08 & 8E01006 & PNNL-AGG-ICP-AES \\
\hline HEIS No. & B1RKW5 & \multicolumn{3}{|c|}{ Lab ID: $\quad$ 0802009-02 } & & & \\
\hline 7429-90-5 & Aluminum & $3.28 \mathrm{E}-1$ & ug/g dry & $8.58 \mathrm{E}-2$ & $2 / 07 / 08$ & 8E01006 & PNNL-AGG-ICP-AES \\
\hline $7440-39-3$ & Barium & $1.50 \mathrm{E}-2$ & ug/g dry & 8.79E-3 & $2 / 07 / 08$ & 8E01006 & PNNL-AGG-ICP-AES \\
\hline $7440-70-2$ & Calcium & 1.05E0 & ug/g dry & 3.87E-1 & $2 / 07 / 08$ & 8E01006 & PNNL-AGG-ICP-AES \\
\hline $7440-48-4$ & Cobalt & $<9.60 \mathrm{E}-2$ & ug/g dry & $9.60 \mathrm{E}-2$ & 2/07/08 & 8E01006 & PNNL-AGG-ICP-AES \\
\hline $7440-47-3$ & Chromium & $<3.33 E-2$ & ug/g dry & $3.33 \mathrm{E}-2$ & 2/07/08 & 8E01006 & PNNL-AGG-ICP-AES \\
\hline $7440-50-8$ & Copper & $<8.04 \mathrm{E}-2$ & ug/g dry & 8.04E-2 & 2/07/08 & 8E01006 & PNNL-AGG-ICP-AES \\
\hline 7440-09-7 & Potassium & 2.59E0 & ug/g dry & 2.33E0 & $2 / 07 / 08$ & 8E01006 & PNNL-AGG-ICP-AES \\
\hline 7439-95-4 & Magnesium & $5.46 \mathrm{E}-1$ & ug/g dry & $8.34 \mathrm{E}-2$ & 2/07/08 & 8E01006 & PNNL-AGG-ICP-AES \\
\hline 7439-96-5 & Manganese & $<1.71 \mathrm{E}-2$ & ug/g dry & $1.71 \mathrm{E}-2$ & 2/07/08 & 8E01006 & PNNL-AGG-ICP-AES \\
\hline $7440-02-0$ & Nickel & $<9.33 \mathrm{E}-2$ & ug/g dry & $9.33 \mathrm{E}-2$ & 2/07/08 & 8E01006 & PNNL-AGG-ICP-AES \\
\hline $7440-62-2$ & Vanadium & $<4.53 \mathrm{E}-2$ & ug/g dry & 4.53E-2 & $2 / 07 / 08$ & 8E01006 & PNNL-AGG-ICP-AES \\
\hline $7440-66-6$ & Zinc & $<9.27 \mathrm{E}-2$ & ug/g dry & $9.27 \mathrm{E}-2$ & 2/07/08 & 8E01006 & PNNL-AGG-ICP-AES \\
\hline $7440-23-5$ & Sodium & 1.47E2 & ug/g dry & $6.69 \mathrm{E}-1$ & $2 / 07 / 08$ & 8E01006 & PNNL-AGG-ICP-AES \\
\hline HEIS No. & B1RKW6 & \multicolumn{3}{|c|}{ Lab ID: $\quad$ 0802009-04 } & & & \\
\hline $7429-90-5$ & Aluminum & $5.11 \mathrm{E}-1$ & ug/g dry & $8.58 \mathrm{E}-2$ & 2/07/08 & 8E01006 & PNNL-AGG-ICP-AES \\
\hline $7440-39-3$ & Barium & $2.48 \mathrm{E}-2$ & ug/g dry & $8.79 \mathrm{E}-3$ & $2 / 07 / 08$ & 8E01006 & PNNL-AGG-ICP-AES \\
\hline $7440-70-2$ & Calcium & $1.97 \mathrm{E} 0$ & ug/g dry & 3.87E-1 & 2/07/08 & 8E01006 & PNNL-AGG-ICP-AES \\
\hline $7440-48-4$ & Cobalt & $<9.60 \mathrm{E}-2$ & ug/g dry & $9.60 \mathrm{E}-2$ & $2 / 07 / 08$ & 8E01006 & PNNL-AGG-ICP-AES \\
\hline $7440-47-3$ & Chromium & $<3.33 \mathrm{E}-2$ & ug/g dry & 3.33E-2 & $2 / 07 / 08$ & 8E01006 & PNNL-AGG-ICP-AES \\
\hline $7440-50-8$ & Copper & $<8.04 \mathrm{E}-2$ & ug/g dry & $8.04 \mathrm{E}-2$ & 2/07/08 & 8E01006 & PNNL-AGG-ICP-AES \\
\hline 7440-09-7 & Potassium & 2.81E0 & ug/g dry & 2.33E0 & 2/07/08 & 8E01006 & PNNL-AGG-ICP-AES \\
\hline 7439-95-4 & Magnesium & $8.67 \mathrm{E}-1$ & ug/g dry & 8.34E-2 & $2 / 07 / 08$ & 8E01006 & PNNL-AGG-ICP-AES \\
\hline 7439-96-5 & Manganese & $3.41 \mathrm{E}-2$ & ug/g dry & $1.71 \mathrm{E}-2$ & $2 / 07 / 08$ & 8E01006 & PNNL-AGG-ICP-AES \\
\hline $7440-02-0$ & Nickel & $<9.33 \mathrm{E}-2$ & ug/g dry & 9.33E-2 & 2/07/08 & 8E01006 & PNNL-AGG-ICP-AES \\
\hline $7440-62-2$ & Vanadium & $<4.53 \mathrm{E}-2$ & ug/g dry & 4.53E-2 & $2 / 07 / 08$ & 8E01006 & PNNL-AGG-ICP-AES \\
\hline $7440-66-6$ & Zinc & $9.31 \mathrm{E}-2$ & ug/g dry & $9.27 \mathrm{E}-2$ & $2 / 07 / 08$ & 8E01006 & PNNL-AGG-ICP-AES \\
\hline $7440-23-5$ & Sodium & $2.32 \mathrm{E} 2$ & ug/g dry & $6.69 \mathrm{E}-1$ & 2/07/08 & 8E01006 & PNNL-AGG-ICP-AES \\
\hline HEIS No. & B1TFV6 & \multicolumn{3}{|c|}{ Lab ID: $\quad$ 0802028-03 } & & & \\
\hline 7429-90-5 & Aluminum & 7.02E-1 & ug/g dry & 1.43E-1 & $5 / 01 / 08$ & 8D28003 & PNNL-AGG-ICP-AES \\
\hline $7440-39-3$ & Barium & $4.21 \mathrm{E}-2$ & ug/g dry & $1.47 \mathrm{E}-2$ & $5 / 01 / 08$ & 8D28003 & PNNL-AGG-ICP-AES \\
\hline $7440-70-2$ & Calcium & 1.69E0 & ug/g dry & $6.47 \mathrm{E}-1$ & $5 / 01 / 08$ & 8D28003 & PNNL-AGG-ICP-AES \\
\hline $7440-48-4$ & Cobalt & $<1.61 \mathrm{E}-1$ & ug/g dry & $1.61 \mathrm{E}-1$ & $5 / 01 / 08$ & 8D28003 & PNNL-AGG-ICP-AES \\
\hline $7440-47-3$ & Chromium & $<5.57 \mathrm{E}-2$ & ug/g dry & 5.57E-2 & $5 / 01 / 08$ & 8D28003 & PNNL-AGG-ICP-AES \\
\hline $7440-50-8$ & Copper & $<1.34 \mathrm{E}-1$ & ug/g dry & $1.34 \mathrm{E}-1$ & $5 / 01 / 08$ & 8D28003 & PNNL-AGG-ICP-AES \\
\hline $7440-09-7$ & Potassium & $<3.89 \mathrm{E} 0$ & ug/g dry & 3.89E0 & $5 / 01 / 08$ & 8D28003 & PNNL-AGG-ICP-AES \\
\hline 7439-95-4 & Magnesium & 7.11E-1 & ug/g dry & $1.39 \mathrm{E}-1$ & $5 / 01 / 08$ & 8D28003 & PNNL-AGG-ICP-AES \\
\hline 7439-96-5 & Manganese & $<2.86 \mathrm{E}-2$ & ug/g dry & $2.86 \mathrm{E}-2$ & $5 / 01 / 08$ & 8D28003 & PNNL-AGG-ICP-AES \\
\hline $7440-02-0$ & Nickel & $<1.56 \mathrm{E}-1$ & ug/g dry & $1.56 \mathrm{E}-1$ & $5 / 01 / 08$ & 8D28003 & PNNL-AGG-ICP-AES \\
\hline $7440-62-2$ & Vanadium & $<7.58 \mathrm{E}-2$ & ug/g dry & $7.58 \mathrm{E}-2$ & $5 / 01 / 08$ & 8D28003 & PNNL-AGG-ICP-AES \\
\hline $7440-66-6$ & Zinc & 4.03E-1 & ug/g dry & $1.55 \mathrm{E}-1$ & $5 / 01 / 08$ & 8D28003 & PNNL-AGG-ICP-AES \\
\hline
\end{tabular}


Total Metals by PNNL-AGG-ICP-AES/Water Extract

\begin{tabular}{|c|c|c|c|c|c|c|c|}
\hline CAS \# & Analyte & Results & Units & EQL & Analyzed & Batch & Method \\
\hline HEIS No. & B1TFV6 & \multicolumn{3}{|c|}{ Lab ID: $\quad$ 0802028-03 } & & & \\
\hline $7440-23-5$ & Sodium & 1.37E2 & ug/g dry & $1.12 \mathrm{E} 0$ & $5 / 01 / 08$ & 8D28003 & PNNL-AGG-ICP-AES \\
\hline HEIS No. & B1TFV8 & \multicolumn{3}{|c|}{ Lab ID: $\quad$ 0802028-05 } & & & \\
\hline 7429-90-5 & Aluminum & $5.34 \mathrm{E}-1$ & ug/g dry & $8.55 \mathrm{E}-2$ & $5 / 01 / 08$ & 8D28003 & PNNL-AGG-ICP-AES \\
\hline $7440-39-3$ & Barium & $2.02 \mathrm{E}-2$ & ug/g dry & 8.76E-3 & $5 / 01 / 08$ & 8D28003 & PNNL-AGG-ICP-AES \\
\hline $7440-70-2$ & Calcium & 7.14E-1 & ug/g dry & 3.86E-1 & $5 / 01 / 08$ & 8D28003 & PNNL-AGG-ICP-AES \\
\hline $7440-48-4$ & Cobalt & $<9.57 \mathrm{E}-2$ & ug/g dry & $9.57 \mathrm{E}-2$ & $5 / 01 / 08$ & 8D28003 & PNNL-AGG-ICP-AES \\
\hline $7440-47-3$ & Chromium & $<3.32 \mathrm{E}-2$ & ug/g dry & 3.32E-2 & $5 / 01 / 08$ & 8D28003 & PNNL-AGG-ICP-AES \\
\hline $7440-50-8$ & Copper & $<8.01 \mathrm{E}-2$ & ug/g dry & 8.01E-2 & $5 / 01 / 08$ & 8D28003 & PNNL-AGG-ICP-AES \\
\hline 7440-09-7 & Potassium & $<2.32 \mathrm{E} 0$ & ug/g dry & $2.32 \mathrm{E} 0$ & $5 / 01 / 08$ & 8D28003 & PNNL-AGG-ICP-AES \\
\hline 7439-95-4 & Magnesium & 3.59E-1 & ug/g dry & 8.31E-2 & $5 / 01 / 08$ & 8D28003 & PNNL-AGG-ICP-AES \\
\hline 7439-96-5 & Manganese & $<1.71 \mathrm{E}-2$ & ug/g dry & $1.71 \mathrm{E}-2$ & $5 / 01 / 08$ & 8D28003 & PNNL-AGG-ICP-AES \\
\hline $7440-02-0$ & Nickel & $<9.30 \mathrm{E}-2$ & ug/g dry & $9.30 \mathrm{E}-2$ & $5 / 01 / 08$ & 8D28003 & PNNL-AGG-ICP-AES \\
\hline $7440-62-2$ & Vanadium & 7.49E-2 & ug/g dry & $4.52 \mathrm{E}-2$ & $5 / 01 / 08$ & 8D28003 & PNNL-AGG-ICP-AES \\
\hline $7440-66-6$ & Zinc & $<9.24 \mathrm{E}-2$ & ug/g dry & $9.24 \mathrm{E}-2$ & $5 / 01 / 08$ & 8D28003 & PNNL-AGG-ICP-AES \\
\hline $7440-23-5$ & Sodium & 2.12E2 & ug/g dry & $6.67 \mathrm{E}-1$ & $5 / 01 / 08$ & 8D28003 & PNNL-AGG-ICP-AES \\
\hline HEIS No. & B1TFW0 & \multicolumn{3}{|c|}{$\begin{array}{ll}\text { Lab ID: } & \text { 0802028-07 }\end{array}$} & & & \\
\hline $7429-90-5$ & Aluminum & $<8.59 \mathrm{E}-2$ & ug/g dry & $8.59 \mathrm{E}-2$ & $5 / 01 / 08$ & 8D28003 & PNNL-AGG-ICP-AES \\
\hline $7440-39-3$ & Barium & $6.95 \mathrm{E}-2$ & ug/g dry & $8.80 \mathrm{E}-3$ & $5 / 01 / 08$ & 8D28003 & PNNL-AGG-ICP-AES \\
\hline $7440-70-2$ & Calcium & $4.05 \mathrm{E} 1$ & ug/g dry & 3.88E-1 & $5 / 01 / 08$ & 8D28003 & PNNL-AGG-ICP-AES \\
\hline $7440-48-4$ & Cobalt & $<9.61 \mathrm{E}-2$ & ug/g dry & $9.61 \mathrm{E}-2$ & $5 / 01 / 08$ & 8D28003 & PNNL-AGG-ICP-AES \\
\hline $7440-47-3$ & Chromium & $<3.33 \mathrm{E}-2$ & ug/g dry & 3.33E-2 & $5 / 01 / 08$ & 8D28003 & PNNL-AGG-ICP-AES \\
\hline $7440-50-8$ & Copper & $<8.05 E-2$ & ug/g dry & 8.05E-2 & $5 / 01 / 08$ & 8D28003 & PNNL-AGG-ICP-AES \\
\hline 7440-09-7 & Potassium & 8.23E0 & ug/g dry & 2.33Е0 & $5 / 01 / 08$ & 8D28003 & PNNL-AGG-ICP-AES \\
\hline 7439-95-4 & Magnesium & $1.72 \mathrm{E} 1$ & ug/g dry & 8.35E-2 & $5 / 01 / 08$ & 8D28003 & PNNL-AGG-ICP-AES \\
\hline $7439-96-5$ & Manganese & $<1.72 \mathrm{E}-2$ & ug/g dry & $1.72 \mathrm{E}-2$ & $5 / 01 / 08$ & 8D28003 & PNNL-AGG-ICP-AES \\
\hline $7440-02-0$ & Nickel & $<9.34 \mathrm{E}-2$ & ug/g dry & $9.34 \mathrm{E}-2$ & $5 / 01 / 08$ & 8D28003 & PNNL-AGG-ICP-AES \\
\hline $7440-62-2$ & Vanadium & $<4.54 \mathrm{E}-2$ & ug/g dry & $4.54 \mathrm{E}-2$ & $5 / 01 / 08$ & 8D28003 & PNNL-AGG-ICP-AES \\
\hline $7440-66-6$ & Zinc & $<9.28 \mathrm{E}-2$ & ug/g dry & $9.28 \mathrm{E}-2$ & $5 / 01 / 08$ & 8D28003 & PNNL-AGG-ICP-AES \\
\hline $7440-23-5$ & Sodium & 8.82E1 & ug/g dry & $6.70 \mathrm{E}-1$ & $5 / 01 / 08$ & 8D28003 & PNNL-AGG-ICP-AES \\
\hline HEIS No. & B1TFW2 & \multicolumn{3}{|c|}{ Lab ID: $\quad$ 0802028-09 } & & & \\
\hline 7429-90-5 & Aluminum & $<8.61 \mathrm{E}-2$ & ug/g dry & $8.61 \mathrm{E}-2$ & $5 / 01 / 08$ & 8D28003 & PNNL-AGG-ICP-AES \\
\hline $7440-39-3$ & Barium & 4.36E-2 & ug/g dry & $8.82 \mathrm{E}-3$ & $5 / 01 / 08$ & 8D28003 & PNNL-AGG-ICP-AES \\
\hline $7440-70-2$ & Calcium & 1.49E2 & ug/g dry & $3.89 \mathrm{E}-1$ & $5 / 01 / 08$ & 8D28003 & PNNL-AGG-ICP-AES \\
\hline $7440-48-4$ & Cobalt & $<9.64 \mathrm{E}-2$ & ug/g dry & $9.64 \mathrm{E}-2$ & $5 / 01 / 08$ & 8D28003 & PNNL-AGG-ICP-AES \\
\hline $7440-47-3$ & Chromium & $<3.34 \mathrm{E}-2$ & ug/g dry & $3.34 \mathrm{E}-2$ & $5 / 01 / 08$ & 8D28003 & PNNL-AGG-ICP-AES \\
\hline $7440-50-8$ & Copper & $<8.07 \mathrm{E}-2$ & ug/g dry & 8.07E-2 & $5 / 01 / 08$ & 8D28003 & PNNL-AGG-ICP-AES \\
\hline $7440-09-7$ & Potassium & $1.30 \mathrm{E} 1$ & ug/g dry & 2.34E0 & $5 / 01 / 08$ & 8D28003 & PNNL-AGG-ICP-AES \\
\hline 7439-95-4 & Magnesium & 6.99E1 & ug/g dry & 8.37E-2 & $5 / 01 / 08$ & 8D28003 & PNNL-AGG-ICP-AES \\
\hline 7439-96-5 & Manganese & $<1.72 \mathrm{E}-2$ & ug/g dry & $1.72 \mathrm{E}-2$ & $5 / 01 / 08$ & 8D28003 & PNNL-AGG-ICP-AES \\
\hline $7440-02-0$ & Nickel & $<9.37 \mathrm{E}-2$ & ug/g dry & $9.37 \mathrm{E}-2$ & $5 / 01 / 08$ & 8D28003 & PNNL-AGG-ICP-AES \\
\hline $7440-62-2$ & Vanadium & $<4.55 \mathrm{E}-2$ & ug/g dry & 4.55E-2 & $5 / 01 / 08$ & 8D28003 & PNNL-AGG-ICP-AES \\
\hline $7440-66-6$ & Zinc & $<9.31 \mathrm{E}-2$ & ug/g dry & $9.31 \mathrm{E}-2$ & $5 / 01 / 08$ & 8D28003 & PNNL-AGG-ICP-AES \\
\hline $7440-23-5$ & Sodium & 1.35E2 & ug/g dry & $6.72 \mathrm{E}-1$ & $5 / 01 / 08$ & 8D28003 & PNNL-AGG-ICP-AES \\
\hline HEIS No. & B1RKX0 & \multicolumn{3}{|c|}{$\begin{array}{ll}\text { Lab ID: } & \text { 0802028-11 }\end{array}$} & & & \\
\hline $7429-90-5$ & Aluminum & $<8.57 \mathrm{E}-2$ & ug/g dry & $8.57 \mathrm{E}-2$ & $5 / 01 / 08$ & 8D28003 & PNNL-AGG-ICP-AES \\
\hline $7440-39-3$ & Barium & $3.36 \mathrm{E}-2$ & ug/g dry & 8.78E-3 & $5 / 01 / 08$ & 8D28003 & PNNL-AGG-ICP-AES \\
\hline $7440-70-2$ & Calcium & 1.32E1 & ug/g dry & $3.86 \mathrm{E}-1$ & $5 / 01 / 08$ & 8D28003 & PNNL-AGG-ICP-AES \\
\hline $7440-48-4$ & Cobalt & $<9.58 \mathrm{E}-2$ & ug/g dry & $9.58 \mathrm{E}-2$ & $5 / 01 / 08$ & 8D28003 & PNNL-AGG-ICP-AES \\
\hline
\end{tabular}


Total Metals by PNNL-AGG-ICP-AES/Water Extract

\begin{tabular}{|c|c|c|c|c|c|c|c|}
\hline CAS \# & Analyte & Results & Units & EQL & Analyzed & Batch & Method \\
\hline HEIS No. & B1RKX0 & \multicolumn{3}{|c|}{ Lab ID: $\quad$ 0802028-11 } & & & \\
\hline $7440-47-3$ & Chromium & $<3.32 \mathrm{E}-2$ & ug/g dry & $3.32 \mathrm{E}-2$ & $5 / 01 / 08$ & 8D28003 & PNNL-AGG-ICP-AES \\
\hline $7440-50-8$ & Copper & $<8.03 E-2$ & ug/g dry & 8.03E-2 & $5 / 01 / 08$ & 8D28003 & PNNL-AGG-ICP-AES \\
\hline 7440-09-7 & Potassium & 6.17E0 & ug/g dry & 2.32E0 & $5 / 01 / 08$ & 8D28003 & PNNL-AGG-ICP-AES \\
\hline 7439-95-4 & Magnesium & $7.55 \mathrm{E} 0$ & ug/g dry & 8.33E-2 & $5 / 01 / 08$ & 8D28003 & PNNL-AGG-ICP-AES \\
\hline 7439-96-5 & Manganese & $<1.71 \mathrm{E}-2$ & ug/g dry & $1.71 \mathrm{E}-2$ & $5 / 01 / 08$ & 8D28003 & PNNL-AGG-ICP-AES \\
\hline 7440-02-0 & Nickel & $<9.31 \mathrm{E}-2$ & ug/g dry & $9.31 \mathrm{E}-2$ & $5 / 01 / 08$ & 8D28003 & PNNL-AGG-ICP-AES \\
\hline $7440-62-2$ & Vanadium & $<4.52 \mathrm{E}-2$ & ug/g dry & $4.52 \mathrm{E}-2$ & $5 / 01 / 08$ & 8D28003 & PNNL-AGG-ICP-AES \\
\hline $7440-66-6$ & Zinc & $<9.25 \mathrm{E}-2$ & ug/g dry & $9.25 \mathrm{E}-2$ & $5 / 01 / 08$ & 8D28003 & PNNL-AGG-ICP-AES \\
\hline $7440-23-5$ & Sodium & 2.32E2 & ug/g dry & $6.68 \mathrm{E}-1$ & $5 / 01 / 08$ & 8D28003 & PNNL-AGG-ICP-AES \\
\hline HEIS No. & B1RKX3 & \multicolumn{3}{|c|}{ Lab ID: $\quad$ 0802028-17 } & & & \\
\hline 7429-90-5 & Aluminum & $1.02 \mathrm{E} 0$ & ug/g dry & 8.58E-2 & $5 / 01 / 08$ & 8D28003 & PNNL-AGG-ICP-AES \\
\hline $7440-39-3$ & Barium & $3.62 \mathrm{E}-2$ & ug/g dry & $8.79 \mathrm{E}-3$ & $5 / 01 / 08$ & 8D28003 & PNNL-AGG-ICP-AES \\
\hline $7440-70-2$ & Calcium & $1.19 \mathrm{E} 0$ & ug/g dry & $3.87 \mathrm{E}-1$ & $5 / 01 / 08$ & 8D28003 & PNNL-AGG-ICP-AES \\
\hline $7440-48-4$ & Cobalt & $<9.60 \mathrm{E}-2$ & ug/g dry & $9.60 \mathrm{E}-2$ & $5 / 01 / 08$ & 8D28003 & PNNL-AGG-ICP-AES \\
\hline $7440-47-3$ & Chromium & 1.22E-1 & ug/g dry & 3.33E-2 & $5 / 01 / 08$ & 8D28003 & PNNL-AGG-ICP-AES \\
\hline $7440-50-8$ & Copper & $<8.04 \mathrm{E}-2$ & ug/g dry & $8.04 \mathrm{E}-2$ & $5 / 01 / 08$ & 8D28003 & PNNL-AGG-ICP-AES \\
\hline 7440-09-7 & Potassium & 2.66E0 & ug/g dry & 2.33E0 & $5 / 01 / 08$ & 8D28003 & PNNL-AGG-ICP-AES \\
\hline 7439-95-4 & Magnesium & $7.51 \mathrm{E}-1$ & ug/g dry & $8.34 \mathrm{E}-2$ & $5 / 01 / 08$ & 8D28003 & PNNL-AGG-ICP-AES \\
\hline $7439-96-5$ & Manganese & 1.80E-2 & ug/g dry & $1.71 \mathrm{E}-2$ & $5 / 01 / 08$ & 8D28003 & PNNL-AGG-ICP-AES \\
\hline $7440-02-0$ & Nickel & $<9.33 \mathrm{E}-2$ & ug/g dry & $9.33 \mathrm{E}-2$ & $5 / 01 / 08$ & 8D28003 & PNNL-AGG-ICP-AES \\
\hline $7440-62-2$ & Vanadium & 6.93E-2 & ug/g dry & 4.53E-2 & $5 / 01 / 08$ & 8D28003 & PNNL-AGG-ICP-AES \\
\hline $7440-66-6$ & Zinc & $<9.27 \mathrm{E}-2$ & ug/g dry & $9.27 \mathrm{E}-2$ & $5 / 01 / 08$ & 8D28003 & PNNL-AGG-ICP-AES \\
\hline $7440-23-5$ & Sodium & 8.33E1 & ug/g dry & $6.69 \mathrm{E}-1$ & $5 / 01 / 08$ & 8D28003 & PNNL-AGG-ICP-AES \\
\hline HEIS No. & B1RKX5 & \multicolumn{3}{|c|}{ Lab ID: $\quad$ 0802028-23 } & & & \\
\hline 7429-90-5 & Aluminum & $1.02 \mathrm{E} 0$ & ug/g dry & $8.60 \mathrm{E}-2$ & $5 / 01 / 08$ & 8D28003 & PNNL-AGG-ICP-AES \\
\hline $7440-39-3$ & Barium & $3.89 \mathrm{E}-2$ & ug/g dry & $8.81 \mathrm{E}-3$ & $5 / 01 / 08$ & 8D28003 & PNNL-AGG-ICP-AES \\
\hline $7440-70-2$ & Calcium & $1.45 \mathrm{E} 0$ & ug/g dry & $3.88 \mathrm{E}-1$ & $5 / 01 / 08$ & 8D28003 & PNNL-AGG-ICP-AES \\
\hline $7440-48-4$ & Cobalt & $<9.62 \mathrm{E}-2$ & ug/g dry & $9.62 \mathrm{E}-2$ & $5 / 01 / 08$ & 8D28003 & PNNL-AGG-ICP-AES \\
\hline $7440-47-3$ & Chromium & $5.00 \mathrm{E}-2$ & ug/g dry & $3.34 \mathrm{E}-2$ & $5 / 01 / 08$ & 8D28003 & PNNL-AGG-ICP-AES \\
\hline $7440-50-8$ & Copper & $<8.06 \mathrm{E}-2$ & ug/g dry & 8.06E-2 & $5 / 01 / 08$ & 8D28003 & PNNL-AGG-ICP-AES \\
\hline $7440-09-7$ & Potassium & 2.68E0 & ug/g dry & 2.33E0 & $5 / 01 / 08$ & 8D28003 & PNNL-AGG-ICP-AES \\
\hline 7439-95-4 & Magnesium & $8.12 \mathrm{E}-1$ & ug/g dry & 8.36E-2 & $5 / 01 / 08$ & 8D28003 & PNNL-AGG-ICP-AES \\
\hline 7439-96-5 & Manganese & 1.73E-2 & ug/g dry & $1.72 \mathrm{E}-2$ & $5 / 01 / 08$ & 8D28003 & PNNL-AGG-ICP-AES \\
\hline $7440-02-0$ & Nickel & $<9.35 \mathrm{E}-2$ & ug/g dry & $9.35 \mathrm{E}-2$ & $5 / 01 / 08$ & 8D28003 & PNNL-AGG-ICP-AES \\
\hline $7440-62-2$ & Vanadium & 6.66E-2 & ug/g dry & $4.54 \mathrm{E}-2$ & $5 / 01 / 08$ & 8D28003 & PNNL-AGG-ICP-AES \\
\hline $7440-66-6$ & Zinc & $<9.29 \mathrm{E}-2$ & ug/g dry & $9.29 \mathrm{E}-2$ & $5 / 01 / 08$ & 8D28003 & PNNL-AGG-ICP-AES \\
\hline $7440-23-5$ & Sodium & 6.77E1 & ug/g dry & $6.71 \mathrm{E}-1$ & $5 / 01 / 08$ & 8D28003 & PNNL-AGG-ICP-AES \\
\hline HEIS No. & B1RKX7 & \multicolumn{3}{|c|}{ Lab ID: $\quad$ 0802028-29 } & & & \\
\hline $7429-90-5$ & Aluminum & $1.91 \mathrm{E}-1$ & ug/g dry & $8.58 \mathrm{E}-2$ & $5 / 01 / 08$ & 8D28003 & PNNL-AGG-ICP-AES \\
\hline $7440-39-3$ & Barium & 3.05E-2 & ug/g dry & 8.79E-3 & $5 / 01 / 08$ & 8D28003 & PNNL-AGG-ICP-AES \\
\hline $7440-70-2$ & Calcium & 8.84E0 & ug/g dry & 3.87E-1 & $5 / 01 / 08$ & 8D28003 & PNNL-AGG-ICP-AES \\
\hline $7440-48-4$ & Cobalt & $<9.60 \mathrm{E}-2$ & ug/g dry & $9.60 \mathrm{E}-2$ & $5 / 01 / 08$ & 8D28003 & PNNL-AGG-ICP-AES \\
\hline $7440-47-3$ & Chromium & $<3.33 \mathrm{E}-2$ & ug/g dry & 3.33E-2 & $5 / 01 / 08$ & 8D28003 & PNNL-AGG-ICP-AES \\
\hline $7440-50-8$ & Copper & $<8.04 \mathrm{E}-2$ & ug/g dry & 8.04E-2 & $5 / 01 / 08$ & 8D28003 & PNNL-AGG-ICP-AES \\
\hline $7440-09-7$ & Potassium & 4.22E0 & ug/g dry & 2.33E0 & $5 / 01 / 08$ & 8D28003 & PNNL-AGG-ICP-AES \\
\hline 7439-95-4 & Magnesium & 2.55E0 & ug/g dry & 8.34E-2 & $5 / 01 / 08$ & 8D28003 & PNNL-AGG-ICP-AES \\
\hline 7439-96-5 & Manganese & $<1.71 \mathrm{E}-2$ & ug/g dry & $1.71 \mathrm{E}-2$ & $5 / 01 / 08$ & 8D28003 & PNNL-AGG-ICP-AES \\
\hline $7440-02-0$ & Nickel & $<9.33 \mathrm{E}-2$ & ug/g dry & $9.33 \mathrm{E}-2$ & $5 / 01 / 08$ & 8D28003 & PNNL-AGG-ICP-AES \\
\hline
\end{tabular}


Total Metals by PNNL-AGG-ICP-AES/Water Extract

\begin{tabular}{|c|c|c|c|c|c|c|c|}
\hline CAS \# & Analyte & Results & Units & EQL & Analyzed & Batch & Method \\
\hline HEIS No. & B1RKX7 & & b ID: & $2028-29$ & & & \\
\hline $7440-62-2$ & Vanadium & $<4.53 \mathrm{E}-2$ & ug/g dry & 4.53E-2 & $5 / 01 / 08$ & 8D28003 & PNNL-AGG-ICP-AES \\
\hline $7440-66-6$ & Zinc & $<9.27 \mathrm{E}-2$ & ug/g dry & 9.27E-2 & $5 / 01 / 08$ & 8D28003 & PNNL-AGG-ICP-AES \\
\hline $7440-23-5$ & Sodium & $1.24 \mathrm{E} 1$ & ug/g dry & 6.69E-1 & $5 / 01 / 08$ & 8D28003 & PNNL-AGG-ICP-AES \\
\hline
\end{tabular}


Total Metals by PNNL-AGG-ICP-AES/Acid Extract

\begin{tabular}{|c|c|c|c|c|c|c|c|}
\hline CAS \# & Analyte & Results & Units & EQL & Analyzed & Batch & Method \\
\hline HEIS No. & B1RKL1 & \multicolumn{3}{|c|}{ Lab ID: $\quad$ 0802002-02 } & & & \\
\hline $7429-90-5$ & Aluminum & $4.41 \mathrm{E} 3$ & ug/g dry & 2.79E1 & 2/20/08 & 8F11003 & PNNL-AGG-ICP-AES \\
\hline $7440-39-3$ & Barium & 4.43E1 & ug/g dry & 2.66E0 & $2 / 20 / 08$ & 8F11003 & PNNL-AGG-ICP-AES \\
\hline $7440-70-2$ & Calcium & 6.99E3 & ug/g dry & $1.04 \mathrm{E} 2$ & 2/20/08 & 8F11003 & PNNL-AGG-ICP-AES \\
\hline $7440-48-4$ & Cobalt & $<5.79 \mathrm{E} 0$ & ug/g dry & 5.79E0 & $2 / 20 / 08$ & 8F11003 & PNNL-AGG-ICP-AES \\
\hline $7440-47-3$ & Chromium & 6.89E0 & ug/g dry & 2.32E0 & 2/20/08 & $8 F 11003$ & PNNL-AGG-ICP-AES \\
\hline $7440-50-8$ & Copper & $<2.47 \mathrm{E} 1$ & ug/g dry & 2.47E1 & $2 / 20 / 08$ & 8F11003 & PNNL-AGG-ICP-AES \\
\hline $7440-09-7$ & Potassium & 8.64E2 & ug/g dry & $6.58 \mathrm{E} 1$ & $2 / 20 / 08$ & 8F11003 & PNNL-AGG-ICP-AES \\
\hline 7439-95-4 & Magnesium & $3.58 \mathrm{E} 3$ & ug/g dry & $2.19 \mathrm{E} 1$ & 2/20/08 & 8F11003 & PNNL-AGG-ICP-AES \\
\hline 7439-96-5 & Manganese & $2.02 \mathrm{E} 2$ & ug/g dry & $8.30 \mathrm{E}-1$ & $2 / 20 / 08$ & 8F11003 & PNNL-AGG-ICP-AES \\
\hline $7440-02-0$ & Nickel & 1.03E1 & ug/g dry & $5.70 \mathrm{E} 0$ & $2 / 20 / 08$ & 8F11003 & PNNL-AGG-ICP-AES \\
\hline $7440-62-2$ & Vanadium & $9.61 \mathrm{E} 0$ & ug/g dry & $2.58 \mathrm{E} 0$ & 2/20/08 & 8F11003 & PNNL-AGG-ICP-AES \\
\hline $7440-66-6$ & Zinc & $2.40 \mathrm{E} 1$ & ug/g dry & 4.35E0 & 2/20/08 & 8F11003 & PNNL-AGG-ICP-AES \\
\hline $7440-23-5$ & Sodium & $<7.43 \mathrm{E} 2$ & ug/g dry & 7.43E2 & 2/20/08 & 8F11003 & PNNL-AGG-ICP-AES \\
\hline HEIS No. & B1RKL6 & \multicolumn{3}{|c|}{ Lab ID: $\quad$ 0802002-09 } & & & \\
\hline $7429-90-5$ & Aluminum & $4.50 \mathrm{E} 3$ & ug/g dry & $2.76 \mathrm{E} 1$ & 2/13/08 & $8 F 10003$ & PNNL-AGG-ICP-AES \\
\hline $7440-39-3$ & Barium & $5.76 \mathrm{E} 1$ & ug/g dry & 2.63E0 & 2/13/08 & 8F10003 & PNNL-AGG-ICP-AES \\
\hline $7440-70-2$ & Calcium & $6.88 \mathrm{E} 3$ & ug/g dry & $1.02 \mathrm{E} 2$ & 2/13/08 & $8 F 10003$ & PNNL-AGG-ICP-AES \\
\hline $7440-48-4$ & Cobalt & 5.87E0 & ug/g dry & 5.72E0 & 2/13/08 & $8 F 10003$ & PNNL-AGG-ICP-AES \\
\hline $7440-47-3$ & Chromium & 7.17E0 & ug/g dry & 2.29E0 & 2/13/08 & 8F10003 & PNNL-AGG-ICP-AES \\
\hline $7440-50-8$ & Copper & $<2.44 \mathrm{E} 1$ & ug/g dry & 2.44E1 & 2/13/08 & $8 F 10003$ & PNNL-AGG-ICP-AES \\
\hline $7440-09-7$ & Potassium & 8.86E2 & ug/g dry & $6.51 \mathrm{E} 1$ & 2/13/08 & 8F10003 & PNNL-AGG-ICP-AES \\
\hline 7439-95-4 & Magnesium & 4.62E3 & ug/g dry & $2.17 \mathrm{E} 1$ & 2/13/08 & $8 \mathrm{~F} 10003$ & PNNL-AGG-ICP-AES \\
\hline $7439-96-5$ & Manganese & $1.92 \mathrm{E} 2$ & ug/g dry & $8.21 \mathrm{E}-1$ & 2/13/08 & 8F10003 & PNNL-AGG-ICP-AES \\
\hline $7440-02-0$ & Nickel & $1.51 \mathrm{E} 1$ & ug/g dry & $5.64 \mathrm{E} 0$ & 2/13/08 & $8 \mathrm{~F} 10003$ & PNNL-AGG-ICP-AES \\
\hline $7440-62-2$ & Vanadium & $1.01 \mathrm{E} 1$ & ug/g dry & $2.55 \mathrm{E} 0$ & 2/13/08 & 8F10003 & PNNL-AGG-ICP-AES \\
\hline $7440-66-6$ & Zinc & $2.59 \mathrm{E} 1$ & ug/g dry & 4.30E0 & 2/13/08 & $8 F 10003$ & PNNL-AGG-ICP-AES \\
\hline $7440-23-5$ & Sodium & $<7.35 \mathrm{E} 2$ & ug/g dry & 7.35E2 & 2/13/08 & $8 \mathrm{~F} 10003$ & PNNL-AGG-ICP-AES \\
\hline HEIS No. & B1RKM1 & \multicolumn{3}{|c|}{$\begin{array}{ll}\text { Lab ID: } & \mathbf{0 8 0 2 0 0 2 - 1 7}\end{array}$} & & & \\
\hline $7429-90-5$ & Aluminum & 4.99E3 & ug/g dry & $2.62 \mathrm{E} 1$ & 2/20/08 & 8F11003 & PNNL-AGG-ICP-AES \\
\hline $7440-39-3$ & Barium & $4.81 \mathrm{E} 1$ & ug/g dry & 2.49E0 & $2 / 20 / 08$ & 8F11003 & PNNL-AGG-ICP-AES \\
\hline $7440-70-2$ & Calcium & 7.76E3 & ug/g dry & 9.73E1 & 2/20/08 & 8F11003 & PNNL-AGG-ICP-AES \\
\hline $7440-48-4$ & Cobalt & 5.88E0 & ug/g dry & 5.43E0 & 2/20/08 & $8 F 11003$ & PNNL-AGG-ICP-AES \\
\hline $7440-47-3$ & Chromium & $8.46 \mathrm{E} 0$ & ug/g dry & $2.18 \mathrm{E} 0$ & 2/20/08 & 8F11003 & PNNL-AGG-ICP-AES \\
\hline $7440-50-8$ & Copper & $<2.32 \mathrm{E} 1$ & ug/g dry & 2.32E1 & 2/20/08 & $8 F 11003$ & PNNL-AGG-ICP-AES \\
\hline 7440-09-7 & Potassium & 8.73E2 & ug/g dry & $6.17 \mathrm{E} 1$ & 2/20/08 & 8F11003 & PNNL-AGG-ICP-AES \\
\hline 7439-95-4 & Magnesium & $4.28 \mathrm{E} 3$ & ug/g dry & $2.06 \mathrm{E} 1$ & 2/20/08 & 8F11003 & PNNL-AGG-ICP-AES \\
\hline $7439-96-5$ & Manganese & $2.12 \mathrm{E} 2$ & ug/g dry & $7.79 \mathrm{E}-1$ & 2/20/08 & 8F11003 & PNNL-AGG-ICP-AES \\
\hline $7440-02-0$ & Nickel & $1.10 \mathrm{E} 1$ & ug/g dry & $5.35 \mathrm{E} 0$ & $2 / 20 / 08$ & 8F11003 & PNNL-AGG-ICP-AES \\
\hline $7440-62-2$ & Vanadium & $1.15 \mathrm{E} 1$ & ug/g dry & 2.42E0 & 2/20/08 & 8F11003 & PNNL-AGG-ICP-AES \\
\hline $7440-66-6$ & Zinc & $2.56 \mathrm{E} 1$ & ug/g dry & 4.08E0 & 2/20/08 & $8 F 11003$ & PNNL-AGG-ICP-AES \\
\hline $7440-23-5$ & Sodium & $<6.97 \mathrm{E} 2$ & ug/g dry & $6.97 \mathrm{E} 2$ & 2/20/08 & 8F11003 & PNNL-AGG-ICP-AES \\
\hline HEIS No. & B1RKM8 & \multicolumn{3}{|c|}{ Lab ID: $\quad 0802002-31$} & & & \\
\hline $7429-90-5$ & Aluminum & 4.95E3 & ug/g dry & $2.65 \mathrm{E} 1$ & 2/13/08 & 8F10003 & PNNL-AGG-ICP-AES \\
\hline $7440-39-3$ & Barium & 4.49E1 & ug/g dry & $2.52 \mathrm{E} 0$ & 2/13/08 & 8F10003 & PNNL-AGG-ICP-AES \\
\hline $7440-70-2$ & Calcium & $6.89 \mathrm{E} 3$ & ug/g dry & 9.84E1 & 2/13/08 & 8F10003 & PNNL-AGG-ICP-AES \\
\hline $7440-48-4$ & Cobalt & $<5.50 \mathrm{E} 0$ & ug/g dry & $5.50 \mathrm{E} 0$ & 2/13/08 & $8 \mathrm{~F} 10003$ & PNNL-AGG-ICP-AES \\
\hline $7440-47-3$ & Chromium & $1.09 \mathrm{E} 1$ & ug/g dry & $2.20 \mathrm{E} 0$ & 2/13/08 & 8F10003 & PNNL-AGG-ICP-AES \\
\hline $7440-50-8$ & Copper & $<2.35 \mathrm{E} 1$ & ug/g dry & $2.35 \mathrm{E} 1$ & 2/13/08 & 8F10003 & PNNL-AGG-ICP-AES \\
\hline
\end{tabular}


Total Metals by PNNL-AGG-ICP-AES/Acid Extract

\begin{tabular}{|c|c|c|c|c|c|c|c|}
\hline CAS \# & Analyte & Results & Units & EQL & Analyzed & Batch & Method \\
\hline HEIS No. & B1RKM8 & \multicolumn{3}{|c|}{ Lab ID: $\quad$ 0802002-31 } & & & \\
\hline 7440-09-7 & Potassium & $9.50 \mathrm{E} 2$ & ug/g dry & $6.25 \mathrm{E} 1$ & 2/13/08 & 8F10003 & PNNL-AGG-ICP-AES \\
\hline 7439-95-4 & Magnesium & 4.05E3 & ug/g dry & 2.08E1 & 2/13/08 & 8F10003 & PNNL-AGG-ICP-AES \\
\hline 7439-96-5 & Manganese & $1.96 \mathrm{E} 2$ & ug/g dry & $7.88 \mathrm{E}-1$ & 2/13/08 & 8F10003 & PNNL-AGG-ICP-AES \\
\hline $7440-02-0$ & Nickel & $1.24 \mathrm{E} 1$ & ug/g dry & 5.42E0 & 2/13/08 & 8F10003 & PNNL-AGG-ICP-AES \\
\hline $7440-62-2$ & Vanadium & $1.14 \mathrm{E} 1$ & ug/g dry & $2.45 \mathrm{E} 0$ & 2/13/08 & 8F10003 & PNNL-AGG-ICP-AES \\
\hline $7440-66-6$ & Zinc & 2.59E1 & ug/g dry & 4.13E0 & 2/13/08 & 8F10003 & PNNL-AGG-ICP-AES \\
\hline $7440-23-5$ & Sodium & $<7.06 \mathrm{E} 2$ & ug/g dry & 7.06E2 & 2/13/08 & 8F10003 & PNNL-AGG-ICP-AES \\
\hline HEIS No. & B1RKW7 & \multicolumn{3}{|c|}{ Lab ID: $\quad$ 0802003-02 } & & & \\
\hline 7429-90-5 & Aluminum & 7.51E3 & ug/g dry & 3.44E1 & 2/20/08 & 8F11003 & PNNL-AGG-ICP-AES \\
\hline $7440-39-3$ & Barium & $9.10 \mathrm{E} 1$ & ug/g dry & 3.27E0 & 2/20/08 & 8F11003 & PNNL-AGG-ICP-AES \\
\hline $7440-41-7$ & Beryllium & $<1.30 \mathrm{E} 0$ & ug/g dry & $1.30 \mathrm{E} 0$ & 2/20/08 & 8F11003 & PNNL-AGG-ICP-AES \\
\hline $7440-70-2$ & Calcium & $1.28 \mathrm{E} 4$ & ug/g dry & $1.28 \mathrm{E} 2$ & $2 / 20 / 08$ & 8F11003 & PNNL-AGG-ICP-AES \\
\hline $7440-48-4$ & Cobalt & 7.49E0 & ug/g dry & 7.13E0 & 2/20/08 & 8F11003 & PNNL-AGG-ICP-AES \\
\hline $7440-47-3$ & Chromium & $1.33 \mathrm{E} 1$ & ug/g dry & $2.86 \mathrm{E} 0$ & 2/20/08 & 8F11003 & PNNL-AGG-ICP-AES \\
\hline $7440-50-8$ & Copper & $<3.05 \mathrm{E} 1$ & ug/g dry & 3.05E1 & 2/20/08 & 8F11003 & PNNL-AGG-ICP-AES \\
\hline $7440-09-7$ & Potassium & $1.68 \mathrm{E} 3$ & ug/g dry & 8.11E1 & 2/20/08 & 8F11003 & PNNL-AGG-ICP-AES \\
\hline 7439-95-4 & Magnesium & $5.02 \mathrm{E} 3$ & ug/g dry & $2.70 \mathrm{E} 1$ & 2/20/08 & 8F11003 & PNNL-AGG-ICP-AES \\
\hline 7439-96-5 & Manganese & $3.21 \mathrm{E} 2$ & ug/g dry & 1.02E0 & 2/20/08 & 8 F11003 & PNNL-AGG-ICP-AES \\
\hline $7440-02-0$ & Nickel & $1.30 \mathrm{E} 1$ & ug/g dry & 7.03E0 & 2/20/08 & 8F11003 & PNNL-AGG-ICP-AES \\
\hline $7440-23-5$ & Sodium & $<9.16 \mathrm{E} 2$ & ug/g dry & $9.16 \mathrm{E} 2$ & 2/20/08 & 8F11003 & PNNL-AGG-ICP-AES \\
\hline HEIS No. & B1RKW8 & \multicolumn{3}{|c|}{ Lab ID: $\quad$ 0802003-04 } & & & \\
\hline $7429-90-5$ & Aluminum & $8.51 \mathrm{E} 3$ & ug/g dry & 3.37E1 & 2/13/08 & 8F10003 & PNNL-AGG-ICP-AES \\
\hline $7440-39-3$ & Barium & 8.80E1 & ug/g dry & $3.21 \mathrm{E} 0$ & 2/13/08 & 8F10003 & PNNL-AGG-ICP-AES \\
\hline $7440-70-2$ & Calcium & $1.60 \mathrm{E} 4$ & ug/g dry & $1.25 \mathrm{E} 2$ & 2/13/08 & 8F10003 & PNNL-AGG-ICP-AES \\
\hline $7440-48-4$ & Cobalt & 7.55E0 & ug/g dry & 7.00E0 & 2/13/08 & 8F10003 & PNNL-AGG-ICP-AES \\
\hline $7440-47-3$ & Chromium & $1.16 \mathrm{E} 1$ & ug/g dry & $2.80 \mathrm{E} 0$ & 2/13/08 & 8F10003 & PNNL-AGG-ICP-AES \\
\hline $7440-50-8$ & Copper & $<2.99 \mathrm{E} 1$ & ug/g dry & 2.99E1 & 2/13/08 & 8F10003 & PNNL-AGG-ICP-AES \\
\hline $7440-09-7$ & Potassium & $1.95 \mathrm{E} 3$ & ug/g dry & 7.95E1 & 2/13/08 & 8F10003 & PNNL-AGG-ICP-AES \\
\hline 7439-95-4 & Magnesium & 5.89E3 & ug/g dry & 2.65E1 & 2/13/08 & 8F10003 & PNNL-AGG-ICP-AES \\
\hline 7439-96-5 & Manganese & $4.25 \mathrm{E} 2$ & ug/g dry & $1.00 \mathrm{E} 0$ & 2/13/08 & 8 F10003 & PNNL-AGG-ICP-AES \\
\hline $7440-02-0$ & Nickel & $1.16 \mathrm{E} 1$ & ug/g dry & $6.89 \mathrm{E} 0$ & 2/13/08 & 8F10003 & PNNL-AGG-ICP-AES \\
\hline $7440-23-5$ & Sodium & $<8.98 \mathrm{E} 2$ & ug/g dry & 8.98E2 & 2/13/08 & 8F10003 & PNNL-AGG-ICP-AES \\
\hline HEIS No. & B1RKN7 & \multicolumn{3}{|c|}{ Lab ID: $\quad$ 0802005-03 } & & & \\
\hline $7429-90-5$ & Aluminum & $5.21 \mathrm{E} 3$ & ug/g dry & $2.48 \mathrm{E} 1$ & 2/20/08 & 8F11003 & PNNL-AGG-ICP-AES \\
\hline $7440-39-3$ & Barium & 4.96E1 & ug/g dry & 2.37E0 & $2 / 20 / 08$ & 8F11003 & PNNL-AGG-ICP-AES \\
\hline $7440-70-2$ & Calcium & 7.53E3 & ug/g dry & $9.22 \mathrm{E} 1$ & 2/20/08 & 8F11003 & PNNL-AGG-ICP-AES \\
\hline 7440-48-4 & Cobalt & 5.22E0 & ug/g dry & 5.15E0 & 2/20/08 & 8F11003 & PNNL-AGG-ICP-AES \\
\hline $7440-47-3$ & Chromium & $1.01 \mathrm{E} 1$ & ug/g dry & 2.06Е0 & 2/20/08 & 8F11003 & PNNL-AGG-ICP-AES \\
\hline $7440-50-8$ & Copper & $<2.20 \mathrm{E} 1$ & ug/g dry & $2.20 \mathrm{E} 1$ & 2/20/08 & 8F11003 & PNNL-AGG-ICP-AES \\
\hline $7440-09-7$ & Potassium & $9.02 \mathrm{E} 2$ & ug/g dry & $5.86 \mathrm{E} 1$ & 2/20/08 & 8F11003 & PNNL-AGG-ICP-AES \\
\hline 7439-95-4 & Magnesium & 4.42E3 & ug/g dry & $1.95 \mathrm{E} 1$ & 2/20/08 & 8F11003 & PNNL-AGG-ICP-AES \\
\hline 7439-96-5 & Manganese & $2.47 \mathrm{E} 2$ & ug/g dry & 7.39E-1 & 2/20/08 & 8F11003 & PNNL-AGG-ICP-AES \\
\hline $7440-02-0$ & Nickel & $1.44 \mathrm{E} 1$ & ug/g dry & $5.08 \mathrm{E} 0$ & 2/20/08 & 8F11003 & PNNL-AGG-ICP-AES \\
\hline $7440-62-2$ & Vanadium & $9.07 \mathrm{E} 0$ & ug/g dry & $2.30 \mathrm{E} 0$ & 2/20/08 & 8F11003 & PNNL-AGG-ICP-AES \\
\hline $7440-66-6$ & Zinc & 2.67E1 & ug/g dry & 3.87E0 & 2/20/08 & 8F11003 & PNNL-AGG-ICP-AES \\
\hline $7440-23-5$ & Sodium & $<6.62 \mathrm{E} 2$ & ug/g dry & 6.62E2 & 2/20/08 & 8F11003 & PNNL-AGG-ICP-AES \\
\hline HEIS No. & B1RKP3 & \multicolumn{3}{|c|}{ Lab ID: $\quad$ 0802005-09 } & & & \\
\hline 7429-90-5 & Aluminum & $5.07 \mathrm{E} 3$ & ug/g dry & 2.76E1 & 2/13/08 & 8F10003 & PNNL-AGG-ICP-AES \\
\hline
\end{tabular}


Total Metals by PNNL-AGG-ICP-AES/Acid Extract

\begin{tabular}{|c|c|c|c|c|c|c|c|}
\hline CAS \# & Analyte & Results & Units & EQL & Analyzed & Batch & Method \\
\hline HEIS No. & B1RKP3 & \multicolumn{3}{|c|}{ Lab ID: $\quad$ 0802005-09 } & & & \\
\hline $7440-39-3$ & Barium & 4.19E1 & ug/g dry & 2.63E0 & 2/13/08 & 8F10003 & PNNL-AGG-ICP-AES \\
\hline $7440-70-2$ & Calcium & $7.48 \mathrm{E} 3$ & ug/g dry & 1.03E2 & 2/13/08 & 8F10003 & PNNL-AGG-ICP-AES \\
\hline $7440-48-4$ & Cobalt & $<5.74 \mathrm{E} 0$ & ug/g dry & 5.74E0 & 2/13/08 & 8F10003 & PNNL-AGG-ICP-AES \\
\hline $7440-47-3$ & Chromium & 1.03E1 & ug/g dry & 2.30E0 & 2/13/08 & 8F10003 & PNNL-AGG-ICP-AES \\
\hline $7440-50-8$ & Copper & $<2.45 \mathrm{E} 1$ & ug/g dry & $2.45 \mathrm{E} 1$ & 2/13/08 & $8 \mathrm{~F} 10003$ & PNNL-AGG-ICP-AES \\
\hline 7440-09-7 & Potassium & $9.38 \mathrm{E} 2$ & ug/g dry & $6.52 \mathrm{E} 1$ & 2/13/08 & 8F10003 & PNNL-AGG-ICP-AES \\
\hline $7439-95-4$ & Magnesium & 4.35E3 & ug/g dry & $2.17 \mathrm{E} 1$ & 2/13/08 & 8F10003 & PNNL-AGG-ICP-AES \\
\hline 7439-96-5 & Manganese & $2.10 \mathrm{E} 2$ & ug/g dry & 8.23E-1 & 2/13/08 & $8 \mathrm{~F} 10003$ & PNNL-AGG-ICP-AES \\
\hline 7440-02-0 & Nickel & 1.39E1 & ug/g dry & $5.65 \mathrm{E} 0$ & 2/13/08 & 8F10003 & PNNL-AGG-ICP-AES \\
\hline $7440-62-2$ & Vanadium & $9.78 \mathrm{E} 0$ & ug/g dry & $2.56 \mathrm{E} 0$ & 2/13/08 & $8 \mathrm{~F} 10003$ & PNNL-AGG-ICP-AES \\
\hline $7440-66-6$ & Zinc & $2.50 \mathrm{E} 1$ & ug/g dry & 4.31E0 & 2/13/08 & 8F10003 & PNNL-AGG-ICP-AES \\
\hline $7440-23-5$ & Sodium & $<7.36 \mathrm{E} 2$ & ug/g dry & 7.36E2 & 2/13/08 & 8F10003 & PNNL-AGG-ICP-AES \\
\hline HEIS No. & B1RKP9 & \multicolumn{3}{|c|}{$\begin{array}{ll}\text { Lab ID: } & \text { 0802005-15 }\end{array}$} & & & \\
\hline $7429-90-5$ & Aluminum & 4.57E3 & ug/g dry & $2.76 \mathrm{E} 1$ & 2/20/08 & 8F11003 & PNNL-AGG-ICP-AES \\
\hline $7440-39-3$ & Barium & 4.98E1 & ug/g dry & 2.63E0 & 2/20/08 & 8F11003 & PNNL-AGG-ICP-AES \\
\hline $7440-70-2$ & Calcium & $6.61 \mathrm{E} 3$ & ug/g dry & $1.03 \mathrm{E} 2$ & $2 / 20 / 08$ & 8F11003 & PNNL-AGG-ICP-AES \\
\hline $7440-48-4$ & Cobalt & $<5.73 \mathrm{E} 0$ & ug/g dry & 5.73E0 & $2 / 20 / 08$ & 8F11003 & PNNL-AGG-ICP-AES \\
\hline $7440-47-3$ & Chromium & 8.88E0 & ug/g dry & 2.29E0 & $2 / 20 / 08$ & 8F11003 & PNNL-AGG-ICP-AES \\
\hline $7440-50-8$ & Copper & $<2.44 \mathrm{E} 1$ & ug/g dry & $2.44 \mathrm{E} 1$ & $2 / 20 / 08$ & 8F11003 & PNNL-AGG-ICP-AES \\
\hline 7440-09-7 & Potassium & $8.08 \mathrm{E} 2$ & ug/g dry & $6.51 \mathrm{E} 1$ & 2/20/08 & 8F11003 & PNNL-AGG-ICP-AES \\
\hline 7439-95-4 & Magnesium & 3.68E3 & ug/g dry & $2.17 \mathrm{E} 1$ & $2 / 20 / 08$ & 8F11003 & PNNL-AGG-ICP-AES \\
\hline $7439-96-5$ & Manganese & $2.20 \mathrm{E} 2$ & ug/g dry & $8.21 \mathrm{E}-1$ & $2 / 20 / 08$ & 8F11003 & PNNL-AGG-ICP-AES \\
\hline $7440-02-0$ & Nickel & $1.15 \mathrm{E} 1$ & ug/g dry & $5.64 \mathrm{E} 0$ & $2 / 20 / 08$ & 8F11003 & PNNL-AGG-ICP-AES \\
\hline $7440-62-2$ & Vanadium & $1.05 \mathrm{E} 1$ & ug/g dry & $2.55 \mathrm{E} 0$ & 2/20/08 & 8F11003 & PNNL-AGG-ICP-AES \\
\hline $7440-66-6$ & Zinc & $2.20 \mathrm{E} 1$ & ug/g dry & $4.30 \mathrm{E} 0$ & 2/20/08 & 8F11003 & PNNL-AGG-ICP-AES \\
\hline $7440-23-5$ & Sodium & $<7.35 \mathrm{E} 2$ & ug/g dry & 7.35E2 & $2 / 20 / 08$ & 8F11003 & PNNL-AGG-ICP-AES \\
\hline HEIS No. & B1RKR5 & \multicolumn{3}{|c|}{ Lab ID: $\quad$ 0802005-21 } & & & \\
\hline $7429-90-5$ & Aluminum & 5.23E3 & ug/g dry & $3.01 \mathrm{E} 1$ & 2/13/08 & 8F10003 & PNNL-AGG-ICP-AES \\
\hline $7440-39-3$ & Barium & $5.41 \mathrm{E} 1$ & ug/g dry & $2.86 \mathrm{E} 0$ & 2/13/08 & 8F10003 & PNNL-AGG-ICP-AES \\
\hline $7440-70-2$ & Calcium & $5.92 \mathrm{E} 3$ & ug/g dry & $1.12 \mathrm{E} 2$ & 2/13/08 & $8 F 10003$ & PNNL-AGG-ICP-AES \\
\hline $7440-48-4$ & Cobalt & $<6.24 \mathrm{E} 0$ & ug/g dry & $6.24 \mathrm{E} 0$ & 2/13/08 & 8F10003 & PNNL-AGG-ICP-AES \\
\hline $7440-47-3$ & Chromium & 1.07E1 & ug/g dry & $2.50 \mathrm{E} 0$ & 2/13/08 & $8 \mathrm{~F} 10003$ & PNNL-AGG-ICP-AES \\
\hline $7440-50-8$ & Copper & $<2.66 \mathrm{E} 1$ & ug/g dry & $2.66 \mathrm{E} 1$ & 2/13/08 & 8F10003 & PNNL-AGG-ICP-AES \\
\hline 7440-09-7 & Potassium & $1.01 \mathrm{E} 3$ & ug/g dry & 7.09E1 & 2/13/08 & $8 F 10003$ & PNNL-AGG-ICP-AES \\
\hline 7439-95-4 & Magnesium & $4.27 \mathrm{E} 3$ & ug/g dry & $2.36 \mathrm{E} 1$ & 2/13/08 & 8F10003 & PNNL-AGG-ICP-AES \\
\hline 7439-96-5 & Manganese & $2.11 \mathrm{E} 2$ & ug/g dry & 8.95E-1 & 2/13/08 & $8 \mathrm{~F} 10003$ & PNNL-AGG-ICP-AES \\
\hline $7440-02-0$ & Nickel & $1.38 \mathrm{E} 1$ & ug/g dry & $6.15 \mathrm{E} 0$ & 2/13/08 & $8 \mathrm{~F} 10003$ & PNNL-AGG-ICP-AES \\
\hline $7440-62-2$ & Vanadium & $1.31 \mathrm{E} 1$ & ug/g dry & $2.78 \mathrm{E} 0$ & 2/13/08 & $8 \mathrm{~F} 10003$ & PNNL-AGG-ICP-AES \\
\hline $7440-66-6$ & Zinc & $2.70 \mathrm{E} 1$ & ug/g dry & 4.69E0 & 2/13/08 & 8F10003 & PNNL-AGG-ICP-AES \\
\hline $7440-23-5$ & Sodium & $<8.01 \mathrm{E} 2$ & ug/g dry & $8.01 \mathrm{E} 2$ & 2/13/08 & $8 F 10003$ & PNNL-AGG-ICP-AES \\
\hline HEIS No. & B1RKR9 & \multicolumn{3}{|c|}{ Lab ID: $\quad$ 0802005-25 } & & & \\
\hline $7429-90-5$ & Aluminum & 4.96E3 & ug/g dry & $2.91 \mathrm{E} 1$ & $2 / 20 / 08$ & 8F11003 & PNNL-AGG-ICP-AES \\
\hline $7440-39-3$ & Barium & 4.37E1 & ug/g dry & 2.77E0 & 2/20/08 & 8F11003 & PNNL-AGG-ICP-AES \\
\hline $7440-70-2$ & Calcium & 6.13E3 & ug/g dry & $1.08 \mathrm{E} 2$ & $2 / 20 / 08$ & $8 F 11003$ & PNNL-AGG-ICP-AES \\
\hline $7440-48-4$ & Cobalt & $<6.03 \mathrm{E} 0$ & ug/g dry & 6.03E0 & $2 / 20 / 08$ & 8F11003 & PNNL-AGG-ICP-AES \\
\hline $7440-47-3$ & Chromium & $9.98 \mathrm{E} 0$ & ug/g dry & 2.42E0 & $2 / 20 / 08$ & 8F11003 & PNNL-AGG-ICP-AES \\
\hline $7440-50-8$ & Copper & $<2.57 \mathrm{E} 1$ & ug/g dry & $2.57 \mathrm{E} 1$ & 2/20/08 & 8F11003 & PNNL-AGG-ICP-AES \\
\hline $7440-09-7$ & Potassium & 9.77E2 & ug/g dry & $6.86 \mathrm{E} 1$ & 2/20/08 & 8F11003 & PNNL-AGG-ICP-AES \\
\hline
\end{tabular}


Total Metals by PNNL-AGG-ICP-AES/Acid Extract

\begin{tabular}{|c|c|c|c|c|c|c|c|}
\hline CAS \# & Analyte & Results & Units & EQL & Analyzed & Batch & Method \\
\hline HEIS No. & B1RKR9 & \multicolumn{3}{|c|}{ Lab ID: $\quad 0802005-25$} & & & \\
\hline 7439-95-4 & Magnesium & $4.01 \mathrm{E} 3$ & ug/g dry & $2.29 \mathrm{E} 1$ & 2/20/08 & 8F11003 & PNNL-AGG-ICP-AES \\
\hline 7439-96-5 & Manganese & 2.19E2 & ug/g dry & 8.65E-1 & $2 / 20 / 08$ & 8F11003 & PNNL-AGG-ICP-AES \\
\hline $7440-02-0$ & Nickel & $1.27 \mathrm{E} 1$ & ug/g dry & 5.94E0 & 2/20/08 & 8F11003 & PNNL-AGG-ICP-AES \\
\hline $7440-62-2$ & Vanadium & $9.28 \mathrm{E} 0$ & ug/g dry & 2.69E0 & $2 / 20 / 08$ & 8F11003 & PNNL-AGG-ICP-AES \\
\hline $7440-66-6$ & Zinc & $2.46 \mathrm{E} 1$ & ug/g dry & 4.53E0 & 2/20/08 & $8 F 11003$ & PNNL-AGG-ICP-AES \\
\hline $7440-23-5$ & Sodium & $<7.74 \mathrm{E} 2$ & ug/g dry & 7.74E2 & $2 / 20 / 08$ & 8F11003 & PNNL-AGG-ICP-AES \\
\hline HEIS No. & B1RKT3 & \multicolumn{3}{|c|}{ Lab ID: $\quad$ 0802005-29 } & & & \\
\hline $7429-90-5$ & Aluminum & $5.50 \mathrm{E} 3$ & ug/g dry & 3.07E1 & $2 / 13 / 08$ & 8F10003 & PNNL-AGG-ICP-AES \\
\hline $7440-39-3$ & Barium & $5.34 \mathrm{E} 1$ & ug/g dry & 2.92E0 & 2/13/08 & $8 \mathrm{~F} 10003$ & PNNL-AGG-ICP-AES \\
\hline $7440-70-2$ & Calcium & 7.14E3 & ug/g dry & $1.14 \mathrm{E} 2$ & 2/13/08 & 8F10003 & PNNL-AGG-ICP-AES \\
\hline $7440-48-4$ & Cobalt & $6.78 \mathrm{E} 0$ & ug/g dry & 6.37E0 & 2/13/08 & 8F10003 & PNNL-AGG-ICP-AES \\
\hline $7440-47-3$ & Chromium & $9.88 \mathrm{E} 0$ & ug/g dry & $2.55 \mathrm{E} 0$ & 2/13/08 & $8 F 10003$ & PNNL-AGG-ICP-AES \\
\hline $7440-50-8$ & Copper & $<2.72 \mathrm{E} 1$ & ug/g dry & $2.72 \mathrm{E} 1$ & 2/13/08 & 8F10003 & PNNL-AGG-ICP-AES \\
\hline 7440-09-7 & Potassium & $8.70 \mathrm{E} 2$ & ug/g dry & $7.24 \mathrm{E} 1$ & 2/13/08 & $8 \mathrm{~F} 10003$ & PNNL-AGG-ICP-AES \\
\hline 7439-95-4 & Magnesium & 4.49E3 & ug/g dry & $2.41 \mathrm{E} 1$ & 2/13/08 & 8F10003 & PNNL-AGG-ICP-AES \\
\hline $7439-96-5$ & Manganese & $2.29 \mathrm{E} 2$ & ug/g dry & $9.13 \mathrm{E}-1$ & 2/13/08 & 8F10003 & PNNL-AGG-ICP-AES \\
\hline $7440-02-0$ & Nickel & $1.38 \mathrm{E} 1$ & ug/g dry & $6.28 \mathrm{E} 0$ & 2/13/08 & 8F10003 & PNNL-AGG-ICP-AES \\
\hline $7440-62-2$ & Vanadium & $1.73 \mathrm{E} 1$ & ug/g dry & 2.84E0 & 2/13/08 & $8 F 10003$ & PNNL-AGG-ICP-AES \\
\hline $7440-66-6$ & Zinc & 2.96E1 & ug/g dry & $4.78 \mathrm{E} 0$ & 2/13/08 & 8F10003 & PNNL-AGG-ICP-AES \\
\hline $7440-23-5$ & Sodium & $<8.18 \mathrm{E} 2$ & ug/g dry & 8.18E2 & 2/13/08 & 8F10003 & PNNL-AGG-ICP-AES \\
\hline HEIS No. & B1RKT7 & \multicolumn{3}{|c|}{ Lab ID: $\quad$ 0802006-07 } & & & \\
\hline 7429-90-5 & Aluminum & 5.19E3 & ug/g dry & $3.00 \mathrm{E} 1$ & $2 / 20 / 08$ & 8F11003 & PNNL-AGG-ICP-AES \\
\hline $7440-39-3$ & Barium & 5.37E1 & ug/g dry & $2.86 \mathrm{E} 0$ & $2 / 20 / 08$ & 8F11003 & PNNL-AGG-ICP-AES \\
\hline $7440-70-2$ & Calcium & 7.07E3 & ug/g dry & $1.12 \mathrm{E} 2$ & $2 / 20 / 08$ & 8F11003 & PNNL-AGG-ICP-AES \\
\hline $7440-48-4$ & Cobalt & $<6.23 \mathrm{E} 0$ & ug/g dry & 6.23E0 & 2/20/08 & 8F11003 & PNNL-AGG-ICP-AES \\
\hline $7440-47-3$ & Chromium & $8.46 \mathrm{E} 0$ & ug/g dry & 2.49E0 & $2 / 20 / 08$ & 8F11003 & PNNL-AGG-ICP-AES \\
\hline $7440-50-8$ & Copper & $<2.66 \mathrm{E} 1$ & ug/g dry & $2.66 \mathrm{E} 1$ & $2 / 20 / 08$ & 8F11003 & PNNL-AGG-ICP-AES \\
\hline 7440-09-7 & Potassium & $1.00 \mathrm{E} 3$ & ug/g dry & $7.08 \mathrm{E} 1$ & 2/20/08 & 8F11003 & PNNL-AGG-ICP-AES \\
\hline 7439-95-4 & Magnesium & 3.85E3 & ug/g dry & $2.36 \mathrm{E} 1$ & 2/20/08 & 8F11003 & PNNL-AGG-ICP-AES \\
\hline 7439-96-5 & Manganese & $2.25 \mathrm{E} 2$ & ug/g dry & 8.93E-1 & $2 / 20 / 08$ & 8F11003 & PNNL-AGG-ICP-AES \\
\hline $7440-02-0$ & Nickel & 9.72E0 & ug/g dry & $6.14 \mathrm{E} 0$ & $2 / 20 / 08$ & 8F11003 & PNNL-AGG-ICP-AES \\
\hline $7440-62-2$ & Vanadium & $1.56 \mathrm{E} 1$ & ug/g dry & $2.78 \mathrm{E} 0$ & 2/20/08 & $8 \mathrm{~F} 11003$ & PNNL-AGG-ICP-AES \\
\hline $7440-66-6$ & Zinc & $2.69 \mathrm{E} 1$ & ug/g dry & 4.68E0 & $2 / 20 / 08$ & 8F11003 & PNNL-AGG-ICP-AES \\
\hline $7440-23-5$ & Sodium & $<8.00 \mathrm{E} 2$ & ug/g dry & $8.00 \mathrm{E} 2$ & $2 / 20 / 08$ & 8F11003 & PNNL-AGG-ICP-AES \\
\hline HEIS No. & B1RKV1 & \multicolumn{3}{|c|}{ Lab ID: $\quad$ 0802006-11 } & & & \\
\hline $7429-90-5$ & Aluminum & $5.05 \mathrm{E} 3$ & ug/g dry & 2.93E1 & 2/13/08 & 8F10003 & PNNL-AGG-ICP-AES \\
\hline $7440-39-3$ & Barium & $5.73 \mathrm{E} 1$ & ug/g dry & 2.79E0 & 2/13/08 & $8 \mathrm{~F} 10003$ & PNNL-AGG-ICP-AES \\
\hline $7440-70-2$ & Calcium & $6.50 \mathrm{E} 3$ & ug/g dry & $1.09 \mathrm{E} 2$ & 2/13/08 & $8 \mathrm{~F} 10003$ & PNNL-AGG-ICP-AES \\
\hline $7440-48-4$ & Cobalt & $<6.07 \mathrm{E} 0$ & ug/g dry & 6.07E0 & 2/13/08 & $8 \mathrm{~F} 10003$ & PNNL-AGG-ICP-AES \\
\hline $7440-47-3$ & Chromium & 8.37E0 & ug/g dry & 2.43E0 & 2/13/08 & 8F10003 & PNNL-AGG-ICP-AES \\
\hline $7440-50-8$ & Copper & $<2.59 \mathrm{E} 1$ & ug/g dry & $2.59 \mathrm{E} 1$ & 2/13/08 & 8F10003 & PNNL-AGG-ICP-AES \\
\hline 7440-09-7 & Potassium & $1.01 \mathrm{E} 3$ & ug/g dry & $6.90 \mathrm{E} 1$ & 2/13/08 & $8 F 10003$ & PNNL-AGG-ICP-AES \\
\hline 7439-95-4 & Magnesium & 3.65E3 & ug/g dry & $2.30 \mathrm{E} 1$ & 2/13/08 & 8F10003 & PNNL-AGG-ICP-AES \\
\hline $7439-96-5$ & Manganese & $2.04 \mathrm{E} 2$ & ug/g dry & $8.71 \mathrm{E}-1$ & 2/13/08 & $8 \mathrm{~F} 10003$ & PNNL-AGG-ICP-AES \\
\hline $7440-02-0$ & Nickel & 8.65E0 & ug/g dry & $5.98 \mathrm{E} 0$ & 2/13/08 & 8F10003 & PNNL-AGG-ICP-AES \\
\hline $7440-62-2$ & Vanadium & $1.43 \mathrm{E} 1$ & ug/g dry & $2.71 \mathrm{E} 0$ & 2/13/08 & $8 \mathrm{~F} 10003$ & PNNL-AGG-ICP-AES \\
\hline $7440-66-6$ & Zinc & $2.60 \mathrm{E} 1$ & ug/g dry & $4.56 \mathrm{E} 0$ & 2/13/08 & 8F10003 & PNNL-AGG-ICP-AES \\
\hline $7440-23-5$ & Sodium & $<7.80 \mathrm{E} 2$ & ug/g dry & $7.80 \mathrm{E} 2$ & 2/13/08 & 8F10003 & PNNL-AGG-ICP-AES \\
\hline
\end{tabular}


Total Metals by PNNL-AGG-ICP-AES/Acid Extract

\begin{tabular}{|c|c|c|c|c|c|c|c|}
\hline CAS \# & Analyte & Results & Units & EQL & Analyzed & Batch & Method \\
\hline HEIS No. & B1RKV5 & \multicolumn{3}{|c|}{ Lab ID: $\quad$ 0802007-04 } & & & \\
\hline $7429-90-5$ & Aluminum & $4.54 \mathrm{E} 3$ & ug/g dry & 2.87E1 & 2/20/08 & 8F11003 & PNNL-AGG-ICP-AES \\
\hline $7440-39-3$ & Barium & 4.92E1 & ug/g dry & 2.74E0 & $2 / 20 / 08$ & 8F11003 & PNNL-AGG-ICP-AES \\
\hline $7440-70-2$ & Calcium & 6.03E3 & ug/g dry & 1.07E2 & 2/20/08 & 8F11003 & PNNL-AGG-ICP-AES \\
\hline $7440-48-4$ & Cobalt & $<5.96 \mathrm{E} 0$ & ug/g dry & 5.96E0 & $2 / 20 / 08$ & 8F11003 & PNNL-AGG-ICP-AES \\
\hline $7440-47-3$ & Chromium & 7.82E0 & ug/g dry & 2.39E0 & 2/20/08 & $8 F 11003$ & PNNL-AGG-ICP-AES \\
\hline $7440-50-8$ & Copper & $<2.55 \mathrm{E} 1$ & ug/g dry & $2.55 \mathrm{E} 1$ & $2 / 20 / 08$ & 8F11003 & PNNL-AGG-ICP-AES \\
\hline $7440-09-7$ & Potassium & 8.03E2 & ug/g dry & $6.78 \mathrm{E} 1$ & $2 / 20 / 08$ & 8F11003 & PNNL-AGG-ICP-AES \\
\hline 7439-95-4 & Magnesium & $3.68 \mathrm{E} 3$ & ug/g dry & $2.26 \mathrm{E} 1$ & 2/20/08 & 8F11003 & PNNL-AGG-ICP-AES \\
\hline 7439-96-5 & Manganese & $2.02 \mathrm{E} 2$ & ug/g dry & 8.55E-1 & $2 / 20 / 08$ & 8F11003 & PNNL-AGG-ICP-AES \\
\hline $7440-02-0$ & Nickel & $1.20 \mathrm{E} 1$ & ug/g dry & $5.88 \mathrm{E} 0$ & $2 / 20 / 08$ & 8F11003 & PNNL-AGG-ICP-AES \\
\hline $7440-62-2$ & Vanadium & $1.46 \mathrm{E} 1$ & ug/g dry & $2.66 \mathrm{E} 0$ & 2/20/08 & 8F11003 & PNNL-AGG-ICP-AES \\
\hline $7440-66-6$ & Zinc & $2.42 \mathrm{E} 1$ & ug/g dry & $4.48 \mathrm{E} 0$ & 2/20/08 & 8F11003 & PNNL-AGG-ICP-AES \\
\hline $7440-23-5$ & Sodium & $<7.66 \mathrm{E} 2$ & ug/g dry & $7.66 \mathrm{E} 2$ & 2/20/08 & 8F11003 & PNNL-AGG-ICP-AES \\
\hline HEIS No. & B1RKV9 & \multicolumn{3}{|c|}{ Lab ID: $\quad$ 0802007-12 } & & & \\
\hline $7429-90-5$ & Aluminum & $4.74 \mathrm{E} 3$ & ug/g dry & $2.78 \mathrm{E} 1$ & 2/13/08 & 8F10003 & PNNL-AGG-ICP-AES \\
\hline $7440-39-3$ & Barium & 7.34E1 & ug/g dry & 2.65E0 & 2/13/08 & 8F10003 & PNNL-AGG-ICP-AES \\
\hline $7440-70-2$ & Calcium & $5.35 \mathrm{E} 3$ & ug/g dry & $1.03 \mathrm{E} 2$ & 2/13/08 & $8 F 10003$ & PNNL-AGG-ICP-AES \\
\hline $7440-48-4$ & Cobalt & $<5.77 \mathrm{E} 0$ & ug/g dry & 5.77E0 & 2/13/08 & $8 F 10003$ & PNNL-AGG-ICP-AES \\
\hline $7440-47-3$ & Chromium & 8.12E0 & ug/g dry & $2.31 \mathrm{E} 0$ & 2/13/08 & 8F10003 & PNNL-AGG-ICP-AES \\
\hline $7440-50-8$ & Copper & $<2.46 \mathrm{E} 1$ & ug/g dry & $2.46 \mathrm{E} 1$ & 2/13/08 & $8 \mathrm{~F} 10003$ & PNNL-AGG-ICP-AES \\
\hline $7440-09-7$ & Potassium & 7.35E2 & ug/g dry & $6.56 \mathrm{E} 1$ & 2/13/08 & 8F10003 & PNNL-AGG-ICP-AES \\
\hline 7439-95-4 & Magnesium & 3.00E3 & ug/g dry & 2.19E1 & 2/13/08 & $8 \mathrm{~F} 10003$ & PNNL-AGG-ICP-AES \\
\hline $7439-96-5$ & Manganese & $1.61 \mathrm{E} 2$ & ug/g dry & $8.27 \mathrm{E}-1$ & 2/13/08 & 8F10003 & PNNL-AGG-ICP-AES \\
\hline $7440-02-0$ & Nickel & 8.79E0 & ug/g dry & 5.69E0 & 2/13/08 & $8 \mathrm{~F} 10003$ & PNNL-AGG-ICP-AES \\
\hline $7440-62-2$ & Vanadium & $1.68 \mathrm{E} 1$ & ug/g dry & 2.57E0 & 2/13/08 & 8F10003 & PNNL-AGG-ICP-AES \\
\hline $7440-66-6$ & Zinc & $2.49 \mathrm{E} 1$ & ug/g dry & 4.33E0 & 2/13/08 & $8 F 10003$ & PNNL-AGG-ICP-AES \\
\hline $7440-23-5$ & Sodium & $<7.41 \mathrm{E} 2$ & ug/g dry & 7.41E2 & 2/13/08 & $8 \mathrm{~F} 10003$ & PNNL-AGG-ICP-AES \\
\hline HEIS No. & B1RKW2 & \multicolumn{3}{|c|}{$\begin{array}{ll}\text { Lab ID: } & \text { 0802008-04 }\end{array}$} & & & \\
\hline $7429-90-5$ & Aluminum & $6.71 \mathrm{E} 3$ & ug/g dry & 3.33E1 & 2/20/08 & 8F11003 & PNNL-AGG-ICP-AES \\
\hline $7440-39-3$ & Barium & $1.05 \mathrm{E} 2$ & ug/g dry & 3.17E0 & $2 / 20 / 08$ & $8 F 11003$ & PNNL-AGG-ICP-AES \\
\hline $7440-70-2$ & Calcium & $1.12 \mathrm{E} 4$ & ug/g dry & $1.24 \mathrm{E} 2$ & 2/20/08 & 8F11003 & PNNL-AGG-ICP-AES \\
\hline $7440-48-4$ & Cobalt & $<6.91 \mathrm{E} 0$ & ug/g dry & 6.91E0 & 2/20/08 & $8 F 11003$ & PNNL-AGG-ICP-AES \\
\hline $7440-47-3$ & Chromium & 1.60E1 & ug/g dry & 2.77E0 & 2/20/08 & 8F11003 & PNNL-AGG-ICP-AES \\
\hline $7440-50-8$ & Copper & $<2.95 \mathrm{E} 1$ & ug/g dry & 2.95E1 & 2/20/08 & $8 F 11003$ & PNNL-AGG-ICP-AES \\
\hline 7440-09-7 & Potassium & 1.33E3 & ug/g dry & $7.86 \mathrm{E} 1$ & 2/20/08 & 8F11003 & PNNL-AGG-ICP-AES \\
\hline 7439-95-4 & Magnesium & 4.68E3 & ug/g dry & $2.62 \mathrm{E} 1$ & 2/20/08 & 8F11003 & PNNL-AGG-ICP-AES \\
\hline $7439-96-5$ & Manganese & $4.94 \mathrm{E} 2$ & ug/g dry & $9.91 \mathrm{E}-1$ & 2/20/08 & 8F11003 & PNNL-AGG-ICP-AES \\
\hline $7440-02-0$ & Nickel & 1.73E1 & ug/g dry & $6.81 \mathrm{E} 0$ & $2 / 20 / 08$ & 8F11003 & PNNL-AGG-ICP-AES \\
\hline $7440-62-2$ & Vanadium & $1.17 \mathrm{E} 1$ & ug/g dry & $3.08 \mathrm{E} 0$ & 2/20/08 & 8F11003 & PNNL-AGG-ICP-AES \\
\hline $7440-66-6$ & Zinc & $3.20 \mathrm{E} 1$ & ug/g dry & 5.19E0 & 2/20/08 & $8 F 11003$ & PNNL-AGG-ICP-AES \\
\hline $7440-23-5$ & Sodium & $<8.87 \mathrm{E} 2$ & ug/g dry & 8.87E2 & $2 / 20 / 08$ & 8F11003 & PNNL-AGG-ICP-AES \\
\hline HEIS No. & B1RKW4 & \multicolumn{3}{|c|}{ Lab ID: $\quad$ 0802008-08 } & & & \\
\hline $7429-90-5$ & Aluminum & $9.66 \mathrm{E} 3$ & ug/g dry & 3.31E1 & 2/13/08 & 8F10003 & PNNL-AGG-ICP-AES \\
\hline $7440-39-3$ & Barium & $1.14 \mathrm{E} 2$ & ug/g dry & $3.16 \mathrm{E} 0$ & 2/13/08 & 8F10003 & PNNL-AGG-ICP-AES \\
\hline $7440-70-2$ & Calcium & $1.20 \mathrm{E} 4$ & ug/g dry & $1.23 \mathrm{E} 2$ & 2/13/08 & 8F10003 & PNNL-AGG-ICP-AES \\
\hline $7440-48-4$ & Cobalt & 9.95E0 & ug/g dry & $6.88 \mathrm{E} 0$ & 2/13/08 & $8 \mathrm{~F} 10003$ & PNNL-AGG-ICP-AES \\
\hline $7440-47-3$ & Chromium & $2.90 \mathrm{E} 1$ & ug/g dry & $2.75 \mathrm{E} 0$ & 2/13/08 & 8F10003 & PNNL-AGG-ICP-AES \\
\hline $7440-50-8$ & Copper & $<2.93 \mathrm{E} 1$ & ug/g dry & 2.93E1 & 2/13/08 & 8F10003 & PNNL-AGG-ICP-AES \\
\hline
\end{tabular}


Total Metals by PNNL-AGG-ICP-AES/Acid Extract

\begin{tabular}{|c|c|c|c|c|c|c|c|}
\hline CAS \# & Analyte & Results & Units & EQL & Analyzed & Batch & Method \\
\hline HEIS No. & B1RKW4 & \multicolumn{3}{|c|}{ Lab ID: $\quad$ 0802008-08 } & & & \\
\hline 7440-09-7 & Potassium & $2.01 \mathrm{E} 3$ & ug/g dry & 7.81E1 & 2/13/08 & 8F10003 & PNNL-AGG-ICP-AES \\
\hline 7439-95-4 & Magnesium & $6.45 \mathrm{E} 3$ & ug/g dry & $2.61 \mathrm{E} 1$ & 2/13/08 & 8F10003 & PNNL-AGG-ICP-AES \\
\hline $7439-96-5$ & Manganese & 3.61E2 & ug/g dry & $9.86 \mathrm{E}-1$ & 2/13/08 & 8F10003 & PNNL-AGG-ICP-AES \\
\hline $7440-02-0$ & Nickel & $2.35 \mathrm{E} 1$ & ug/g dry & 6.77E0 & 2/13/08 & 8F10003 & PNNL-AGG-ICP-AES \\
\hline $7440-62-2$ & Vanadium & $2.06 \mathrm{E} 1$ & ug/g dry & 3.07E0 & 2/13/08 & $8 \mathrm{~F} 10003$ & PNNL-AGG-ICP-AES \\
\hline $7440-66-6$ & Zinc & 4.63E1 & ug/g dry & 5.16E0 & 2/13/08 & 8F10003 & PNNL-AGG-ICP-AES \\
\hline $7440-23-5$ & Sodium & 1.97E3 & ug/g dry & 8.83E2 & 2/13/08 & 8F10003 & PNNL-AGG-ICP-AES \\
\hline HEIS No. & B1RKW5 & \multicolumn{3}{|c|}{ Lab ID: $\quad$ 0802009-02 } & & & \\
\hline 7429-90-5 & Aluminum & $5.87 \mathrm{E} 3$ & ug/g dry & $3.21 \mathrm{E} 1$ & 2/13/08 & $8 F 10003$ & PNNL-AGG-ICP-AES \\
\hline $7440-39-3$ & Barium & 6.62E1 & ug/g dry & 3.06E0 & 2/13/08 & 8F10003 & PNNL-AGG-ICP-AES \\
\hline $7440-70-2$ & Calcium & $9.91 \mathrm{E} 3$ & ug/g dry & 1.19E2 & 2/13/08 & 8F10003 & PNNL-AGG-ICP-AES \\
\hline $7440-48-4$ & Cobalt & $<6.67 \mathrm{E} 0$ & ug/g dry & 6.67E0 & 2/13/08 & 8F10003 & PNNL-AGG-ICP-AES \\
\hline $7440-47-3$ & Chromium & 1.46E1 & ug/g dry & 2.67E0 & 2/13/08 & 8F10003 & PNNL-AGG-ICP-AES \\
\hline $7440-50-8$ & Copper & $<2.85 \mathrm{E} 1$ & ug/g dry & $2.85 \mathrm{E} 1$ & 2/13/08 & $8 \mathrm{~F} 10003$ & PNNL-AGG-ICP-AES \\
\hline 7440-09-7 & Potassium & $1.23 \mathrm{E} 3$ & ug/g dry & $7.58 \mathrm{E} 1$ & 2/13/08 & 8F10003 & PNNL-AGG-ICP-AES \\
\hline 7439-95-4 & Magnesium & 4.39E3 & ug/g dry & $2.53 \mathrm{E} 1$ & 2/13/08 & 8F10003 & PNNL-AGG-ICP-AES \\
\hline $7439-96-5$ & Manganese & $2.42 \mathrm{E} 2$ & ug/g dry & $9.56 \mathrm{E}-1$ & 2/13/08 & 8F10003 & PNNL-AGG-ICP-AES \\
\hline 7440-02-0 & Nickel & $1.47 \mathrm{E} 1$ & ug/g dry & $6.57 \mathrm{E} 0$ & 2/13/08 & $8 F 10003$ & PNNL-AGG-ICP-AES \\
\hline $7440-62-2$ & Vanadium & $1.08 \mathrm{E} 1$ & ug/g dry & 2.97E0 & 2/13/08 & 8F10003 & PNNL-AGG-ICP-AES \\
\hline $7440-66-6$ & Zinc & 2.99E1 & ug/g dry & $5.01 \mathrm{E} 0$ & 2/13/08 & $8 \mathrm{~F} 10003$ & PNNL-AGG-ICP-AES \\
\hline $7440-23-5$ & Sodium & $<8.56 \mathrm{E} 2$ & ug/g dry & 8.56E2 & 2/13/08 & 8F10003 & PNNL-AGG-ICP-AES \\
\hline HEIS No. & B1RKW6 & \multicolumn{3}{|c|}{ Lab ID: $\quad$ 0802009-04 } & & & \\
\hline $7429-90-5$ & Aluminum & 5.53E3 & ug/g dry & $3.44 \mathrm{E} 1$ & 2/13/08 & $8 \mathrm{~F} 10003$ & PNNL-AGG-ICP-AES \\
\hline $7440-39-3$ & Barium & $6.15 \mathrm{E} 1$ & ug/g dry & 3.27E0 & 2/13/08 & 8F10003 & PNNL-AGG-ICP-AES \\
\hline $7440-70-2$ & Calcium & $9.61 \mathrm{E} 3$ & ug/g dry & $1.28 \mathrm{E} 2$ & 2/13/08 & 8F10003 & PNNL-AGG-ICP-AES \\
\hline $7440-48-4$ & Cobalt & $<7.13 \mathrm{E} 0$ & ug/g dry & 7.13E0 & 2/13/08 & $8 \mathrm{~F} 10003$ & PNNL-AGG-ICP-AES \\
\hline $7440-47-3$ & Chromium & $1.55 \mathrm{E} 1$ & ug/g dry & $2.86 \mathrm{E} 0$ & 2/13/08 & $8 \mathrm{~F} 10003$ & PNNL-AGG-ICP-AES \\
\hline $7440-50-8$ & Copper & $<3.05 \mathrm{E} 1$ & ug/g dry & $3.05 \mathrm{E} 1$ & 2/13/08 & 8F10003 & PNNL-AGG-ICP-AES \\
\hline 7440-09-7 & Potassium & $1.18 \mathrm{E} 3$ & ug/g dry & 8.11E1 & 2/13/08 & 8F10003 & PNNL-AGG-ICP-AES \\
\hline 7439-95-4 & Magnesium & $4.20 \mathrm{E} 3$ & ug/g dry & $2.70 \mathrm{E} 1$ & 2/13/08 & $8 F 10003$ & PNNL-AGG-ICP-AES \\
\hline $7439-96-5$ & Manganese & $2.46 \mathrm{E} 2$ & ug/g dry & $1.02 \mathrm{E} 0$ & 2/13/08 & 8F10003 & PNNL-AGG-ICP-AES \\
\hline $7440-02-0$ & Nickel & $1.41 \mathrm{E} 1$ & ug/g dry & 7.03E0 & 2/13/08 & $8 \mathrm{~F} 10003$ & PNNL-AGG-ICP-AES \\
\hline $7440-62-2$ & Vanadium & 1.03E1 & ug/g dry & $3.18 \mathrm{E} 0$ & 2/13/08 & 8F10003 & PNNL-AGG-ICP-AES \\
\hline $7440-66-6$ & Zinc & 2.92E1 & ug/g dry & 5.36E0 & 2/13/08 & $8 \mathrm{~F} 10003$ & PNNL-AGG-ICP-AES \\
\hline $7440-23-5$ & Sodium & $<9.16 \mathrm{E} 2$ & ug/g dry & $9.16 \mathrm{E} 2$ & 2/13/08 & 8F10003 & PNNL-AGG-ICP-AES \\
\hline HEIS No. & B1TFV6 & \multicolumn{3}{|c|}{ Lab ID: $\quad$ 0802028-03 } & & & \\
\hline $7429-90-5$ & Aluminum & $9.98 \mathrm{E} 3$ & ug/g dry & 4.36E1 & 6/13/08 & 8E12002 & PNNL-AGG-ICP-AES \\
\hline $7440-39-3$ & Barium & 7.27E1 & ug/g dry & 4.16E0 & 6/13/08 & 8E12002 & PNNL-AGG-ICP-AES \\
\hline $7440-70-2$ & Calcium & $9.80 \mathrm{E} 3$ & ug/g dry & 1.62E2 & 6/13/08 & 8E12002 & PNNL-AGG-ICP-AES \\
\hline $7440-48-4$ & Cobalt & $<9.05 \mathrm{E} 0$ & ug/g dry & $9.05 \mathrm{E} 0$ & 6/13/08 & 8E12002 & PNNL-AGG-ICP-AES \\
\hline $7440-47-3$ & Chromium & $1.82 \mathrm{E} 1$ & ug/g dry & 3.63E0 & 6/13/08 & 8E12002 & PNNL-AGG-ICP-AES \\
\hline $7440-50-8$ & Copper & $<3.86 \mathrm{E} 1$ & ug/g dry & 3.86E1 & 6/13/08 & 8E12002 & PNNL-AGG-ICP-AES \\
\hline $7440-09-7$ & Potassium & $1.84 \mathrm{E} 3$ & ug/g dry & $1.03 \mathrm{E} 2$ & 6/13/08 & 8E12002 & PNNL-AGG-ICP-AES \\
\hline 7439-95-4 & Magnesium & $6.06 \mathrm{E} 3$ & ug/g dry & 3.43E1 & 6/13/08 & 8E12002 & PNNL-AGG-ICP-AES \\
\hline $7439-96-5$ & Manganese & $4.00 \mathrm{E} 2$ & ug/g dry & $1.30 \mathrm{E} 0$ & 6/13/08 & 8E12002 & PNNL-AGG-ICP-AES \\
\hline $7440-02-0$ & Nickel & $2.15 \mathrm{E} 1$ & ug/g dry & 8.92E0 & 6/13/08 & 8E12002 & PNNL-AGG-ICP-AES \\
\hline $7440-62-2$ & Vanadium & $1.91 \mathrm{E} 1$ & ug/g dry & 4.04E0 & 6/13/08 & 8E12002 & PNNL-AGG-ICP-AES \\
\hline $7440-66-6$ & Zinc & 4.61E1 & ug/g dry & $6.80 \mathrm{E} 0$ & 6/13/08 & 8E12002 & PNNL-AGG-ICP-AES \\
\hline
\end{tabular}


Total Metals by PNNL-AGG-ICP-AES/Acid Extract

\begin{tabular}{|c|c|c|c|c|c|c|c|}
\hline CAS \# & Analyte & Results & Units & EQL & Analyzed & Batch & Method \\
\hline HEIS No. & B1TFV6 & \multicolumn{3}{|c|}{ Lab ID: $\quad$ 0802028-03 } & & & \\
\hline $7440-23-5$ & Sodium & $<1.16 \mathrm{E} 3$ & ug/g dry & $1.16 \mathrm{E} 3$ & 6/13/08 & 8E12002 & PNNL-AGG-ICP-AES \\
\hline HEIS No. & B1TFV8 & \multicolumn{3}{|c|}{ Lab ID: $\quad$ 0802028-05 } & & & \\
\hline 7429-90-5 & Aluminum & 6.13E3 & ug/g dry & 4.10E1 & 6/13/08 & 8E12002 & PNNL-AGG-ICP-AES \\
\hline $7440-39-3$ & Barium & 6.75E1 & ug/g dry & $3.90 \mathrm{E} 0$ & 6/13/08 & 8E12002 & PNNL-AGG-ICP-AES \\
\hline $7440-70-2$ & Calcium & 8.77E3 & ug/g dry & $1.52 \mathrm{E} 2$ & 6/13/08 & 8E12002 & PNNL-AGG-ICP-AES \\
\hline $7440-48-4$ & Cobalt & $<8.50 \mathrm{E} 0$ & ug/g dry & $8.50 \mathrm{E} 0$ & 6/13/08 & 8E12002 & PNNL-AGG-ICP-AES \\
\hline $7440-47-3$ & Chromium & $1.25 \mathrm{E} 1$ & ug/g dry & 3.41E0 & 6/13/08 & 8E12002 & PNNL-AGG-ICP-AES \\
\hline $7440-50-8$ & Copper & $<3.63 \mathrm{E} 1$ & ug/g dry & 3.63E1 & 6/13/08 & 8E12002 & PNNL-AGG-ICP-AES \\
\hline 7440-09-7 & Potassium & $1.25 \mathrm{E} 3$ & ug/g dry & $9.66 \mathrm{E} 1$ & 6/13/08 & 8E12002 & PNNL-AGG-ICP-AES \\
\hline 7439-95-4 & Magnesium & 4.27E3 & ug/g dry & $3.22 \mathrm{E} 1$ & 6/13/08 & 8E12002 & PNNL-AGG-ICP-AES \\
\hline 7439-96-5 & Manganese & 2.98E2 & ug/g dry & $1.22 \mathrm{E} 0$ & 6/13/08 & 8E12002 & PNNL-AGG-ICP-AES \\
\hline $7440-02-0$ & Nickel & 1.37E1 & ug/g dry & 8.38E0 & 6/13/08 & 8E12002 & PNNL-AGG-ICP-AES \\
\hline $7440-62-2$ & Vanadium & $1.40 \mathrm{E} 1$ & ug/g dry & 3.79E0 & 6/13/08 & 8E12002 & PNNL-AGG-ICP-AES \\
\hline 7440-66-6 & Zinc & $2.82 \mathrm{E} 1$ & ug/g dry & $6.39 \mathrm{E} 0$ & 6/13/08 & 8E12002 & PNNL-AGG-ICP-AES \\
\hline $7440-23-5$ & Sodium & $<1.09 \mathrm{E} 3$ & ug/g dry & $1.09 \mathrm{E} 3$ & 6/13/08 & 8E12002 & PNNL-AGG-ICP-AES \\
\hline HEIS No. & B1TFW0 & \multicolumn{3}{|c|}{ Lab ID: $\quad$ 0802028-07 } & & & \\
\hline 7429-90-5 & Aluminum & 5.64E3 & ug/g dry & 4.27E1 & 6/13/08 & 8E12002 & PNNL-AGG-ICP-AES \\
\hline 7440-39-3 & Barium & $6.26 \mathrm{E} 1$ & ug/g dry & 4.07E0 & 6/13/08 & 8E12002 & PNNL-AGG-ICP-AES \\
\hline $7440-70-2$ & Calcium & $1.02 \mathrm{E} 4$ & ug/g dry & $1.59 \mathrm{E} 2$ & 6/13/08 & 8E12002 & PNNL-AGG-ICP-AES \\
\hline $7440-48-4$ & Cobalt & $<8.86 \mathrm{E} 0$ & ug/g dry & 8.86E0 & 6/13/08 & 8E12002 & PNNL-AGG-ICP-AES \\
\hline $7440-47-3$ & Chromium & $1.00 \mathrm{E} 1$ & ug/g dry & 3.55E0 & 6/13/08 & 8E12002 & PNNL-AGG-ICP-AES \\
\hline $7440-50-8$ & Copper & $<3.78 \mathrm{E} 1$ & ug/g dry & 3.78E1 & 6/13/08 & 8E12002 & PNNL-AGG-ICP-AES \\
\hline $7440-09-7$ & Potassium & $1.24 \mathrm{E} 3$ & ug/g dry & $1.01 \mathrm{E} 2$ & 6/13/08 & 8E12002 & PNNL-AGG-ICP-AES \\
\hline 7439-95-4 & Magnesium & $4.41 \mathrm{E} 3$ & ug/g dry & 3.36E1 & 6/13/08 & 8E12002 & PNNL-AGG-ICP-AES \\
\hline 7439-96-5 & Manganese & $2.31 \mathrm{E} 2$ & ug/g dry & $1.27 \mathrm{E} 0$ & 6/13/08 & 8E12002 & PNNL-AGG-ICP-AES \\
\hline 7440-02-0 & Nickel & $1.32 \mathrm{E} 1$ & ug/g dry & 8.73E0 & 6/13/08 & 8E12002 & PNNL-AGG-ICP-AES \\
\hline $7440-62-2$ & Vanadium & 1.19E1 & ug/g dry & 3.95E0 & 6/13/08 & 8E12002 & PNNL-AGG-ICP-AES \\
\hline $7440-66-6$ & Zinc & $2.79 \mathrm{E} 1$ & ug/g dry & $6.66 \mathrm{E} 0$ & 6/13/08 & 8E12002 & PNNL-AGG-ICP-AES \\
\hline $7440-23-5$ & Sodium & $<1.14 \mathrm{E} 3$ & ug/g dry & $1.14 \mathrm{E} 3$ & 6/13/08 & 8E12002 & PNNL-AGG-ICP-AES \\
\hline HEIS No. & B1TFW2 & \multicolumn{3}{|c|}{ Lab ID: $\quad$ 0802028-09 } & & & \\
\hline 7429-90-5 & Aluminum & $9.69 \mathrm{E} 3$ & ug/g dry & 4.42E1 & 6/13/08 & 8E12002 & PNNL-AGG-ICP-AES \\
\hline $7440-39-3$ & Barium & 1.19E2 & ug/g dry & $4.21 \mathrm{E} 0$ & 6/13/08 & 8E12002 & PNNL-AGG-ICP-AES \\
\hline $7440-70-2$ & Calcium & $1.06 \mathrm{E} 4$ & ug/g dry & $1.64 \mathrm{E} 2$ & 6/13/08 & 8E12002 & PNNL-AGG-ICP-AES \\
\hline $7440-48-4$ & Cobalt & $<9.18 \mathrm{E} 0$ & ug/g dry & $9.18 \mathrm{E} 0$ & 6/13/08 & 8E12002 & PNNL-AGG-ICP-AES \\
\hline $7440-47-3$ & Chromium & $1.42 \mathrm{E} 1$ & ug/g dry & $3.68 \mathrm{E} 0$ & 6/13/08 & 8E12002 & PNNL-AGG-ICP-AES \\
\hline $7440-50-8$ & Copper & $<3.92 \mathrm{E} 1$ & ug/g dry & 3.92E1 & 6/13/08 & 8E12002 & PNNL-AGG-ICP-AES \\
\hline 7440-09-7 & Potassium & $2.00 \mathrm{E} 3$ & ug/g dry & $1.04 \mathrm{E} 2$ & 6/13/08 & 8E12002 & PNNL-AGG-ICP-AES \\
\hline 7439-95-4 & Magnesium & $6.12 \mathrm{E} 3$ & ug/g dry & 3.48E1 & 6/13/08 & 8E12002 & PNNL-AGG-ICP-AES \\
\hline 7439-96-5 & Manganese & 4.27E2 & ug/g dry & 1.32E0 & 6/13/08 & 8E12002 & PNNL-AGG-ICP-AES \\
\hline $7440-02-0$ & Nickel & $1.77 \mathrm{E} 1$ & ug/g dry & $9.05 \mathrm{E} 0$ & 6/13/08 & 8E12002 & PNNL-AGG-ICP-AES \\
\hline $7440-62-2$ & Vanadium & $1.66 \mathrm{E} 1$ & ug/g dry & 4.09E0 & 6/13/08 & 8E12002 & PNNL-AGG-ICP-AES \\
\hline 7440-66-6 & Zinc & $4.37 \mathrm{E} 1$ & ug/g dry & $6.90 \mathrm{E} 0$ & 6/13/08 & 8E12002 & PNNL-AGG-ICP-AES \\
\hline $7440-23-5$ & Sodium & $<1.18 \mathrm{E} 3$ & ug/g dry & $1.18 \mathrm{E} 3$ & 6/13/08 & 8E12002 & PNNL-AGG-ICP-AES \\
\hline HEIS No. & B1RKX0 & \multicolumn{3}{|c|}{ Lab ID: $\quad$ 0802028-11 } & & & \\
\hline 7429-90-5 & Aluminum & 7.95E3 & ug/g dry & 4.33E1 & 6/13/08 & 8E12002 & PNNL-AGG-ICP-AES \\
\hline $7440-39-3$ & Barium & 8.38E1 & ug/g dry & 4.13E0 & 6/13/08 & 8E12002 & PNNL-AGG-ICP-AES \\
\hline $7440-70-2$ & Calcium & $1.42 \mathrm{E} 4$ & ug/g dry & $1.61 \mathrm{E} 2$ & 6/13/08 & 8E12002 & PNNL-AGG-ICP-AES \\
\hline $7440-48-4$ & Cobalt & $<8.99 \mathrm{E} 0$ & ug/g dry & 8.99E0 & 6/13/08 & 8E12002 & PNNL-AGG-ICP-AES \\
\hline
\end{tabular}


Total Metals by PNNL-AGG-ICP-AES/Acid Extract

\begin{tabular}{|c|c|c|c|c|c|c|c|}
\hline CAS \# & Analyte & Results & Units & EQL & Analyzed & Batch & Method \\
\hline HEIS No. & B1RKX0 & \multicolumn{3}{|c|}{ Lab ID: $\quad$ 0802028-11 } & & & \\
\hline $7440-47-3$ & Chromium & $1.20 \mathrm{E} 1$ & ug/g dry & $3.60 \mathrm{E} 0$ & 6/13/08 & 8E12002 & PNNL-AGG-ICP-AES \\
\hline $7440-50-8$ & Copper & $<3.84 \mathrm{E} 1$ & ug/g dry & 3.84E1 & 6/13/08 & 8E12002 & PNNL-AGG-ICP-AES \\
\hline 7440-09-7 & Potassium & 1.67E3 & ug/g dry & $1.02 \mathrm{E} 2$ & 6/13/08 & 8E12002 & PNNL-AGG-ICP-AES \\
\hline 7439-95-4 & Magnesium & $5.44 \mathrm{E} 3$ & ug/g dry & $3.41 \mathrm{E} 1$ & 6/13/08 & 8E12002 & PNNL-AGG-ICP-AES \\
\hline $7439-96-5$ & Manganese & $2.32 \mathrm{E} 2$ & ug/g dry & $1.29 \mathrm{E} 0$ & 6/13/08 & 8E12002 & PNNL-AGG-ICP-AES \\
\hline 7440-02-0 & Nickel & 1.03E1 & ug/g dry & 8.86E0 & 6/13/08 & 8E12002 & PNNL-AGG-ICP-AES \\
\hline $7440-62-2$ & Vanadium & $1.22 \mathrm{E} 1$ & ug/g dry & 4.01E0 & 6/13/08 & 8E12002 & PNNL-AGG-ICP-AES \\
\hline $7440-66-6$ & Zinc & $4.08 \mathrm{E} 1$ & ug/g dry & $6.75 \mathrm{E} 0$ & 6/13/08 & 8E12002 & PNNL-AGG-ICP-AES \\
\hline $7440-23-5$ & Sodium & $<1.15 \mathrm{E} 3$ & ug/g dry & $1.15 \mathrm{E} 3$ & 6/13/08 & 8E12002 & PNNL-AGG-ICP-AES \\
\hline HEIS No. & B1RKX3 & \multicolumn{3}{|c|}{ Lab ID: $\quad$ 0802028-17 } & & & \\
\hline $7429-90-5$ & Aluminum & 4.75E3 & ug/g dry & 3.67E1 & 6/13/08 & 8E12002 & PNNL-AGG-ICP-AES \\
\hline $7440-39-3$ & Barium & $6.29 \mathrm{E} 1$ & ug/g dry & $3.49 \mathrm{E} 0$ & 6/13/08 & 8E12002 & PNNL-AGG-ICP-AES \\
\hline $7440-70-2$ & Calcium & $4.98 \mathrm{E} 3$ & ug/g dry & $1.36 \mathrm{E} 2$ & 6/13/08 & 8E12002 & PNNL-AGG-ICP-AES \\
\hline $7440-48-4$ & Cobalt & $<7.61 \mathrm{E} 0$ & ug/g dry & 7.61E0 & 6/13/08 & 8E12002 & PNNL-AGG-ICP-AES \\
\hline $7440-47-3$ & Chromium & 5.18E0 & ug/g dry & 3.05E0 & 6/13/08 & 8E12002 & PNNL-AGG-ICP-AES \\
\hline $7440-50-8$ & Copper & $<3.25 \mathrm{E} 1$ & ug/g dry & $3.25 \mathrm{E} 1$ & 6/13/08 & 8E12002 & PNNL-AGG-ICP-AES \\
\hline 7440-09-7 & Potassium & 8.35E2 & ug/g dry & 8.65E1 & 6/13/08 & 8E12002 & PNNL-AGG-ICP-AES \\
\hline 7439-95-4 & Magnesium & 3.30E3 & ug/g dry & $2.88 \mathrm{E} 1$ & 6/13/08 & 8E12002 & PNNL-AGG-ICP-AES \\
\hline $7439-96-5$ & Manganese & 2.16E2 & ug/g dry & $1.09 \mathrm{E} 0$ & 6/13/08 & 8E12002 & PNNL-AGG-ICP-AES \\
\hline $7440-02-0$ & Nickel & $<7.49 \mathrm{E} 0$ & ug/g dry & 7.49E0 & 6/13/08 & 8E12002 & PNNL-AGG-ICP-AES \\
\hline $7440-62-2$ & Vanadium & $1.78 \mathrm{E} 1$ & ug/g dry & 3.39E0 & 6/13/08 & 8E12002 & PNNL-AGG-ICP-AES \\
\hline $7440-66-6$ & Zinc & $2.59 \mathrm{E} 1$ & ug/g dry & 5.71E0 & 6/13/08 & 8E12002 & PNNL-AGG-ICP-AES \\
\hline $7440-23-5$ & Sodium & $<9.77 \mathrm{E} 2$ & ug/g dry & $9.77 \mathrm{E} 2$ & 6/13/08 & 8E12002 & PNNL-AGG-ICP-AES \\
\hline HEIS No. & B1RKX5 & \multicolumn{3}{|c|}{ Lab ID: $\quad$ 0802028-23 } & & & \\
\hline 7429-90-5 & Aluminum & 4.43E3 & ug/g dry & 3.69E1 & 6/13/08 & 8E12002 & PNNL-AGG-ICP-AES \\
\hline $7440-39-3$ & Barium & $5.21 \mathrm{E} 1$ & ug/g dry & 3.51E0 & 6/13/08 & 8E12002 & PNNL-AGG-ICP-AES \\
\hline $7440-70-2$ & Calcium & $4.86 \mathrm{E} 3$ & ug/g dry & $1.37 \mathrm{E} 2$ & 6/13/08 & 8E12002 & PNNL-AGG-ICP-AES \\
\hline $7440-48-4$ & Cobalt & $<7.65 \mathrm{E} 0$ & ug/g dry & 7.65E0 & 6/13/08 & 8E12002 & PNNL-AGG-ICP-AES \\
\hline $7440-47-3$ & Chromium & 5.93E0 & ug/g dry & $3.06 \mathrm{E} 0$ & 6/13/08 & 8E12002 & PNNL-AGG-ICP-AES \\
\hline $7440-50-8$ & Copper & $<3.26 \mathrm{E} 1$ & ug/g dry & $3.26 \mathrm{E} 1$ & 6/13/08 & 8E12002 & PNNL-AGG-ICP-AES \\
\hline 7440-09-7 & Potassium & 8.16E2 & ug/g dry & 8.69E1 & 6/13/08 & 8E12002 & PNNL-AGG-ICP-AES \\
\hline 7439-95-4 & Magnesium & 3.00E3 & ug/g dry & $2.90 \mathrm{E} 1$ & 6/13/08 & 8E12002 & PNNL-AGG-ICP-AES \\
\hline $7439-96-5$ & Manganese & $1.98 \mathrm{E} 2$ & ug/g dry & $1.10 \mathrm{E} 0$ & 6/13/08 & 8E12002 & PNNL-AGG-ICP-AES \\
\hline $7440-02-0$ & Nickel & $<7.54 \mathrm{E} 0$ & ug/g dry & $7.54 \mathrm{E} 0$ & 6/13/08 & 8E12002 & PNNL-AGG-ICP-AES \\
\hline $7440-62-2$ & Vanadium & $1.90 \mathrm{E} 1$ & ug/g dry & $3.41 \mathrm{E} 0$ & 6/13/08 & 8E12002 & PNNL-AGG-ICP-AES \\
\hline $7440-66-6$ & Zinc & $2.32 \mathrm{E} 1$ & ug/g dry & $5.75 \mathrm{E} 0$ & 6/13/08 & 8E12002 & PNNL-AGG-ICP-AES \\
\hline $7440-23-5$ & Sodium & $<9.82 \mathrm{E} 2$ & ug/g dry & $9.82 \mathrm{E} 2$ & 6/13/08 & 8E12002 & PNNL-AGG-ICP-AES \\
\hline HEIS No. & B1RKX7 & \multicolumn{3}{|c|}{ Lab ID: $\quad$ 0802028-29 } & & & \\
\hline $7429-90-5$ & Aluminum & 3.29E3 & ug/g dry & $3.70 \mathrm{E} 1$ & 6/13/08 & 8E12002 & PNNL-AGG-ICP-AES \\
\hline $7440-39-3$ & Barium & $4.31 \mathrm{E} 1$ & ug/g dry & 3.52E0 & 6/13/08 & 8E12002 & PNNL-AGG-ICP-AES \\
\hline $7440-70-2$ & Calcium & $3.74 \mathrm{E} 3$ & ug/g dry & $1.37 \mathrm{E} 2$ & 6/13/08 & 8E12002 & PNNL-AGG-ICP-AES \\
\hline $7440-48-4$ & Cobalt & $<7.67 \mathrm{E} 0$ & ug/g dry & 7.67E0 & 6/13/08 & 8E12002 & PNNL-AGG-ICP-AES \\
\hline $7440-47-3$ & Chromium & $5.26 \mathrm{E} 0$ & ug/g dry & 3.07E0 & 6/13/08 & 8E12002 & PNNL-AGG-ICP-AES \\
\hline $7440-50-8$ & Copper & $<3.27 \mathrm{E} 1$ & ug/g dry & $3.27 \mathrm{E} 1$ & 6/13/08 & 8E12002 & PNNL-AGG-ICP-AES \\
\hline $7440-09-7$ & Potassium & 4.77E2 & ug/g dry & 8.72E1 & 6/13/08 & 8E12002 & PNNL-AGG-ICP-AES \\
\hline 7439-95-4 & Magnesium & $2.40 \mathrm{E} 3$ & ug/g dry & 2.91E1 & 6/13/08 & 8E12002 & PNNL-AGG-ICP-AES \\
\hline $7439-96-5$ & Manganese & $1.62 \mathrm{E} 2$ & ug/g dry & $1.10 \mathrm{E} 0$ & 6/13/08 & 8E12002 & PNNL-AGG-ICP-AES \\
\hline $7440-02-0$ & Nickel & $<7.56 \mathrm{E} 0$ & ug/g dry & $7.56 \mathrm{E} 0$ & 6/13/08 & 8E12002 & PNNL-AGG-ICP-AES \\
\hline
\end{tabular}


Total Metals by PNNL-AGG-ICP-AES/Acid Extract

\begin{tabular}{|c|c|c|c|c|c|c|c|}
\hline CAS \# & Analyte & Results & Units & EQL & Analyzed & Batch & Method \\
\hline HEIS No. & B1RKX7 & \multicolumn{3}{|c|}{ Lab ID: $\quad$ 0802028-29 } & & & \\
\hline $7440-62-2$ & Vanadium & $1.53 \mathrm{E} 1$ & ug/g dry & $3.42 \mathrm{E} 0$ & 6/13/08 & 8E12002 & PNNL-AGG-ICP-AES \\
\hline $7440-66-6$ & Zinc & 2.06E1 & ug/g dry & 5.76E0 & $6 / 13 / 08$ & 8E12002 & PNNL-AGG-ICP-AES \\
\hline $7440-23-5$ & Sodium & $<9.85 \mathrm{E} 2$ & ug/g dry & $9.85 \mathrm{E} 2$ & $6 / 13 / 08$ & 8E12002 & PNNL-AGG-ICP-AES \\
\hline
\end{tabular}




\section{Radionuclides by ICP-MS/Acid Extract}

\begin{tabular}{|c|c|c|c|c|c|c|c|}
\hline CAS \# & Analyte & Results & Units & EQL & Analyzed & Batch & Method \\
\hline \multirow{3}{*}{$\begin{array}{l}\text { HEIS No. } \\
14133-76-7\end{array}$} & B1RKL1 & \multicolumn{3}{|c|}{ Lab ID: $\quad$ 0802002-02 } & & & \\
\hline & Technetium-99 & $<9.60 \mathrm{E}-4$ & ug/g dry & $9.60 \mathrm{E}-4$ & 2/20/08 & 8E01017 & PNNL-AGG-415 \\
\hline & Uranium 238 & 3.43E-1 & ug/g dry & 6.97E-3 & $2 / 20 / 08$ & 8E01017 & PNNL-AGG-415 \\
\hline \multirow{3}{*}{$\begin{array}{l}\text { HEIS No. } \\
\text { 14133-76-7 }\end{array}$} & B1RKL6 & \multicolumn{3}{|c|}{ Lab ID: $\quad$ 0802002-09 } & & & \\
\hline & Technetium-99 & $<9.63 \mathrm{E}-4$ & ug/g dry & $9.63 \mathrm{E}-4$ & 2/12/08 & 8E01013 & PNNL-AGG-415 \\
\hline & Uranium 238 & 3.99E-1 & ug/g dry & $6.99 \mathrm{E}-3$ & $2 / 12 / 08$ & 8E01013 & PNNL-AGG-415 \\
\hline \multirow{3}{*}{$\begin{array}{l}\text { HEIS No. } \\
14133-76-7\end{array}$} & B1RKM1 & \multicolumn{3}{|c|}{ Lab ID: $\quad$ 0802002-17 } & & & \\
\hline & Technetium-99 & $<9.01 \mathrm{E}-4$ & ug/g dry & $9.01 \mathrm{E}-4$ & $2 / 20 / 08$ & 8E01017 & PNNL-AGG-415 \\
\hline & Uranium 238 & 3.81E-1 & ug/g dry & $6.54 \mathrm{E}-3$ & $2 / 20 / 08$ & 8E01017 & PNNL-AGG-415 \\
\hline \multirow{3}{*}{$\begin{array}{l}\text { HEIS No. } \\
14133-76-7\end{array}$} & B1RKM8 & \multicolumn{3}{|c|}{ Lab ID: $\quad$ 0802002-31 } & & & \\
\hline & Technetium-99 & $<9.12 \mathrm{E}-4$ & ug/g dry & $9.12 \mathrm{E}-4$ & $2 / 12 / 08$ & 8E01013 & PNNL-AGG-415 \\
\hline & Uranium 238 & $3.50 \mathrm{E}-1$ & ug/g dry & $6.62 \mathrm{E}-3$ & $2 / 12 / 08$ & 8E01013 & PNNL-AGG-415 \\
\hline \multirow{3}{*}{$\begin{array}{l}\text { HEIS No. } \\
14133-76-7\end{array}$} & B1RKW7 & \multicolumn{3}{|c|}{ Lab ID: $\quad$ 0802003-02 } & & & \\
\hline & Technetium-99 & $1.31 \mathrm{E}-3$ & ug/g dry & $1.18 \mathrm{E}-3$ & $2 / 20 / 08$ & 8E01017 & PNNL-AGG-415 \\
\hline & Uranium 238 & $4.81 \mathrm{E} 0$ & ug/g dry & 8.59E-3 & $2 / 20 / 08$ & 8E01017 & PNNL-AGG-415 \\
\hline \multirow{3}{*}{$\begin{array}{l}\text { HEIS No. } \\
14133-76-7\end{array}$} & B1RKW8 & \multicolumn{3}{|c|}{ Lab ID: $\quad$ 0802003-04 } & & & \\
\hline & Technetium-99 & $1.36 \mathrm{E}-3$ & ug/g dry & $1.16 \mathrm{E}-3$ & $2 / 12 / 08$ & 8E01013 & PNNL-AGG-415 \\
\hline & Uranium 238 & $1.24 \mathrm{E} 0$ & ug/g dry & $8.42 \mathrm{E}-3$ & $2 / 12 / 08$ & 8E01013 & PNNL-AGG-415 \\
\hline \multirow{3}{*}{$\begin{array}{l}\text { HEIS No. } \\
14133-76-7\end{array}$} & B1RKN7 & \multicolumn{3}{|c|}{ Lab ID: $\quad$ 0802005-03 } & & & \\
\hline & Technetium-99 & $<8.55 \mathrm{E}-4$ & ug/g dry & 8.55E-4 & $2 / 20 / 08$ & 8E01017 & PNNL-AGG-415 \\
\hline & Uranium 238 & 3.09E-1 & ug/g dry & $6.20 \mathrm{E}-3$ & $2 / 20 / 08$ & 8E01017 & PNNL-AGG-415 \\
\hline \multirow{3}{*}{$\begin{array}{l}\text { HEIS No. } \\
\text { 14133-76-7 }\end{array}$} & B1RKP3 & \multicolumn{3}{|c|}{ Lab ID: $\quad$ 0802005-09 } & & & \\
\hline & Technetium-99 & $<9.51 \mathrm{E}-4$ & ug/g dry & $9.51 \mathrm{E}-4$ & 2/12/08 & 8E01013 & PNNL-AGG-415 \\
\hline & Uranium 238 & 3.09E-1 & ug/g dry & $6.91 \mathrm{E}-3$ & $2 / 12 / 08$ & 8E01013 & PNNL-AGG-415 \\
\hline \multirow{3}{*}{$\begin{array}{l}\text { HEIS No. } \\
14133-76-7\end{array}$} & B1RKP9 & \multicolumn{3}{|c|}{ Lab ID: $\quad$ 0802005-15 } & & & \\
\hline & Technetium-99 & $<9.50 \mathrm{E}-4$ & ug/g dry & $9.50 \mathrm{E}-4$ & $2 / 20 / 08$ & 8E01017 & PNNL-AGG-415 \\
\hline & Uranium 238 & $3.84 \mathrm{E}-1$ & ug/g dry & $6.90 \mathrm{E}-3$ & $2 / 20 / 08$ & 8E01017 & PNNL-AGG-415 \\
\hline \multirow{3}{*}{$\begin{array}{l}\text { HEIS No. } \\
14133-76-7\end{array}$} & B1RKR5 & \multicolumn{3}{|c|}{ Lab ID: $\quad$ 0802005-21 } & & & \\
\hline & Technetium-99 & $<1.03 \mathrm{E}-3$ & ug/g dry & $1.03 \mathrm{E}-3$ & $2 / 12 / 08$ & 8E01013 & PNNL-AGG-415 \\
\hline & Uranium 238 & 3.63E-1 & ug/g dry & $7.51 \mathrm{E}-3$ & $2 / 12 / 08$ & 8E01013 & PNNL-AGG-415 \\
\hline \multirow{3}{*}{$\begin{array}{l}\text { HEIS No. } \\
14133-76-7\end{array}$} & B1RKR9 & \multicolumn{3}{|c|}{ Lab ID: $\quad$ 0802005-25 } & & & \\
\hline & Technetium-99 & $<1.00 \mathrm{E}-3$ & ug/g dry & $1.00 \mathrm{E}-3$ & $2 / 20 / 08$ & 8E01017 & PNNL-AGG-415 \\
\hline & Uranium 238 & 3.20E-1 & ug/g dry & $7.26 \mathrm{E}-3$ & $2 / 20 / 08$ & 8E01017 & PNNL-AGG-415 \\
\hline \multirow{3}{*}{$\begin{array}{l}\text { HEIS No. } \\
14133-76-7\end{array}$} & B1RKT3 & \multicolumn{3}{|c|}{ Lab ID: $\quad$ 0802005-29 } & & & \\
\hline & Technetium-99 & $<1.06 \mathrm{E}-3$ & ug/g dry & $1.06 \mathrm{E}-3$ & $2 / 12 / 08$ & 8E01013 & PNNL-AGG-415 \\
\hline & Uranium 238 & $3.45 \mathrm{E}-1$ & ug/g dry & 7.67E-3 & $2 / 12 / 08$ & 8E01013 & PNNL-AGG-415 \\
\hline HEIS No. & B1RKT7 & & b ID: & 2006-07 & & & \\
\hline 14133-76-7 & Technetium-99 & $<1.03 E-3$ & ug/g dry & $1.03 \mathrm{E}-3$ & $2 / 20 / 08$ & 8E01017 & PNNL-AGG-415 \\
\hline & Uranium 238 & $3.56 \mathrm{E}-1$ & ug/g dry & $7.50 \mathrm{E}-3$ & 2/20/08 & 8E01017 & PNNL-AGG-415 \\
\hline HEIS No. & B1RKV1 & & b ID: & 2006-11 & & & \\
\hline $14133-76-7$ & Technetium-99 & $<1.01 \mathrm{E}-3$ & ug/g dry & $1.01 \mathrm{E}-3$ & $2 / 12 / 08$ & 8E01013 & PNNL-AGG-415 \\
\hline
\end{tabular}




\section{Radionuclides by ICP-MS/Acid Extract}

\begin{tabular}{|c|c|c|c|c|c|c|c|}
\hline CAS \# & Analyte & Results & Units & EQL & Analyzed & Batch & Method \\
\hline \multirow[t]{2}{*}{ HEIS No. } & B1RKV1 & \multicolumn{3}{|c|}{ Lab ID: $\quad$ 0802006-11 } & & & \\
\hline & Uranium 238 & 3.37E-1 & ug/g dry & $7.31 \mathrm{E}-3$ & $2 / 12 / 08$ & 8E01013 & PNNL-AGG-415 \\
\hline \multirow{3}{*}{$\begin{array}{l}\text { HEIS No. } \\
\text { 14133-76-7 }\end{array}$} & B1RKV5 & \multicolumn{3}{|c|}{ Lab ID: $\quad$ 0802007-04 } & & & \\
\hline & Technetium-99 & $<9.89 \mathrm{E}-4$ & ug/g dry & 9.89E-4 & $2 / 20 / 08$ & 8E01017 & PNNL-AGG-415 \\
\hline & Uranium 238 & 3.93E-1 & ug/g dry & $7.18 \mathrm{E}-3$ & $2 / 20 / 08$ & 8E01017 & PNNL-AGG-415 \\
\hline \multirow{3}{*}{$\begin{array}{l}\text { HEIS No. } \\
14133-76-7\end{array}$} & B1RKV9 & \multicolumn{3}{|c|}{ Lab ID: $\quad$ 0802007-12 } & & & \\
\hline & Technetium-99 & $<9.57 \mathrm{E}-4$ & ug/g dry & $9.57 \mathrm{E}-4$ & $2 / 12 / 08$ & 8E01013 & PNNL-AGG-415 \\
\hline & Uranium 238 & $3.85 \mathrm{E}-1$ & ug/g dry & $6.95 \mathrm{E}-3$ & $2 / 12 / 08$ & 8E01013 & PNNL-AGG-415 \\
\hline \multirow{3}{*}{$\begin{array}{l}\text { HEIS No. } \\
\text { 14133-76-7 }\end{array}$} & B1RKW2 & \multicolumn{3}{|c|}{ Lab ID: $\quad$ 0802008-04 } & & & \\
\hline & Technetium-99 & $<1.15 \mathrm{E}-3$ & ug/g dry & $1.15 \mathrm{E}-3$ & $2 / 20 / 08$ & 8E01017 & PNNL-AGG-415 \\
\hline & Uranium 238 & 1.10E0 & ug/g dry & 8.32E-3 & $2 / 20 / 08$ & 8E01017 & PNNL-AGG-415 \\
\hline \multirow{3}{*}{$\begin{array}{l}\text { HEIS No. } \\
14133-76-7\end{array}$} & B1RKW4 & \multicolumn{3}{|c|}{ Lab ID: $\quad$ 0802008-08 } & & & \\
\hline & Technetium-99 & $<1.14 \mathrm{E}-3$ & ug/g dry & $1.14 \mathrm{E}-3$ & 2/12/08 & 8E01013 & PNNL-AGG-415 \\
\hline & Uranium 238 & 6.37E-1 & ug/g dry & $8.28 \mathrm{E}-3$ & $2 / 12 / 08$ & 8E01013 & PNNL-AGG-415 \\
\hline \multirow{3}{*}{$\begin{array}{l}\text { HEIS No. } \\
14133-76-7\end{array}$} & B1RKW5 & \multicolumn{3}{|c|}{ Lab ID: $\quad$ 0802009-02 } & & & \\
\hline & Technetium-99 & $<1.11 \mathrm{E}-3$ & ug/g dry & $1.11 \mathrm{E}-3$ & $2 / 12 / 08$ & 8E01013 & PNNL-AGG-415 \\
\hline & Uranium 238 & 1.04E0 & ug/g dry & 8.03E-3 & $2 / 12 / 08$ & 8E01013 & PNNL-AGG-415 \\
\hline \multirow{3}{*}{$\begin{array}{l}\text { HEIS No. } \\
14133-76-7\end{array}$} & B1RKW6 & \multicolumn{3}{|c|}{ Lab ID: $\quad$ 0802009-04 } & & & \\
\hline & Technetium-99 & $<1.18 \mathrm{E}-3$ & ug/g dry & $1.18 \mathrm{E}-3$ & $2 / 12 / 08$ & 8E01013 & PNNL-AGG-415 \\
\hline & Uranium 238 & 9.45E0 & ug/g dry & $8.59 E-3$ & 2/12/08 & 8E01013 & PNNL-AGG-415 \\
\hline \multirow{3}{*}{$\begin{array}{l}\text { HEIS No. } \\
14133-76-7\end{array}$} & B1TFV6 & \multicolumn{3}{|c|}{ Lab ID: $\quad$ 0802028-03 } & & & \\
\hline & Technetium-99 & $<5.26 \mathrm{E}-3$ & ug/g dry & $5.26 \mathrm{E}-3$ & $5 / 16 / 08$ & 8E15002 & PNNL-AGG-415 \\
\hline & Uranium 238 & 6.67E-1 & ug/g dry & $3.82 \mathrm{E}-2$ & $5 / 16 / 08$ & 8E15002 & PNNL-AGG-415 \\
\hline \multirow{3}{*}{$\begin{array}{l}\text { HEIS No. } \\
14133-76-7\end{array}$} & B1TFV8 & \multicolumn{3}{|c|}{ Lab ID: $\quad$ 0802028-05 } & & & \\
\hline & Technetium-99 & $<4.94 \mathrm{E}-3$ & ug/g dry & $4.94 \mathrm{E}-3$ & $5 / 16 / 08$ & 8E15002 & PNNL-AGG-415 \\
\hline & Uranium 238 & $4.88 \mathrm{E}-1$ & ug/g dry & $3.58 \mathrm{E}-2$ & $5 / 16 / 08$ & 8E15002 & PNNL-AGG-415 \\
\hline \multirow{3}{*}{$\begin{array}{l}\text { HEIS No. } \\
\text { 14133-76-7 }\end{array}$} & B1TFW0 & \multicolumn{3}{|c|}{ Lab ID: $\quad$ 0802028-07 } & & & \\
\hline & Technetium-99 & $<5.14 \mathrm{E}-3$ & ug/g dry & $5.14 \mathrm{E}-3$ & $5 / 16 / 08$ & 8E15002 & PNNL-AGG-415 \\
\hline & Uranium 238 & 4.64E1 & ug/g dry & 3.73E0 & $5 / 16 / 08$ & 8E15002 & PNNL-AGG-415 \\
\hline \multirow{3}{*}{$\begin{array}{l}\text { HEIS No. } \\
14133-76-7\end{array}$} & B1TFW2 & \multicolumn{3}{|c|}{ Lab ID: $\quad$ 0802028-09 } & & & \\
\hline & Technetium-99 & $6.72 \mathrm{E}-3$ & ug/g dry & 5.33E-3 & $5 / 16 / 08$ & 8E15002 & PNNL-AGG-415 \\
\hline & Uranium 238 & 1.19E2 & ug/g dry & 3.87E0 & $5 / 16 / 08$ & 8E15002 & PNNL-AGG-415 \\
\hline \multirow{3}{*}{$\begin{array}{l}\text { HEIS No. } \\
14133-76-7\end{array}$} & B1RKX0 & & b ID: & 2028-11 & & & \\
\hline & Technetium-99 & $<5.22 \mathrm{E}-3$ & ug/g dry & $5.22 \mathrm{E}-3$ & $5 / 16 / 08$ & 8E15002 & PNNL-AGG-415 \\
\hline & Uranium 238 & $1.32 \mathrm{E} 0$ & ug/g dry & $3.79 \mathrm{E}-2$ & $5 / 16 / 08$ & 8E15002 & PNNL-AGG-415 \\
\hline HEIS No. & B1RKX3 & & b ID: & 2028-17 & & & \\
\hline 14133-76-7 & Technetium-99 & $<4.42 \mathrm{E}-3$ & ug/g dry & $4.42 \mathrm{E}-3$ & $5 / 16 / 08$ & 8E15002 & PNNL-AGG-415 \\
\hline & Uranium 238 & 4.19E-1 & ug/g dry & $3.21 \mathrm{E}-2$ & 5/16/08 & 8E15002 & PNNL-AGG-415 \\
\hline HEIS No. & B1RKX5 & & b ID: & $2028-23$ & & & \\
\hline $14133-76-7$ & Technetium-99 & $<4.44 \mathrm{E}-3$ & ug/g dry & $4.44 \mathrm{E}-3$ & $5 / 16 / 08$ & 8E15002 & PNNL-AGG-415 \\
\hline & Uranium 238 & $3.91 \mathrm{E}-1$ & ug/g dry & $3.22 \mathrm{E}-2$ & $5 / 16 / 08$ & 8E15002 & PNNL-AGG-415 \\
\hline HEIS No. & B1RKX7 & & b ID: & 2028-29 & & & \\
\hline $14133-76-7$ & Technetium-99 & $<4.45 \mathrm{E}-3$ & ug/g dry & $4.45 E-3$ & $5 / 16 / 08$ & 8E15002 & PNNL-AGG-415 \\
\hline & Uranium 238 & 3.43E-1 & ug/g dry & $3.23 E-2$ & $5 / 16 / 08$ & 8E15002 & PNNL-AGG-415 \\
\hline
\end{tabular}




\section{Radionuclides by ICP-MS/Water Extract}

\begin{tabular}{|c|c|c|c|c|c|c|c|}
\hline CAS \# & Analyte & Results & Units & EQL & Analyzed & Batch & Method \\
\hline \multirow{3}{*}{$\begin{array}{l}\text { HEIS No. } \\
14133-76-7\end{array}$} & B1RKL1 & \multicolumn{3}{|c|}{ Lab ID: $\quad$ 0802002-02 } & & & \\
\hline & Technetium-99 & $<2.31 \mathrm{E}-5$ & ug/g dry & $2.31 \mathrm{E}-5$ & $2 / 11 / 08$ & 8D24001 & PNNL-AGG-415 \\
\hline & Uranium 238 & $<5.65 \mathrm{E}-4$ & ug/g dry & 5.65E-4 & $2 / 11 / 08$ & 8D24001 & PNNL-AGG-415 \\
\hline \multirow{3}{*}{$\begin{array}{l}\text { HEIS No. } \\
\text { 14133-76-7 }\end{array}$} & B1RKL6 & \multicolumn{3}{|c|}{ Lab ID: $\quad$ 0802002-09 } & & & \\
\hline & Technetium-99 & $<2.31 \mathrm{E}-5$ & ug/g dry & $2.31 \mathrm{E}-5$ & 2/11/08 & 8D24001 & PNNL-AGG-415 \\
\hline & Uranium 238 & $<5.67 \mathrm{E}-4$ & ug/g dry & 5.67E-4 & $2 / 11 / 08$ & 8D24001 & PNNL-AGG-415 \\
\hline \multirow{3}{*}{$\begin{array}{l}\text { HEIS No. } \\
14133-76-7\end{array}$} & B1RKM1 & \multicolumn{3}{|c|}{ Lab ID: $\quad$ 0802002-17 } & & & \\
\hline & Technetium-99 & $<2.30 \mathrm{E}-5$ & ug/g dry & $2.30 \mathrm{E}-5$ & 2/11/08 & 8D24001 & PNNL-AGG-415 \\
\hline & Uranium 238 & $<5.64 \mathrm{E}-4$ & ug/g dry & $5.64 \mathrm{E}-4$ & $2 / 11 / 08$ & 8D24001 & PNNL-AGG-415 \\
\hline \multirow{3}{*}{$\begin{array}{l}\text { HEIS No. } \\
14133-76-7\end{array}$} & B1RKM8 & \multicolumn{3}{|c|}{ Lab ID: $\quad$ 0802002-31 } & & & \\
\hline & Technetium-99 & $<2.30 \mathrm{E}-5$ & ug/g dry & $2.30 \mathrm{E}-5$ & $2 / 11 / 08$ & 8D24001 & PNNL-AGG-415 \\
\hline & Uranium 238 & $<5.64 \mathrm{E}-4$ & ug/g dry & $5.64 \mathrm{E}-4$ & $2 / 11 / 08$ & 8D24001 & PNNL-AGG-415 \\
\hline \multirow{3}{*}{$\begin{array}{l}\text { HEIS No. } \\
14133-76-7\end{array}$} & B1RKW7 & \multicolumn{3}{|c|}{ Lab ID: $\quad$ 0802003-02 } & & & \\
\hline & Technetium-99 & 4.52E-4 & ug/g dry & $2.45 \mathrm{E}-5$ & $2 / 11 / 08$ & 8D24001 & PNNL-AGG-415 \\
\hline & Uranium 238 & $9.76 \mathrm{E}-1$ & ug/g dry & $1.20 \mathrm{E}-1$ & $2 / 11 / 08$ & 8D24001 & PNNL-AGG-415 \\
\hline \multirow{3}{*}{$\begin{array}{l}\text { HEIS No. } \\
\text { 14133-76-7 }\end{array}$} & B1RKW8 & \multicolumn{3}{|c|}{ Lab ID: $\quad$ 0802003-04 } & & & \\
\hline & Technetium-99 & $1.04 \mathrm{E}-3$ & ug/g dry & $2.30 \mathrm{E}-5$ & $2 / 11 / 08$ & 8D24001 & PNNL-AGG-415 \\
\hline & Uranium 238 & $3.44 \mathrm{E}-2$ & ug/g dry & $5.64 \mathrm{E}-4$ & $2 / 11 / 08$ & 8D24001 & PNNL-AGG-415 \\
\hline \multirow{3}{*}{$\begin{array}{l}\text { HEIS No. } \\
14133-76-7\end{array}$} & B1RKN7 & \multicolumn{3}{|c|}{ Lab ID: $\quad$ 0802005-03 } & & & \\
\hline & Technetium-99 & $<2.31 \mathrm{E}-5$ & ug/g dry & $2.31 \mathrm{E}-5$ & $2 / 11 / 08$ & 8D24001 & PNNL-AGG-415 \\
\hline & Uranium 238 & $<5.65 \mathrm{E}-4$ & ug/g dry & 5.65E-4 & $2 / 11 / 08$ & 8D24001 & PNNL-AGG-415 \\
\hline \multirow{3}{*}{$\begin{array}{l}\text { HEIS No. } \\
\text { 14133-76-7 }\end{array}$} & B1RKP3 & \multicolumn{3}{|c|}{ Lab ID: $\quad$ 0802005-09 } & & & \\
\hline & Technetium-99 & $<2.30 \mathrm{E}-5$ & ug/g dry & $2.30 \mathrm{E}-5$ & $2 / 11 / 08$ & 8D24001 & PNNL-AGG-415 \\
\hline & Uranium 238 & $<5.64 \mathrm{E}-4$ & ug/g dry & $5.64 \mathrm{E}-4$ & 2/11/08 & 8D24001 & PNNL-AGG-415 \\
\hline \multirow{3}{*}{$\begin{array}{l}\text { HEIS No. } \\
14133-76-7\end{array}$} & B1RKP9 & \multicolumn{3}{|c|}{ Lab ID: $\quad$ 0802005-15 } & & & \\
\hline & Technetium-99 & $<2.30 \mathrm{E}-5$ & ug/g dry & $2.30 \mathrm{E}-5$ & $2 / 11 / 08$ & 8D24001 & PNNL-AGG-415 \\
\hline & Uranium 238 & $<5.64 \mathrm{E}-4$ & ug/g dry & $5.64 \mathrm{E}-4$ & 2/11/08 & 8D24001 & PNNL-AGG-415 \\
\hline \multirow{3}{*}{$\begin{array}{l}\text { HEIS No. } \\
14133-76-7\end{array}$} & B1RKR5 & \multicolumn{3}{|c|}{ Lab ID: $\quad$ 0802005-21 } & & & \\
\hline & Technetium-99 & $<2.30 \mathrm{E}-5$ & ug/g dry & $2.30 \mathrm{E}-5$ & $2 / 11 / 08$ & 8D24001 & PNNL-AGG-415 \\
\hline & Uranium 238 & $<5.63 \mathrm{E}-4$ & ug/g dry & 5.63E-4 & $2 / 11 / 08$ & 8D24001 & PNNL-AGG-415 \\
\hline \multirow{3}{*}{$\begin{array}{l}\text { HEIS No. } \\
14133-76-7\end{array}$} & B1RKR9 & \multicolumn{3}{|c|}{ Lab ID: $\quad$ 0802005-25 } & & & \\
\hline & Technetium-99 & $<2.30 \mathrm{E}-5$ & ug/g dry & $2.30 \mathrm{E}-5$ & 2/11/08 & 8D24001 & PNNL-AGG-415 \\
\hline & Uranium 238 & $<5.64 \mathrm{E}-4$ & ug/g dry & $5.64 \mathrm{E}-4$ & 2/11/08 & 8D24001 & PNNL-AGG-415 \\
\hline \multirow{3}{*}{$\begin{array}{l}\text { HEIS No. } \\
14133-76-7\end{array}$} & B1RKT3 & \multicolumn{3}{|c|}{ Lab ID: $\quad$ 0802005-29 } & & & \\
\hline & Technetium-99 & $<2.30 \mathrm{E}-5$ & ug/g dry & $2.30 \mathrm{E}-5$ & $2 / 11 / 08$ & 8D24001 & PNNL-AGG-415 \\
\hline & Uranium 238 & $<5.64 \mathrm{E}-4$ & ug/g dry & $5.64 \mathrm{E}-4$ & $2 / 11 / 08$ & 8D24001 & PNNL-AGG-415 \\
\hline HEIS No. & B1RKT7 & & b ID: & $2006-07$ & & & \\
\hline $14133-76-7$ & Technetium-99 & $<2.30 \mathrm{E}-5$ & ug/g dry & $2.30 \mathrm{E}-5$ & 2/11/08 & 8D24001 & PNNL-AGG-415 \\
\hline & Uranium 238 & $<5.64 \mathrm{E}-4$ & ug/g dry & $5.64 \mathrm{E}-4$ & 2/11/08 & 8D24001 & PNNL-AGG-415 \\
\hline HEIS No. & B1RKV1 & & b ID: & $2006-11$ & & & \\
\hline $14133-76-7$ & Technetium-99 & $<2.56 \mathrm{E}-5$ & ug/g dry & $2.56 \mathrm{E}-5$ & 2/11/08 & 8D24001 & PNNL-AGG-415 \\
\hline
\end{tabular}




\section{Radionuclides by ICP-MS/Water Extract}

\begin{tabular}{|c|c|c|c|c|c|c|c|}
\hline CAS \# & Analyte & Results & Units & EQL & Analyzed & Batch & Method \\
\hline \multirow[t]{2}{*}{ HEIS No. } & B1RKV1 & \multicolumn{3}{|c|}{ Lab ID: $\quad$ 0802006-11 } & & & \\
\hline & Uranium 238 & $<6.28 \mathrm{E}-4$ & ug/g dry & $6.28 \mathrm{E}-4$ & 2/11/08 & 8D24001 & PNNL-AGG-415 \\
\hline \multirow{3}{*}{$\begin{array}{l}\text { HEIS No. } \\
14133-76-7\end{array}$} & B1RKV5 & \multicolumn{3}{|c|}{ Lab ID: $\quad$ 0802007-04 } & & & \\
\hline & Technetium-99 & $<2.30 \mathrm{E}-5$ & ug/g dry & $2.30 \mathrm{E}-5$ & $2 / 11 / 08$ & 8D24001 & PNNL-AGG-415 \\
\hline & Uranium 238 & $<5.63 \mathrm{E}-4$ & ug/g dry & 5.63E-4 & $2 / 11 / 08$ & 8D24001 & PNNL-AGG-415 \\
\hline \multirow{3}{*}{$\begin{array}{l}\text { HEIS No. } \\
14133-76-7\end{array}$} & B1RKV9 & \multicolumn{3}{|c|}{ Lab ID: $\quad$ 0802007-12 } & & & \\
\hline & Technetium-99 & $<2.30 \mathrm{E}-5$ & ug/g dry & $2.30 \mathrm{E}-5$ & $2 / 11 / 08$ & 8D24001 & PNNL-AGG-415 \\
\hline & Uranium 238 & $<5.64 \mathrm{E}-4$ & ug/g dry & $5.64 \mathrm{E}-4$ & $2 / 11 / 08$ & 8D24001 & PNNL-AGG-415 \\
\hline \multirow{3}{*}{$\begin{array}{l}\text { HEIS No. } \\
\text { 14133-76-7 }\end{array}$} & B1RKW2 & \multicolumn{3}{|c|}{ Lab ID: $\quad$ 0802008-04 } & & & \\
\hline & Technetium-99 & $<2.30 \mathrm{E}-5$ & ug/g dry & $2.30 \mathrm{E}-5$ & $2 / 11 / 08$ & 8D24001 & PNNL-AGG-415 \\
\hline & Uranium 238 & $1.05 \mathrm{E}-2$ & ug/g dry & 5.63E-4 & $2 / 11 / 08$ & 8D24001 & PNNL-AGG-415 \\
\hline \multirow{3}{*}{$\begin{array}{l}\text { HEIS No. } \\
\text { 14133-76-7 }\end{array}$} & B1RKW4 & \multicolumn{3}{|c|}{ Lab ID: $\quad$ 0802008-08 } & & & \\
\hline & Technetium-99 & $3.64 \mathrm{E}-5$ & ug/g dry & $2.30 \mathrm{E}-5$ & $2 / 11 / 08$ & 8D24001 & PNNL-AGG-415 \\
\hline & Uranium 238 & $1.00 \mathrm{E}-1$ & ug/g dry & $5.64 \mathrm{E}-4$ & $2 / 11 / 08$ & 8D24001 & PNNL-AGG-415 \\
\hline \multirow{3}{*}{$\begin{array}{l}\text { HEIS No. } \\
14133-76-7\end{array}$} & B1RKW5 & \multicolumn{3}{|c|}{ Lab ID: $\quad$ 0802009-02 } & & & \\
\hline & Technetium-99 & $2.76 \mathrm{E}-5$ & ug/g dry & $2.30 \mathrm{E}-5$ & $2 / 11 / 08$ & 8D24001 & PNNL-AGG-415 \\
\hline & Uranium 238 & $9.49 \mathrm{E}-3$ & ug/g dry & $5.64 \mathrm{E}-4$ & $2 / 11 / 08$ & 8D24001 & PNNL-AGG-415 \\
\hline \multirow{3}{*}{$\begin{array}{l}\text { HEIS No. } \\
14133-76-7\end{array}$} & B1RKW6 & \multicolumn{3}{|c|}{ Lab ID: $\quad$ 0802009-04 } & & & \\
\hline & Technetium-99 & 4.55E-5 & ug/g dry & $2.30 \mathrm{E}-5$ & $2 / 11 / 08$ & 8D24001 & PNNL-AGG-415 \\
\hline & Uranium 238 & 3.94E0 & ug/g dry & $1.13 \mathrm{E}-1$ & $2 / 11 / 08$ & 8D24001 & PNNL-AGG-415 \\
\hline \multirow{3}{*}{$\begin{array}{l}\text { HEIS No. } \\
\text { 14133-76-7 }\end{array}$} & B1TFV6 & \multicolumn{3}{|c|}{ Lab ID: $\quad$ 0802028-03 } & & & \\
\hline & Technetium-99 & $<9.23 \mathrm{E}-5$ & ug/g dry & $9.23 \mathrm{E}-5$ & $4 / 15 / 08$ & 8D15001 & PNNL-AGG-415 \\
\hline & Uranium 238 & $2.64 \mathrm{E}-3$ & ug/g dry & $2.26 \mathrm{E}-3$ & $4 / 15 / 08$ & 8D15001 & PNNL-AGG-415 \\
\hline \multirow{3}{*}{$\begin{array}{l}\text { HEIS No. } \\
14133-76-7\end{array}$} & B1TFV8 & \multicolumn{3}{|c|}{ Lab ID: $\quad$ 0802028-05 } & & & \\
\hline & Technetium-99 & $<2.29 \mathrm{E}-5$ & ug/g dry & $2.29 \mathrm{E}-5$ & $4 / 15 / 08$ & 8D15001 & PNNL-AGG-415 \\
\hline & Uranium 238 & 1.17E-2 & ug/g dry & $5.62 \mathrm{E}-4$ & $4 / 15 / 08$ & 8D15001 & PNNL-AGG-415 \\
\hline \multirow{3}{*}{$\begin{array}{l}\text { HEIS No. } \\
14133-76-7\end{array}$} & B1TFW0 & \multicolumn{3}{|c|}{ Lab ID: $\quad$ 0802028-07 } & & & \\
\hline & Technetium-99 & $6.17 \mathrm{E}-4$ & ug/g dry & $2.30 \mathrm{E}-5$ & $4 / 15 / 08$ & 8D15001 & PNNL-AGG-415 \\
\hline & Uranium 238 & $1.26 \mathrm{E} 1$ & ug/g dry & 1.13E0 & $4 / 15 / 08$ & 8D15001 & PNNL-AGG-415 \\
\hline \multirow{3}{*}{$\begin{array}{l}\text { HEIS No. } \\
\text { 14133-76-7 }\end{array}$} & B1TFW2 & \multicolumn{3}{|c|}{ Lab ID: $\quad$ 0802028-09 } & & & \\
\hline & Technetium-99 & $5.32 \mathrm{E}-3$ & ug/g dry & $2.31 \mathrm{E}-5$ & $4 / 15 / 08$ & 8D15001 & PNNL-AGG-415 \\
\hline & Uranium 238 & $1.82 \mathrm{E} 1$ & ug/g dry & 1.13E0 & $4 / 15 / 08$ & 8D15001 & PNNL-AGG-415 \\
\hline \multirow{3}{*}{$\begin{array}{l}\text { HEIS No. } \\
\text { 14133-76-7 }\end{array}$} & B1RKX0 & & b ID: & $2028-11$ & & & \\
\hline & Technetium-99 & $2.75 E-3$ & ug/g dry & $2.30 \mathrm{E}-5$ & $4 / 15 / 08$ & 8D15001 & PNNL-AGG-415 \\
\hline & Uranium 238 & 3.37E-2 & ug/g dry & $5.63 \mathrm{E}-4$ & $4 / 15 / 08$ & 8D15001 & PNNL-AGG-415 \\
\hline HEIS No. & B1RKX3 & & b ID: & $2028-17$ & & & \\
\hline $14133-76-7$ & Technetium-99 & $1.10 \mathrm{E}-4$ & ug/g dry & $2.30 \mathrm{E}-5$ & $4 / 15 / 08$ & 8D15001 & PNNL-AGG-415 \\
\hline & Uranium 238 & 3.23E-3 & ug/g dry & 5.63E-4 & $4 / 15 / 08$ & 8D15001 & PNNL-AGG-415 \\
\hline HEIS No. & B1RKX5 & & b ID: & $2028-23$ & & & \\
\hline $14133-76-7$ & Technetium-99 & $1.13 E-4$ & ug/g dry & $2.31 E-5$ & 4/15/08 & 8D15001 & PNNL-AGG-415 \\
\hline & Uranium 238 & $1.46 \mathrm{E}-3$ & ug/g dry & $5.65 \mathrm{E}-4$ & $4 / 15 / 08$ & 8D15001 & PNNL-AGG-415 \\
\hline HEIS No. & B1RKX7 & & b ID: & $2028-29$ & & & \\
\hline $14133-76-7$ & Technetium-99 & $<2.30 \mathrm{E}-5$ & ug/g dry & $2.30 \mathrm{E}-5$ & $4 / 15 / 08$ & 8D15001 & PNNL-AGG-415 \\
\hline & Uranium 238 & $1.12 \mathrm{E}-3$ & ug/g dry & $5.63 \mathrm{E}-4$ & 4/15/08 & 8D15001 & PNNL-AGG-415 \\
\hline
\end{tabular}


RCRA Metals By PNNL-AGG-415/Water Extract

\begin{tabular}{|c|c|c|c|c|c|c|c|}
\hline CAS \# & Analyte & Results & Units & EQL & Analyzed & Batch & Method \\
\hline HEIS No. & B1RKL1 & \multicolumn{3}{|c|}{ Lab ID: $\quad$ 0802002-02 } & & & \\
\hline $14378-38-2$ & Silver & $<1.07 \mathrm{E}-3$ & ug/g dry & $1.07 \mathrm{E}-3$ & 2/07/08 & 8E01009 & PNNL-AGG-415 \\
\hline 14336-64-2 & Cadmium & $<2.96 \mathrm{E}-4$ & ug/g dry & 2.96E-4 & $2 / 07 / 08$ & 8E01009 & PNNL-AGG-415 \\
\hline $14265-72-6$ & Antimony & $<5.42 \mathrm{E}-4$ & ug/g dry & $5.42 \mathrm{E}-4$ & $2 / 07 / 08$ & 8E01009 & PNNL-AGG-415 \\
\hline 13966-28-4 & Lead & $<5.62 \mathrm{E}-4$ & ug/g dry & $5.62 \mathrm{E}-4$ & $2 / 07 / 08$ & 8E01009 & PNNL-AGG-415 \\
\hline HEIS No. & B1RKL6 & \multicolumn{3}{|c|}{$\begin{array}{ll}\text { Lab ID: } & \text { 0802002-09 }\end{array}$} & & & \\
\hline $14378-38-2$ & Silver & $<1.08 \mathrm{E}-3$ & ug/g dry & $1.08 \mathrm{E}-3$ & $2 / 07 / 08$ & 8E01009 & PNNL-AGG-415 \\
\hline 14336-64-2 & Cadmium & $<2.97 \mathrm{E}-4$ & ug/g dry & 2.97E-4 & $2 / 07 / 08$ & 8E01009 & PNNL-AGG-415 \\
\hline $14265-72-6$ & Antimony & $<5.43 \mathrm{E}-4$ & ug/g dry & 5.43E-4 & $2 / 07 / 08$ & 8E01009 & PNNL-AGG-415 \\
\hline 13966-28-4 & Lead & $<5.63 \mathrm{E}-4$ & ug/g dry & 5.63E-4 & $2 / 07 / 08$ & 8E01009 & PNNL-AGG-415 \\
\hline HEIS No. & B1RKM1 & \multicolumn{3}{|c|}{$\begin{array}{ll}\text { Lab ID: } & 0802002-17\end{array}$} & & & \\
\hline $14378-38-2$ & Silver & $<1.07 E-3$ & ug/g dry & $1.07 \mathrm{E}-3$ & 2/07/08 & 8E01009 & PNNL-AGG-415 \\
\hline 14336-64-2 & Cadmium & $<2.95 \mathrm{E}-4$ & ug/g dry & 2.95E-4 & 2/07/08 & 8E01009 & PNNL-AGG-415 \\
\hline $14265-72-6$ & Antimony & $<5.40 \mathrm{E}-4$ & ug/g dry & $5.40 \mathrm{E}-4$ & 2/07/08 & 8E01009 & PNNL-AGG-415 \\
\hline 13966-28-4 & Lead & $<5.60 \mathrm{E}-4$ & ug/g dry & $5.60 \mathrm{E}-4$ & $2 / 07 / 08$ & 8E01009 & PNNL-AGG-415 \\
\hline HEIS No. & B1RKM8 & \multicolumn{3}{|c|}{$\begin{array}{ll}\text { Lab ID: } & \text { 0802002-31 }\end{array}$} & & & \\
\hline $14378-38-2$ & Silver & $<1.07 \mathrm{E}-3$ & ug/g dry & $1.07 \mathrm{E}-3$ & $2 / 07 / 08$ & 8E01009 & PNNL-AGG-415 \\
\hline $14336-64-2$ & Cadmium & $<2.95 \mathrm{E}-4$ & ug/g dry & 2.95E-4 & 2/07/08 & 8E01009 & PNNL-AGG-415 \\
\hline $14265-72-6$ & Antimony & $<5.40 \mathrm{E}-4$ & ug/g dry & $5.40 \mathrm{E}-4$ & 2/07/08 & 8E01009 & PNNL-AGG-415 \\
\hline 13966-28-4 & Lead & $<5.60 \mathrm{E}-4$ & ug/g dry & $5.60 \mathrm{E}-4$ & 2/07/08 & 8E01009 & PNNL-AGG-415 \\
\hline HEIS No. & B1RKW7 & \multicolumn{3}{|c|}{$\begin{array}{ll}\text { Lab ID: } & \text { 0802003-02 }\end{array}$} & & & \\
\hline $14378-38-2$ & Silver & $<1.14 \mathrm{E}-3$ & ug/g dry & $1.14 \mathrm{E}-3$ & $2 / 07 / 08$ & 8E01009 & PNNL-AGG-415 \\
\hline 14336-64-2 & Cadmium & $5.81 \mathrm{E}-4$ & ug/g dry & 3.15E-4 & 2/07/08 & 8E01009 & PNNL-AGG-415 \\
\hline $14265-72-6$ & Antimony & $9.68 \mathrm{E}-4$ & ug/g dry & 5.76E-4 & 2/07/08 & 8E01009 & PNNL-AGG-415 \\
\hline 13966-28-4 & Lead & $<5.98 \mathrm{E}-4$ & ug/g dry & $5.98 \mathrm{E}-4$ & $2 / 07 / 08$ & 8E01009 & PNNL-AGG-415 \\
\hline HEIS No. & B1RKW8 & \multicolumn{3}{|c|}{$\begin{array}{ll}\text { Lab ID: } & \text { 0802003-04 }\end{array}$} & & & \\
\hline $14378-38-2$ & Silver & $<1.07 \mathrm{E}-3$ & ug/g dry & $1.07 \mathrm{E}-3$ & $2 / 07 / 08$ & 8E01009 & PNNL-AGG-415 \\
\hline 14336-64-2 & Cadmium & $3.74 \mathrm{E}-4$ & ug/g dry & 2.95E-4 & 2/07/08 & 8E01009 & PNNL-AGG-415 \\
\hline $14265-72-6$ & Antimony & $<5.40 \mathrm{E}-4$ & ug/g dry & $5.40 \mathrm{E}-4$ & 2/07/08 & 8E01009 & PNNL-AGG-415 \\
\hline 13966-28-4 & Lead & $<5.60 \mathrm{E}-4$ & ug/g dry & $5.60 \mathrm{E}-4$ & $2 / 07 / 08$ & 8E01009 & PNNL-AGG-415 \\
\hline HEIS No. & B1RKN7 & \multicolumn{3}{|c|}{$\begin{array}{ll}\text { Lab ID: } & 0802005-03\end{array}$} & & & \\
\hline $14378-38-2$ & Silver & $<1.07 \mathrm{E}-3$ & ug/g dry & $1.07 \mathrm{E}-3$ & 2/07/08 & 8E01009 & PNNL-AGG-415 \\
\hline $14336-64-2$ & Cadmium & $<2.96 \mathrm{E}-4$ & ug/g dry & 2.96E-4 & 2/07/08 & 8E01009 & PNNL-AGG-415 \\
\hline $14265-72-6$ & Antimony & $<5.42 \mathrm{E}-4$ & ug/g dry & $5.42 \mathrm{E}-4$ & 2/07/08 & 8E01009 & PNNL-AGG-415 \\
\hline 13966-28-4 & Lead & $<5.62 \mathrm{E}-4$ & ug/g dry & $5.62 \mathrm{E}-4$ & 2/07/08 & 8E01009 & PNNL-AGG-415 \\
\hline HEIS No. & B1RKP3 & \multicolumn{3}{|c|}{$\begin{array}{ll}\text { Lab ID: } & \text { 0802005-09 }\end{array}$} & & & \\
\hline $14378-38-2$ & Silver & $<1.07 \mathrm{E}-3$ & ug/g dry & $1.07 \mathrm{E}-3$ & 2/07/08 & 8E01009 & PNNL-AGG-415 \\
\hline 14336-64-2 & Cadmium & $<2.95 \mathrm{E}-4$ & ug/g dry & 2.95E-4 & 2/07/08 & 8E01009 & PNNL-AGG-415 \\
\hline $14265-72-6$ & Antimony & $<5.40 \mathrm{E}-4$ & ug/g dry & $5.40 \mathrm{E}-4$ & 2/07/08 & 8E01009 & PNNL-AGG-415 \\
\hline 13966-28-4 & Lead & $<5.60 \mathrm{E}-4$ & ug/g dry & $5.60 \mathrm{E}-4$ & 2/07/08 & 8E01009 & PNNL-AGG-415 \\
\hline HEIS No. & B1RKP9 & \multicolumn{3}{|c|}{$\begin{array}{ll}\text { Lab ID: } & 0802005-15\end{array}$} & & & \\
\hline $14378-38-2$ & Silver & $<1.07 \mathrm{E}-3$ & ug/g dry & $1.07 \mathrm{E}-3$ & 2/07/08 & 8E01009 & PNNL-AGG-415 \\
\hline 14336-64-2 & Cadmium & $<2.95 \mathrm{E}-4$ & ug/g dry & 2.95E-4 & 2/07/08 & 8E01009 & PNNL-AGG-415 \\
\hline $14265-72-6$ & Antimony & $<5.41 \mathrm{E}-4$ & ug/g dry & $5.41 \mathrm{E}-4$ & 2/07/08 & 8E01009 & PNNL-AGG-415 \\
\hline 13966-28-4 & Lead & $<5.61 \mathrm{E}-4$ & ug/g dry & $5.61 \mathrm{E}-4$ & 2/07/08 & 8E01009 & PNNL-AGG-415 \\
\hline HEIS No. & B1RKR5 & \multicolumn{3}{|c|}{$\begin{array}{ll}\text { Lab ID: } & 0802005-21\end{array}$} & & & \\
\hline 14378-38-2 & Silver & $<1.07 \mathrm{E}-3$ & ug/g dry & $1.07 \mathrm{E}-3$ & 2/07/08 & 8E01009 & PNNL-AGG-415 \\
\hline 14336-64-2 & Cadmium & $<2.95 \mathrm{E}-4$ & ug/g dry & 2.95E-4 & 2/07/08 & 8E01009 & PNNL-AGG-415 \\
\hline
\end{tabular}


RCRA Metals By PNNL-AGG-415/Water Extract

\begin{tabular}{|c|c|c|c|c|c|c|c|}
\hline CAS \# & Analyte & Results & Units & EQL & Analyzed & Batch & Method \\
\hline HEIS No. & B1RKR5 & \multicolumn{3}{|c|}{ Lab ID: $\quad$ 0802005-21 } & & & \\
\hline $14265-72-6$ & Antimony & $<5.40 \mathrm{E}-4$ & ug/g dry & $5.40 \mathrm{E}-4$ & 2/07/08 & 8E01009 & PNNL-AGG-415 \\
\hline 13966-28-4 & Lead & $<5.60 \mathrm{E}-4$ & ug/g dry & $5.60 \mathrm{E}-4$ & 2/07/08 & 8E01009 & PNNL-AGG-415 \\
\hline HEIS No. & B1RKR9 & \multicolumn{3}{|c|}{ Lab ID: $\quad 0802005-25$} & & & \\
\hline $14378-38-2$ & Silver & $<1.07 \mathrm{E}-3$ & ug/g dry & $1.07 \mathrm{E}-3$ & $2 / 07 / 08$ & 8E01009 & PNNL-AGG-415 \\
\hline 14336-64-2 & Cadmium & $<2.95 \mathrm{E}-4$ & ug/g dry & 2.95E-4 & $2 / 07 / 08$ & 8E01009 & PNNL-AGG-415 \\
\hline $14265-72-6$ & Antimony & $<5.40 \mathrm{E}-4$ & ug/g dry & $5.40 \mathrm{E}-4$ & 2/07/08 & 8E01009 & PNNL-AGG-415 \\
\hline 13966-28-4 & Lead & $<5.60 \mathrm{E}-4$ & ug/g dry & $5.60 \mathrm{E}-4$ & $2 / 07 / 08$ & 8E01009 & PNNL-AGG-415 \\
\hline HEIS No. & B1RKT3 & \multicolumn{3}{|c|}{ Lab ID: $\quad$ 0802005-29 } & & & \\
\hline $14378-38-2$ & Silver & $<1.07 \mathrm{E}-3$ & ug/g dry & $1.07 \mathrm{E}-3$ & $2 / 07 / 08$ & 8E01009 & PNNL-AGG-415 \\
\hline $14336-64-2$ & Cadmium & $<2.95 \mathrm{E}-4$ & ug/g dry & 2.95E-4 & 2/07/08 & 8E01009 & PNNL-AGG-415 \\
\hline $14265-72-6$ & Antimony & $<5.40 \mathrm{E}-4$ & ug/g dry & $5.40 \mathrm{E}-4$ & 2/07/08 & 8E01009 & PNNL-AGG-415 \\
\hline 13966-28-4 & Lead & $<5.60 \mathrm{E}-4$ & ug/g dry & $5.60 \mathrm{E}-4$ & 2/07/08 & 8E01009 & PNNL-AGG-415 \\
\hline HEIS No. & B1RKT7 & \multicolumn{3}{|c|}{$\begin{array}{ll}\text { Lab ID: } & \text { 0802006-07 }\end{array}$} & & & \\
\hline $14378-38-2$ & Silver & $<1.07 \mathrm{E}-3$ & ug/g dry & $1.07 \mathrm{E}-3$ & $2 / 07 / 08$ & 8E01009 & PNNL-AGG-415 \\
\hline 14336-64-2 & Cadmium & $<2.95 \mathrm{E}-4$ & ug/g dry & 2.95E-4 & 2/07/08 & 8E01009 & PNNL-AGG-415 \\
\hline $14265-72-6$ & Antimony & $<5.40 \mathrm{E}-4$ & ug/g dry & $5.40 \mathrm{E}-4$ & 2/07/08 & 8E01009 & PNNL-AGG-415 \\
\hline 13966-28-4 & Lead & $<5.60 \mathrm{E}-4$ & ug/g dry & $5.60 \mathrm{E}-4$ & 2/07/08 & 8E01009 & PNNL-AGG-415 \\
\hline HEIS No. & B1RKV1 & \multicolumn{3}{|c|}{$\begin{array}{ll}\text { Lab ID: } & \text { 0802006-11 }\end{array}$} & & & \\
\hline $14378-38-2$ & Silver & $<1.19 \mathrm{E}-3$ & ug/g dry & $1.19 \mathrm{E}-3$ & $2 / 07 / 08$ & 8E01009 & PNNL-AGG-415 \\
\hline $14336-64-2$ & Cadmium & $<3.29 \mathrm{E}-4$ & ug/g dry & $3.29 \mathrm{E}-4$ & 2/07/08 & 8E01009 & PNNL-AGG-415 \\
\hline $14265-72-6$ & Antimony & $<6.02 \mathrm{E}-4$ & ug/g dry & $6.02 \mathrm{E}-4$ & $2 / 07 / 08$ & 8E01009 & PNNL-AGG-415 \\
\hline 13966-28-4 & Lead & $<6.24 \mathrm{E}-4$ & ug/g dry & $6.24 \mathrm{E}-4$ & 2/07/08 & 8E01009 & PNNL-AGG-415 \\
\hline HEIS No. & B1RKV5 & \multicolumn{3}{|c|}{$\begin{array}{ll}\text { Lab ID: } & \text { 0802007-04 }\end{array}$} & & & \\
\hline $14378-38-2$ & Silver & $<1.07 \mathrm{E}-3$ & ug/g dry & $1.07 \mathrm{E}-3$ & 2/07/08 & 8E01009 & PNNL-AGG-415 \\
\hline 14336-64-2 & Cadmium & $<2.95 \mathrm{E}-4$ & ug/g dry & 2.95E-4 & 2/07/08 & 8E01009 & PNNL-AGG-415 \\
\hline $14265-72-6$ & Antimony & $<5.40 \mathrm{E}-4$ & ug/g dry & $5.40 \mathrm{E}-4$ & $2 / 07 / 08$ & 8E01009 & PNNL-AGG-415 \\
\hline 13966-28-4 & Lead & $<5.60 \mathrm{E}-4$ & ug/g dry & $5.60 \mathrm{E}-4$ & 2/07/08 & 8E01009 & PNNL-AGG-415 \\
\hline HEIS No. & B1RKV9 & \multicolumn{3}{|c|}{ Lab ID: $\quad$ 0802007-12 } & & & \\
\hline $14378-38-2$ & Silver & $<1.07 \mathrm{E}-3$ & ug/g dry & $1.07 \mathrm{E}-3$ & 2/07/08 & 8E01009 & PNNL-AGG-415 \\
\hline 14336-64-2 & Cadmium & $<2.95 \mathrm{E}-4$ & ug/g dry & 2.95E-4 & $2 / 07 / 08$ & 8E01009 & PNNL-AGG-415 \\
\hline $14265-72-6$ & Antimony & $<5.40 \mathrm{E}-4$ & ug/g dry & $5.40 \mathrm{E}-4$ & 2/07/08 & 8E01009 & PNNL-AGG-415 \\
\hline 13966-28-4 & Lead & $<5.60 \mathrm{E}-4$ & ug/g dry & $5.60 \mathrm{E}-4$ & $2 / 07 / 08$ & 8E01009 & PNNL-AGG-415 \\
\hline HEIS No. & B1RKW2 & \multicolumn{3}{|c|}{$\begin{array}{ll}\text { Lab ID: } & \text { 0802008-04 }\end{array}$} & & & \\
\hline $14378-38-2$ & Silver & $<1.07 \mathrm{E}-3$ & ug/g dry & $1.07 \mathrm{E}-3$ & $2 / 07 / 08$ & 8E01009 & PNNL-AGG-415 \\
\hline 14336-64-2 & Cadmium & $<2.95 \mathrm{E}-4$ & ug/g dry & 2.95E-4 & 2/07/08 & 8E01009 & PNNL-AGG-415 \\
\hline $14265-72-6$ & Antimony & $6.11 \mathrm{E}-4$ & ug/g dry & $5.40 \mathrm{E}-4$ & 2/07/08 & 8E01009 & PNNL-AGG-415 \\
\hline 13966-28-4 & Lead & $<5.60 \mathrm{E}-4$ & ug/g dry & $5.60 \mathrm{E}-4$ & 2/07/08 & 8E01009 & PNNL-AGG-415 \\
\hline HEIS No. & B1RKW4 & \multicolumn{3}{|c|}{ Lab ID: $\quad$ 0802008-08 } & & & \\
\hline $14378-38-2$ & Silver & 2.95E-3 & ug/g dry & $1.07 \mathrm{E}-3$ & $2 / 07 / 08$ & 8E01009 & PNNL-AGG-415 \\
\hline 14336-64-2 & Cadmium & 7.07E-4 & ug/g dry & 2.95E-4 & $2 / 07 / 08$ & 8E01009 & PNNL-AGG-415 \\
\hline $14265-72-6$ & Antimony & $1.79 \mathrm{E}-3$ & ug/g dry & $5.40 \mathrm{E}-4$ & 2/07/08 & 8E01009 & PNNL-AGG-415 \\
\hline 13966-28-4 & Lead & $2.39 \mathrm{E}-2$ & ug/g dry & $5.60 \mathrm{E}-4$ & 2/07/08 & 8E01009 & PNNL-AGG-415 \\
\hline HEIS No. & B1RKW5 & \multicolumn{3}{|c|}{$\begin{array}{ll}\text { Lab ID: } & \text { 0802009-02 }\end{array}$} & & & \\
\hline $14378-38-2$ & Silver & $<1.07 \mathrm{E}-3$ & ug/g dry & $1.07 \mathrm{E}-3$ & $2 / 07 / 08$ & 8E01009 & PNNL-AGG-415 \\
\hline 14336-64-2 & Cadmium & $4.08 \mathrm{E}-4$ & ug/g dry & 2.95E-4 & 2/07/08 & 8E01009 & PNNL-AGG-415 \\
\hline $14265-72-6$ & Antimony & $6.29 \mathrm{E}-4$ & ug/g dry & $5.40 \mathrm{E}-4$ & 2/07/08 & 8E01009 & PNNL-AGG-415 \\
\hline $13966-28-4$ & Lead & $9.08 \mathrm{E}-4$ & ug/g dry & $5.60 \mathrm{E}-4$ & 2/07/08 & 8E01009 & PNNL-AGG-415 \\
\hline
\end{tabular}


RCRA Metals By PNNL-AGG-415/Water Extract

\begin{tabular}{|c|c|c|c|c|c|c|c|}
\hline CAS \# & Analyte & Results & Units & EQL & Analyzed & Batch & Method \\
\hline HEIS No. & B1RKW6 & \multicolumn{3}{|c|}{ Lab ID: $\quad$ 0802009-04 } & & & \\
\hline $14378-38-2$ & Silver & $<1.07 \mathrm{E}-3$ & ug/g dry & $1.07 \mathrm{E}-3$ & 2/07/08 & 8E01009 & PNNL-AGG-415 \\
\hline $14336-64-2$ & Cadmium & $6.79 \mathrm{E}-4$ & ug/g dry & $2.95 \mathrm{E}-4$ & 2/07/08 & 8E01009 & PNNL-AGG-415 \\
\hline $14265-72-6$ & Antimony & $1.07 \mathrm{E}-3$ & ug/g dry & $5.40 \mathrm{E}-4$ & 2/07/08 & 8E01009 & PNNL-AGG-415 \\
\hline $13966-28-4$ & Lead & 2.67E-3 & ug/g dry & $5.60 \mathrm{E}-4$ & $2 / 07 / 08$ & 8E01009 & PNNL-AGG-415 \\
\hline HEIS No. & B1TFV6 & \multicolumn{3}{|c|}{ Lab ID: $\quad$ 0802028-03 } & & & \\
\hline $14378-38-2$ & Silver & $<4.29 \mathrm{E}-3$ & ug/g dry & $4.29 \mathrm{E}-3$ & $4 / 14 / 08$ & 8D07004 & PNNL-AGG-415 \\
\hline $14336-64-2$ & Cadmium & $<1.18 \mathrm{E}-3$ & ug/g dry & $1.18 \mathrm{E}-3$ & 4/14/08 & 8D07004 & PNNL-AGG-415 \\
\hline $14265-72-6$ & Antimony & $<2.17 \mathrm{E}-3$ & ug/g dry & 2.17E-3 & $4 / 14 / 08$ & 8D07004 & PNNL-AGG-415 \\
\hline $13966-28-4$ & Lead & $<2.25 \mathrm{E}-3$ & ug/g dry & $2.25 E-3$ & $4 / 14 / 08$ & 8D07004 & PNNL-AGG-415 \\
\hline HEIS No. & B1TFV8 & \multicolumn{3}{|c|}{ Lab ID: $\quad$ 0802028-05 } & & & \\
\hline $14378-38-2$ & Silver & $<1.07 \mathrm{E}-3$ & ug/g dry & $1.07 \mathrm{E}-3$ & $4 / 14 / 08$ & 8D07004 & PNNL-AGG-415 \\
\hline $14336-64-2$ & Cadmium & $<2.94 \mathrm{E}-4$ & ug/g dry & $2.94 \mathrm{E}-4$ & $4 / 14 / 08$ & 8D07004 & PNNL-AGG-415 \\
\hline $14265-72-6$ & Antimony & 2.11E-3 & ug/g dry & $5.38 \mathrm{E}-4$ & $4 / 14 / 08$ & 8D07004 & PNNL-AGG-415 \\
\hline $13966-28-4$ & Lead & $7.32 \mathrm{E}-4$ & ug/g dry & $5.58 \mathrm{E}-4$ & $4 / 14 / 08$ & 8D07004 & PNNL-AGG-415 \\
\hline HEIS No. & B1TFW0 & \multicolumn{3}{|c|}{ Lab ID: $\quad$ 0802028-07 } & & & \\
\hline $14378-38-2$ & Silver & $<1.07 \mathrm{E}-3$ & ug/g dry & $1.07 \mathrm{E}-3$ & $4 / 14 / 08$ & 8D07004 & PNNL-AGG-415 \\
\hline 14336-64-2 & Cadmium & $<2.95 \mathrm{E}-4$ & ug/g dry & $2.95 E-4$ & $4 / 14 / 08$ & 8D07004 & PNNL-AGG-415 \\
\hline $14265-72-6$ & Antimony & $<5.41 \mathrm{E}-4$ & ug/g dry & $5.41 \mathrm{E}-4$ & $4 / 14 / 08$ & 8D07004 & PNNL-AGG-415 \\
\hline 13966-28-4 & Lead & $<5.61 \mathrm{E}-4$ & ug/g dry & $5.61 \mathrm{E}-4$ & $4 / 14 / 08$ & 8D07004 & PNNL-AGG-415 \\
\hline HEIS No. & B1TFW2 & \multicolumn{3}{|c|}{ Lab ID: $\quad$ 0802028-09 } & & & \\
\hline $14378-38-2$ & Silver & $<1.07 \mathrm{E}-3$ & ug/g dry & $1.07 \mathrm{E}-3$ & 4/14/08 & 8D07004 & PNNL-AGG-415 \\
\hline 14336-64-2 & Cadmium & $<2.96 \mathrm{E}-4$ & ug/g dry & $2.96 \mathrm{E}-4$ & $4 / 14 / 08$ & 8D07004 & PNNL-AGG-415 \\
\hline $14265-72-6$ & Antimony & $<5.42 \mathrm{E}-4$ & ug/g dry & $5.42 \mathrm{E}-4$ & $4 / 14 / 08$ & 8D07004 & PNNL-AGG-415 \\
\hline 13966-28-4 & Lead & $<5.62 \mathrm{E}-4$ & ug/g dry & $5.62 \mathrm{E}-4$ & $4 / 14 / 08$ & 8D07004 & PNNL-AGG-415 \\
\hline HEIS No. & B1RKX0 & \multicolumn{3}{|c|}{ Lab ID: $\quad$ 0802028-11 } & & & \\
\hline $14378-38-2$ & Silver & $<1.07 \mathrm{E}-3$ & ug/g dry & $1.07 \mathrm{E}-3$ & $4 / 14 / 08$ & 8D07004 & PNNL-AGG-415 \\
\hline $14336-64-2$ & Cadmium & $<2.94 \mathrm{E}-4$ & ug/g dry & $2.94 \mathrm{E}-4$ & $4 / 14 / 08$ & 8D07004 & PNNL-AGG-415 \\
\hline $14265-72-6$ & Antimony & $<5.39 \mathrm{E}-4$ & ug/g dry & $5.39 \mathrm{E}-4$ & $4 / 14 / 08$ & 8D07004 & PNNL-AGG-415 \\
\hline $13966-28-4$ & Lead & $<5.59 \mathrm{E}-4$ & ug/g dry & $5.59 \mathrm{E}-4$ & $4 / 14 / 08$ & 8D07004 & PNNL-AGG-415 \\
\hline HEIS No. & B1RKX3 & \multicolumn{3}{|c|}{ Lab ID: $\quad$ 0802028-17 } & & & \\
\hline $14378-38-2$ & Silver & $<1.07 \mathrm{E}-3$ & ug/g dry & $1.07 \mathrm{E}-3$ & $4 / 14 / 08$ & 8D07004 & PNNL-AGG-415 \\
\hline 14336-64-2 & Cadmium & $<2.95 \mathrm{E}-4$ & ug/g dry & $2.95 \mathrm{E}-4$ & $4 / 14 / 08$ & 8D07004 & PNNL-AGG-415 \\
\hline $14265-72-6$ & Antimony & $<5.40 \mathrm{E}-4$ & ug/g dry & $5.40 \mathrm{E}-4$ & $4 / 14 / 08$ & 8D07004 & PNNL-AGG-415 \\
\hline 13966-28-4 & Lead & $<5.60 \mathrm{E}-4$ & ug/g dry & $5.60 \mathrm{E}-4$ & $4 / 14 / 08$ & 8D07004 & PNNL-AGG-415 \\
\hline HEIS No. & B1RKX5 & \multicolumn{3}{|c|}{ Lab ID: $\quad$ 0802028-23 } & & & \\
\hline $14378-38-2$ & Silver & $<1.07 \mathrm{E}-3$ & ug/g dry & $1.07 \mathrm{E}-3$ & $4 / 14 / 08$ & 8D07004 & PNNL-AGG-415 \\
\hline 14336-64-2 & Cadmium & $<2.96 \mathrm{E}-4$ & ug/g dry & $2.96 \mathrm{E}-4$ & $4 / 14 / 08$ & 8D07004 & PNNL-AGG-415 \\
\hline $14265-72-6$ & Antimony & $5.82 \mathrm{E}-4$ & ug/g dry & $5.41 \mathrm{E}-4$ & $4 / 14 / 08$ & 8D07004 & PNNL-AGG-415 \\
\hline 13966-28-4 & Lead & $<5.61 \mathrm{E}-4$ & ug/g dry & $5.61 \mathrm{E}-4$ & $4 / 14 / 08$ & 8D07004 & PNNL-AGG-415 \\
\hline HEIS No. & B1RKX7 & \multicolumn{3}{|c|}{ Lab ID: $\quad$ 0802028-29 } & & & \\
\hline $14378-38-2$ & Silver & $<1.07 \mathrm{E}-3$ & ug/g dry & $1.07 \mathrm{E}-3$ & $4 / 14 / 08$ & 8D07004 & PNNL-AGG-415 \\
\hline $14336-64-2$ & Cadmium & $<2.95 \mathrm{E}-4$ & ug/g dry & $2.95 \mathrm{E}-4$ & $4 / 14 / 08$ & 8D07004 & PNNL-AGG-415 \\
\hline $14265-72-6$ & Antimony & $6.44 \mathrm{E}-4$ & ug/g dry & $5.40 \mathrm{E}-4$ & $4 / 14 / 08$ & 8D07004 & PNNL-AGG-415 \\
\hline $13966-28-4$ & Lead & $<5.60 \mathrm{E}-4$ & ug/g dry & $5.60 \mathrm{E}-4$ & $4 / 14 / 08$ & 8D07004 & PNNL-AGG-415 \\
\hline
\end{tabular}


RCRA Metals By PNNL-AGG-415/Acid Extract

\begin{tabular}{|c|c|c|c|c|c|c|c|}
\hline CAS \# & Analyte & Results & Units & EQL & Analyzed & Batch & Method \\
\hline HEIS No. & B1RKL1 & \multicolumn{3}{|c|}{ Lab ID: $\quad$ 0802002-02 } & & & \\
\hline $14336-64-2$ & Cadmium & 4.43E-2 & ug/g dry & $1.14 \mathrm{E}-2$ & 2/19/08 & 8E01016 & PNNL-AGG-415 \\
\hline $14265-72-6$ & Antimony & $<1.84 \mathrm{E}-2$ & ug/g dry & $1.84 \mathrm{E}-2$ & 2/19/08 & 8E01016 & PNNL-AGG-415 \\
\hline 13966-28-4 & Lead & 2.15E0 & ug/g dry & $8.55 \mathrm{E}-3$ & 2/19/08 & 8E01016 & PNNL-AGG-415 \\
\hline HEIS No. & B1RKL6 & \multicolumn{3}{|c|}{ Lab ID: $\quad$ 0802002-09 } & & & \\
\hline $14336-64-2$ & Cadmium & $5.23 \mathrm{E}-2$ & ug/g dry & $1.14 \mathrm{E}-2$ & 2/13/08 & 8E01015 & PNNL-AGG-415 \\
\hline $14265-72-6$ & Antimony & $<1.84 \mathrm{E}-2$ & ug/g dry & $1.84 \mathrm{E}-2$ & 2/13/08 & 8E01015 & PNNL-AGG-415 \\
\hline 13966-28-4 & Lead & 2.37E0 & ug/g dry & $8.57 \mathrm{E}-3$ & 2/13/08 & 8E01015 & PNNL-AGG-415 \\
\hline HEIS No. & B1RKM1 & \multicolumn{3}{|c|}{ Lab ID: $\quad$ 0802002-17 } & & & \\
\hline $14336-64-2$ & Cadmium & $5.38 \mathrm{E}-2$ & ug/g dry & $1.07 \mathrm{E}-2$ & 2/19/08 & 8E01016 & PNNL-AGG-415 \\
\hline $14265-72-6$ & Antimony & $<1.73 \mathrm{E}-2$ & ug/g dry & $1.73 \mathrm{E}-2$ & 2/19/08 & 8E01016 & PNNL-AGG-415 \\
\hline $13966-28-4$ & Lead & $2.50 \mathrm{E} 0$ & ug/g dry & 8.03E-3 & 2/19/08 & 8E01016 & PNNL-AGG-415 \\
\hline HEIS No. & B1RKM8 & \multicolumn{3}{|c|}{ Lab ID: $\quad$ 0802002-31 } & & & \\
\hline $14336-64-2$ & Cadmium & $4.64 \mathrm{E}-2$ & ug/g dry & $1.08 \mathrm{E}-2$ & $2 / 13 / 08$ & 8E01015 & PNNL-AGG-415 \\
\hline $14265-72-6$ & Antimony & $<1.75 \mathrm{E}-2$ & ug/g dry & $1.75 \mathrm{E}-2$ & 2/13/08 & 8E01015 & PNNL-AGG-415 \\
\hline 13966-28-4 & Lead & 2.13E0 & ug/g dry & $8.12 \mathrm{E}-3$ & 2/13/08 & 8E01015 & PNNL-AGG-415 \\
\hline HEIS No. & B1RKW7 & \multicolumn{3}{|c|}{ Lab ID: $\quad$ 0802003-02 } & & & \\
\hline $14336-64-2$ & Cadmium & 1.03E-1 & ug/g dry & $1.40 \mathrm{E}-2$ & 2/19/08 & 8E01016 & PNNL-AGG-415 \\
\hline $14265-72-6$ & Antimony & $<2.27 \mathrm{E}-2$ & ug/g dry & $2.27 \mathrm{E}-2$ & 2/19/08 & 8E01016 & PNNL-AGG-415 \\
\hline 13966-28-4 & Lead & 4.78E0 & ug/g dry & $1.05 \mathrm{E}-2$ & 2/19/08 & 8E01016 & PNNL-AGG-415 \\
\hline HEIS No. & B1RKW8 & \multicolumn{3}{|c|}{ Lab ID: $\quad$ 0802003-04 } & & & \\
\hline $14336-64-2$ & Cadmium & $1.25 \mathrm{E}-1$ & ug/g dry & $1.37 \mathrm{E}-2$ & 2/13/08 & 8E01015 & PNNL-AGG-415 \\
\hline $14265-72-6$ & Antimony & $<2.22 \mathrm{E}-2$ & ug/g dry & $2.22 \mathrm{E}-2$ & 2/13/08 & 8E01015 & PNNL-AGG-415 \\
\hline $13966-28-4$ & Lead & $6.46 \mathrm{E} 0$ & ug/g dry & $1.03 \mathrm{E}-2$ & 2/13/08 & 8E01015 & PNNL-AGG-415 \\
\hline HEIS No. & B1RKN7 & \multicolumn{3}{|c|}{ Lab ID: $\quad$ 0802005-03 } & & & \\
\hline $14336-64-2$ & Cadmium & 4.55E-2 & ug/g dry & $1.01 \mathrm{E}-2$ & 2/19/08 & 8E01016 & PNNL-AGG-415 \\
\hline $14265-72-6$ & Antimony & $<1.64 \mathrm{E}-2$ & ug/g dry & $1.64 \mathrm{E}-2$ & 2/19/08 & 8E01016 & PNNL-AGG-415 \\
\hline 13966-28-4 & Lead & 2.19E0 & ug/g dry & $7.61 \mathrm{E}-3$ & 2/19/08 & 8E01016 & PNNL-AGG-415 \\
\hline HEIS No. & B1RKP3 & \multicolumn{3}{|c|}{ Lab ID: $\quad$ 0802005-09 } & & & \\
\hline $14336-64-2$ & Cadmium & $5.58 \mathrm{E}-2$ & ug/g dry & $1.13 \mathrm{E}-2$ & 2/13/08 & 8E01015 & PNNL-AGG-415 \\
\hline $14265-72-6$ & Antimony & $<1.82 \mathrm{E}-2$ & ug/g dry & $1.82 \mathrm{E}-2$ & 2/13/08 & 8E01015 & PNNL-AGG-415 \\
\hline 13966-28-4 & Lead & $2.40 \mathrm{E} 0$ & ug/g dry & $8.47 \mathrm{E}-3$ & 2/13/08 & 8E01015 & PNNL-AGG-415 \\
\hline HEIS No. & B1RKP9 & \multicolumn{3}{|c|}{ Lab ID: $\quad$ 0802005-15 } & & & \\
\hline $14336-64-2$ & Cadmium & 4.37E-2 & ug/g dry & $1.13 \mathrm{E}-2$ & 2/19/08 & 8E01016 & PNNL-AGG-415 \\
\hline $14265-72-6$ & Antimony & $<1.82 \mathrm{E}-2$ & ug/g dry & $1.82 \mathrm{E}-2$ & 2/19/08 & 8E01016 & PNNL-AGG-415 \\
\hline $13966-28-4$ & Lead & $1.81 \mathrm{E} 0$ & ug/g dry & $8.46 \mathrm{E}-3$ & $2 / 19 / 08$ & 8E01016 & PNNL-AGG-415 \\
\hline HEIS No. & B1RKR5 & \multicolumn{3}{|c|}{ Lab ID: $\quad$ 0802005-21 } & & & \\
\hline $14336-64-2$ & Cadmium & $5.56 \mathrm{E}-2$ & ug/g dry & $1.23 \mathrm{E}-2$ & 2/13/08 & 8E01015 & PNNL-AGG-415 \\
\hline $14265-72-6$ & Antimony & $<1.98 \mathrm{E}-2$ & ug/g dry & $1.98 \mathrm{E}-2$ & 2/13/08 & 8E01015 & PNNL-AGG-415 \\
\hline 13966-28-4 & Lead & $1.93 \mathrm{E} 0$ & ug/g dry & $9.22 \mathrm{E}-3$ & $2 / 13 / 08$ & 8E01015 & PNNL-AGG-415 \\
\hline HEIS No. & B1RKR9 & \multicolumn{3}{|c|}{ Lab ID: $\quad$ 0802005-25 } & & & \\
\hline $14336-64-2$ & Cadmium & 4.75E-2 & ug/g dry & $1.19 \mathrm{E}-2$ & $2 / 19 / 08$ & 8E01016 & PNNL-AGG-415 \\
\hline $14265-72-6$ & Antimony & $<1.92 \mathrm{E}-2$ & ug/g dry & $1.92 \mathrm{E}-2$ & $2 / 19 / 08$ & 8E01016 & PNNL-AGG-415 \\
\hline 13966-28-4 & Lead & 2.05E0 & ug/g dry & $8.91 \mathrm{E}-3$ & 2/19/08 & 8E01016 & PNNL-AGG-415 \\
\hline HEIS No. & B1RKT3 & \multicolumn{3}{|c|}{ Lab ID: $\quad$ 0802005-29 } & & & \\
\hline 14336-64-2 & Cadmium & $5.85 \mathrm{E}-2$ & ug/g dry & $1.25 \mathrm{E}-2$ & 2/13/08 & 8E01015 & PNNL-AGG-415 \\
\hline $14265-72-6$ & Antimony & $<2.02 \mathrm{E}-2$ & ug/g dry & 2.02E-2 & 2/13/08 & 8E01015 & PNNL-AGG-415 \\
\hline 13966-28-4 & Lead & 2.26E0 & ug/g dry & $9.41 \mathrm{E}-3$ & 2/13/08 & 8E01015 & PNNL-AGG-415 \\
\hline
\end{tabular}


RCRA Metals By PNNL-AGG-415/Acid Extract

\begin{tabular}{|c|c|c|c|c|c|c|c|}
\hline CAS \# & Analyte & Results & Units & EQL & Analyzed & Batch & Method \\
\hline HEIS No. & B1RKT7 & \multicolumn{3}{|c|}{ Lab ID: $\quad$ 0802006-07 } & & & \\
\hline $14336-64-2$ & Cadmium & $4.71 \mathrm{E}-2$ & ug/g dry & $1.22 \mathrm{E}-2$ & 2/19/08 & 8E01016 & PNNL-AGG-415 \\
\hline $14265-72-6$ & Antimony & $<1.98 \mathrm{E}-2$ & ug/g dry & $1.98 \mathrm{E}-2$ & 2/19/08 & 8E01016 & PNNL-AGG-415 \\
\hline 13966-28-4 & Lead & 2.09E0 & ug/g dry & $9.20 \mathrm{E}-3$ & 2/19/08 & 8E01016 & PNNL-AGG-415 \\
\hline HEIS No. & B1RKV1 & \multicolumn{3}{|c|}{ Lab ID: $\quad$ 0802006-11 } & & & \\
\hline $14336-64-2$ & Cadmium & $5.18 \mathrm{E}-2$ & ug/g dry & $1.19 \mathrm{E}-2$ & 2/13/08 & 8E01015 & PNNL-AGG-415 \\
\hline $14265-72-6$ & Antimony & $<1.93 \mathrm{E}-2$ & ug/g dry & $1.93 \mathrm{E}-2$ & 2/13/08 & 8E01015 & PNNL-AGG-415 \\
\hline 13966-28-4 & Lead & 2.12E0 & ug/g dry & 8.97E-3 & 2/13/08 & 8E01015 & PNNL-AGG-415 \\
\hline HEIS No. & B1RKV5 & \multicolumn{3}{|c|}{ Lab ID: $\quad$ 0802007-04 } & & & \\
\hline $14336-64-2$ & Cadmium & $5.02 \mathrm{E}-2$ & ug/g dry & $1.17 \mathrm{E}-2$ & 2/19/08 & 8E01016 & PNNL-AGG-415 \\
\hline $14265-72-6$ & Antimony & $<1.90 \mathrm{E}-2$ & ug/g dry & $1.90 \mathrm{E}-2$ & 2/19/08 & 8E01016 & PNNL-AGG-415 \\
\hline $13966-28-4$ & Lead & $1.72 \mathrm{E} 0$ & ug/g dry & $8.81 \mathrm{E}-3$ & 2/19/08 & 8E01016 & PNNL-AGG-415 \\
\hline HEIS No. & B1RKV9 & \multicolumn{3}{|c|}{ Lab ID: $\quad$ 0802007-12 } & & & \\
\hline $14336-64-2$ & Cadmium & $5.86 \mathrm{E}-2$ & ug/g dry & $1.13 \mathrm{E}-2$ & $2 / 13 / 08$ & 8E01015 & PNNL-AGG-415 \\
\hline $14265-72-6$ & Antimony & $<1.83 \mathrm{E}-2$ & ug/g dry & $1.83 \mathrm{E}-2$ & 2/13/08 & 8E01015 & PNNL-AGG-415 \\
\hline 13966-28-4 & Lead & 2.02E0 & ug/g dry & $8.52 \mathrm{E}-3$ & 2/13/08 & 8E01015 & PNNL-AGG-415 \\
\hline HEIS No. & B1RKW2 & \multicolumn{3}{|c|}{ Lab ID: $\quad$ 0802008-04 } & & & \\
\hline $14336-64-2$ & Cadmium & $1.21 \mathrm{E}-1$ & ug/g dry & $1.36 \mathrm{E}-2$ & 2/19/08 & 8E01016 & PNNL-AGG-415 \\
\hline $14265-72-6$ & Antimony & $<2.20 \mathrm{E}-2$ & ug/g dry & $2.20 \mathrm{E}-2$ & 2/19/08 & 8E01016 & PNNL-AGG-415 \\
\hline 13966-28-4 & Lead & 3.55E0 & ug/g dry & $1.02 \mathrm{E}-2$ & 2/19/08 & 8E01016 & PNNL-AGG-415 \\
\hline HEIS No. & B1RKW4 & \multicolumn{3}{|c|}{ Lab ID: $\quad$ 0802008-08 } & & & \\
\hline $14336-64-2$ & Cadmium & $1.51 \mathrm{E}-1$ & ug/g dry & $1.35 \mathrm{E}-2$ & 2/13/08 & 8E01015 & PNNL-AGG-415 \\
\hline $14265-72-6$ & Antimony & $<2.18 \mathrm{E}-2$ & ug/g dry & $2.18 \mathrm{E}-2$ & 2/13/08 & 8E01015 & PNNL-AGG-415 \\
\hline $13966-28-4$ & Lead & $4.08 \mathrm{E} 0$ & ug/g dry & $1.02 \mathrm{E}-2$ & 2/13/08 & 8E01015 & PNNL-AGG-415 \\
\hline HEIS No. & B1RKW5 & \multicolumn{3}{|c|}{ Lab ID: $\quad$ 0802009-02 } & & & \\
\hline $14336-64-2$ & Cadmium & 9.77E-2 & ug/g dry & $1.31 \mathrm{E}-2$ & 2/13/08 & 8E01015 & PNNL-AGG-415 \\
\hline $14265-72-6$ & Antimony & $<2.12 \mathrm{E}-2$ & ug/g dry & $2.12 \mathrm{E}-2$ & 2/13/08 & 8E01015 & PNNL-AGG-415 \\
\hline 13966-28-4 & Lead & 2.83E0 & ug/g dry & $9.85 \mathrm{E}-3$ & 2/13/08 & 8E01015 & PNNL-AGG-415 \\
\hline HEIS No. & B1RKW6 & \multicolumn{3}{|c|}{ Lab ID: $\quad$ 0802009-04 } & & & \\
\hline 14336-64-2 & Cadmium & $9.40 \mathrm{E}-2$ & ug/g dry & $1.40 \mathrm{E}-2$ & 2/13/08 & 8E01015 & PNNL-AGG-415 \\
\hline $14265-72-6$ & Antimony & $<2.27 \mathrm{E}-2$ & ug/g dry & $2.27 \mathrm{E}-2$ & 2/13/08 & 8E01015 & PNNL-AGG-415 \\
\hline 13966-28-4 & Lead & 2.70E0 & ug/g dry & $1.05 \mathrm{E}-2$ & 2/13/08 & 8E01015 & PNNL-AGG-415 \\
\hline HEIS No. & B1TFV6 & \multicolumn{3}{|c|}{ Lab ID: $\quad$ 0802028-03 } & & & \\
\hline $14336-64-2$ & Cadmium & 1.64E-1 & ug/g dry & $6.23 \mathrm{E}-2$ & 6/03/08 & 8E15001 & PNNL-AGG-415 \\
\hline $14265-72-6$ & Antimony & $<1.01 \mathrm{E}-1$ & ug/g dry & $1.01 \mathrm{E}-1$ & 6/03/08 & 8E15001 & PNNL-AGG-415 \\
\hline $13966-28-4$ & Lead & 5.57E0 & ug/g dry & $4.68 \mathrm{E}-2$ & 6/03/08 & 8E15001 & PNNL-AGG-415 \\
\hline HEIS No. & B1TFV8 & \multicolumn{3}{|c|}{ Lab ID: $\quad$ 0802028-05 } & & & \\
\hline 14336-64-2 & Cadmium & $9.62 \mathrm{E}-2$ & ug/g dry & $5.85 \mathrm{E}-2$ & 6/03/08 & 8E15001 & PNNL-AGG-415 \\
\hline $14265-72-6$ & Antimony & $<9.46 \mathrm{E}-2$ & ug/g dry & $9.46 \mathrm{E}-2$ & 6/03/08 & 8E15001 & PNNL-AGG-415 \\
\hline $13966-28-4$ & Lead & 3.31E0 & ug/g dry & $4.40 \mathrm{E}-2$ & 6/03/08 & 8E15001 & PNNL-AGG-415 \\
\hline HEIS No. & B1TFW0 & \multicolumn{3}{|c|}{ Lab ID: $\quad$ 0802028-07 } & & & \\
\hline $14336-64-2$ & Cadmium & 9.73E-2 & ug/g dry & $6.09 \mathrm{E}-2$ & 6/03/08 & 8E15001 & PNNL-AGG-415 \\
\hline $14265-72-6$ & Antimony & $<9.85 \mathrm{E}-2$ & ug/g dry & $9.85 \mathrm{E}-2$ & 6/03/08 & 8E15001 & PNNL-AGG-415 \\
\hline 13966-28-4 & Lead & 3.17E0 & ug/g dry & $4.58 \mathrm{E}-2$ & 6/03/08 & 8E15001 & PNNL-AGG-415 \\
\hline HEIS No. & B1TFW2 & \multicolumn{3}{|c|}{ Lab ID: $\quad$ 0802028-09 } & & & \\
\hline 14336-64-2 & Cadmium & $1.51 \mathrm{E}-1$ & ug/g dry & $6.31 \mathrm{E}-2$ & 6/03/08 & 8E15001 & PNNL-AGG-415 \\
\hline $14265-72-6$ & Antimony & $<1.02 \mathrm{E}-1$ & ug/g dry & $1.02 \mathrm{E}-1$ & 6/03/08 & 8E15001 & PNNL-AGG-415 \\
\hline 13966-28-4 & Lead & 6.64E0 & ug/g dry & 4.75E-2 & 6/03/08 & 8E15001 & PNNL-AGG-415 \\
\hline
\end{tabular}


RCRA Metals By PNNL-AGG-415/Acid Extract

\begin{tabular}{|c|c|c|c|c|c|c|c|}
\hline CAS \# & Analyte & Results & Units & EQL & Analyzed & Batch & Method \\
\hline HEIS No. & B1RKX0 & \multicolumn{3}{|c|}{ Lab ID: $\quad$ 0802028-11 } & & & \\
\hline 14336-64-2 & Cadmium & $1.16 \mathrm{E}-1$ & ug/g dry & $6.18 \mathrm{E}-2$ & $6 / 03 / 08$ & 8E15001 & PNNL-AGG-415 \\
\hline $14265-72-6$ & Antimony & $<1.00 \mathrm{E}-1$ & ug/g dry & $1.00 \mathrm{E}-1$ & 6/03/08 & 8E15001 & PNNL-AGG-415 \\
\hline 13966-28-4 & Lead & 6.93E0 & ug/g dry & 4.65E-2 & 6/03/08 & 8E15001 & PNNL-AGG-415 \\
\hline HEIS No. & B1RKX3 & \multicolumn{3}{|c|}{ Lab ID: $\quad$ 0802028-17 } & & & \\
\hline 14336-64-2 & Cadmium & 5.35E-2 & ug/g dry & 5.23E-2 & 6/03/08 & 8E15001 & PNNL-AGG-415 \\
\hline $14265-72-6$ & Antimony & $<8.46 \mathrm{E}-2$ & ug/g dry & $8.46 \mathrm{E}-2$ & 6/03/08 & 8E15001 & PNNL-AGG-415 \\
\hline 13966-28-4 & Lead & 2.05E0 & ug/g dry & 3.93E-2 & $6 / 03 / 08$ & 8E15001 & PNNL-AGG-415 \\
\hline HEIS No. & B1RKX5 & \multicolumn{3}{|c|}{ Lab ID: $\quad$ 0802028-23 } & & & \\
\hline 14336-64-2 & Cadmium & 5.51E-2 & ug/g dry & $5.26 \mathrm{E}-2$ & 6/03/08 & 8E15001 & PNNL-AGG-415 \\
\hline $14265-72-6$ & Antimony & $<8.51 \mathrm{E}-2$ & ug/g dry & 8.51E-2 & 6/03/08 & 8E15001 & PNNL-AGG-415 \\
\hline $13966-28-4$ & Lead & $1.86 \mathrm{E} 0$ & ug/g dry & $3.95 \mathrm{E}-2$ & 6/03/08 & 8E15001 & PNNL-AGG-415 \\
\hline HEIS No. & B1RKX7 & \multicolumn{3}{|c|}{ Lab ID: $\quad$ 0802028-29 } & & & \\
\hline $14336-64-2$ & Cadmium & $<5.27 \mathrm{E}-2$ & ug/g dry & $5.27 \mathrm{E}-2$ & 6/03/08 & 8E15001 & PNNL-AGG-415 \\
\hline $14265-72-6$ & Antimony & $<8.53 \mathrm{E}-2$ & ug/g dry & 8.53E-2 & 6/03/08 & 8E15001 & PNNL-AGG-415 \\
\hline $13966-28-4$ & Lead & $1.81 \mathrm{E} 0$ & ug/g dry & $3.97 \mathrm{E}-2$ & 6/03/08 & 8E15001 & PNNL-AGG-415 \\
\hline
\end{tabular}




\section{GEA/Soil}

\begin{tabular}{|c|c|c|c|c|c|c|c|c|}
\hline CAS \# & Analyte & Results & Units & MDA & UNC & Analyzed & Batch & Method \\
\hline HEIS No. & B1RKL1 & \multicolumn{4}{|c|}{ Lab ID: $\quad$ 0802002-02 } & & & \\
\hline 10198-40-0 & Cobalt-60 & $<1.93 \mathrm{E}-1$ & pCi/g dry & 1.93E-1 & & 2/18/08 & 8D28002 & AGG-RRL-001 \\
\hline $10045-97-3$ & Cesium-137 & $<2.31 \mathrm{E}-1$ & pCi/g dry & $2.31 \mathrm{E}-1$ & & 2/18/08 & 8D28002 & AGG-RRL-001 \\
\hline 14683-23-9 & Europium-152 & $<7.85 \mathrm{E}-1$ & pCi/g dry & $7.85 \mathrm{E}-1$ & & 2/18/08 & 8D28002 & AGG-RRL-001 \\
\hline 15585-10-1 & Europium-154 & $<4.90 \mathrm{E}-1$ & pCi/g dry & 4.90E-1 & & 2/18/08 & 8D28002 & AGG-RRL-001 \\
\hline 14391-16-3 & Europium-155 & $<7.55 \mathrm{E}-1$ & pCi/g dry & 7.55E-1 & & 2/18/08 & 8D28002 & AGG-RRL-001 \\
\hline HEIS No. & B1RKL6 & \multicolumn{4}{|c|}{$\begin{array}{ll}\text { Lab ID: } & 0802002-09\end{array}$} & & & \\
\hline 10198-40-0 & Cobalt-60 & $<2.03 \mathrm{E}-1$ & pCi/g dry & 2.03E-1 & & 2/18/08 & 8D28002 & AGG-RRL-001 \\
\hline $10045-97-3$ & Cesium-137 & $<2.29 \mathrm{E}-1$ & pCi/g dry & $2.29 \mathrm{E}-1$ & & 2/18/08 & 8D28002 & AGG-RRL-001 \\
\hline 14683-23-9 & Europium-152 & $<8.96 \mathrm{E}-1$ & pCi/g dry & 8.96E-1 & & 2/18/08 & 8D28002 & AGG-RRL-001 \\
\hline 15585-10-1 & Europium-154 & $<4.95 \mathrm{E}-1$ & pCi/g dry & 4.95E-1 & & 2/18/08 & 8D28002 & AGG-RRL-001 \\
\hline 14391-16-3 & Europium-155 & $<8.39 \mathrm{E}-1$ & pCi/g dry & 8.39E-1 & & 2/18/08 & 8D28002 & AGG-RRL-001 \\
\hline HEIS No. & B1RKM1 & \multicolumn{4}{|c|}{ Lab ID: $\quad$ 0802002-17 } & & & \\
\hline 10198-40-0 & Cobalt-60 & $<1.95 \mathrm{E}-1$ & pCi/g dry & $1.95 \mathrm{E}-1$ & & 2/18/08 & 8D28002 & AGG-RRL-001 \\
\hline $10045-97-3$ & Cesium-137 & $<2.24 \mathrm{E}-1$ & pCi/g dry & $2.24 \mathrm{E}-1$ & & 2/18/08 & 8D28002 & AGG-RRL-001 \\
\hline $14683-23-9$ & Europium-152 & $<8.22 \mathrm{E}-1$ & pCi/g dry & $8.22 \mathrm{E}-1$ & & $2 / 18 / 08$ & 8D28002 & AGG-RRL-001 \\
\hline 15585-10-1 & Europium-154 & $<4.80 \mathrm{E}-1$ & pCi/g dry & $4.80 \mathrm{E}-1$ & & 2/18/08 & 8D28002 & AGG-RRL-001 \\
\hline 14391-16-3 & Europium-155 & $<7.54 \mathrm{E}-1$ & pCi/g dry & 7.54E-1 & & 2/18/08 & 8D28002 & AGG-RRL-001 \\
\hline HEIS No. & B1RKM8 & \multicolumn{4}{|c|}{ Lab ID: $\quad$ 0802002-31 } & & & \\
\hline 10198-40-0 & Cobalt-60 & $<2.31 \mathrm{E}-1$ & pCi/g dry & $2.31 \mathrm{E}-1$ & & 2/18/08 & 8D28002 & AGG-RRL-001 \\
\hline $10045-97-3$ & Cesium-137 & $<2.57 \mathrm{E}-1$ & pCi/g dry & $2.57 \mathrm{E}-1$ & & 2/18/08 & 8D28002 & AGG-RRL-001 \\
\hline 14683-23-9 & Europium-152 & $<1.01 \mathrm{E} 0$ & pCi/g dry & $1.01 \mathrm{E} 0$ & & 2/18/08 & 8D28002 & AGG-RRL-001 \\
\hline 15585-10-1 & Europium-154 & $<5.60 \mathrm{E}-1$ & pCi/g dry & $5.60 \mathrm{E}-1$ & & 2/18/08 & 8D28002 & AGG-RRL-001 \\
\hline 14391-16-3 & Europium-155 & $<9.33 \mathrm{E}-1$ & pCi/g dry & 9.33E-1 & & 2/18/08 & 8D28002 & AGG-RRL-001 \\
\hline HEIS No. & B1RKW7 & \multicolumn{4}{|c|}{$\begin{array}{ll}\text { Lab ID: } & \text { 0802003-02 }\end{array}$} & & & \\
\hline $10198-40-0$ & Cobalt-60 & $<2.73 \mathrm{E}-1$ & pCi/g dry & 2.73E-1 & & $2 / 19 / 08$ & 8D28002 & AGG-RRL-001 \\
\hline $10045-97-3$ & Cesium-137 & $<3.22 \mathrm{E}-1$ & pCi/g dry & 3.22E-1 & & 2/19/08 & 8D28002 & AGG-RRL-001 \\
\hline $14683-23-9$ & Europium-152 & $<1.18 \mathrm{E} 0$ & pCi/g dry & $1.18 \mathrm{E} 0$ & & 2/19/08 & 8D28002 & AGG-RRL-001 \\
\hline $15585-10-1$ & Europium-154 & $<7.11 \mathrm{E}-1$ & pCi/g dry & 7.11E-1 & & 2/19/08 & 8D28002 & AGG-RRL-001 \\
\hline 14391-16-3 & Europium-155 & $<1.19 \mathrm{E} 0$ & pCi/g dry & 1.19E0 & & 2/19/08 & 8D28002 & AGG-RRL-001 \\
\hline HEIS No. & B1RKW8 & \multicolumn{4}{|c|}{ Lab ID: $\quad$ 0802003-04 } & & & \\
\hline 10198-40-0 & Cobalt-60 & $<2.97 \mathrm{E}-1$ & pCi/g dry & 2.97E-1 & & 2/19/08 & 8D28002 & AGG-RRL-001 \\
\hline $10045-97-3$ & Cesium-137 & $<3.33 \mathrm{E}-1$ & pCi/g dry & 3.33E-1 & & 2/19/08 & 8D28002 & AGG-RRL-001 \\
\hline 14683-23-9 & Europium-152 & $<1.20 \mathrm{E} 0$ & pCi/g dry & $1.20 \mathrm{E} 0$ & & 2/19/08 & 8D28002 & AGG-RRL-001 \\
\hline 15585-10-1 & Europium-154 & $<7.52 \mathrm{E}-1$ & pCi/g dry & $7.52 \mathrm{E}-1$ & & 2/19/08 & 8D28002 & AGG-RRL-001 \\
\hline 14391-16-3 & Europium-155 & $<1.18 \mathrm{E} 0$ & pCi/g dry & $1.18 \mathrm{E} 0$ & & 2/19/08 & 8D28002 & AGG-RRL-001 \\
\hline HEIS No. & B1RKN7 & \multicolumn{4}{|c|}{$\begin{array}{ll}\text { Lab ID: } & 0802005-03\end{array}$} & & & \\
\hline $10198-40-0$ & Cobalt-60 & $<2.22 \mathrm{E}-1$ & pCi/g dry & $2.22 \mathrm{E}-1$ & & 2/19/08 & 8D28002 & AGG-RRL-001 \\
\hline $10045-97-3$ & Cesium-137 & $<2.68 \mathrm{E}-1$ & pCi/g dry & $2.68 \mathrm{E}-1$ & & 2/19/08 & 8D28002 & AGG-RRL-001 \\
\hline 14683-23-9 & Europium-152 & $<1.03 \mathrm{E} 0$ & pCi/g dry & 1.03E0 & & 2/19/08 & 8D28002 & AGG-RRL-001 \\
\hline $15585-10-1$ & Europium-154 & $<5.79 \mathrm{E}-1$ & pCi/g dry & $5.79 \mathrm{E}-1$ & & 2/19/08 & 8D28002 & AGG-RRL-001 \\
\hline 14391-16-3 & Europium-155 & $<9.41 \mathrm{E}-1$ & pCi/g dry & $9.41 \mathrm{E}-1$ & & 2/19/08 & 8D28002 & AGG-RRL-001 \\
\hline HEIS No. & B1RKP3 & \multicolumn{4}{|c|}{$\begin{array}{ll}\text { Lab ID: } & \text { 0802005-09 }\end{array}$} & & & \\
\hline 10198-40-0 & Cobalt-60 & $<1.67 \mathrm{E}-1$ & pCi/g dry & $1.67 \mathrm{E}-1$ & & $2 / 20 / 08$ & 8D28002 & AGG-RRL-001 \\
\hline $10045-97-3$ & Cesium-137 & $<2.21 \mathrm{E}-1$ & pCi/g dry & $2.21 \mathrm{E}-1$ & & 2/20/08 & 8D28002 & AGG-RRL-001 \\
\hline $14683-23-9$ & Europium-152 & $<7.08 \mathrm{E}-1$ & pCi/g dry & $7.08 \mathrm{E}-1$ & & 2/20/08 & 8D28002 & AGG-RRL-001 \\
\hline 15585-10-1 & Europium-154 & $<4.49 \mathrm{E}-1$ & pCi/g dry & 4.49E-1 & & 2/20/08 & 8D28002 & AGG-RRL-001 \\
\hline 14391-16-3 & Europium-155 & $<6.91 \mathrm{E}-1$ & pCi/g dry & $6.91 \mathrm{E}-1$ & & $2 / 20 / 08$ & 8D28002 & AGG-RRL-001 \\
\hline
\end{tabular}




\section{GEA/Soil}

\begin{tabular}{|c|c|c|c|c|c|c|c|c|}
\hline CAS \# & Analyte & Results & Units & MDA & UNC & Analyzed & Batch & Method \\
\hline HEIS No. & B1RKP9 & \multicolumn{3}{|c|}{ Lab ID: $\quad$ 0802005-15 } & & & & \\
\hline 10198-40-0 & Cobalt-60 & $<2.05 \mathrm{E}-1$ & pCi/g dry & 2.05E-1 & & 2/20/08 & 8D28002 & AGG-RRL-001 \\
\hline $10045-97-3$ & Cesium-137 & $<2.31 \mathrm{E}-1$ & pCi/g dry & $2.31 \mathrm{E}-1$ & & $2 / 20 / 08$ & 8D28002 & AGG-RRL-001 \\
\hline 14683-23-9 & Europium-152 & $<9.00 \mathrm{E}-1$ & pCi/g dry & $9.00 \mathrm{E}-1$ & & $2 / 20 / 08$ & 8D28002 & AGG-RRL-001 \\
\hline 15585-10-1 & Europium-154 & $<5.19 \mathrm{E}-1$ & pCi/g dry & 5.19E-1 & & 2/20/08 & 8D28002 & AGG-RRL-001 \\
\hline 14391-16-3 & Europium-155 & $<8.51 \mathrm{E}-1$ & pCi/g dry & $8.51 \mathrm{E}-1$ & & 2/20/08 & 8D28002 & AGG-RRL-001 \\
\hline HEIS No. & B1RKR5 & \multicolumn{3}{|c|}{$\begin{array}{ll}\text { Lab ID: } & 0802005-21\end{array}$} & & & & \\
\hline 10198-40-0 & Cobalt-60 & $<1.68 \mathrm{E}-1$ & pCi/g dry & $1.68 \mathrm{E}-1$ & & 2/20/08 & 8D28002 & AGG-RRL-001 \\
\hline $10045-97-3$ & Cesium-137 & $<2.04 \mathrm{E}-1$ & pCi/g dry & $2.04 \mathrm{E}-1$ & & 2/20/08 & 8D28002 & AGG-RRL-001 \\
\hline 14683-23-9 & Europium-152 & $<6.96 \mathrm{E}-1$ & pCi/g dry & $6.96 \mathrm{E}-1$ & & 2/20/08 & 8D28002 & AGG-RRL-001 \\
\hline $15585-10-1$ & Europium-154 & $<4.27 \mathrm{E}-1$ & pCi/g dry & $4.27 \mathrm{E}-1$ & & 2/20/08 & 8D28002 & AGG-RRL-001 \\
\hline 14391-16-3 & Europium-155 & $<6.56 \mathrm{E}-1$ & pCi/g dry & $6.56 \mathrm{E}-1$ & & 2/20/08 & 8D28002 & AGG-RRL-001 \\
\hline HEIS No. & B1RKR9 & \multicolumn{3}{|c|}{ Lab ID: $\quad$ 0802005-25 } & & & & \\
\hline 10198-40-0 & Cobalt-60 & $<2.31 \mathrm{E}-1$ & pCi/g dry & $2.31 \mathrm{E}-1$ & & 2/20/08 & 8D28002 & AGG-RRL-001 \\
\hline $10045-97-3$ & Cesium-137 & $<2.78 \mathrm{E}-1$ & pCi/g dry & $2.78 \mathrm{E}-1$ & & 2/20/08 & 8D28002 & AGG-RRL-001 \\
\hline 14683-23-9 & Europium-152 & $<1.01 \mathrm{E} 0$ & pCi/g dry & $1.01 \mathrm{E} 0$ & & $2 / 20 / 08$ & 8D28002 & AGG-RRL-001 \\
\hline 15585-10-1 & Europium-154 & $<5.80 \mathrm{E}-1$ & pCi/g dry & $5.80 \mathrm{E}-1$ & & $2 / 20 / 08$ & 8D28002 & AGG-RRL-001 \\
\hline 14391-16-3 & Europium-155 & $<9.58 \mathrm{E}-1$ & pCi/g dry & $9.58 \mathrm{E}-1$ & & 2/20/08 & 8D28002 & AGG-RRL-001 \\
\hline HEIS No. & B1RKT3 & \multicolumn{3}{|c|}{ Lab ID: $\quad$ 0802005-29 } & & & & \\
\hline 10198-40-0 & Cobalt-60 & $<1.95 \mathrm{E}-1$ & pCi/g dry & 1.95E-1 & & $2 / 20 / 08$ & 8D28002 & AGG-RRL-001 \\
\hline $10045-97-3$ & Cesium-137 & $<2.05 \mathrm{E}-1$ & pCi/g dry & 2.05E-1 & & $2 / 20 / 08$ & 8D28002 & AGG-RRL-001 \\
\hline 14683-23-9 & Europium-152 & $<7.37 \mathrm{E}-1$ & pCi/g dry & 7.37E-1 & & $2 / 20 / 08$ & 8D28002 & AGG-RRL-001 \\
\hline 15585-10-1 & Europium-154 & $<4.59 \mathrm{E}-1$ & pCi/g dry & $4.59 \mathrm{E}-1$ & & 2/20/08 & 8D28002 & AGG-RRL-001 \\
\hline 14391-16-3 & Europium-155 & $<7.00 \mathrm{E}-1$ & pCi/g dry & $7.00 \mathrm{E}-1$ & & 2/20/08 & 8D28002 & AGG-RRL-001 \\
\hline HEIS No. & B1RKT7 & \multicolumn{3}{|c|}{$\begin{array}{ll}\text { Lab ID: } & \text { 0802006-07 }\end{array}$} & & & & \\
\hline $10198-40-0$ & Cobalt-60 & $<1.97 \mathrm{E}-1$ & pCi/g dry & $1.97 \mathrm{E}-1$ & & $2 / 20 / 08$ & 8D28002 & AGG-RRL-001 \\
\hline $10045-97-3$ & Cesium-137 & $<2.40 \mathrm{E}-1$ & pCi/g dry & $2.40 \mathrm{E}-1$ & & 2/20/08 & 8D28002 & AGG-RRL-001 \\
\hline $14683-23-9$ & Europium-152 & $<8.86 \mathrm{E}-1$ & pCi/g dry & 8.86E-1 & & 2/20/08 & 8D28002 & AGG-RRL-001 \\
\hline $15585-10-1$ & Europium-154 & $<5.00 \mathrm{E}-1$ & pCi/g dry & $5.00 \mathrm{E}-1$ & & 2/20/08 & 8D28002 & AGG-RRL-001 \\
\hline 14391-16-3 & Europium-155 & $<8.35 \mathrm{E}-1$ & pCi/g dry & 8.35E-1 & & 2/20/08 & 8D28002 & AGG-RRL-001 \\
\hline HEIS No. & B1RKV1 & \multicolumn{3}{|c|}{ Lab ID: $\quad$ 0802006-11 } & & & & \\
\hline 10198-40-0 & Cobalt-60 & $<1.82 \mathrm{E}-1$ & pCi/g dry & $1.82 \mathrm{E}-1$ & & 2/21/08 & 8D28002 & AGG-RRL-001 \\
\hline $10045-97-3$ & Cesium-137 & $<2.14 \mathrm{E}-1$ & pCi/g dry & $2.14 \mathrm{E}-1$ & & 2/21/08 & 8D28002 & AGG-RRL-001 \\
\hline 14683-23-9 & Europium-152 & $<7.25 \mathrm{E}-1$ & pCi/g dry & $7.25 \mathrm{E}-1$ & & $2 / 21 / 08$ & 8D28002 & AGG-RRL-001 \\
\hline 15585-10-1 & Europium-154 & $<4.47 \mathrm{E}-1$ & pCi/g dry & 4.47E-1 & & $2 / 21 / 08$ & 8D28002 & AGG-RRL-001 \\
\hline 14391-16-3 & Europium-155 & $<6.83 \mathrm{E}-1$ & pCi/g dry & 6.83E-1 & & $2 / 21 / 08$ & 8D28002 & AGG-RRL-001 \\
\hline HEIS No. & B1RKV5 & \multicolumn{3}{|c|}{ Lab ID: $\quad$ 0802007-04 } & & & & \\
\hline $10198-40-0$ & Cobalt-60 & $<2.15 \mathrm{E}-1$ & pCi/g dry & $2.15 \mathrm{E}-1$ & & 2/21/08 & 8D28002 & AGG-RRL-001 \\
\hline $10045-97-3$ & Cesium-137 & $<2.46 \mathrm{E}-1$ & pCi/g dry & $2.46 \mathrm{E}-1$ & & $2 / 21 / 08$ & 8D28002 & AGG-RRL-001 \\
\hline 14683-23-9 & Europium-152 & $<9.18 \mathrm{E}-1$ & pCi/g dry & $9.18 \mathrm{E}-1$ & & $2 / 21 / 08$ & 8D28002 & AGG-RRL-001 \\
\hline $15585-10-1$ & Europium-154 & $<5.31 \mathrm{E}-1$ & pCi/g dry & $5.31 \mathrm{E}-1$ & & 2/21/08 & 8D28002 & AGG-RRL-001 \\
\hline 14391-16-3 & Europium-155 & $<8.84 \mathrm{E}-1$ & pCi/g dry & 8.84E-1 & & $2 / 21 / 08$ & 8D28002 & AGG-RRL-001 \\
\hline HEIS No. & B1RKV9 & \multicolumn{3}{|c|}{$\begin{array}{ll}\text { Lab ID: } & \text { 0802007-12 }\end{array}$} & & & & \\
\hline 10198-40-0 & Cobalt-60 & $<1.51 \mathrm{E}-1$ & pCi/g dry & $1.51 \mathrm{E}-1$ & & $2 / 21 / 08$ & 8D28002 & AGG-RRL-001 \\
\hline $10045-97-3$ & Cesium-137 & $<1.82 \mathrm{E}-1$ & pCi/g dry & $1.82 \mathrm{E}-1$ & & $2 / 21 / 08$ & 8D28002 & AGG-RRL-001 \\
\hline $14683-23-9$ & Europium-152 & $<6.21 \mathrm{E}-1$ & pCi/g dry & $6.21 \mathrm{E}-1$ & & 2/21/08 & 8D28002 & AGG-RRL-001 \\
\hline 15585-10-1 & Europium-154 & $<3.86 \mathrm{E}-1$ & pCi/g dry & 3.86E-1 & & 2/21/08 & 8D28002 & AGG-RRL-001 \\
\hline 14391-16-3 & Europium-155 & $<6.00 \mathrm{E}-1$ & pCi/g dry & $6.00 \mathrm{E}-1$ & & $2 / 21 / 08$ & 8D28002 & AGG-RRL-001 \\
\hline
\end{tabular}




\section{GEA/Soil}

\begin{tabular}{|c|c|c|c|c|c|c|c|c|}
\hline CAS \# & Analyte & Results & Units & MDA & UNC & Analyzed & Batch & Method \\
\hline HEIS No. & B1RKW2 & \multicolumn{3}{|c|}{ Lab ID: $\quad$ 0802008-04 } & & & & \\
\hline 10198-40-0 & Cobalt-60 & $<3.03 \mathrm{E}-1$ & pCi/g dry & 3.03E-1 & & $2 / 21 / 08$ & 8D28002 & AGG-RRL-001 \\
\hline $10045-97-3$ & Cesium-137 & $<3.49 \mathrm{E}-1$ & pCi/g dry & 3.49E-1 & & $2 / 21 / 08$ & 8D28002 & AGG-RRL-001 \\
\hline 14683-23-9 & Europium-152 & $<1.27 \mathrm{E} 0$ & pCi/g dry & 1.27E0 & & $2 / 21 / 08$ & 8D28002 & AGG-RRL-001 \\
\hline 15585-10-1 & Europium-154 & $<7.28 \mathrm{E}-1$ & pCi/g dry & $7.28 \mathrm{E}-1$ & & $2 / 21 / 08$ & 8D28002 & AGG-RRL-001 \\
\hline 14391-16-3 & Europium-155 & $<1.22 \mathrm{E} 0$ & pCi/g dry & $1.22 \mathrm{E} 0$ & & $2 / 21 / 08$ & 8D28002 & AGG-RRL-001 \\
\hline HEIS No. & B1RKW4 & \multicolumn{3}{|c|}{ Lab ID: $\quad$ 0802008-08 } & & & & \\
\hline 10198-40-0 & Cobalt-60 & $<2.91 \mathrm{E}-1$ & pCi/g dry & 2.91E-1 & & $2 / 21 / 08$ & 8D28002 & AGG-RRL-001 \\
\hline $10045-97-3$ & Cesium-137 & $<3.31 \mathrm{E}-1$ & pCi/g dry & $3.31 \mathrm{E}-1$ & & $2 / 21 / 08$ & 8D28002 & AGG-RRL-001 \\
\hline 14683-23-9 & Europium-152 & $<1.11 \mathrm{E} 0$ & pCi/g dry & $1.11 \mathrm{E} 0$ & & $2 / 21 / 08$ & 8D28002 & AGG-RRL-001 \\
\hline $15585-10-1$ & Europium-154 & $<7.02 \mathrm{E}-1$ & pCi/g dry & $7.02 \mathrm{E}-1$ & & 2/21/08 & 8D28002 & AGG-RRL-001 \\
\hline 14391-16-3 & Europium-155 & $<1.13 \mathrm{E} 0$ & pCi/g dry & 1.13E0 & & $2 / 21 / 08$ & 8D28002 & AGG-RRL-001 \\
\hline HEIS No. & B1RKW5 & \multicolumn{3}{|c|}{ Lab ID: $\quad$ 0802009-02 } & & & & \\
\hline 10198-40-0 & Cobalt-60 & $<3.03 \mathrm{E}-1$ & pCi/g dry & 3.03E-1 & & $2 / 21 / 08$ & 8D28002 & AGG-RRL-001 \\
\hline $10045-97-3$ & Cesium-137 & $<3.53 \mathrm{E}-1$ & pCi/g dry & $3.53 \mathrm{E}-1$ & & 2/21/08 & 8D28002 & AGG-RRL-001 \\
\hline 14683-23-9 & Europium-152 & $<1.34 \mathrm{E} 0$ & pCi/g dry & $1.34 \mathrm{E} 0$ & & $2 / 21 / 08$ & 8D28002 & AGG-RRL-001 \\
\hline 15585-10-1 & Europium-154 & $<7.58 \mathrm{E}-1$ & pCi/g dry & $7.58 \mathrm{E}-1$ & & $2 / 21 / 08$ & 8D28002 & AGG-RRL-001 \\
\hline 14391-16-3 & Europium-155 & $<1.28 \mathrm{E} 0$ & pCi/g dry & $1.28 \mathrm{E} 0$ & & $2 / 21 / 08$ & 8D28002 & AGG-RRL-001 \\
\hline HEIS No. & B1RKW6 & \multicolumn{3}{|c|}{ Lab ID: $\quad$ 0802009-04 } & & & & \\
\hline 10198-40-0 & Cobalt-60 & $<2.71 \mathrm{E}-1$ & pCi/g dry & $2.71 \mathrm{E}-1$ & & 2/21/08 & 8D28002 & AGG-RRL-001 \\
\hline $10045-97-3$ & Cesium-137 & $<3.06 \mathrm{E}-1$ & pCi/g dry & 3.06E-1 & & $2 / 21 / 08$ & 8D28002 & AGG-RRL-001 \\
\hline 14683-23-9 & Europium-152 & $<1.08 \mathrm{E} 0$ & pCi/g dry & $1.08 \mathrm{E} 0$ & & $2 / 21 / 08$ & 8D28002 & AGG-RRL-001 \\
\hline 15585-10-1 & Europium-154 & $<6.58 \mathrm{E}-1$ & pCi/g dry & $6.58 \mathrm{E}-1$ & & 2/21/08 & 8D28002 & AGG-RRL-001 \\
\hline 14391-16-3 & Europium-155 & $<9.91 \mathrm{E}-1$ & pCi/g dry & $9.91 \mathrm{E}-1$ & & 2/21/08 & 8D28002 & AGG-RRL-001 \\
\hline HEIS No. & B1TFV6 & \multicolumn{3}{|c|}{$\begin{array}{ll}\text { Lab ID: } & \text { 0802028-03 }\end{array}$} & & & & \\
\hline $10198-40-0$ & Cobalt-60 & $<2.28 \mathrm{E}-1$ & pCi/g dry & $2.28 \mathrm{E}-1$ & & $4 / 02 / 08$ & $8 C 25005$ & AGG-RRL-001 \\
\hline $10045-97-3$ & Cesium-137 & $<2.96 \mathrm{E}-1$ & pCi/g dry & 2.96E-1 & & $4 / 02 / 08$ & 8C25005 & AGG-RRL-001 \\
\hline $14683-23-9$ & Europium-152 & $<1.00 \mathrm{E} 0$ & pCi/g dry & $1.00 \mathrm{E} 0$ & & $4 / 02 / 08$ & 8C25005 & AGG-RRL-001 \\
\hline $15585-10-1$ & Europium-154 & $<6.18 \mathrm{E}-1$ & pCi/g dry & $6.18 \mathrm{E}-1$ & & $4 / 02 / 08$ & $8 C 25005$ & AGG-RRL-001 \\
\hline 14391-16-3 & Europium-155 & $<1.02 \mathrm{E} 0$ & pCi/g dry & $1.02 \mathrm{E} 0$ & & $4 / 02 / 08$ & 8C25005 & AGG-RRL-001 \\
\hline HEIS No. & B1TFV8 & \multicolumn{3}{|c|}{$\begin{array}{ll}\text { Lab ID: } & \text { 0802028-05 }\end{array}$} & & & & \\
\hline 10198-40-0 & Cobalt-60 & $<2.73 \mathrm{E}-1$ & pCi/g dry & 2.73E-1 & & $4 / 02 / 08$ & $8 C 25005$ & AGG-RRL-001 \\
\hline $10045-97-3$ & Cesium-137 & $<3.54 \mathrm{E}-1$ & pCi/g dry & $3.54 \mathrm{E}-1$ & & $4 / 02 / 08$ & $8 C 25005$ & AGG-RRL-001 \\
\hline 14683-23-9 & Europium-152 & $<1.23 \mathrm{E} 0$ & pCi/g dry & $1.23 \mathrm{E} 0$ & & $4 / 02 / 08$ & $8 C 25005$ & AGG-RRL-001 \\
\hline 15585-10-1 & Europium-154 & $<7.55 \mathrm{E}-1$ & pCi/g dry & 7.55E-1 & & $4 / 02 / 08$ & $8 C 25005$ & AGG-RRL-001 \\
\hline 14391-16-3 & Europium-155 & $<1.31 \mathrm{E} 0$ & pCi/g dry & $1.31 \mathrm{E} 0$ & & $4 / 02 / 08$ & 8C25005 & AGG-RRL-001 \\
\hline HEIS No. & B1TFW0 & \multicolumn{3}{|c|}{ Lab ID: $\quad$ 0802028-07 } & & & & \\
\hline $10198-40-0$ & Cobalt-60 & $<2.38 \mathrm{E}-1$ & pCi/g dry & $2.38 \mathrm{E}-1$ & & $4 / 02 / 08$ & $8 C 25005$ & AGG-RRL-001 \\
\hline $10045-97-3$ & Cesium-137 & $<3.29 \mathrm{E}-1$ & pCi/g dry & $3.29 \mathrm{E}-1$ & & $4 / 02 / 08$ & $8 C 25005$ & AGG-RRL-001 \\
\hline 14683-23-9 & Europium-152 & $<1.06 \mathrm{E} 0$ & pCi/g dry & 1.06E0 & & $4 / 02 / 08$ & $8 C 25005$ & AGG-RRL-001 \\
\hline $15585-10-1$ & Europium-154 & $<6.99 \mathrm{E}-1$ & pCi/g dry & $6.99 \mathrm{E}-1$ & & $4 / 02 / 08$ & $8 C 25005$ & AGG-RRL-001 \\
\hline 14391-16-3 & Europium-155 & $<1.26 \mathrm{E} 0$ & pCi/g dry & $1.26 \mathrm{E} 0$ & & $4 / 02 / 08$ & 8C25005 & AGG-RRL-001 \\
\hline HEIS No. & B1TFW2 & \multicolumn{3}{|c|}{$\begin{array}{ll}\text { Lab ID: } & \text { 0802028-09 }\end{array}$} & & & & \\
\hline 10198-40-0 & Cobalt-60 & $<3.48 \mathrm{E}-1$ & pCi/g dry & $3.48 \mathrm{E}-1$ & & $4 / 02 / 08$ & 8C25005 & AGG-RRL-001 \\
\hline $10045-97-3$ & Cesium-137 & $<4.33 \mathrm{E}-1$ & pCi/g dry & 4.33E-1 & & $4 / 02 / 08$ & $8 C 25005$ & AGG-RRL-001 \\
\hline $14683-23-9$ & Europium-152 & $<1.53 \mathrm{E} 0$ & pCi/g dry & $1.53 \mathrm{E} 0$ & & $4 / 02 / 08$ & 8C25005 & AGG-RRL-001 \\
\hline 15585-10-1 & Europium-154 & $<1.03 \mathrm{E} 0$ & pCi/g dry & $1.03 \mathrm{E} 0$ & & $4 / 02 / 08$ & 8C25005 & AGG-RRL-001 \\
\hline 14391-16-3 & Europium-155 & $<2.36 \mathrm{E} 0$ & pCi/g dry & 2.36E0 & & $4 / 02 / 08$ & 8C25005 & AGG-RRL-001 \\
\hline
\end{tabular}




\section{GEA/Soil}

\begin{tabular}{|c|c|c|c|c|c|c|c|c|}
\hline CAS \# & Analyte & Results & Units & MDA & UNC & Analyzed & Batch & Method \\
\hline HEIS No. & B1RKX0 & \multicolumn{4}{|c|}{ Lab ID: $\quad$ 0802028-11 } & & & \\
\hline $10198-40-0$ & Cobalt-60 & $<2.24 \mathrm{E}-1$ & pCi/g dry & 2.24E-1 & & 4/03/08 & $8 C 25005$ & AGG-RRL-001 \\
\hline $10045-97-3$ & Cesium-137 & $<3.10 \mathrm{E}-1$ & pCi/g dry & 3.10E-1 & & $4 / 03 / 08$ & $8 C 25005$ & AGG-RRL-001 \\
\hline 14683-23-9 & Europium-152 & $<1.01 \mathrm{E} 0$ & $\mathrm{pCi} / \mathrm{g}$ dry & $1.01 \mathrm{E} 0$ & & 4/03/08 & 8C25005 & AGG-RRL-001 \\
\hline $15585-10-1$ & Europium-154 & $<6.46 \mathrm{E}-1$ & pCi/g dry & $6.46 \mathrm{E}-1$ & & $4 / 03 / 08$ & $8 C 25005$ & AGG-RRL-001 \\
\hline $14391-16-3$ & Europium-155 & $<1.04 \mathrm{E} 0$ & pCi/g dry & $1.04 \mathrm{E} 0$ & & $4 / 03 / 08$ & $8 C 25005$ & AGG-RRL-001 \\
\hline HEIS No. & B1RKX7 & \multicolumn{4}{|c|}{ Lab ID: $\quad$ 0802028-29 } & & & \\
\hline $10198-40-0$ & Cobalt-60 & $<2.05 \mathrm{E}-1$ & pCi/g dry & 2.05E-1 & & $4 / 03 / 08$ & $8 C 25005$ & AGG-RRL-001 \\
\hline $10045-97-3$ & Cesium-137 & $<2.39 \mathrm{E}-1$ & pCi/g dry & 2.39E-1 & & $4 / 03 / 08$ & $8 C 25005$ & AGG-RRL-001 \\
\hline $14683-23-9$ & Europium-152 & $<8.40 \mathrm{E}-1$ & pCi/g dry & 8.40E-1 & & 4/03/08 & $8 C 25005$ & AGG-RRL-001 \\
\hline 15585-10-1 & Europium-154 & $<5.09 \mathrm{E}-1$ & pCi/g dry & 5.09E-1 & & 4/03/08 & 8C25005 & AGG-RRL-001 \\
\hline $14391-16-3$ & Europium-155 & $<8.66 \mathrm{E}-1$ & pCi/g dry & 8.66E-1 & & 4/03/08 & $8 C 25005$ & AGG-RRL-001 \\
\hline
\end{tabular}


Total Alpha Total Beta/Acid Extract

\begin{tabular}{|c|c|c|c|c|c|c|c|c|}
\hline CAS \# & Analyte & Results & Units & MDA & UNC & Analyzed & Batch & Method \\
\hline HEIS No. & B1TFV6 & \multicolumn{4}{|c|}{ Lab ID: $\quad$ 0802028-03 } & & & \\
\hline $12587-47-2$ & Gross Beta & $<6.34 \mathrm{E} 1$ & pCi/g dry & $6.34 \mathrm{E} 1$ & & $4 / 23 / 08$ & $8 \mathrm{I} 28001$ & AGG-RRL-002 \\
\hline $12587-46-1$ & Gross Alpha & $<2.11 \mathrm{E} 1$ & pCi/g dry & 2.11E1 & & $4 / 23 / 08$ & $8 \mathrm{I} 28001$ & AGG-RRL-002 \\
\hline HEIS No. & B1TFV8 & \multicolumn{4}{|c|}{ Lab ID: $\quad$ 0802028-05 } & & & \\
\hline $12587-47-2$ & Gross Beta & $<5.95 \mathrm{E} 1$ & pCi/g dry & $5.95 \mathrm{E} 1$ & & $4 / 23 / 08$ & $8 \mathrm{I} 28001$ & AGG-RRL-002 \\
\hline $12587-46-1$ & Gross Alpha & $<1.98 \mathrm{E} 1$ & pCi/g dry & $1.98 \mathrm{E} 1$ & & $4 / 23 / 08$ & $8 \mathrm{I} 28001$ & AGG-RRL-002 \\
\hline HEIS No. & B1TFW0 & \multicolumn{4}{|c|}{ Lab ID: $\quad$ 0802028-07 } & & & \\
\hline $12587-47-2$ & Gross Beta & $<6.24 \mathrm{E} 1$ & pCi/g dry & $6.24 \mathrm{E} 1$ & & $4 / 23 / 08$ & $8 \mathrm{I} 28001$ & AGG-RRL-002 \\
\hline $12587-46-1$ & Gross Alpha & 2.78E1 & pCi/g dry & $2.08 \mathrm{E} 1$ & 2.16E0 & $4 / 23 / 08$ & $8 \mathrm{I} 28001$ & AGG-RRL-002 \\
\hline HEIS No. & B1TFW2 & \multicolumn{4}{|c|}{ Lab ID: $\quad$ 0802028-09 } & & & \\
\hline $12587-47-2$ & Gross Beta & $1.49 \mathrm{E} 2$ & pCi/g dry & $6.44 \mathrm{E} 1$ & $6.24 \mathrm{E} 0$ & $4 / 23 / 08$ & $8 \mathrm{I} 28001$ & AGG-RRL-002 \\
\hline $12587-46-1$ & Gross Alpha & 7.23E1 & pCi/g dry & $2.15 \mathrm{E} 1$ & $3.00 \mathrm{E} 0$ & $4 / 23 / 08$ & $8 \mathrm{I} 28001$ & AGG-RRL-002 \\
\hline HEIS No. & B1RKX0 & \multicolumn{4}{|c|}{ Lab ID: $\quad$ 0802028-11 } & & & \\
\hline $12587-47-2$ & Gross Beta & $<6.31 \mathrm{E} 1$ & pCi/g dry & 6.31E1 & & $4 / 23 / 08$ & $8 \mathrm{I} 28001$ & AGG-RRL-002 \\
\hline $12587-46-1$ & Gross Alpha & $<2.10 \mathrm{E} 1$ & pCi/g dry & $2.10 \mathrm{E} 1$ & & $4 / 23 / 08$ & $8 \mathrm{I} 28001$ & AGG-RRL-002 \\
\hline HEIS No. & B1RKX3 & \multicolumn{4}{|c|}{ Lab ID: $\quad$ 0802028-17 } & & & \\
\hline $12587-47-2$ & Gross Beta & $<5.35 \mathrm{E} 1$ & pCi/g dry & 5.35E1 & & $4 / 23 / 08$ & $8 \mathrm{I} 28001$ & AGG-RRL-002 \\
\hline $12587-46-1$ & Gross Alpha & $<1.78 \mathrm{E} 1$ & pCi/g dry & $1.78 \mathrm{E} 1$ & & $4 / 23 / 08$ & $8 \mathrm{I} 28001$ & AGG-RRL-002 \\
\hline HEIS No. & B1RKX5 & \multicolumn{4}{|c|}{$\begin{array}{ll}\text { Lab ID: } & \text { 0802028-23 }\end{array}$} & & & \\
\hline $12587-47-2$ & Gross Beta & $<5.36 \mathrm{E} 1$ & pCi/g dry & $5.36 \mathrm{E} 1$ & & $4 / 23 / 08$ & $8 \mathrm{I} 28001$ & AGG-RRL-002 \\
\hline $12587-46-1$ & Gross Alpha & $<1.78 \mathrm{E} 1$ & pCi/g dry & $1.78 \mathrm{E} 1$ & & $4 / 23 / 08$ & $8 \mathrm{I} 28001$ & AGG-RRL-002 \\
\hline HEIS No. & B1RKX7 & \multicolumn{4}{|c|}{ Lab ID: $\quad$ 0802028-29 } & & & \\
\hline $12587-47-2$ & Gross Beta & $<5.39 \mathrm{E} 1$ & pCi/g dry & 5.39E1 & & $4 / 23 / 08$ & $8 \mathrm{I} 28001$ & AGG-RRL-002 \\
\hline $12587-46-1$ & Gross Alpha & $<1.80 \mathrm{E} 1$ & pCi/g dry & $1.80 \mathrm{E} 1$ & & $4 / 23 / 08$ & $8 \mathrm{I} 28001$ & AGG-RRL-002 \\
\hline
\end{tabular}


Wet Chemistry - Quality Control

Environmental Science Laboratory

\begin{tabular}{|lrrrrrrrrrr}
\hline & & Reporting & & Spike & Source & & \%REC & & RPD & \\
Analyte & Result & Limit & Units & Level & Result & \%REC & Limits & RPD & Limit & Notes \\
\hline
\end{tabular}

Batch 8B22001 - 1:1 Water Extract Initial

\begin{tabular}{|c|c|c|c|c|c|c|}
\hline Duplicate (8B22001-DUP1) & \multicolumn{3}{|c|}{ Source: 0802008-08 } & \multicolumn{3}{|l|}{ Prepared \& Analyzed: 02/06/08 } \\
\hline Specific Conductance (EC) & $1.55 \mathrm{E} 0$ & $1.00 \mathrm{E}-3$ & $\mathrm{mS} / \mathrm{cm}$ & $1.57 \mathrm{E} 0$ & 1.47 & 35 \\
\hline Duplicate (8B22001-DUP2) & \multicolumn{3}{|c|}{ Source: 0802005-15 } & Prepared \& Analyzed: 02/06/08 & & \\
\hline Specific Conductance (EC) & $<1.00 \mathrm{E}-3$ & $1.00 \mathrm{E}-3$ & $\mathrm{mS} / \mathrm{cm}$ & $1.22 \mathrm{E}-1$ & & 35 \\
\hline
\end{tabular}

Batch 8B28005 - 1:1 Water Extract (pH_EC_Alk)

\begin{tabular}{|c|c|c|c|c|c|c|}
\hline \multirow{2}{*}{$\frac{\text { Blank (8B28005-BLK1) }}{\text { Specific Conductance (EC) }}$} & & & & \multicolumn{3}{|l|}{ Prepared \& Analyzed: 02/06/08 } \\
\hline & $<1.00 \mathrm{E}-2$ & $1.00 \mathrm{E}-2$ & $\mathrm{mS} / \mathrm{cm}$ & & & \\
\hline Duplicate (8B28005-DUP1) & \multicolumn{3}{|c|}{ Source: 0802008-08 } & \multicolumn{3}{|l|}{ Prepared \& Analyzed: 02/06/08 } \\
\hline $\mathrm{pH}$ & $9.30 \mathrm{E} 0$ & N/A & pH Units & 9.31E0 & 0.107 & 35 \\
\hline Specific Conductance (EC) & $1.55 \mathrm{E} 0$ & $1.00 \mathrm{E}-2$ & $\mathrm{mS} / \mathrm{cm}$ & $1.57 \mathrm{E} 0$ & 1.47 & 35 \\
\hline Duplicate (8B28005-DUP2) & \multicolumn{3}{|c|}{ Source: 0802005-15 } & \multicolumn{3}{|l|}{ Prepared \& Analyzed: 02/06/08 } \\
\hline Specific Conductance (EC) & $<1.00 \mathrm{E}-2$ & $1.00 \mathrm{E}-2$ & $\mathrm{mS} / \mathrm{cm}$ & $1.22 \mathrm{E}-1$ & & 35 \\
\hline $\mathrm{pH}$ & $7.88 \mathrm{E} 0$ & N/A & $\mathrm{pH}$ Units & $7.98 \mathrm{E} 0$ & 1.26 & 35 \\
\hline \multicolumn{7}{|c|}{ Batch 8B29001 - 1:1 Water Extract (pH_EC_Alk) } \\
\hline Blank (8B29001-BLK1) & & & & Prepared \& Analyzed: 02/14/08 & & \\
\hline Alkalinity as $\mathrm{CaCO} 3$ & $<2.53 \mathrm{E} 1$ & $2.53 \mathrm{E} 1$ & ug/g wet & & & \\
\hline Duplicate (8B29001-DUP1) & \multicolumn{3}{|c|}{ Source: 0802008-08 } & \multicolumn{3}{|l|}{ Prepared \& Analyzed: 02/14/08 } \\
\hline Alkalinity as $\mathrm{CaCO} 3$ & $3.81 \mathrm{E} 2$ & $2.53 \mathrm{E} 1$ & ug/g dry & $3.88 \mathrm{E} 2$ & 1.95 & 35 \\
\hline
\end{tabular}


Wet Chemistry - Quality Control

Environmental Science Laboratory

\begin{tabular}{|lrrrrrrrrrr}
\hline & & Reporting & & Spike & Source & & \%REC & & RPD & \\
Analyte & Result & Limit & Units & Level & Result & \%REC & Limits & RPD & Limit & Notes \\
\hline
\end{tabular}

Batch 8C28002 - 1:1 Water Extract (pH_EC_Alk)

\begin{tabular}{|c|c|c|c|c|c|c|}
\hline \multirow{2}{*}{$\begin{array}{l}\text { Blank (8C28002-BLK1) } \\
\text { Specific Conductance (EC) }\end{array}$} & \multicolumn{6}{|c|}{ Prepared \& Analyzed: 03/28/08 } \\
\hline & $<1.00 \mathrm{E}-2$ & $1.00 \mathrm{E}-2$ & $\mathrm{mS} / \mathrm{cm}$ & & & \\
\hline Duplicate (8C28002-DUP1) & \multicolumn{3}{|c|}{ Source: 0802028-03 } & \multicolumn{3}{|l|}{ Prepared \& Analyzed: 03/28/08 } \\
\hline Specific Conductance (EC) & $7.18 \mathrm{E}-1$ & $1.00 \mathrm{E}-2$ & $\mathrm{mS} / \mathrm{cm}$ & $6.95 \mathrm{E}-1$ & 3.26 & 35 \\
\hline $\mathrm{pH}$ & $8.72 \mathrm{E} 0$ & N/A & $\mathrm{pH}$ Units & 8.84E0 & 1.37 & 35 \\
\hline
\end{tabular}

Batch 8C31002 - 1:1 Water Extract (pH_EC_Alk)

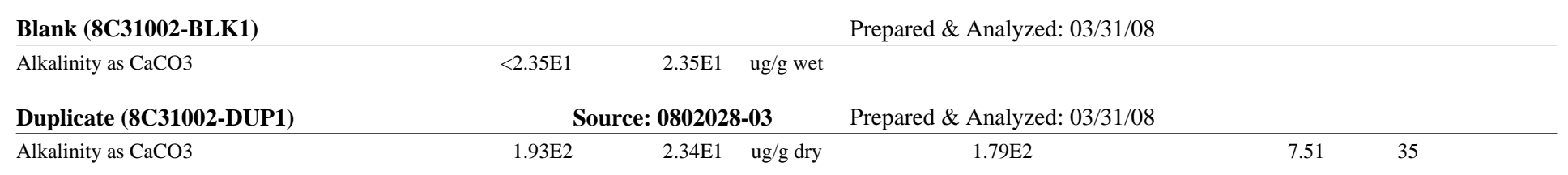


Anions by Ion Chromatography - Quality Control

Environmental Science Laboratory

\begin{tabular}{|lrrrrrrrrrrr}
\hline & & Reporting & & Spike & Source & & & \%REC & & RPD & \\
Analyte & Result & Limit & Units & Level & Result & \%REC & Limits & RPD & Limit & Notes \\
\hline
\end{tabular}

Batch 8C28006 - 1:1 Water Extract (IC)

\section{Blank (8C28006-BLK1)}

Fluoride

Chloride

Nitrite

Nitrate

Sulfate

Phosphate

LCS (8C28006-BS1)

Fluoride

Chloride

Nitrite

Nitrate

Sulfate

Phosphate

Duplicate (8C28006-DUP1)

Fluoride

Chloride

Nitrite

Nitrate

Sulfate

Phosphate

$\begin{array}{lrc}<2.00 \mathrm{E}-1 & 2.00 \mathrm{E}-1 & \text { ug/g wet } \\ <5.00 \mathrm{E}-1 & 5.00 \mathrm{E}-1 & " \\ <1.00 \mathrm{E} 0 & 1.00 \mathrm{E} 0 & " \\ <1.00 \mathrm{E} 0 & 1.00 \mathrm{E} 0 & " \\ <1.50 \mathrm{E} 0 & 1.50 \mathrm{E} 0 & " \\ <1.50 \mathrm{E} 0 & 1.50 \mathrm{E} 0 & "\end{array}$

Prepared: 03/28/08 Analyzed: 03/29/08

Prepared: 03/28/08 Analyzed: 03/29/08

$\begin{array}{lccccc}2.05 \mathrm{E} 0 & 2.00 \mathrm{E}-1 & \text { ug/g wet } & 2.00 \mathrm{E} 0 & 103 & 80-120 \\ 4.88 \mathrm{E} 0 & 5.00 \mathrm{E}-1 & " & 5.00 \mathrm{E} 0 & 97.7 & 80-120 \\ 1.05 \mathrm{E} 1 & 1.00 \mathrm{E} 0 & " & 1.00 \mathrm{E} 1 & 105 & 80-120 \\ 1.05 \mathrm{E} 1 & 1.00 \mathrm{E} 0 & " & 1.00 \mathrm{E} 1 & 105 & 80-120 \\ 1.52 \mathrm{E} 1 & 1.50 \mathrm{E} 0 & " & 1.51 \mathrm{E} 1 & 101 & 80-120 \\ 1.46 \mathrm{E} 1 & 1.50 \mathrm{E} 0 & " & 1.51 \mathrm{E} 1 & 97.2 & 80-120\end{array}$

Source: 0802028-03 Prepared: 03/28/08 Analyzed: 03/29/08

\begin{tabular}{|c|c|c|c|c|c|}
\hline $1.30 \mathrm{E} 0$ & $2.01 \mathrm{E}-1$ & ug/g dry & $1.48 \mathrm{E} 0$ & 13.0 & 35 \\
\hline 9.59E0 & $5.02 \mathrm{E}-1$ & " & $9.17 \mathrm{E} 0$ & 4.46 & 35 \\
\hline$<1.00 \mathrm{E} 0$ & $1.00 \mathrm{E} 0$ & " & ND & & 35 \\
\hline 4.84E1 & $1.00 \mathrm{E} 0$ & $"$ & $4.70 \mathrm{E} 1$ & 2.92 & 35 \\
\hline $1.10 \mathrm{E} 2$ & $1.51 \mathrm{E} 0$ & $"$ & 1.07E2 & 2.91 & 35 \\
\hline 3.53E0 & $1.51 \mathrm{E} 0$ & $"$ & $3.24 \mathrm{E} 0$ & 8.42 & 35 \\
\hline
\end{tabular}

Batch 8E05002 - 1:1 Water Extract (IC)

\begin{tabular}{lccc} 
Blank (8E05002-BLK1) & \multicolumn{2}{c}{ Prepared: 02/06/08 Analyzed: 02/07/08 } \\
\hline Fluoride & $<2.00 \mathrm{E}-1$ & $2.00 \mathrm{E}-1$ & ug/g wet \\
Chloride & $<5.00 \mathrm{E}-1$ & $5.00 \mathrm{E}-1$ & $"$ \\
Nitrite & $<1.00 \mathrm{E} 0$ & $1.00 \mathrm{E} 0$ & $"$ \\
Nitrate & $<1.00 \mathrm{E} 0$ & $1.00 \mathrm{E} 0$ & $"$ \\
Sulfate & $<1.50 \mathrm{E} 0$ & $1.50 \mathrm{E} 0$ & $"$ \\
Phosphate & $<1.50 \mathrm{E} 0$ & $1.50 \mathrm{E} 0$ & $"$
\end{tabular}


Anions by Ion Chromatography - Quality Control

Environmental Science Laboratory

\begin{tabular}{|c|c|c|c|c|c|c|c|c|c|c|}
\hline Analyte & Result & $\begin{array}{c}\text { orting } \\
\text { Limit }\end{array}$ & Units & $\begin{array}{l}\text { Spike } \\
\text { Level }\end{array}$ & $\begin{array}{l}\text { Source } \\
\text { Result }\end{array}$ & \%REC & $\begin{array}{l}\text { \%REC } \\
\text { Limits }\end{array}$ & RPD & $\begin{array}{l}\text { RPD } \\
\text { Limit }\end{array}$ & Notes \\
\hline
\end{tabular}

Batch 8E05002 - 1:1 Water Extract (IC)

\begin{tabular}{|c|c|c|c|c|c|c|c|c|c|}
\hline \multirow{2}{*}{$\begin{array}{l}\text { LCS (8E05002-BS1) } \\
\text { Fluoride }\end{array}$} & \multicolumn{9}{|c|}{ Prepared: 02/06/08 Analyzed: 02/08/08 } \\
\hline & $2.04 \mathrm{E} 0$ & $2.00 \mathrm{E}-1$ & ug/g wet & $2.00 \mathrm{E} 0$ & & 102 & $80-120$ & & \\
\hline Chloride & $4.80 \mathrm{E} 0$ & $5.00 \mathrm{E}-1$ & $"$ & $5.00 \mathrm{E} 0$ & & 96.0 & $80-120$ & & \\
\hline Nitrite & $1.16 \mathrm{E} 1$ & $1.00 \mathrm{E} 0$ & $"$ & $1.00 \mathrm{E} 1$ & & 116 & $80-120$ & & \\
\hline Nitrate & 1.07E1 & $1.00 \mathrm{E} 0$ & $"$ & $1.00 \mathrm{E} 1$ & & 106 & $80-120$ & & \\
\hline Sulfate & $1.56 \mathrm{E} 1$ & $1.50 \mathrm{E} 0$ & $"$ & $1.51 \mathrm{E} 1$ & & 104 & $80-120$ & & \\
\hline Phosphate & $1.54 \mathrm{E} 1$ & $1.50 \mathrm{E} 0$ & $"$ & $1.51 \mathrm{E} 1$ & & 102 & $80-120$ & & \\
\hline Duplicate (8E05002-DUP1) & \multicolumn{3}{|c|}{ Source: 0802008-08 } & \multicolumn{6}{|c|}{ Prepared: 02/06/08 Analyzed: 02/08/08 } \\
\hline Fluoride & $5.60 \mathrm{E} 1$ & $2.00 \mathrm{E} 0$ & ug/g dry & & $5.24 \mathrm{E} 1$ & & & 6.62 & 35 \\
\hline Chloride & 1.37E1 & $5.00 \mathrm{E} 0$ & $"$ & & $1.24 \mathrm{E} 1$ & & & 10.1 & 35 \\
\hline Nitrite & $<1.00 \mathrm{E} 1$ & $1.00 \mathrm{E} 1$ & $"$ & & ND & & & & 35 \\
\hline Nitrate & $1.42 \mathrm{E} 2$ & $1.00 \mathrm{E} 1$ & $"$ & & $1.30 \mathrm{E} 2$ & & & 8.55 & 35 \\
\hline Sulfate & $1.61 \mathrm{E} 2$ & $1.50 \mathrm{E} 1$ & $"$ & & $1.48 \mathrm{E} 2$ & & & 8.30 & 35 \\
\hline Phosphate & $<1.50 \mathrm{E} 1$ & $1.50 \mathrm{E} 1$ & $"$ & & ND & & & & 35 \\
\hline Post Spike (8E05002-PS1) & \multicolumn{3}{|c|}{ Source: 0802005-29 } & \multicolumn{4}{|c|}{ Prepared \& Analyzed: 02/06/08 } & & \\
\hline Fluoride & $4.10 \mathrm{E} 0$ & N/A & $\mathrm{ug} / \mathrm{mL}$ & $4.00 \mathrm{E} 0$ & $2.07 \mathrm{E}-1$ & 97.3 & $75-125$ & & \\
\hline Chloride & $1.03 \mathrm{E} 1$ & N/A & $"$ & $1.00 \mathrm{E} 1$ & $4.00 \mathrm{E}-1$ & 98.9 & $75-125$ & & \\
\hline Nitrite & $2.13 \mathrm{E} 1$ & N/A & $"$ & $2.00 \mathrm{E} 1$ & ND & 106 & $75-125$ & & \\
\hline Nitrate & $2.08 \mathrm{E} 1$ & N/A & $"$ & $2.00 \mathrm{E} 1$ & $1.02 \mathrm{E} 0$ & 99.1 & $75-125$ & & \\
\hline Sulfate & $4.14 \mathrm{E} 1$ & N/A & $"$ & $3.00 \mathrm{E} 1$ & $9.04 \mathrm{E} 0$ & 108 & $75-125$ & & \\
\hline Phosphate & 3.14E1 & N/A & $"$ & $3.00 \mathrm{E} 1$ & 2.82E-1 & 104 & $75-125$ & & \\
\hline
\end{tabular}


Total Metals by PNNL-AGG-ICP-AES/Water Extract - Quality Control

Environmental Science Laboratory

\begin{tabular}{|c|c|c|c|c|c|c|c|c|c|c|}
\hline Analyte & Result & $\begin{array}{c}\text { orting } \\
\text { Limit }\end{array}$ & Units & $\begin{array}{l}\text { Spike } \\
\text { Level }\end{array}$ & $\begin{array}{l}\text { Source } \\
\text { Result }\end{array}$ & \%REC & $\begin{array}{l}\text { \%REC } \\
\text { Limits }\end{array}$ & RPD & $\begin{array}{l}\text { RPD } \\
\text { Limit }\end{array}$ & Notes \\
\hline
\end{tabular}

Batch 8D28003 - 1:1 Water Extract (ICP/ICPMS)

\begin{tabular}{|c|c|c|c|c|c|c|}
\hline \multirow{2}{*}{$\begin{array}{l}\text { Blank (8D28003-BLK1) } \\
\text { Aluminum }\end{array}$} & \multicolumn{6}{|c|}{ Prepared: 04/28/08 Analyzed: 05/01/08 } \\
\hline & $<8.58 \mathrm{E}-2$ & $8.58 \mathrm{E}-2$ & ug/g wet & & & \\
\hline Barium & $3.12 \mathrm{E}-2$ & $8.79 \mathrm{E}-3$ & " & & & \\
\hline Calcium & $<3.87 \mathrm{E}-1$ & $3.87 \mathrm{E}-1$ & $"$ & & & \\
\hline Cobalt & $<9.60 \mathrm{E}-2$ & $9.60 \mathrm{E}-2$ & $"$ & & & \\
\hline Chromium & $<3.33 \mathrm{E}-2$ & $3.33 \mathrm{E}-2$ & $"$ & & & \\
\hline Copper & $<8.04 \mathrm{E}-2$ & $8.04 \mathrm{E}-2$ & " & & & \\
\hline Potassium & $<2.33 \mathrm{E} 0$ & 2.33E0 & " & & & \\
\hline Magnesium & $<8.34 \mathrm{E}-2$ & 8.34E-2 & $"$ & & & \\
\hline Manganese & $<1.71 \mathrm{E}-2$ & $1.71 \mathrm{E}-2$ & $"$ & & & \\
\hline Nickel & $<9.33 \mathrm{E}-2$ & $9.33 \mathrm{E}-2$ & " & & & \\
\hline Vanadium & $<4.53 \mathrm{E}-2$ & $4.53 \mathrm{E}-2$ & " & & & \\
\hline Zinc & $<9.27 \mathrm{E}-2$ & $9.27 \mathrm{E}-2$ & " & & & \\
\hline Sodium & $<6.69 \mathrm{E}-1$ & $6.69 \mathrm{E}-1$ & " & & & \\
\hline LCS (8D28003-BS1) & \multicolumn{6}{|c|}{ Prepared: 04/28/08 Analyzed: 05/01/08 } \\
\hline Aluminum & $4.86 \mathrm{E} 0$ & $8.58 \mathrm{E}-2$ & ug/g wet & $5.00 \mathrm{E} 0$ & 97.1 & $80-120$ \\
\hline Barium & 4.67E0 & 8.79E-3 & $"$ & $5.00 \mathrm{E} 0$ & 93.4 & $80-120$ \\
\hline Calcium & 4.62E0 & $3.87 \mathrm{E}-1$ & $"$ & $5.00 \mathrm{E} 0$ & 92.4 & $80-120$ \\
\hline Cobalt & $4.91 \mathrm{E} 0$ & $9.60 \mathrm{E}-2$ & " & $5.00 \mathrm{E} 0$ & 98.2 & $80-120$ \\
\hline Chromium & $5.20 \mathrm{E} 0$ & $3.33 E-2$ & " & $5.00 \mathrm{E} 0$ & 104 & $80-120$ \\
\hline Copper & $4.86 \mathrm{E} 0$ & $8.04 \mathrm{E}-2$ & $"$ & $5.00 \mathrm{E} 0$ & 97.1 & $80-120$ \\
\hline Potassium & $4.78 \mathrm{E} 1$ & 2.33E0 & " & $5.00 \mathrm{E} 1$ & 95.5 & $80-120$ \\
\hline Magnesium & $4.91 \mathrm{E} 0$ & $8.34 \mathrm{E}-2$ & " & $5.00 \mathrm{E} 0$ & 98.1 & $80-120$ \\
\hline Manganese & $4.98 \mathrm{E} 0$ & $1.71 \mathrm{E}-2$ & $"$ & 5.00E0 & 99.6 & $80-120$ \\
\hline Nickel & 5.03E0 & $9.33 \mathrm{E}-2$ & $"$ & $5.00 \mathrm{E} 0$ & 101 & $80-120$ \\
\hline Vanadium & 4.95E0 & $4.53 \mathrm{E}-2$ & " & $5.00 \mathrm{E} 0$ & 99.0 & $80-120$ \\
\hline Zinc & 4.99E0 & $9.27 \mathrm{E}-2$ & " & $5.00 \mathrm{E} 0$ & 99.8 & $80-120$ \\
\hline Sodium & $5.01 \mathrm{E} 0$ & $6.69 \mathrm{E}-1$ & $"$ & $5.00 \mathrm{E} 0$ & 100 & $80-120$ \\
\hline
\end{tabular}


Total Metals by PNNL-AGG-ICP-AES/Water Extract - Quality Control

Environmental Science Laboratory

\begin{tabular}{|lrrrrrrrrrrr}
\hline & & Reporting & & Spike & Source & & & \%REC & & RPD & \\
Analyte & Result & Limit & Units & Level & Result & \%REC & Limits & RPD & Limit & Notes \\
\hline
\end{tabular}

Batch 8D28003 - 1:1 Water Extract (ICP/ICPMS)

\begin{tabular}{|c|c|c|c|c|c|c|c|c|c|}
\hline \multirow{2}{*}{$\begin{array}{l}\text { Duplicate (8D28003-DUP1) } \\
\text { Aluminum }\end{array}$} & \multicolumn{3}{|c|}{ Source: 0802028-03 } & \multicolumn{4}{|c|}{ Prepared: 04/28/08 Analyzed: 05/01/08 } & \multirow[b]{2}{*}{46.4} & \multirow[b]{2}{*}{35} \\
\hline & $4.38 \mathrm{E}-1$ & $8.55 \mathrm{E}-2$ & ug/g dry & & $7.02 \mathrm{E}-1$ & & & & \\
\hline Barium & $2.29 \mathrm{E}-2$ & 8.76E-3 & $"$ & & $4.21 \mathrm{E}-2$ & & & 59.3 & 35 \\
\hline Calcium & $1.89 \mathrm{E} 0$ & $3.86 \mathrm{E}-1$ & $"$ & & 1.69E0 & & & 11.2 & 35 \\
\hline Cobalt & $<9.57 \mathrm{E}-2$ & $9.57 \mathrm{E}-2$ & " & & ND & & & & 35 \\
\hline Chromium & $<3.32 \mathrm{E}-2$ & $3.32 \mathrm{E}-2$ & $"$ & & ND & & & & 35 \\
\hline Copper & $<8.02 \mathrm{E}-2$ & 8.02E-2 & $"$ & & ND & & & & 35 \\
\hline Potassium & 2.43E0 & 2.32E0 & $"$ & & ND & & & & 35 \\
\hline Magnesium & 7.55E-1 & $8.31 \mathrm{E}-2$ & $"$ & & $7.11 \mathrm{E}-1$ & & & 6.02 & 35 \\
\hline Manganese & $<1.71 \mathrm{E}-2$ & $1.71 \mathrm{E}-2$ & $"$ & & ND & & & & 35 \\
\hline Nickel & $<9.30 \mathrm{E}-2$ & $9.30 \mathrm{E}-2$ & $"$ & & ND & & & & 35 \\
\hline Vanadium & $5.92 \mathrm{E}-2$ & $4.52 \mathrm{E}-2$ & $"$ & & ND & & & & 35 \\
\hline Zinc & $<9.24 \mathrm{E}-2$ & $9.24 \mathrm{E}-2$ & $"$ & & 4.03E-1 & & & & 35 \\
\hline Sodium & $1.41 \mathrm{E} 2$ & 6.67E-1 & $"$ & & 1.37E2 & & & 3.10 & 35 \\
\hline Post Spike (8D28003-PS1) & \multicolumn{3}{|c|}{ Source: 0802028-11 } & \multicolumn{2}{|c|}{ Prepared: 04/28/08 } & \multicolumn{2}{|c|}{ Analyzed: 05/01/08 } & & \\
\hline Aluminum & 4.78E2 & N/A & $\mathrm{ug} / \mathrm{L}$ & $5.00 \mathrm{E} 2$ & $1.42 \mathrm{E} 1$ & 92.7 & $75-125$ & & \\
\hline Barium & $2.40 \mathrm{E} 2$ & N/A & $"$ & $2.50 \mathrm{E} 2$ & $1.12 \mathrm{E} 1$ & 91.4 & $75-125$ & & \\
\hline Calcium & 4.74E3 & N/A & $"$ & 5.00E2 & 4.39E3 & 70.1 & $75-125$ & & \\
\hline Cobalt & $2.26 \mathrm{E} 2$ & N/A & $"$ & $2.50 \mathrm{E} 2$ & $2.02 \mathrm{E}-1$ & 90.2 & $75-125$ & & \\
\hline Chromium & 1.17E2 & N/A & $"$ & $1.25 \mathrm{E} 2$ & ND & 94.4 & $75-125$ & & \\
\hline Copper & 4.70E2 & N/A & $"$ & $5.00 \mathrm{E} 2$ & $6.70 \mathrm{E}-1$ & 94 & $75-125$ & & \\
\hline Potassium & 3.13E3 & N/A & $"$ & $1.25 \mathrm{E} 3$ & 2.06E3 & 85.7 & $75-125$ & & \\
\hline Magnesium & 2.87E3 & N/A & $"$ & $5.00 \mathrm{E} 2$ & 2.52E3 & 70.7 & $75-125$ & & \\
\hline Manganese & 2.35E2 & N/A & $"$ & $2.50 \mathrm{E} 2$ & ND & 94 & $75-125$ & & \\
\hline Nickel & 4.64E2 & N/A & $"$ & $5.00 \mathrm{E} 2$ & ND & 93 & $75-125$ & & \\
\hline Vanadium & $2.28 \mathrm{E} 2$ & N/A & " & $2.50 \mathrm{E} 2$ & ND & 91.7 & $75-125$ & & \\
\hline Zinc & 2.67E2 & N/A & $"$ & $2.50 \mathrm{E} 2$ & 1.27E1 & 102 & $75-125$ & & \\
\hline Sodium & $7.59 \mathrm{E} 4$ & N/A & $"$ & $5.00 \mathrm{E} 2$ & 7.73E4 & NR & $75-125$ & & \\
\hline
\end{tabular}


Total Metals by PNNL-AGG-ICP-AES/Water Extract - Quality Control

Environmental Science Laboratory

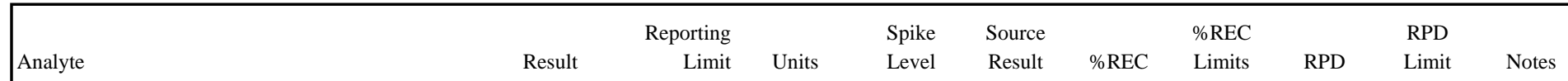

Batch 8E01006 - 1:1 Water Extract (ICP/ICPMS)

\section{Blank (8E01006-BLK1)}

Aluminum

Barium

Calcium

Cobalt

Chromium

Copper

Potassium

Magnesium

Manganese

Nickel

Vanadium

Zinc

Sodium

Blank (8E01006-BLK2)

Aluminum

Barium

Calcium

Cobalt

Chromium

Copper

Potassium

Magnesium

Manganese

Nickel

Vanadium

Zinc

Sodium
Prepared: 02/06/08 Analyzed: 02/07/08

$\begin{array}{lrc}<8.58 \mathrm{E}-2 & 8.58 \mathrm{E}-2 & \text { ug/g wet } \\ <8.79 \mathrm{E}-3 & 8.79 \mathrm{E}-3 & " \\ <3.87 \mathrm{E}-1 & 3.87 \mathrm{E}-1 & " \\ <9.60 \mathrm{E}-2 & 9.60 \mathrm{E}-2 & " \\ <3.33 \mathrm{E}-2 & 3.33 \mathrm{E}-2 & " \\ <8.04 \mathrm{E}-2 & 8.04 \mathrm{E}-2 & " \\ <2.33 \mathrm{E} 0 & 2.33 \mathrm{E} 0 & " \\ <8.34 \mathrm{E}-2 & 8.34 \mathrm{E}-2 & " \\ <1.71 \mathrm{E}-2 & 1.71 \mathrm{E}-2 & " \\ <9.33 \mathrm{E}-2 & 9.33 \mathrm{E}-2 & " \\ <4.53 \mathrm{E}-2 & 4.53 \mathrm{E}-2 & " \\ <9.27 \mathrm{E}-2 & 9.27 \mathrm{E}-2 & " \\ <6.69 \mathrm{E}-1 & 6.69 \mathrm{E}-1 & "\end{array}$

Prepared: 02/06/08 Analyzed: 02/07/08 
Total Metals by PNNL-AGG-ICP-AES/Water Extract - Quality Control

Environmental Science Laboratory

\begin{tabular}{|lrrrrrrrrrrr}
\hline & & Reporting & & Spike & Source & & & \%REC & & RPD & \\
Analyte & Result & Limit & Units & Level & Result & \%REC & Limits & RPD & Limit & Notes \\
\hline
\end{tabular}

Batch 8E01006 - 1:1 Water Extract (ICP/ICPMS)

\begin{tabular}{|c|c|c|c|c|c|c|}
\hline \multirow{2}{*}{$\begin{array}{l}\text { LCS (8E01006-BS1) } \\
\text { Aluminum }\end{array}$} & \multicolumn{6}{|c|}{ Prepared: 02/06/08 Analyzed: 02/07/08 } \\
\hline & 4.73E0 & 8.58E-2 & ug/g wet & $5.00 \mathrm{E} 0$ & 94.6 & $80-120$ \\
\hline Barium & 4.96E0 & 8.79E-3 & $"$ & $5.00 \mathrm{E} 0$ & 99.1 & $80-120$ \\
\hline Calcium & $4.98 \mathrm{E} 0$ & 3.87E-1 & $"$ & $5.00 \mathrm{E} 0$ & 99.6 & $80-120$ \\
\hline Cobalt & 4.96E0 & $9.60 \mathrm{E}-2$ & $"$ & $5.00 \mathrm{E} 0$ & 99.2 & $80-120$ \\
\hline Chromium & 4.93E0 & 3.33E-2 & $"$ & $5.00 \mathrm{E} 0$ & 98.6 & $80-120$ \\
\hline Copper & 4.88E0 & $8.04 \mathrm{E}-2$ & $"$ & $5.00 \mathrm{E} 0$ & 97.6 & $80-120$ \\
\hline Potassium & 4.91E1 & 2.33E0 & $"$ & $5.00 \mathrm{E} 1$ & 98.2 & $80-120$ \\
\hline Magnesium & 4.78E0 & $8.34 \mathrm{E}-2$ & $"$ & $5.00 \mathrm{E} 0$ & 95.6 & $80-120$ \\
\hline Manganese & 4.94E0 & $1.71 \mathrm{E}-2$ & $"$ & 5.00E0 & 98.9 & $80-120$ \\
\hline Nickel & 4.94E0 & $9.33 \mathrm{E}-2$ & $"$ & 5.00E0 & 98.9 & $80-120$ \\
\hline Vanadium & 4.85E0 & 4.53E-2 & $"$ & $5.00 \mathrm{E} 0$ & 97.0 & $80-120$ \\
\hline Zinc & $4.76 \mathrm{E} 0$ & $9.27 \mathrm{E}-2$ & $"$ & $5.00 \mathrm{E} 0$ & 95.2 & $80-120$ \\
\hline Sodium & $6.70 \mathrm{E} 0$ & $6.69 \mathrm{E}-1$ & $"$ & $5.00 \mathrm{E} 0$ & 134 & $80-120$ \\
\hline LCS (8E01006-BS2) & \multicolumn{6}{|c|}{ Prepared: 02/06/08 Analyzed: 02/07/08 } \\
\hline Aluminum & 4.64E0 & $8.58 \mathrm{E}-2$ & ug/g wet & $5.00 \mathrm{E} 0$ & 93.0 & $80-120$ \\
\hline Barium & 4.91E0 & 8.79E-3 & $"$ & $5.00 \mathrm{E} 0$ & 98.3 & $80-120$ \\
\hline Calcium & 4.93E0 & 3.87E-1 & $"$ & $5.00 \mathrm{E} 0$ & 98.8 & $80-120$ \\
\hline Cobalt & 4.94E0 & $9.60 \mathrm{E}-2$ & $"$ & $5.00 \mathrm{E} 0$ & 98.9 & $80-120$ \\
\hline Chromium & 4.91E0 & 3.33E-2 & $"$ & $5.00 \mathrm{E} 0$ & 98.3 & $80-120$ \\
\hline Copper & $4.84 \mathrm{E} 0$ & 8.04E-2 & $"$ & $5.00 \mathrm{E} 0$ & 96.8 & $80-120$ \\
\hline Potassium & 4.85E1 & 2.33E0 & $"$ & $5.00 \mathrm{E} 1$ & 97.2 & $80-120$ \\
\hline Magnesium & 4.75E0 & 8.34E-2 & $"$ & $5.00 \mathrm{E} 0$ & 95.1 & $80-120$ \\
\hline Manganese & 4.92E0 & $1.71 \mathrm{E}-2$ & $"$ & 5.00E0 & 98.6 & $80-120$ \\
\hline Nickel & 4.93E0 & 9.33E-2 & $"$ & $5.00 \mathrm{E} 0$ & 98.7 & $80-120$ \\
\hline Vanadium & 4.83E0 & 4.53E-2 & $"$ & $5.00 \mathrm{E} 0$ & 96.7 & $80-120$ \\
\hline Zinc & 4.78E0 & $9.27 \mathrm{E}-2$ & $"$ & $5.00 \mathrm{E} 0$ & 95.7 & $80-120$ \\
\hline Sodium & 5.03E0 & 6.69E-1 & $"$ & $5.00 \mathrm{E} 0$ & 101 & $80-120$ \\
\hline
\end{tabular}


Total Metals by PNNL-AGG-ICP-AES/Water Extract - Quality Control

Environmental Science Laboratory

\begin{tabular}{|lrrrrrrrrrrr}
\hline & & Reporting & & Spike & Source & & & \%REC & & RPD & \\
Analyte & Result & Limit & Units & Level & Result & \%REC & Limits & RPD & Limit & Notes \\
\hline
\end{tabular}

Batch 8E01006 - 1:1 Water Extract (ICP/ICPMS)

\begin{tabular}{|c|c|c|c|c|c|c|}
\hline Duplicate (8E01006-DUP1) & \multicolumn{3}{|c|}{ Source: 0802008-08 } & \multirow{2}{*}{$\begin{array}{c}\text { Prepared: 02/06/08 Analyzed: 02/07/08 } \\
\text { 3.57E0 }\end{array}$} & \multirow[b]{2}{*}{102} & \multirow[b]{2}{*}{35} \\
\hline Aluminum & $1.16 \mathrm{E} 0$ & $8.58 \mathrm{E}-2$ & ug/g dry & & & \\
\hline Barium & 6.63E-2 & 8.79E-3 & $"$ & $9.20 \mathrm{E}-2$ & 32.4 & 35 \\
\hline Calcium & $1.45 \mathrm{E} 0$ & 3.87E-1 & $"$ & 2.25E0 & 43.3 & 35 \\
\hline Cobalt & $<9.60 \mathrm{E}-2$ & $9.60 \mathrm{E}-2$ & $"$ & ND & & 35 \\
\hline Chromium & $6.67 \mathrm{E}-2$ & 3.33E-2 & $"$ & 7.07E-2 & 5.74 & 35 \\
\hline Copper & $<8.04 \mathrm{E}-2$ & 8.04E-2 & $"$ & ND & & 35 \\
\hline Potassium & $2.41 \mathrm{E} 0$ & 2.33E0 & $"$ & 2.73E0 & 12.4 & 35 \\
\hline Magnesium & $6.74 \mathrm{E}-1$ & 8.34E-2 & $"$ & 7.23E-1 & 6.98 & 35 \\
\hline Manganese & 8.49E-2 & $1.71 \mathrm{E}-2$ & $"$ & $1.10 \mathrm{E}-1$ & 25.8 & 35 \\
\hline Nickel & $<9.33 \mathrm{E}-2$ & 9.33E-2 & $"$ & ND & & 35 \\
\hline Vanadium & $1.33 \mathrm{E}-1$ & $4.53 \mathrm{E}-2$ & $"$ & $1.36 \mathrm{E}-1$ & 1.74 & 35 \\
\hline Zinc & $<9.27 \mathrm{E}-2$ & $9.27 \mathrm{E}-2$ & $"$ & ND & & 35 \\
\hline Sodium & $3.56 \mathrm{E} 2$ & 6.69E-1 & $"$ & 3.64E2 & 2.21 & 35 \\
\hline Duplicate (8E01006-DUP2) & \multicolumn{3}{|c|}{ Source: 0802011-04 } & Prepared: 02/06/08 Analyzed: 02/07/08 & & \\
\hline Aluminum & $<8.58 \mathrm{E}-2$ & $8.58 \mathrm{E}-2$ & ug/g dry & ND & & 35 \\
\hline Barium & 4.87E-2 & 8.79E-3 & $"$ & $4.24 \mathrm{E}-2$ & 13.7 & 35 \\
\hline Calcium & $1.94 \mathrm{E} 1$ & 3.87E-1 & $"$ & $2.04 \mathrm{E} 1$ & 4.78 & 35 \\
\hline Cobalt & $<9.60 \mathrm{E}-2$ & $9.60 \mathrm{E}-2$ & $"$ & ND & & 35 \\
\hline Chromium & $<3.33 \mathrm{E}-2$ & 3.33E-2 & " & ND & & 35 \\
\hline Copper & $<8.04 \mathrm{E}-2$ & 8.04E-2 & $"$ & ND & & 35 \\
\hline Potassium & 8.01E0 & 2.33E0 & $"$ & 8.47E0 & 5.68 & 35 \\
\hline Magnesium & 6.17E0 & 8.34E-2 & $"$ & $6.56 \mathrm{E} 0$ & 6.17 & 35 \\
\hline Manganese & $<1.71 \mathrm{E}-2$ & $1.71 \mathrm{E}-2$ & " & ND & & 35 \\
\hline Nickel & $<9.33 \mathrm{E}-2$ & $9.33 \mathrm{E}-2$ & $"$ & ND & & 35 \\
\hline Vanadium & $<4.53 \mathrm{E}-2$ & $4.53 \mathrm{E}-2$ & " & ND & & 35 \\
\hline Zinc & $<9.27 \mathrm{E}-2$ & $9.27 \mathrm{E}-2$ & $"$ & ND & & 35 \\
\hline Sodium & $2.25 \mathrm{E} 1$ & 6.69E-1 & $"$ & $2.29 \mathrm{E} 1$ & 1.57 & 35 \\
\hline
\end{tabular}


Total Metals by PNNL-AGG-ICP-AES/Water Extract - Quality Control

Environmental Science Laboratory

\begin{tabular}{|lrrrrrrrrrrr}
\hline & & Reporting & & Spike & Source & & \%REC & & RPD & & \\
Analyte & Result & Limit & Units & Level & Result & \%REC & Limits & RPD & Limit & Notes \\
\hline
\end{tabular}

Batch 8E01006 - 1:1 Water Extract (ICP/ICPMS)

\begin{tabular}{|c|c|c|c|c|c|c|c|}
\hline \multirow{2}{*}{$\begin{array}{l}\text { Post Spike (8E01006-PS1) } \\
\text { Aluminum }\end{array}$} & \multicolumn{3}{|c|}{ Source: 0802006-11 } & \multicolumn{2}{|c|}{ Prepared: 02/06/08 } & \multicolumn{2}{|c|}{ Analyzed: 02/07/08 } \\
\hline & $5.22 \mathrm{E} 2$ & N/A & $\mathrm{ug} / \mathrm{L}$ & 4.73E2 & $9.49 \mathrm{E} 1$ & 90.2 & $75-125$ \\
\hline Barium & $2.52 \mathrm{E} 2$ & N/A & $"$ & 2.37E2 & $5.65 \mathrm{E} 0$ & 104 & $75-125$ \\
\hline Calcium & $2.84 \mathrm{E} 3$ & N/A & $"$ & 4.73E2 & 3.79E3 & NR & $75-125$ \\
\hline Cobalt & $2.53 \mathrm{E} 2$ & N/A & $"$ & 2.37E2 & $1.11 \mathrm{E} 0$ & 106 & $75-125$ \\
\hline Chromium & $1.25 \mathrm{E} 2$ & N/A & $"$ & $1.18 \mathrm{E} 2$ & 3.92E-3 & 106 & $75-125$ \\
\hline Copper & $5.08 \mathrm{E} 2$ & N/A & $"$ & 4.73E2 & $3.74 \mathrm{E} 0$ & 106 & $75-125$ \\
\hline Potassium & $2.41 \mathrm{E} 3$ & N/A & $"$ & $1.18 \mathrm{E} 3$ & $1.89 \mathrm{E} 3$ & 43.3 & $75-125$ \\
\hline Magnesium & $1.18 \mathrm{E} 3$ & N/A & $"$ & 4.73E2 & $1.14 \mathrm{E} 3$ & 7.79 & $75-125$ \\
\hline Manganese & $2.60 \mathrm{E} 2$ & N/A & $"$ & 2.37E2 & $1.54 \mathrm{E} 0$ & 109 & $75-125$ \\
\hline Nickel & $5.05 \mathrm{E} 2$ & N/A & $"$ & 4.73E2 & $1.04 \mathrm{E} 0$ & 106 & $75-125$ \\
\hline Vanadium & 2.43E2 & N/A & $"$ & 2.37E2 & $1.95 \mathrm{E} 0$ & 102 & $75-125$ \\
\hline Zinc & $2.47 \mathrm{E} 2$ & N/A & $"$ & $2.37 \mathrm{E} 2$ & $1.16 \mathrm{E} 1$ & 99.4 & $75-125$ \\
\hline Sodium & $3.56 \mathrm{E} 3$ & N/A & $"$ & 4.73E2 & $4.96 \mathrm{E} 3$ & NR & $75-125$ \\
\hline
\end{tabular}


Total Metals by PNNL-AGG-ICP-AES/Acid Extract - Quality Control

Environmental Science Laboratory

\begin{tabular}{|c|c|c|c|c|c|c|c|c|c|c|}
\hline Analyte & Result & $\begin{array}{c}\text { orting } \\
\text { Limit }\end{array}$ & Units & $\begin{array}{l}\text { Spike } \\
\text { Level }\end{array}$ & $\begin{array}{l}\text { Source } \\
\text { Result }\end{array}$ & \%REC & $\begin{array}{l}\text { \%REC } \\
\text { Limits }\end{array}$ & RPD & $\begin{array}{l}\text { RPD } \\
\text { Limit }\end{array}$ & Notes \\
\hline
\end{tabular}

Batch 8E12002 - ASTM D 5198 (ICP/ICPMS)

\begin{tabular}{|c|c|c|c|c|}
\hline Blank (8E12002-BLK1) & & & & Prepared: 05/12/08 Analyzed: 06/13/08 \\
\hline Aluminum & $<4.94 \mathrm{E}-1$ & $4.94 \mathrm{E}-1$ & ug/g wet & \\
\hline Barium & $<4.70 \mathrm{E}-2$ & 4.70E-2 & $"$ & \\
\hline Calcium & $<1.84 \mathrm{E} 0$ & $1.84 \mathrm{E} 0$ & $"$ & \\
\hline Cobalt & $<1.02 \mathrm{E}-1$ & $1.02 \mathrm{E}-1$ & $"$ & \\
\hline Chromium & $<4.10 \mathrm{E}-2$ & 4.10E-2 & $"$ & \\
\hline Copper & $<4.38 \mathrm{E}-1$ & 4.38E-1 & $"$ & \\
\hline Potassium & $<1.16 \mathrm{E} 0$ & $1.16 \mathrm{E} 0$ & " & \\
\hline Magnesium & $<3.88 \mathrm{E}-1$ & $3.88 \mathrm{E}-1$ & $"$ & \\
\hline Manganese & $<1.47 \mathrm{E}-2$ & $1.47 \mathrm{E}-2$ & $"$ & \\
\hline Nickel & $<1.01 \mathrm{E}-1$ & $1.01 \mathrm{E}-1$ & $"$ & \\
\hline Vanadium & $<4.57 \mathrm{E}-2$ & $4.57 \mathrm{E}-2$ & " & \\
\hline Zinc & $<7.70 \mathrm{E}-2$ & $7.70 \mathrm{E}-2$ & " & \\
\hline Sodium & $<1.32 \mathrm{E} 1$ & $1.32 \mathrm{E} 1$ & $"$ & \\
\hline Blank (8E12002-BLK2) & & & & Prepared: 05/12/08 Analyzed: 06/13/08 \\
\hline Aluminum & $<4.94 \mathrm{E}-1$ & $4.94 \mathrm{E}-1$ & ug/g wet & \\
\hline Barium & $<4.70 \mathrm{E}-2$ & 4.70E-2 & $"$ & \\
\hline Calcium & $<1.84 \mathrm{E} 0$ & $1.84 \mathrm{E} 0$ & $"$ & \\
\hline Cobalt & $<1.02 \mathrm{E}-1$ & $1.02 \mathrm{E}-1$ & $"$ & \\
\hline Chromium & $<4.10 \mathrm{E}-2$ & $4.10 \mathrm{E}-2$ & " & \\
\hline Copper & $<4.38 \mathrm{E}-1$ & 4.38E-1 & $"$ & \\
\hline Potassium & $<1.16 \mathrm{E} 0$ & $1.16 \mathrm{E} 0$ & " & \\
\hline Magnesium & $<3.88 \mathrm{E}-1$ & $3.88 \mathrm{E}-1$ & $"$ & \\
\hline Manganese & $<1.47 \mathrm{E}-2$ & $1.47 \mathrm{E}-2$ & $"$ & \\
\hline Nickel & $<1.01 \mathrm{E}-1$ & $1.01 \mathrm{E}-1$ & $"$ & \\
\hline Vanadium & $<4.57 \mathrm{E}-2$ & $4.57 \mathrm{E}-2$ & " & \\
\hline Zinc & $<7.70 \mathrm{E}-2$ & $7.70 \mathrm{E}-2$ & " & \\
\hline Sodium & $<1.32 \mathrm{E} 1$ & 1.32E1 & $"$ & \\
\hline
\end{tabular}


Total Metals by PNNL-AGG-ICP-AES/Acid Extract - Quality Control

Environmental Science Laboratory

\begin{tabular}{|c|c|c|c|c|c|c|c|c|c|c|}
\hline & & Reporting & & Spike & Source & & \%REC & & RPD & \\
\hline Analyte & Result & Limit & Units & Level & Result & \%REC & Limits & RPD & Limit & Notes \\
\hline
\end{tabular}

Batch 8E12002 - ASTM D 5198 (ICP/ICPMS)

\begin{tabular}{|c|c|c|c|c|c|c|}
\hline \multirow{2}{*}{$\frac{\text { LCS (8E12002-BS1) }}{\text { Aluminum }}$} & \multicolumn{6}{|c|}{ Prepared: 05/12/08 Analyzed: 06/13/08 } \\
\hline & 4.84E0 & $1.41 \mathrm{E}-1$ & ug/g wet & 4.94E0 & 98.1 & $80-120$ \\
\hline Barium & 5.19E0 & $1.34 \mathrm{E}-2$ & $"$ & 4.94E0 & 105 & $80-120$ \\
\hline Calcium & 5.04E0 & $5.24 \mathrm{E}-1$ & $"$ & 4.94E0 & 102 & $80-120$ \\
\hline Cobalt & $5.11 \mathrm{E} 0$ & 2.93E-2 & $"$ & 4.94E0 & 104 & $80-120$ \\
\hline Chromium & 5.19E0 & $1.17 \mathrm{E}-2$ & $"$ & 4.94E0 & 105 & $80-120$ \\
\hline Copper & $5.22 \mathrm{E} 0$ & $1.25 \mathrm{E}-1$ & $"$ & 4.94E0 & 106 & $80-120$ \\
\hline Potassium & 5.19E1 & 3.33E-1 & $"$ & 4.94E1 & 105 & $80-120$ \\
\hline Magnesium & $4.84 \mathrm{E} 0$ & $1.11 \mathrm{E}-1$ & $"$ & 4.94E0 & 98.0 & $80-120$ \\
\hline Manganese & 5.27E0 & $4.20 \mathrm{E}-3$ & $"$ & 4.94E0 & 107 & $80-120$ \\
\hline Nickel & $5.22 \mathrm{E} 0$ & $2.89 \mathrm{E}-2$ & $"$ & $4.94 \mathrm{E} 0$ & 106 & $80-120$ \\
\hline Vanadium & 5.23E0 & $1.31 \mathrm{E}-2$ & $"$ & 4.94E0 & 106 & $80-120$ \\
\hline Zinc & 4.77E0 & $2.20 \mathrm{E}-2$ & $"$ & $4.94 \mathrm{E} 0$ & 96.7 & $80-120$ \\
\hline Sodium & 5.67E0 & 3.76E0 & $"$ & 4.94E0 & 115 & $80-120$ \\
\hline LCS (8E12002-BS2) & \multicolumn{6}{|c|}{ Prepared: 05/12/08 Analyzed: 06/13/08 } \\
\hline Aluminum & 4.75E0 & $1.41 \mathrm{E}-1$ & ug/g wet & 4.92E0 & 96.6 & $80-120$ \\
\hline Barium & $4.98 \mathrm{E} 0$ & $1.34 \mathrm{E}-2$ & $"$ & 4.92E0 & 101 & $80-120$ \\
\hline Calcium & 4.82E0 & $5.24 \mathrm{E}-1$ & $"$ & 4.92E0 & 98.0 & $80-120$ \\
\hline Cobalt & 5.00E0 & 2.93E-2 & $"$ & 4.92E0 & 102 & $80-120$ \\
\hline Chromium & 5.14E0 & $1.17 \mathrm{E}-2$ & $"$ & 4.92E0 & 105 & $80-120$ \\
\hline Copper & $5.10 \mathrm{E} 0$ & $1.25 \mathrm{E}-1$ & $"$ & 4.92E0 & 104 & $80-120$ \\
\hline Potassium & $4.97 \mathrm{E} 1$ & 3.33E-1 & $"$ & 4.92E1 & 101 & $80-120$ \\
\hline Magnesium & 4.74E0 & $1.11 \mathrm{E}-1$ & $"$ & $4.92 \mathrm{E} 0$ & 96.4 & $80-120$ \\
\hline Manganese & 5.15E0 & $4.20 \mathrm{E}-3$ & $"$ & $4.92 \mathrm{E} 0$ & 105 & $80-120$ \\
\hline Nickel & $5.09 \mathrm{E} 0$ & $2.89 \mathrm{E}-2$ & $"$ & 4.92E0 & 103 & $80-120$ \\
\hline Vanadium & $5.12 \mathrm{E} 0$ & $1.31 \mathrm{E}-2$ & $"$ & 4.92E0 & 104 & $80-120$ \\
\hline Zinc & 4.73E0 & $2.20 \mathrm{E}-2$ & $"$ & 4.92E0 & 96.3 & $80-120$ \\
\hline Sodium & 5.37E0 & $3.76 \mathrm{E} 0$ & $"$ & 4.92E0 & 109 & $80-120$ \\
\hline
\end{tabular}


Total Metals by PNNL-AGG-ICP-AES/Acid Extract - Quality Control

Environmental Science Laboratory

\begin{tabular}{|c|c|c|c|c|c|c|c|c|c|c|}
\hline Analyte & Result & $\begin{array}{c}\text { orting } \\
\text { Limit }\end{array}$ & Units & $\begin{array}{l}\text { Spike } \\
\text { Level }\end{array}$ & $\begin{array}{l}\text { Source } \\
\text { Result }\end{array}$ & \%REC & $\begin{array}{l}\text { \%REC } \\
\text { Limits }\end{array}$ & RPD & $\begin{array}{l}\text { RPD } \\
\text { Limit }\end{array}$ & Notes \\
\hline
\end{tabular}

Batch 8E12002 - ASTM D 5198 (ICP/ICPMS)

\begin{tabular}{|c|c|c|c|c|c|c|}
\hline Duplicate (8E12002-DUP1) & \multicolumn{3}{|c|}{ Source: 0802024-18 } & \multirow{2}{*}{$\begin{array}{r}\text { Prepared: 05/12/08 } \\
7.84 \mathrm{E} 3\end{array}$} & \multicolumn{2}{|l|}{ Analyzed: 06/13/08 } \\
\hline Aluminum & 8.00E3 & 4.27E1 & ug/g dry & & 2.02 & 35 \\
\hline Barium & $6.76 \mathrm{E} 1$ & 4.07E0 & $"$ & 6.77E1 & 0.147 & 35 \\
\hline Calcium & $7.76 \mathrm{E} 3$ & 1.59E2 & $"$ & 7.08E3 & 9.07 & 35 \\
\hline Cobalt & $<8.86 \mathrm{E} 0$ & 8.86E0 & $"$ & ND & & 35 \\
\hline Chromium & $1.45 \mathrm{E} 1$ & $3.55 \mathrm{E} 0$ & $"$ & $1.50 \mathrm{E} 1$ & 3.42 & 35 \\
\hline Copper & $<3.78 \mathrm{E} 1$ & 3.78E1 & $"$ & ND & & 35 \\
\hline Potassium & $1.57 \mathrm{E} 3$ & $1.01 \mathrm{E} 2$ & $"$ & $1.54 \mathrm{E} 3$ & 1.91 & 35 \\
\hline Magnesium & $5.24 \mathrm{E} 3$ & 3.36E1 & $"$ & 5.12E3 & 2.40 & 35 \\
\hline Manganese & $3.69 \mathrm{E} 2$ & $1.27 \mathrm{E} 0$ & $"$ & $3.34 \mathrm{E} 2$ & 10.1 & 35 \\
\hline Nickel & $1.79 \mathrm{E} 1$ & 8.73E0 & $"$ & $1.76 \mathrm{E} 1$ & 1.79 & 35 \\
\hline Vanadium & $1.60 \mathrm{E} 1$ & 3.95E0 & $"$ & $1.58 \mathrm{E} 1$ & 1.01 & 35 \\
\hline Zinc & $3.75 \mathrm{E} 1$ & 6.66E0 & $"$ & 3.70E1 & 1.33 & 35 \\
\hline Sodium & $<1.14 \mathrm{E} 3$ & $1.14 \mathrm{E} 3$ & $"$ & ND & & 35 \\
\hline Duplicate (8E12002-DUP2) & \multicolumn{3}{|c|}{ Source: 0802028-03 } & Prepared: 05/12/08 & Analyzed: 06/13/08 & \\
\hline Aluminum & $1.08 \mathrm{E} 4$ & 4.32E1 & ug/g dry & 9.98E3 & 7.44 & 35 \\
\hline Barium & 8.15E1 & 4.12E0 & " & 7.27E1 & 11.4 & 35 \\
\hline Calcium & $1.02 \mathrm{E} 4$ & $1.61 \mathrm{E} 2$ & $"$ & $9.80 \mathrm{E} 3$ & 4.49 & 35 \\
\hline Cobalt & $9.71 \mathrm{E} 0$ & 8.97E0 & $"$ & ND & & 35 \\
\hline Chromium & $2.06 \mathrm{E} 1$ & 3.59E0 & $"$ & $1.82 \mathrm{E} 1$ & 12.0 & 35 \\
\hline Copper & $<3.83 \mathrm{E} 1$ & 3.83E1 & $"$ & ND & & 35 \\
\hline Potassium & $1.94 \mathrm{E} 3$ & $1.02 \mathrm{E} 2$ & $"$ & $1.84 \mathrm{E} 3$ & 5.48 & 35 \\
\hline Magnesium & $6.56 \mathrm{E} 3$ & $3.40 \mathrm{E} 1$ & $"$ & 6.06E3 & 8.00 & 35 \\
\hline Manganese & 4.13E2 & 1.29E0 & $"$ & 4.00E2 & 3.24 & 35 \\
\hline Nickel & $2.38 \mathrm{E} 1$ & 8.84E0 & $"$ & $2.15 \mathrm{E} 1$ & 10.3 & 35 \\
\hline Vanadium & $1.95 \mathrm{E} 1$ & $4.00 \mathrm{E} 0$ & $"$ & $1.91 \mathrm{E} 1$ & 1.96 & 35 \\
\hline Zinc & 4.67E1 & $6.74 \mathrm{E} 0$ & $"$ & 4.61E1 & 1.27 & 35 \\
\hline Sodium & $<1.15 \mathrm{E} 3$ & 1.15E3 & $"$ & ND & & 35 \\
\hline
\end{tabular}


Total Metals by PNNL-AGG-ICP-AES/Acid Extract - Quality Control

Environmental Science Laboratory

\begin{tabular}{|c|c|c|c|c|c|c|c|c|c|c|}
\hline Analyte & Result & $\begin{array}{c}\text { orting } \\
\text { Limit }\end{array}$ & Units & $\begin{array}{l}\text { Spike } \\
\text { Level }\end{array}$ & $\begin{array}{l}\text { Source } \\
\text { Result }\end{array}$ & \%REC & $\begin{array}{l}\text { \%REC } \\
\text { Limits }\end{array}$ & RPD & $\begin{array}{l}\text { RPD } \\
\text { Limit }\end{array}$ & Notes \\
\hline
\end{tabular}

Batch 8E12002 - ASTM D 5198 (ICP/ICPMS)

\begin{tabular}{lllllllrr}
\hline Post Spike (8E12002-PS2) & \multicolumn{3}{c}{ Source: 0802028-03 } & \multicolumn{2}{c}{ Prepared: 05/12/08 } & Analyzed: 06/13/08 \\
\hline Aluminum & $2.38 \mathrm{E} 4$ & N/A & ug/L & $5.00 \mathrm{E} 2$ & $2.26 \mathrm{E} 4$ & 241 & $75-125$ \\
Barium & $4.07 \mathrm{E} 2$ & N/A & $"$ & $2.50 \mathrm{E} 2$ & $1.65 \mathrm{E} 2$ & 97 & $75-125$ \\
Calcium & $2.39 \mathrm{E} 4$ & N/A & $"$ & $5.00 \mathrm{E} 2$ & $2.22 \mathrm{E} 4$ & 350 & $75-125$ \\
Cobalt & $2.59 \mathrm{E} 2$ & N/A & $"$ & $2.50 \mathrm{E} 2$ & $2.00 \mathrm{E} 1$ & 95.7 & $75-125$ \\
Chromium & $1.64 \mathrm{E} 2$ & N/A & $"$ & $1.25 \mathrm{E} 2$ & $4.13 \mathrm{E} 1$ & 98.2 & $75-125$ \\
Copper & $5.24 \mathrm{E} 2$ & N/A & $"$ & $5.00 \mathrm{E} 2$ & $3.88 \mathrm{E} 1$ & 97.1 & $75-125$ \\
Potassium & $5.48 \mathrm{E} 3$ & N/A & $"$ & $1.25 \mathrm{E} 3$ & $4.16 \mathrm{E} 3$ & 105 & $75-125$ \\
Magnesium & $1.47 \mathrm{E} 4$ & N/A & $"$ & $5.00 \mathrm{E} 2$ & $1.37 \mathrm{E} 4$ & 199 & $75-125$ \\
Manganese & $1.20 \mathrm{E} 3$ & N/A & $"$ & $2.50 \mathrm{E} 2$ & $9.05 \mathrm{E} 2$ & 116 & $75-125$ \\
Nickel & $5.36 \mathrm{E} 2$ & N/A & $"$ & $5.00 \mathrm{E} 2$ & $4.87 \mathrm{E} 1$ & 97.5 & $75-125$ \\
Vanadium & $2.92 \mathrm{E} 2$ & N/A & $"$ & $2.50 \mathrm{E} 2$ & $4.33 \mathrm{E} 1$ & 99.3 & $75-125$ \\
Zinc & $3.49 \mathrm{E} 2$ & N/A & $"$ & $2.50 \mathrm{E} 2$ & $1.04 \mathrm{E} 2$ & 98 & $75-125$ \\
Sodium & $2.45 \mathrm{E} 3$ & N/A & $"$ & $5.00 \mathrm{E} 2$ & $1.93 \mathrm{E} 3$ & 104 & $75-125$
\end{tabular}

Batch 8F10003 - ASTM D 5198 (ICP/ICPMS)

\begin{tabular}{lccc} 
Blank (8F10003-BLK1) & & & \\
\hline Aluminum & $<4.94 \mathrm{E}-1$ & $4.94 \mathrm{E}-1$ & ug/g wet \\
Barium & $<4.70 \mathrm{E}-2$ & $4.70 \mathrm{E}-2$ & $"$ \\
Calcium & $<1.83 \mathrm{E} 0$ & $1.83 \mathrm{E} 0$ & $"$ \\
Cobalt & $<1.02 \mathrm{E}-1$ & $1.02 \mathrm{E}-1$ & $"$ \\
Chromium & $<4.10 \mathrm{E}-2$ & $4.10 \mathrm{E}-2$ & $"$ \\
Copper & $<4.37 \mathrm{E}-1$ & $4.37 \mathrm{E}-1$ & $"$ \\
Potassium & $<1.16 \mathrm{E} 0$ & $1.16 \mathrm{E} 0$ & $"$ \\
Magnesium & $<3.88 \mathrm{E}-1$ & $3.88 \mathrm{E}-1$ & $"$ \\
Manganese & $<1.47 \mathrm{E}-2$ & $1.47 \mathrm{E}-2$ & $"$ \\
Nickel & $<1.01 \mathrm{E}-1$ & $1.01 \mathrm{E}-1$ & $"$ \\
Vanadium & $<4.57 \mathrm{E}-2$ & $4.57 \mathrm{E}-2$ & $"$ \\
Zinc & $<7.70 \mathrm{E}-2$ & $7.70 \mathrm{E}-2$ & $"$ \\
Sodium & $<1.32 \mathrm{E} 1$ & $1.32 \mathrm{E} 1$ & $"$
\end{tabular}


Total Metals by PNNL-AGG-ICP-AES/Acid Extract - Quality Control

Environmental Science Laboratory

\begin{tabular}{|lrrrrrrrrrrr}
\hline & & Reporting & & Spike & Source & & & \%REC & & RPD & \\
Analyte & Result & Limit & Units & Level & Result & \%REC & Limits & RPD & Limit & Notes \\
\hline
\end{tabular}

Batch 8F10003 - ASTM D 5198 (ICP/ICPMS)

\begin{tabular}{|c|c|c|c|c|c|c|c|c|}
\hline \multirow{2}{*}{$\begin{array}{l}\text { LCS (8F10003-BS1) } \\
\text { Aluminum }\end{array}$} & \multicolumn{8}{|c|}{ Prepared \& Analyzed: 02/13/08 } \\
\hline & 6.43E0 & $1.41 \mathrm{E}-1$ & ug/g wet & $6.42 \mathrm{E} 0$ & 100 & $80-120$ & & \\
\hline Barium & $6.95 \mathrm{E} 0$ & $1.34 \mathrm{E}-2$ & " & $6.42 \mathrm{E} 0$ & 108 & $80-120$ & & \\
\hline Calcium & $6.91 \mathrm{E} 0$ & $5.24 \mathrm{E}-1$ & $"$ & $6.42 \mathrm{E} 0$ & 108 & $80-120$ & & \\
\hline Cobalt & 7.02E0 & 2.93E-2 & $"$ & $6.42 \mathrm{E} 0$ & 109 & $80-120$ & & \\
\hline Chromium & 7.08E0 & $1.17 \mathrm{E}-2$ & " & $6.42 \mathrm{E} 0$ & 110 & $80-120$ & & \\
\hline Copper & $7.40 \mathrm{E} 0$ & $1.25 \mathrm{E}-1$ & $"$ & $6.42 \mathrm{E} 0$ & 115 & $80-120$ & & \\
\hline Potassium & $6.90 \mathrm{E} 1$ & 3.33E-1 & $"$ & $6.42 \mathrm{E} 1$ & 107 & $80-120$ & & \\
\hline Magnesium & $6.54 \mathrm{E} 0$ & $1.11 \mathrm{E}-1$ & $"$ & $6.42 \mathrm{E} 0$ & 102 & $80-120$ & & \\
\hline Manganese & 7.15E0 & $4.20 \mathrm{E}-3$ & $"$ & $6.42 \mathrm{E} 0$ & 111 & $80-120$ & & \\
\hline Nickel & 6.93E0 & $2.89 \mathrm{E}-2$ & " & $6.42 \mathrm{E} 0$ & 108 & $80-120$ & & \\
\hline Vanadium & 7.06E0 & $1.31 \mathrm{E}-2$ & $"$ & $6.42 \mathrm{E} 0$ & 110 & $80-120$ & & \\
\hline Zinc & $6.72 \mathrm{E} 0$ & $2.20 \mathrm{E}-2$ & " & $6.42 \mathrm{E} 0$ & 105 & $80-120$ & & \\
\hline Sodium & 7.10E0 & 3.76E0 & $"$ & $6.42 \mathrm{E} 0$ & 110 & $80-120$ & & \\
\hline Duplicate (8F10003-DUP1) & \multicolumn{3}{|c|}{ Source: 0802005-09 } & \multicolumn{5}{|c|}{ Prepared \& Analyzed: 02/13/08 } \\
\hline Aluminum & $5.16 \mathrm{E} 3$ & $2.89 \mathrm{E} 1$ & ug/g dry & & & & 1.74 & 35 \\
\hline Barium & 4.65E1 & 2.75E0 & " & & & & 10.6 & 35 \\
\hline Calcium & 7.13E3 & $1.07 \mathrm{E} 2$ & " & & & & 4.89 & 35 \\
\hline Cobalt & $<6.00 \mathrm{E} 0$ & 6.00E0 & $"$ & & & & & 35 \\
\hline Chromium & $1.01 \mathrm{E} 1$ & $2.40 \mathrm{E} 0$ & $"$ & & & & 2.33 & 35 \\
\hline Copper & $<2.56 \mathrm{E} 1$ & $2.56 \mathrm{E} 1$ & $"$ & & & & & 35 \\
\hline Potassium & $9.60 \mathrm{E} 2$ & $6.82 \mathrm{E} 1$ & $"$ & & & & 2.34 & 35 \\
\hline Magnesium & 4.31E3 & $2.27 \mathrm{E} 1$ & $"$ & & & & 0.949 & 35 \\
\hline Manganese & 2.31E2 & $8.60 \mathrm{E}-1$ & $"$ & & & & 9.63 & 35 \\
\hline Nickel & $1.21 \mathrm{E} 1$ & $5.91 \mathrm{E} 0$ & " & & & & 13.6 & 35 \\
\hline Vanadium & $1.03 \mathrm{E} 1$ & 2.67E0 & " & & & & 5.48 & 35 \\
\hline Zinc & 2.65E1 & $4.51 \mathrm{E} 0$ & " & & & & 5.88 & 35 \\
\hline Sodium & $<7.70 \mathrm{E} 2$ & 7.70E2 & $"$ & & & & & 35 \\
\hline
\end{tabular}


Total Metals by PNNL-AGG-ICP-AES/Acid Extract - Quality Control

Environmental Science Laboratory

\begin{tabular}{|c|c|c|c|c|c|c|c|c|c|c|}
\hline Analyte & Result & $\begin{array}{c}\text { orting } \\
\text { Limit }\end{array}$ & Units & $\begin{array}{l}\text { Spike } \\
\text { Level }\end{array}$ & $\begin{array}{l}\text { Source } \\
\text { Result }\end{array}$ & \%REC & $\begin{array}{l}\text { \%REC } \\
\text { Limits }\end{array}$ & RPD & $\begin{array}{l}\text { RPD } \\
\text { Limit }\end{array}$ & Notes \\
\hline
\end{tabular}

Batch 8F10003 - ASTM D 5198 (ICP/ICPMS)

\begin{tabular}{|c|c|c|c|c|c|c|c|}
\hline \multirow{2}{*}{$\begin{array}{l}\text { Post Spike (8F10003-PS1) } \\
\text { Aluminum }\end{array}$} & \multicolumn{3}{|c|}{ Source: 0802005-29 } & \multicolumn{4}{|c|}{ Prepared \& Analyzed: 02/13/08 } \\
\hline & 8.64E3 & N/A & ug/L & $5.00 \mathrm{E} 2$ & $1.77 \mathrm{E} 4$ & NR & $75-125$ \\
\hline Barium & $3.38 \mathrm{E} 2$ & N/A & $"$ & $2.50 \mathrm{E} 2$ & $1.72 \mathrm{E} 2$ & 66.6 & $75-125$ \\
\hline Calcium & 1.15E4 & N/A & $"$ & $5.00 \mathrm{E} 2$ & 2.30E4 & NR & $75-125$ \\
\hline Cobalt & 2.68E2 & N/A & $"$ & $2.50 \mathrm{E} 2$ & 2.18E1 & 98.5 & $75-125$ \\
\hline Chromium & 1.43E2 & N/A & $"$ & $1.25 \mathrm{E} 2$ & 3.18E1 & 89.3 & $75-125$ \\
\hline Copper & 5.25E2 & N/A & $"$ & $5.00 \mathrm{E} 2$ & 3.47E1 & 98 & $75-125$ \\
\hline Potassium & $2.64 \mathrm{E} 3$ & N/A & $"$ & $1.25 \mathrm{E} 3$ & $2.80 \mathrm{E} 3$ & NR & $75-125$ \\
\hline Magnesium & 7.43E3 & N/A & $"$ & $5.00 \mathrm{E} 2$ & $1.44 \mathrm{E} 4$ & NR & $75-125$ \\
\hline Manganese & $6.20 \mathrm{E} 2$ & N/A & $"$ & $2.50 \mathrm{E} 2$ & 7.37E2 & NR & $75-125$ \\
\hline Nickel & 5.27E2 & N/A & $"$ & $5.00 \mathrm{E} 2$ & 4.44E1 & 96.5 & $75-125$ \\
\hline Vanadium & $2.81 \mathrm{E} 2$ & N/A & $"$ & $2.50 \mathrm{E} 2$ & $5.58 \mathrm{E} 1$ & 90.2 & $75-125$ \\
\hline Zinc & 3.07E2 & N/A & $"$ & $2.50 \mathrm{E} 2$ & $9.54 \mathrm{E} 1$ & 84.8 & $75-125$ \\
\hline Sodium & 8.62E2 & N/A & $"$ & $5.00 \mathrm{E} 2$ & 7.06E2 & 31.1 & $75-125$ \\
\hline
\end{tabular}

Batch 8F11003 - ASTM D 5198 (ICP/ICPMS)

\begin{tabular}{lccc} 
Blank (8F11003-BLK1) & & & \\
\hline Aluminum & $<4.94 \mathrm{E}-1$ & $4.94 \mathrm{E}-1$ & ug/g wet \\
Barium & $<4.70 \mathrm{E}-2$ & $4.70 \mathrm{E}-2$ & $"$ \\
Calcium & $<1.84 \mathrm{E} 0$ & $1.84 \mathrm{E} 0$ & $"$ \\
Cobalt & $<1.02 \mathrm{E}-1$ & $1.02 \mathrm{E}-1$ & $"$ \\
Chromium & $<4.10 \mathrm{E}-2$ & $4.10 \mathrm{E}-2$ & $"$ \\
Copper & $<4.38 \mathrm{E}-1$ & $4.38 \mathrm{E}-1$ & $"$ \\
Potassium & $<1.16 \mathrm{E} 0$ & $1.16 \mathrm{E} 0$ & $"$ \\
Magnesium & $<3.88 \mathrm{E}-1$ & $3.88 \mathrm{E}-1$ & $"$ \\
Manganese & $<1.47 \mathrm{E}-2$ & $1.47 \mathrm{E}-2$ & $"$ \\
Nickel & $<1.01 \mathrm{E}-1$ & $1.01 \mathrm{E}-1$ & $"$ \\
Vanadium & $<4.57 \mathrm{E}-2$ & $4.57 \mathrm{E}-2$ & $"$ \\
Zinc & $1.15 \mathrm{E}-1$ & $7.70 \mathrm{E}-2$ & $"$ \\
Sodium & $<1.32 \mathrm{E} 1$ & $1.32 \mathrm{E} 1$ & $"$
\end{tabular}


Total Metals by PNNL-AGG-ICP-AES/Acid Extract - Quality Control

Environmental Science Laboratory

\begin{tabular}{|c|c|c|c|c|c|c|c|c|c|c|}
\hline & & Reporting & & Spike & Source & & \%REC & & RPD & \\
\hline Analyte & Result & Limit & Units & Level & Result & \%REC & Limits & RPD & Limit & Notes \\
\hline
\end{tabular}

Batch 8F11003 - ASTM D 5198 (ICP/ICPMS)

\begin{tabular}{|c|c|c|c|c|c|c|c|}
\hline \multirow{2}{*}{$\begin{array}{l}\text { LCS (8F11003-BS1) } \\
\text { Aluminum }\end{array}$} & \multicolumn{7}{|c|}{ Prepared: 02/04/08 Analyzed: 02/20/08 } \\
\hline & 6.39E0 & $1.41 \mathrm{E}-1$ & ug/g wet & 6.42E0 & & 99.5 & $80-120$ \\
\hline Barium & $6.88 \mathrm{E} 0$ & $1.34 \mathrm{E}-2$ & $"$ & $6.42 \mathrm{E} 0$ & & 107 & $80-120$ \\
\hline Calcium & $6.73 \mathrm{E} 0$ & $5.24 \mathrm{E}-1$ & $"$ & $6.42 \mathrm{E} 0$ & & 105 & $80-120$ \\
\hline Cobalt & $7.04 \mathrm{E} 0$ & 2.93E-2 & $"$ & $6.42 \mathrm{E} 0$ & & 110 & $80-120$ \\
\hline Chromium & 7.05E0 & $1.17 \mathrm{E}-2$ & $"$ & 6.42E0 & & 110 & $80-120$ \\
\hline Copper & $7.21 \mathrm{E} 0$ & $1.25 \mathrm{E}-1$ & $"$ & $6.42 \mathrm{E} 0$ & & 112 & $80-120$ \\
\hline Potassium & 6.61E1 & 3.33E-1 & $"$ & $6.42 \mathrm{E} 1$ & & 103 & $80-120$ \\
\hline Magnesium & $6.43 \mathrm{E} 0$ & $1.11 \mathrm{E}-1$ & $"$ & $6.42 \mathrm{E} 0$ & & 100 & $80-120$ \\
\hline Manganese & 7.05E0 & $4.20 \mathrm{E}-3$ & $"$ & 6.42E0 & & 110 & $80-120$ \\
\hline Nickel & $6.94 \mathrm{E} 0$ & $2.89 \mathrm{E}-2$ & $"$ & $6.42 \mathrm{E} 0$ & & 108 & $80-120$ \\
\hline Vanadium & 6.93E0 & $1.31 \mathrm{E}-2$ & $"$ & 6.42E0 & & 108 & $80-120$ \\
\hline Zinc & $6.66 \mathrm{E} 0$ & $2.20 \mathrm{E}-2$ & $"$ & $6.42 \mathrm{E} 0$ & & 104 & $80-120$ \\
\hline Sodium & 7.03E0 & $3.76 \mathrm{E} 0$ & $"$ & $6.42 \mathrm{E} 0$ & & 109 & $80-120$ \\
\hline Post Spike (8F11003-PS1) & \multicolumn{3}{|c|}{ Source: 0802005-25 } & \multicolumn{4}{|c|}{ Prepared: 02/04/08 Analyzed: 02/20/08 } \\
\hline Aluminum & $6.06 \mathrm{E} 3$ & N/A & ug/L & $5.00 \mathrm{E} 2$ & $1.69 \mathrm{E} 4$ & NR & $75-125$ \\
\hline Barium & 3.10E2 & N/A & $"$ & $2.50 \mathrm{E} 2$ & $1.48 \mathrm{E} 2$ & 64.6 & $75-125$ \\
\hline Calcium & $7.60 \mathrm{E} 3$ & N/A & $"$ & $5.00 \mathrm{E} 2$ & $2.08 \mathrm{E} 4$ & NR & $75-125$ \\
\hline Cobalt & 2.61E2 & N/A & $"$ & $2.50 \mathrm{E} 2$ & $1.48 \mathrm{E} 1$ & 98.6 & $75-125$ \\
\hline Chromium & $1.39 \mathrm{E} 2$ & N/A & $"$ & 1.25E2 & 3.39E1 & 84.2 & $75-125$ \\
\hline Copper & 5.18E2 & N/A & $"$ & $5.00 \mathrm{E} 2$ & 2.66E1 & 98.3 & $75-125$ \\
\hline Potassium & 2.42E3 & N/A & $"$ & $1.25 \mathrm{E} 3$ & 3.32E3 & NR & $75-125$ \\
\hline Magnesium & $5.18 \mathrm{E} 3$ & N/A & $"$ & $5.00 \mathrm{E} 2$ & $1.36 \mathrm{E} 4$ & NR & $75-125$ \\
\hline Manganese & 5.17E2 & N/A & $"$ & $2.50 \mathrm{E} 2$ & $7.45 \mathrm{E} 2$ & NR & $75-125$ \\
\hline Nickel & $5.16 \mathrm{E} 2$ & N/A & $"$ & $5.00 \mathrm{E} 2$ & 4.33E1 & 94.6 & $75-125$ \\
\hline Vanadium & $2.65 \mathrm{E} 2$ & N/A & $"$ & 2.50E2 & $3.16 \mathrm{E} 1$ & 93.5 & $75-125$ \\
\hline Zinc & 2.95E2 & N/A & $"$ & 2.50E2 & 8.36E1 & 84.6 & $75-125$ \\
\hline Sodium & 6.99E2 & N/A & $"$ & $5.00 \mathrm{E} 2$ & 5.61E2 & 27.6 & $75-125$ \\
\hline
\end{tabular}


Radionuclides by ICP-MS/Acid Extract - Quality Control

Environmental Science Laboratory

\begin{tabular}{|lccccccccccc}
\hline & & Reporting & & Spike & Source & & \%REC & & RPD & & \\
Analyte & Result & Limit & Units & Level & Result & \%REC & Limits & RPD & Limit & Notes
\end{tabular}

Batch 8E15002 - ASTM D 5198 (ICP/ICPMS)

Blank (8E15002-BLK2)

Technetium-99

Uranium 238

Duplicate (8E15002-DUP1)

Technetium-99

Uranium 238

Duplicate (8E15002-DUP2)

Technetium-99

Uranium 238

Post Spike (8E15002-PS2)

Technetium-99

Uranium 238

$\begin{array}{ccc}<1.19 \mathrm{E}-3 & 1.19 \mathrm{E}-3 & \text { ug/g wet } \\ <8.64 \mathrm{E}-3 & 8.64 \mathrm{E}-3 & "\end{array}$

Prepared: 05/15/08 Analyzed: 05/16/08

Source: 0802024-18

$<5.14 \mathrm{E}-3 \quad 5.14 \mathrm{E}-3 \quad$ ug/g dry

$6.80 \mathrm{E}-1$

3.74E-2

"

Prepared: 05/15/08 Analyzed: 05/16/08

\begin{tabular}{|c|c|}
\hline ND & \\
\hline $6.99 \mathrm{E}-1$ & 2.66 \\
\hline
\end{tabular}

Source: 0802028-03

Prepared: 05/15/08 Analyzed: 05/16/08

$\begin{array}{rccccc}<5.21 \mathrm{E}-3 & 5.21 \mathrm{E}-3 & \text { ug/g dry } & \text { ND } & & 35 \\ 7.10 \mathrm{E}-1 & 3.78 \mathrm{E}-2 & " & 6.67 \mathrm{E}-1 & 6.23 & 35\end{array}$

Source: 0802028-03

Prepared: 05/15/08 Analyzed: 05/16/08

$\begin{array}{lllllll}4.88 \mathrm{E}-1 & \text { N/A } & \text { ug/L } & 5.00 \mathrm{E}-1 & 3.33 \mathrm{E}-3 & 96.9 & 75-125\end{array}$

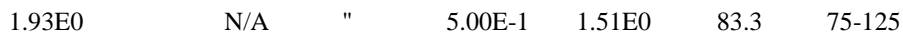




\section{Radionuclides by ICP-MS/Water Extract - Quality Control \\ Environmental Science Laboratory}

\begin{tabular}{|c|c|c|c|c|c|c|c|c|c|c|}
\hline Analyte & Result & $\begin{array}{c}\text { orting } \\
\text { Limit }\end{array}$ & Units & $\begin{array}{l}\text { Spike } \\
\text { Level }\end{array}$ & $\begin{array}{l}\text { Source } \\
\text { Result }\end{array}$ & \%REC & $\begin{array}{l}\text { \%REC } \\
\text { Limits }\end{array}$ & RPD & $\begin{array}{l}\text { RPD } \\
\text { Limit }\end{array}$ & Notes \\
\hline
\end{tabular}

Batch 8D15001 - 1:1 Water Extract (ICP/ICPMS)

Blank (8D15001-BLK1)

Technetium-99

Uranium 238

Blank (8D15001-BLK2)

Technetium-99

Uranium 238

Duplicate (8D15001-DUP1)

Technetium-99

Uranium 238

Duplicate (8D15001-DUP2)

Technetium-99

Uranium 238

Post Spike (8D15001-PS1)

Technetium-99

Uranium 238

B.59E-1

Batch 8D24001 - 1:1 Water Extract (ICP/ICPMS)

\section{Blank (8D24001-BLK1)}

Technetium-99

Uranium 238

Blank (8D24001-BLK2)

Technetium-99

Uranium 238

$\begin{array}{ccc}<2.30 \mathrm{E}-5 & 2.30 \mathrm{E}-5 & \text { ug/g wet } \\ <5.64 \mathrm{E}-4 & 5.64 \mathrm{E}-4 & "\end{array}$

Prepared \& Analyzed: 04/15/08

Prepared \& Analyzed: 04/15/08

$<2.30 \mathrm{E}-5 \quad 2.30 \mathrm{E}-5 \quad \mathrm{ug} / \mathrm{g}$ wet

$<5.64 \mathrm{E}-4 \quad 5.64 \mathrm{E}-4 \quad "$

Source: 0802028-03

Prepared \& Analyzed: 04/15/08

$\begin{array}{lcccc}<9.17 \mathrm{E}-5 & 9.17 \mathrm{E}-5 & \text { ug/g dry } & \text { ND } & 35 \\ <2.25 \mathrm{E}-3 & 2.25 \mathrm{E}-3 & " & 2.64 \mathrm{E}-3 & 35\end{array}$

$<2.25 \mathrm{E}-3 \quad 2.25 \mathrm{E}-3 \quad "$

Source: 0802024-18

Prepared \& Analyzed: 04/15/08

$\begin{array}{cccccc}<2.30 \mathrm{E}-5 & 2.30 \mathrm{E}-5 & \text { ug/g dry } & \text { ND } & & 35 \\ 7.68 \mathrm{E}-3 & 5.64 \mathrm{E}-4 & " & 1.16 \mathrm{E}-2 & 40.8 & 35\end{array}$

Source: 0802028-03

Prepared \& Analyzed: 04/15/08

$\begin{array}{lcccccc}5.12 \mathrm{E}-1 & \text { N/A } & \text { ug/L } & 5.00 \mathrm{E}-1 & 1.42 \mathrm{E}-3 & 102 & 75-125 \\ 5.59 \mathrm{E}-1 & \text { N/A } & " & 5.00 \mathrm{E}-1 & 1.31 \mathrm{E}-1 & 85.5 & 75-125\end{array}$




\section{Radionuclides by ICP-MS/Water Extract - Quality Control \\ Environmental Science Laboratory}

\begin{tabular}{|lrrrrrrrrrrr}
\hline & & Reporting & & Spike & Source & & \%REC & & RPD \\
Analyte & Result & Limit & Units & Level & Result & \%REC & Limits & RPD & Limit & Notes \\
\hline
\end{tabular}

Batch 8D24001 - 1:1 Water Extract (ICP/ICPMS)

\begin{tabular}{|c|c|c|c|c|c|c|c|c|c|}
\hline \multirow{2}{*}{$\begin{array}{l}\text { Duplicate (8D24001-DUP1) } \\
\text { Technetium-99 }\end{array}$} & \multicolumn{3}{|c|}{ Source: $0802008-08$} & \multicolumn{6}{|c|}{ Prepared \& Analyzed: 02/11/08 } \\
\hline & $3.50 \mathrm{E}-5$ & $2.30 \mathrm{E}-5$ & ug/g dry & & 3.64E-5 & & & 4.14 & 35 \\
\hline Uranium 238 & $9.52 \mathrm{E}-2$ & $5.64 \mathrm{E}-4$ & $"$ & & $1.00 \mathrm{E}-1$ & & & 4.99 & 35 \\
\hline Duplicate (8D24001-DUP2) & \multicolumn{3}{|c|}{ Source: 0802011-04 } & \multicolumn{6}{|c|}{ Prepared \& Analyzed: 02/11/08 } \\
\hline Technetium-99 & $<2.30 \mathrm{E}-5$ & $2.30 \mathrm{E}-5$ & ug/g dry & & ND & & & & 35 \\
\hline Uranium 238 & $1.18 \mathrm{E}-3$ & $5.64 \mathrm{E}-4$ & $"$ & & $1.30 \mathrm{E}-3$ & & & 9.63 & 35 \\
\hline Post Spike (8D24001-PS1) & \multicolumn{3}{|c|}{ Source: 0802008-08 } & \multicolumn{4}{|c|}{ Prepared \& Analyzed: 02/11/08 } & & \\
\hline Technetium-99 & $4.50 \mathrm{E}-1$ & N/A & $\mathrm{ug} / \mathrm{L}$ & $5.00 \mathrm{E}-1$ & $7.29 \mathrm{E}-3$ & 88.5 & $75-125$ & & \\
\hline Uranium 238 & 1.95E1 & N/A & $"$ & $5.00 \mathrm{E}-1$ & $2.00 \mathrm{E} 1$ & NR & $75-125$ & & \\
\hline
\end{tabular}


RCRA Metals By PNNL-AGG-415/Water Extract - Quality Control

Environmental Science Laboratory

\begin{tabular}{|c|c|c|c|c|c|c|c|c|c|c|}
\hline Analyte & Result & $\begin{array}{c}\text { orting } \\
\text { Limit }\end{array}$ & Units & $\begin{array}{l}\text { Spike } \\
\text { Level }\end{array}$ & $\begin{array}{l}\text { Source } \\
\text { Result }\end{array}$ & \%REC & $\begin{array}{l}\text { \%REC } \\
\text { Limits }\end{array}$ & RPD & $\begin{array}{l}\text { RPD } \\
\text { Limit }\end{array}$ & Notes \\
\hline
\end{tabular}

Batch 8D07004 - 1:1 Water Extract (ICP/ICPMS)

\begin{tabular}{|c|c|c|c|c|c|c|}
\hline \multirow{2}{*}{$\begin{array}{l}\text { Blank (8D07004-BLK1) } \\
\text { Silver }\end{array}$} & \multicolumn{6}{|c|}{ Prepared: 04/07/08 Analyzed: 04/14/08 } \\
\hline & $<1.07 \mathrm{E}-3$ & $1.07 \mathrm{E}-3$ & ug/g wet & & & \\
\hline Cadmium & $<2.95 \mathrm{E}-4$ & 2.95E-4 & $"$ & & & \\
\hline Antimony & $<5.40 \mathrm{E}-4$ & $5.40 \mathrm{E}-4$ & $"$ & & & \\
\hline Lead & $<5.60 \mathrm{E}-4$ & $5.60 \mathrm{E}-4$ & $"$ & & & \\
\hline Blank (8D07004-BLK2) & \multicolumn{6}{|c|}{ Prepared: 04/07/08 Analyzed: 04/24/08 } \\
\hline Silver & $<1.07 \mathrm{E}-3$ & $1.07 \mathrm{E}-3$ & ug/g wet & & & \\
\hline Cadmium & $<2.95 \mathrm{E}-4$ & 2.95E-4 & $"$ & & & \\
\hline Antimony & $<5.40 \mathrm{E}-4$ & $5.40 \mathrm{E}-4$ & $"$ & & & \\
\hline Lead & $<5.60 \mathrm{E}-4$ & $5.60 \mathrm{E}-4$ & $"$ & & & \\
\hline LCS (8D07004-BS1) & \multicolumn{6}{|c|}{ Prepared: 04/07/08 Analyzed: 04/14/08 } \\
\hline Silver & 4.01E0 & $1.07 \mathrm{E}-1$ & ug/g wet & $5.00 \mathrm{E} 0$ & $80-120$ & \\
\hline Cadmium & 4.42E0 & $2.95 \mathrm{E}-2$ & $"$ & $5.00 \mathrm{E} 0$ & $80-120$ & \\
\hline Antimony & 4.39E0 & $5.40 \mathrm{E}-2$ & $"$ & $5.00 \mathrm{E} 0$ & $80-120$ & \\
\hline Lead & $4.52 \mathrm{E} 0$ & $5.60 \mathrm{E}-2$ & $"$ & $5.00 \mathrm{E} 0$ & $80-120$ & \\
\hline LCS (8D07004-BS2) & \multicolumn{6}{|c|}{ Prepared: 04/07/08 Analyzed: 04/24/08 } \\
\hline Silver & $4.81 \mathrm{E} 0$ & $1.07 \mathrm{E}-1$ & ug/g wet & $5.00 \mathrm{E} 0$ & $80-120$ & \\
\hline Cadmium & 4.89E0 & $2.95 \mathrm{E}-2$ & " & $5.00 \mathrm{E} 0$ & $80-120$ & \\
\hline Antimony & $4.62 \mathrm{E} 0$ & $5.40 \mathrm{E}-2$ & $"$ & $5.00 \mathrm{E} 0$ & $80-120$ & \\
\hline Lead & $4.72 \mathrm{E} 0$ & $5.60 \mathrm{E}-2$ & $"$ & $5.00 \mathrm{E} 0$ & $80-120$ & \\
\hline Duplicate (8D07004-DUP1) & \multicolumn{3}{|c|}{ Source: $0802028-03$} & \multicolumn{3}{|c|}{ Prepared: 04/07/08 Analyzed: 04/14/08 } \\
\hline Silver & $<4.27 \mathrm{E}-3$ & $4.27 \mathrm{E}-3$ & ug/g dry & ND & & 35 \\
\hline Cadmium & $<1.18 \mathrm{E}-3$ & $1.18 \mathrm{E}-3$ & $"$ & ND & & 35 \\
\hline Antimony & $<2.15 \mathrm{E}-3$ & $2.15 \mathrm{E}-3$ & " & ND & & 35 \\
\hline Lead & $<2.23 E-3$ & $2.23 \mathrm{E}-3$ & $"$ & ND & & 35 \\
\hline
\end{tabular}


RCRA Metals By PNNL-AGG-415/Water Extract - Quality Control

Environmental Science Laboratory

\begin{tabular}{|lrrrrrrrrrrr}
\hline & & Reporting & & Spike & Source & & & \%REC & & RPD & \\
Analyte & Result & Limit & Units & Level & Result & \%REC & Limits & RPD & Limit & Notes \\
\hline
\end{tabular}

Batch 8D07004 - 1:1 Water Extract (ICP/ICPMS)

\begin{tabular}{|c|c|c|c|c|c|c|c|}
\hline Duplicate (8D07004-DUP2) & \multicolumn{3}{|c|}{ Source: 0802024-18 } & \multicolumn{2}{|c|}{ Prepared: 04/07/08 } & \multicolumn{2}{|c|}{ Analyzed: 04/24/08 } \\
\hline Silver & $<1.26 \mathrm{E}-3$ & $1.26 \mathrm{E}-3$ & ug/g dry & & ND & & \\
\hline Cadmium & $<3.47 \mathrm{E}-4$ & $3.47 \mathrm{E}-4$ & $"$ & & ND & & \\
\hline Antimony & $<6.36 \mathrm{E}-4$ & $6.36 \mathrm{E}-4$ & $"$ & & ND & & \\
\hline Lead & $<6.59 \mathrm{E}-4$ & $6.59 \mathrm{E}-4$ & $"$ & & 7.10E-4 & & \\
\hline Post Spike (8D07004-PS1) & \multicolumn{3}{|c|}{ Source: 0802028-03 } & \multicolumn{2}{|c|}{ Prepared: 04/07/08 } & Analyzed & $4 / 14 / 08$ \\
\hline Silver & 4.61E0 & N/A & $\mathrm{ug} / \mathrm{L}$ & 5.00E0 & 6.47E-3 & 92 & $75-125$ \\
\hline Cadmium & 4.67E0 & N/A & $"$ & 5.00E0 & $2.20 \mathrm{E}-2$ & 92.9 & $75-125$ \\
\hline Antimony & 4.78E0 & N/A & $"$ & 5.00E0 & 9.83E-2 & 93.5 & $75-125$ \\
\hline Lead & 4.74E0 & N/A & $"$ & $5.00 \mathrm{E} 0$ & 5.43E-2 & 93.8 & $75-125$ \\
\hline
\end{tabular}

Batch 8E01009 - 1:1 Water Extract (ICP/ICPMS)

\begin{tabular}{|c|c|c|c|c|c|c|}
\hline Blank (8E01009-BLK1) & \multicolumn{6}{|c|}{ Prepared \& Analyzed: 02/07/08 } \\
\hline Silver & $<1.07 \mathrm{E}-3$ & $1.07 \mathrm{E}-3$ & ug/g wet & & & \\
\hline Cadmium & $<2.95 \mathrm{E}-4$ & $2.95 \mathrm{E}-4$ & $"$ & & & \\
\hline Antimony & $<5.40 \mathrm{E}-4$ & $5.40 \mathrm{E}-4$ & $"$ & & & \\
\hline Lead & $<5.60 \mathrm{E}-4$ & $5.60 \mathrm{E}-4$ & $"$ & & & \\
\hline Blank (8E01009-BLK2) & \multicolumn{6}{|c|}{ Prepared \& Analyzed: 02/07/08 } \\
\hline Silver & $<1.07 \mathrm{E}-3$ & $1.07 \mathrm{E}-3$ & ug/g wet & & & \\
\hline Cadmium & $<2.95 \mathrm{E}-4$ & $2.95 \mathrm{E}-4$ & $"$ & & & \\
\hline Antimony & $<5.40 \mathrm{E}-4$ & $5.40 \mathrm{E}-4$ & $"$ & & & \\
\hline Lead & $<5.60 \mathrm{E}-4$ & $5.60 \mathrm{E}-4$ & $"$ & & & \\
\hline LCS (8E01009-BS1) & \multicolumn{6}{|c|}{ Prepared \& Analyzed: 02/07/08 } \\
\hline Silver & $4.48 \mathrm{E} 0$ & $1.07 \mathrm{E}-1$ & ug/g wet & $5.00 \mathrm{E} 0$ & 89.7 & $80-120$ \\
\hline Cadmium & 4.95E0 & 2.95E-2 & $"$ & $5.00 \mathrm{E} 0$ & 99.1 & $80-120$ \\
\hline Antimony & 4.49E0 & $5.40 \mathrm{E}-2$ & $"$ & $5.00 \mathrm{E} 0$ & 89.8 & $80-120$ \\
\hline Lead & 4.92E0 & $5.60 \mathrm{E}-2$ & $"$ & $5.00 \mathrm{E} 0$ & 98.3 & $80-120$ \\
\hline
\end{tabular}


RCRA Metals By PNNL-AGG-415/Water Extract - Quality Control

Environmental Science Laboratory

\begin{tabular}{|c|c|c|c|c|c|c|c|c|c|c|}
\hline Analyte & Result & $\begin{array}{c}\text { orting } \\
\text { Limit }\end{array}$ & Units & $\begin{array}{l}\text { Spike } \\
\text { Level }\end{array}$ & $\begin{array}{l}\text { Source } \\
\text { Result }\end{array}$ & \%REC & $\begin{array}{l}\text { \%REC } \\
\text { Limits }\end{array}$ & RPD & $\begin{array}{l}\text { RPD } \\
\text { Limit }\end{array}$ & Notes \\
\hline
\end{tabular}

Batch 8E01009 - 1:1 Water Extract (ICP/ICPMS)

\begin{tabular}{|c|c|c|c|c|c|c|c|c|c|}
\hline \multirow{2}{*}{$\begin{array}{l}\text { LCS (8E01009-BS2) } \\
\text { Silver }\end{array}$} & \multicolumn{9}{|c|}{ Prepared \& Analyzed: 02/07/08 } \\
\hline & 4.33E0 & $1.07 \mathrm{E}-1$ & ug/g wet & $5.00 \mathrm{E} 0$ & & 86.8 & $80-120$ & & \\
\hline Cadmium & $4.49 \mathrm{E} 0$ & $2.95 \mathrm{E}-2$ & $"$ & 5.00E0 & & 89.9 & $80-120$ & & \\
\hline Antimony & 4.00E0 & $5.40 \mathrm{E}-2$ & $"$ & $5.00 \mathrm{E} 0$ & & 80.1 & $80-120$ & & \\
\hline Lead & 4.43E0 & $5.60 \mathrm{E}-2$ & $"$ & 5.00E0 & & 88.7 & $80-120$ & & \\
\hline Duplicate (8E01009-DUP1) & \multicolumn{3}{|c|}{ Source: 0802008-08 } & \multicolumn{6}{|c|}{ Prepared \& Analyzed: 02/07/08 } \\
\hline Silver & $2.14 \mathrm{E}-3$ & $1.07 \mathrm{E}-3$ & ug/g dry & & $2.95 \mathrm{E}-3$ & & & 31.8 & 35 \\
\hline Cadmium & $6.70 \mathrm{E}-4$ & $2.95 \mathrm{E}-4$ & $"$ & & 7.07E-4 & & & 5.41 & 35 \\
\hline Antimony & $1.79 \mathrm{E}-3$ & $5.40 \mathrm{E}-4$ & $"$ & & $1.79 \mathrm{E}-3$ & & & 0.117 & 35 \\
\hline Lead & $2.18 \mathrm{E}-2$ & $5.60 \mathrm{E}-4$ & $"$ & & $2.39 \mathrm{E}-2$ & & & 9.42 & 35 \\
\hline Duplicate (8E01009-DUP2) & \multicolumn{3}{|c|}{ Source: 0802011-04 } & \multicolumn{6}{|c|}{ Prepared \& Analyzed: 02/07/08 } \\
\hline Silver & $<1.07 \mathrm{E}-3$ & $1.07 \mathrm{E}-3$ & ug/g dry & & ND & & & & 35 \\
\hline Cadmium & $3.94 \mathrm{E}-4$ & $2.95 \mathrm{E}-4$ & $"$ & & $3.92 \mathrm{E}-4$ & & & 0.535 & 35 \\
\hline Antimony & $1.07 \mathrm{E}-3$ & $5.40 \mathrm{E}-4$ & $"$ & & $1.65 \mathrm{E}-3$ & & & 42.3 & 35 \\
\hline Lead & $<5.60 \mathrm{E}-4$ & $5.60 \mathrm{E}-4$ & $"$ & & ND & & & & 35 \\
\hline Post Spike (8E01009-PS1) & \multicolumn{3}{|c|}{ Source: 0802008-08 } & \multicolumn{4}{|c|}{ Prepared \& Analyzed: 02/07/08 } & & \\
\hline Silver & 2.66E0 & N/A & ug/L & $2.50 \mathrm{E} 0$ & $5.90 \mathrm{E}-1$ & 82.7 & $75-125$ & & \\
\hline Cadmium & 2.49E0 & N/A & $"$ & $2.50 \mathrm{E} 0$ & $1.41 \mathrm{E}-1$ & 93.9 & $75-125$ & & \\
\hline Antimony & 2.63E0 & N/A & $"$ & 2.50E0 & 3.57E-1 & 91.1 & $75-125$ & & \\
\hline Lead & 7.19E0 & N/A & $"$ & $2.50 \mathrm{E} 0$ & 4.78E0 & 96.4 & $75-125$ & & \\
\hline
\end{tabular}


RCRA Metals By PNNL-AGG-415/Acid Extract - Quality Control

Environmental Science Laboratory

\begin{tabular}{|c|c|c|c|c|c|c|c|c|c|c|}
\hline Analyte & Result & $\begin{array}{c}\text { orting } \\
\text { Limit }\end{array}$ & Units & $\begin{array}{l}\text { Spike } \\
\text { Level }\end{array}$ & $\begin{array}{l}\text { Source } \\
\text { Result }\end{array}$ & \%REC & $\begin{array}{l}\text { \%REC } \\
\text { Limits }\end{array}$ & RPD & $\begin{array}{l}\text { RPD } \\
\text { Limit }\end{array}$ & Notes \\
\hline
\end{tabular}

Batch 8E01016 - ASTM D 5198 (ICP/ICPMS)

Duplicate (8E01016-DUP1)

Source: 0802003-02 Prepared \& Analyzed: 02/19/08

Cadmium

1.39E-1 7.01E-2 ug/g dry

1.03E-1

ND

$30.5 \quad 35$

Antimony

$<1.13 \mathrm{E}-1$

$1.13 \mathrm{E}-1$

4.78E0

26.1

35

Lead

$6.22 \mathrm{E} 0$

5.27E-2

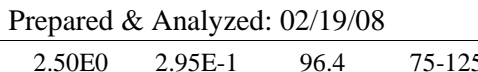

Post Spike (8E01016-PS1)

Source: 0802003-02

Cadmium

2.70E0

N/A ug/

$2.50 \mathrm{E} 0 \quad 3.14 \mathrm{E}-2 \quad 95.0 \quad 75-125$

Antimony

$2.41 \mathrm{E} 0$

N/A

95.0

75-125

Batch 8E15001 - ASTM D 5198 (ICP/ICPMS)

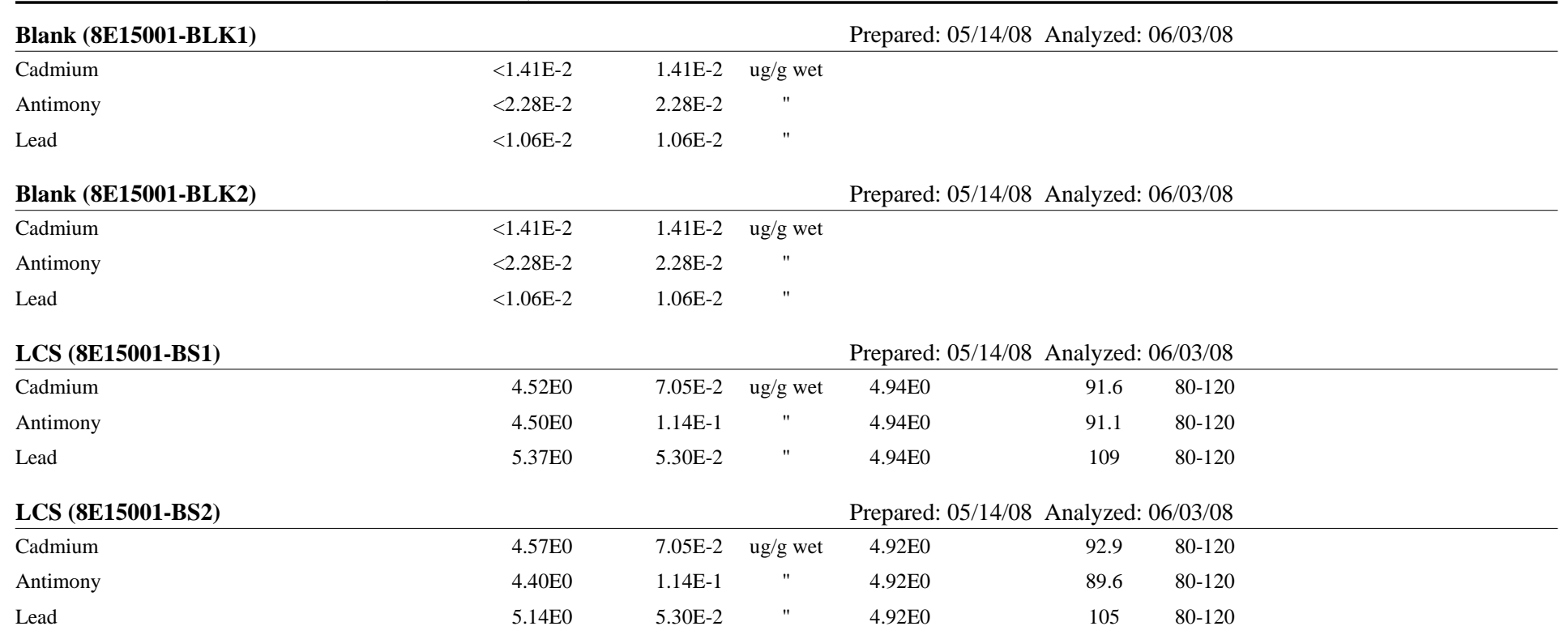


RCRA Metals By PNNL-AGG-415/Acid Extract - Quality Control

Environmental Science Laboratory

\begin{tabular}{|c|c|c|c|c|c|c|c|c|c|c|}
\hline Analyte & Result & $\begin{array}{r}\text { porting } \\
\text { Limit }\end{array}$ & Units & $\begin{array}{l}\text { Spike } \\
\text { Level }\end{array}$ & $\begin{array}{l}\text { Source } \\
\text { Result }\end{array}$ & \%REC & $\begin{array}{l}\text { \%REC } \\
\text { Limits }\end{array}$ & RPD & $\begin{array}{l}\text { RPD } \\
\text { Limit }\end{array}$ & Notes \\
\hline
\end{tabular}

Batch 8E15001 - ASTM D 5198 (ICP/ICPMS)

\begin{tabular}{|c|c|c|c|c|c|c|c|c|c|}
\hline \multirow{2}{*}{$\begin{array}{l}\text { Duplicate (8E15001-DUP1) } \\
\text { Cadmium }\end{array}$} & \multicolumn{3}{|c|}{ Source: 0802024-18 } & \multicolumn{4}{|c|}{ Prepared: 05/14/08 Analyzed: 06/03/08 } & \multirow[b]{2}{*}{7.80} & \multirow[b]{2}{*}{35} \\
\hline & $1.07 \mathrm{E}-1$ & 6.10E-2 & ug/g dry & & 1.15E-1 & & & & \\
\hline Antimony & $<9.86 \mathrm{E}-2$ & $9.86 \mathrm{E}-2$ & $"$ & & ND & & & & 35 \\
\hline Lead & $4.28 \mathrm{E} 0$ & $4.58 \mathrm{E}-2$ & $"$ & & 4.24E0 & & & 0.964 & 35 \\
\hline Duplicate (8E15001-DUP2) & \multicolumn{3}{|c|}{ Source: 0802028-03 } & \multicolumn{4}{|c|}{ Prepared: 05/14/08 Analyzed: 06/03/08 } & & \\
\hline Cadmium & $1.58 \mathrm{E}-1$ & $6.17 \mathrm{E}-2$ & ug/g dry & & $1.64 \mathrm{E}-1$ & & & 4.01 & 35 \\
\hline Antimony & $<9.97 \mathrm{E}-2$ & $9.97 \mathrm{E}-2$ & $"$ & & ND & & & & 35 \\
\hline Lead & 5.55E0 & $4.64 \mathrm{E}-2$ & $"$ & & 5.57E0 & & & 0.207 & 35 \\
\hline Post Spike (8E15001-PS2) & \multicolumn{3}{|c|}{ Source: 0802028-03 } & \multicolumn{4}{|c|}{ Prepared: 05/14/08 Analyzed: 06/03/08 } & & \\
\hline Cadmium & 4.66E0 & N/A & ug/L & $5.00 \mathrm{E} 0$ & $3.71 \mathrm{E}-1$ & 85.8 & $75-125$ & & \\
\hline Antimony & 3.93E0 & N/A & " & $5.00 \mathrm{E} 0$ & $3.47 \mathrm{E}-2$ & 78 & $75-125$ & & \\
\hline Lead & 1.67E1 & N/A & $"$ & $5.00 \mathrm{E} 0$ & $1.26 \mathrm{E} 1$ & 81.1 & $75-125$ & & \\
\hline
\end{tabular}


GEA/Soil - Quality Control

Environmental Science Laboratory

\begin{tabular}{|c|c|c|c|c|c|c|c|c|c|c|}
\hline Analyte & Result & $\begin{array}{r}\text { orting } \\
\text { Limit }\end{array}$ & Units & $\begin{array}{l}\text { Spike } \\
\text { Level }\end{array}$ & $\begin{array}{l}\text { Source } \\
\text { Result }\end{array}$ & \%REC & $\begin{array}{l}\text { \%REC } \\
\text { Limits }\end{array}$ & RPD & $\begin{array}{l}\text { RPD } \\
\text { Limit }\end{array}$ & Notes \\
\hline
\end{tabular}

Batch 8D28002 - NO PREP (Radchem)

\begin{tabular}{|c|c|c|c|c|c|c|}
\hline \multirow{2}{*}{$\begin{array}{l}\text { Duplicate (8D28002-DUP1) } \\
\text { Cobalt-60 }\end{array}$} & \multicolumn{3}{|c|}{ Source: 0802008-08 } & \multirow{2}{*}{\multicolumn{2}{|c|}{$\begin{array}{c}\text { Prepared: 02/17/08 Analyzed: 02/21/08 } \\
\text { ND }\end{array}$}} & \multirow[b]{2}{*}{20} \\
\hline & $<3.45 \mathrm{E}-1$ & $3.45 \mathrm{E}-1$ & pCi/g dry & & & \\
\hline Cesium-137 & $<3.99 \mathrm{E}-1$ & 3.99E-1 & $"$ & ND & & 20 \\
\hline Europium-152 & $<1.53 \mathrm{E} 0$ & $1.53 \mathrm{E} 0$ & $"$ & ND & & 20 \\
\hline Europium-154 & $<8.93 \mathrm{E}-1$ & 8.93E-1 & $"$ & ND & & 20 \\
\hline Europium-155 & $<1.51 \mathrm{E} 0$ & $1.51 \mathrm{E} 0$ & $"$ & ND & & 20 \\
\hline
\end{tabular}


Total Alpha Total Beta/Acid Extract - Quality Control

Environmental Science Laboratory

\begin{tabular}{|lrrrrrrrrrr}
\hline & & Reporting & & Spike & Source & & \%REC & & RPD & \\
Analyte & Result & Limit & Units & Level & Result & \%REC & Limits & RPD & Limit & Notes \\
\hline
\end{tabular}

Batch 8I28001 - ASTM D 5198 (RadChem)

\begin{tabular}{|c|c|c|c|c|c|c|}
\hline Blank (8I28001-BLK1) & \multicolumn{6}{|c|}{ Prepared: 04/16/08 Analyzed: 04/23/08 } \\
\hline Gross Beta & $<5.03 \mathrm{E} 1$ & 5.03E1 & $\mathrm{pCi} / \mathrm{g}$ wet & & & \\
\hline Gross Alpha & $<1.68 \mathrm{E} 1$ & $1.68 \mathrm{E} 1$ & $"$ & & & \\
\hline Duplicate (8I28001-DUP1) & \multicolumn{3}{|c|}{ Source: 0802028-03 } & Prepared: 04/16/08 & Analyzed: 04/23/08 & \\
\hline Gross Beta & $<6.29 \mathrm{E} 1$ & $6.29 \mathrm{E} 1$ & pCi/g dry & ND & & 35 \\
\hline Gross Alpha & $<2.10 \mathrm{E} 1$ & $2.10 \mathrm{E} 1$ & $"$ & ND & & 35 \\
\hline
\end{tabular}




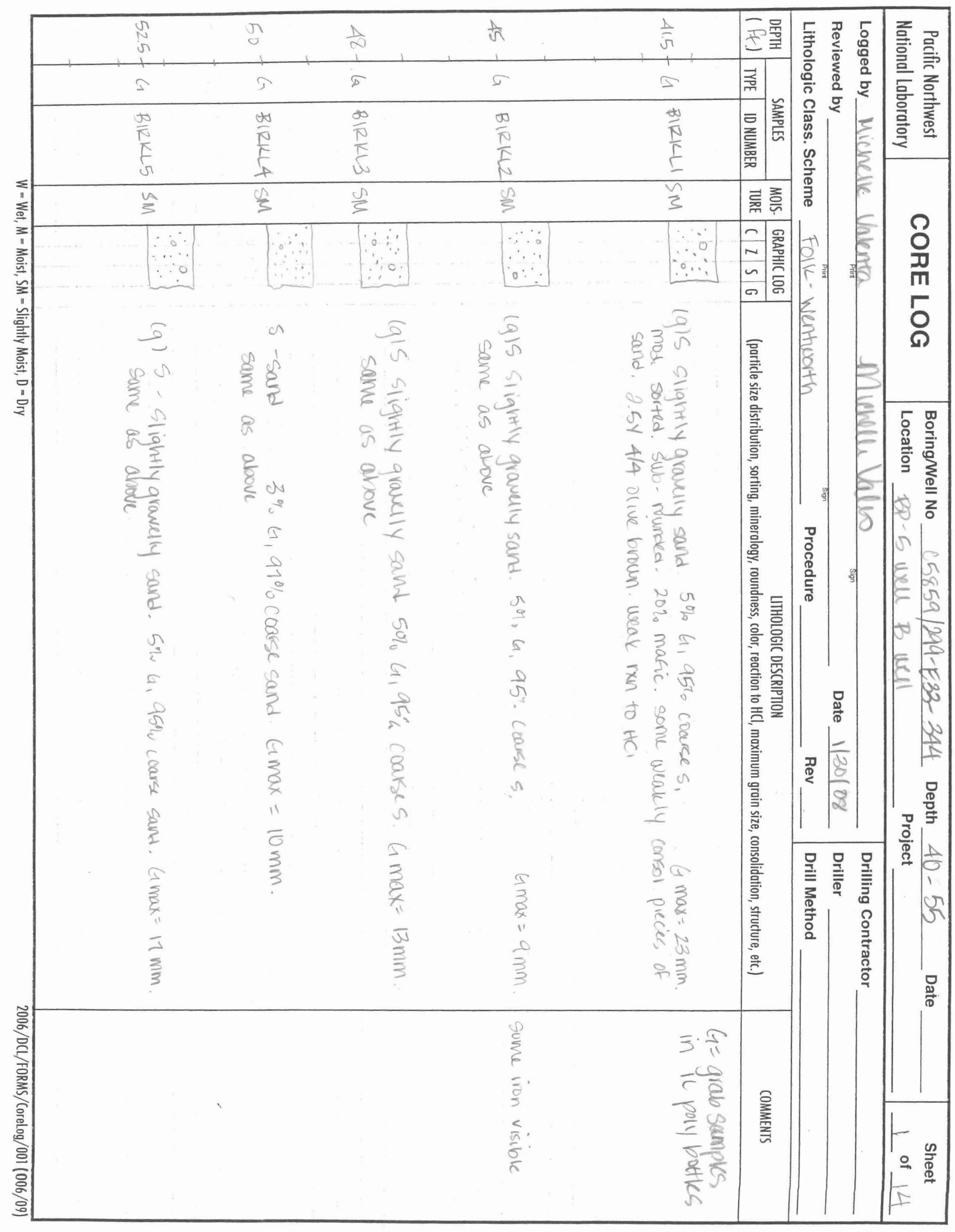




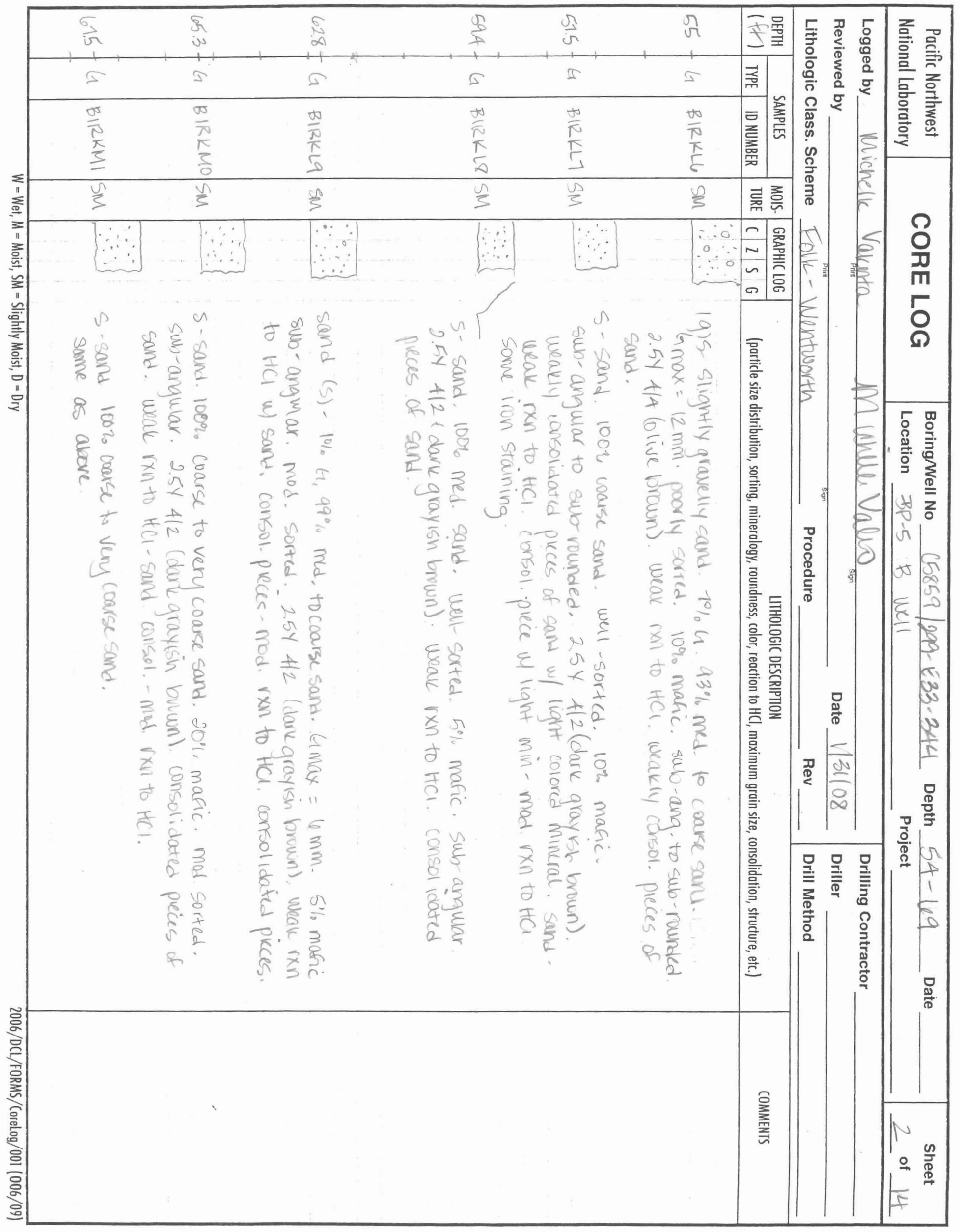




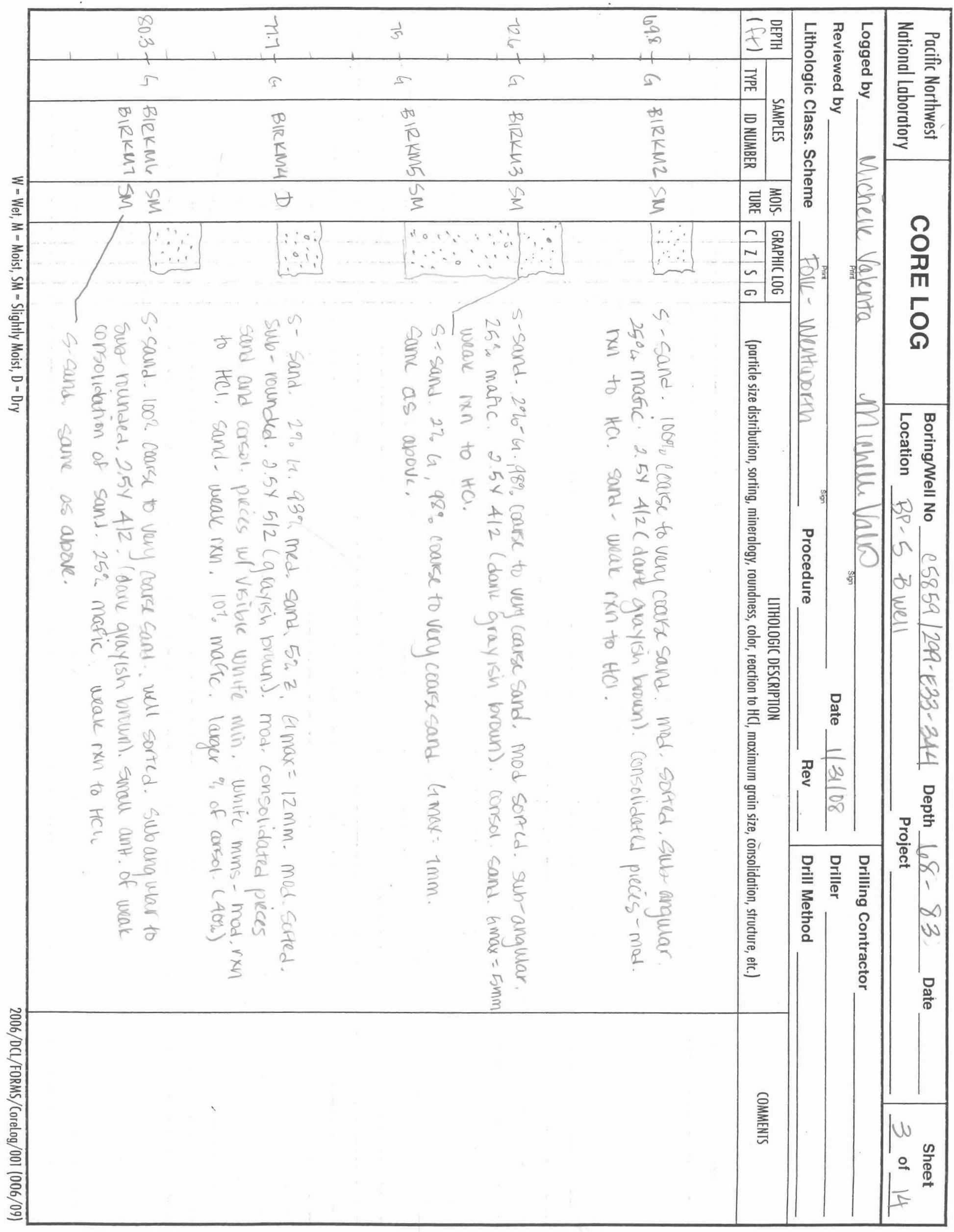




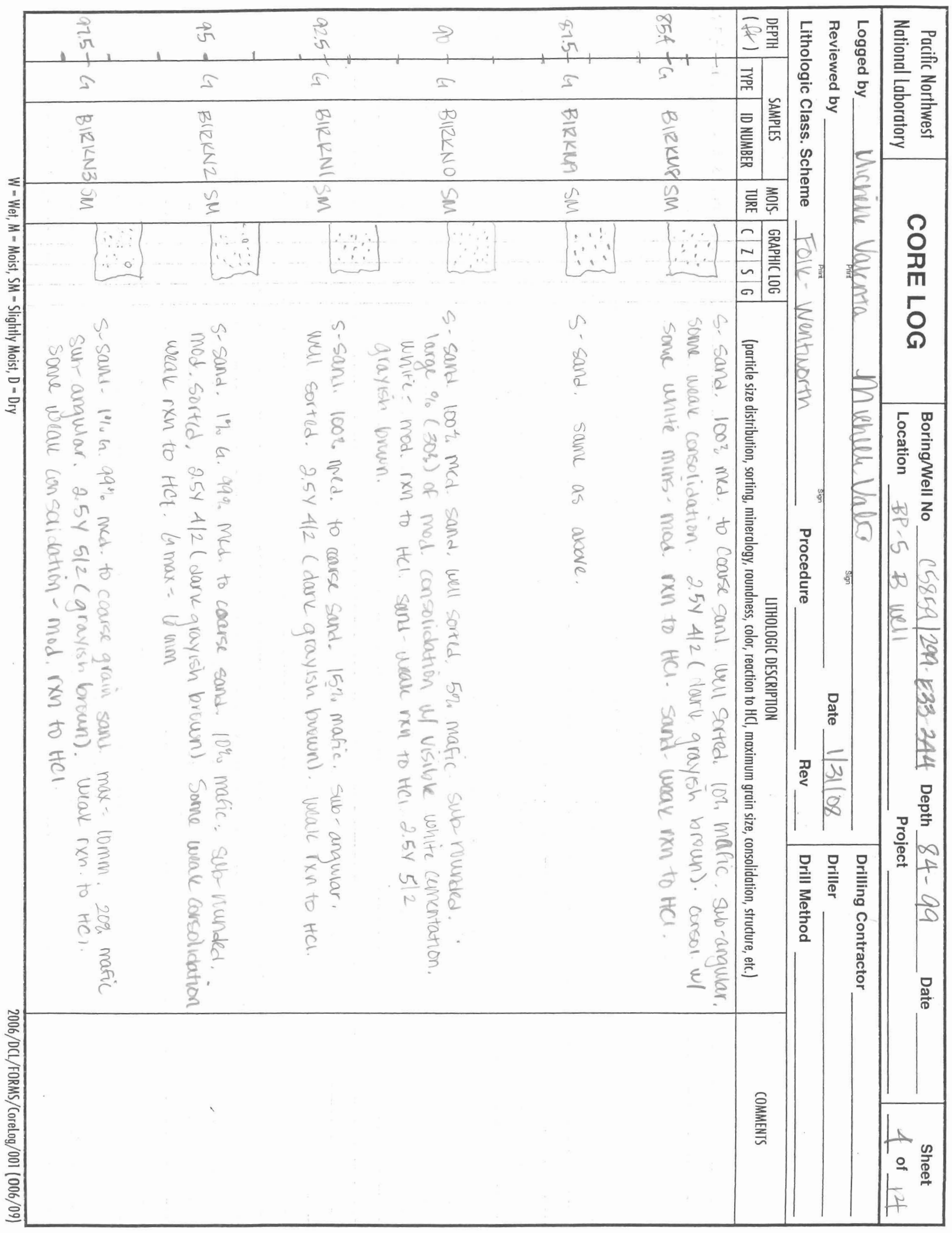




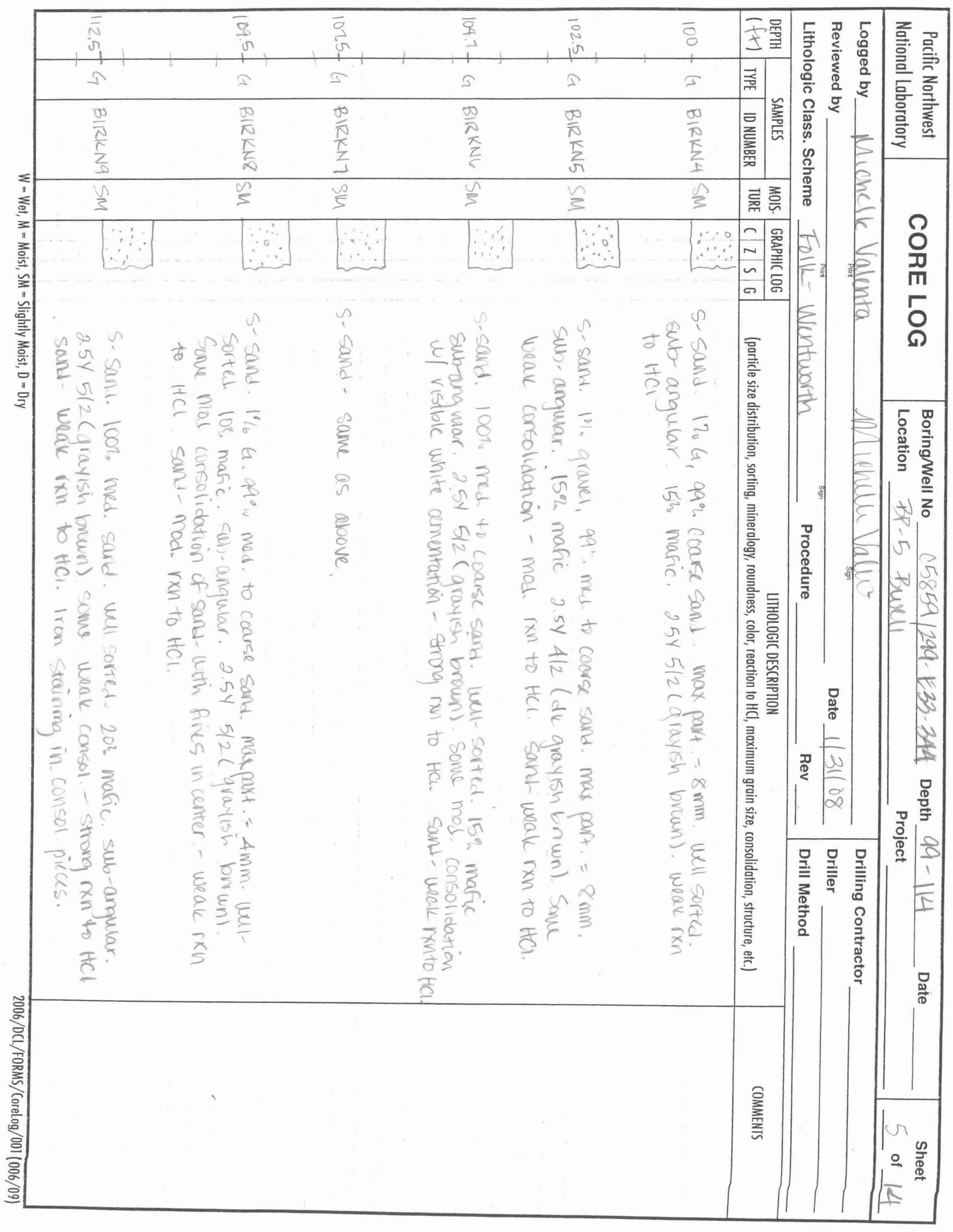




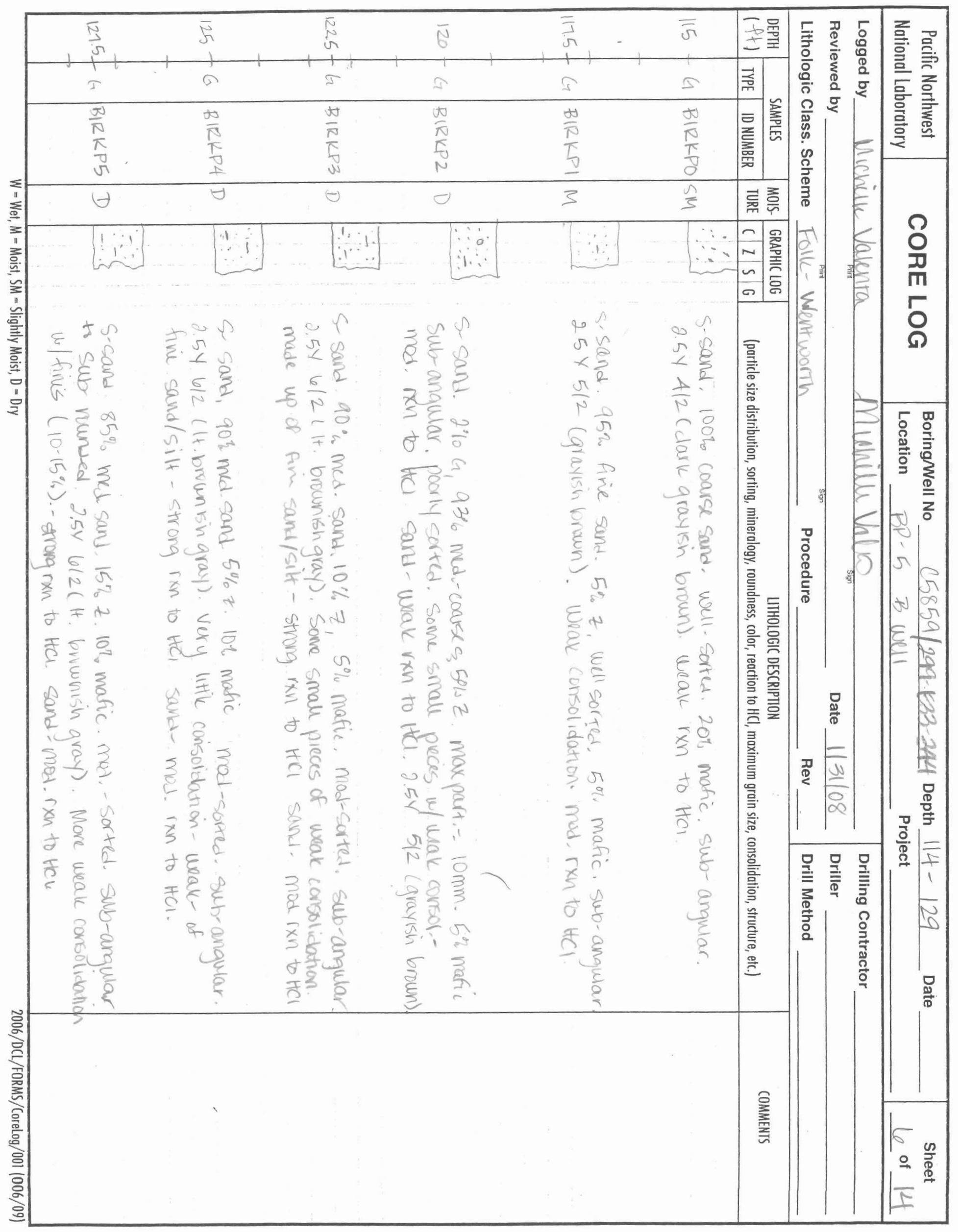




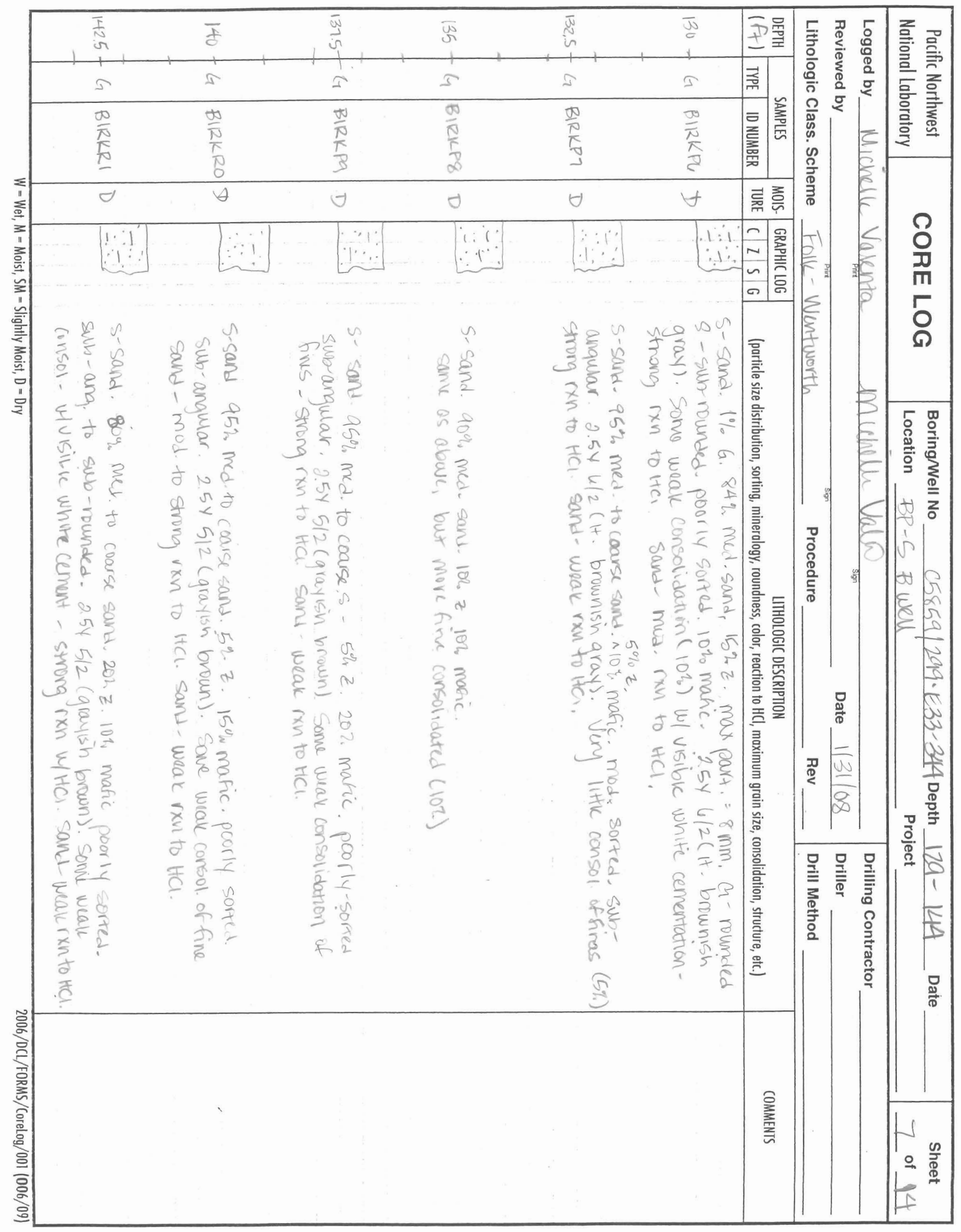




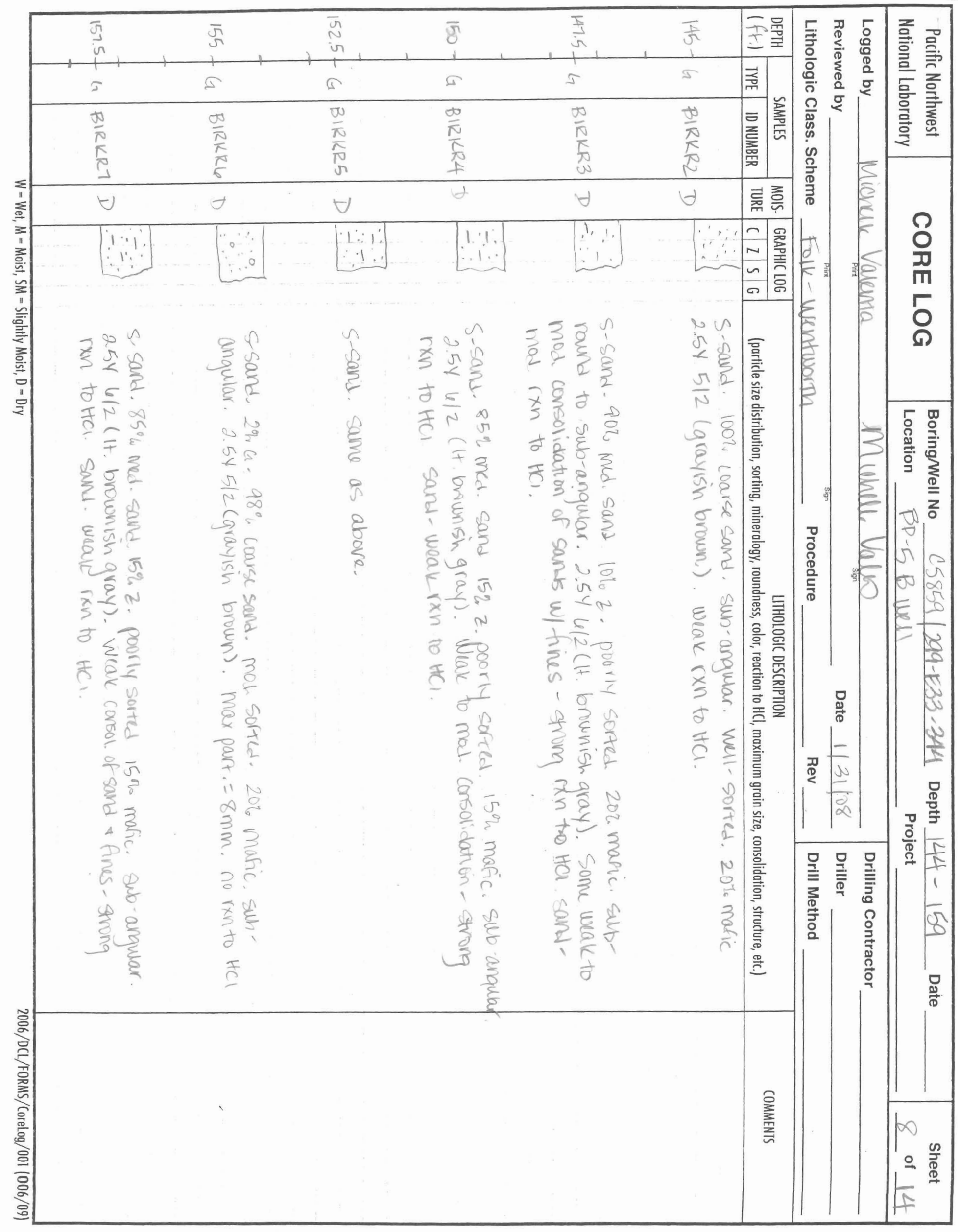




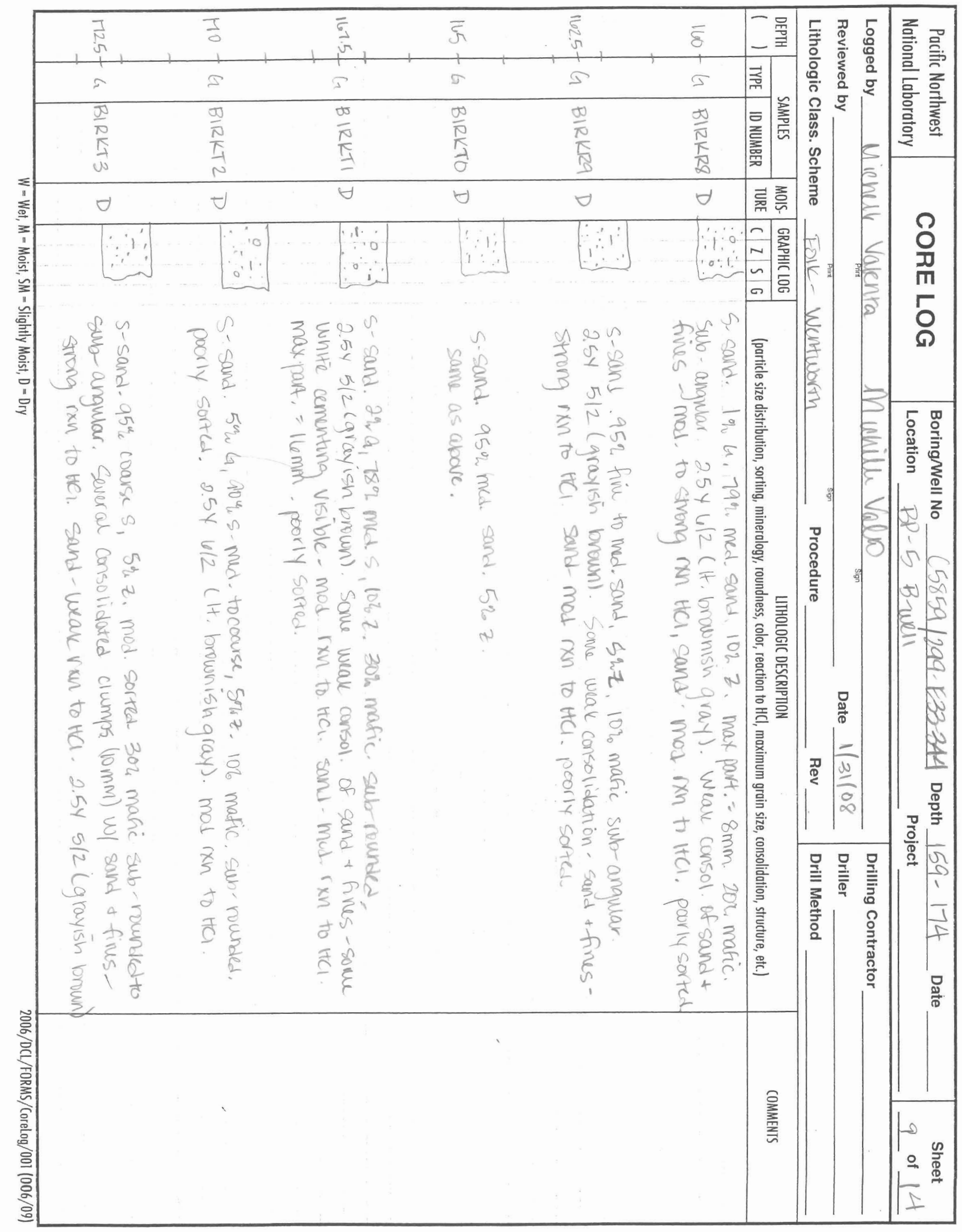




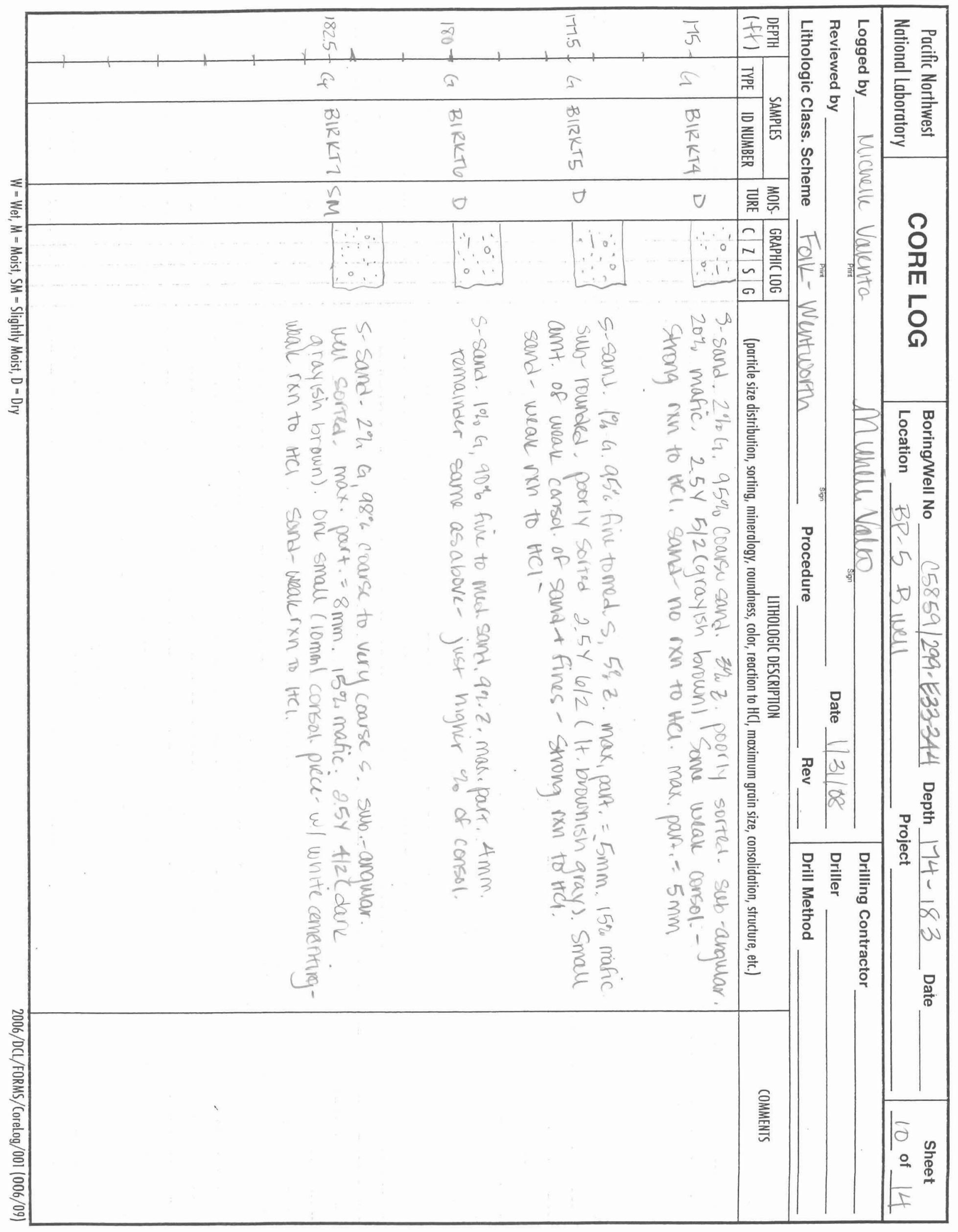




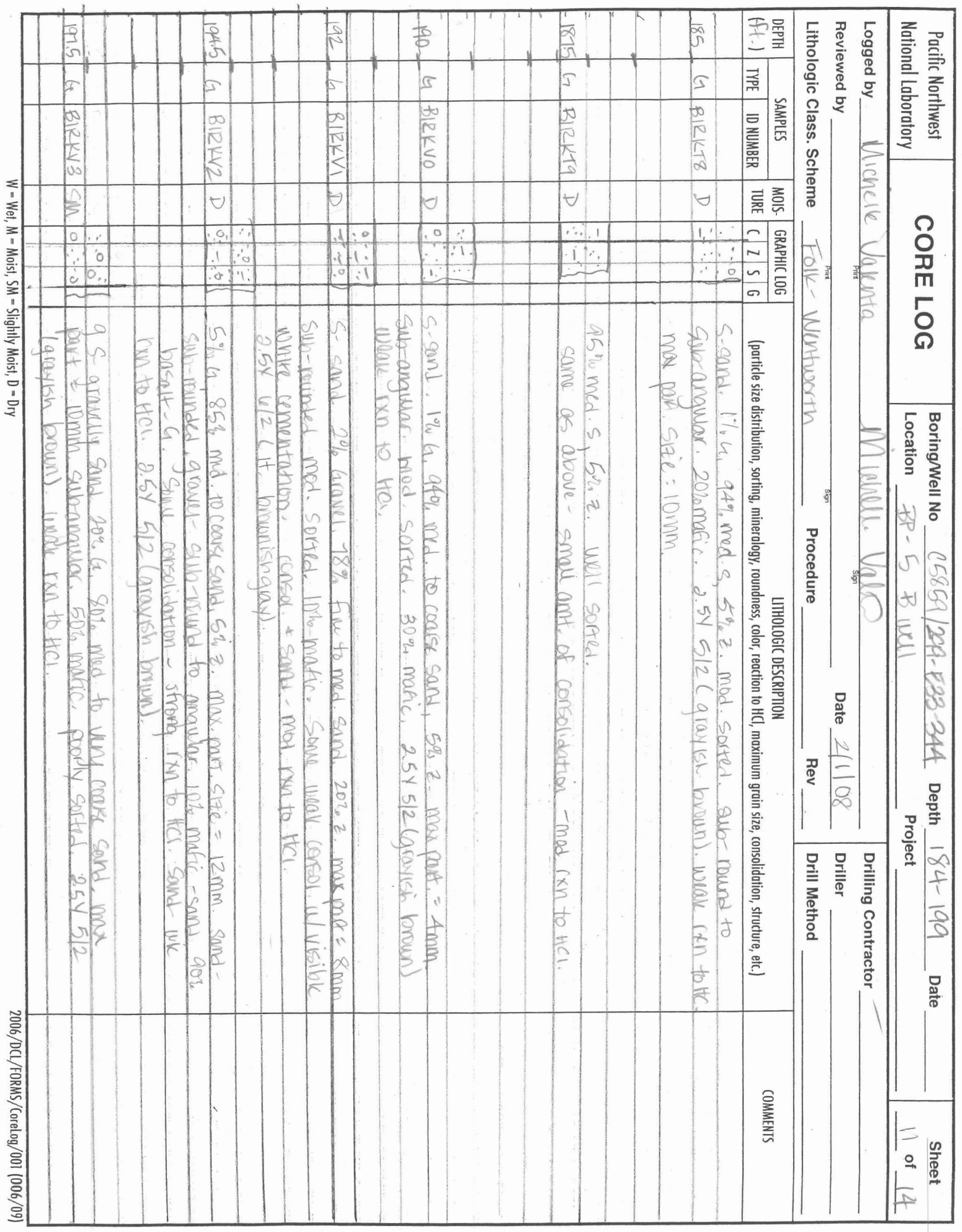




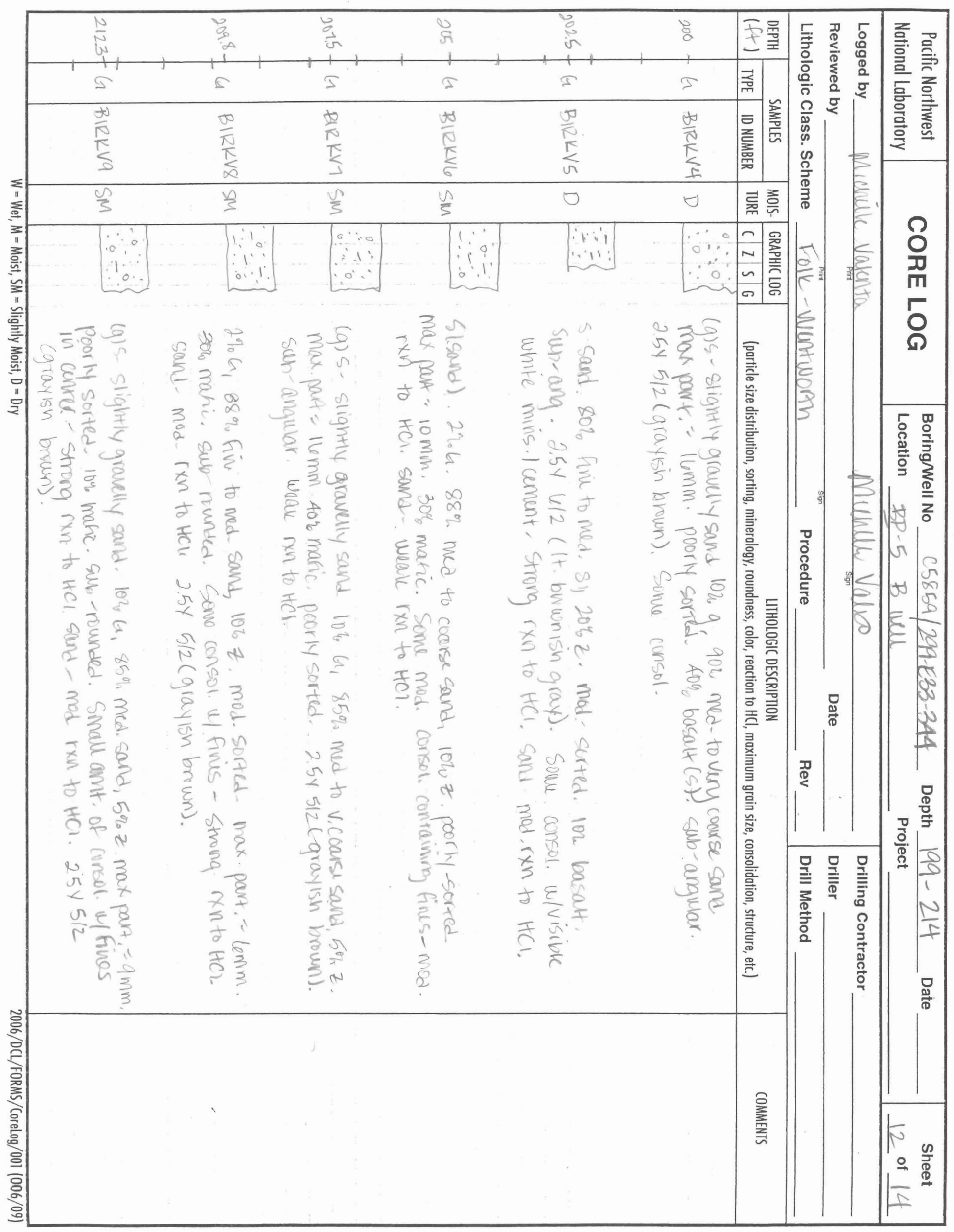




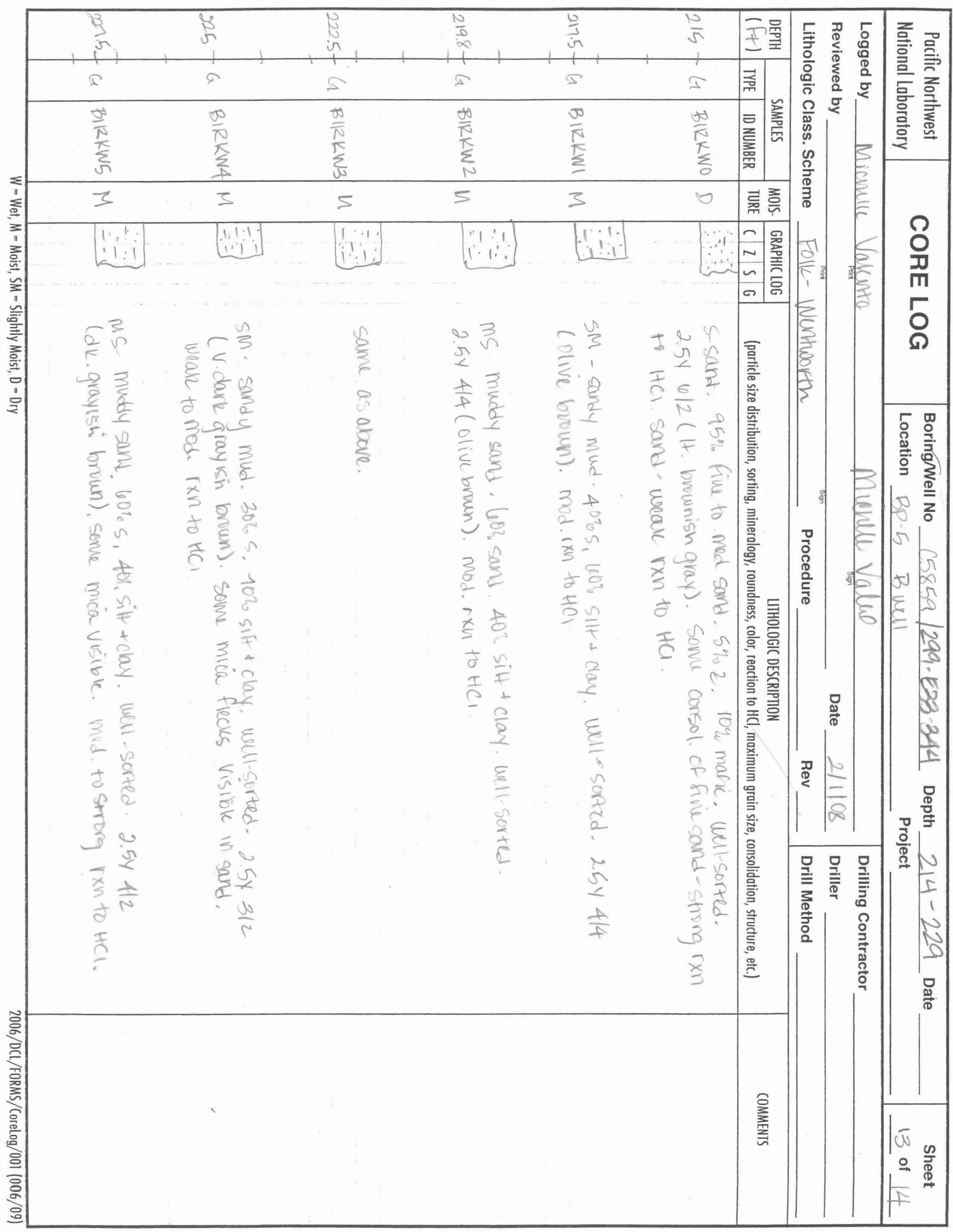




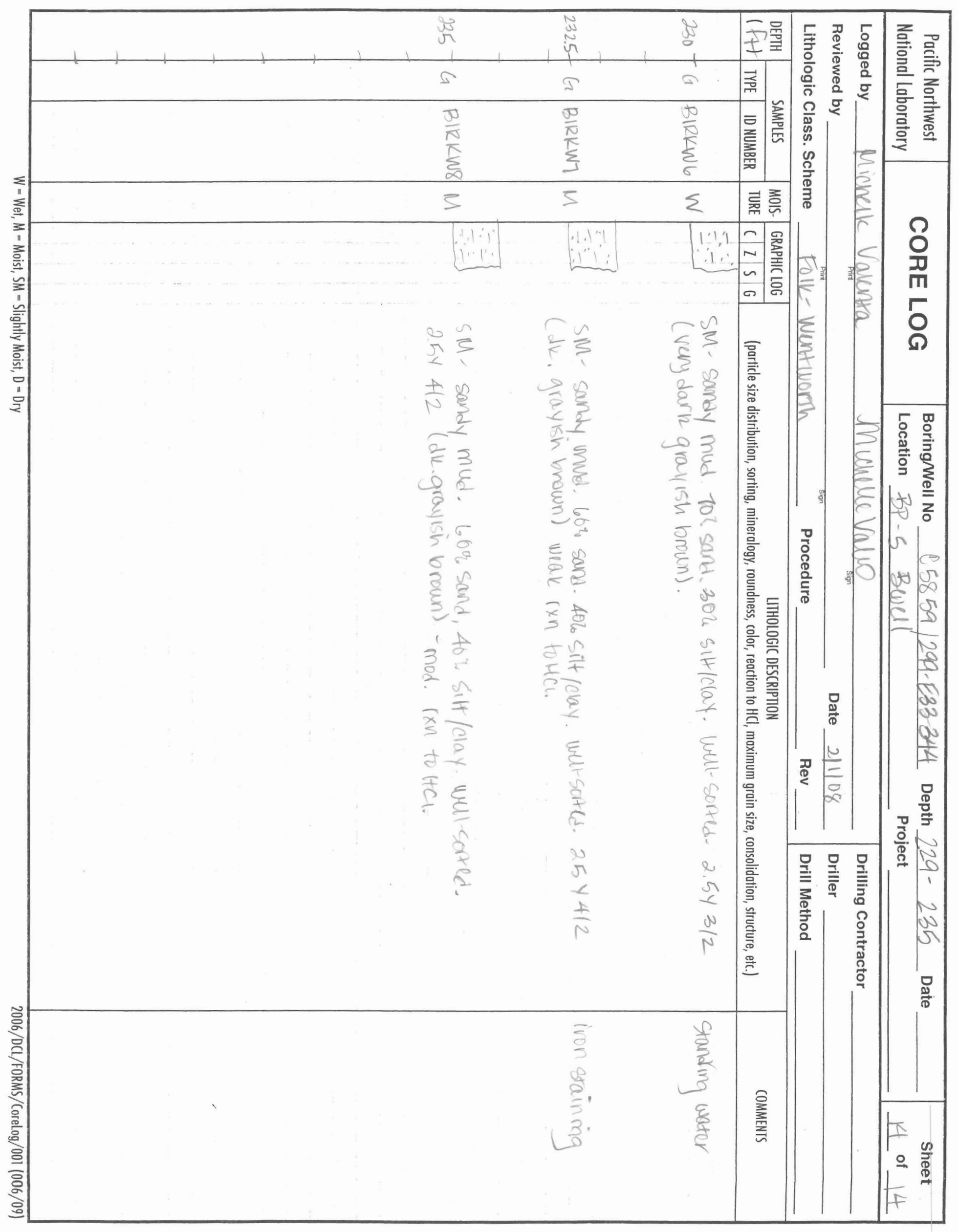




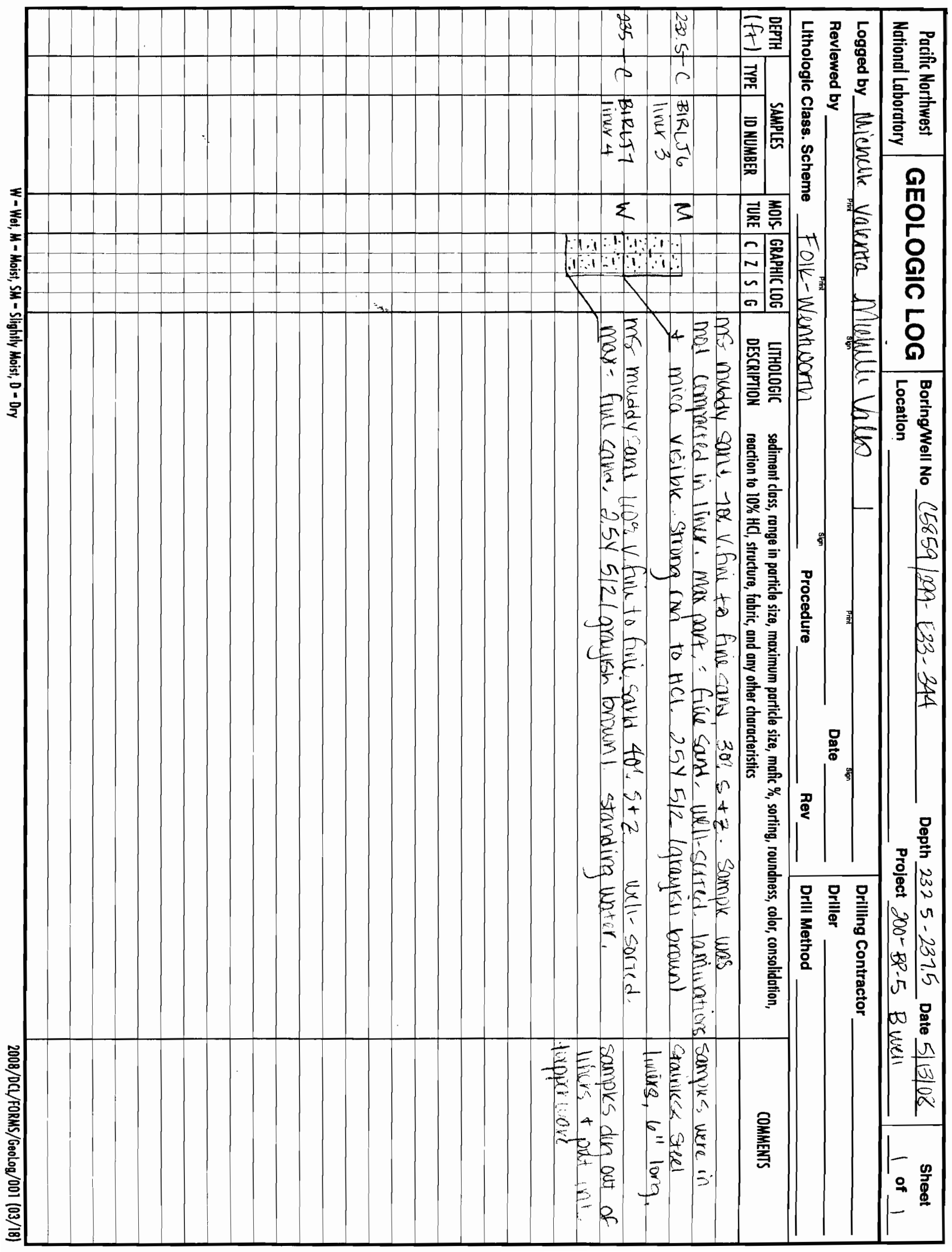




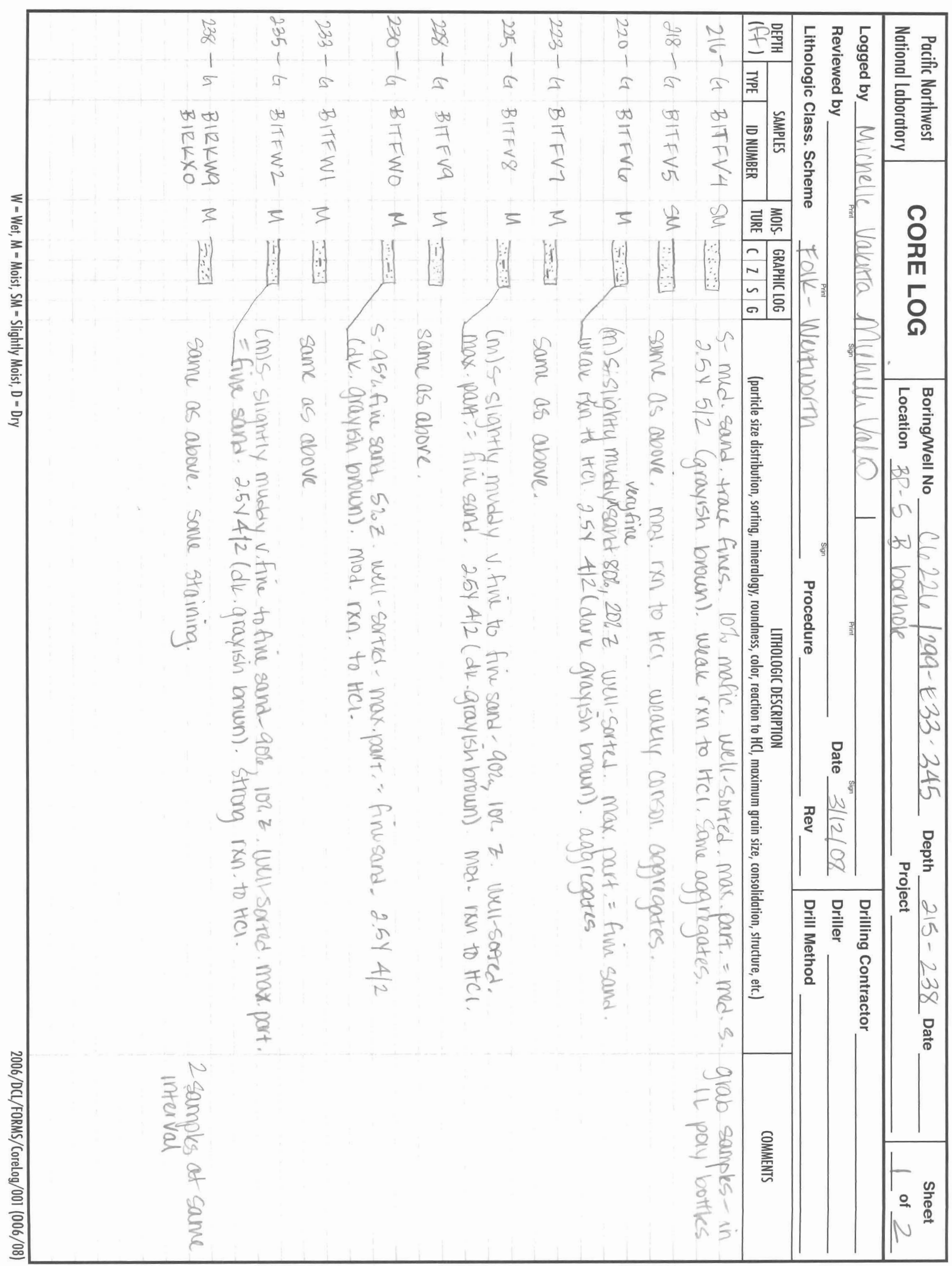




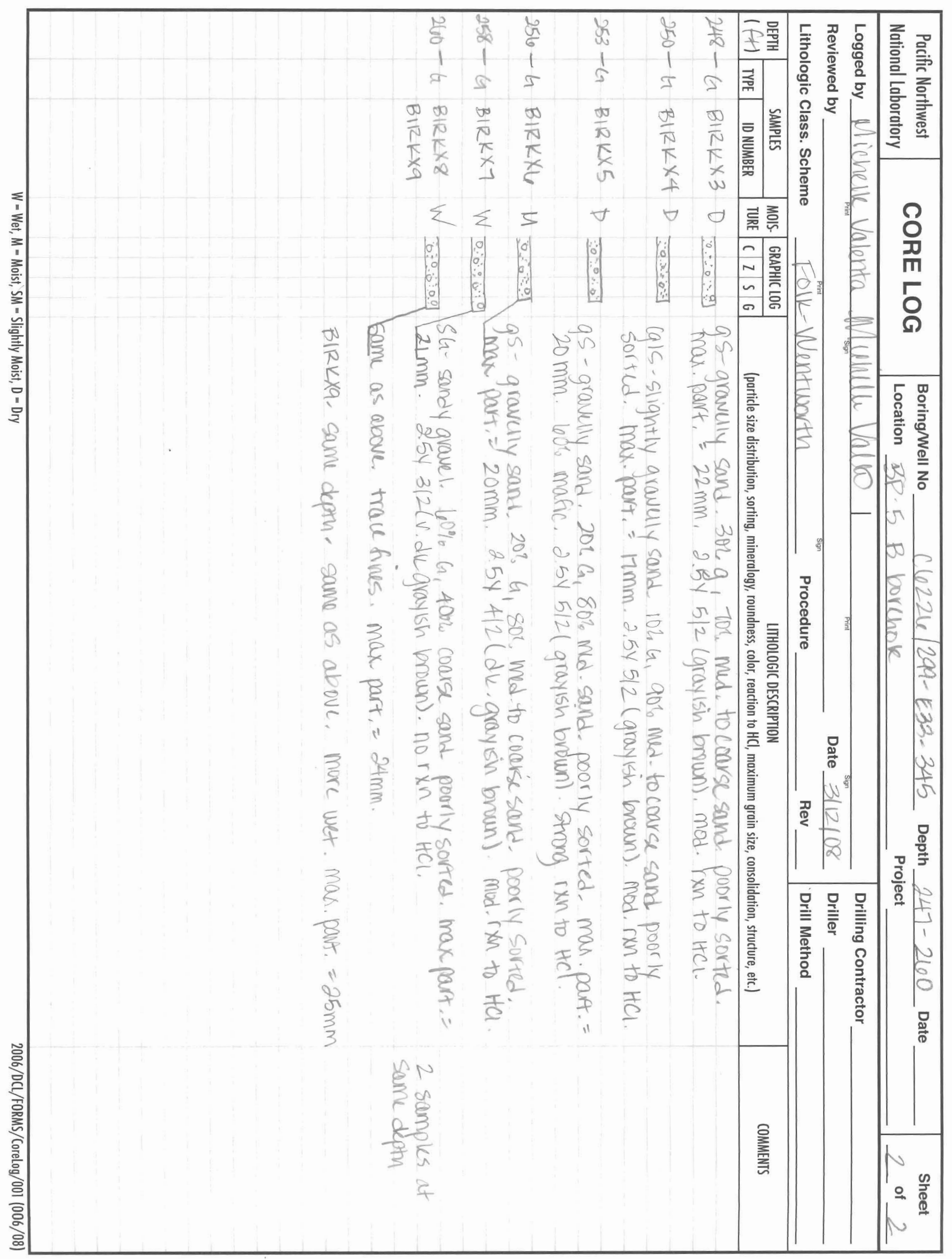




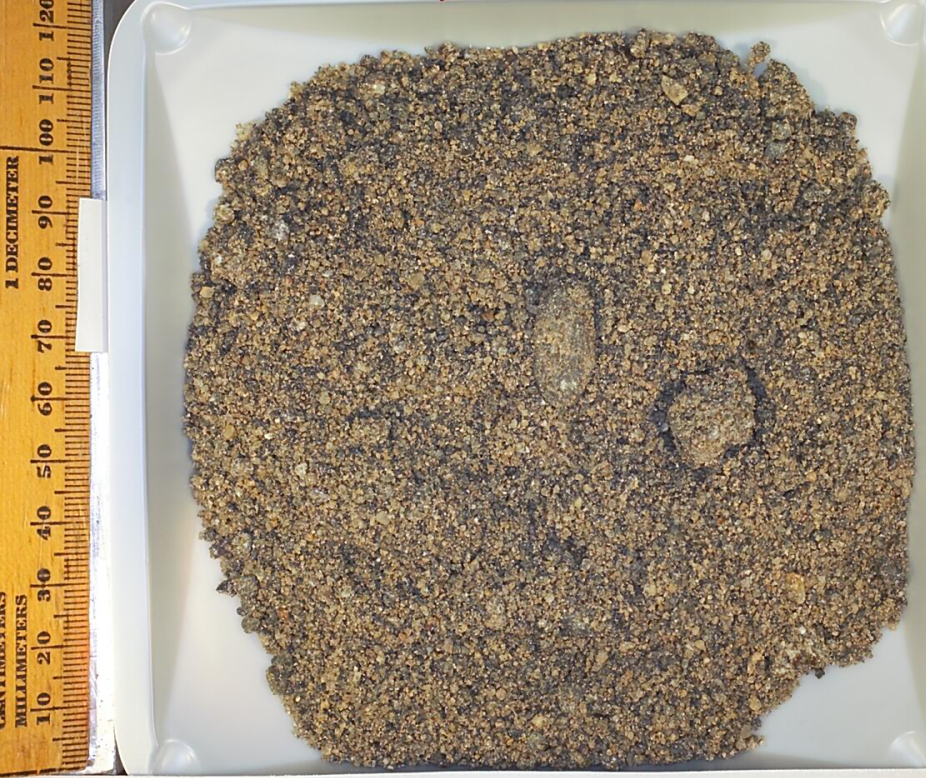
C5859
B1RKL2
$45 \mathrm{ft}$ Grab Sample

Depth from Chain-of-Custody 


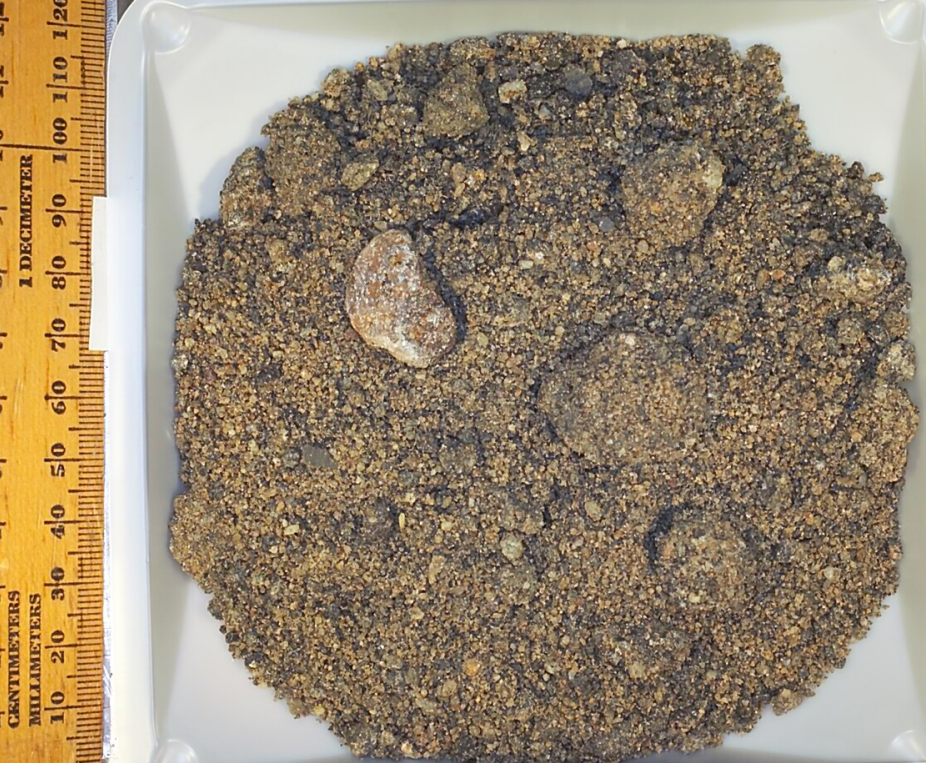

\section{C5859 B1RKL3 \\ $48 \mathrm{ft}$ Grab Sample} Depth from Chain-of-Custody 


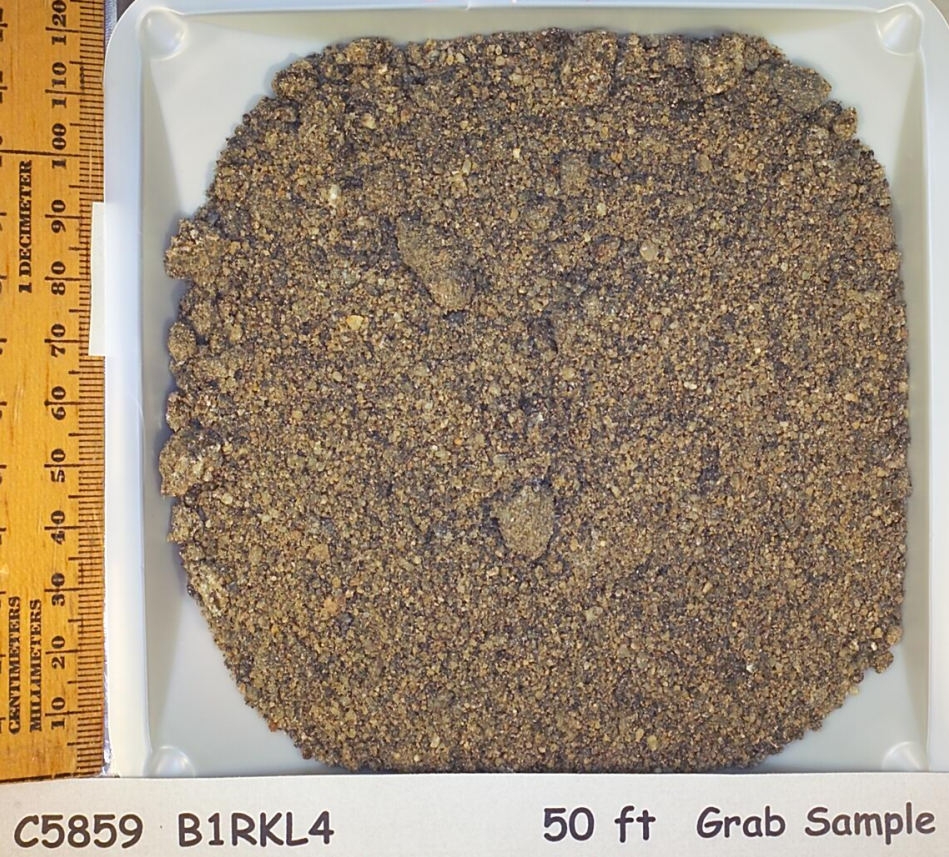

Depth from Chain-of-Custody 


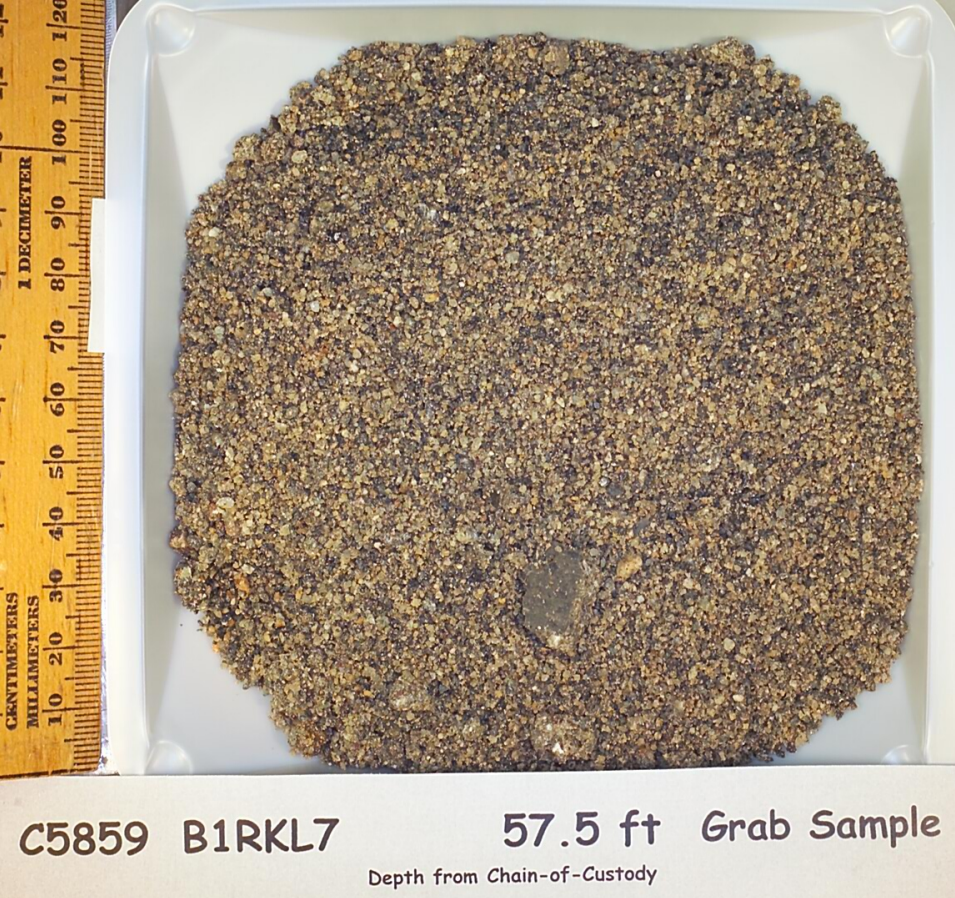




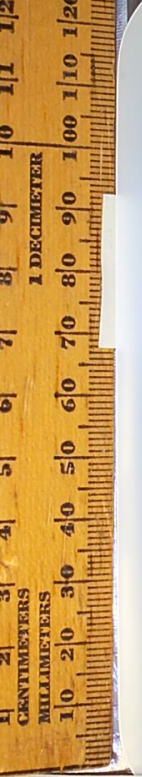

$60^{3}$

3.

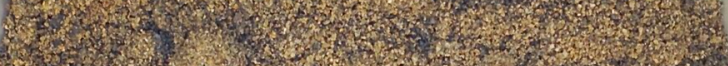

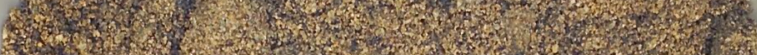

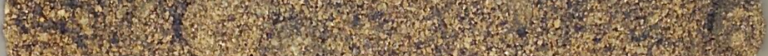

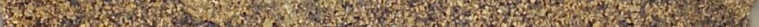

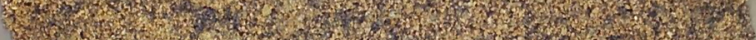

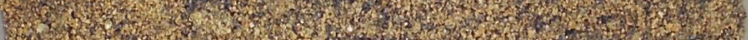

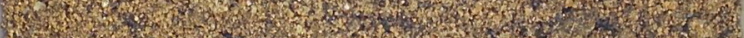

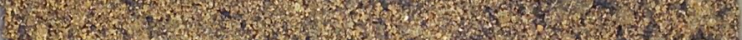

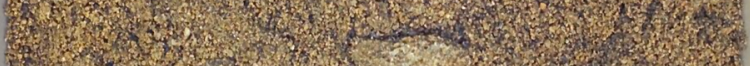
50 (5)

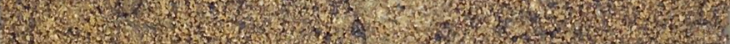
3.

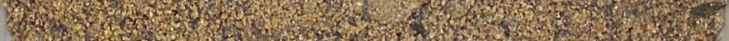

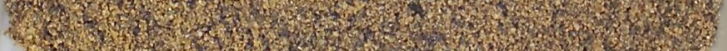

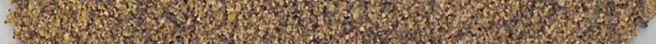
W. Ho

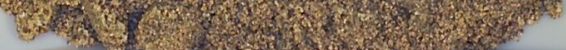

C5859 B1RKL8 $59.4 \mathrm{ft}$ Grab Sample Depth from Chain-of-Custody 


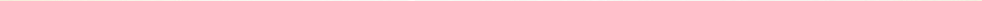




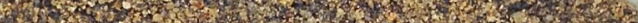

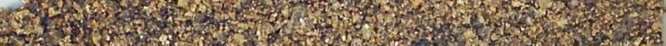

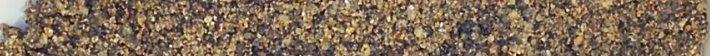

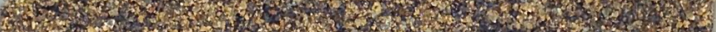

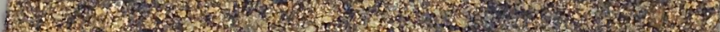

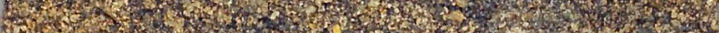

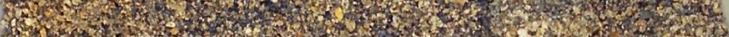

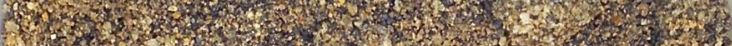

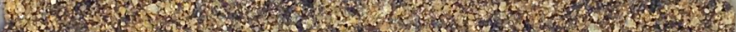

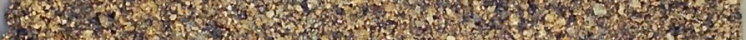

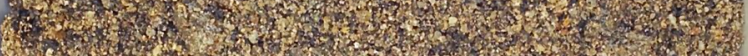

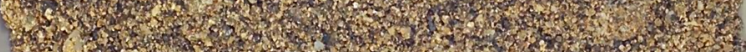

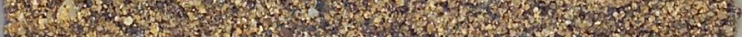

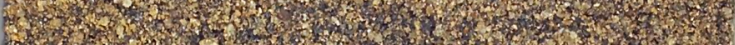
10.5.

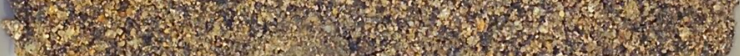

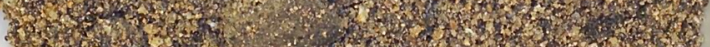

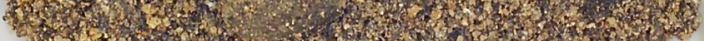

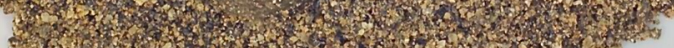

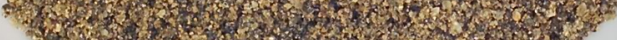

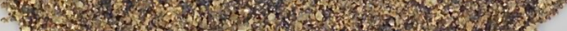




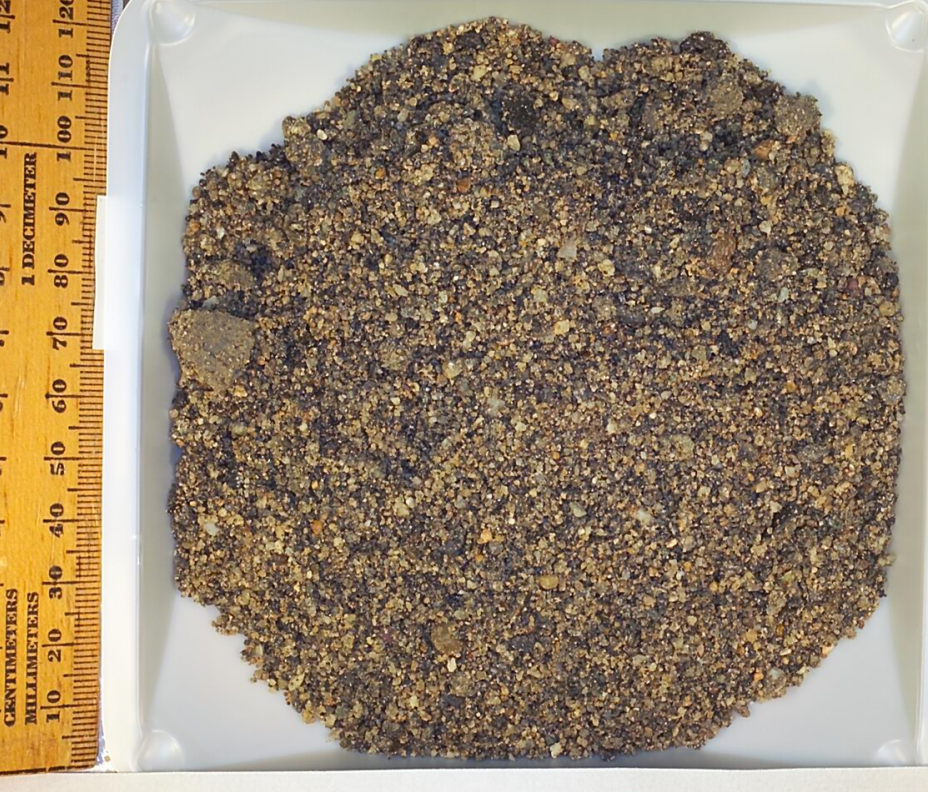

C5859 B1RKM3 $\quad 72.6 \mathrm{ft}$ Grab Sample

Depth from Chain-of-Custody 


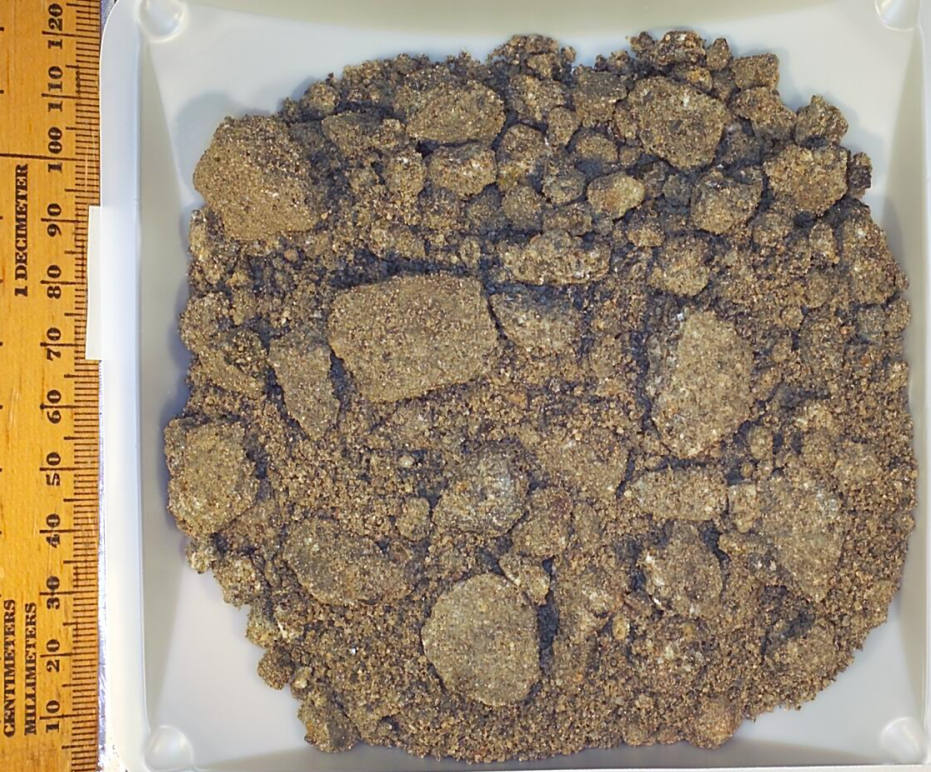

C5859 B1RKM4 $\quad 77.7 \mathrm{ft}$ Grab Sample Depth from Chain-of-Custody 


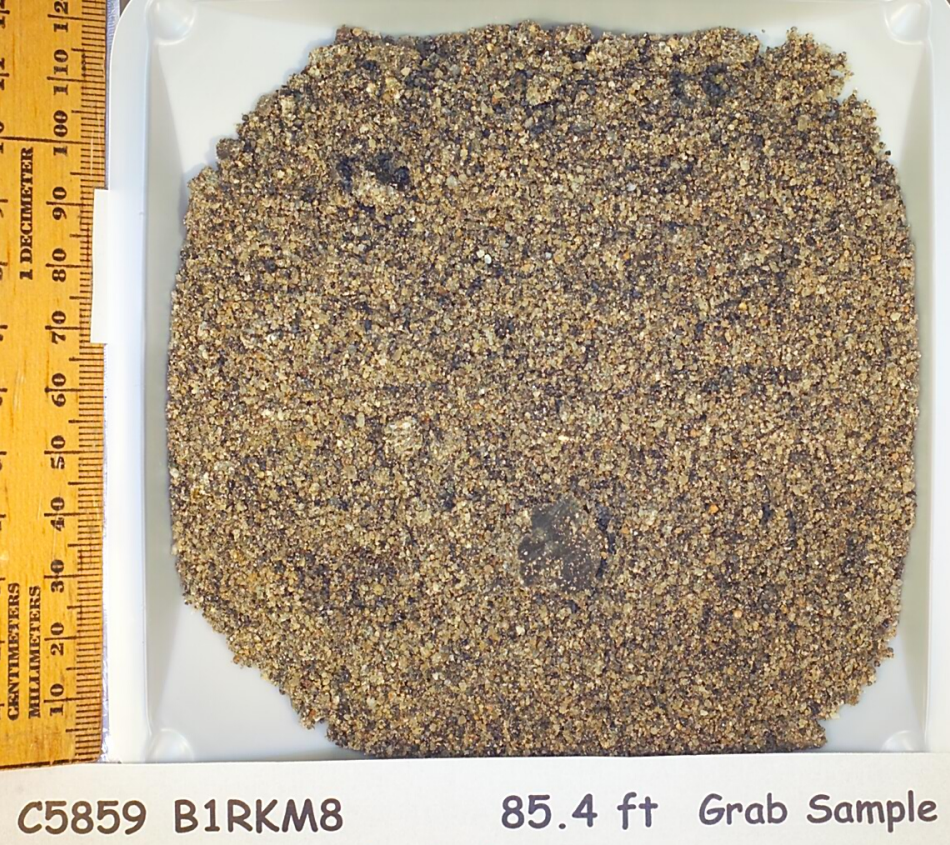

Depth from Chain-of-Custody 


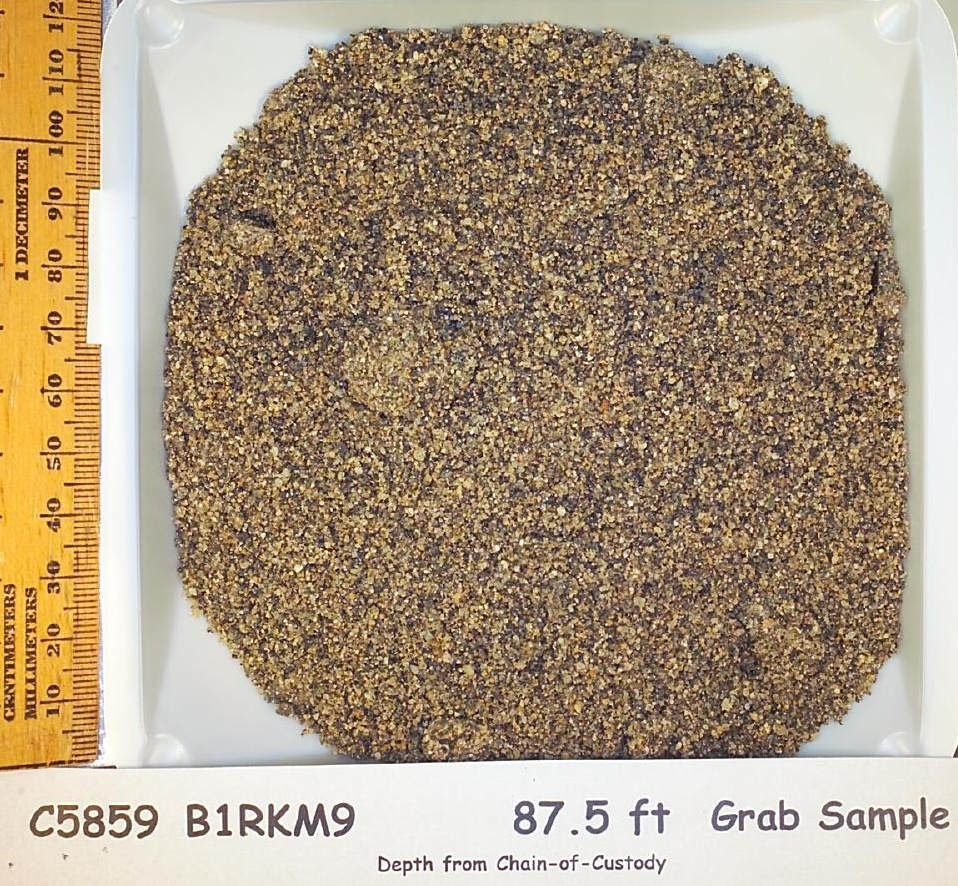




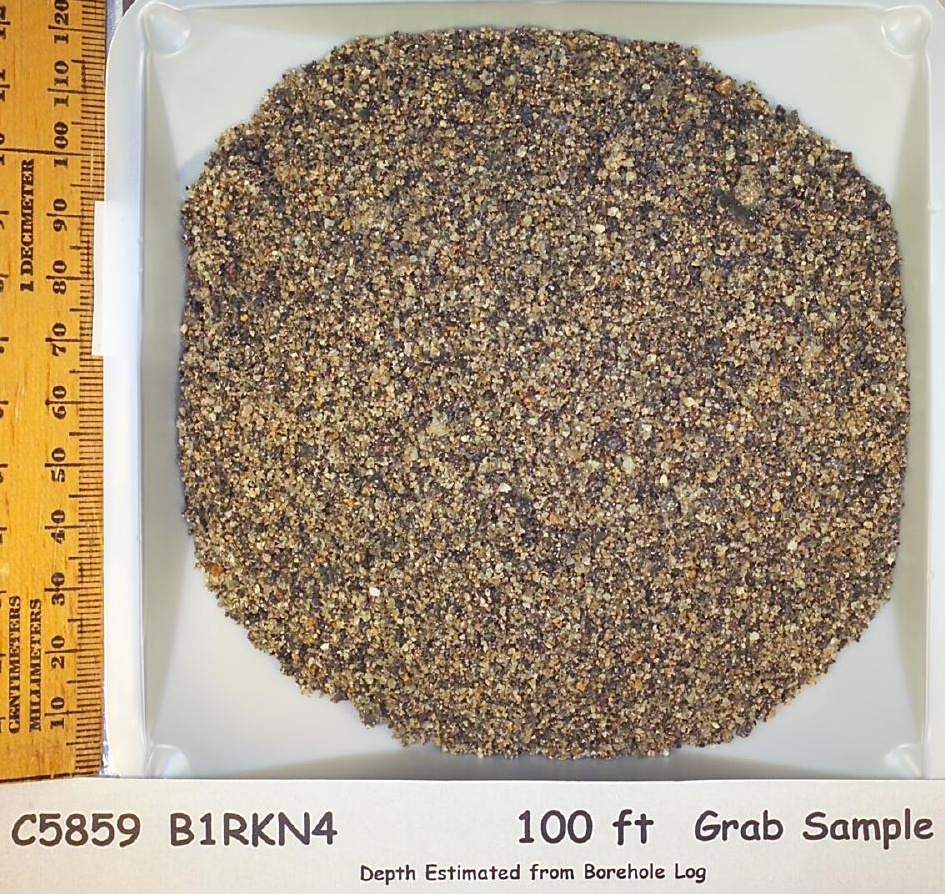




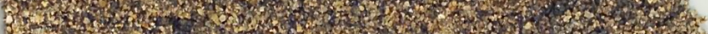

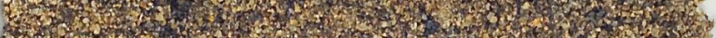

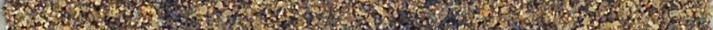

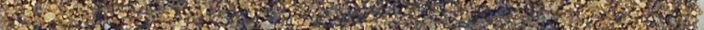

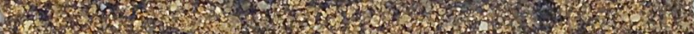

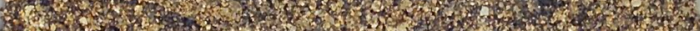

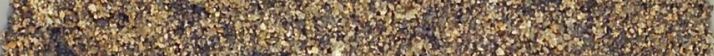

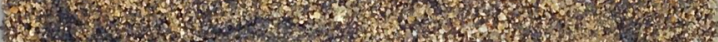

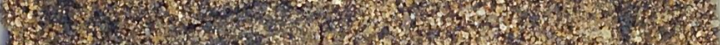

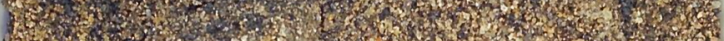

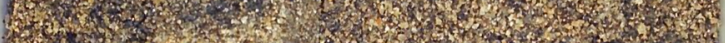

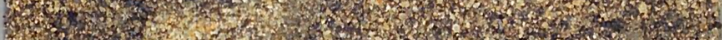

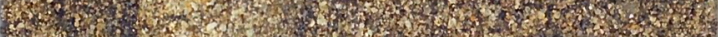

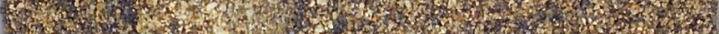

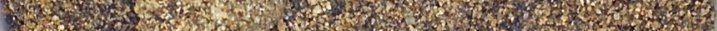

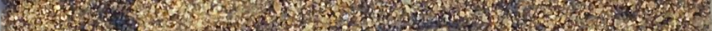

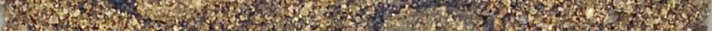

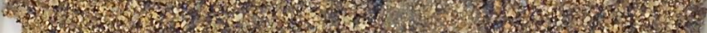
4.6.

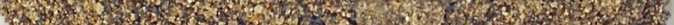
If 3 the

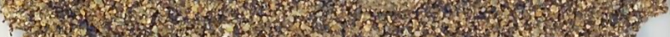

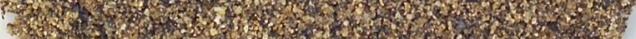

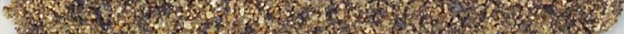
T3t

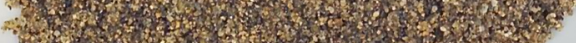

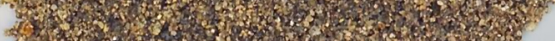

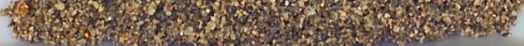

Grab Sample 


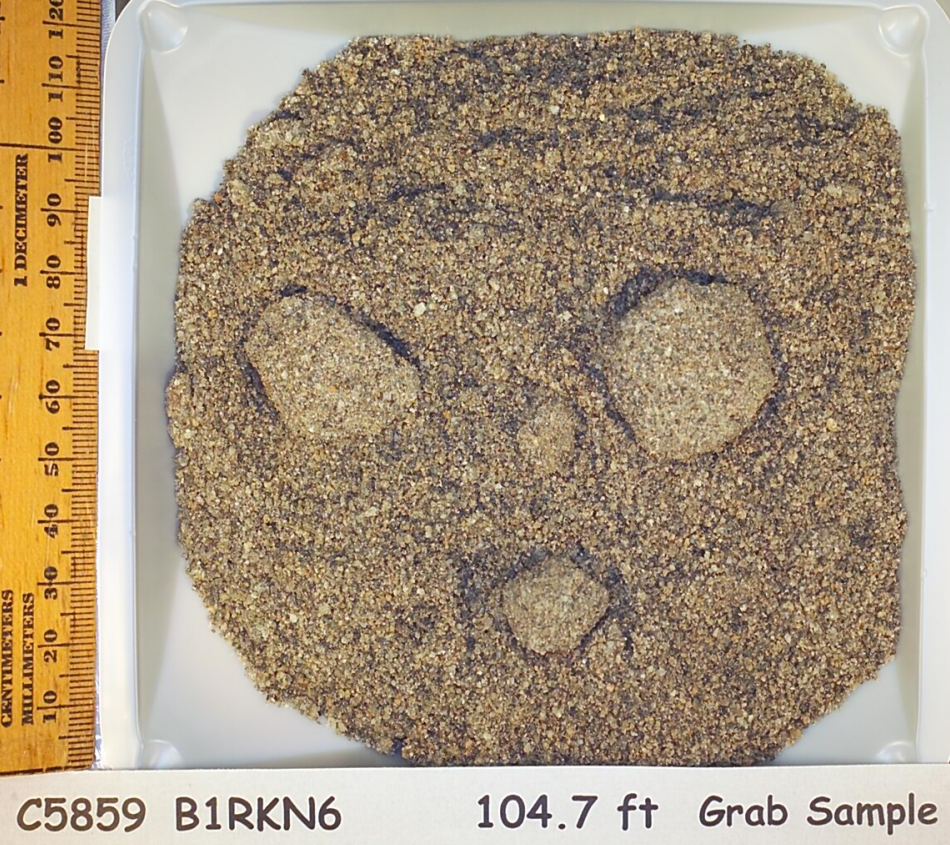

Depth from Chain-of-Custody 


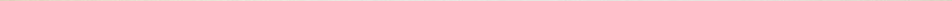




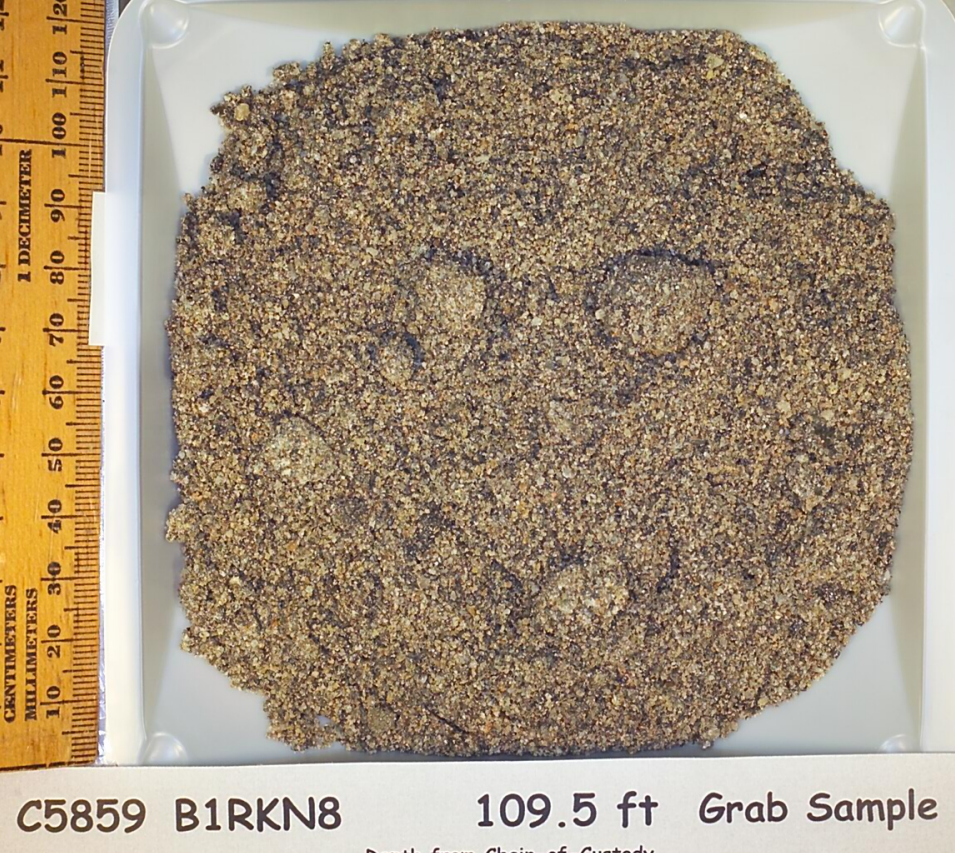

Depth from Chain-of-Custody 


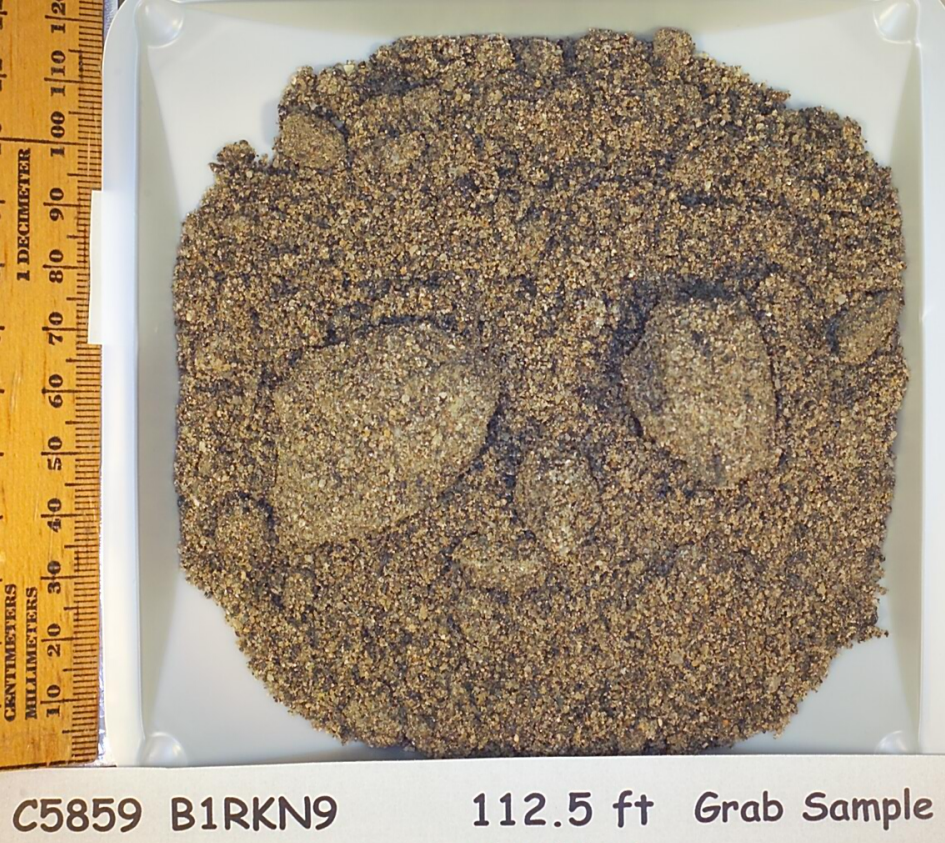

Depth from Chain-of-Custody 


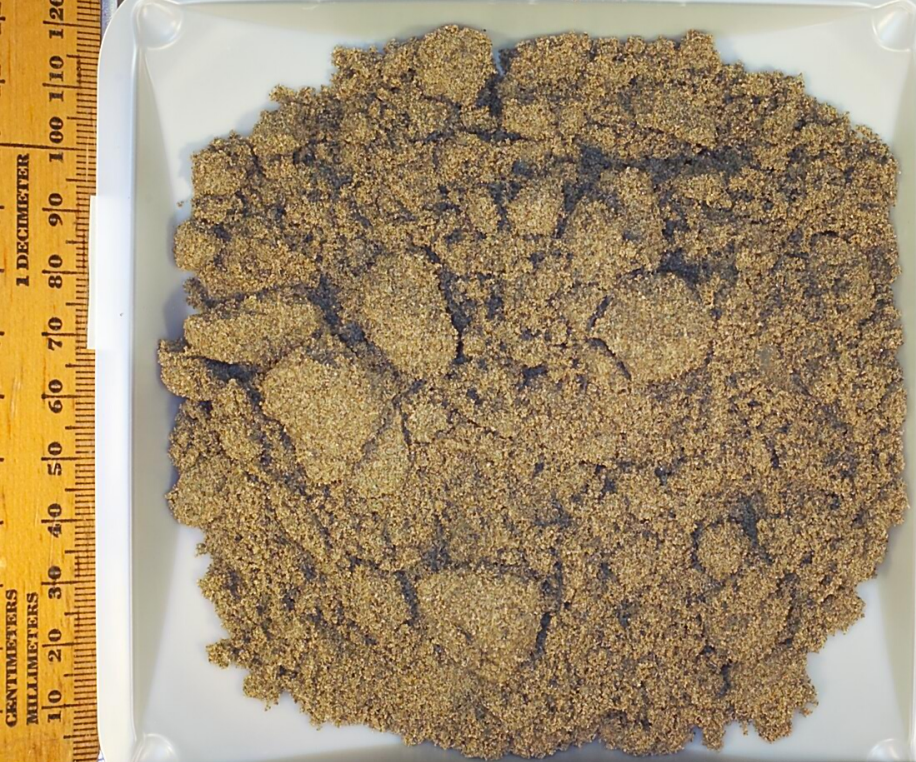

C5859 B1RKP1

$117.5 \mathrm{ft}$ Grab Sample Depth from Chain-of-Custody 


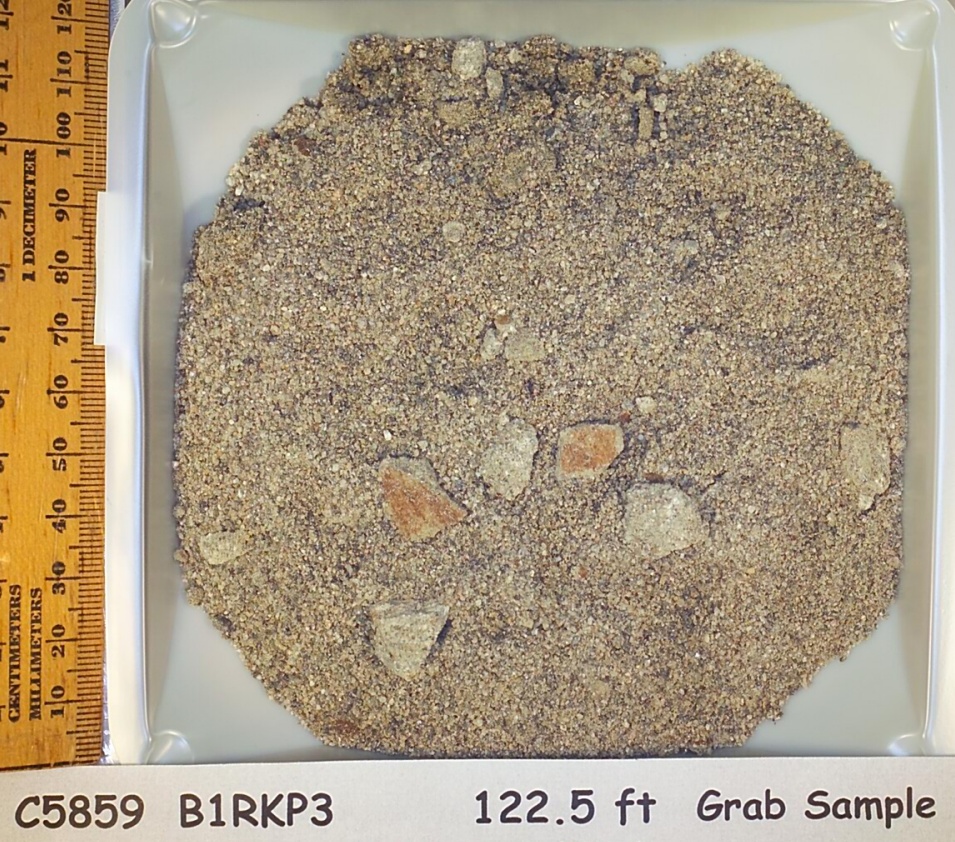

Depth from Chain-of-Custody 


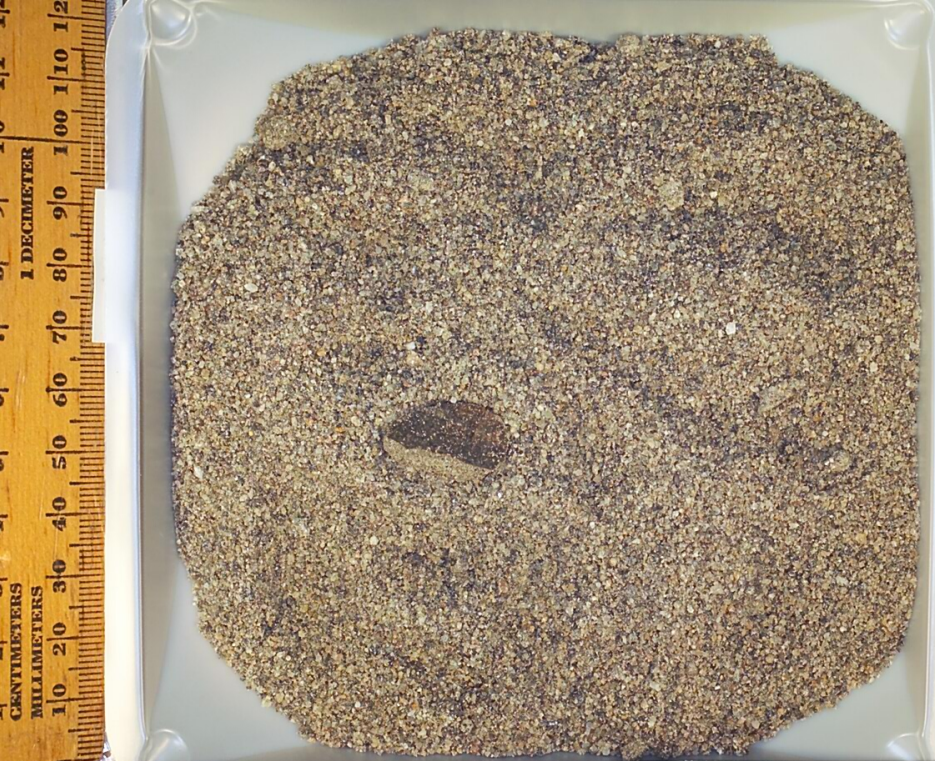

C5859 B1RKP4

$125 \mathrm{ft}$ Grab Sample

Depth from Chain-of-Custody 


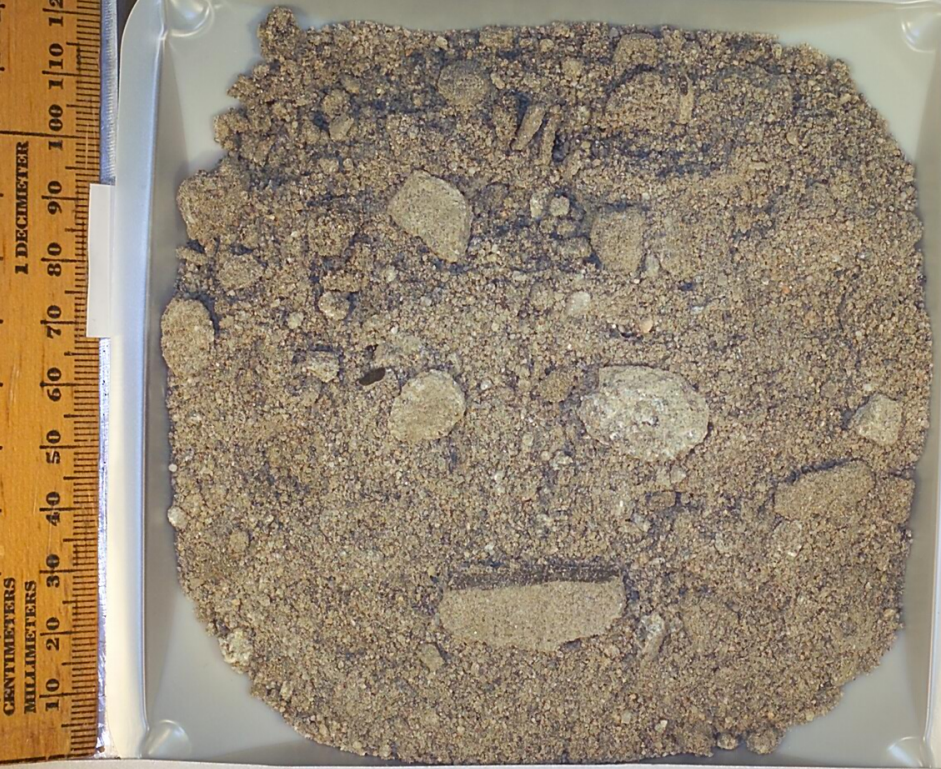

\section{C5859 B1RKP5 $\quad 127.5 \mathrm{ft}$ Grab Sample Depth from Chain-of-Custody}




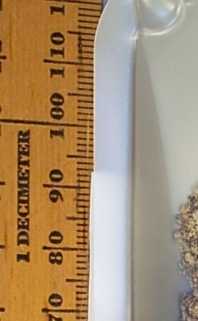

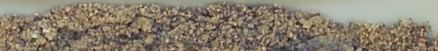

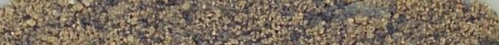
U.

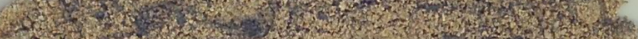
L.1. Wh

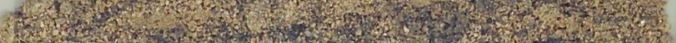

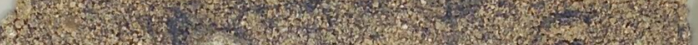
1.9.

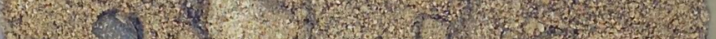

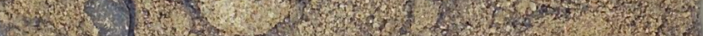

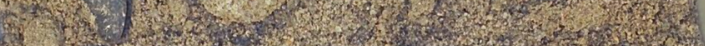

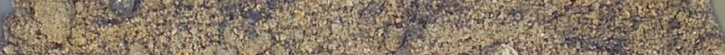

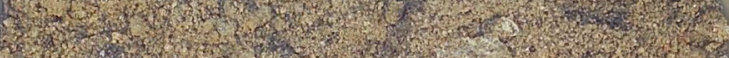

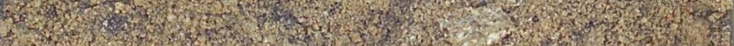

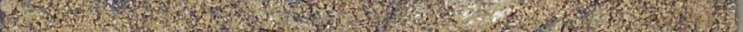
5.

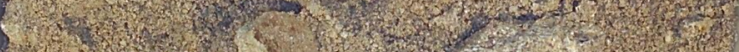

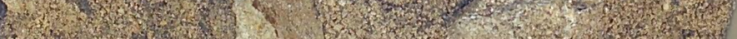
3.

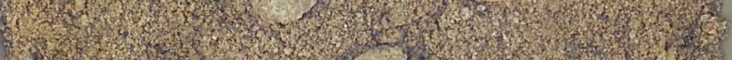

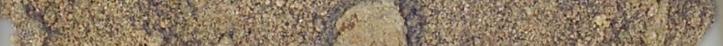

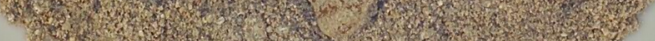

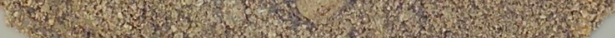

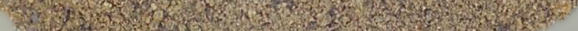

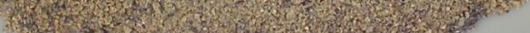
x. $x^{2}=5$. 


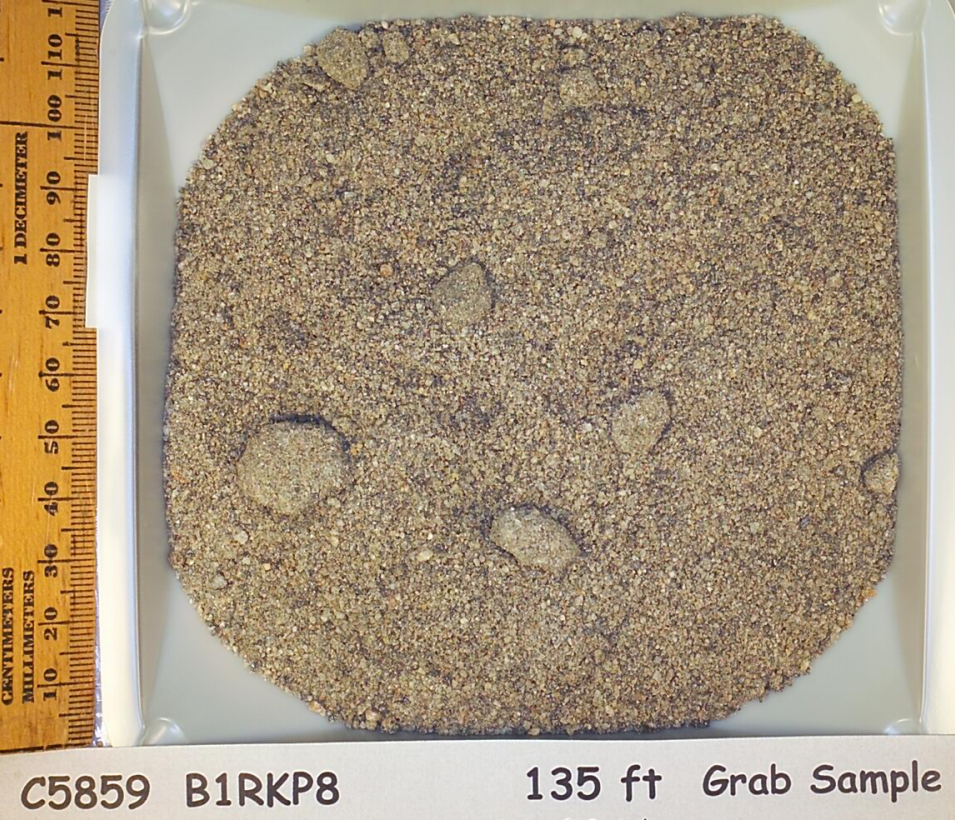

Depth from Chain-of-Custody 


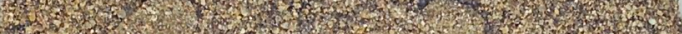

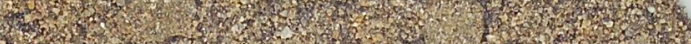

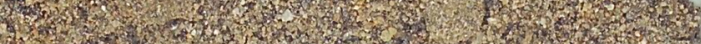

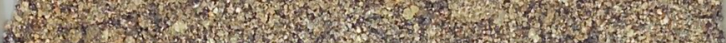

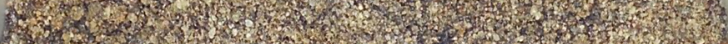

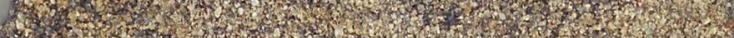

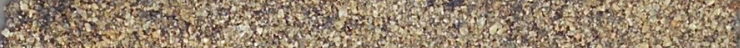

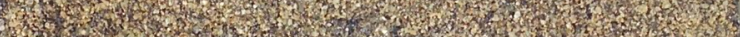

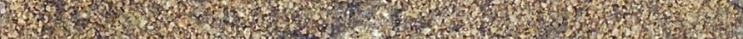
40
8

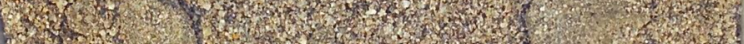

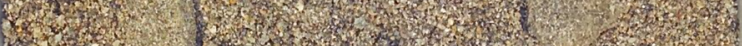

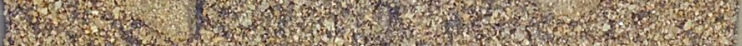
E.2.

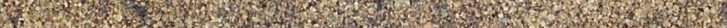

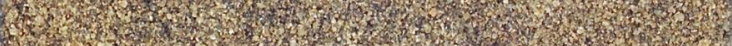

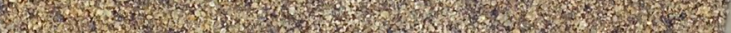

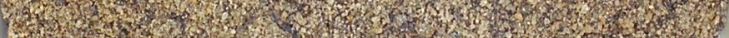
Anth

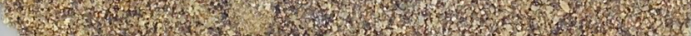

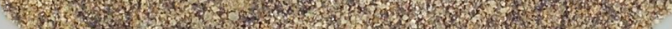

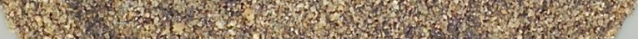

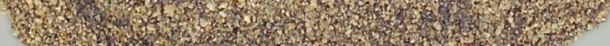

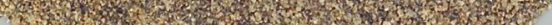
Fis 20 s.

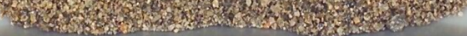

\section{C5859 B1RKP9}

\section{$137.5 \mathrm{ft}$ Grab Sample}

Depth from Chain-of-Custody 


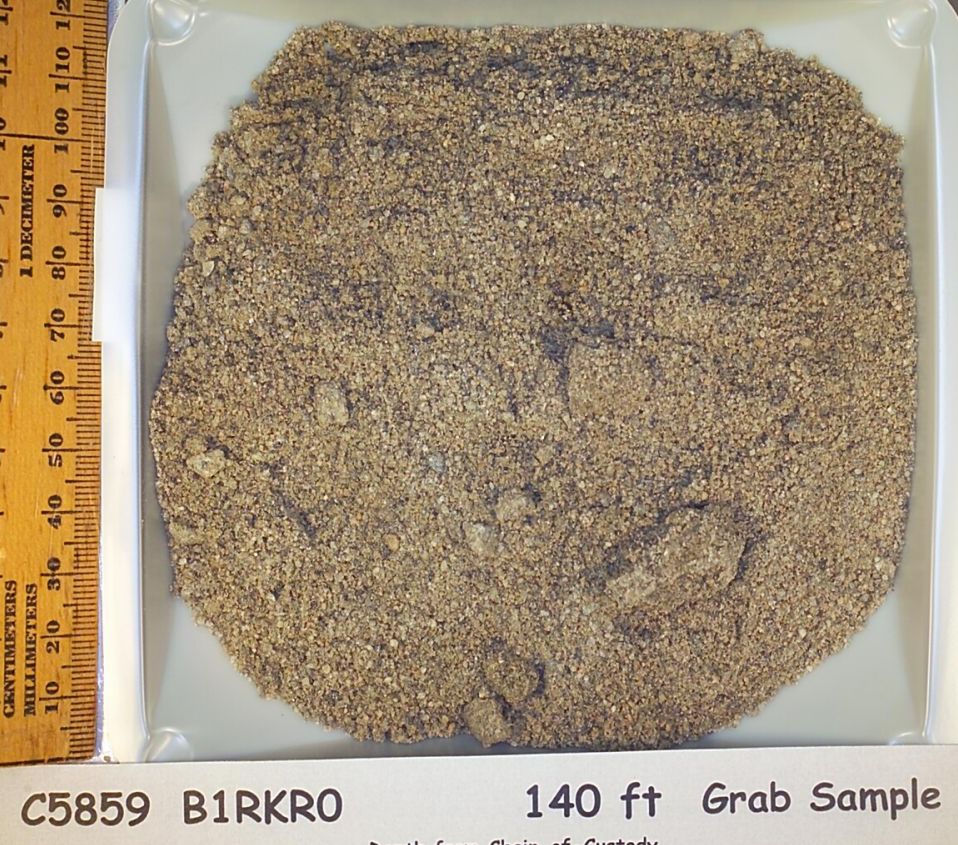

Depth from Chain-of-Custody 



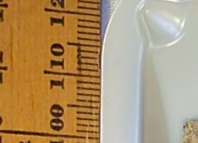

W.

ats

is.

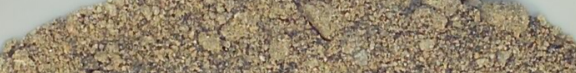

45.

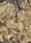

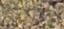

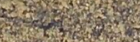

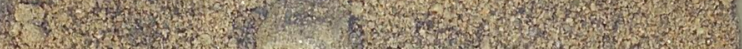
3.5. 3.5.

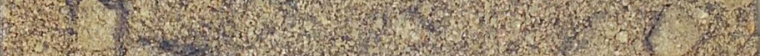

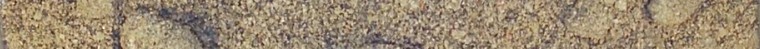

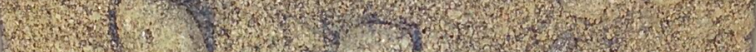

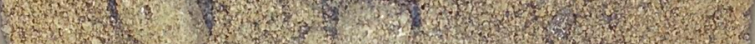

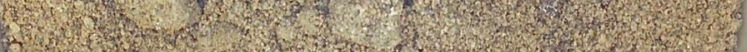

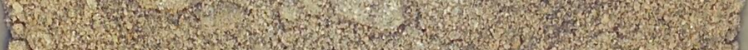

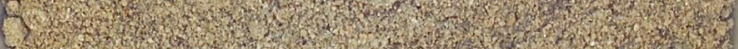

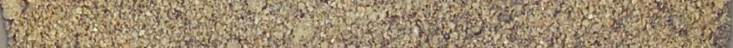

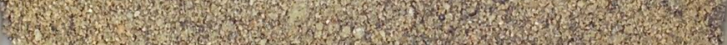

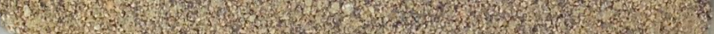

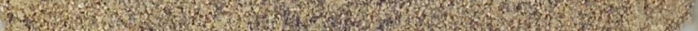

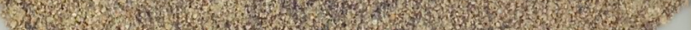

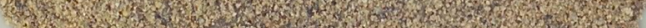

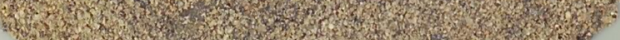
thy 


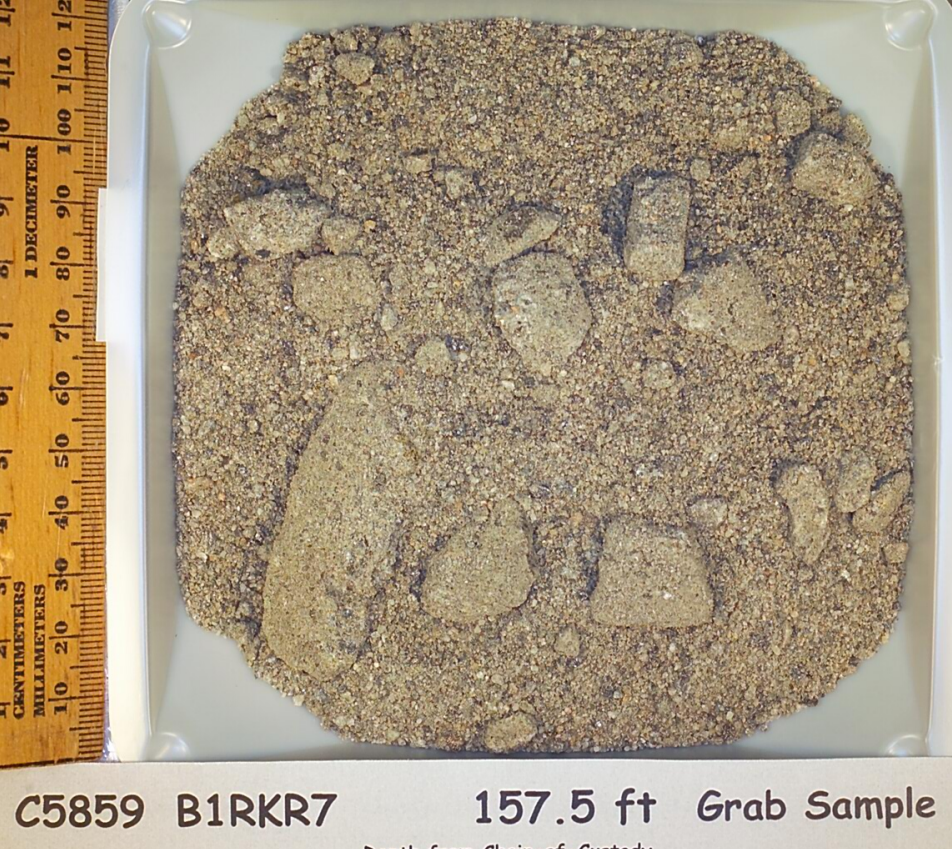

Depth from Chain-of-Custody 


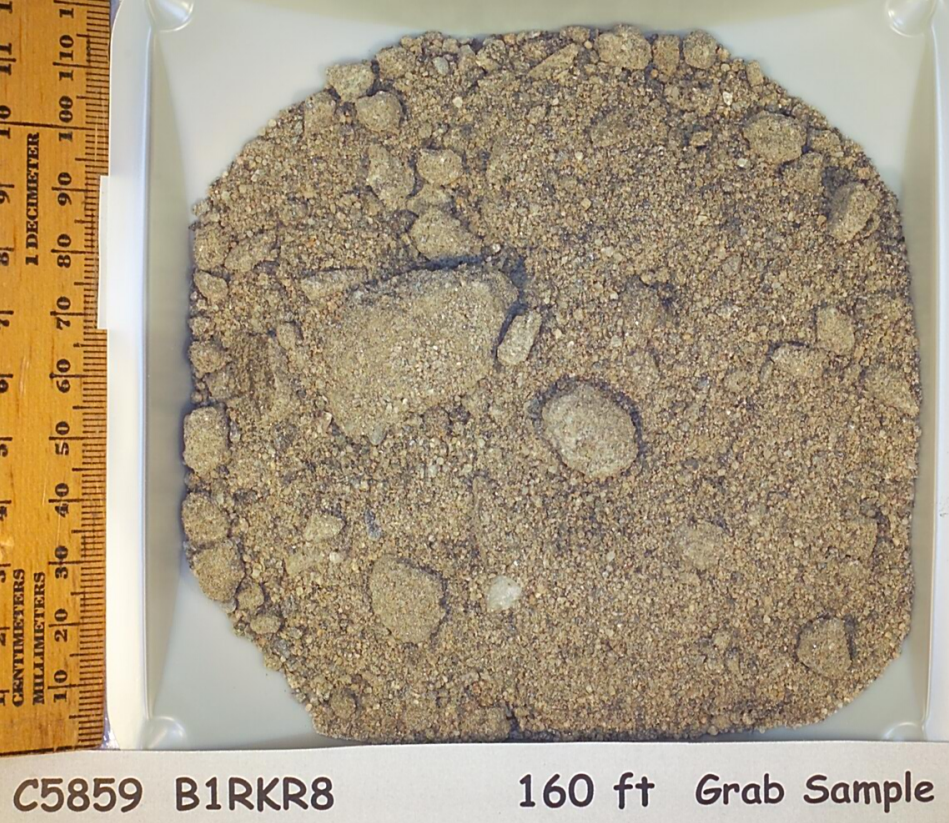

Depth from Chain-of-Custody 


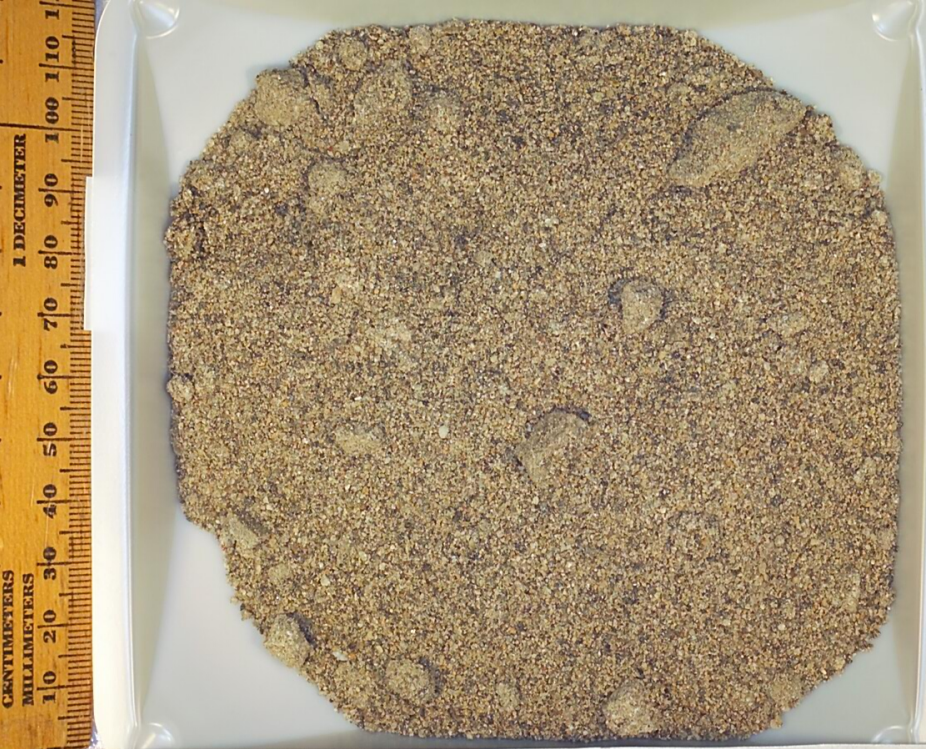

C5859 B1RKR9 $\quad 162.5 \mathrm{ft}$ Grab Sample Depth from Chain-of-Custody 



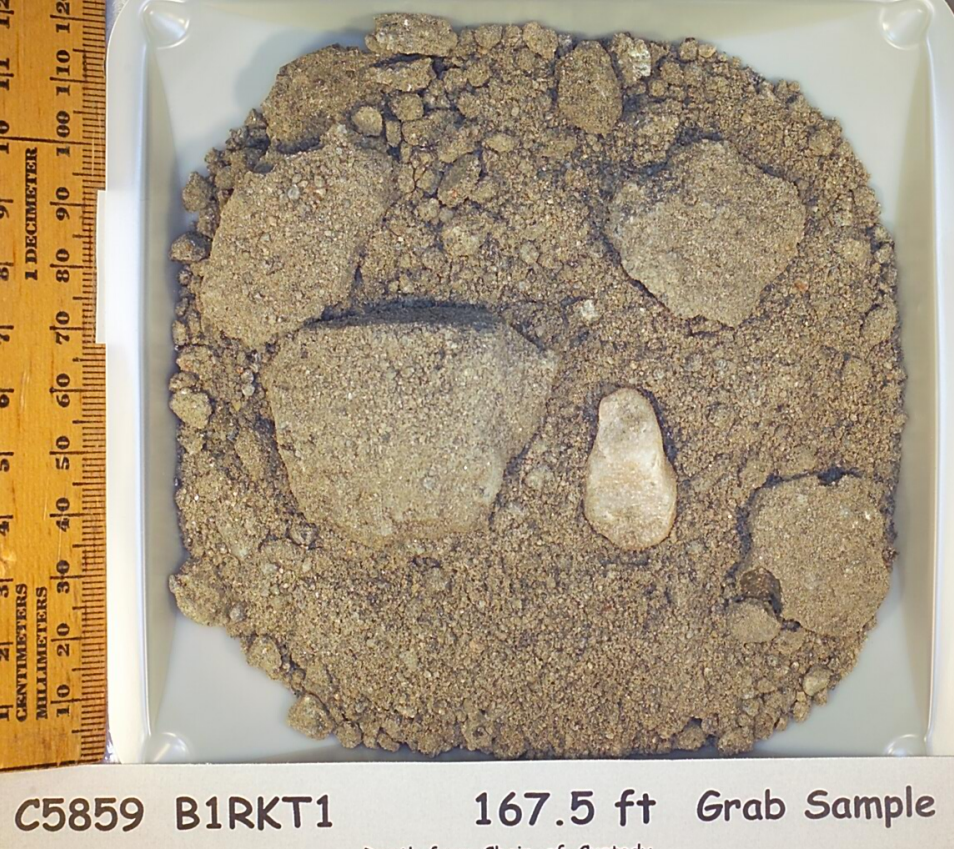

Depth from Chain-of-Custody 


\section{C5859 B1RKT2 $\quad 170 \mathrm{ft}$ Grab Sample}

Depth Estimated from Borehole Log 


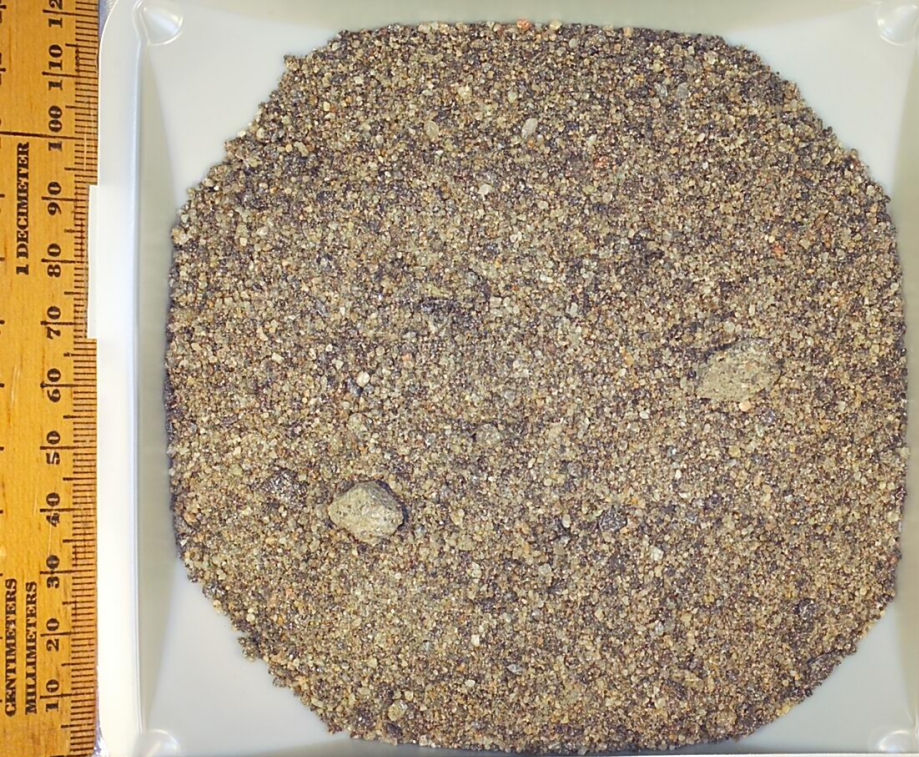

C5859 B1RKT3 $\quad 172.5 \mathrm{ft}$ Grab Sample Depth from Chain-of-Custody 


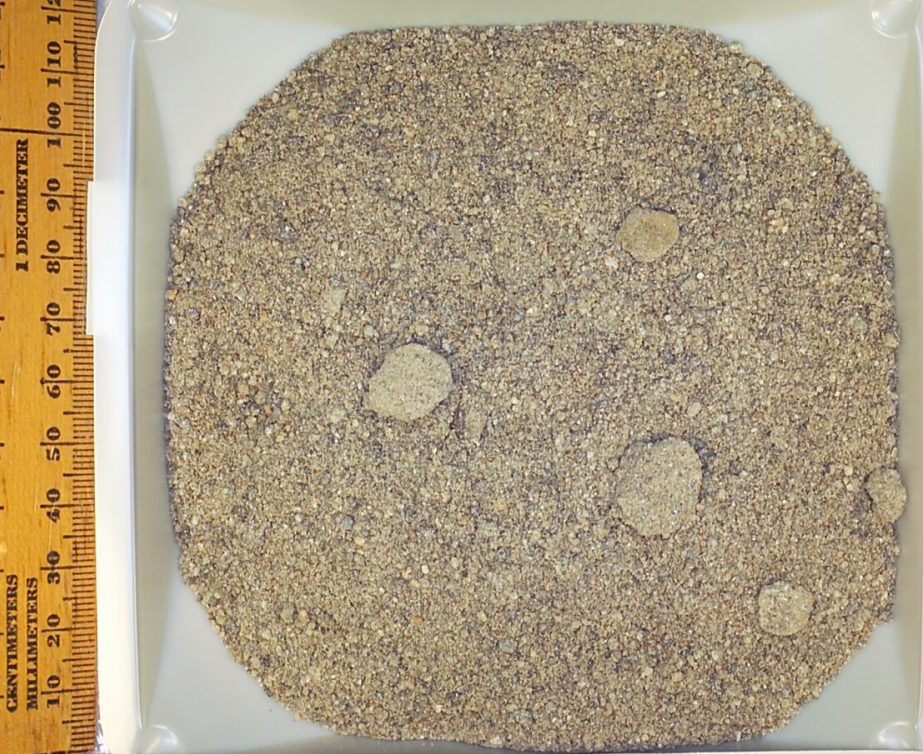

C5859 B1RKT5 $\quad 177.5 \mathrm{ft}$ Grab Sample Depth from Chain-of-Custody 


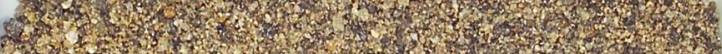

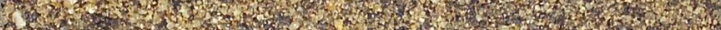

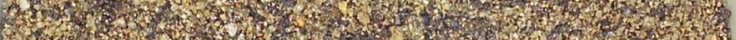

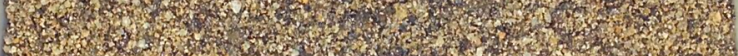

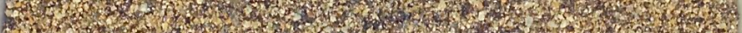

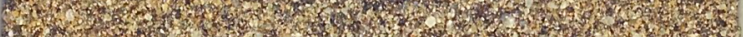

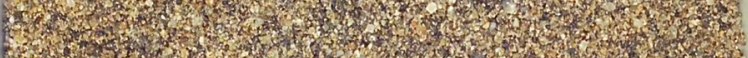

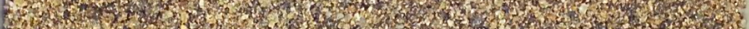

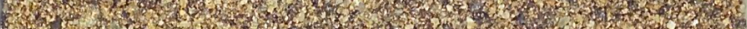

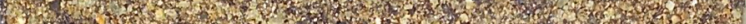

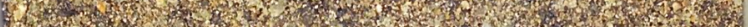

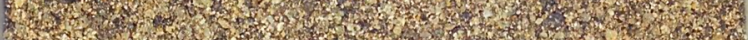

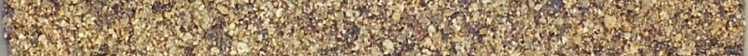
3 3.

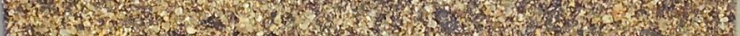

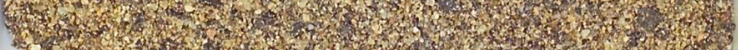

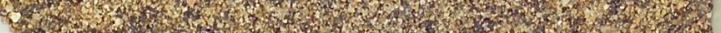

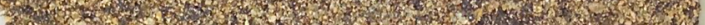

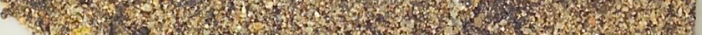

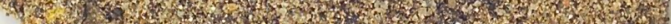

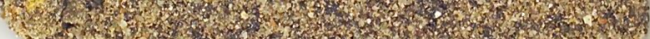

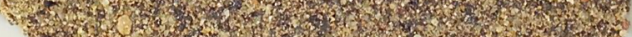

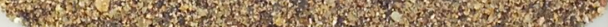
7.

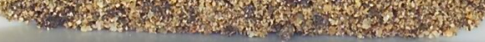

B1RKT7 $\quad 182.5 \mathrm{ft}$ Grab Sample Depth from Chain-of-Custody 


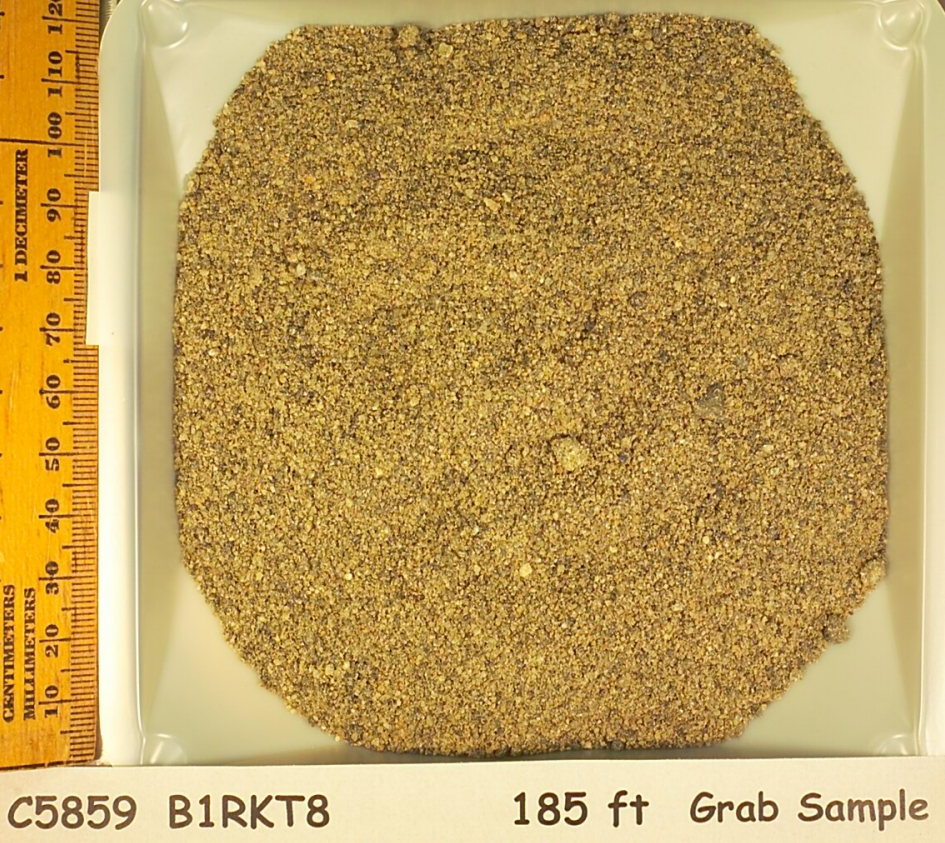

Depth from Chain-of-Custody 


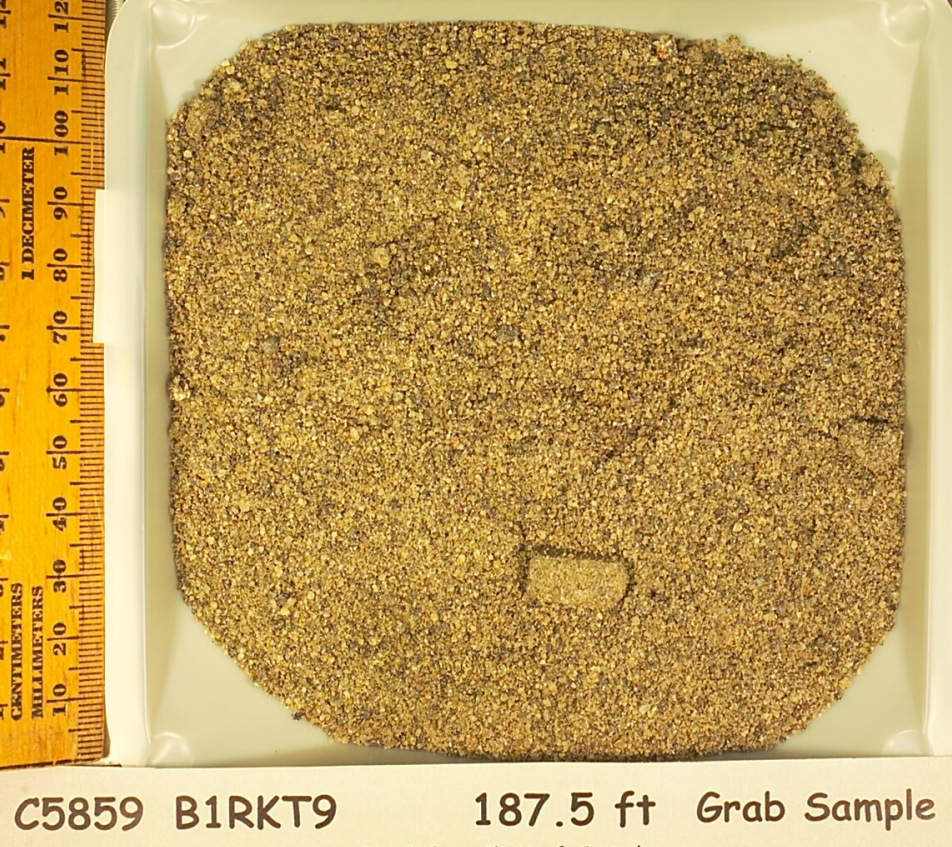

Depth from Chain-of-Custody 


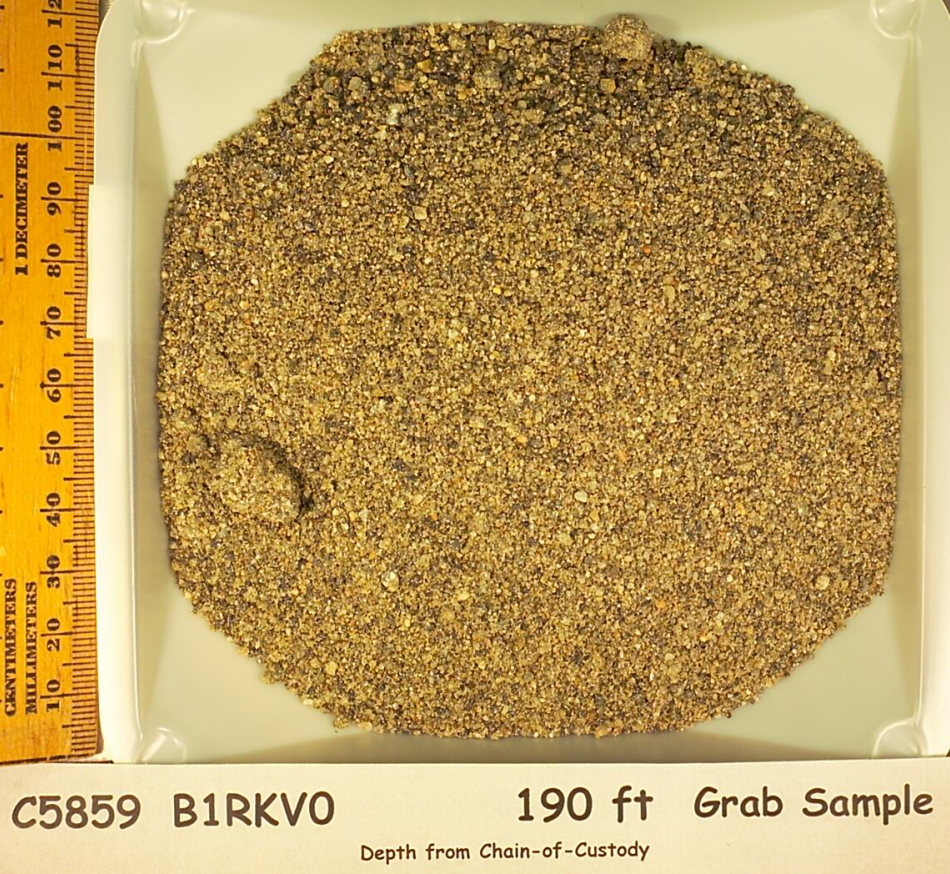




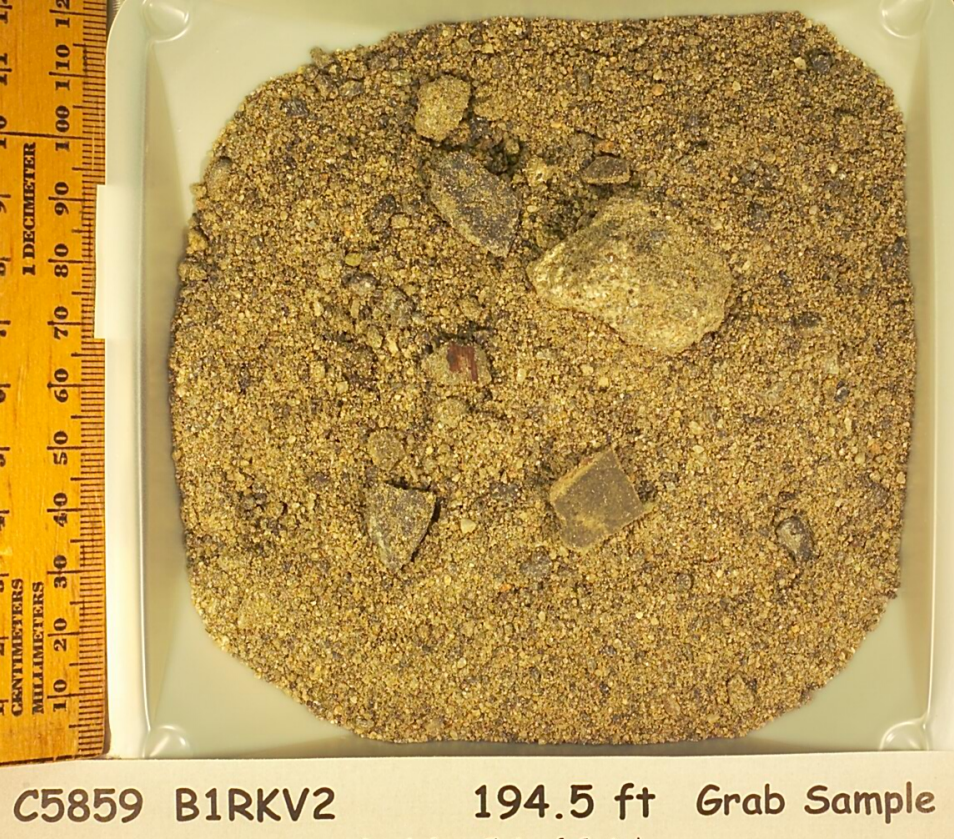

Depth from Chain-of-Custody 


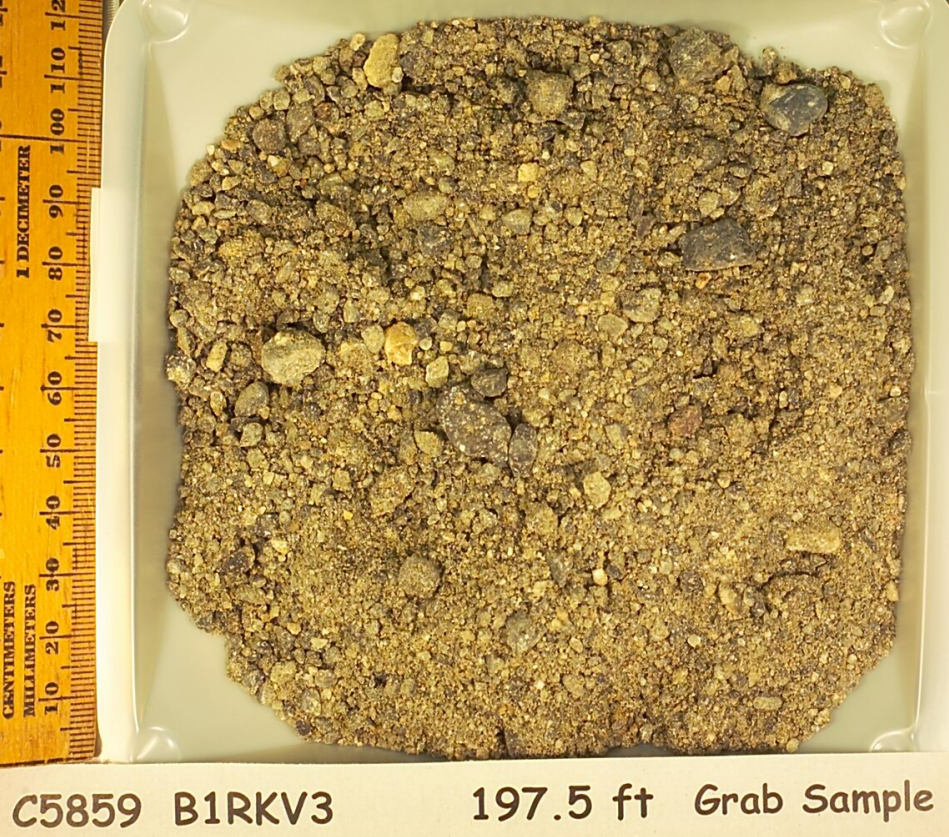

Depth from Chain-of-Custody 


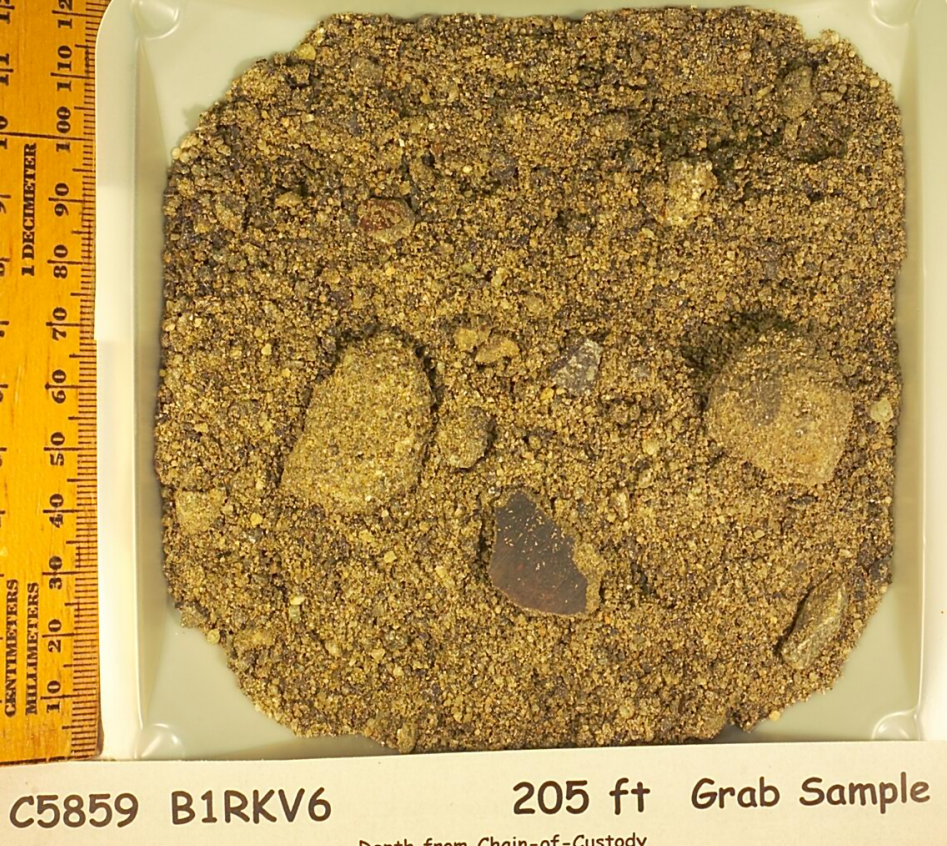

Depth from Chain-of-Custody 
3.

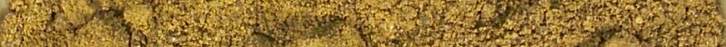

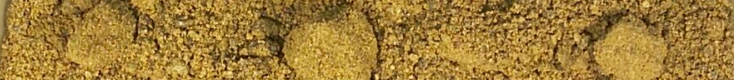

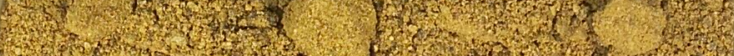
3.

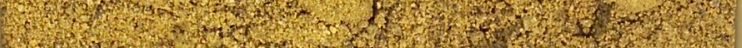
T.7.

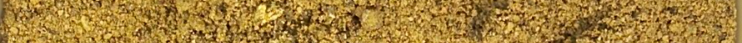

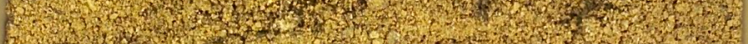

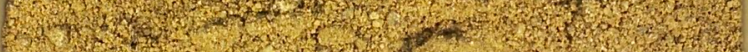
H. Q.

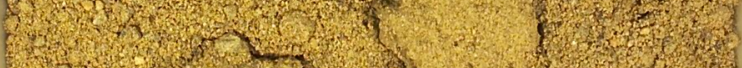

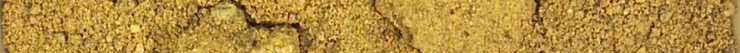

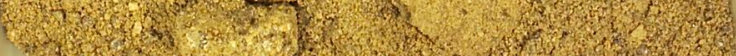

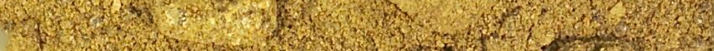
3)

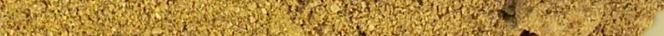

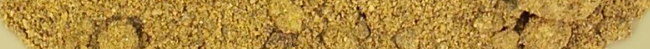

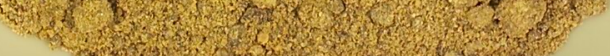




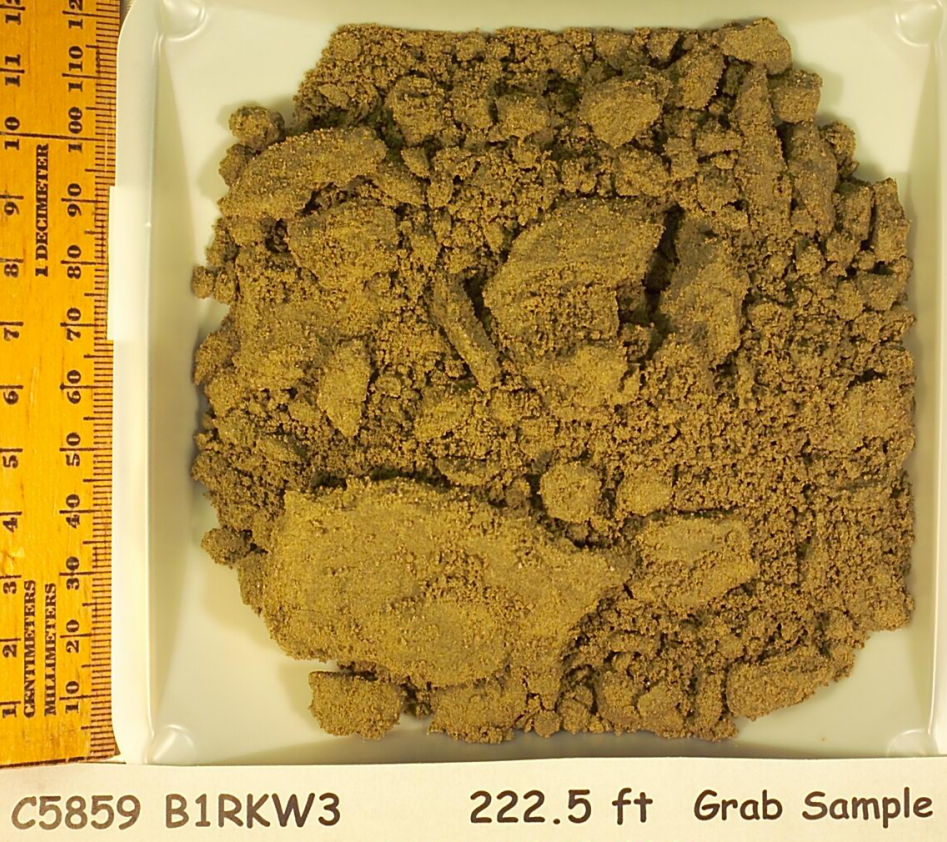

Depth Estimated from Borehole Log 


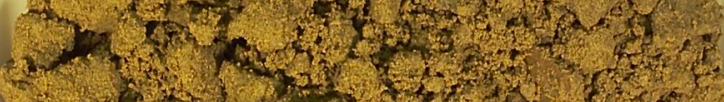

a

- 을

官

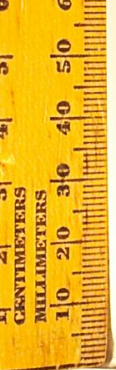

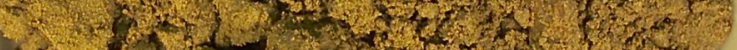

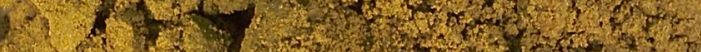

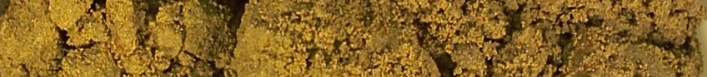

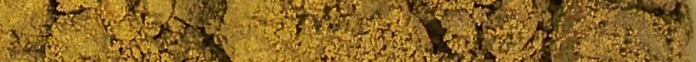

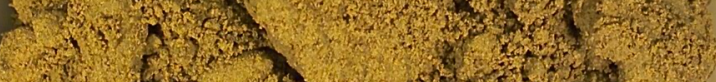

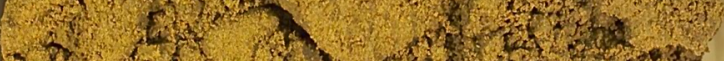

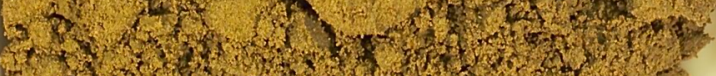

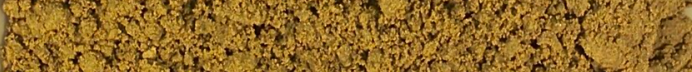

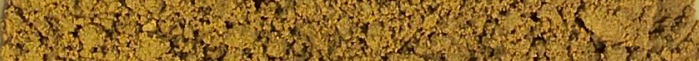

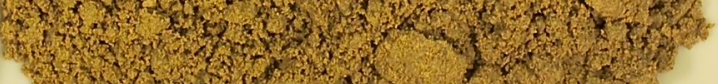




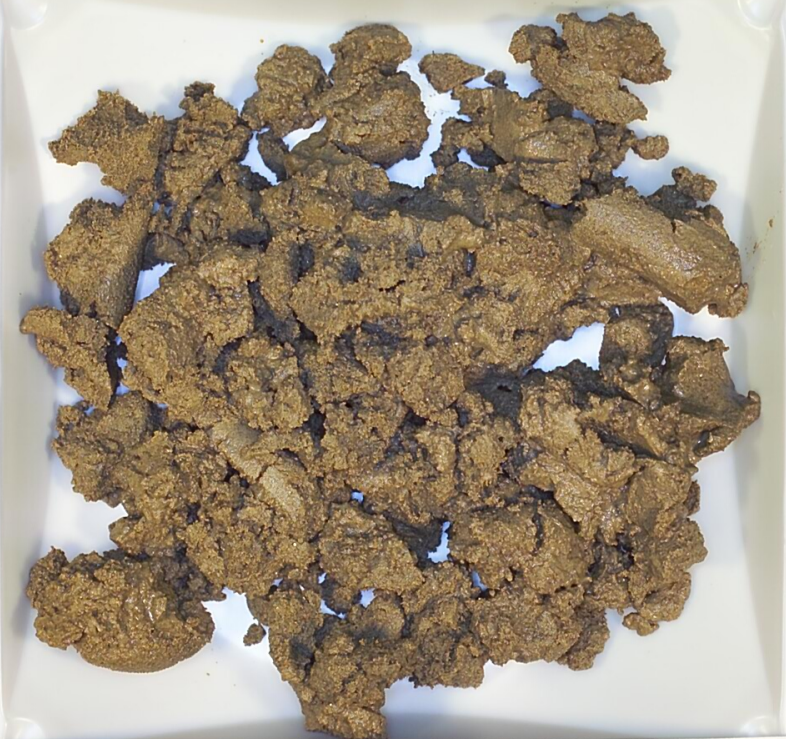




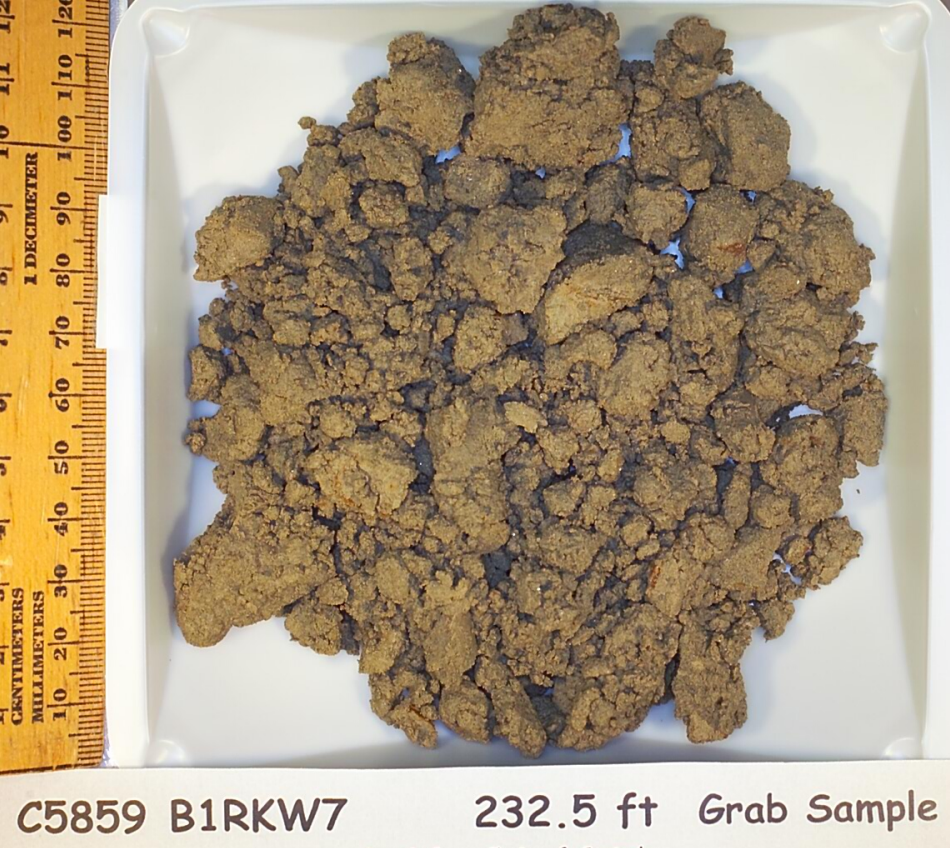

Depth from Chain-of-Custody 


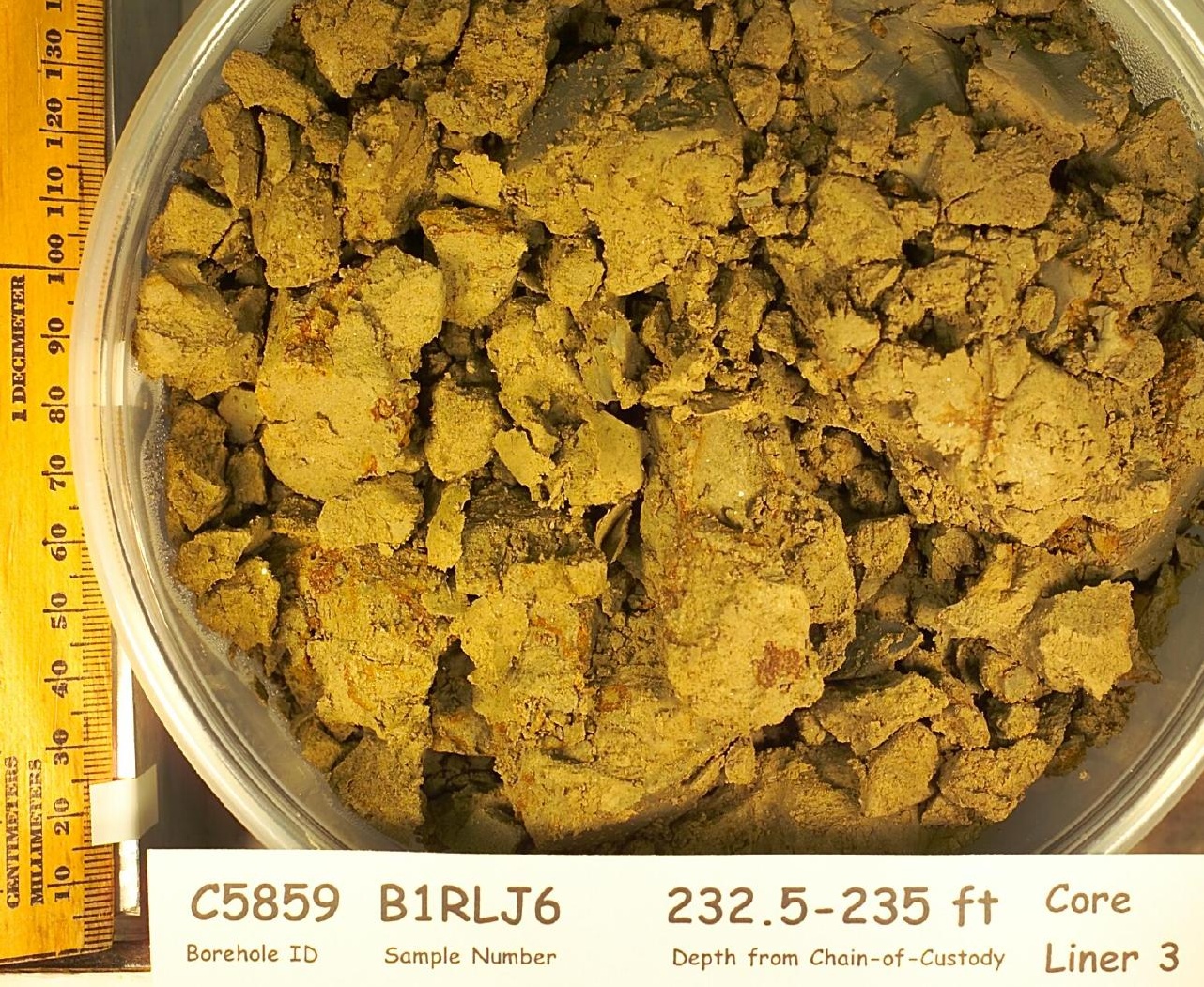




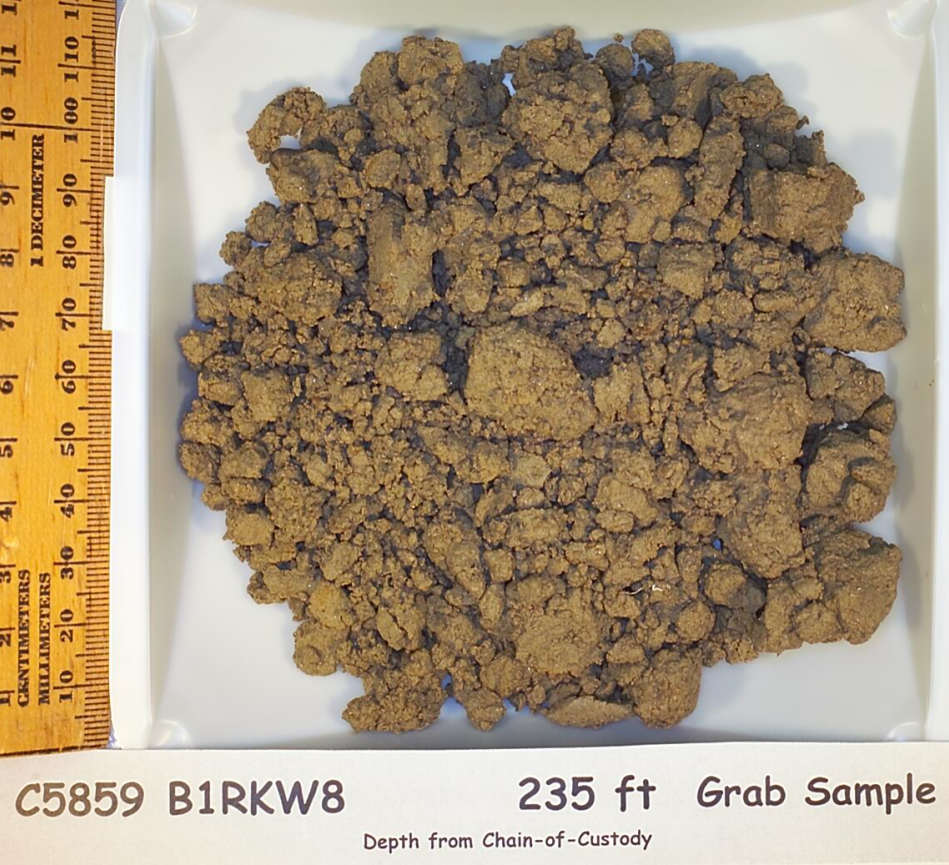




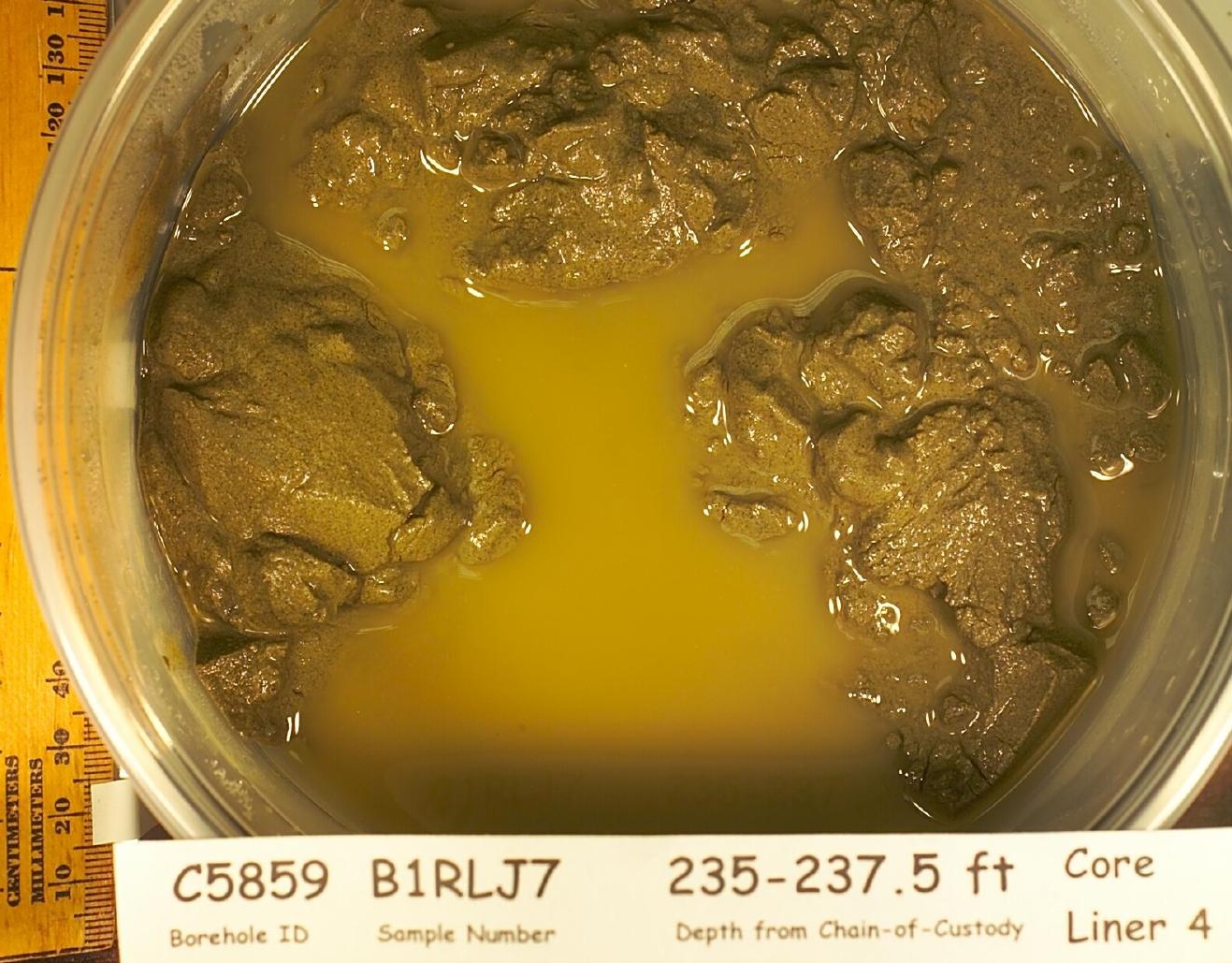




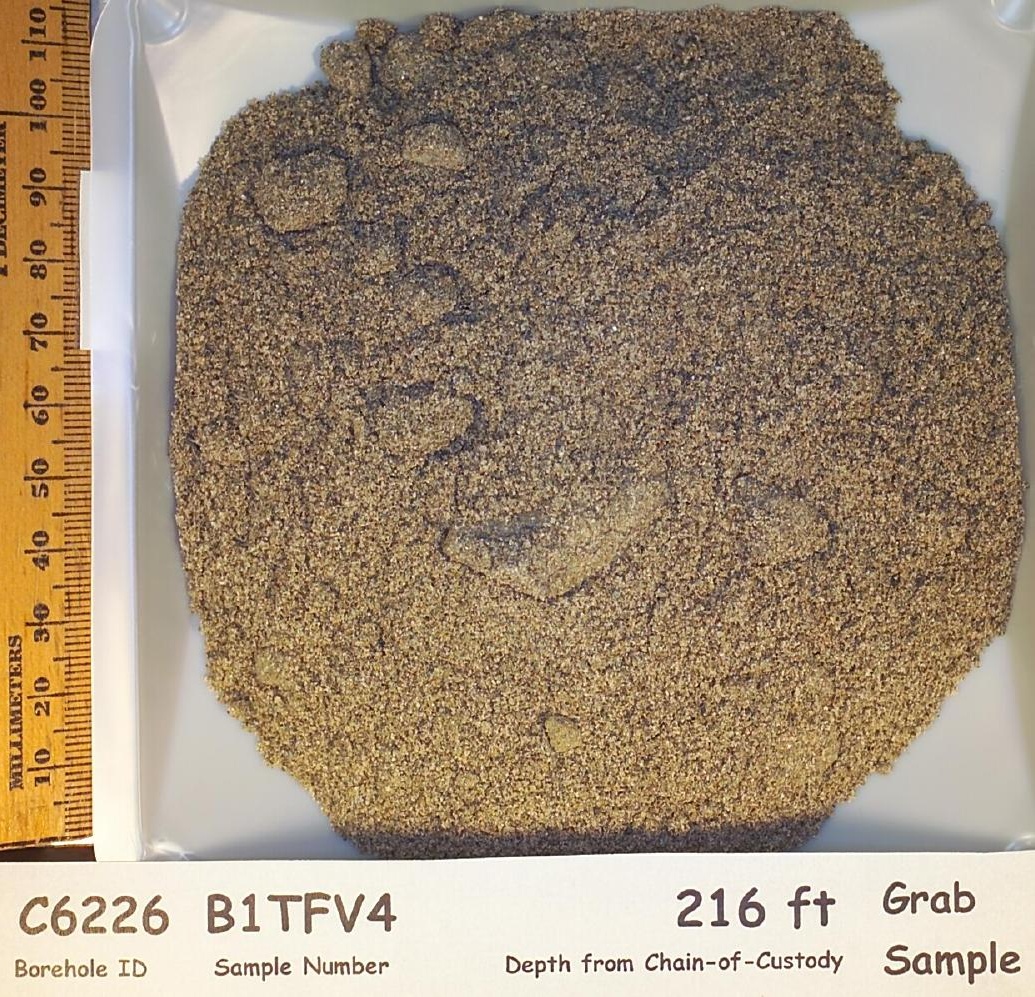




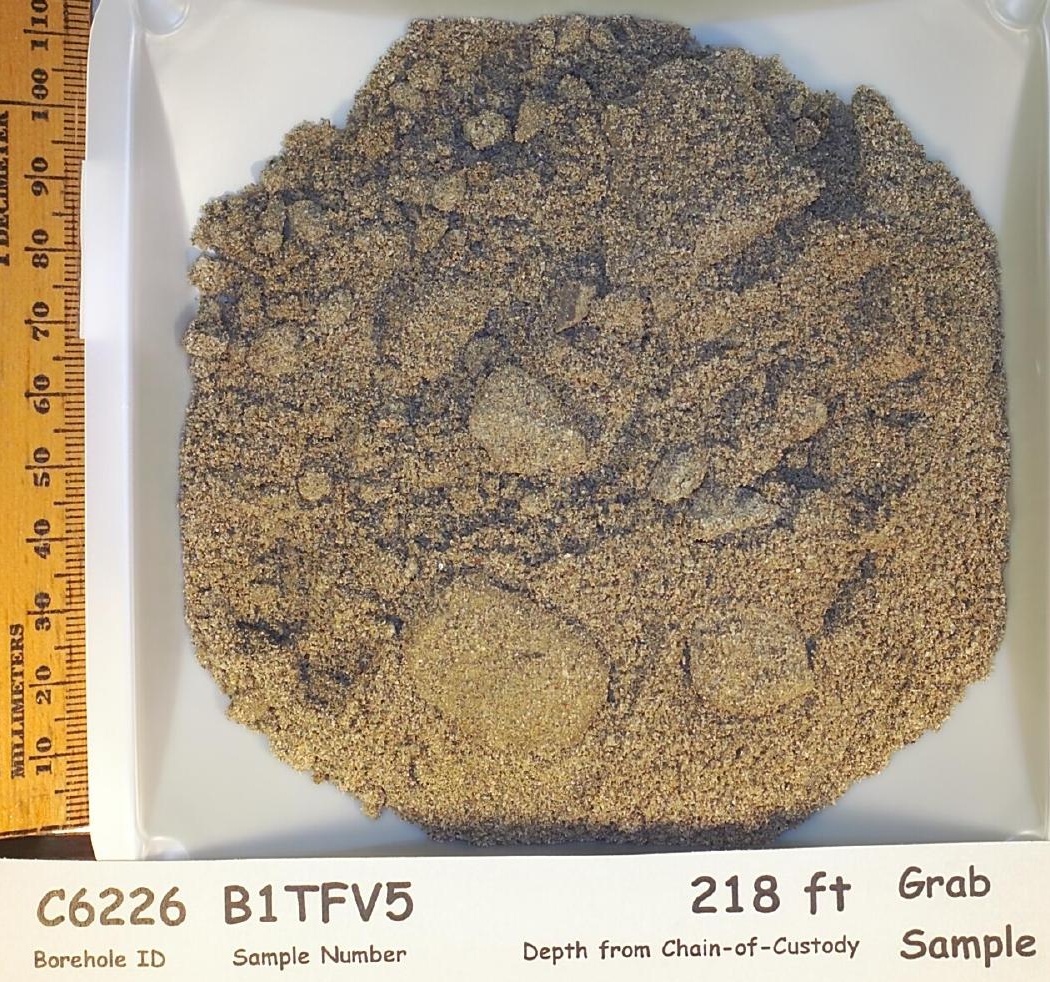




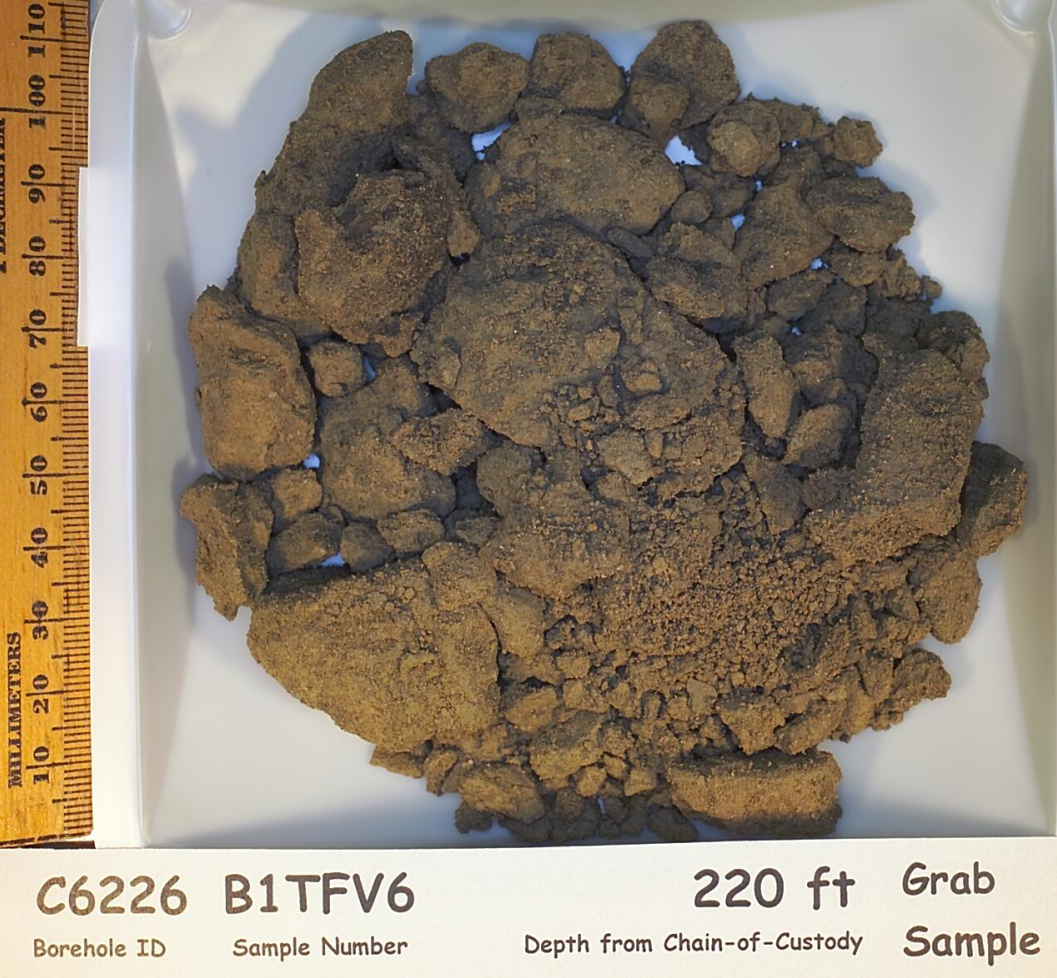




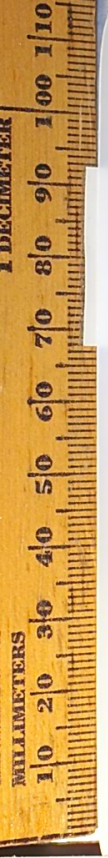

\section{C6226}

Borehole ID
Sample Number
$223 \mathrm{ft}$

Depth from Chain-of-Custody
Grab Sample 


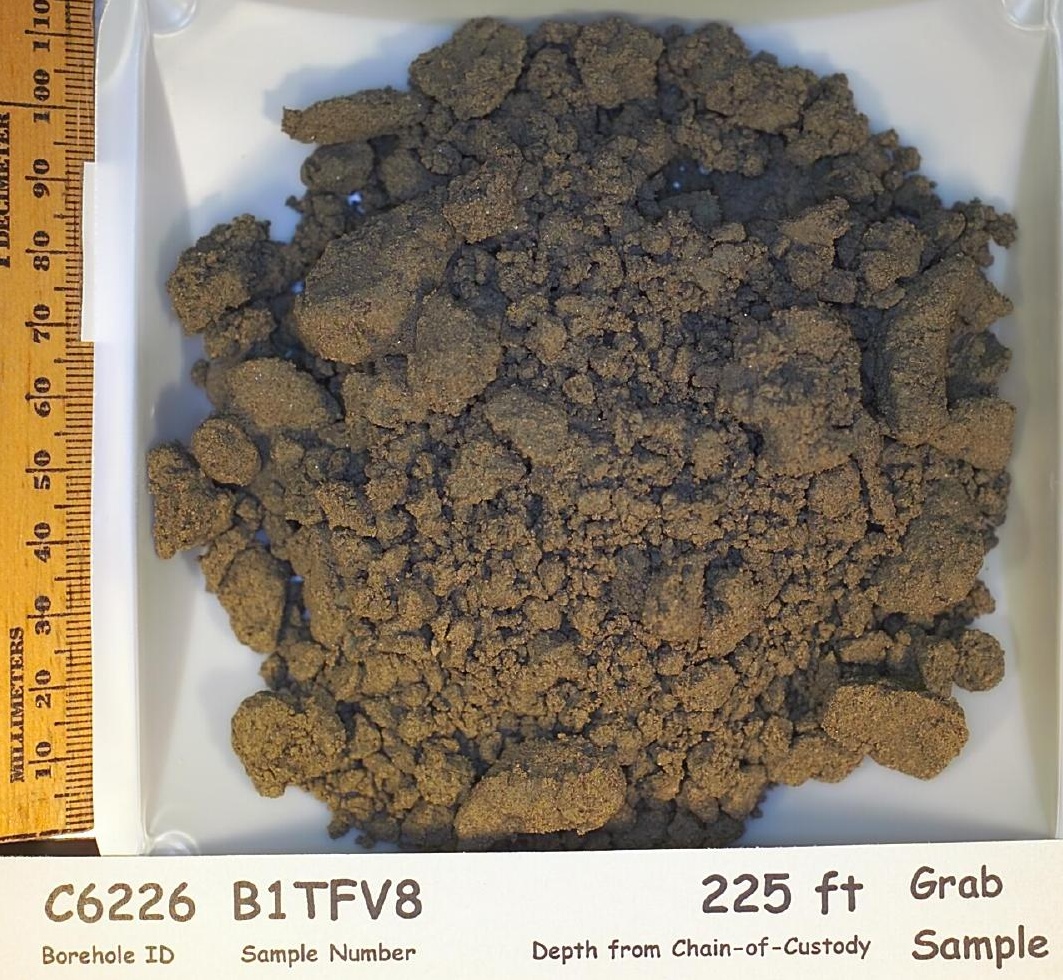




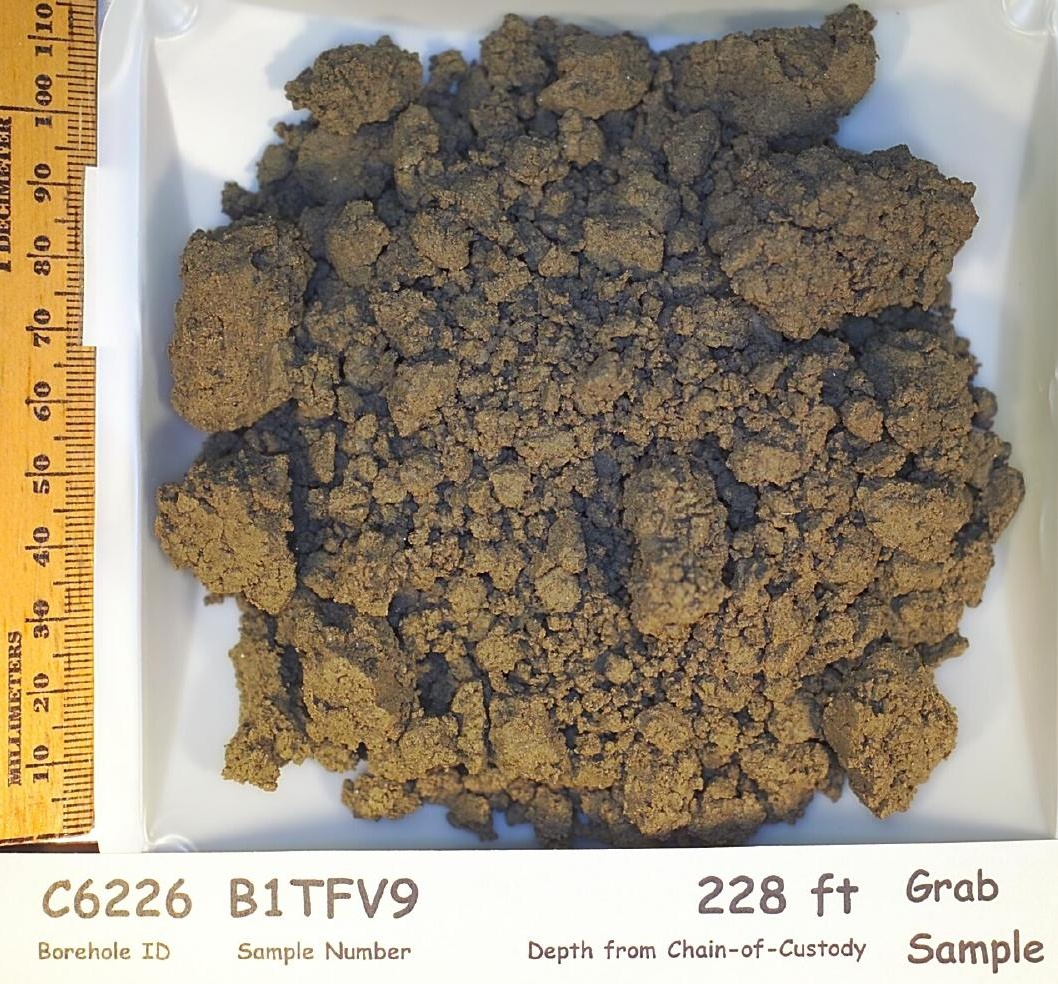




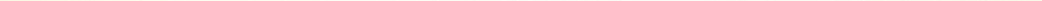




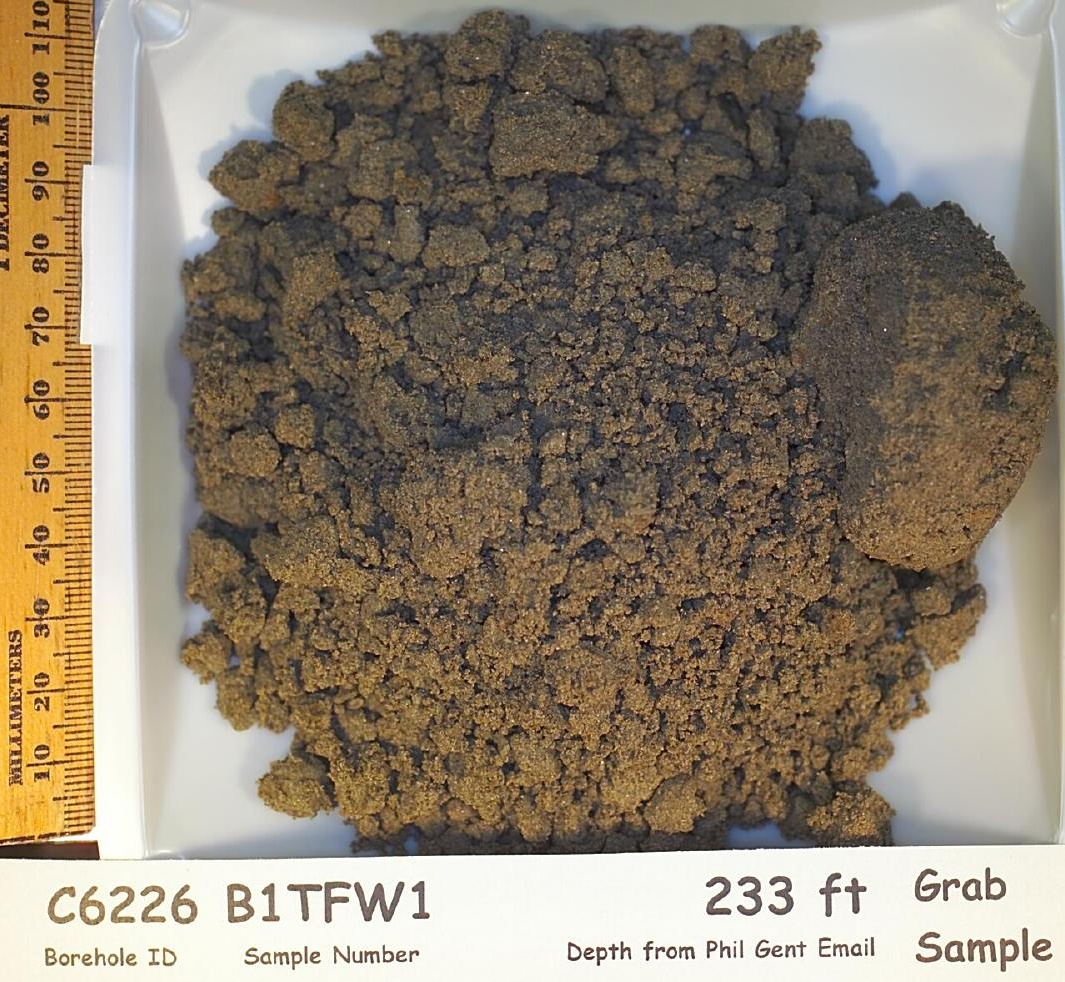




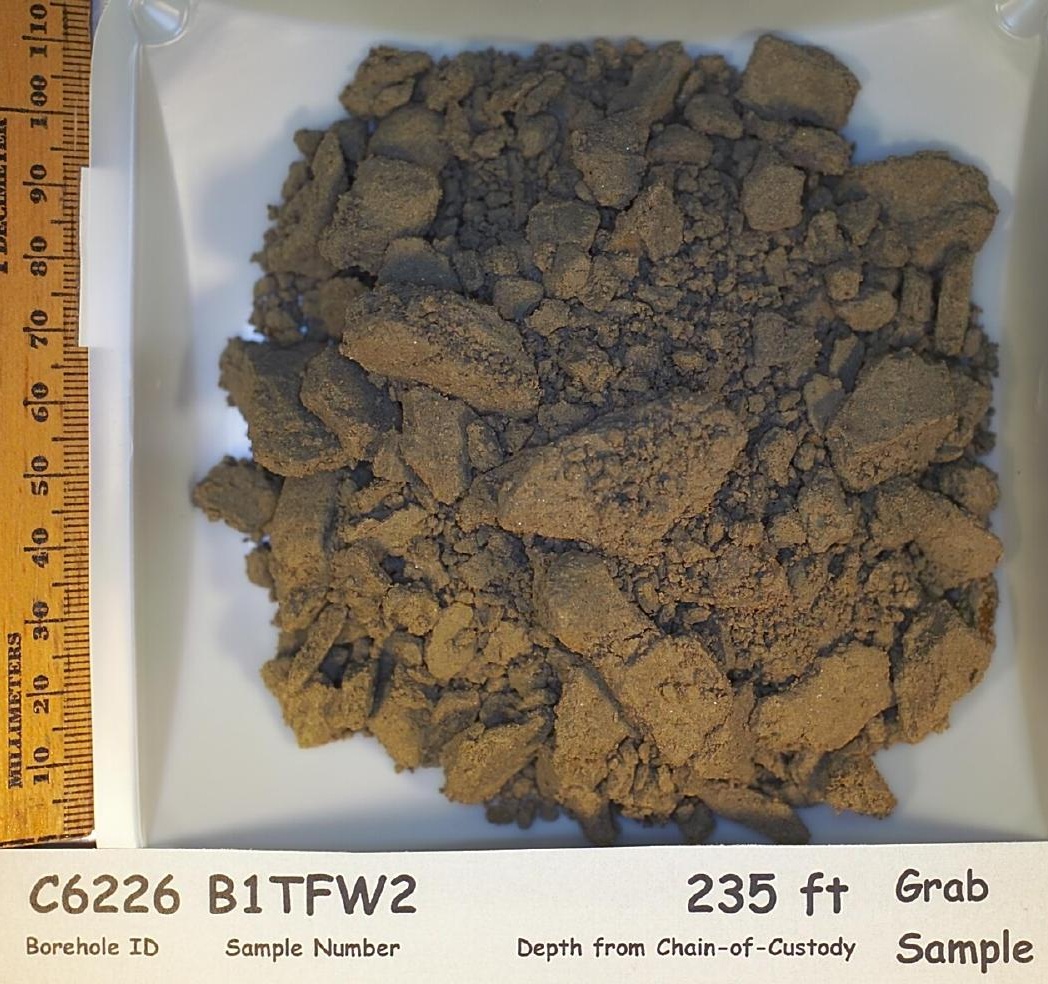




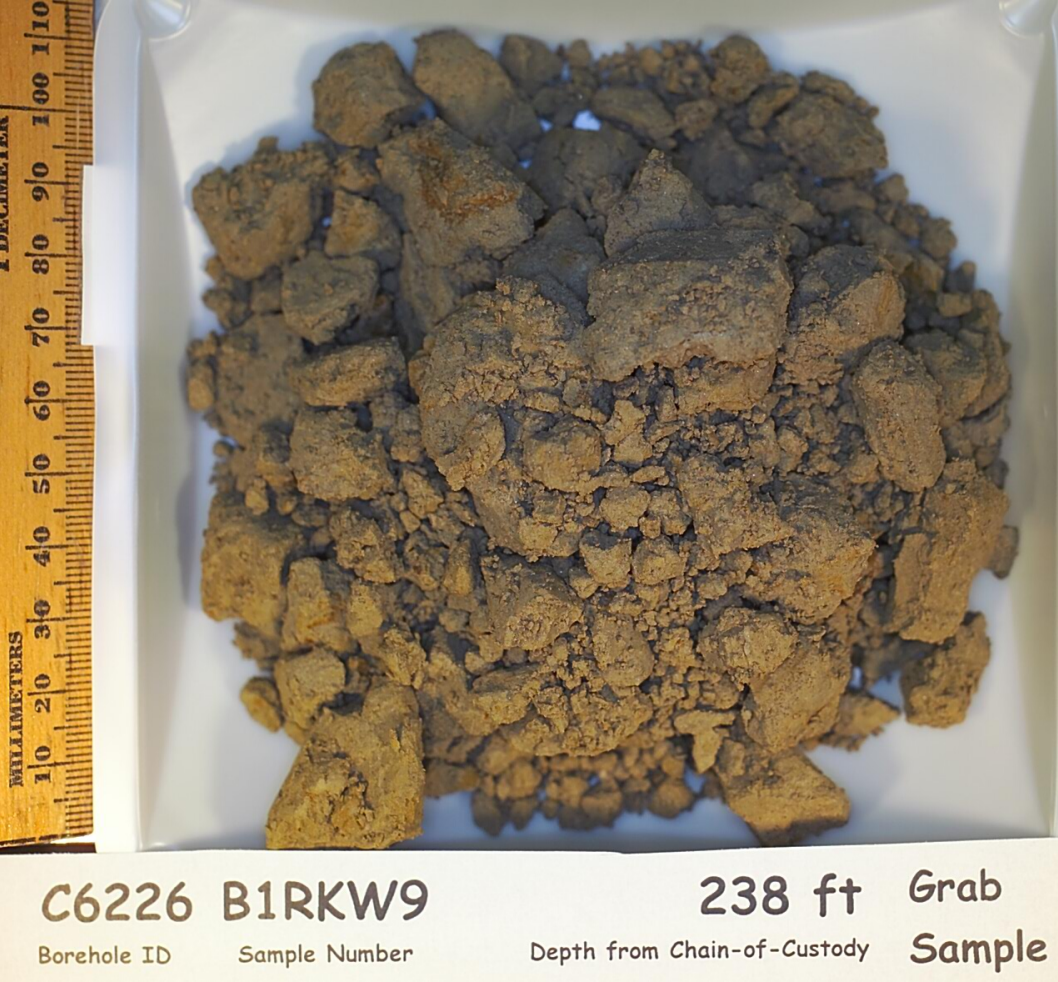




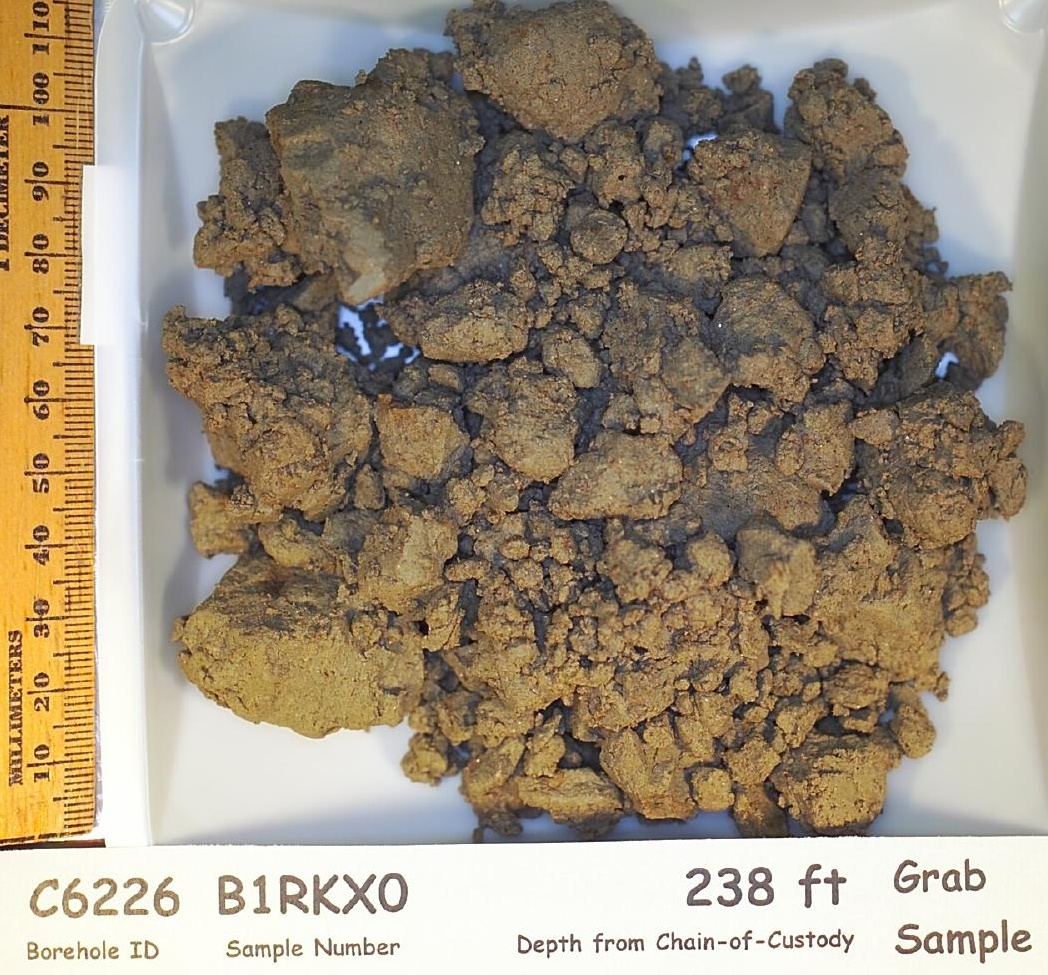




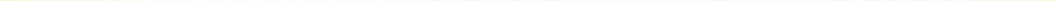




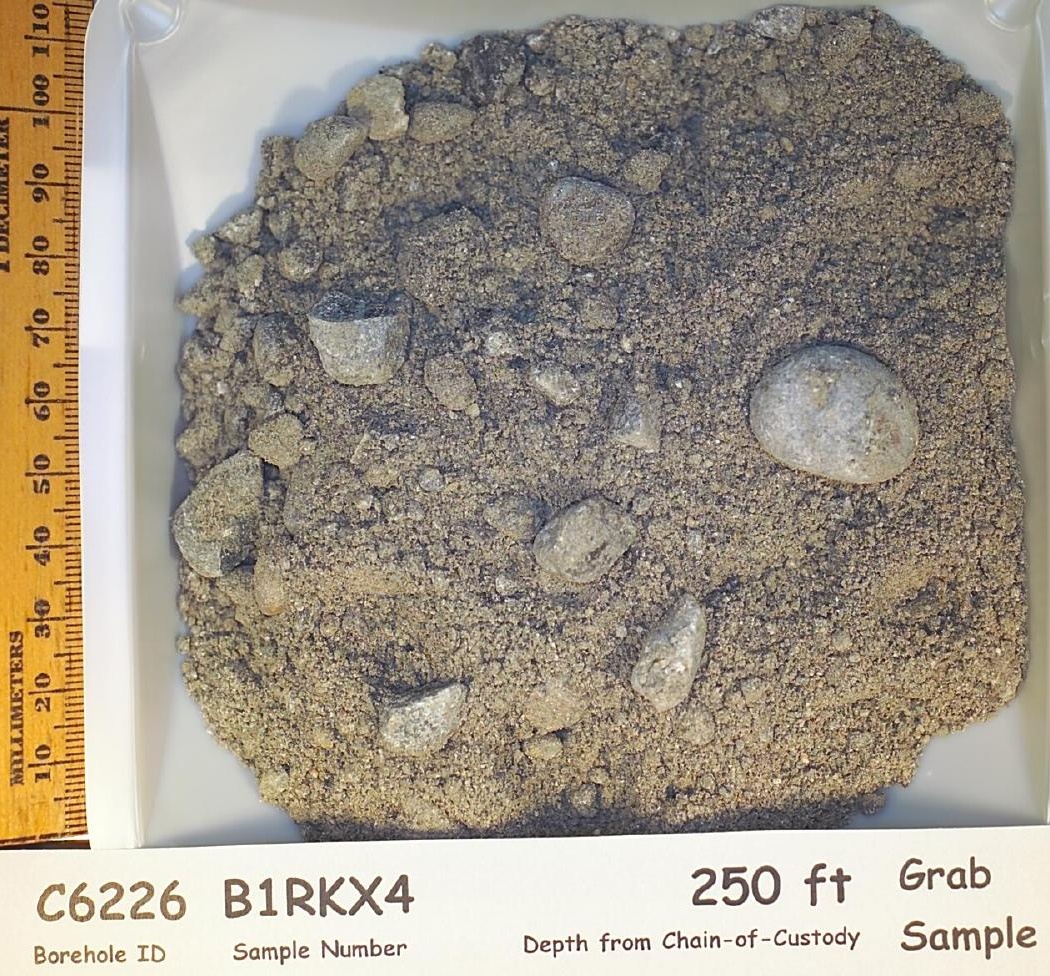




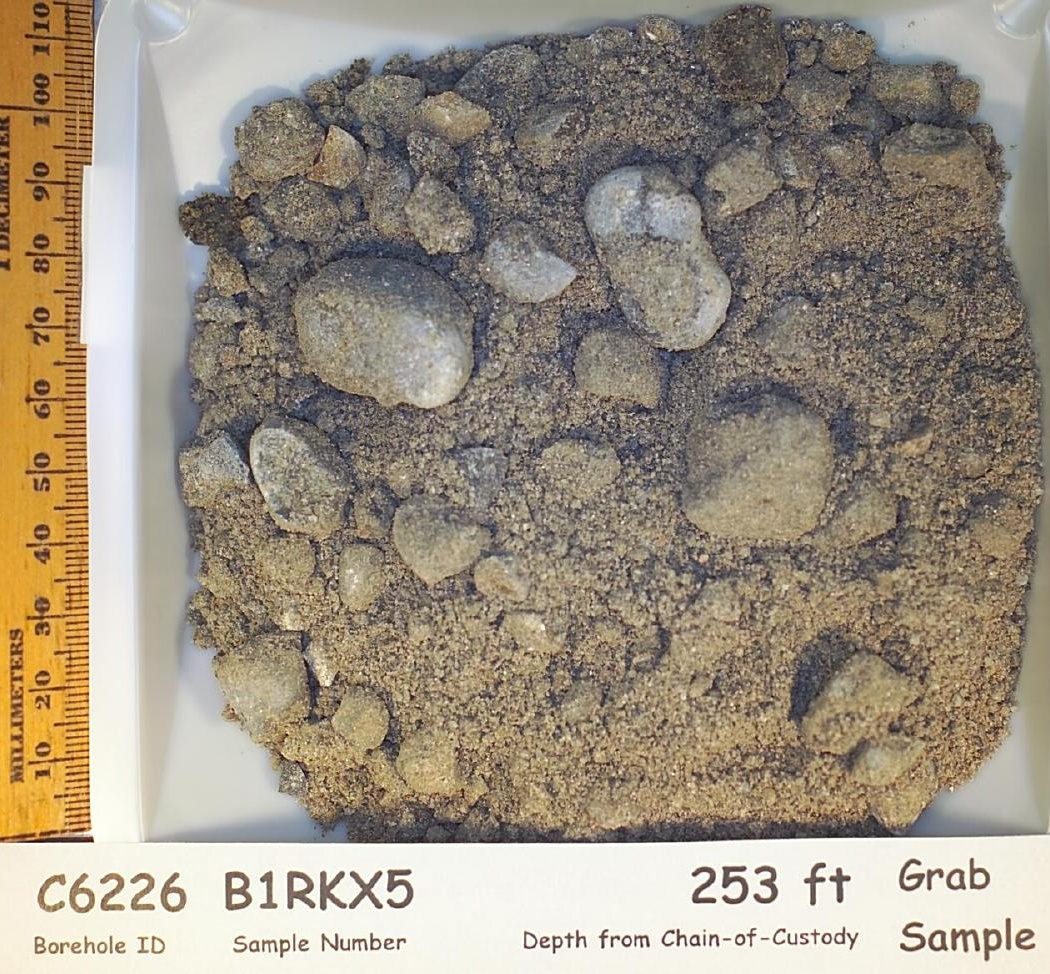




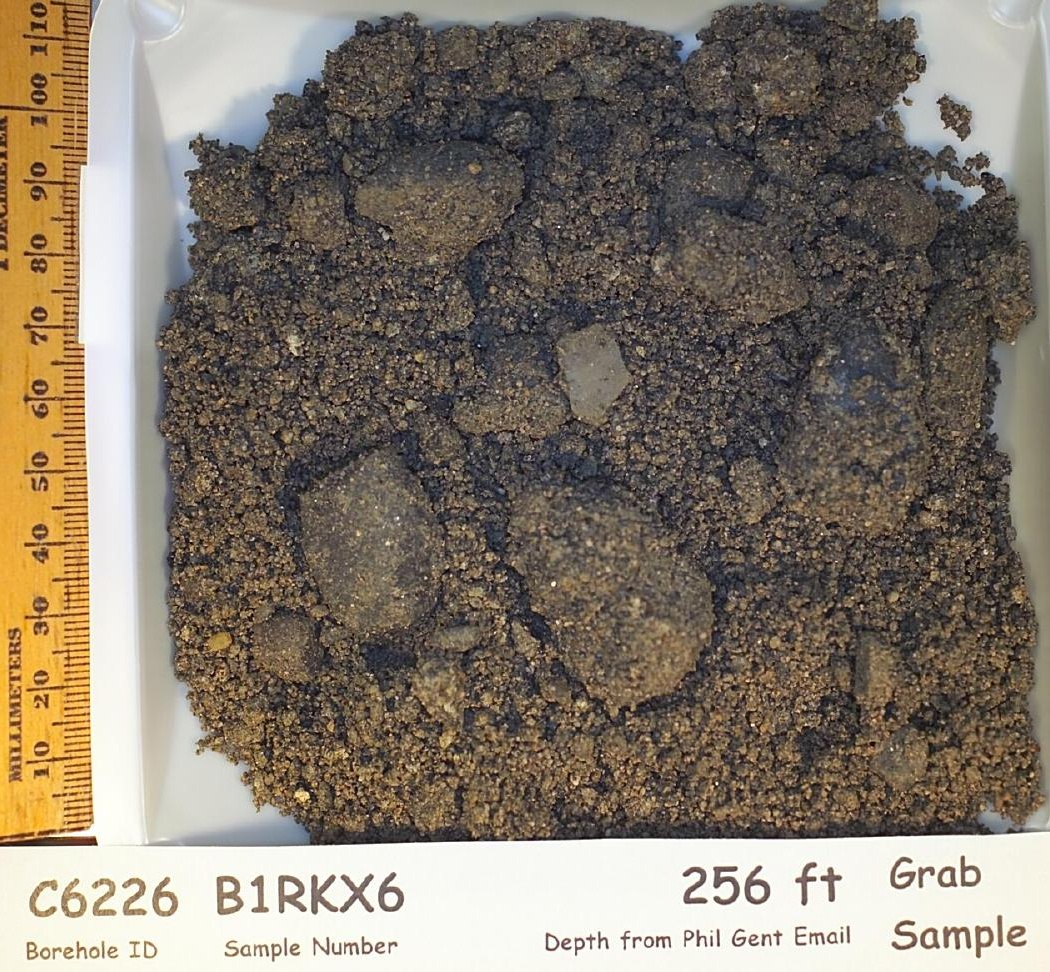




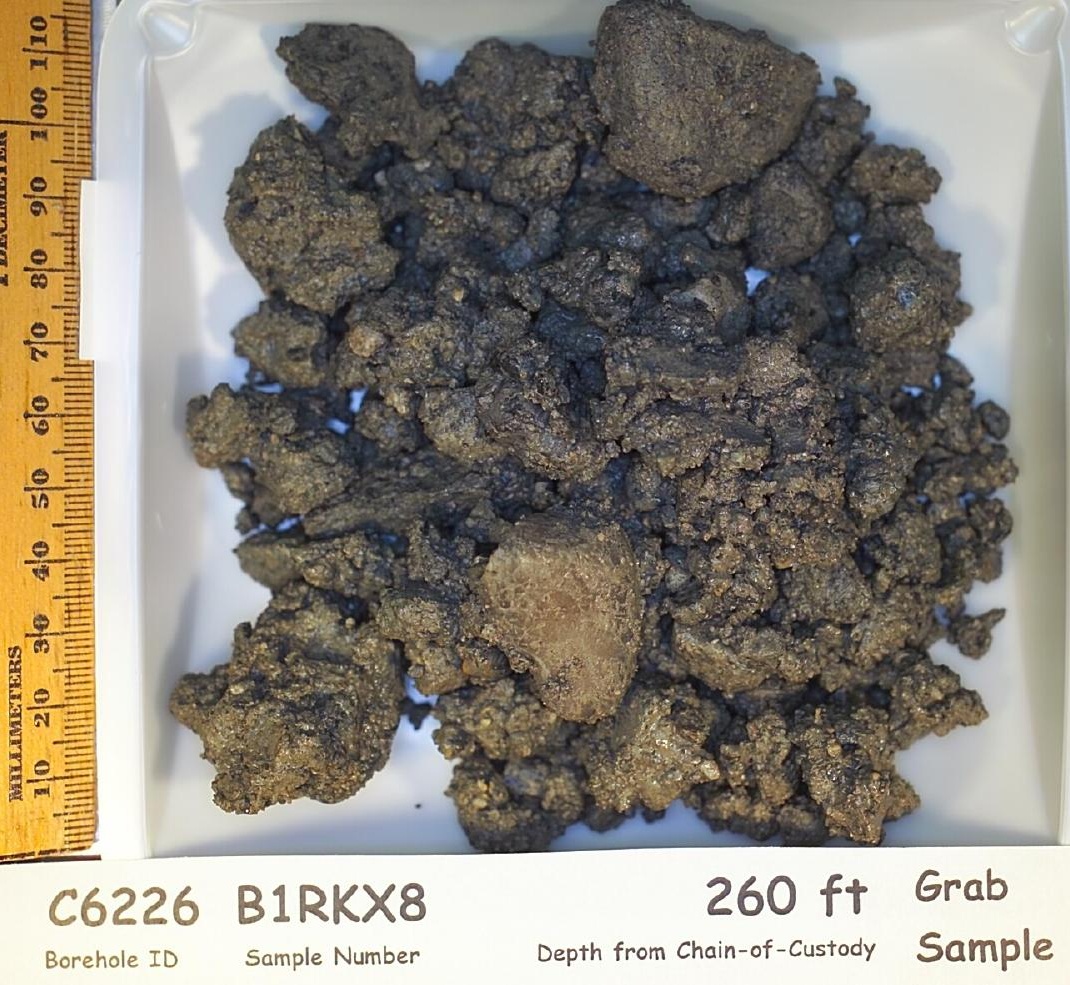




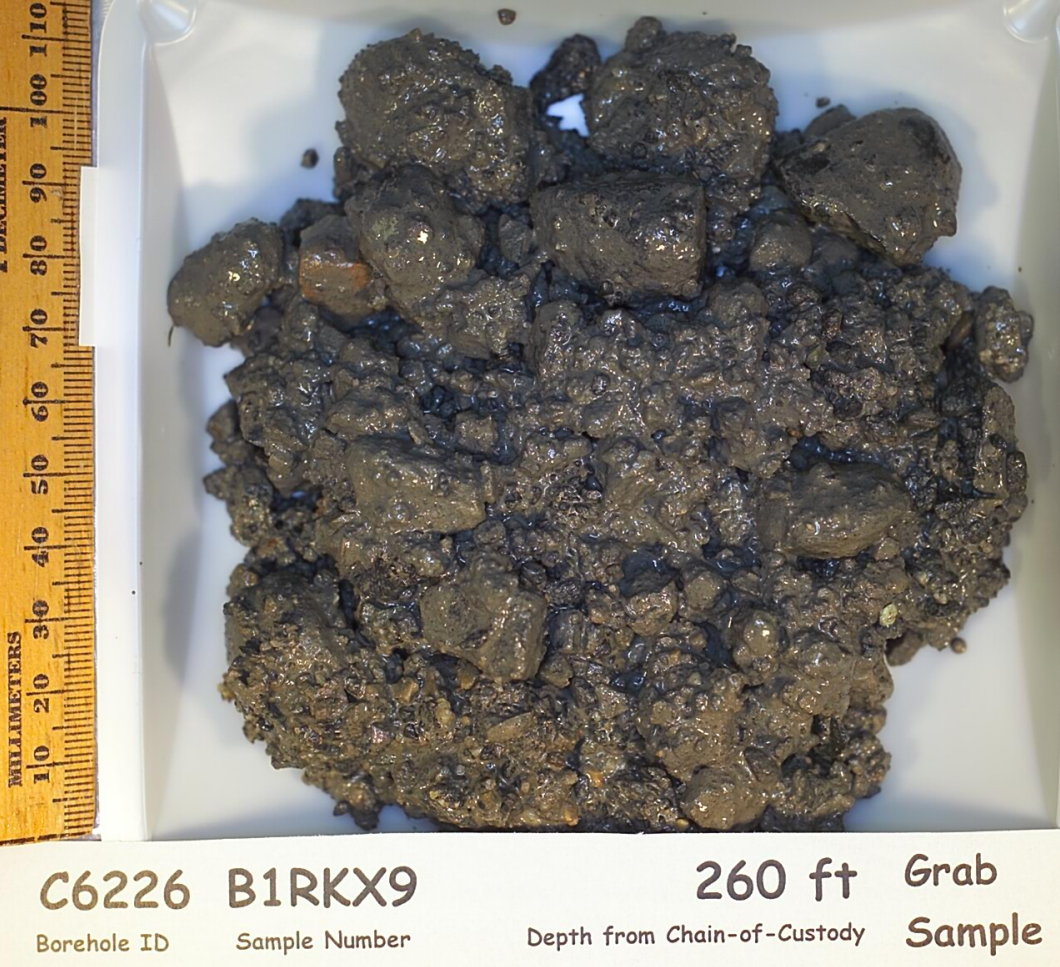




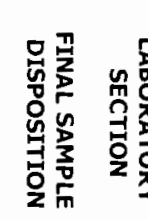

W"

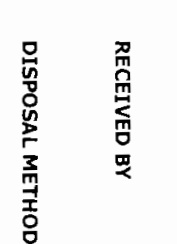

$\underset{\tilde{U}}{N}$

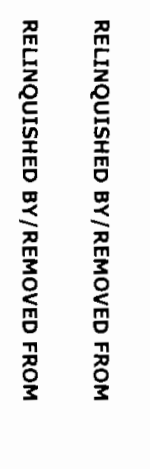

备

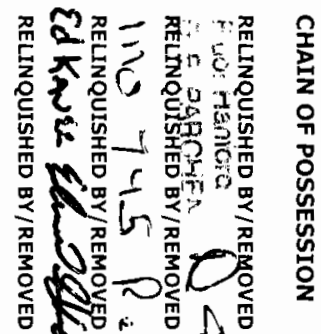

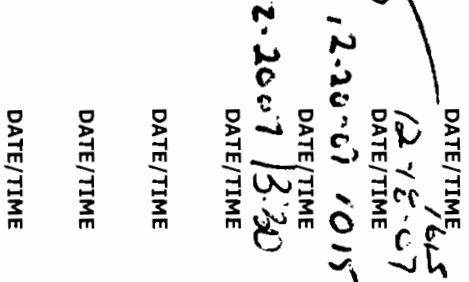

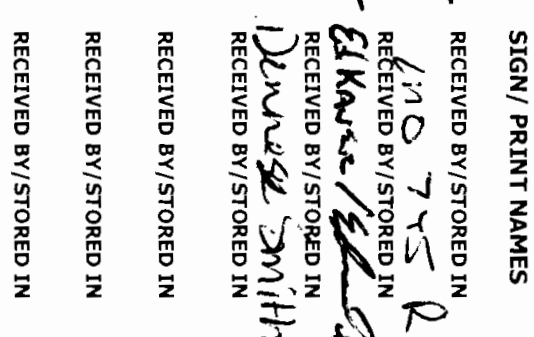

2.

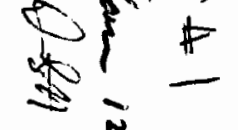

Q $\stackrel{\sim}{\sim}$

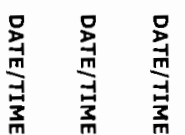

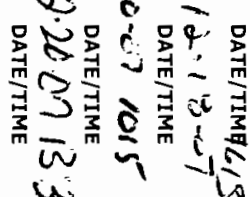

$\underset{m}{\overrightarrow{7}}$

$\ddot{\Xi}$
D
屶
品

$\stackrel{0}{\circ}$

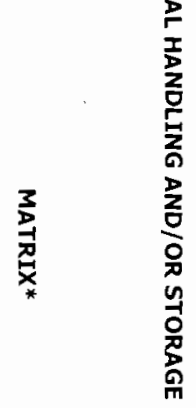

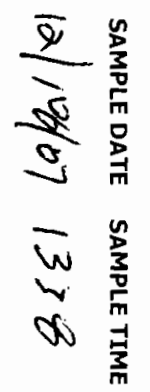

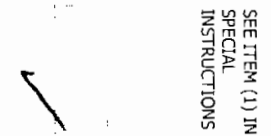

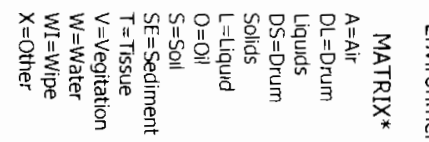

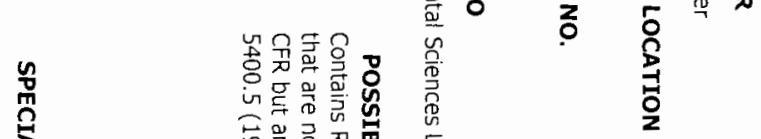

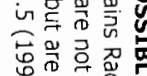

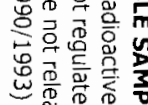

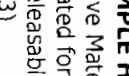

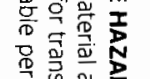

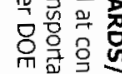

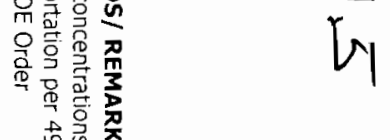

W

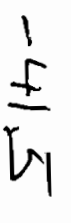

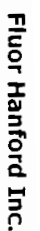

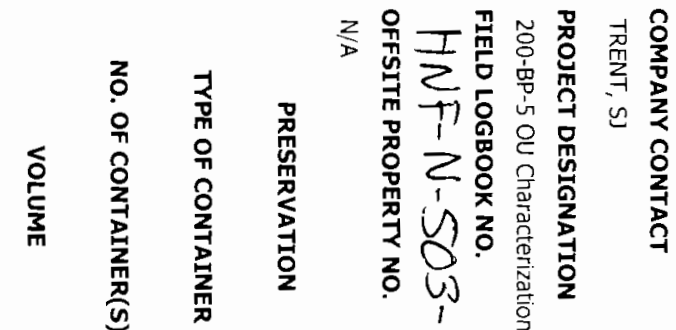

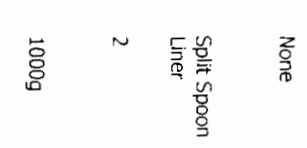

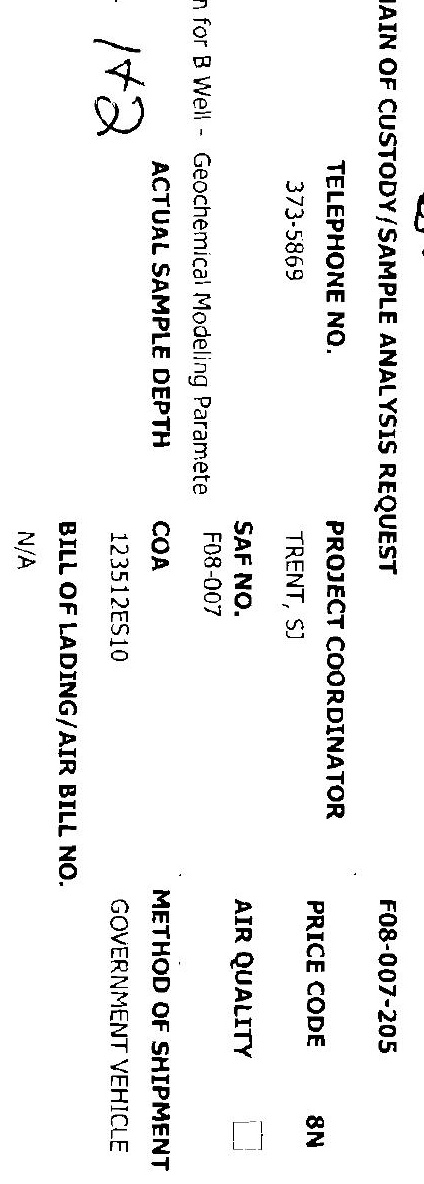



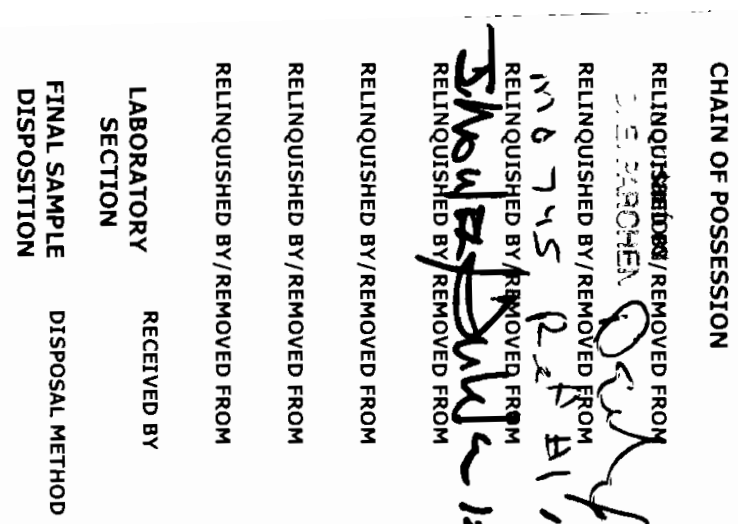

管

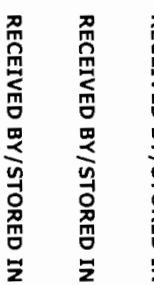

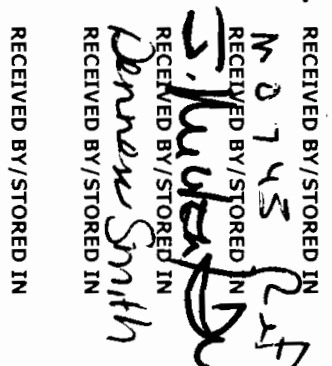

हE

है

जे

总品

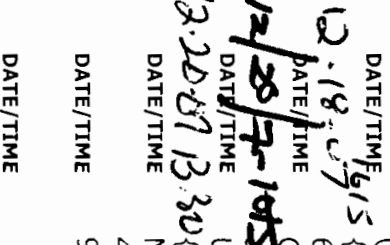

$\begin{array}{ll}0 & -1 \\ 0 & \exists \\ 0 & \text { m } \\ 0 & 0 \\ 0 & \\ 0 & \\ 0 & \end{array}$

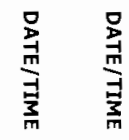

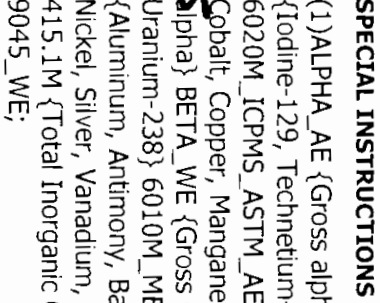

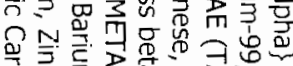

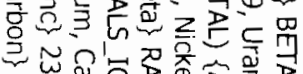

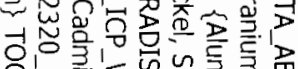

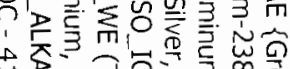

$\overrightarrow{2}$

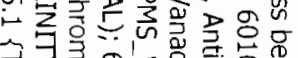

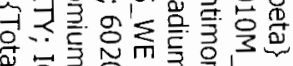

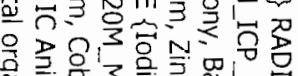

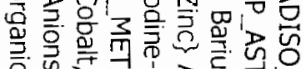

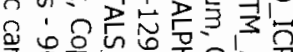

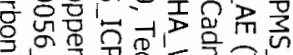

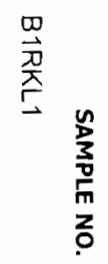

$\stackrel{\infty}{\stackrel{\rho}{\rightleftharpoons}}$

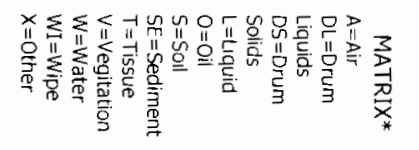

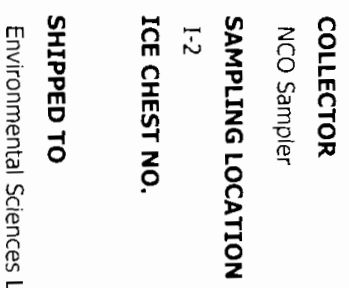

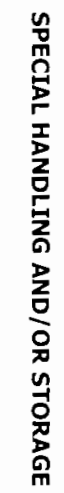

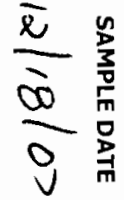

$\begin{array}{cc}- & \frac{n}{3} \\ - & \frac{3}{0} \\ 0 & \frac{5}{3} \\ 0 & \frac{1}{3}\end{array}$

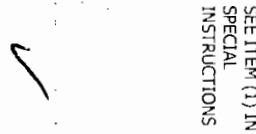

S
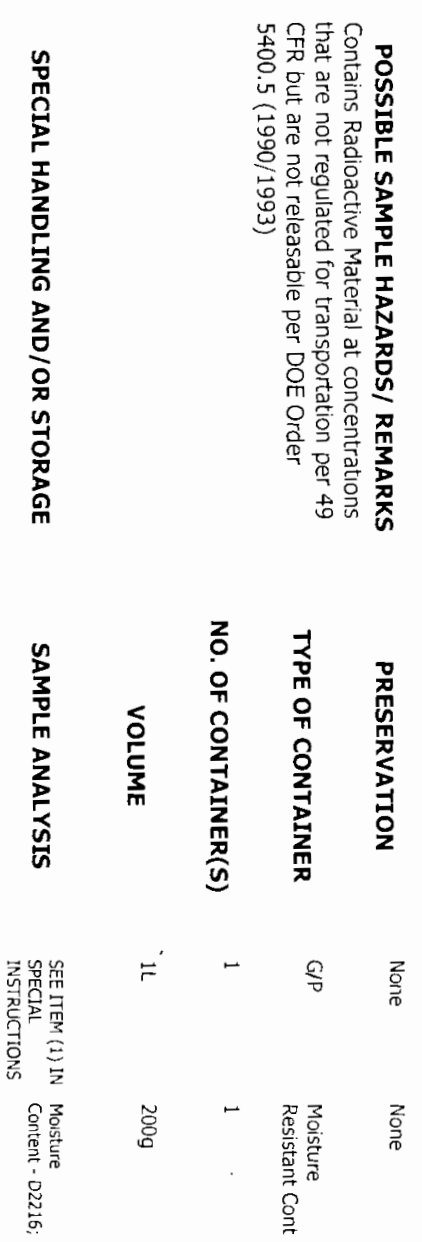

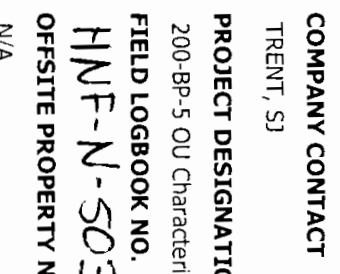

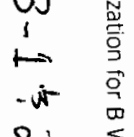

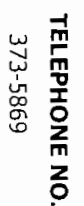

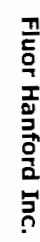
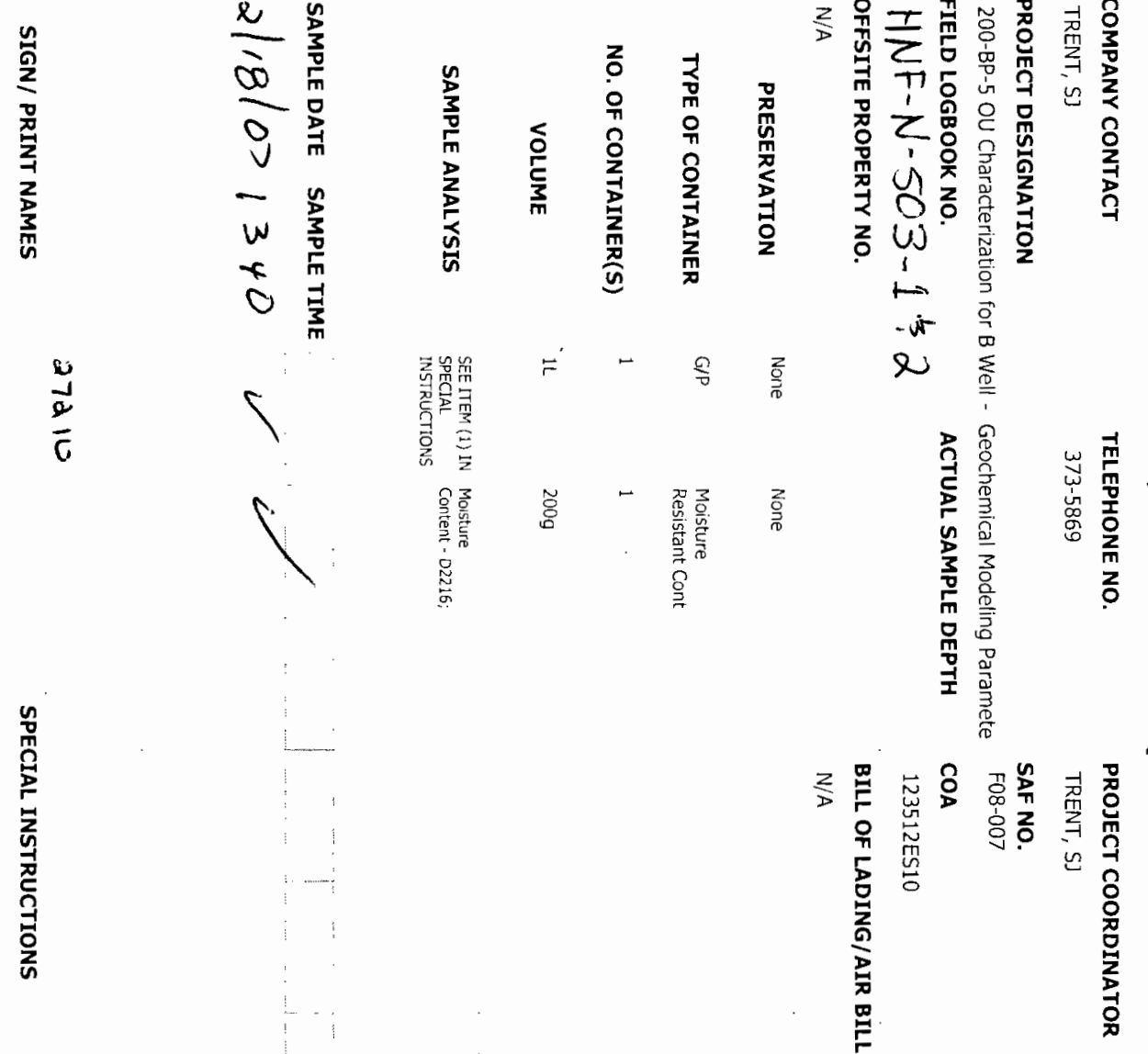

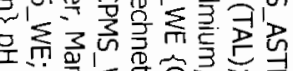

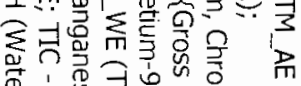

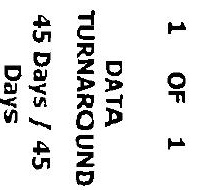



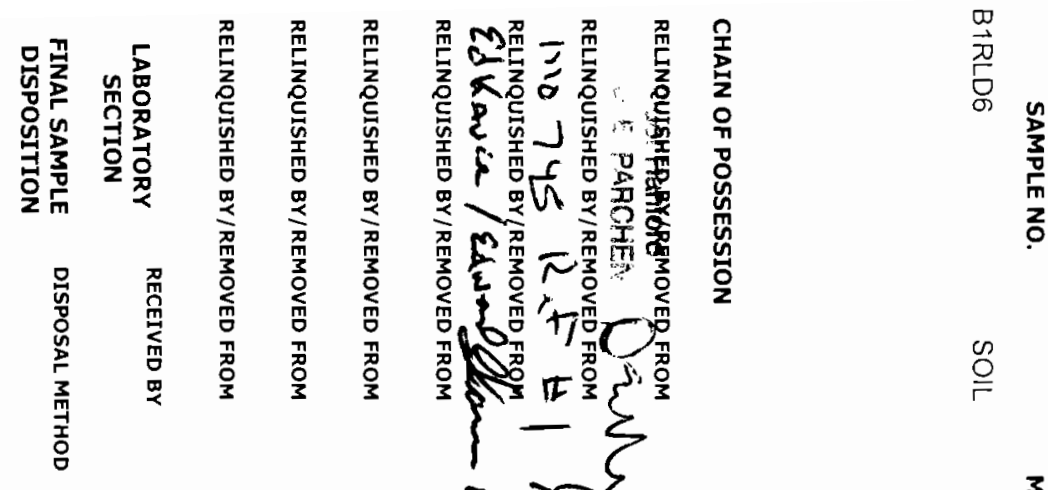

$N$
$\sigma$
$\sigma$

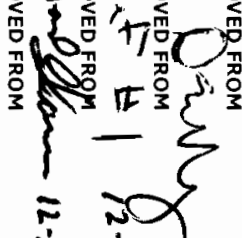

نे

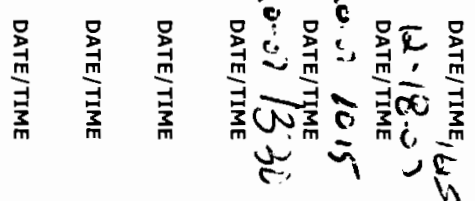
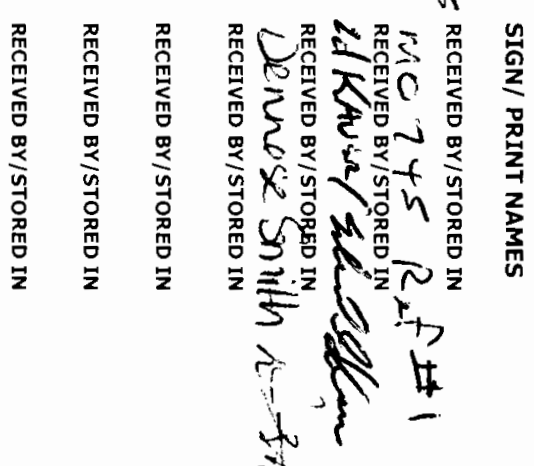

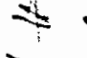

凶

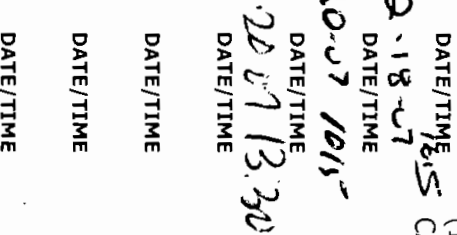

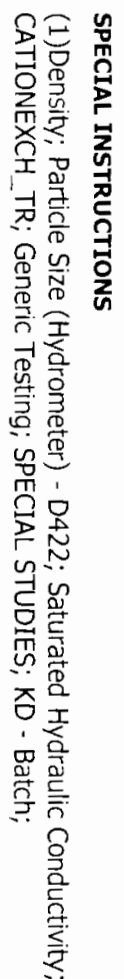

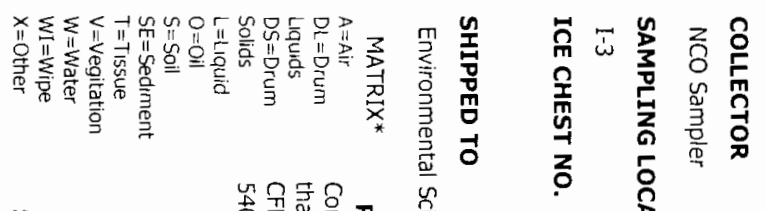

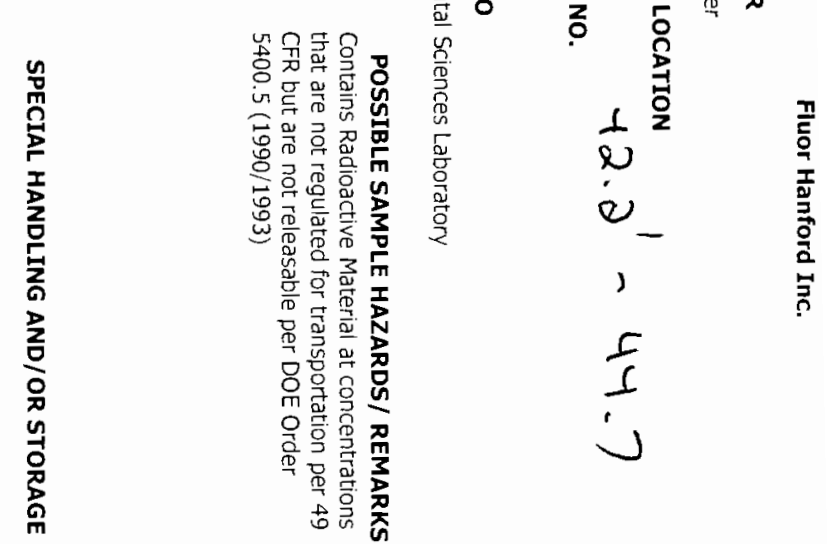

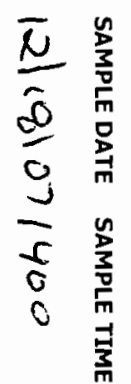

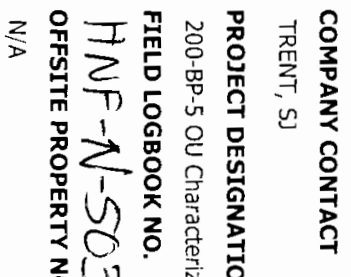
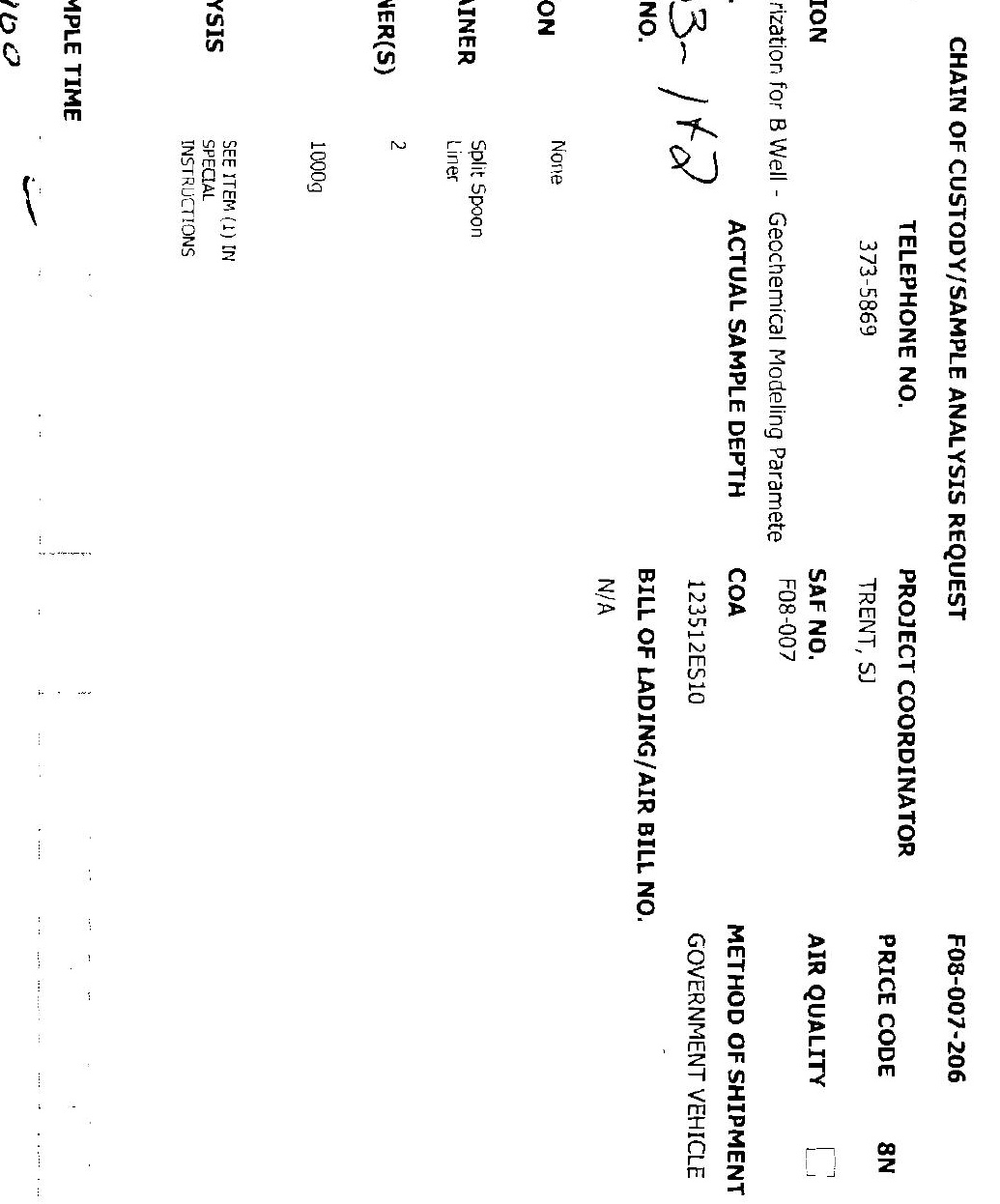

旁

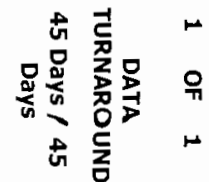




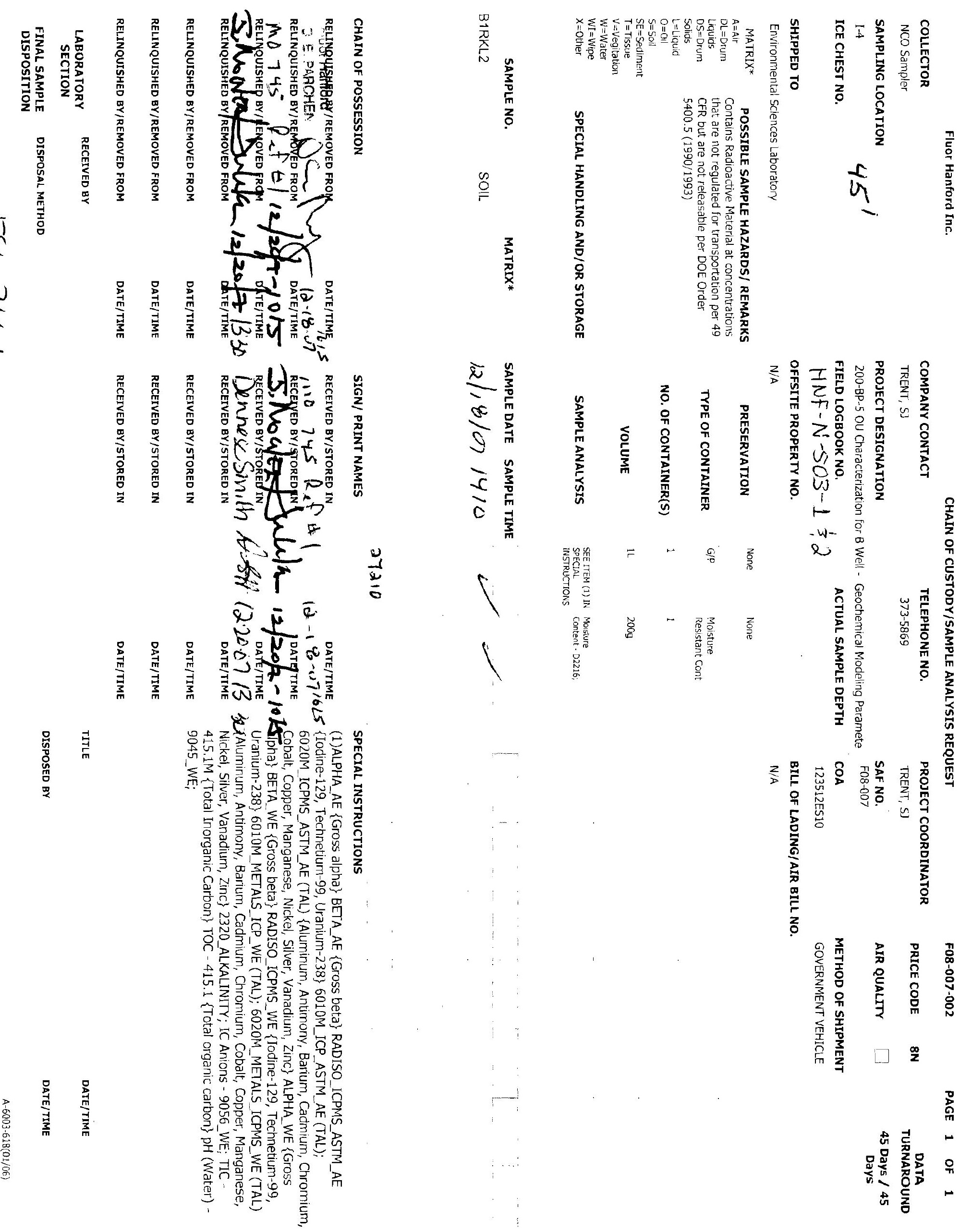




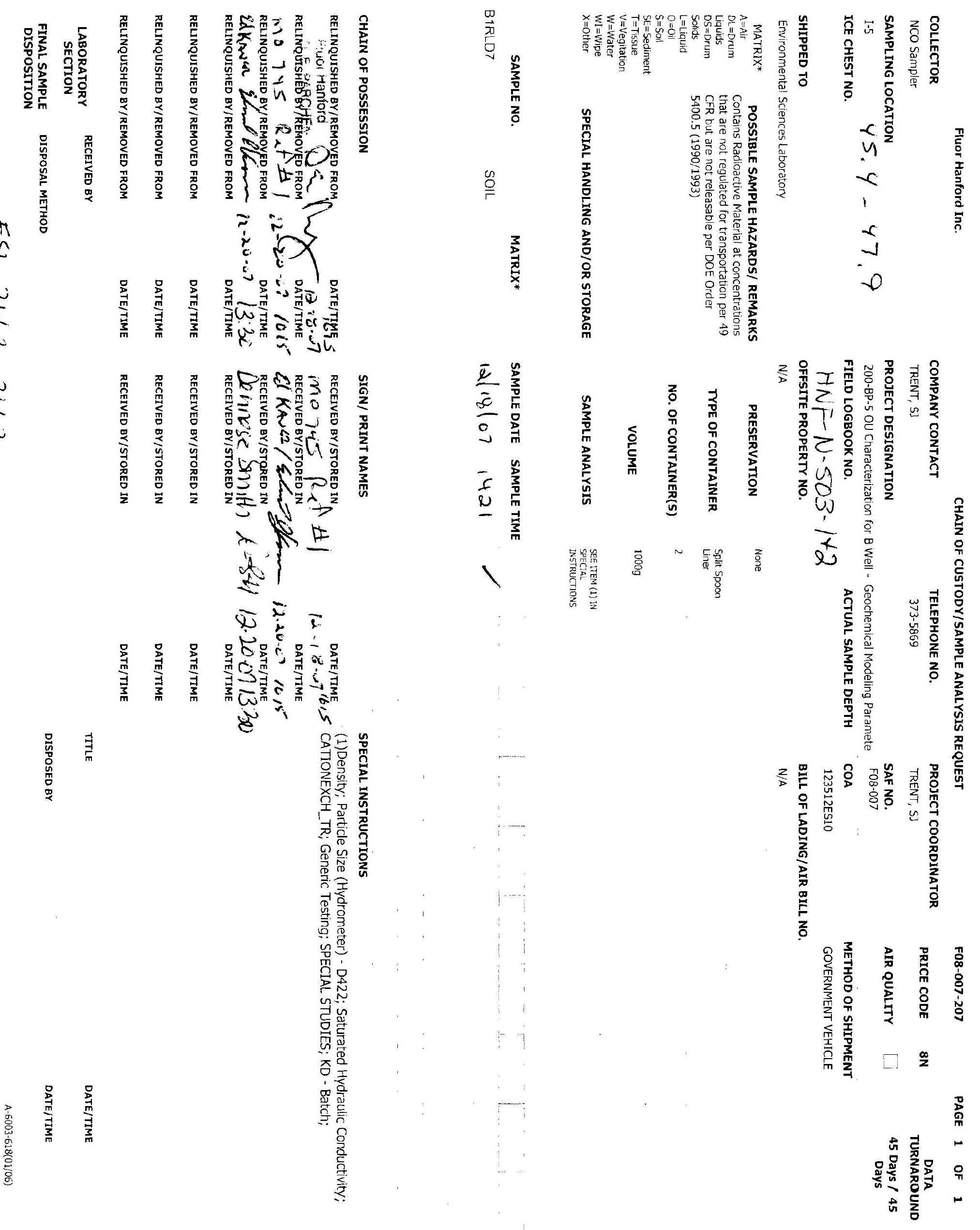



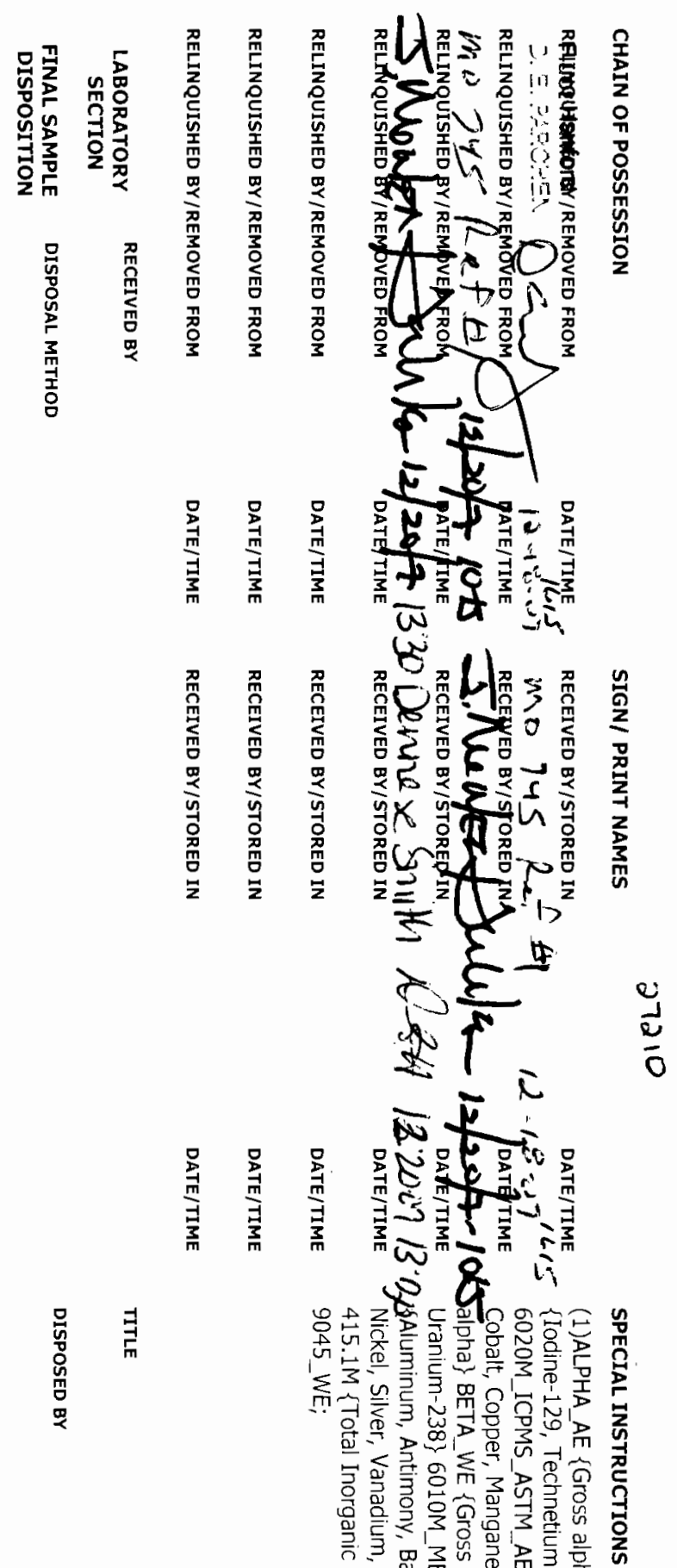

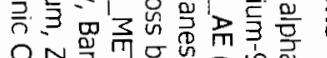

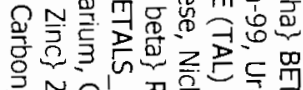

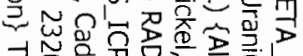

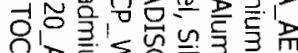

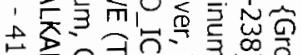

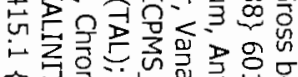

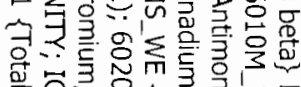

응

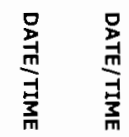

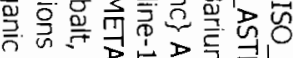

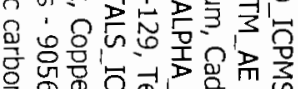

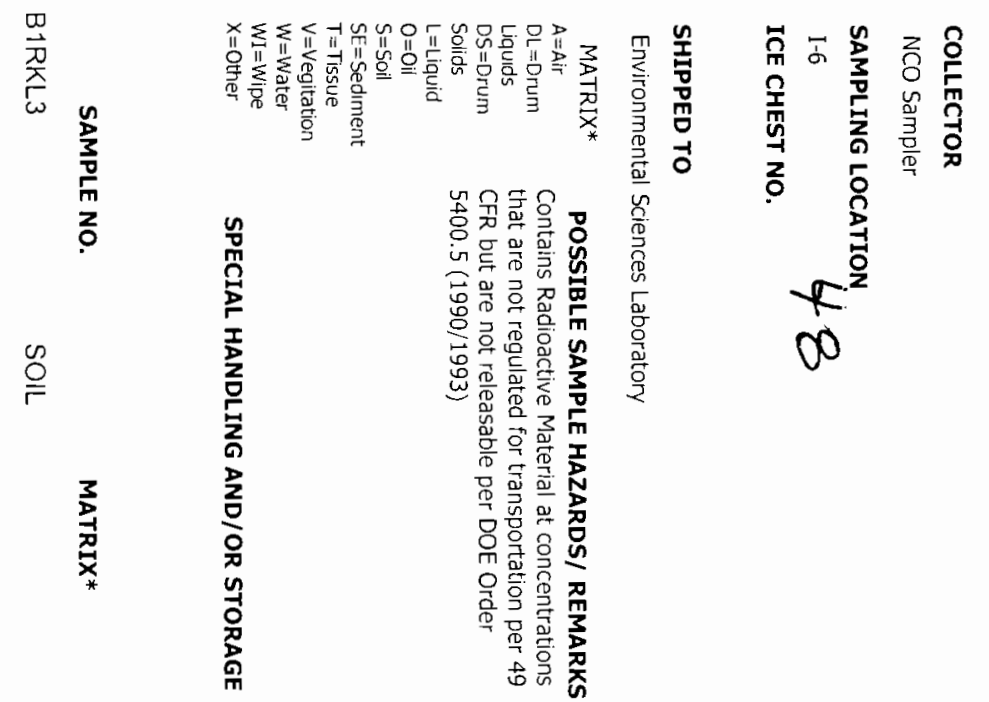

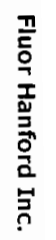
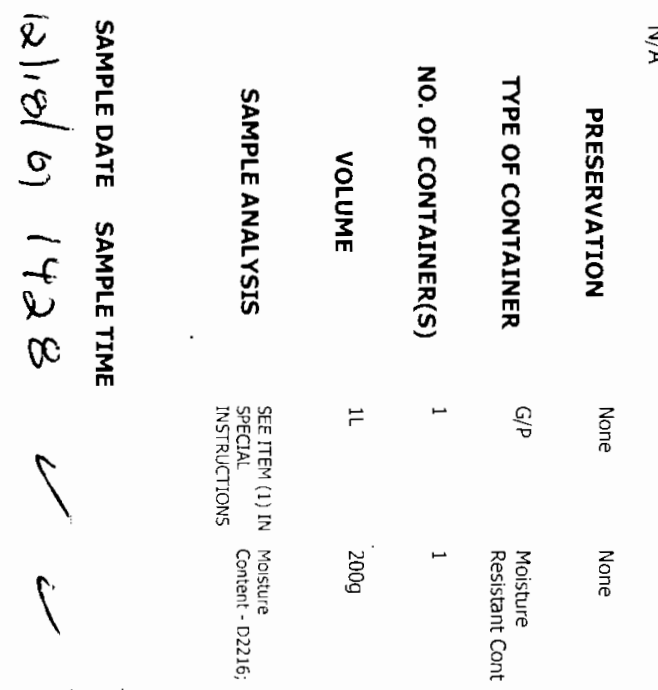

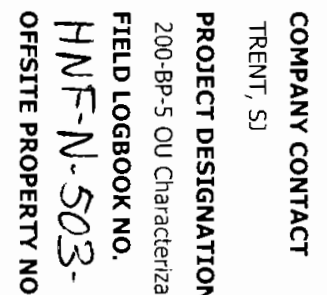
- w

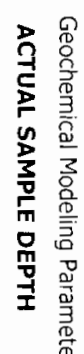

롱

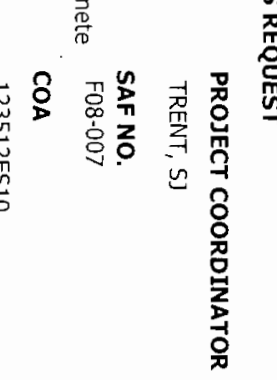

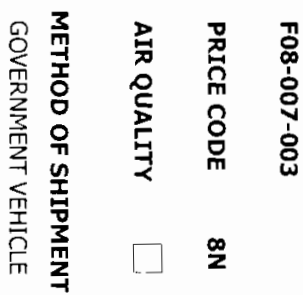

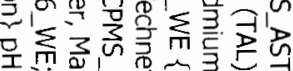

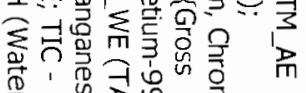

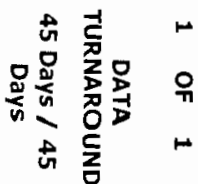



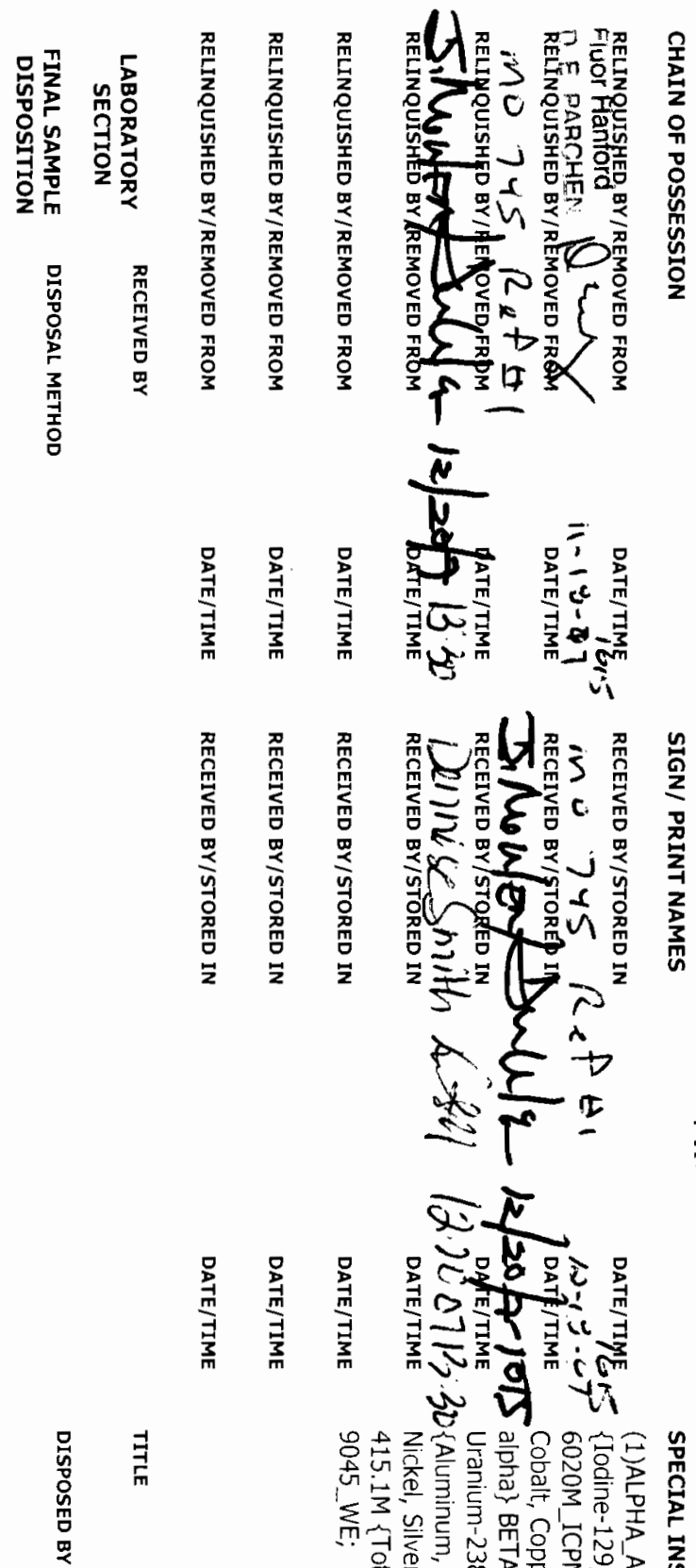

寻

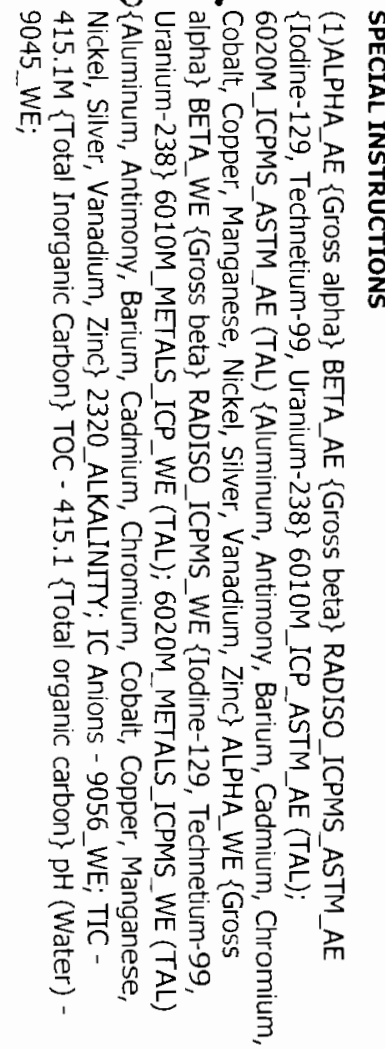

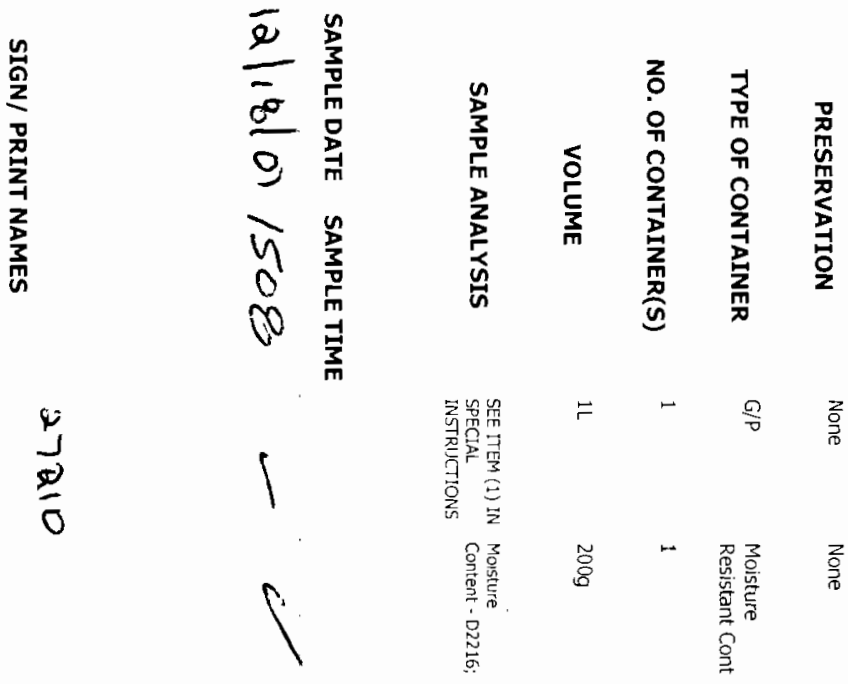
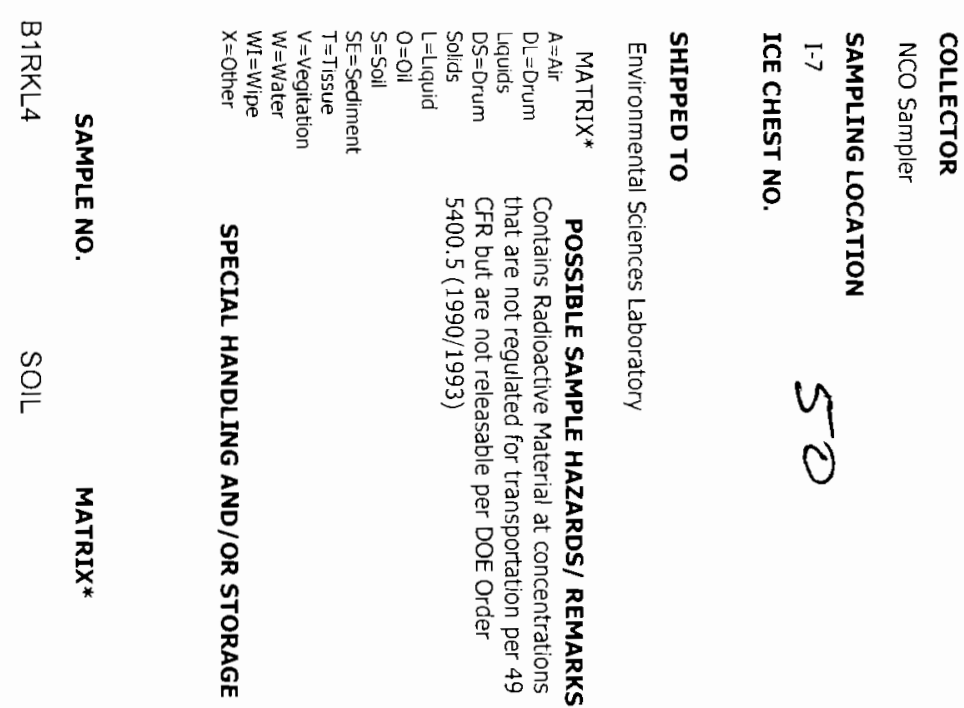

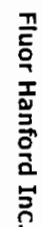

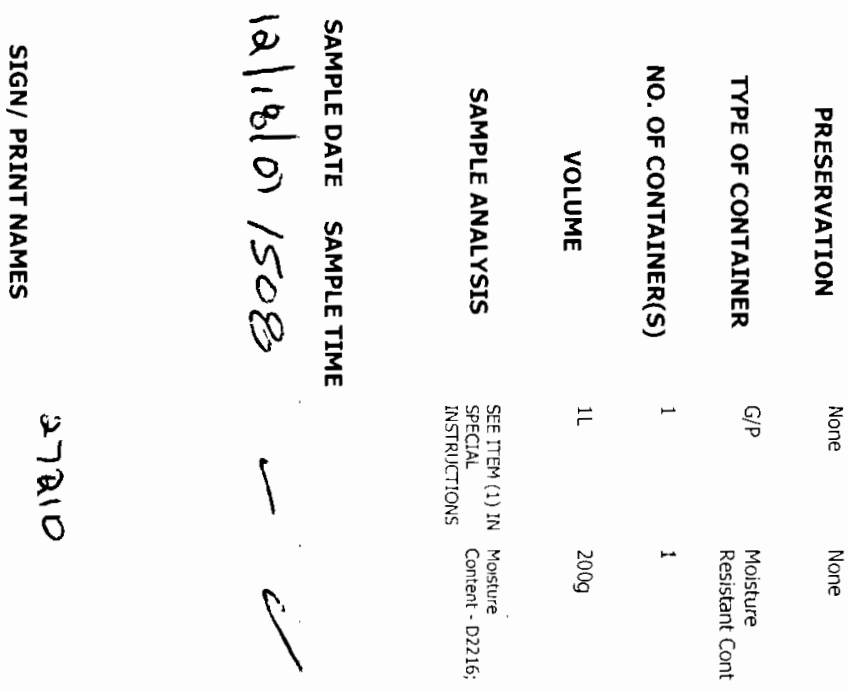

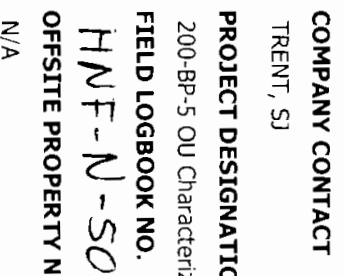

㝞 뭉

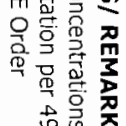




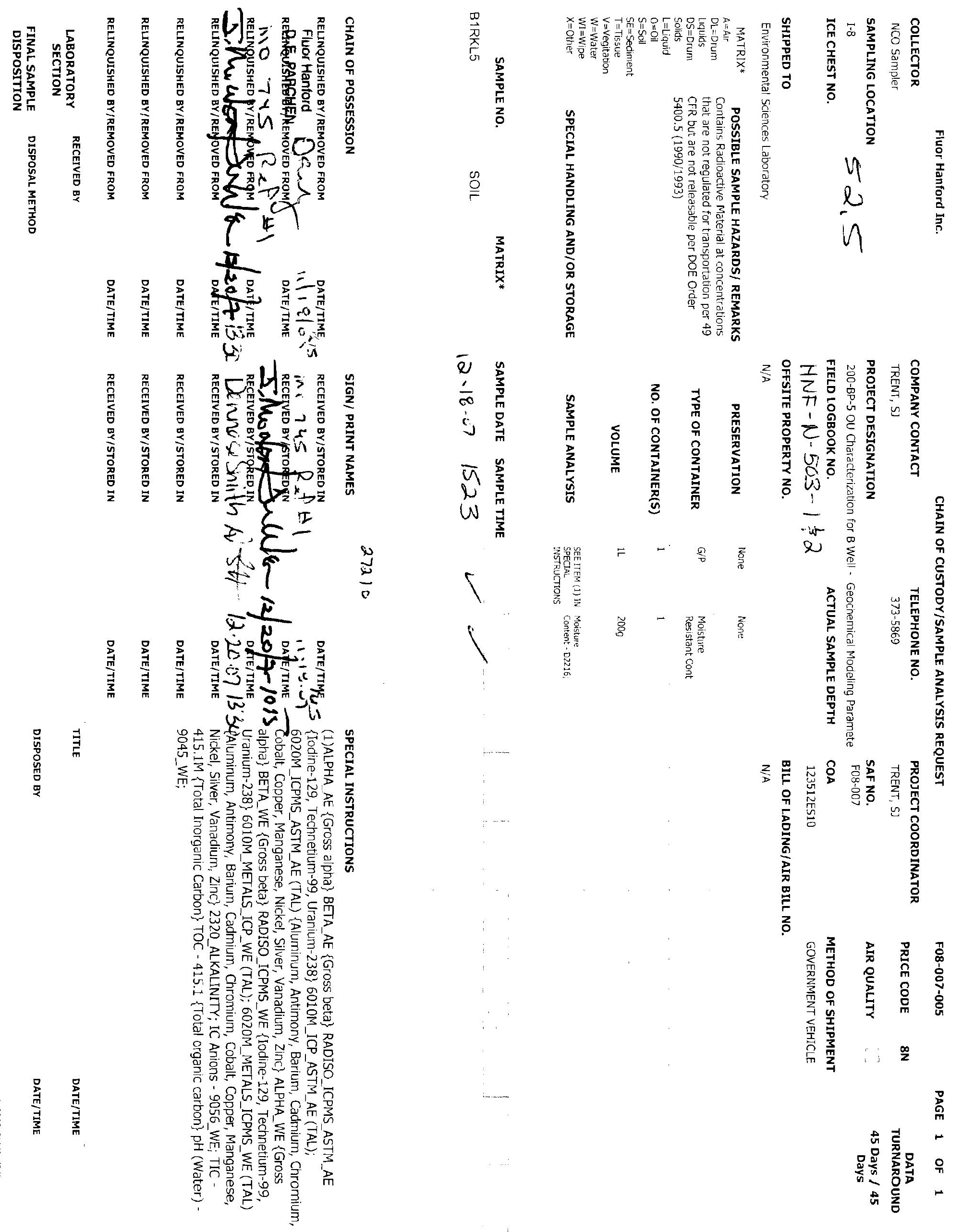



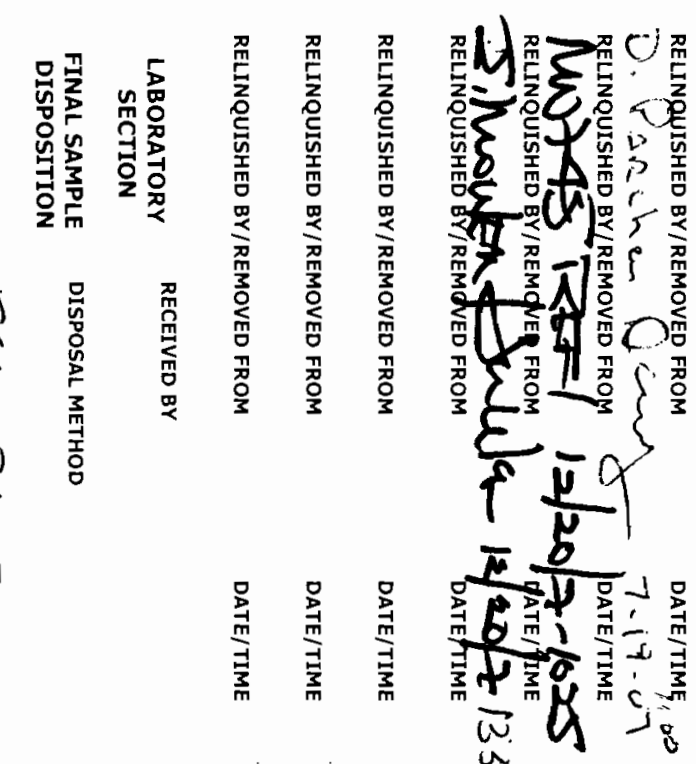

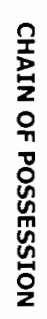

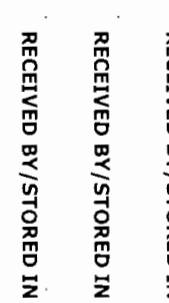

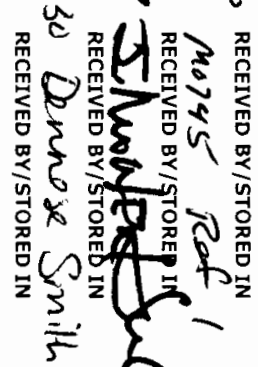

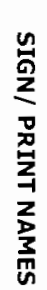

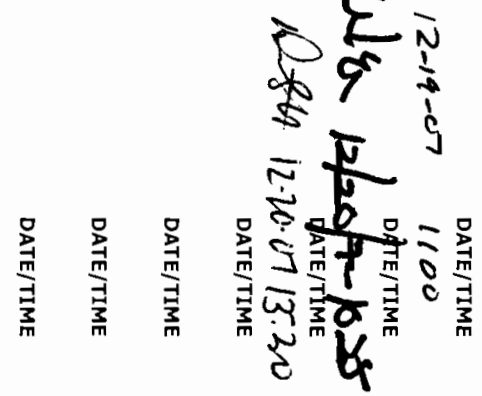

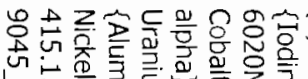

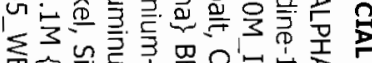

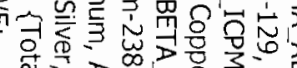

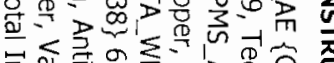

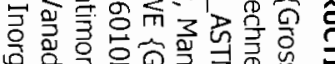

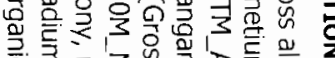

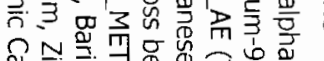

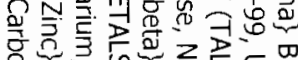

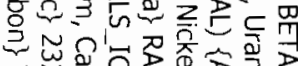

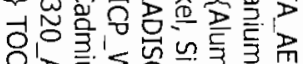

1

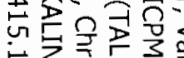

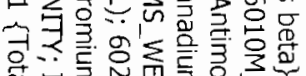

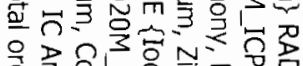

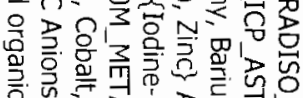

品是

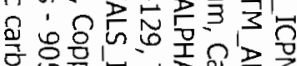

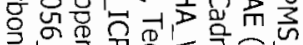

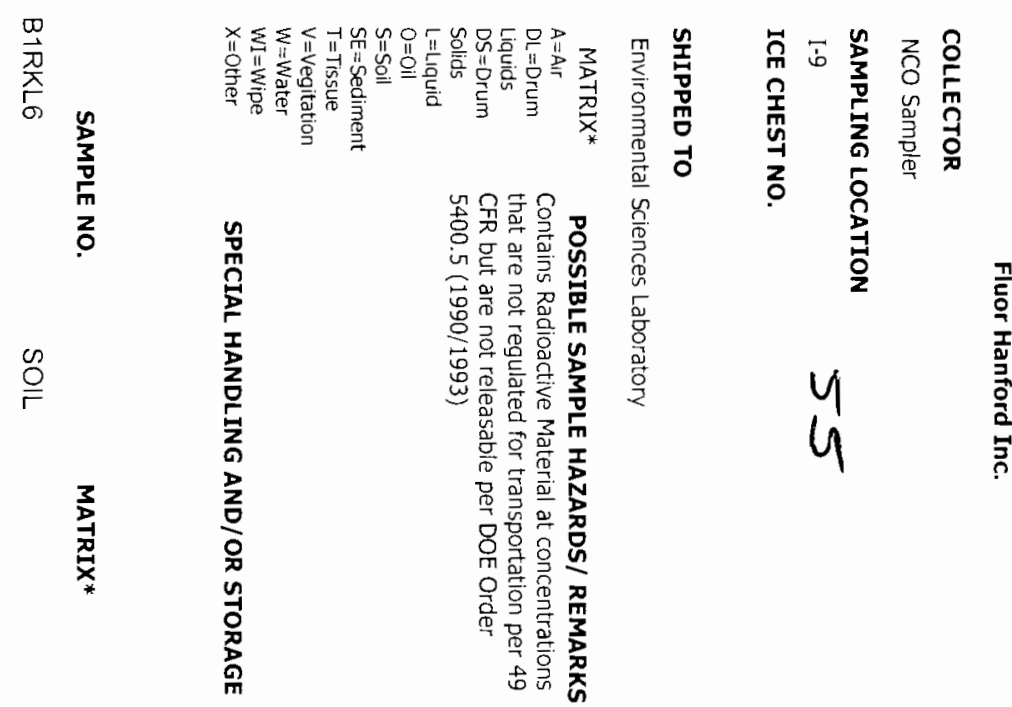

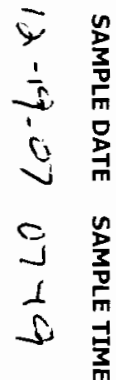

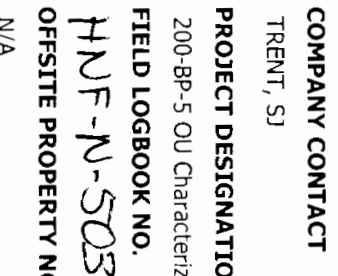
1 2

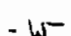

$-w$

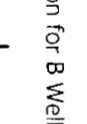

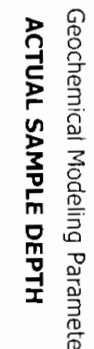

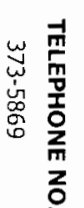

雍

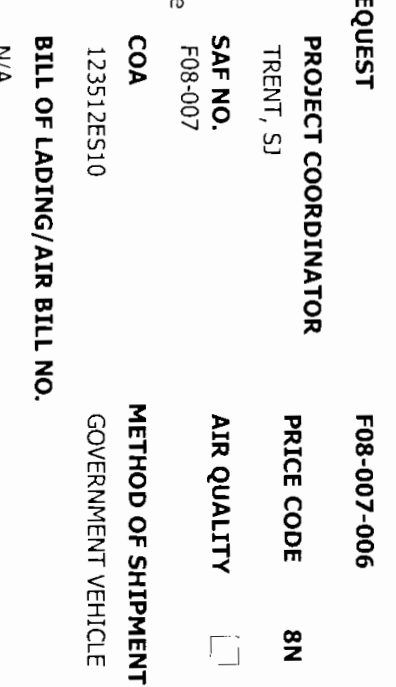

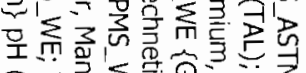

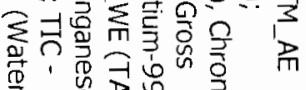




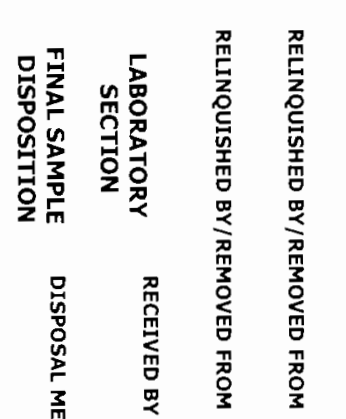

त)

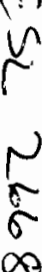

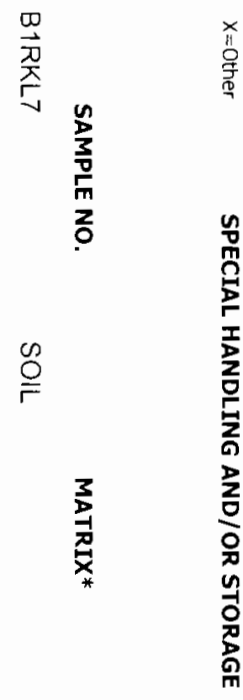

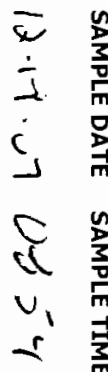

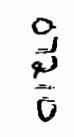

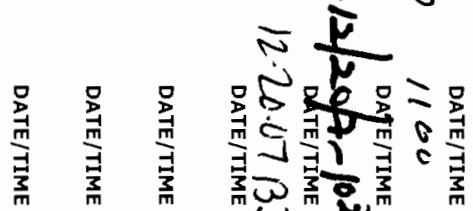

咅

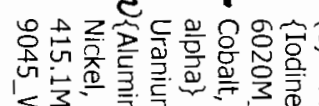

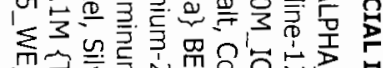

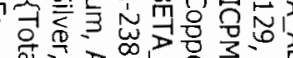

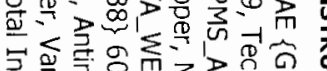

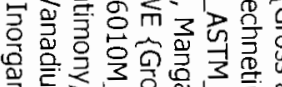

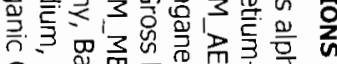

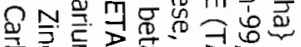

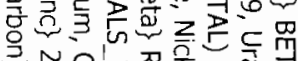

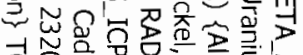

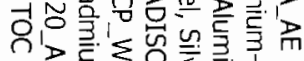

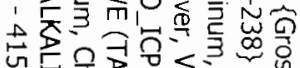

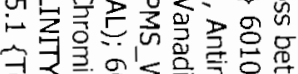

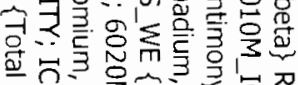

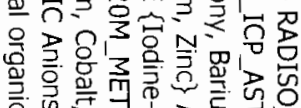

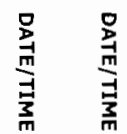

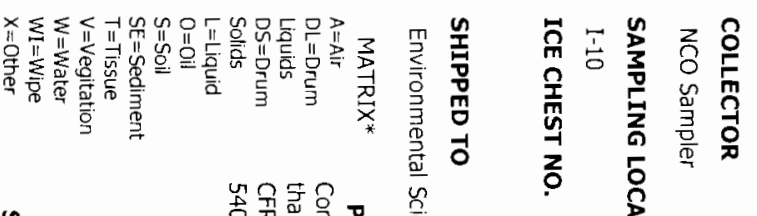

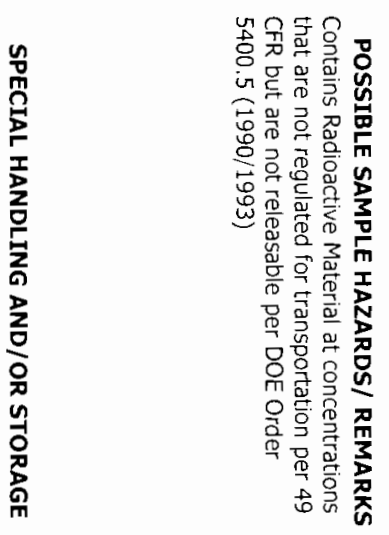

荝.

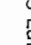

范

$M$

$-1$

in

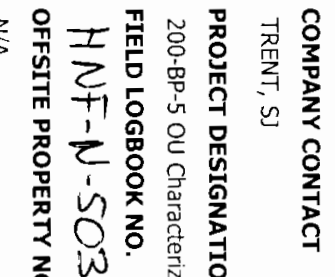

$\therefore$ 음

N

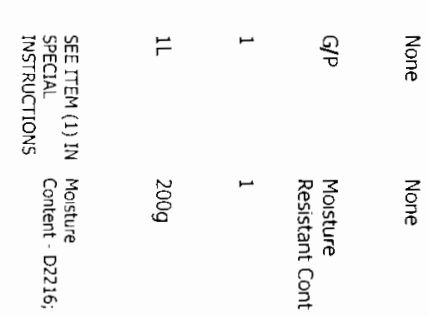

$\frac{\pi}{5}$ 


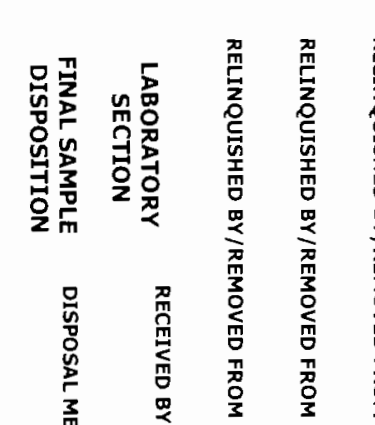
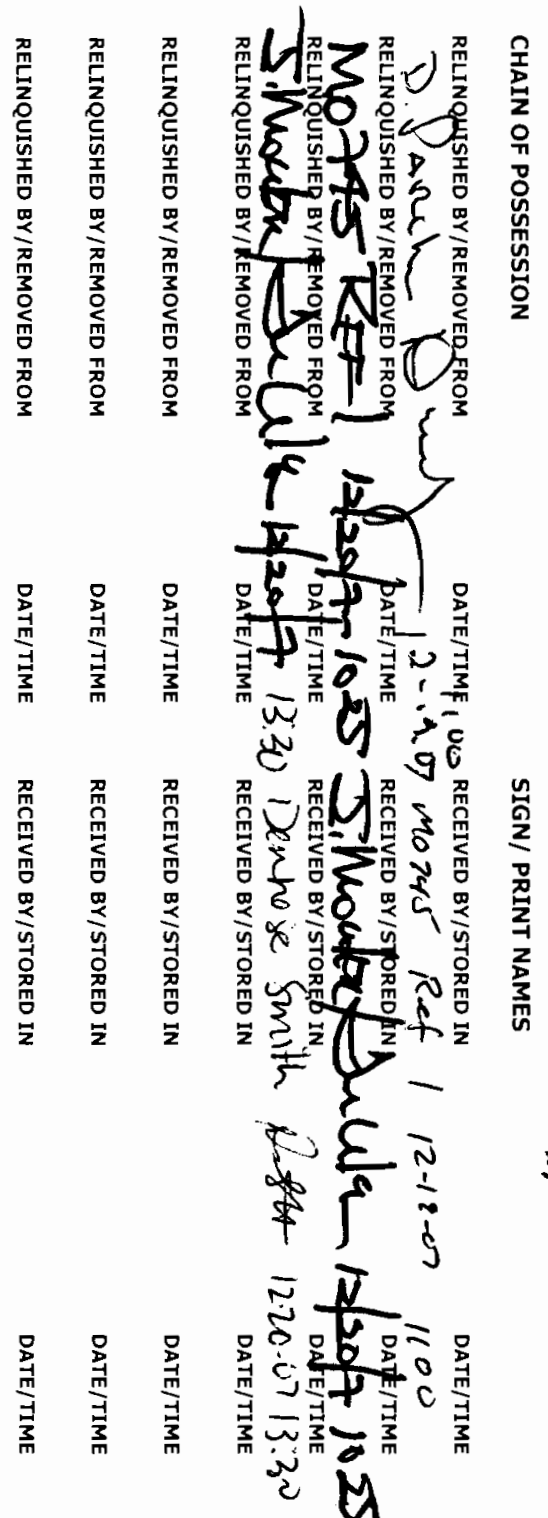

몸

$\underset{7}{7}$

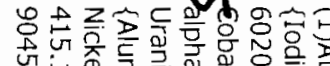

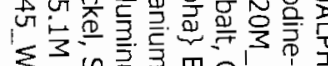

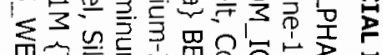

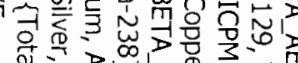

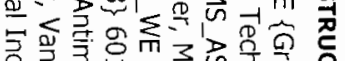

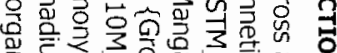

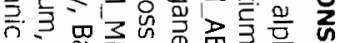

@Nㅡㄹ

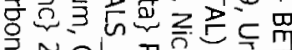

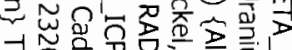

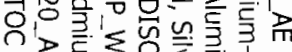

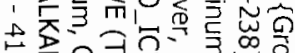

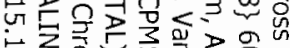

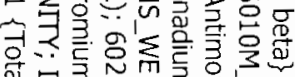

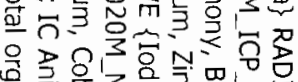

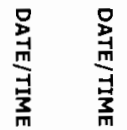
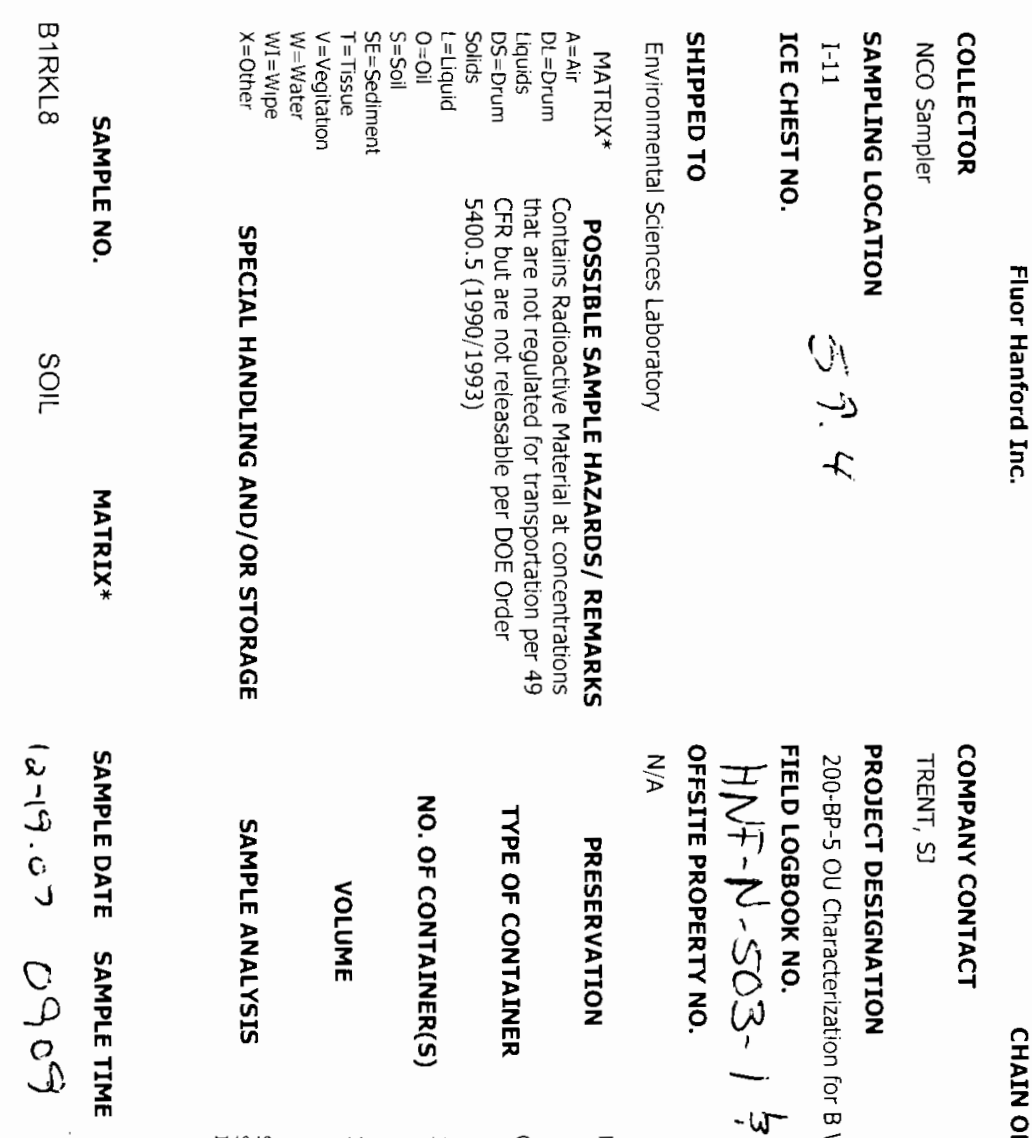

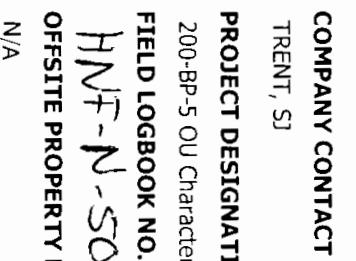

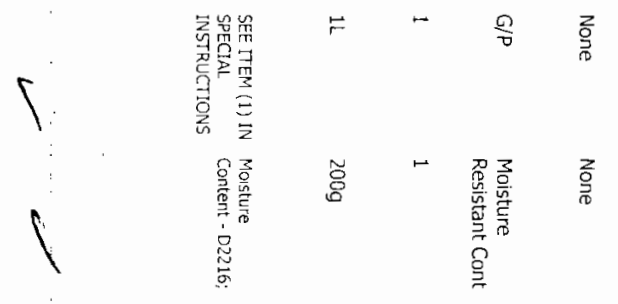

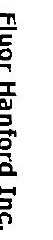

$\frac{9}{2}$

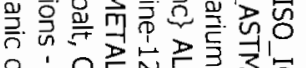

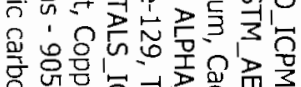

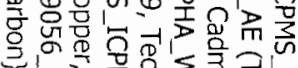

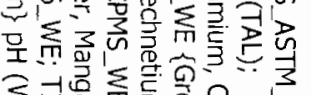

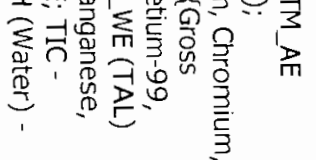

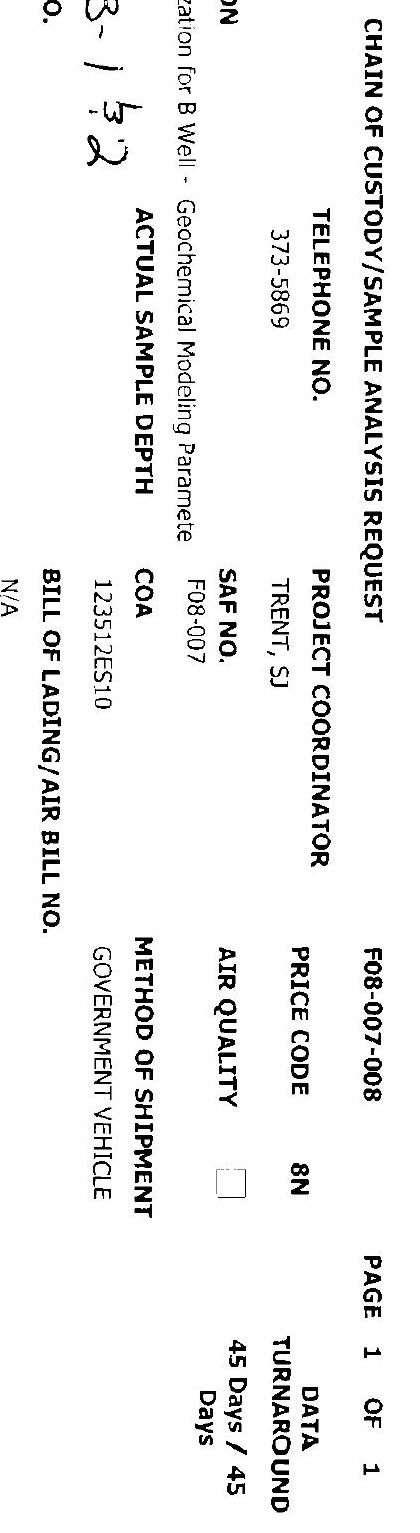




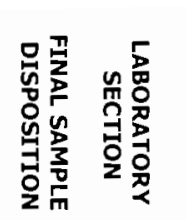
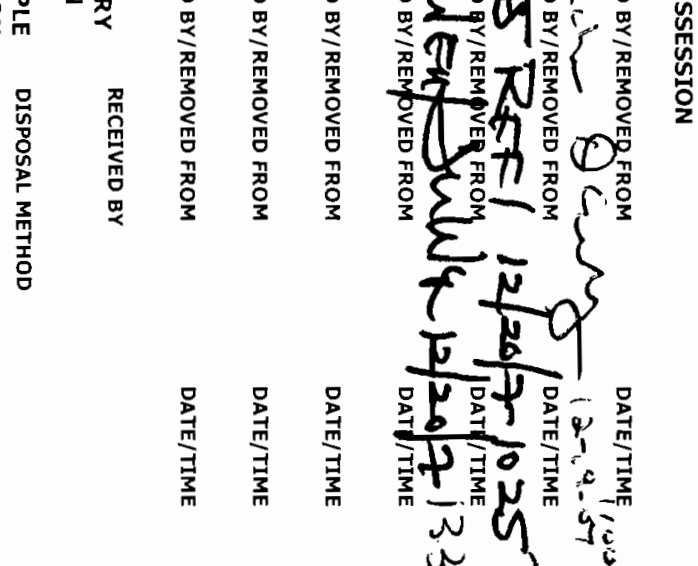

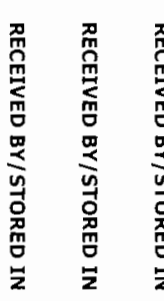

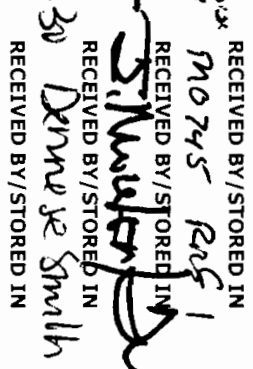

这

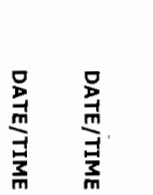

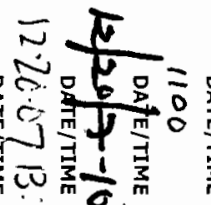

$$
\begin{aligned}
& \text { ن }
\end{aligned}
$$

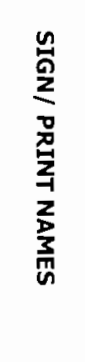

옹

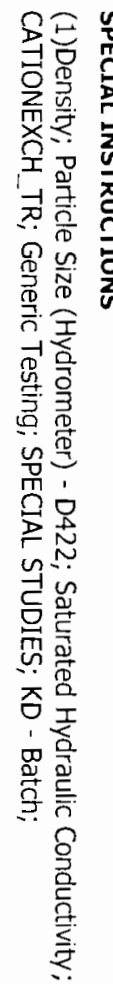

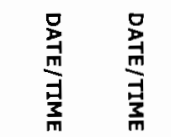

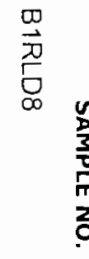

$\stackrel{\infty}{\risingdotseq}$

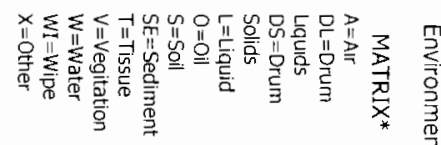

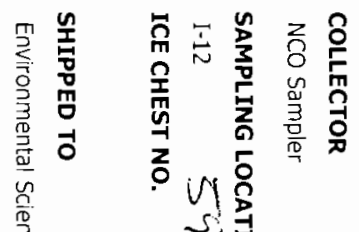

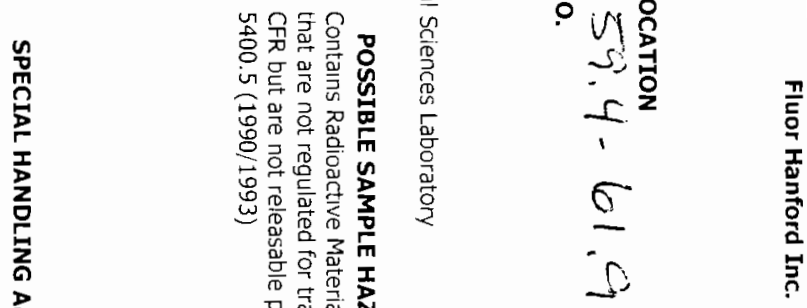

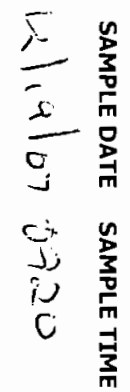

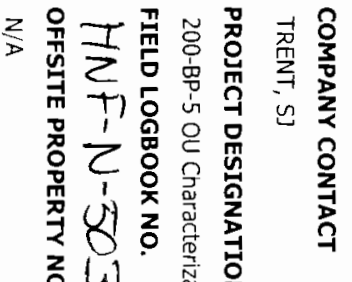

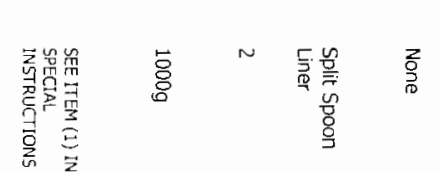

空 总

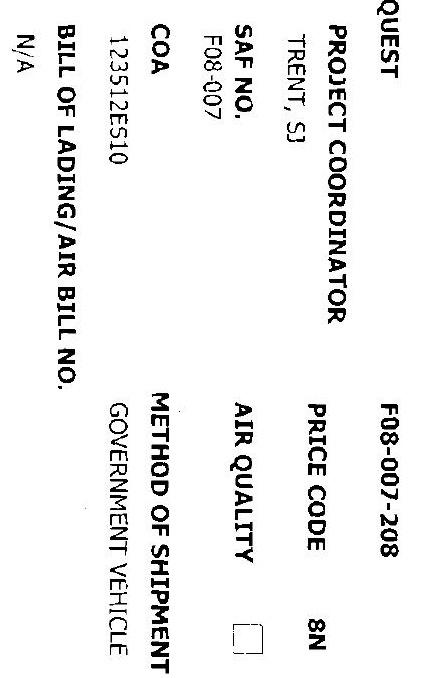




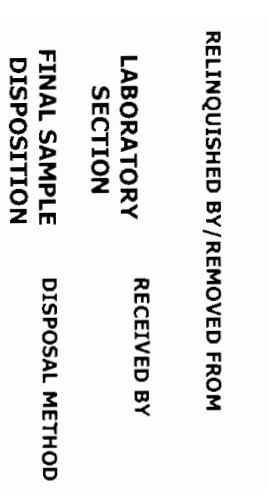
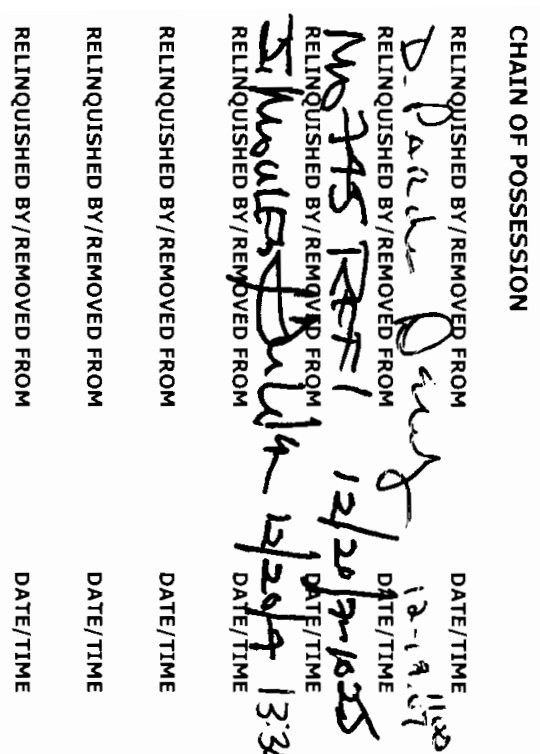

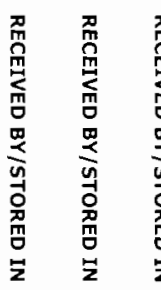
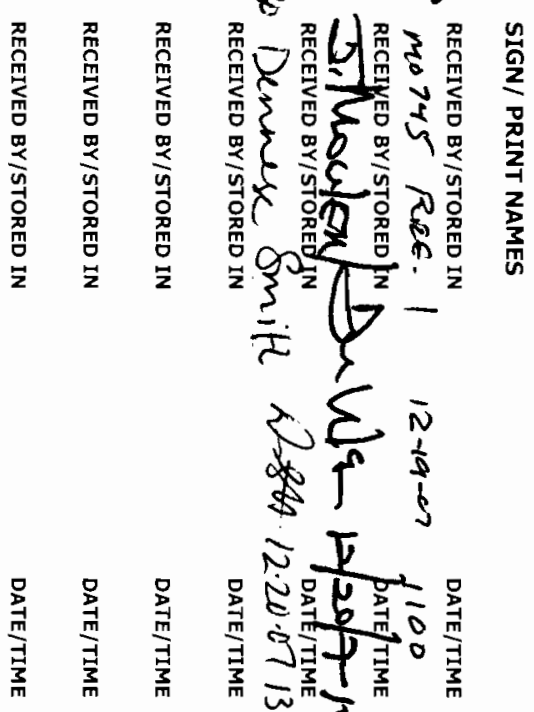

$\stackrel{\mathscr{O}}{\mathrm{O}}$

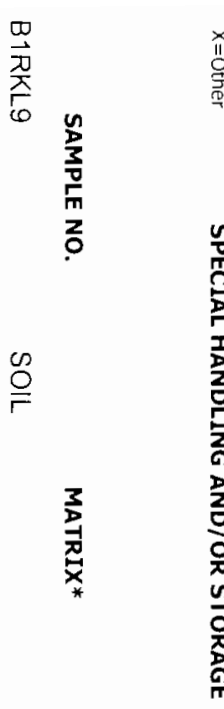

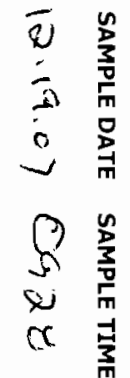

总

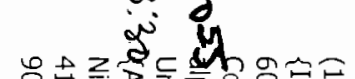

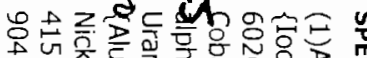

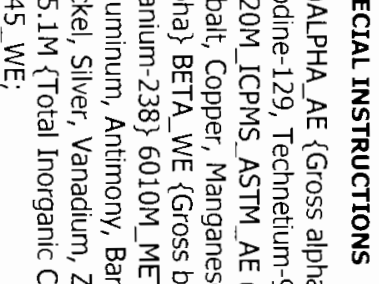

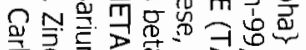

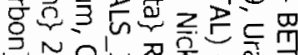

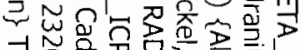

우의. 10

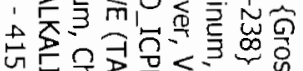

记引思思

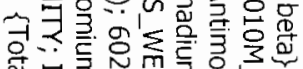

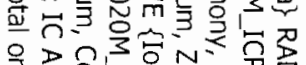

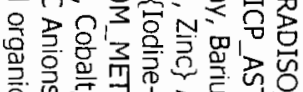

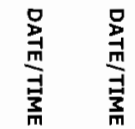

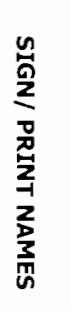

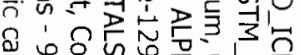

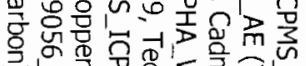

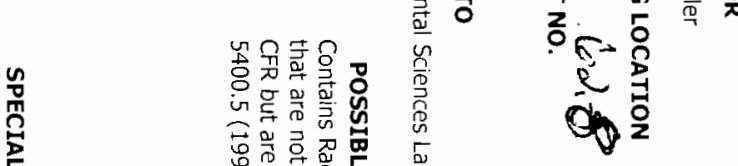

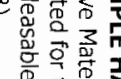

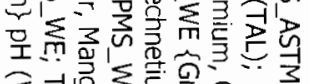

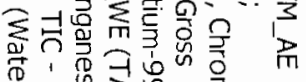

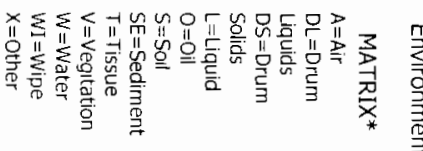

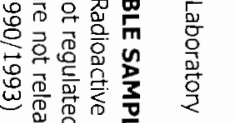

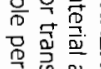

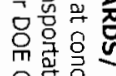

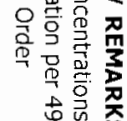

कै口

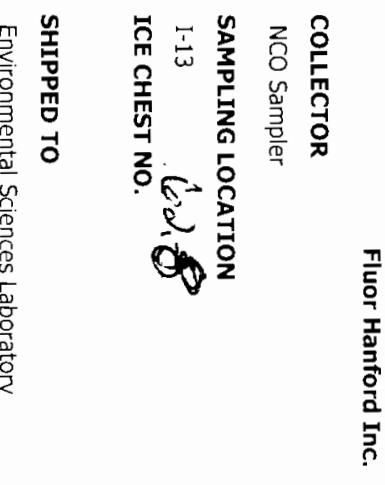

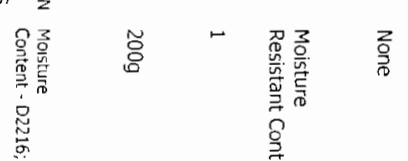

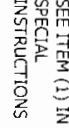

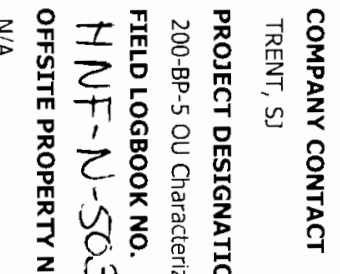

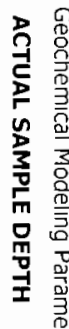

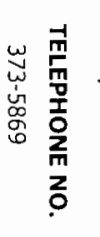

$\frac{1}{-w} \stackrel{\overrightarrow{0}}{\stackrel{5}{\circ}}$

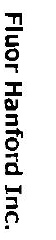

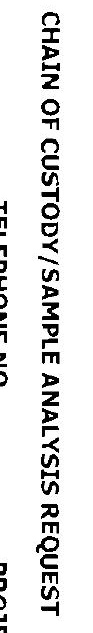
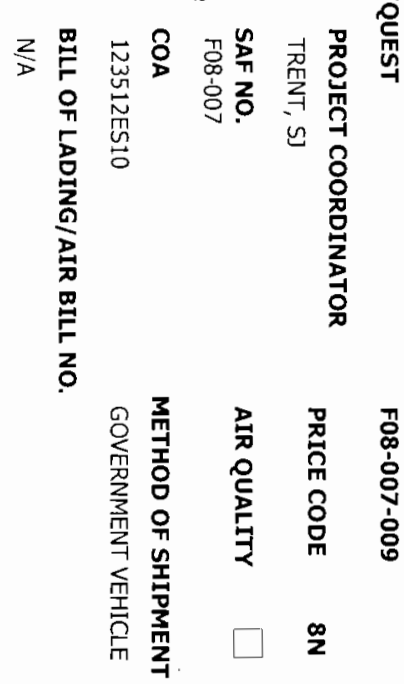

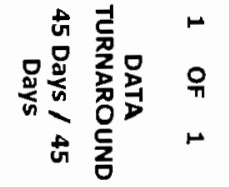




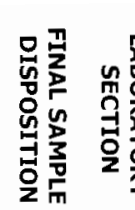

,

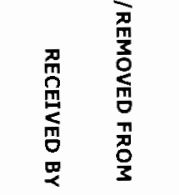

总总

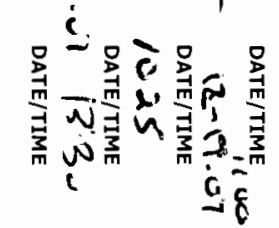

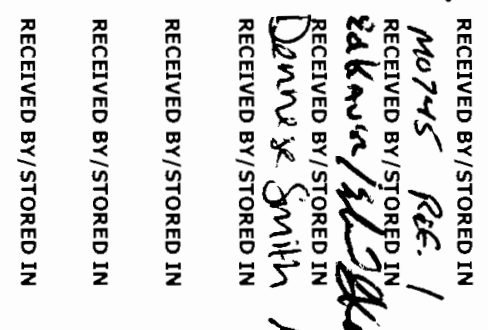

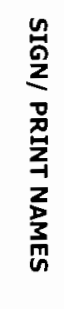

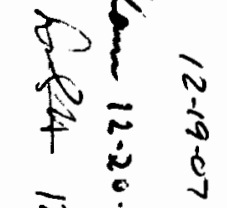

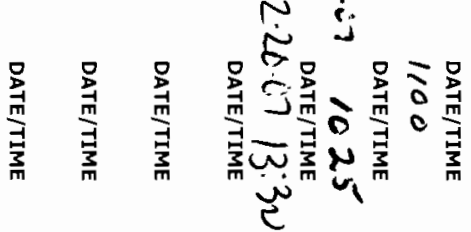

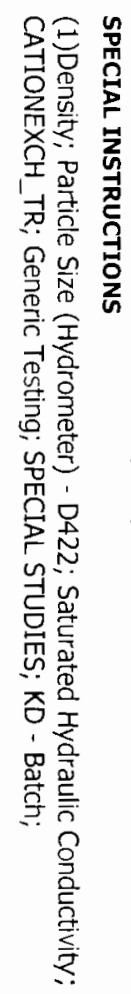

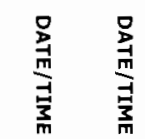

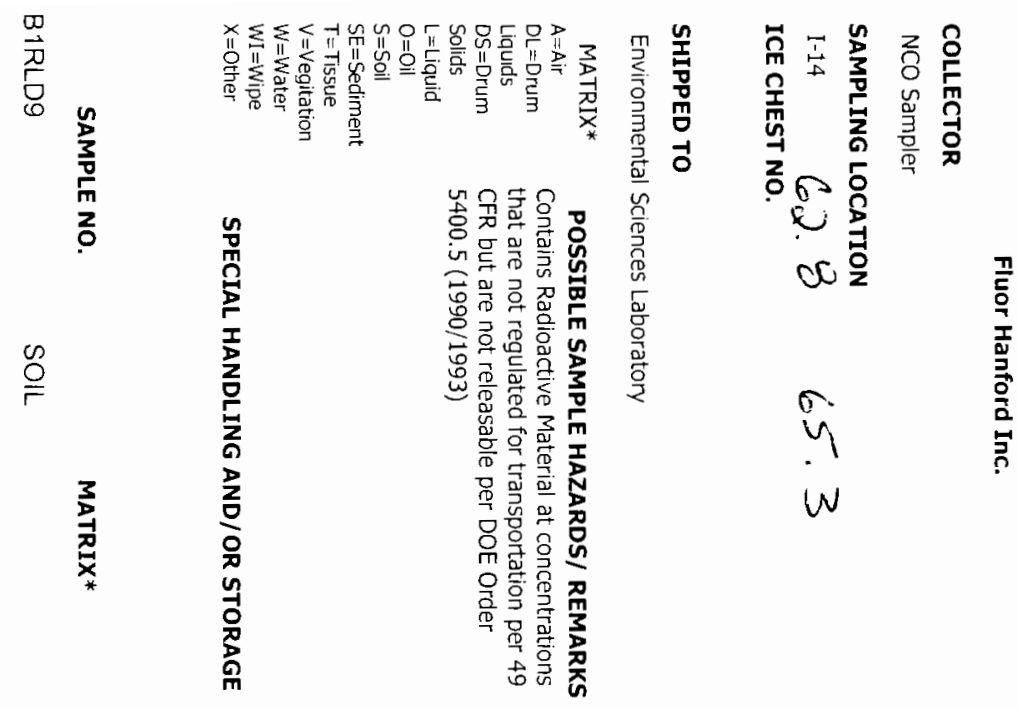

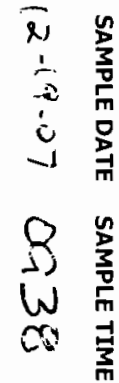

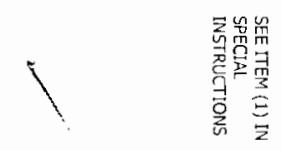

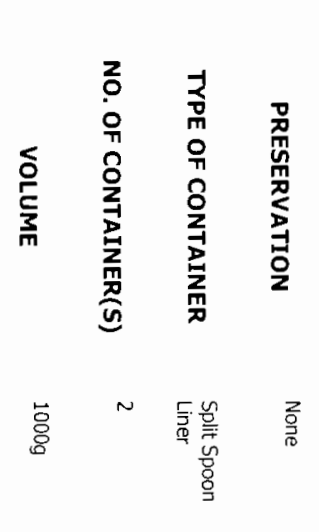

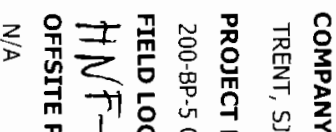

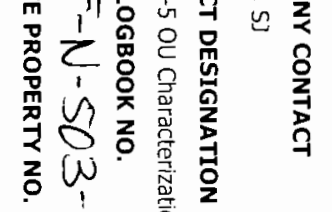

$-\frac{\overline{\bar{s}}}{3}$

少

วัㅇํㅇ

岕营

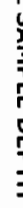

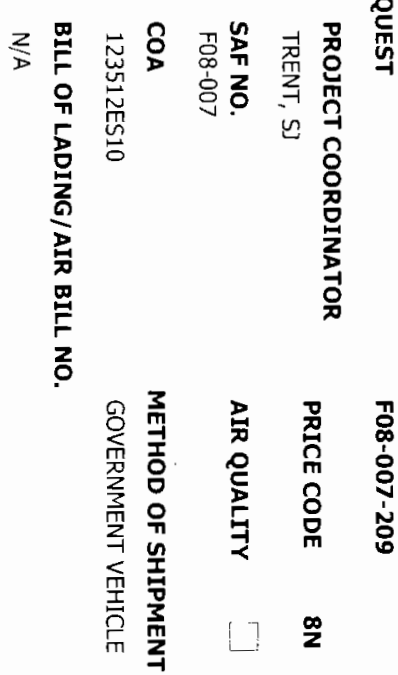

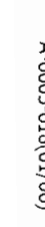

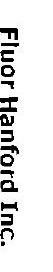




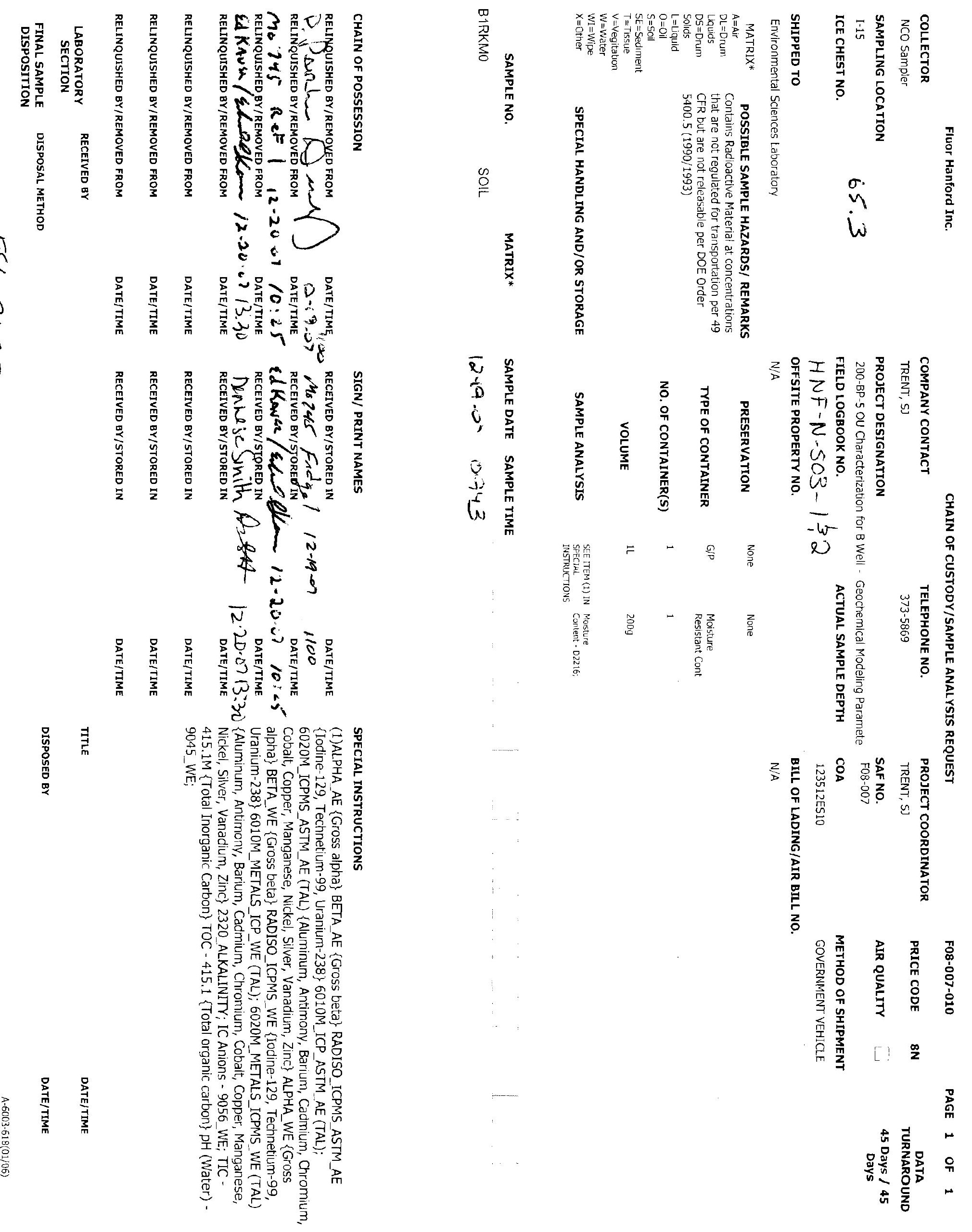



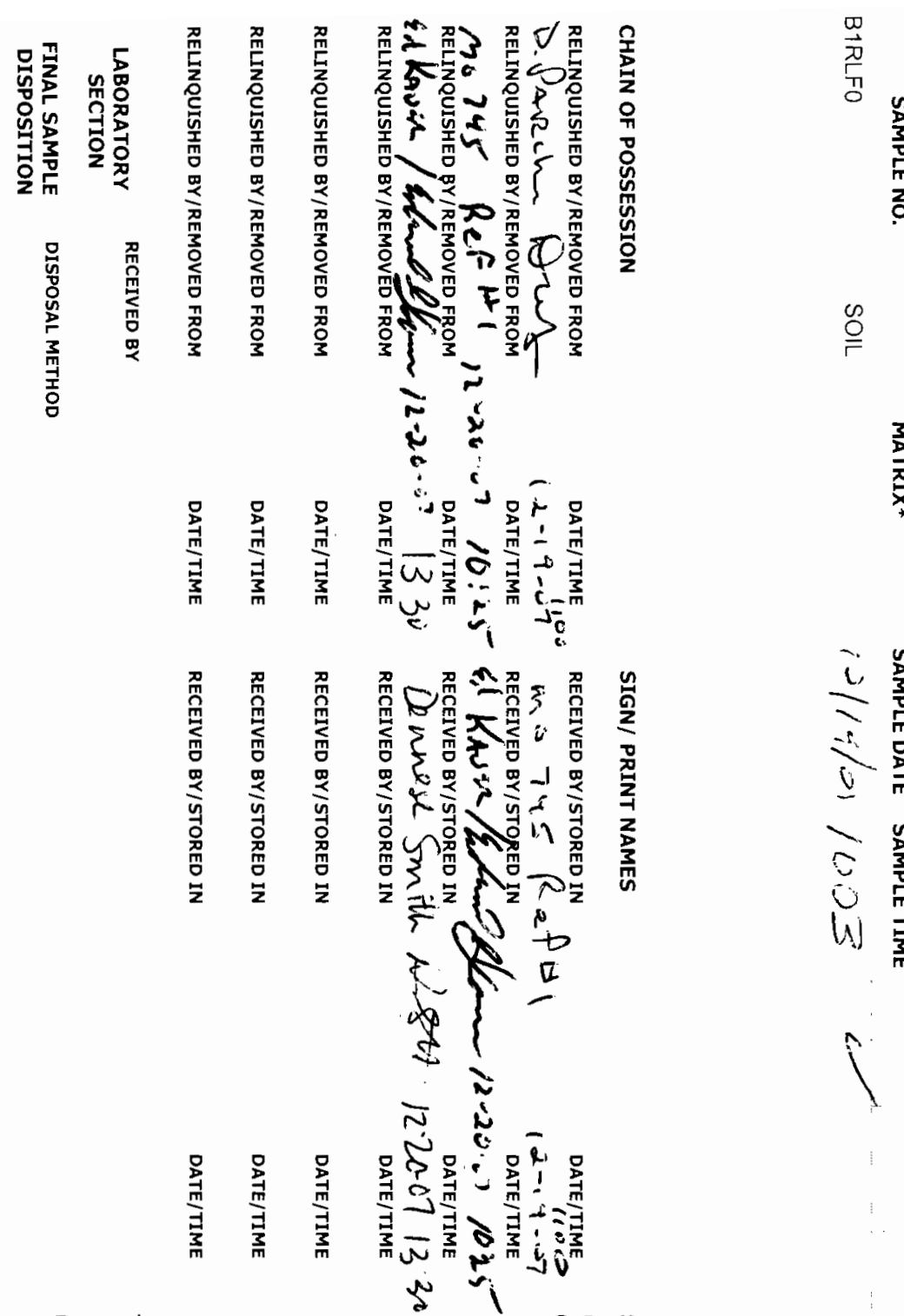

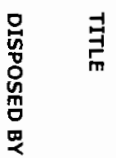

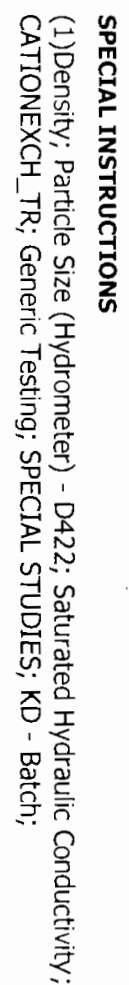

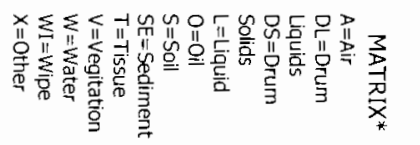

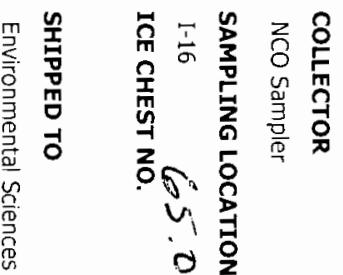

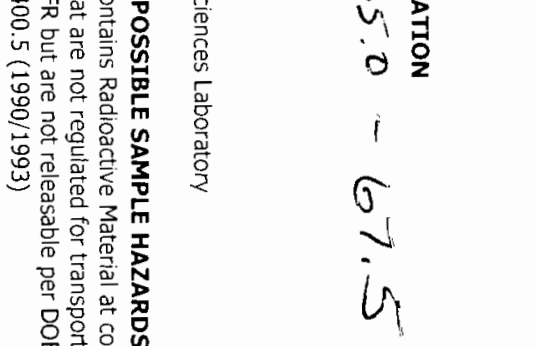

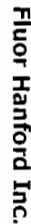

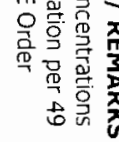

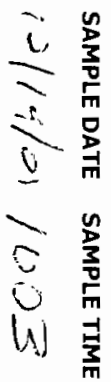

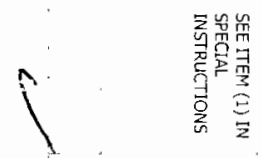

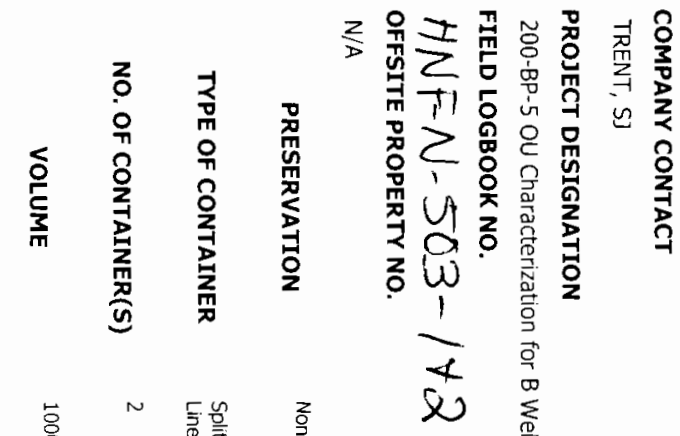

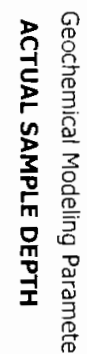

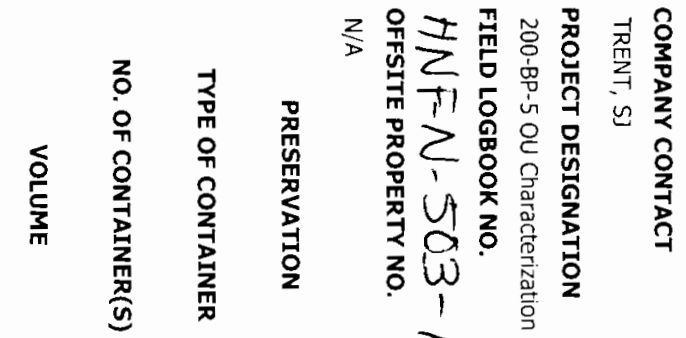

w

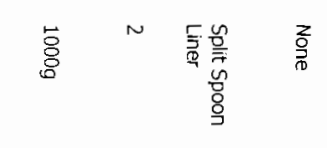

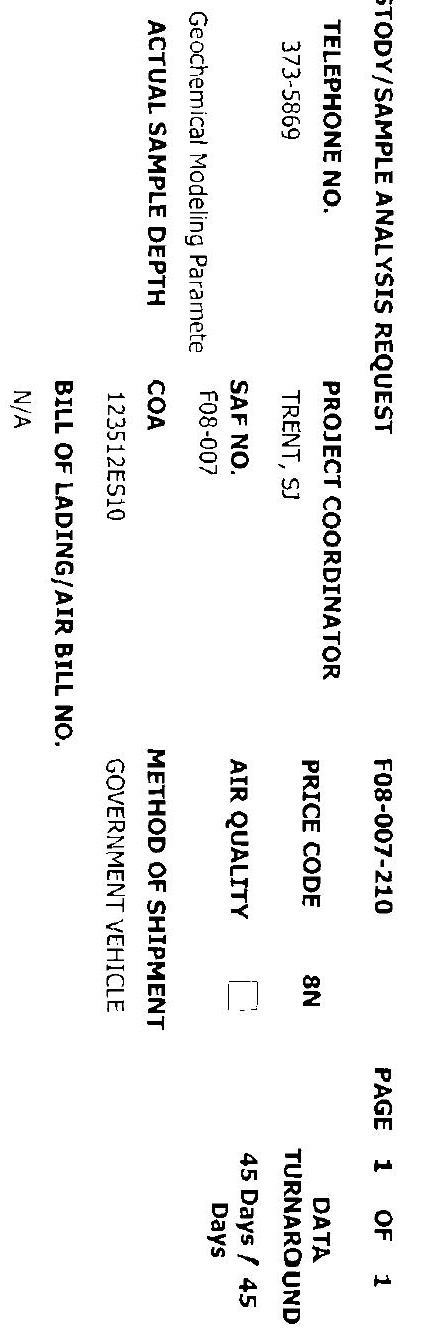


唯毁

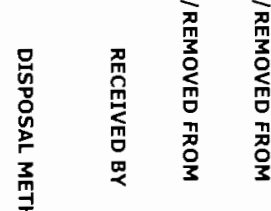

$\infty$
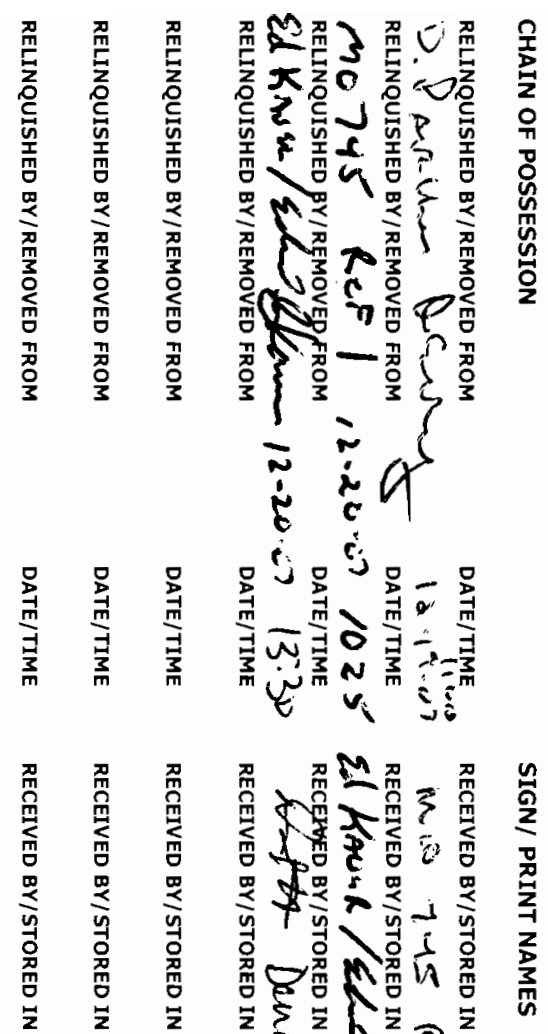

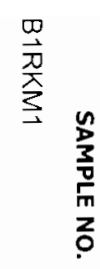

$\stackrel{\mathscr{O}}{\rightleftharpoons}$

$\underset{*}{\stackrel{3}{3}}$

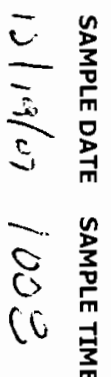

1

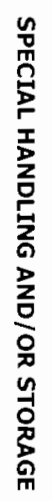

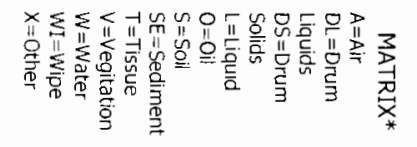

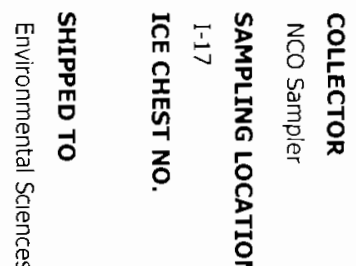

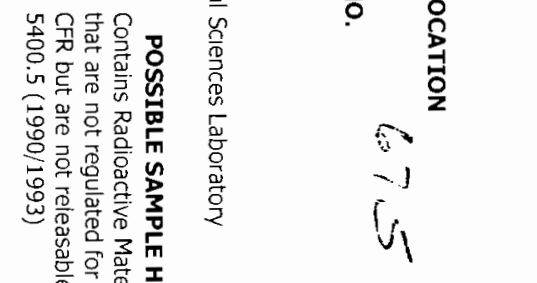

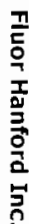

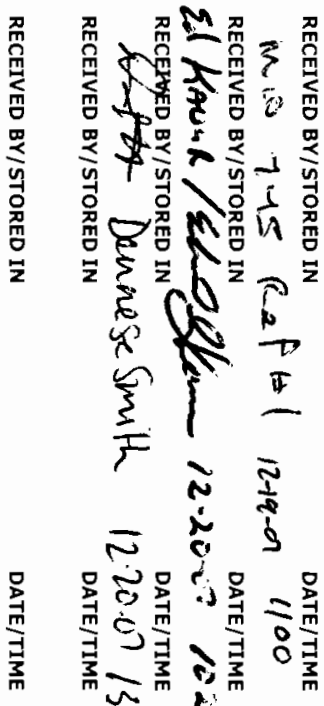

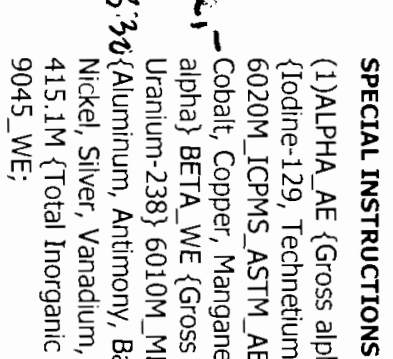

N

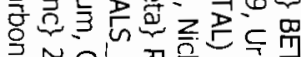

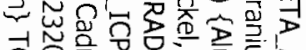

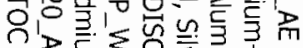

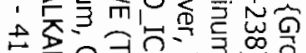

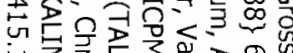

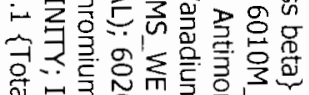

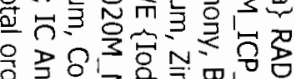

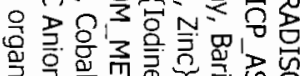

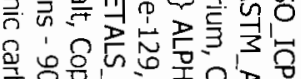

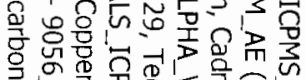

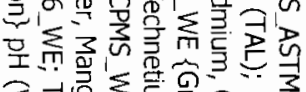

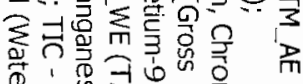

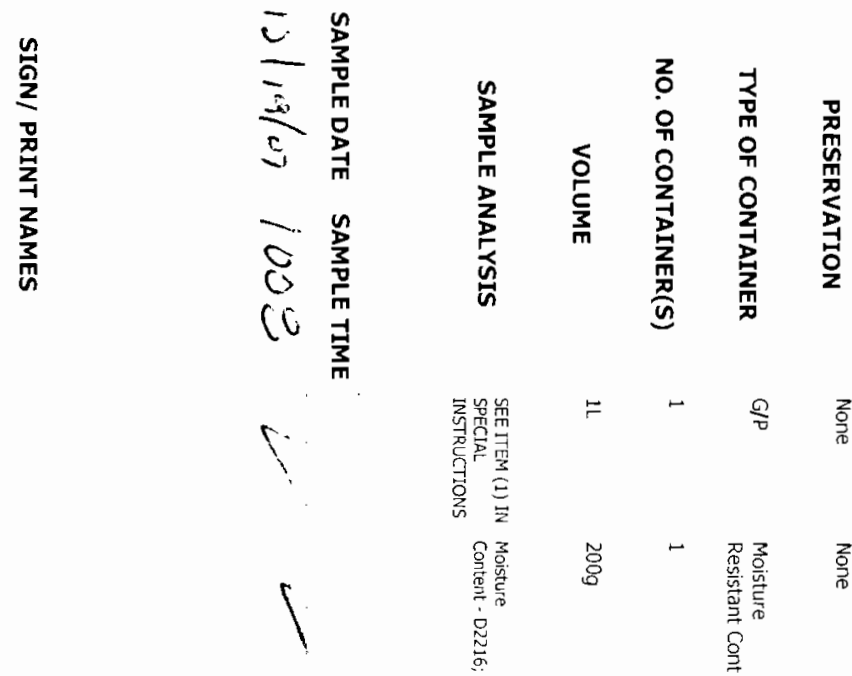

2

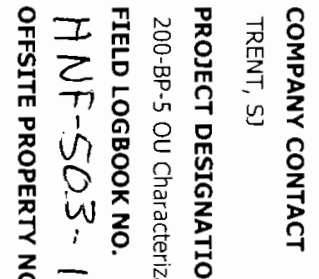

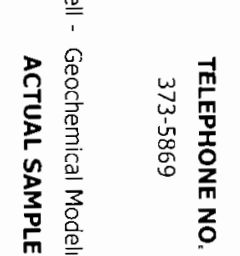

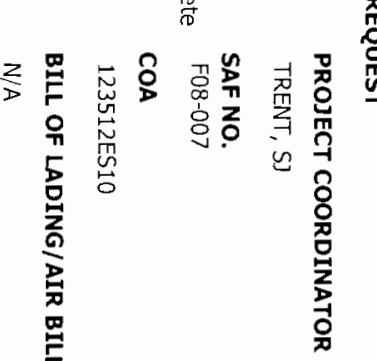

z

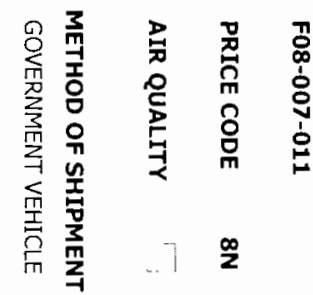

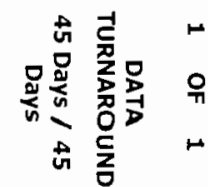




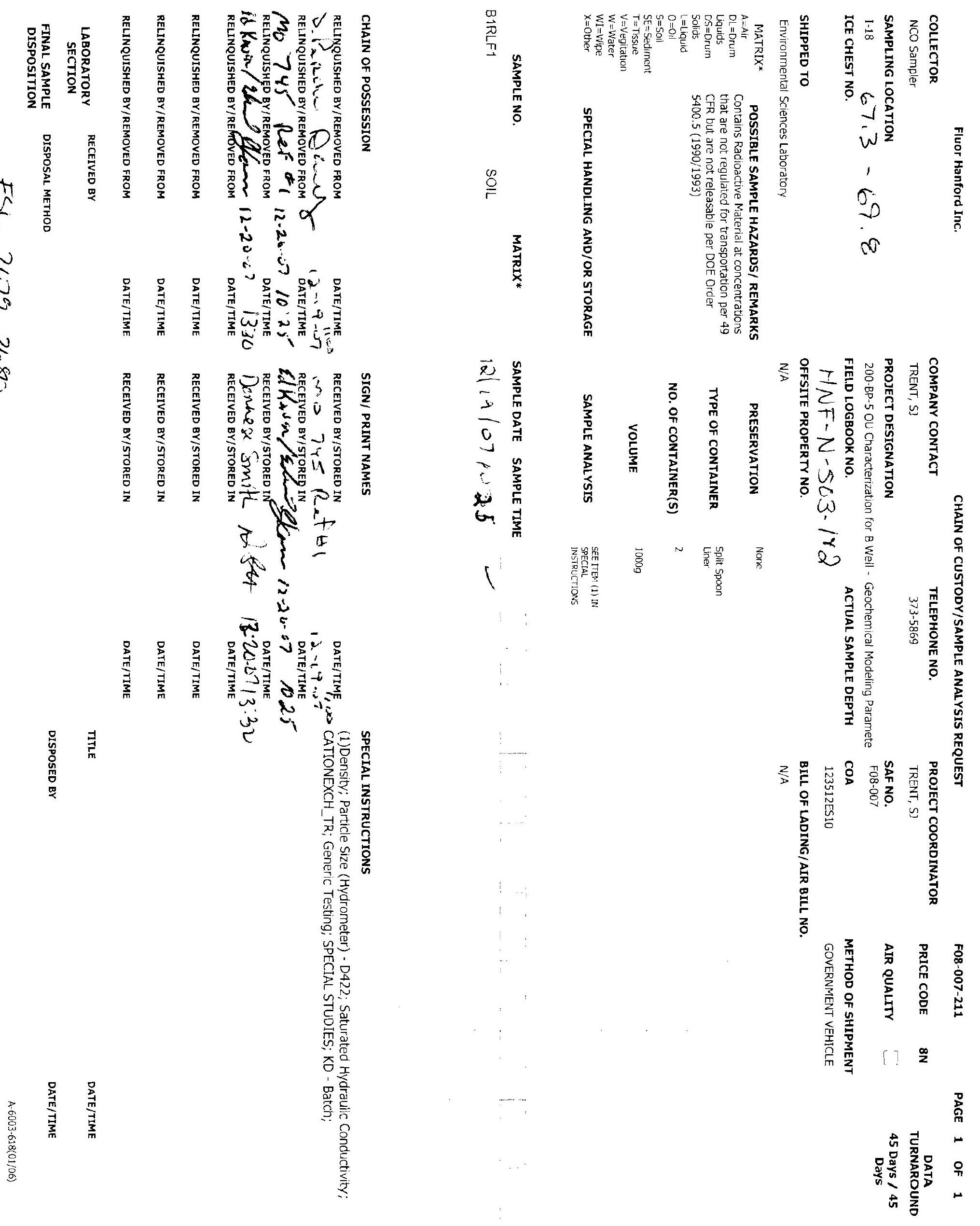




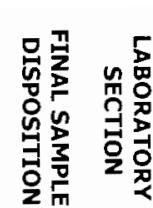

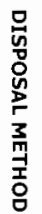

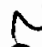

6

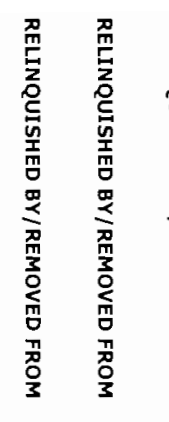

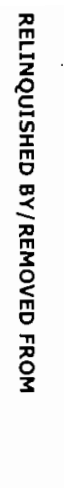

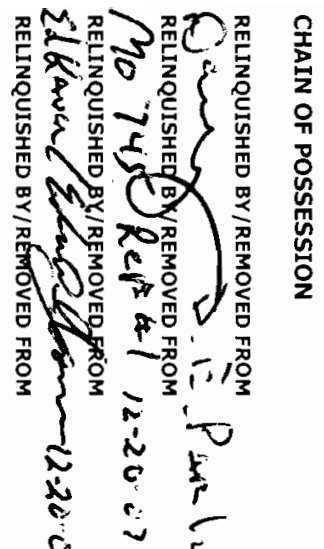

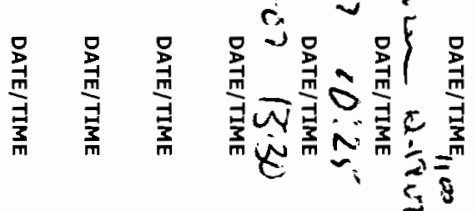

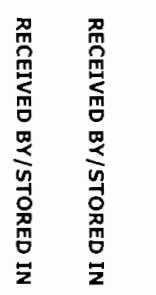

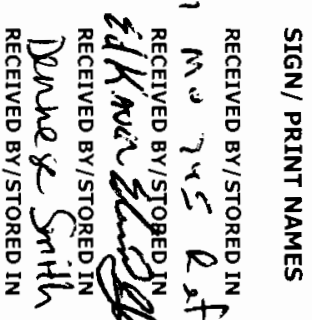

$\sum_{i}^{\infty}$

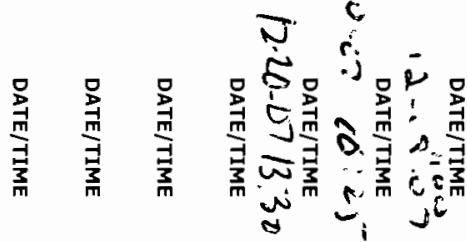

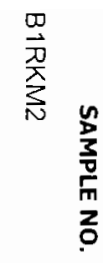

$\stackrel{\mathscr{O}}{\stackrel{2}{ }}$

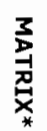

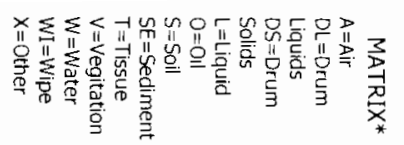

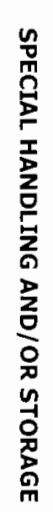

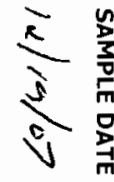

$0 \sum_{\frac{3}{5}}^{n} \frac{2}{\frac{2}{2}}$

O)

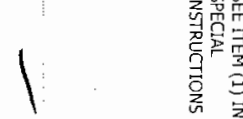

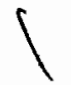

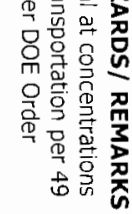

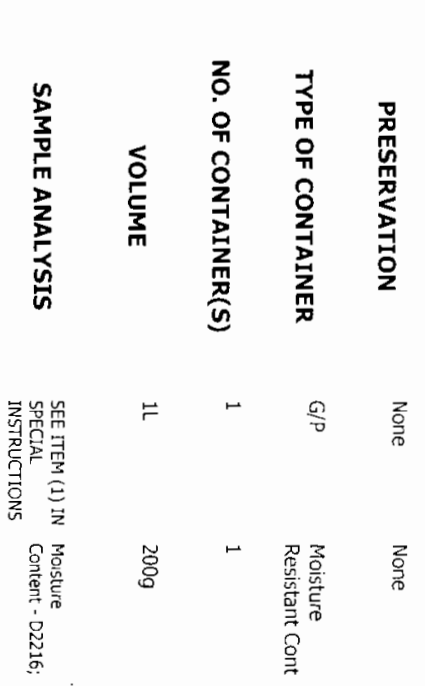

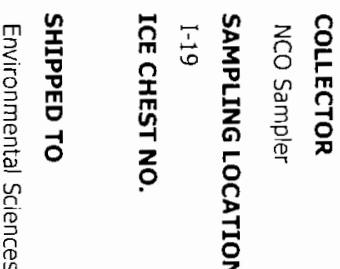

0

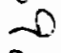

ar

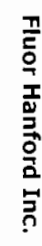

z 윢

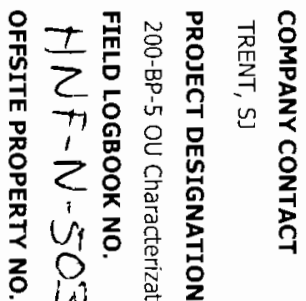

국

e)

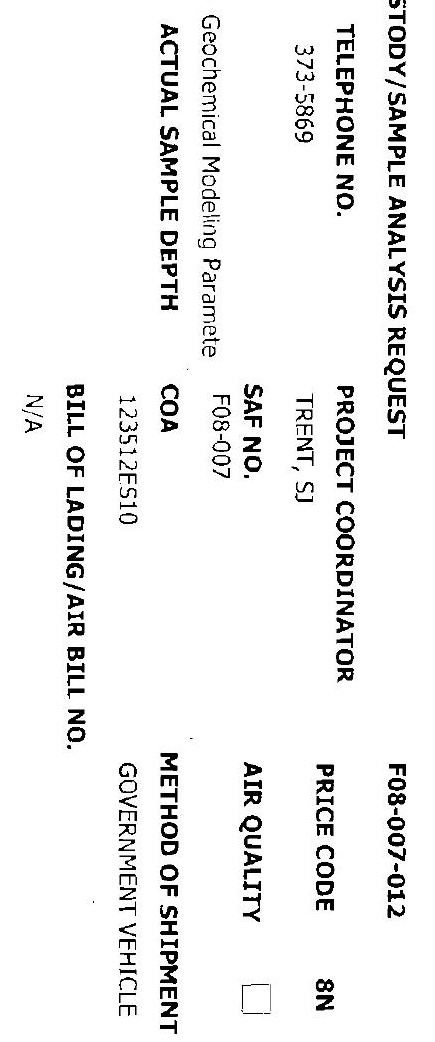

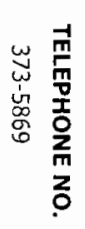

iㅣㅇ

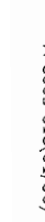

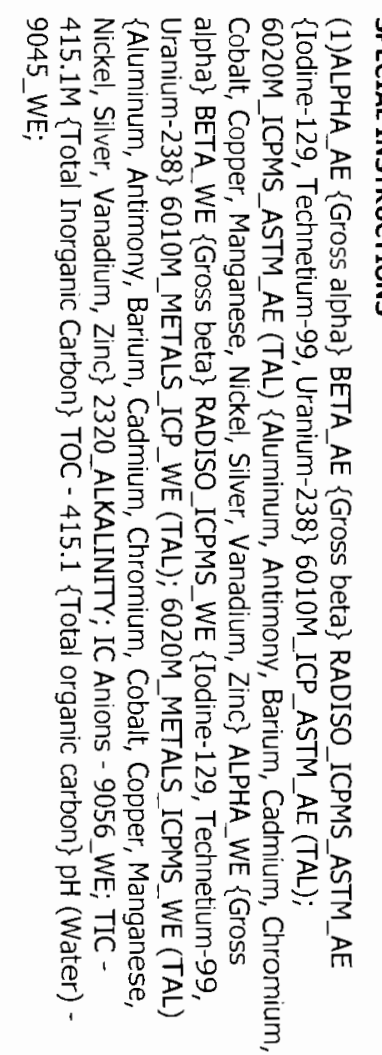




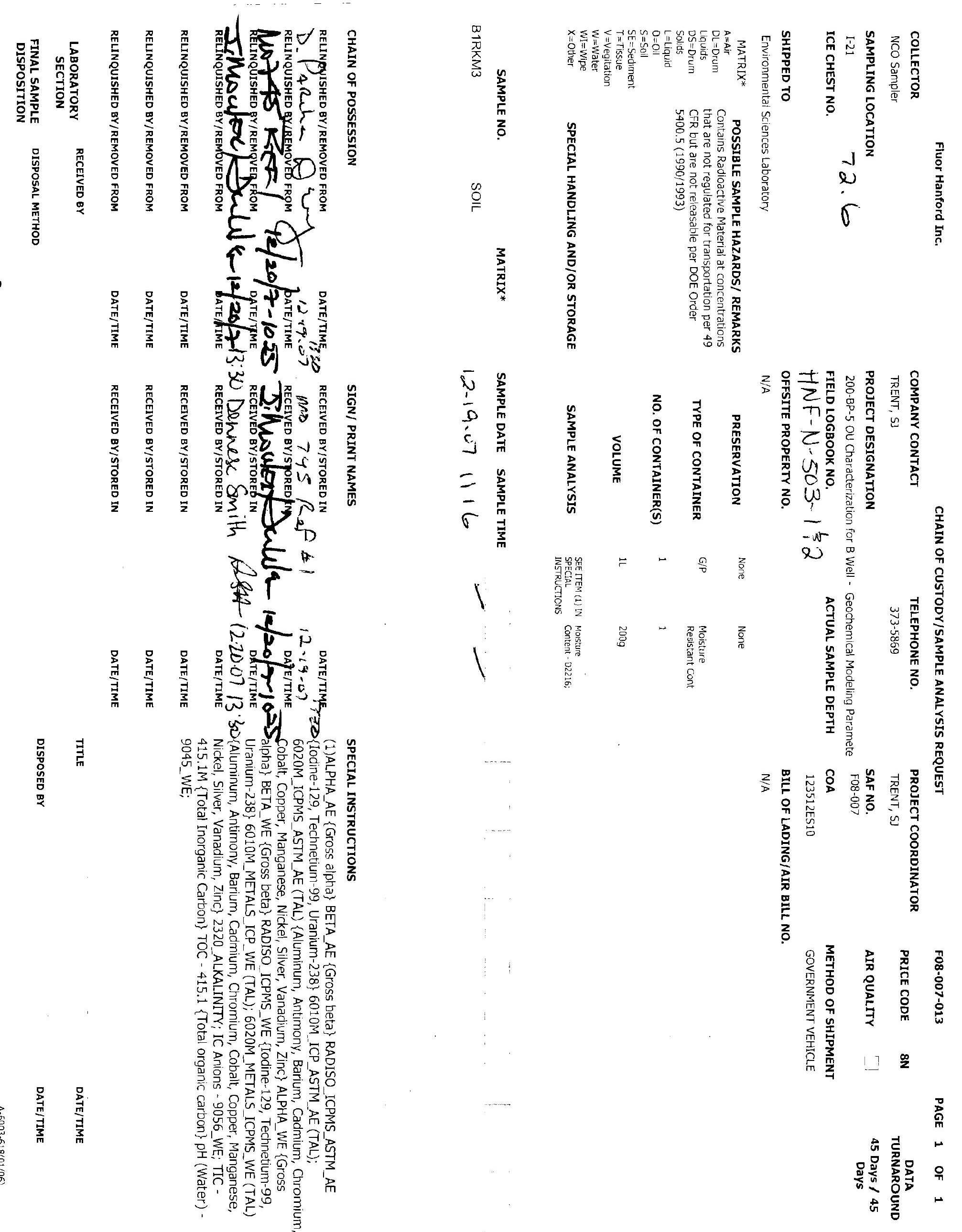



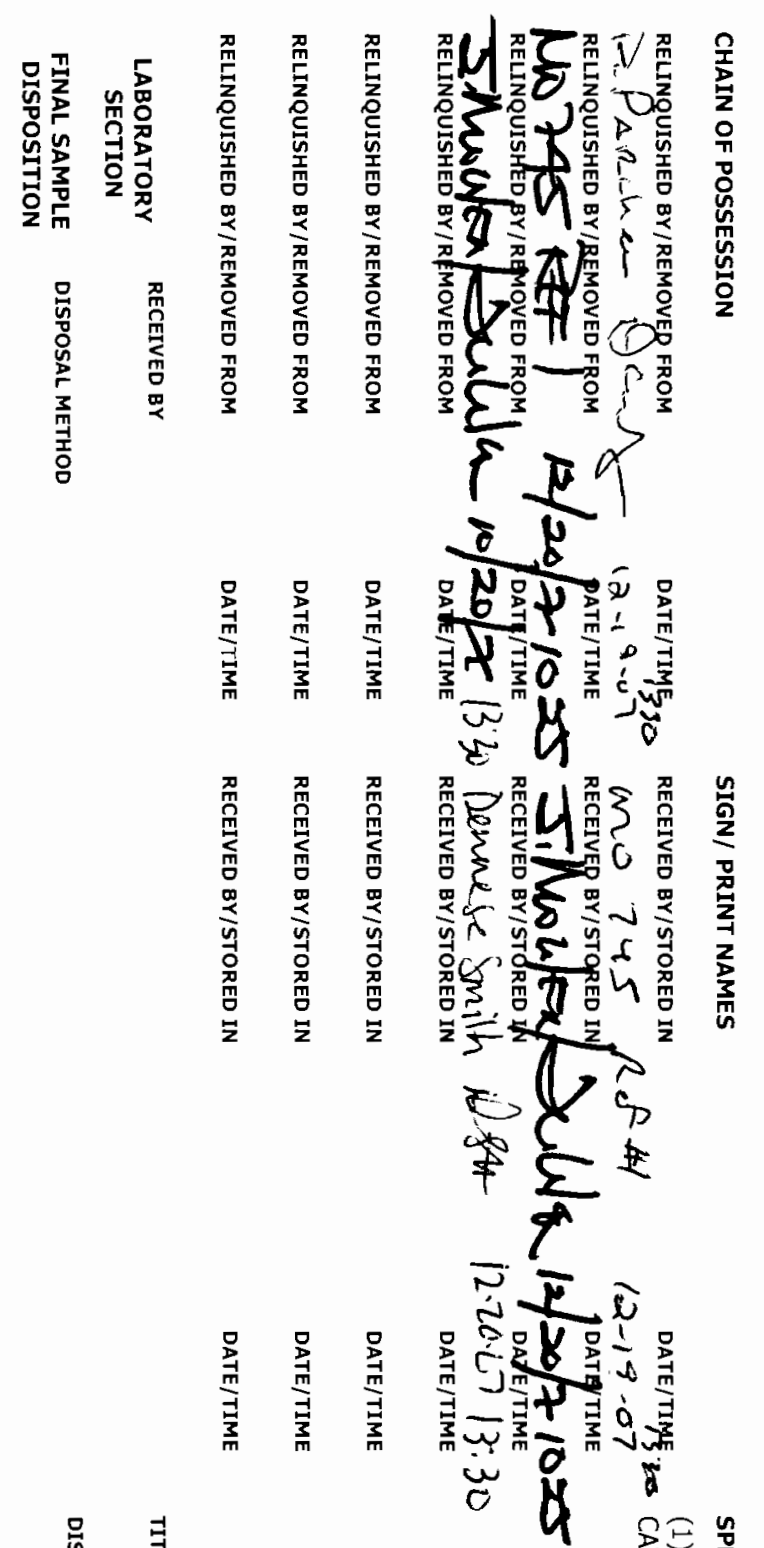

옹

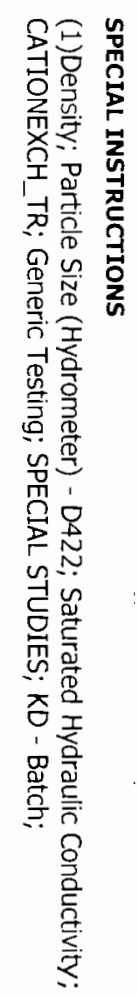

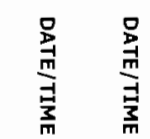

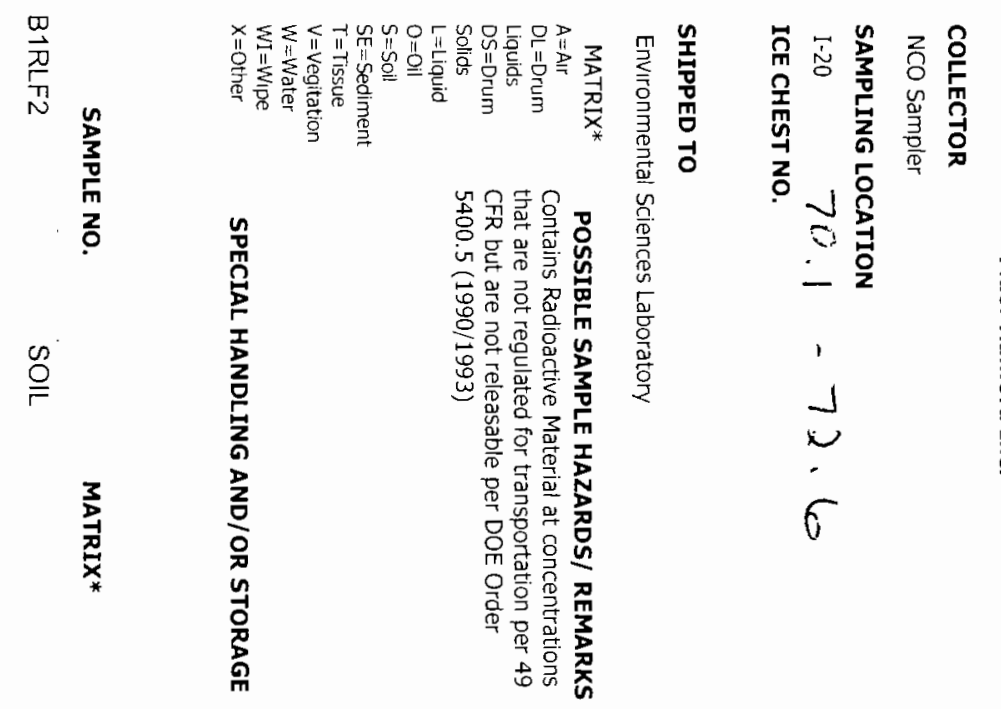

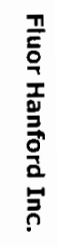

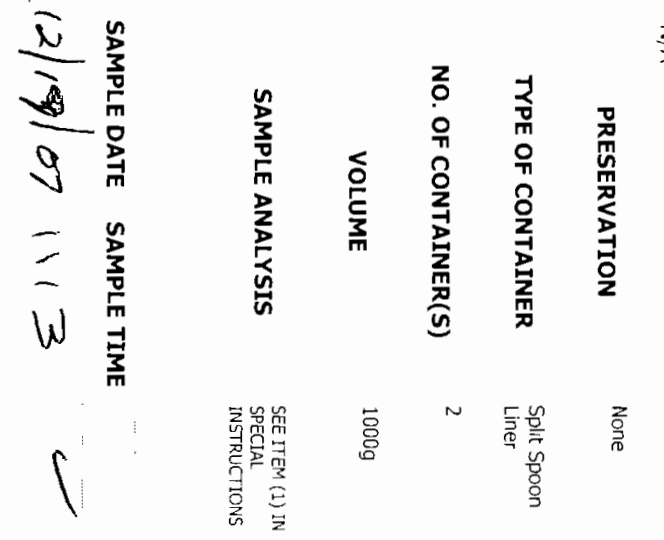

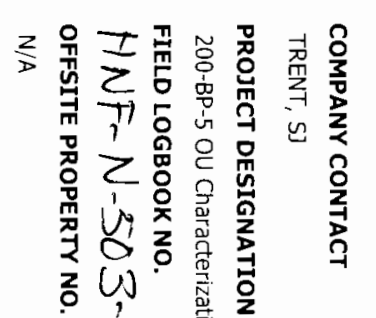

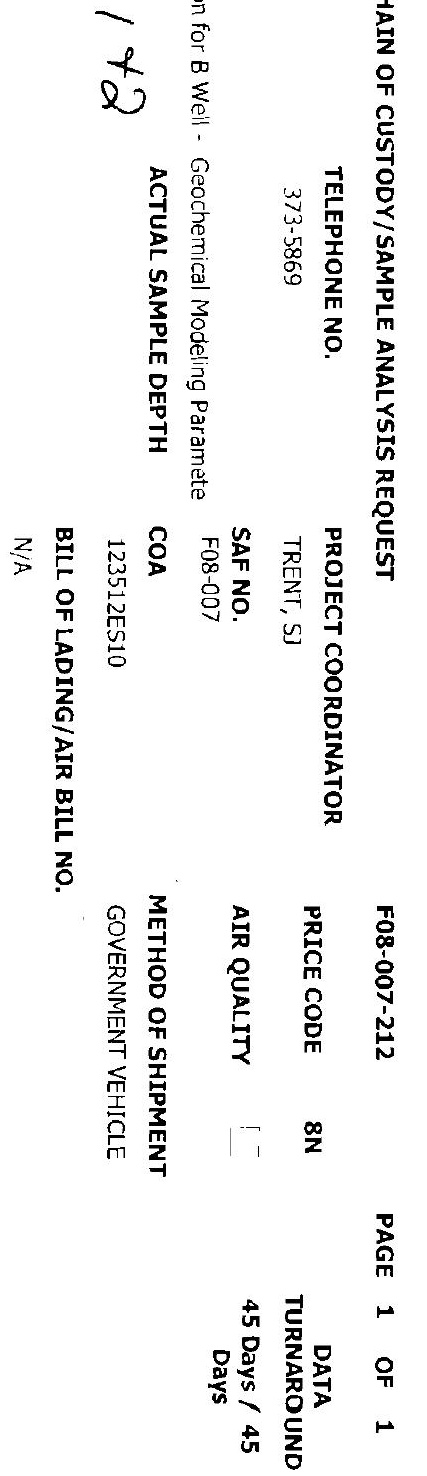




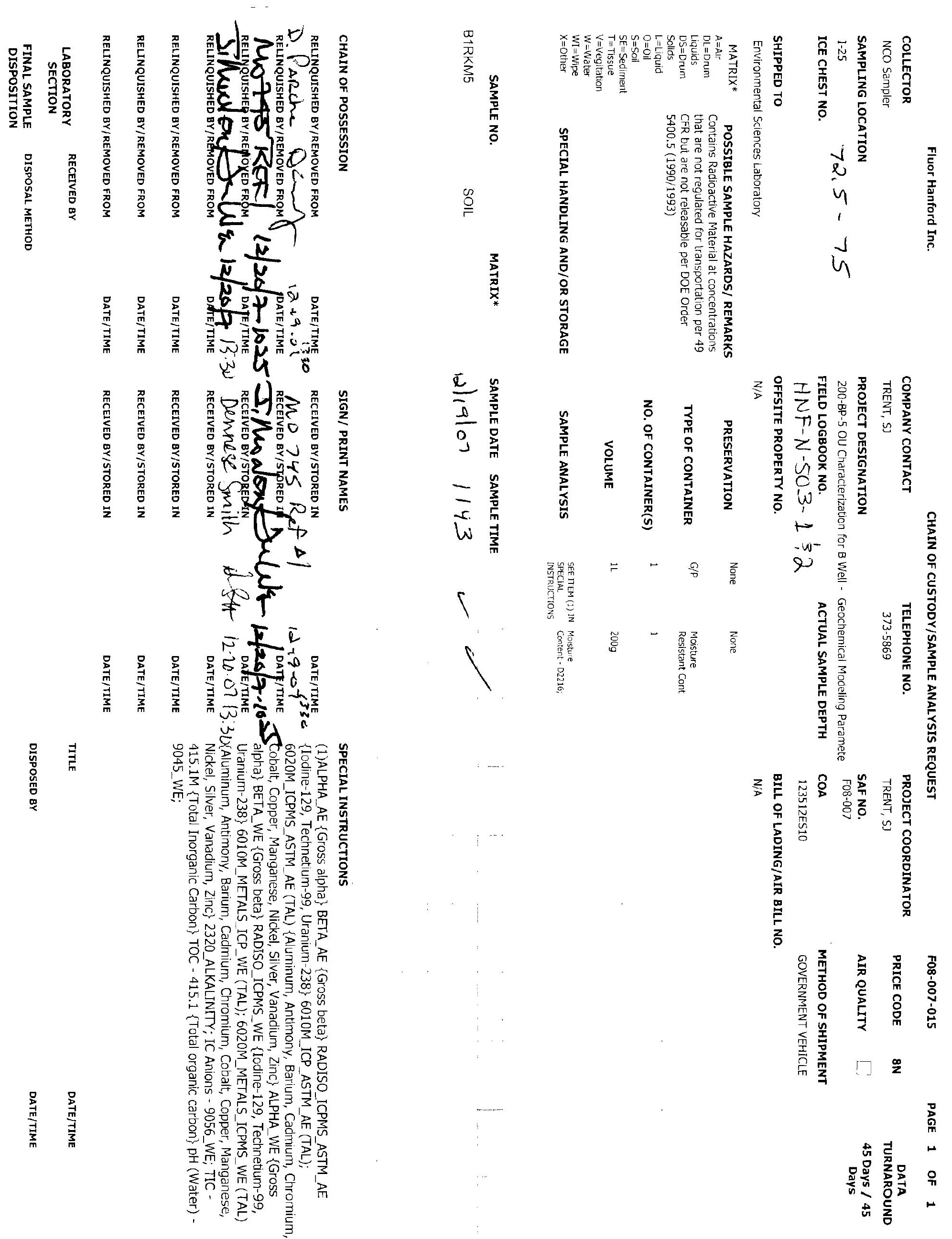




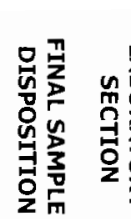
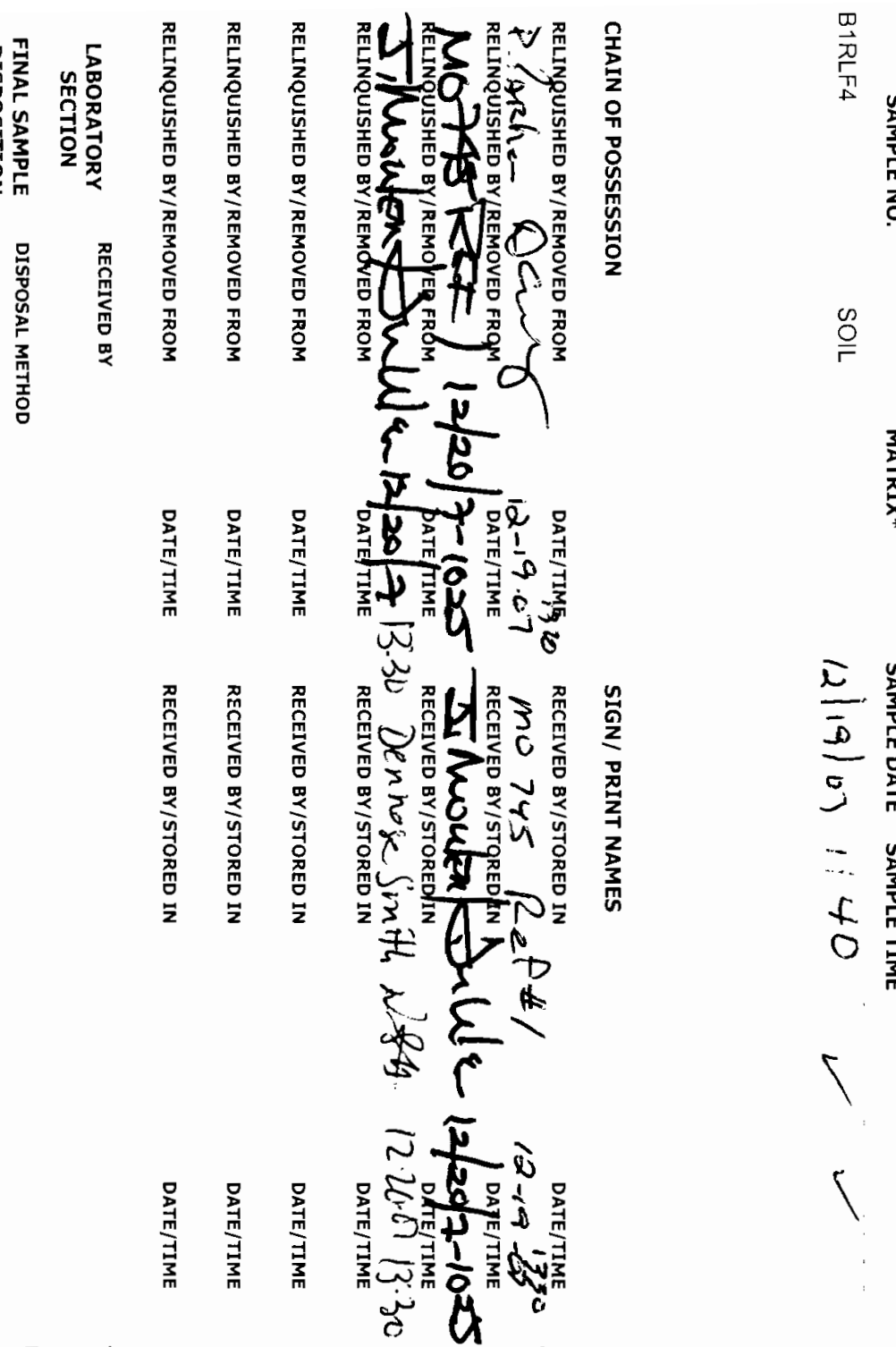

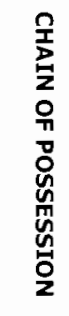

$\stackrel{0}{\circ}$

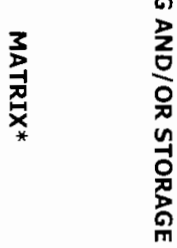

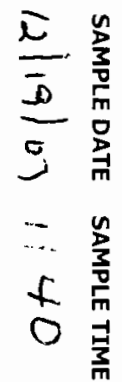

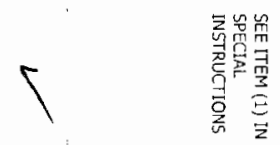

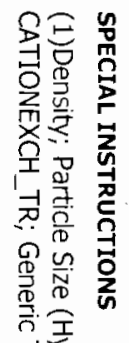

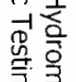

热

㖞

罗品

굴

需

즘 목

迩

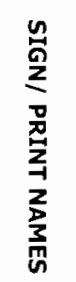

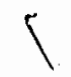

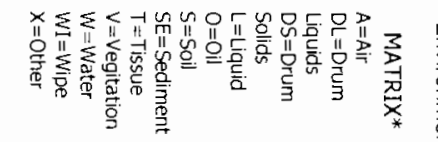

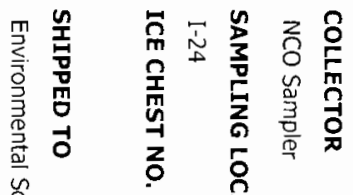
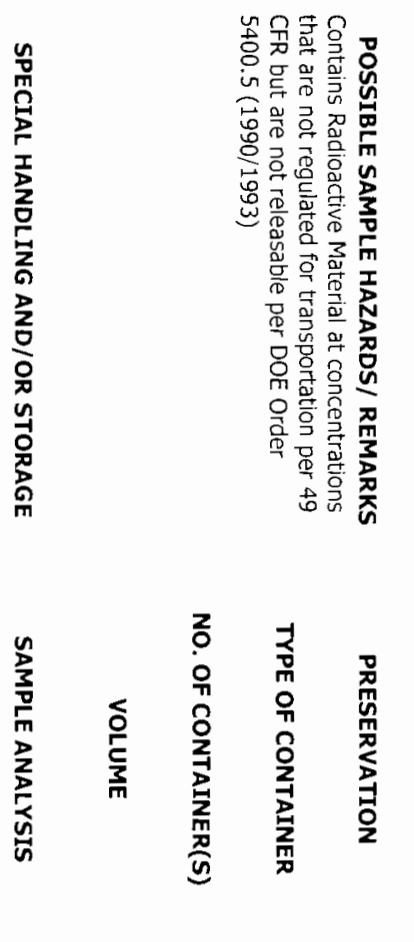

z

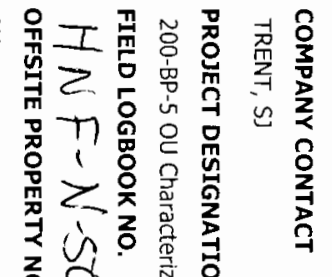

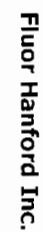

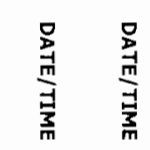

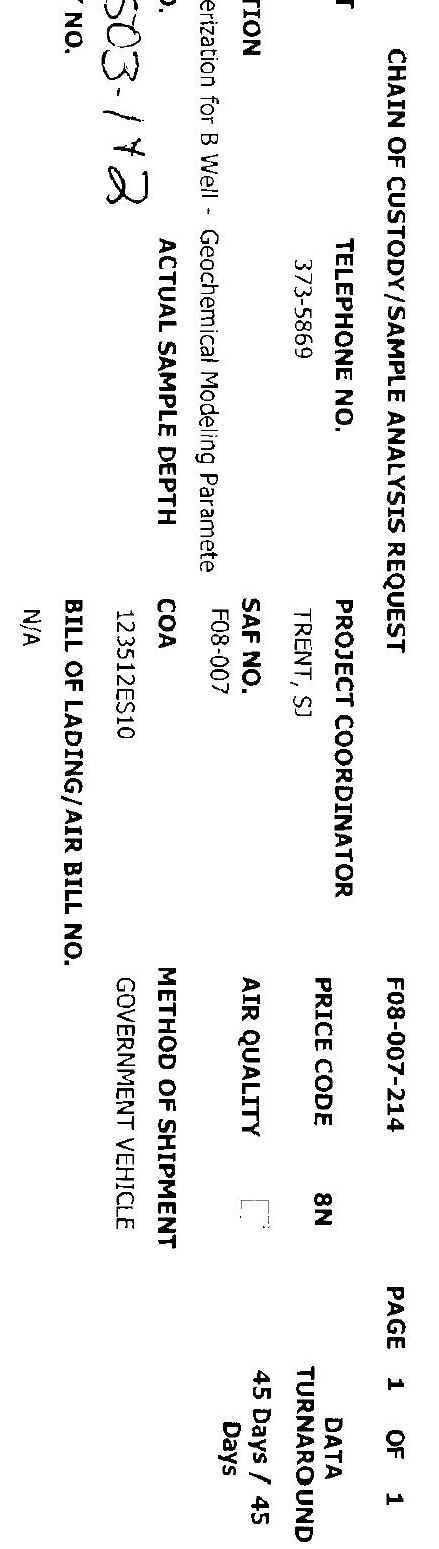




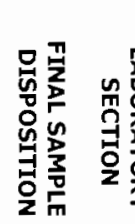 \\ 竞 竞}
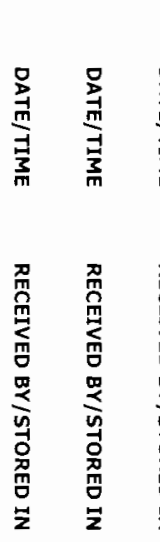

罗
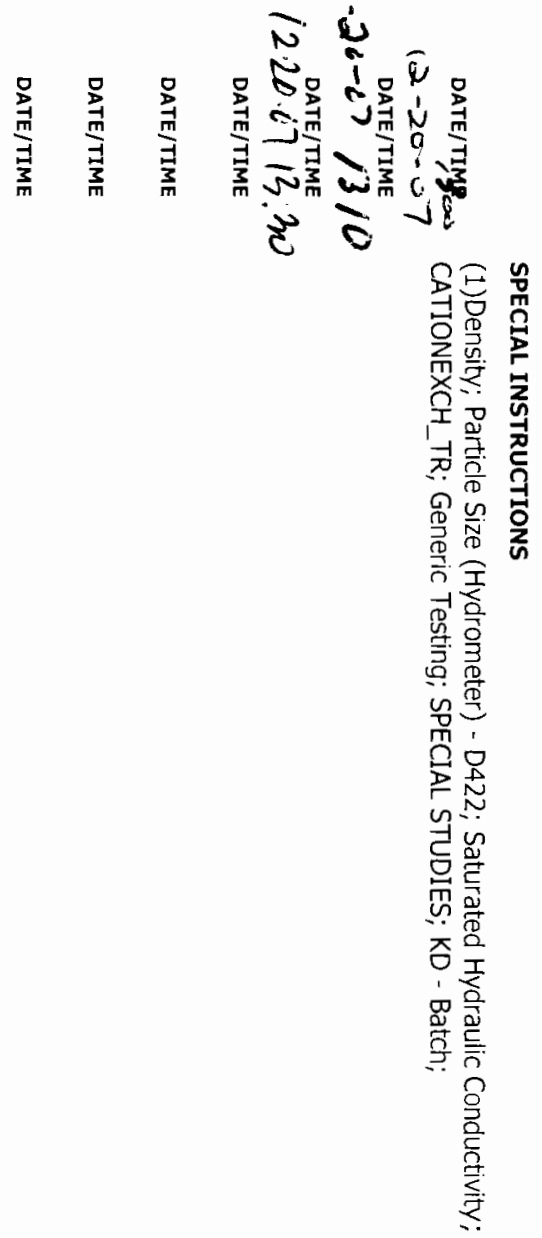

㕝

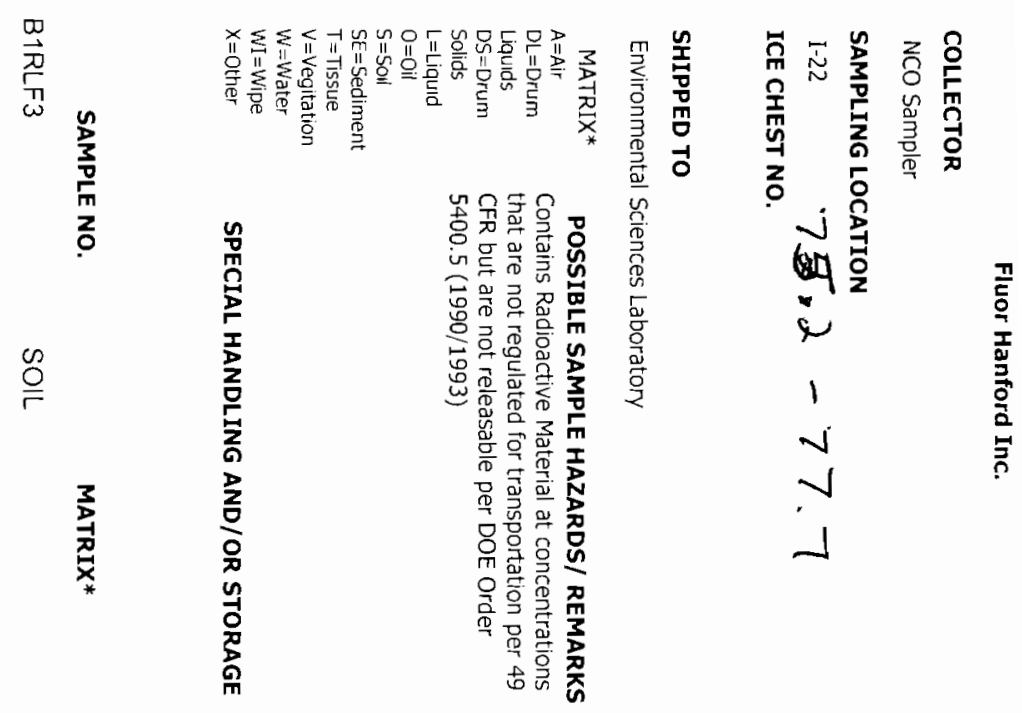

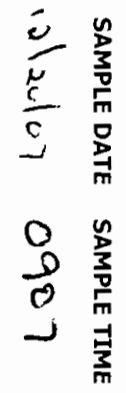

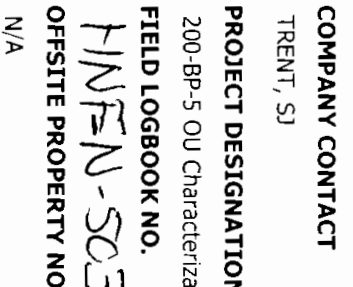

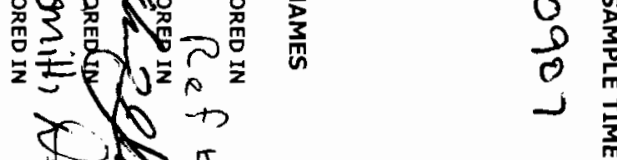

\

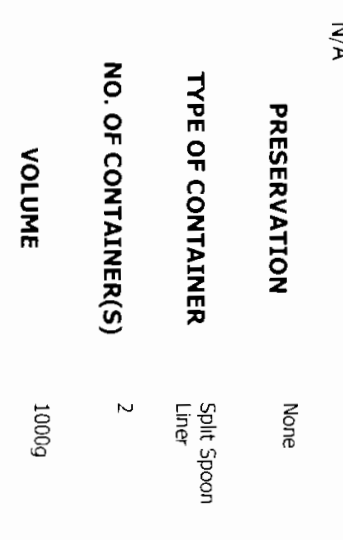

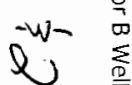

学

w
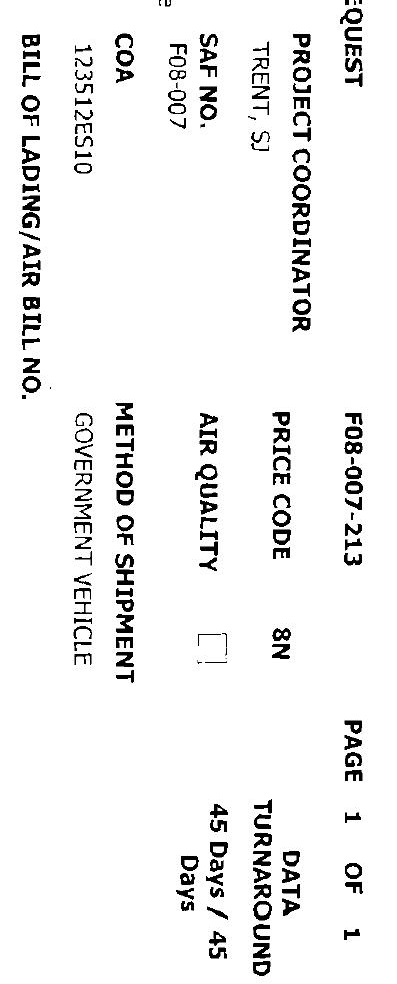

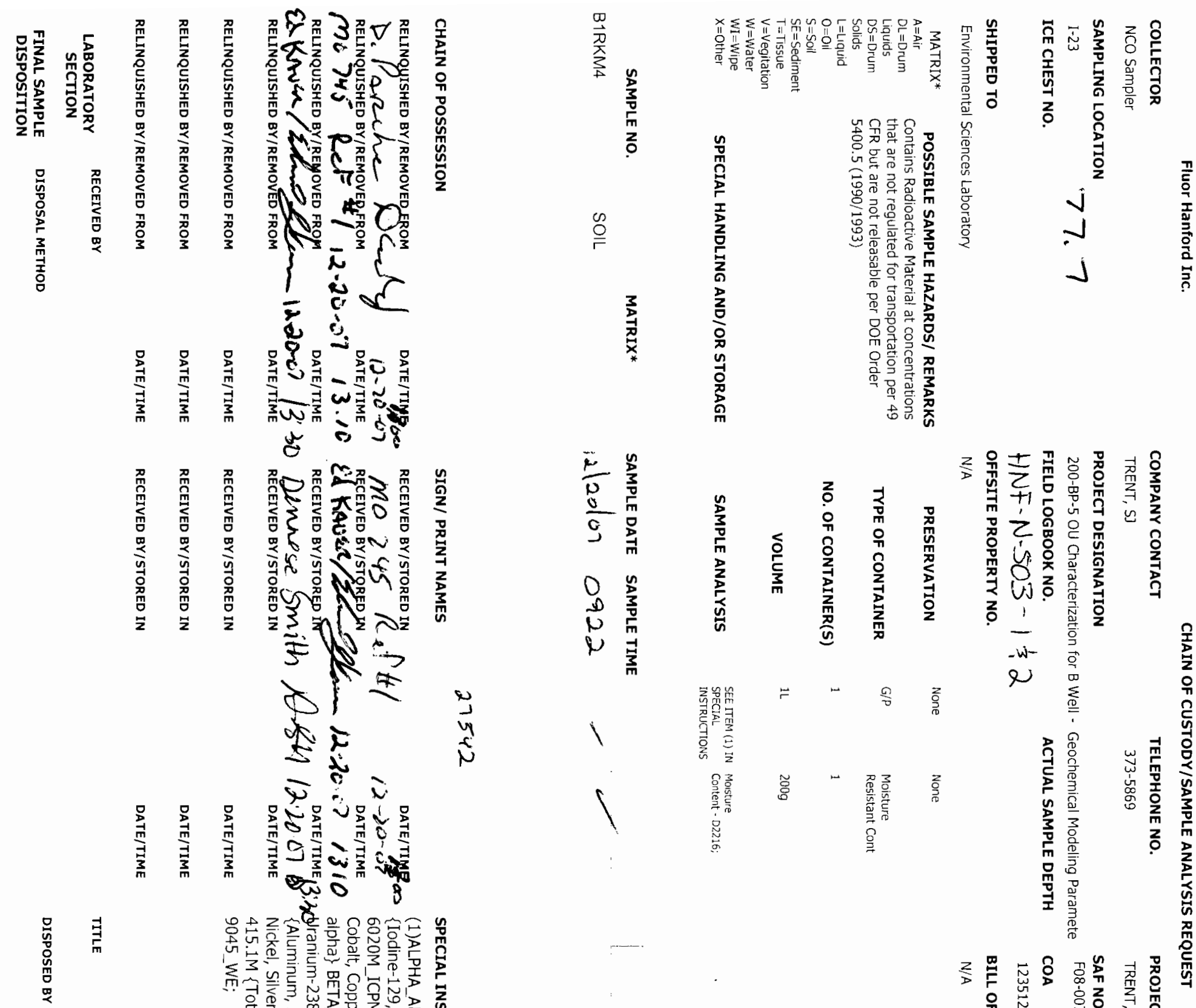

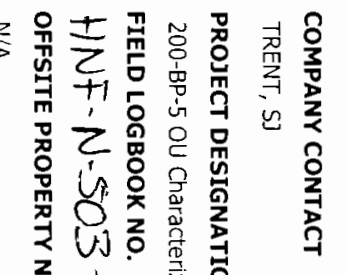

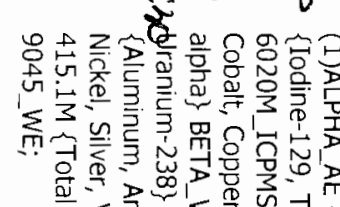
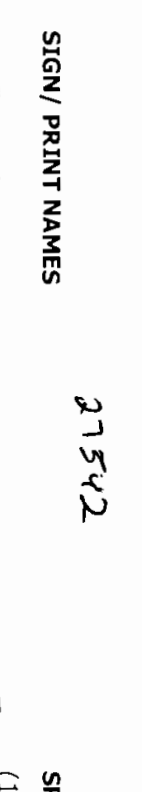

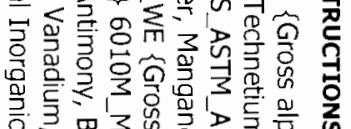

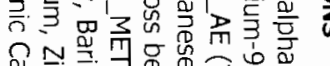

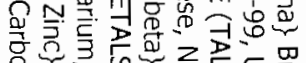

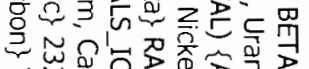

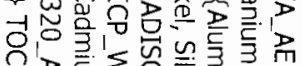

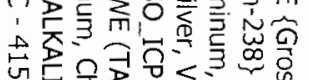

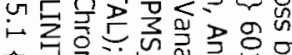

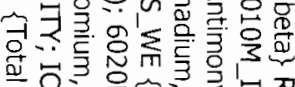

응

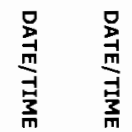
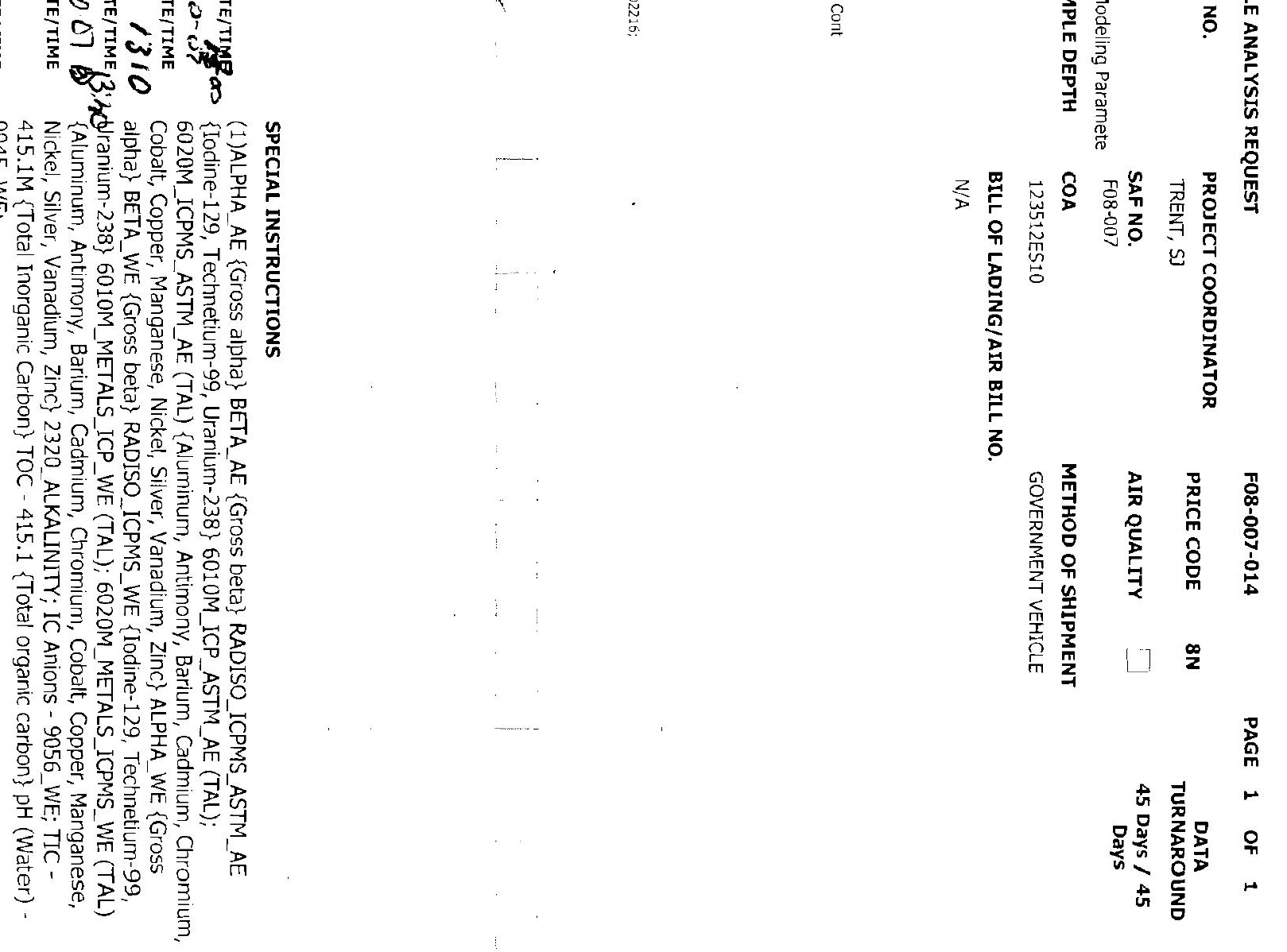


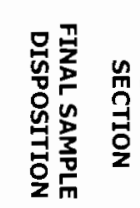
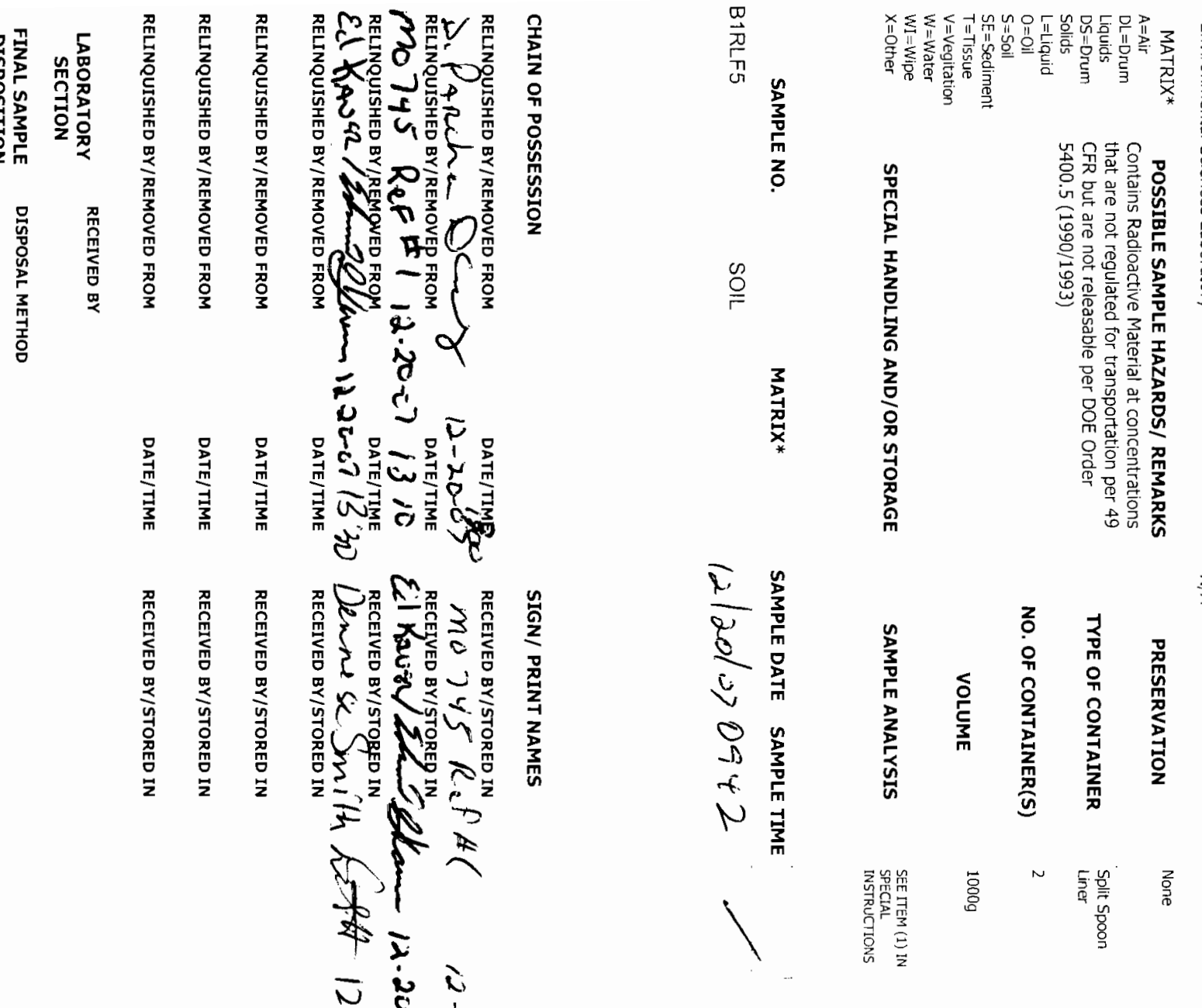

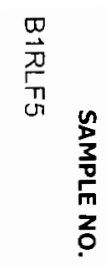

$\stackrel{9}{ }$

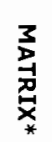

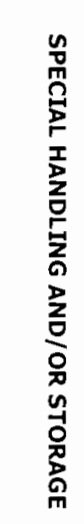

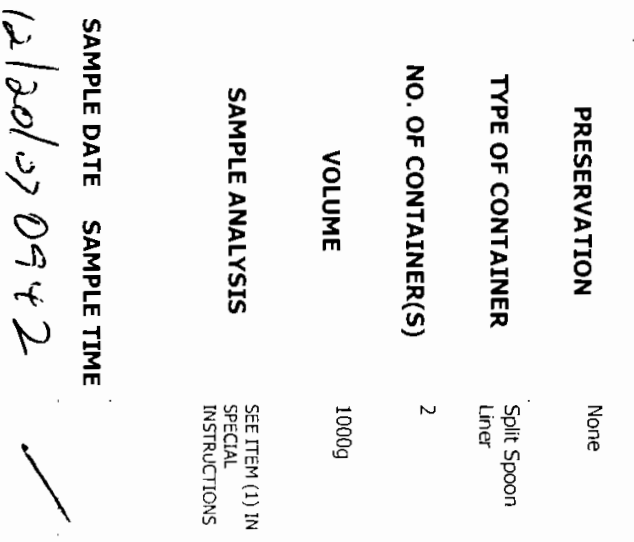

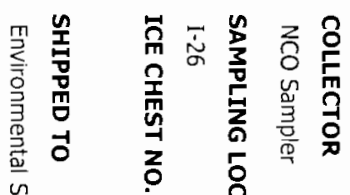

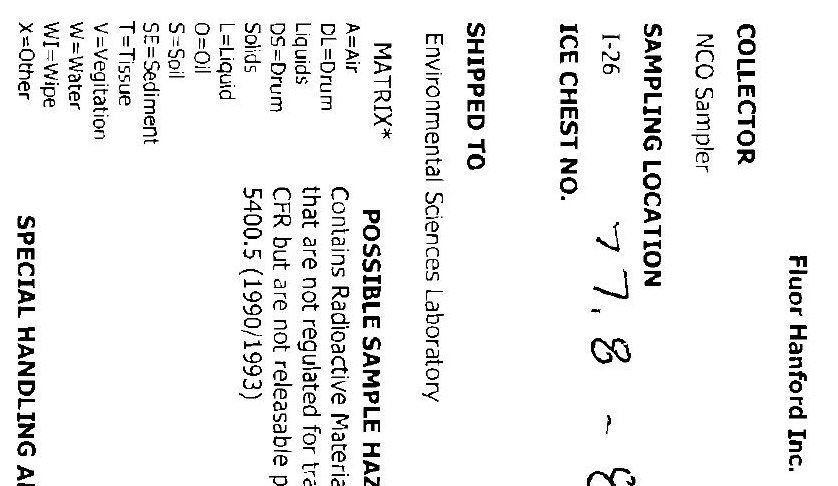

oo<smiles>C1CCCCC1</smiles>

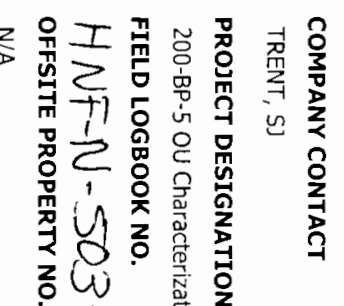
$\stackrel{1}{4} \stackrel{\overrightarrow{\bar{O}}}{\stackrel{\overrightarrow{0}}{0}}$

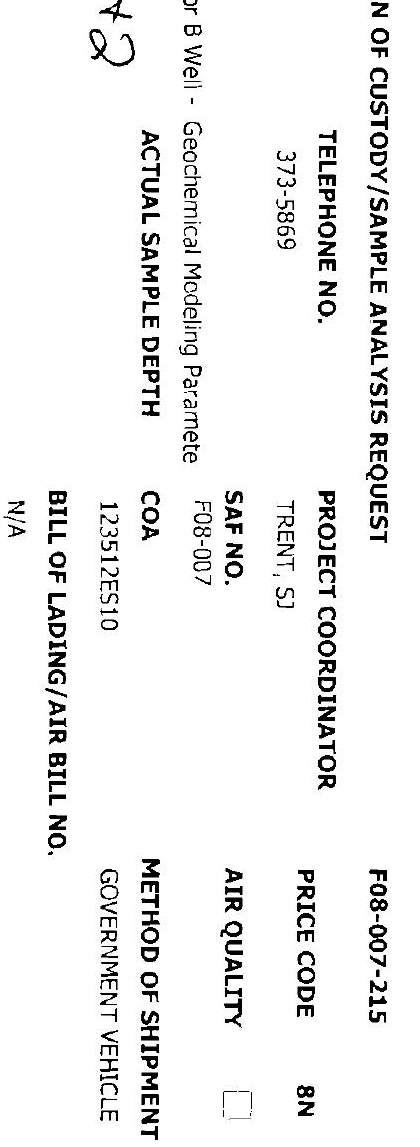

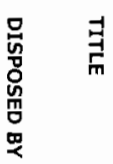

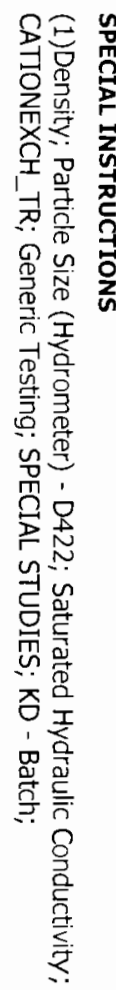

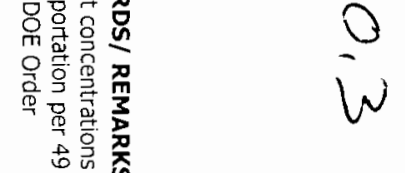

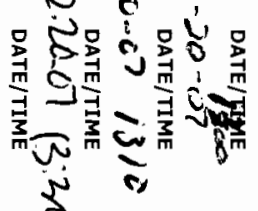

¿ 


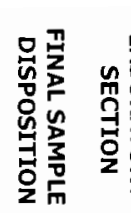

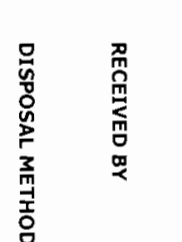

$\frac{\rho}{\infty}$

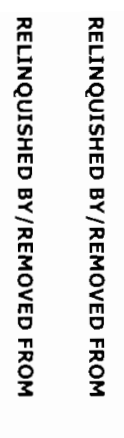

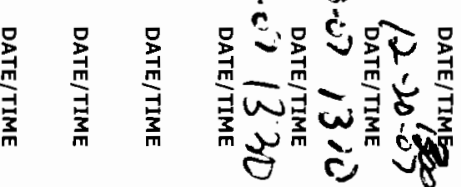

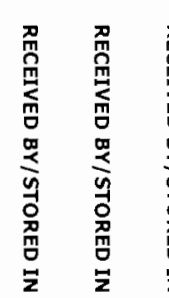

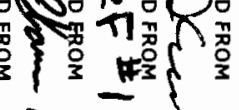

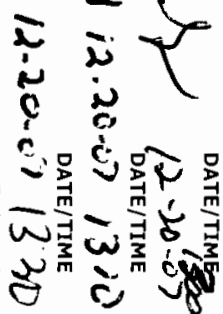

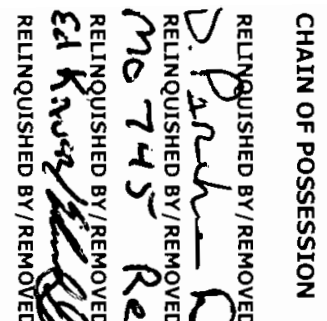

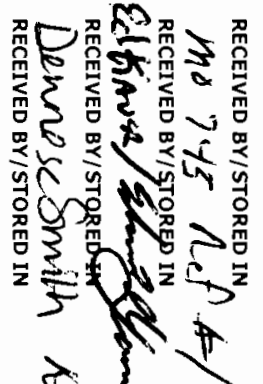

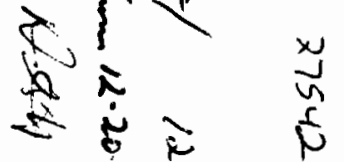

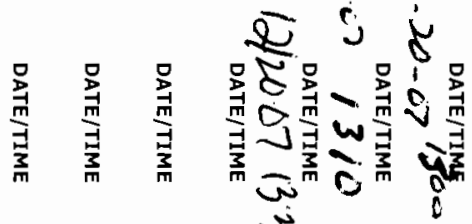

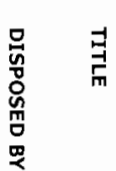

量罯

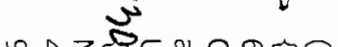

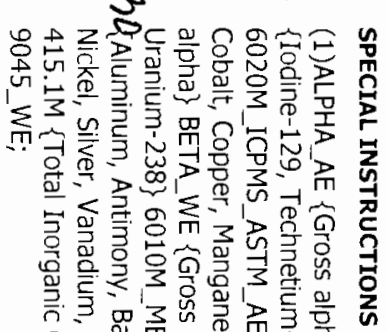

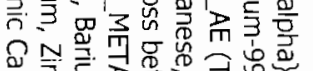

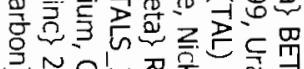

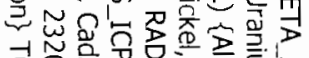

ㅇㅇ일

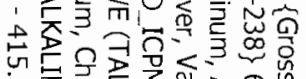

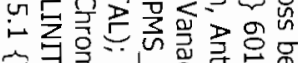

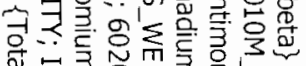

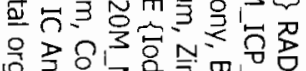

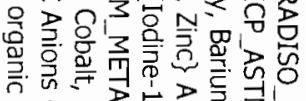

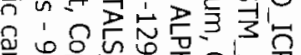

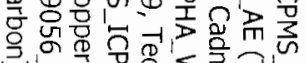

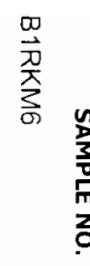

$\stackrel{\infty}{\circ}$
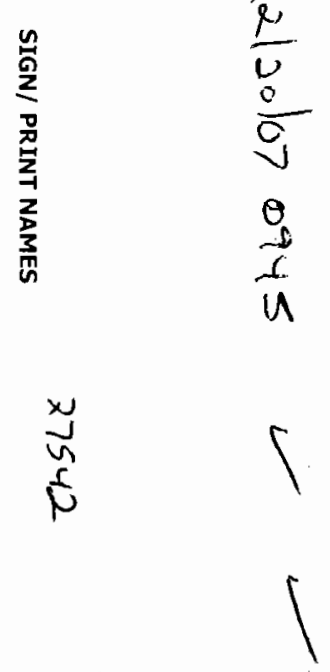

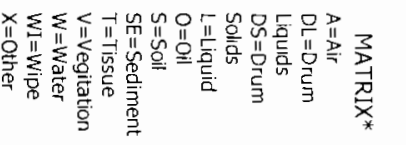

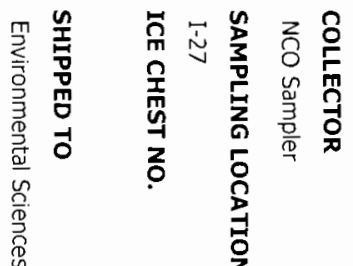

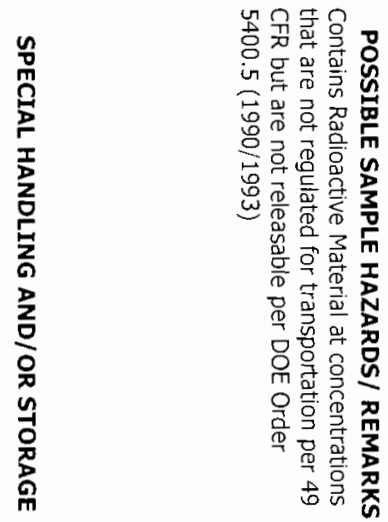

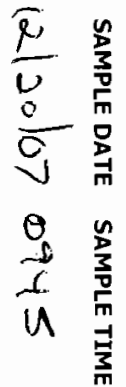

2

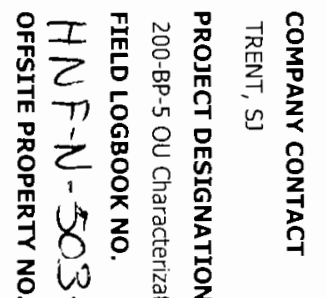

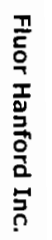

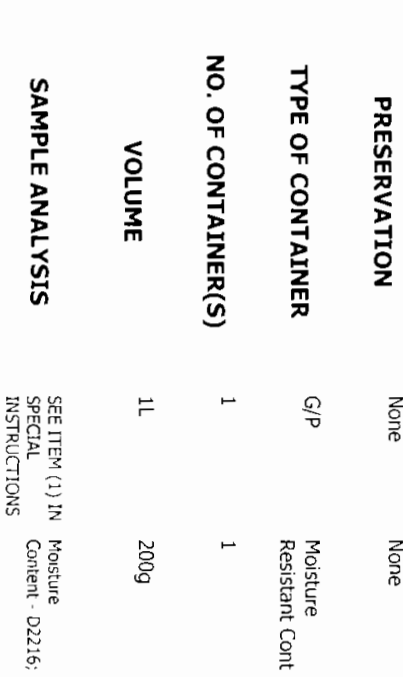

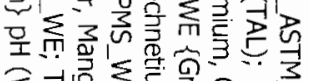

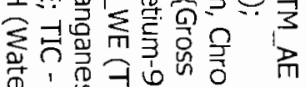

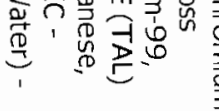




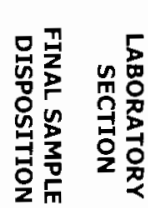

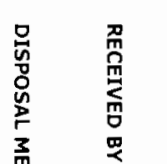

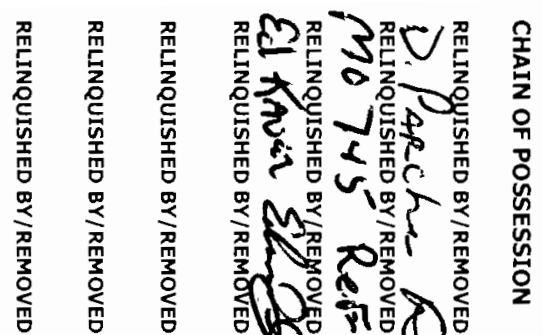

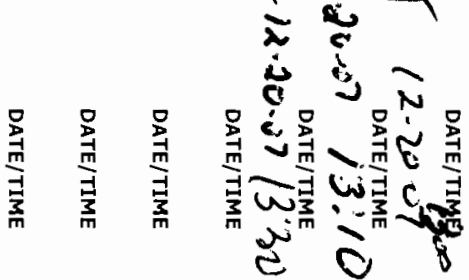
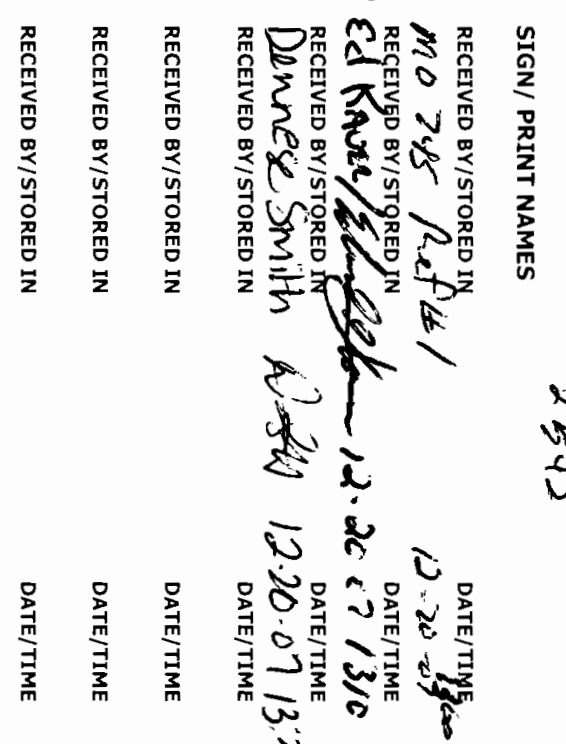

$\frac{d}{d}$

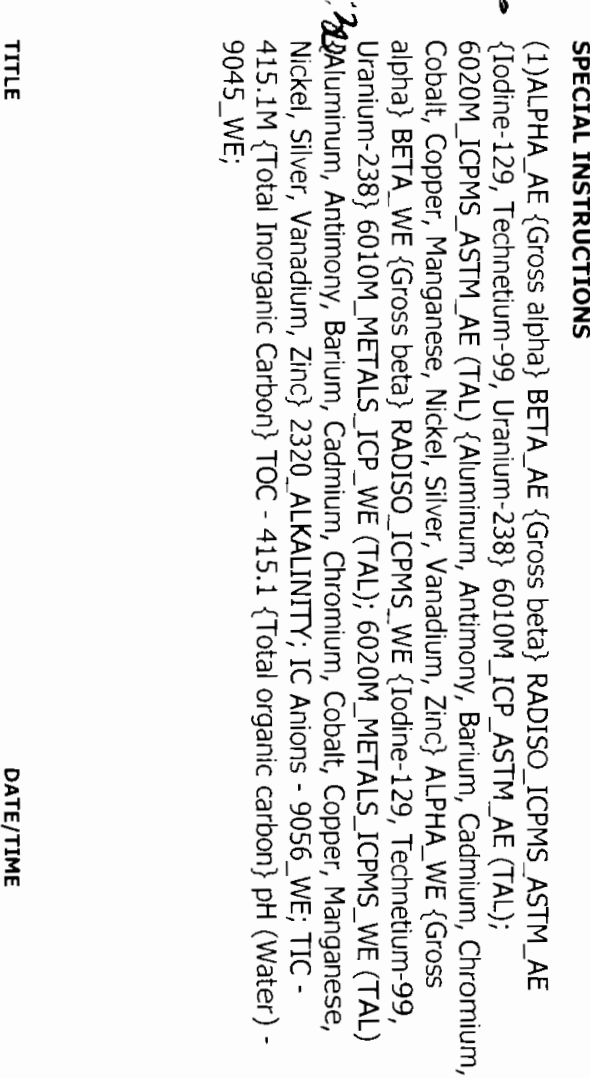

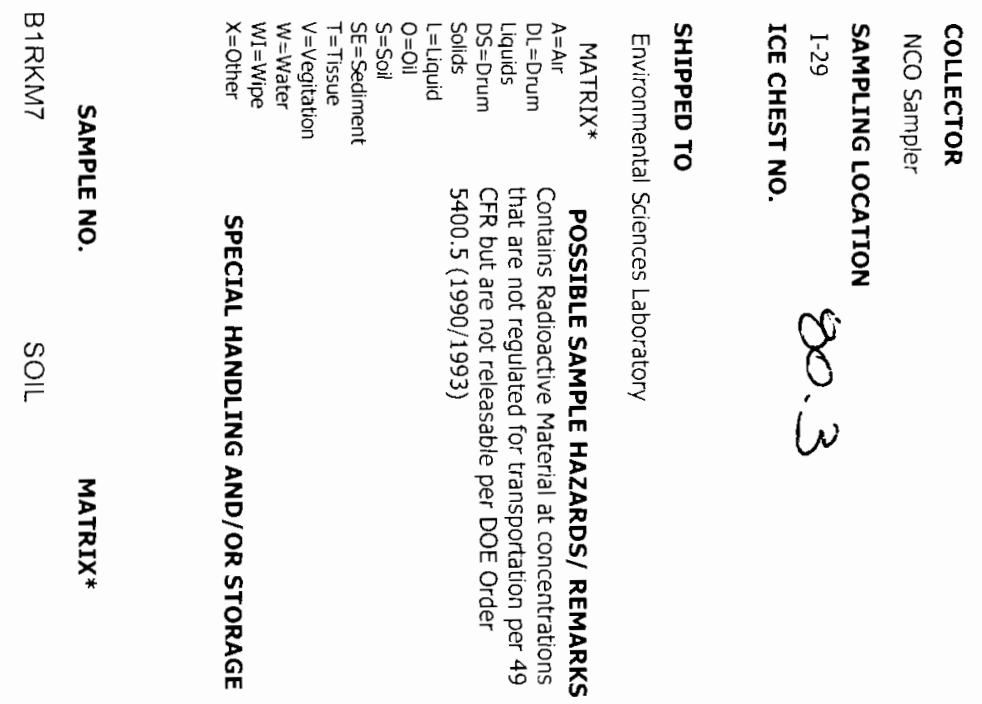

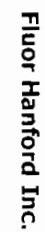

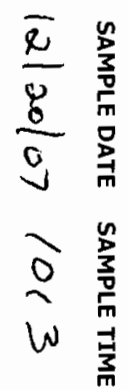

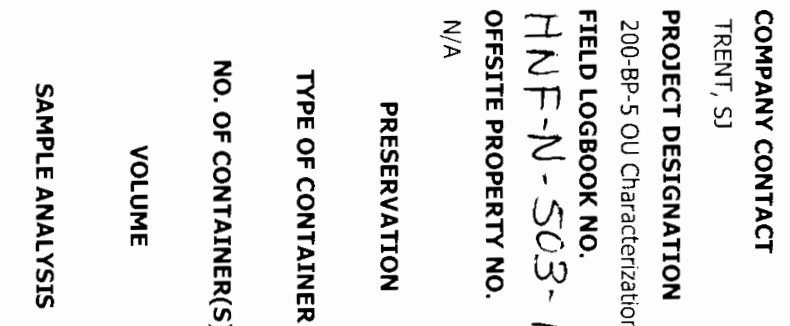

- v-

)

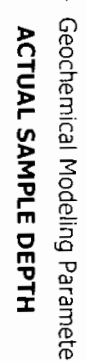

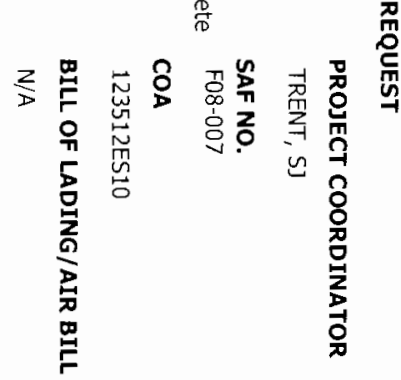

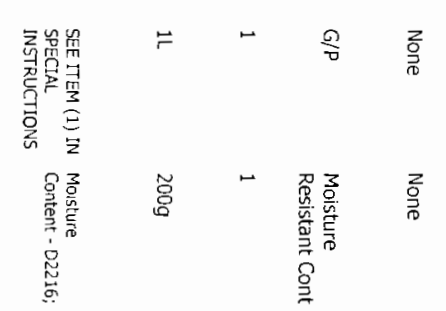

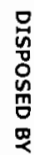

$\vec{\exists}$

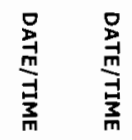

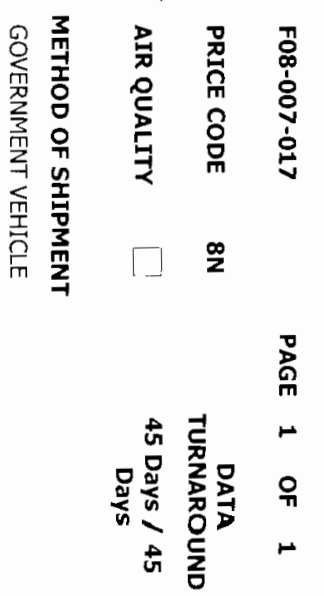




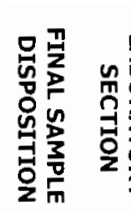

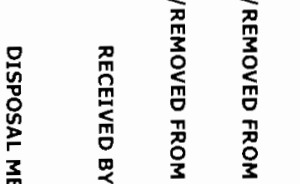

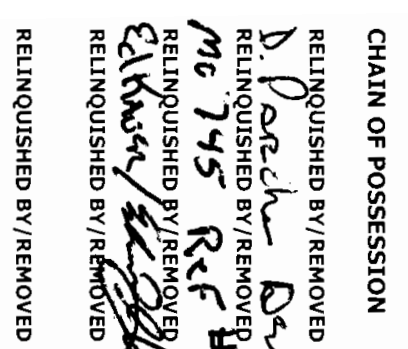
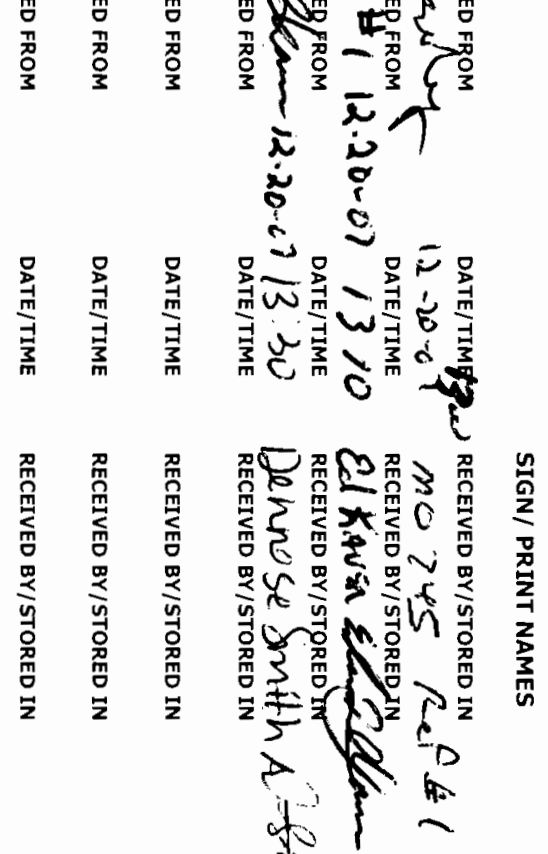

善
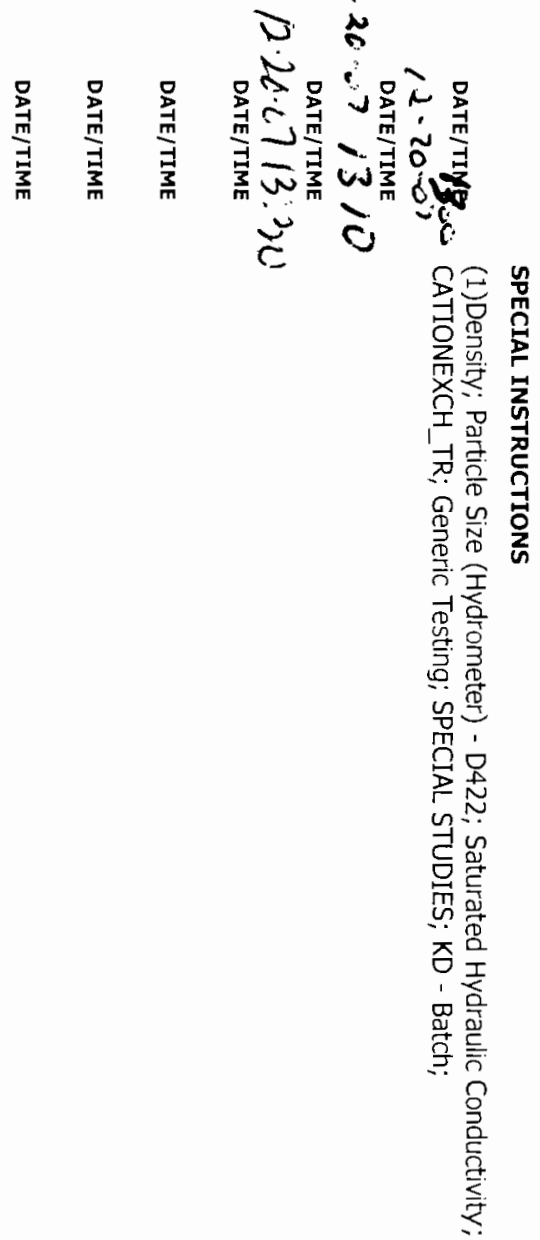

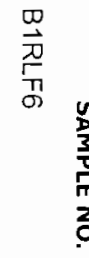

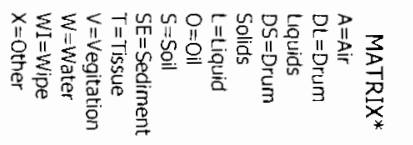

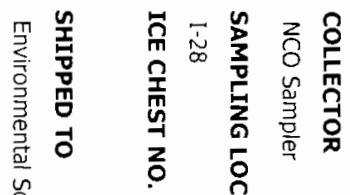

$\stackrel{\infty}{1}$

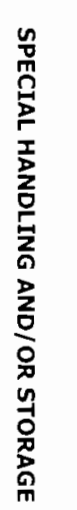

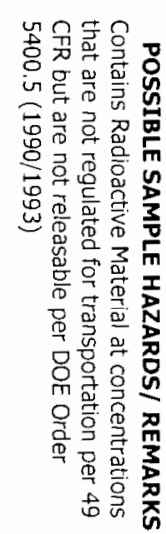

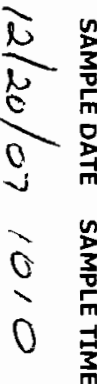

눙

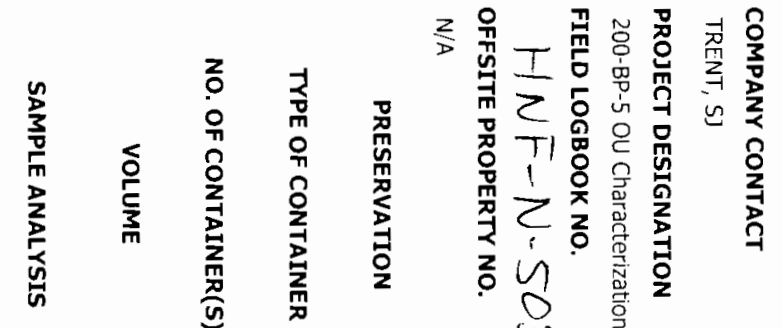

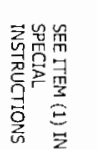

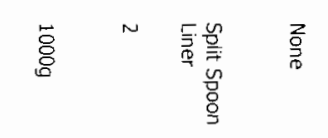

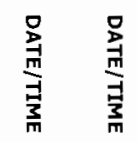

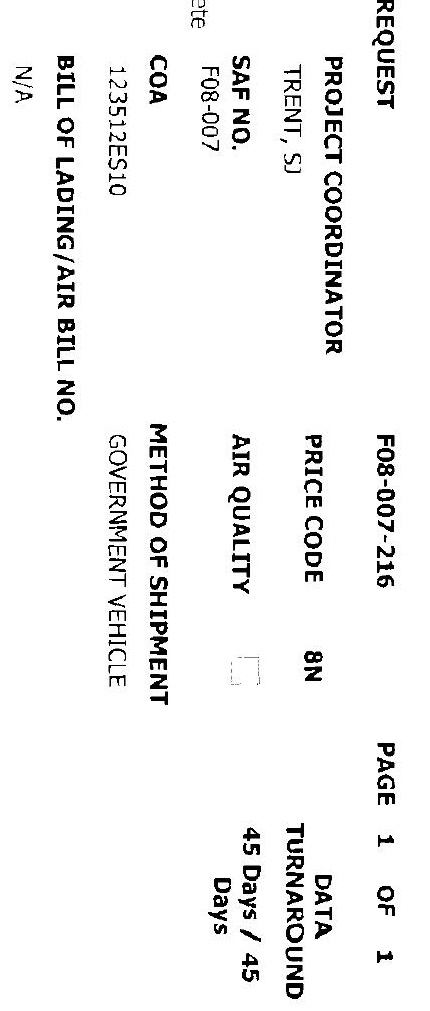



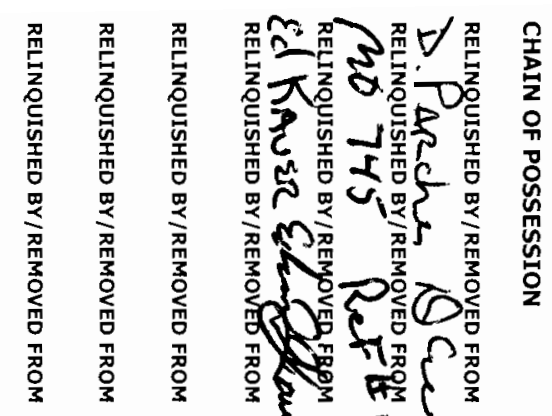

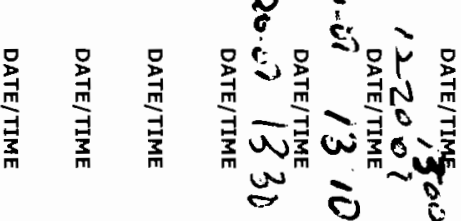
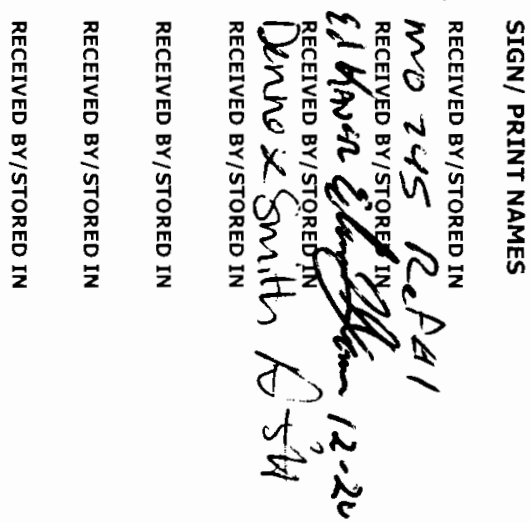

足品

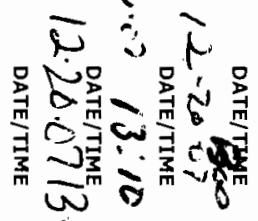

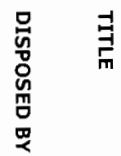

$\ddot{c}$

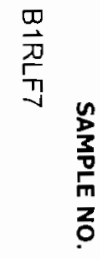

$\stackrel{0}{\circ}$

$\underset{3}{3}$

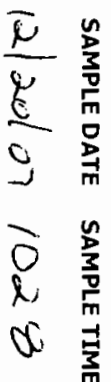

〔

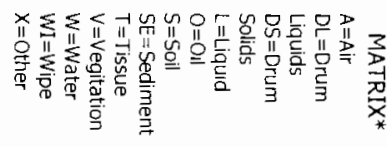

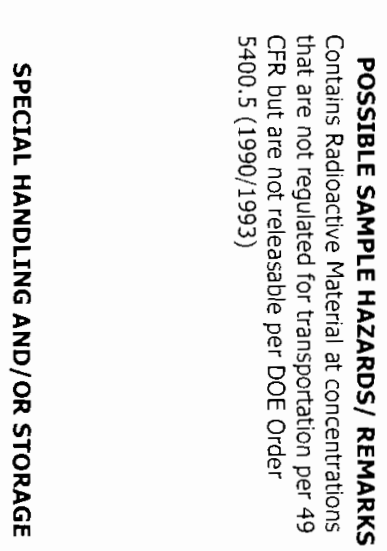

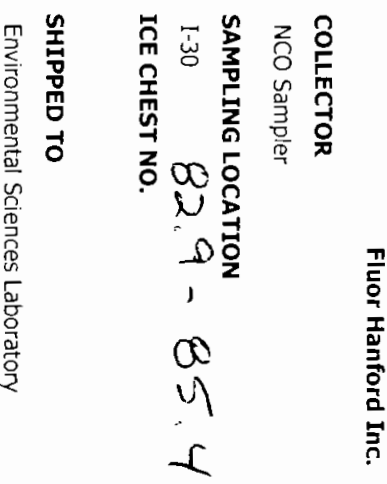

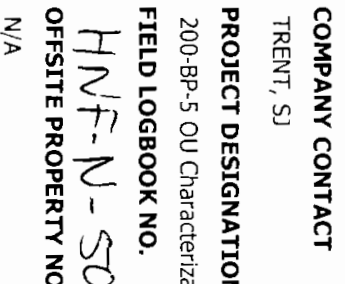

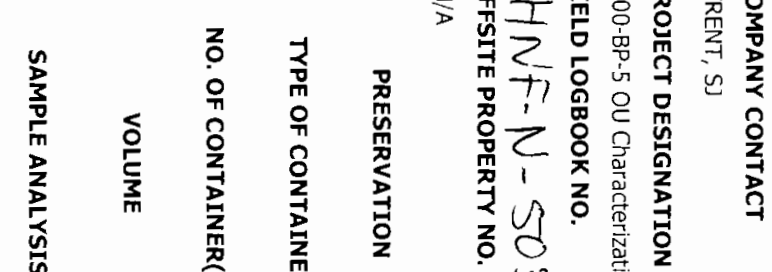

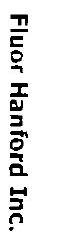

爱

(4)

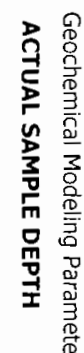

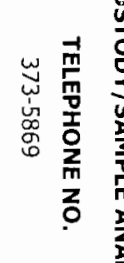

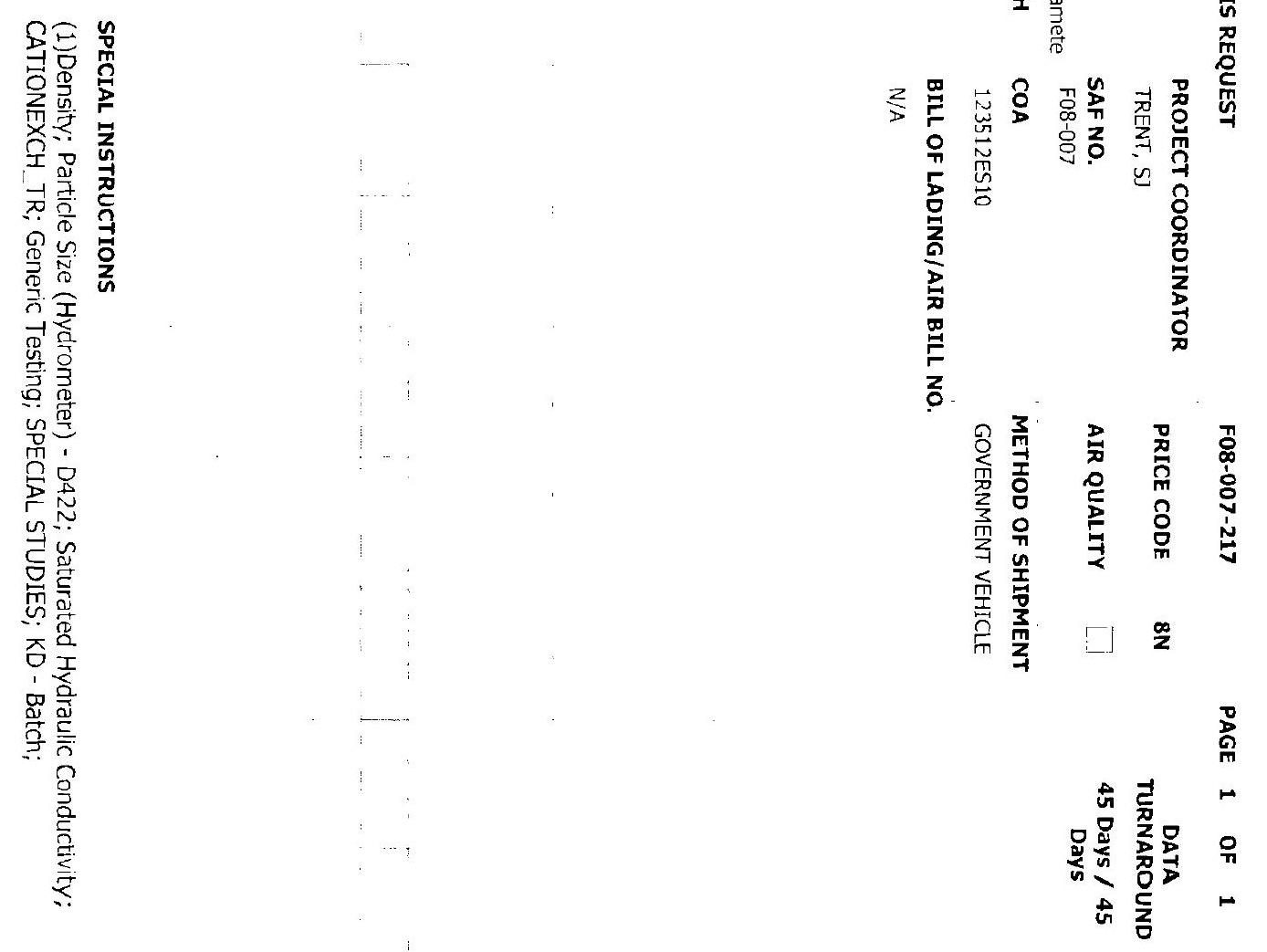



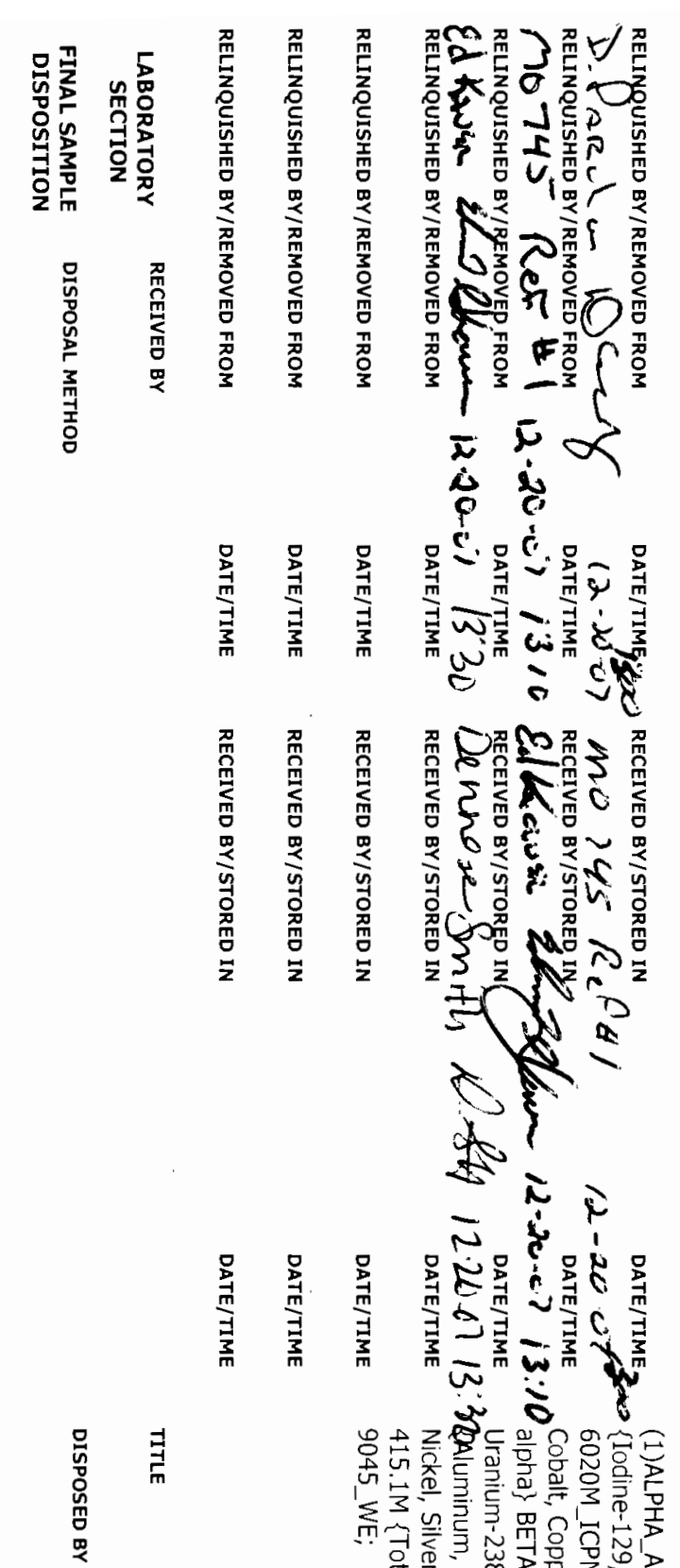

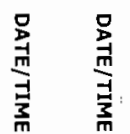

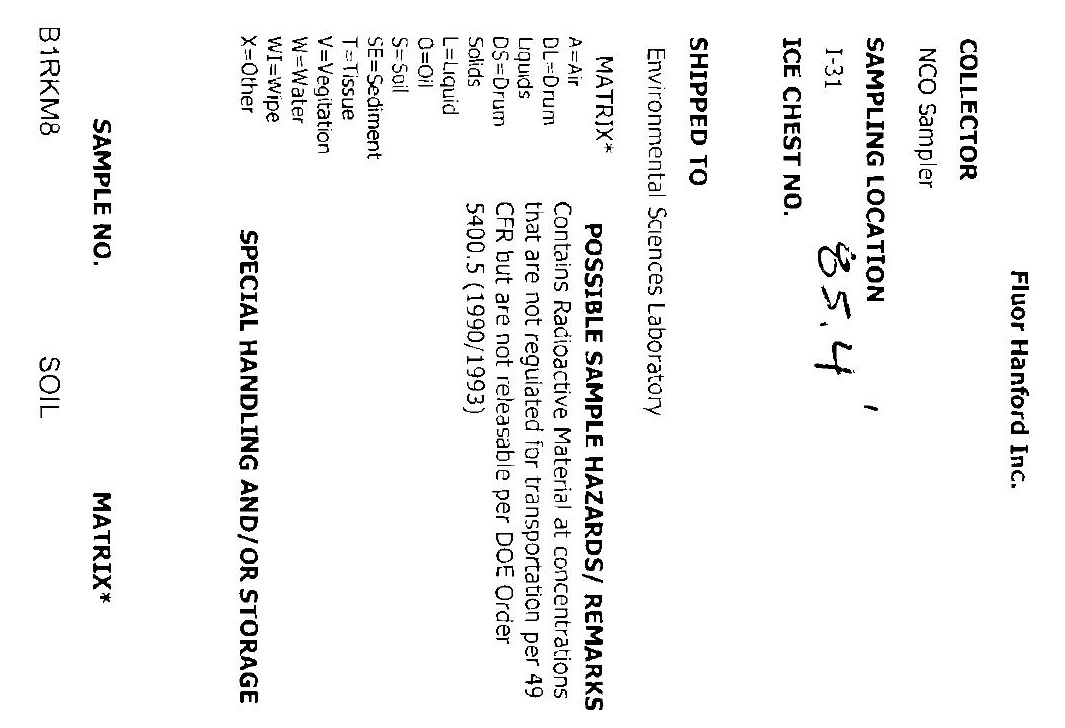

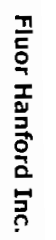

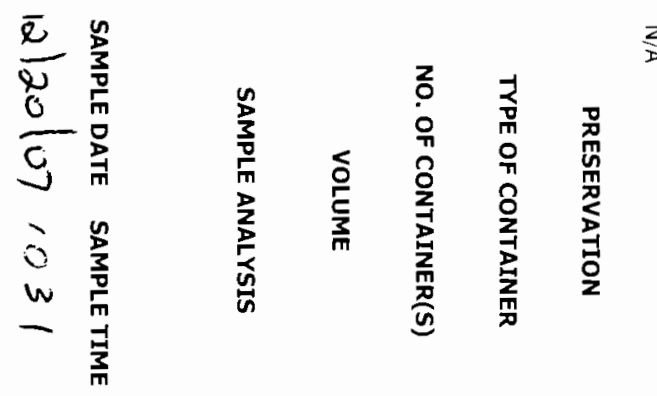

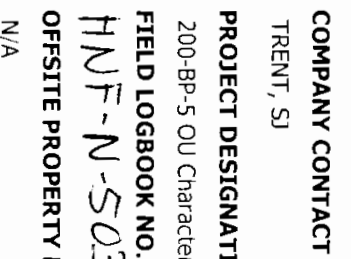
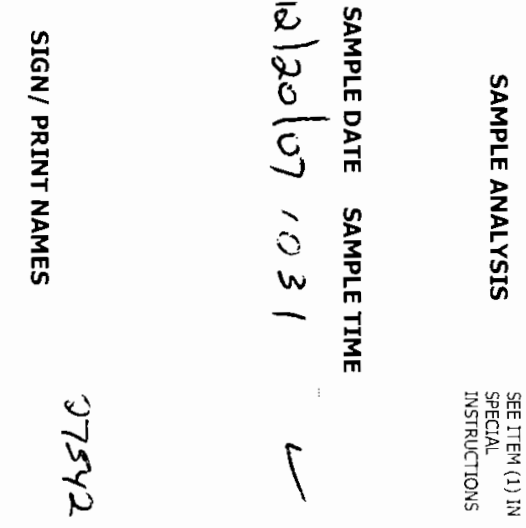

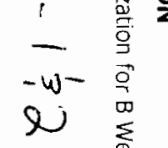
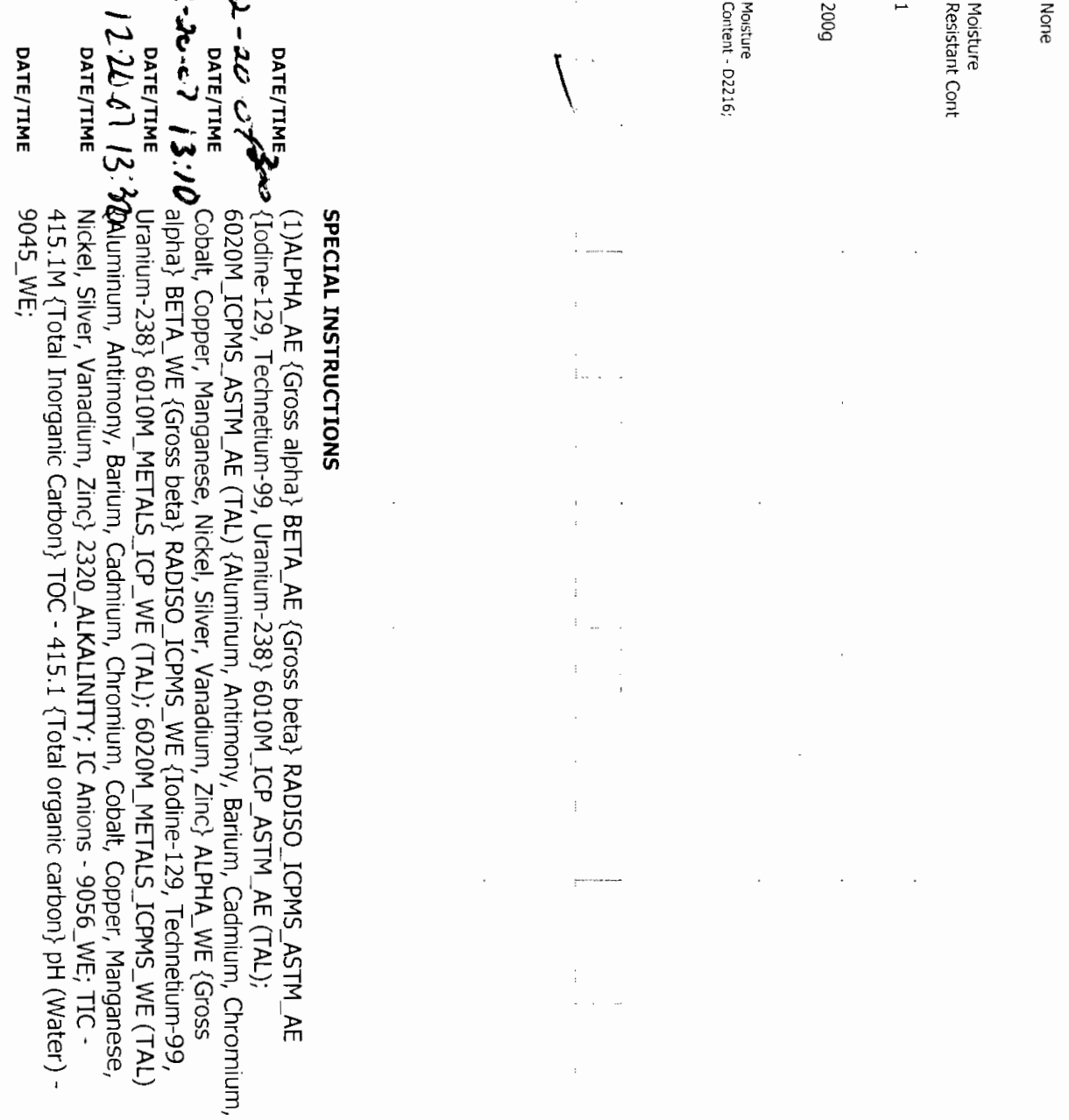


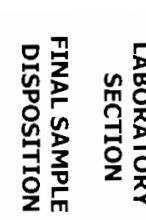 \\ 高}

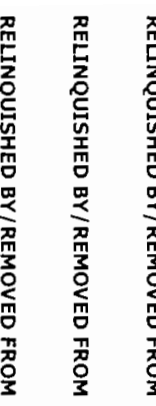

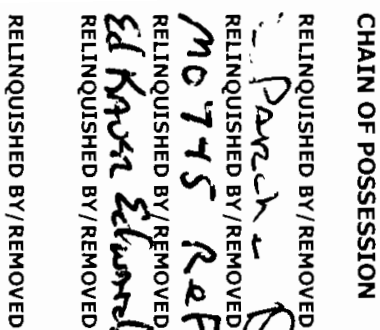

$x \quad x \quad D^{\infty}$

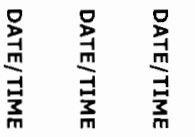

$\overbrace{}^{m}$

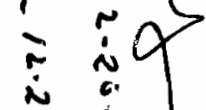

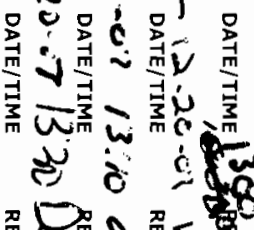

总羔量

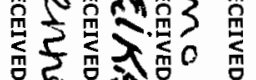

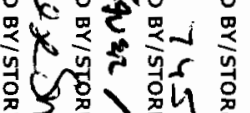

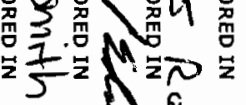

2. 次 4
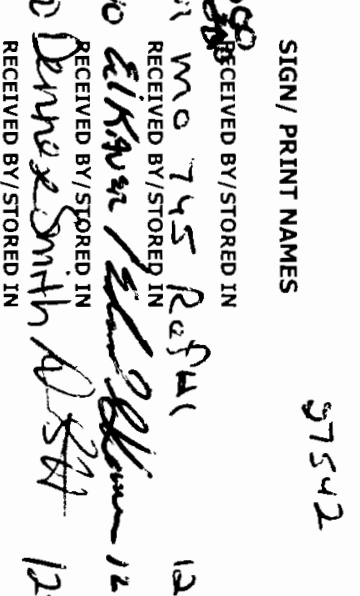

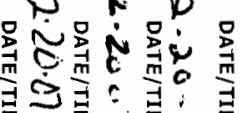

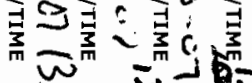

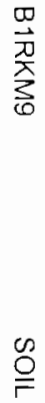

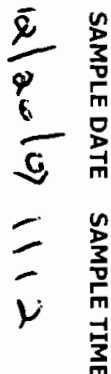

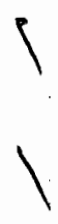

量

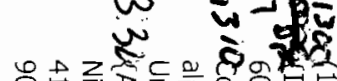

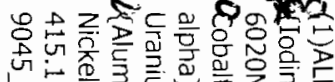

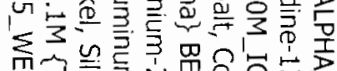

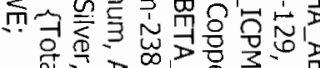

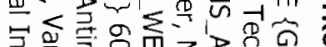

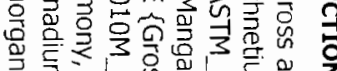

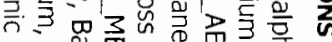

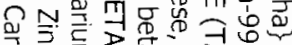

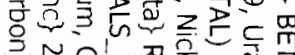

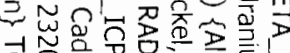

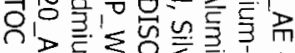

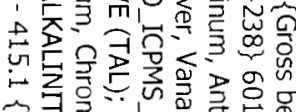

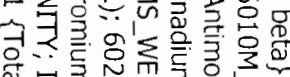

응

品是

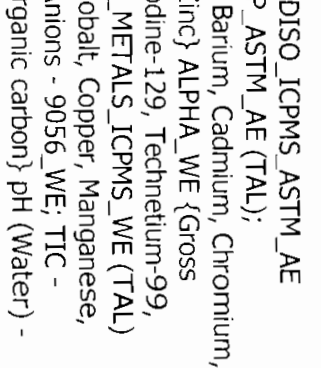

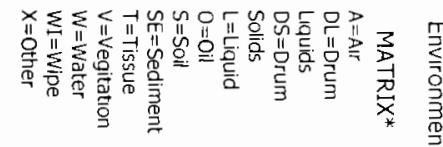

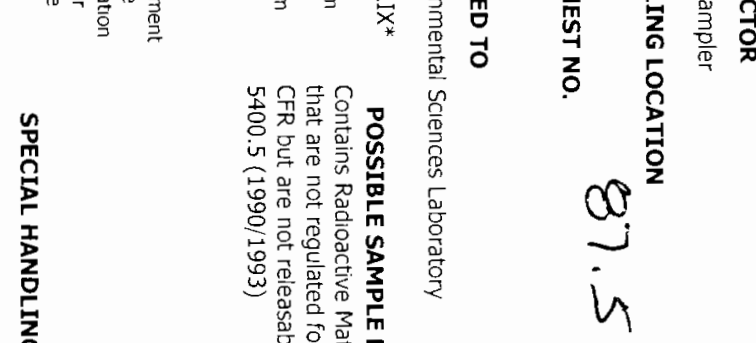

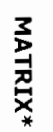

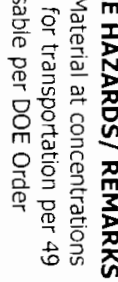

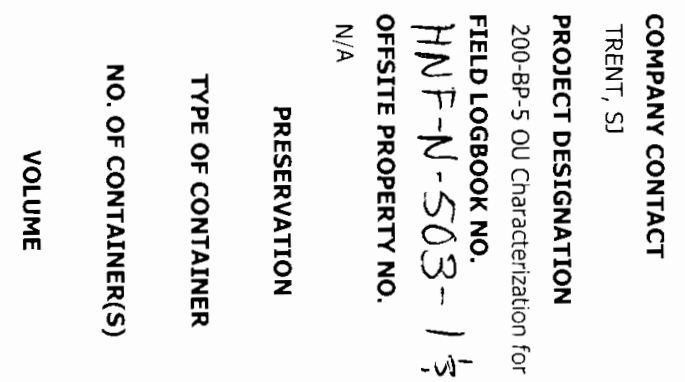

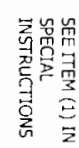

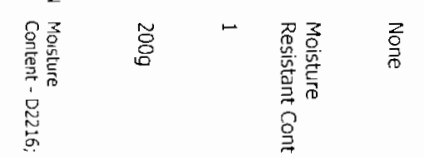

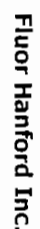

$\frac{9}{2}$

$\infty$

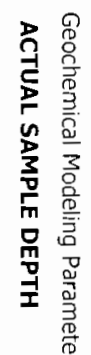

岕

管

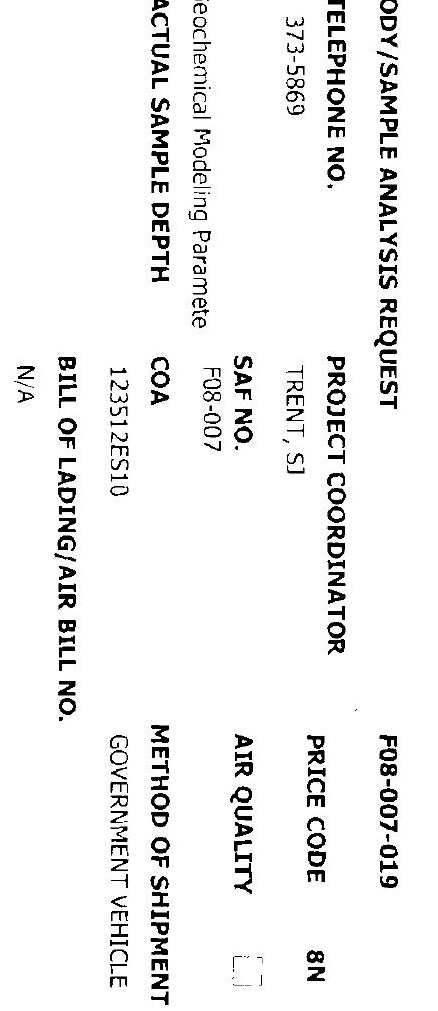

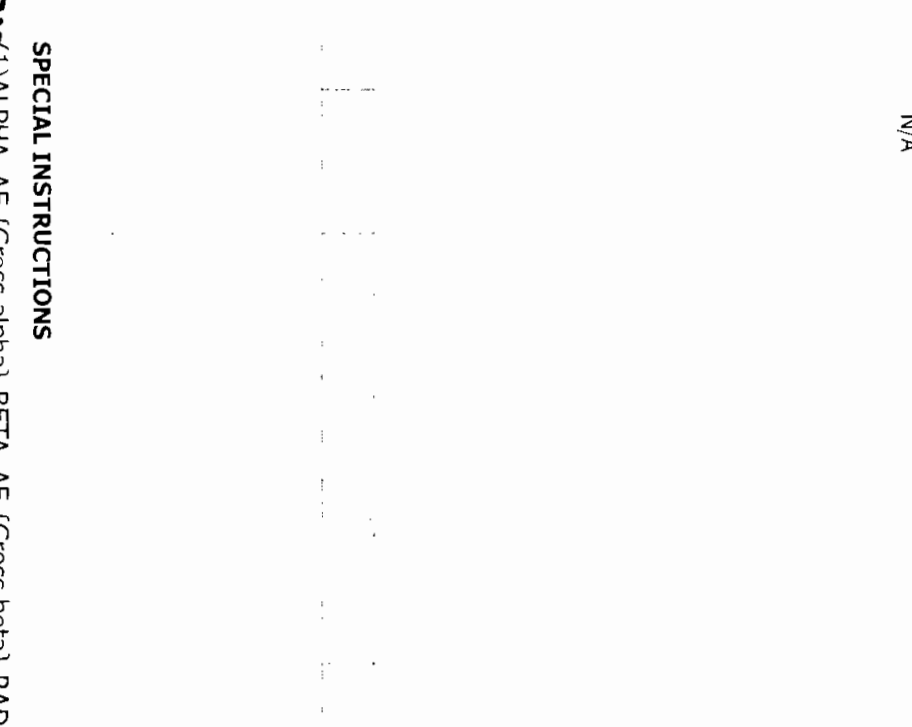




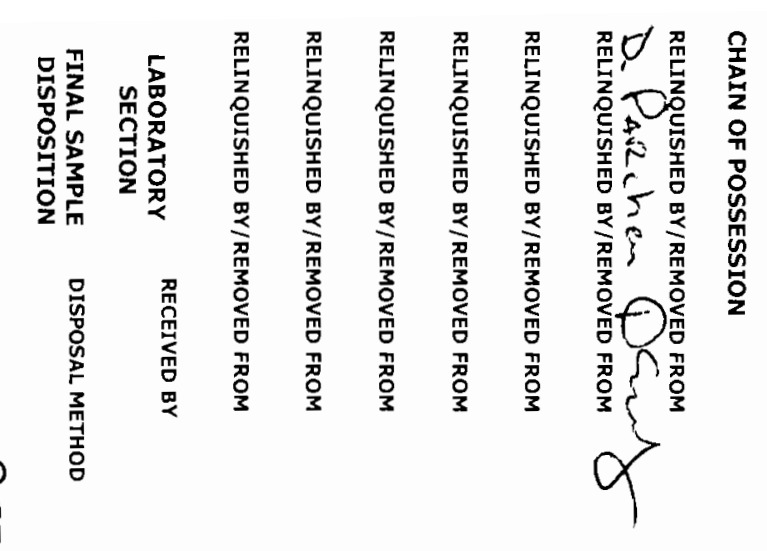

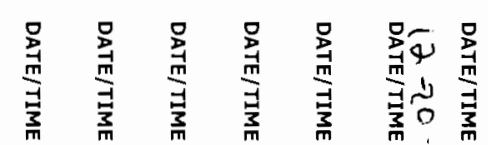
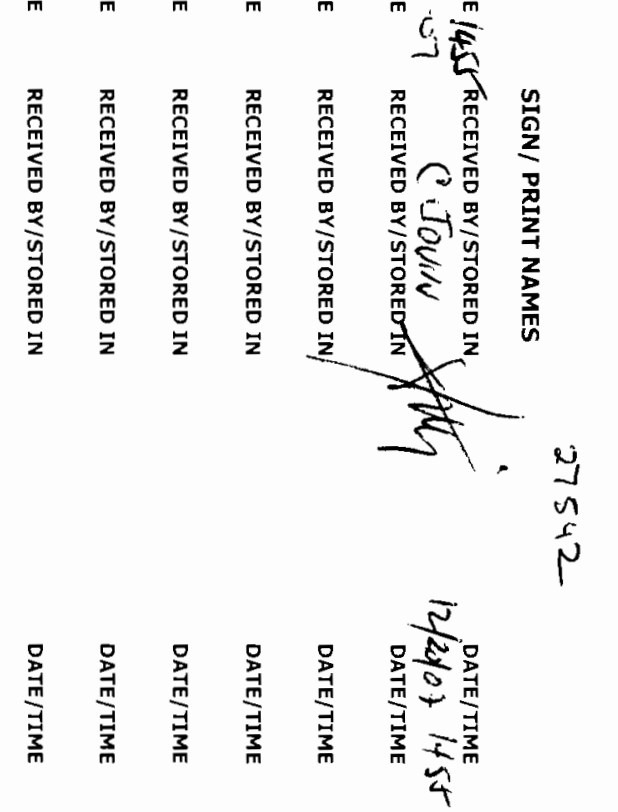

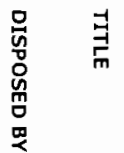

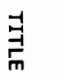

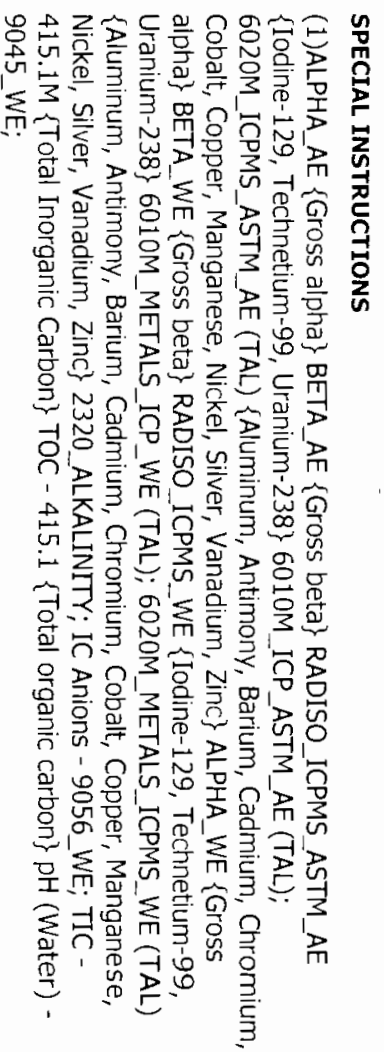

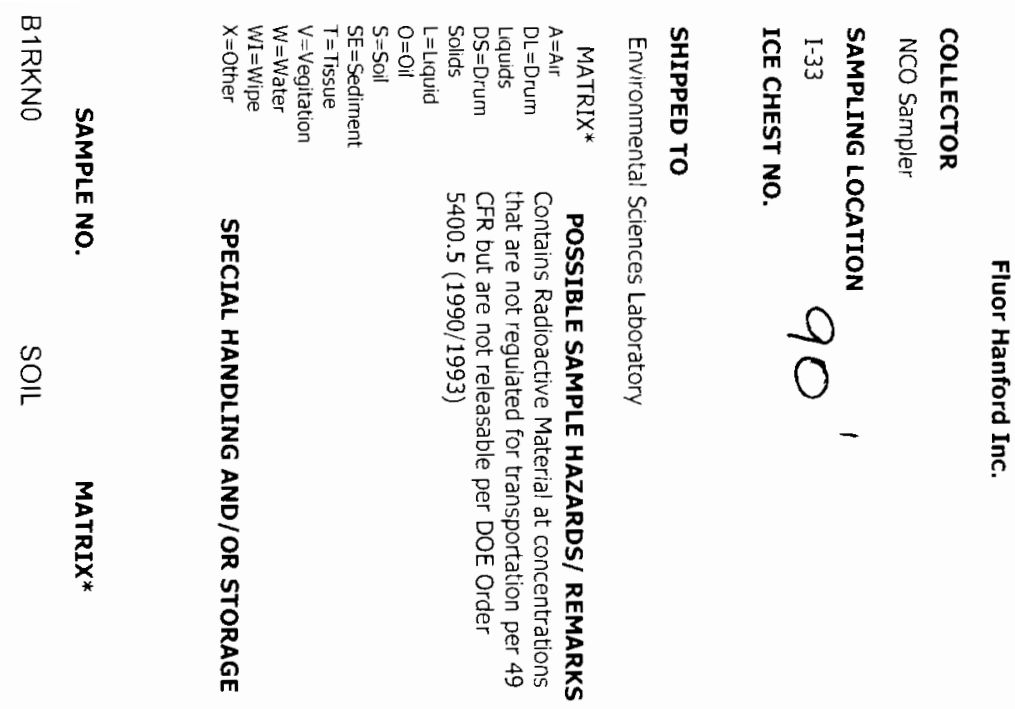
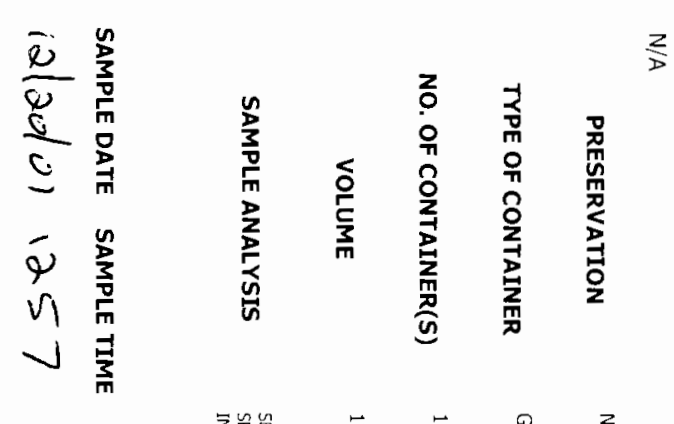

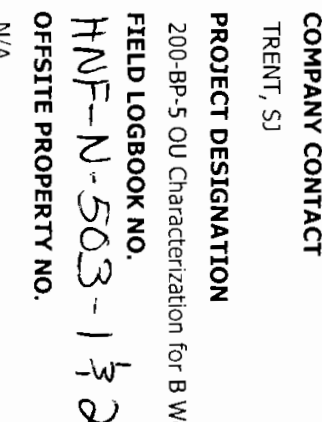
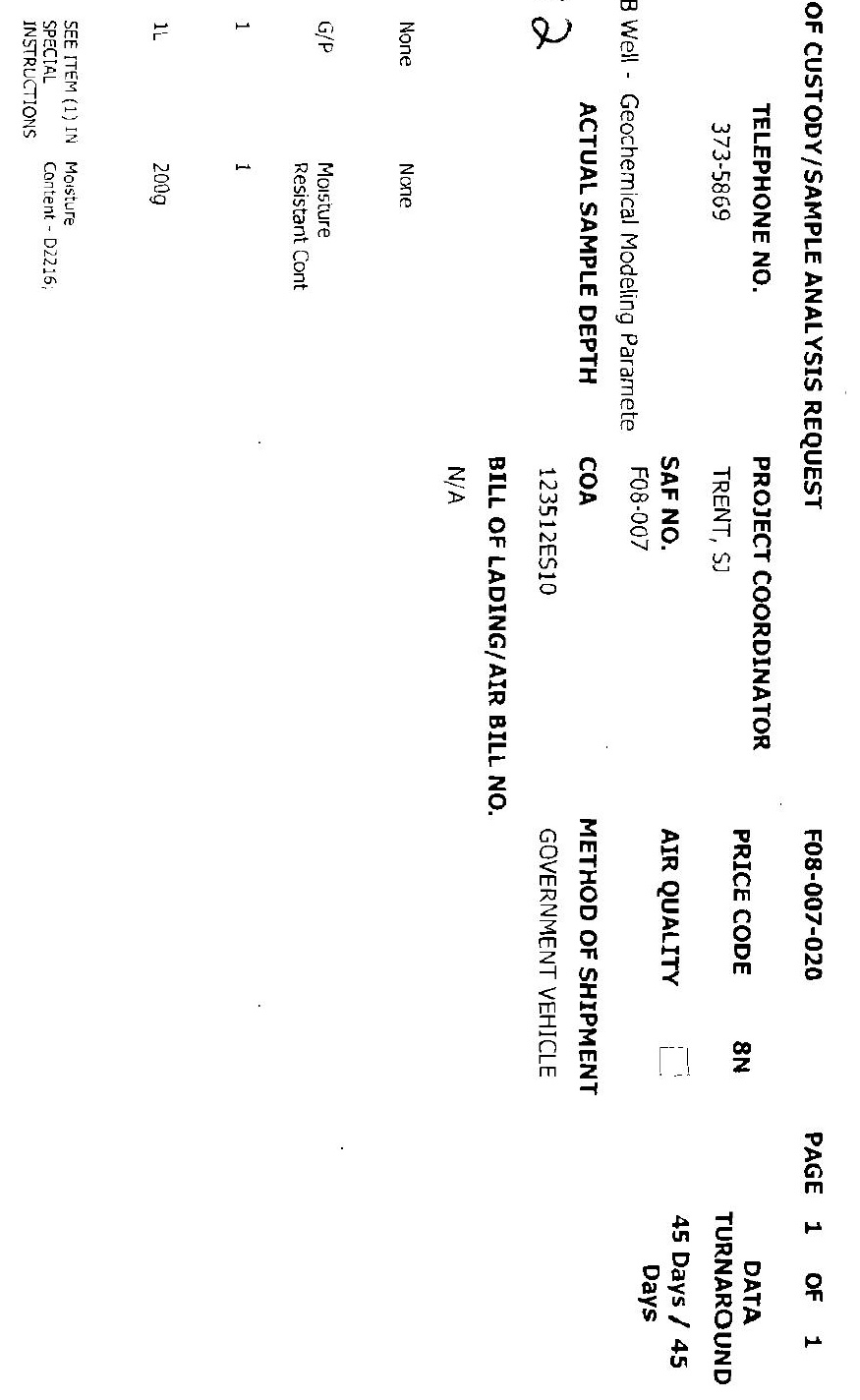

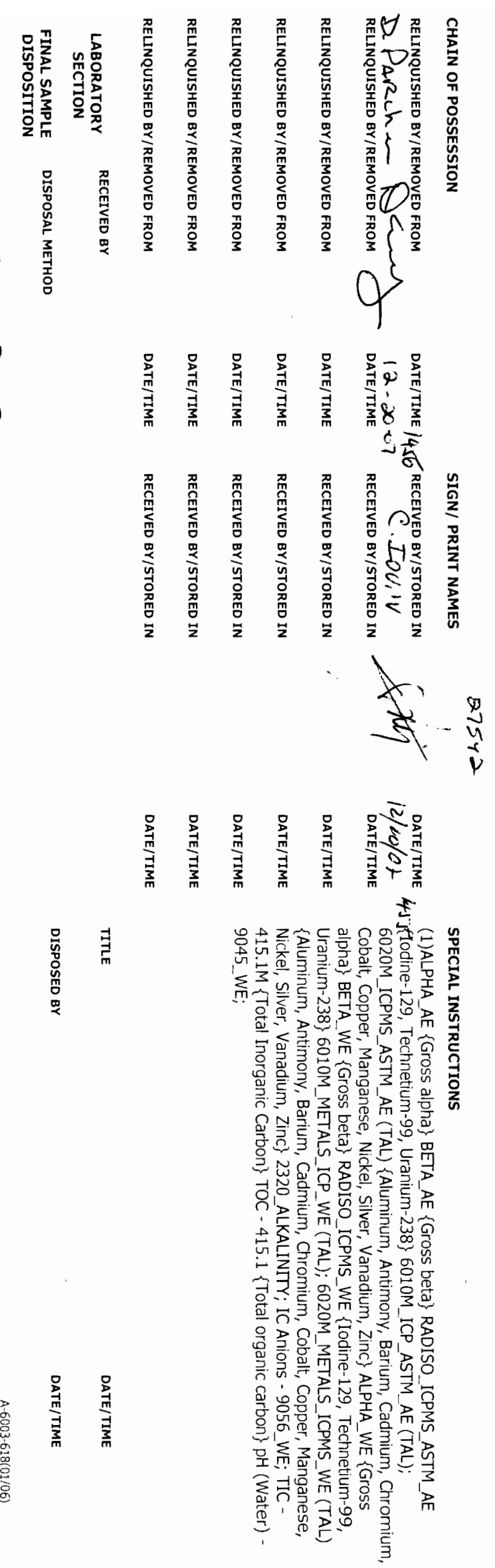
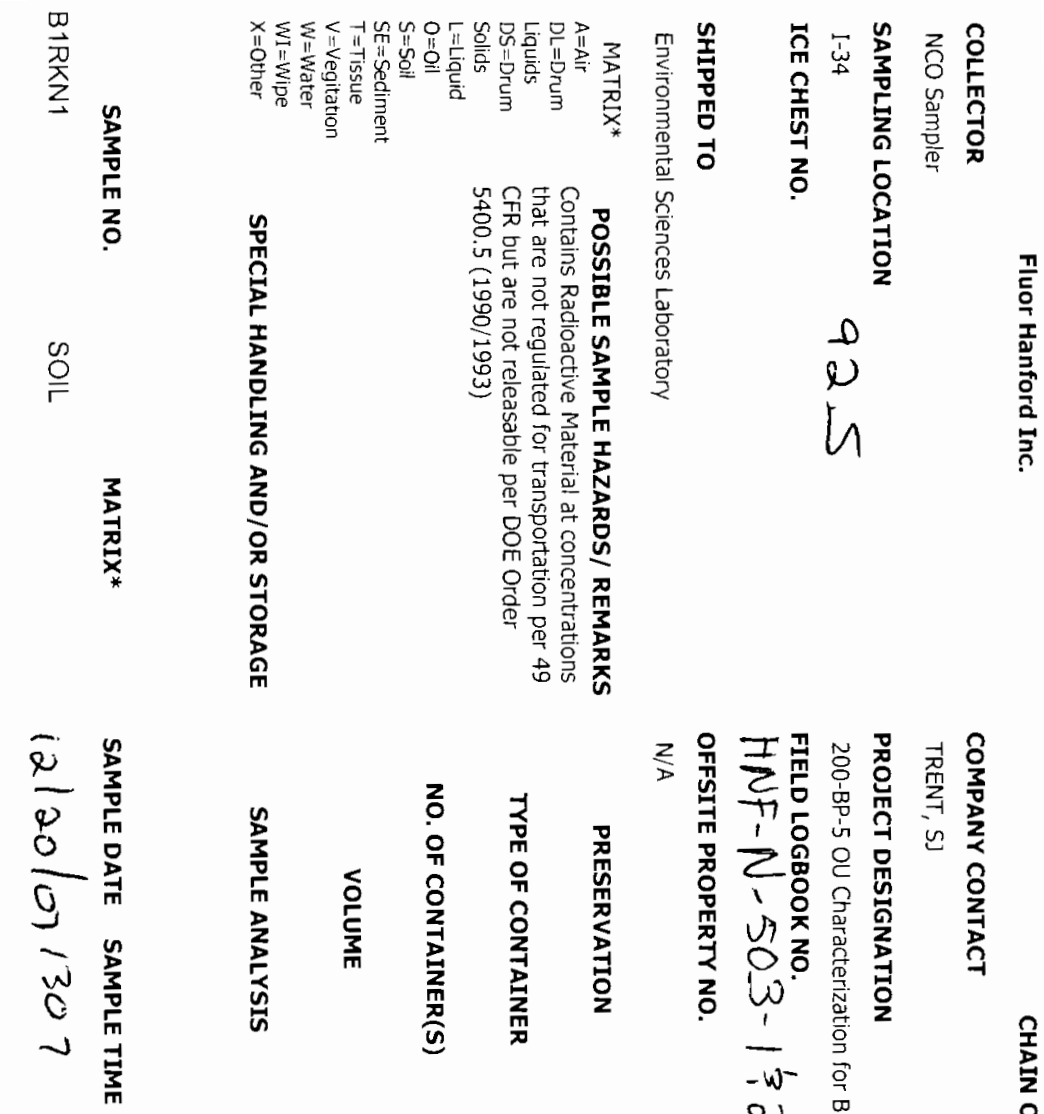

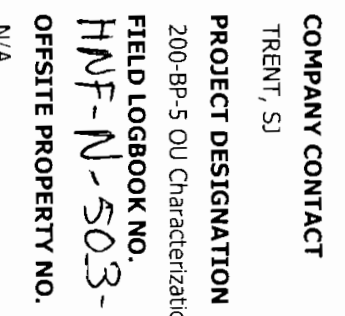
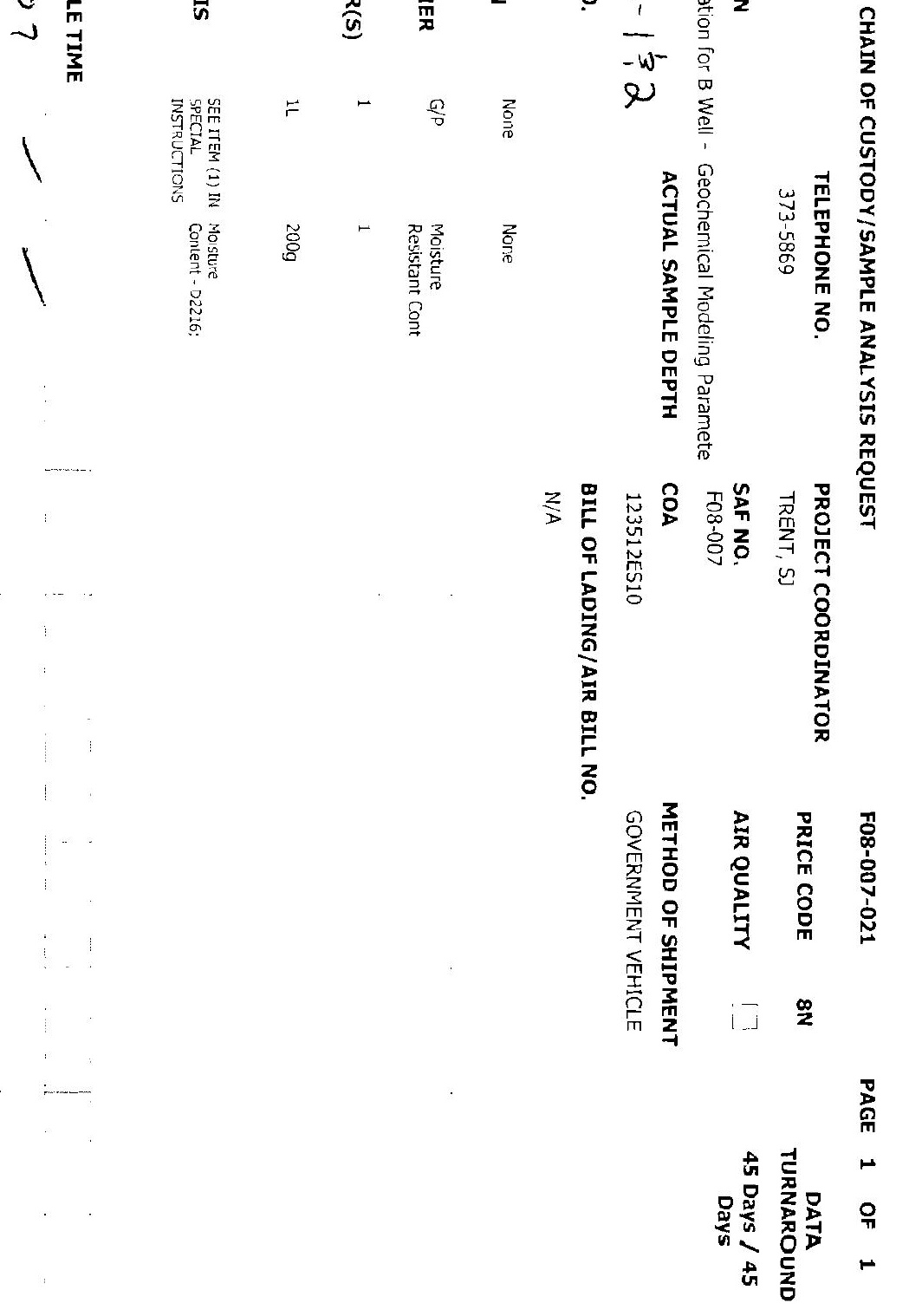


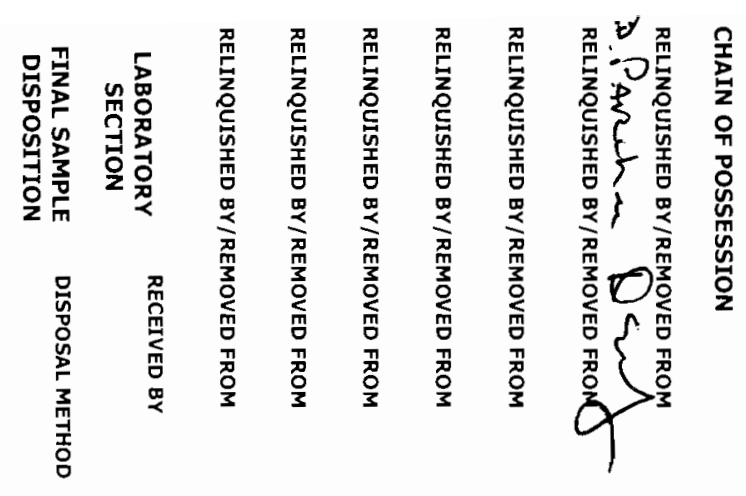

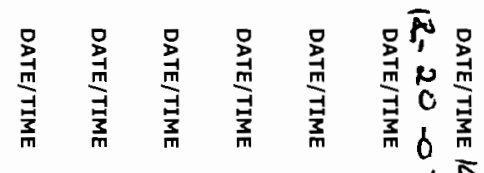

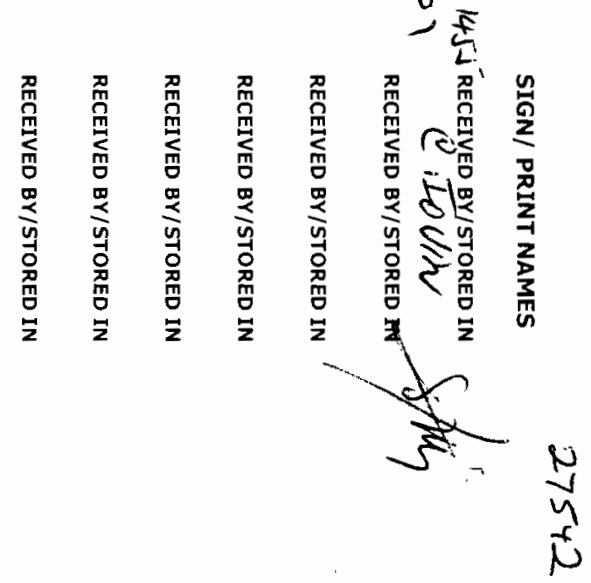

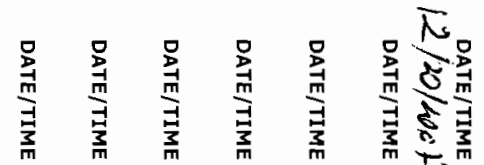

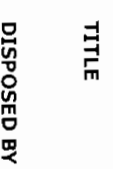

品总

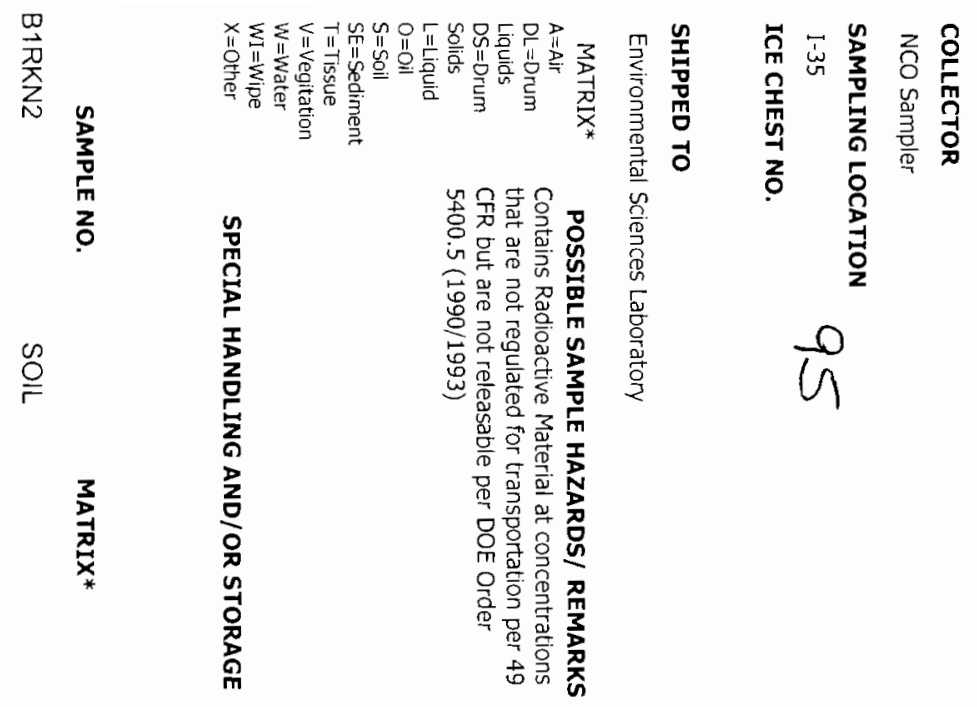

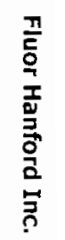

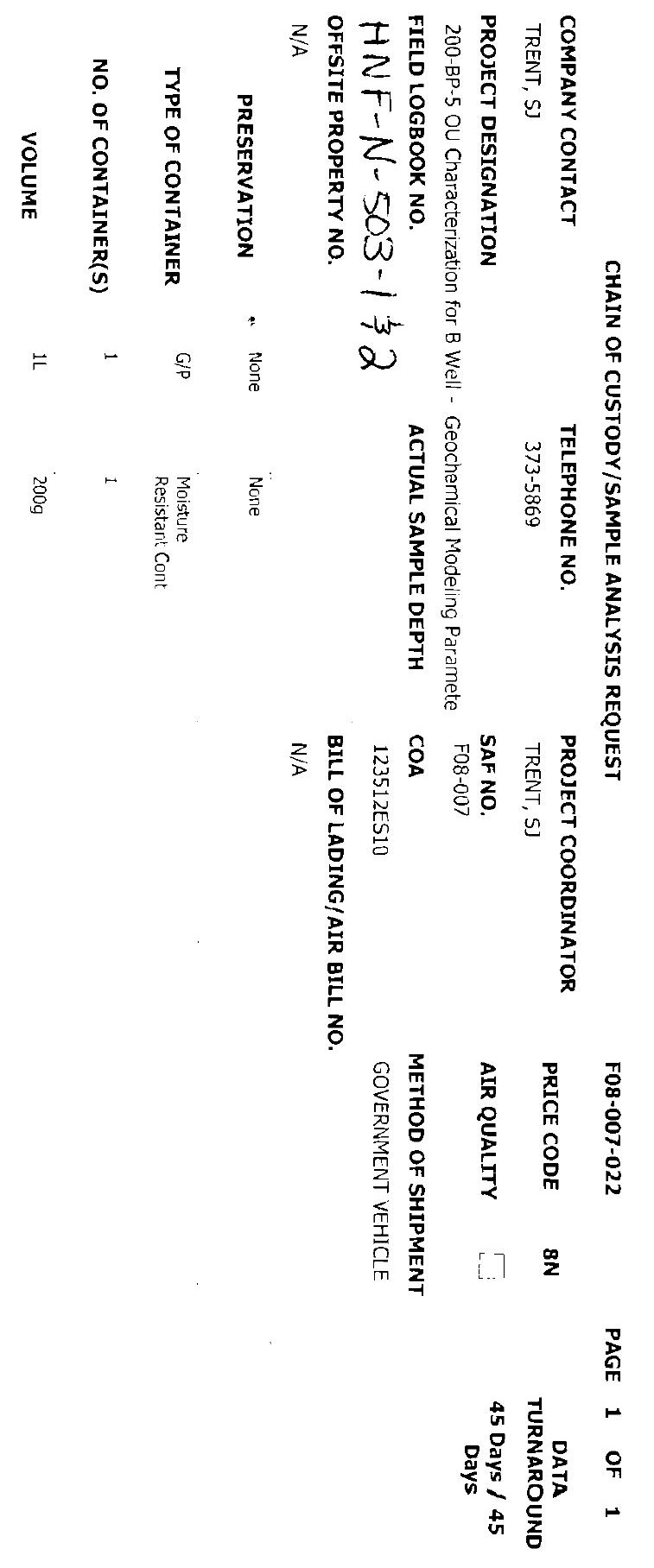




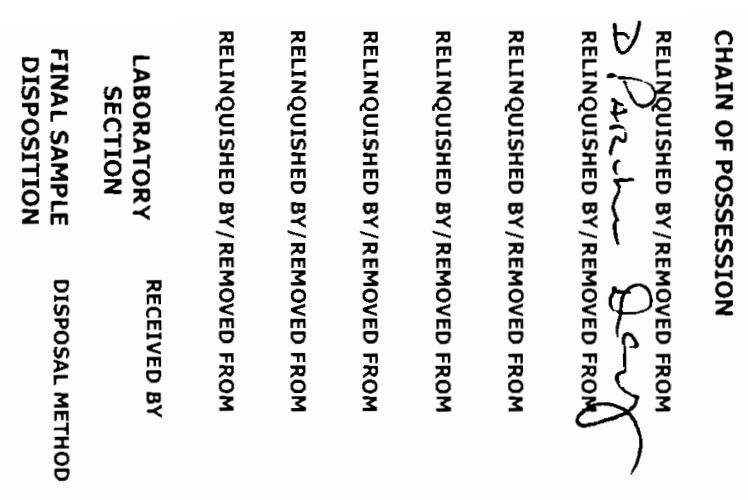

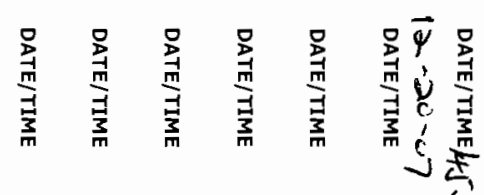

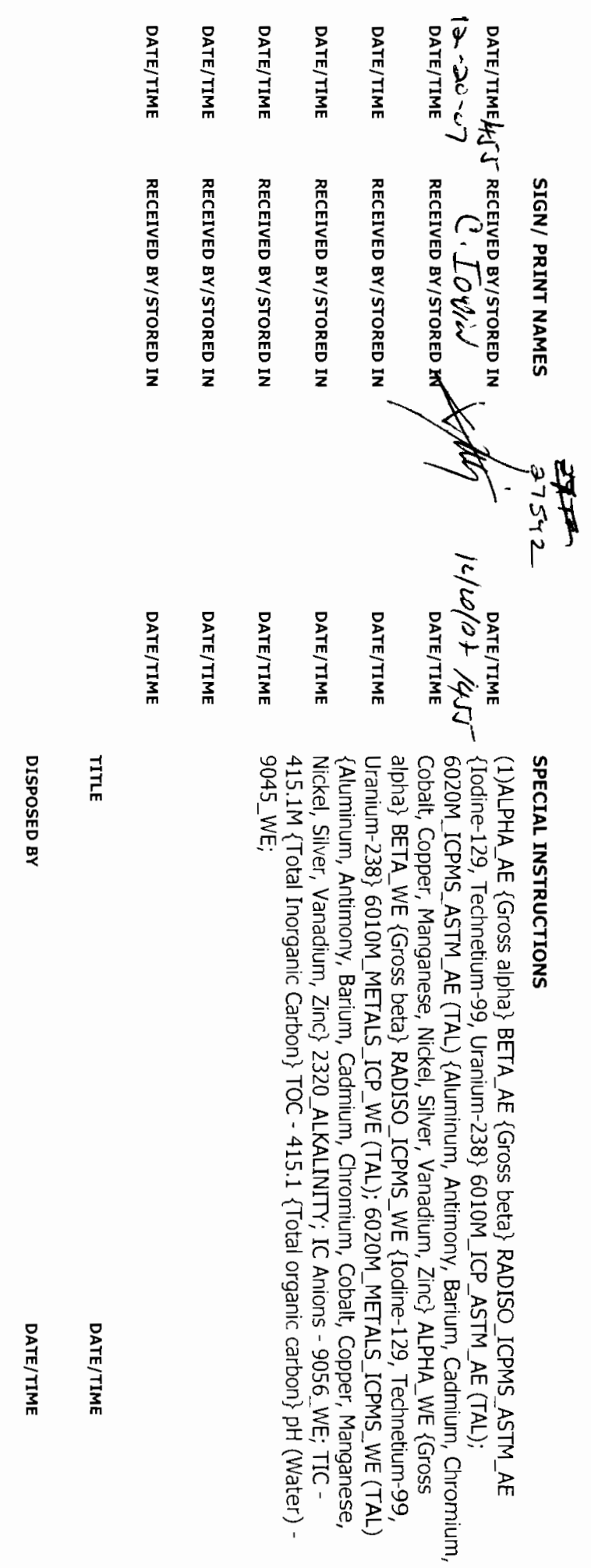

$\stackrel{\infty}{\circ}$
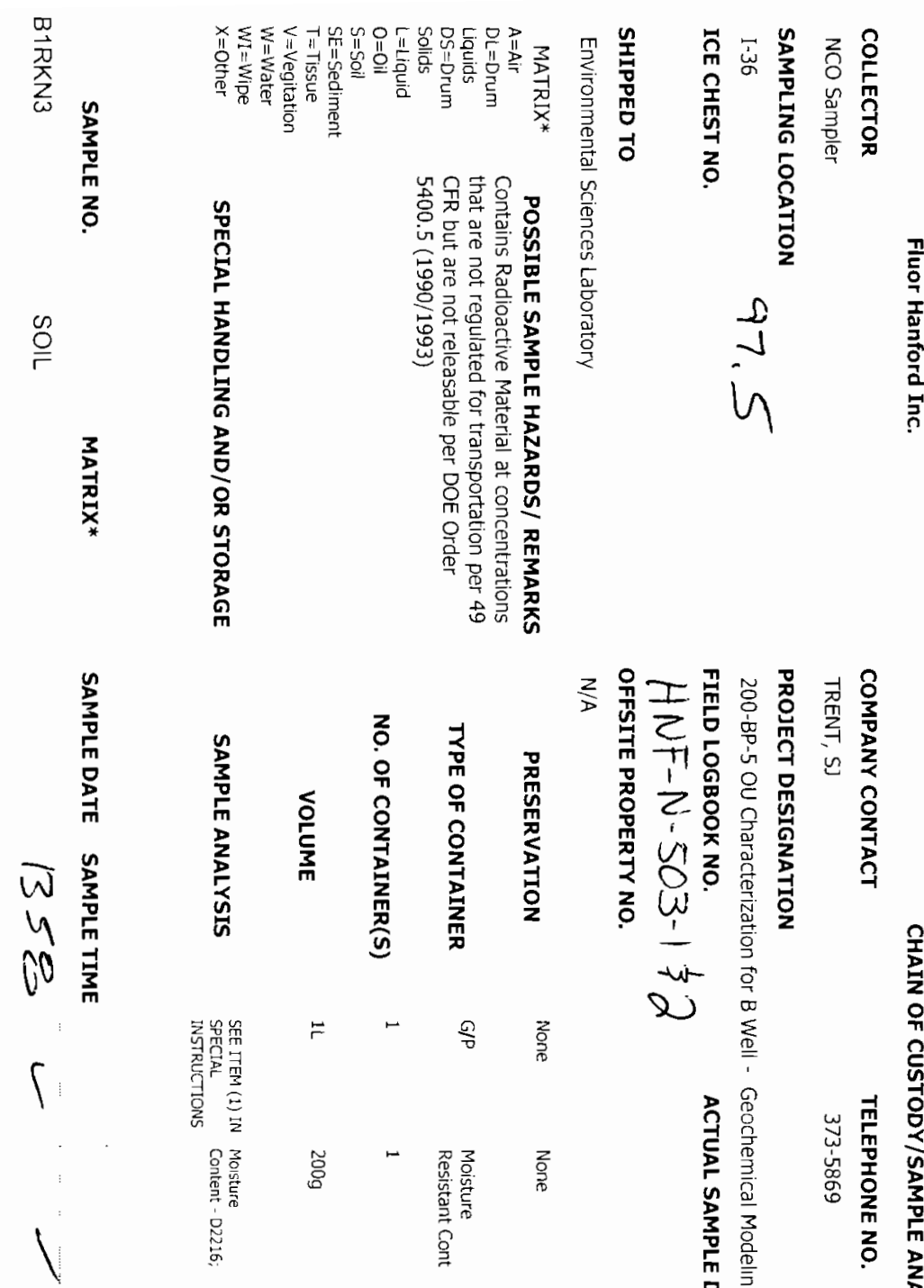

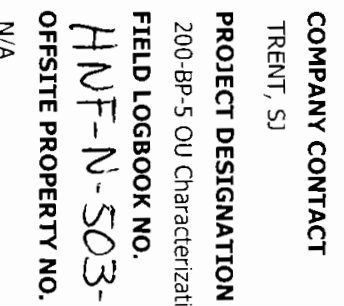

$\stackrel{1}{-i} \frac{\overrightarrow{0}}{2 j} \sum_{0}^{\infty}$

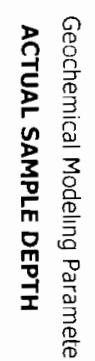

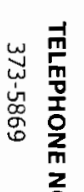

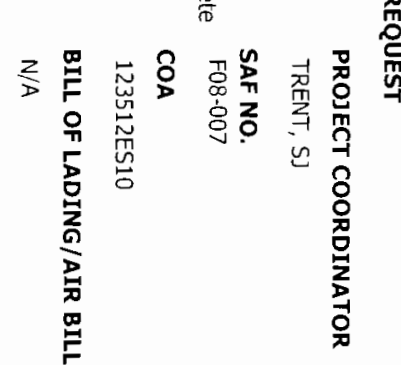

z
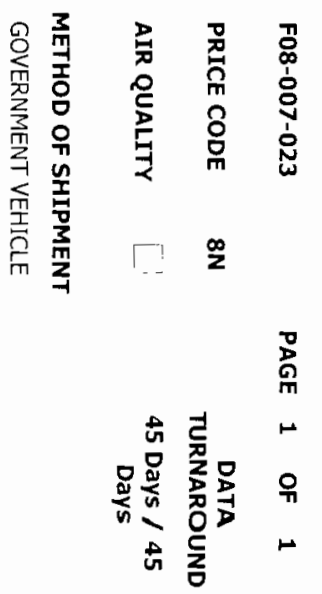


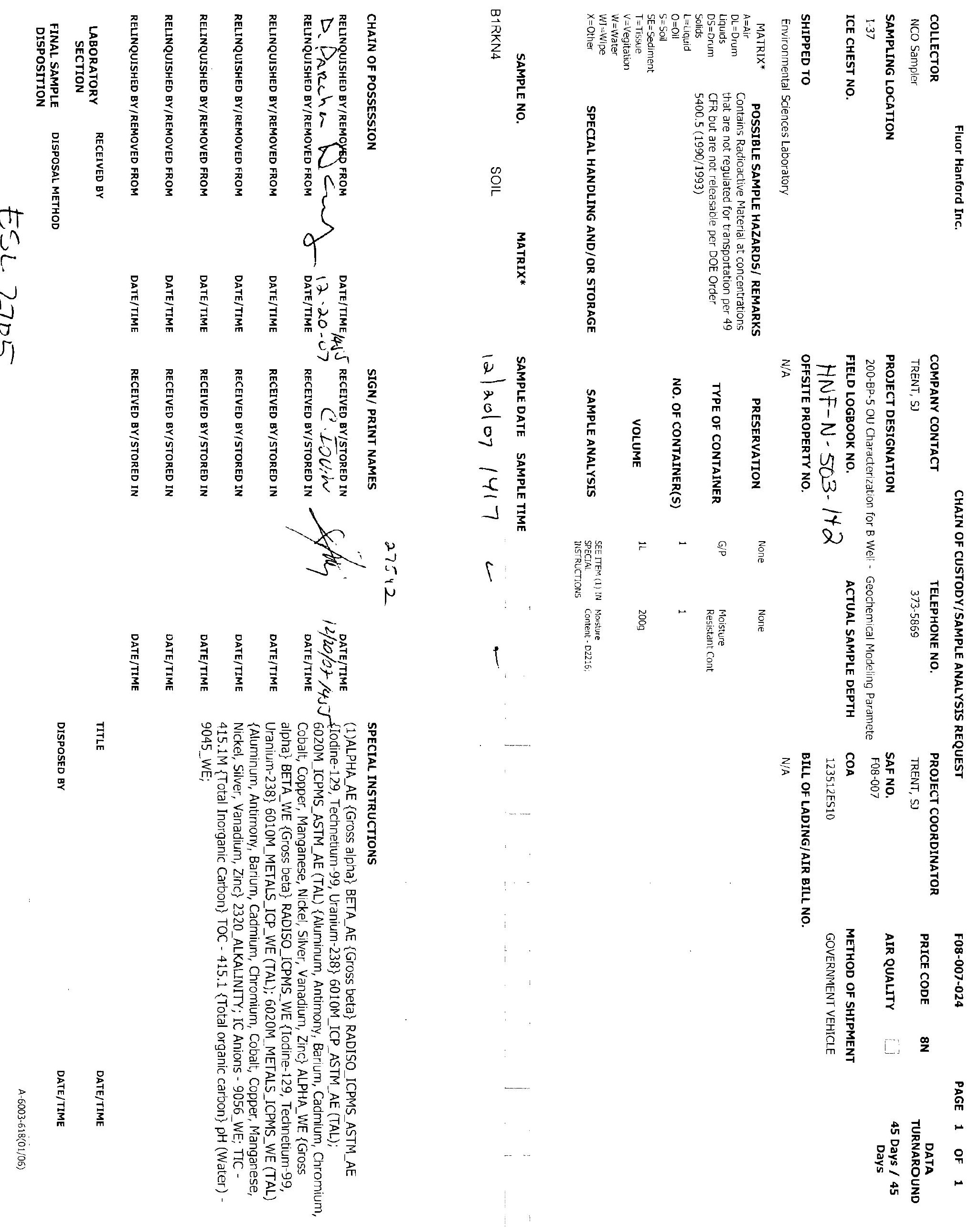



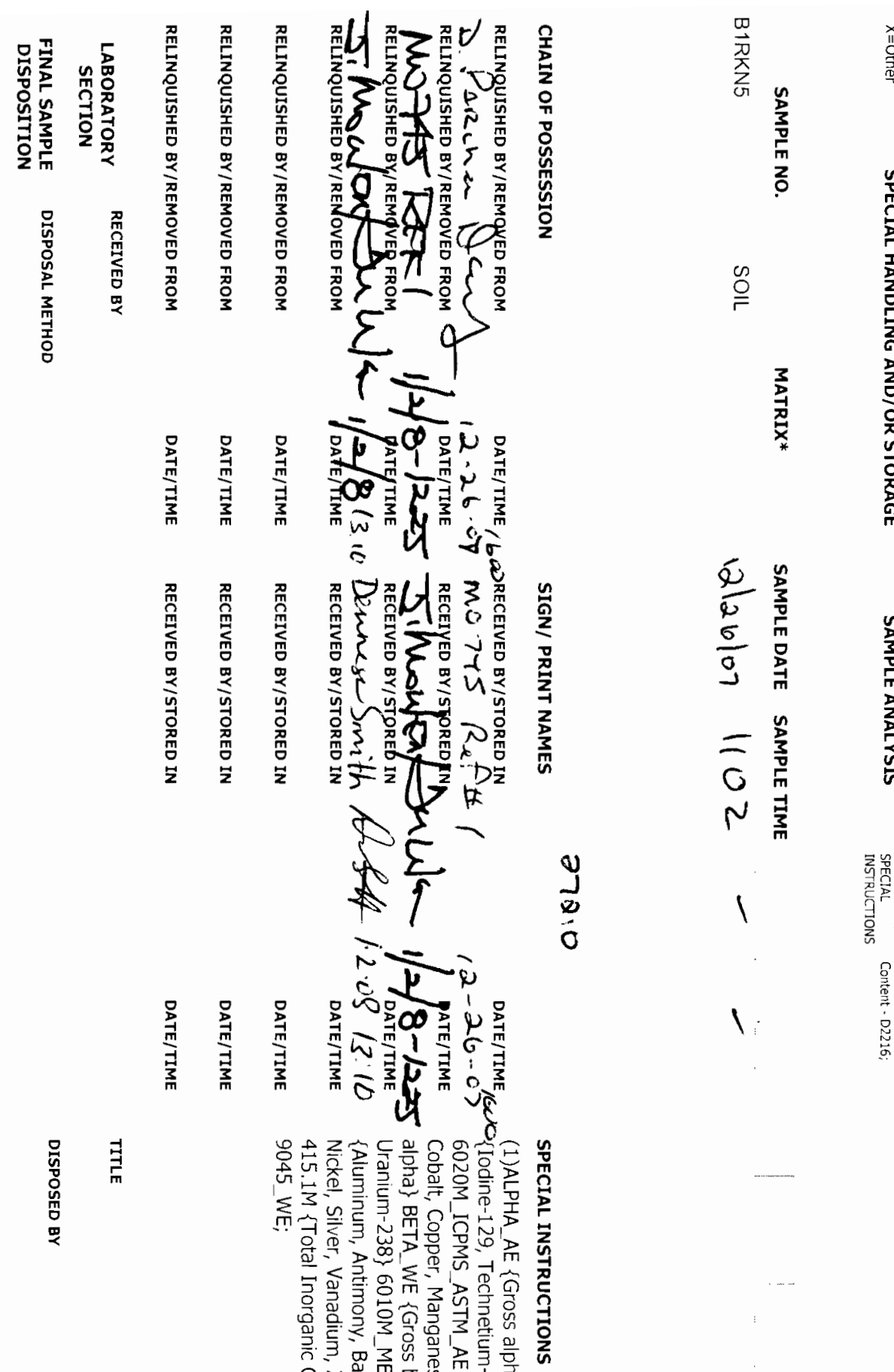

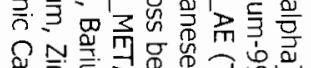

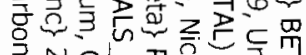

㟧

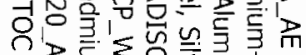

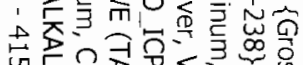

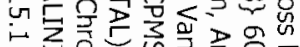

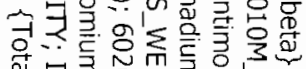

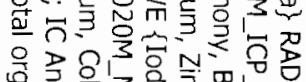

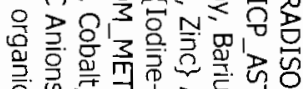

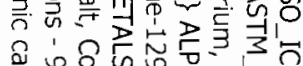

ฏ

$\frac{\sigma}{5}$

$\begin{array}{ll}= & \frac{n}{3} \\ 0 & \frac{3}{5} \\ N & \frac{7}{3}\end{array}$

$\underset{0}{0}$

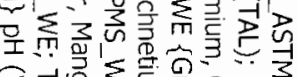

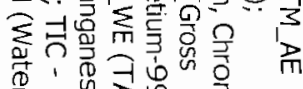

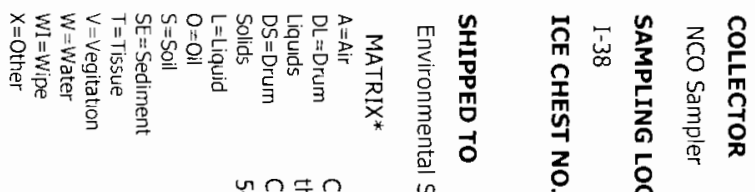
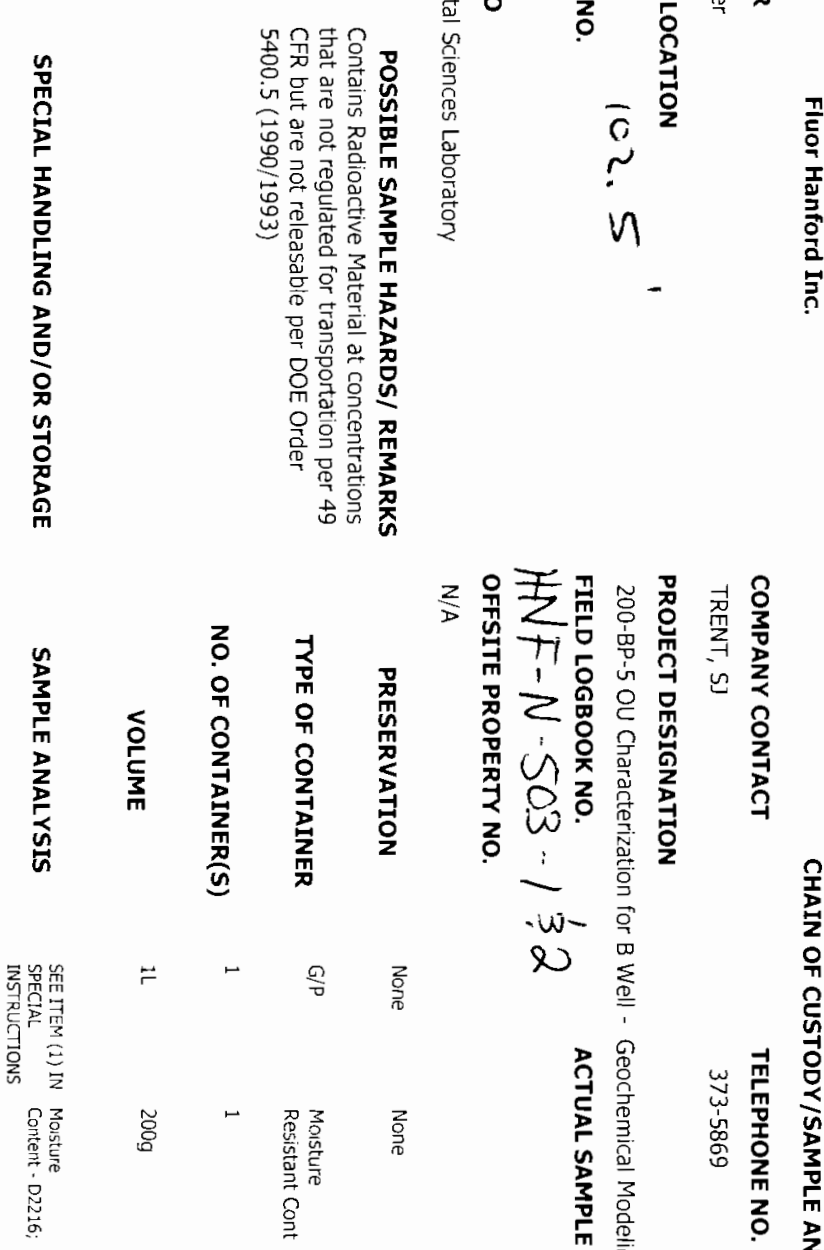

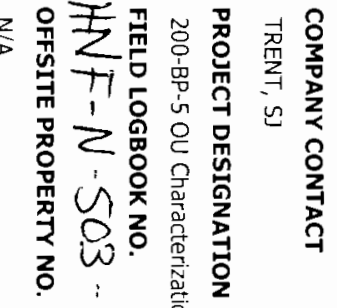

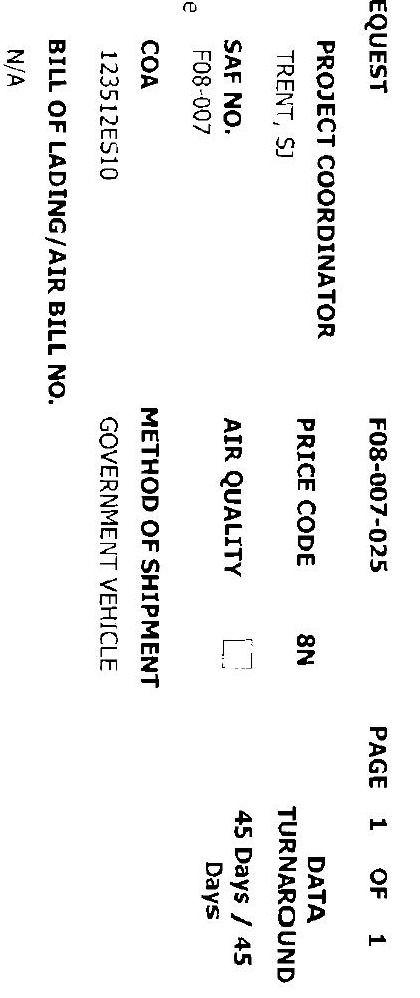




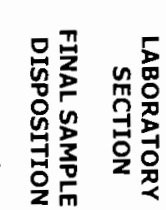 \\ 高章

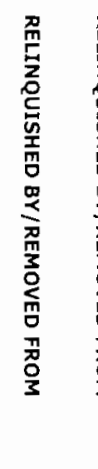

$\|$
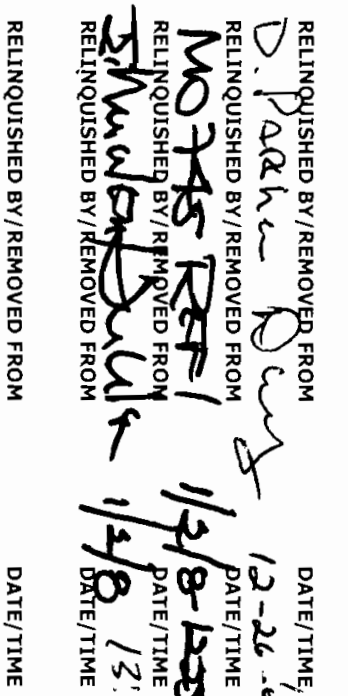

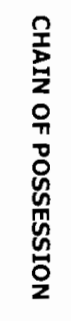

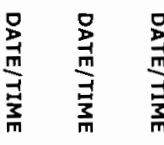

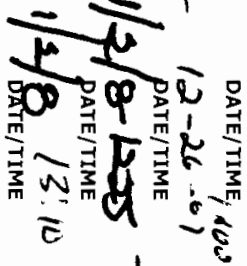

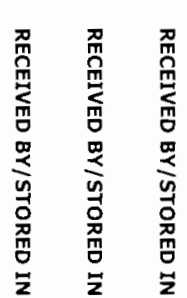

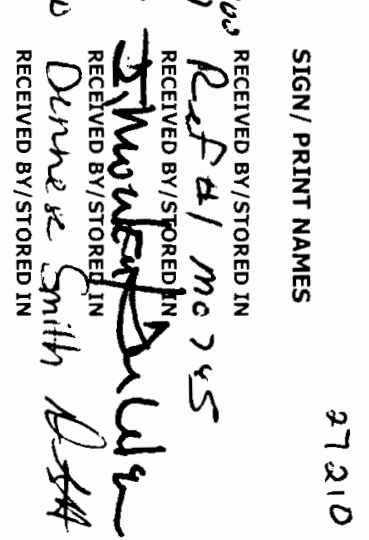

尊是

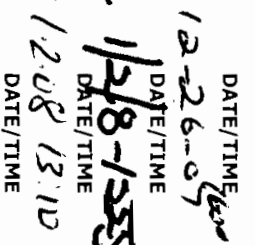

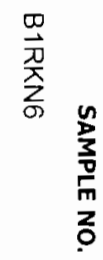

$\stackrel{\infty}{\rightleftharpoons}$

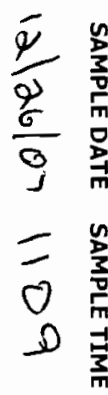

I

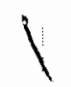

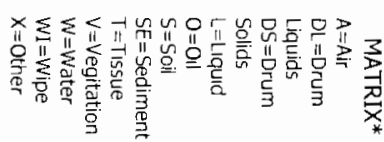

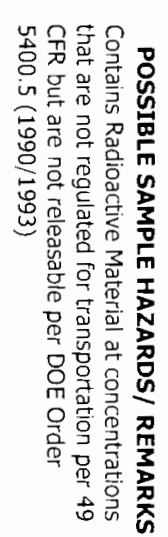

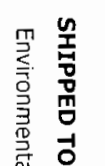

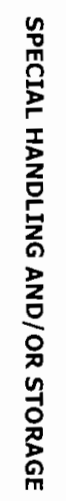

$\frac{\bar{n}}{\frac{1}{0}}$

on

站

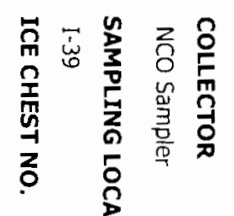

$\hat{b}^{2}$

1
$\vdots$
$\vdots$

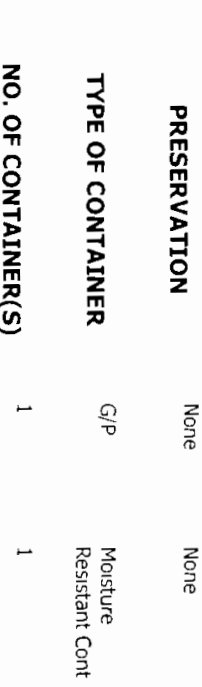

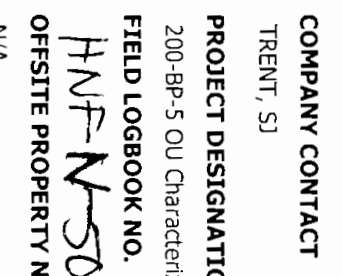$$
7
$$

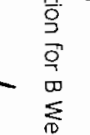

$\underset{m}{-1}$

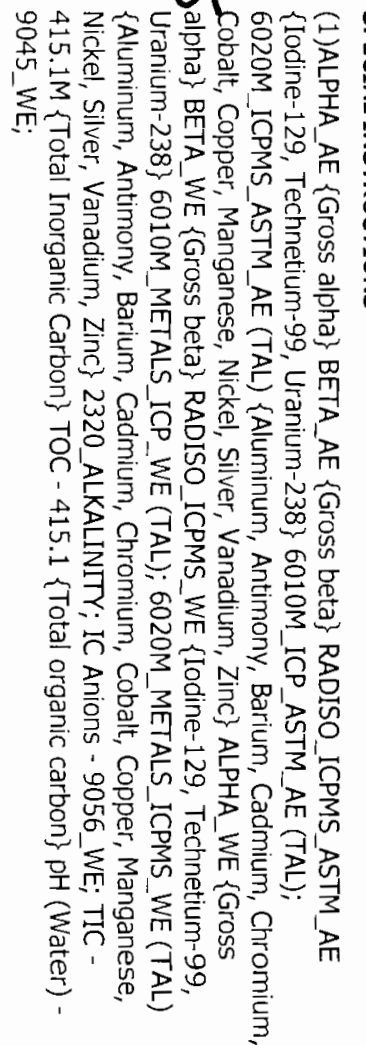

z

Q)

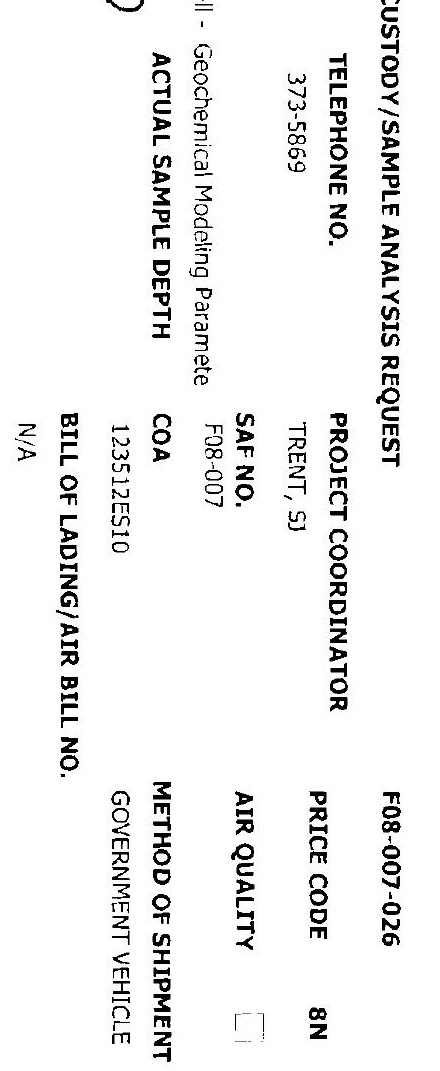

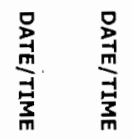




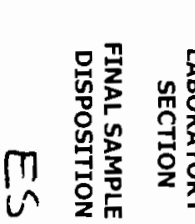
量
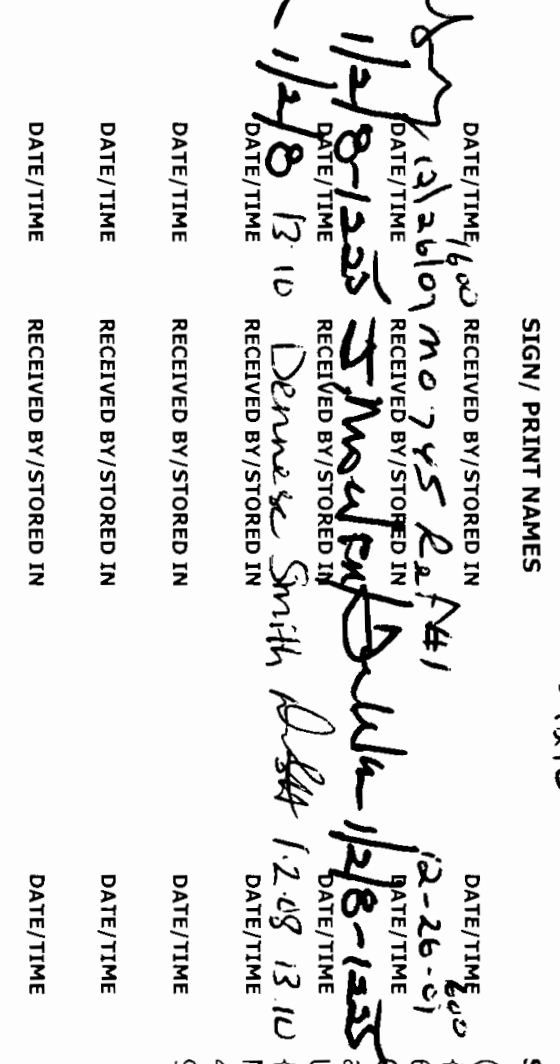

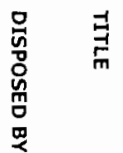

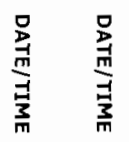

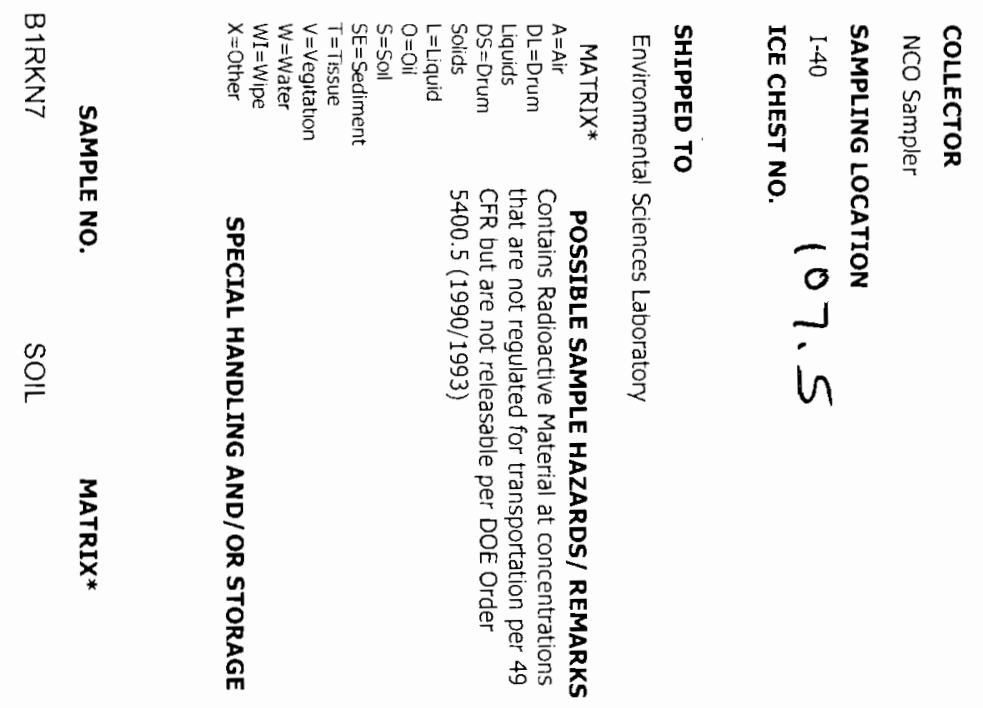

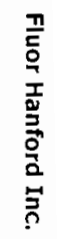

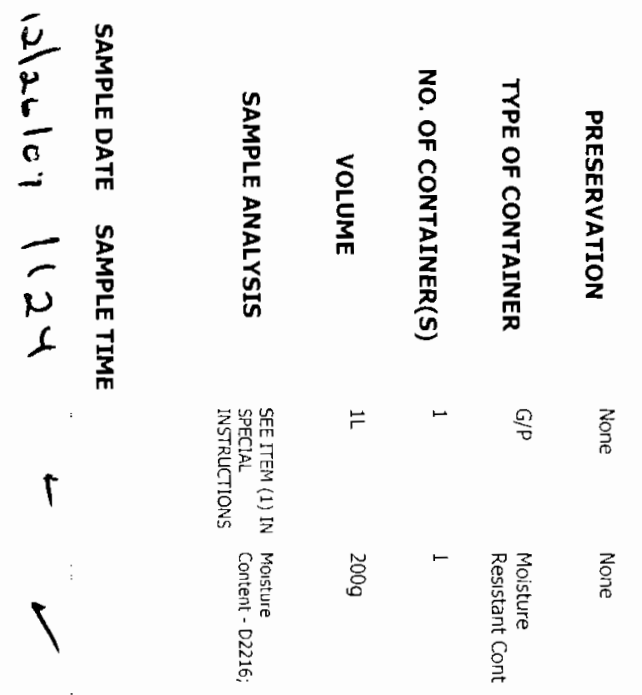

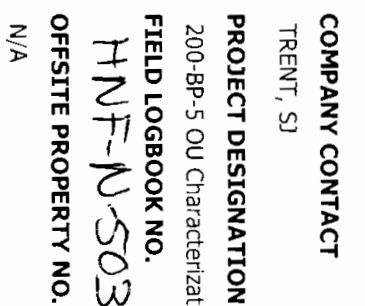

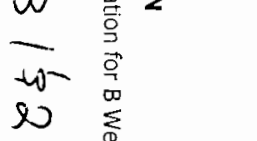

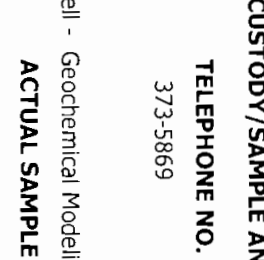

登

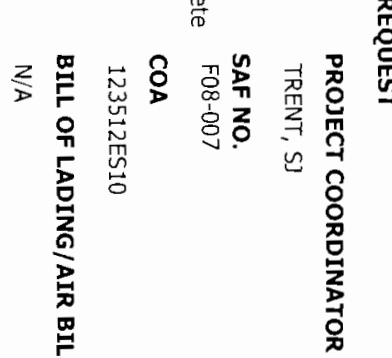

의

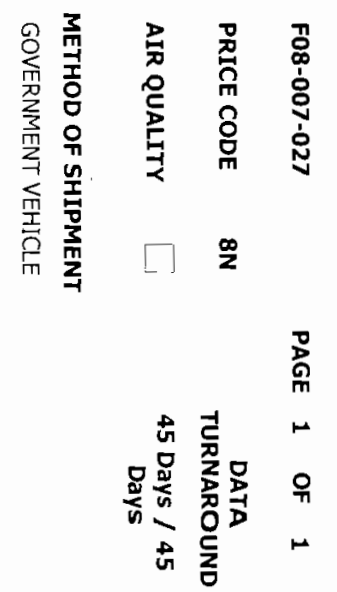




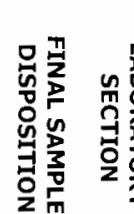

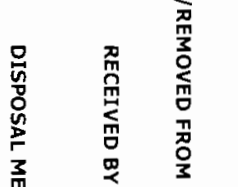

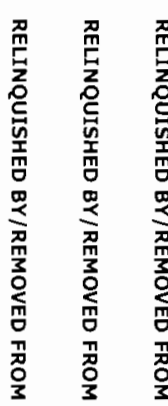
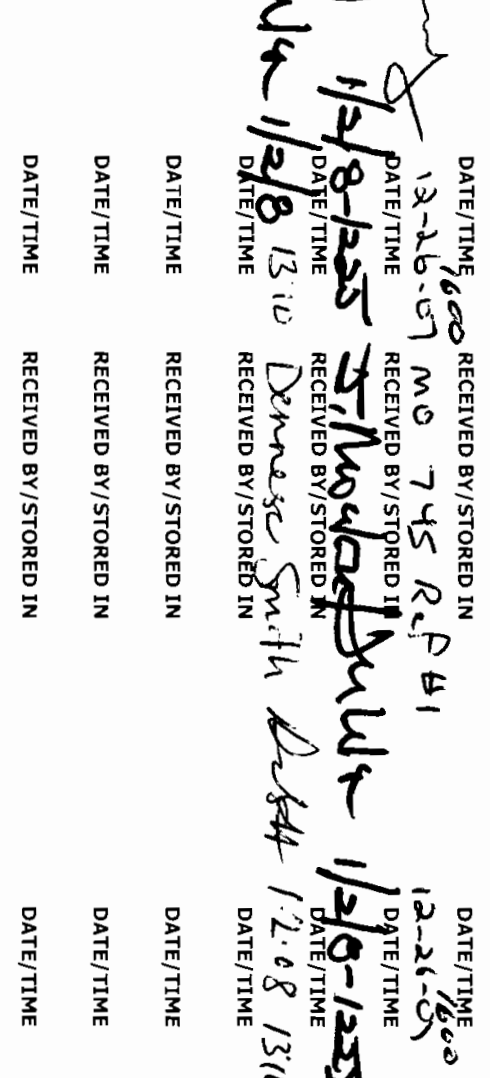

$\frac{y}{a}$

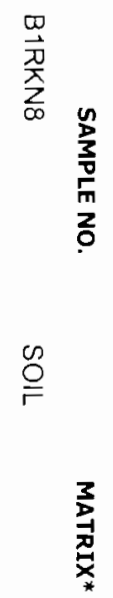

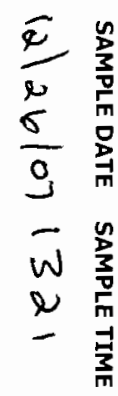

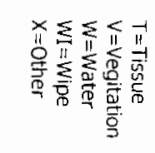

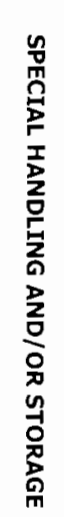

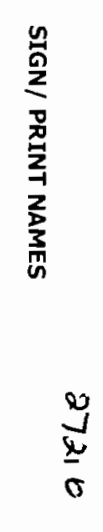

个 总

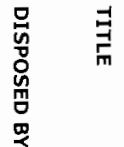

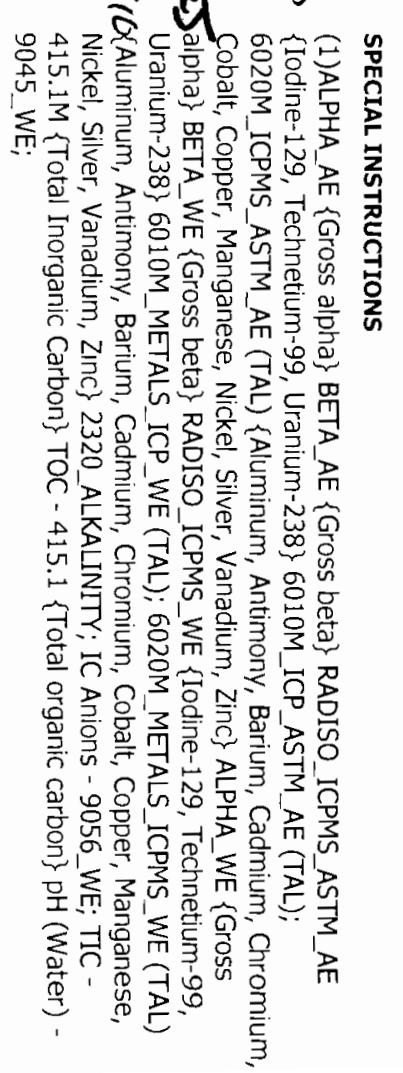

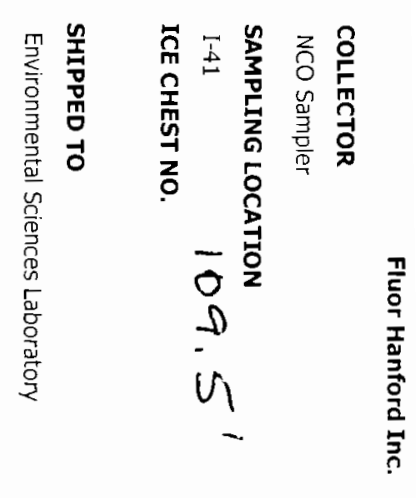
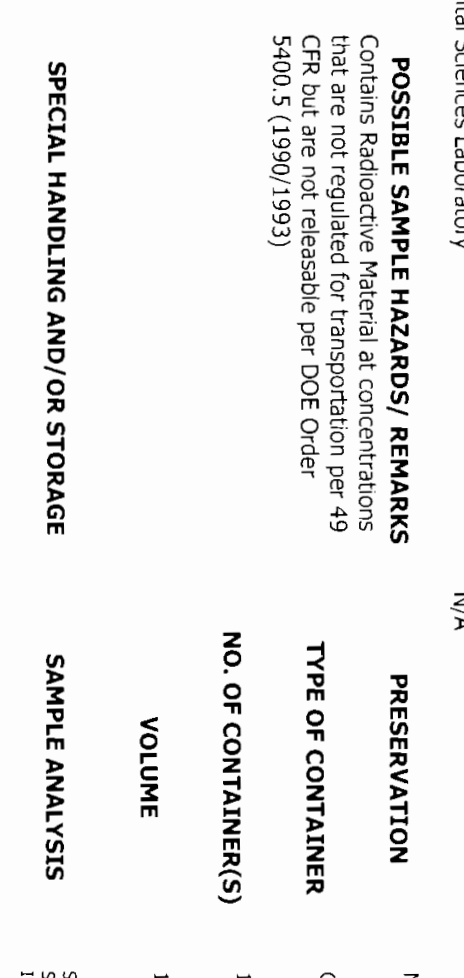

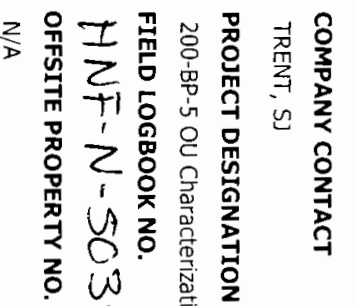

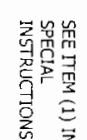
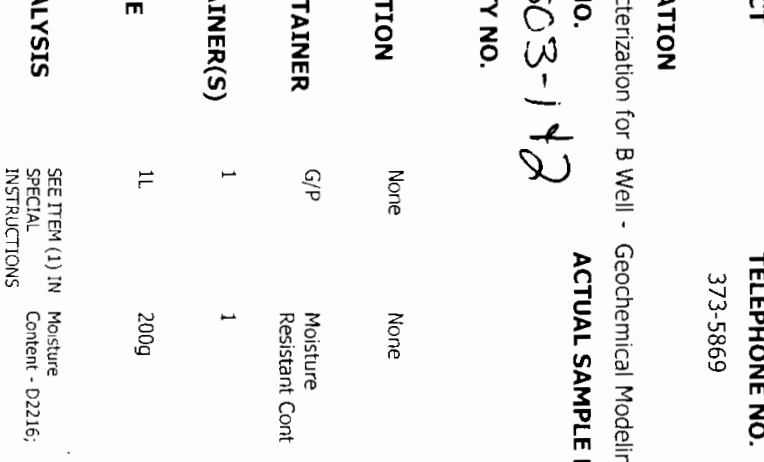

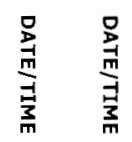




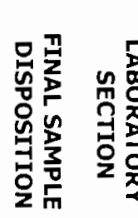

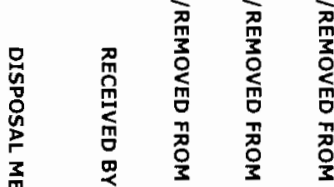

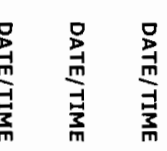

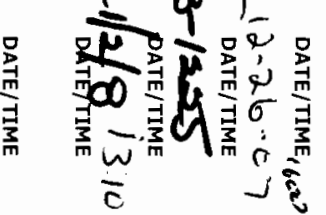

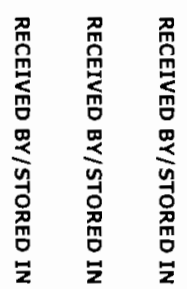

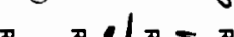

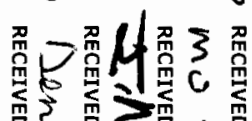

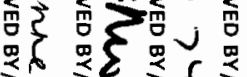

ज़้

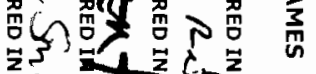

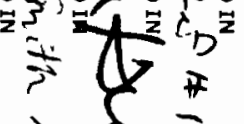

迹

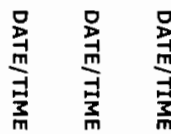

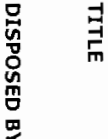

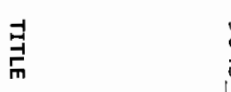

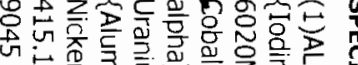

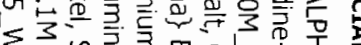

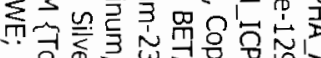

iे $\overline{0 र}$
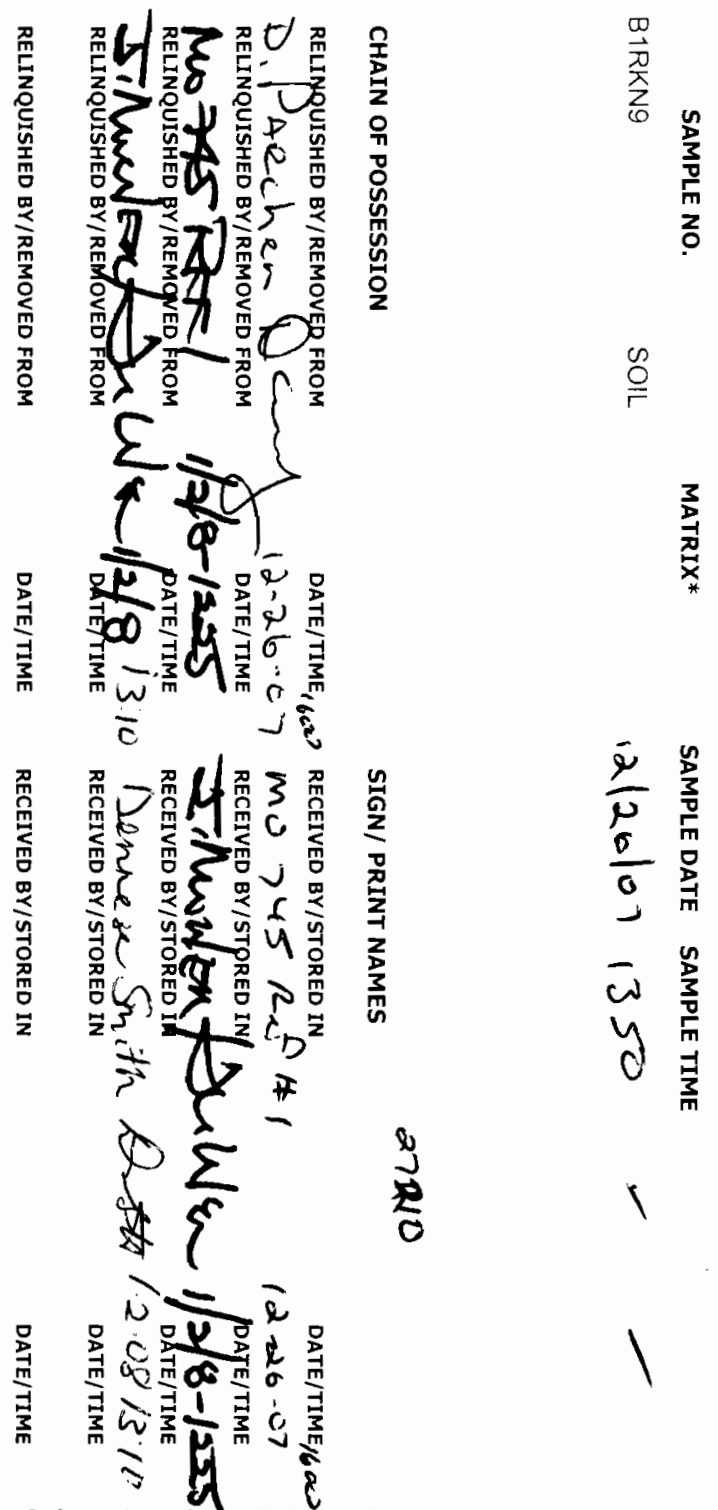

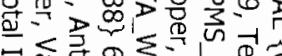

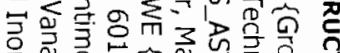

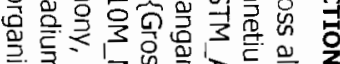

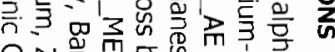

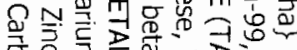

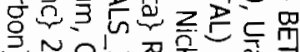

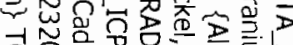

웅.

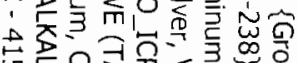

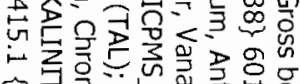

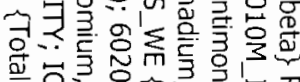

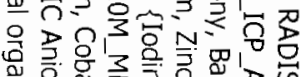

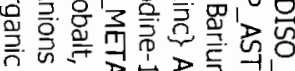

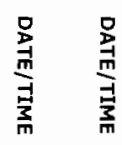

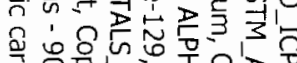

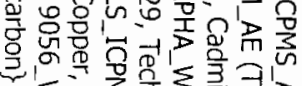

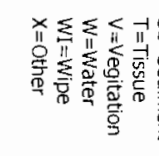

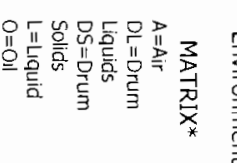

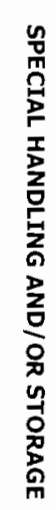

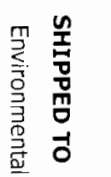

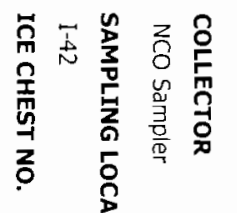

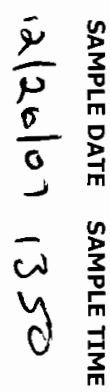

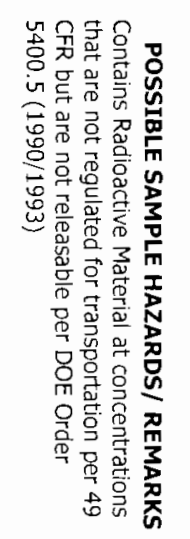

品

a

西
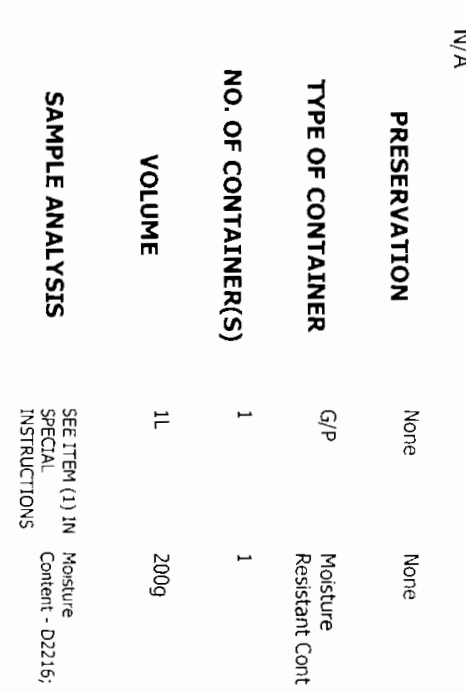

$-\frac{7}{2}$

eे

is

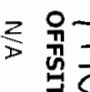

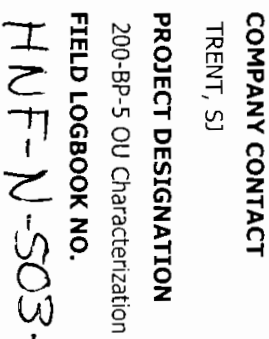

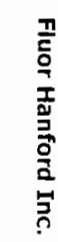

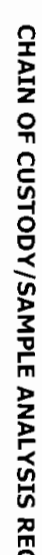

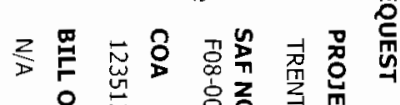

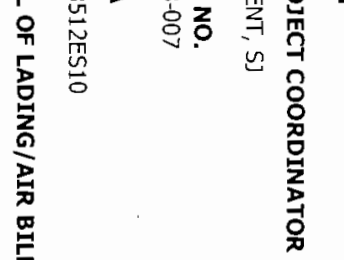

z

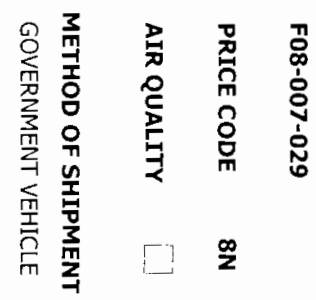

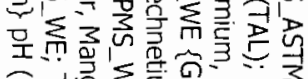

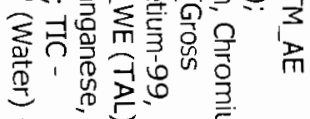

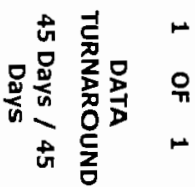




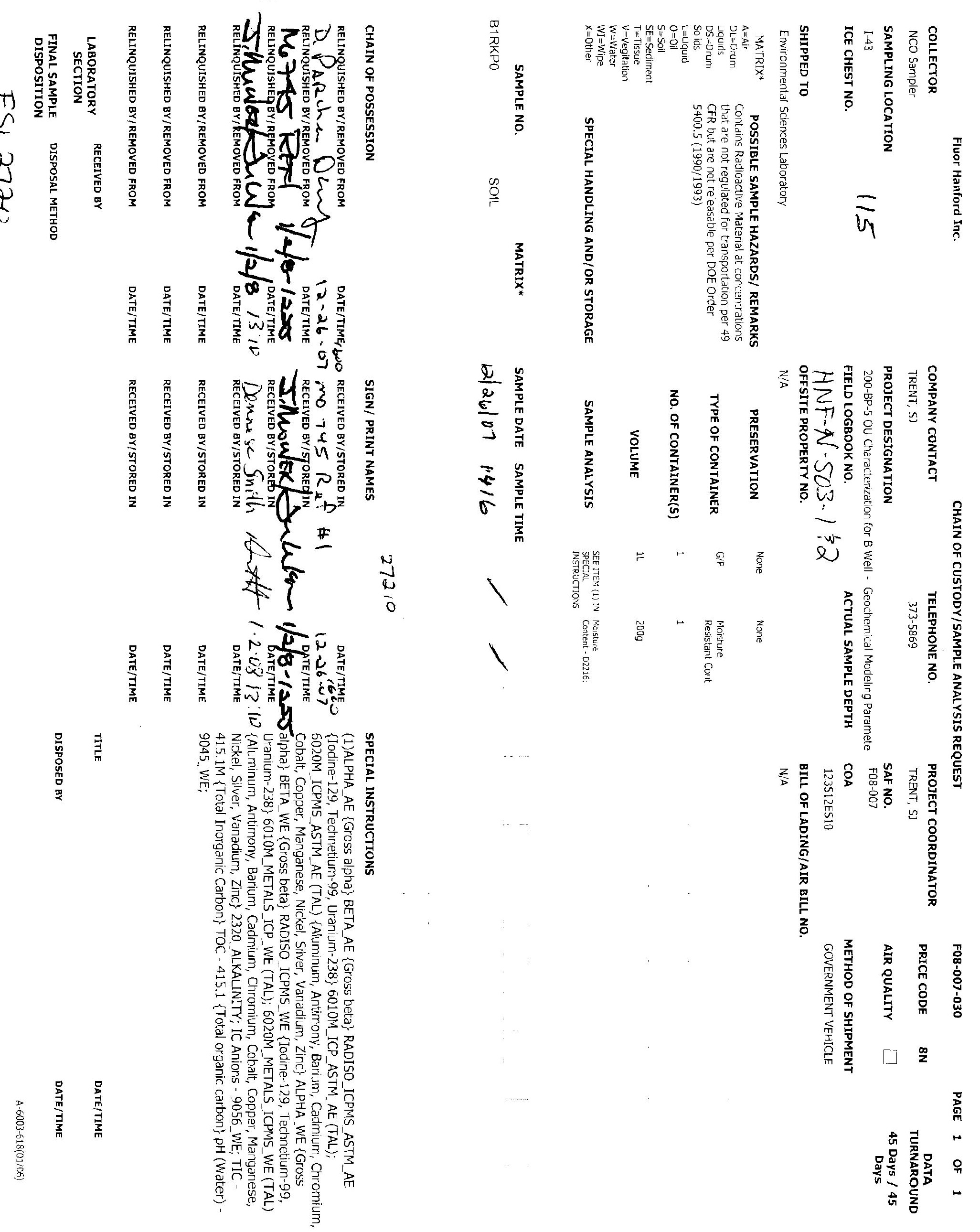



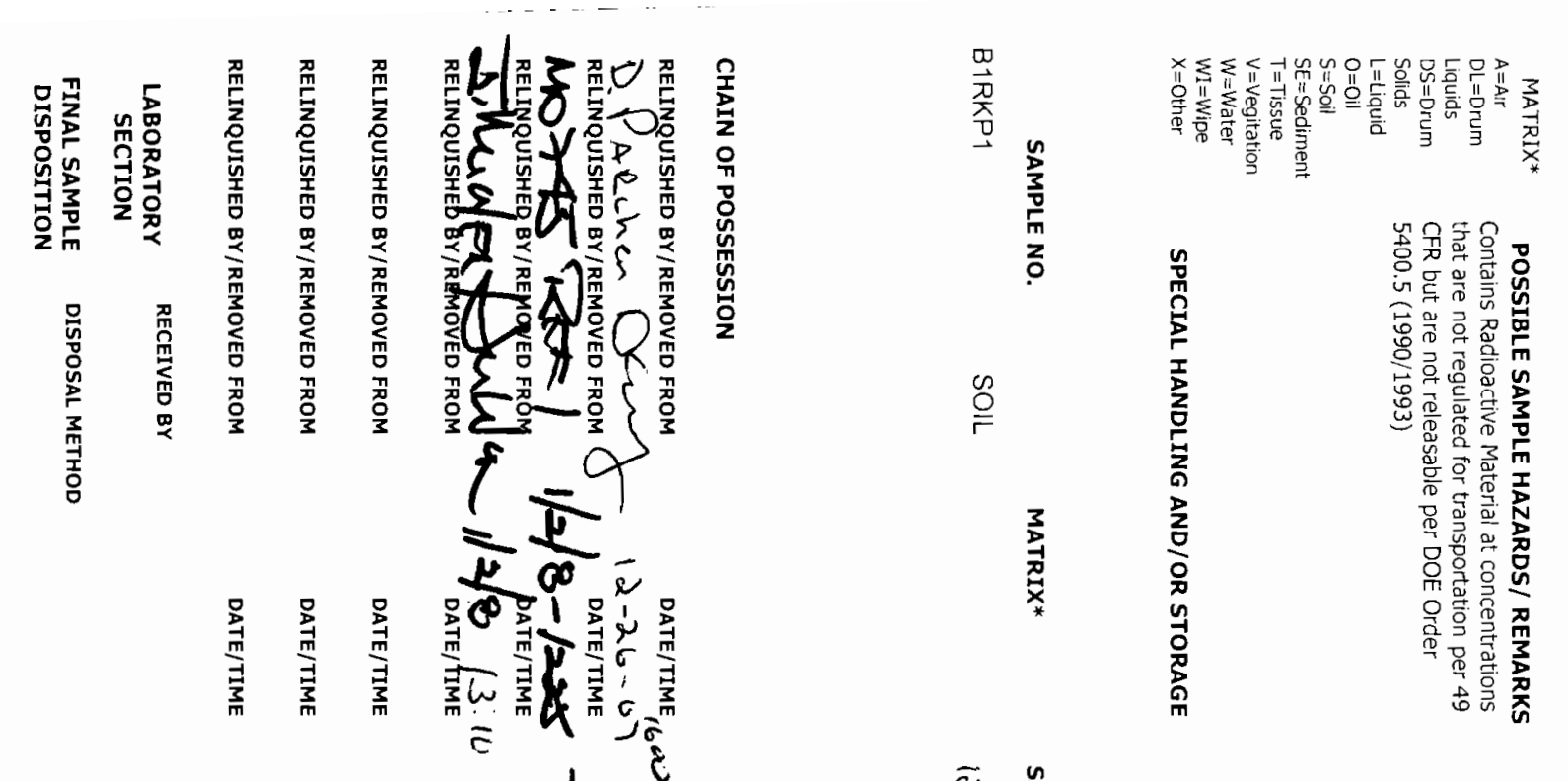

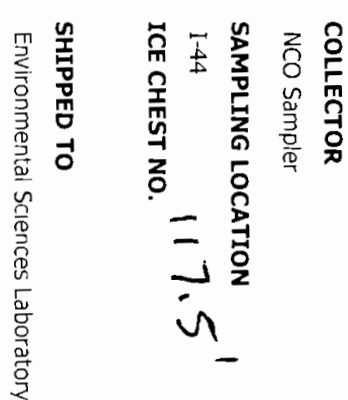

$\stackrel{\infty}{\Gamma}$

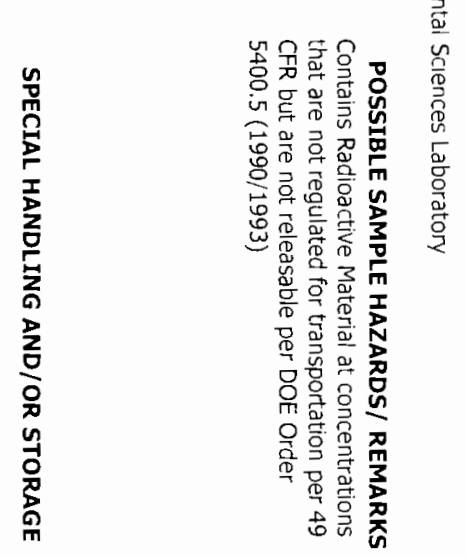

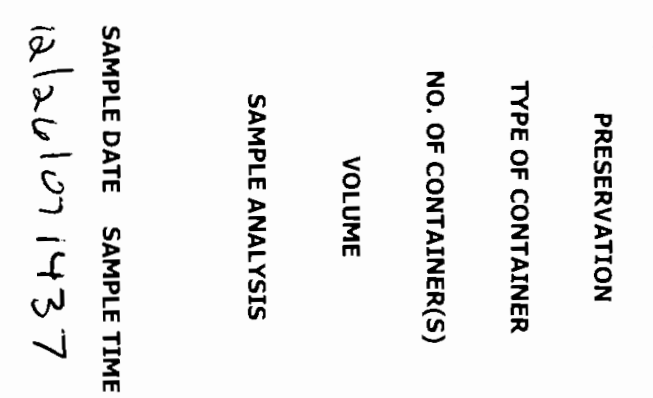

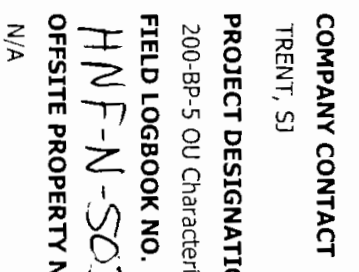

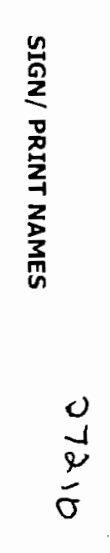

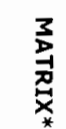

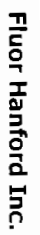

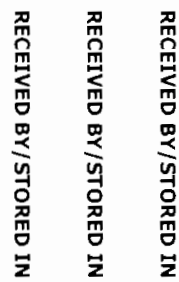

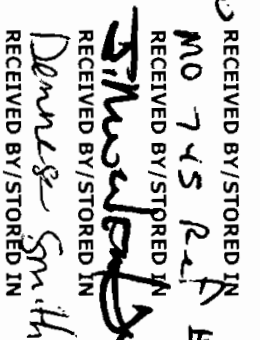

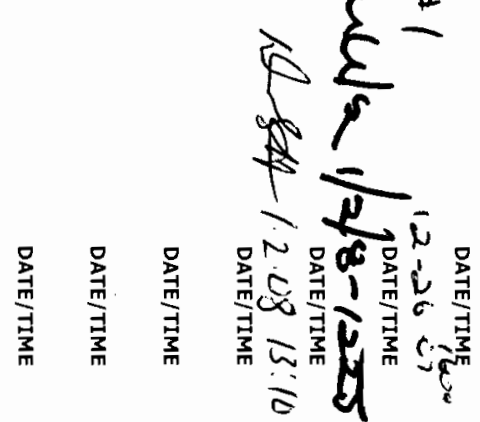

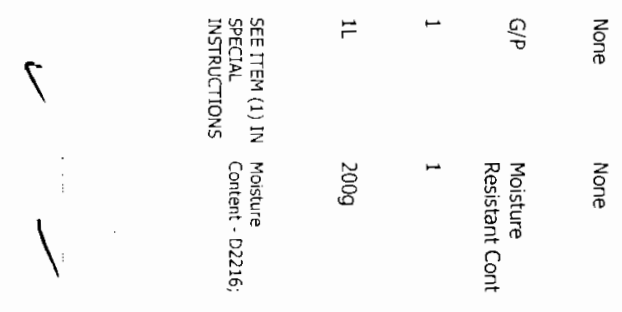

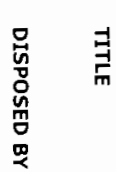

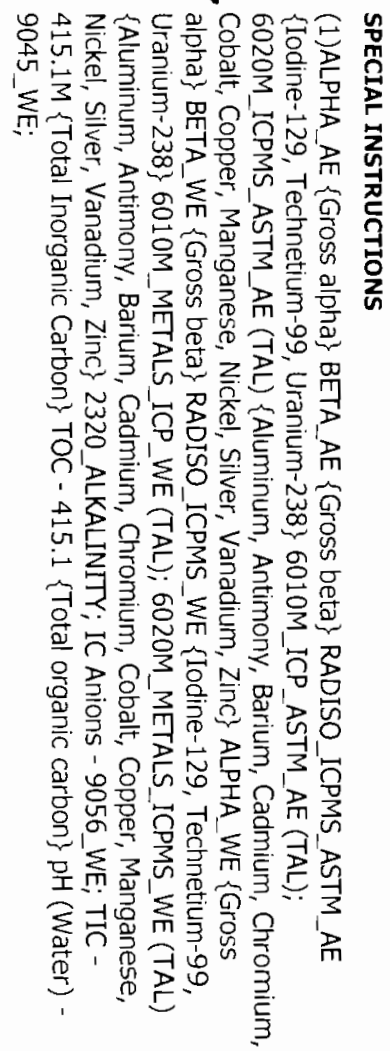

总

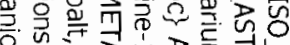
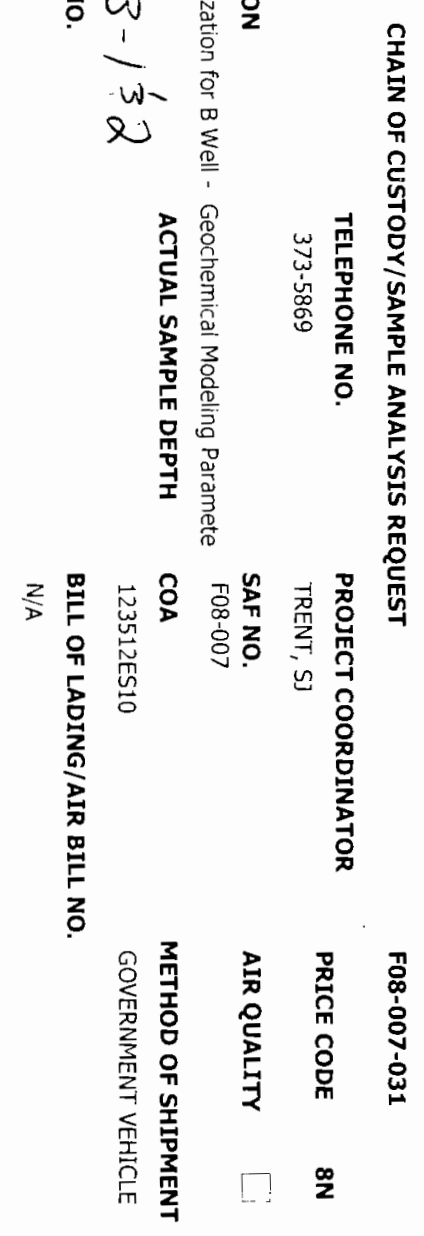

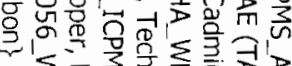

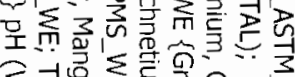

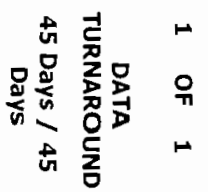


In
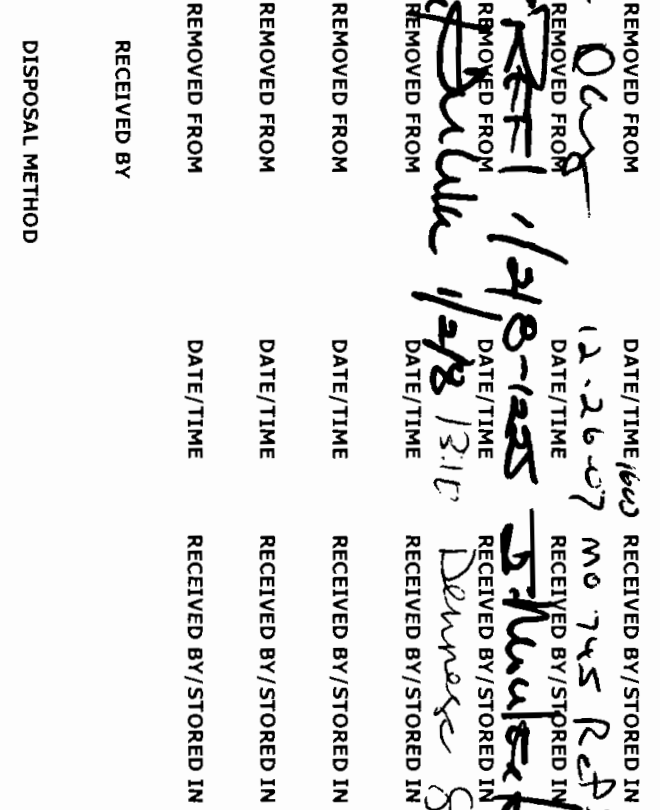

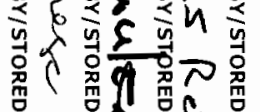

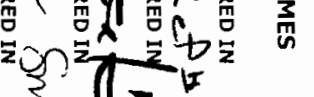
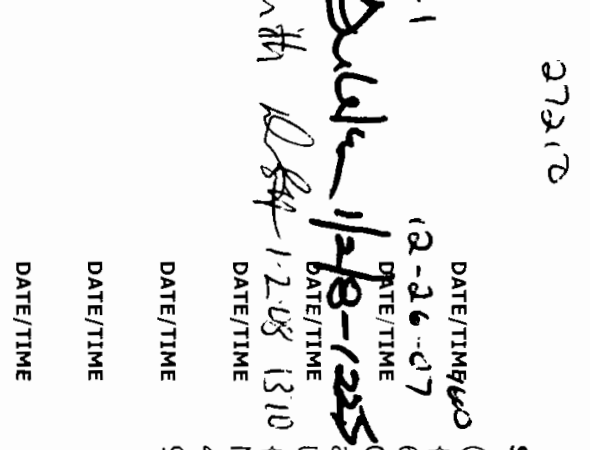

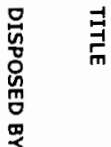

品量

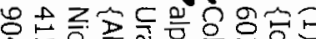

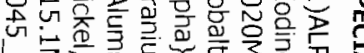

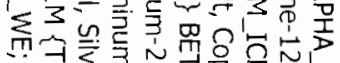

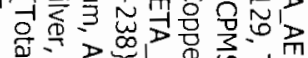

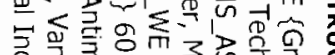

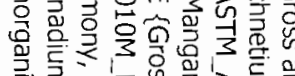

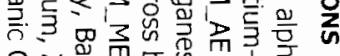
을 월

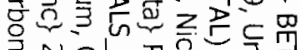

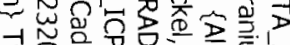

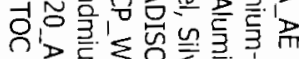
1

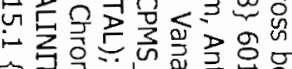

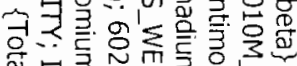

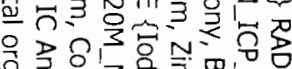

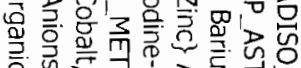

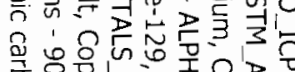

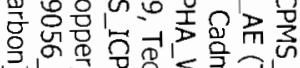

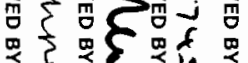
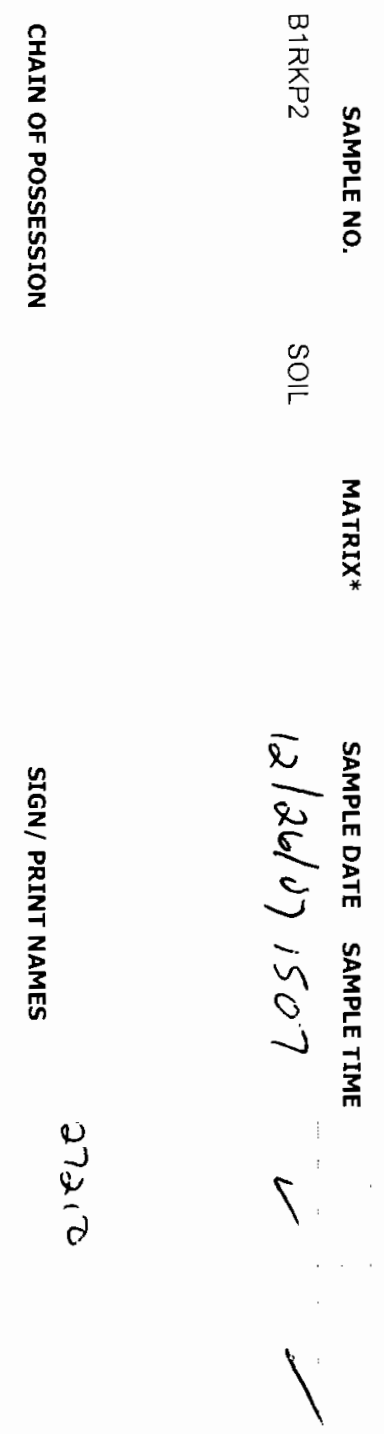

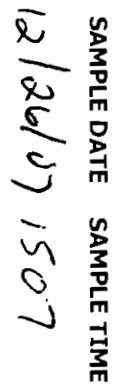

$\stackrel{\infty}{\square}$

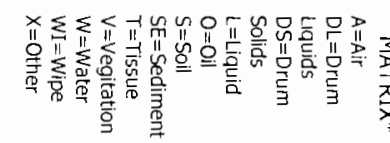

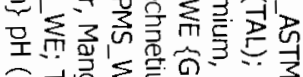

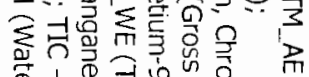

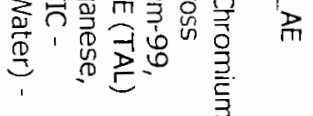
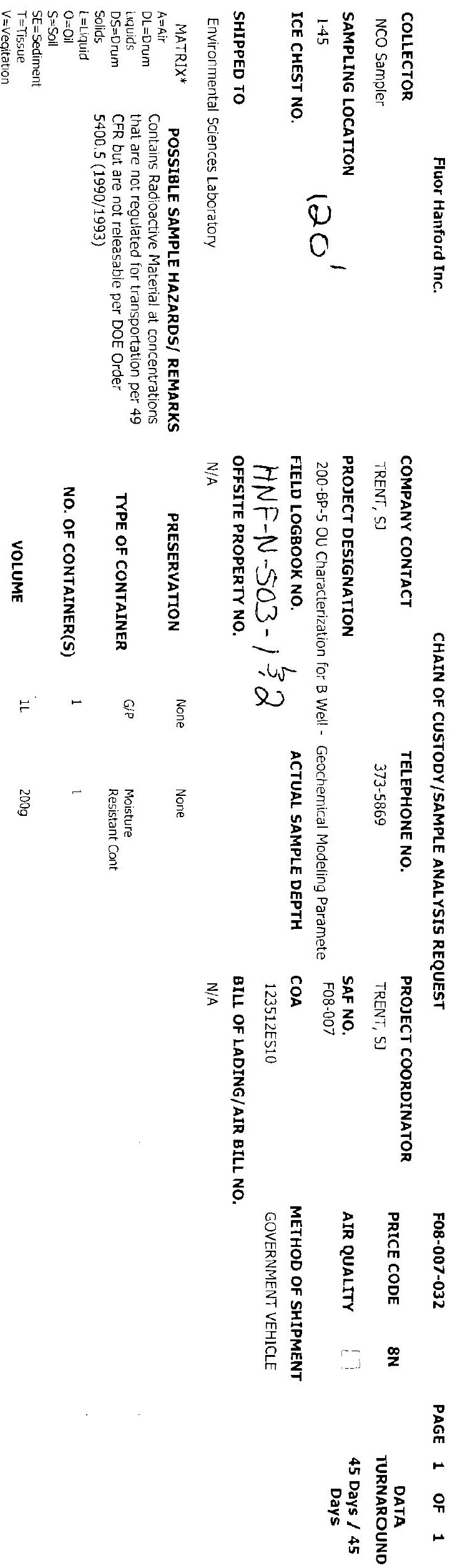


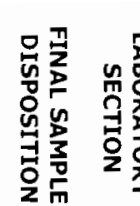

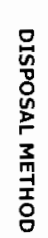

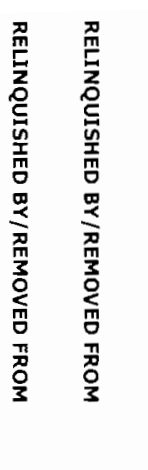

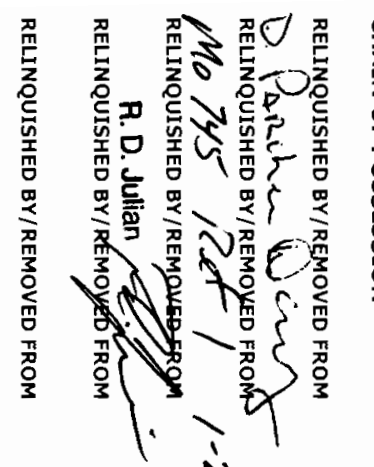

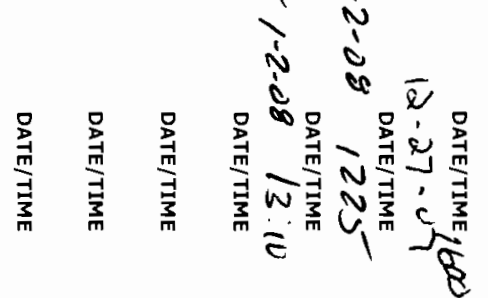

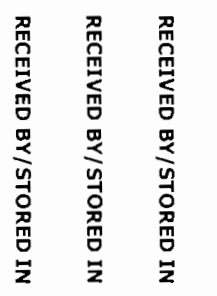

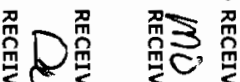
感 0 学

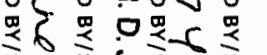

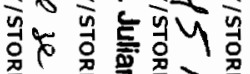

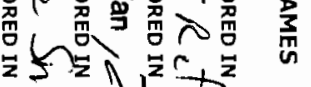

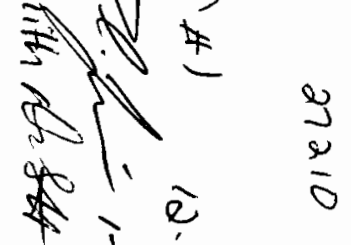

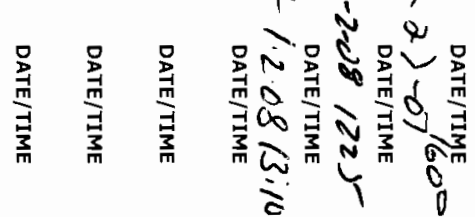

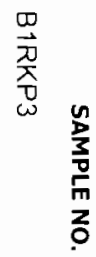

$\stackrel{\infty}{\stackrel{0}{\Gamma}}$
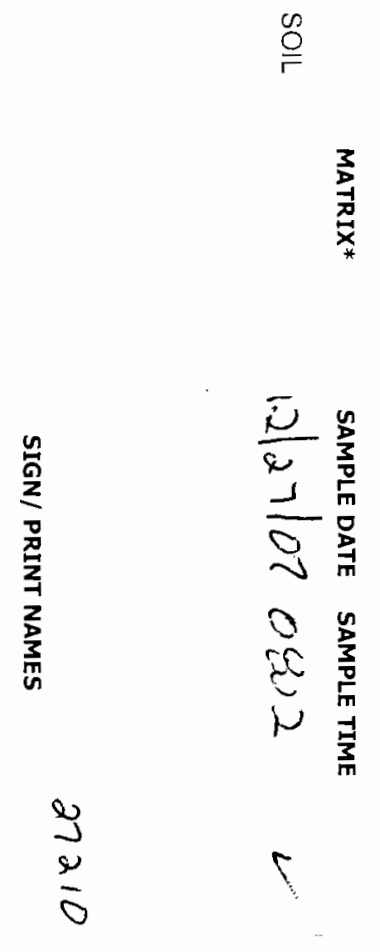

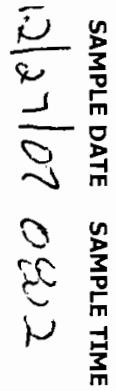

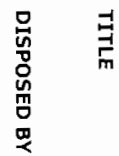

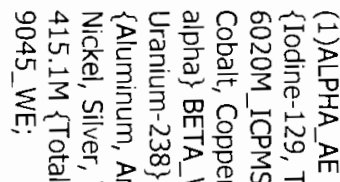

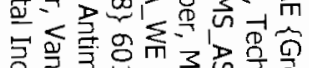

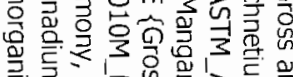

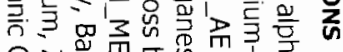

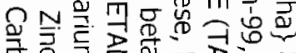

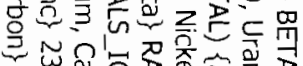

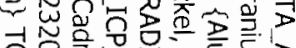

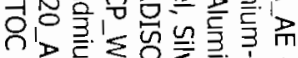

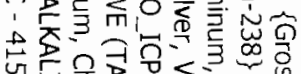

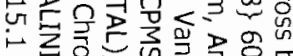

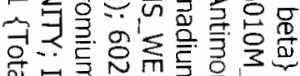

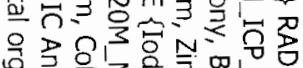

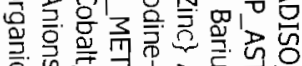

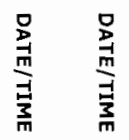

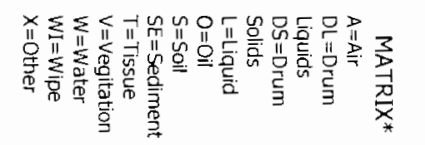

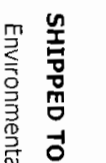

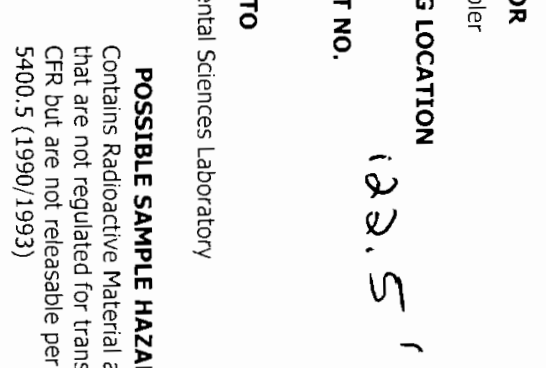

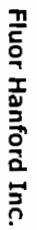

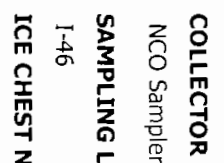
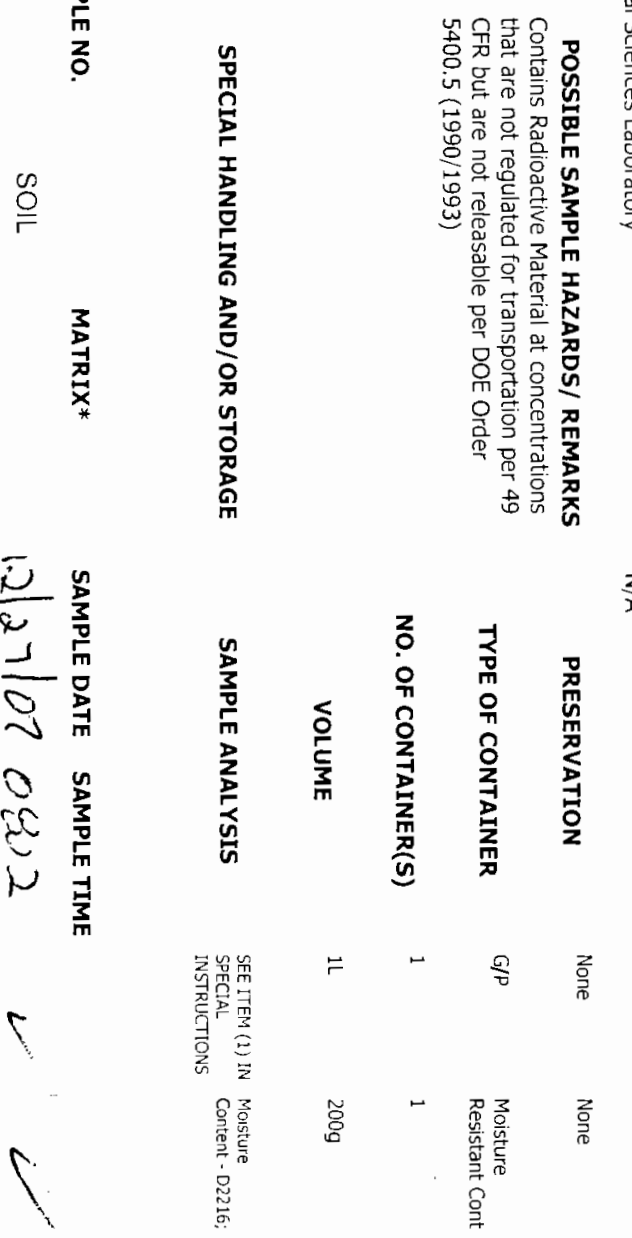

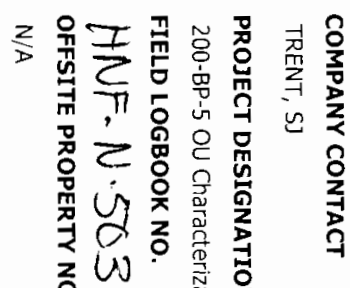
$-\omega$
$-\omega$

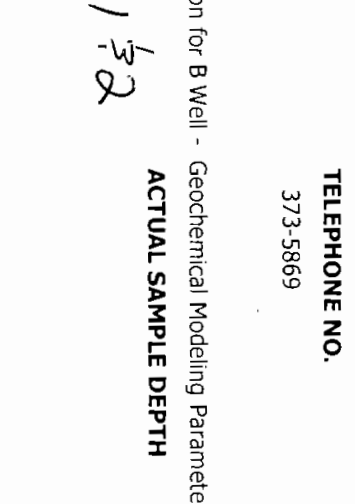

爱
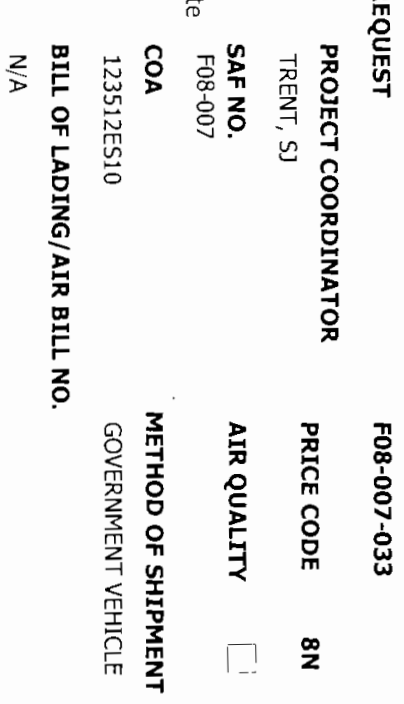

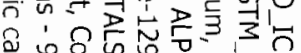

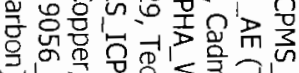

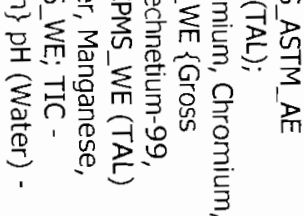


111

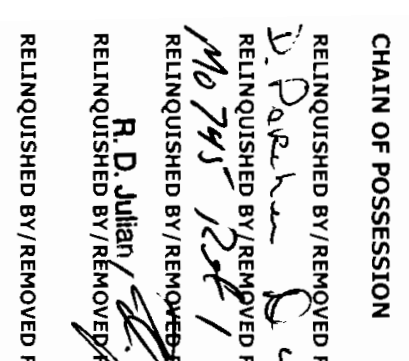

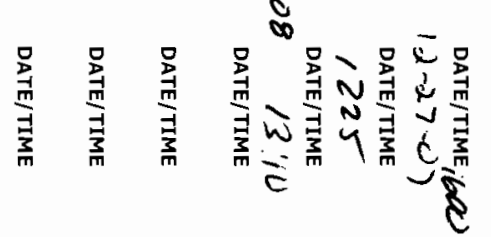

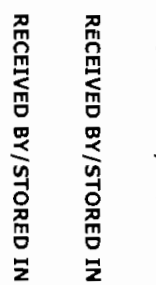

镸

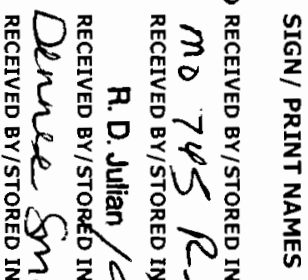

$=\sqrt{2} x^{2}$

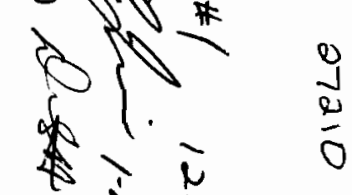

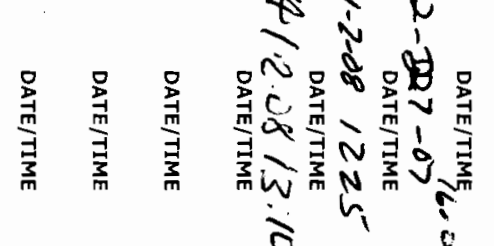

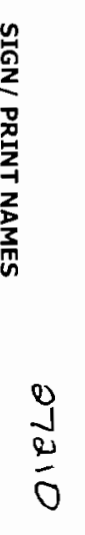

要

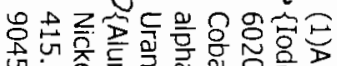

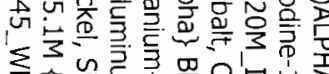

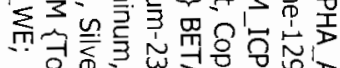

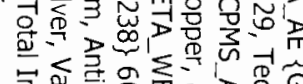

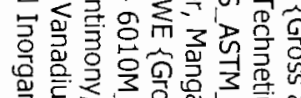

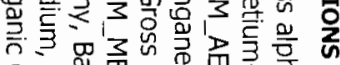

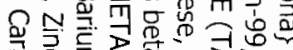

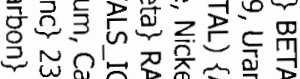

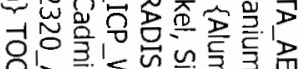

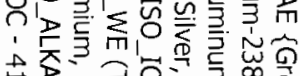

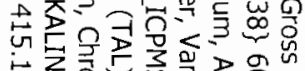

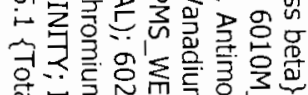

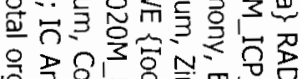

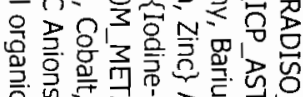

品品

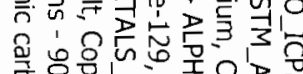

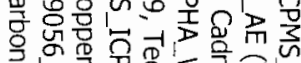

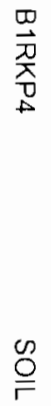

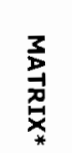

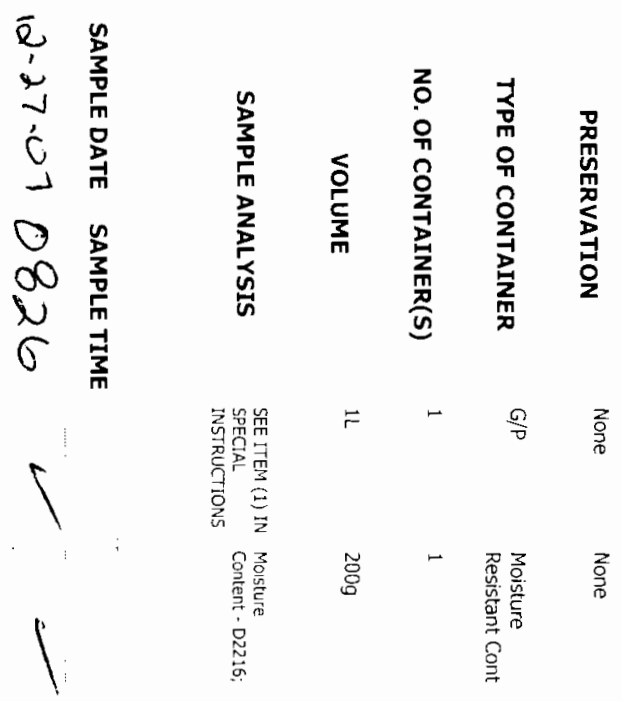

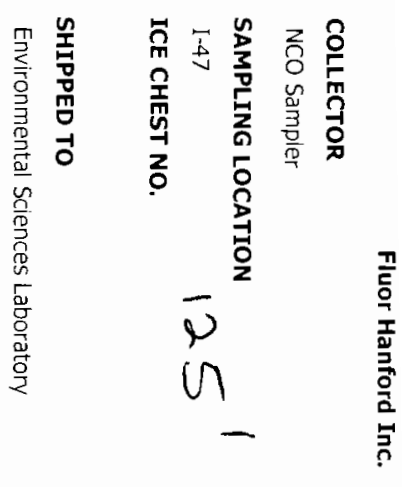

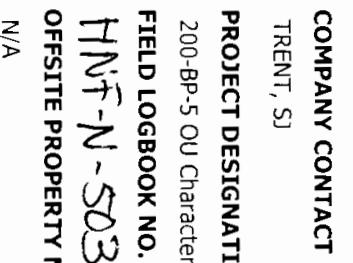
$\frac{1}{e^{-w}}$

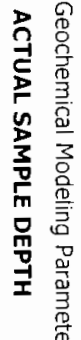

㟧

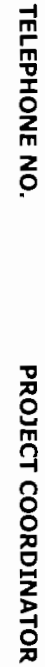

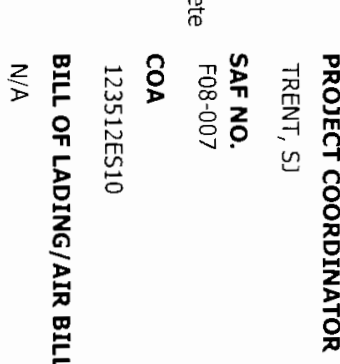

ำ

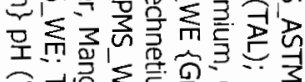

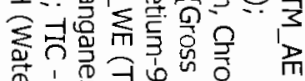

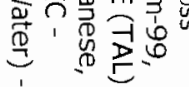




\section{IIt

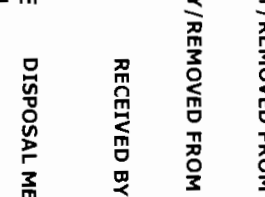

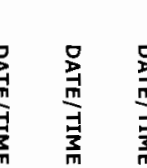

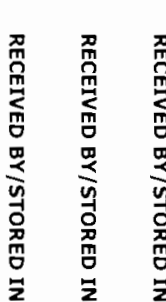

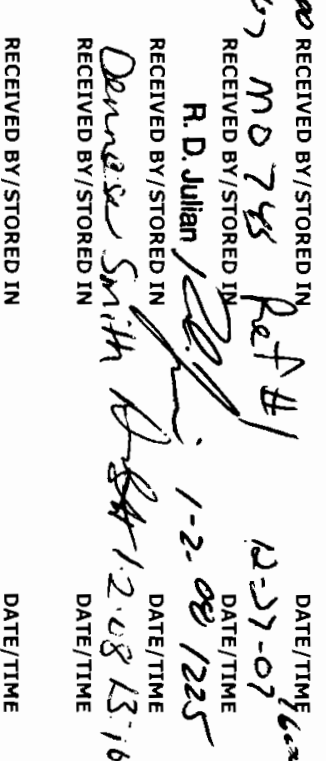

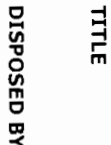

阮

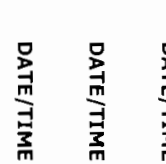

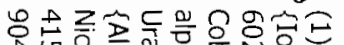

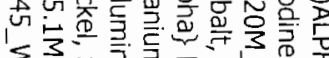

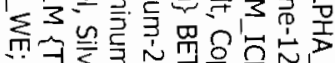

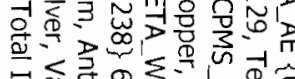

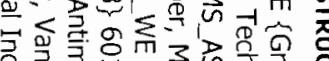

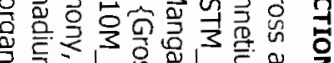

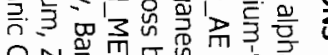

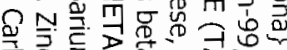

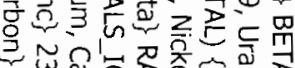

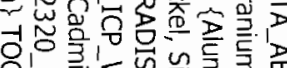

记运设

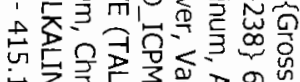

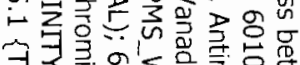

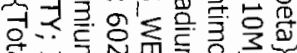

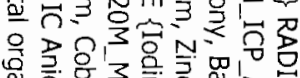

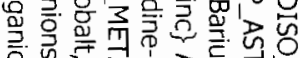

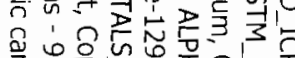

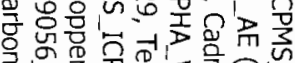

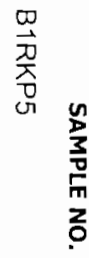

$\stackrel{\infty}{\rightleftharpoons}$

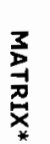

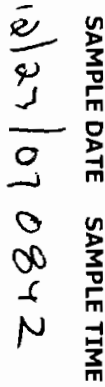

$\frac{0}{2}$

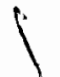

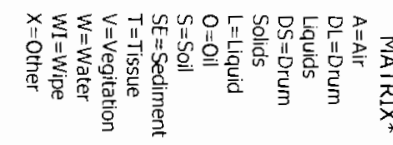

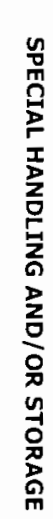
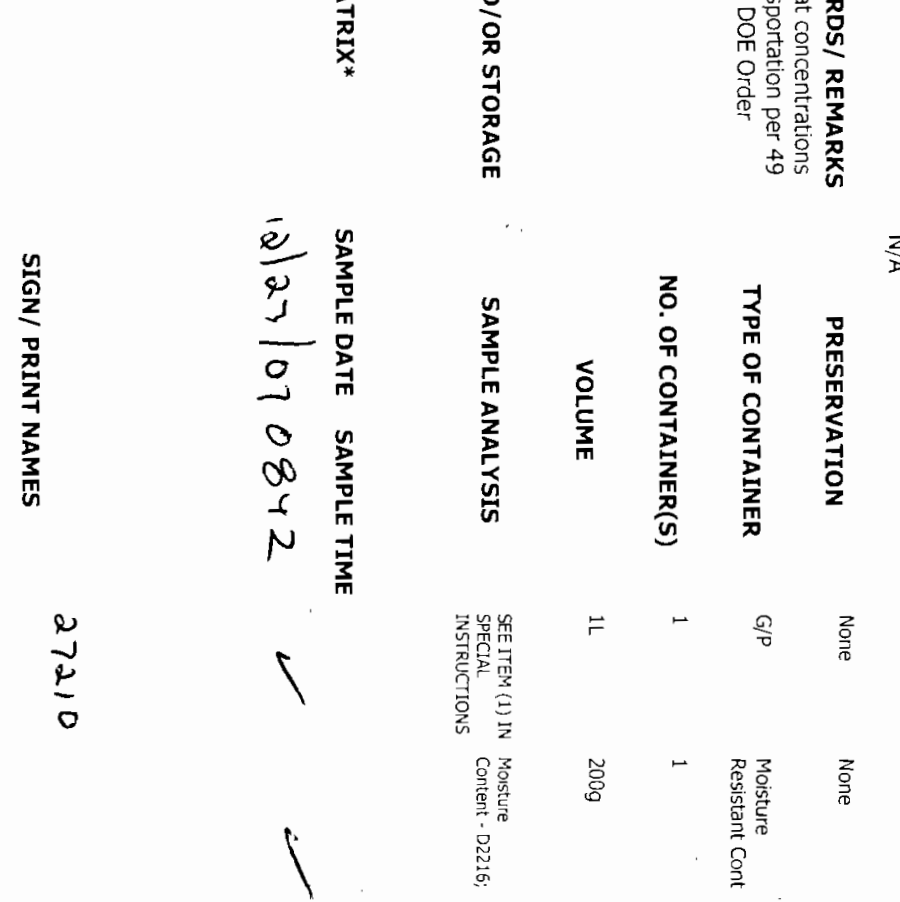

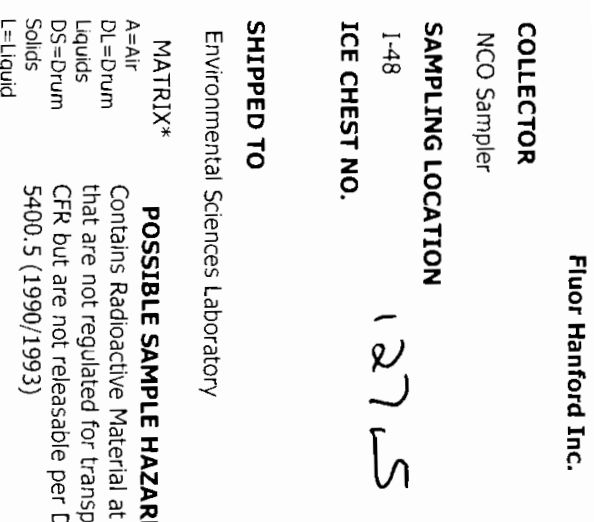

.

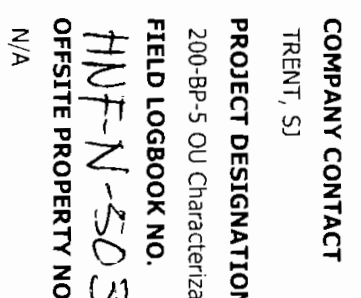

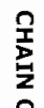

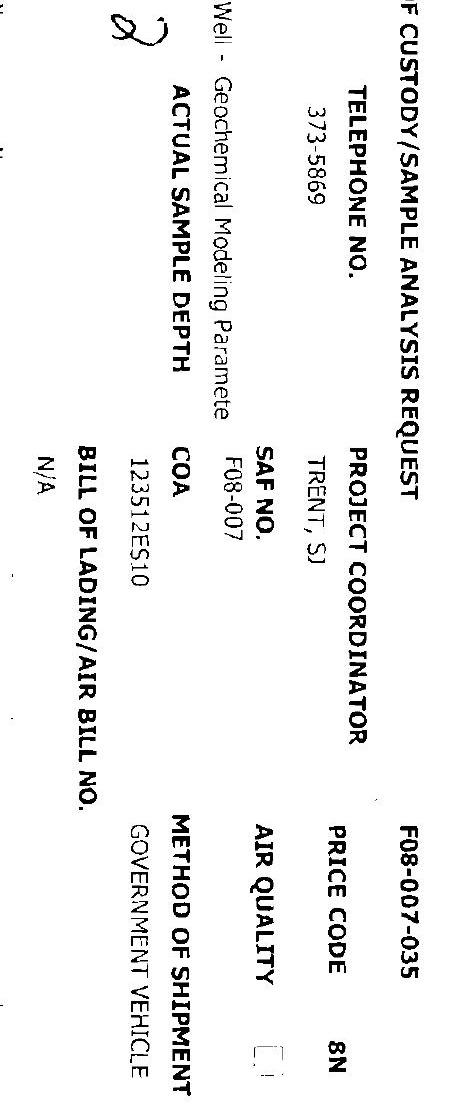

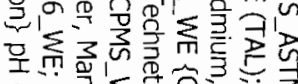

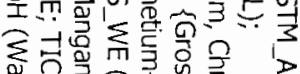

劳

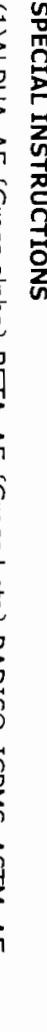




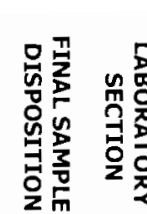

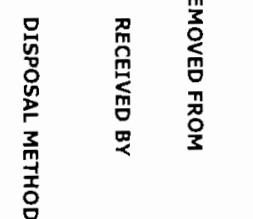

总号

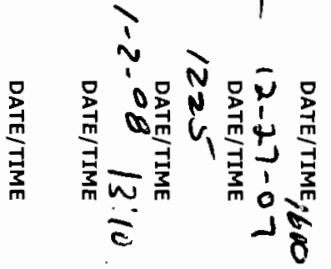

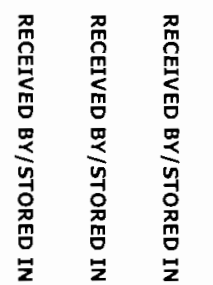

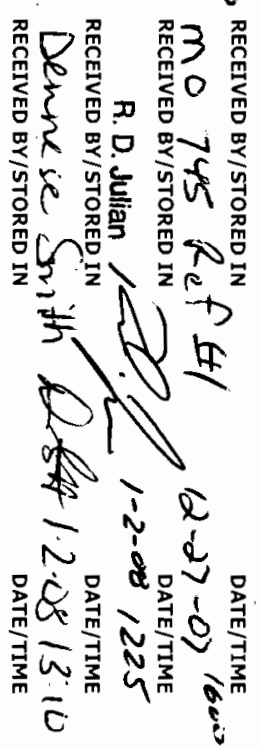

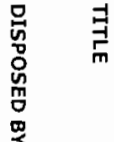

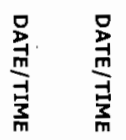

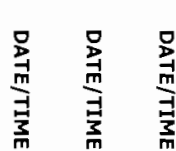
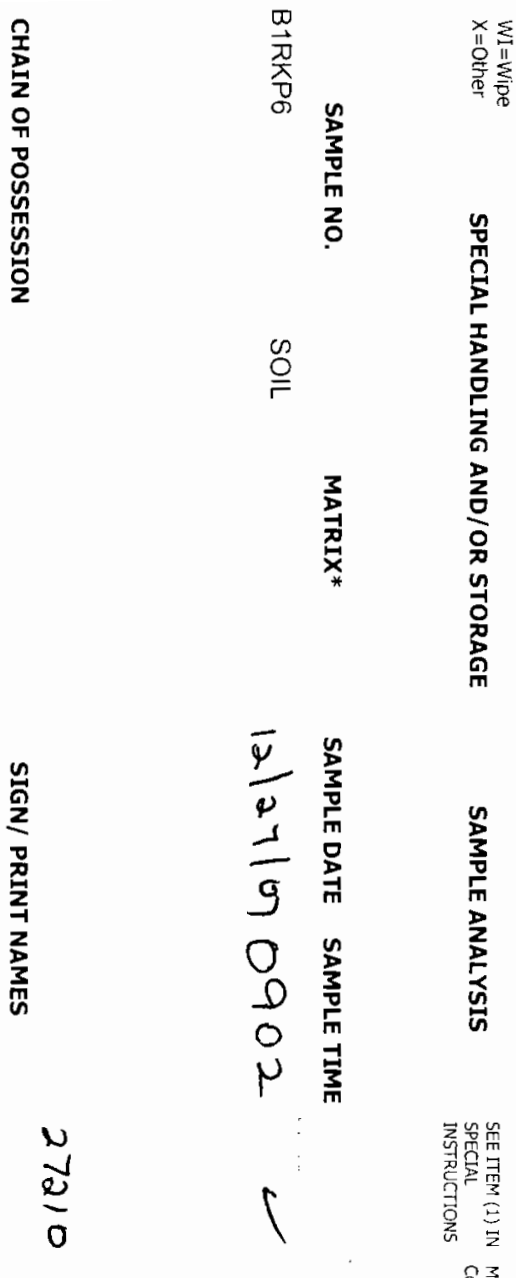

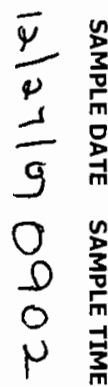

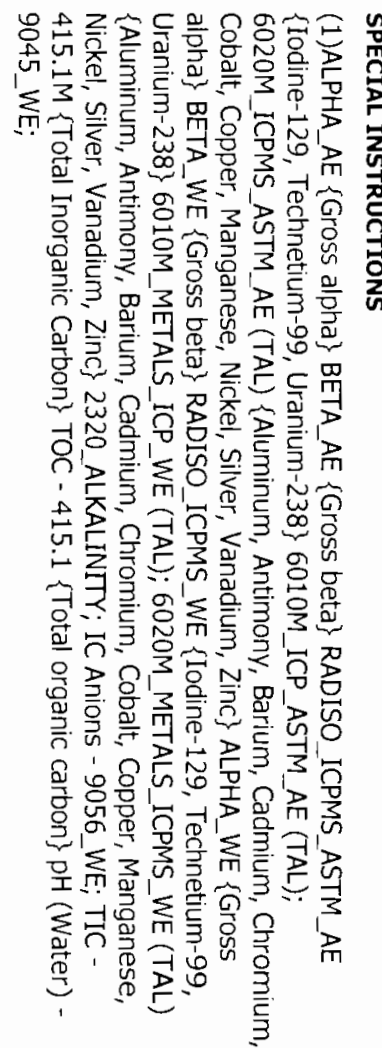

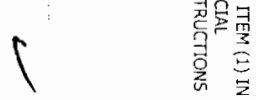

m

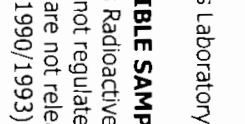

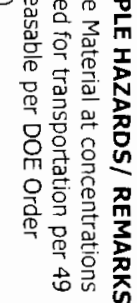

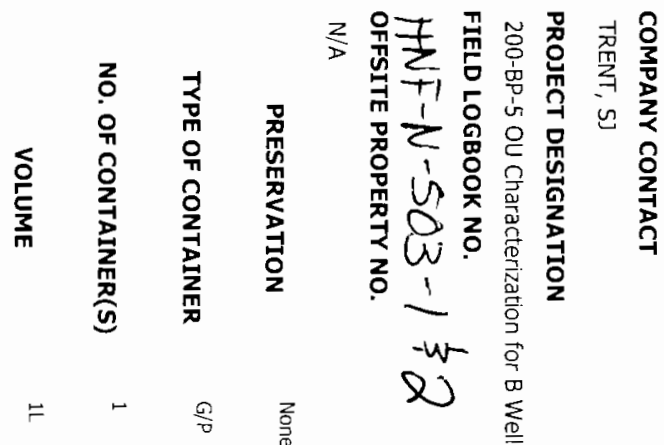

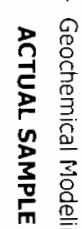

贺
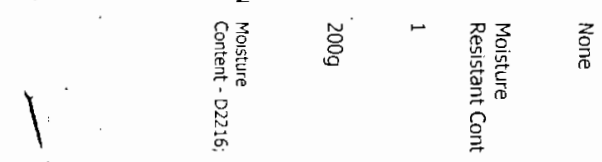


\section{III

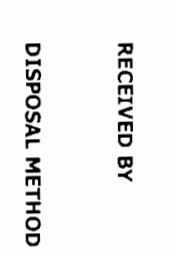

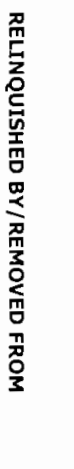

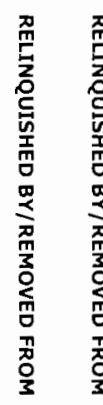

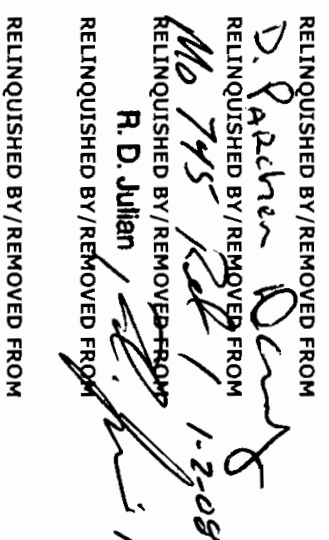

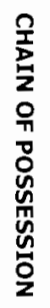

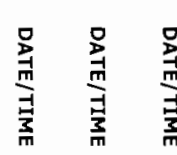

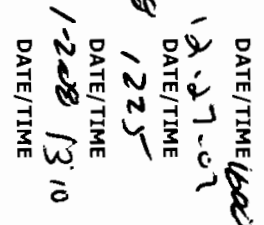

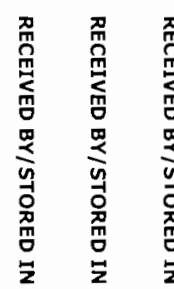
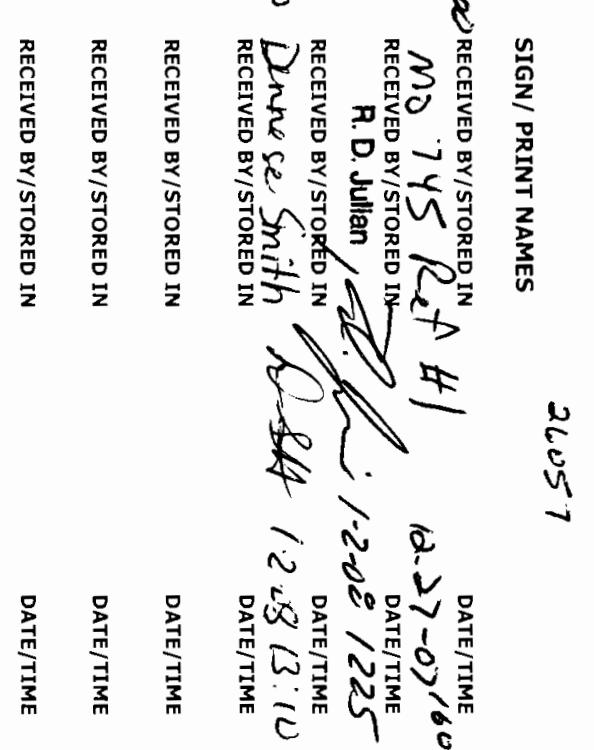

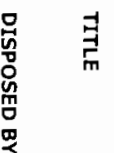
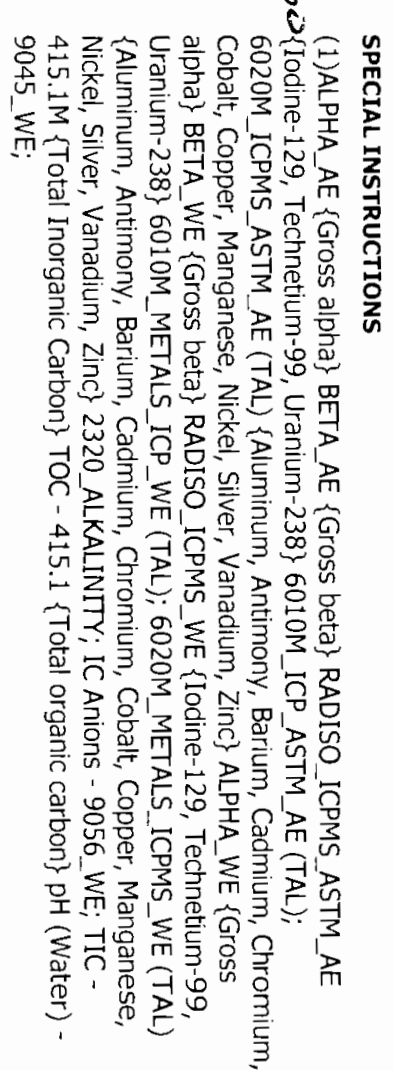
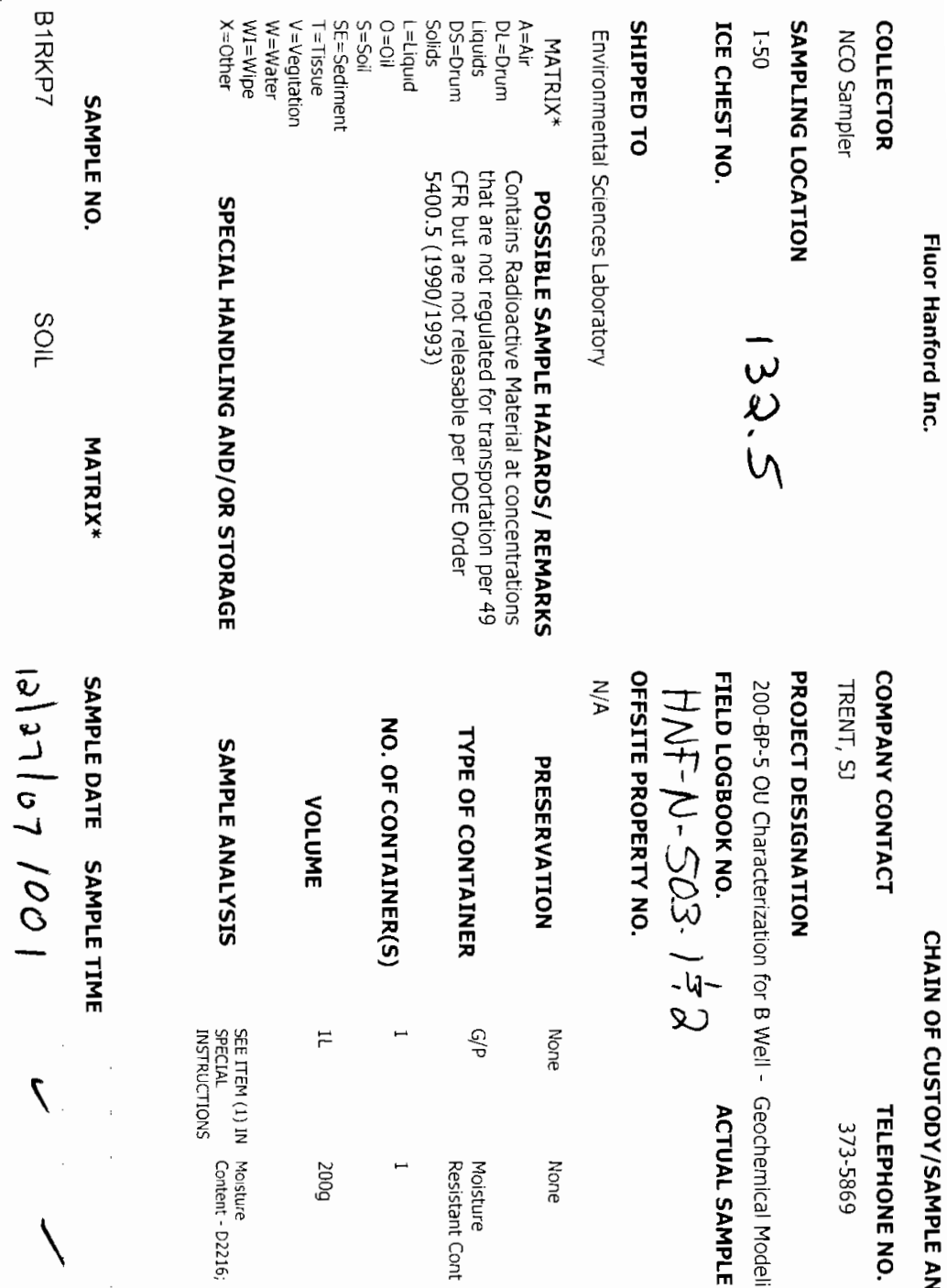

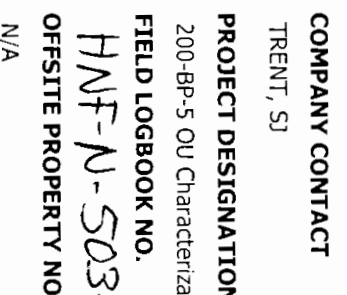

- $\begin{array}{ll}1 \\ 2\end{array}$

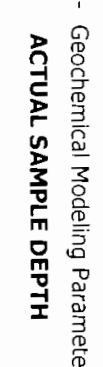

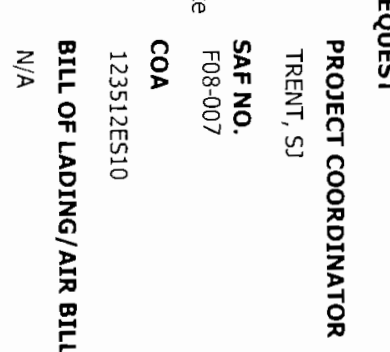

으
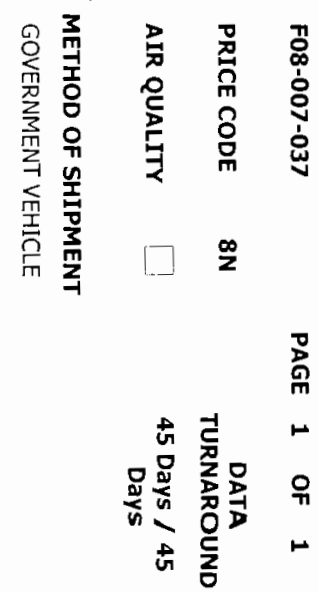


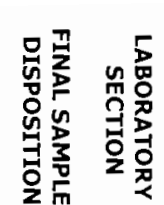

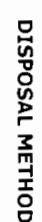

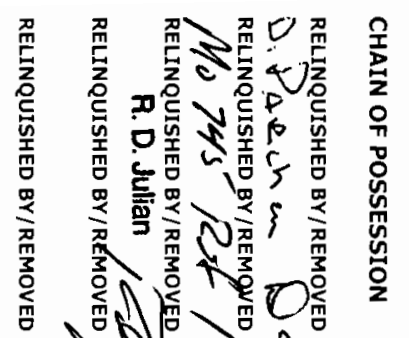

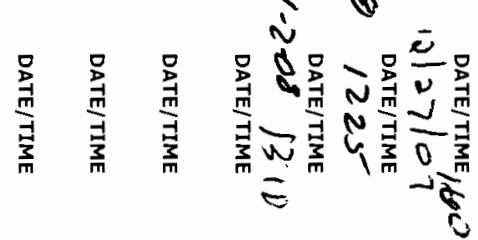

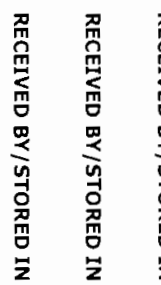

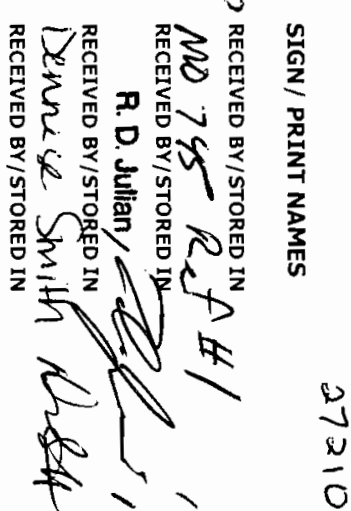

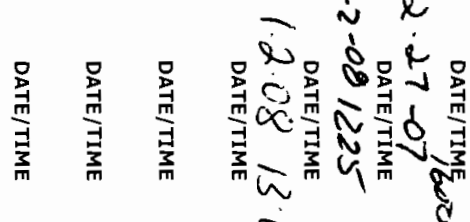

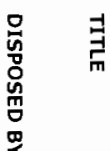

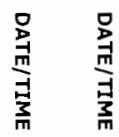

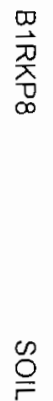

$\underset{\substack{3 \\ \hdashline}}{3}$

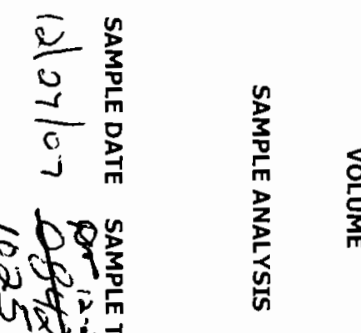

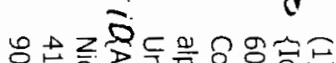

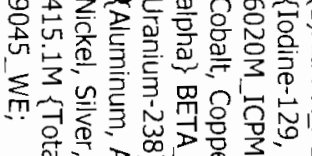

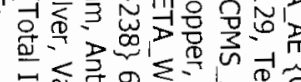

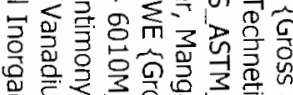

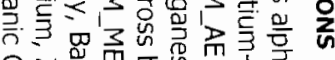

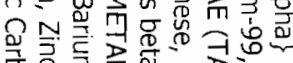

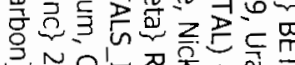

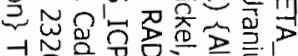

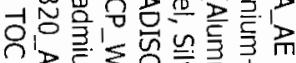

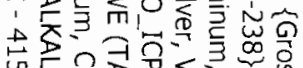

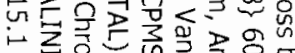

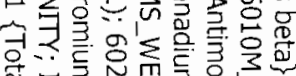

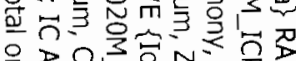

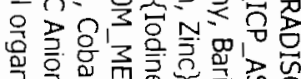

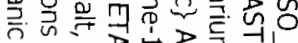

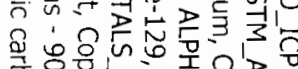

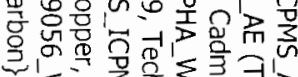

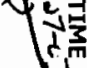<smiles>CCCCCC</smiles><smiles>CCC</smiles>

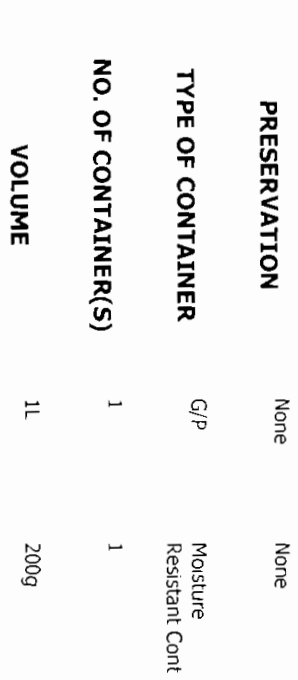<smiles>C1CCCCC1</smiles>

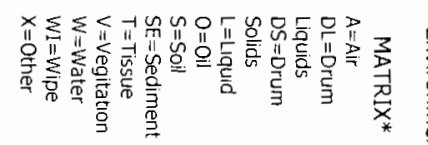

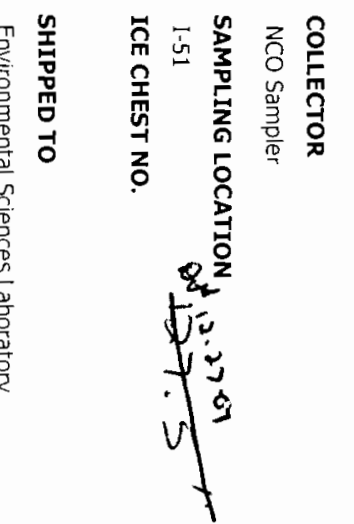

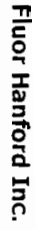

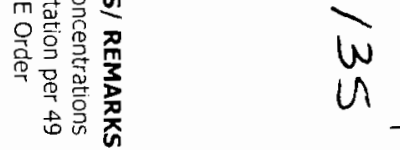

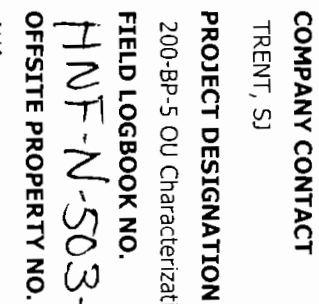
i $\frac{\text { i }}{\text { D }}$

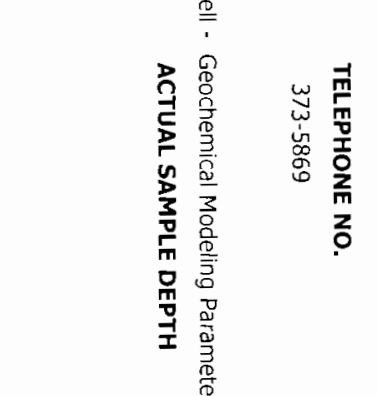

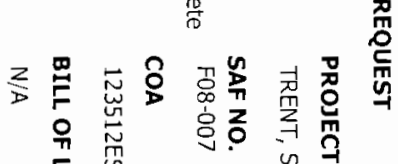

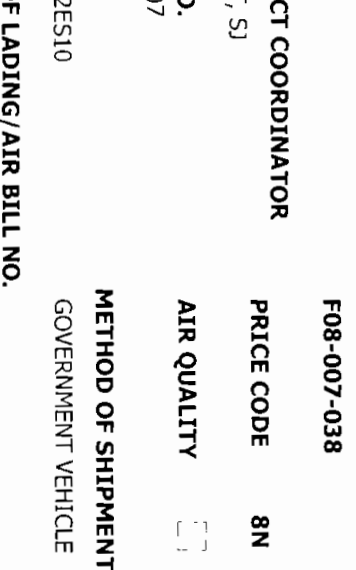

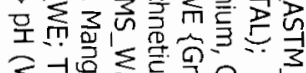

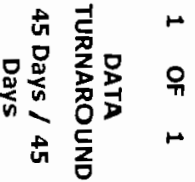




\section{II}
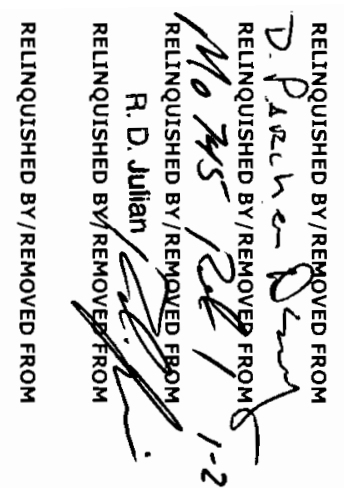

ஸे ó

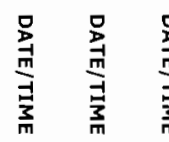

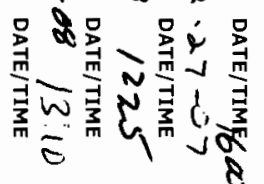

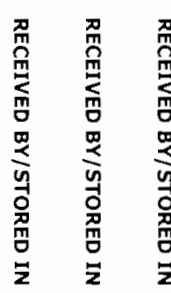

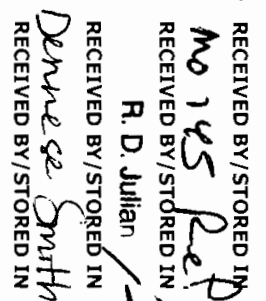

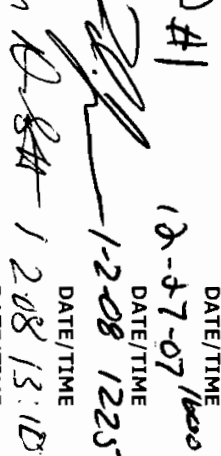

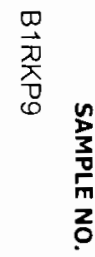

$\stackrel{\infty}{\circ}$

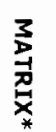

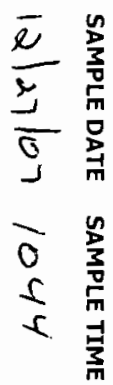

$u$
$\sigma$
$g$
$y$

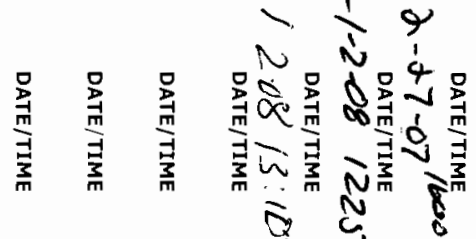

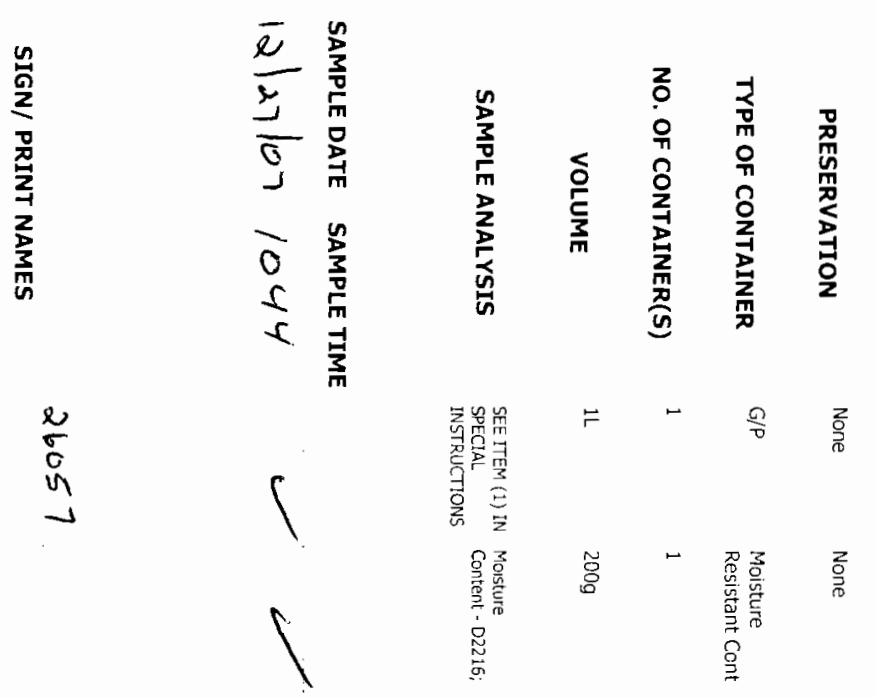
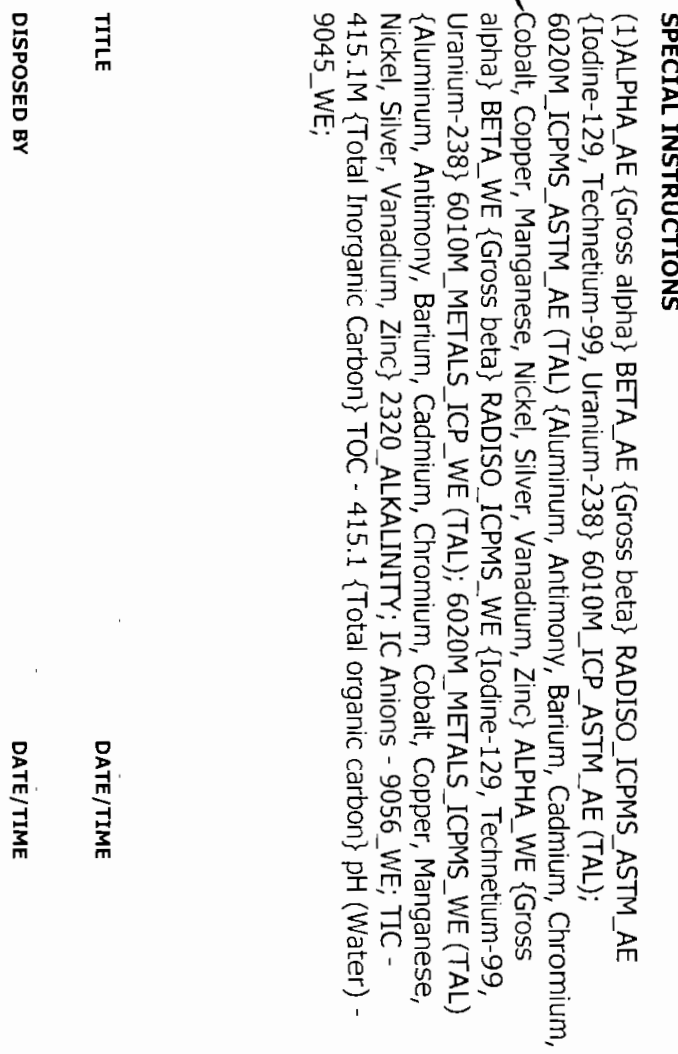

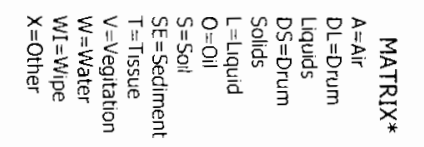

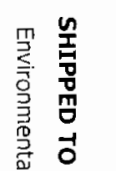

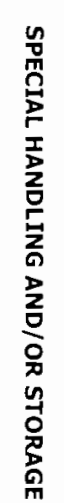

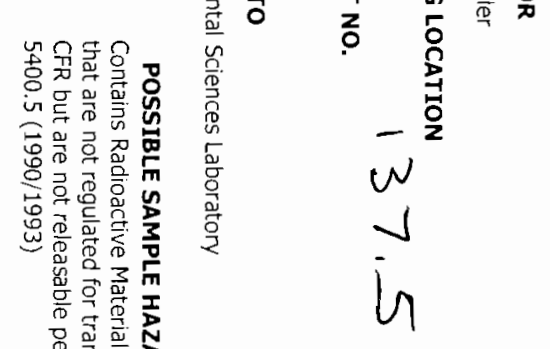

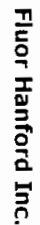
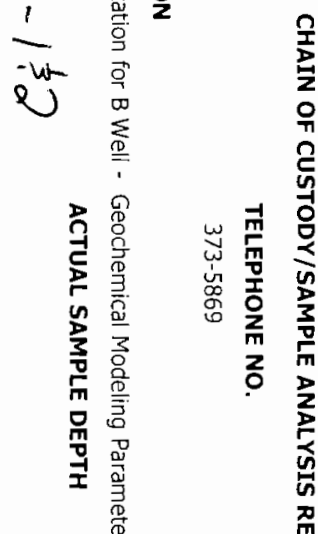

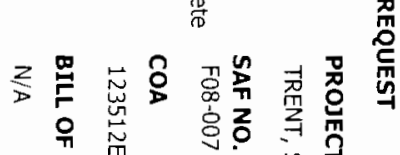

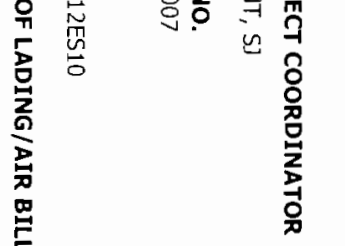

总咞

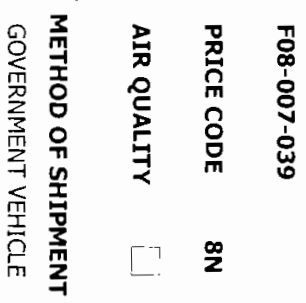

总

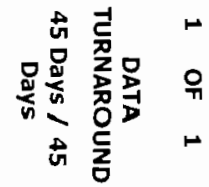




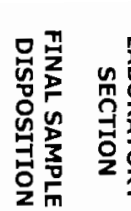

量

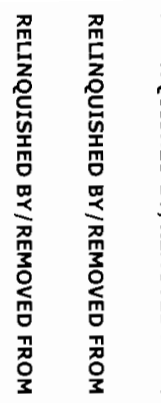

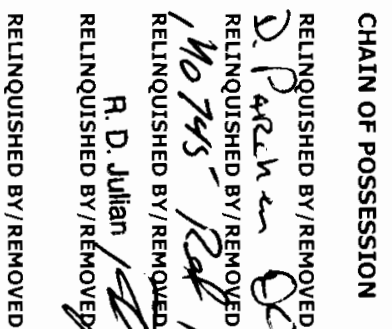

ग $>$ है
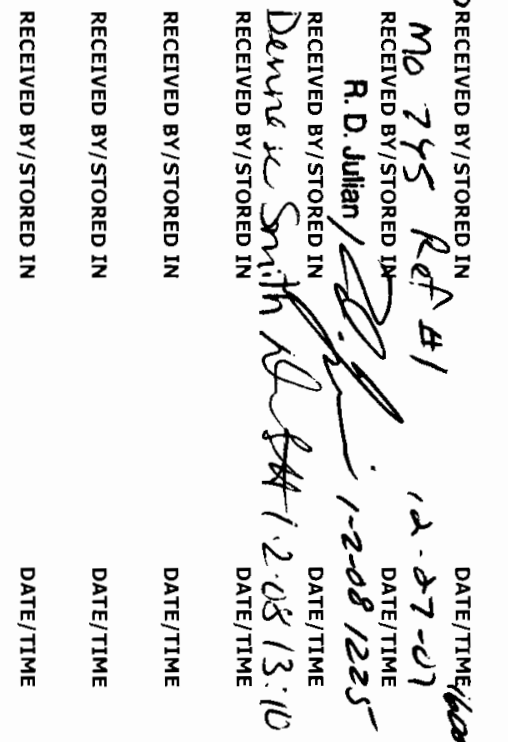

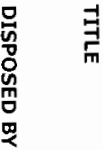

亲

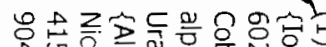

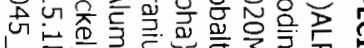

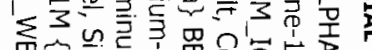

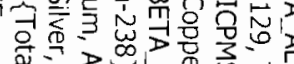

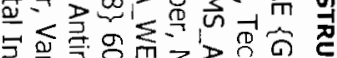

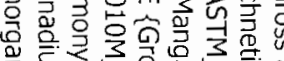

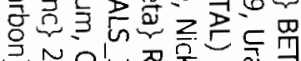

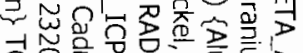

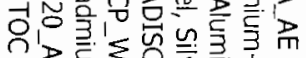

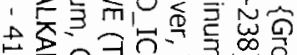

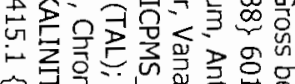

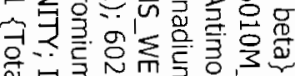

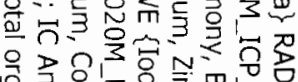

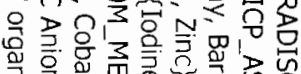

$\begin{array}{ll}\frac{g}{3} & \frac{g}{3} \\ \frac{m}{3} & \frac{m}{3} \\ \frac{3}{m} & \frac{3}{m}\end{array}$

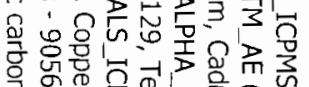

$\rightarrow>2$

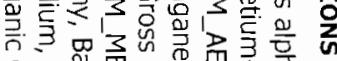

$\odot \cong$

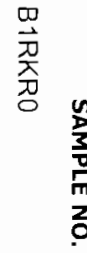

$\stackrel{\infty}{\stackrel{9}{ }}$

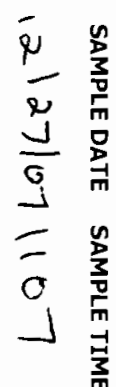

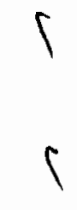

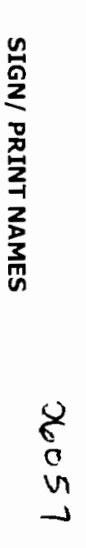

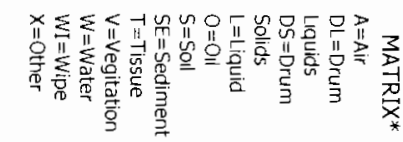

$\frac{0}{2}$

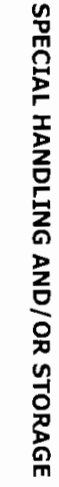

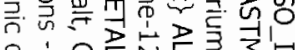

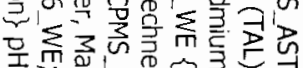

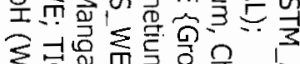

谣.

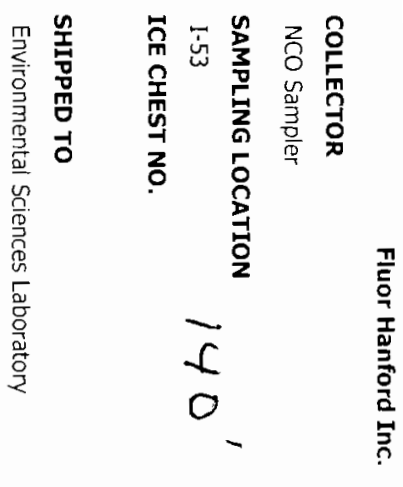

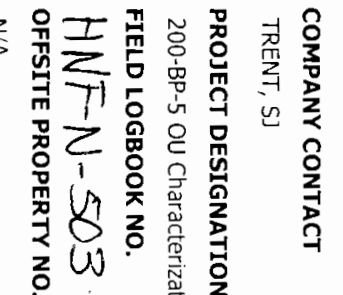

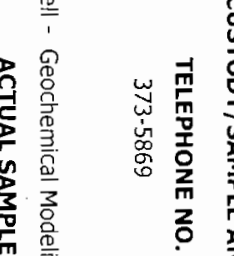

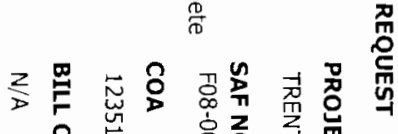

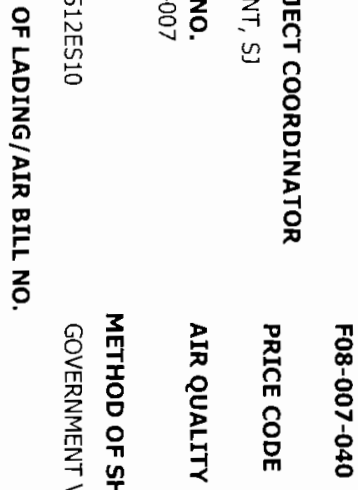

$\stackrel{\infty}{2}$

莖 


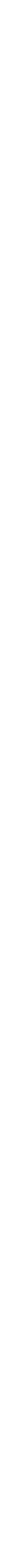


\|\|

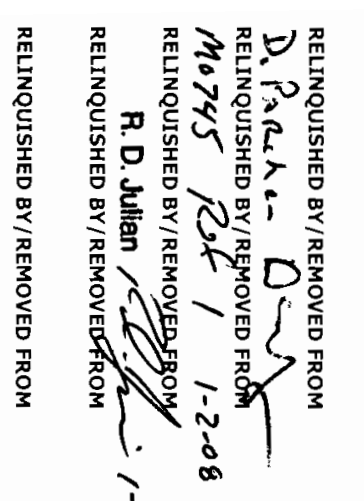

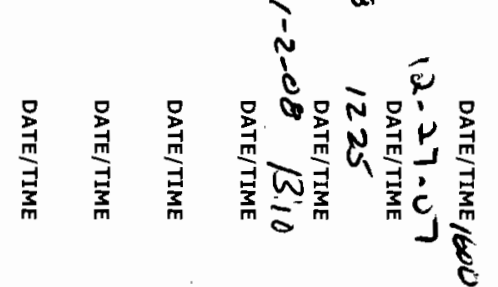

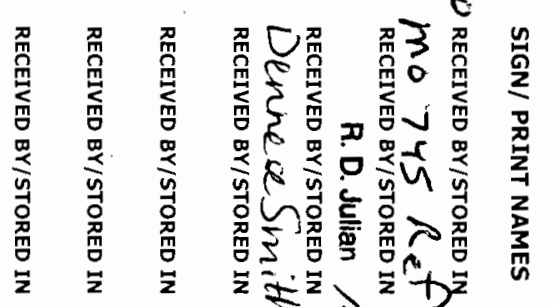

a ivis

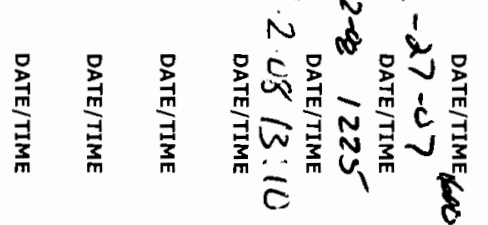

唄

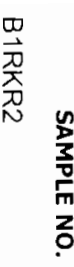

$\stackrel{\infty}{\stackrel{0}{\rightleftharpoons}}$

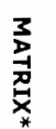

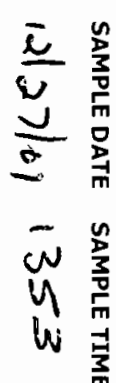

个:

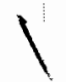

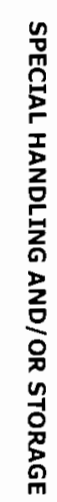

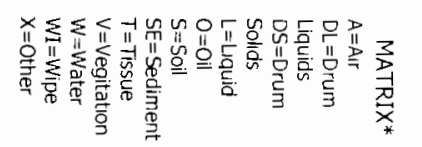

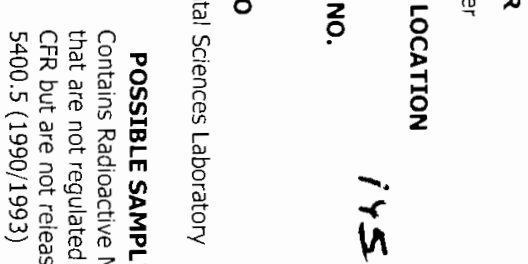

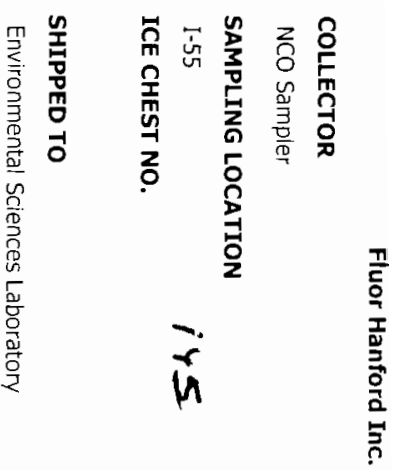

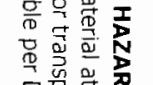

8 영

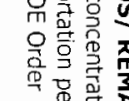

范管

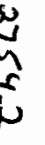

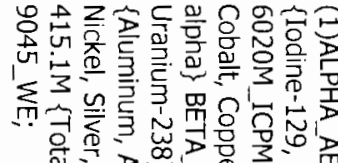

果

劳

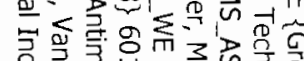

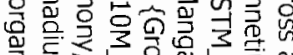

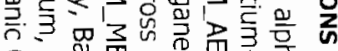

@Nㅜㄹ 굴

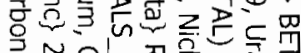

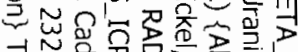

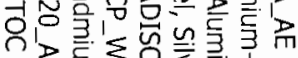

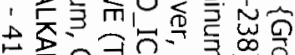

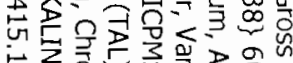

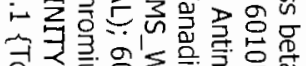

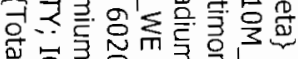

等

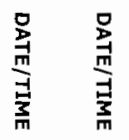
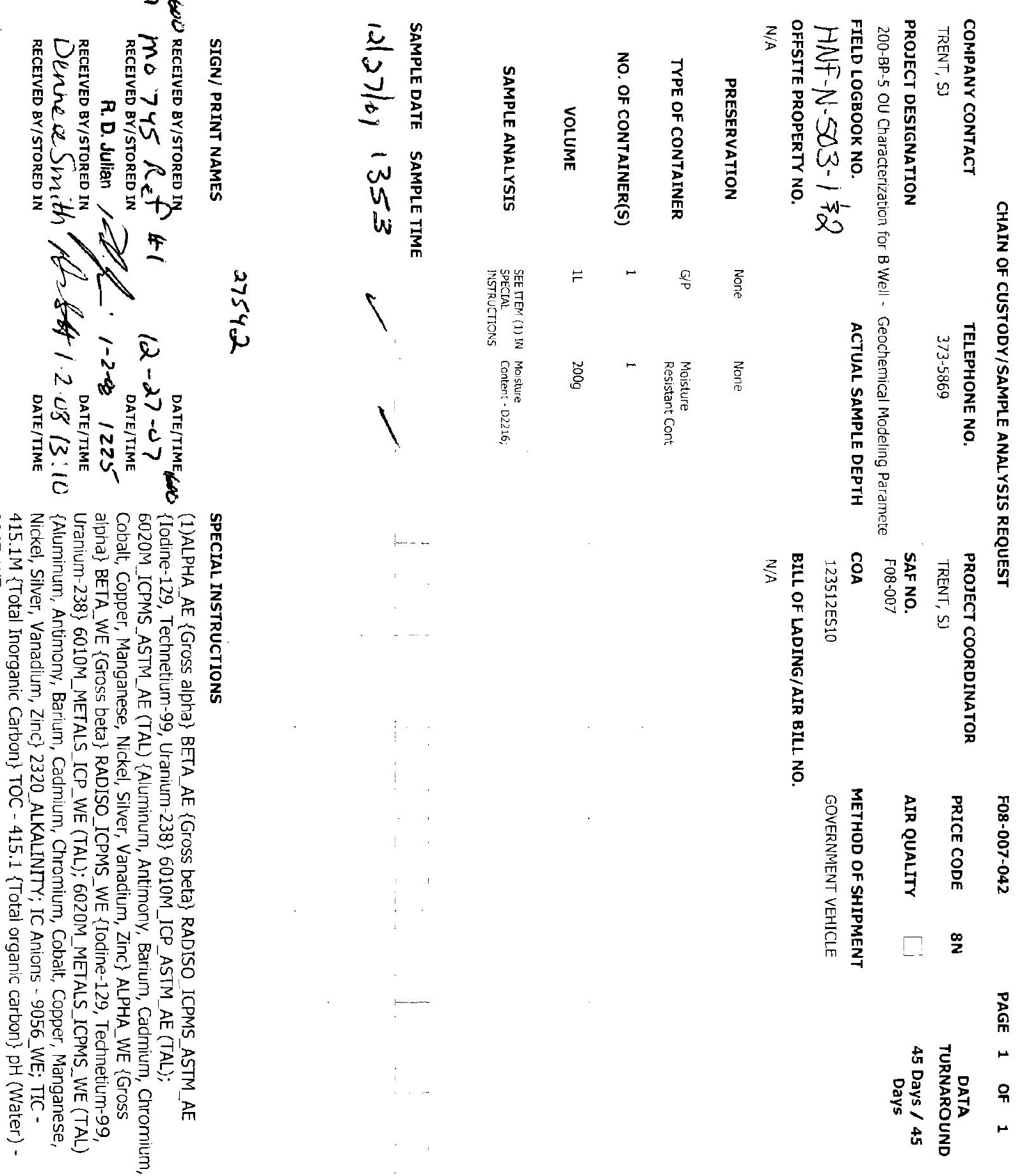


\section{III

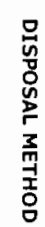

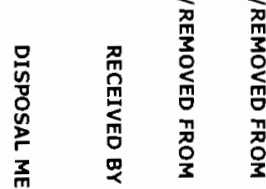

总量

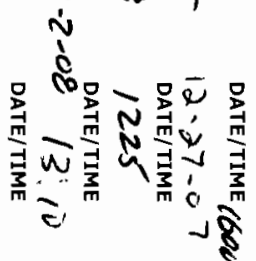
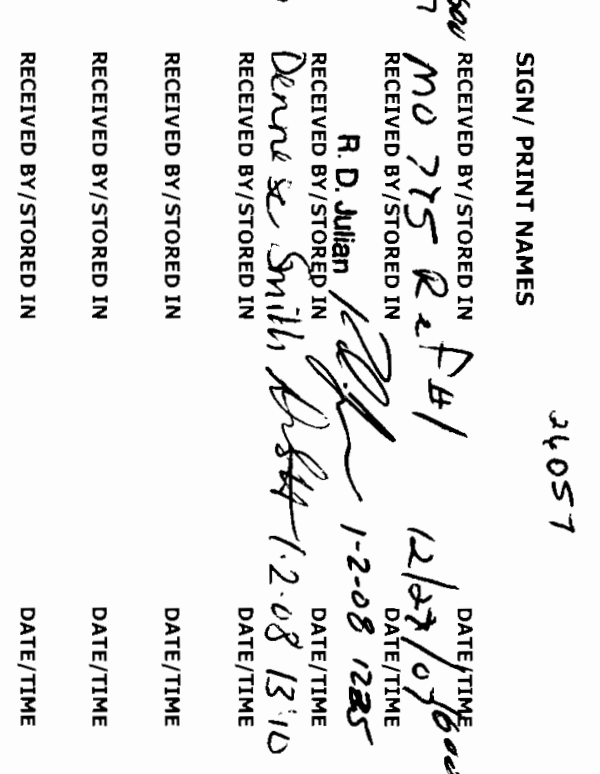

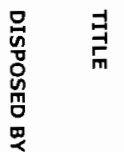

$\underset{\frac{\pi}{3}}{\stackrel{8}{\frac{8}{m}}}$

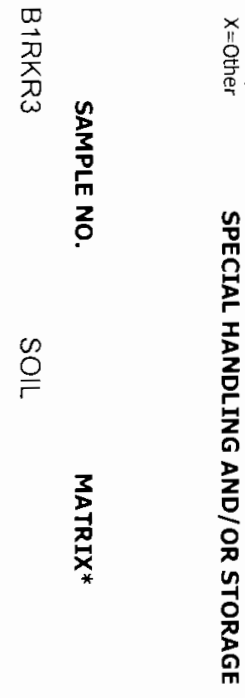

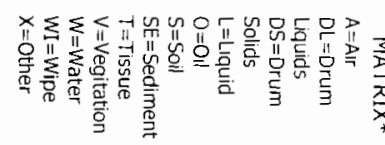

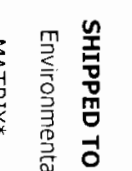

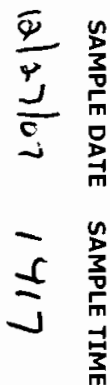

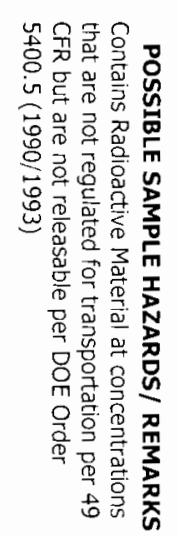

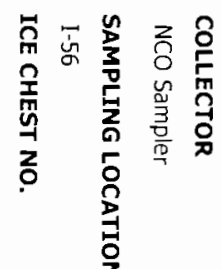

c

-

U

$\checkmark$

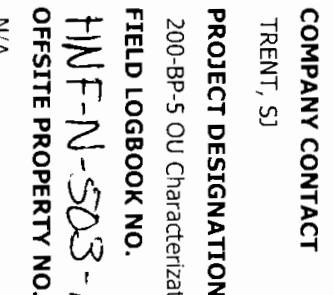

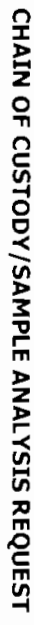

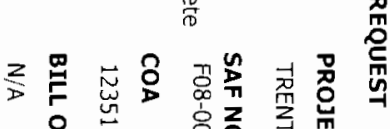

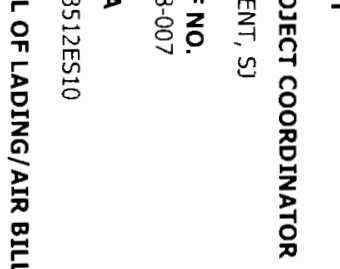

z

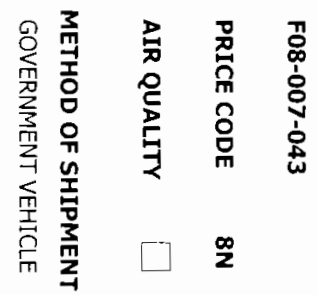

莕

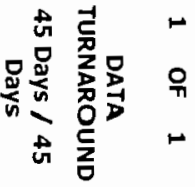




\section{嘿 \\ $\|$}

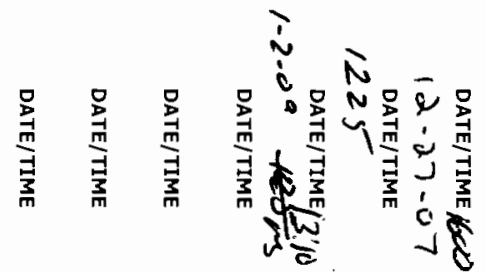

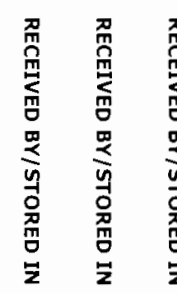
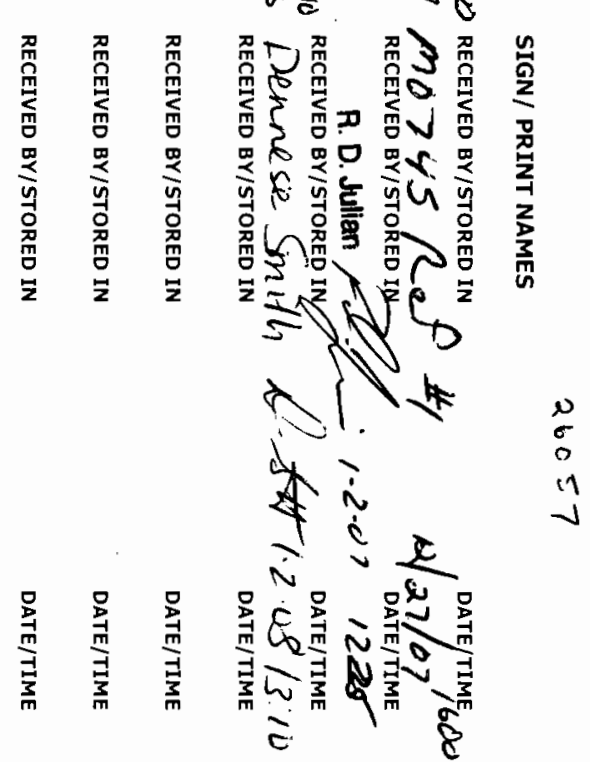

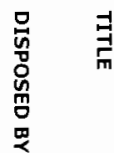

总暑

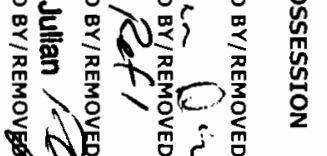

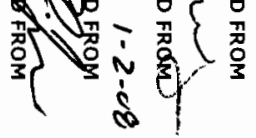

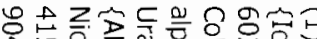

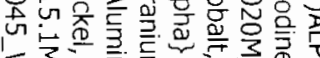

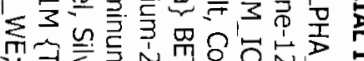

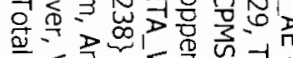

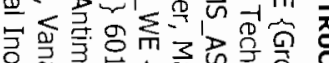
응

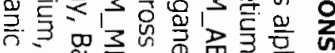

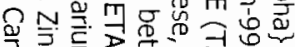

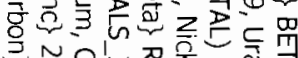

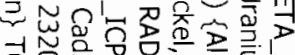

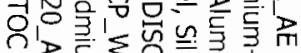

1

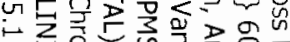

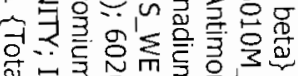

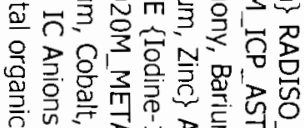

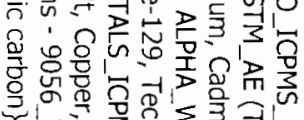

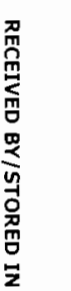

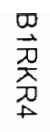

$\stackrel{?}{\rightleftharpoons}$

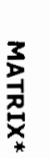

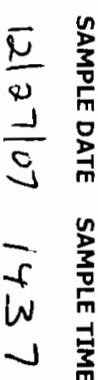

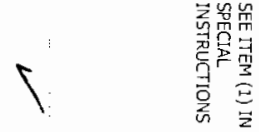

1

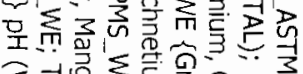

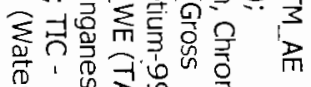

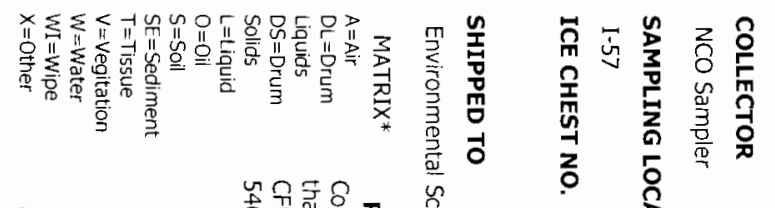

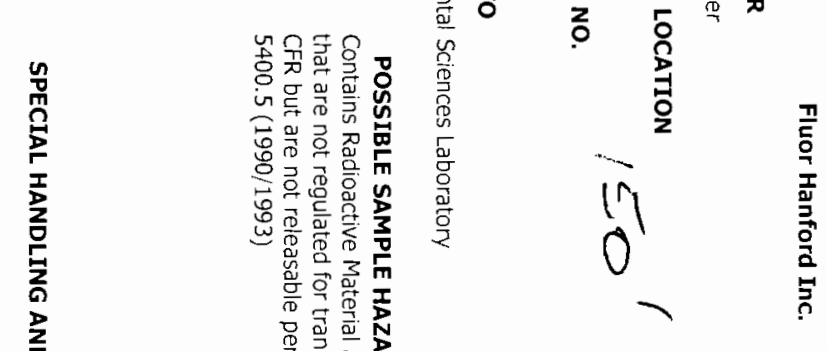

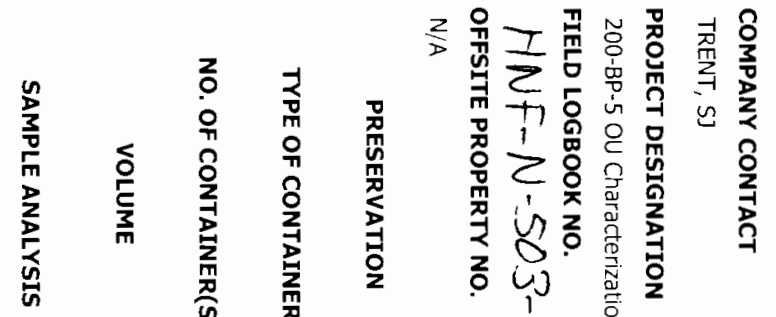

莖

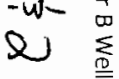

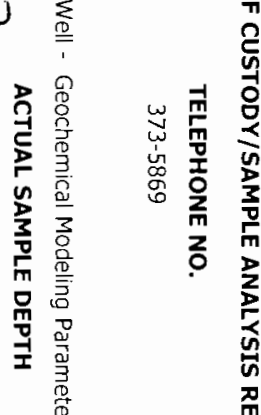

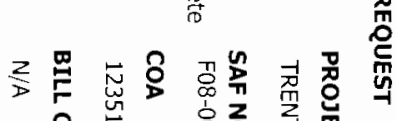

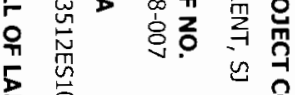

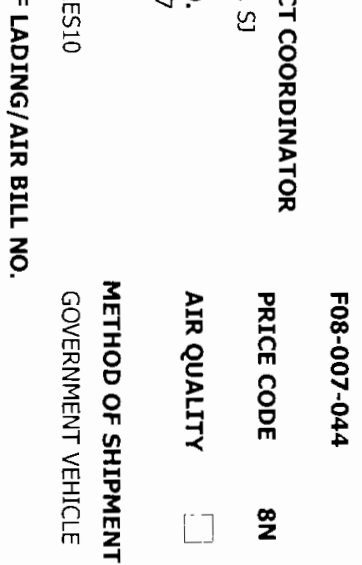

为

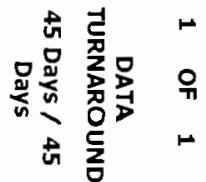




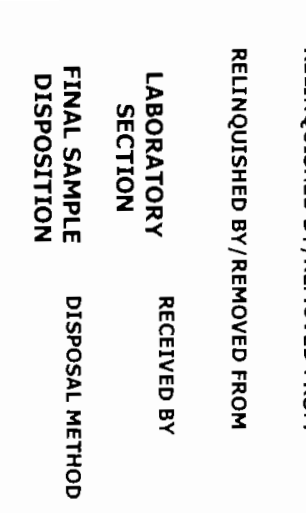
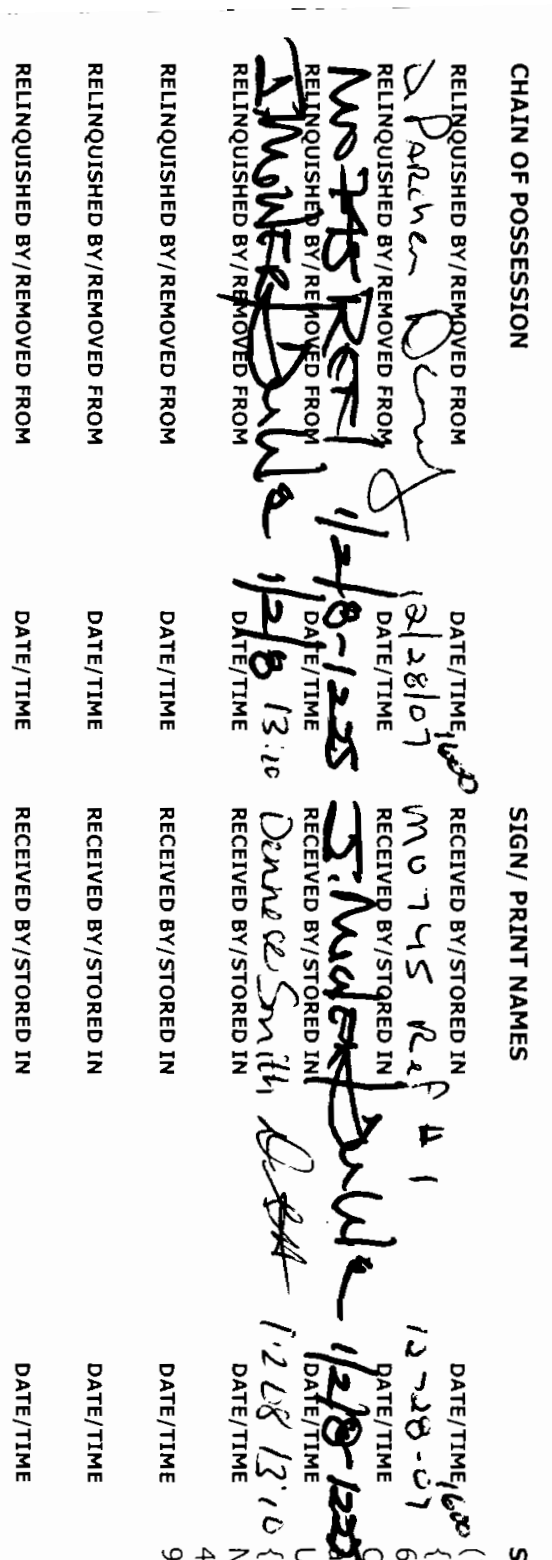

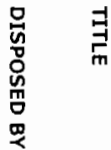

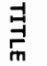

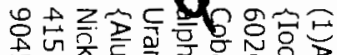

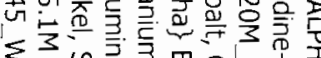

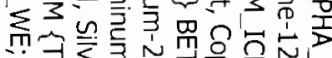

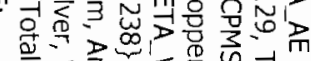

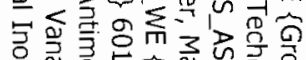

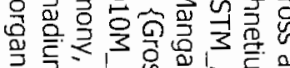

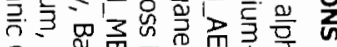

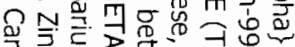

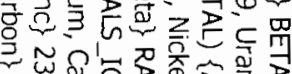

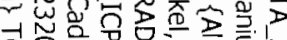

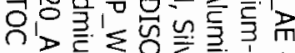

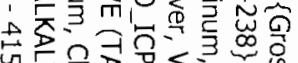

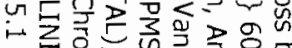

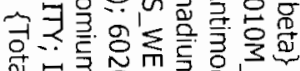

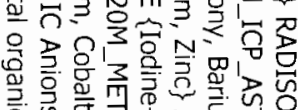

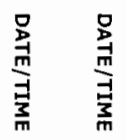

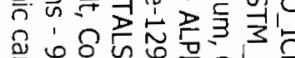

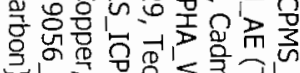

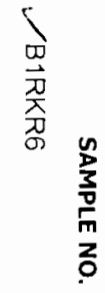

$\stackrel{0}{\circ}$

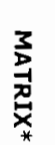

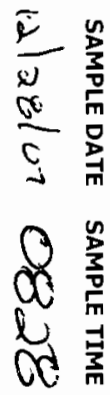

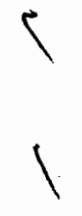

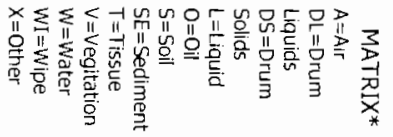

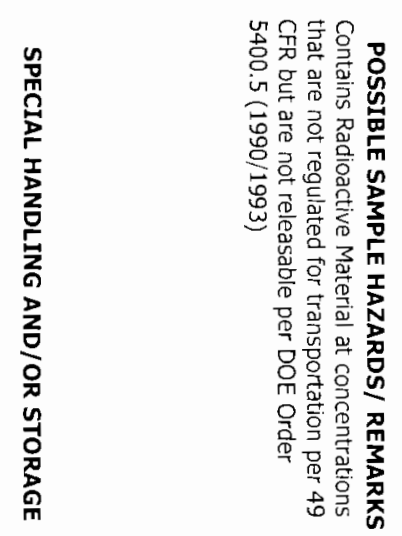

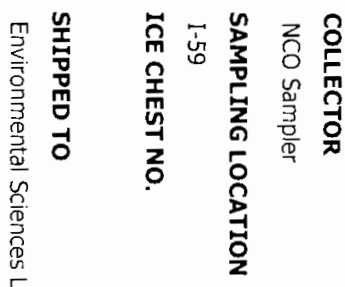

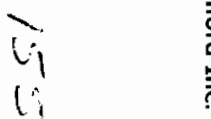

2 욱

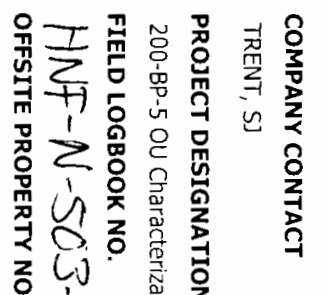

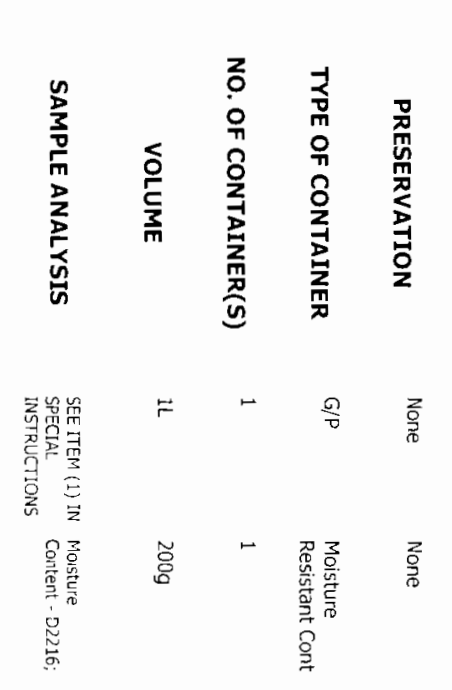

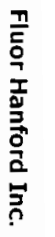

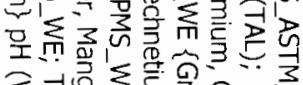

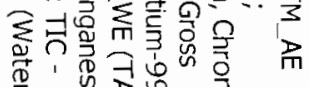




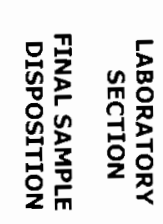

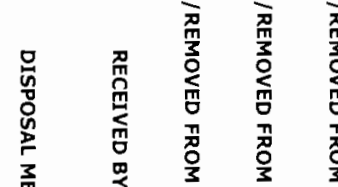

wi

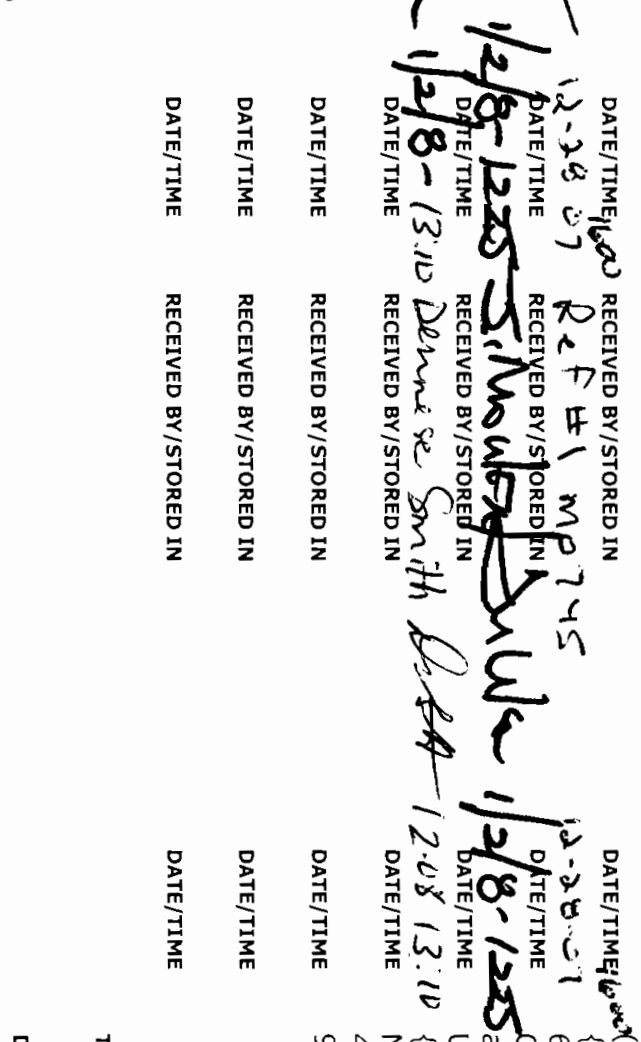

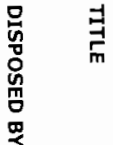

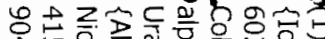

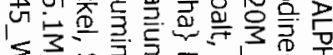

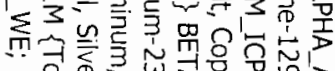

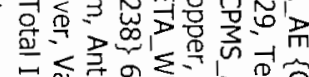

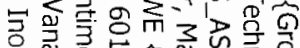

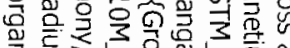

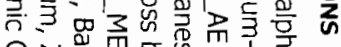

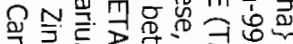

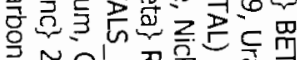

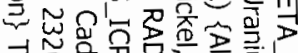

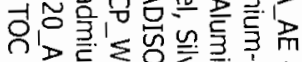

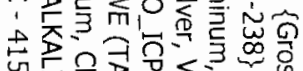

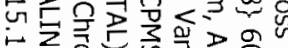

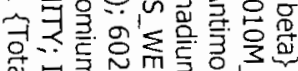

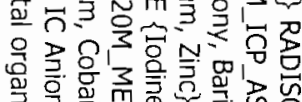

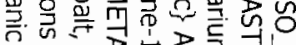
量

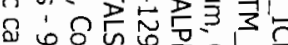

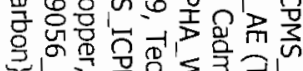
.
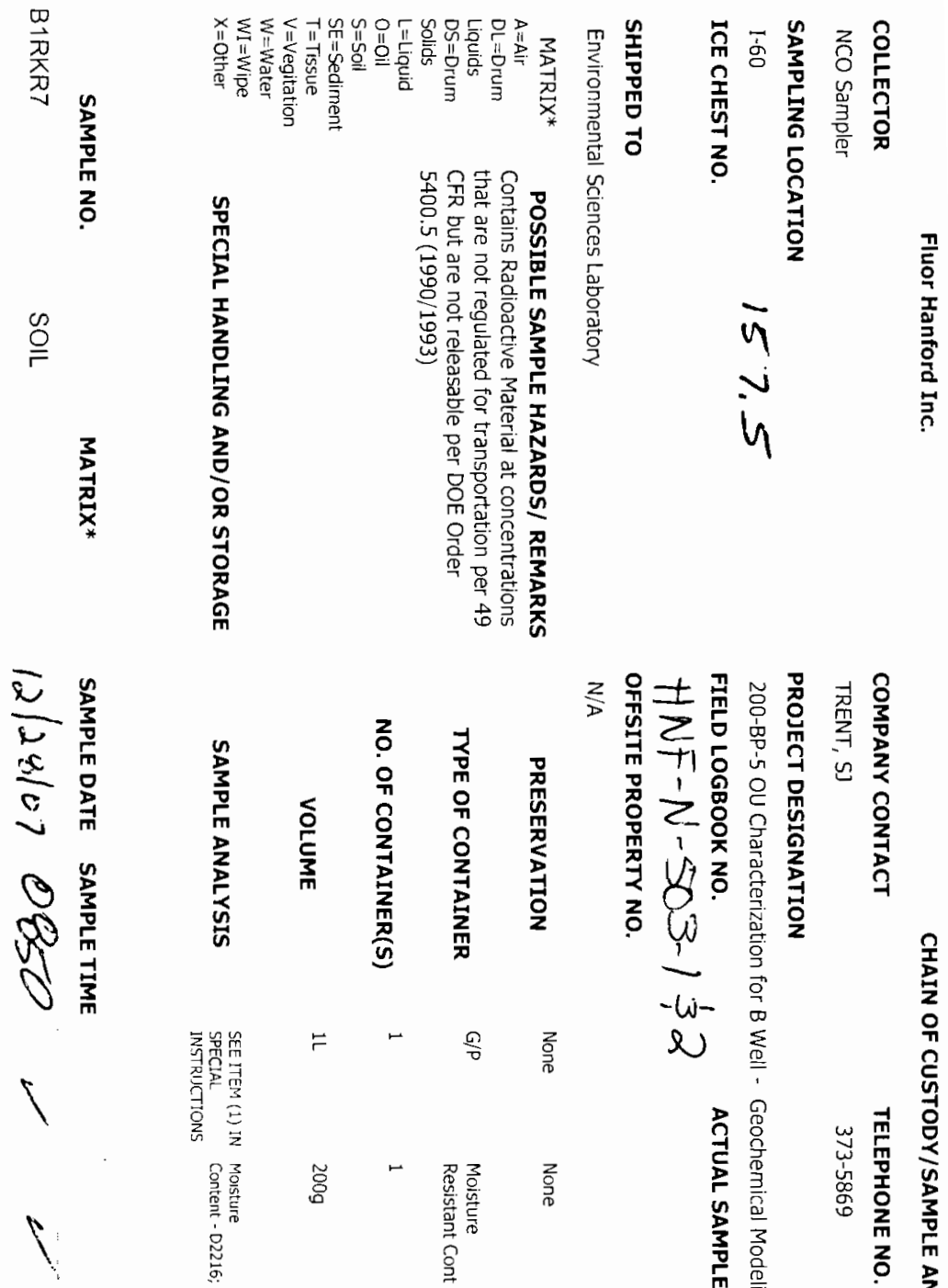

品

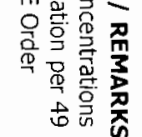

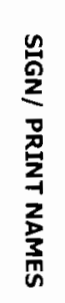

n

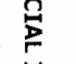

\section{임}

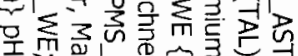

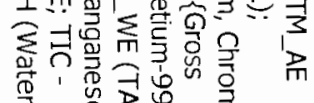

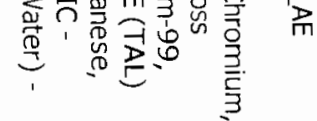




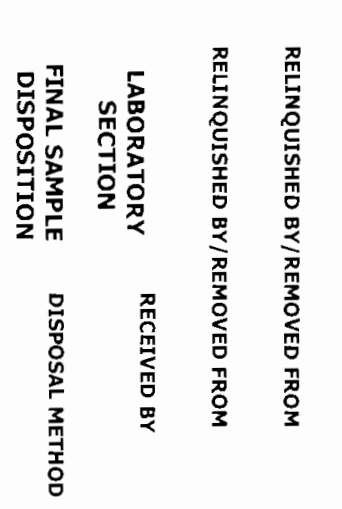
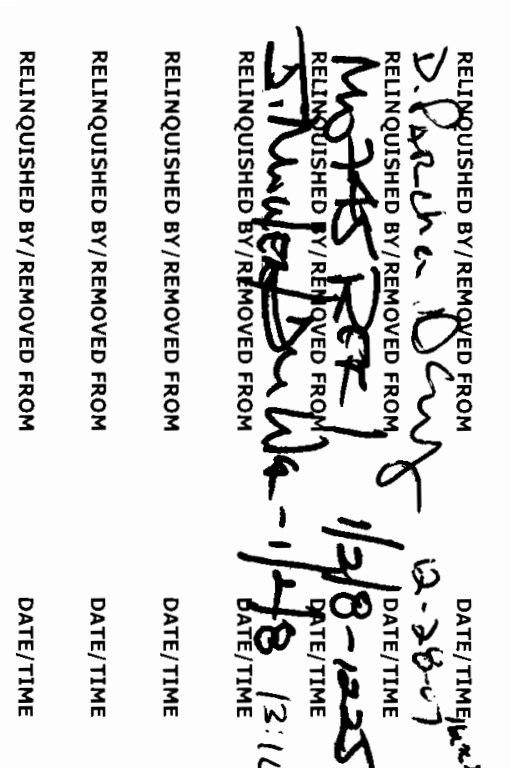

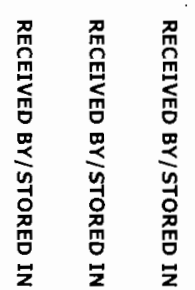
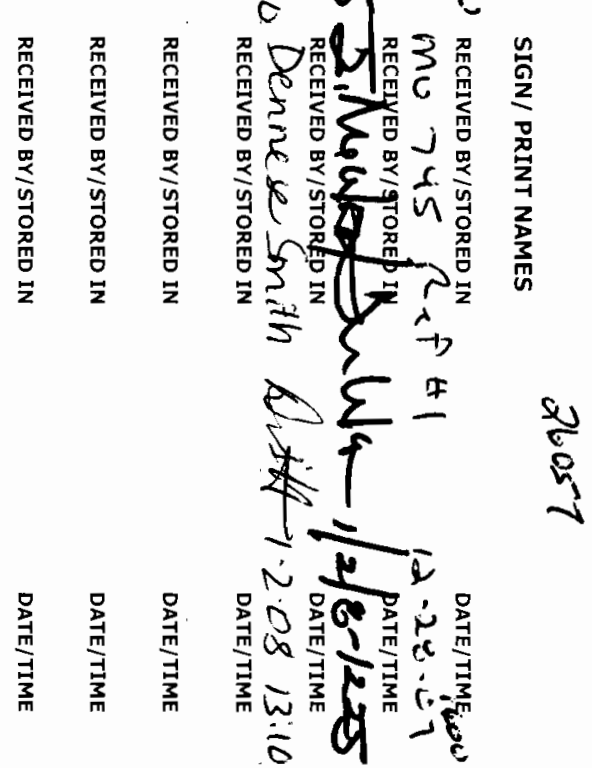

ปั

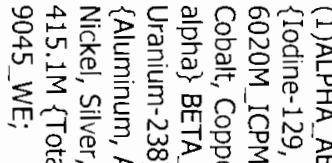

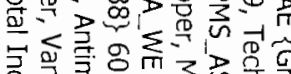

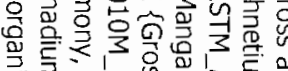

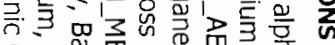

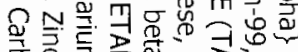

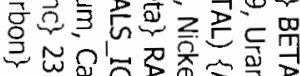

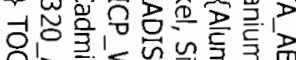

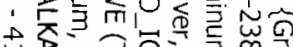

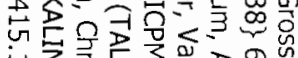

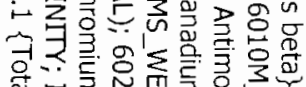

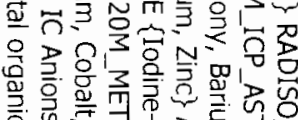

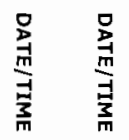

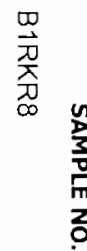

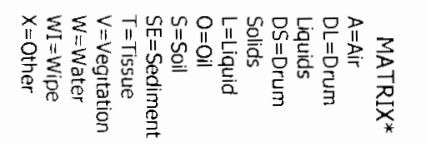

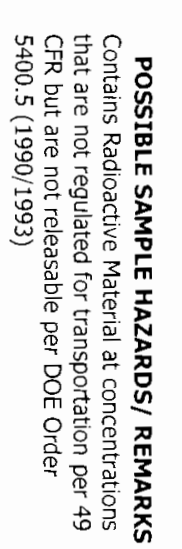

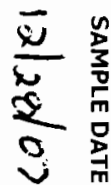

( ) $\frac{1}{3}$ 辛

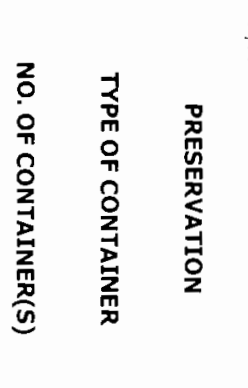

$\rightleftarrows$
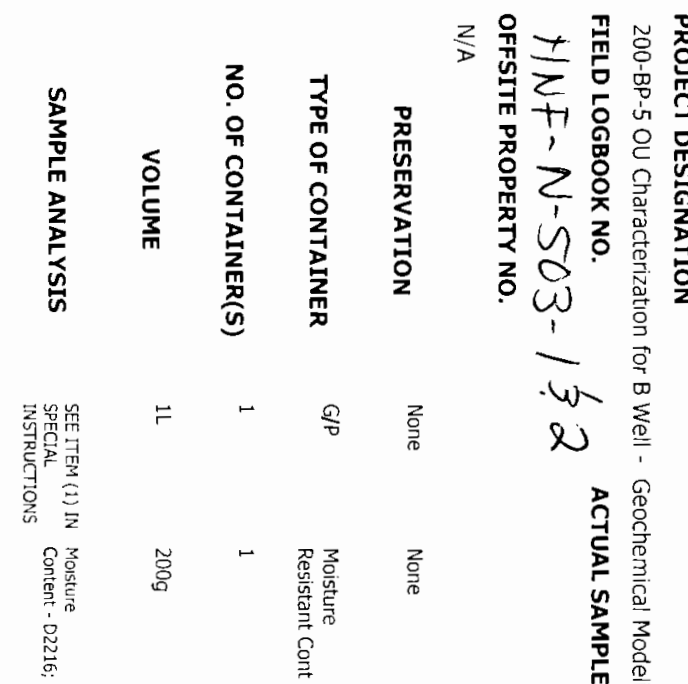

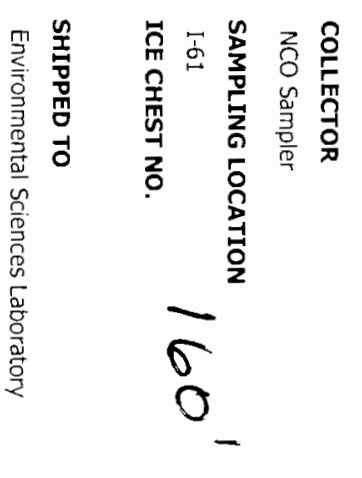

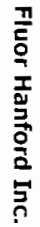

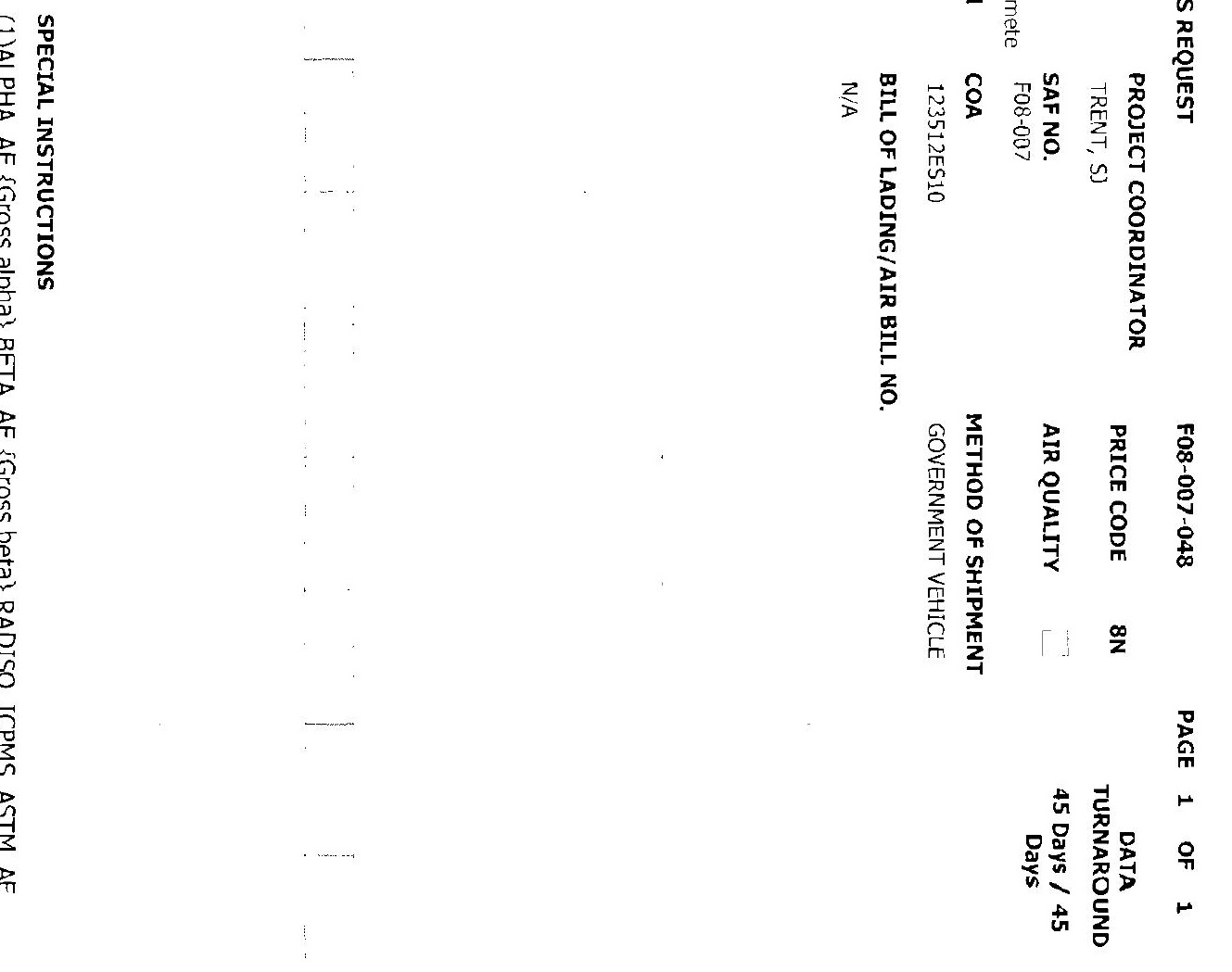


\|\|
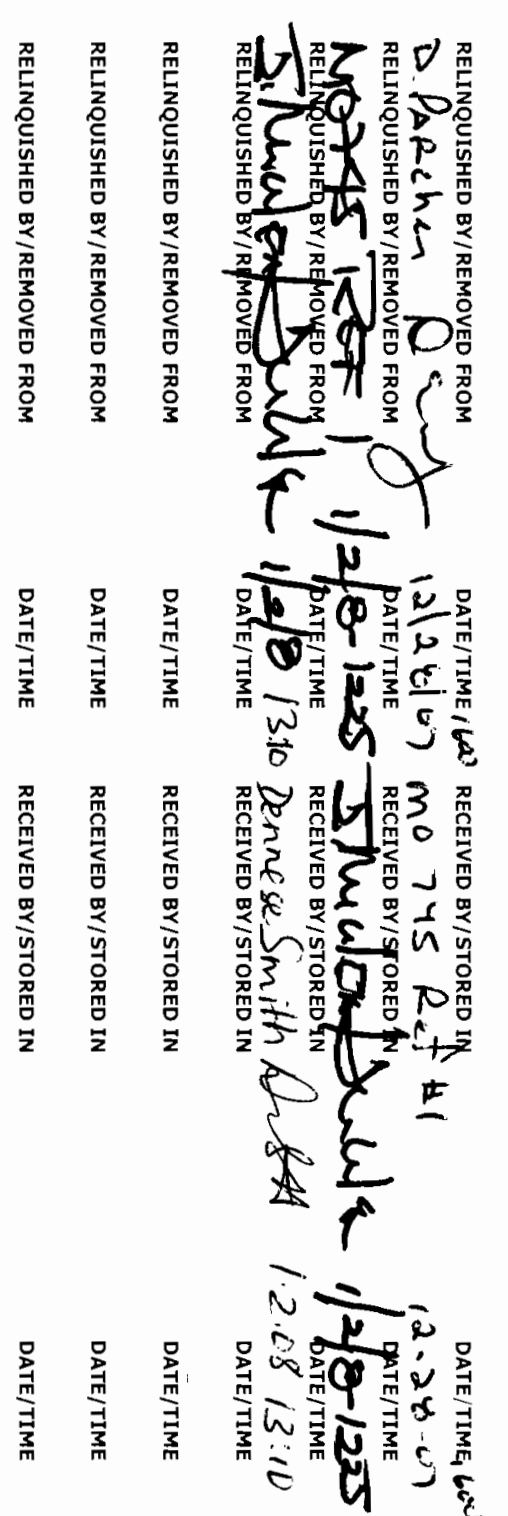

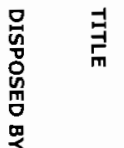

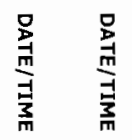

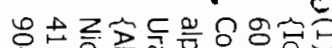

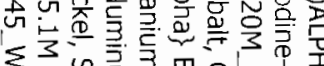

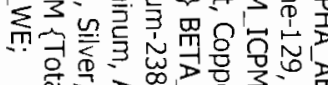

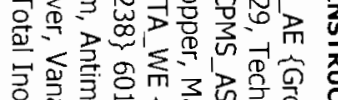

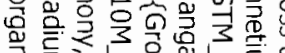

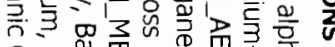

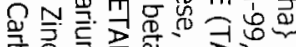

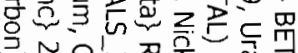
过 0

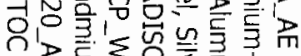

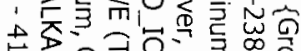

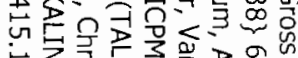

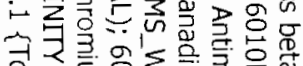

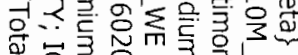
응

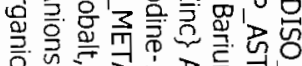

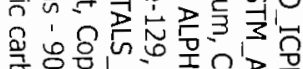

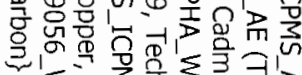
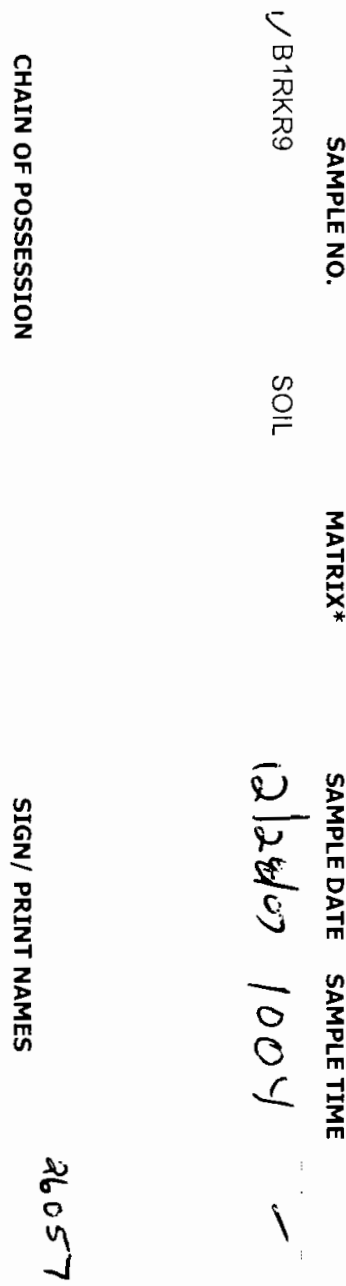

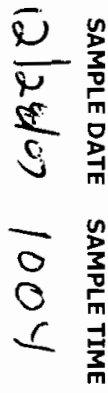
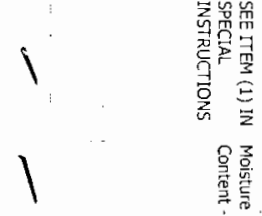

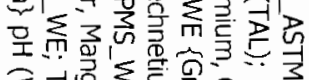

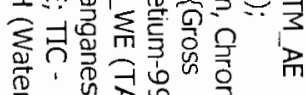

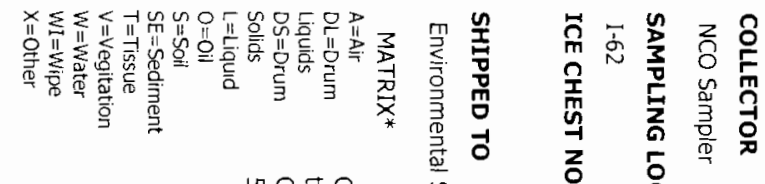

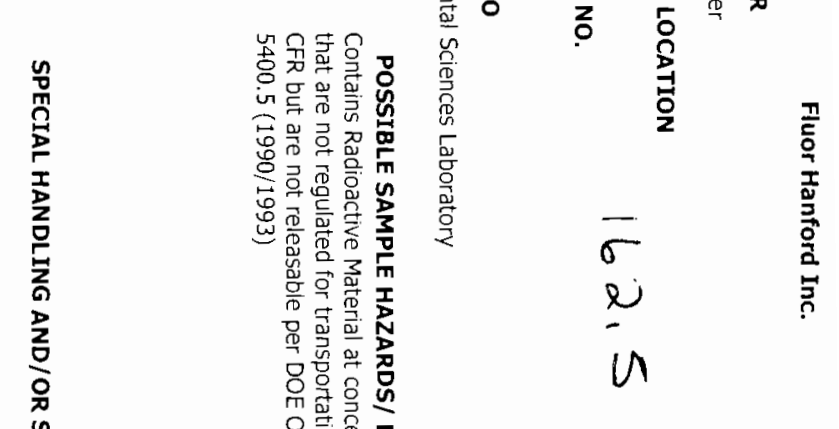

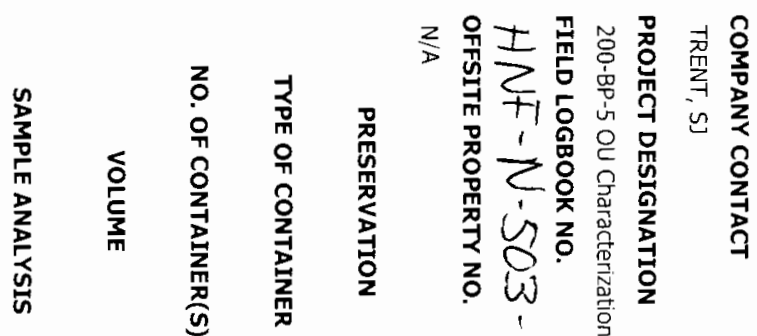

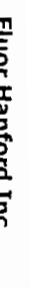



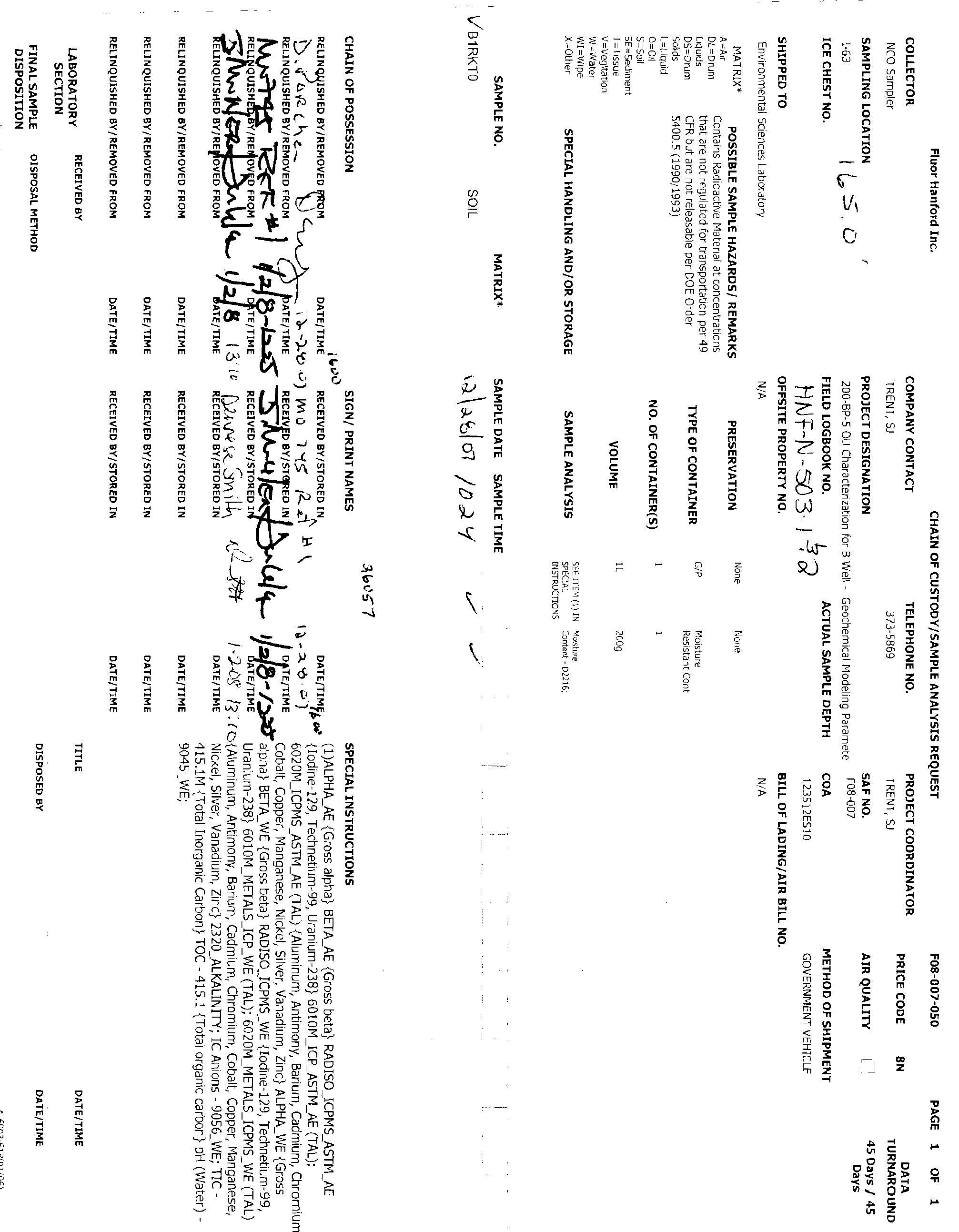


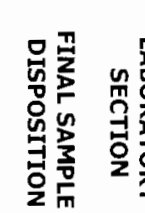

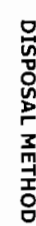
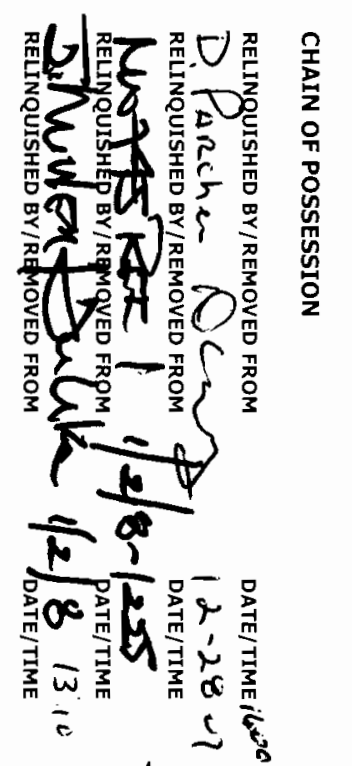

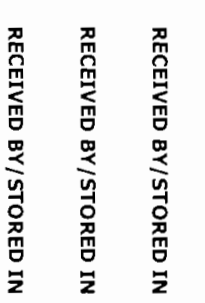

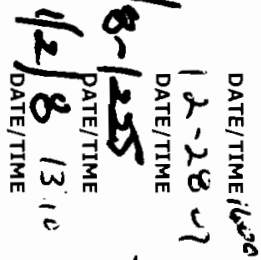

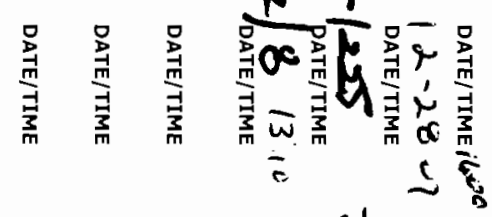

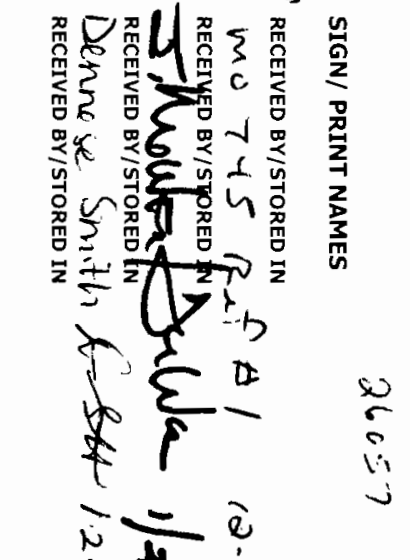

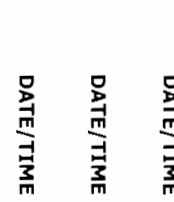

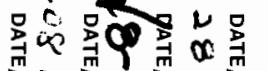

min

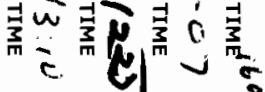

量

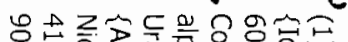

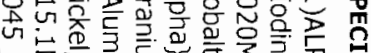

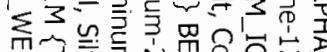

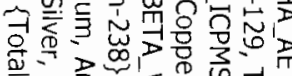

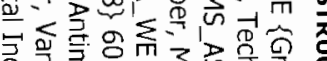

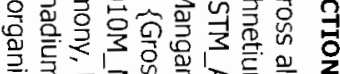

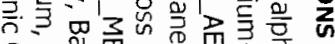

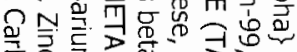

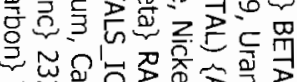

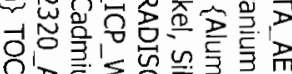

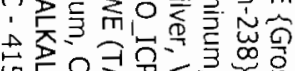

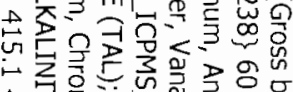

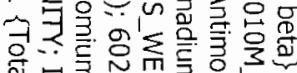

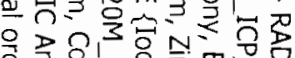

足是

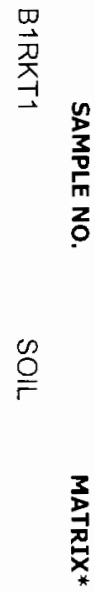

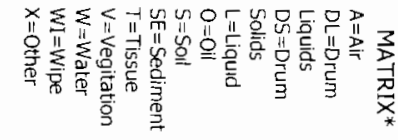

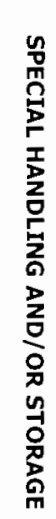

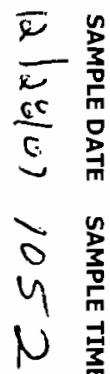

(

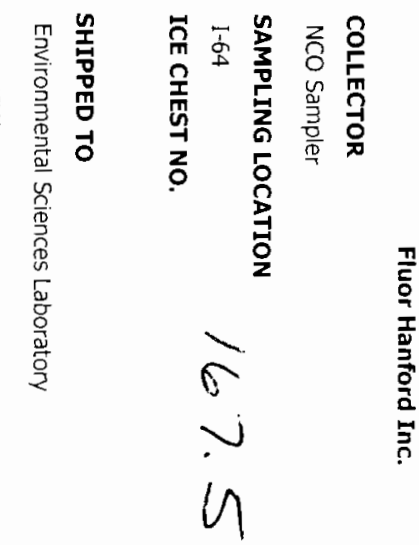

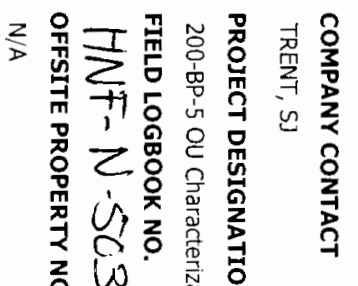
$\frac{1}{2} \stackrel{\frac{0}{3}}{\circ}$ ע $\sum_{0}^{\infty}$

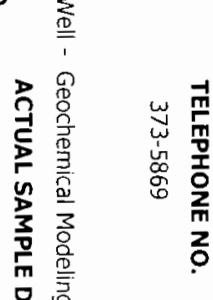

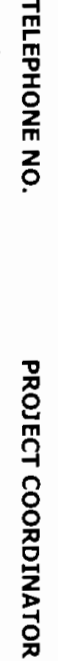

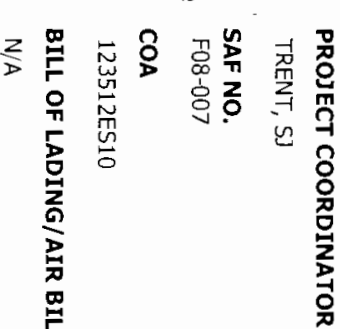

z

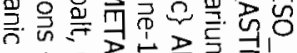

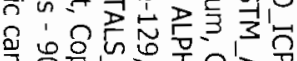

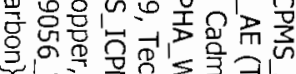

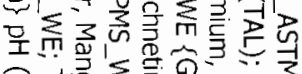

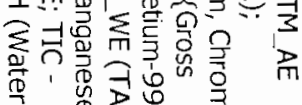

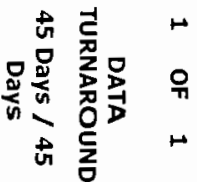




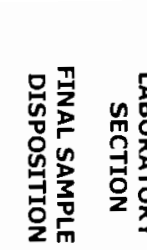

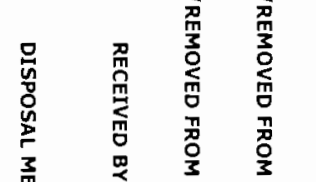

훔

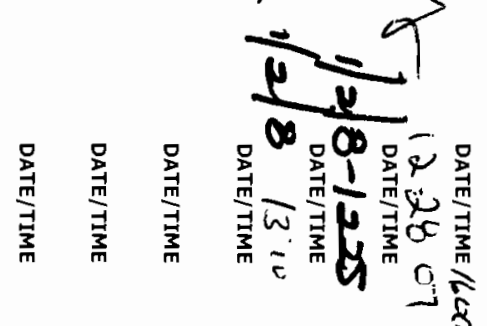

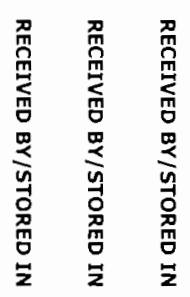

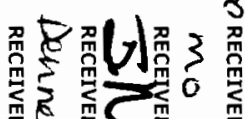

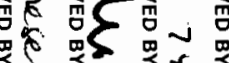

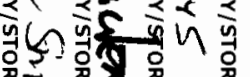

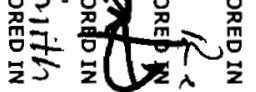

$>\varepsilon$

0
5
5
5

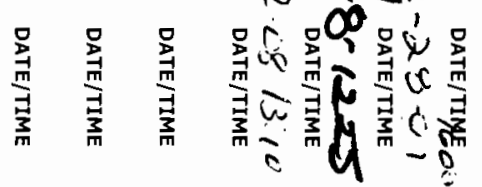

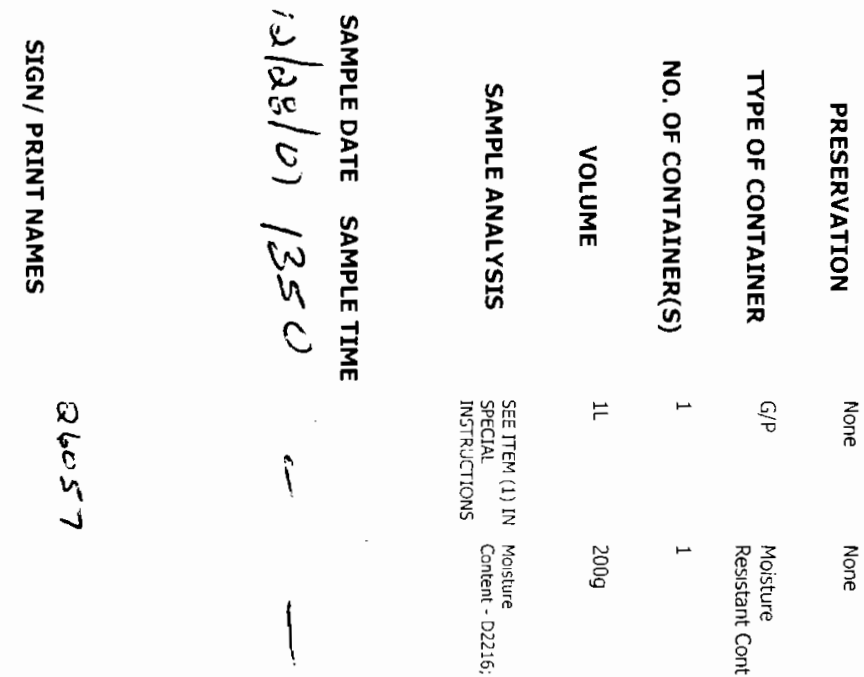

$\stackrel{0}{\circ}$

$\underset{*}{\stackrel{3}{x}}$

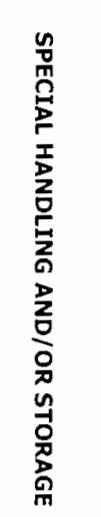

容孛蛋

更

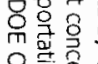

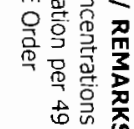

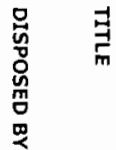

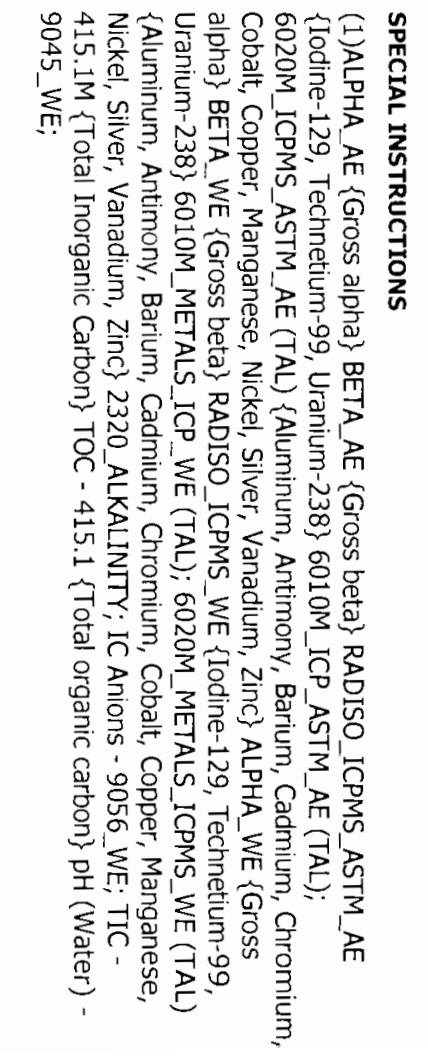

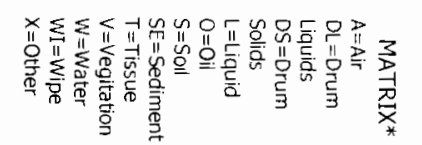

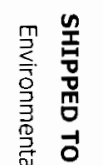

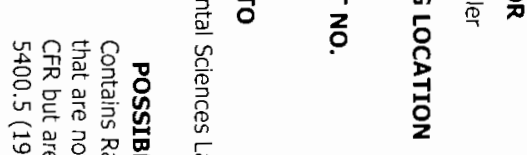

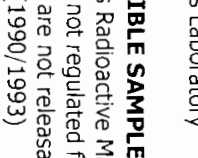

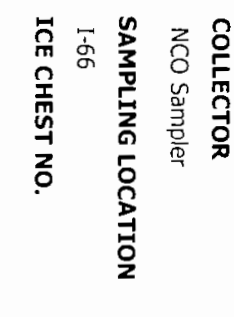

$\bar{j}$

n

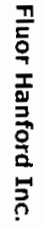

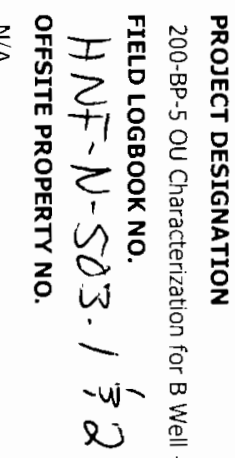

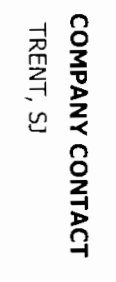

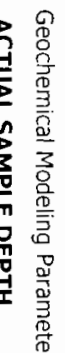

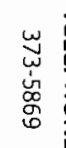

沗

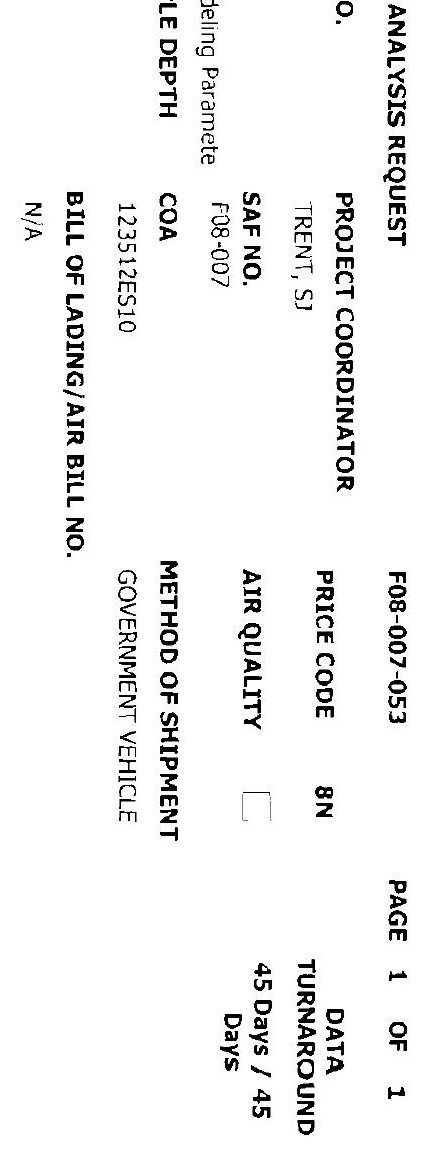

3
0
9
0
0
0
0
0
0
0

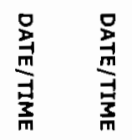




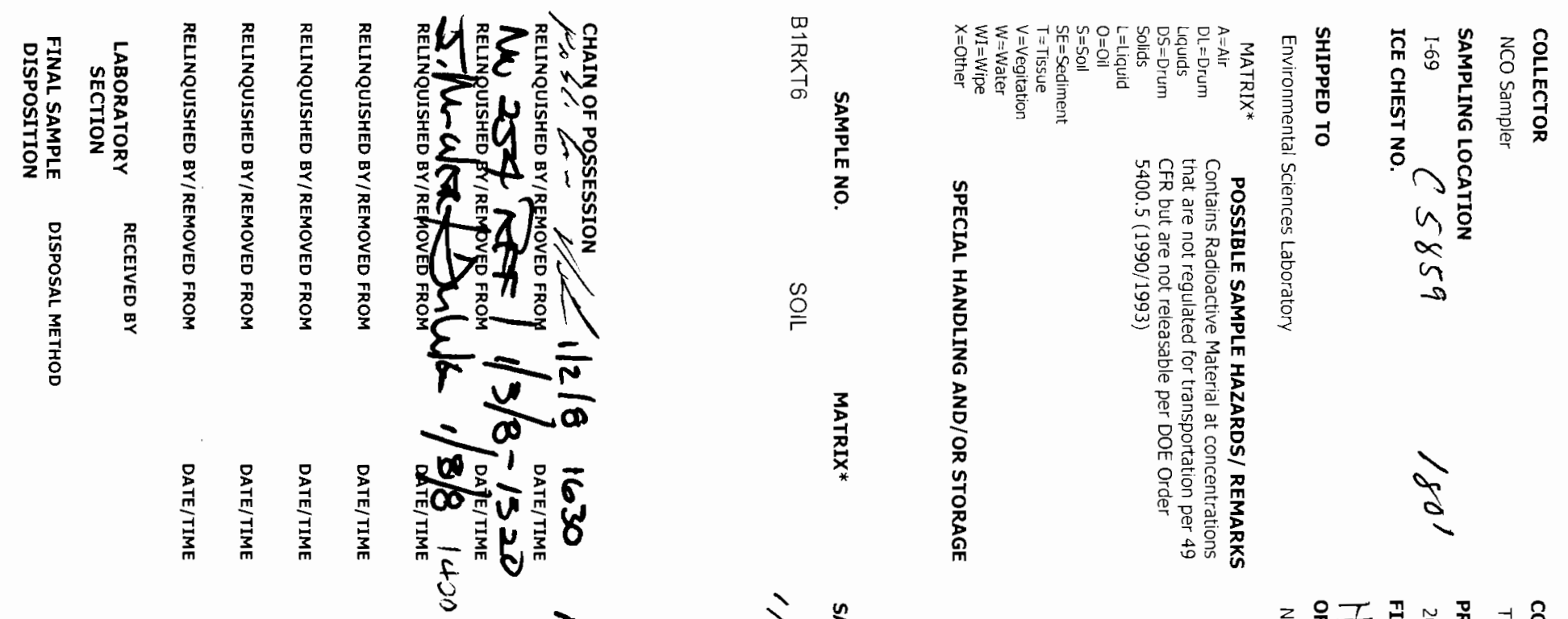

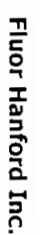
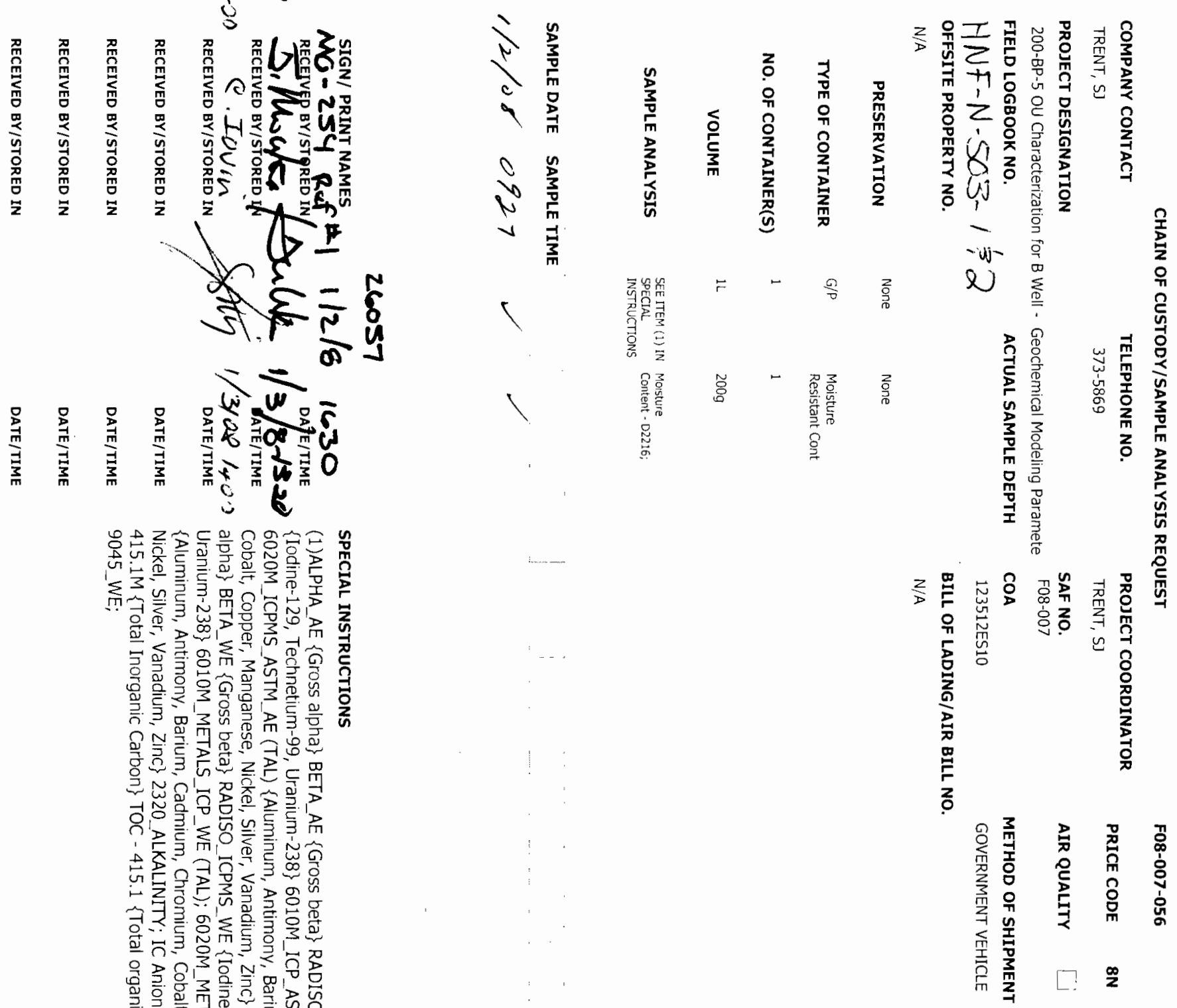

是暑
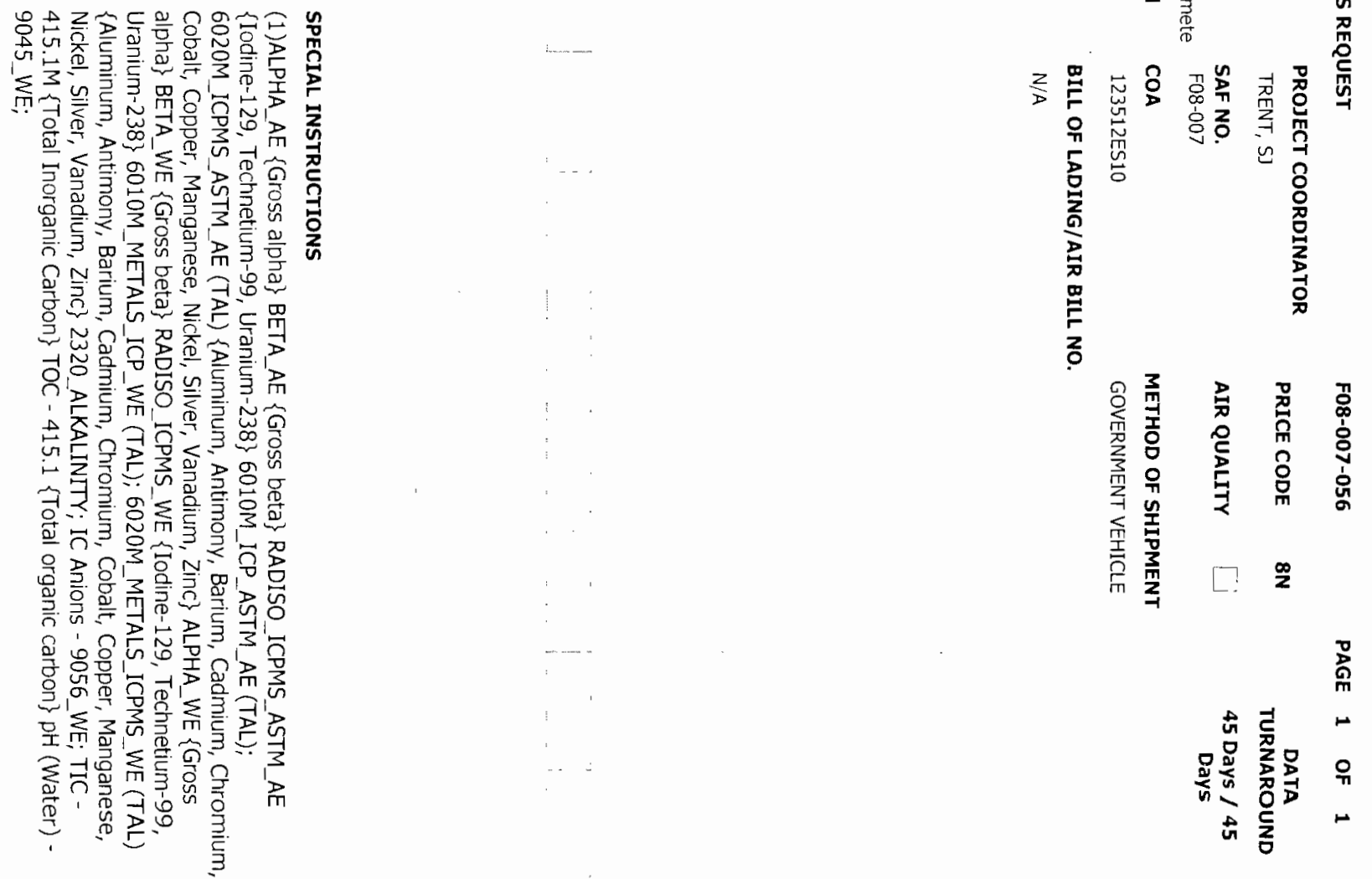


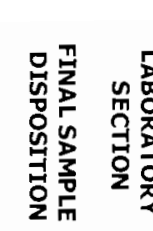

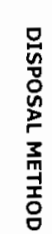

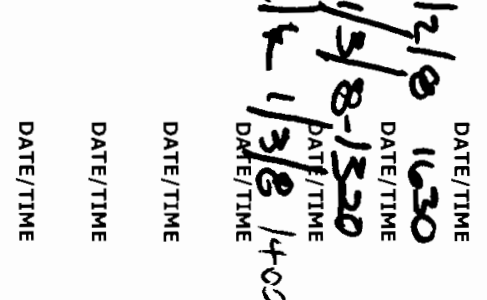

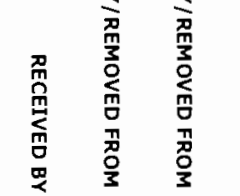

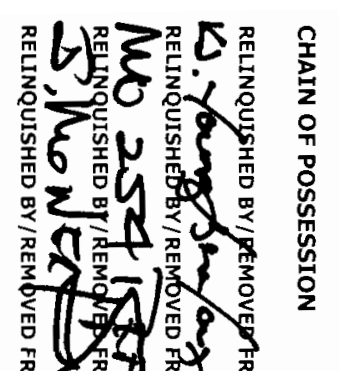

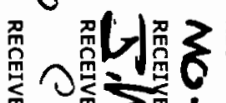

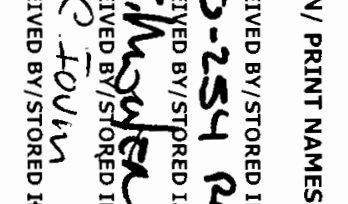
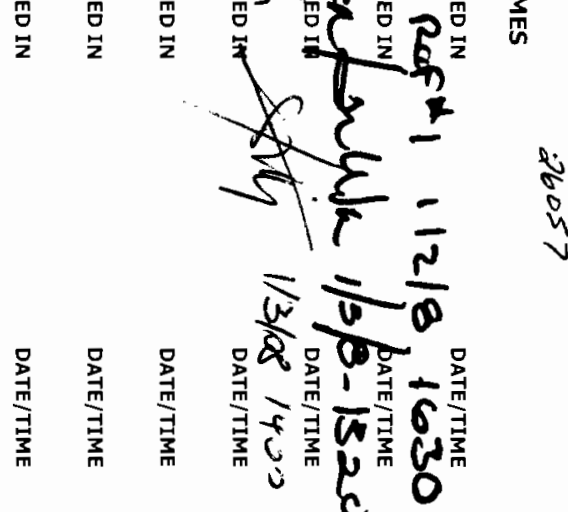

$\underset{\substack{0 \\ \vec{x}}}{\stackrel{0}{\lambda}}$

$\stackrel{\infty}{O}$
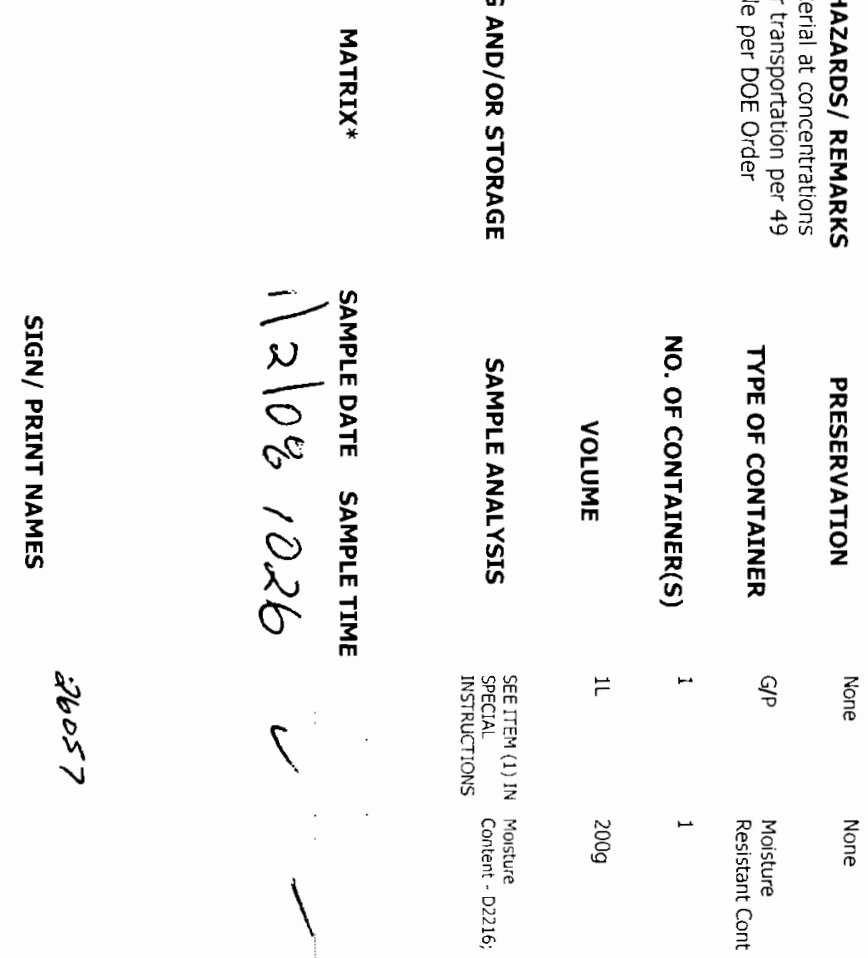

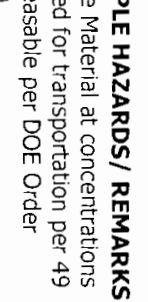

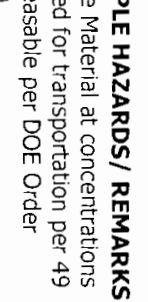

콕

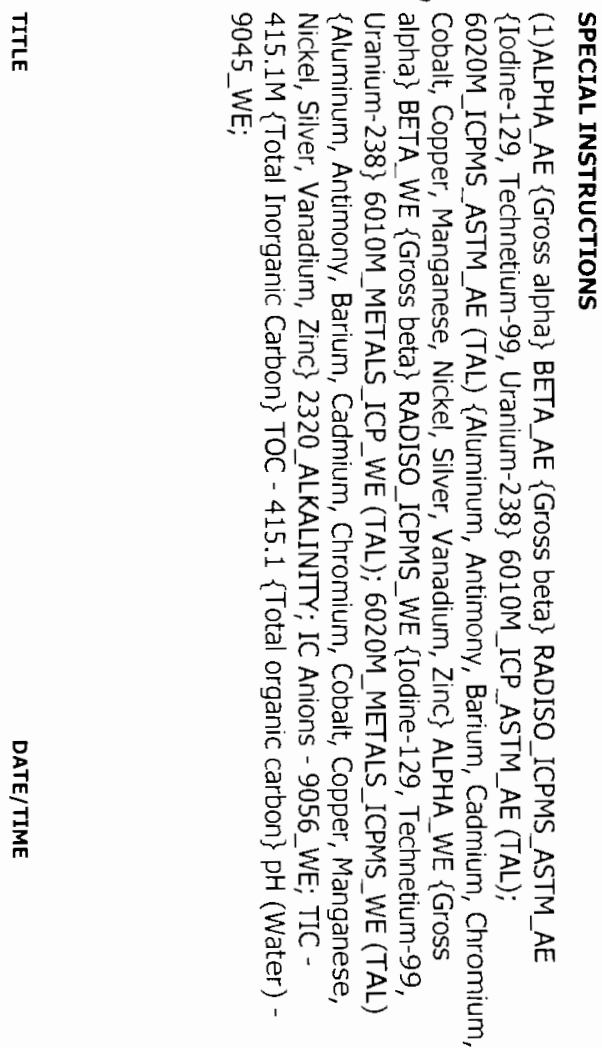

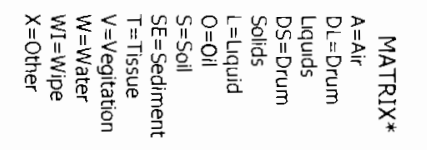

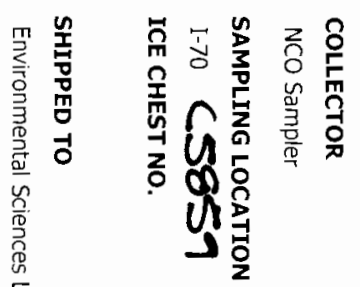

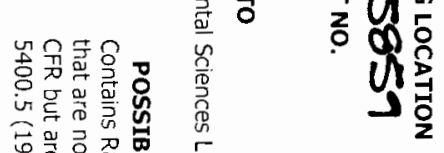

0
$b$
0

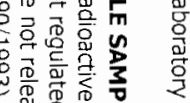

$z$

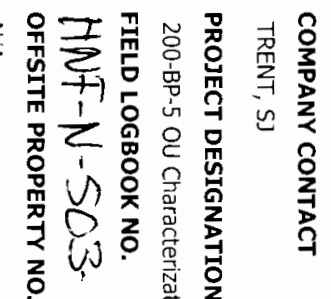
- $\overline{\mathrm{g}}$

-

$\infty$

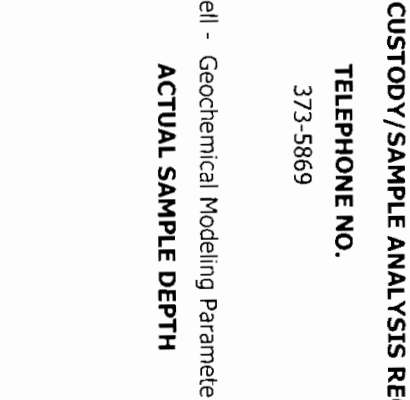

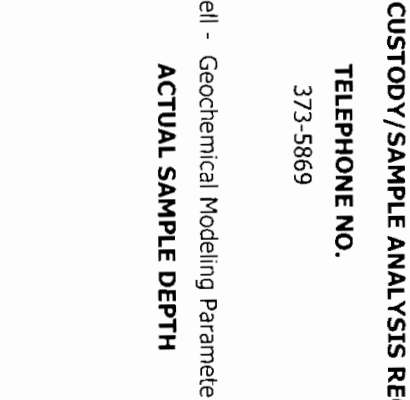

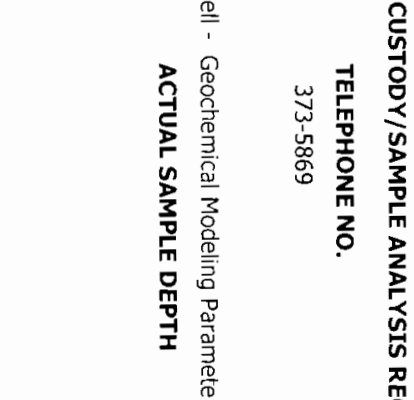

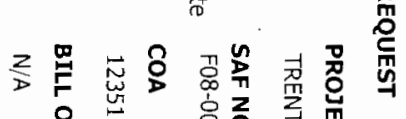

空

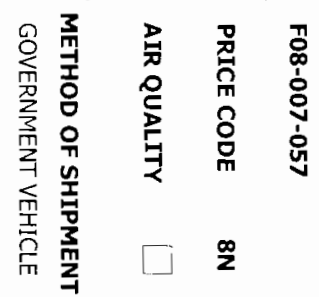

品

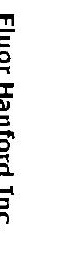




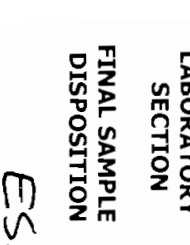

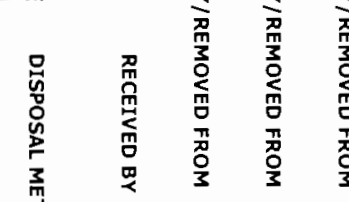
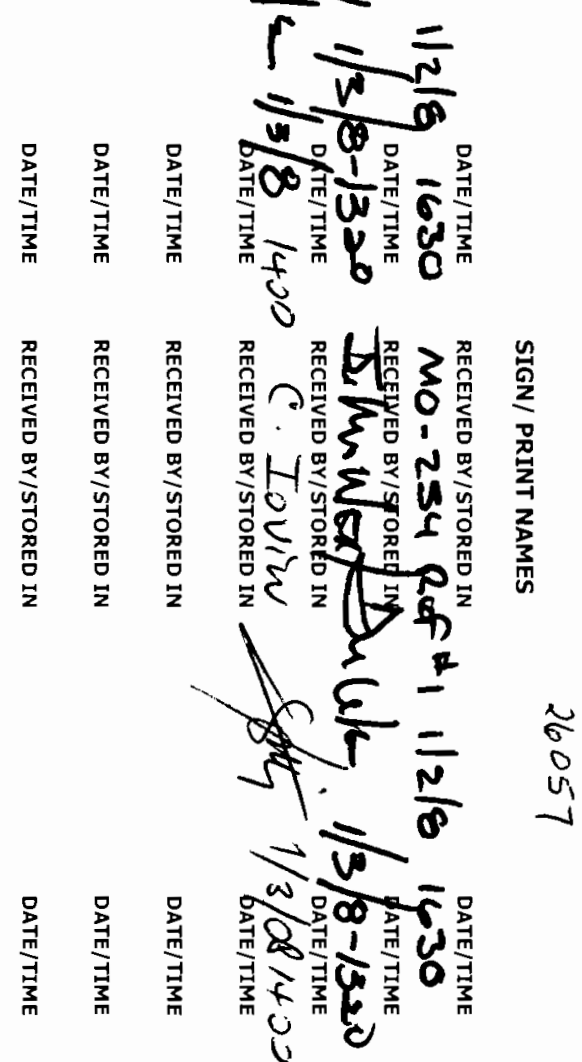

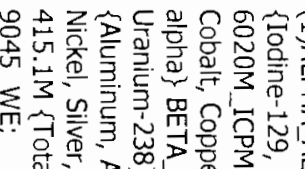

Iㄴ.

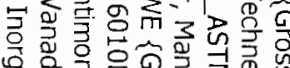

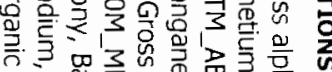

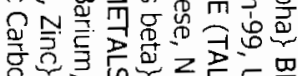

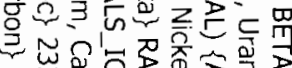

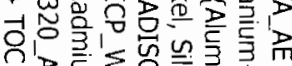

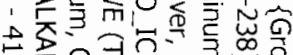

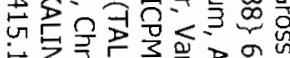

的当亭

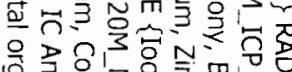

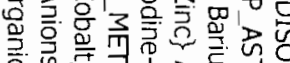

$\begin{array}{ll}\frac{9}{2} & \frac{0}{2} \\ \frac{7}{m} & \frac{7}{3} \\ \frac{7}{3} & \frac{3}{3} \\ \frac{3}{m} & \frac{3}{m}\end{array}$ $\frac{\sqrt{x_{3}^{2}}}{2}$

$\stackrel{\mathscr{\varrho}}{\risingdotseq}$

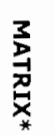

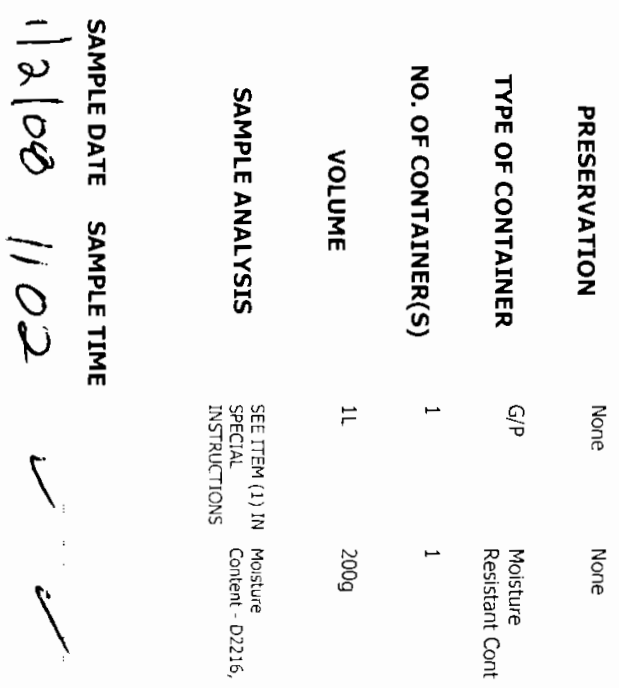

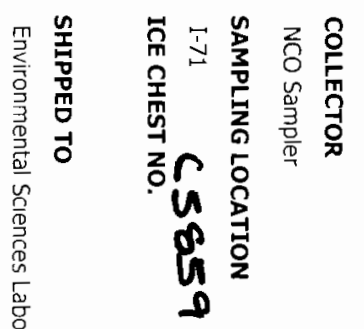

n

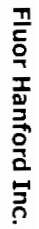

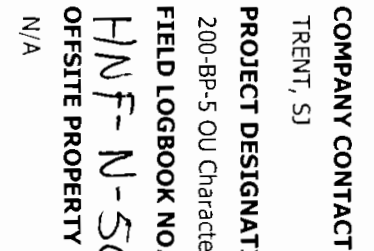

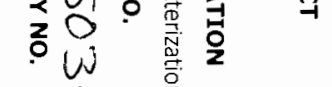



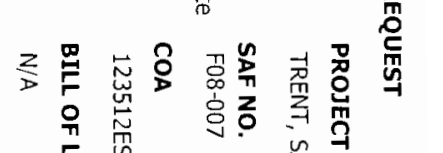

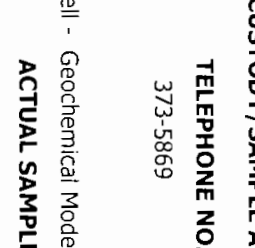

品

学

心

z

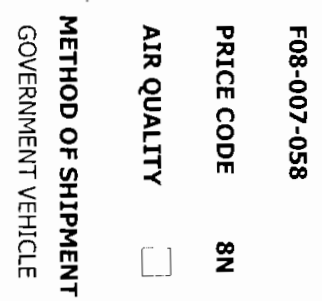

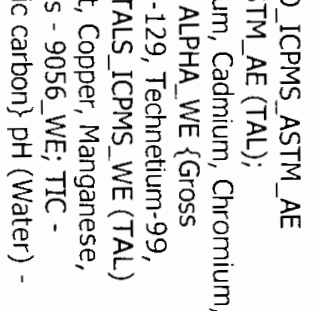




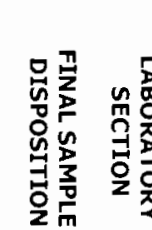

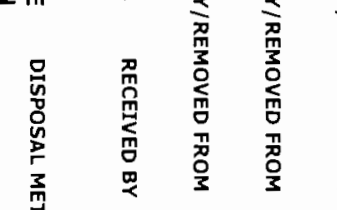

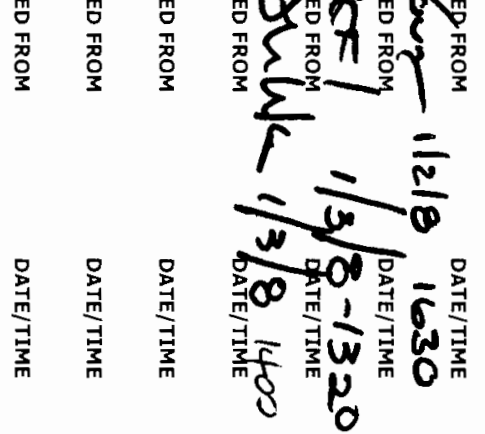

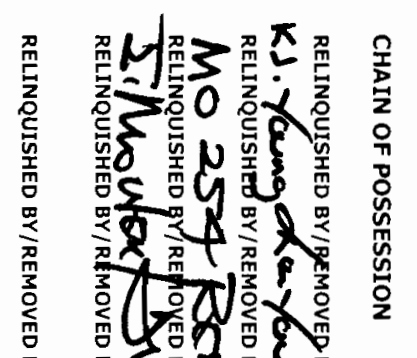
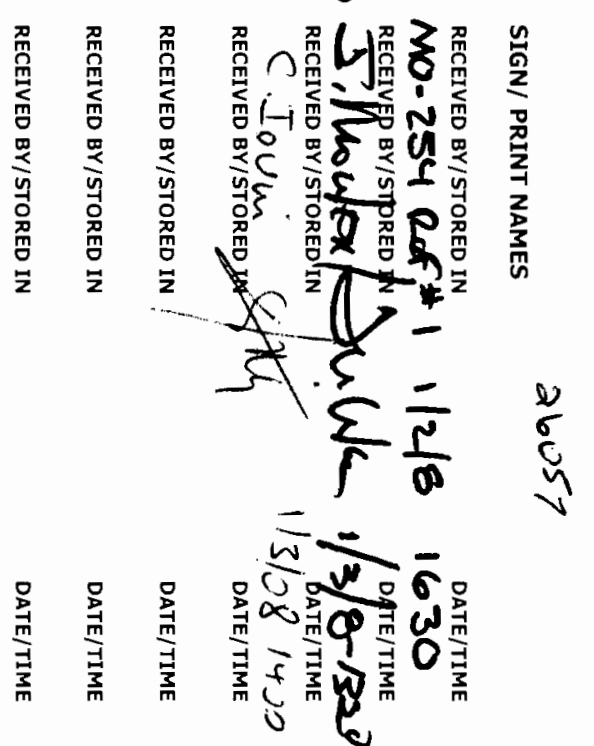

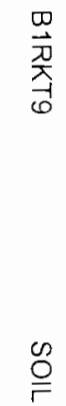

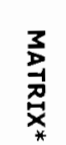

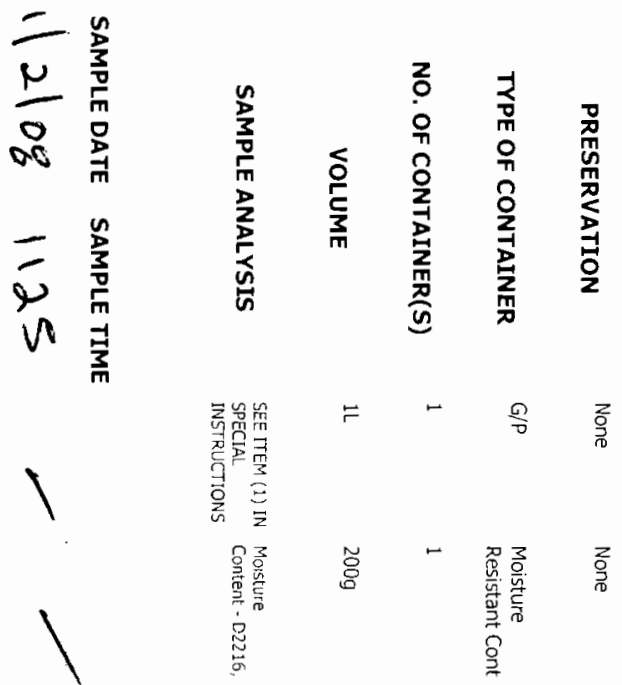

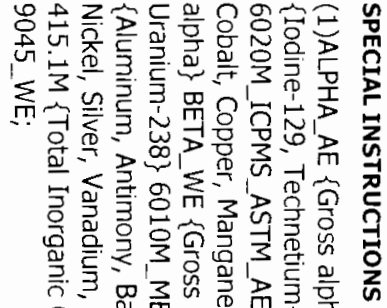

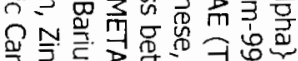

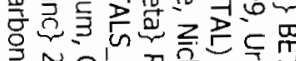

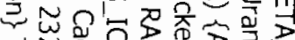

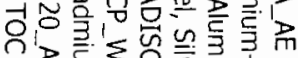

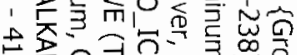

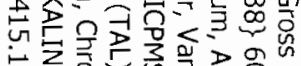

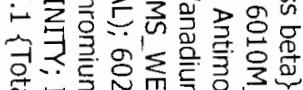

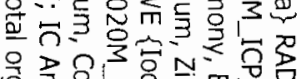

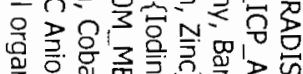

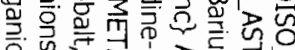

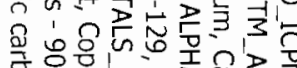

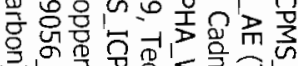

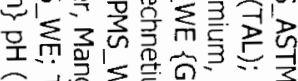

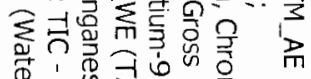

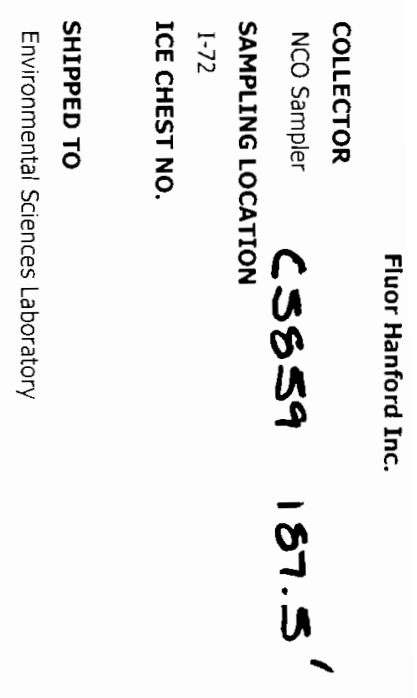

2

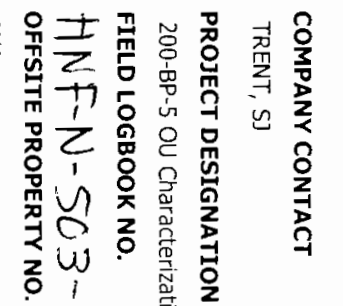

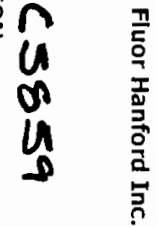

0
$\frac{0}{0}$
$\frac{1}{2}$
$\delta$
$\frac{\delta}{2}$
$\frac{1}{2}$
$\frac{1}{1}$

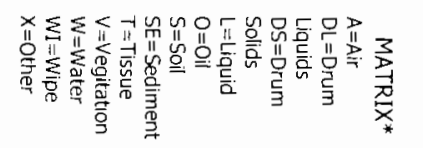

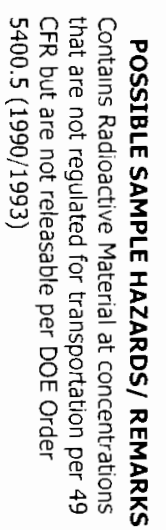

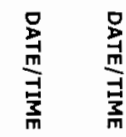

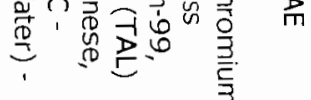



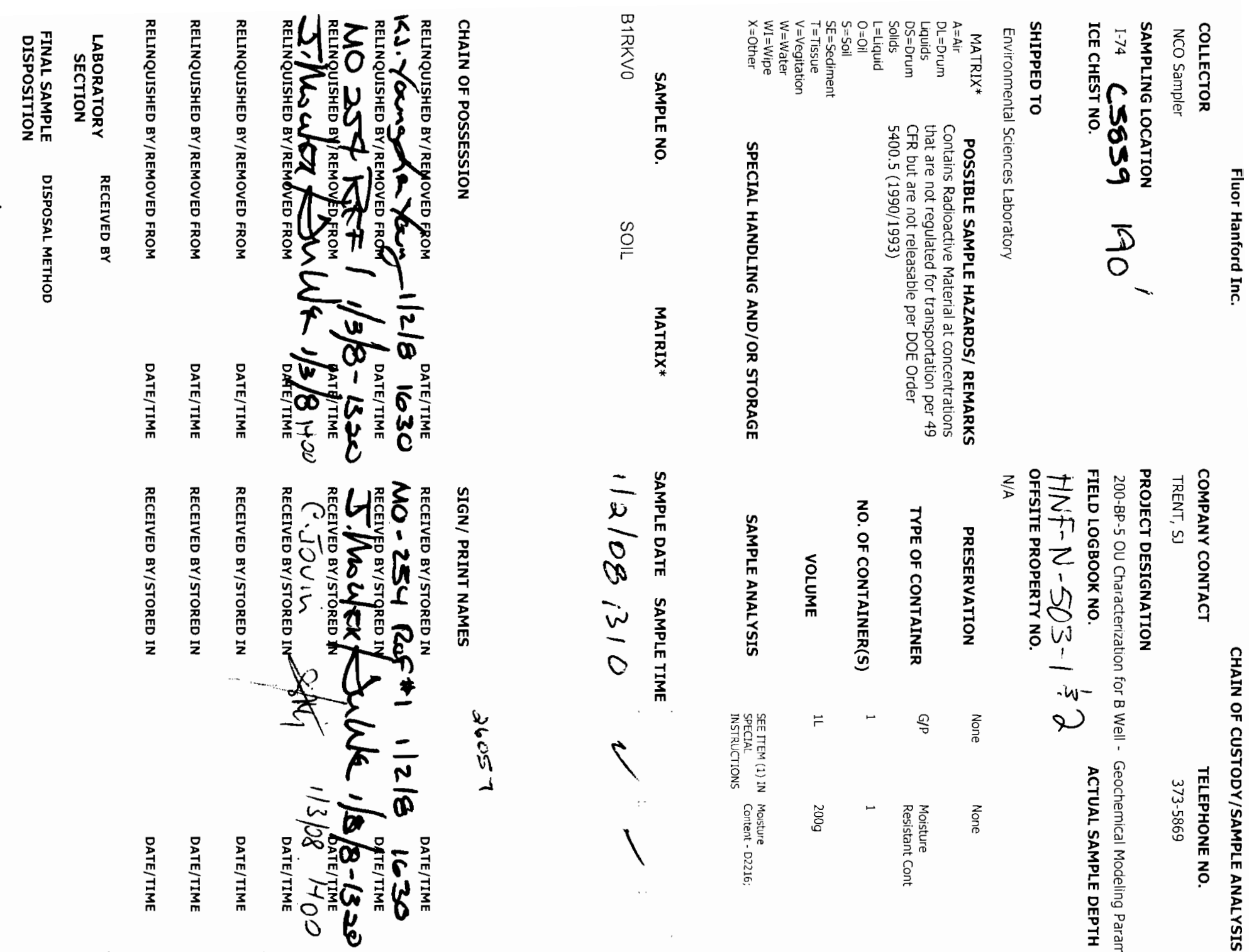

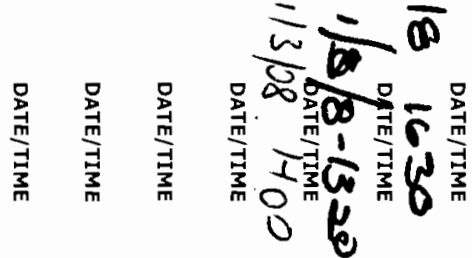
$\frac{1}{-w} \stackrel{\substack{\overline{9} \\ 0 \\ 0}}{0}$

2

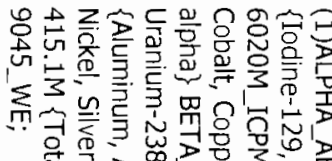

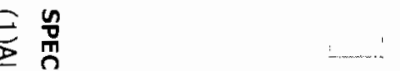

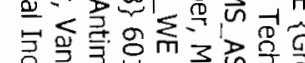

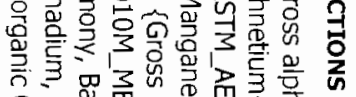

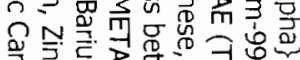

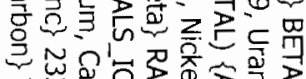

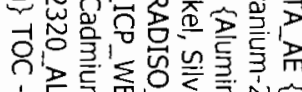

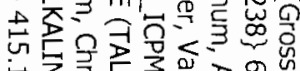

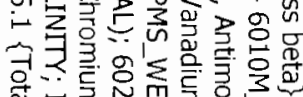

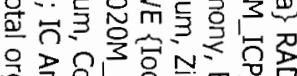

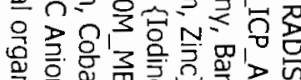

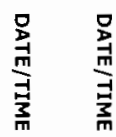

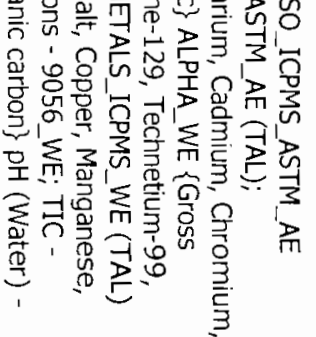




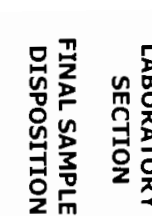

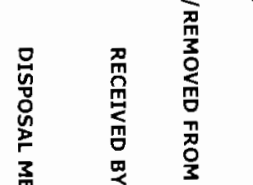

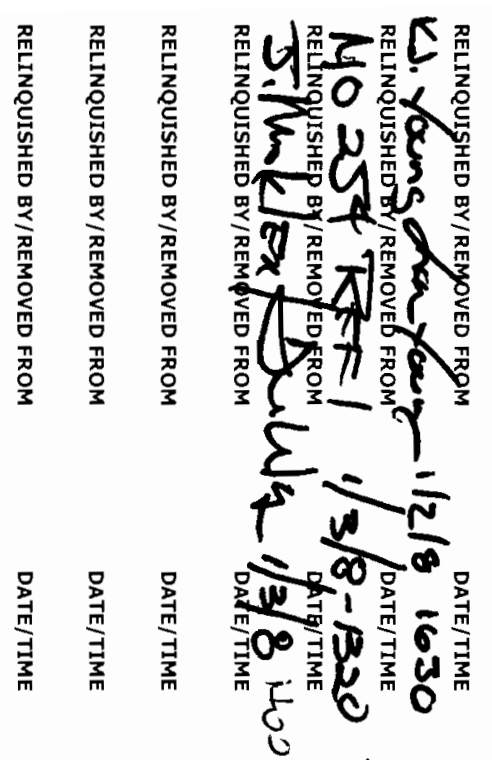

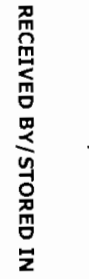
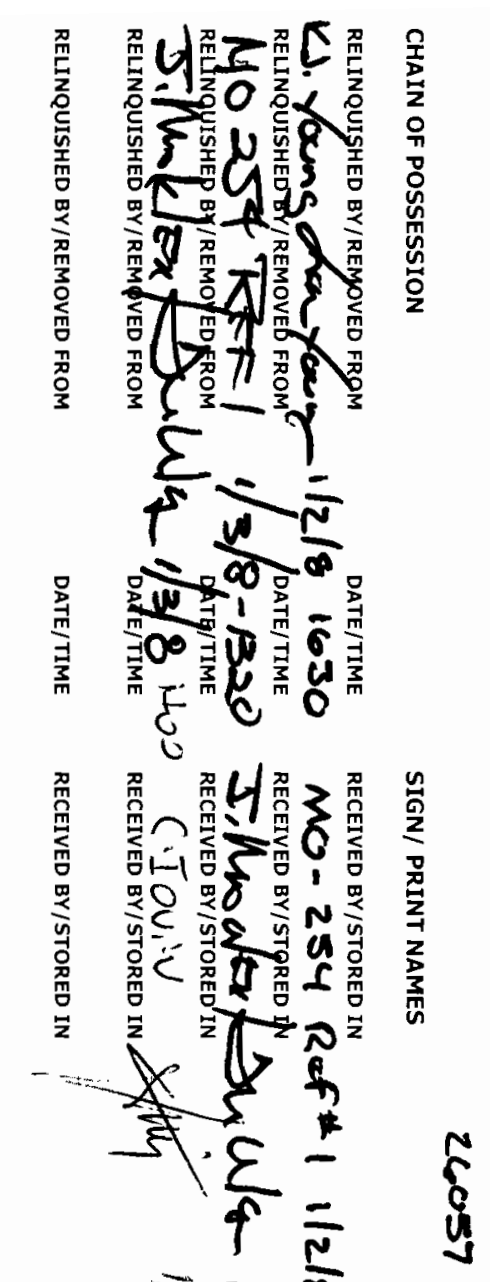

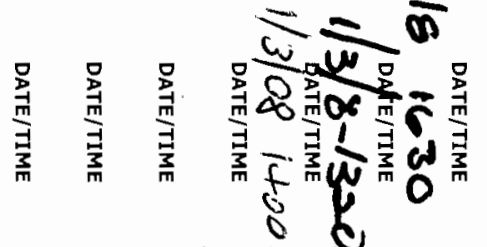

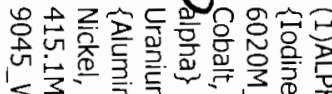

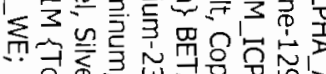

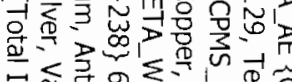

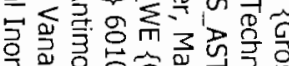

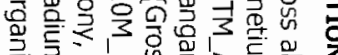

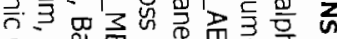

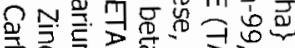

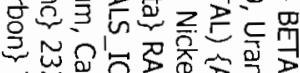

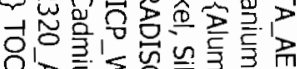

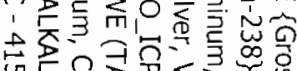

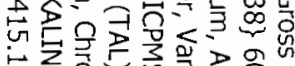

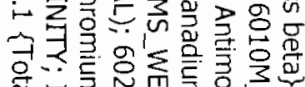

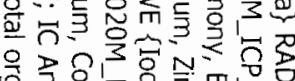

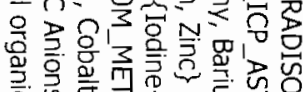
䛨咅

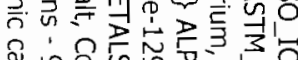
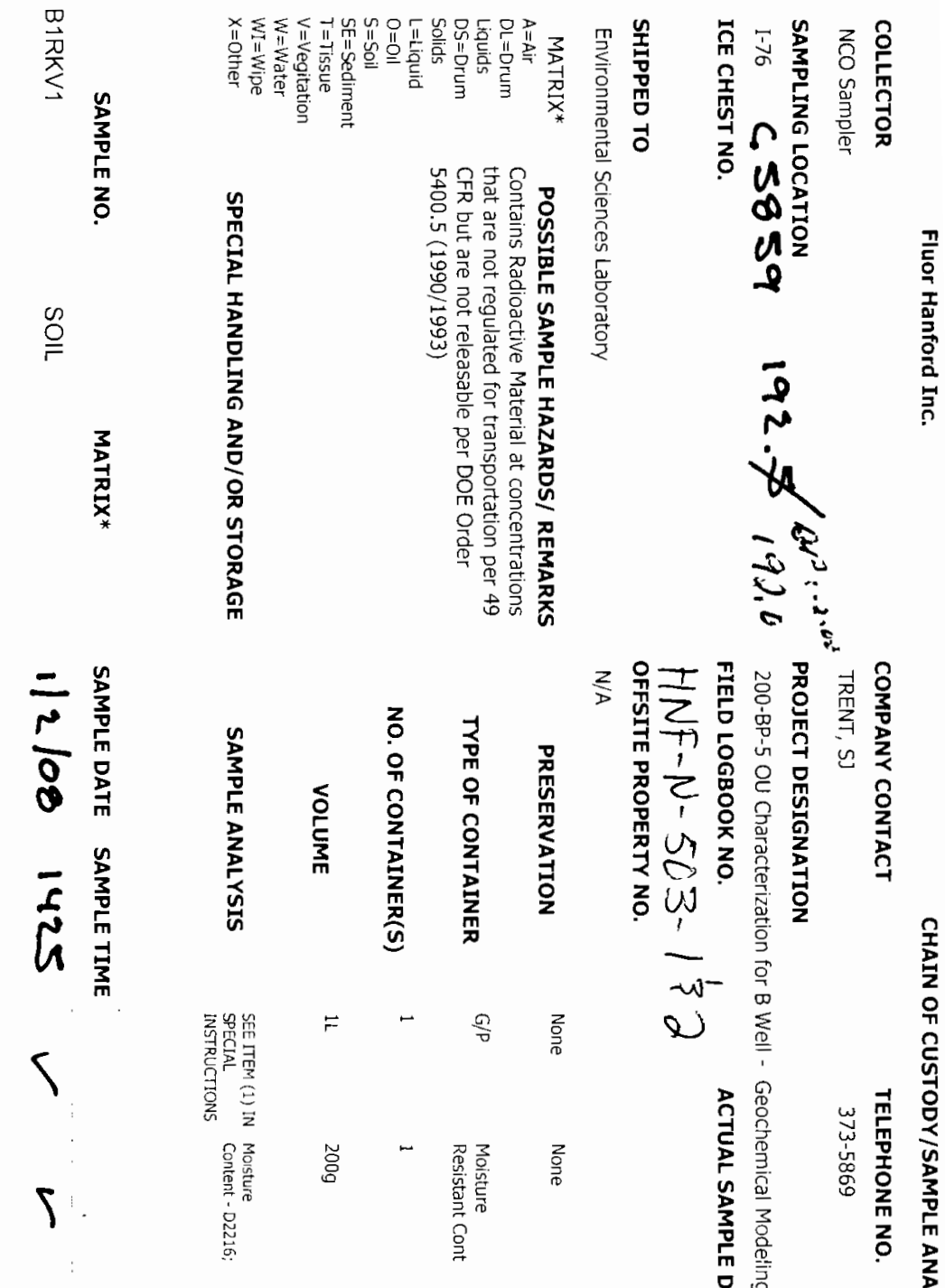

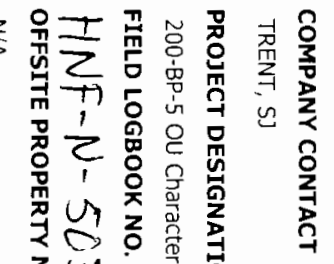

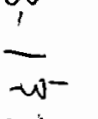

Q
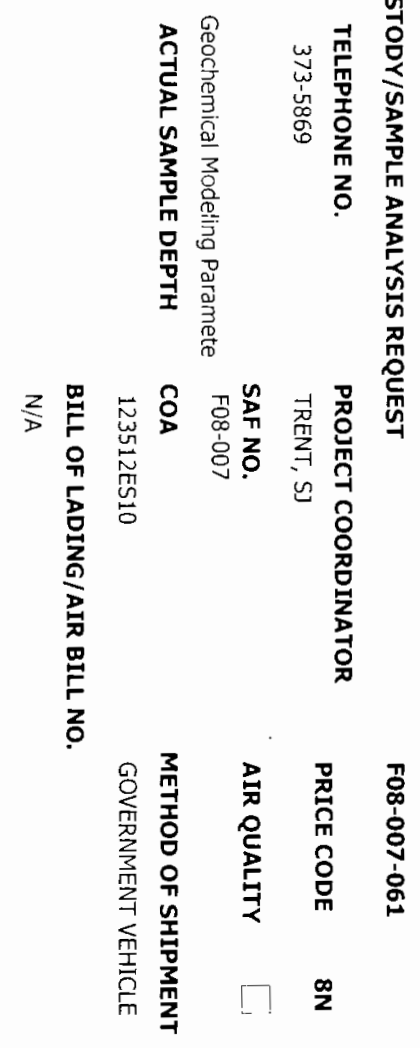

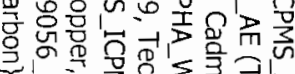

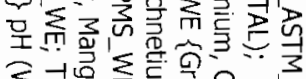

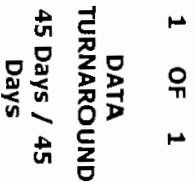


II

11
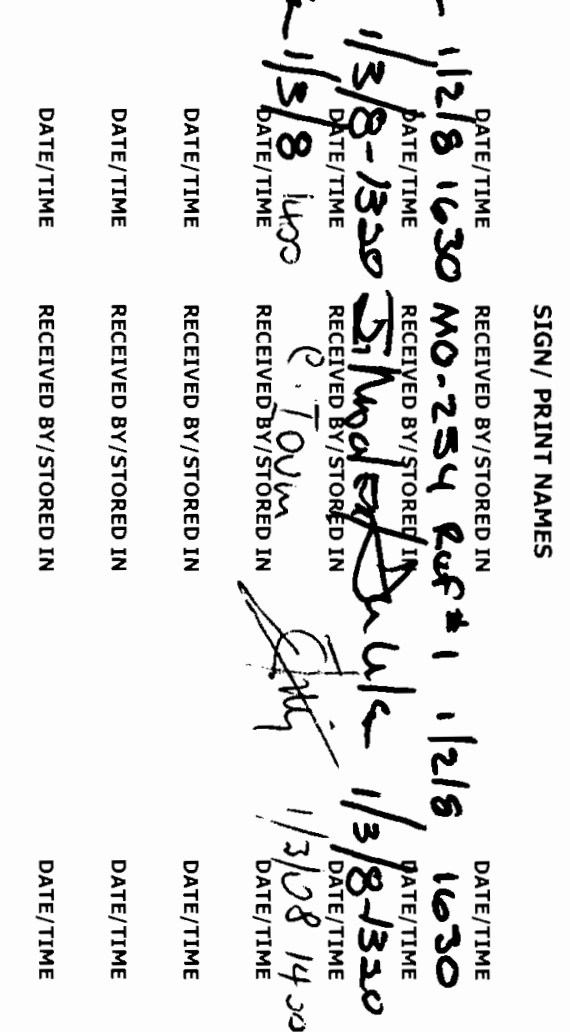

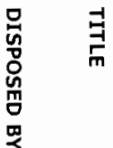

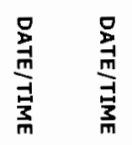

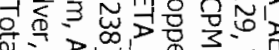

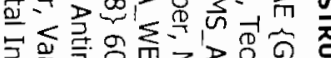

웅 을을

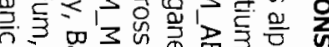

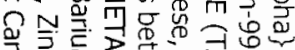

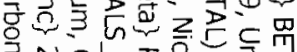

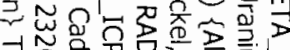

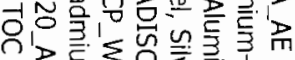

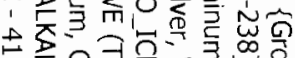

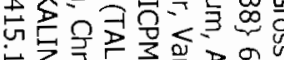

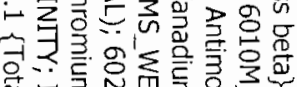

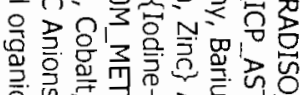

की

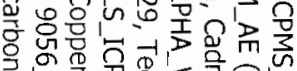

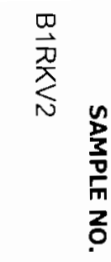

$\stackrel{\infty}{\stackrel{c}{c}}$

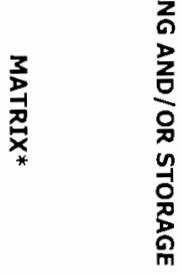

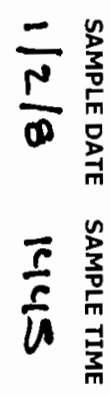

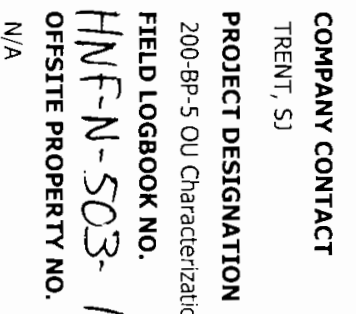

$-\omega-\stackrel{\overrightarrow{0}}{\circ}$

) $\sum_{\underline{0}}^{\infty}$

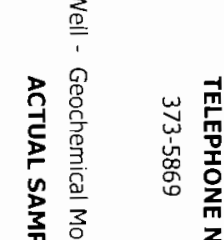

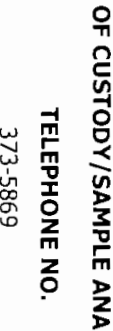

营

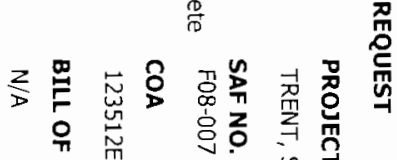

咞莕

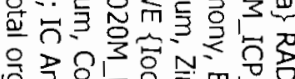

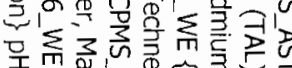

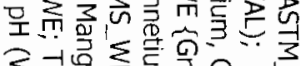

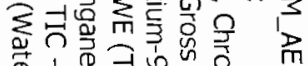

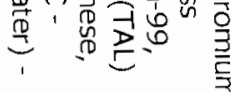

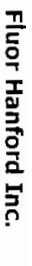

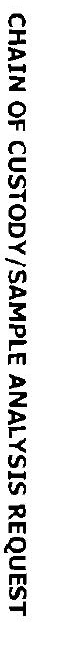




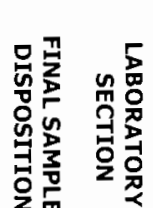

.

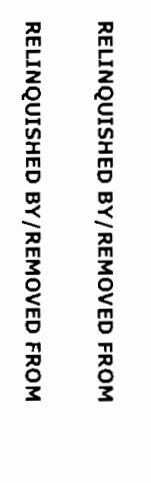

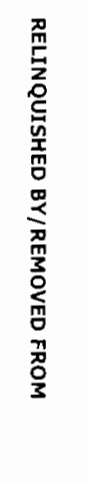

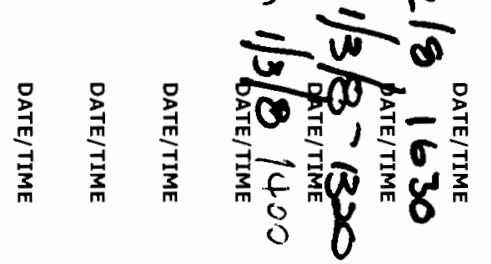

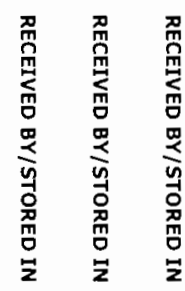
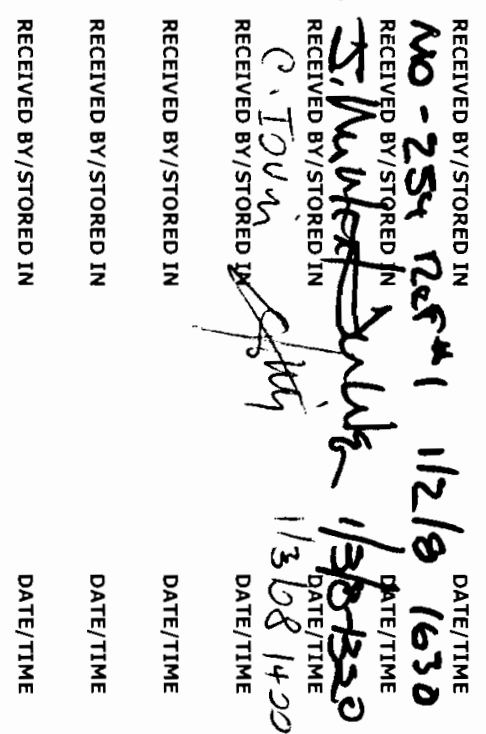

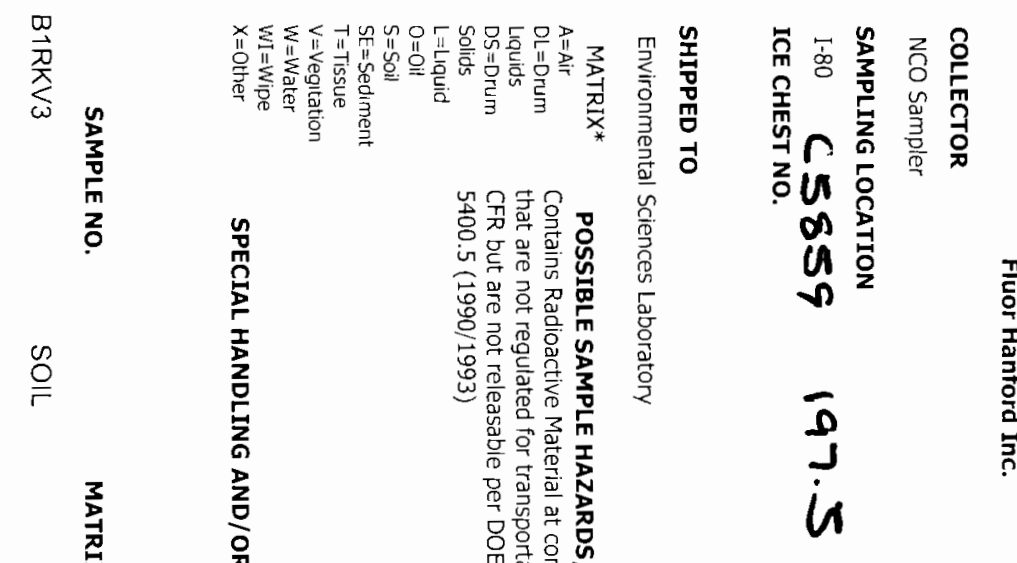

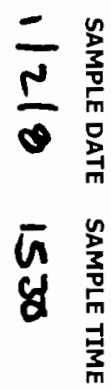

S

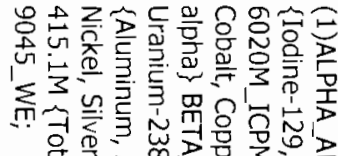

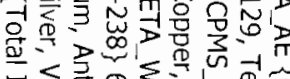

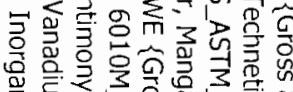

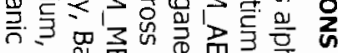

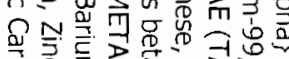

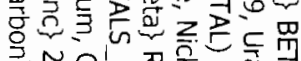

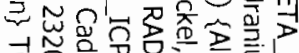

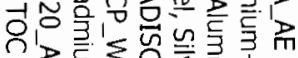

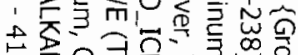

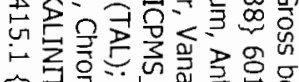

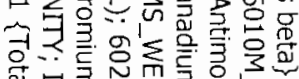

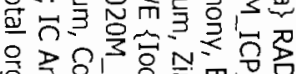

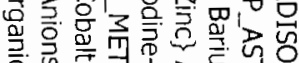

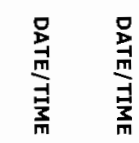

â

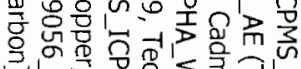

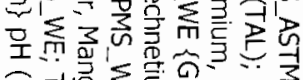

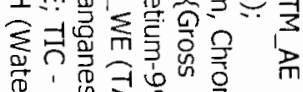

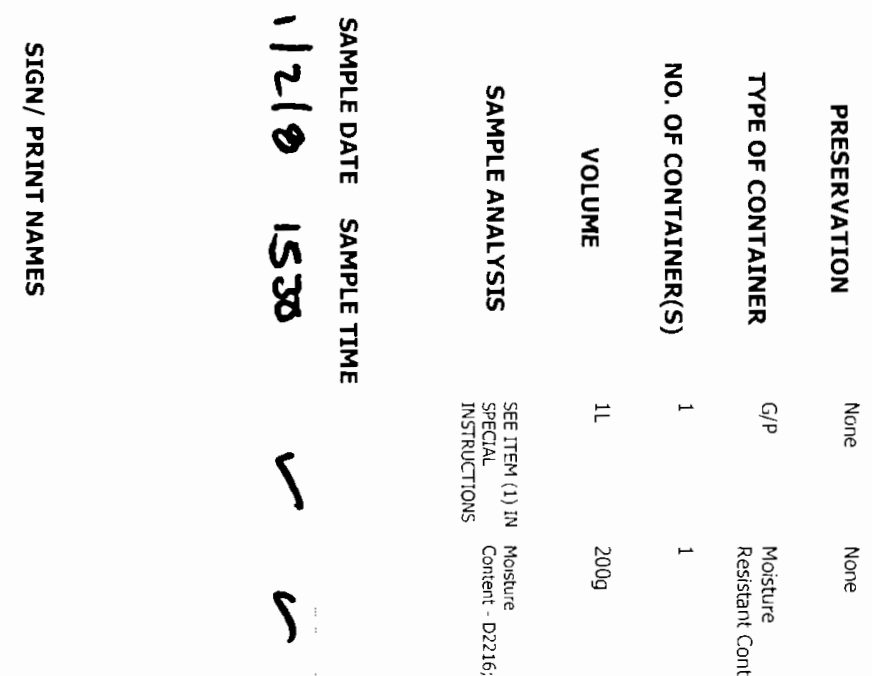

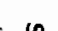

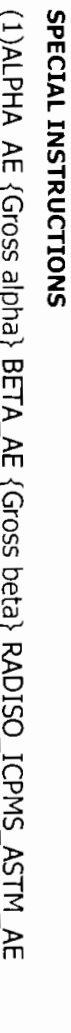

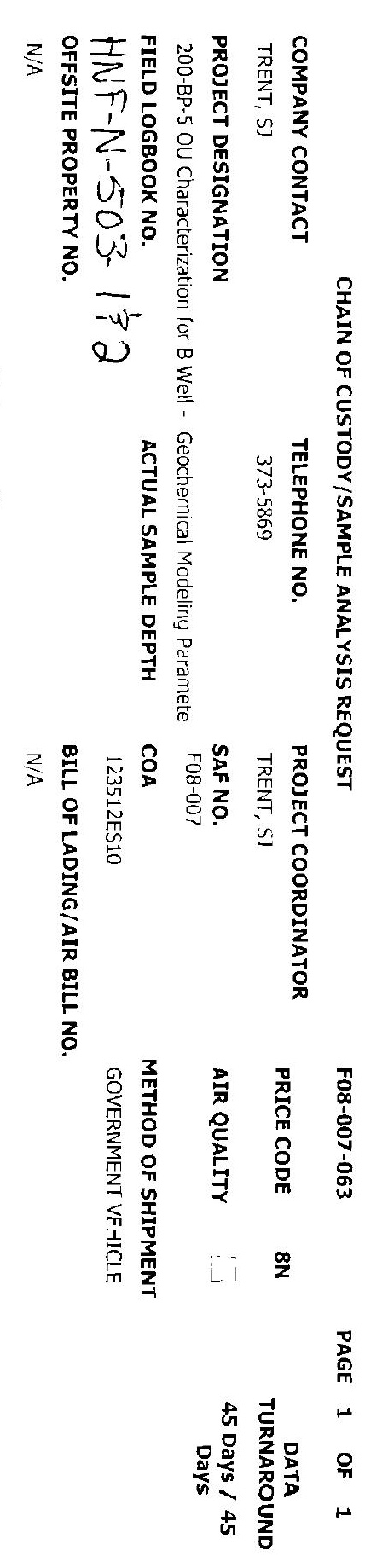




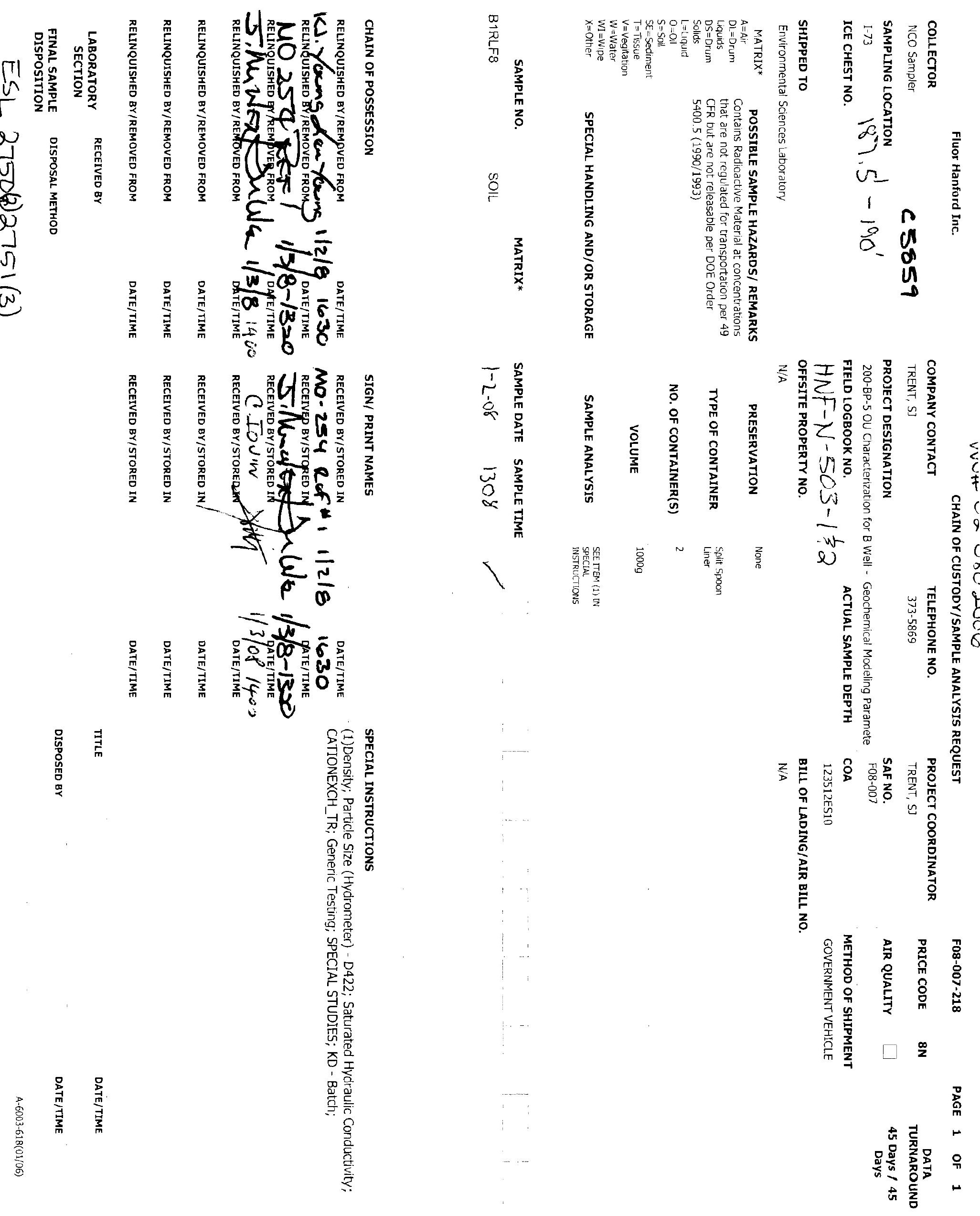




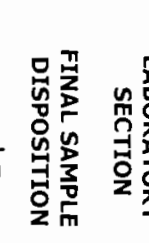
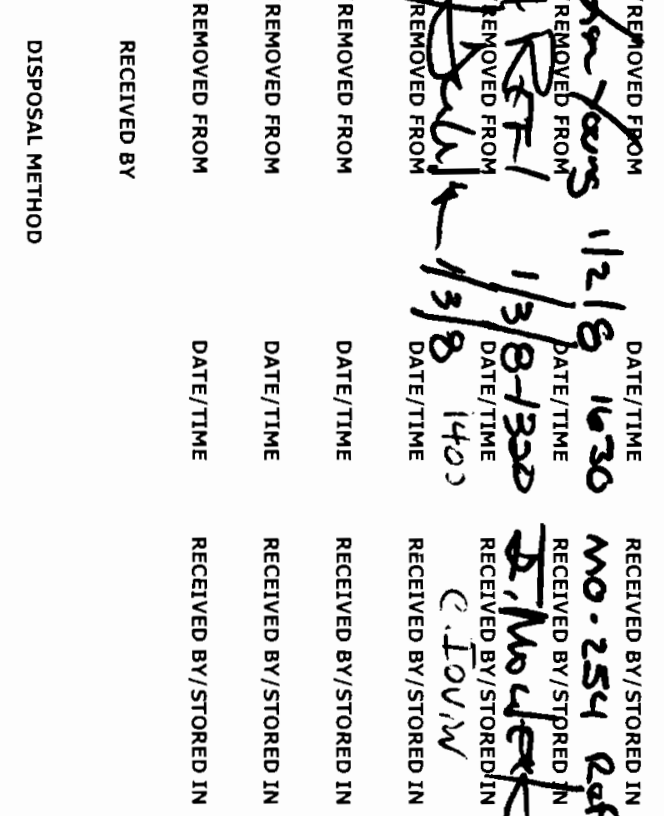

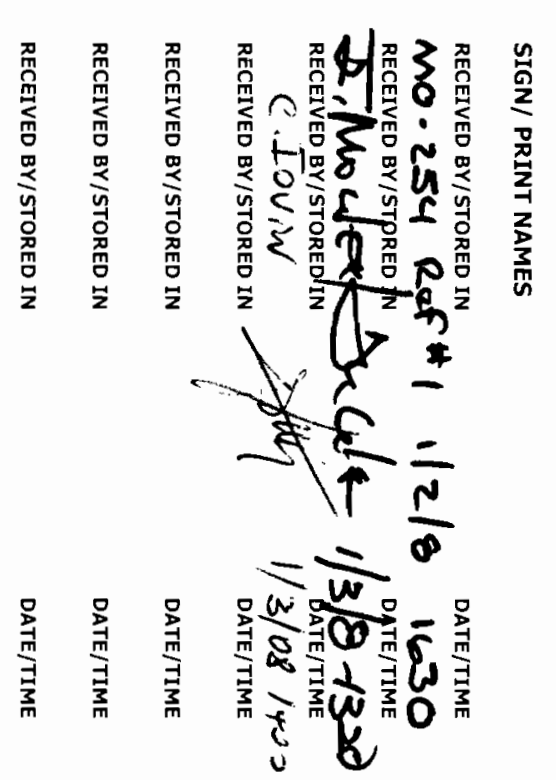

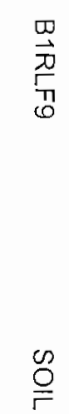

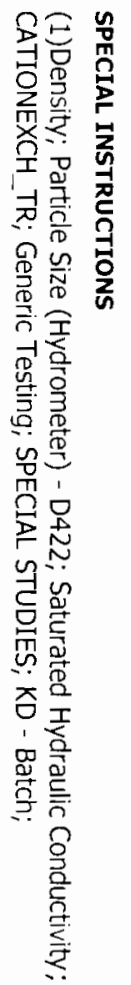

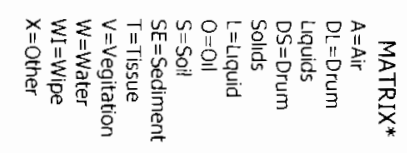

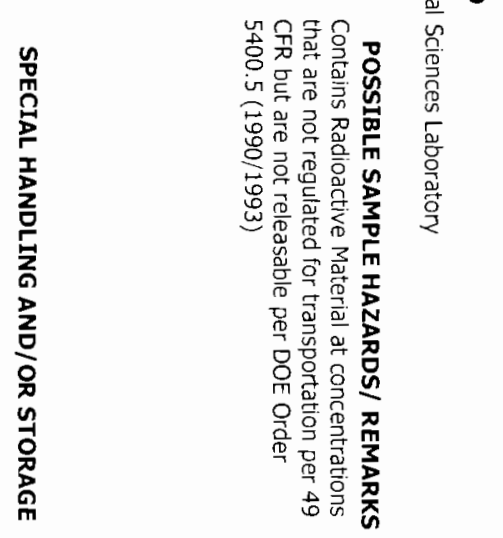

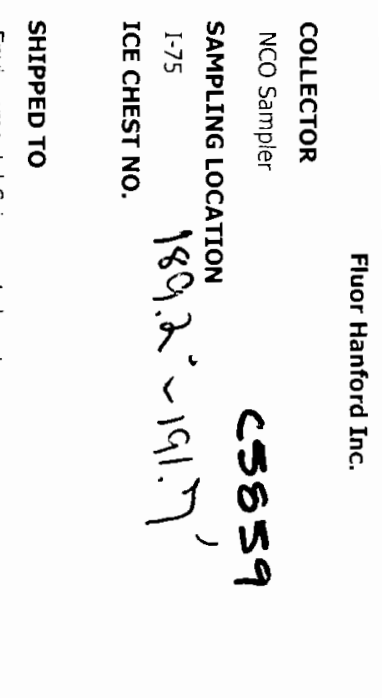

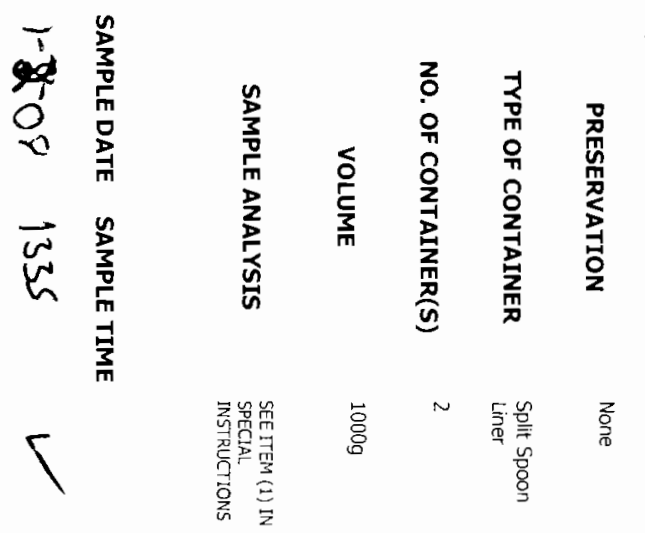

옥

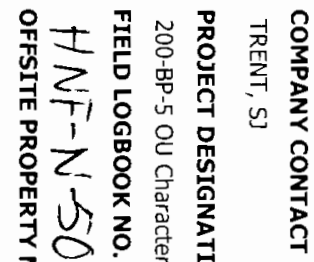

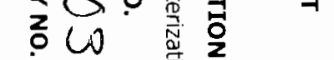

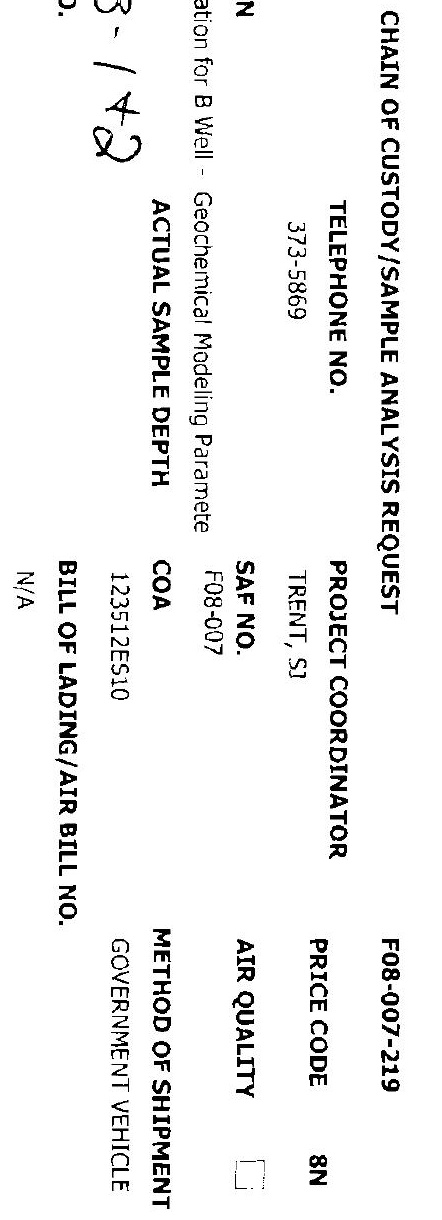

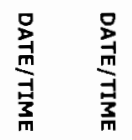




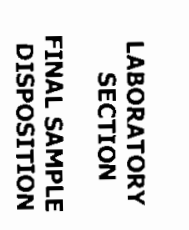

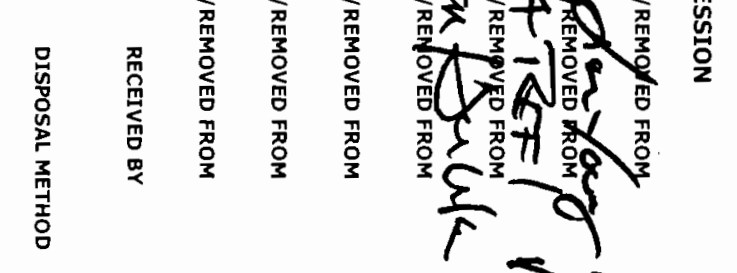

美

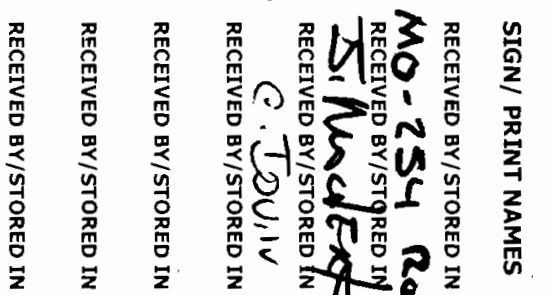

录
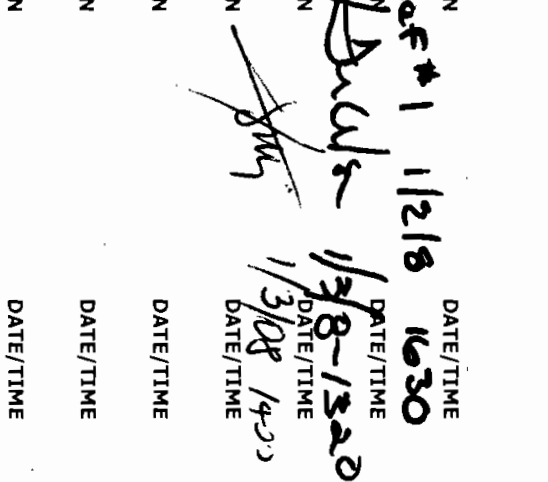
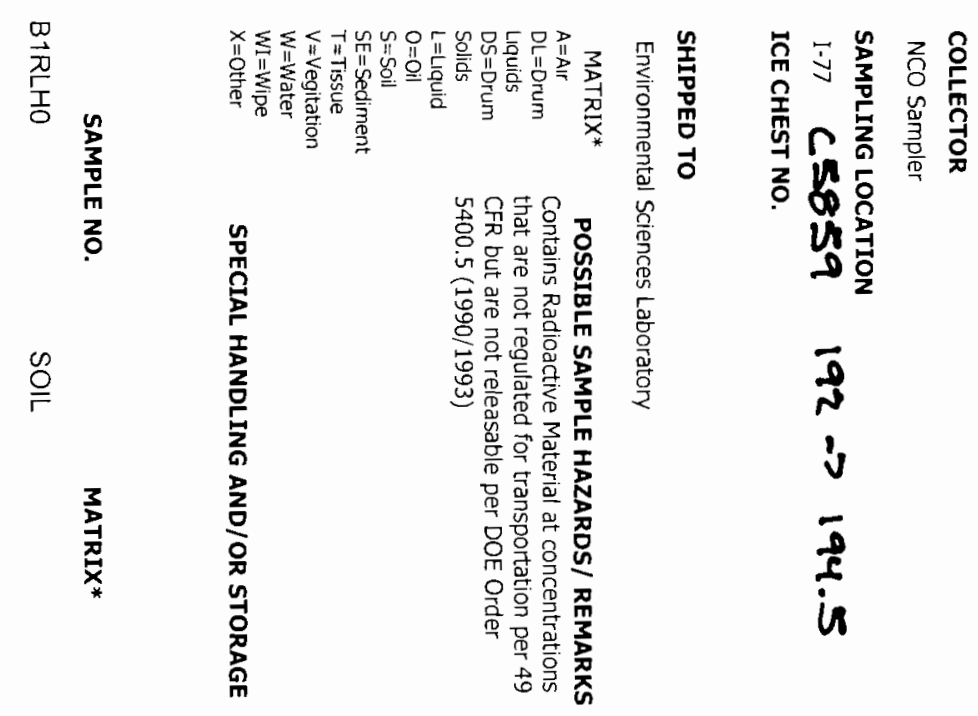

is

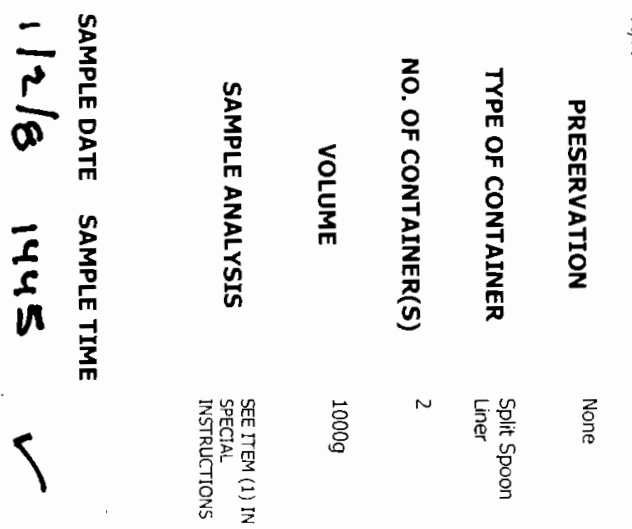

2

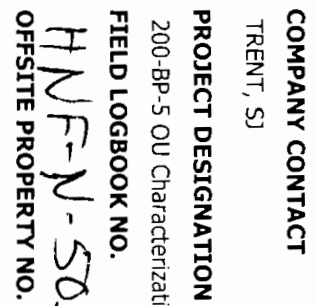
w

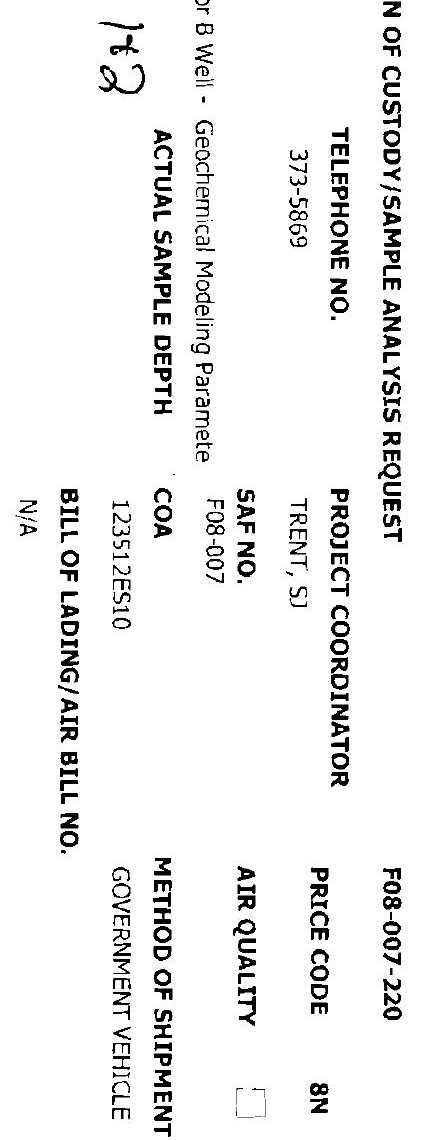

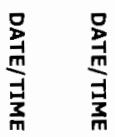

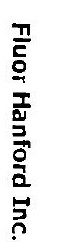

ํㅗㄹ

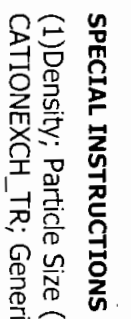

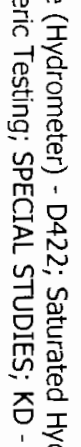

몀 몸 

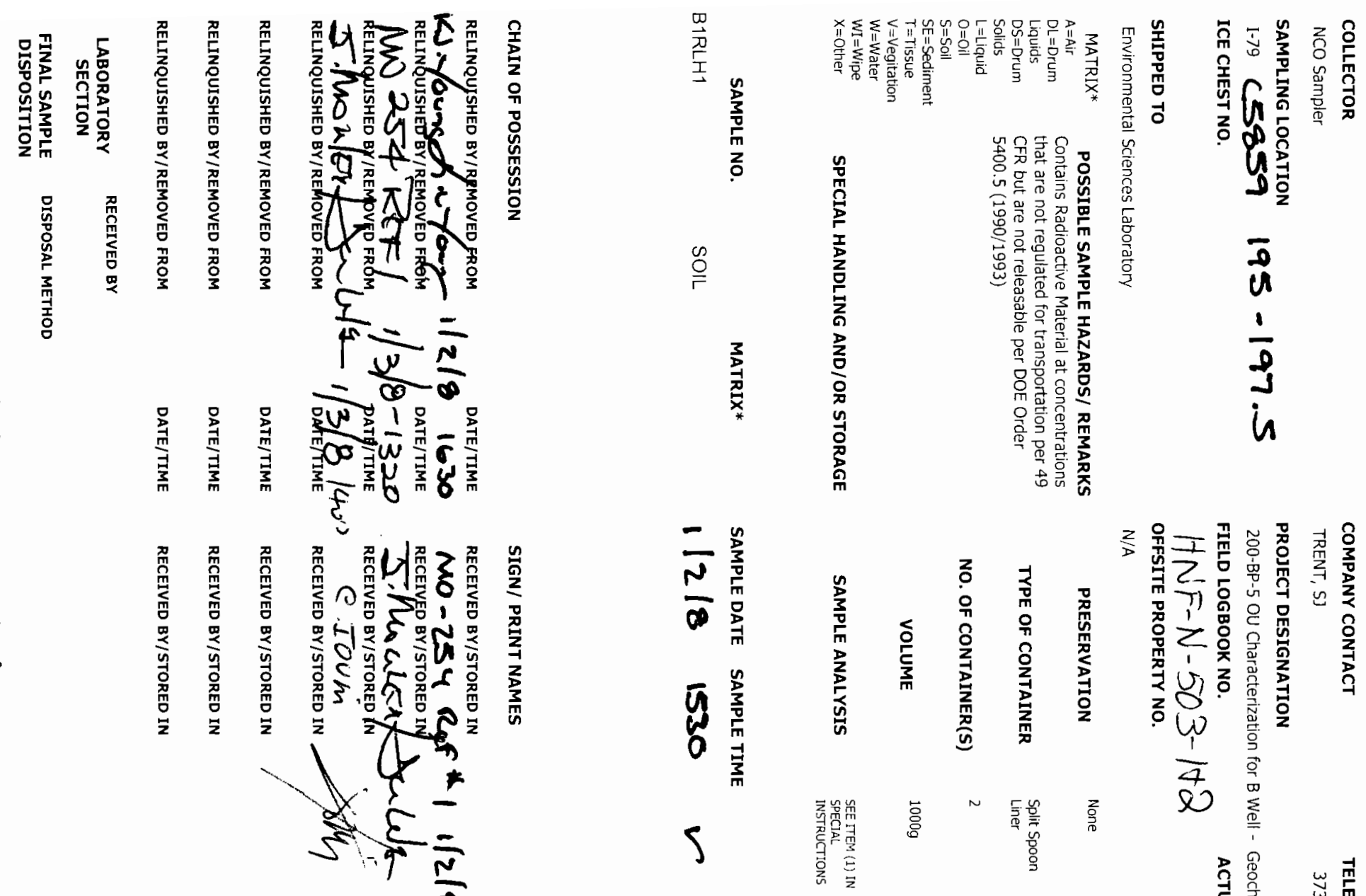

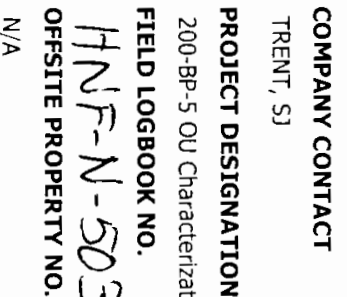
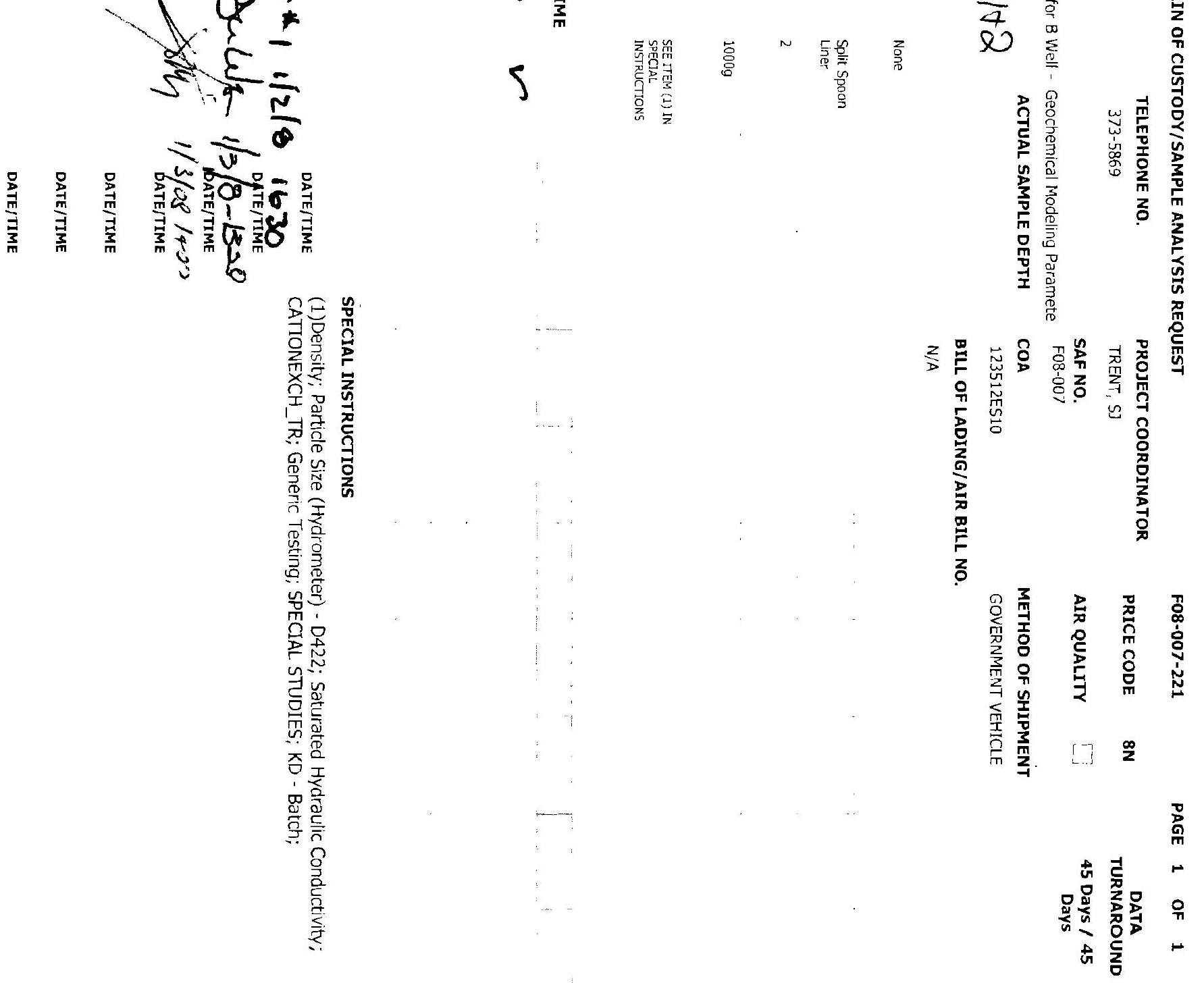


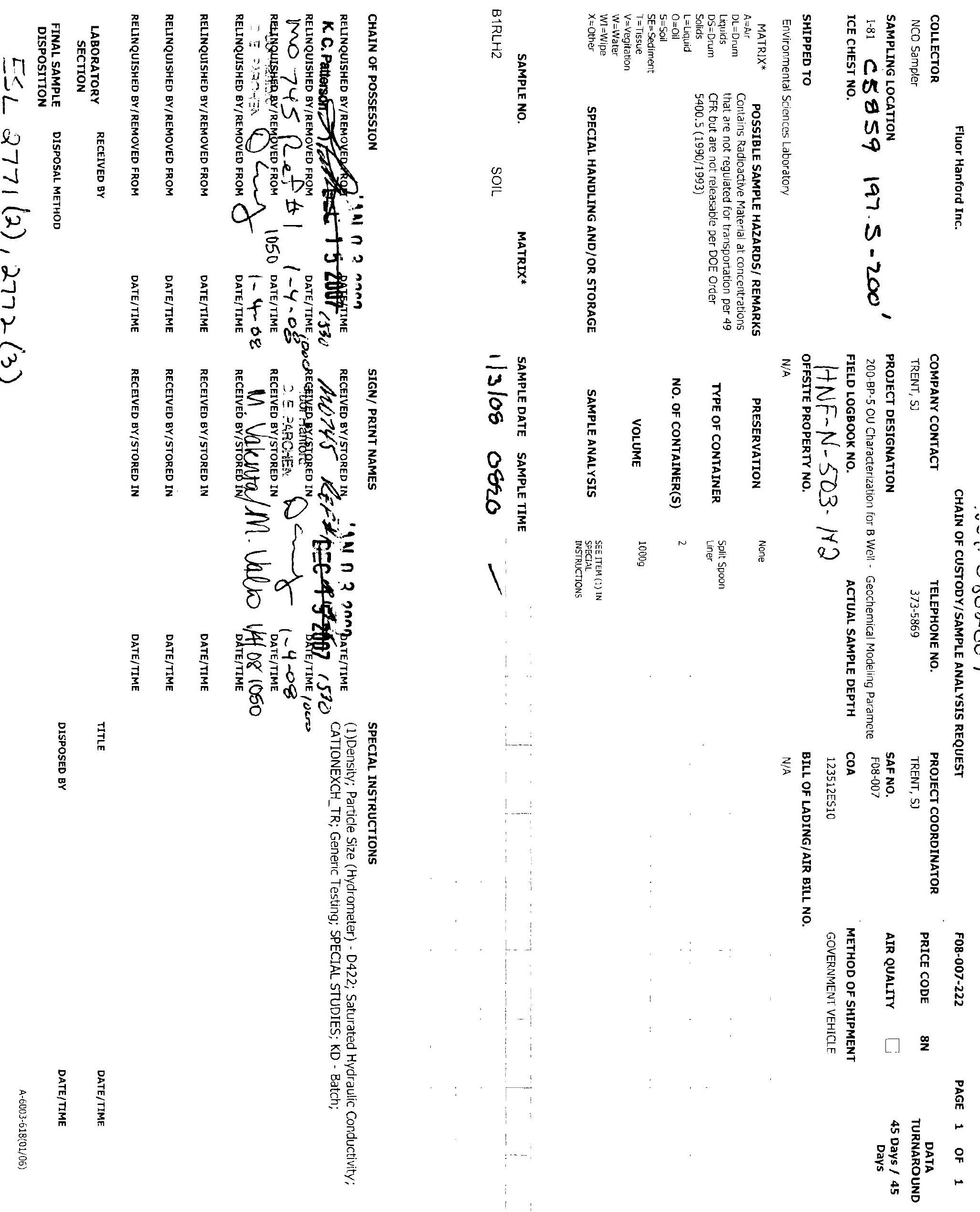




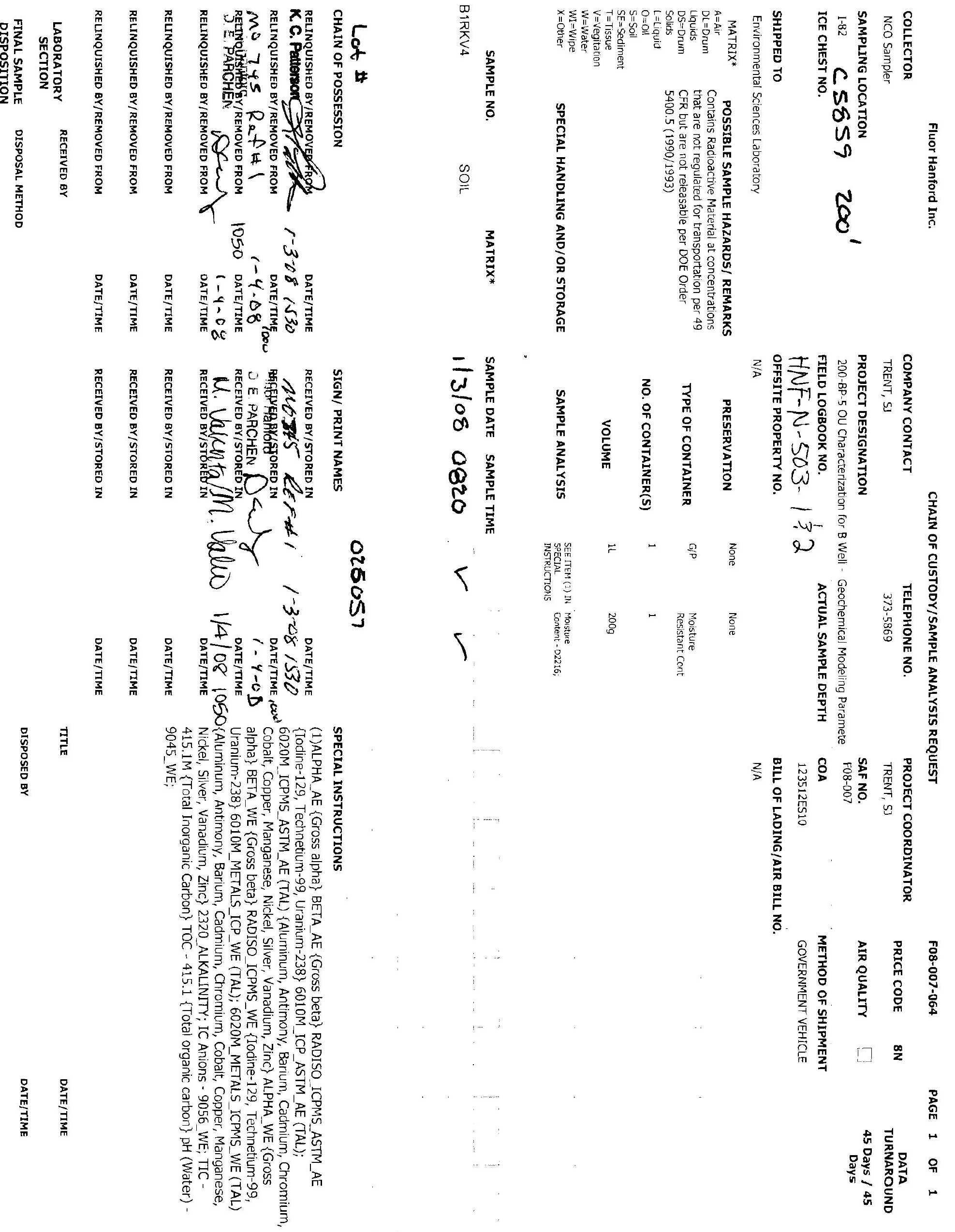



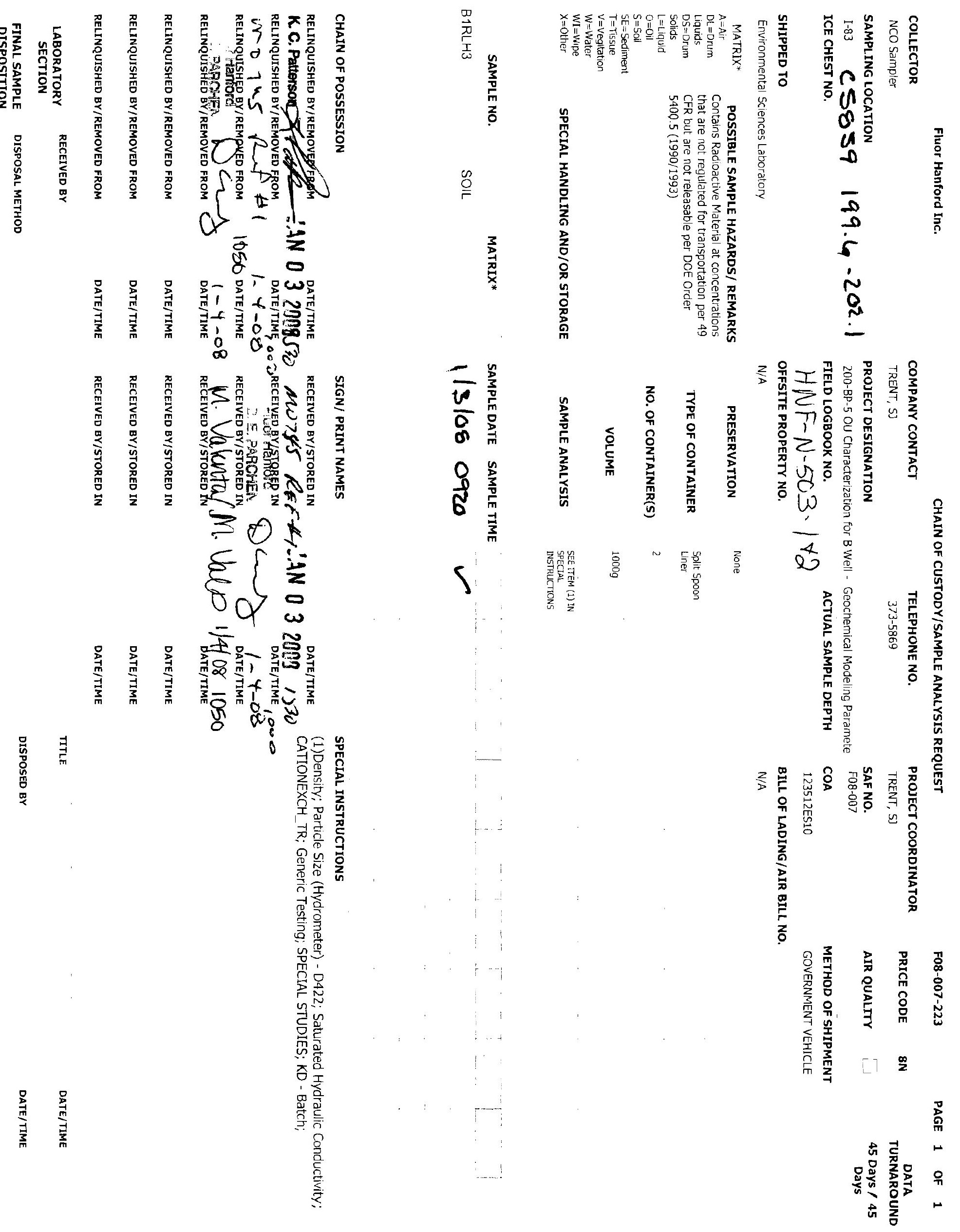


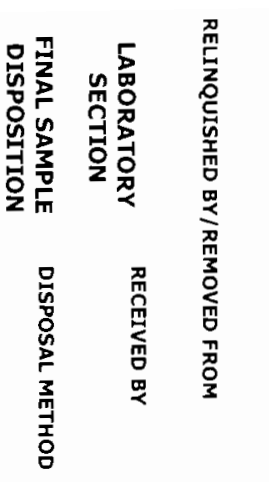

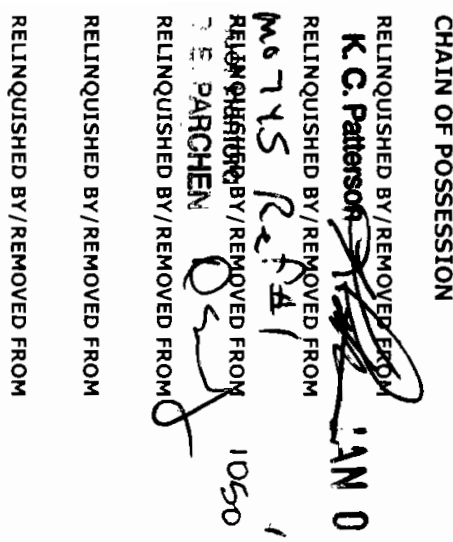

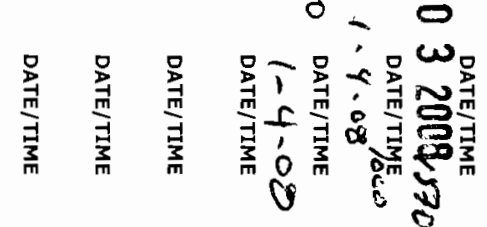
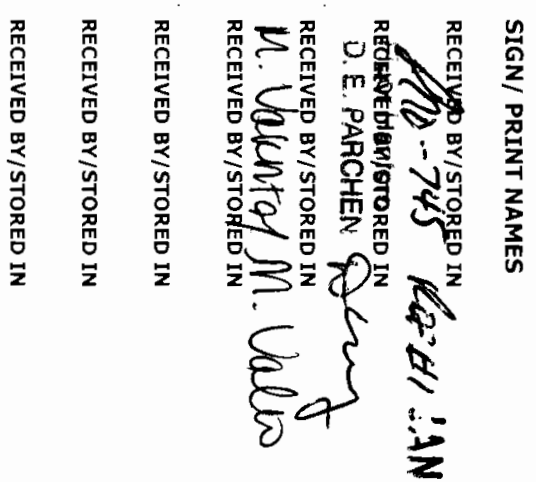

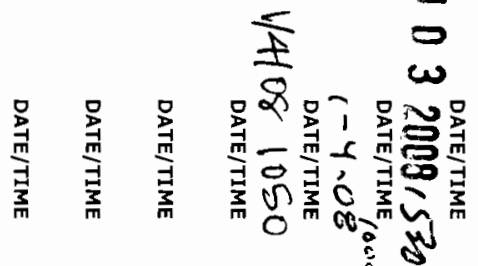

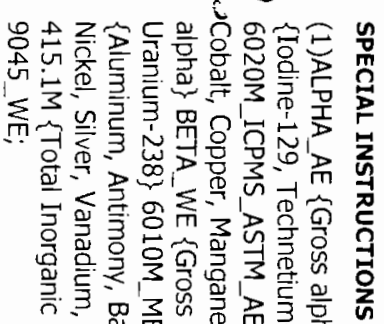

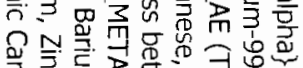

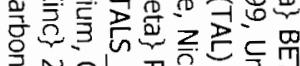

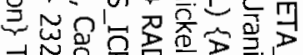

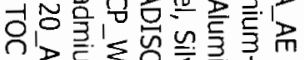

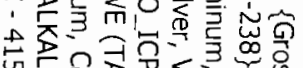

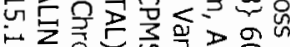

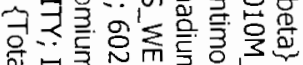

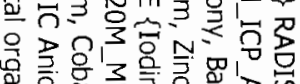

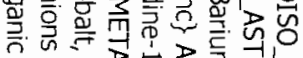

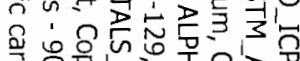

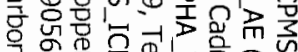

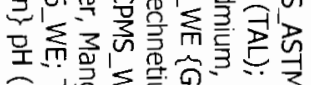

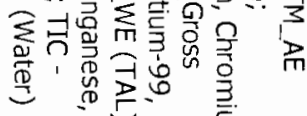

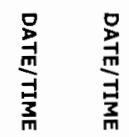
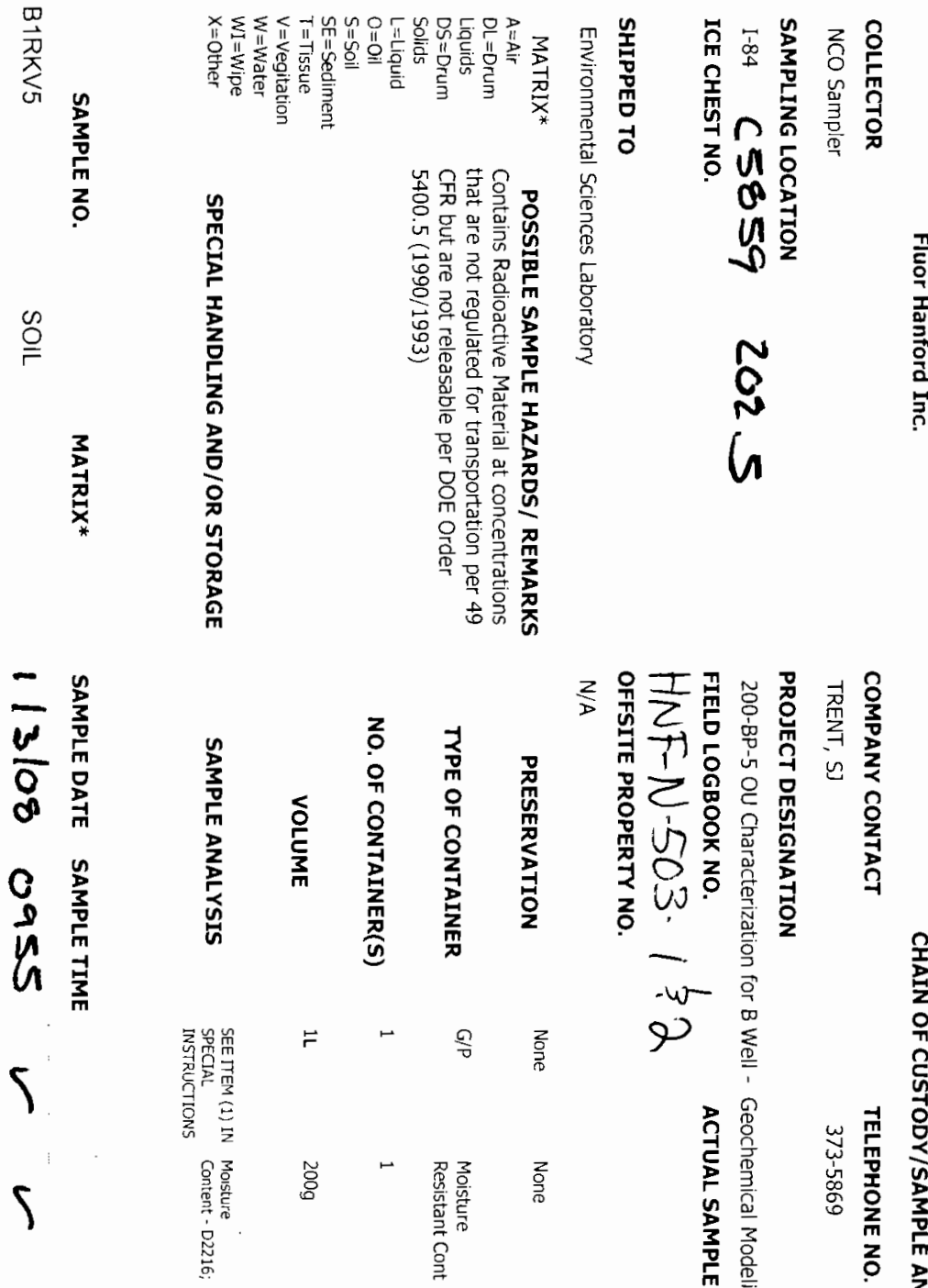

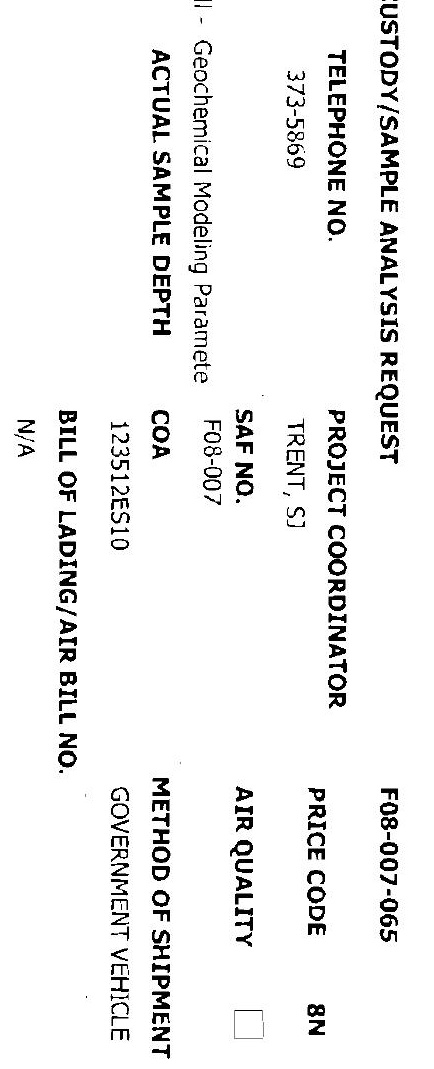




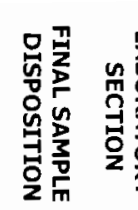

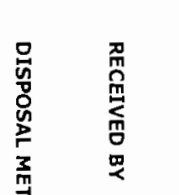

尊
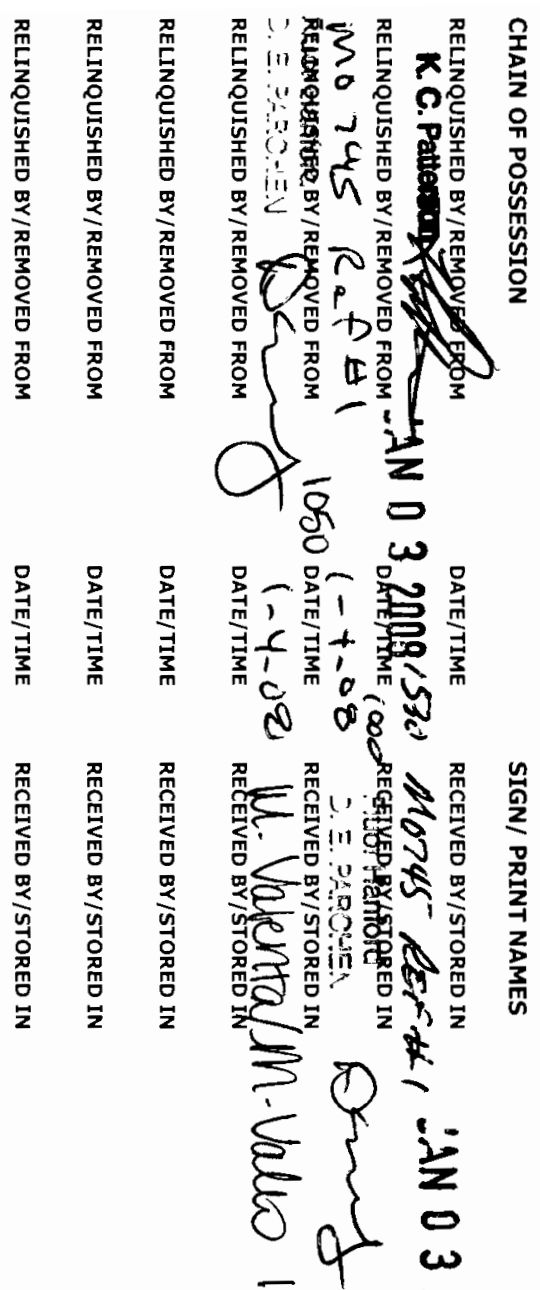

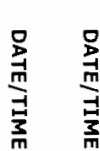

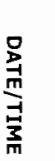

굴을 $\stackrel{\infty}{\circ}$

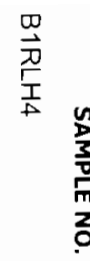

$\stackrel{2}{\stackrel{0}{=}}$

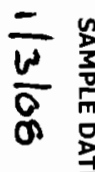

$\frac{\overline{0}}{0} \frac{\frac{n}{3}}{0}$

5

용<smiles>[Ni]=[AsH]</smiles>

点

离量

要

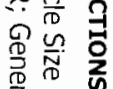

근 곤

욕음

㲅

号号

$\rightarrow N$

总

증 䍃

品

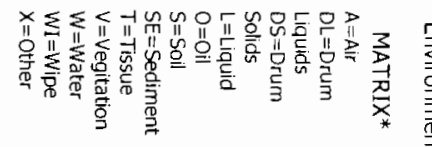

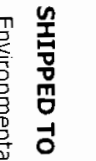

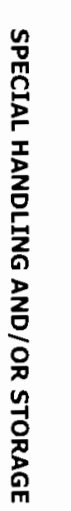

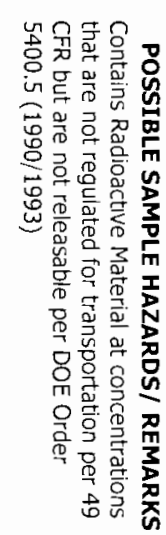

啇

a

莡
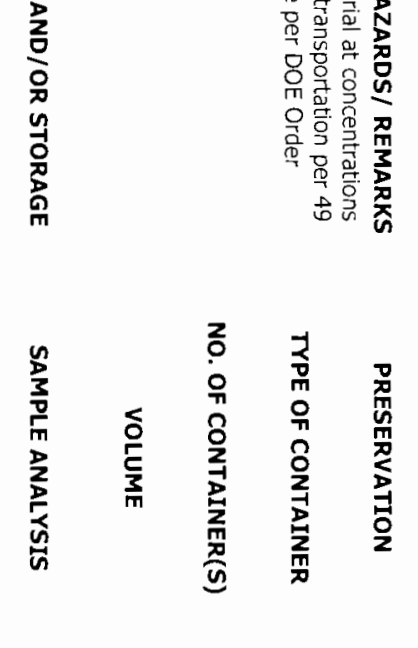

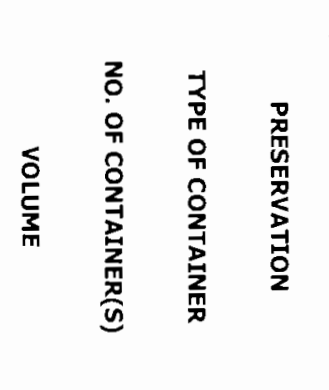

z

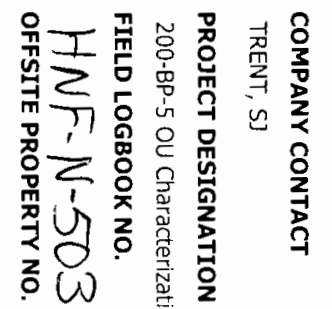

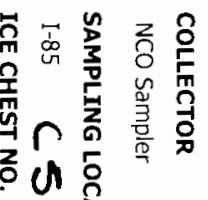

(1)

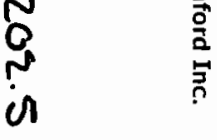

N

en

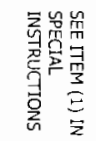

$\frac{1}{\sqrt{0}}$

공용

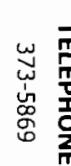

䎡

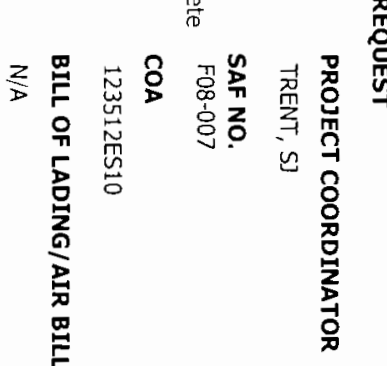

z

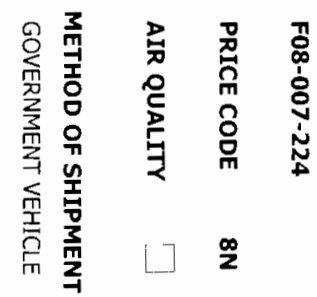

疍

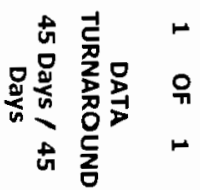



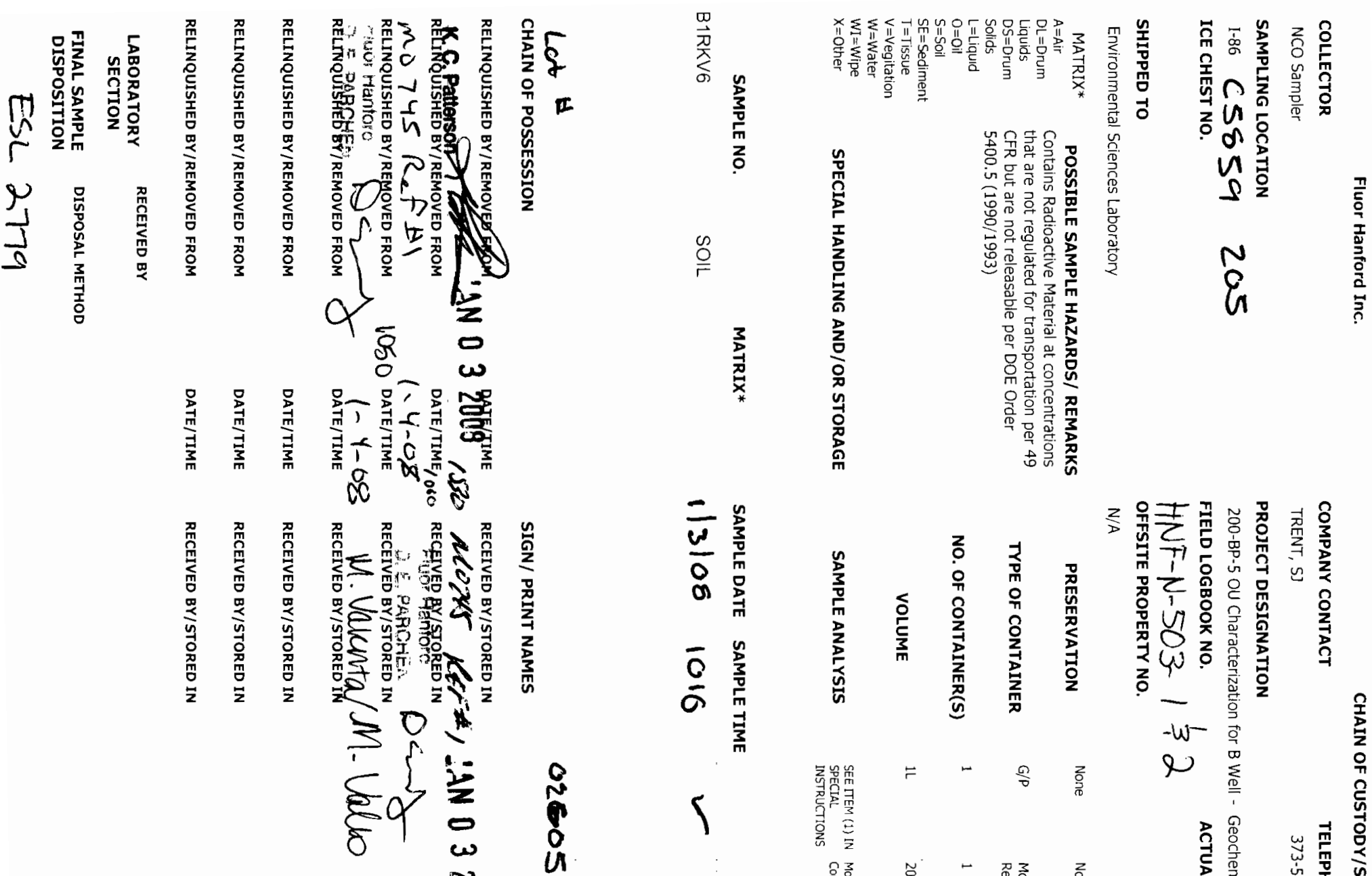

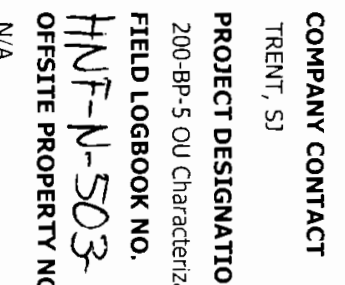
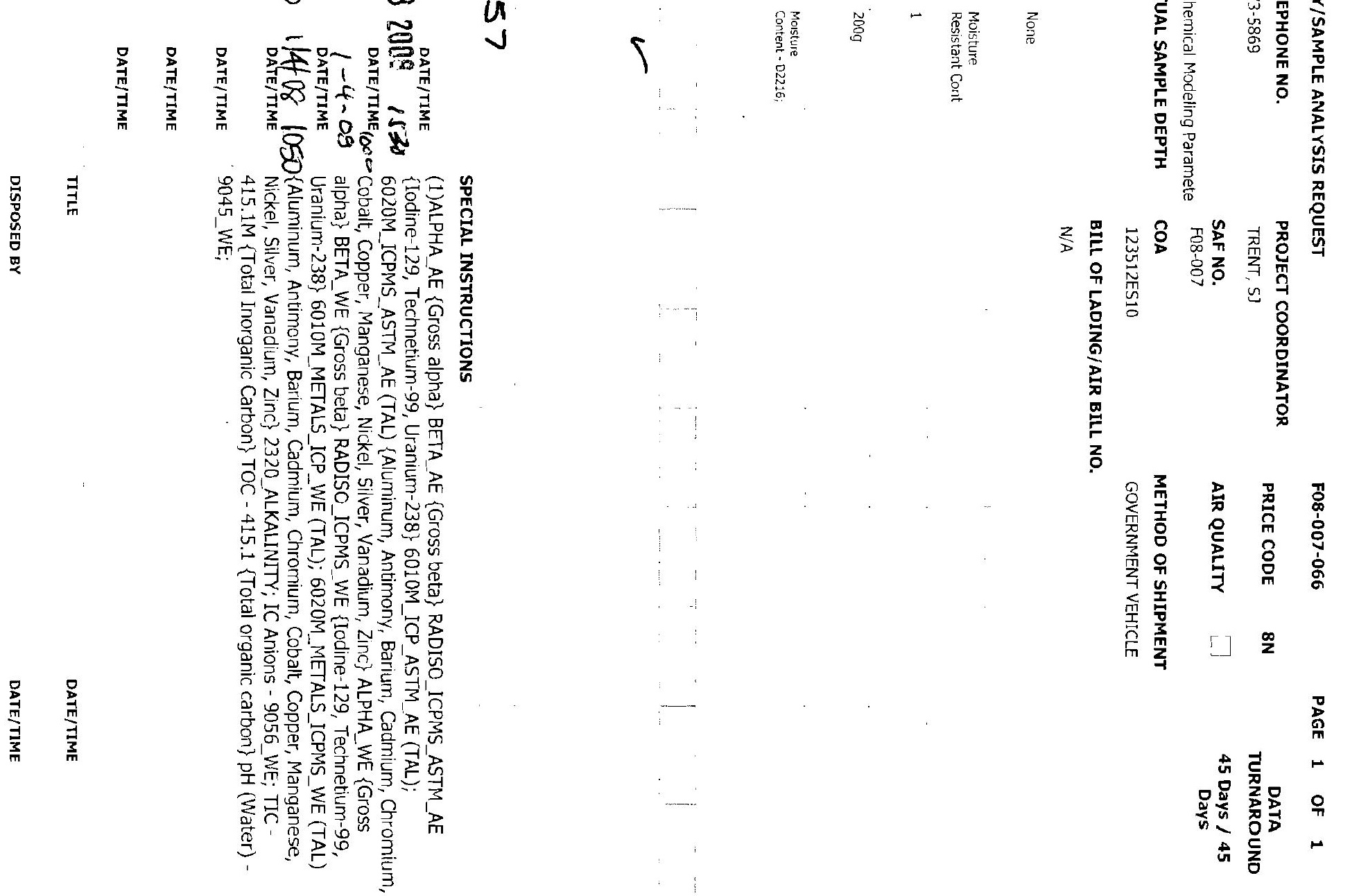


\section{\|\|}
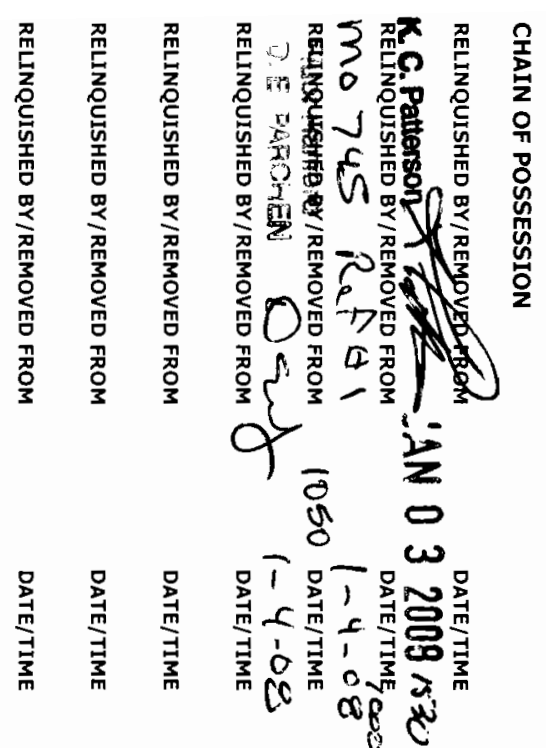

$\stackrel{\infty}{\circ}$
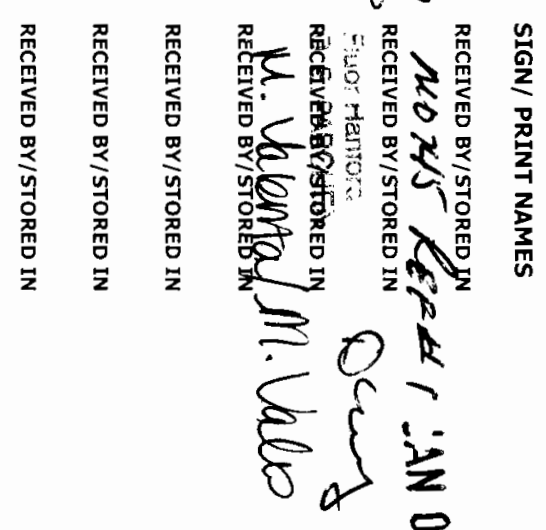

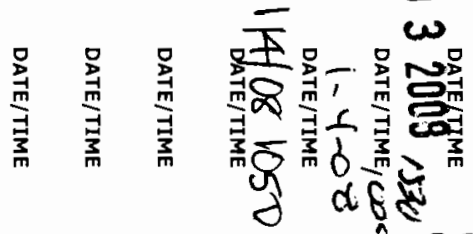

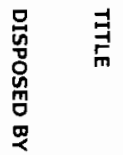

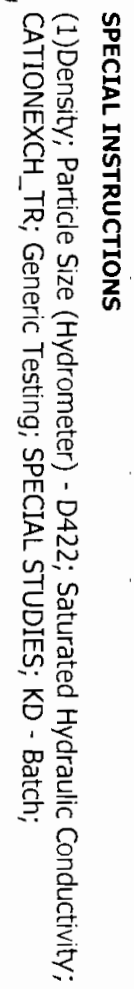

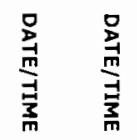

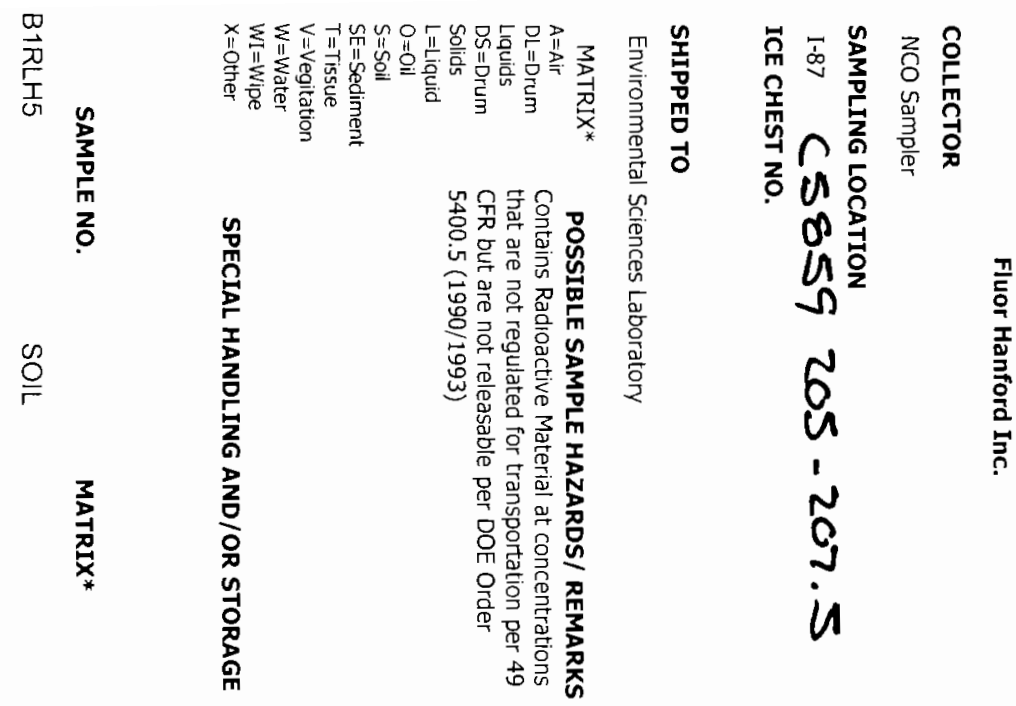

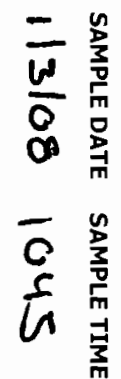
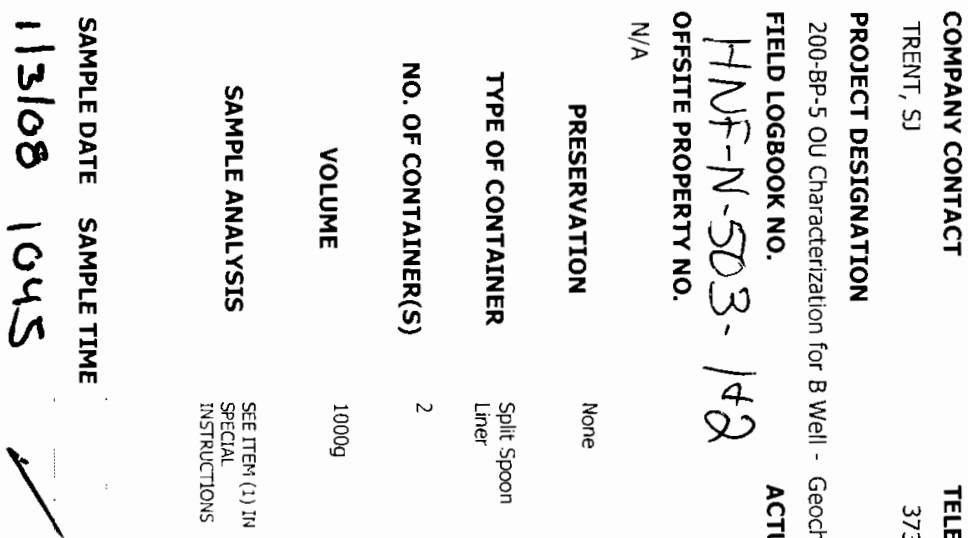


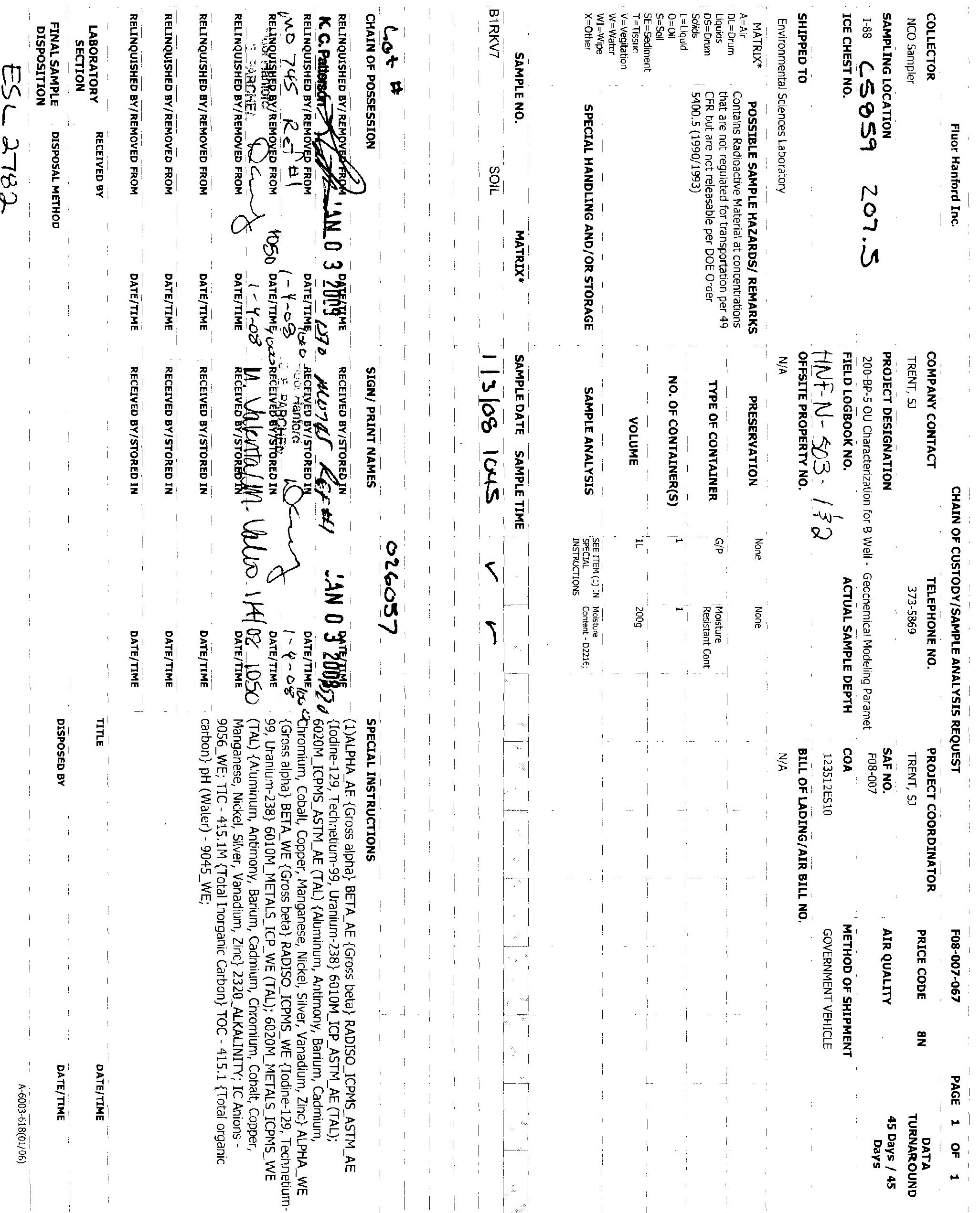



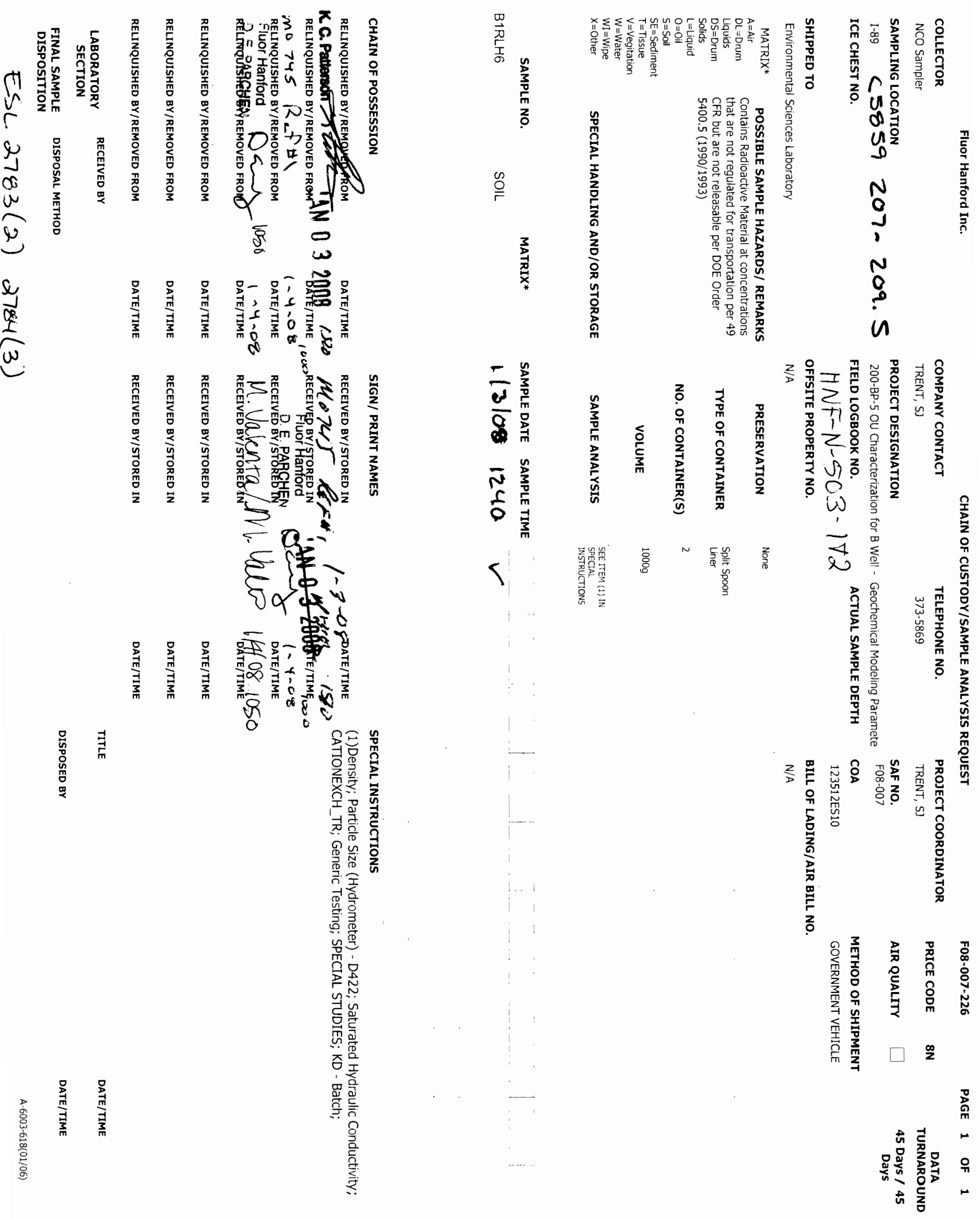

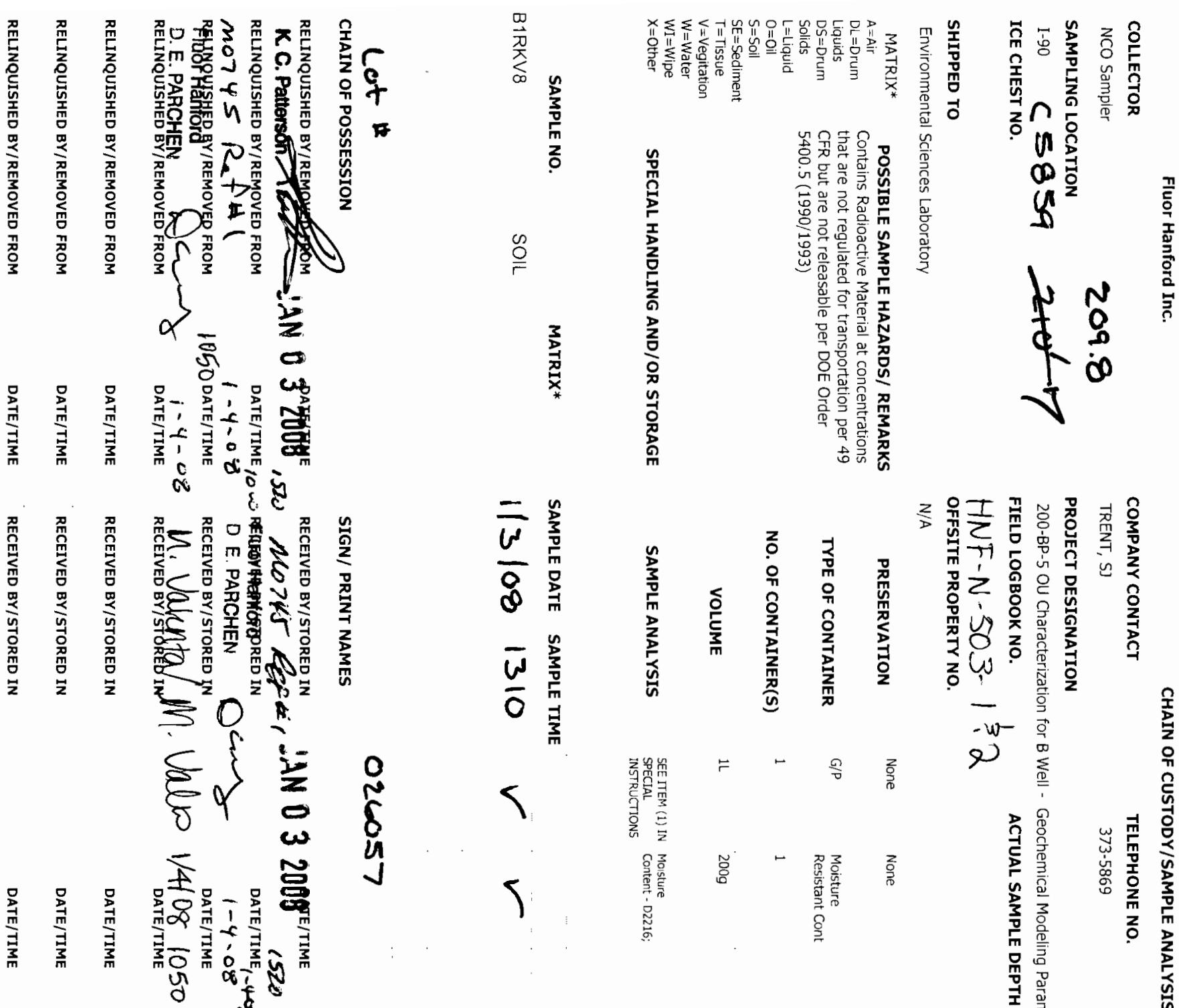

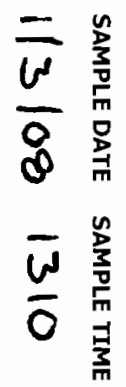
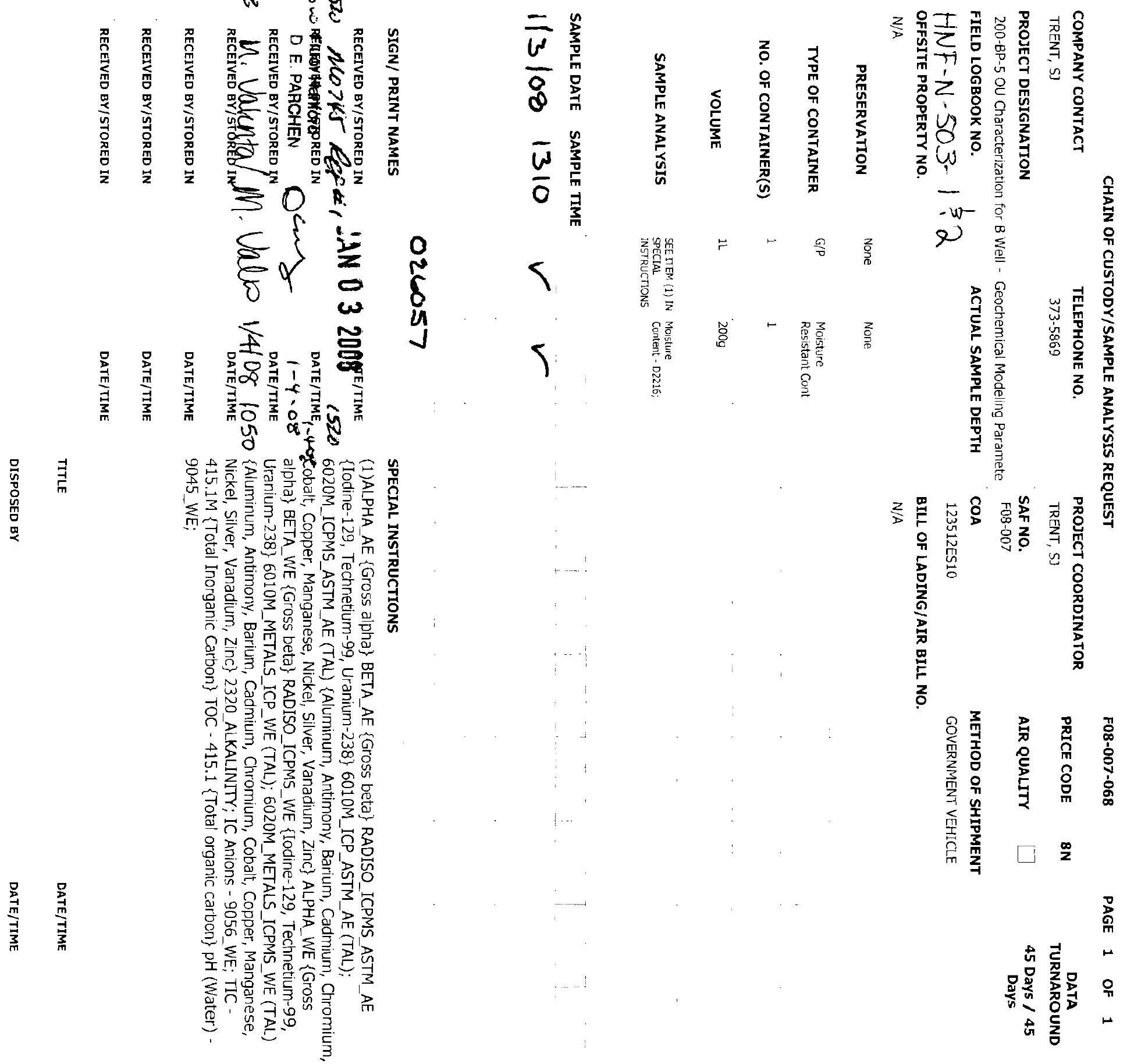


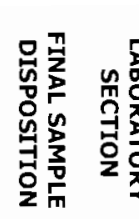
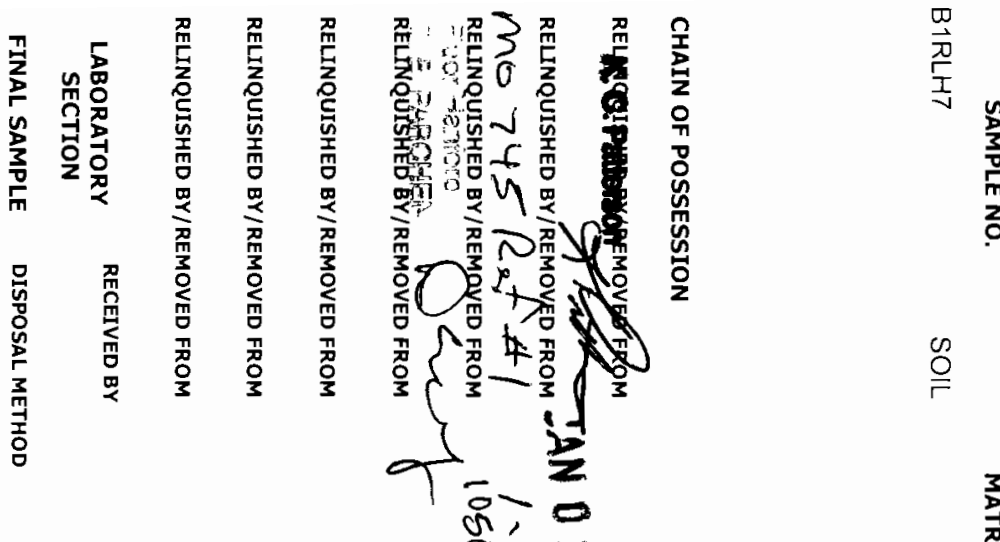

品品
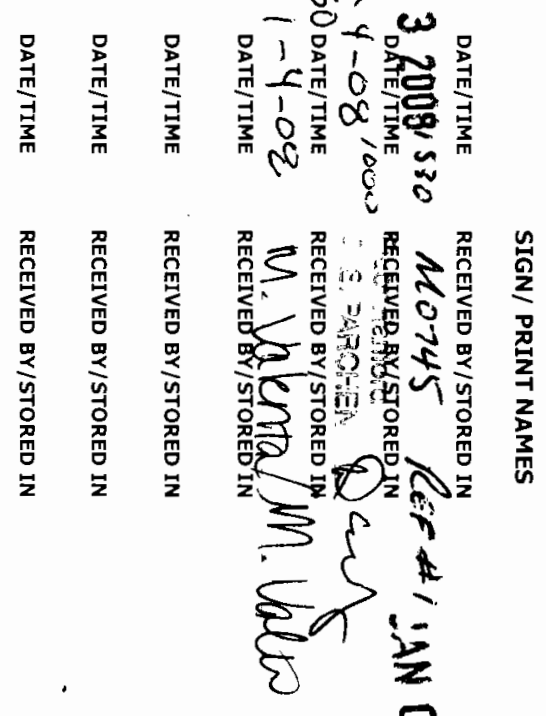

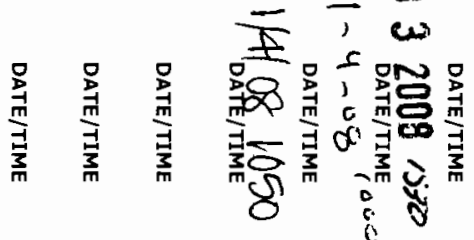

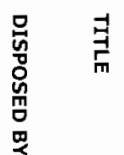

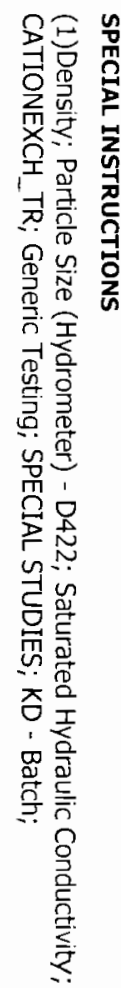

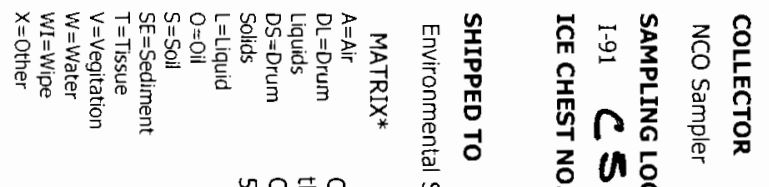

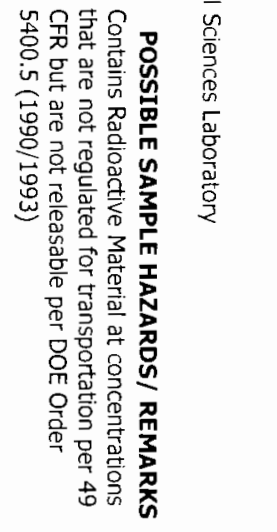

48을

$\stackrel{?}{\stackrel{0}{ }}$

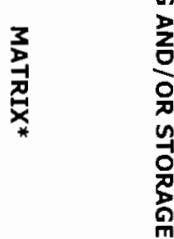

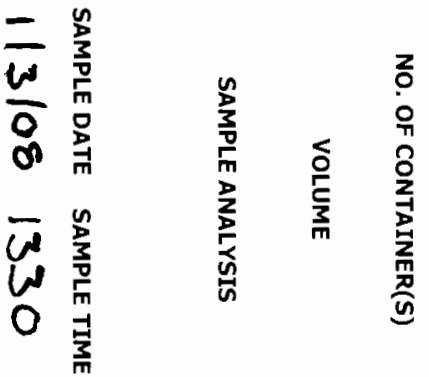

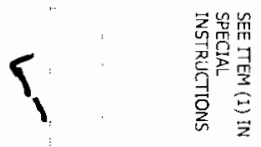

2

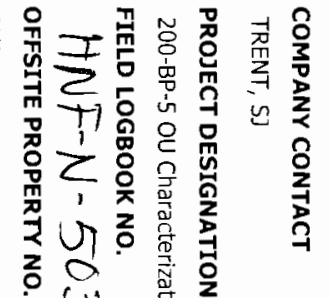

(u) $\frac{2}{5} 2$

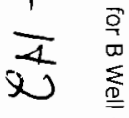

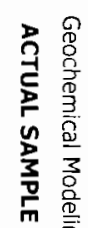

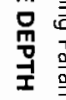

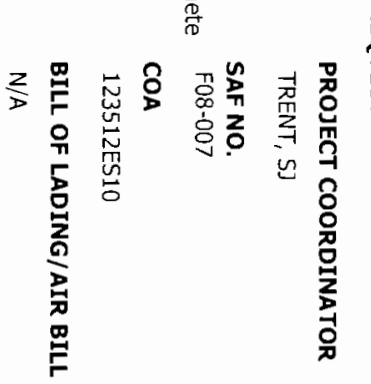

중
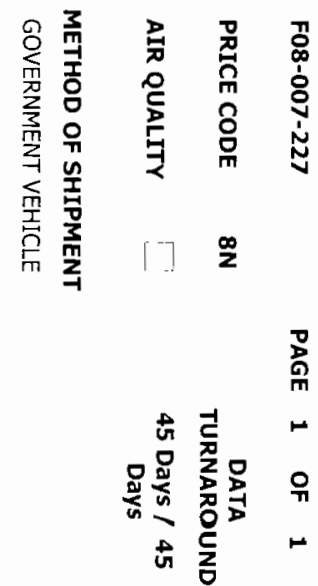


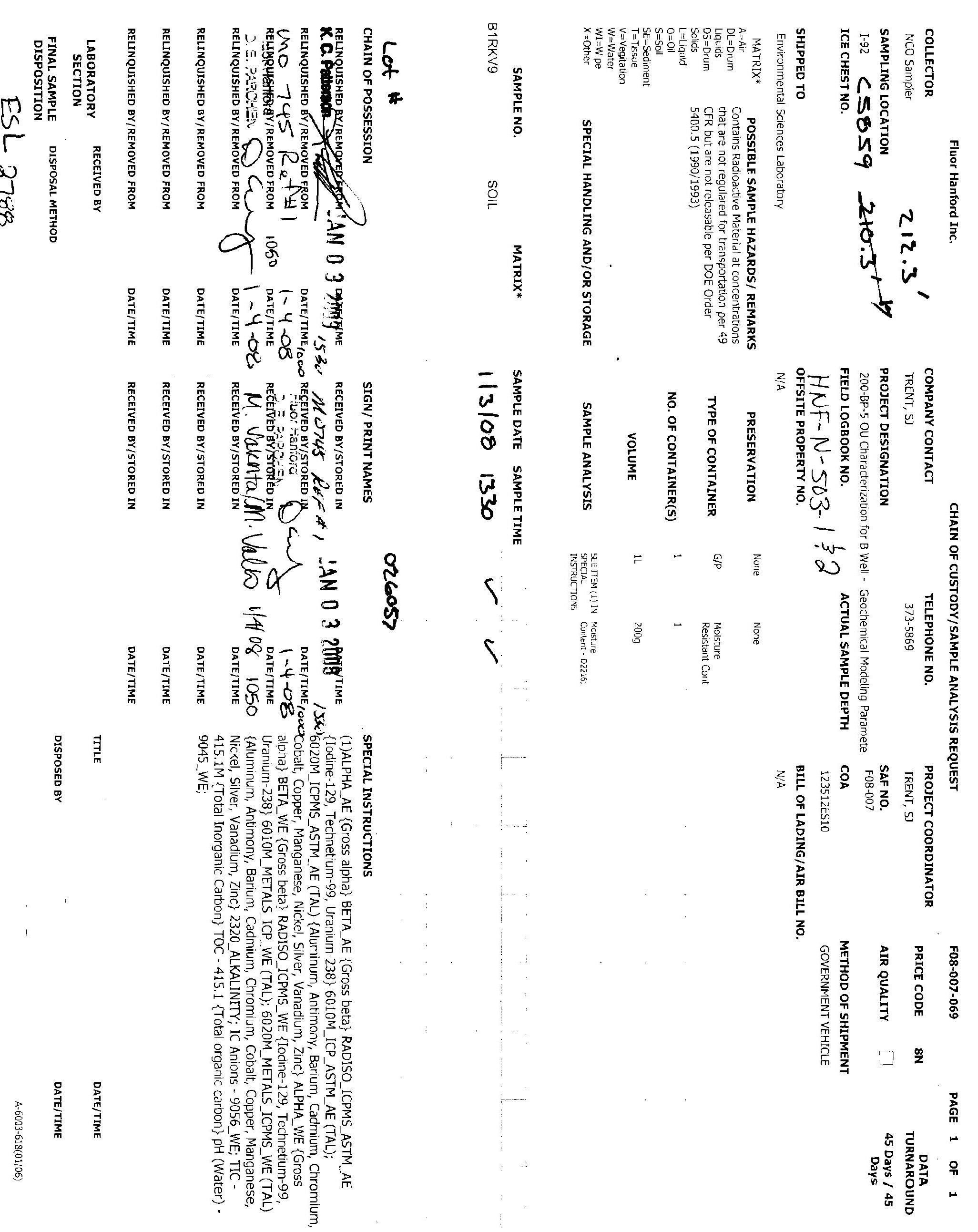




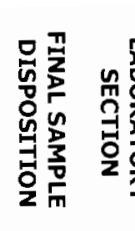

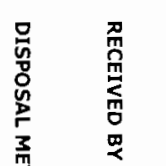

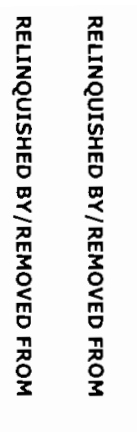

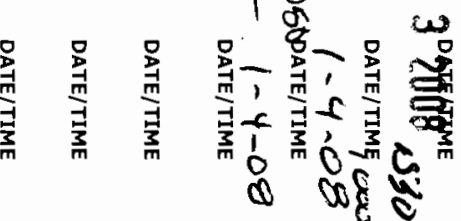

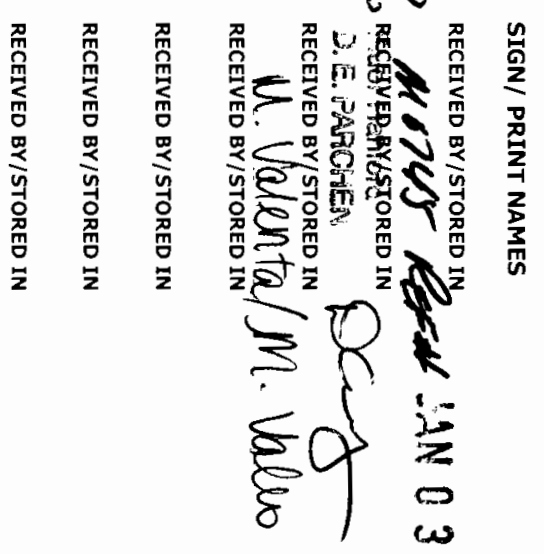

苗

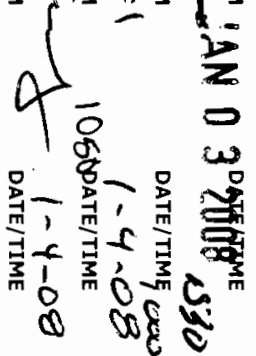

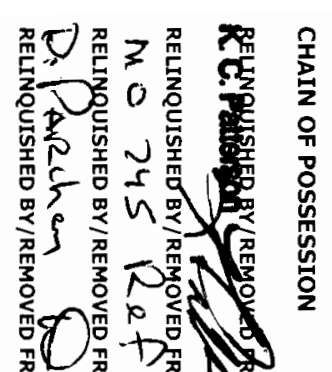

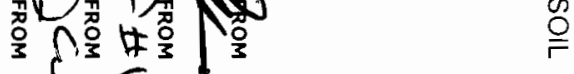

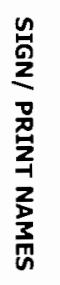

용

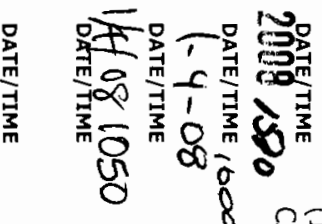

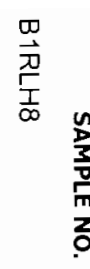

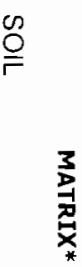

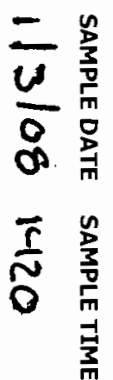

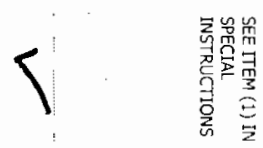



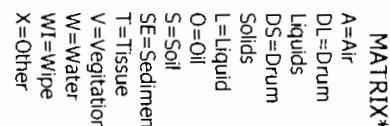

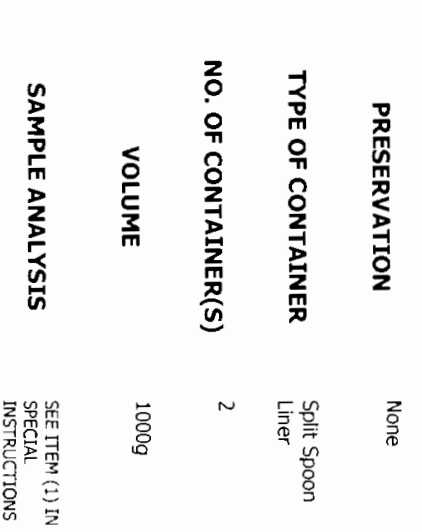

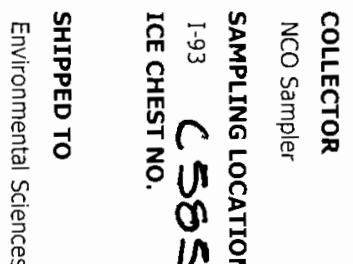

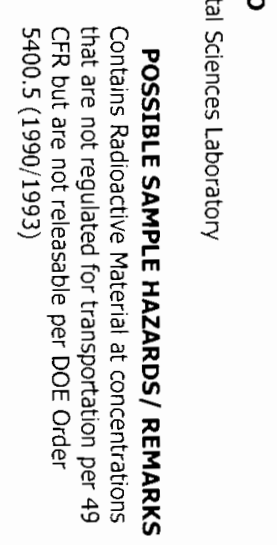

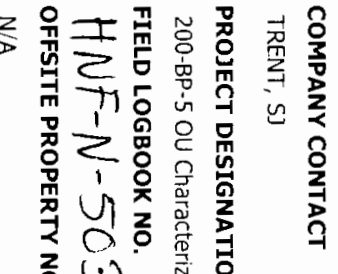

n

u

$\frac{1}{n}$

$I$

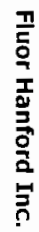

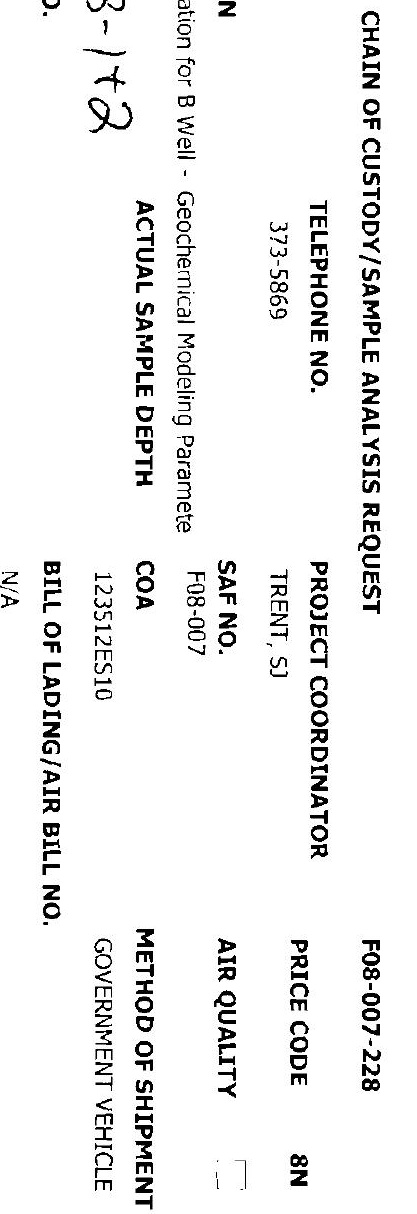

总量
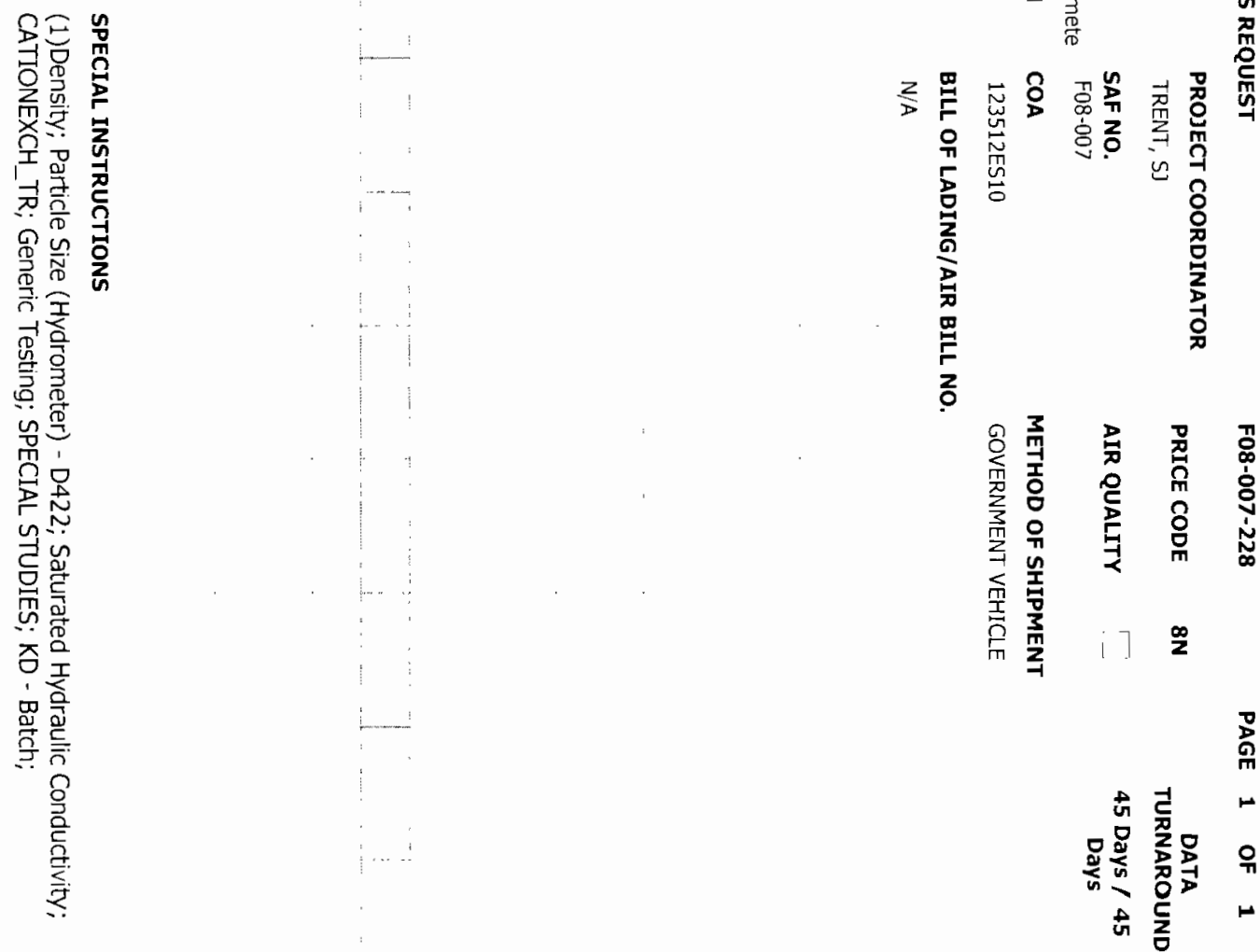

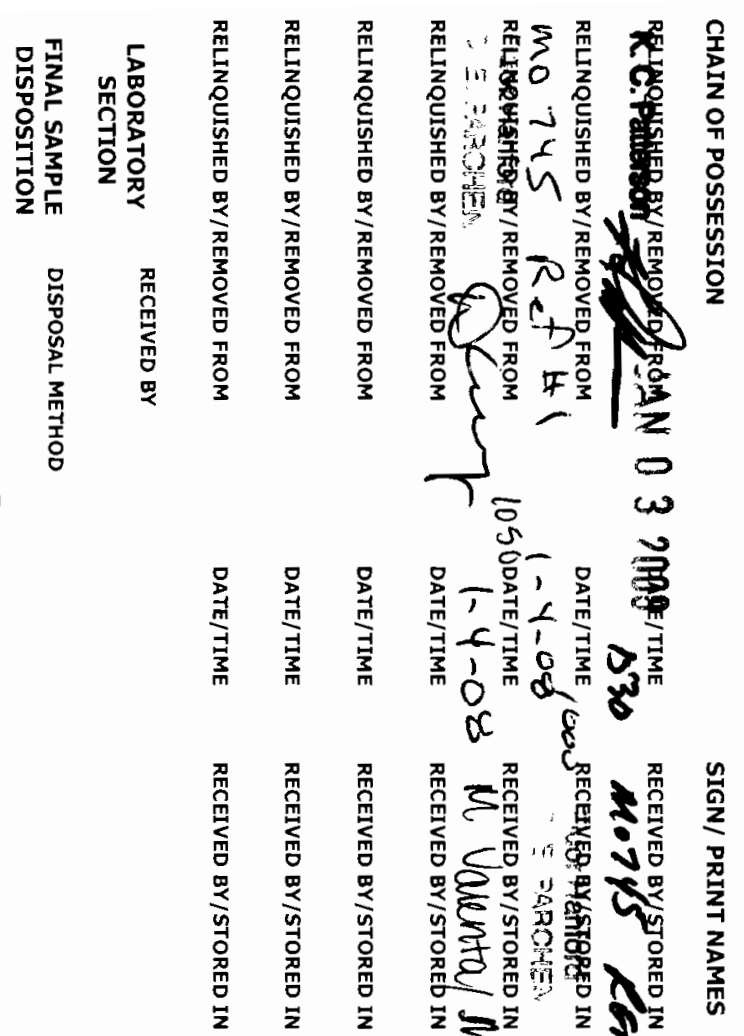

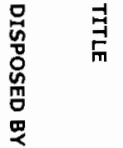

品是

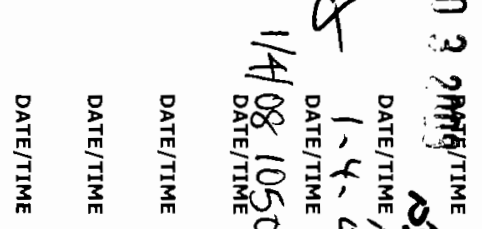

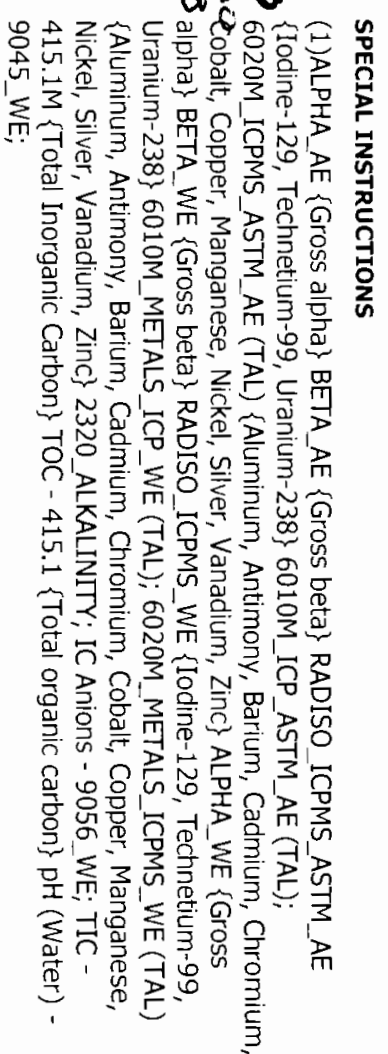

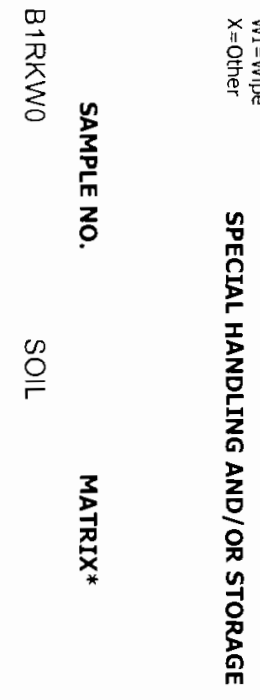

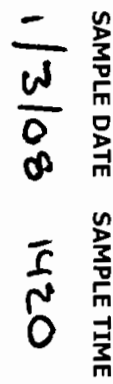

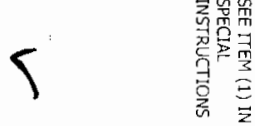

i

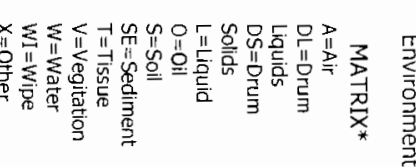

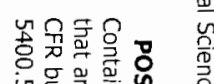

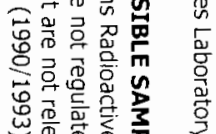

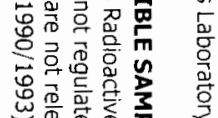

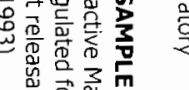

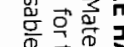

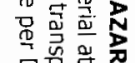

咞整号

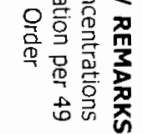
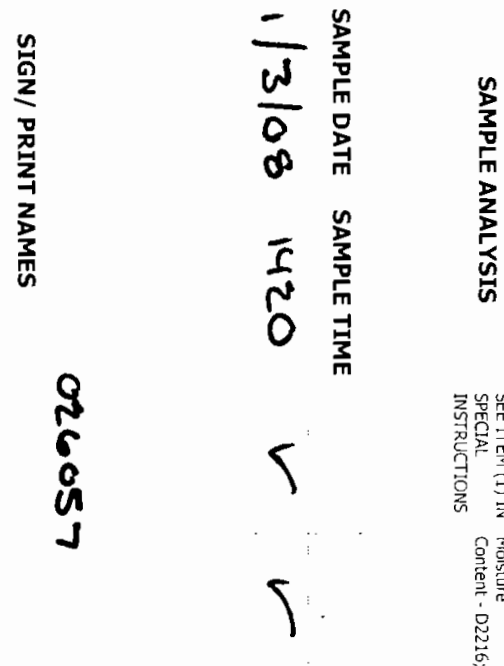

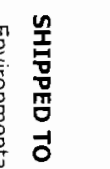

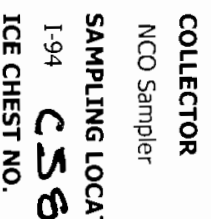

U

$\frac{N}{n}$

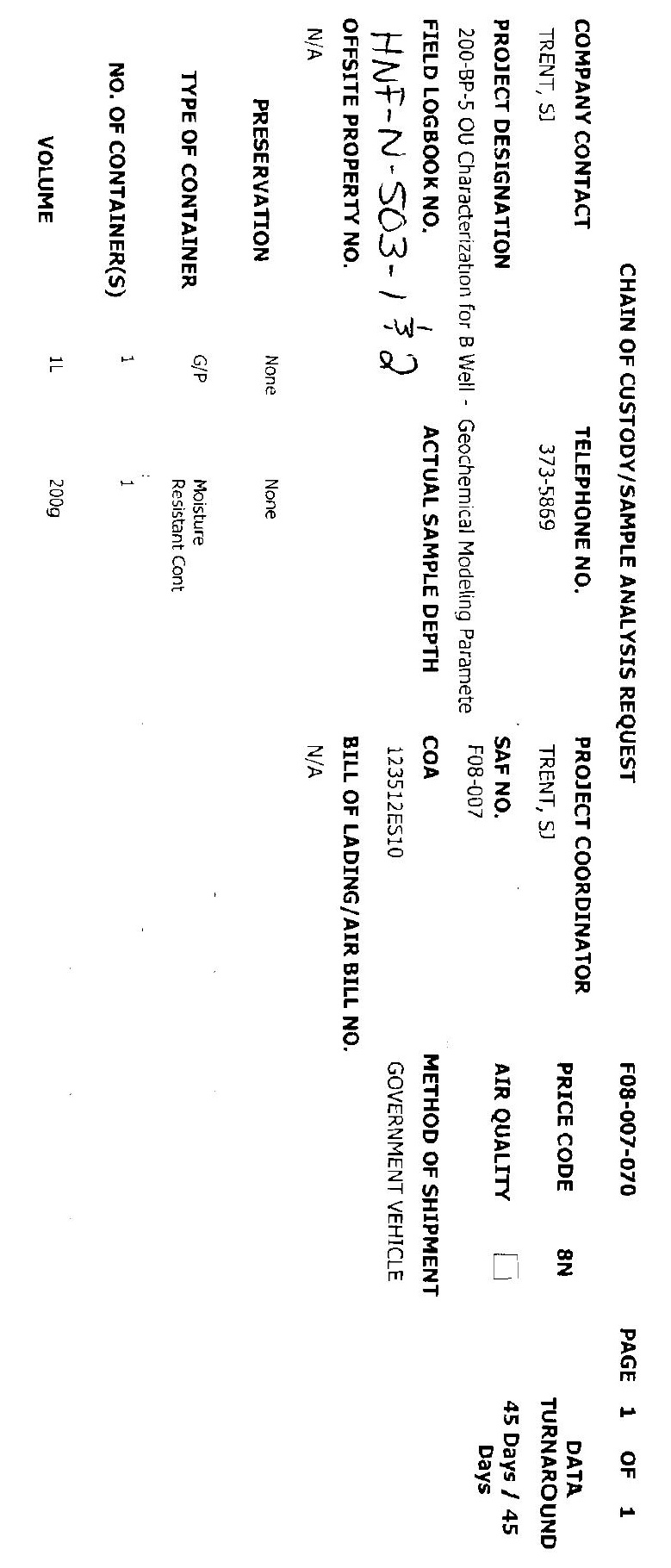




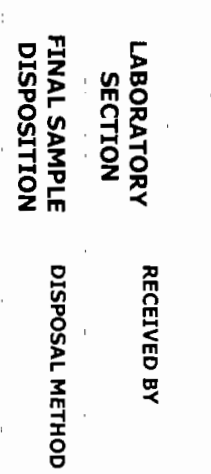

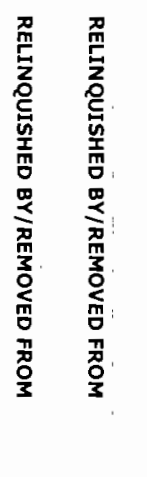

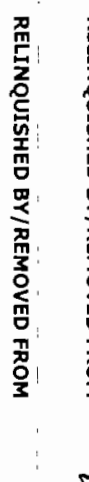

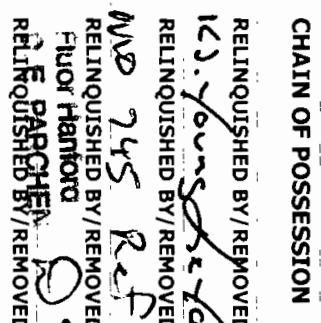

号

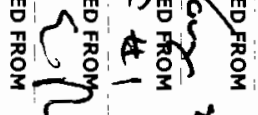

$\underbrace{}_{\frac{\pi}{\pi}} \frac{n}{\bar{n}}$

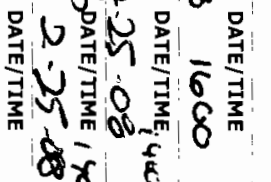

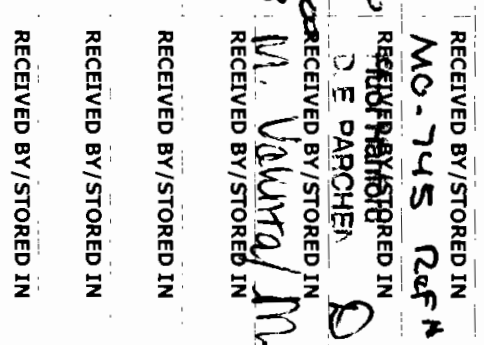

$\sum_{0}^{\infty} \frac{r}{2}$

善:

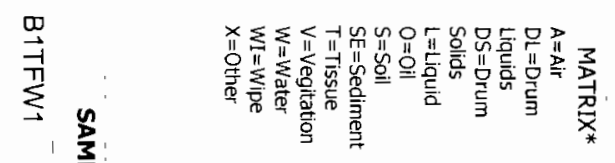

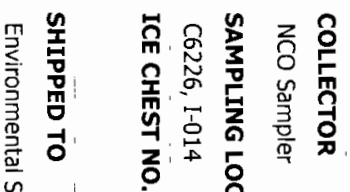

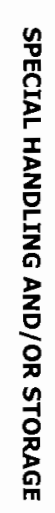

$\frac{n}{2} \frac{n}{\infty}$

$\bar{w}: \frac{n}{3}$

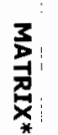

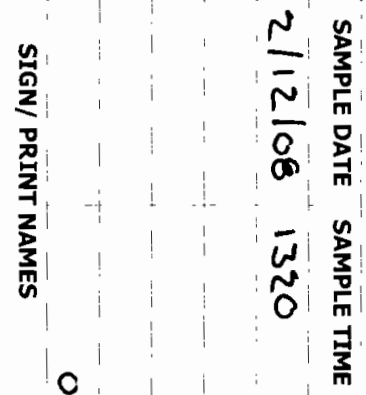

15

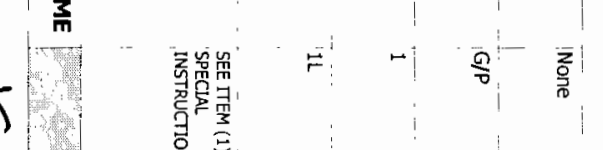

5

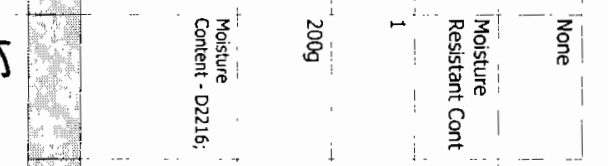

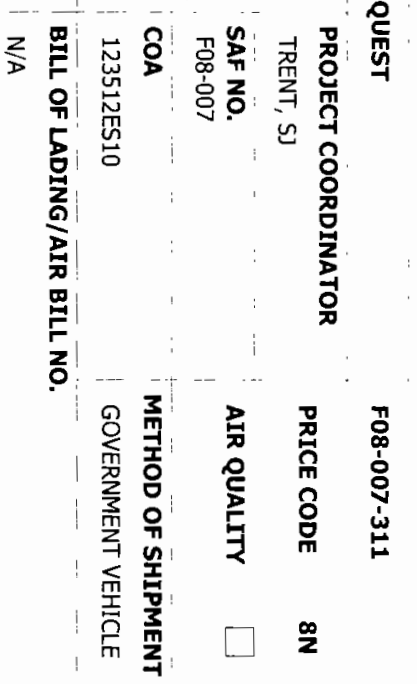




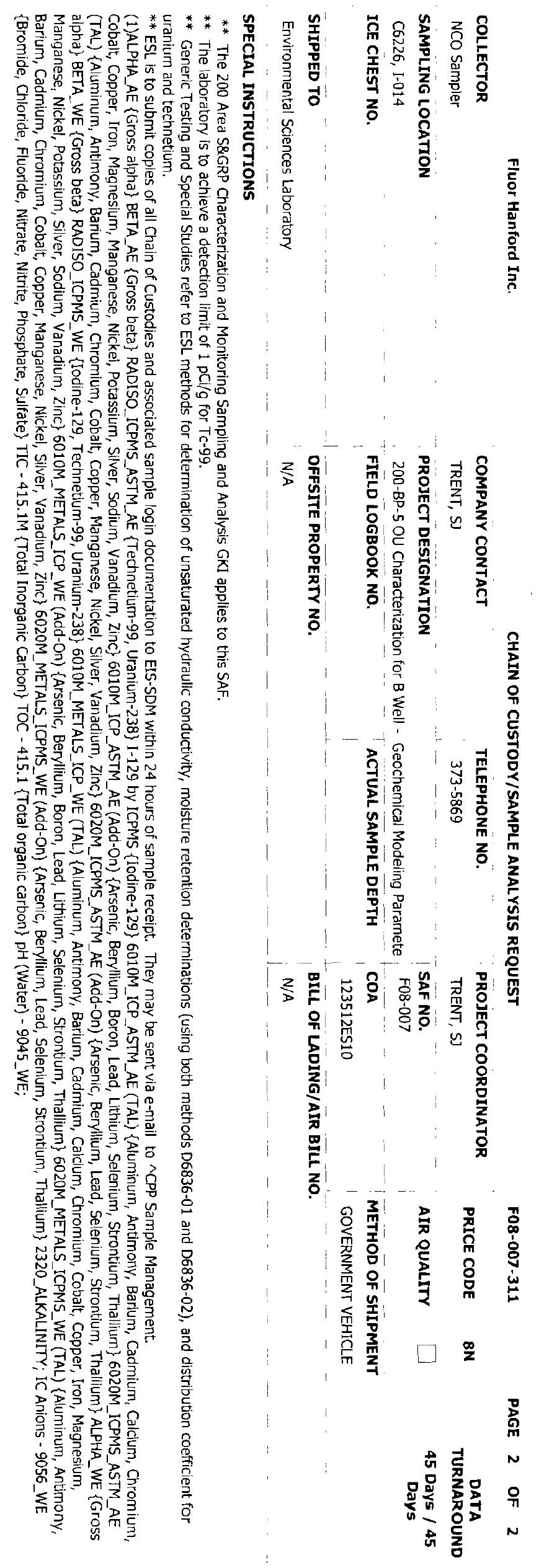




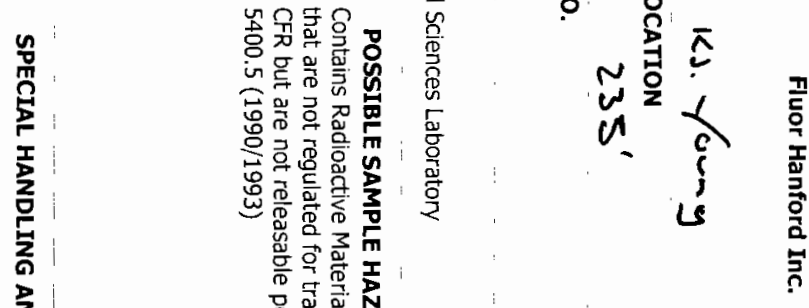
$\sum_{0} \sum_{\substack{n \\ n}}^{\infty} \sum_{0}^{n}$

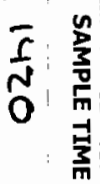

2:

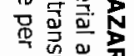

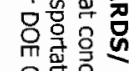

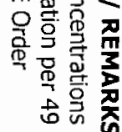

\section{紊}

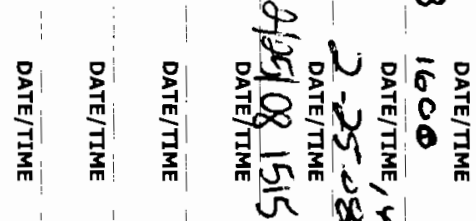
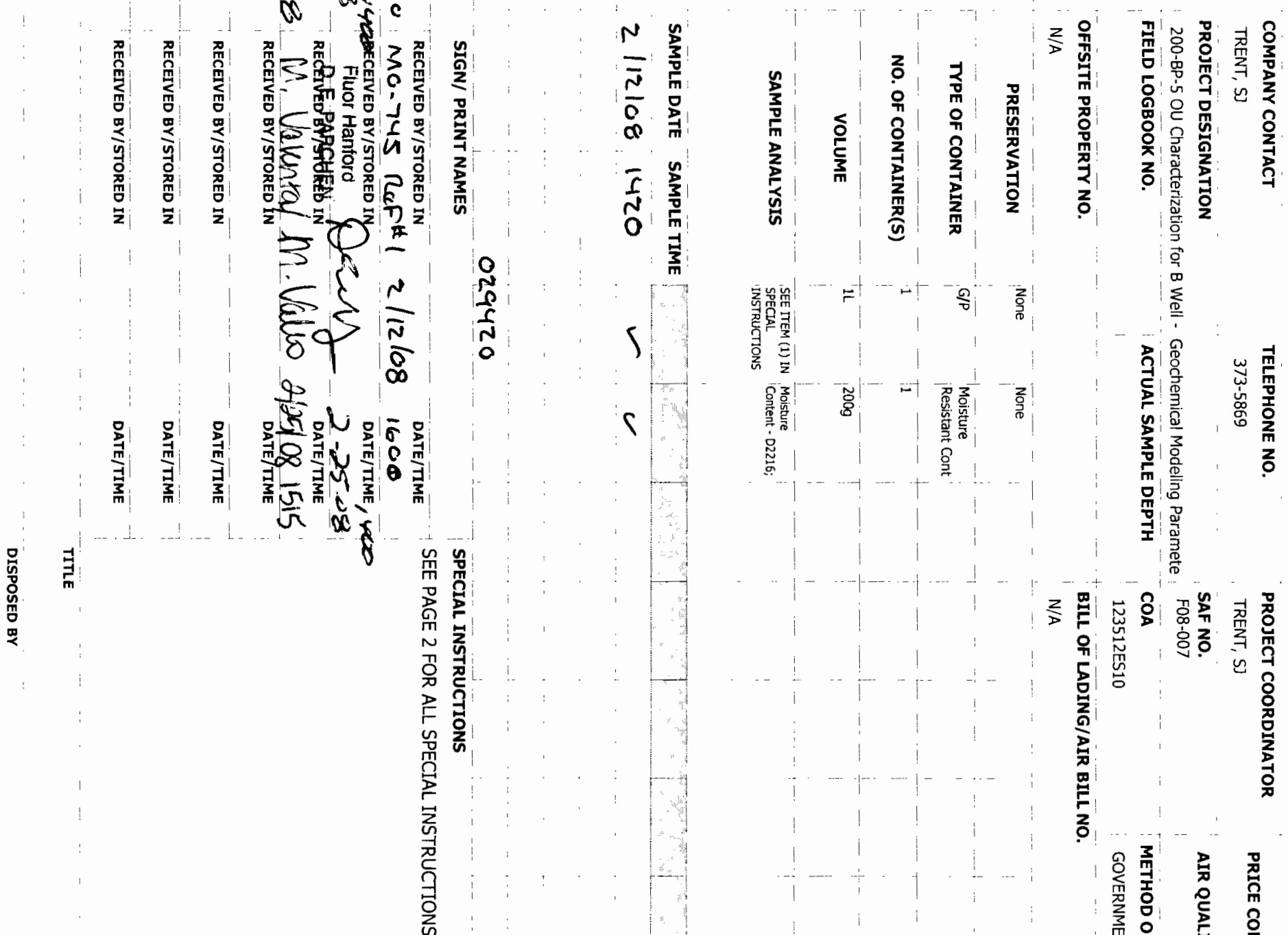

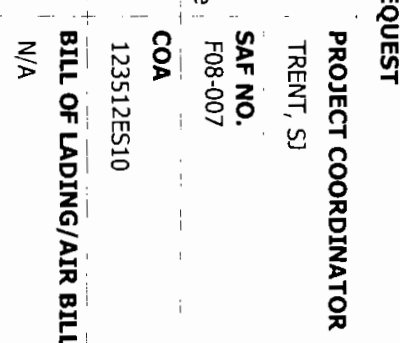

$$
\begin{aligned}
& \text { ํㅣㄴ }
\end{aligned}
$$

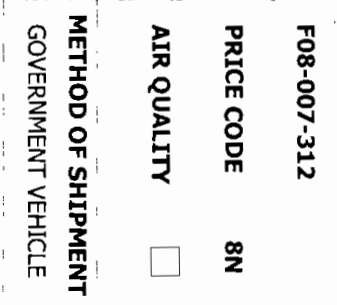

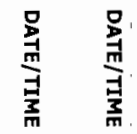




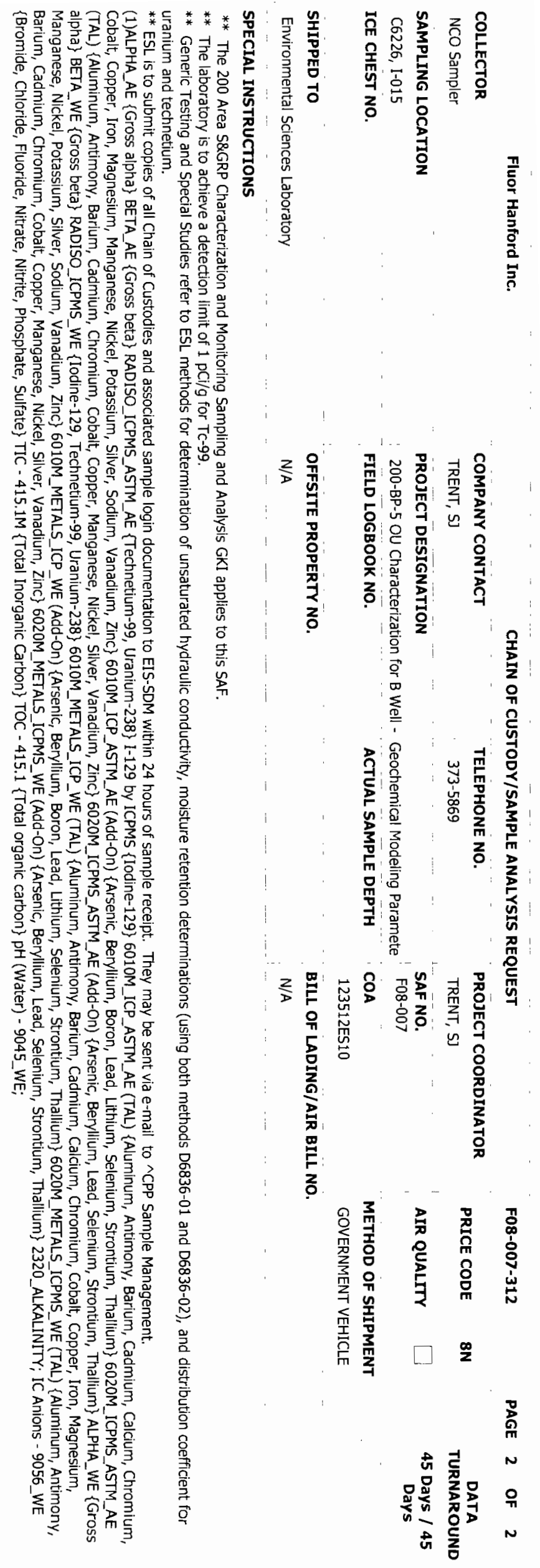




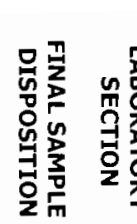

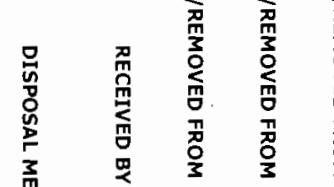
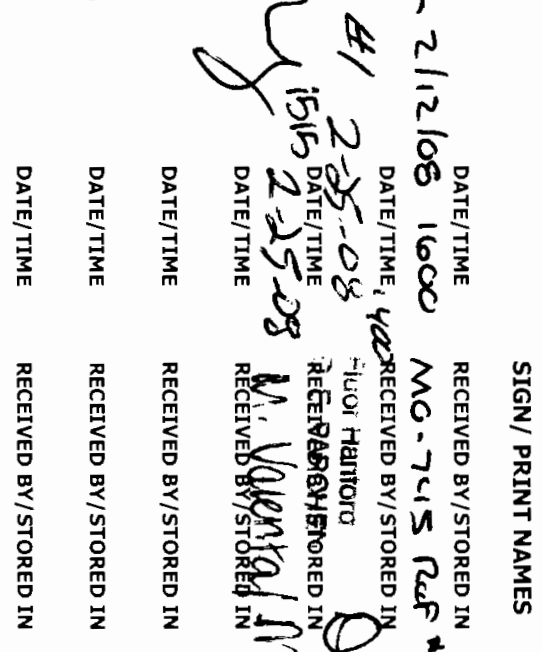

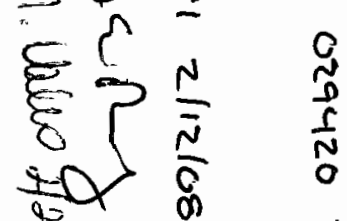

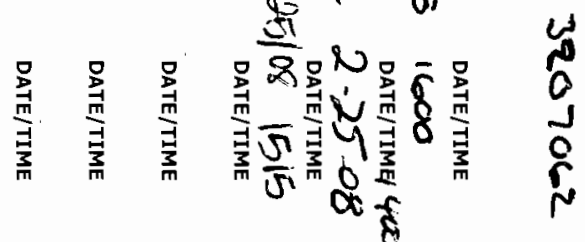

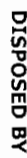

录

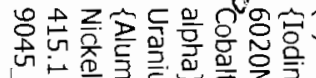

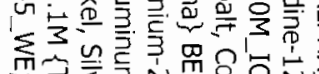

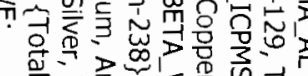

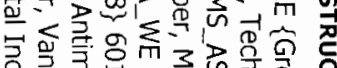

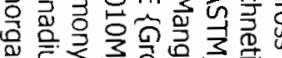

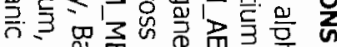

N

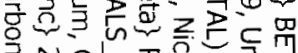

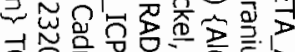

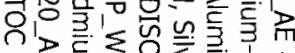

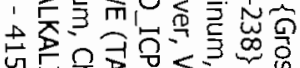

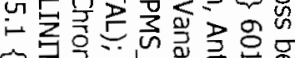

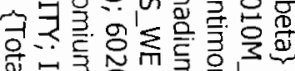

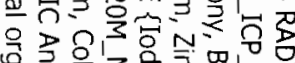

竞是

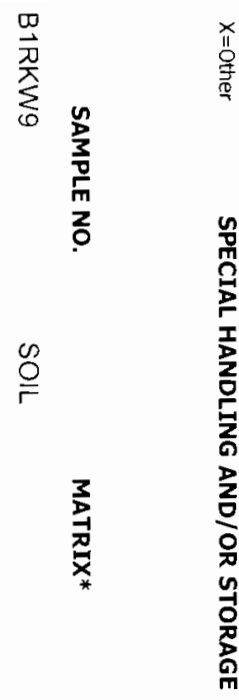

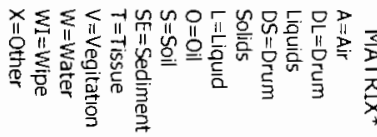

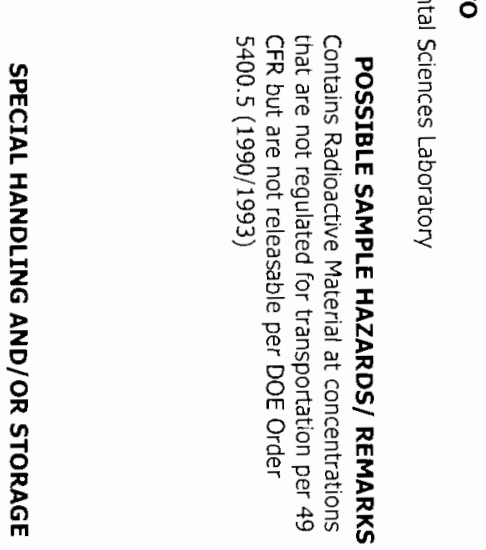

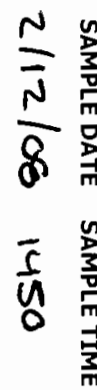

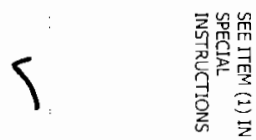

I.

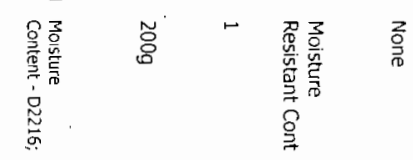

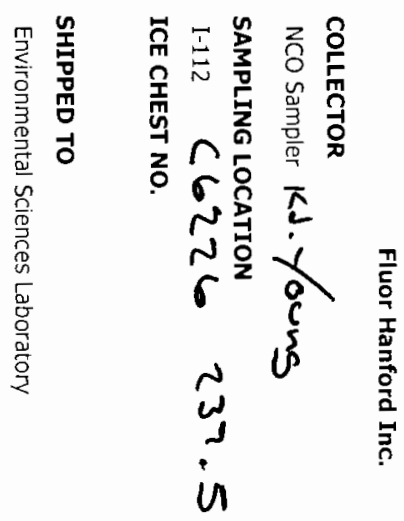

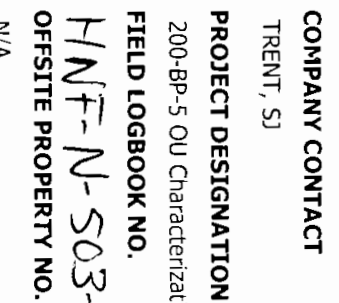

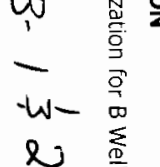

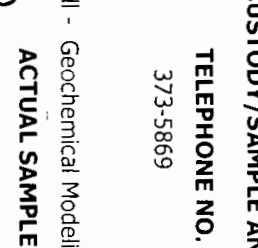

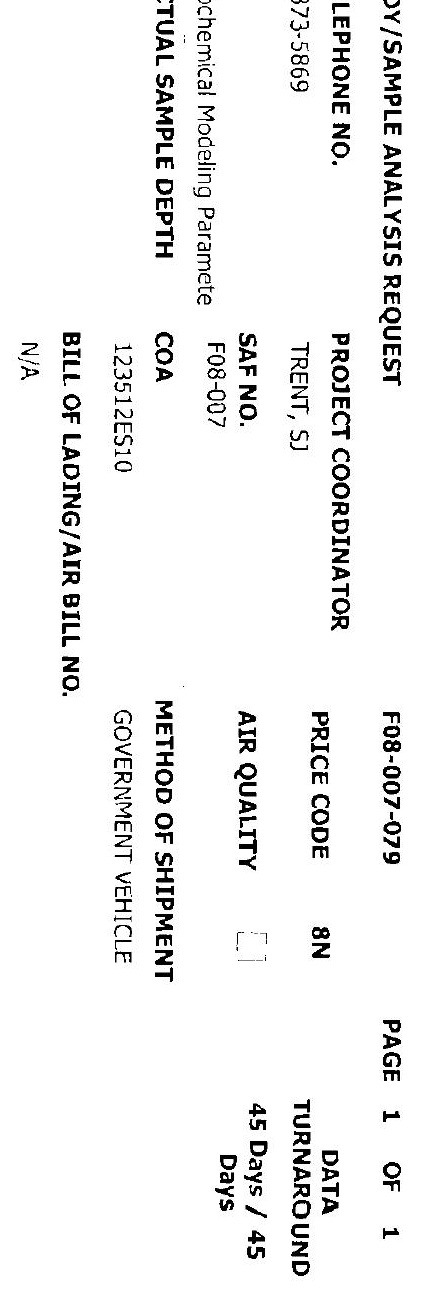




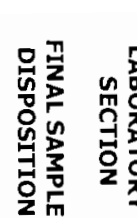

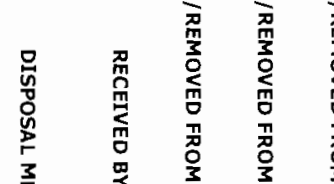

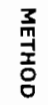

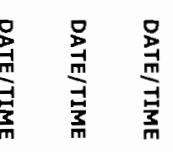

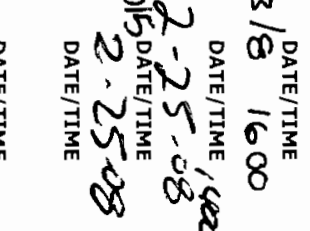

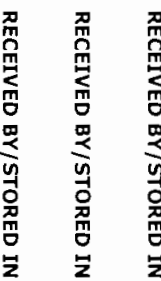

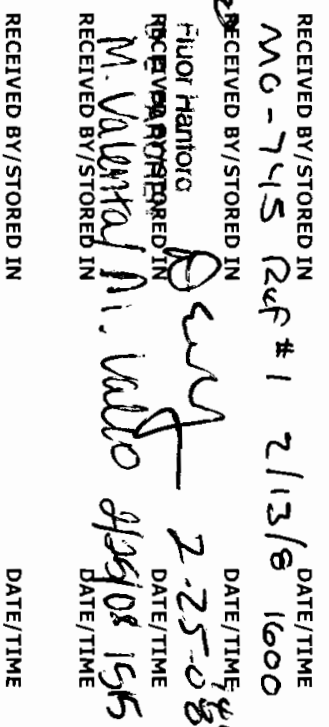

劳 $\quad$ 音

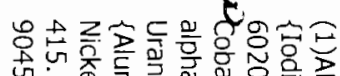

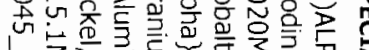

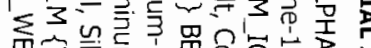

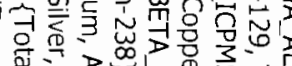

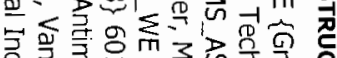

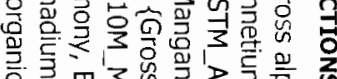

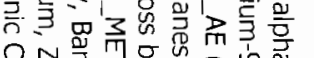

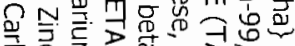

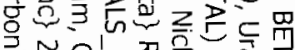

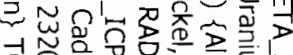

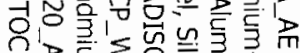

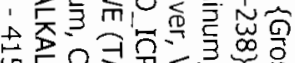

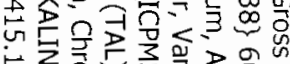

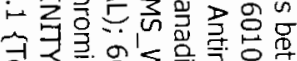

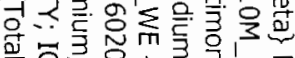

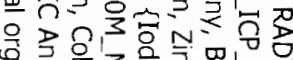

竞量量
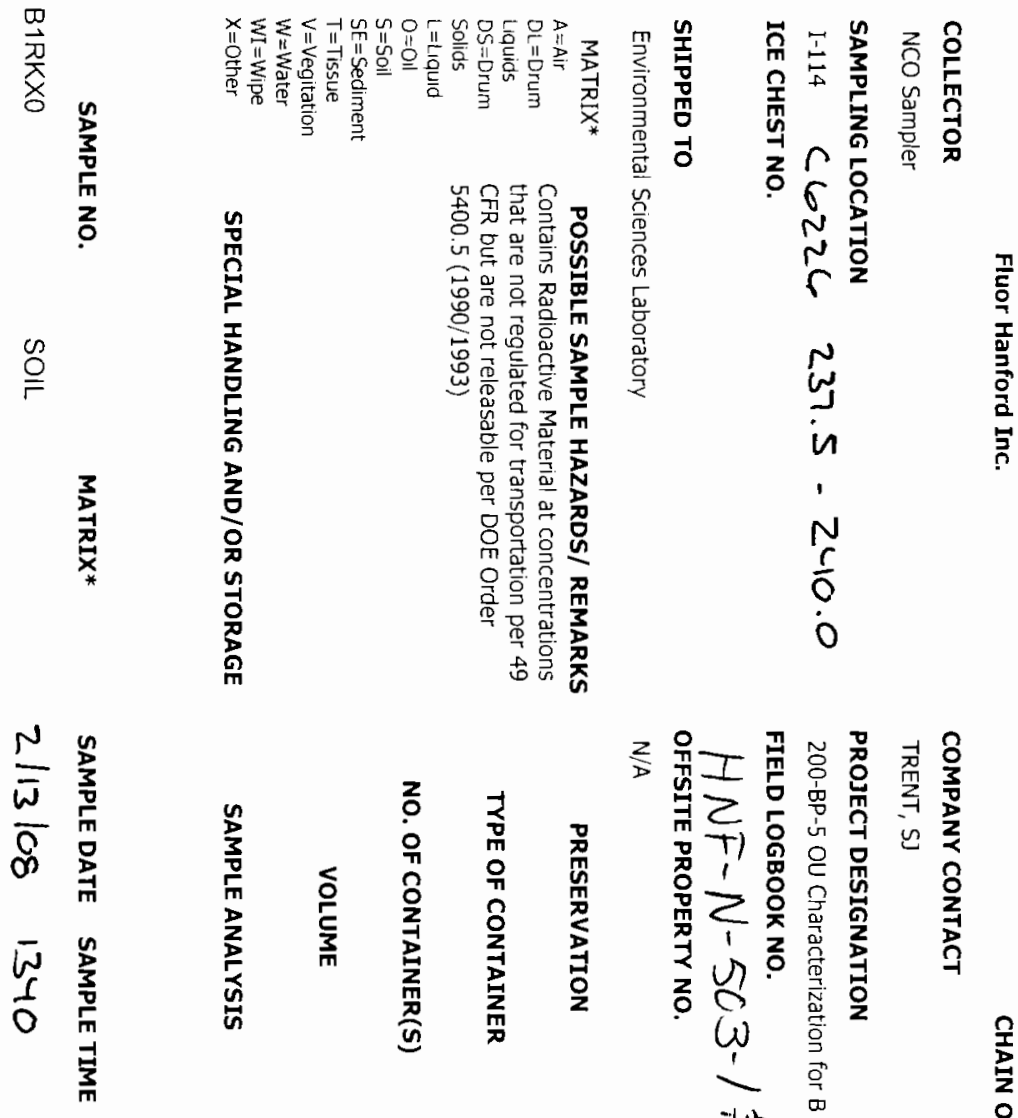

2 육금

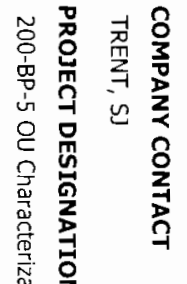

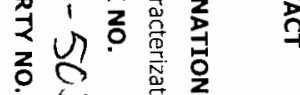

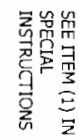

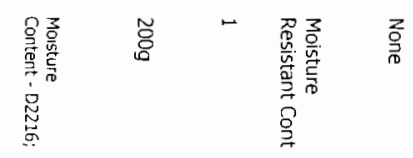




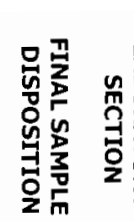

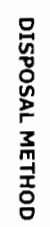 \\ II}

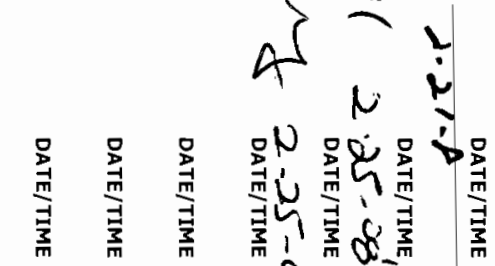

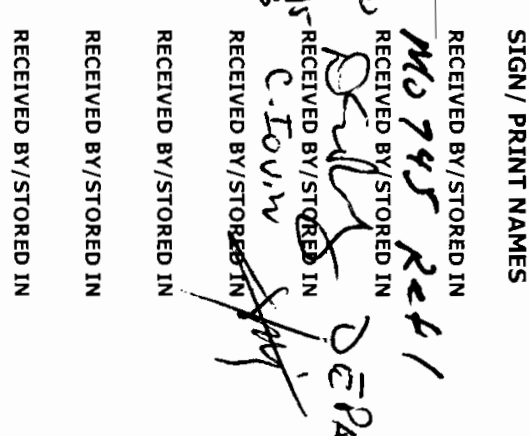

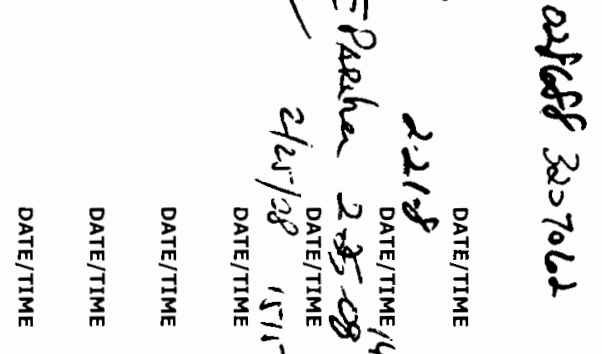

吾

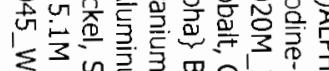

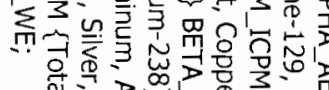

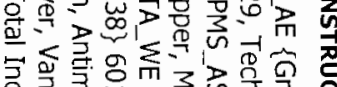

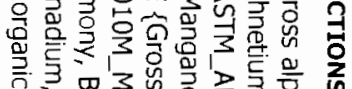

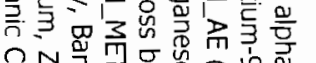

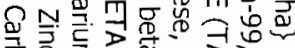

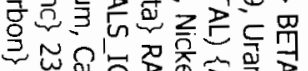

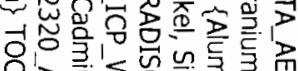

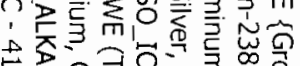

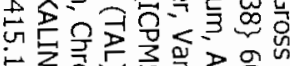

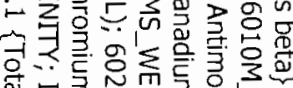

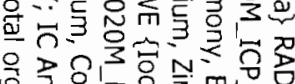

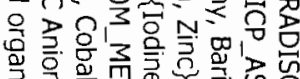

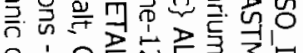

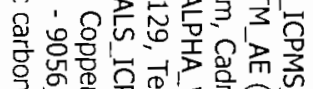

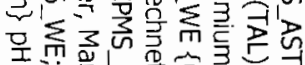

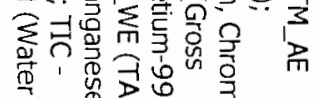

疍

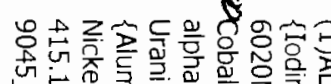

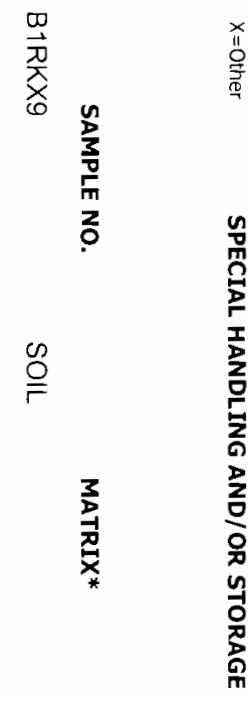

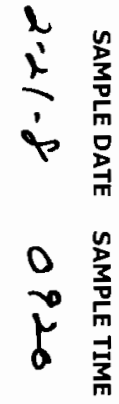
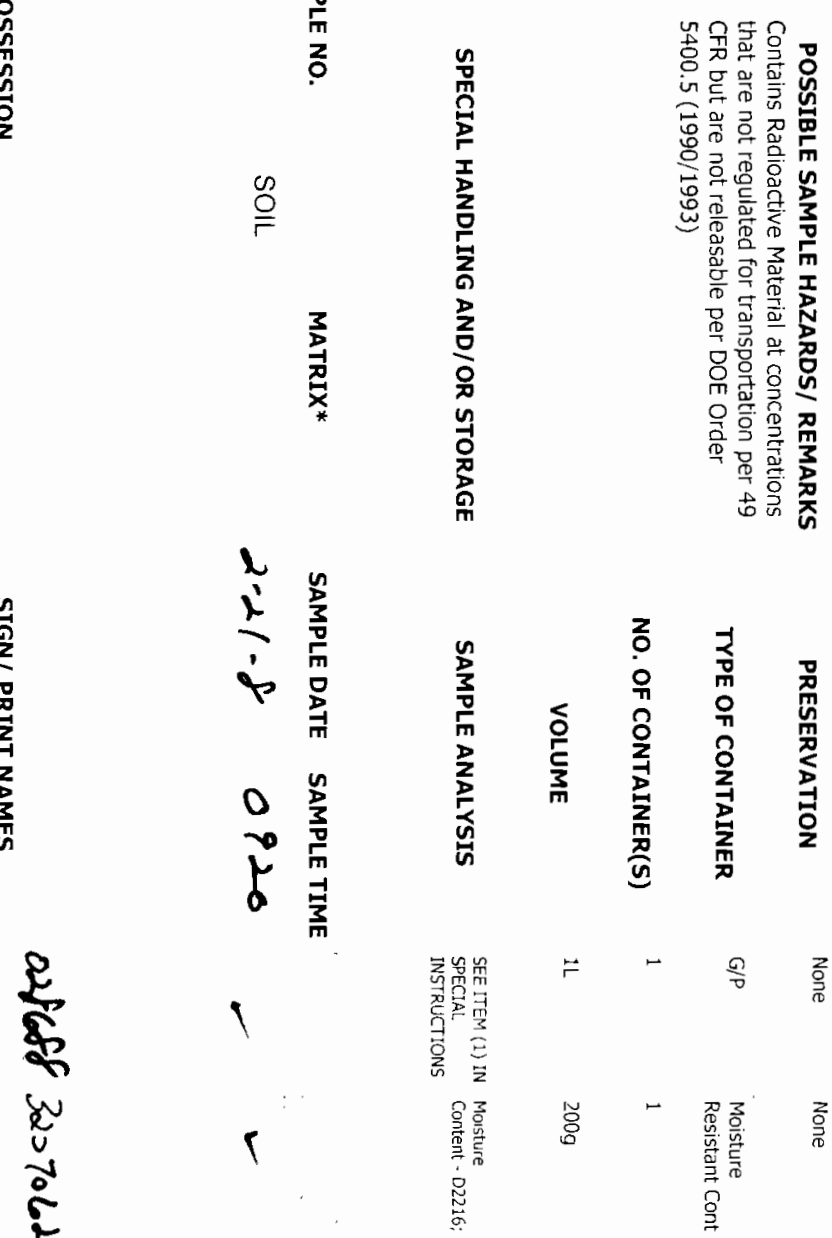

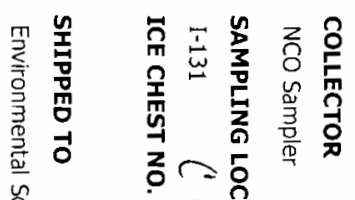
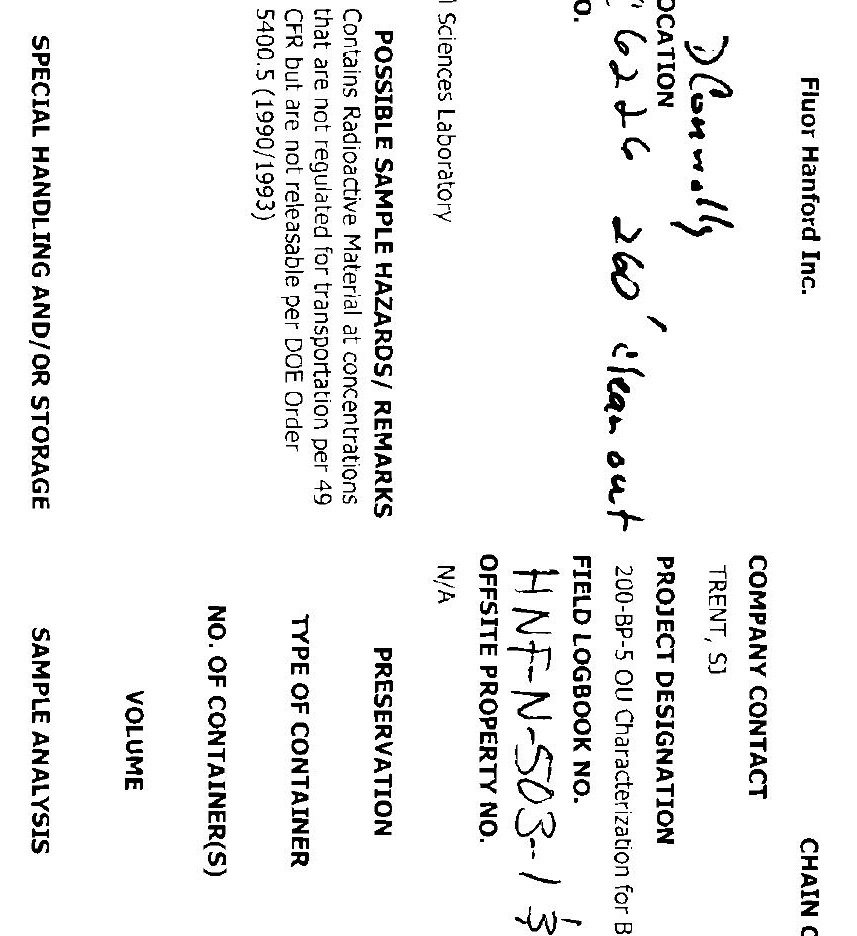

2 윢

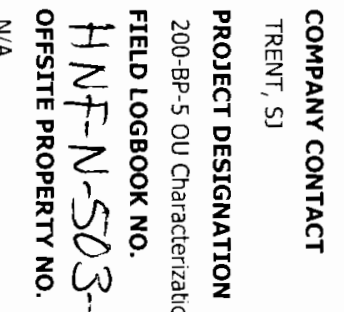

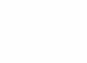

꼴

孛

w 금

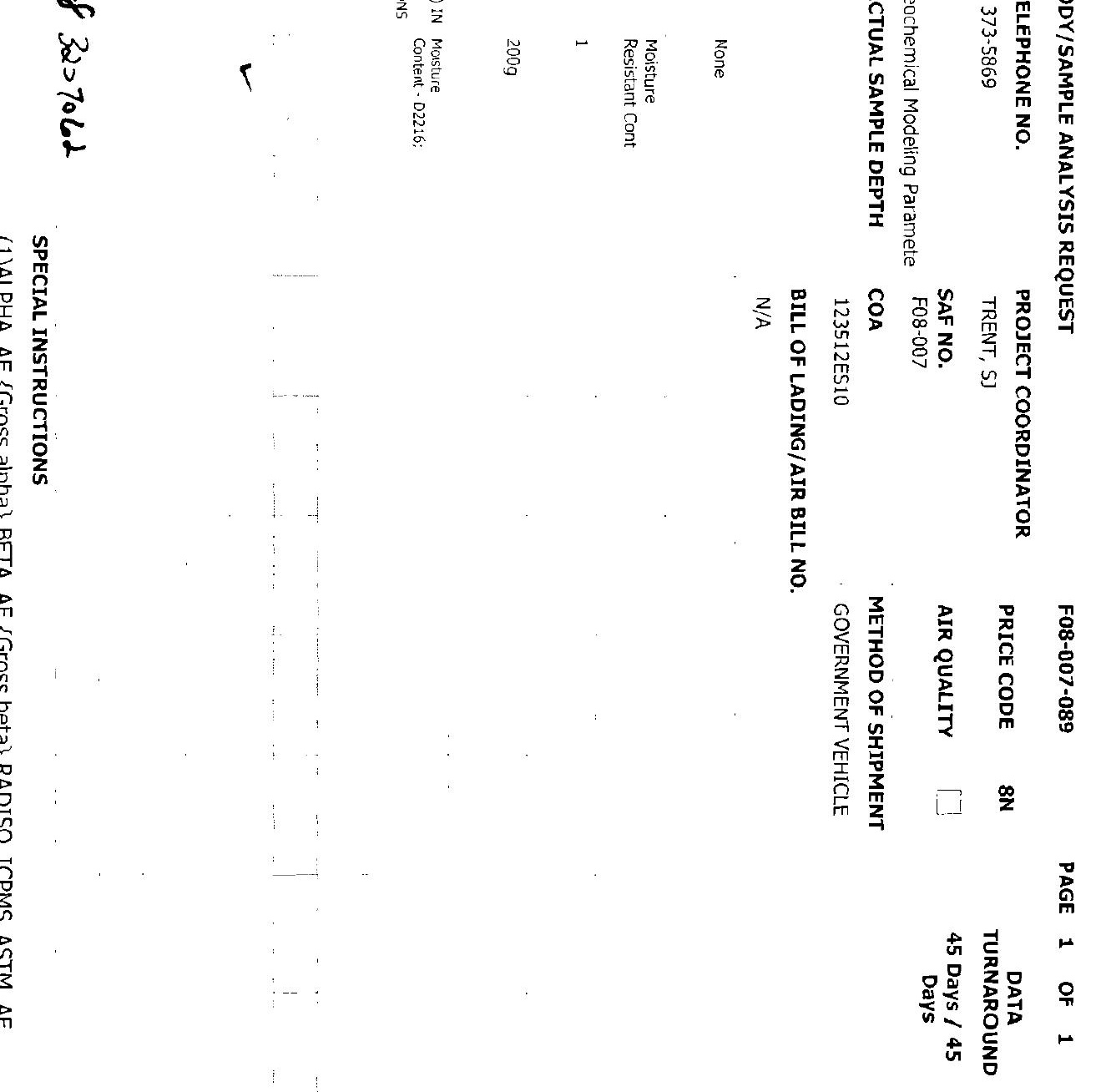



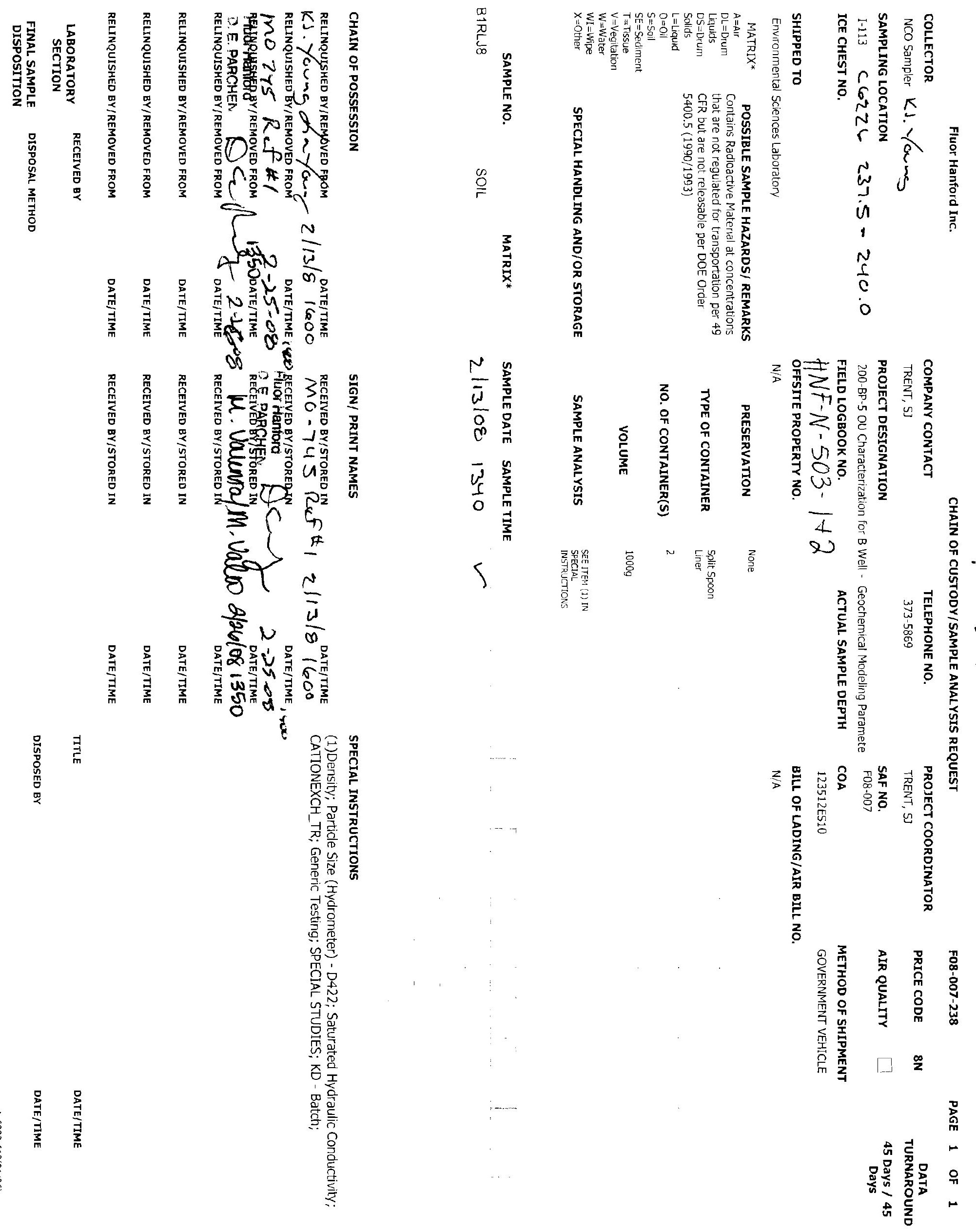

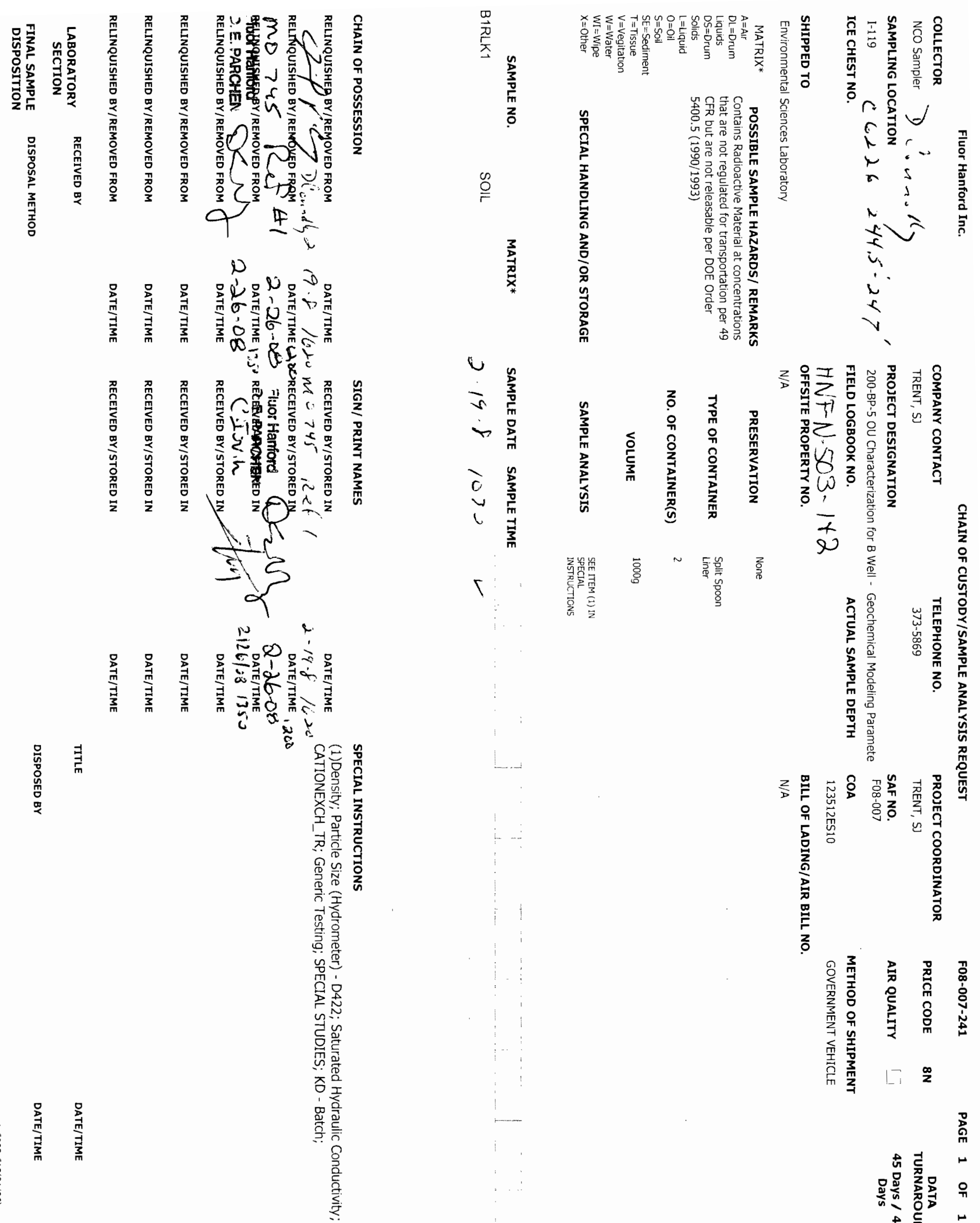

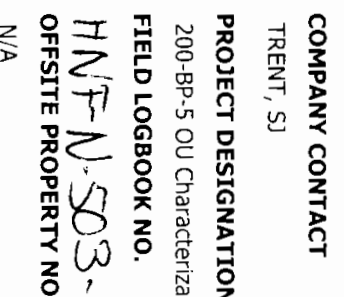
क

窟

w

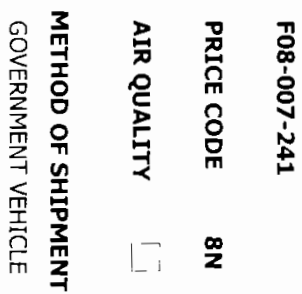



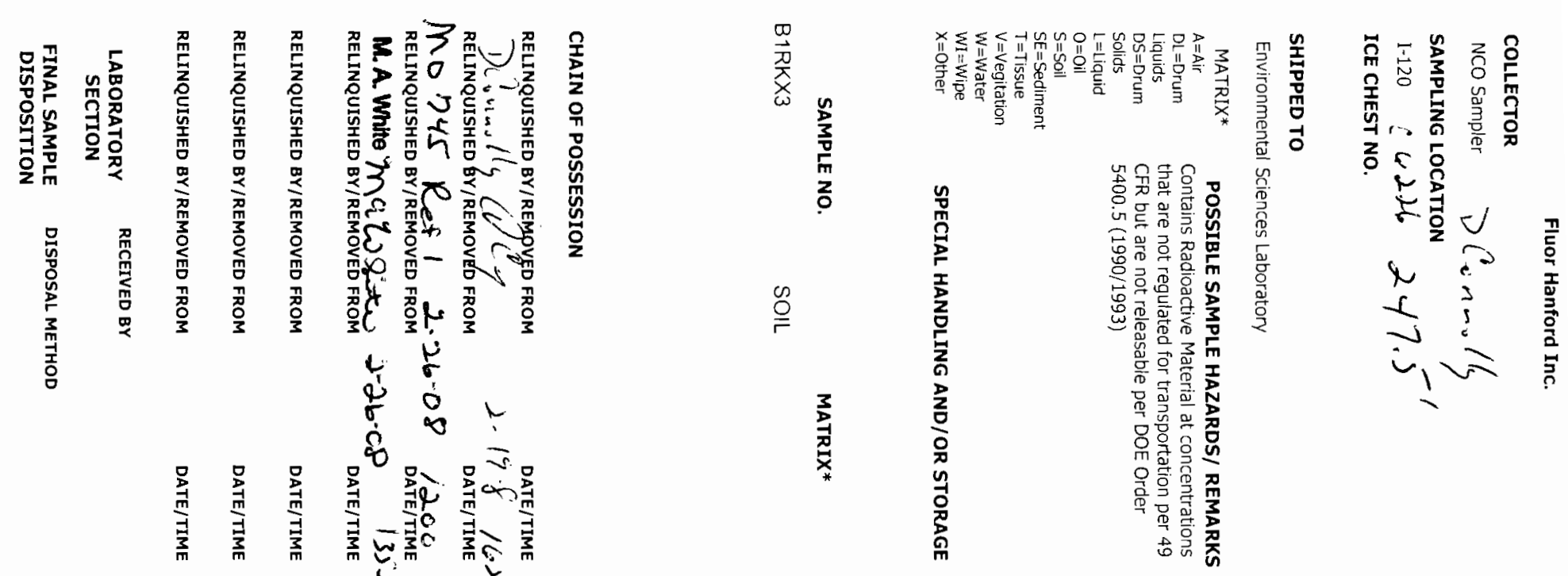

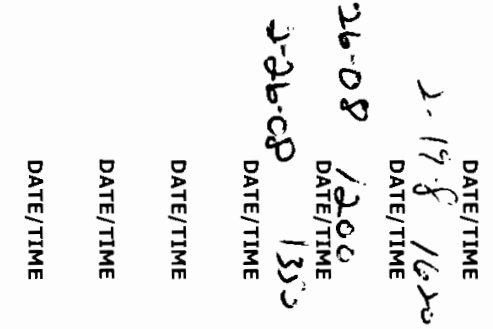
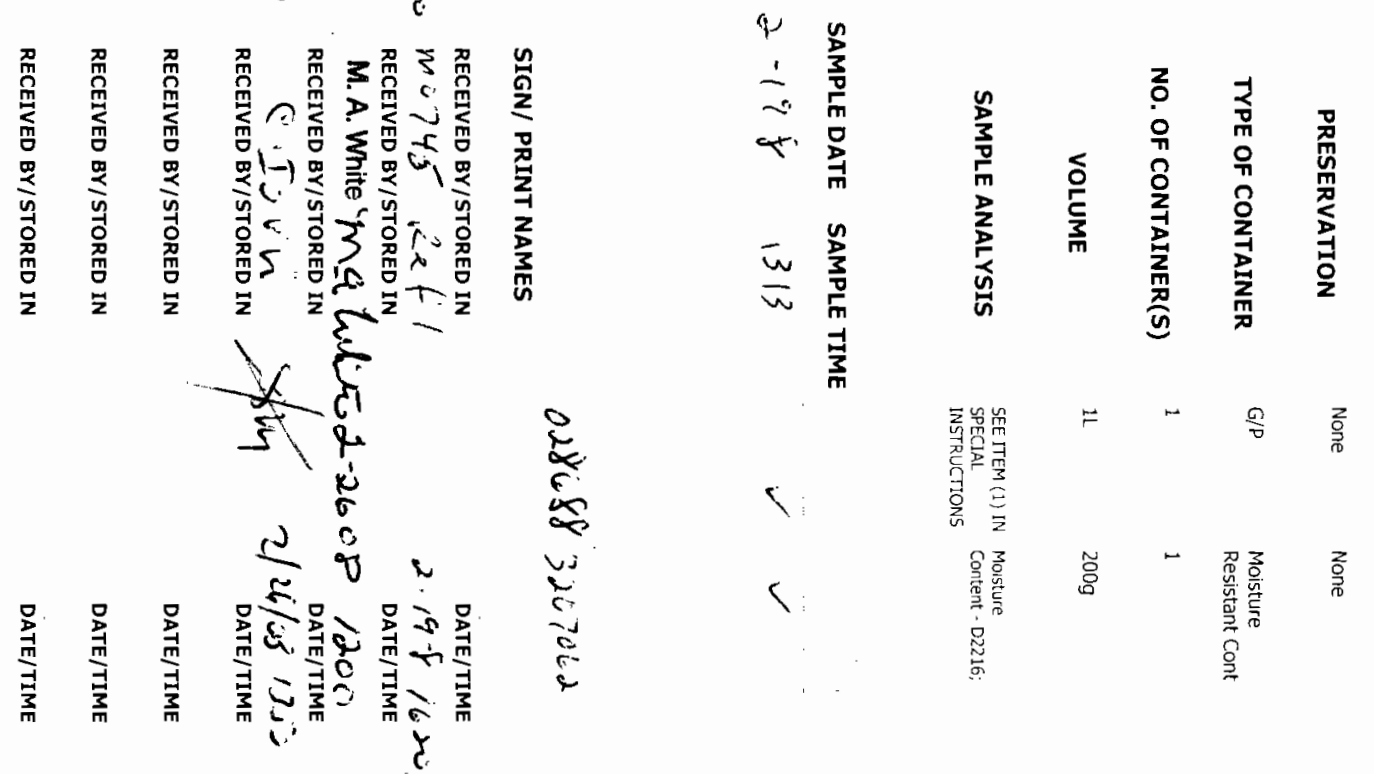

$$
\text { 촉육 }
$$

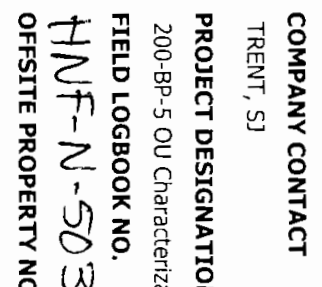
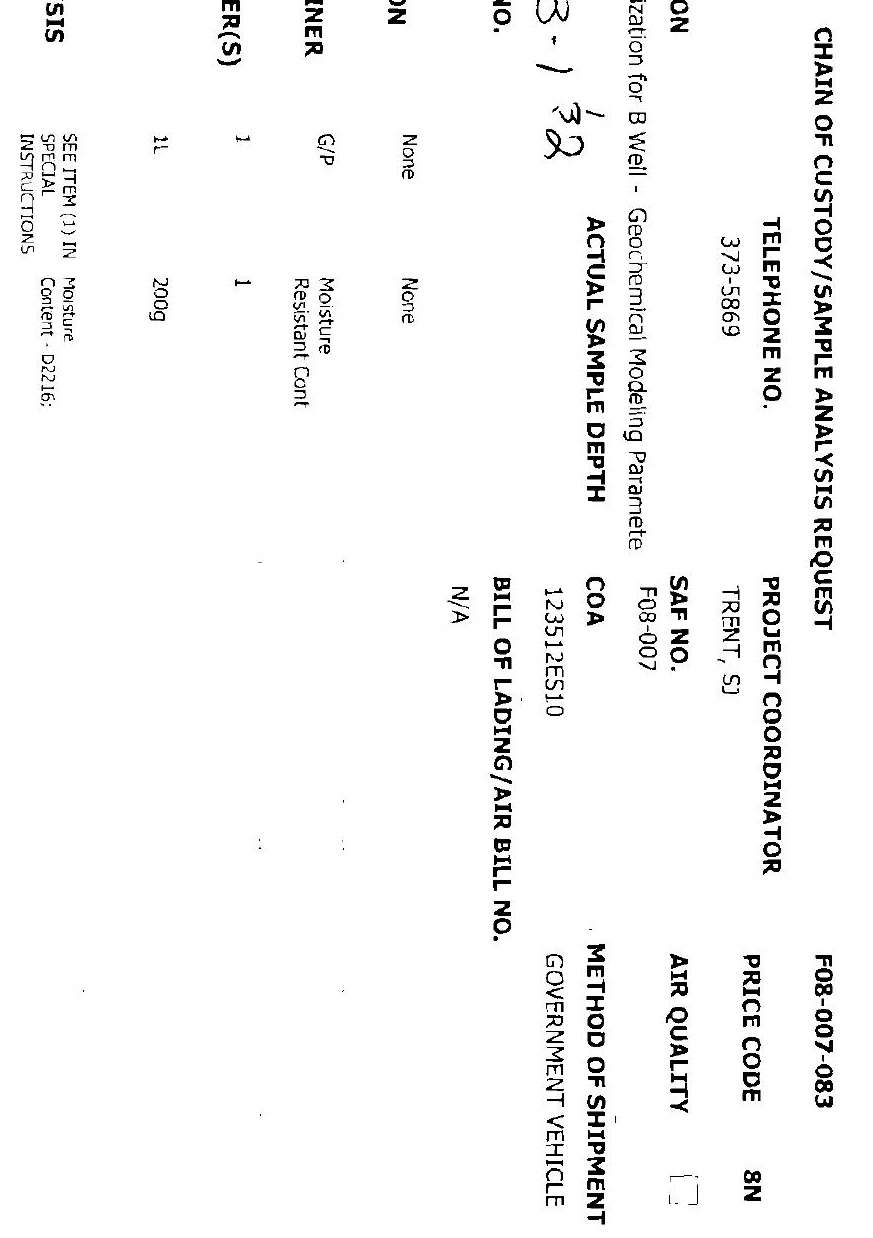

量
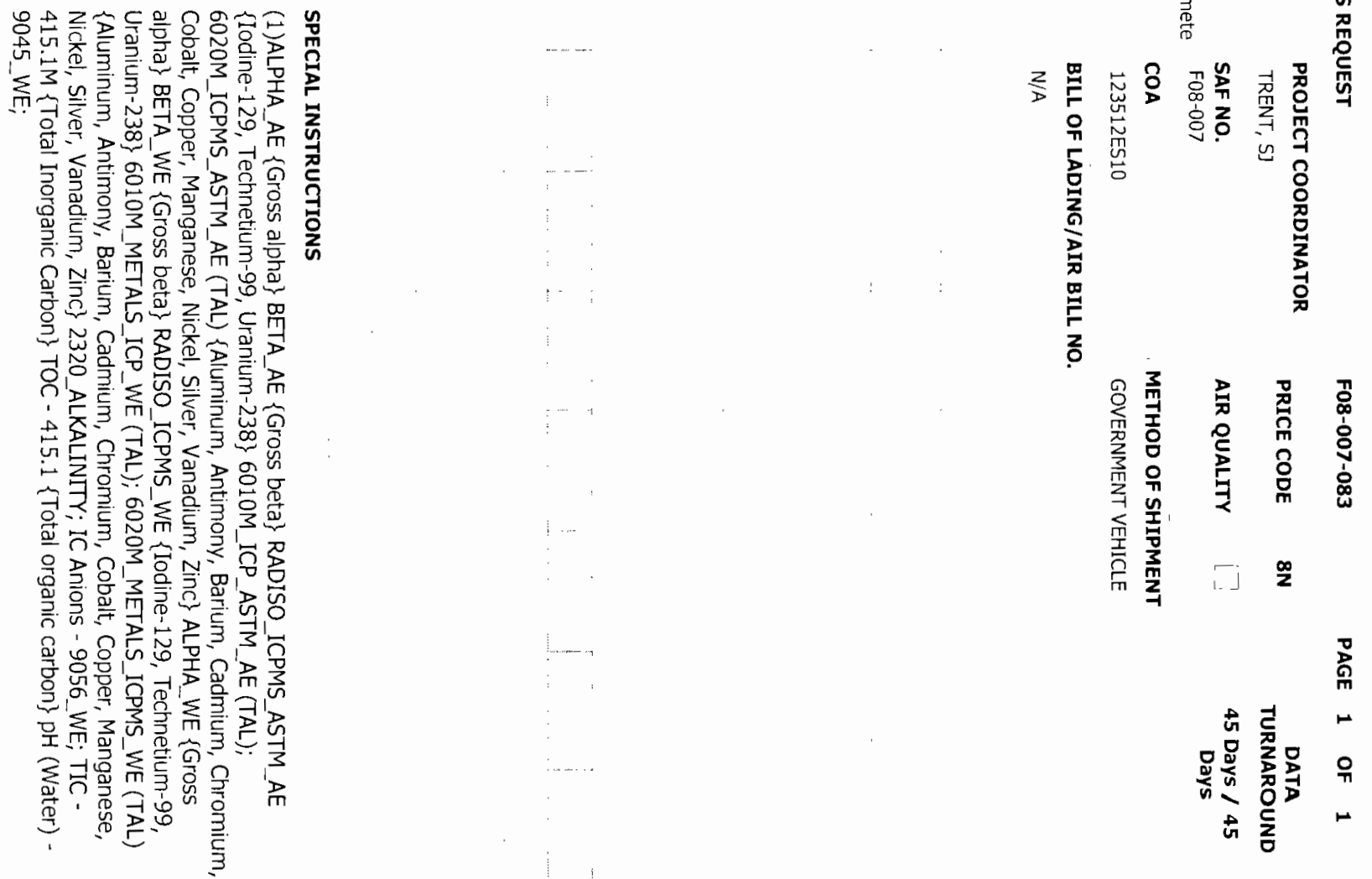


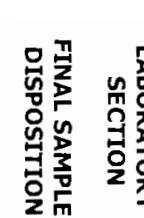

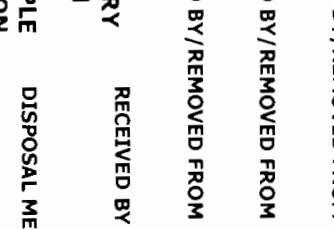
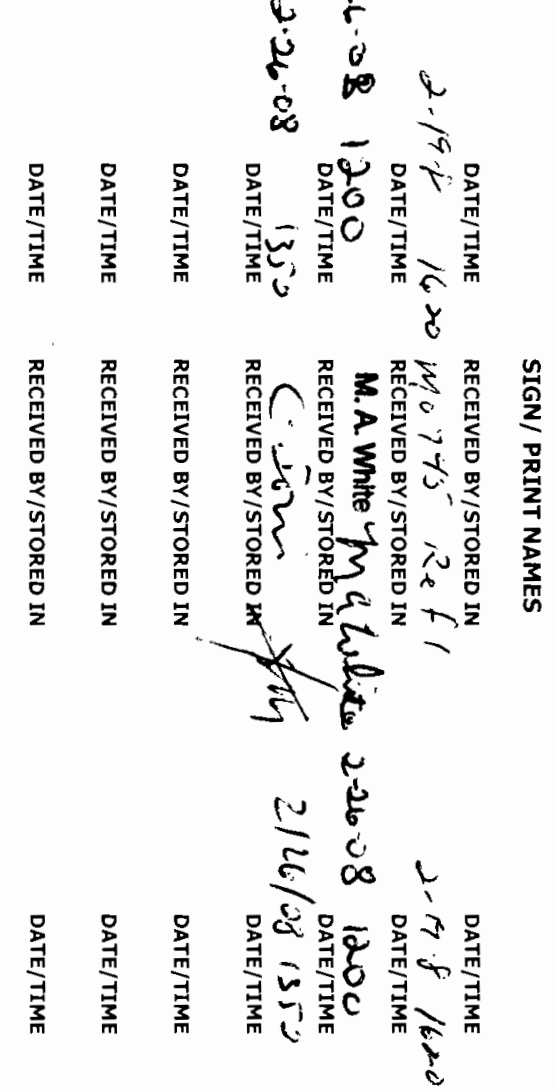

I:

章
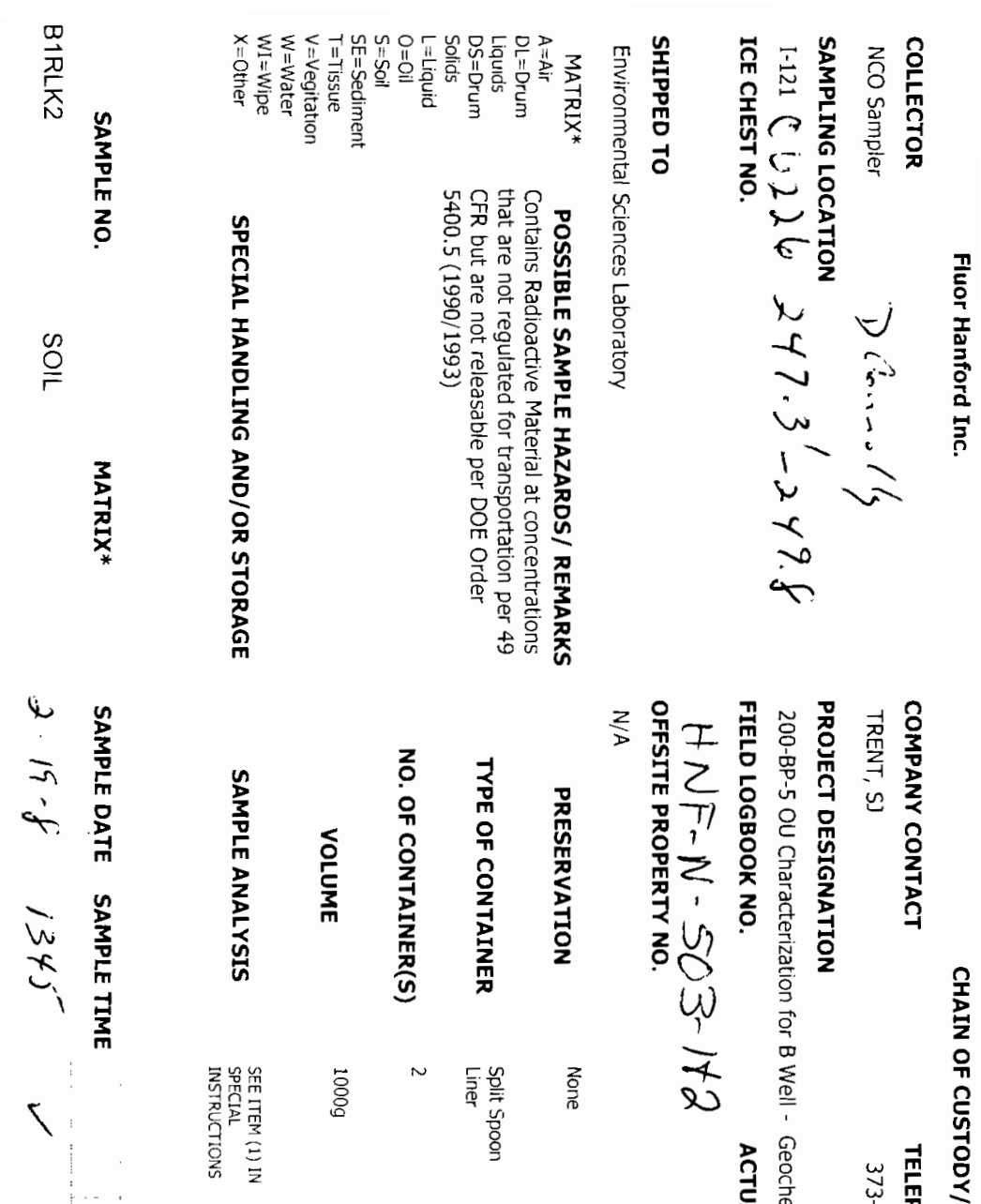

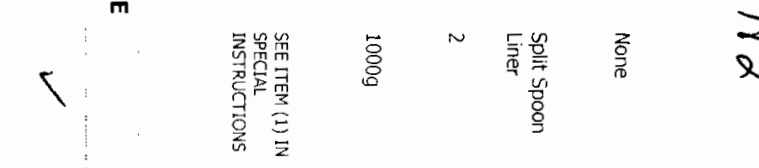
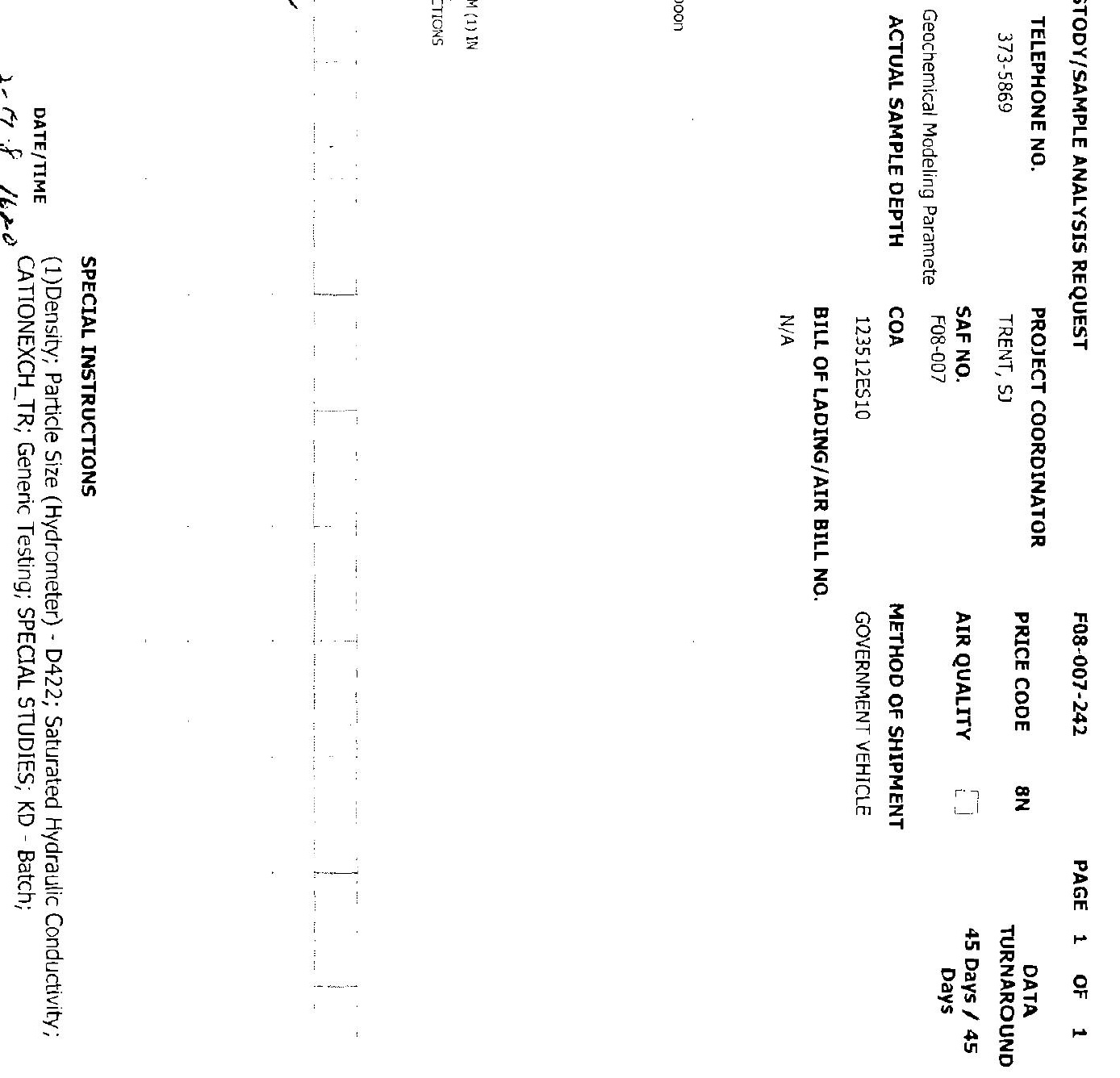


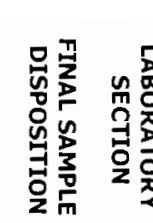

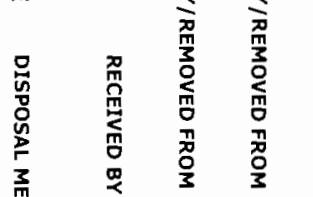

言

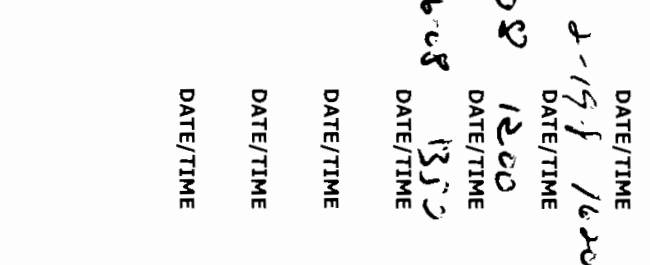

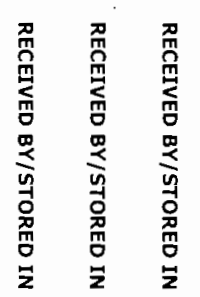

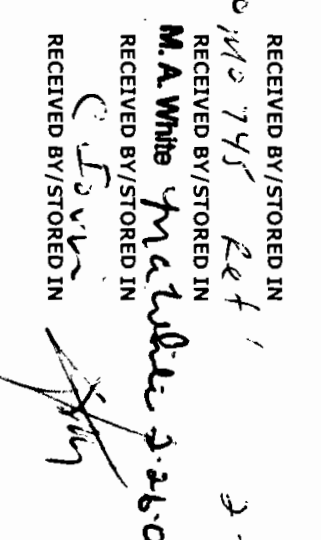

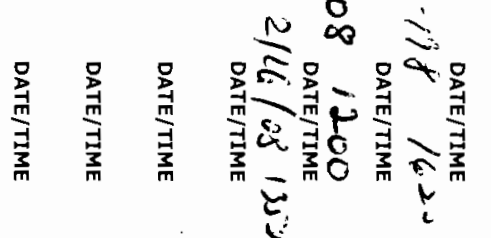

高

11 $\stackrel{2}{2}$

$\stackrel{\square}{\stackrel{\square}{\pi}}$

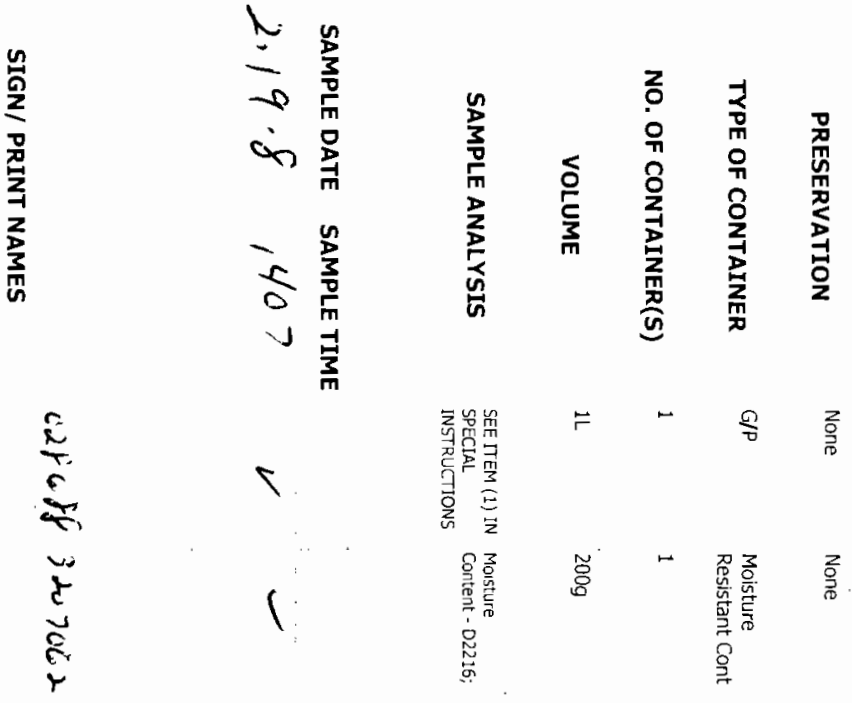

$\stackrel{8}{\circ}$

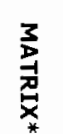

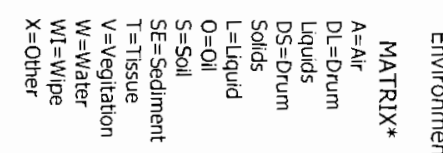

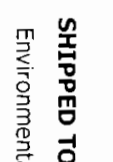

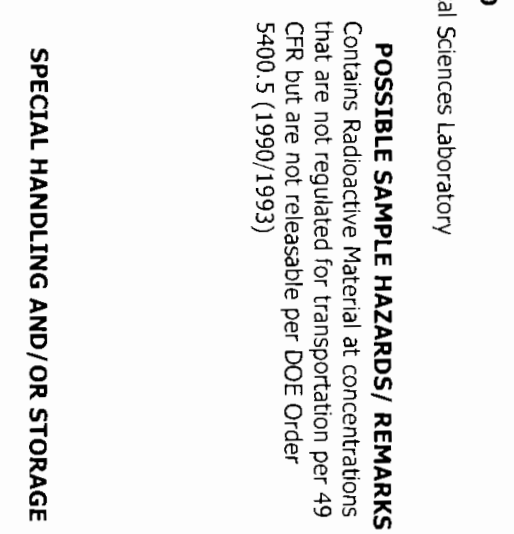

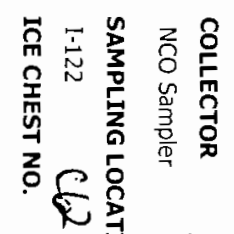

$e^{2} \underset{c}{2}$

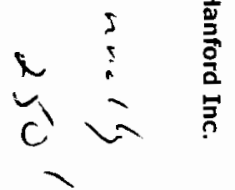

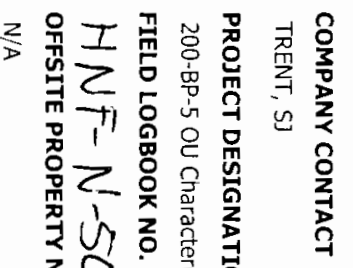

co

- $w-\sum_{\substack{0 \\ 0}}^{\infty}$

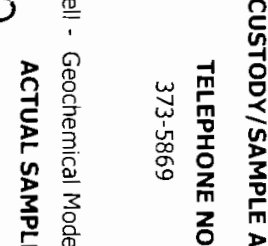

而

署

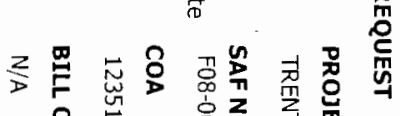

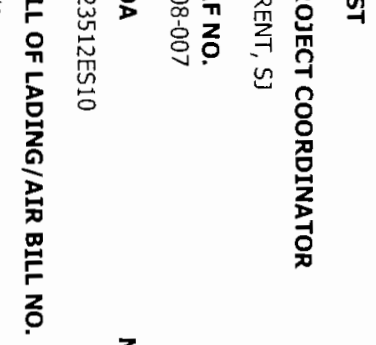

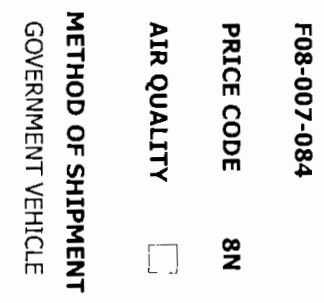

㝵

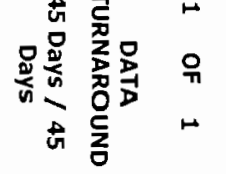




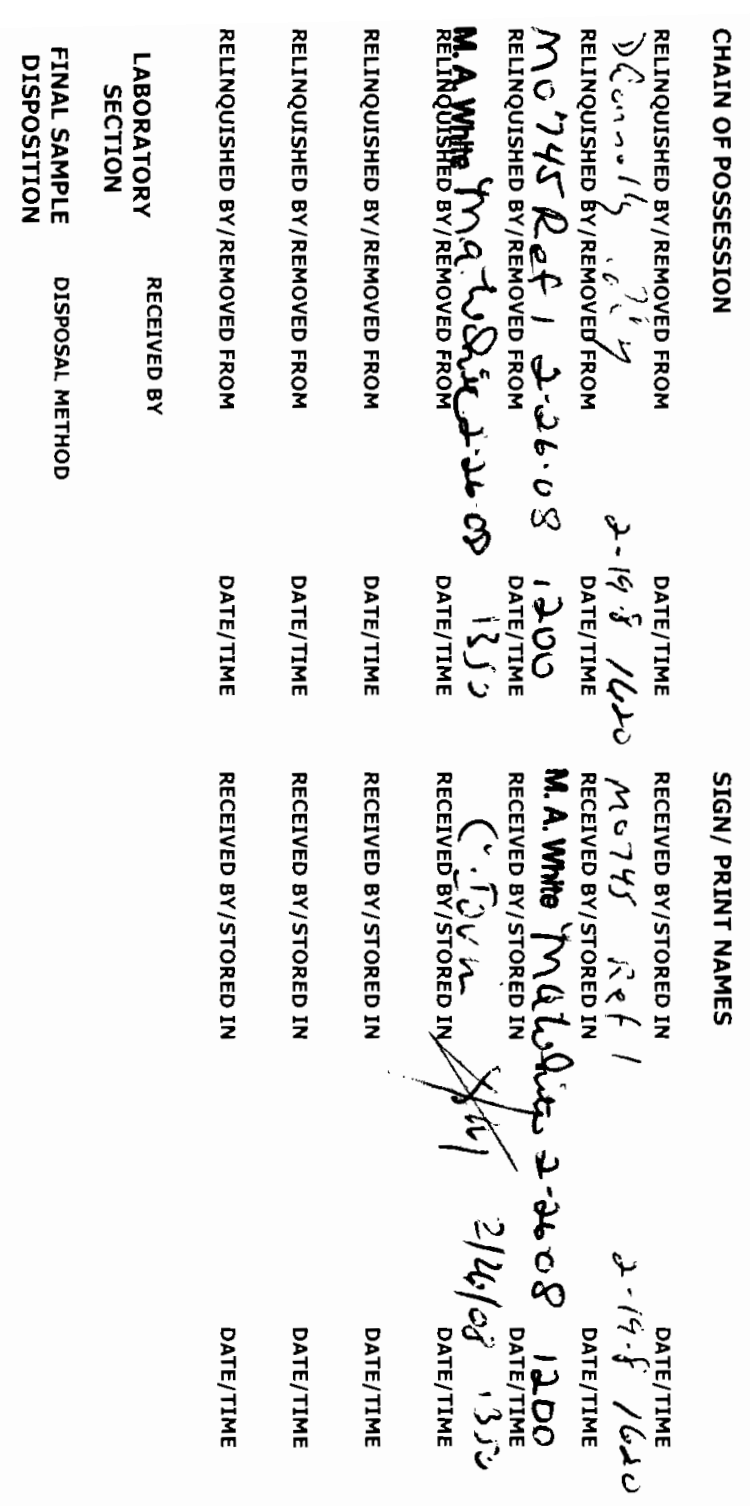

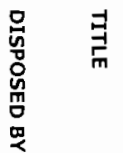

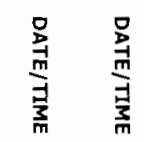
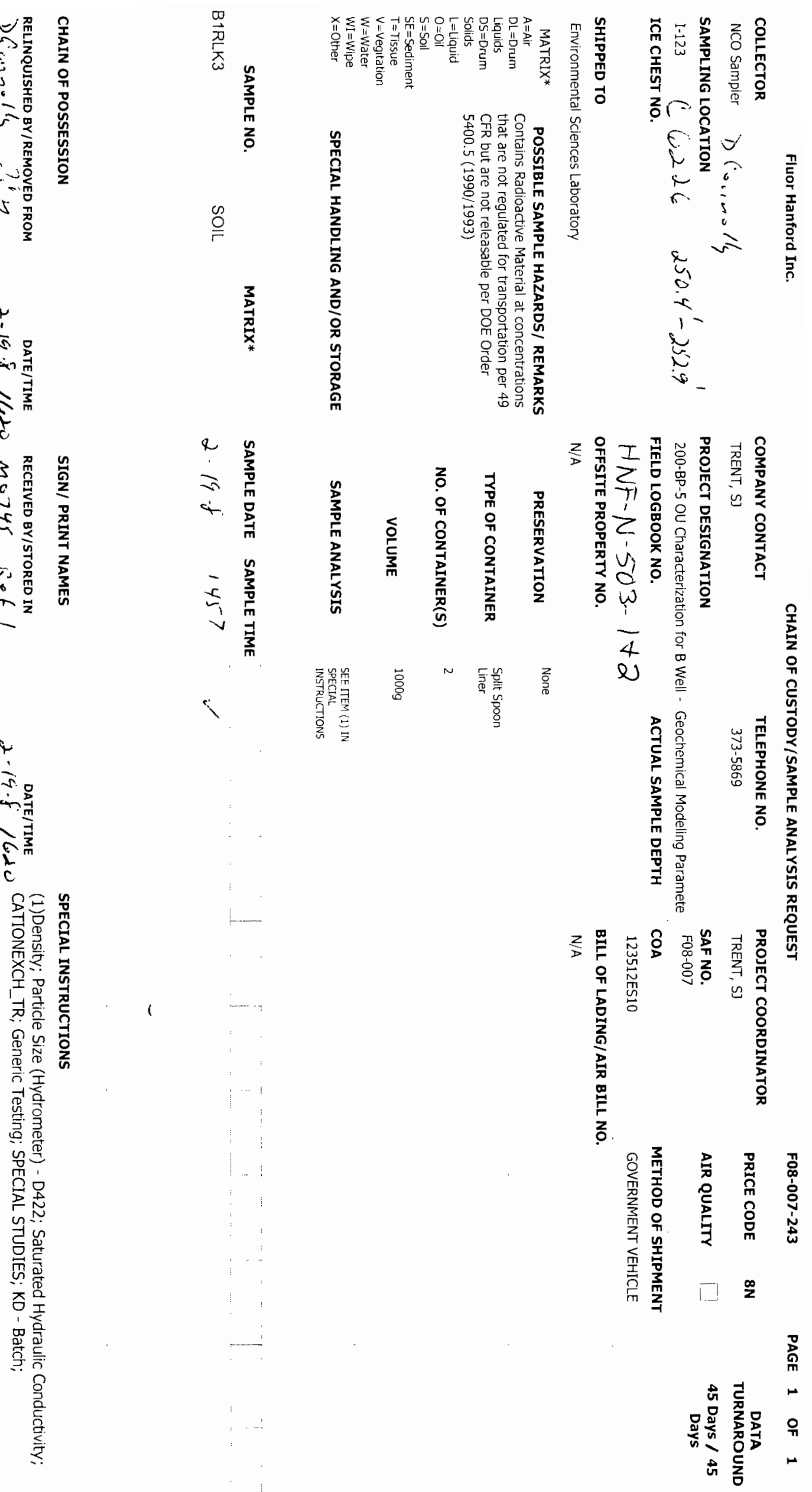


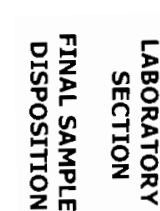

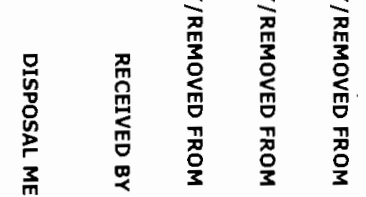

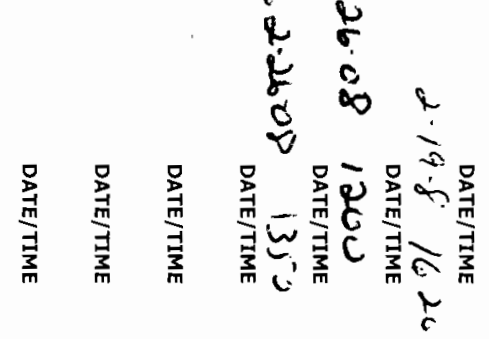

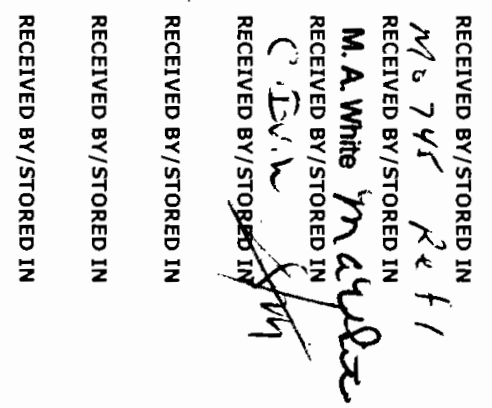

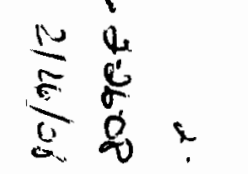

总

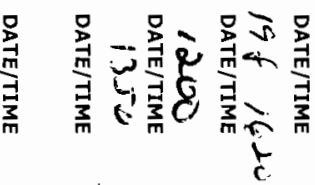

है

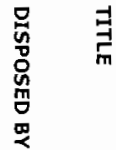

$\begin{array}{ll}\frac{D}{2} & \frac{D}{7} \\ \frac{m}{3} & \frac{7}{3} \\ \frac{3}{3} & \frac{1}{3}\end{array}$

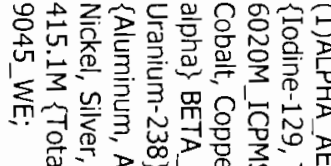

은

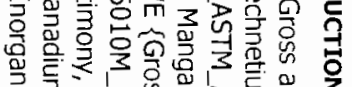

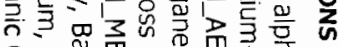

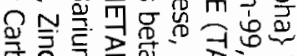

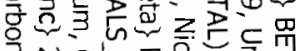

岕 $Q$

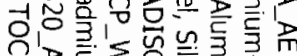

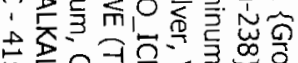

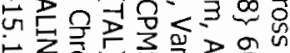

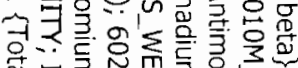

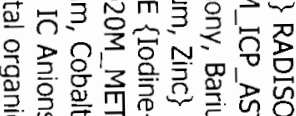

ते कि

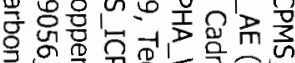

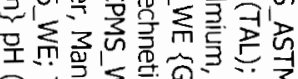

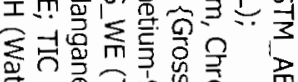

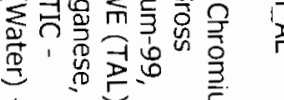

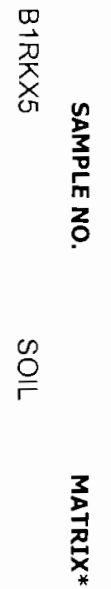

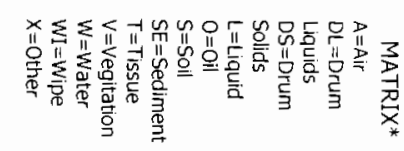

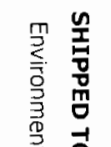

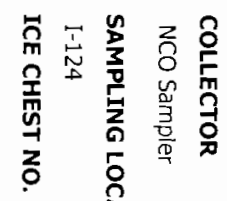

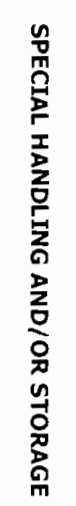
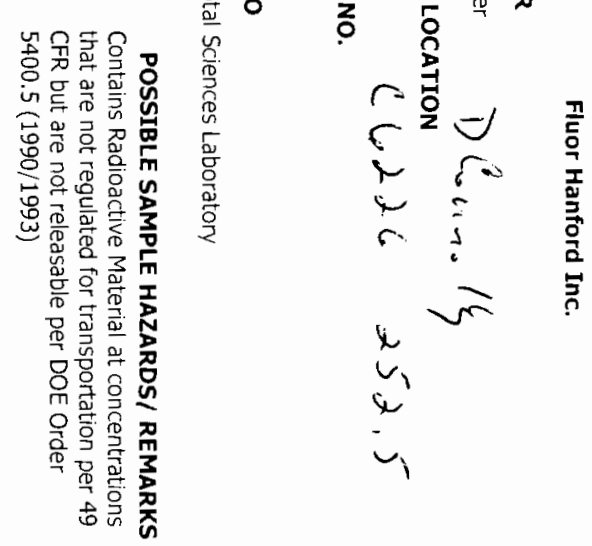

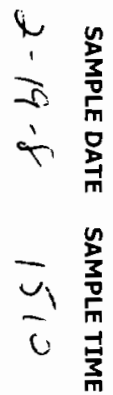

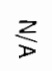

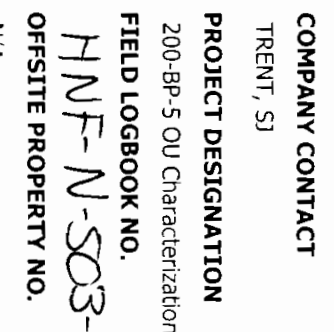

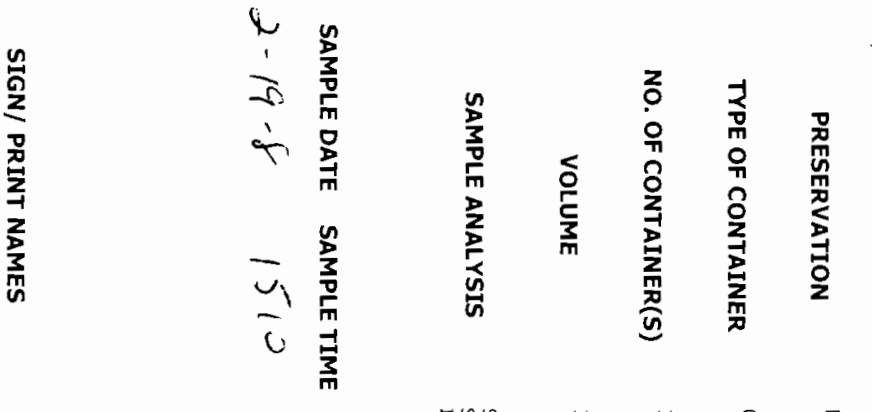

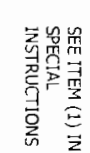

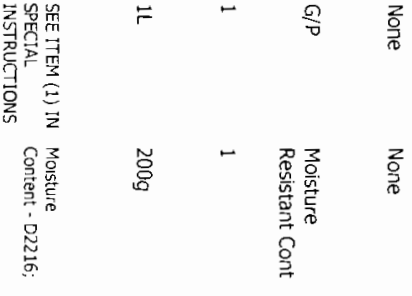

8 

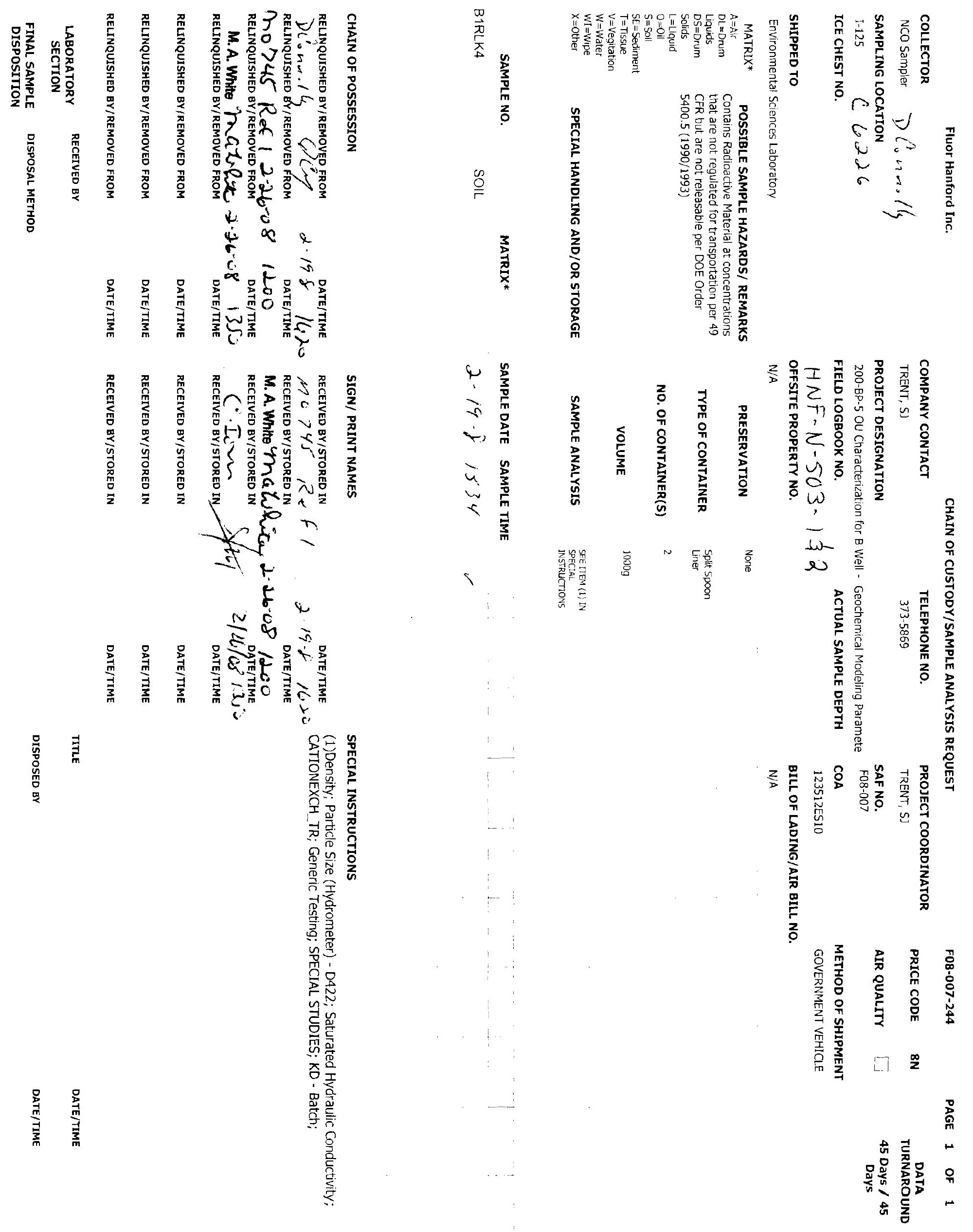


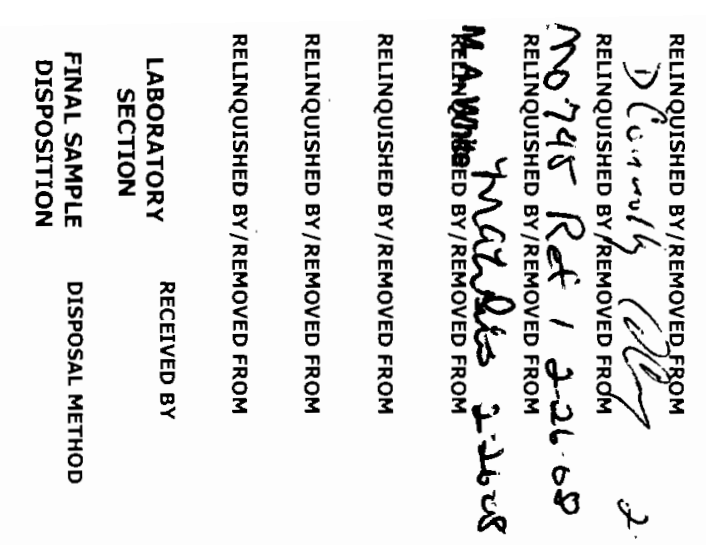

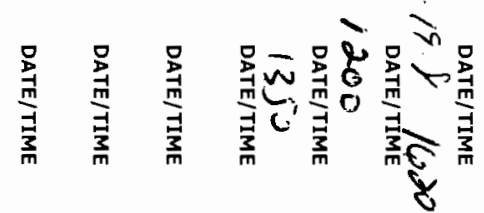

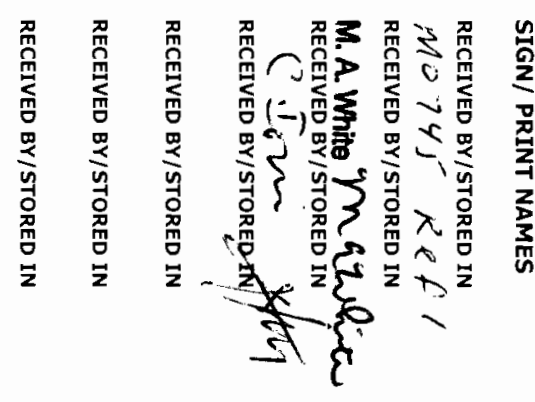

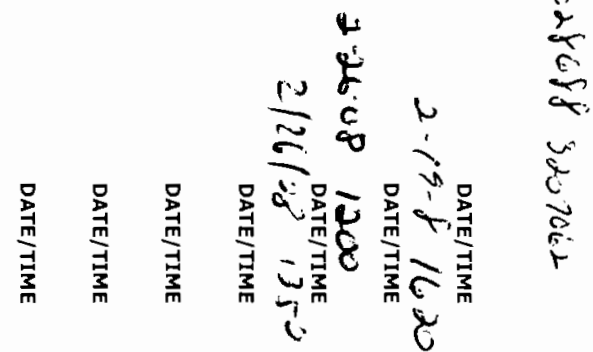

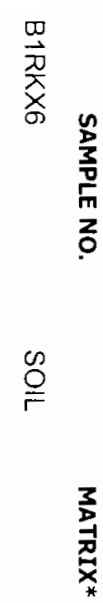

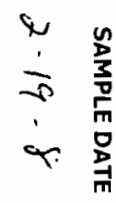

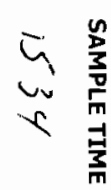
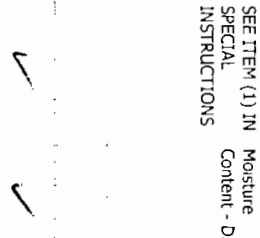

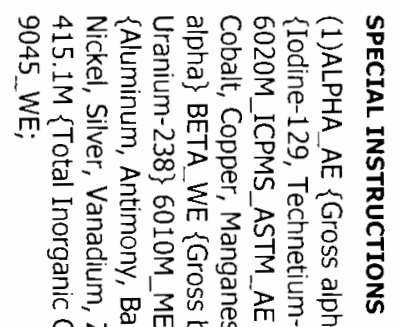

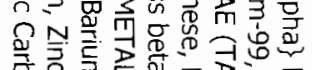

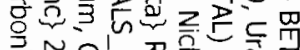

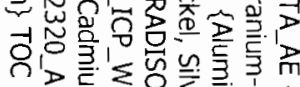

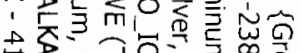

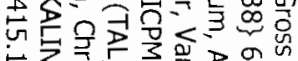

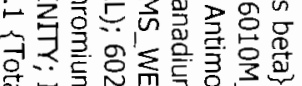

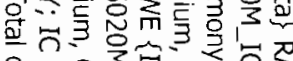

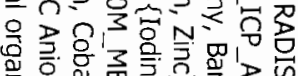

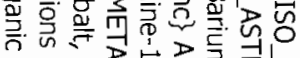

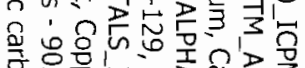

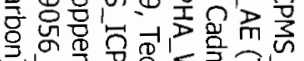

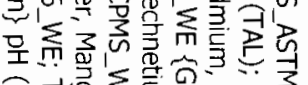

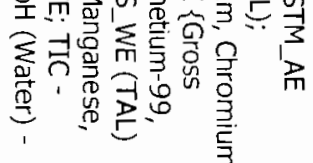

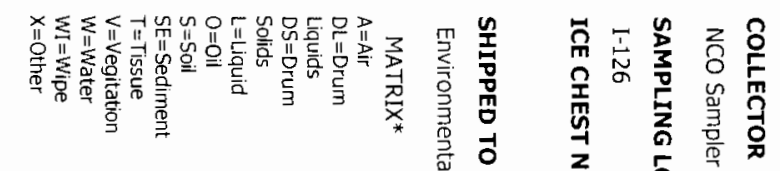
융

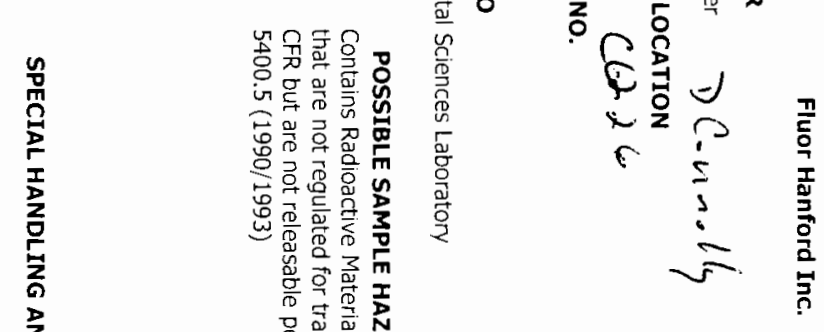

号 讪

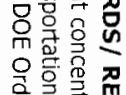

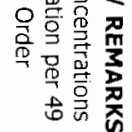
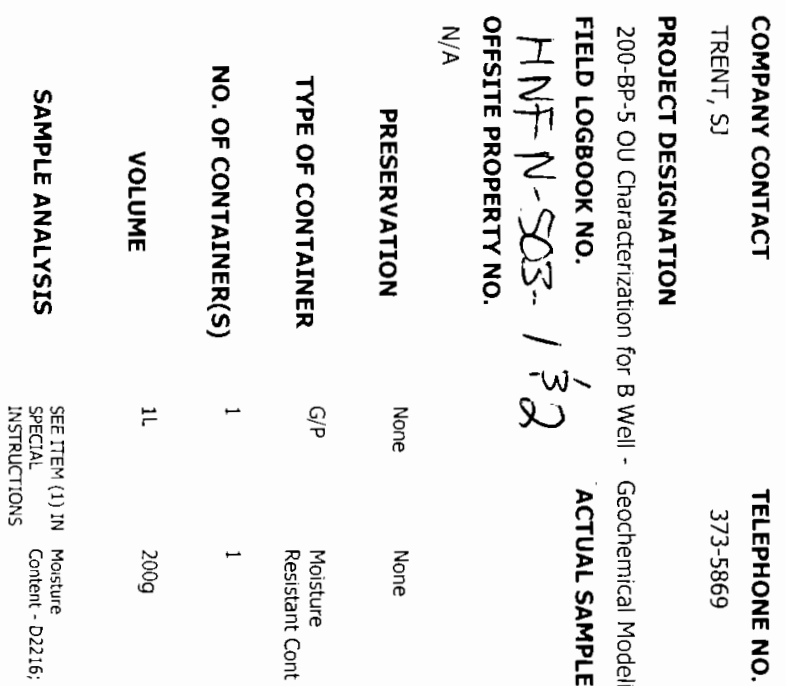

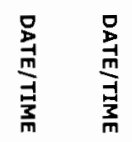




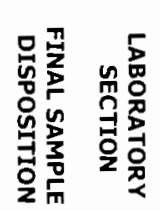

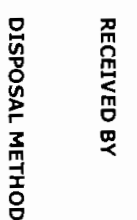
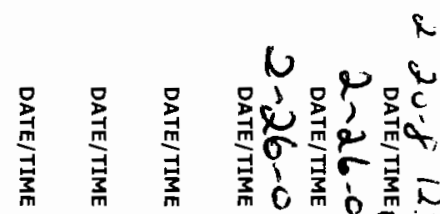

1

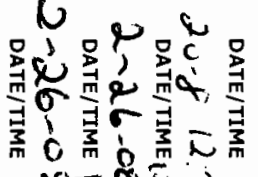

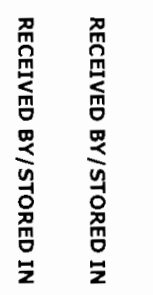

10 $=0$

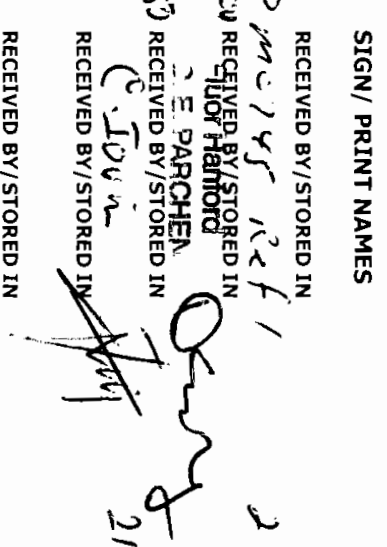

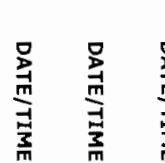
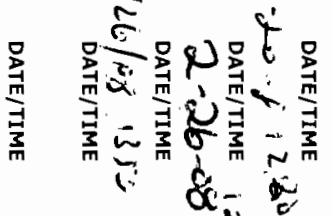

$\underset{\mathrm{m}}{\mathrm{g}} \stackrel{\overrightarrow{\mathrm{G}}}{\mathrm{m}}$
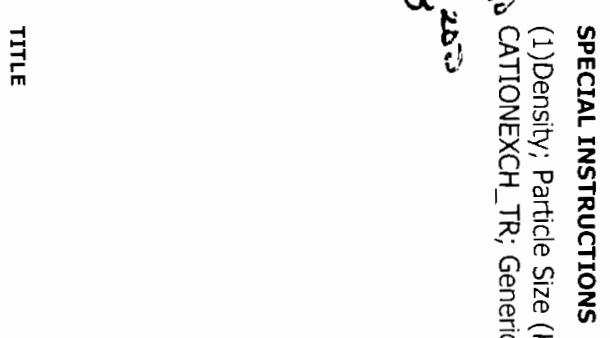

言王

需高

i⿱

空足

瓷管

茴

증 음

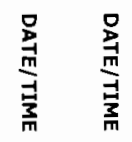

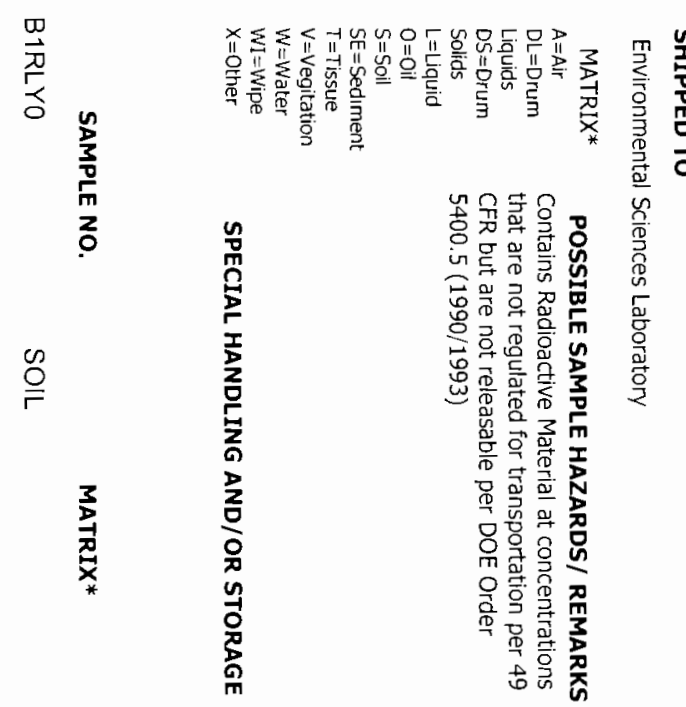

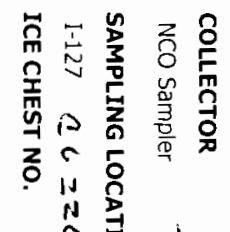

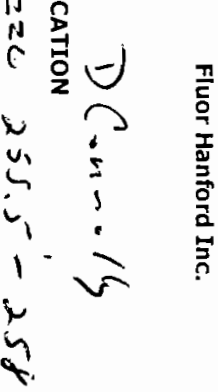

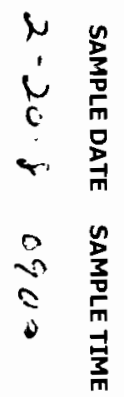

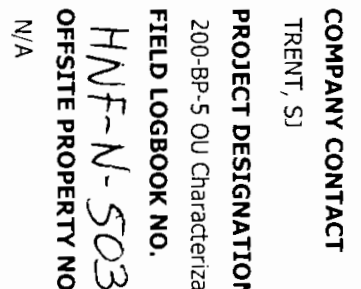

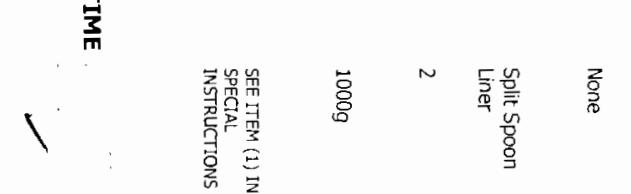

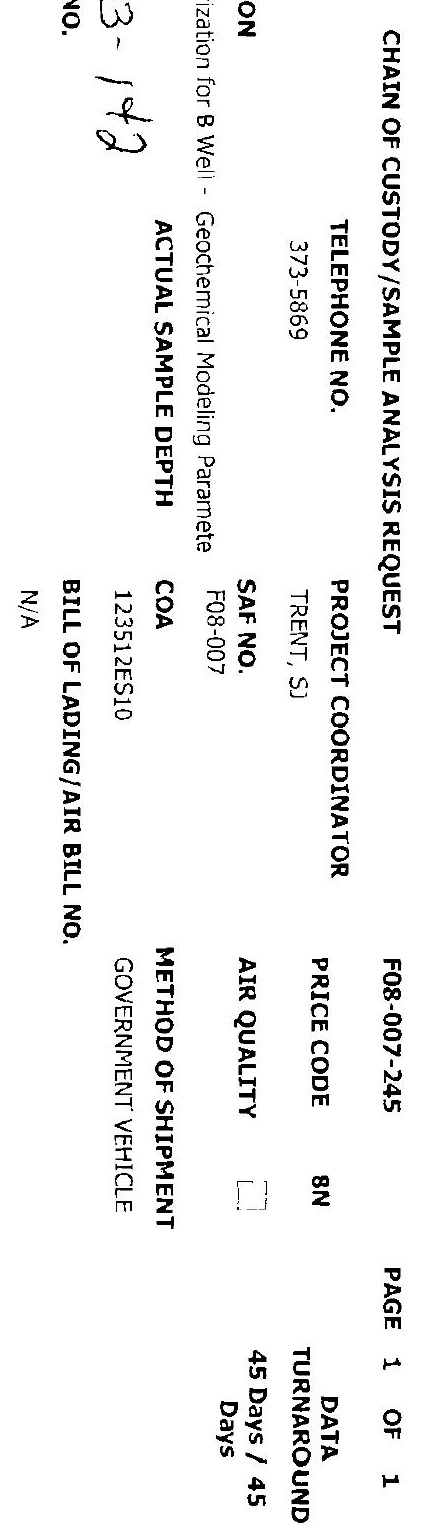




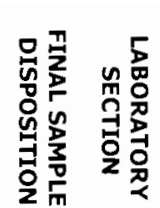

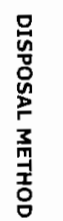
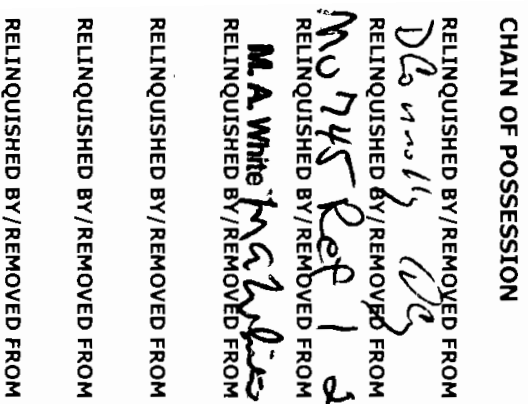

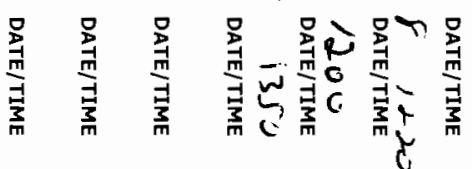
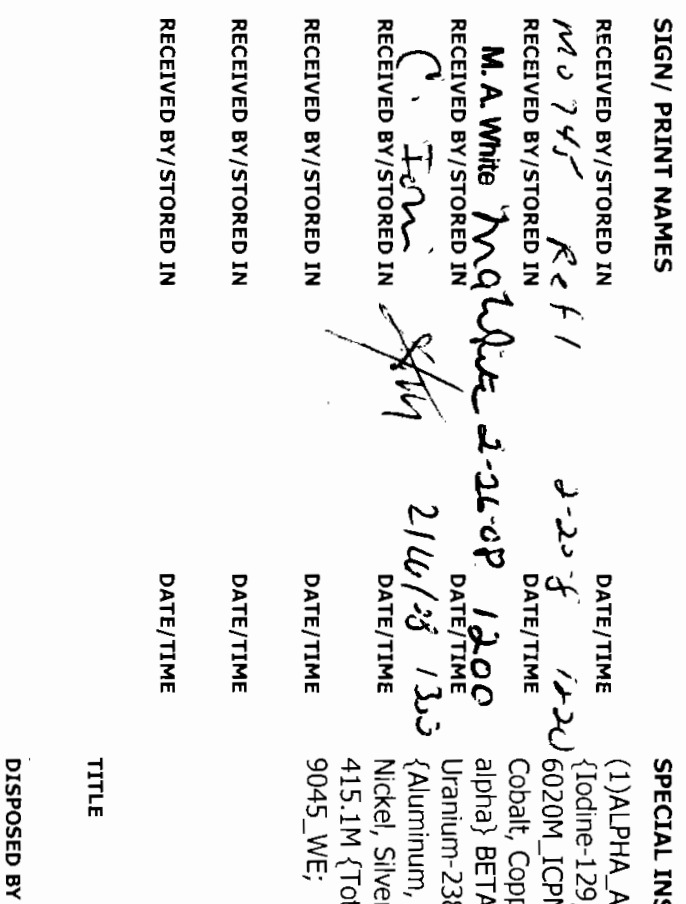

站
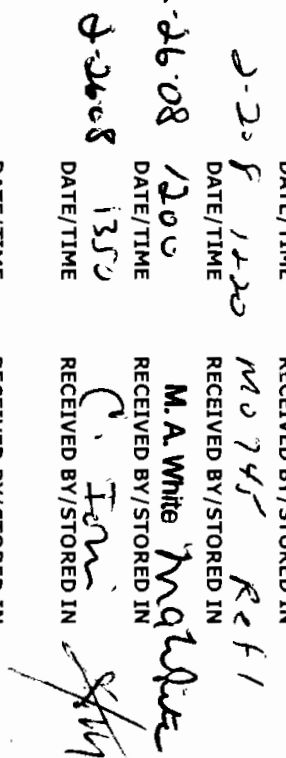

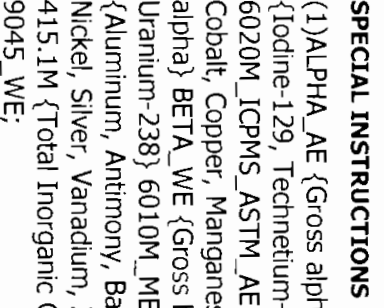

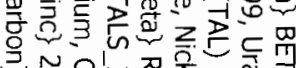

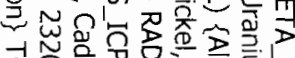

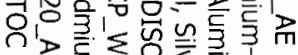

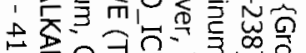

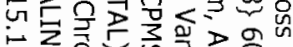

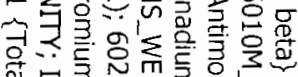

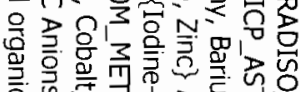

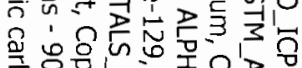

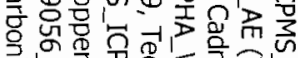

Q

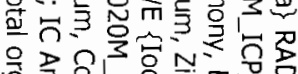

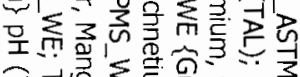

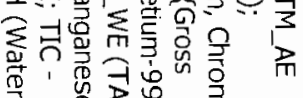

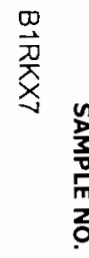

$\stackrel{\varrho}{\circ}$

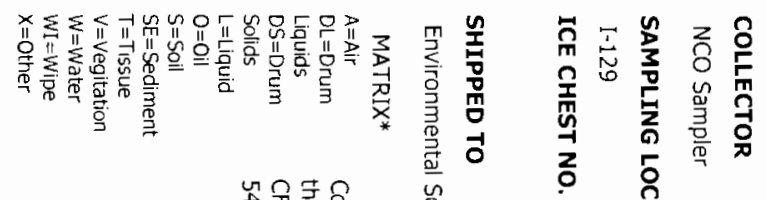

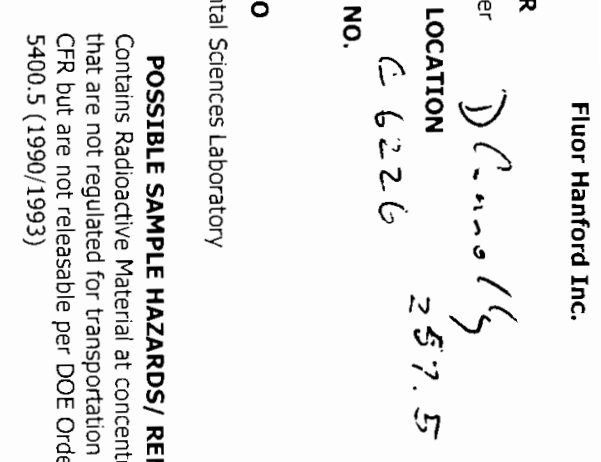

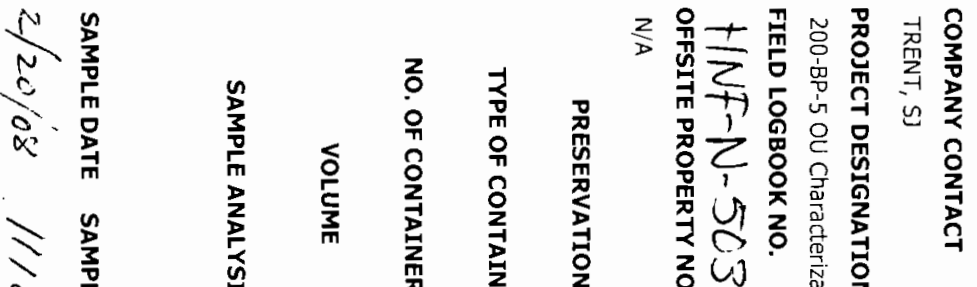

¿ $\frac{2}{\frac{2}{m}}$

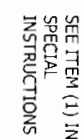<smiles>C#CCCCCCCCC#C</smiles> 


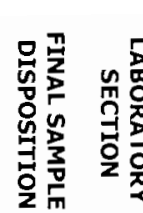

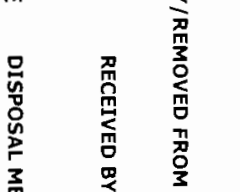

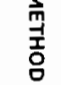

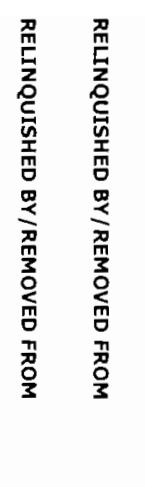

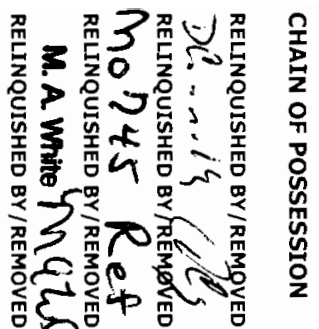

臂量

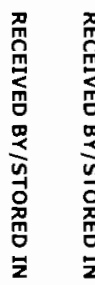

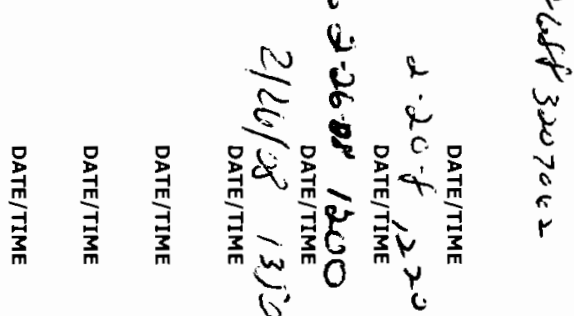

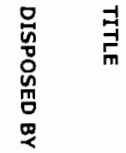

$\begin{array}{ll}\frac{0}{2} & \frac{g}{3} \\ \frac{1}{m} & \frac{1}{3} \\ \frac{1}{3} & \frac{1}{3} \\ \frac{m}{m}\end{array}$

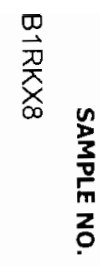

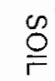

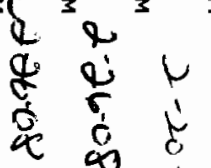

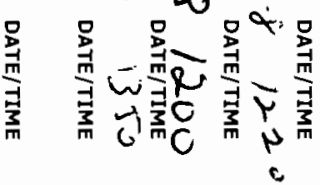

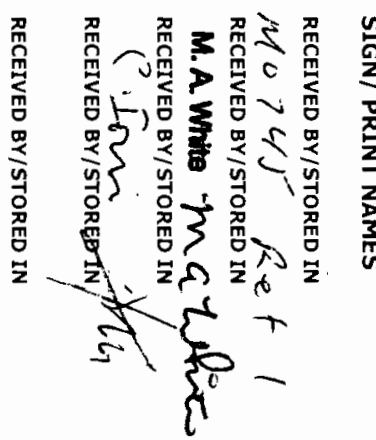<smiles>[Li][14CH3]</smiles>

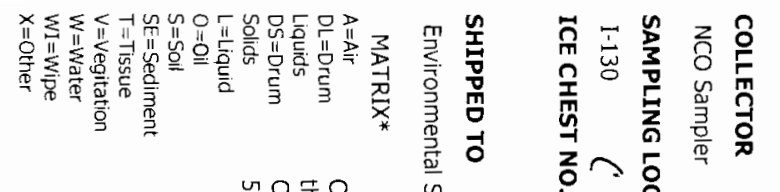

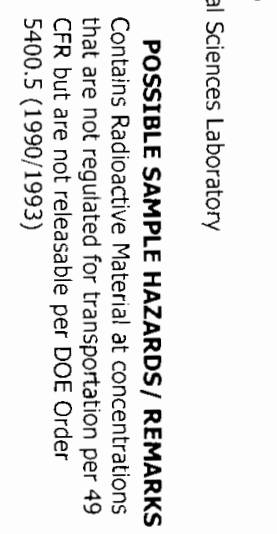

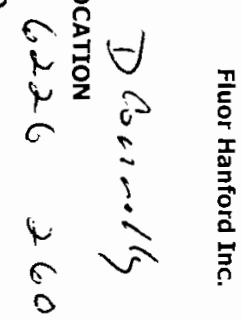

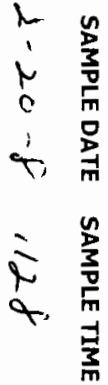

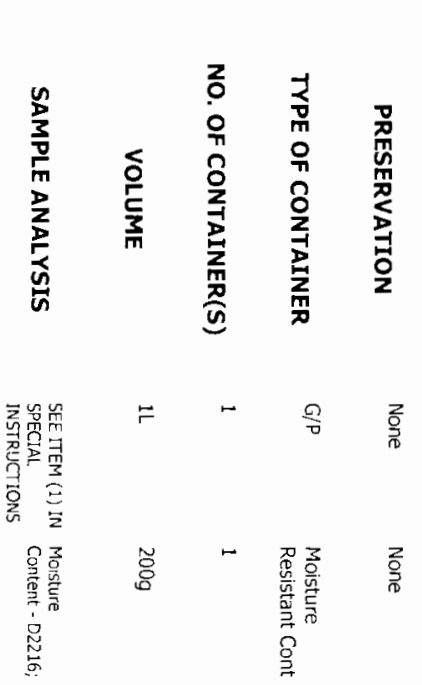

$z$ 우

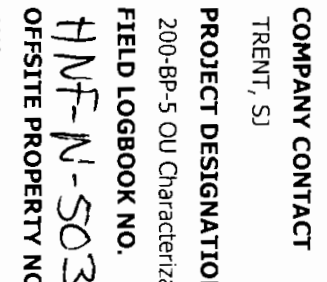

-W

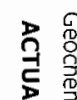

7 응

w

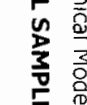

紊

脶 궁

就

营

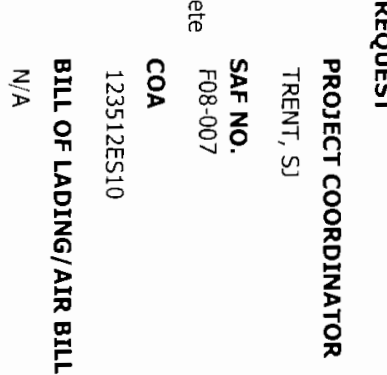

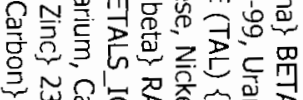

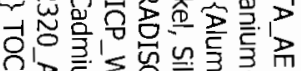

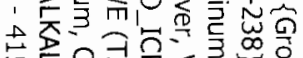

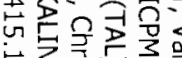

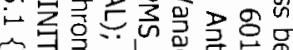

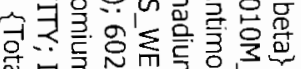

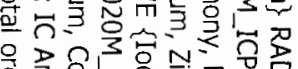

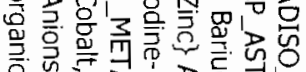

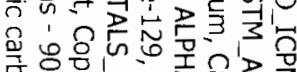

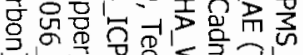

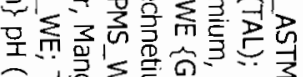

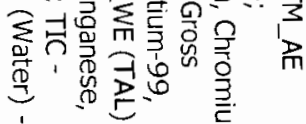



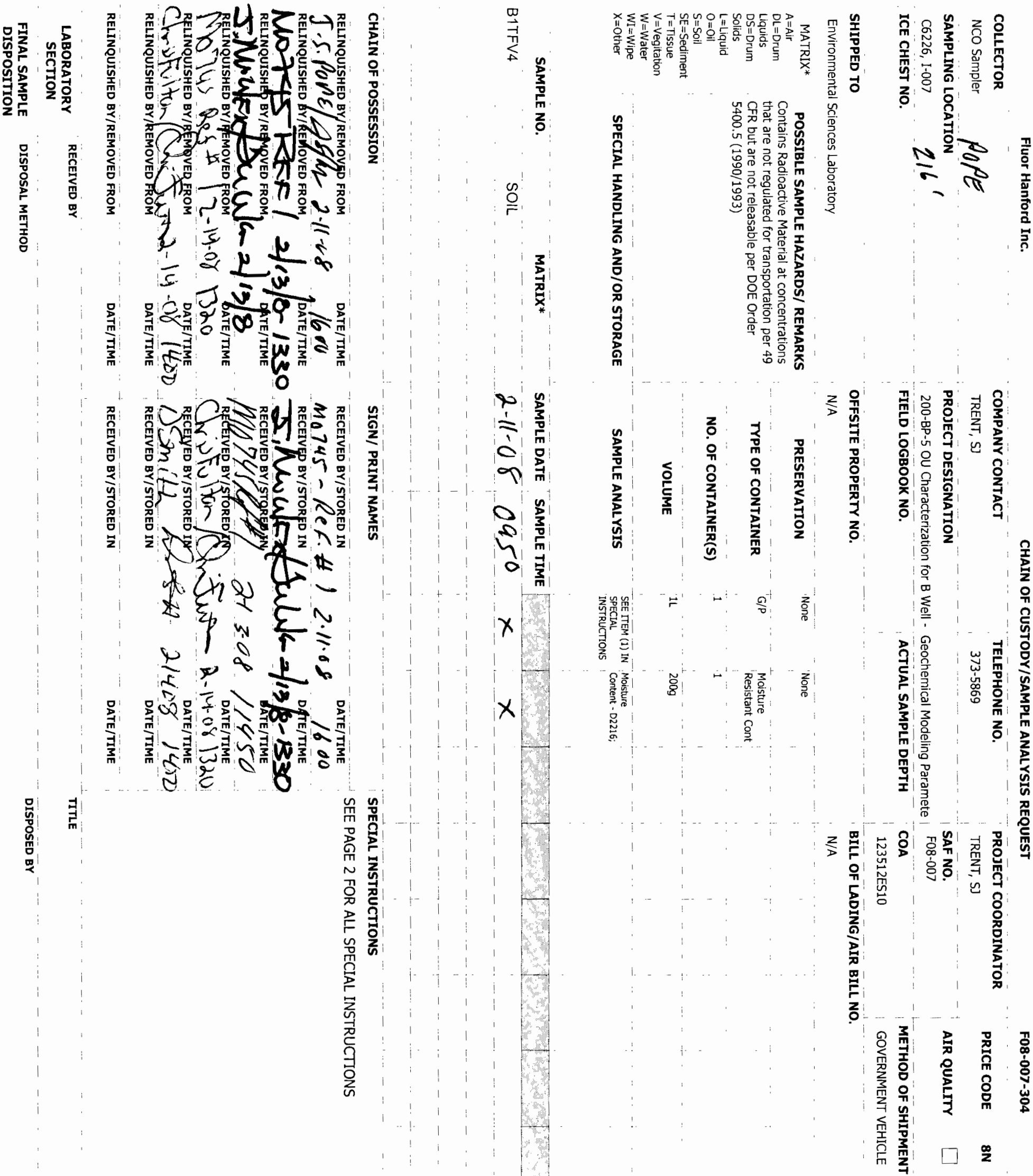

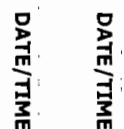

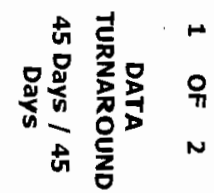




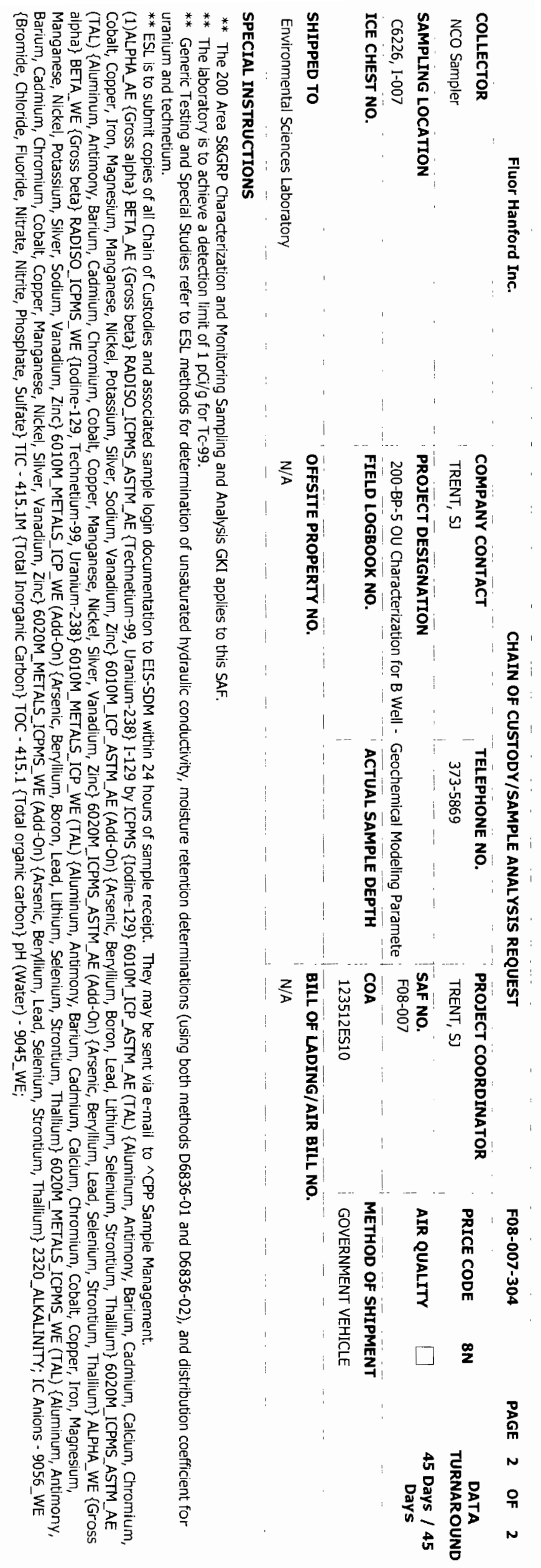




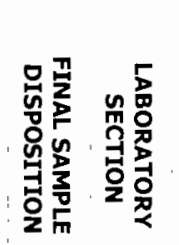

U:

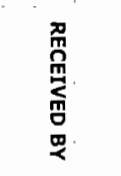

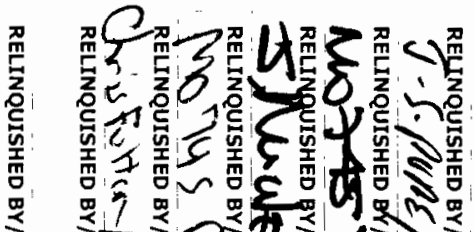

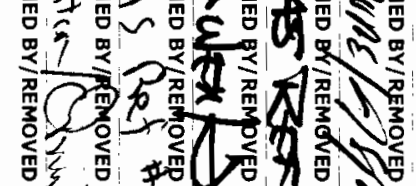

ग्र

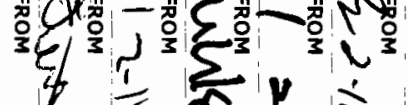

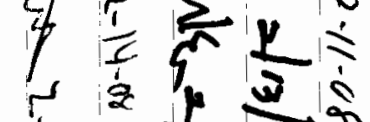

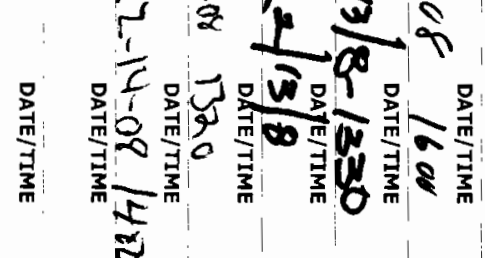

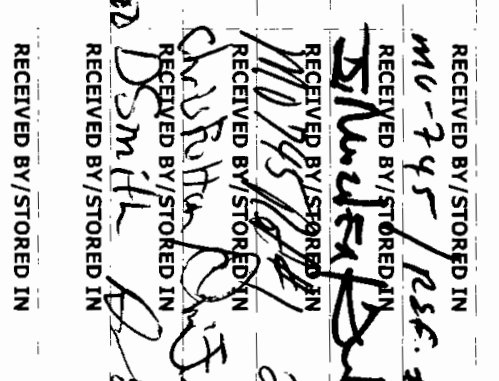

$2 \frac{1}{2}$ \&

$\frac{0}{n} \sum_{2}^{2}$

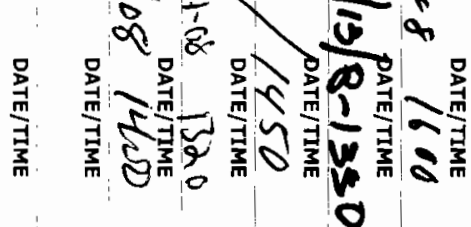

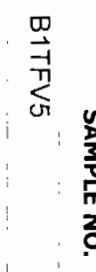

$\stackrel{0}{0}$

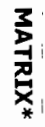

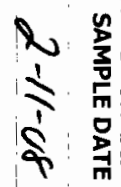

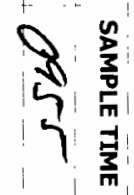

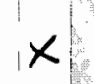

$\times$

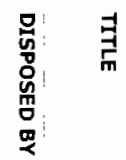

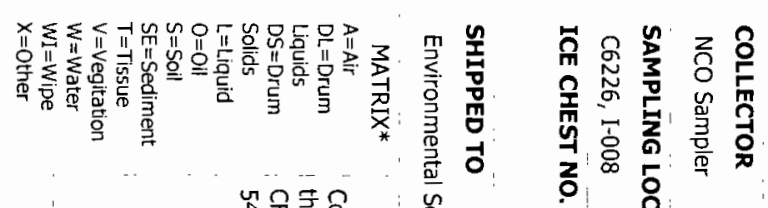

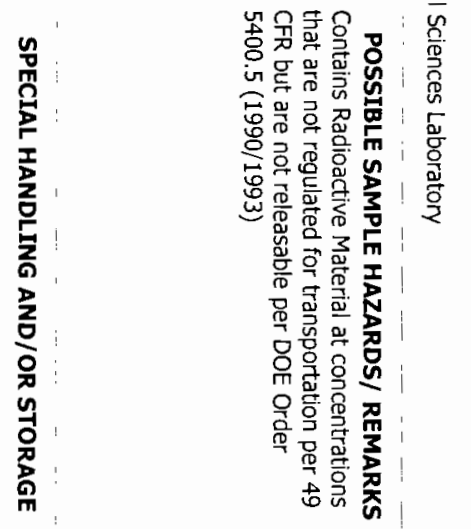

$\underbrace{2} \frac{2}{2}$

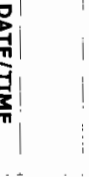

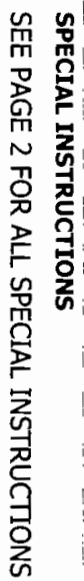

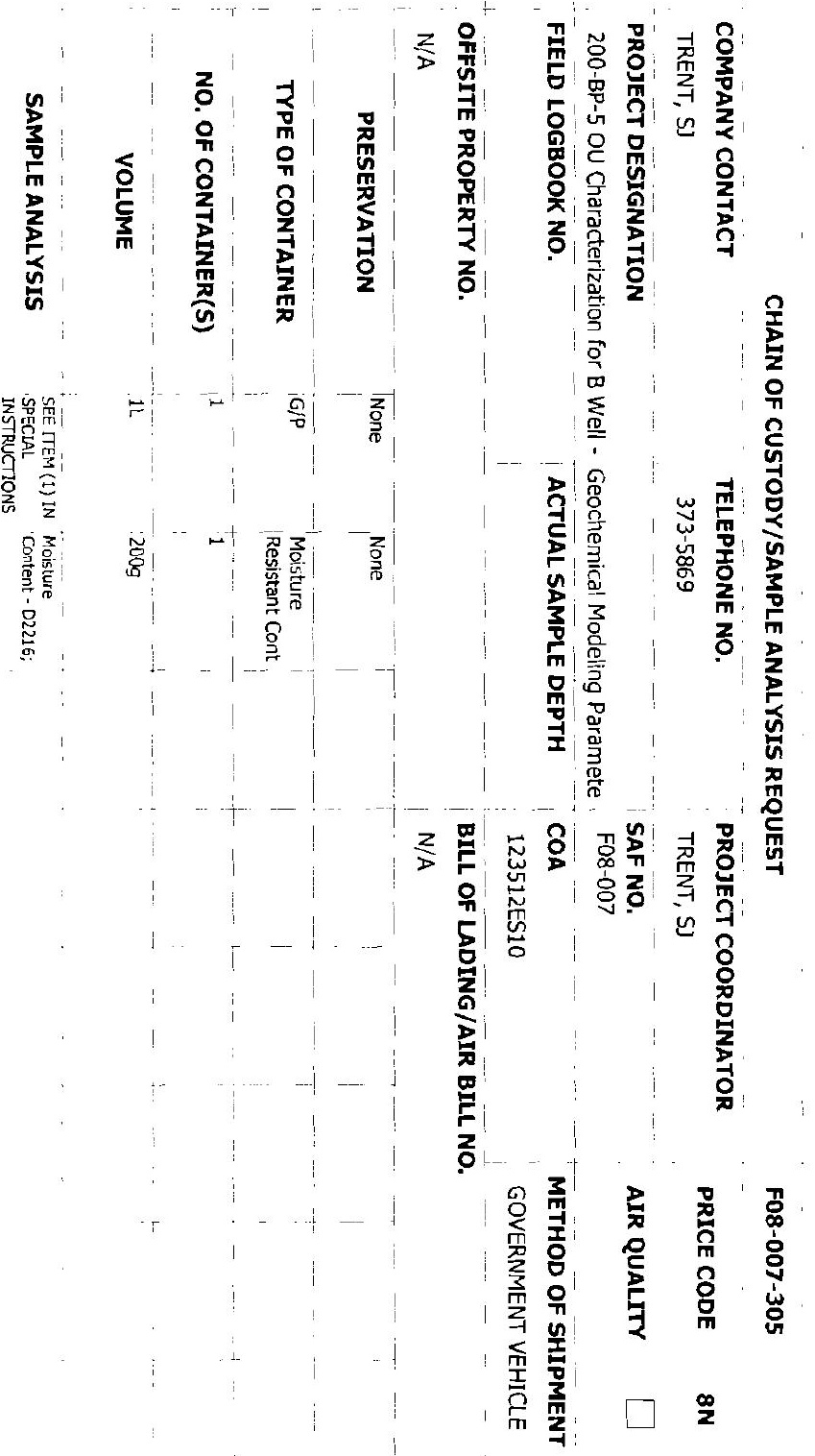




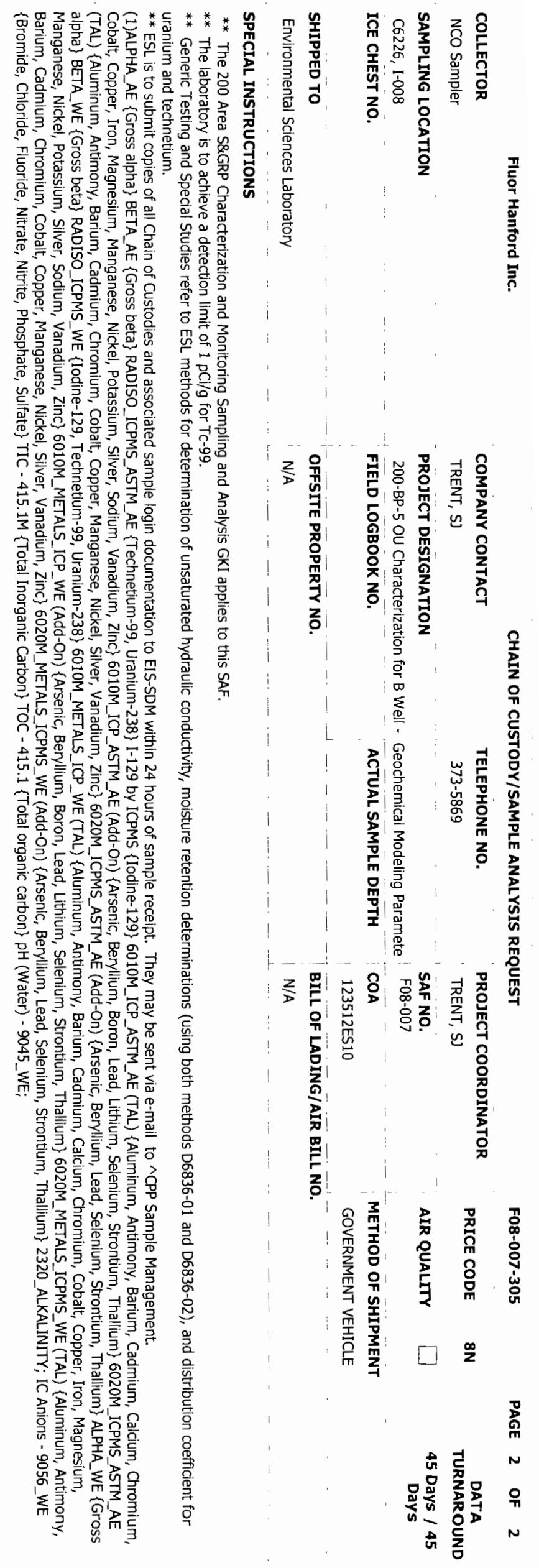



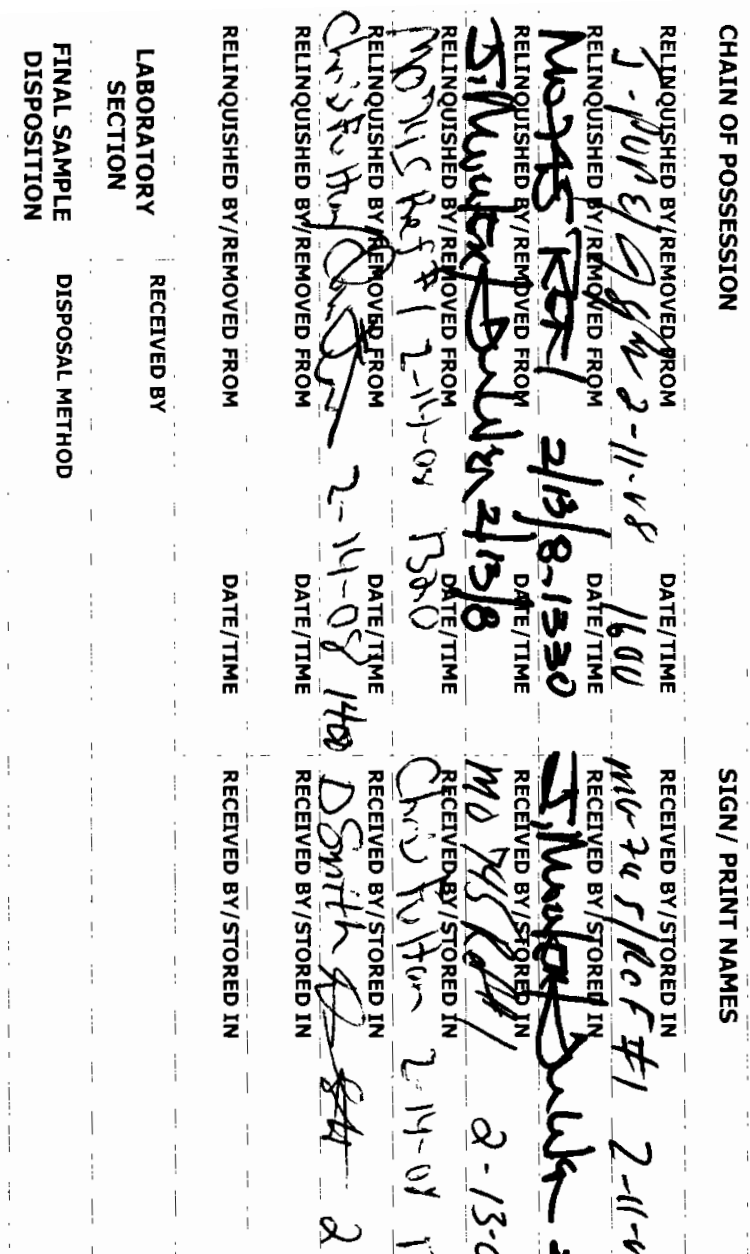

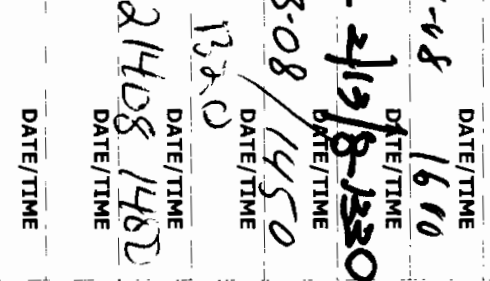

0
5
8
0
0

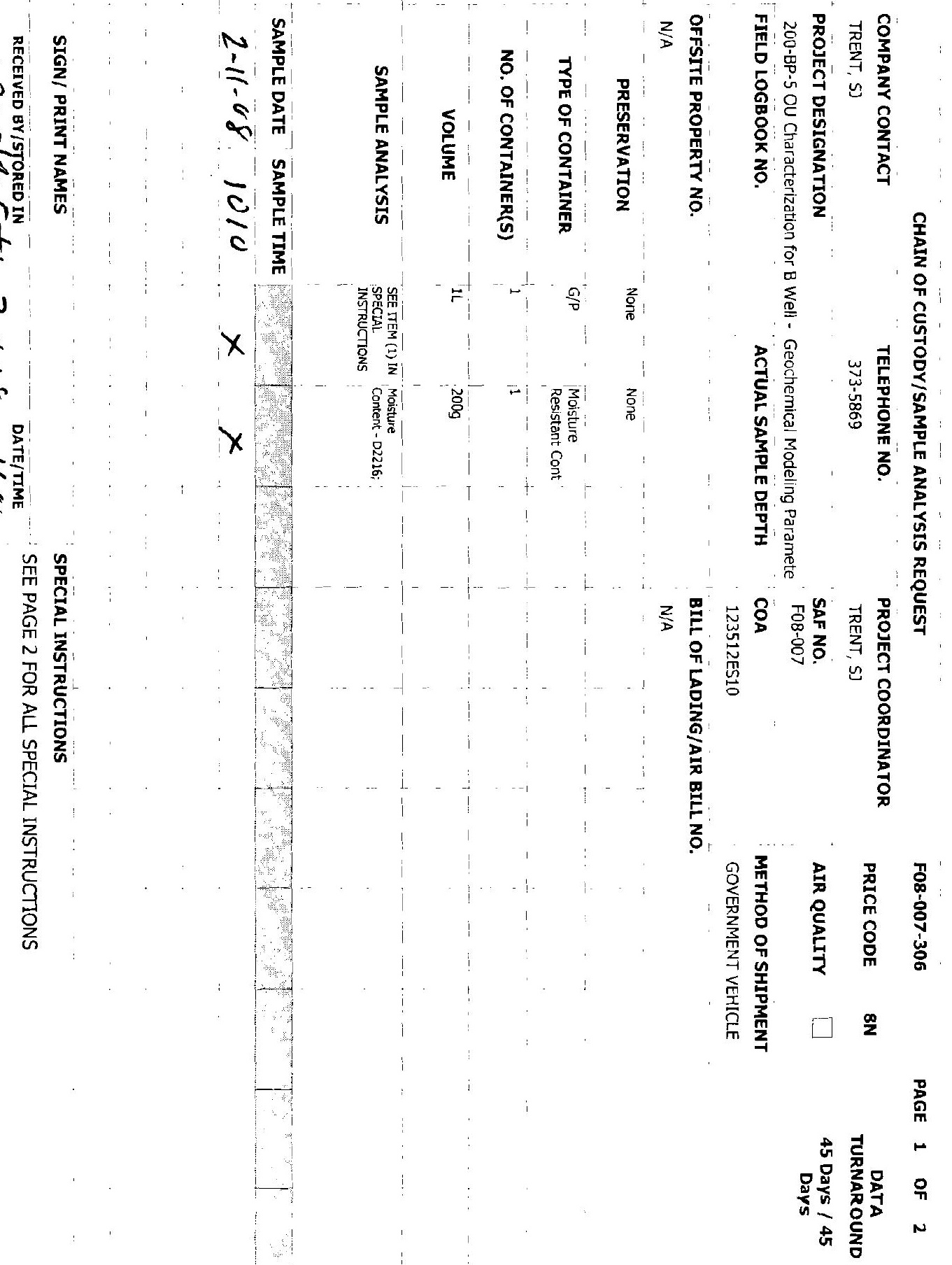

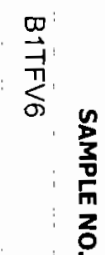

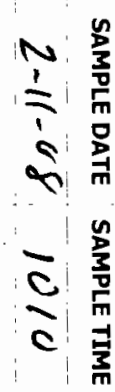

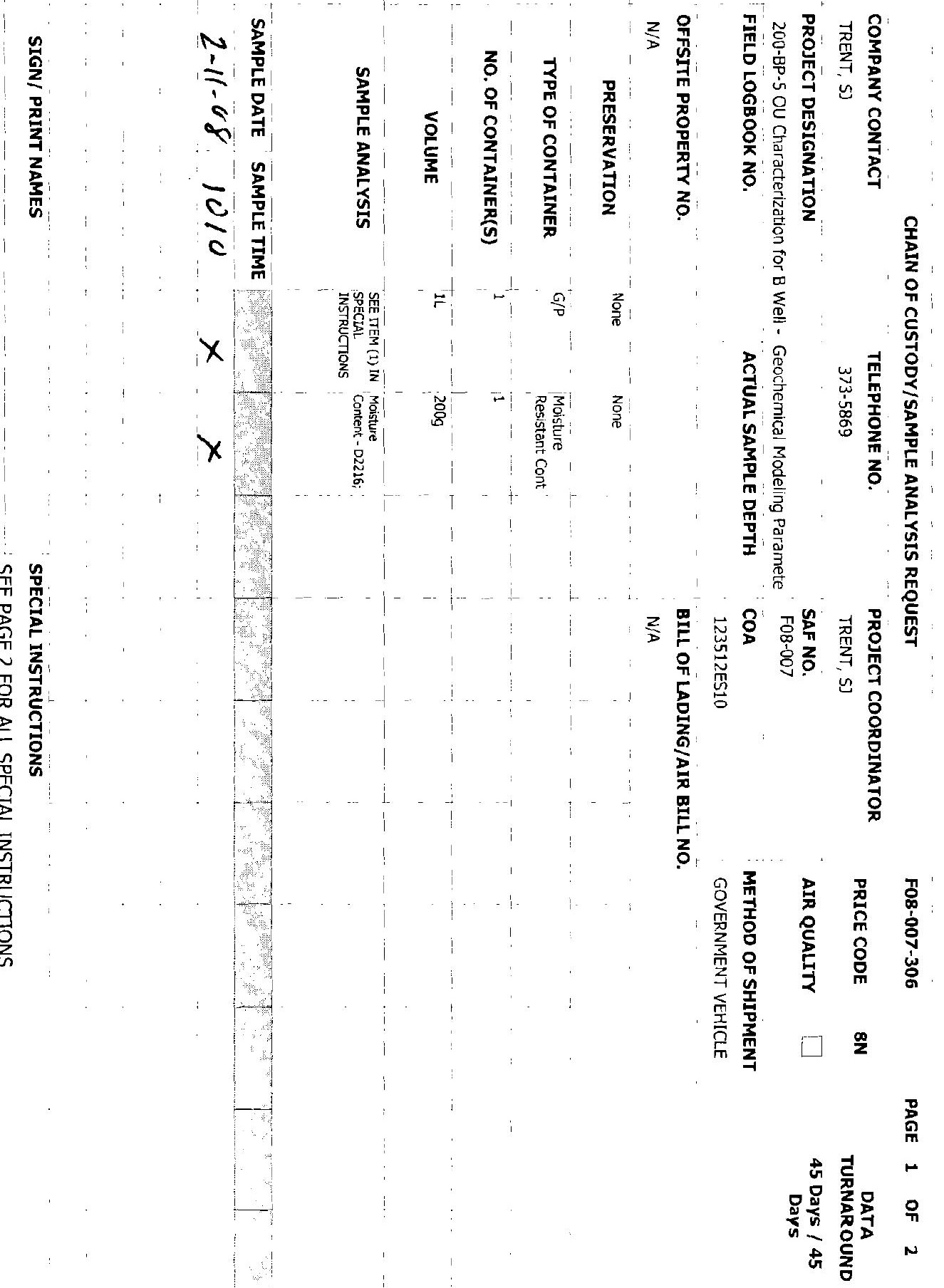

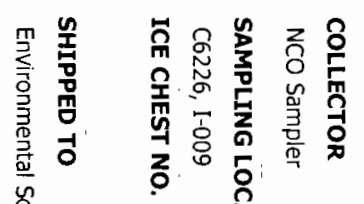

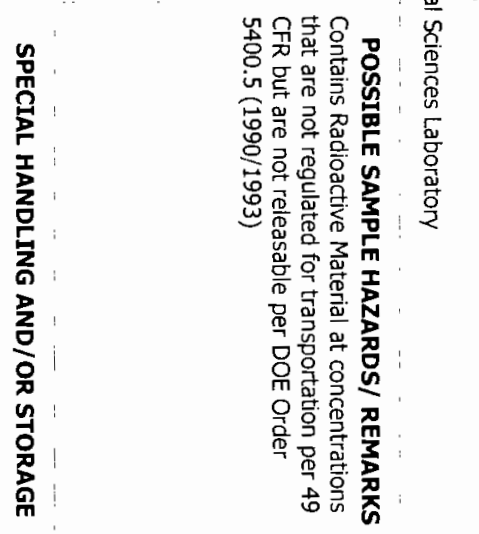

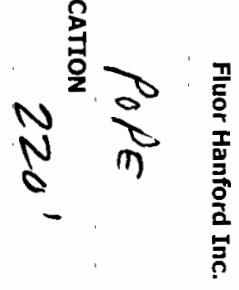




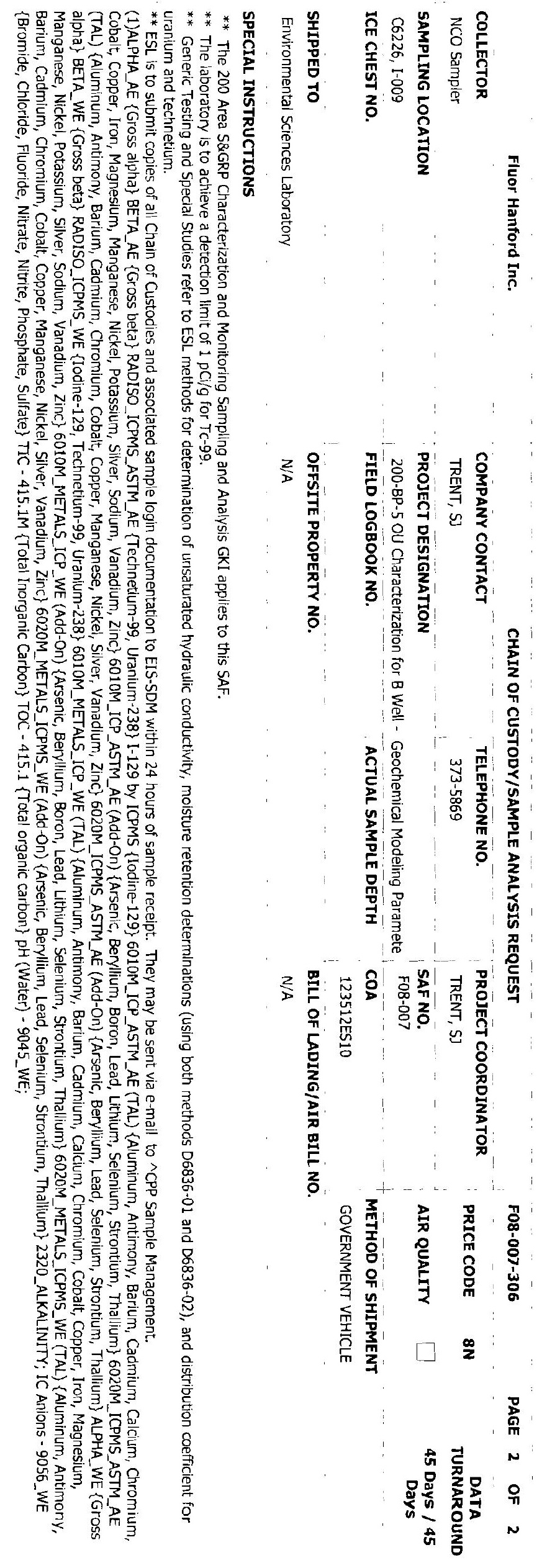


焉

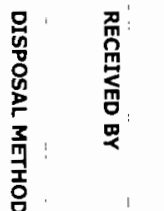

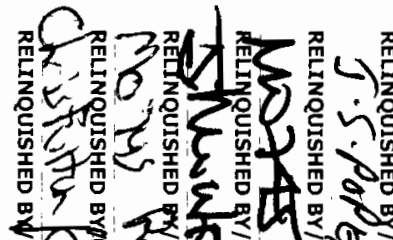

确 $\frac{\pi}{3} \pi_{0}^{\pi}$

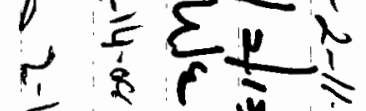

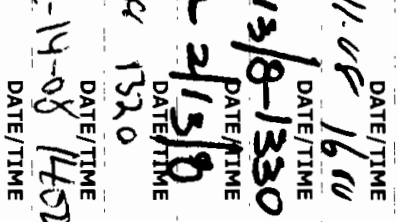

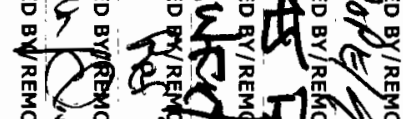

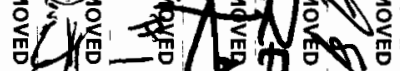

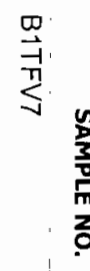

$\stackrel{\mathscr{O}}{\stackrel{\circ}{ }}$

$\frac{3}{3}$

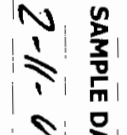

की

$=\frac{2}{2}$

X

岳

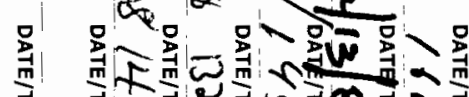

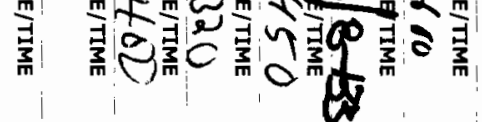

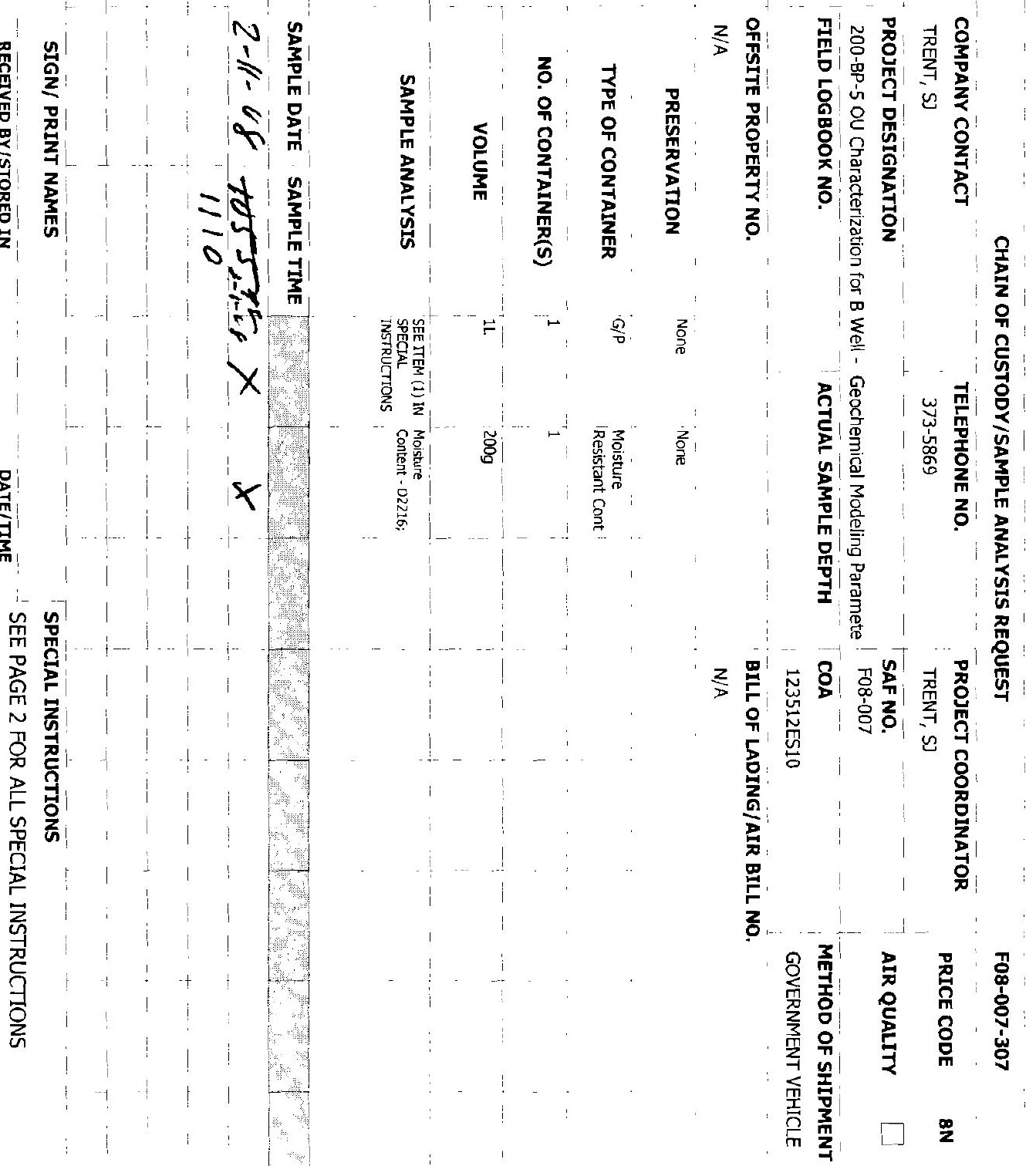

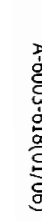

号兴 


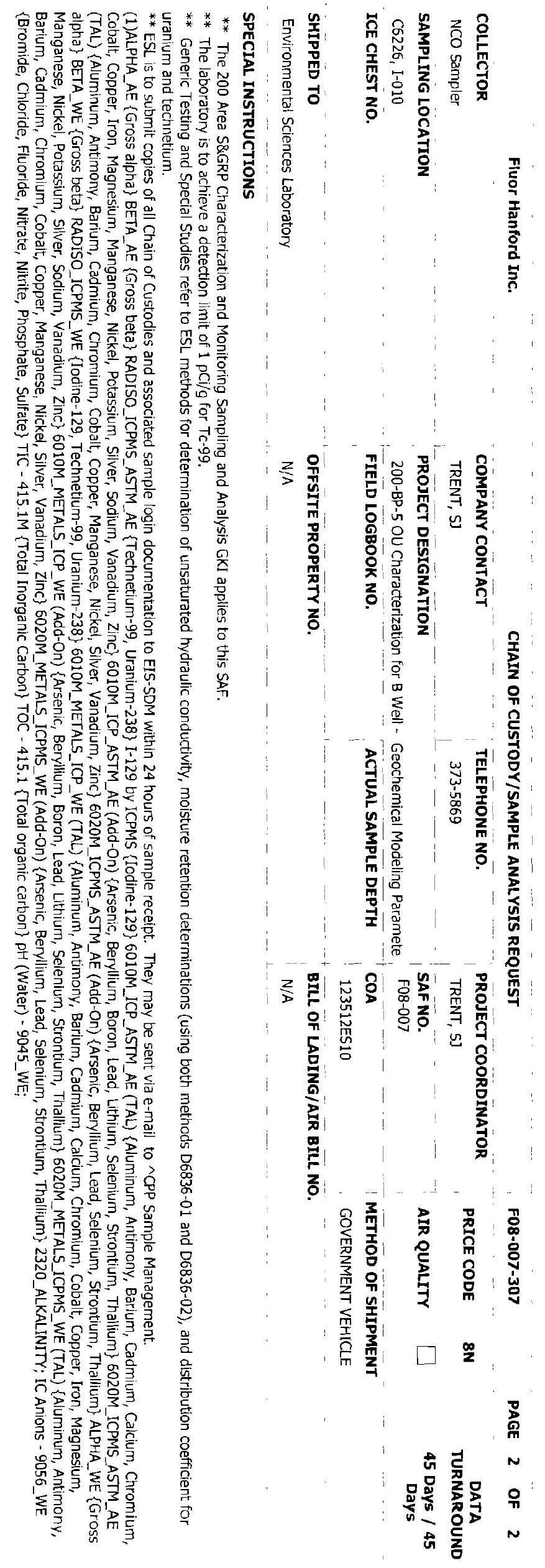




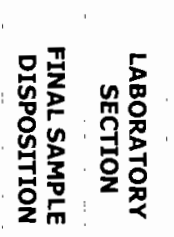

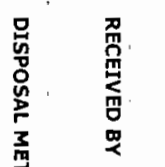

w
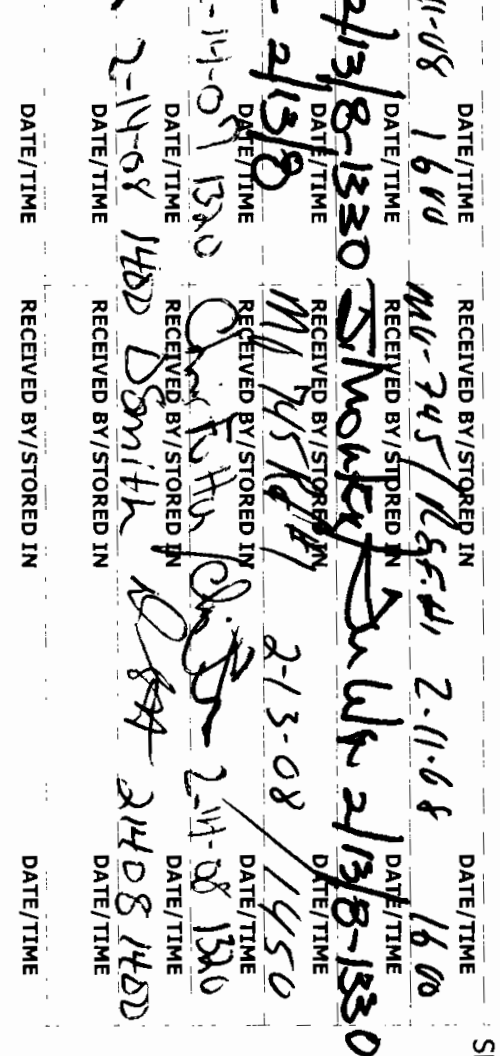

옹

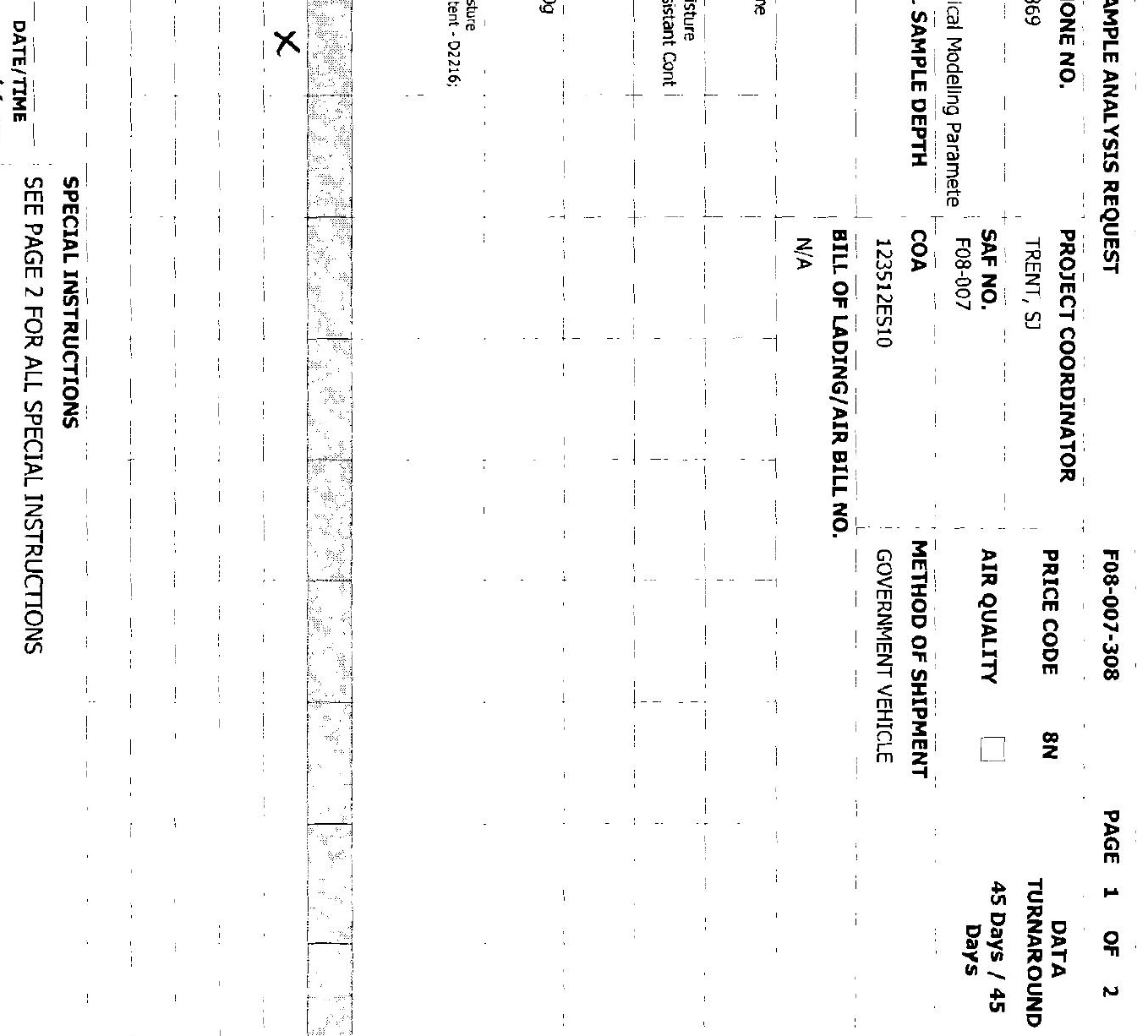

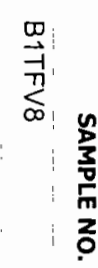

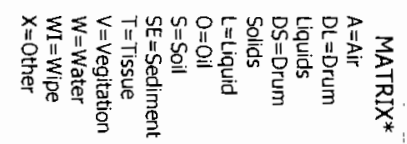

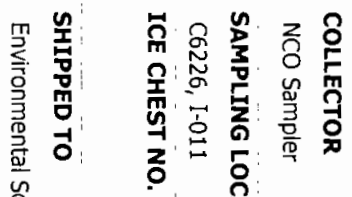

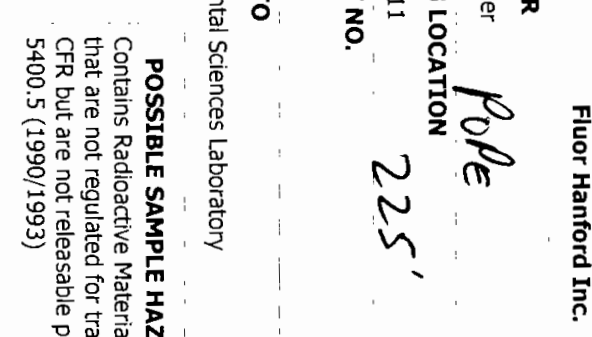

疍

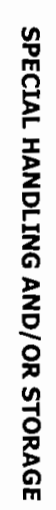

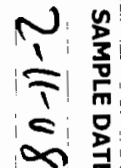

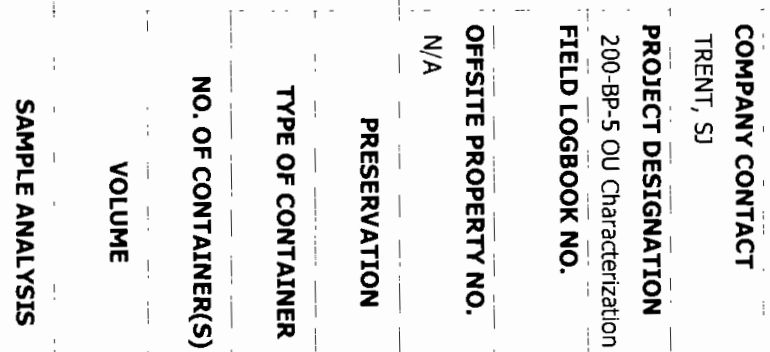

年

$x$

$x$

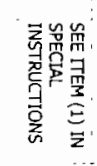

售

m.

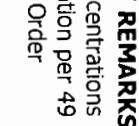

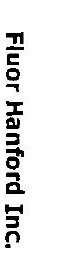




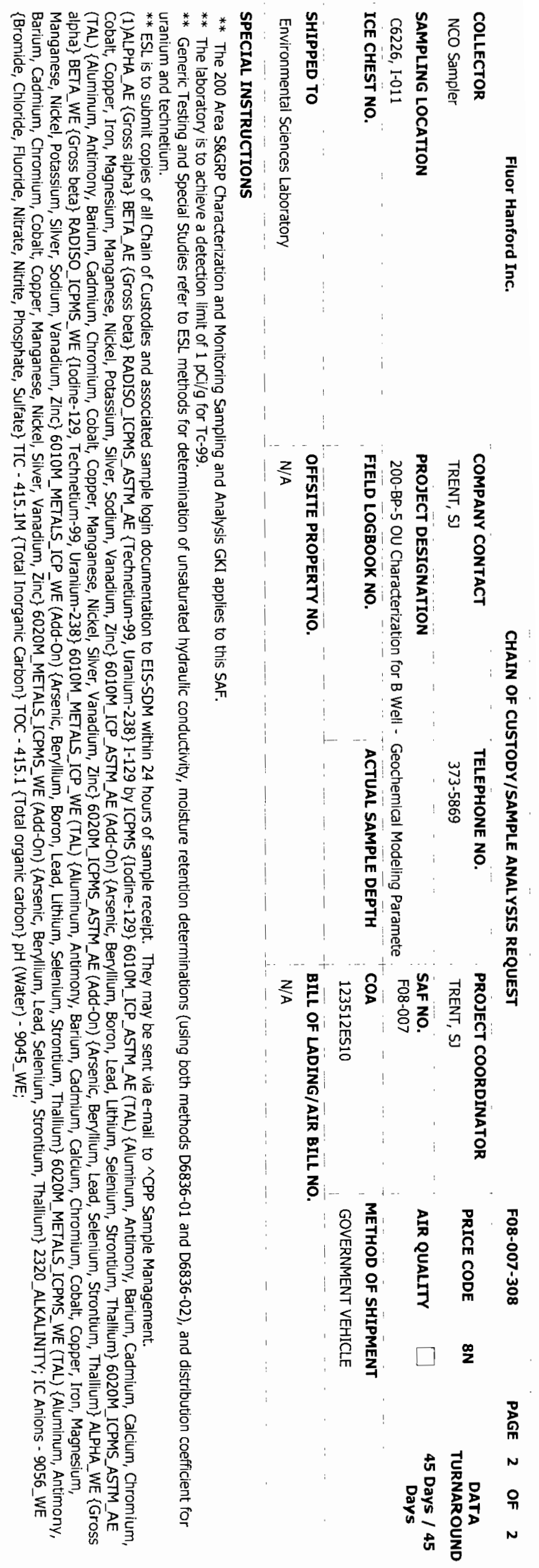




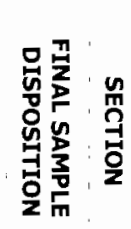$$
\text { . }
$$

量 


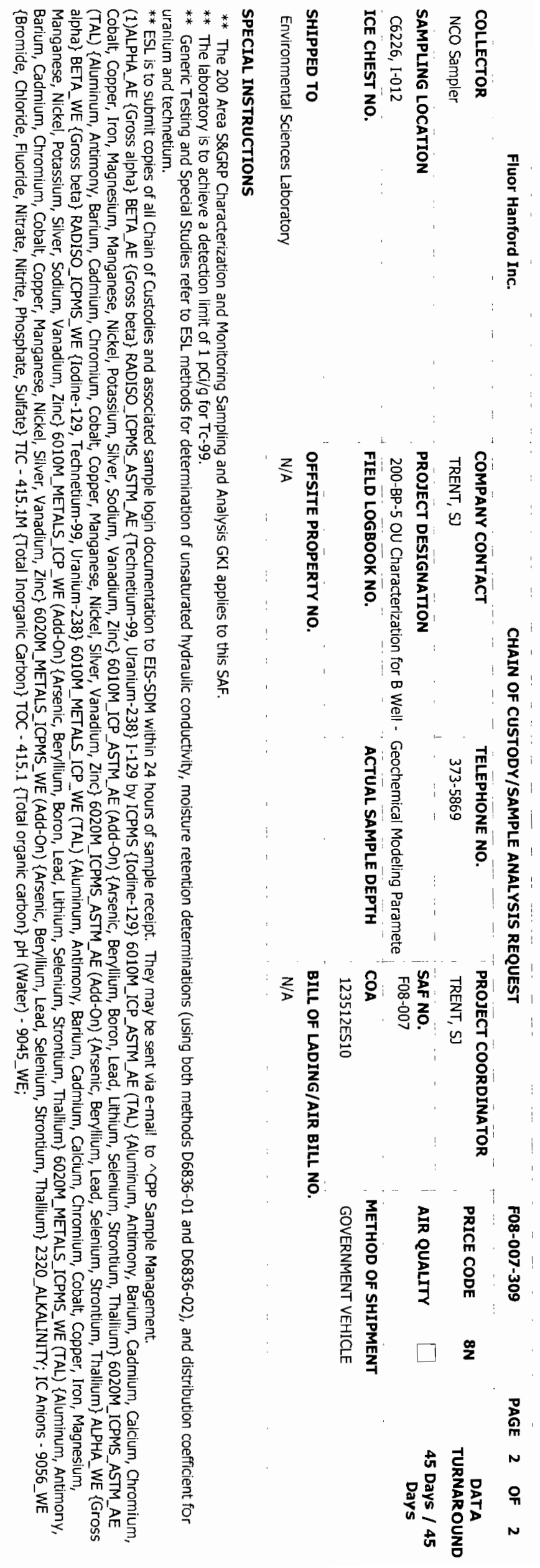




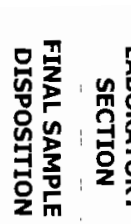

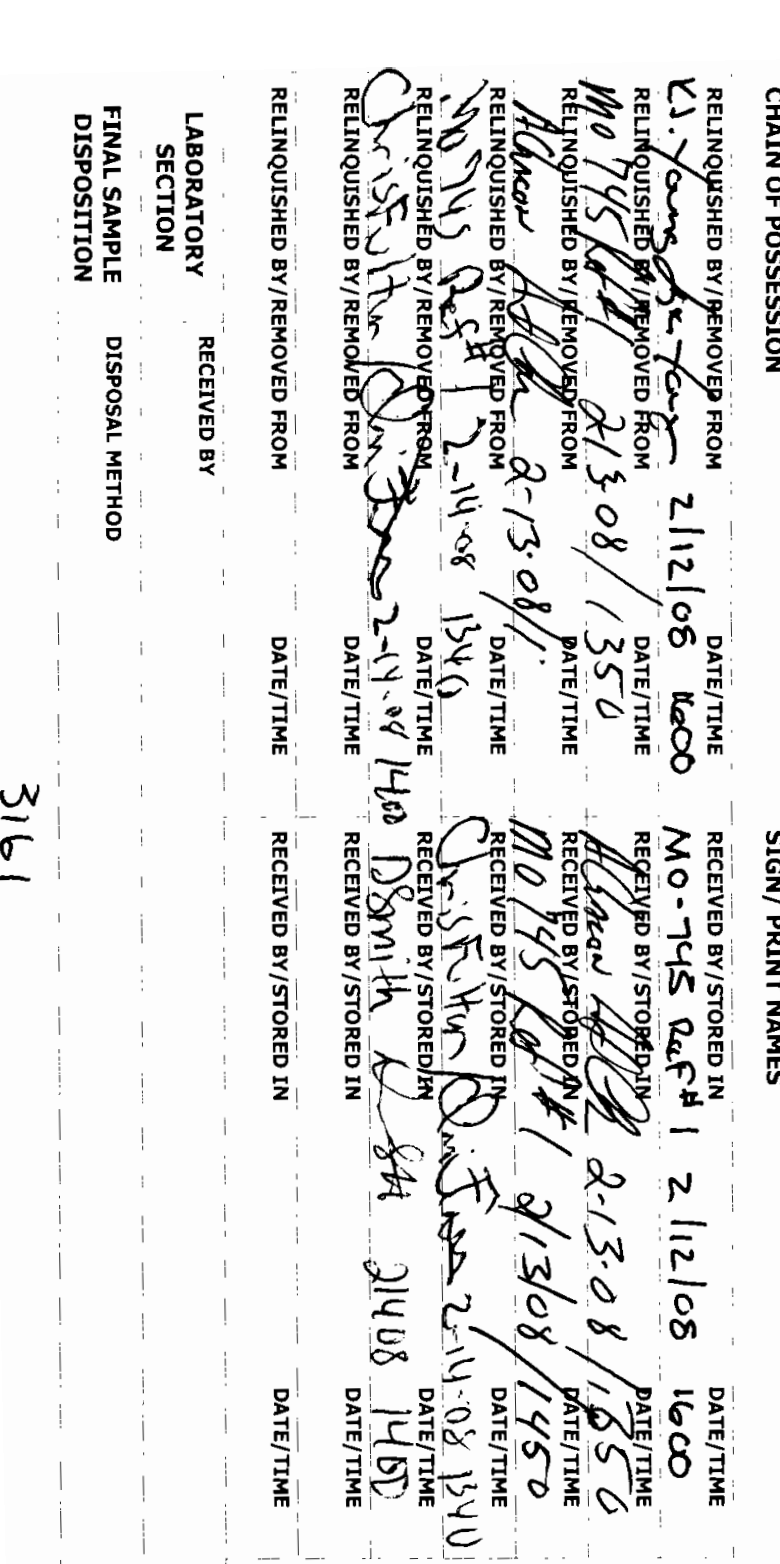

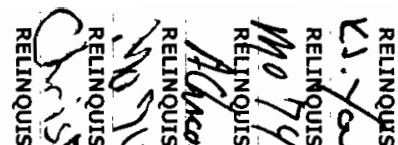

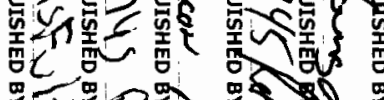

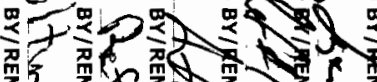

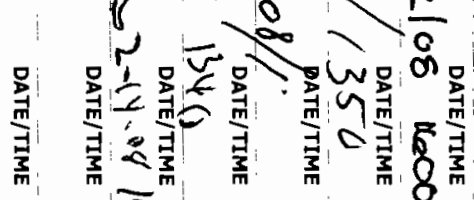
$\frac{T}{3}$

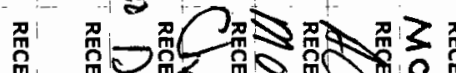
解

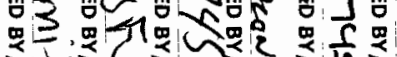
y

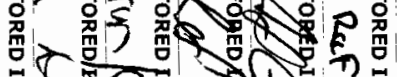
$=62 N=$

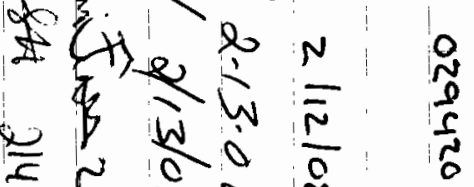

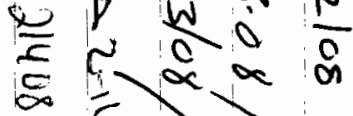
竞
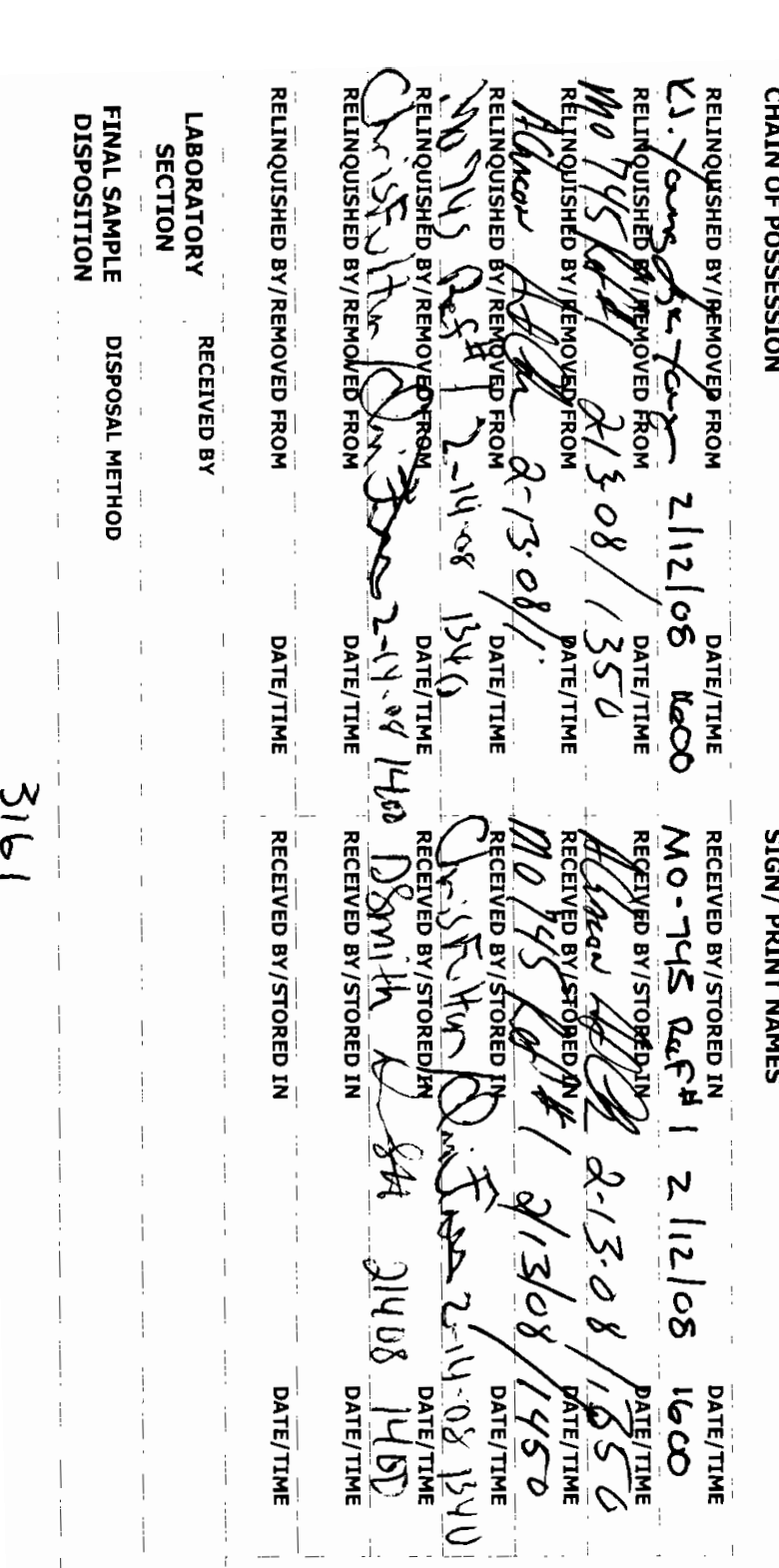

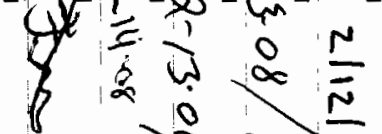

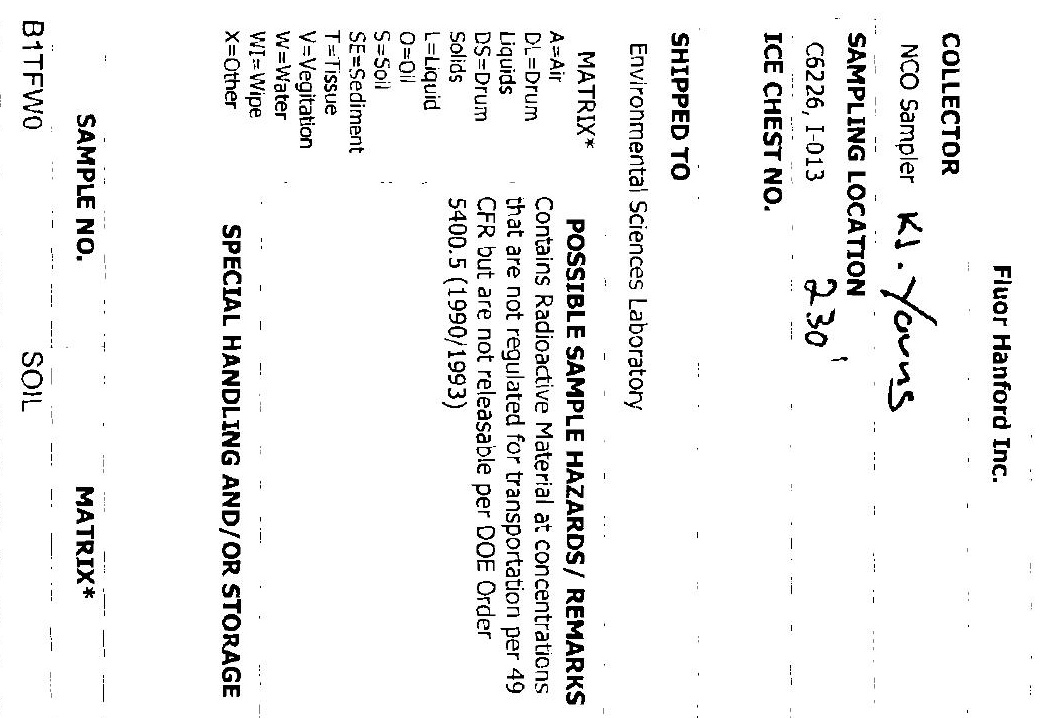

要

空

욲 공

긍

号

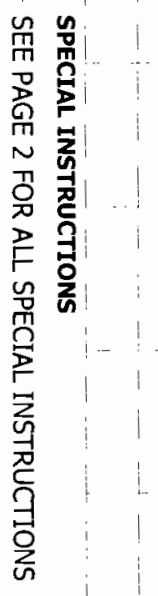

5
5

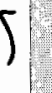

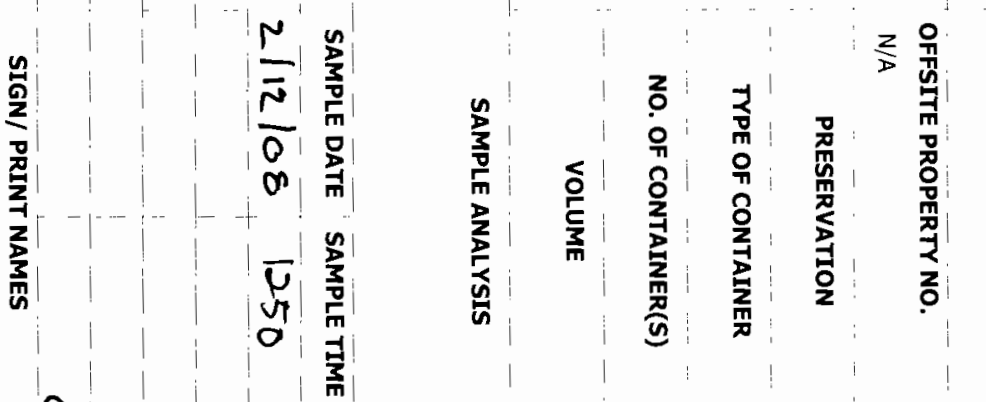

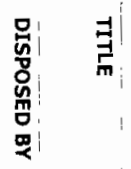
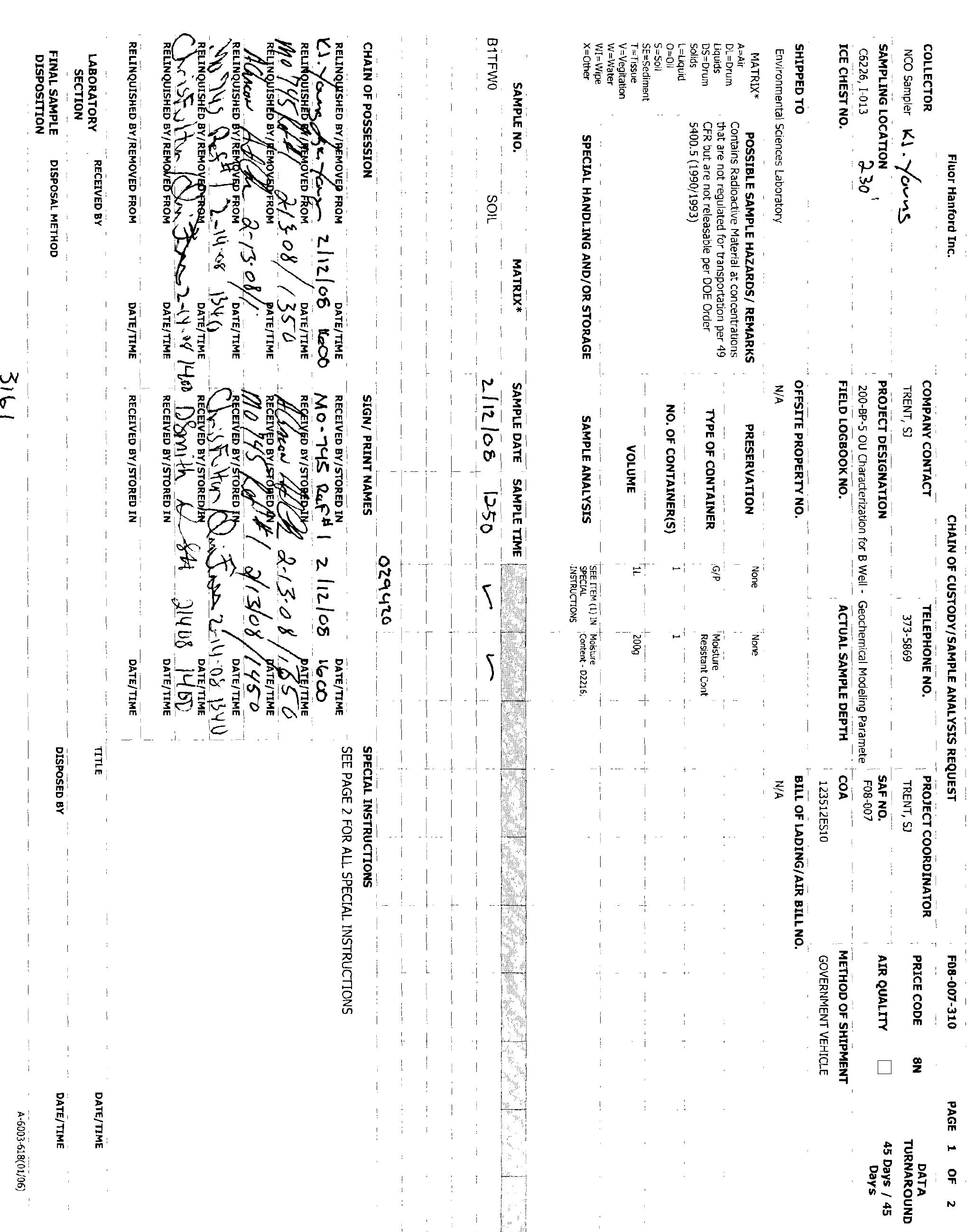

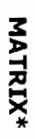

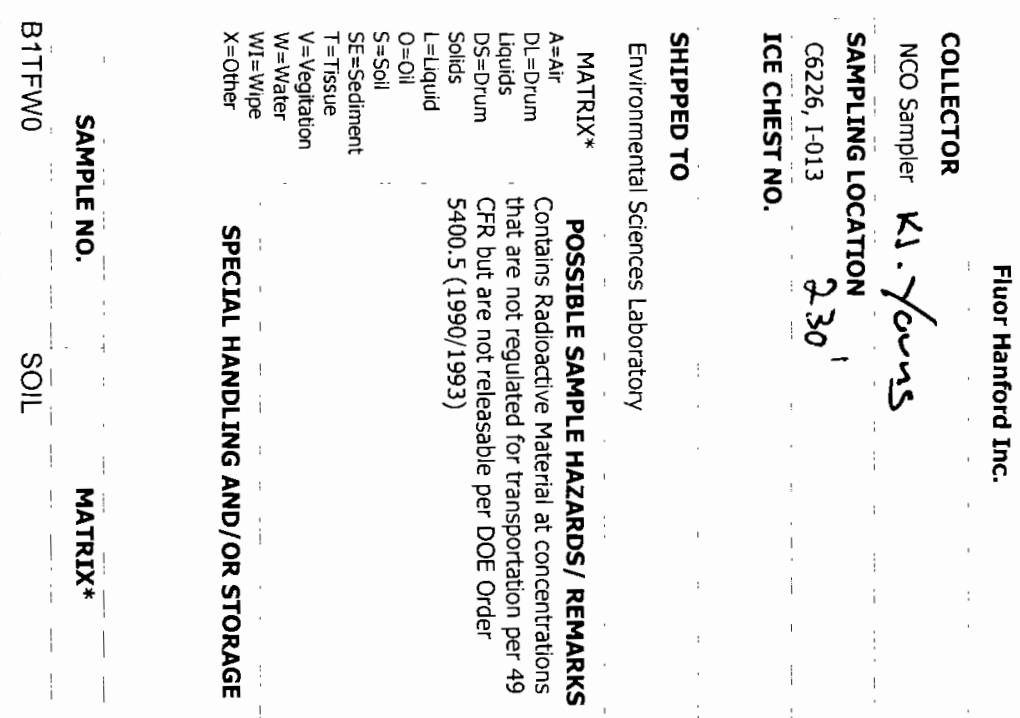

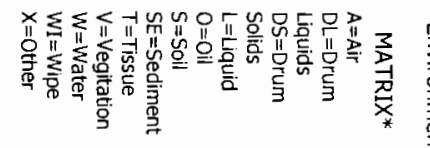

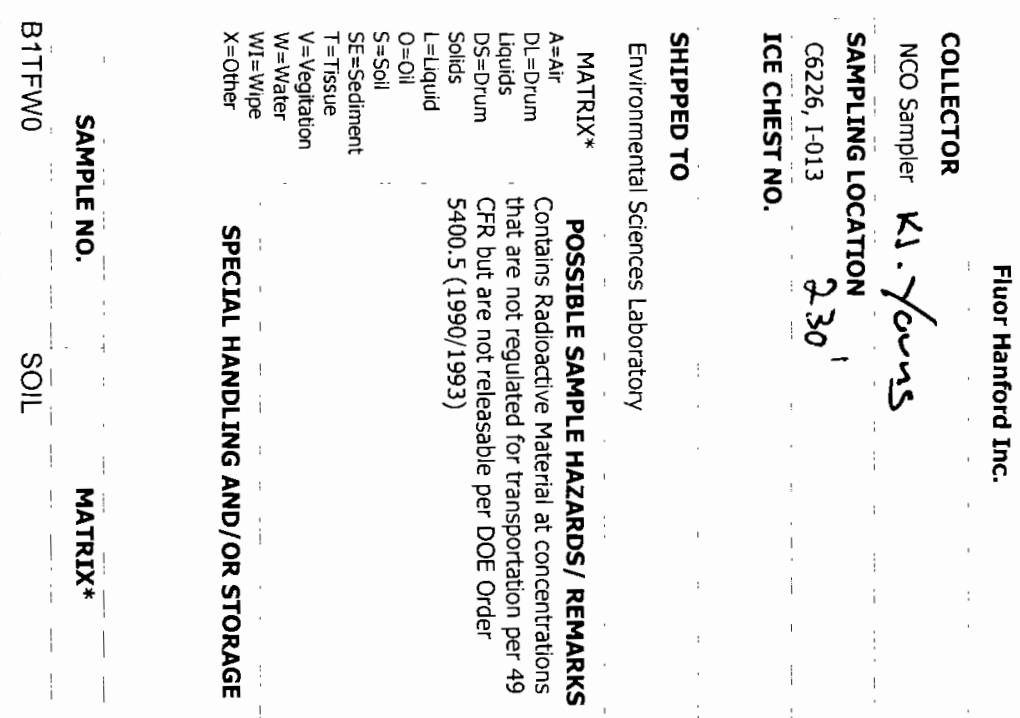

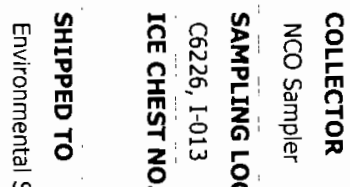
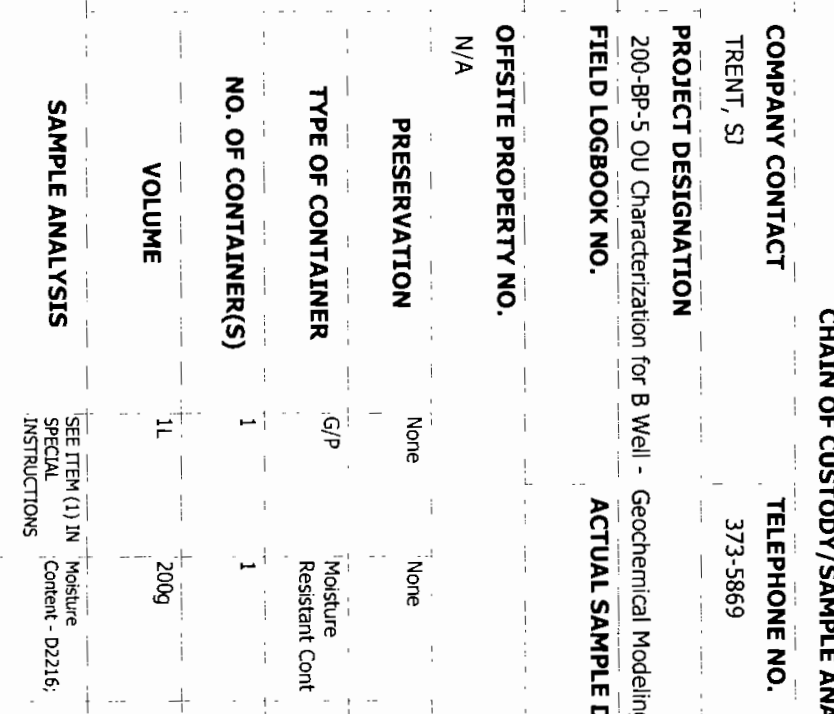

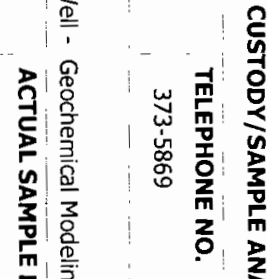
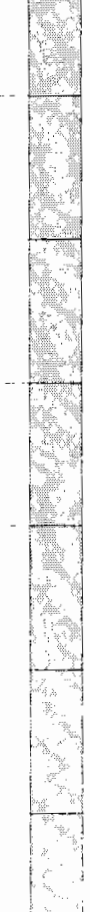

0
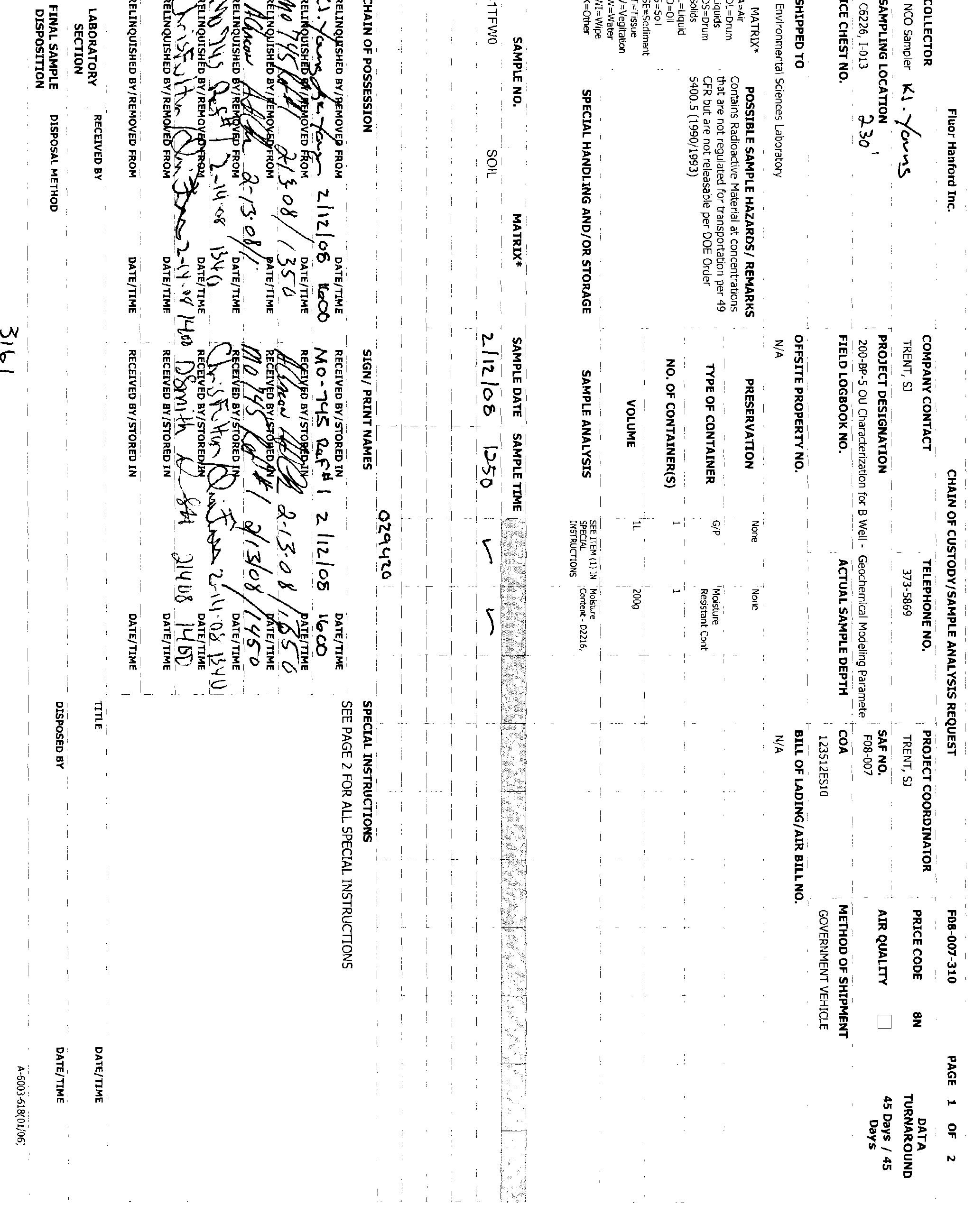


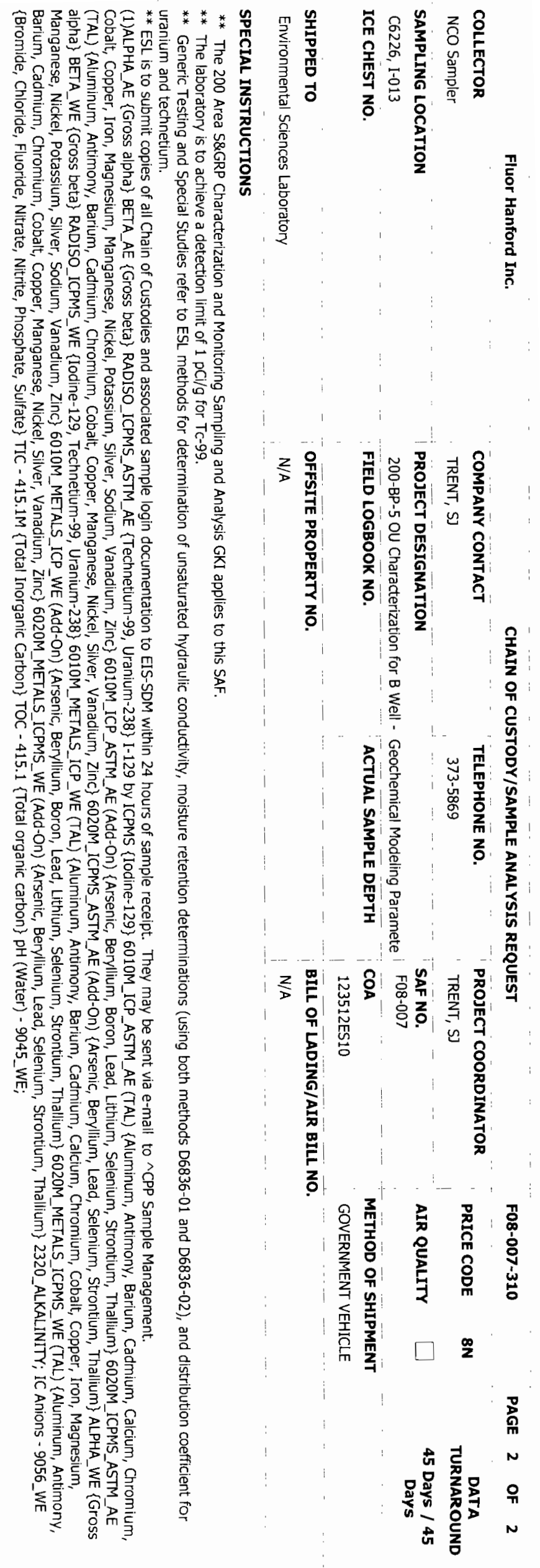




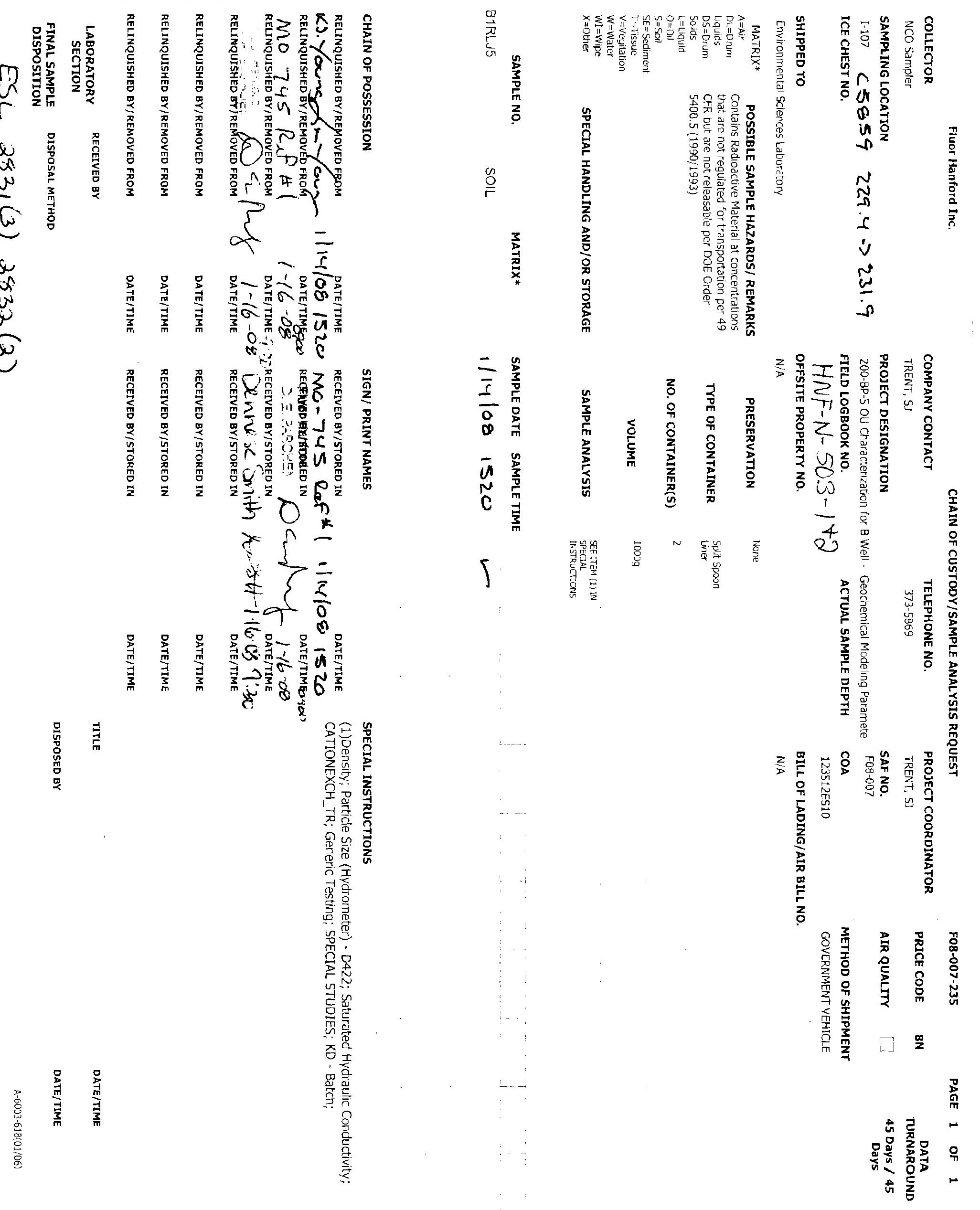




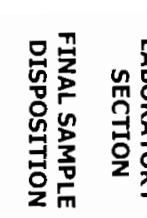

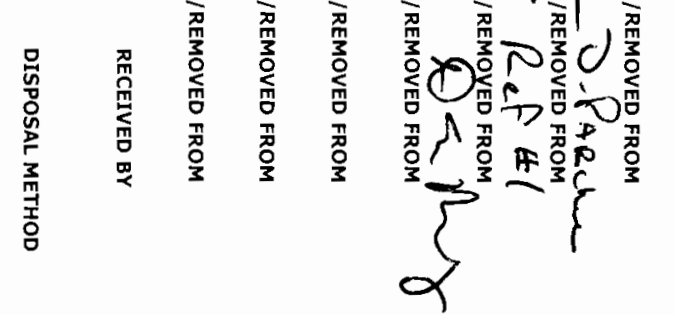

量兾产

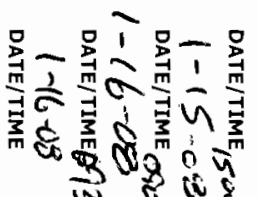
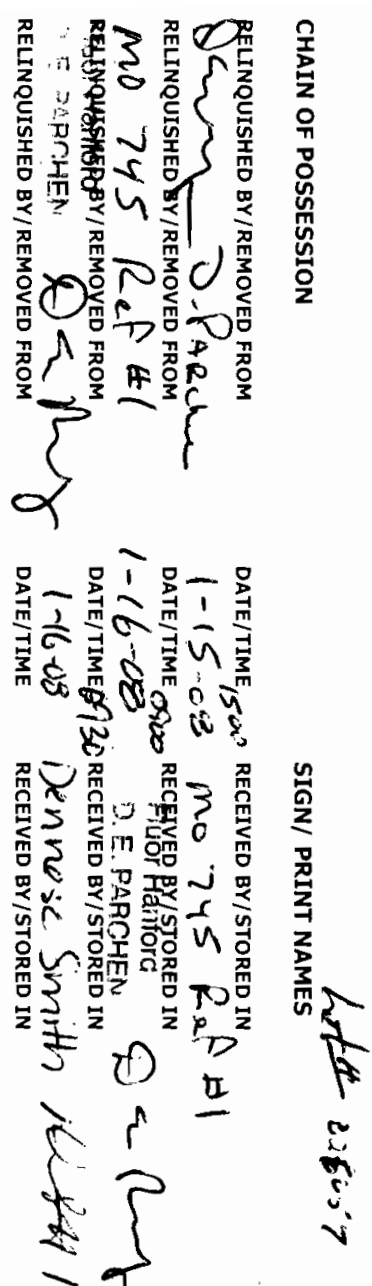

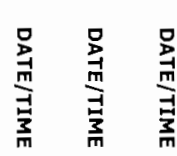

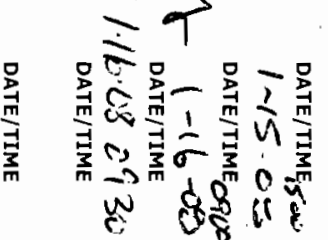

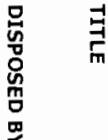

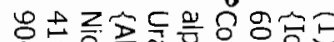

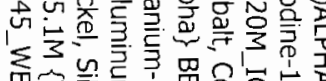

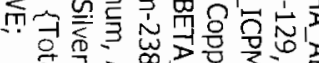

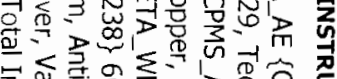

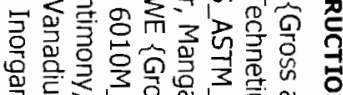

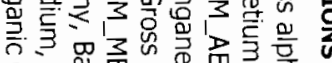

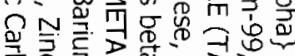

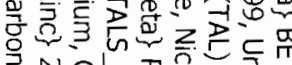

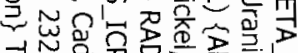

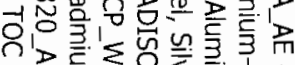

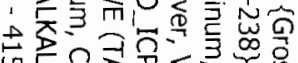

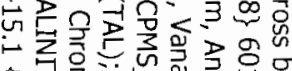

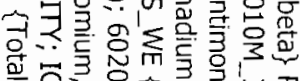

응

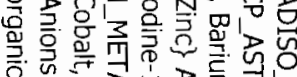

品

3.

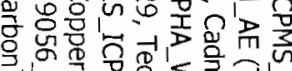

$\stackrel{\infty}{\stackrel{0}{\Gamma}}$

$\underset{*}{\stackrel{3}{3}}$

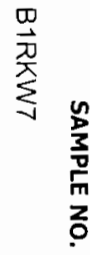

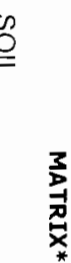

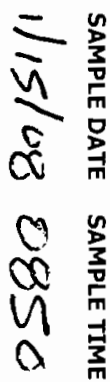
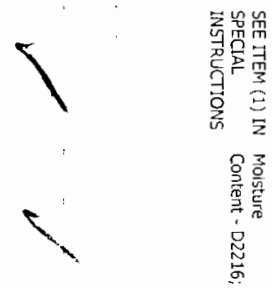

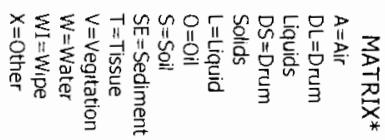

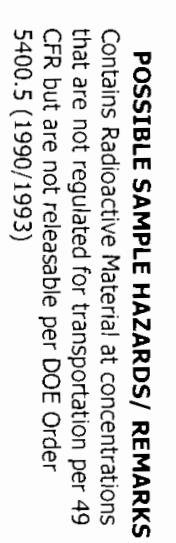

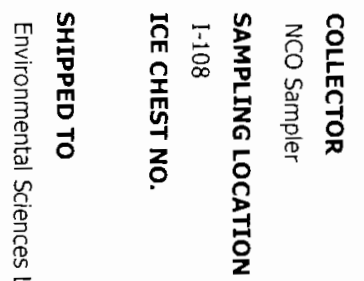

is

W

U

$v$

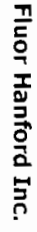

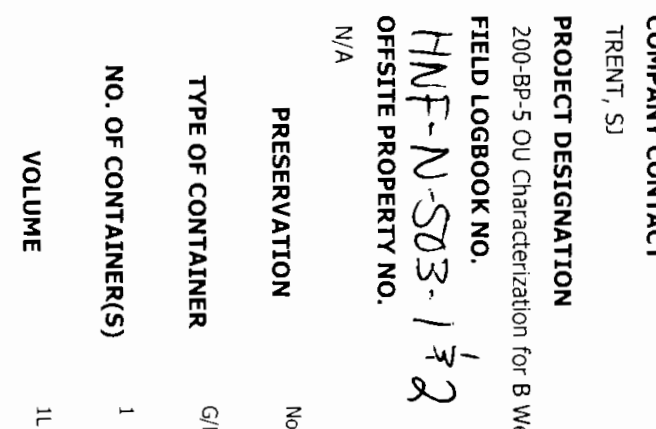

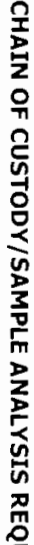

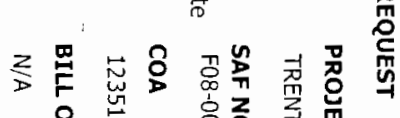

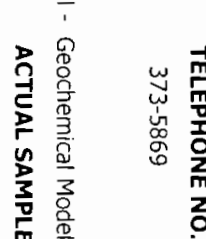

is

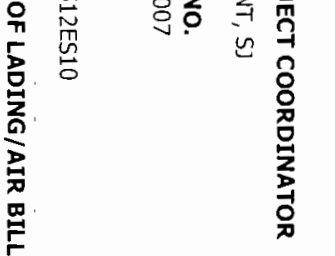

zo

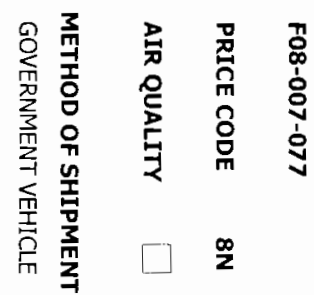

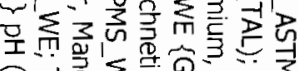

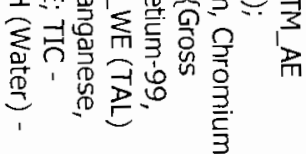




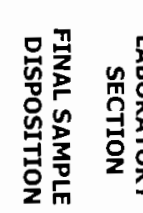

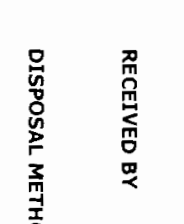

$\pm$

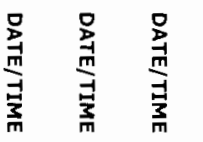

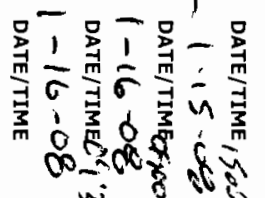

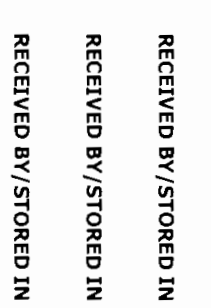

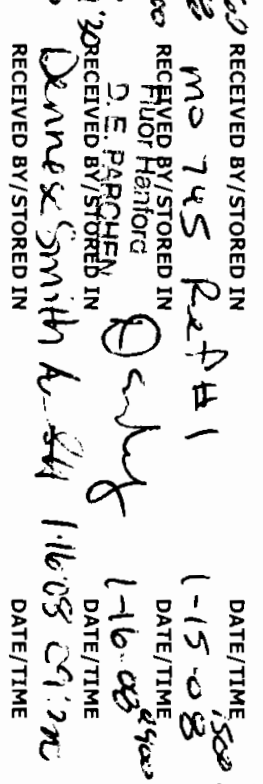

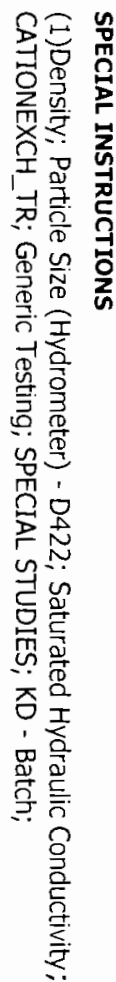

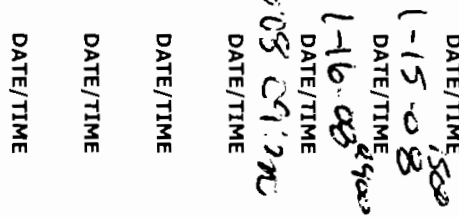

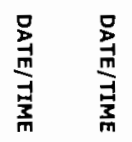

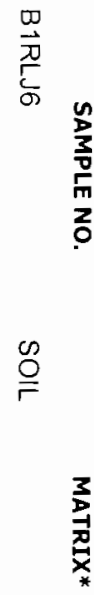

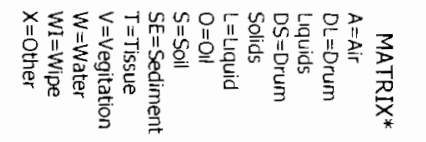

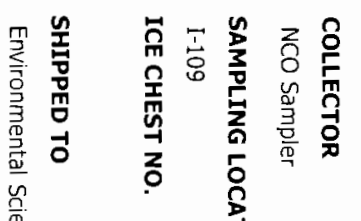

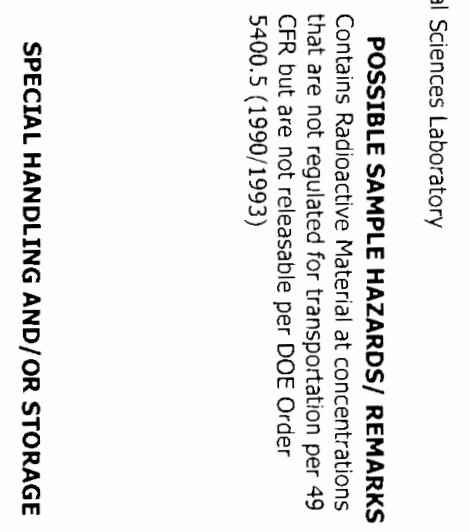

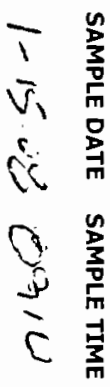

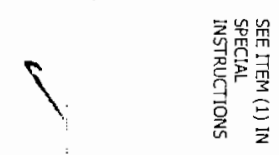

$u_{0}^{\infty}$

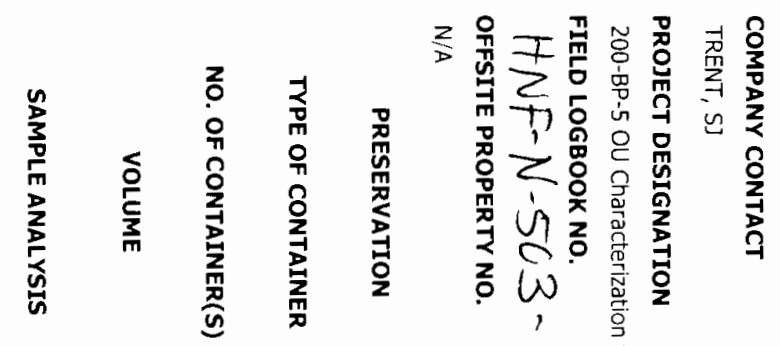

ए

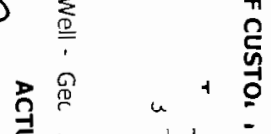

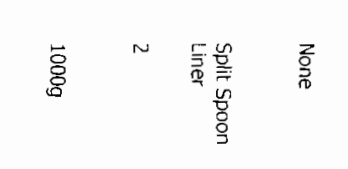

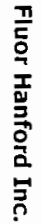
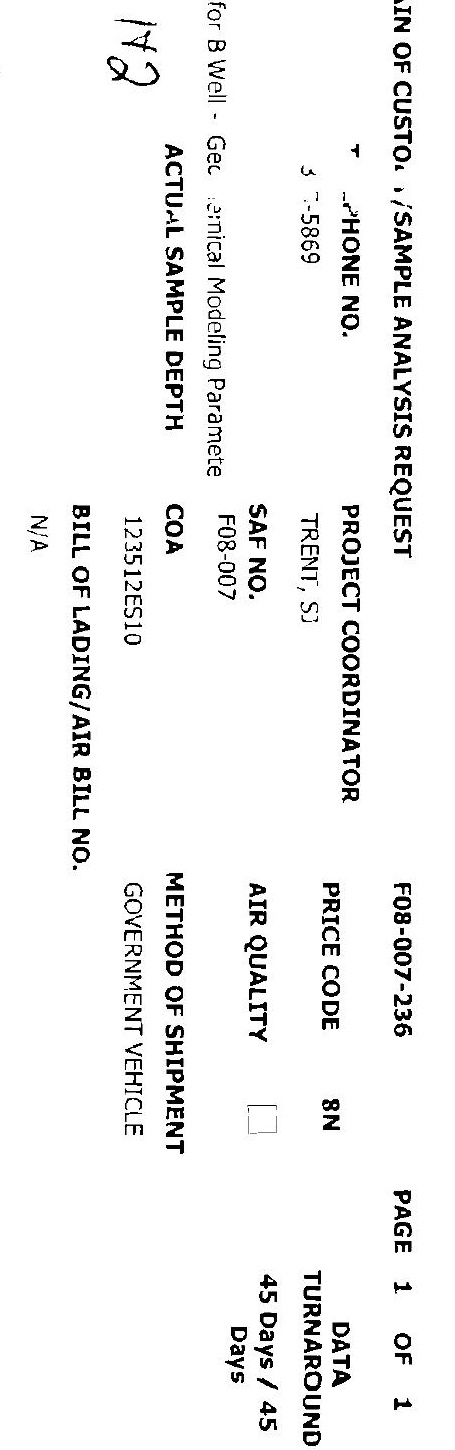

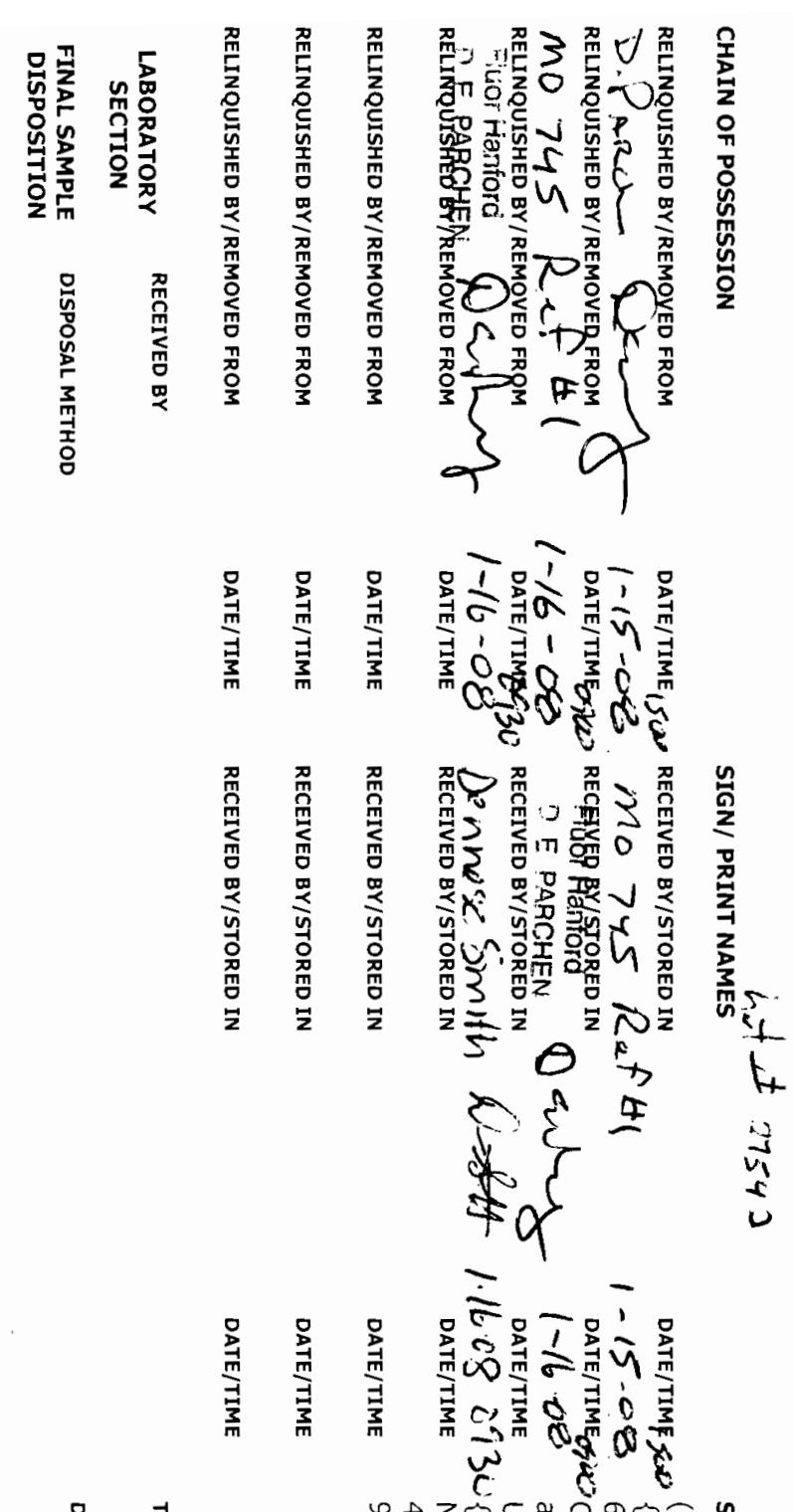

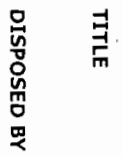

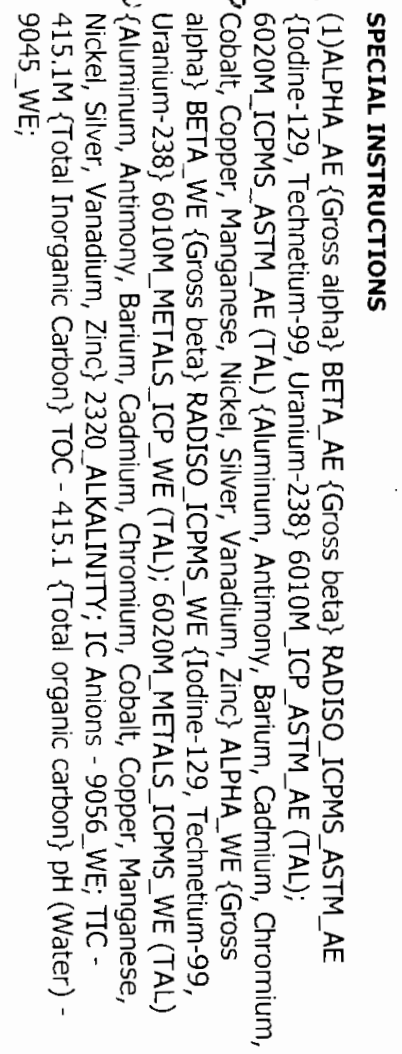

总兽
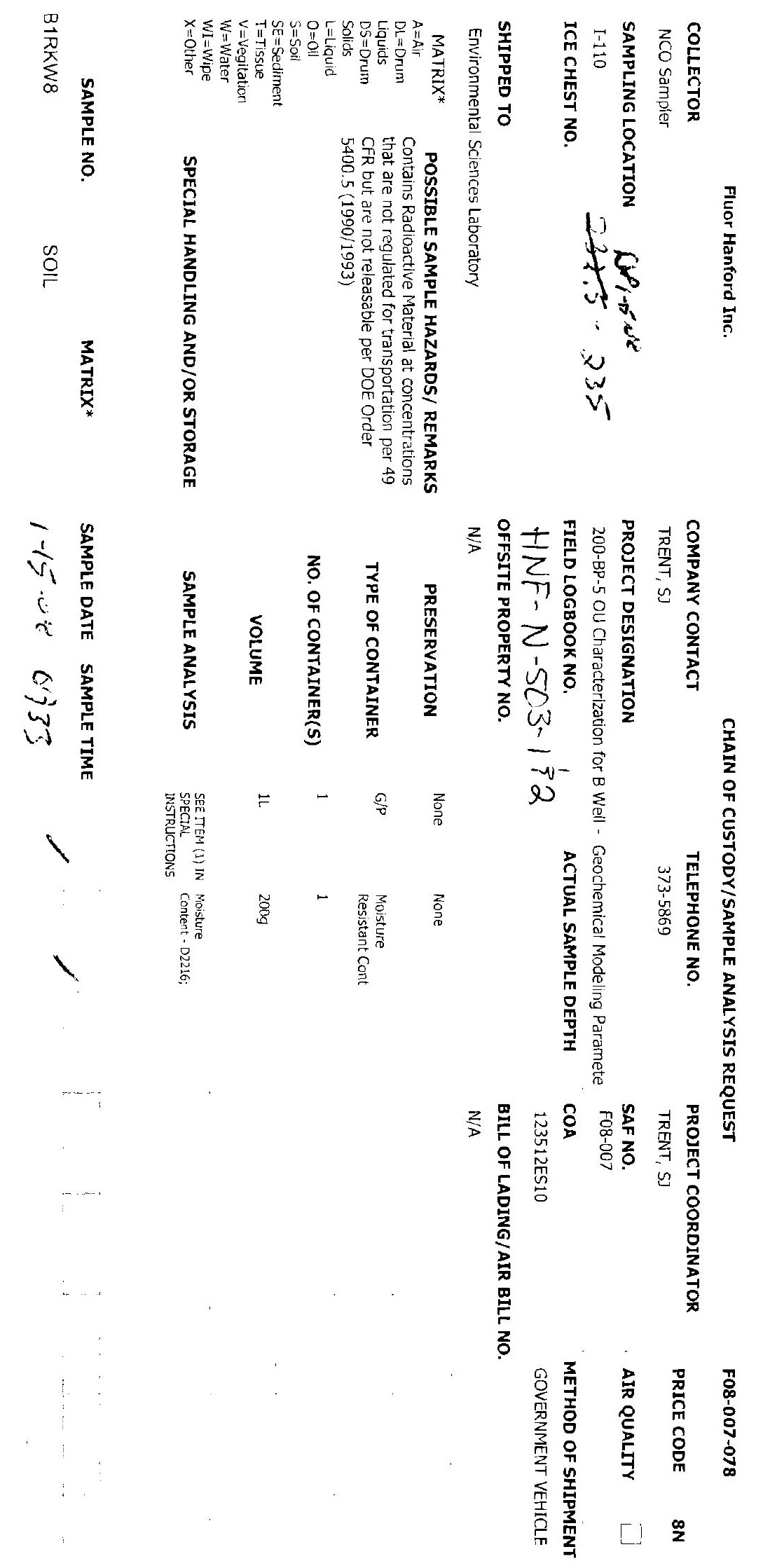

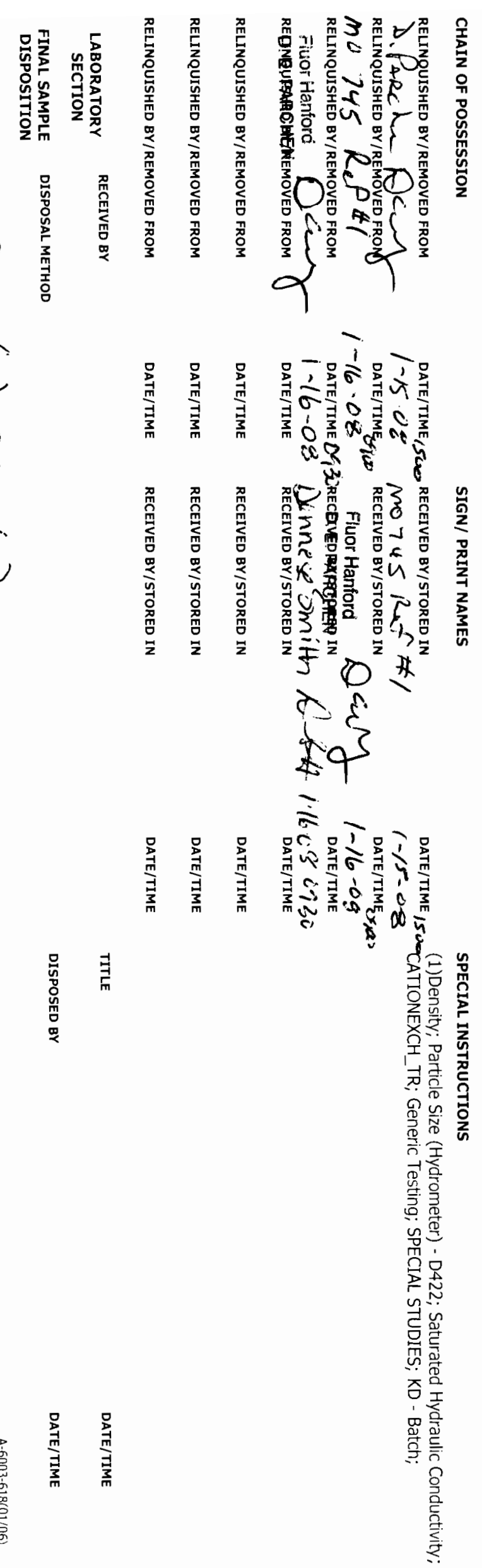

I

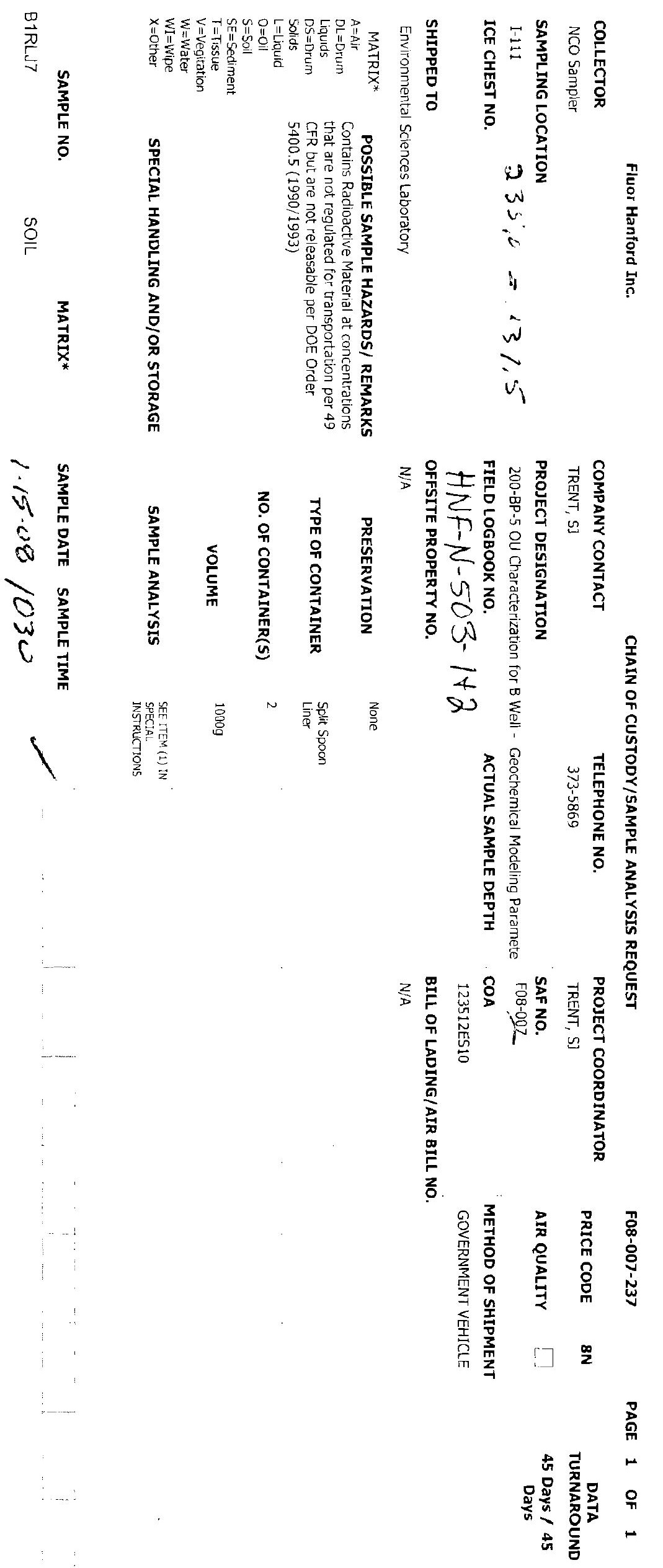



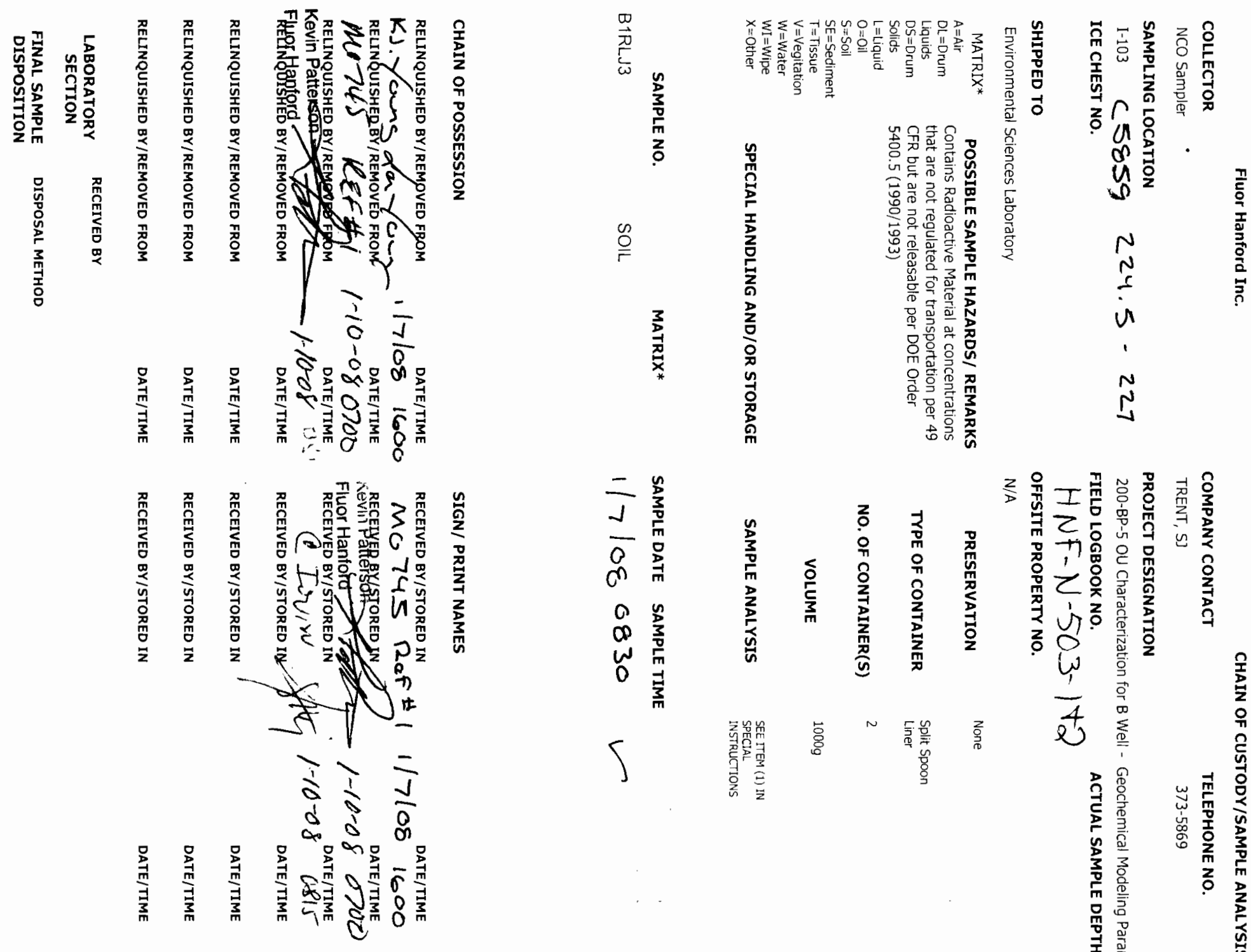

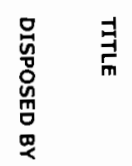
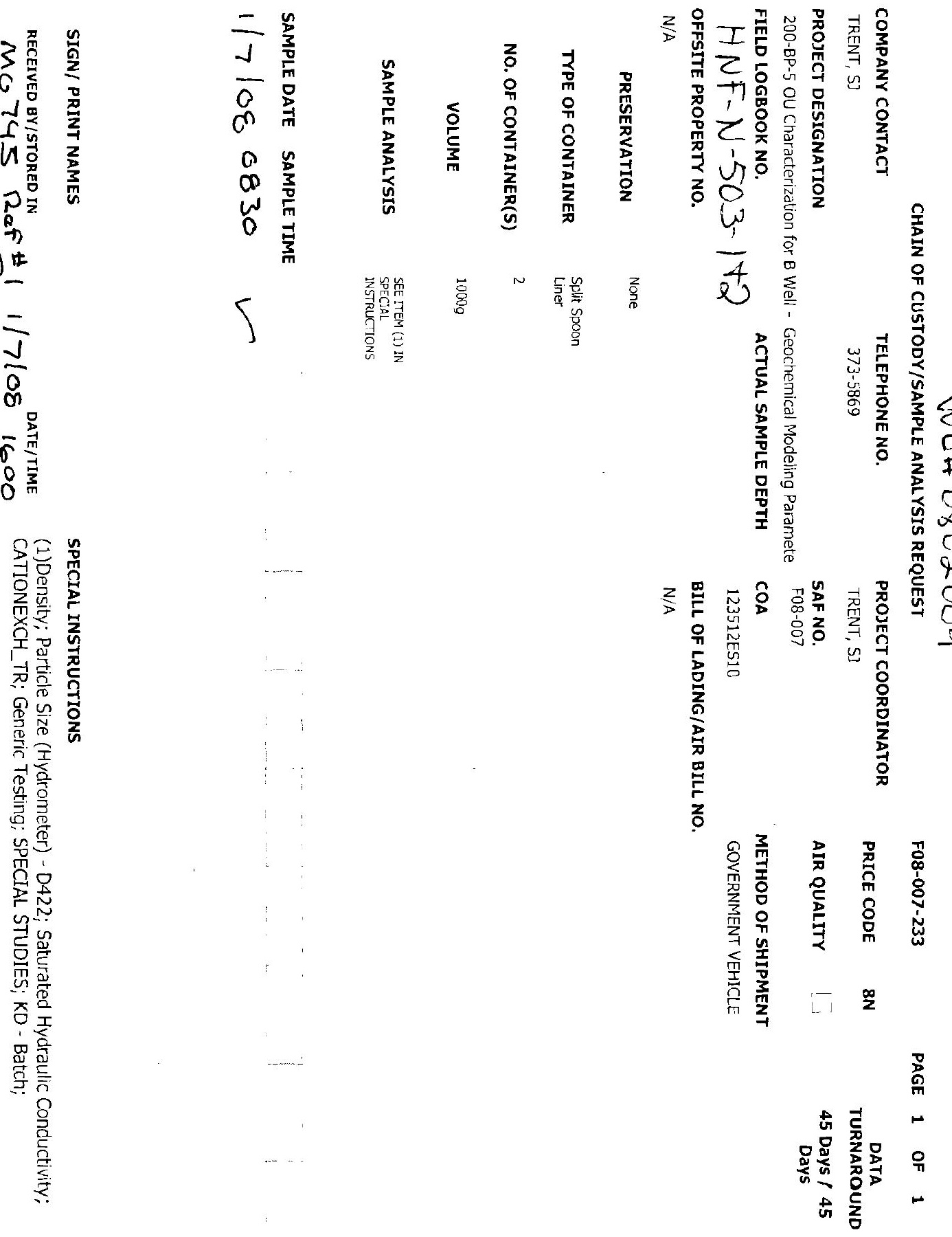


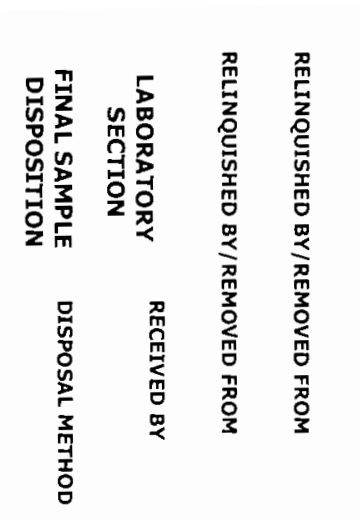

nx

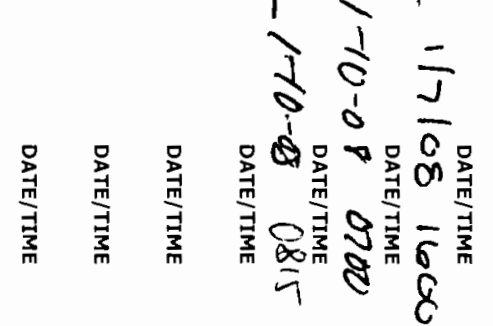

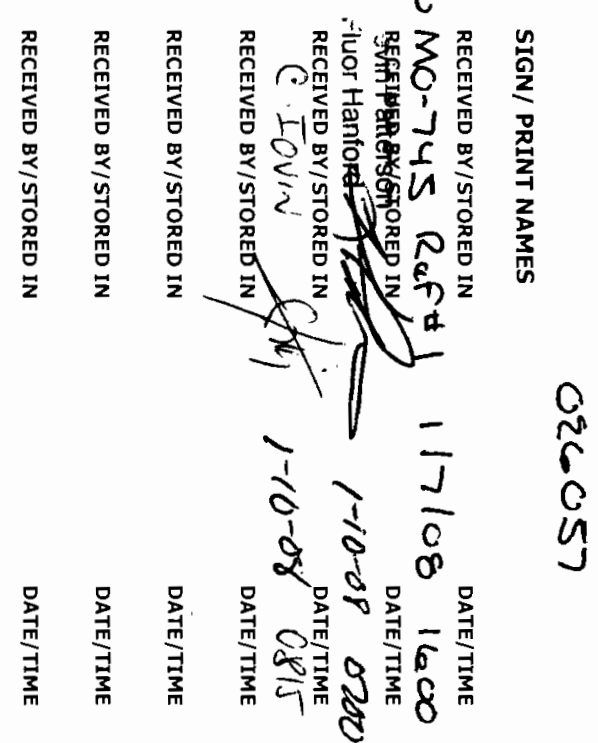

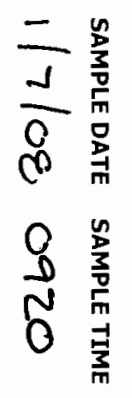

i

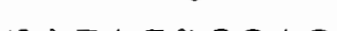

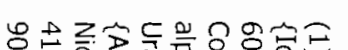

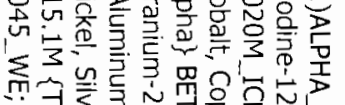

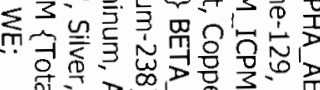

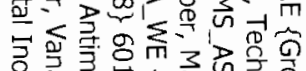

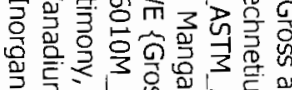

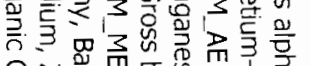

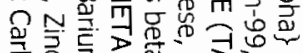

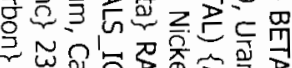

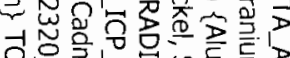

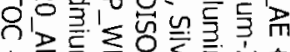

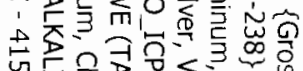

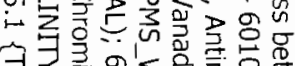

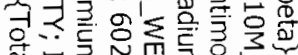

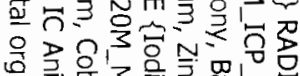

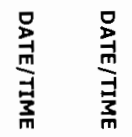

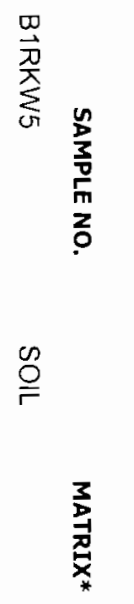

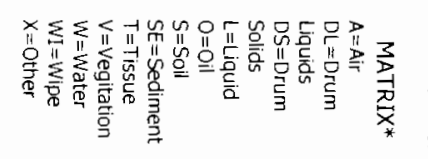

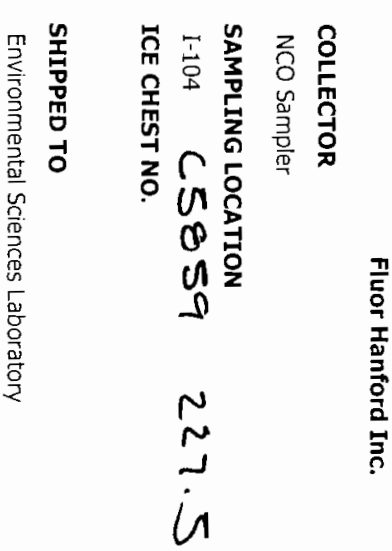

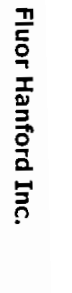

5
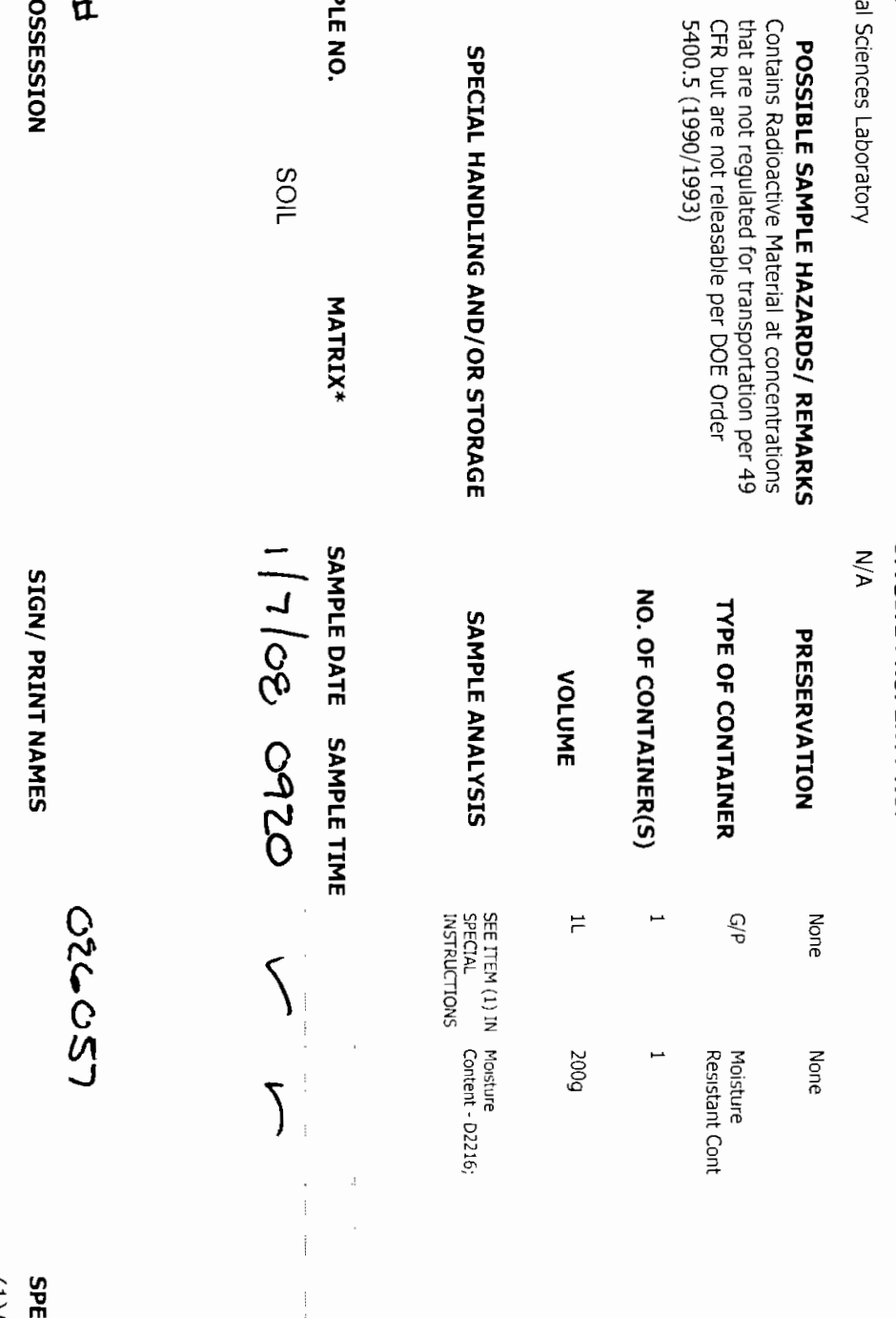

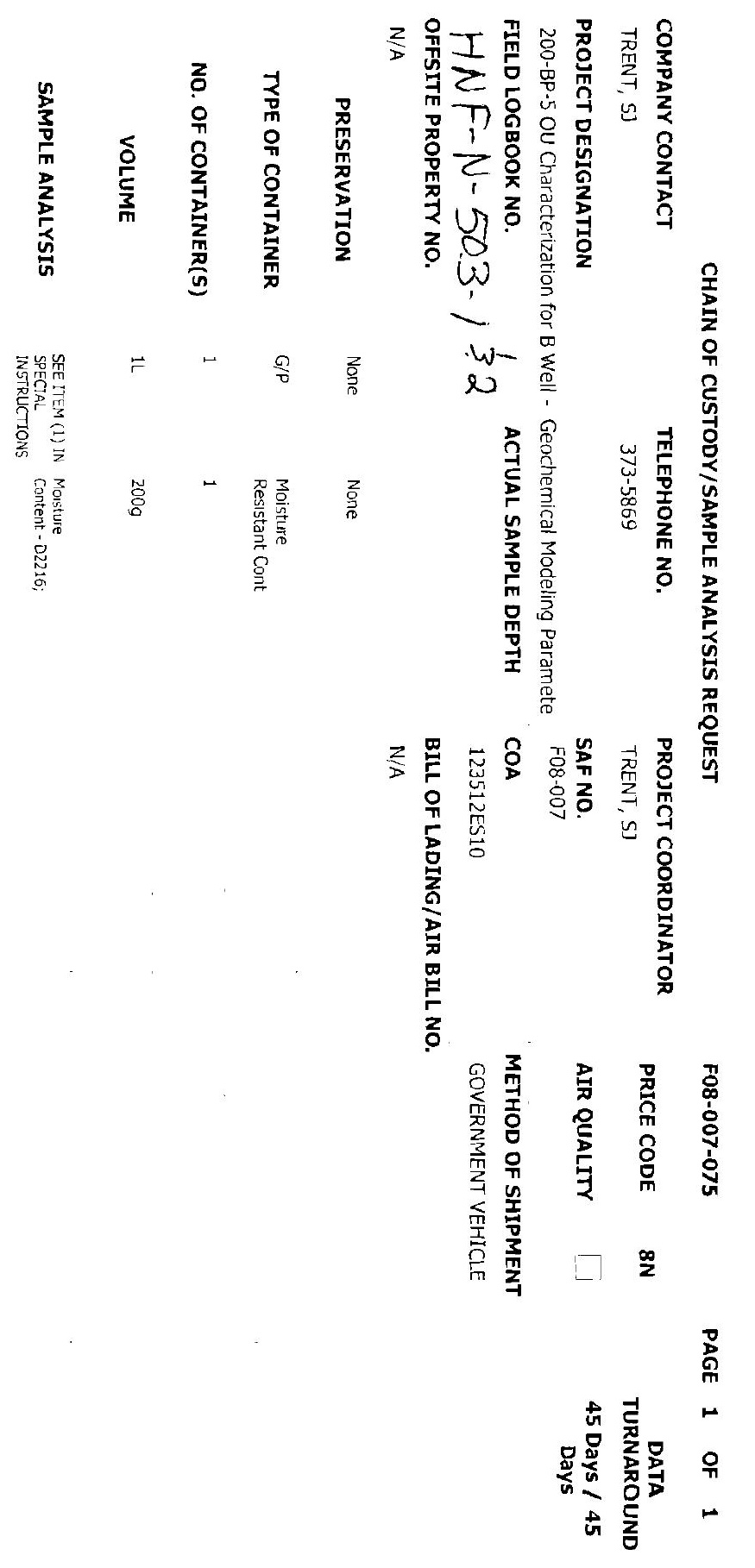




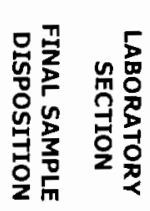
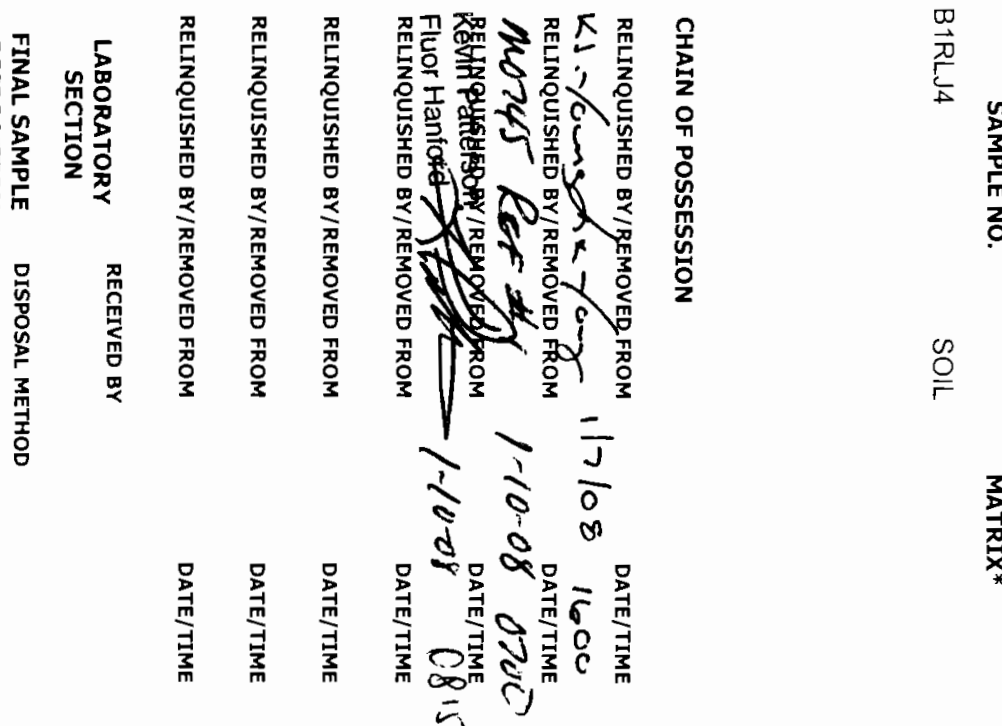

$\stackrel{\infty}{\circ}$
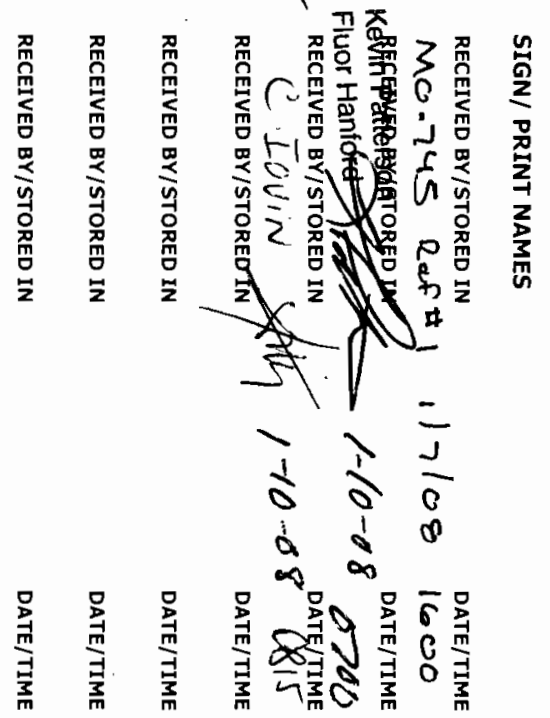

$\frac{-}{0}$
0
$=$
$\overline{0}$
5

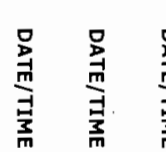

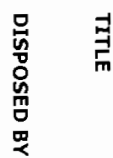

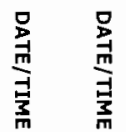

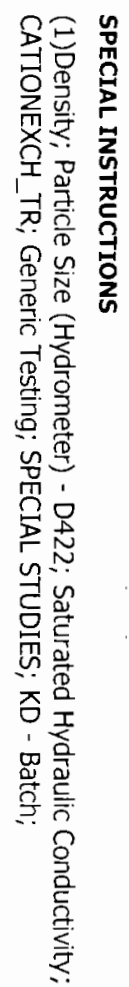

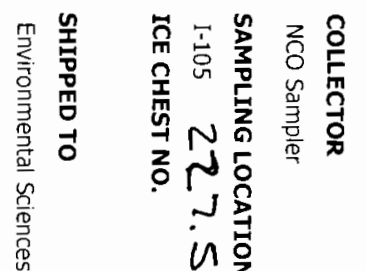

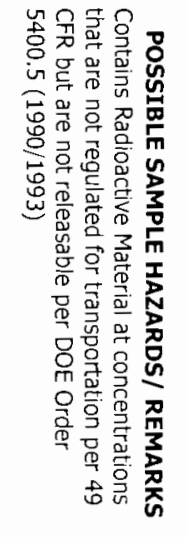

in 훈

$N$
0
w
on
n

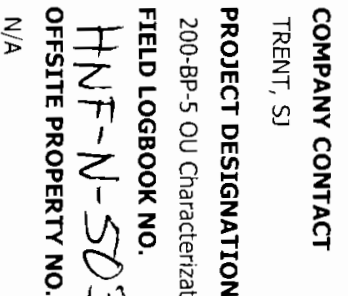

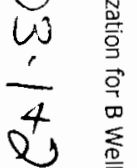

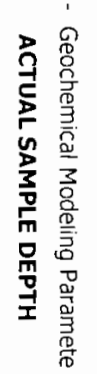

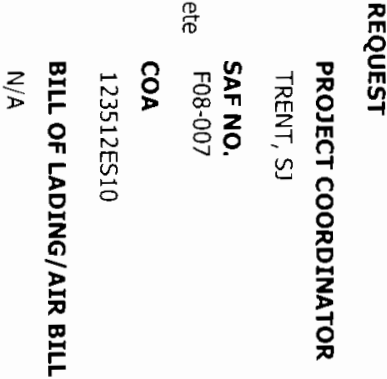

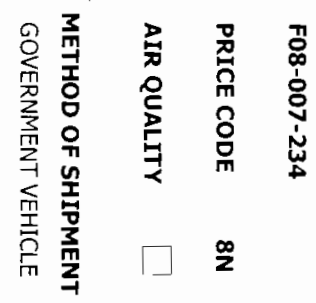

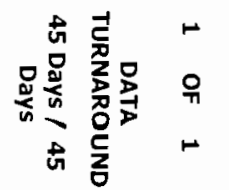




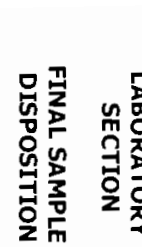

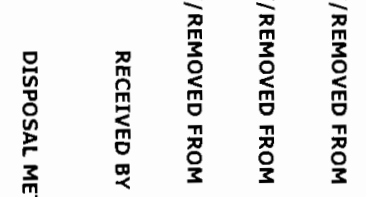

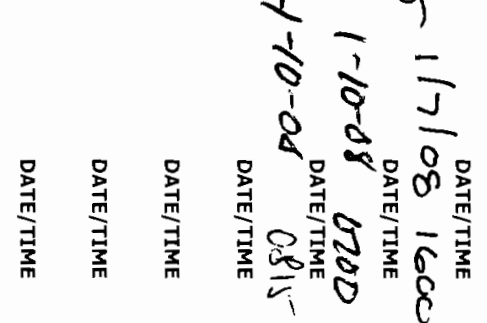

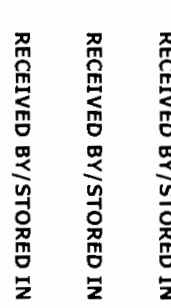

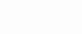

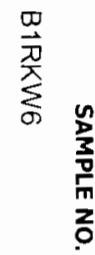

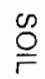

좇

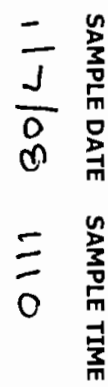

\.

$S$

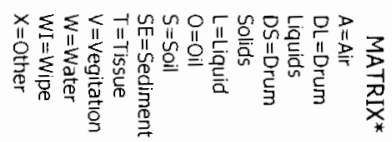

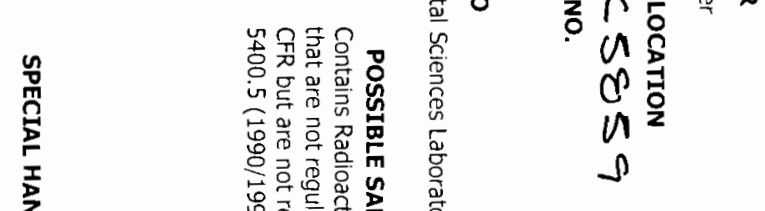

总

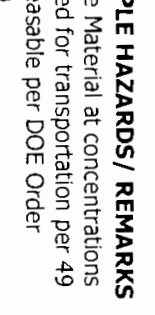

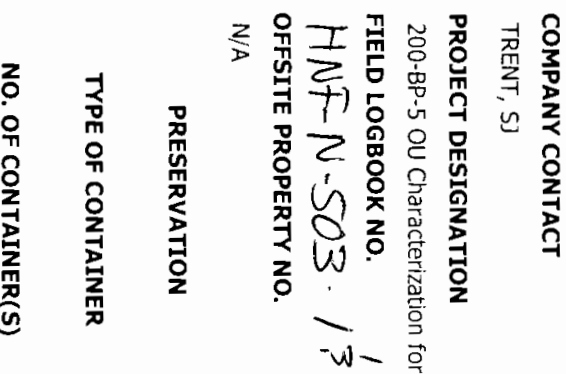

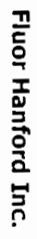

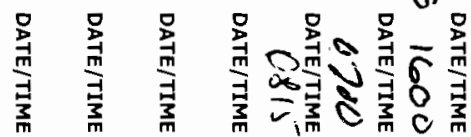

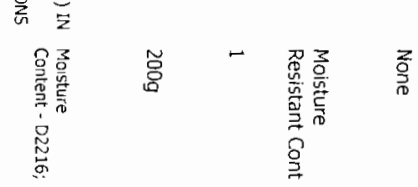

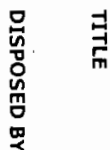
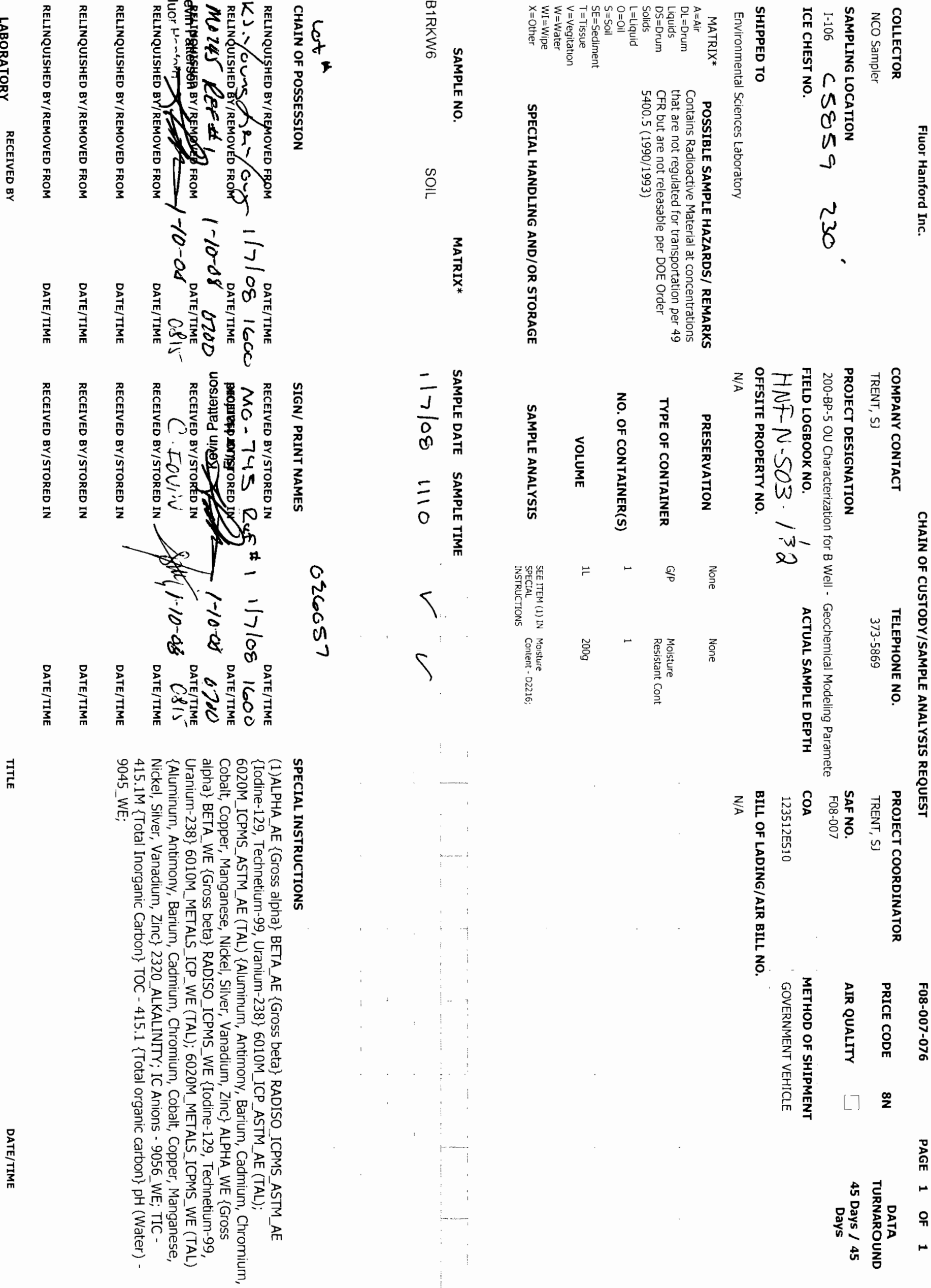

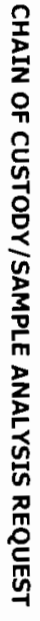

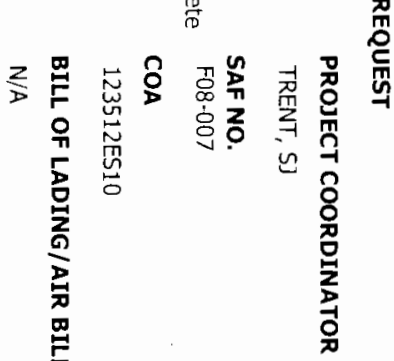

zz.

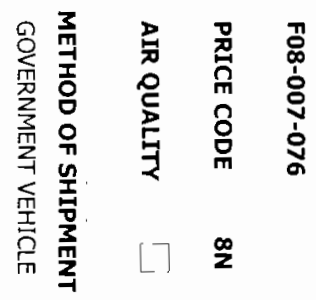

疍

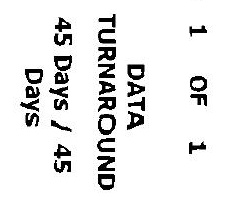

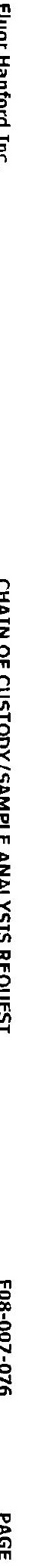




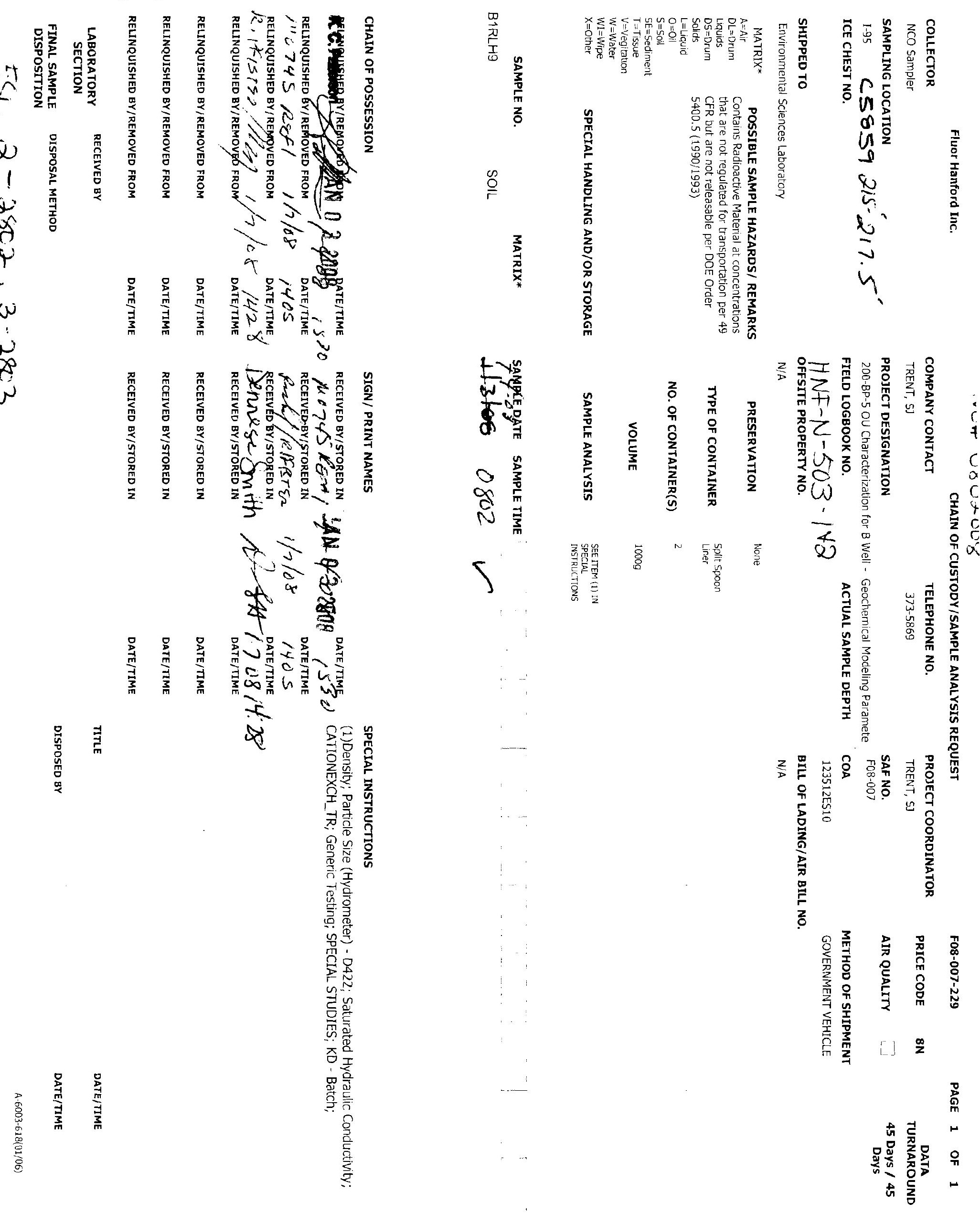




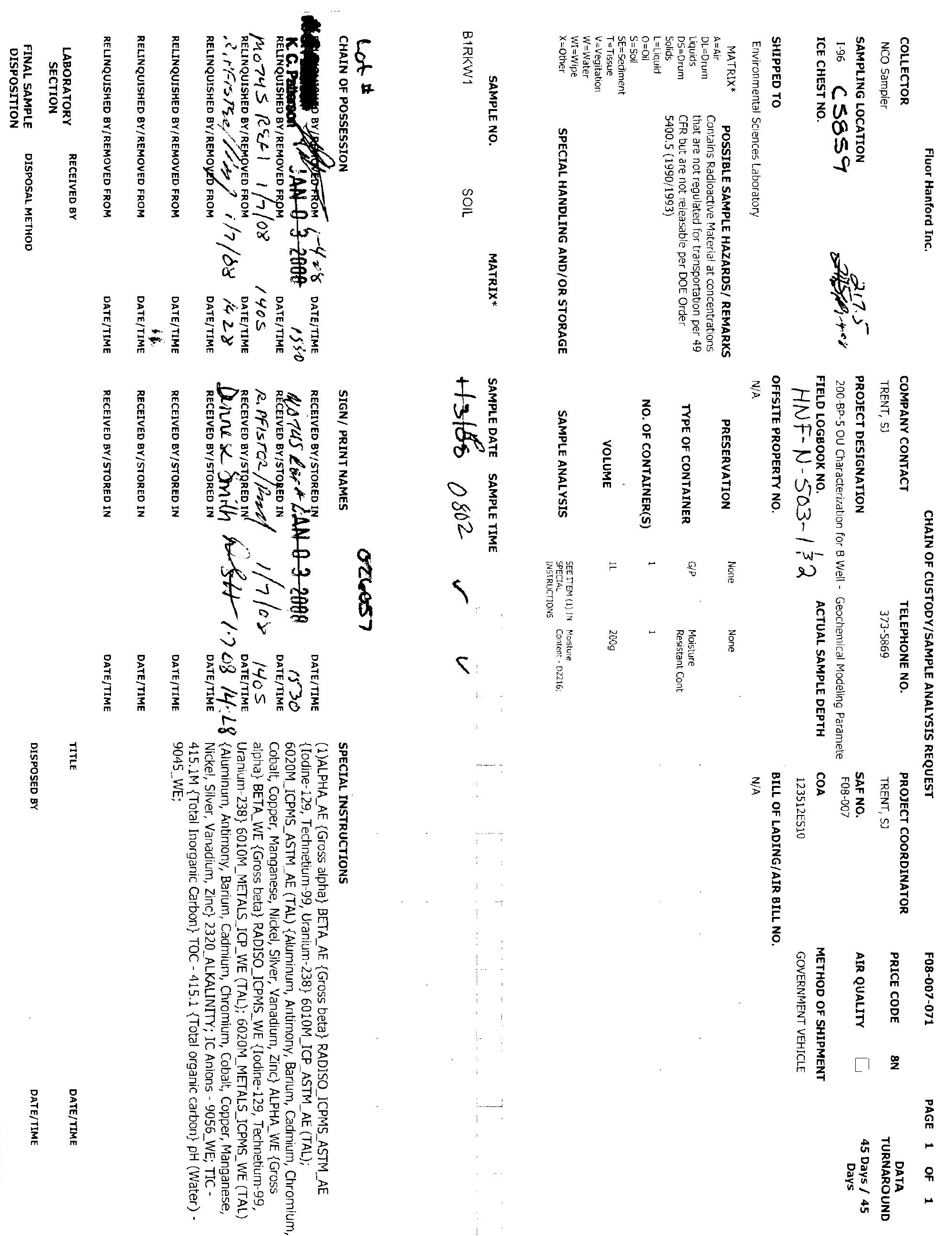




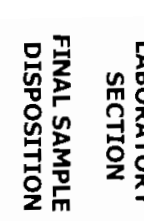
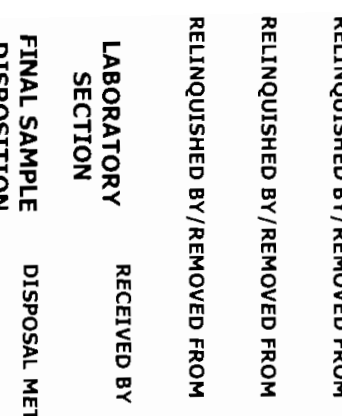

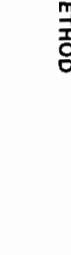

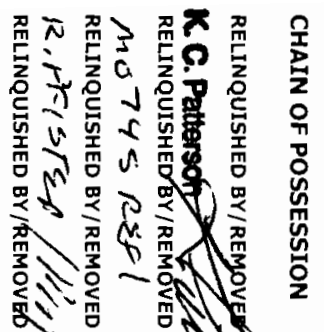

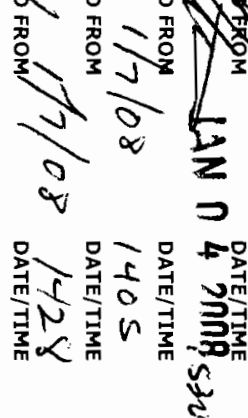
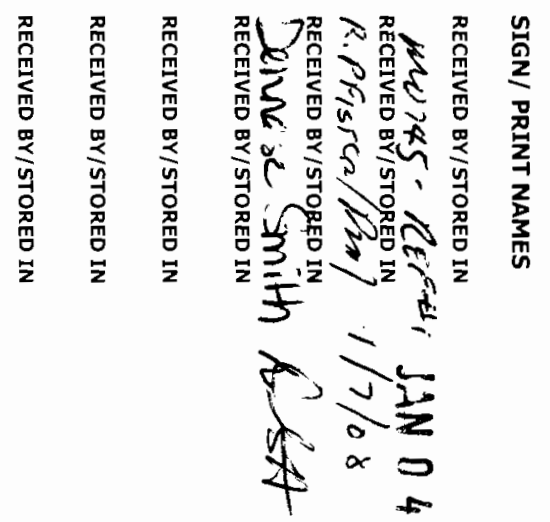

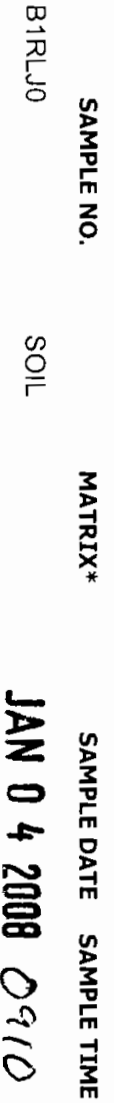

唯

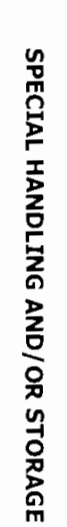

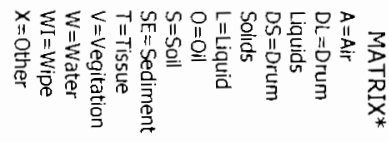

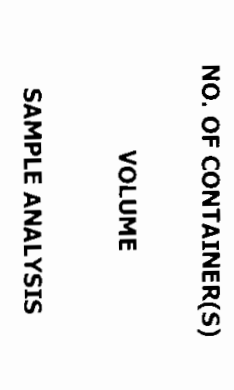

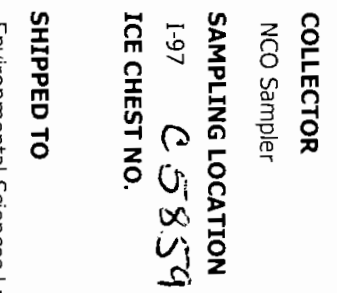

$\frac{1}{i j}$

$\frac{i}{\infty}$

之

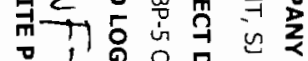

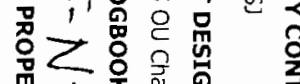

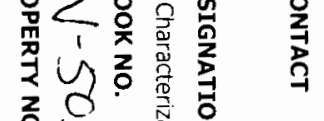
क क

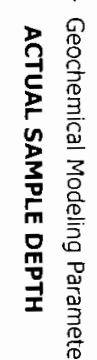

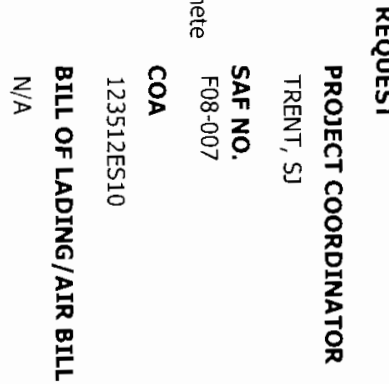

$S E$

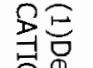

离

II

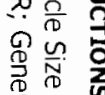

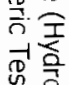

복

곤

足

늘 资

惫空

즘ำ

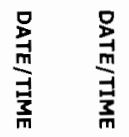

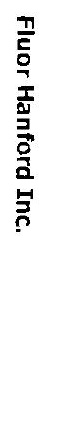




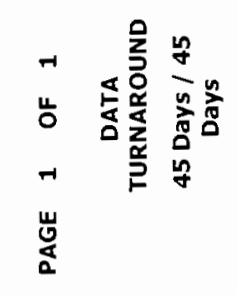

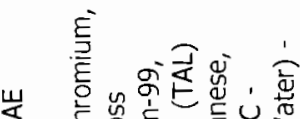

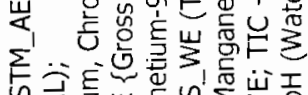

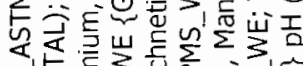

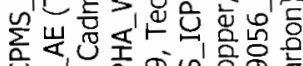

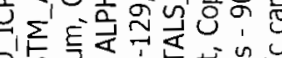

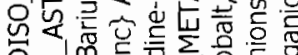

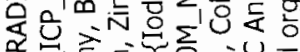

结

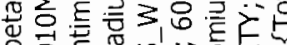

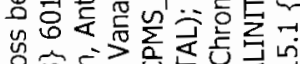

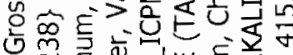

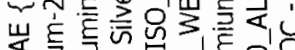

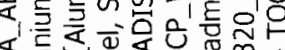

एँ

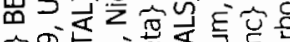

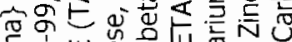

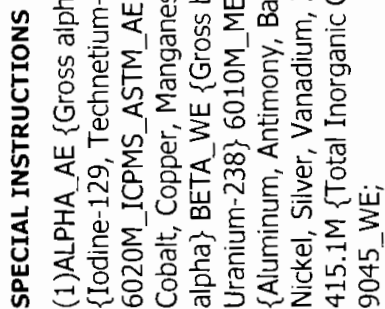

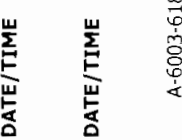

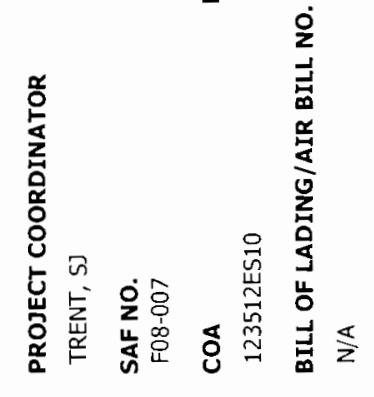

II

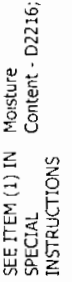

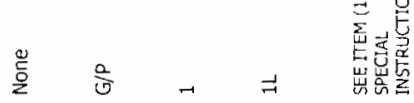

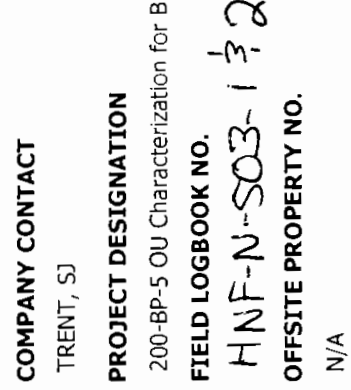
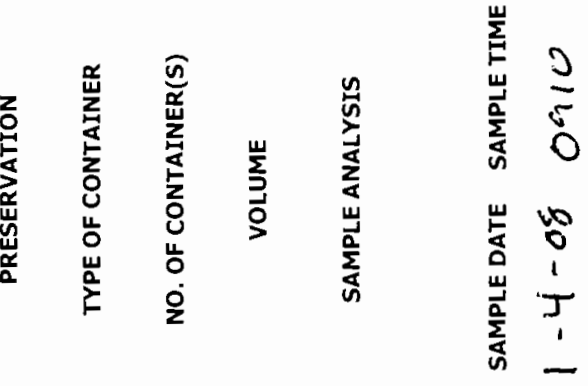

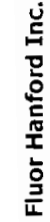

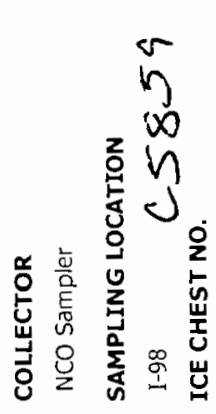

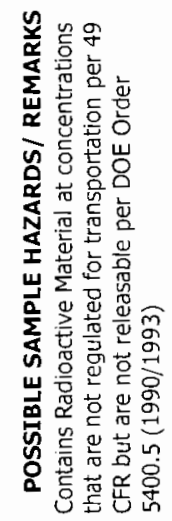

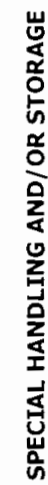

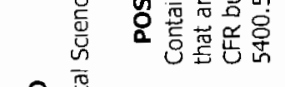

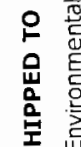

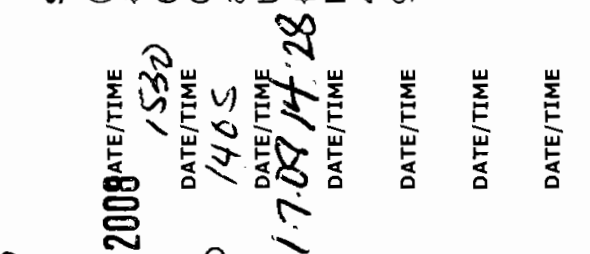

$\begin{array}{ll}5 & 5 \\ 0 & 0 \\ 3 & 2\end{array}$
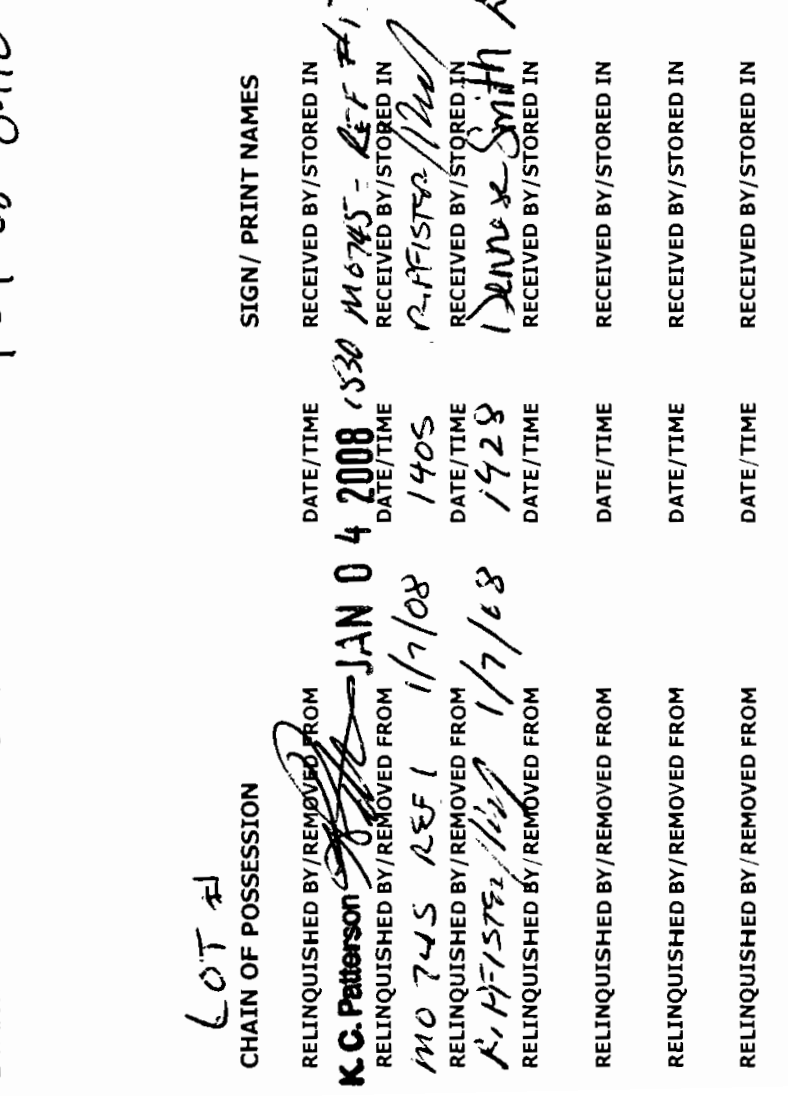

产

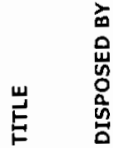




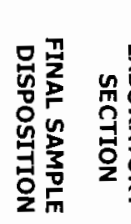
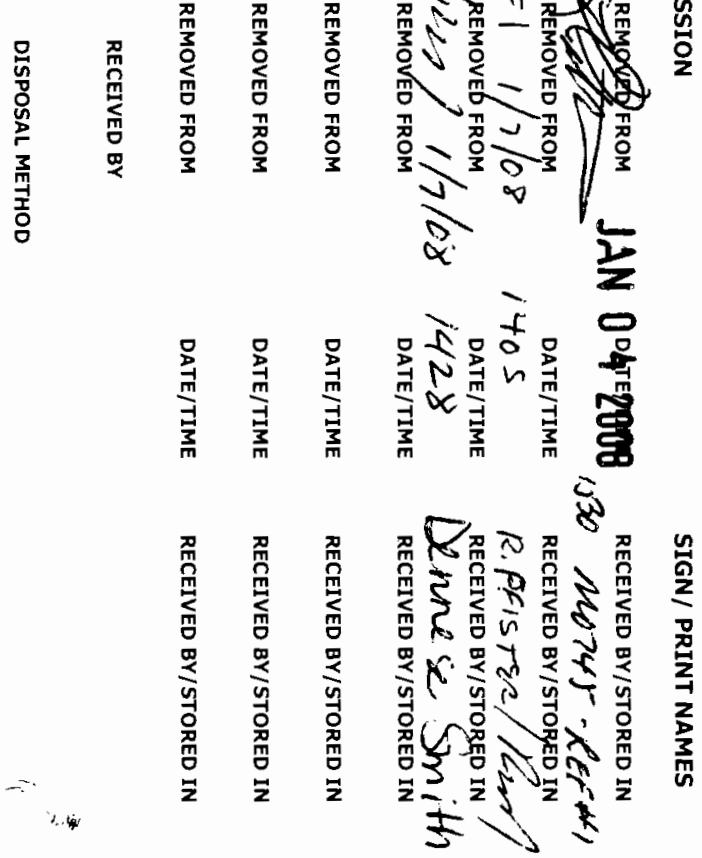

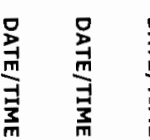
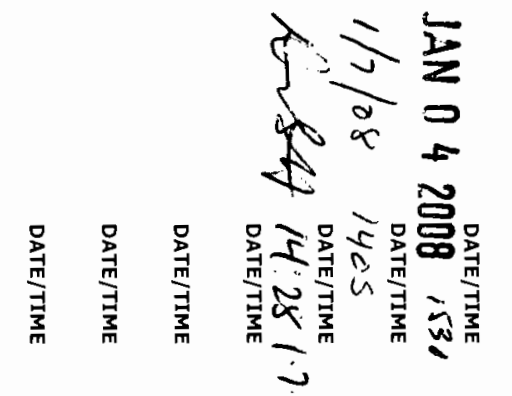

善

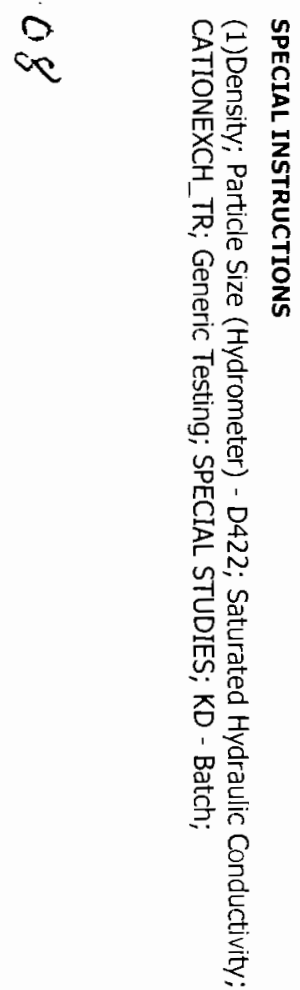

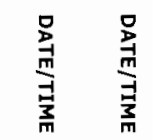

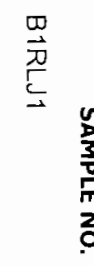

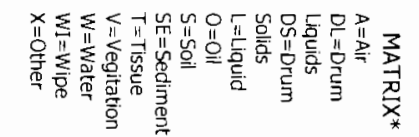

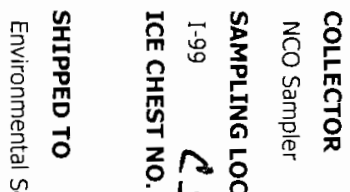

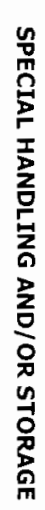

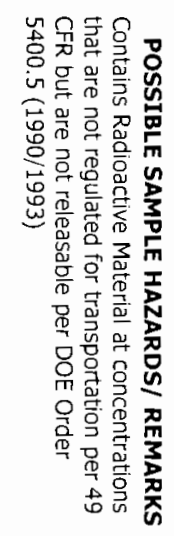

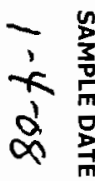

$\frac{2}{4} \frac{\substack{3 \\ \frac{\pi}{m}}}{\frac{1}{3}}$

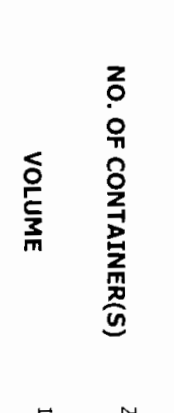

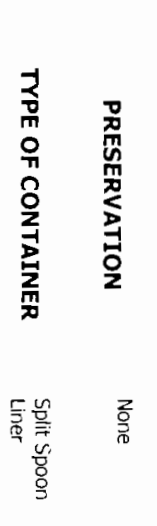

록윢

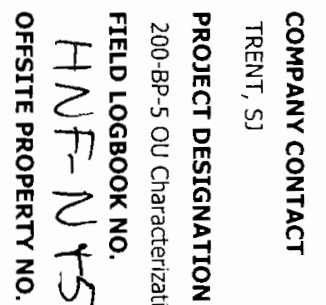

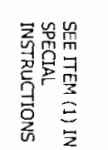

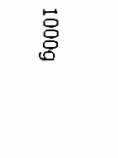

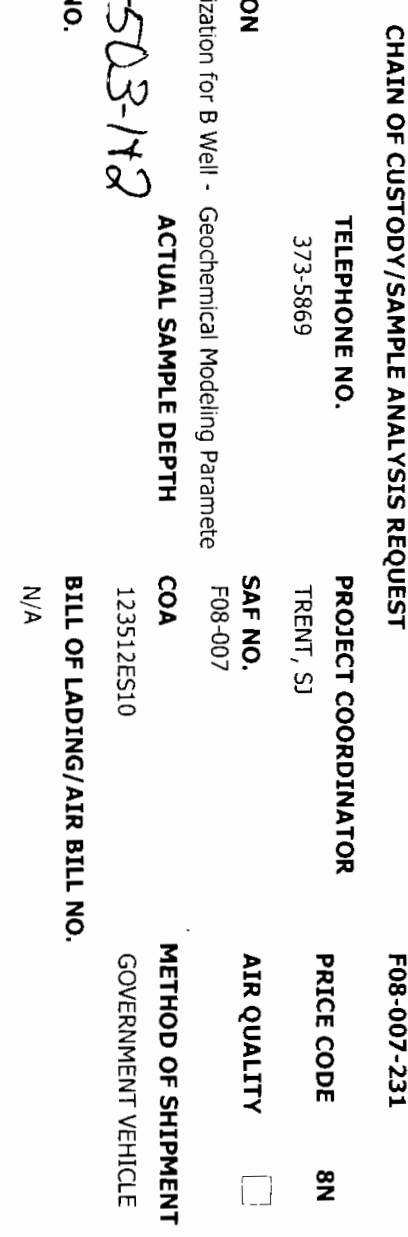

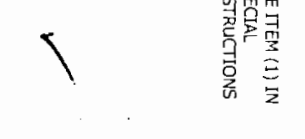



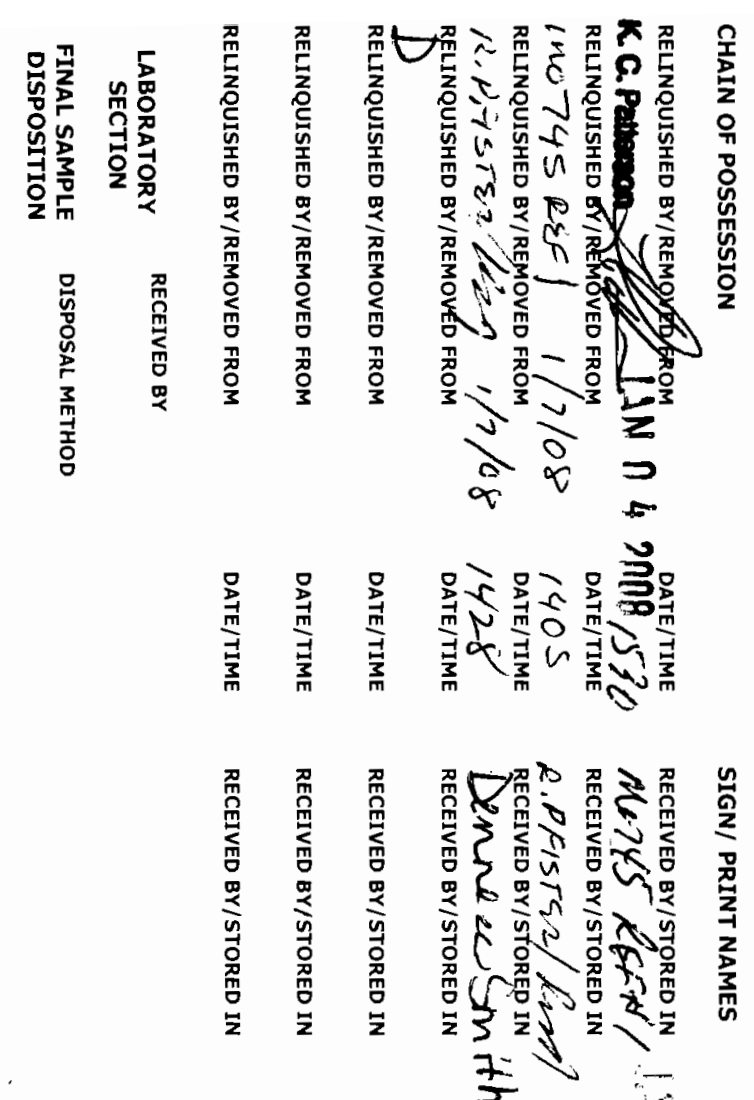

哭
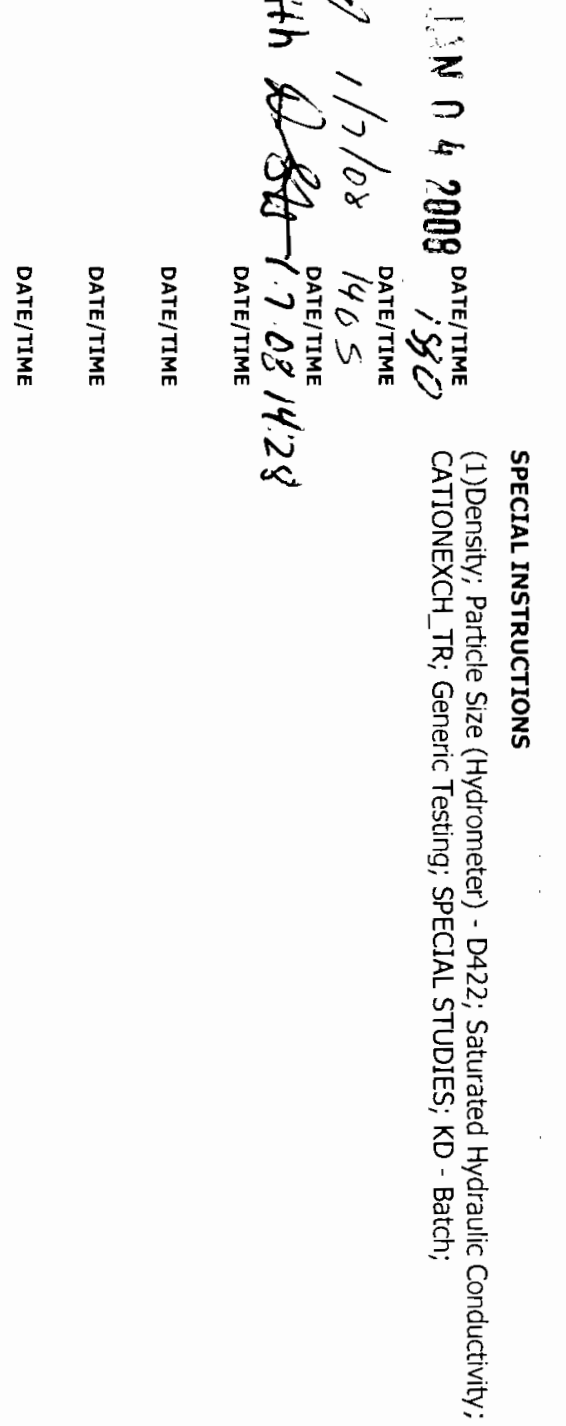

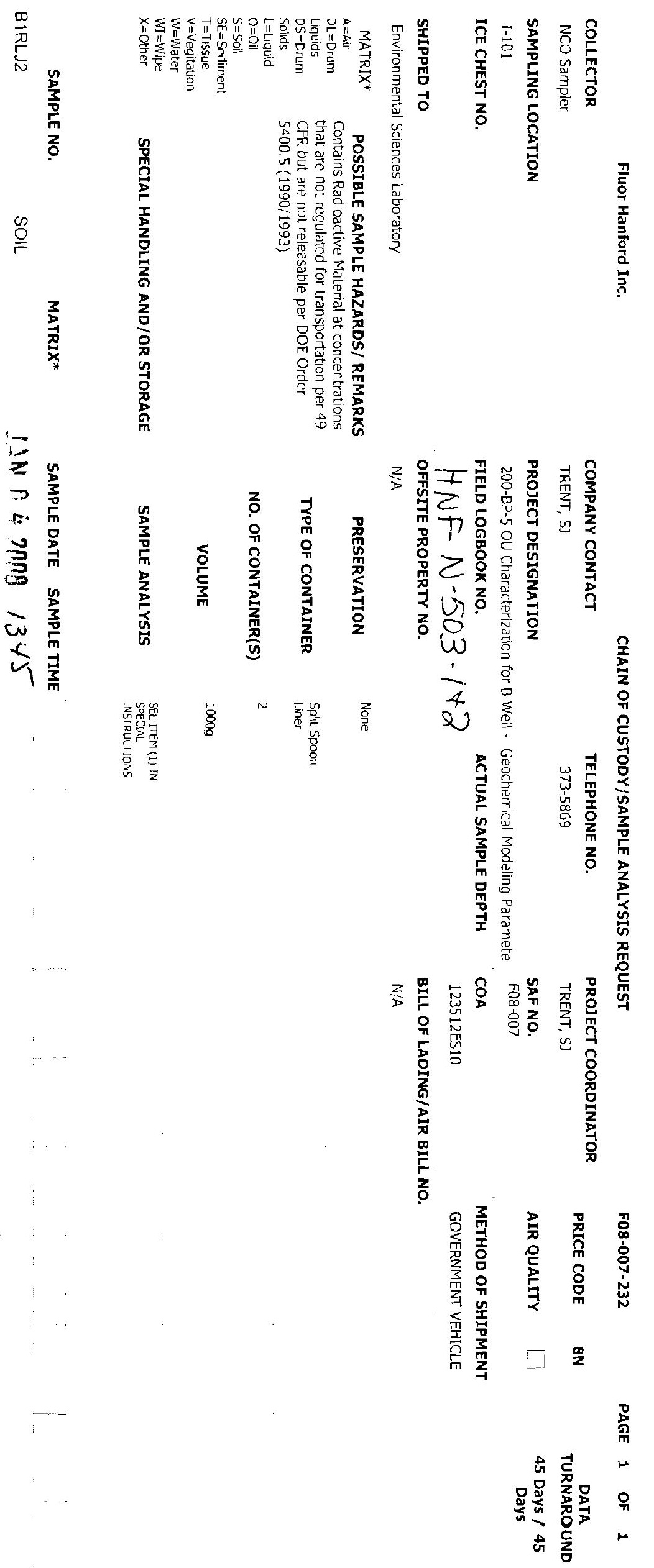




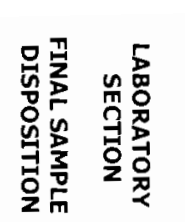

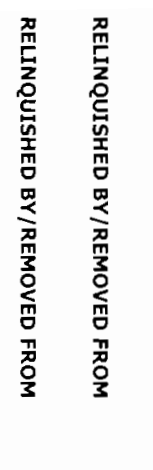

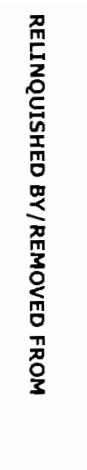

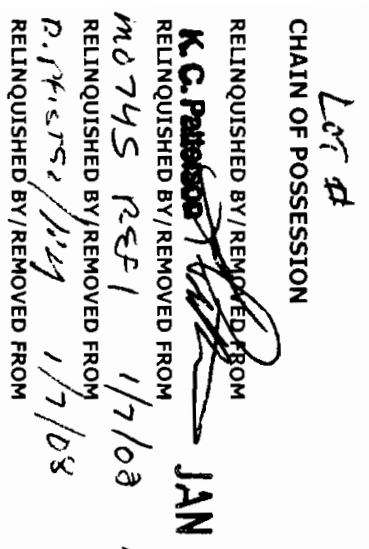

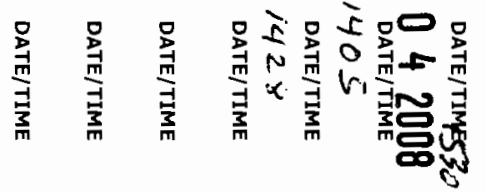

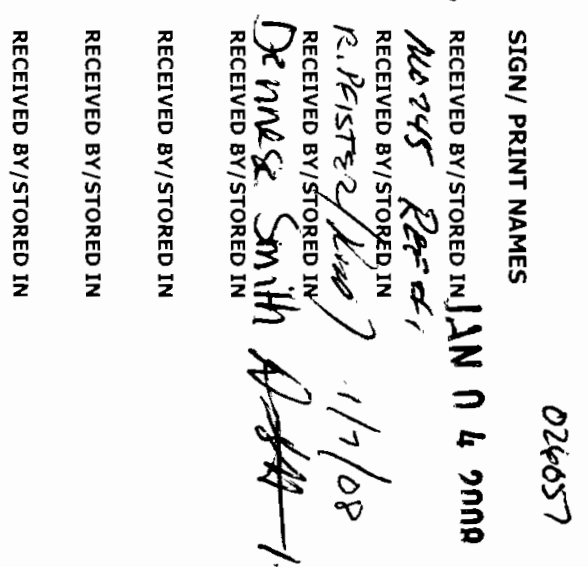

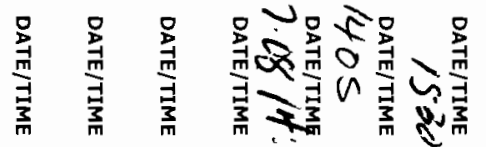

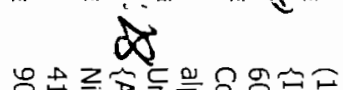

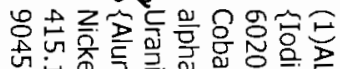

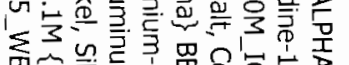

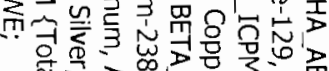

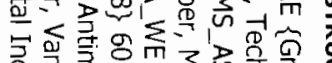

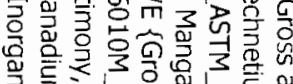

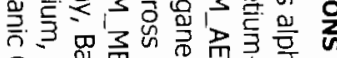

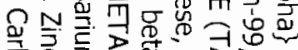

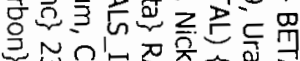

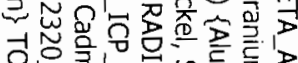

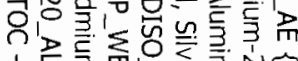

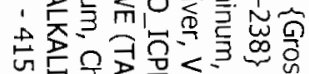

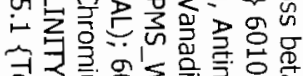

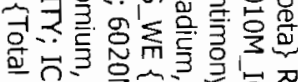

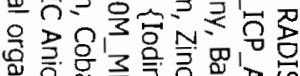

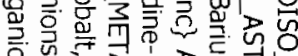

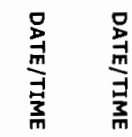

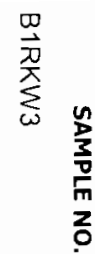

$\stackrel{\infty}{\circ}$

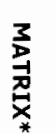

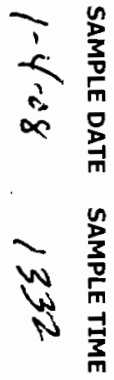

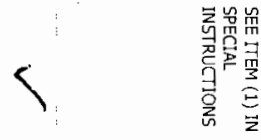

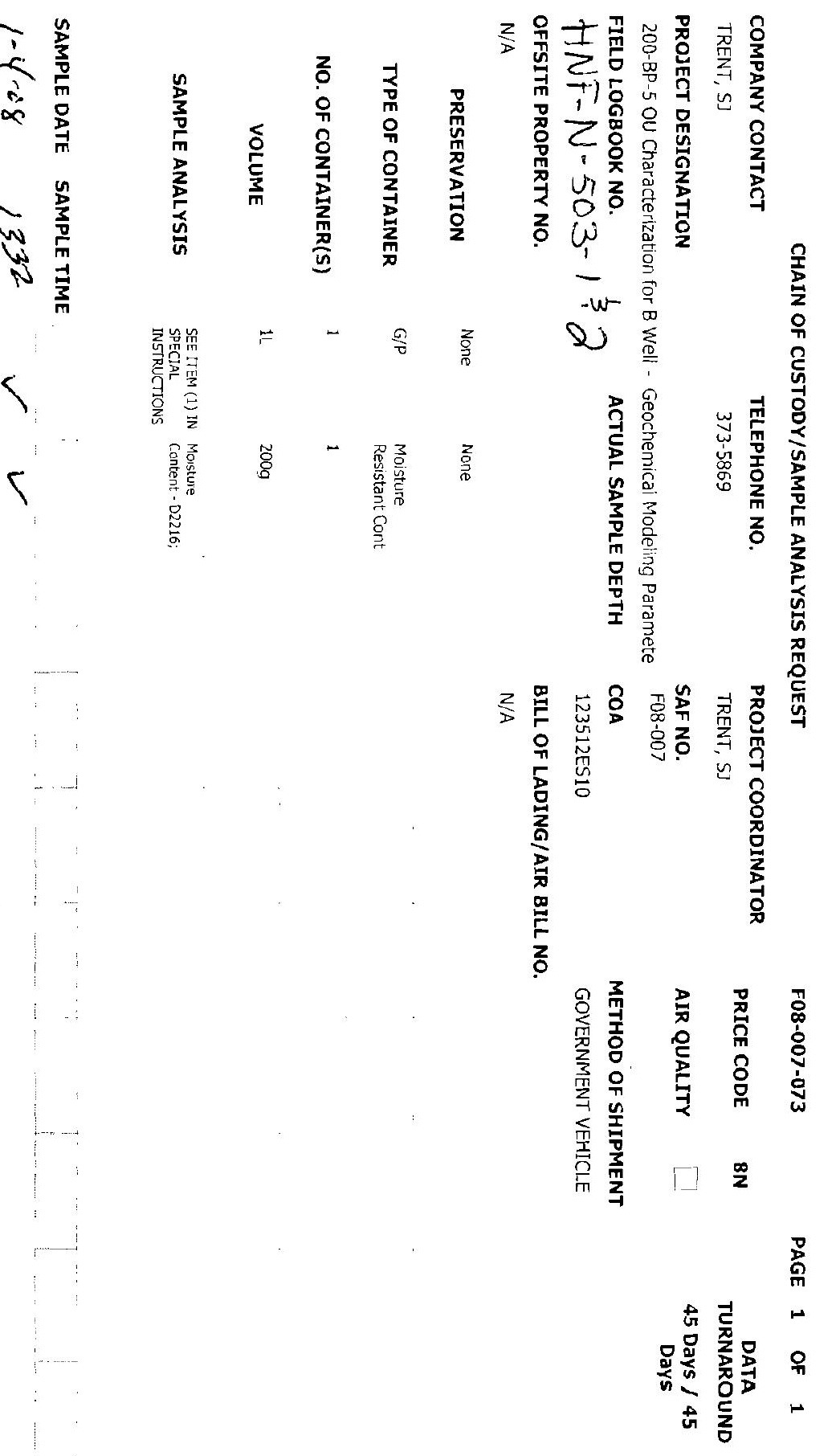

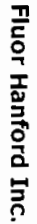




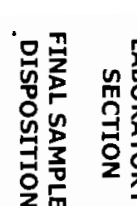

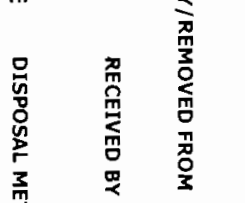

矛

$x$

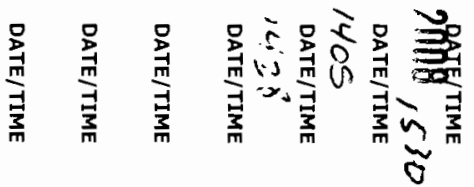

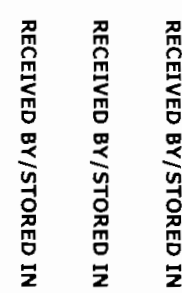

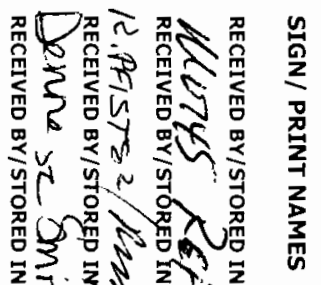

赏
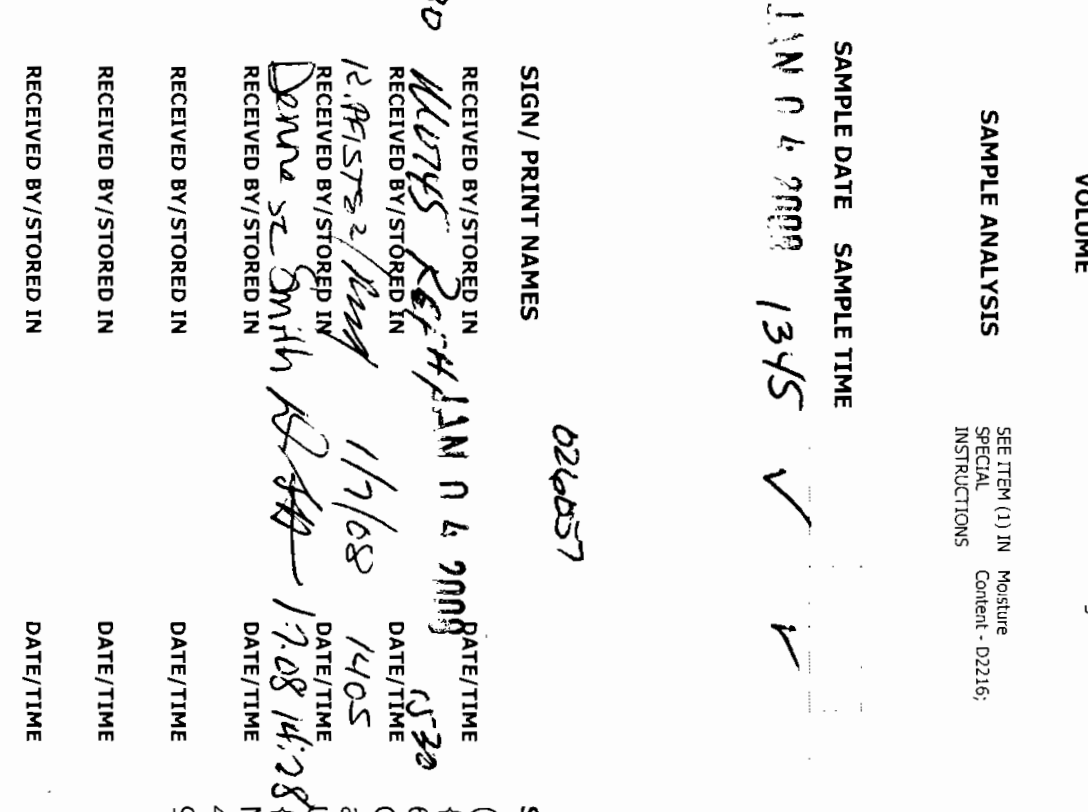

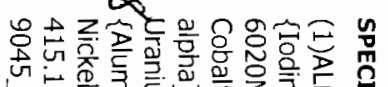

जे

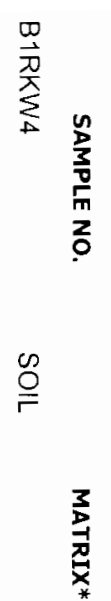

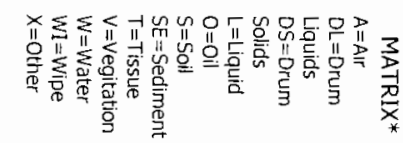

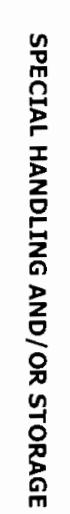

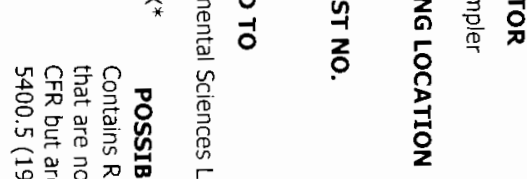

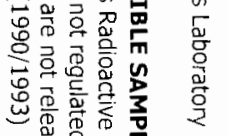

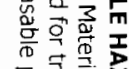

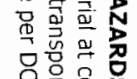

而高焉

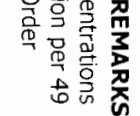

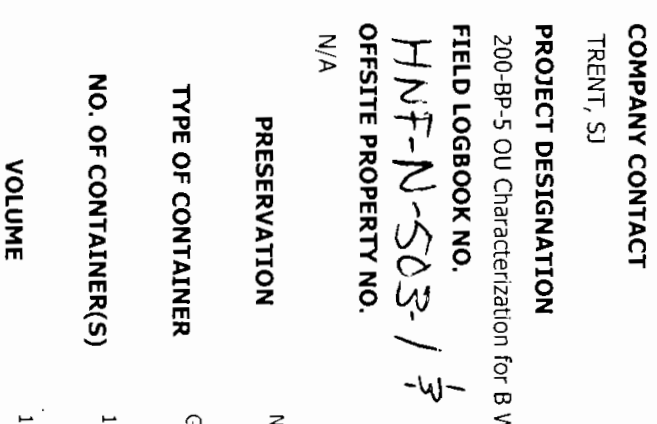

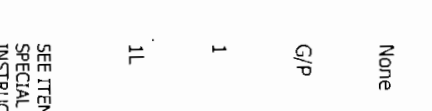

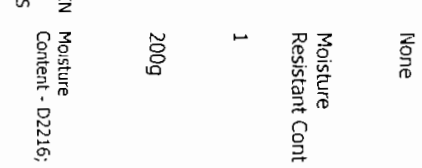

$53 \stackrel{0}{3}$

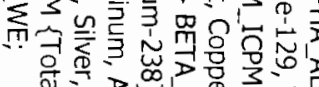

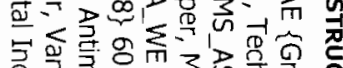

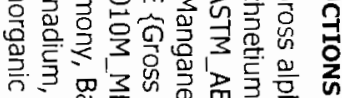

Q

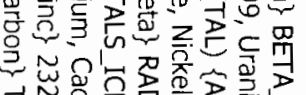

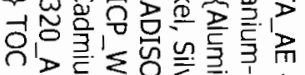

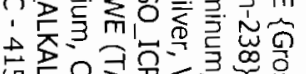

ज记

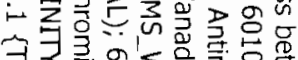

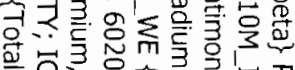

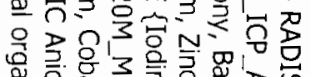

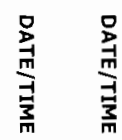

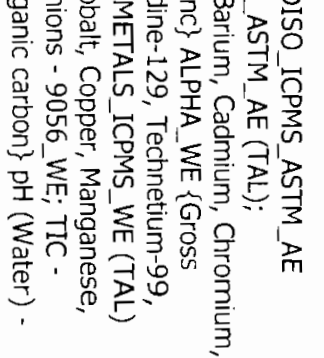

